HX00026026 


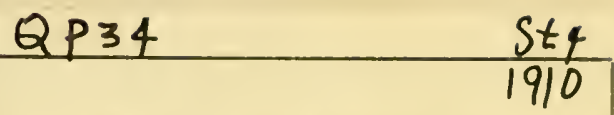

Columbia Ênimersity inthecitpof Irw Dork

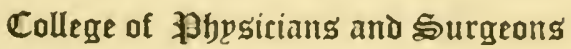

Zibrary

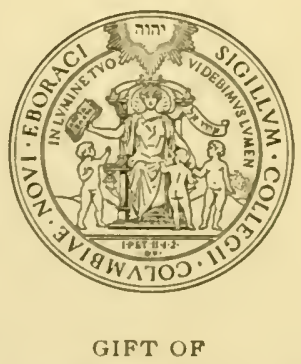

Frederick S. LeE 


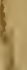




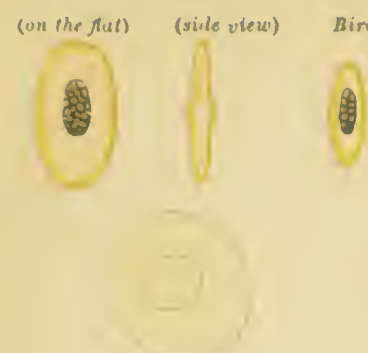

Frog's lorpusicle

ufer adulision of waler
Fish
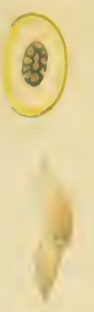

Mammaliun

afeer addition of syrup

1. Red blood-corpuscles.

Mammal

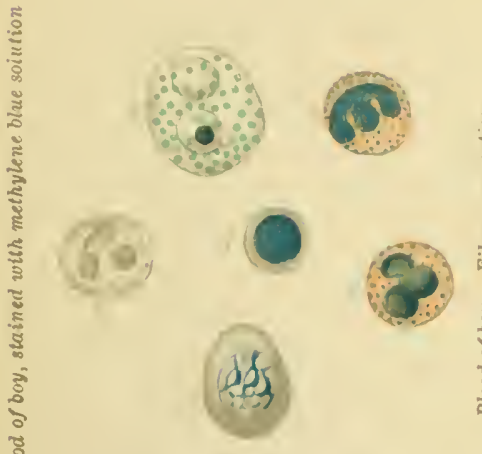

I imel

Bloerl of mammul
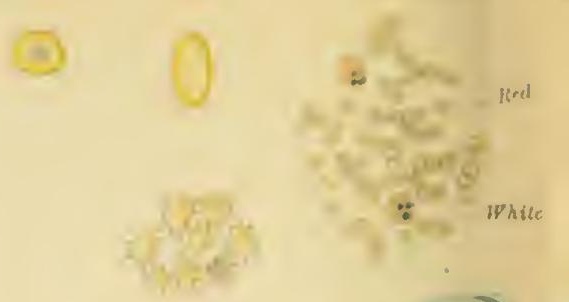

Munamuluan

afer addition of sult

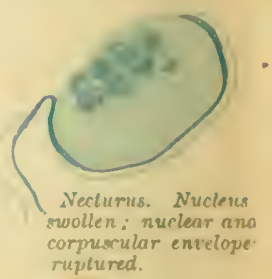
ruptured.
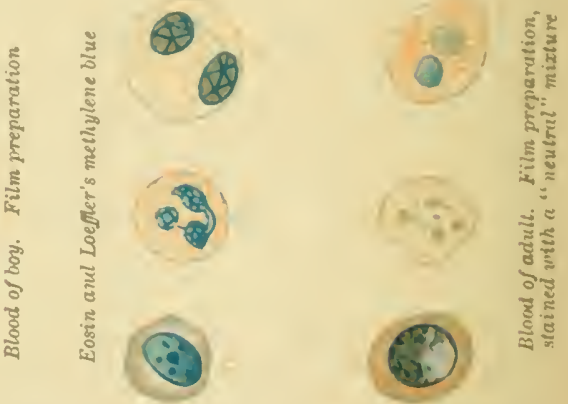

2. The colourless corpuscles of human blood, $\times 1000 . \quad a$, eоsinophile cells;

$b$, finely granular oxyphile cells: $c$, hyaline cells; $d$, lymphocyte;

e, polymorphonuclear neutrophile cells (Kanthack and Hardy). The magnification is much greater than in 1.

3. Cover-glass preparation of spinsl cord of ox, $\times 250$.

(Stained with methylene blue).

Dendritic processes
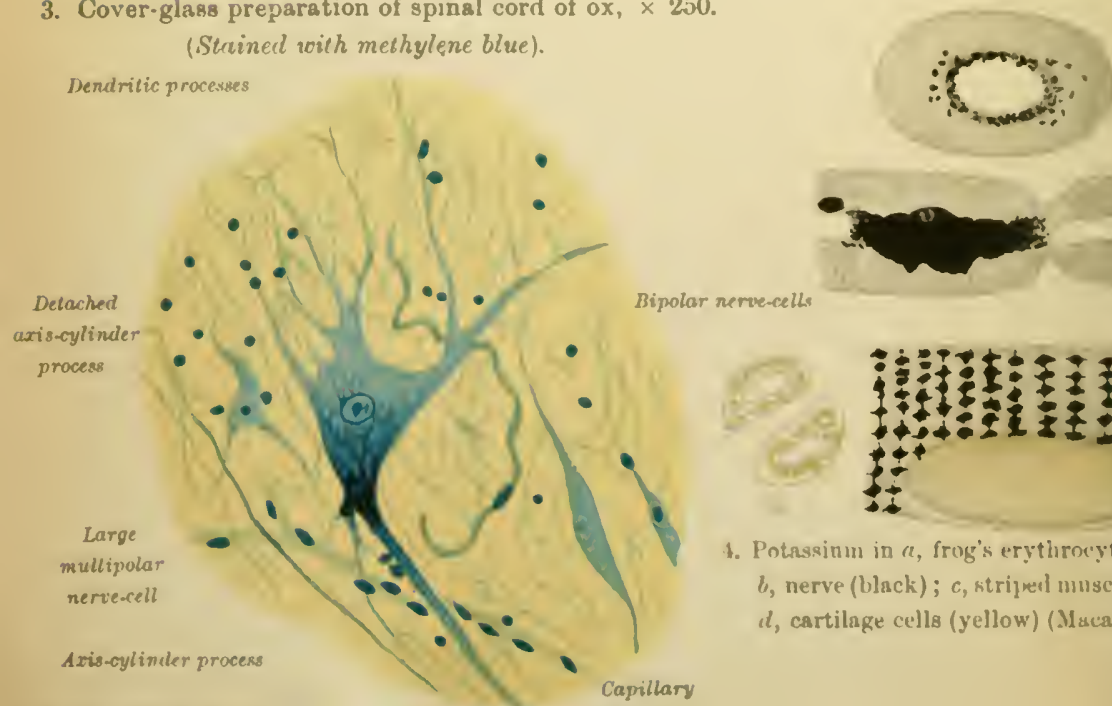

Bipolar nerve-cells

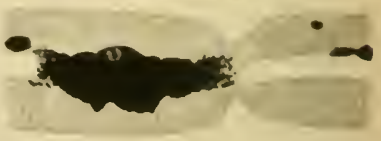

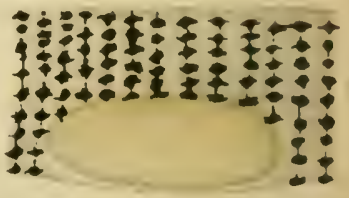

4. Pothssinu in a, frog's erythrocyte (lilack $b$, nerve (black); c, strijnetl muscle (llack ¿t, cartilage cells (yellow) (Mscallum). 
A

\section{MANUAL OF PHYSIOLOGY}

\section{rchitb Practical Excrciscs}

BY

G. N. STEIVART, M A., D.Sc., M.D. EnIN., D.P.H. CAMB. PROFFSSOK OF EXPERINEN TAL MEDICINE IN WESTERN RESERVE UNIVERSITY, CLFVELAND; FORMERLY PROFESSOR OF PHYSIOLOGY IN THE UNIVERSITY OF CHICAGO;

PROFESSOR OF PHYSIOIOGV IN THE IVESTERN RESERVE. UNIVIERSITY; GEORGE HENRY LEWES STUDENT;

FXAMINER IN PIYSIOLOGY IN THE UNIVERSITY OF ABERDEEN :

SENIOR DEMONSTRATOR OF PHYSIOLOGV IN THE OWENS COLLEGE, VICTORIA UNIVEKSITY, ETC.

WITH COLOLRED PLATES AND 450 OTHER ILLUSTRATIONS

SIXTH EDITION

NEW YORK

WILLIAM WOOD \& COMPANY

$\operatorname{MDCCCCX}$ 


$$
\begin{aligned}
& 25=1 \\
& 5-4 \\
& 1910
\end{aligned}
$$

Digitized by the Internet Archive in 2010 with funding from Columbia University Libraries 


\section{PREFACE TO THE SIXTH EDITION}

Is the present edition the book has been extensively revised, and in many parts rewritten. A considerable amount of new matter has been added, and an increase in the bulk of the volume has been found unavoidable.

G. N. STEWART.

Cleveland,

September, I9Io. 



\section{EXTRAC'T FROM THE PREFACE TO THE FIRST EDITION}

IN this book an attempt has been made to interweave formal exposition with practical work, according to a programme which I have followed for some time past in teaching Physiology to medical students on the other side of the Atlantic, and which has, it is believed, proved to be well adapted to their needs and opportunities. It ought, however, to be explained that. for various reasons, a somewhat wider range of experiment is open to the student in America than in this country. But as nobody will use this book except in a regular laboratory and under responsible guidance, it has not been thought necessary to mark in any special manner the parts of the exercises which the English student must do by proxy (that is, learn from demonstrations), and the parts he ought to perform for himself.

An arrangement of the exercises with reference to the systematic course has this advantage-that by a little care it is possible to secure that practical work on a given subject shall actually be going on at the time it is being expounded in the lectures. Cross-reference from lecture-room to laboratory, and from laboratory to lecture-room, from the detailed discussion of the relations of a phenomenon to the living fact itself, is thus rendered easy, natural, and fruitful.

As some teachers may wish to know how a course such as that described in the Practical Exercises may be conducted for a fairly large class, a few words on the method we lave followed may not be out of place. It is obvious that many of the exercises require more than one person for their per- 
formance; and it maty be said that, exeept in the case of the simpler experiments and the chemical work ats at whole. which each student does for himself, it has been found convenient to divide the class into groups of four, each group remaining together throughout the session. It is possible that some may find a group of four too large a unit, and it is certain that three, or perhaps even two, would be better; but in a large school so minute a subdivision is hardly possible, without entailing excessive labour on the teachers.

The systematic portion of the book is so arranged that it can equally well be used independently of the practical work, and aims at being in itself a complete exposition of the subject, adapted to the requirements of the student of medicine.

As to the matter of the text, it is hardly necessary to say that this book does not aspire to the dubious distinction of originality; and it is literally impossible to acknowledge all the sources from which information has been derived. In many cases names have been quoted, but names no less worthy of mention have often been of necessity omitted.

\section{G. N. STEWART.}

CAMBRIDGE,

September, 1895. 


\section{CONTENTS}

INTRODUCTION

The proteins

Carbo-hydrates

Fats

Structure of living matter

Functions of living matter

\section{CHAPTER I.}

The Circulating Liguids of the Body

Blood-corpuscles

Life-history of the corpuscles -

Viscosity of blood

Reaction of blood

Specific gravity of blood

Electrical conductivity of blood -

Relative volume of corpuscles and plasma

Hæmolysis

Precipitins

Coagulation of blood

Chemical composition of blood -

Hæmoglobin and its derivatives

Quantity of blood

Lymph and chyle

Functions of blood and lymph -

CHAPTER II.

The Circulation of the Blood and Lymph Physiological anatomy of vascular system

Flow of a liquid through tubes -

The beat of the heart

The sounds of the heart

The cardiac impulse

Endocardiac pressure

The arterial pulse 
The Circulation of THF Blood axd L fimpl (continued)-

Arterial blood-pressure -

PAGL

Measurement of the blood-pressure in man - - Iuf

Velocity of the blood - _ _ _ _ _ - Ios

Measurement of velocity of blood - - $\quad$ - III

The volume-pulse - $\quad$ - $\quad$ - $\quad$ - $\quad$ - IIG

The circulation in the capillaries - - $\quad$ - 118

The circulation in the veins - $\quad$ - $\quad$ - $\quad$ - $1 \geq 1$

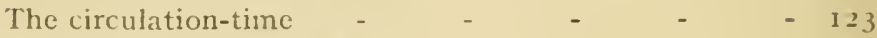

Work and output of heart - - $\quad$ - $\quad 127$

The relation of the nervous system to the circulation - 128

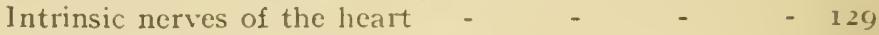

Cause of the heart-beat - $\quad-\quad \quad-\quad-\quad-\quad-129$

Conduction and co-ordination in heart - _ - $\quad$ - 134

Auriculo-ventricular bundle - $\quad$ - $\quad$ - $\quad 135$

Chemical conditions of heart-beat - _ - I39)

Refractory period of heart - _ - - _ - $\mathbf{r}_{4} \mathbf{I}$

Extrinsic nerves of the heart - - _ - $\quad 1+3$

Action of poisons on the heart - - - $\quad$ - 150

Normal excitation of cardiac nervous mechanism - 152

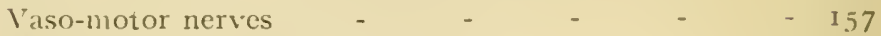

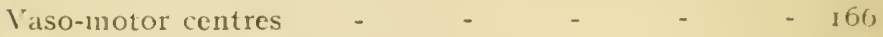

Vaso-motor reflexes - $\quad$ - $\quad$ - $\quad$ - $\quad$ - 160

Influence of gravity on the circulation - $\quad-\quad 173$

The lymphatic circulation - $\quad$ - $\quad$ - $\quad-176$

CHAPTER 11 .

RESPRATION -
Blood-supply of lungs
Mechanical phenomena of respiration


RESPIRATION (continued)-

PAGE

Respiration of muscle - $\quad$ - $\quad$ - $\quad$ - $\quad-261$

Nature of the oxidative process _ - $\quad$ - $\quad-264$

lnfluence of respiration on blood-pressurc - $\quad-265$

Effects of breathing condensed and rarefied air $\quad-272$

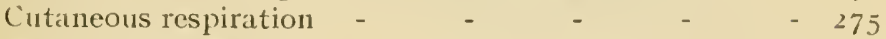

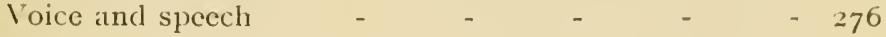

CHAPTEIR IV.

Digestion

Mechanical phenomena of digestion

Deglutition

Movements of stomach and intestines

Influence of central nervous system on gastro-intestinal

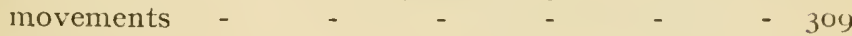

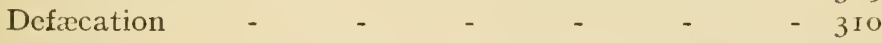

Vomiting

Chemical phenomena of digestion - $\quad-\quad-314$

Ferments

Chemistry of the digestive juices

Saliva

Gastric juice -

Antiseptic function of the gastric juice - $\quad-328$

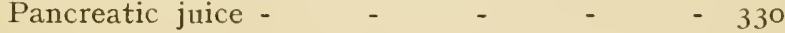

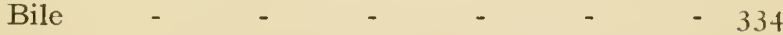

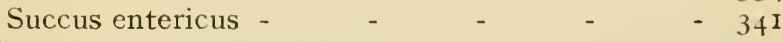

Secretion of the digestive juices $\quad-\quad-\quad-\quad-344$

Changes in pancreas and parotid during secretion $34^{6}$

Changes in gastric glands during secretion - 347

Changes in mucous glands during secretion - 352

Mode of formation of the digestive juices - 354

Why the stomach does not digest itself - $\quad 359$

Influence of the nervous system on the salivary

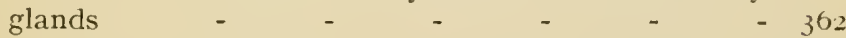

Reflex secretion of saliva $\quad-\quad-\quad-\quad-\quad-36$,

Influence of the nervous system on the gastric glands - 37 ?

Influence of the nervous system on the pancreas - 378

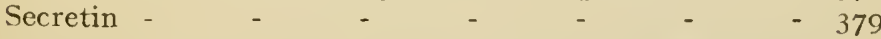

Influence of the nervous system on the secretion of bile 383

Influence of the nervous system on the secretion of intes-

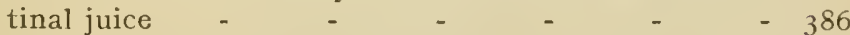

Action of drugs on digestive secretions - $\quad$ - $\quad-387$

Secretion of the digestive juices (summary) - $\quad-388$

Digestion as a whole $\quad-\quad\left[\begin{array}{lllllll} & - & - & - & & - & 389\end{array}\right.$

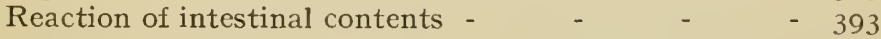

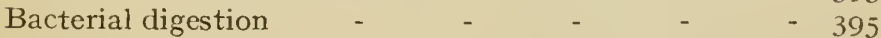

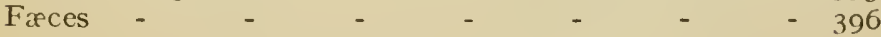




\section{CHAPTER V}

Absorptioy

Imbibition, diffusion, and osmosis - $\quad-\quad-398$

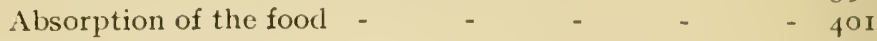

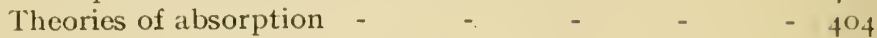

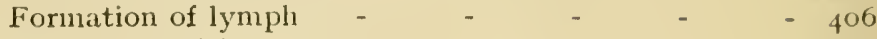

Absorption of fat - - - - $\quad$ - $\quad-412$

Absorption of carbo-hydrates - - $\quad$ - $\quad-416$

Absorption of water and salts - _ - $\quad$ - $\quad-417$

Absorption of proteins - $\quad$ - $\quad$ - $\quad$ - $\quad-418$

\section{CHAPTER VI.}

ExcRetion -
Excretion by the kidneys

\section{CHAPTER VII.}

Metabolism, Nutrition and Dietetics

Metabolism of proteins -

Formation of urea

Formation of uric acid -

Formation of hippuric acid

Formation of kreatinin -

Autolysis

Metabolism of carbo-hydrates-glycogen

Glycogen-formers

Extra-hepatic glycogen -

Fate of the glycogen

Glycolysis

Diabetes

Metabolism of fat

Formation of fat from carbo-liyclrates -

Formation of fat from protein

Income and expenditure of the body

Nitrogenous equilibrium

Laws of nitrogenous metabolism

Carbon equilibrium

$-496$

$-496$

- 500

- 505

- 508

- 509

- 509

- 5 II

- 513

$-514$

$-515$

$-\quad 516$

- 518

- 522

- 524

- 525

- 528

- 529

The oxygen deficit

- 535

- 539

- $54 \mathrm{I}$ 
Metabolism. Nutrition and Dietetics (contmued)Inorganic salts in metabolism -

Dictetics

Stimulants

Internal secretion

of thyroid and parathyroid

CHAPTER VIII.

Animal Heat -

Calorimetry

Heat-loss

Heat-production

Seats of heat-production

Thermotaxis

Heat centres

Fever

Distribution of heat

Temperature topography

Normal variations in body temperature

- 572

- 573

- 578

- 579

$-\quad 583$

- 587

- 595

- 597

- 602

- 604

- 605

\section{CHAPTER IX.}

Muscle -

Physical introduction -

Physical properties of muscle

Stimulation of muscle

Direct excitability of muscle

The muscular contraction

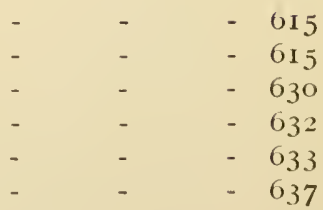

Optical phenomena of (and structure of muscle) - 638

Mechanical phenomena of

Influence of fatigue on -

Electrical tetanus

- 642

Voluntary contractio

Thermal phenomena of -

- 648

Chemical phenomena of -

- 656

- 660

- 663

- 666

Source of the energy of muscular contraction - $\quad-669$

Rigor mortis

- 671

\section{CHAPTER X.}

Nerve

The nerve-impulse $\cdot$ its initiation and conduction 
NERVE (continued) -

Electrotonus

Conduction in the nerve -

Velocity of the nerve-impulse

- 686

Chemistry of nerve

Degeneration of nerve

Regeneration of nerve -

Trophic nerves

Classification of nerves

\section{CHAPTER XI.}

\begin{tabular}{|c|c|c|c|c|c|c|}
\hline ELECTRO-PHÝSIOLOGY & - & - & - & - & & 717 \\
\hline Currents of rest & and action & - & - & - & - & 718 \\
\hline Relation betweer & n action cur & t a & inc & & & 2 \\
\hline Polarization of $n$ & muscle and & & - & - & . & 6 \\
\hline Electrotonic cur & rents & - & - & - & 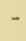 & \\
\hline Heart-currents & - & - & - & - & 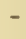 & 0 \\
\hline Human electro-c & ardiogram & - & - & - & - & 73 \\
\hline Glandular curren & ats & - & - & - & 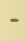 & 37 \\
\hline Eye-currents & - & - & - & - & 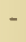 & 34 \\
\hline Electric fishes & - & - & - & - & - & \\
\hline
\end{tabular}

\section{CHAPTER NII.}

The Cextral Nervous System

\section{Structure}

Development

Histology

Nutrition of the neuron -

-
-
-
-

\begin{tabular}{|c|c|c|}
\hline - & - & - \\
\hline - & - & - \\
\hline - & - & - \\
\hline & - & - \\
\hline & - & - \\
\hline
\end{tabular}

General arrangement of grey and white natter in the central nervous system

Arrangement of grey and white matter in the spinal cord 762

Arrangement of grey and white matter in the upper part

of the cercbro-spinal axis

Functions of the central nervous system

Functions of the spinal cord - _ -

Decussation of the sensory paths

Reflex action

Influence of brain on spinal reflexes

Automatism of the spinal cord -

The cranial nerves

The functions of the brain

Functions of the cercbellum

Co-ordination of movements

Functions of the cerebral cortex

' IIotor' areas

Sensory areas

Aphasia -
- 767

- 786

- $78 S$

$-792$

$-790$

- 806

- SI2

- 815

- 827

- 830

- $\$ 37$

- $S_{40}$

- 844

- 858

- 862 
The Central Nervous System (continued)-

Localization of function in central nervous system

Reaction tine

Slecp and fatigue

Hypnosis

Cerebral circulation

Resuscitation of central nervous system

Chemistry of nervous activity -

Cerebro-spinal fluid

Autonomic nervous system

\section{CHAPTER XIII.}

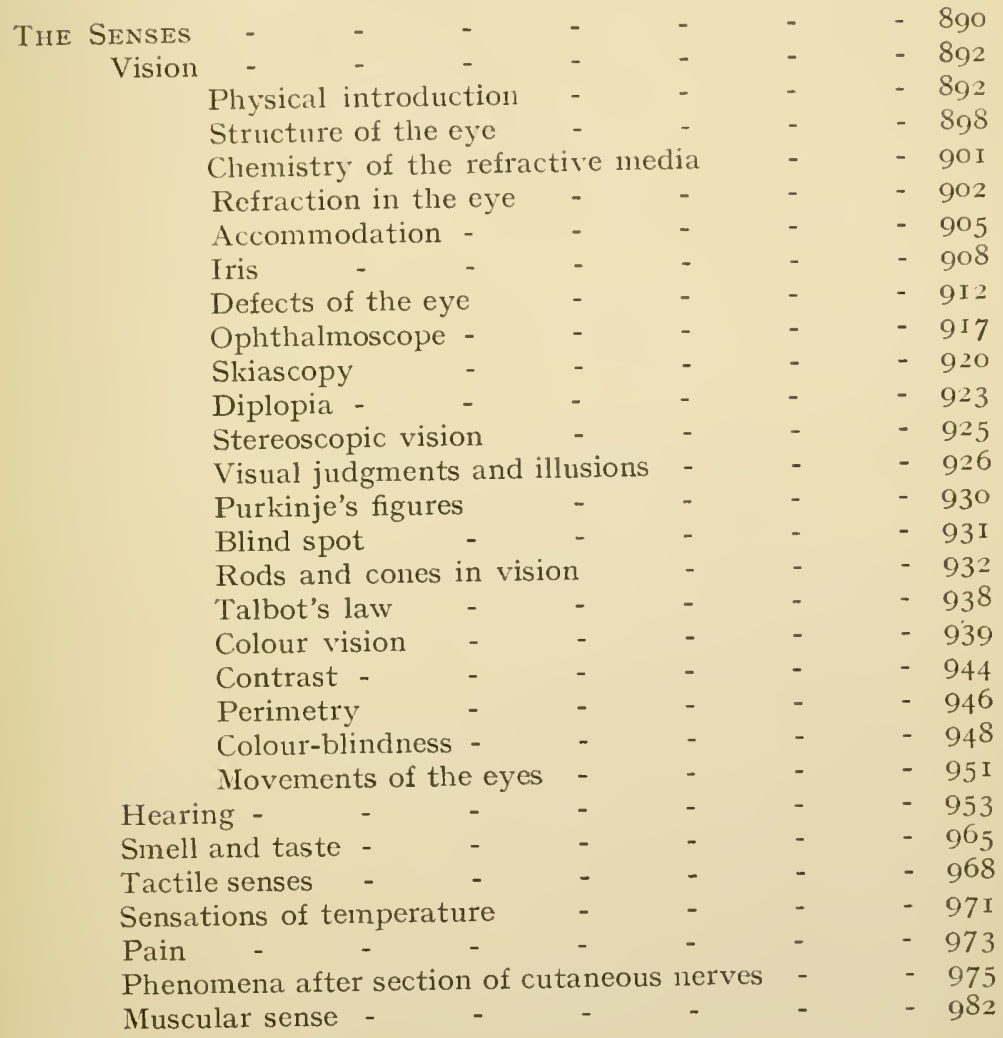

\section{CHAPTER XIV.}

REPRODUCTION -

Regeneration of tissues -

Reproduction in the higher animals 
REPRODUCTION (continued)-

Development of the ov'um

Physiology of the embryo

Exchange of materials in the placenta

$-1010$

Parturition

Milk

Transplantation of tissues

Parabiosis

$-1013$

- $101 \%$

- I02I

$-1023$

$-1024$

APPENDIX

$-$

- 1027

INDEX -

\section{PRACTICAL EXERCISES}

\section{INTRODLC'TION}

\section{General reactions of proteins - -}

\section{Colour reactions of proteins}

Special reactions of groups of proteins -

Precipitation reactions of proteins

Reactions of derivatives of proteins

Carbo-hydrates -

Fats

Scheme for testing for proteins and carbo-hydrates

\section{CHAPTER I.}

I. Reaction of blood -

2. Specific gravity of blood

3. Coagulation of blood

4. Preparation of fibrin-ferment

5. Preparation of extracts containing thrombokinase - $\quad-\quad 57$

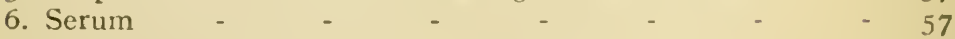

7. Enumeration of the blood-corpuscles - $\quad-\quad 58$

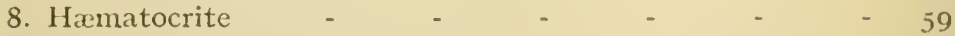

9. Electrical conductivity of blood - $\quad-\quad-\quad-60$

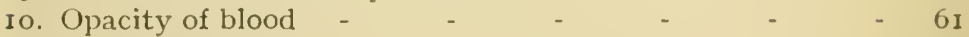

11. Laking of blood - $\quad$ - $\quad$ - $\quad$ - $\quad$ - 6 I

12. Haxmolysis and agglutination - - $\quad-63$

I3. Osmotic resistance of colonred corpuscles - _ - 64

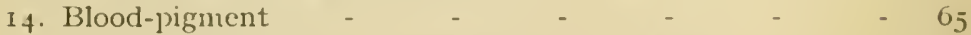

(1) Preparation of hemoglobin crystals - - 65

(2) Spectroscopic examination of hemoglobin and its lerivatives $\quad-\quad \quad-\quad 6 \quad-65-67$

(3) Gualacum test for blood - $\quad$ - 68

(4) Quantitative estimation of liamoglolin - 68

(5) Hæmin test for blood-pigment - 7 I 
CHAPTER II.

1. Microscopic examination of the circulating blood -

2. Anatomy of the frog's heart

- 177

3. The beat of the heart

- 177

4. Apex of the heart -

$-178$

5. Heart tracings

$-178$

6. Dissection of vagus and cardiac sympathetic in frog

- 179

7. Stimulation of the vagus in the frog

- 181

S. Stimulation of the junction of the sinus and auricles

- 182

9. Action of muscarine and atropia on the heart

$-\quad 183$

Io. Stannius' experiment

I I. Stimulation of cardiac sympathetic in frog

12. Action of inorganic salts on heart-muscle -

I3. The action of the mammalian heart

I4. Action of the valves of the heart -

15 . Sounds of the heart

I6. Cardiogram

I7. Sphygmographic tracings

I 8. Venous pulse tracing from jugular -

I9. Polygraph tracings -

20. Plethysmographic tracings -

- 183

- 183

- $\quad$ 84

- $\quad$ I85

- $\quad$ I86

2I. Pulse-rate -

22. Blood-pressure tracing

23. Estimation of arterial pressure in man

24. Influcnce of position of the body on blood-pressure

25. Effects of hæmorrhage and transfusion on blood-pressure -

26. The influence of albumoses on blood-pressure

27. Effect of suparenal extract on blood-pressure - $\quad 20 \mathrm{I}$

28. Section and stimulation of cervical sympathetic in rabbit 202

29. Determination of the circulation-time

\section{CHAPTER III.}

r. Tracing of the respiratory movements in man - $\quad-\quad 287$

2. Production of apnœa and periodic breathing in man $\quad 288$

3. Tracing of the respiratory movements in animals - $\quad-288$

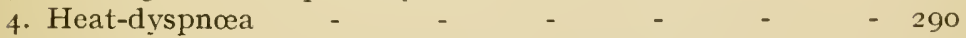

5. Measurement of volume of air inspircd and expired - $29 \mathrm{I}$

6. Cardio-pneumatic movements - - - - $\quad 29 \mathrm{I}$

7. Auscultation of the lungs - $\quad$ - $\quad-\quad-\quad$ - 29 I

8. Measurement of the respiratory pressure - $\quad-\quad 292$

9. Determination of carbon dioxide and oxygen in inspired

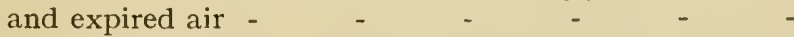

I0. Estimation of carbon dioxide and water given off by an

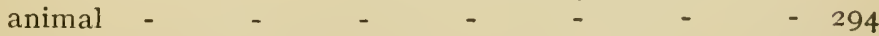

II. Muscular contraction in the absence of free oxygen $\quad 295$

I2. Oxidizing ferments - $\quad$ - $\quad$ - $\quad-\quad 295$ 
CHAPTERS IV. AND V.

I. Chemistry and digestive action of saliva -

2. Stimulation of the chorda troupani -

3. liffect of clrugs on the secretion of saliva -

1. Digestive action of gastric juice -

5. To obtain chyme and gastric juice -

6. Digestive action of pancreatic juice

7. Chemistry of bile

8. Nicroscopical examination of freces

9. Absorption of fat - -

Io. Time required for digestion and alsorption of food substances

I I. Quantity of cane-sugar inverted and absorber in a given time

I 2. Auto-rligestion of the stomacl

CHAPTER VI.

I. Specific gravity of urine

2. Reaction of urine -

3. Chlorides in urine -

4. Phosphates in urine

5. Sulphates in urine -

6. Indoxyl in urine

7. Urea

8. Total nitrogen in urine

9. Uric acid

Io. Kreatinin - -

I I. Hippuric acid

I2. Proteins in urine

I3. Sugar in urine Pentoses in urine

Acetone in urine

Determination of the freezing-point of urine

Examination of urine

Urinary sediments -

CHAPTERS VII. AND VIII.

I. Glycogen

2. Catheterism

3. Experimental glycosuria

(I) Injection of sugar into the blood

(2) Phloridzin glycosuria

(3) Alimentary glycosuria

4. Milk

5. Cheese

6. Flour 
7. Breall - - - - - -

8. Excretion of utea (and total nitrogen) and proteins in food $6 \mathrm{I}_{2}$

9. Measurement of the heat given off in respiration - - 613

\section{CHAPTERS IX. AND X.}

1. Difference of make and breal induction shocks - $\quad 702$

2. Stimulation by the voltaic current - $\quad-\quad-\quad-704$

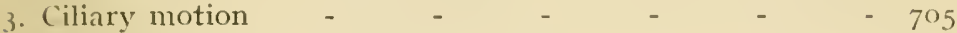

4. Direct excitability of muscle-curara - _ - $\quad$ - 706

5. Craphic record of 'twitch' - - - $\quad$ - $\quad$ - 706

6. Influence of temperature on the muscle-curve - $\quad-706$

7. Influence of load on the muscle-curve - $\quad-\quad$ - 708

8. Influence of fatigue on the muscle-curve - - $\quad$ - 708

9. Seat of exhaustion in fatigne of the muscle-nerve prepara-

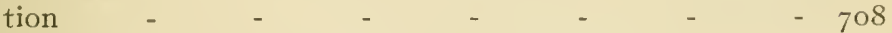

10. Seat of exhaustion in fatigue for voluntary contraction - 709

11. Influence of veratrine on muscular contraction - $\quad 709$

12. Measurement of the latent period of muscular contrac-

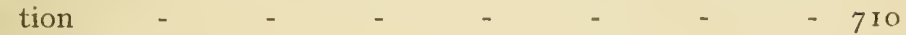

13. Summation of stimuli $\quad-\quad-\quad-\quad-\quad-7$ II

I4. Superposition of contractions - $\quad-\quad-\quad-7$ II

I5. Composition of tetanus - $\quad-\quad \ldots \quad-\quad$ - 7 II

I6. Contraction of smooth muscles - - - - $\quad-7$ I2

17. Velocity of the nerve-impulse - - - $\quad-\quad$ 7r 3

I8. Chemistry of muscle - $\quad-\quad-\quad-\quad-\quad 4$ - 713

19. Reaction of muscle in rest, activity, and rigor - $\quad-715$

\section{CHAPTER XI.}

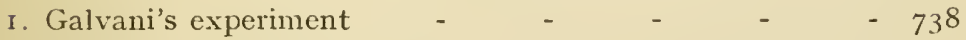

2. Contraction without metals - $\quad-\quad-\quad-\quad 438$

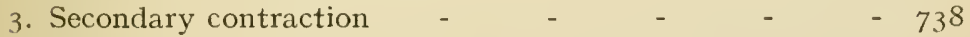

4. Demarcation and action currents with capillary electro-

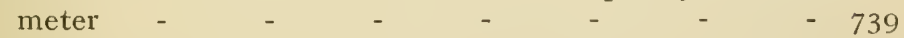

5. Action-current of the heart $\quad-\quad-\quad-\quad-740$

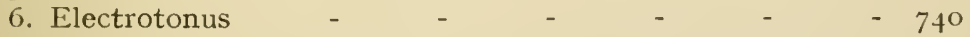

7. Paradoxical contraction - - - - - - 74 I

8. Alterations in excitability and conductivity produced in

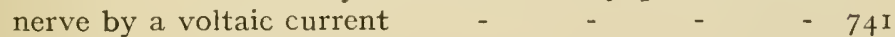

9. Formula of contraction - $\quad$ - $\quad-\quad-\quad$ - $\quad$ - 742

I0. Formula of contraction for (human) nerves in situ - $\quad$ - 743

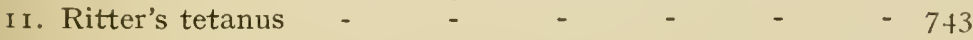

\section{CHAPTER XII.}

I. Section and stimulation of nerve-roots - $\quad-\quad-884$

2. Reflex action in the 'spinal' frog - - _ - $\quad$ - 885

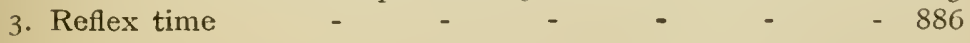


4. Inhibition of the reflexes

5. Spinal cord and muscular tonus

6. Spinil cord and tomus of the bloodvessels -

7. Action of strychnine

8. Mimmalian spinal preparation

9. Reflexes in man

10. Excision of (ercbral hemisplueres (frog)

11. Excision of cerebral hemispheres (pigeon) -

12. Stimulition of the motor areas in the dog -

\section{CHAPTER NIII.}

I. Dissection of the eye

2. Formation of inverted image on retina

3. Phakoscope

4. Scheiner's experiment

5. Kühne's artificial eye

6. Astigmatism (ophthalmometer)

7. Spherical aberration

8. Chromatic aberration

9. Measurement of the field of vision -

Io. Mapping the blind spot

I I. The yellow spot

I 2. Ophthalmoscope

I 3. Retinoscopy

I 4. Pupillo-dilator and constrictor fibres

I 5. Colour-mixing

I6. After-images

17. Retinal fatiguc

I 8. Visual acuity

19. Colour-blindness

20. 'Talbot's law

21. Purkinje's figures

22. Relation of pitch and vibration frequency -

23. Beats

24. Sympathetic vibration

25. Galton's whistle

26. Cranial conduction of sound

27. Taste

28. Smell

29. Touch and pressure -

30. Temperature sensations

31. Pain

CHAPTER NIV. 


\section{A MANUAL OI: PHYSIOLOGY}

\section{INTRODUCTION}

LiviNe matter, whether it is studied in plants or in animals, has certain peculiarities of chemical composition and structure, but especially certain peculiarities of action or function, which mark it off from the unorganized material of the dead world around it.

Chemical Composition of Living Matter. - Although we cannot analyze the living substance as such, we can to a certain, but limited, extent reconstruct it, so to speak, from its ruins. When subjected to analytical processes, which necessarily kill it, living matter invariably yields bodies of the class of proteins, exceedingly complex substances, which have approximately the following composition: Carbon, $5 \mathrm{I}^{\circ} 5$ to $54^{\circ} 5$ per cent.; oxygen, $20^{\circ} 9$ to $23^{\circ} 5$ per cent. ; nitrogen, $I 5^{\cdot} 2$ to $I 7$ per cent. ; hydrogen, 6.9 to 7.3 per cent., with small quantities of sulphur. Nucleoproteins, which are compounds of ordinary proteins with nucleic acids, a series of sulphur-free organic acids rich in phosphorus, are constantly met with. Certain carbo-hydrates, composed of carbon, hydrogen, and oxygen (the last two in the proportions necessary to form water), of which glycogen $\left(\mathrm{C}_{6} \mathrm{H}_{10} \mathrm{O}_{5}\right)_{n}$ may be taken as a type, appear to be always present. Fats, which consist of carbon, hydrogen, and oxygen, and of which tristearin, a compound of stearic acid with glycerin, of the formula $\mathrm{C}_{3} \mathrm{H}_{5}, 3\left(\mathrm{C}_{13} \mathrm{H}_{35} \mathrm{O}_{2}\right)$, may be given as an example, are often, and certain liquids, e.g., lecithin (p. 4), are always, found. Finally, water and certain inorganic salts, such as the chlorides and phosphates of sodium, potassium, and calcium, are constantly present.

The Proteins.- The constitution of the protein molecule is still unknown; but when proteins are broken down by the action of ferments, such as exist in gastric and in pancreatic juice, or by chemical methods-for example, by boiling with dilute acids - the most important of the cleavage products are various amino-acids (p. 332). It has therefore been suggested that proteins are built up by the linking together of amino-acids, the different proteins differing quantitatively or qualitatively as regards the amino-acids present (E. Fischer). Thus serum-albumin and egg-albumin yield 
no glycin or glycocoll (amino-acetic acid, $\mathrm{CH}_{2} . \mathrm{NH}_{\text {.... }} \mathrm{COOH}$ ), while glycin is constantly found among the cleavage proflucts of sermmglobulin. And while leucin (a-aminoisobutylacetic acid) is present to the extent of about 20.5 per cent. in the cleavage products of (horse's) scrum-albumin, (hen's) cgg-albmin yiclds only 7 ' I per cent.

On the other hand, egg-albumin yiclds 8. I per cent. of alanin (amino-propionic acid, $\left.\mathrm{C}_{2} \mathrm{H}_{1} . \mathrm{NH}_{3} . \mathrm{COOH}\right)$, while sermm-albumin yiclds only 2.7 per cent. Of the aromatic amino-acids-that is, amino-acids united to the benzenc ring-phenyl-alanin (aminopropionic acid in which one atom of $\mathrm{H}$ is replaced by phenyl, $\left.\mathrm{C}_{6} \mathrm{H}_{3}\right)$ is obtained to the extent of +4 per cent. from egg-albumin, and a little over 3 per cent. from serum-albumin. Tyrosin or oxyphenylalanin (amino-propionic acid in which a $\mathrm{H}$ atom is replaced by oxyplienyl, $\left.\mathrm{C}_{1 \mathrm{i}} \mathrm{H}_{4} . \mathrm{OH}\right)$ appears to the amount of $1^{\prime} 5$ per cent. among the cleavage products of egg-albumin, and to the amount of 2.1 per cent. among those of serum-albumin. It is an interesting point in this connection that gelatin, which yielrls 16.5 per cent. of glycin. yields no tyrosin at all; tryptophane, an aromatic amino-acid still more complex than tyrosin, is also absent. These facts afford an explanation of certain colour reactions of proteins long known cmpirically, but only recently understood (p. 7). The process by which the protein molecule is thus decomposed is called hydrolysis - that is, the molecule takes up water, and then splits into smaller molecules. The hydrolysis occurs in various stages, bodics like acid-or alkali-albumin (meta- or infra-proteins) being first formed, then proteoses. then peptones. The peptones are further split into bodics containing a relatively small number of anino-acids linked together. These bodies are called pepticles or polypeptides, which finally are decomposed so as to yield the individual amino-acids. The inverse process can also be carried on to a certain extent, and Fischer has taken an important step towards the eventual synthesis of proteins by showing how polypeptides of increasing complexity can be built up by linking amino-acids together. Bodies may thus be formed in the laboratory which give sonic of the characteristic reactions of peptones.

The numerous substances included in the group of proteins may be classified as follows, beginning with the simplest:

I. Protamins, such as the bodics called salmin and sturin present in fish-sperm.

2. Histones, bodies separated from blood-corpuscles. Globin, the protein constituent of hremoglobin, is one of them. Unlike the other groups of proteins, they arc precipitated by ammonia.

3. Albumins.

4. Globulins.

5. Sclero-protcins or albuminoids, such as gelatin and keratin.

6. Phospho-proteins, including such substances as vitcllin. a borly obtainable from cgg-yolk, and caseinogen, the chicf protein of milk. They are rich in phosphorus, but are to be distinguished from nucleoproteins, which also contain a relatively large amount of phosphorus, by the fact that they do not yicld the purin bases, the characteristic products of the dccomposition of nucleo-proteins.

7. Conjugated proteins, substances in which the protein moleculc is united to another constituent, usually spoken of as a 'prosthetic ' group. Thus the nucleo-protcins consist of protcin united with nucleic acid, the chromo-proteins (e.g., haemoglobin) of protein united with a pigment, and the gluco-proteins (e.g., mucin) of protein united with a carbo-hydrate group. 
Among the derivatives of proteins, the most important are those already mentioned as being produced in protein-hydrolysis, viz. :

(a) Meta-proteins.

(b) Proteoses, including albumose, the proteose derived from albumin; globulose, that derived from globulin; gelatose, that derived from gelatin, ete. The proteoses may be furtlier subdivided, according to the order in which they are formed in digestion into proto-proteoses, hetero-proteoses, and deutero-proteoses.

(c) Peptones.

in (d) Polypeptides. The majority of these are artificial products, formed by the synthesis of amino-acids, although some can be obtained from proteins by hydrolysis. Only a few of those hitherto prepared give the binret test.

However formidable the above list may appear to the student, it gives an inalequate idlea of the extreme complexity of the protein class and its richness in individuals. For, apart from the fact that the list has been purposely left incomplete, especially as regards the numerous vegetable proteins, there is the best evidence that proteins of the same name from different animal species have certain properties which distinguish them from each other. The serumalbumins can be crystallized much more easily in some animals than in others. The same is conspicuomsly true of the hæmoglobins, which differ also in certain animals in the relative proportion of sulphur and iron in the molecule, as well as in the crystalline form. Even when no chemical or physical differences have as yet been made out, proteins of the same name from the blood or organs of different species show notable 'specific' differences when subjected to certain biological tests (see, e.g., the paragraph on 'Precipitins,' p. 30).

Carbo-hydrates. - The most important carbo-hydrates in their physiological relations are dextrose, levulose, galactose, lactose, maltose, sucrose (cane-sugar), starch, and glycogen. As regards their chemical constitution, the simplest carbo-hydrates are aldehydes or ketones-that is, the first oxidation products of primary and secondary alcohols respectively. Thus dextrose is the aldehyde of sorbite, a hexatomic alcohol (an alcohol containing six $\mathrm{OH}$ groups), while levulose is the ketone of the isomeric alcohol called mannite, and galactose the aldehyde of the isomeric alcohol called dulcite. The sugars containing six carbon atoms are termed hexoses. They include dextrose, levulose, and galactose. The empirical formula of these three simple sugars (or monosaccharides) is the same $\left(\mathrm{C}_{6} \mathrm{H}_{12} \mathrm{O}_{6 \mathrm{~b}}\right)$, but, owing to the different arrangement of the atoms or groups of atoms, they have each their characteristic propertics by which they can be easily distinguished. For example, dextrose rotates the plane of polarization to the right, levulose to the left. By the union or 'condensation' of two molecules of a monosaccharide, with loss of a molecule of water, a disaccharide is formed. Cane-sugar, maltose, and lactose, all with the same empirical formula, $\left(\mathrm{C}_{12} \mathrm{H}_{22} \mathrm{O}_{11}\right)$, are disaccharides. Cane-sugar yields on hydrolysis a mixture of equal parts of dextrose and levulose; lactose, a mixture of dextrose and galactose; while maltose is converted into dextrose. By the condensation of more than two molecules of monosaccharide polysaccharides are formed, such as starch, dextrin, and glycogen. The exact molecular weights of these substances are unknown. Their general formula can be written $\left(\mathrm{C}_{6} \mathrm{H}_{10} \mathrm{O}_{5}\right)_{n}$, where $n$ represents the number of monosaccharide molecules condensed to form the polysaccharide, in the case of starch probably some hundreds. 
Fats and Lipoids. - The fats are compounds of higher fatty acids witl glycerin (glycerin esters). The ordinary boly-fat consists of a mixture of three neutral fats (palmitin, stearin, and olein) wlich differ both chemically and physically from each other-e.g., in melting-point and in the so-called iodine value, the number which represents the amount of iodine taken up from a stanclard solution. Olein melts at $-5^{\circ} \mathrm{C}$, palmitin at $45^{\circ} \mathrm{C}$, and stearin at a still higher temperature. It is, therefore, the presence of olein which keeps the body-fat liquid at the temperature of the body. The fats are soluble in ether, in hot alcohol, and in many other liquids, but insoluble in water. Besides the ordinary fats, the tissues and liquids of the body contain lecithin $\left(\mathrm{C}_{40} \mathrm{H}_{44} \mathrm{NPO}_{4}\right)$, a fat-like compound which yields on decomposition, in addition to glycerin, and a fatty acid, phosphoric acicl and a nitrogen-containing substance called cholin (p. 337). Lecithin, though found in all cells, is especially abunclant in nervous tissues. It is associated with cholesterin and with other substances which, like lecithin and cholesterin, are soluble in ether and similar solvents of fat. For this reason these substances are often grouped together as lipoids, although some of them are chemically quite different from fat. Cholesterin, for instance, is an alcohol. Although usually present only in small amount, the lipoicls play a very important part in the structure and in the economy of the cell.

Structure of Living Matter-The Cell.-Protoplasm or living substance, when examined in its most primitive, undifferentiated condition in such cells as the amceba or the white blood-corpuscles, appears on first view a homogeneous, structureless mass, except for certain granules embedded in it, and consisting either of products formed by its activity or of food materials. But even here more careful study reveals a certain complexity of structure. At the very least, an external layer, or ectoplasm, can be distinguished from the interior mass, or endoplasm. There is reason to believe that even where no histological demonstration of an ectoplasmic layer or a definite envelope is possible, the surface of the cell is physiologically different from its interior. In many cells the protoplasm presents the appearance of a honeycomb or network, with granules usually situated at the nodes, and holding in its vesicles or meshes a fluid, perhaps containing pabulum, from which the waste of the living framework is made good, or material upon which it works, and which it is its business to transform. Some observers, however, maintain that the network is an artificial appearance produced by the precipitation of the colloid constituents of the protoplasm by the fixing reagent, or even by the coagulative processes associated with the act of dying, and that the unaltered living substance is a homogeneous fluid or jelly. In certain respects it behaves like a liquid, and in others like a solid, a peculiarity which is undoubtedly associated with its richness in colloids, as experiments with such substances as gelatin and agar have shown. In building up our typical cell we start with a piece of protoplasm. Somewhere in the midst of this we find a body which, if not 
absolutely different in kind from the protoplasm of the rest of the cell or cytoplasm, is yet marked off from it by very definite morplological and chemical characters.

This is the mucleus, generally of round or oval shape, and bounded by an envelope. Within the envelope lies a second network of fine threads, which do not themselves stain with nuclear dyes such as hrematoxylin. But in or on these 'achromatic' tilaments lic small, highly refractive particles, staining readily and deeply with dyes, and therefore described as consisting of chromatin. This chromatin is either made up of nucleins (nucleoproteins particularly rich in nucleic acid, and therefore in phosphorus), or yields nucleins by its decomposition; and it seems to owe its affinity for certain staining substances to the presence of nucleic acid. The meshes of the nuclear reticulum contain a semi-fluid material, which does not readily stain. The nucleus is distinguished from the cytoplasm, even as regards its inorganic constituents, by the absence of potassium.* Besides the nucleus, another much smaller structure, the centrosome, is differentiater from the protoplasm of the cell. This is a minute dot staining deeply with such dyes as hrematoxylin, and generally situated near the nucleus. Surrounding it is a clear area, the attraction sphere, in and beyond which fine fibrils radiate out into the cytoplasm. Both the attraction sphere and the nucleus play an important part in division of the cell by the process known as karyokinesis, or mitosis, or indirect division, which is by far the most common mode.

When the nucleus is about to divide, the chromatin granules arrange themselves into one or more coiled filaments or skeins, which then break up into a number of separate portions called chromosomes. These undergo a remarkable series of transformations, leading eventually to the segregation of the nuclear chromatin in two separate daughter nuclei, each surrounded by a portion of the original cytoplasm. Apart from its rôle in the division, and therefore in the multiplication, of the cell, the nucleus is now known to exert an influence perhaps not less important upon those chemical changes in the cytoplasm which are necessary for its normal nutrition and function. $\rightarrow$ It is doubtful whether any portion of protoplasm can permanently survive

* This has been shown microchemically. The potassium is precipitated by a solution of hexanitrite of sodium and cobalt as orange yellow crystals of the triple salt, hexanitrite of potassium, sodium and cobalt. Where very minute traces of potassium are present, ammonium sulphide must be added, after washing out the excess of the cobalt reagent. Black cobalt sulphide is thus formed from the triple salt (Macallum, Frontispiece).

$\dagger$ According to Hertwig, a precursor of chromatin, 'procliromatin,' a substance without characteristic staining reaction, is formed in the cytoplasm, taken up by the nucleus, and there elaborated into chromatin. From the nucleus chromatin and its derivatives return to the cytoplasm to be used in its function. 
the loss of its nuclear material. It must be remembered, however, that nuclear material may sometimes be present in diffuse form in cells which do not show a nucleus in the histological sense.

When we carry back the analysis of an organized body as far as we can, we find that every organ of it is made up of cells, which upon the whole conform to the type we have been describing, although there are many differences in details. Some organisms there are, low down in the scale, whose whole activity is confined within the narrow limits of a single cell. The amceba sets up in life as a cell split off from its parent. It divides in its turn, and each half is a complete amœba. When we come a little higher than the amceba, we find organisms which consist of several cells, and 'specialization of function' begins to appear. Thus the hydra, the 'common fresh-water polyp' of our ponds and marshes, has an outer set of cells, the ectoderm, and an inner set, the endoderm. Through the superficial portions of the former it learns what is going on in the world; by the contraction of their deeply-placed processes it shapes its life to its environment. As we mount in the animal scale, specialization of structure and of function are found continually advancing, and the various kinds of cells are grouped together into colonies or organs. In some organs and tissues the bond of union is simple juxtaposition and similarity of function of the constituent cells. But in others the union is protoplasmic, processes of the cytoplasm actually passing from cell to cell. This is seen in certain epithelial tissues, and conspicuously in the cardiac muscle.

The Functions of Living Matter.-The peculiar functions of living matter as exhibited in the animal body will form the subject of the main portion of this book; and we need only say here: (I) That in all living organisms certain chemical changes go on, the sum total of which constitutes the motabolism of the body. These may be divided into (a) integrative or anabolic changes, by which complex substances (including the living matter jtself) are built up from simpler materials; and (b) disintegrative or katabolic changes, in which complex bodies (including the living substance) are broken down into comparatively simple products. In plants, upon the whole, it is integration which predominates; from substances so simple as the carbon dioxide of the air and the nitrates of the soil the plant builds up its carbo-hydrates and its proteins. In animals the main drift of the metabolic current is from the complex to the simple; no animal can construct its own protoplasm from the inorganic materials that lie around it; it must have ready-made protein in its food. But in all plants there is some disintegration; in all animals there is some synthesis. (2) The living substance is excitable-tlat is, it responds to certain ex- 
terual impressions, or stimuli, by actions peculiar to each kind of cell. (3) The living substance reproduces itself. All the manifold activities included under these three heads have but one source, the transformation of the energy of the food. It is not, however, upon the whole, peculiarities in food, but in molecular structure, that underlic the peculiarities of function of different living cells. A locomotive is fed with coal; a steampmimp is fed with coal. The one carries the mail, and the other keeps a mine from being flooded. Wherein lies the difference of action? Clearly in the build, the structure of the mechanism, which determines the manner in which energy shall be transformed within it, not in any difference in the source of the energy. So one animal cell, when it is stimulated, shortens or contracts; another, fed perhaps with the same food, selects certain constituents from the blood or lymph and passes them through its substance, changing them, it may be, on the way; and a third sets up impulses which, when transmitted to the other two, initiate the contraction or secretion. In the living body the cell is the machine; the transformation of the energy of the food is the process which 'runs' it. The structure and arrangement of cells and the steps by which energy is transformed within them sum up the whole of biology.

\section{PRACTICAL EXERCISES.}

\section{Reactions of Proteins.}

I. General Reactions of Proteins.-Egg-albumin may be taken as a type. Prepare a solution of it by adding water to white of egg. which consists mainly of egg-albumin with a little globulin. In breaking the egg, take care that none of the yolk gets mixed with the white. Snip the white up with scissors in a large capsule, then add ten or fifteen times its volume of distilled water. The solution becomes turbid from the precipitation of traces of globulin, since globulins are insoluble in distilled water. Stir thoroughly, strain through several layers of muslin, and then filter through paper.

\section{Colour Reactions.}

(I) Add to a little of the solution in a test-tubc a few drops of strong nitric acid. A precipitate is thrown down, which becomes ycllow on boiling. Cool, and add strong ammonia; the colour changes to orange (xantho-proteic reaction). The reaction depends upon the presence of aromatic groups in the protein molecule, which are converted into nitro-compounds.

(2) To a third portion add a drop or two of very dilute cupric sulphate and excess of sodium or potassium hydroxide; a violet colour appears (Piotrowski's test). Peptones and proteoses (albumoses) give a pink (biuret reaction).* See p. 426 .

* The reaction is also given, although more faintly, with the hydroxides of lithium, strontium, and barium. It is given by all substances containing at least two $\mathrm{CONH}_{2}$ groups attached to one another (as in oxamide), or to the same nitrogen atom (as in biuret), or to the same carbon atom. 
(3) To another portion add Millon's reagent; ; white precipitate comes down, which is turned reddish on boiling. If only traces of protein are present, no precipitate is caused, but the liguid takes on a red tinge. The reaction is clue to tyrosin. It is given by all aromatic substances which contain the group $\mathrm{C}_{60} \mathrm{H}_{6}$ with at least one H replaced by OH.

(4) Adamkiewicz's reaction (Hopkins's modification).- To a small quantity of the albumin solution add the same bulk of dilute glyoxylic acid. Mix, and to the mixture add an equal volume of strong pure sulphuric acid. A purple colour is obtained. The substance in the protein molecule which gives the reaction is tryptophane (p. 332).

(5) The Formaldehyde Reaction.-Add to the albumin solution a few drops of a very dilute solution of formaldehyde $(1: 2,500)$, and then allow some strong (commercial) sulphuric acid to run from a pipette into the bottom of the test-tube. A purple ring appears at the surface of contact. This reaction depends on the presence of tryptophane in the protein.

\section{Precipitation Reactions.}

(6) Acidify another portion strongly with acetic acid, and add a few drops of a solution of potassium ferrocyanide. A white precipitate is obtained. Peptones do not give this reation.

(7) Hleat a portion to $30^{\circ} \mathrm{C}$. on a water-batl. Saturate with crystals of anmonium sulphate ; the albumin is precipitated. Filter, and test the filtrate for proteins by (2). None, or only slight traces, will be found. The sodium hydroxide must be adeled in more than sufficient quantity to lecompose all the ammonimm sulphate. It will be best to add a piece of the solid hydroxide. Peptones are not precipitated by ammonium sulphate, but all other proteins arc.

(S) Add alcohol to a small quantity of the solution. The protein is precipitated. It can be redissolved at first, but rapidly becomes insoluble.

2. Special Reactions of Certain Proteins-(i) Heat-Coagulable Proteins: (a) Albumins.-(a) Heat a little of the solution of eggalbumin in a test-tube; it coagulates. With another sample cletermine the temperature of coagulation, first very slightly acidulating with a 2 per cent. solution of acetic acid.

To determine the Temperature of Coagulation.-Support a beaker by a ring which just grips it at the rim. Nearly fill the beaker with water, and slide the ring on the stand till the lower part of the beaker is immersed in a small water-bath (a tin can will do (puite well). In this beaker place a test-tube. and in the test-tube a thermometer, both supported by rings or clamps attached to the same stand. Put into the test-tube at least enough of the albumin solution to completely cover the bulb of the thermometer, and heat the bath. stirring

* Millon's reagent consists of a mixture of the nitrates of mercury with nitric acid in excess, and some nitrous acid. To make it, lissolve mercury in its own weight of strong nitric acicl, and add to the solution thus obtained twice its volume of water. Let it stand for a short time, and then decant the clear liquid, which is the reagent.

$\dagger \Lambda$ solution containing glyoxylic acid in the repuisite strength can be prepared by treating half a litre of a saturated solution of oxalic acid with 4) grammes of 2 per cent. sodium amalgam in a tall cylincler. When all the hydrogen has been evolved, the solution is filtered, and diluted with twice its volume of water. Oxalic acid and sodium binoxalate are also present in the solution. 
the water in the beaker occasionally with a feather or a splinter of wood, or a glass rod, the end of which is guarded with a piece of incliarubber tubing. Note the temperature at which the solution becomes turbicl, and then the temperature at which a clistinct congulum or precipitate is formed. Repeat with the unacilulated albumin solution.

( $\beta$ ) A similar experiment may be performed with serum-albumin obtainerl as on p. 57.

(b) Globulins. - Use serum-globulin (p. 57), or myosinogen (p. 672). Fibrinogen is also a globulin, but cannot casily be obtained in quantity. Verify the following properties of globulins :

(a) They coagulate on heating.

( $\beta$ ) They are insoluble in distilled water ( 1 . 5\%).

( $\gamma)$ They are precipitated by saturation with magnesium sulphate or sodium chloricle (p.57).

They give the general protein tests (I) to (8).

Both the heat-coagulated proteins and such proteins as the solid fibrin which is formed from fibrinogen in the clotting of blood give such of the general protein tests, (1), (2), (3) (p. 7), as with suitable modifications can be instituted on solid substances. Thus, in performing (2), a flake of fibrin or a small piece of the boiled egg-white should be soaked for a few minutes in a dilnte solution of cupric sulphate. Then the excess of the cupric sulphate should be poured off, and sodium hydroxide added, when the coagulated protein will become violet. Heat-coagulated proteins are insoluble in water, weak acids and alkalies, and saline solutions; fibrin is slightly soluble in the latter.

(2) Gelatin.-Add some pieces of gelatin to cold water in a testtube. It does not dissolve. Immerse the tube in a boiling waterbath till the gelatin goes into solution. Then cool the test-tube under the tap; the solution sets into a jelly. On heating it redissolves.

Try the general protein reactions (p. 7) on a dilute solution. In Piotrowski's test a violet colour is obtained. The tests which depend on the presence of tryosin or tryptophanc are not given by a solution of pure gelatin, since these amino-acids are absent from the gelatin molecule. Commercial gelatin may give a slight reaction due to traces of other proteins.

3. Reactions of Certain Derivatives of Native Proteins-(I) MetaProteins: (a) Acid-albumin.- To a solution of egg-albumin add a little $0^{\circ} 4$ per cent. hydrochloric acid, and heat to about body temperature-say $40^{\circ} \mathrm{C}$.- for a few minutes. Acid-albumin is formed. It can be produced from all albumins and globulins by the action of dilute acid. Make the following tests :

(a) Add to a portion of the solution in a test-tube a few drops of a solution of litmus; the colour becomes red. Now add drop by drop sodium carbonate or dilute sodium hydroxide solution till the tint just begins to change to blue. A precipitate of acid-albumin is thrown down. Add a little more of the alkali, and the precipitate is redissolved. It can be again brought down by neutralizing with acid.

( $\beta$ ) Heat a portion of the solution to boiling; no precipitate is formed.

$(\gamma)$ Add strong nitric acirl; a precipitate appears, which dissolves on heating, and the liquid becomes yellow.

(b) Alkali-albumin.-To a solution of egg-albumin acld a little sodium hydroxide, and heat gently for a few minutes. Alkali-albumin 
is produced. It can be derived by similar treatment from any albumin or globulin.

(a) Nentralize, after colouring with litmus solution, by the aldition of dilute hydrochloric or acetic acid. Alkali-albumin is precipitated when neutralization has been reached. It is reclissolvel in exeess of the acid.

( $\beta$ ) $T$ o anotles portion of the solntion of alkali-illoumin aclel a few drops of sodium phosplatte solution, then litmus, and then dilnte aciel till the alkali-allsumin is precipitated. More of the dilute acid slombl now be required to precipitate the alkali-albmin, since the sodium phosphate must first be changerl into acid solim phosphite.

$(\gamma)$ On heating the solution of alkali-albumin there is no coaterulation.

(2) Proteoses. - For preparation and reactions, see p. 426. They differ from albumins and globulins in not being coagulated by heat, and from meta-proteins in not being precipitated by neutralization. They are soluble (with the execption of hetero-albumose) in clistilled water, and are not precipitated by saturation of their solutions with magnesium sulpliate or sodium chloricle. Saturation with anmonium sulphate precipitates them. With a solution of commercial "peptone,' which consists chiefly of albumoses, and contains only a little true peptone, perform the following tests :

(a) Boil the slightly acidulated solution; there is no coagulation.

(ß) Biuret reaction, p. 7 .

$(\gamma)$ To a portion of the solution add its own volume of saturated ammonium sulphate solution. The primary albumoses (proto- and hetero-albumose) are precipitated. Filter. Add a drop of sulphuric acid to the filtrate and saturate it with ammonium sulphate crystals. The secondary or deutero-albumoses are precipitated. Filter. The filtrate still contains peptones. Use it for (3).

(3) Peptones.-For preparation and tests, see p. 426. They differ from heat-coagulable proteins and meta-proteins in the same way as proteoses, and they differ from proteoses in not being precipitated by ammonium sulphate. On the filtrate from (2) perform the biuret test, as described in (7), p. 8 ; and note that the pink colour is the same as that given by proteoses.

\section{Carbo-hydrates.}

1. Glucose or Dextrose.-Make a solution of dextrose in water, and apply to it Trommer's test for reducing sugar. l'ut some of the dextrose solution in a test-tube, then a few drops of cupric sulphate, and then excess of sodium or potassium hydroxide. The bluc precipitate of cupric hydroxide which is first thrown down is immediately dissolved in the presence of dextrose and many other organic substances. Now boil the blue liquid, and a yellow or recl precipitate (cuprous hylroxide or oxide) is formed.

2. Cane-sugar.-Perform Trommer's test with a sample of a solution. A blue liquid is obtained, which is not cluanged on boiling. Now put the rest of the solution in a flask. Add $\ldots$ th of its bulk of strong hyclrochloric acid, and boil for a quarter of an hour. Again perform 'Trommer's test. Remember that excess of alkali must be present after the acid is neutralizel. The test now shows much reducing sugar. The cane-sugitr has been 'inverted'-i.e., changed into a mixture of dextrose and levulose.

3. Starch.-(1) Cut a slice from at well-washed potato; talk a scraping from it with a knife, and examine with the microscope. 
Note the statch granules with their concentric markings, using a small diaphragm. Run a drop) of dilute iodine solution under the cover-slip, and olserve that the granules become bluish. Examine also with a polarization microscope, (2) Rub up al little starch in a mortar with cold water, then atd boiling water and stir thoroughly. Decint into a capsule or beaker, and boil for a few minutes. After the licpuid has cooled, perform the following experiments:

(a) Ada a few drops of ioline solution to a little of the thin starch mucilage in a test-tube. $A$ blue colour is produced, which (isappears on heating, returns on cooling, is bleached by the addition of a little sodium hydroxide, and restored by dilute acid.

(b) Test the starch solution for reducing sugar by 'Trommer's test. If none is found, boil some of the mucilage with a little clilute sulphuric acid in a flask for twenty minutes, and again perform Trommer's test. Abundance of reducing sugar will now be present.

4. Dextrin.-Dissolve some dextrin in boiling water. Cool. Acicl iodine solution to a portion; a reclelish-brown (port-wine) colour results, which (lisappears on heating. As a control, the same amount of iodine should be adeled to an equal quantity of water in another test-tube. The colour returns on cooling. The colour is also bleached by alkali, restored by acid. Excess of iodine should be arlded for the bleaching experiment (i.e., more than enough to give the maximum depth of tint). If too little iodine has been arlded there may be no restoration of the colour by the acid. The addition of a little more iodine to the acid solution will then cause the portwine colour to return, and this may be again bleached by alkali, and will now be restored by acid.

5. Glycogen.-Sec p. 608.

6. Molisch's Test for Carbo-hydrates. - This is a general test for carbo-hydrates. It is also given by proteins which contain a carbohydrate group. Put a drop of dextrose solution in a test-tube. Add a clrop of a 10 per cent. solution of $a$-naphthol in methyl alcohol, and then $0^{\circ} 5$ c.c. of water. Then cautiously allow I c.c. of pure concentrated sulphuric acid to run under the mixture, and shake gently. A violet or reddish colour appears.

\section{Fats.}

I. Take a little lard or olive-oil, and observe that fat is soluble in ether or warm alcohol, but not in water. Put a drop of the ethereal solution of fat on a piece of paper, and note that it leaves a greasy stain.

2. Put a little alcohol in a test-tube, and then a drop of phenolphthalein solution and a drop or two of dilute sodium hydroxide to give the solution a red colour. Add a few drops of an ethereal solution of the lard or olive-oil. If the red colour persists the fat is neutral ; if it disappears the fat contains free fatty acids.

3. Saponification.-Mclt some lard in a porcelain dish, and pour it into an alcoholic solution of potassium hydroxide previously heated on a water-bath nearly to boiling. Mix well, and keep the mixture gently boiling on the bath till saponification is complete. This only takes a short time. Remove a little of the soap solution, and drop it into distilled water in a test-tube. If unsaponified fat is present it will rise to the top as drops of oil. In this case boiling should be continued. If all the fat has been saponified the soap solution will mix with the water and no oil-drops will separate. 
न. Falty Acids.-Heat sone 20 per cent. sulplunric acid in a small flask nearly to boiling, and (rop into it sone of the soat) obtained in 3. The fatty acids separate ont and rise to the top ats an oily layer. Cool, skim off the fatty acid, and wash it with distilled water till the wash-water is no longer acicl.

(a) Dissolve a little of the washed fatty acis in ether. Arld a few drops of an alkaline solution of phenolphthatein to a few c.c. of water in a test-tube. Drop into this the ethereal solution of fatty acid. The red colour is discharged.

(b) Put a small portion of the fatty acid on a glass slicle resting on a piece of white paper. Place on it al drop or two of at per cent. solution of osmic acid (osminm tetroxide). The osmic acid is reduced to a lower oxide (which is black) by the action of oleic acicl present in the fatty acid mixture, which abstracts some of the oxygen. Any fat which contains olcin or oleic acid, as body-fat does, is therefore blackened by osmic acirl.

(c) Arld to a portion of the fatty acid some sodium hydroxide solution, and warm. Solium soap is formed. Add warn water and shake up. A lather is produced. Keep the soal) solution for 6 . Keep a little of the fatty acid for 5 (b) and 6 , (b).

5. Clycerin.-(a) Aild to a little glycerin in a dry test-tube a few crystals of potassium bisulphate (kilS() $)_{4}$, and heat over the free flame. Acrolein is given off, which is recognised by its pungent odour, and by blackening a picce of filter-paper moistened with ammoniacal silver nitrate solution, and held over the mouth of the test-tube. The paper is blackened owing to the reducing action of the vapour on the silver nitrate.

(b) Repeat this test with lard, and with a portion of the fatty acid fronı 4. Acrolcin will be given off by the lard because glycerin is contained in neutral fat, but not by the fatty acid if it lias been properly separated from the glycerin.

6. Emulsification.--(a) Take three test-tubes and label them $A$, B, and C. Put a few c.c. of water in A, a solution of soap in B, and a dilute solution of sodium carbonate or sodium hydroxide in C. To each auld a few drops of fresh olive-oil and shake. An cunulsion will be formed in $B$, but not in $\Lambda$. Probably there will be some emulsification in $\mathrm{C}$ also, owing to the presence in the oil of sonce fatty acirl, which forms soap with the alkali. But if the oil is frec from fatty acid 110 enulsion will be formed.

(b) Repeat $(a)$ with rancid olive-oil, which contains much fatty acil, or with fresh olive-oil to which some of the fatty acicl obtained in 4 has been adked. A good cmulsion will be produced in $C$ : as well as in $B$.

7. Melting-point of Fat.-Put into a very narrow test-tube or a short piece of narrow glass tubing some fincly divided mutton fat. freed as far as possible from connective tissue. Fasten the test-tube on to the bulb of a thermometer with a rubber band, and immerse the thermometer and tube in a beaker filled with water and standing on a water-bath which is gradually heated. Observe the temperature at which the fat melts. Repeat the experiment with hog's lard and (log's fat. 
SCHEME FOR TESTING A SOLUTION FOR THE MORE COMMON PROTEINS AND PROTEIN-DERIVATIVIS, AND FOR CARBO-HYDRATES.

I. Note the reaction, and whether the liquid is coloured or colourless, clear or opalescent. A redclish colour suggests blood; opalescence suggests glycogen or starch Try one or more of the general protein tests (e.g., the xantho-proteic or liuret). If the result is positive, proceed as in 2 ; if negative, pass to 3 .

2. Test for Proteins.-(I) If the reaction is acid or alkaline, nentralize with very dilute soclium carbonate or sulphuric acid. A precipitate $=$ acid- or alkali-albumin, according as the original reaction is acicl or alkaline. If the original reaction is neutral, no acicl-or alkali-allumin can be present in solution. Filter off the precipitate, if any.

(2) Boil some of the filtrate from (1) (or some of the original solution if it is neutral), acidulating slightly with dilute acetic acid. $\Lambda$ precipitate $=$ albumin or globulin. Filter, and keep the filtrate.

(3) If a precipitate has been obtainerl in (2), (a) saturate some of the original solution with magnesium sulphate, or half saturate it with ammonium sulphate (i.e., add to it an equal volume of saturated ammonium sulphate solution). If there is no precipitate, globulin is absent, and therefore the precipitate obtained in (2) must be albumin. A precipitate $=$ globulin. But albumin may also be present in the solution. To see whether this is so, filter off the globulin and boil the filtrate after acidulation with acetic acid. A precipitate $=$ albumin.

(b) Half saturate the filtrate from (2) with ammonium sulphate (i.e., add its own volume of a saturated solution of the salt). A precipitate= primary proteoses. Filter.

(c) Saturate the filtrate from $(b)$ with ammonium sulphate crystals. A precipitate $=$ secondary proteoses. Filter.

(d) To the filtrate from $(c)$ add excess of solid sodium hydroxide in small pieces at a time. Much ammonia is given off. Allow the test-tube to stand fifteen minutes, shaking it at intervals. Then add dilute cupric sulphate, and if much of the sodium sulphate formed remains undissolved, add water to dissolve it. A well-marked rose colour=peptone.

(4) If no precipitate has been obtained in (2), the solution contains neither albumin nor globulin. To test whether primary or secondary proteose or peptone is present, apply (3) (b), (c), and $(d)$.

3. Test for Carbo-hydrates.-Use the original solution, freed from coagulable proteins, if such have been found, by acidulation and boiling.

(1) Add iodine. If the solution is alkaline neutralize it before adding the iodine. A blue colour=starch. Confirm by boiling with dilute sulphuric acid and testing for reducing sugar. A reddish-brown colour with iodine $=$ glycogen or dextrin.

Glycogen gives an opalescent, dextrin a clear, solution. Glycogen is precipitated by basic lead acetate, dextrin is not (p. 608). Both are changed into reducing sugar by boiling with dilute acid.

(2) Add to some of the original solution cupric sulphate and excess of sodium hydroxide, and boil. Yellow or red precipitate=reducing sugar.

(3) If (1) and (2) are negative, boil some of the liquid with one-twentieth of its volume of strong hydrochloric acid for fifteen minutes, and test as in (2). A red or yellow precipitate shows that cane-sugar was originally present, and lias been inverted. 


\section{CHAPTER I}

\section{THE CIRCULATING LIQUIDS OF THE BODY}

Is the living cells of the animal body chemical changes are constantly going on ; energy, on the whole, is rumning down; complex substances are being broken up into simpler combinations. So long as life lasts, food must be brought to the tissues, and waste products carried away from them. In lowly forms like the amoba these functions are performed by interchange at the surface of the animal without any special mechanism; but in all complex organisms they are the business of special liquids, which circulate in finely branching channels, and are brought into close relation at various parts of their course with absorbing organs, with eliminating organs, and with the tissue elements in general.

In the higher animals three circulating liquids have been distinguished: blood, lymph, and chyle. But it is to be remarked that chyle is only lymph derived from the walls of the alimentary canal, and therefore, during digestion, containing certain freshly-absorbed constituents of the food; while both ordinary lymph and chyle ultimately find their way into the blood, and are in their turn recruited from it. The blood contains at one time or another everything which is about to become part of the tissues, and everything which has ceased to belong to them. It is at once the scavenger and the foodprovider of the cell. But no bloodvessel enters any cell ;* and if we could unravel the complex mass of tissue elements which essentially constitute what we call an organ, we should see a sheet of cells, with capillaries in very close relation to them, but everywhere separated from them by a thin layer of lymph. And to describe in a word the circulation of the food substances we may say that the blood feeds the lymph, and the lymph feeds the cell.

* Fine intracellular canaliculi, communicating with the blood-capillaries, and probably performing a nutritive function, since they seem to contain blood-plasma, have been described by Schäfer and others in the liver cells. 


\section{Morphology of the Blood.}

The blood consists essentially of a liquid part, the plasma, in which are suspended cellular elements, the corpuscles. When the circulation in a frog's web or lung or in the tail of a tadpole is examined under the microscope, the bloodvessels are seen to be crowded with oval bodies - of a yellowish tinge in a thin layer, but in thick layers crimson-which move with varying velocity, now in single file, now jostling each other two or three abreast, as they are borne along in the axis of an apparently scanty stream of transparent liquid. Nearer the walls of the vessels, sometimes clinging to them for a little and then being washed away again, may be seen, especially as the blood-flow slackens, a few comparatively small, round, colourless cells. The oval bodies are the red or coloured corpuscles, or erythrocytes; the colourless elements are the white blood-corpuscles, or lencocytes; the liquid in which they float is the plasma (Practical Exercises, p. I77).

The Red Blood-corpuscles, or Erythrocytes, differ in shape and size and in other respects in different animal groups. In amphibians, such as the frog and the newt, they are flattened ellipsoids containing a nucleus, and the same is true of nearly all the other vertebrates, except mammals. In mammals they are discs, hollowed out on both the flat surfaces, or biconcave, and possess no nucleus. But the red corpuscles of the llama and the camel,

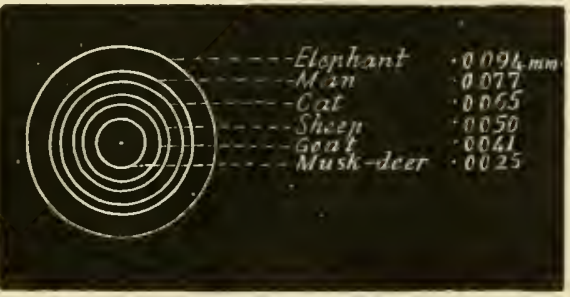

Fig. I.-Diagram showing Relative Size of Red Corpuscles of Various Animals. although non-nucleated, are ellipsoidal in shape like those of the lower vertebrates. As to size, the average diameter in man is between 7 and $8 \mu^{*}{ }^{*}$ In the frog the long diameter is about $22 \mu$, while in Proteus it is as much as $60 \mu$, and in Amphiuma, the corpuscles of which can be seen with the naked eye, nearly $80 \mu$ (Plate I. frontispiece).

As regards the structure of the red corpuscles, the most probable view is that they are solid bodies, with a spongy and elastic structureless framework, denser at the surface of the corpuscle than in its centre, but continuous throughout its whole mass (Rollett). The denser peripheral layer constitutes a physiological envelope which permits the passage of certain substances into or out of the corpuscles, and hinders the passage of others.

* A micro-millimetre, represented by symbol $\mu$, is $\frac{1}{1000}$ millimetre. 
Envelope and spongework are sometimes spoken of as the stroma of the corpuscle, in contradistinction to its most important constituent, a highly complex pigment, the hamoglobin, which, not in solution as such, but either in solution as a compound with some other unknown substance, or more probably bound in some solid or semi-solid combination to the stroma, fills up the space within the envelope in the interstices of the spongework. Since there is good reason to believe that the lamoglobin as obtained artificially from the corpuscles is not quite the same substance as the native blood-pigment within them, the latter is sometimes distinguished by a separate name-hamochrome. To the physical properties of the stroma it is usual to attribute the great elasticity of the corpuscles-that is, the power of recovering their original shape after distortion-for their elasticity is no wise impaired by the removal of the hemoglobin.

Rouleaux Formation. - When blood with disc-shaped corpuscles is shed, there is a great tendency for the corpuscles to run together into groups resenbling vouleaux, or piles of coin. No satisfactory cxplanation of this curious fact has yet been given.

Crenation of the corpuscles, a condition in which they become studded with fine projections, is caused by the addition of moderately strong salt solution, by the passage of shocks of electricity at high potential, as from a Leyden jar, or by simple exposure to the air. Concentrated saline solutions, which abstract water from the corpuscles and cause then to shrink, make the colour of blood a brighter red, because more light is now reflected from the crumpled surfaces. On the other hand, the addition of water renders the corpuscles spherical; more of the light passes through them, less is reflected, and the colour becomes dark crimson (Plate I.. frontispiece).

The White Blood-corpuscles, or Leucocytes.-The red corpuscles are peculiar to blood. The white corpuscles may be looked upon as peripatetic portions of the mesoderm (see Chap. XIV.), and some of them ought not in strictness to be called blood-corpuscles. They are more truly body corpuscles. Similar cells are found in many situations, and wander everywhere in the spaces of the connective tissue. They pass into the bloodvessels with the lymph, and may pass out of them again in virtue of their amœboid power. They consist of protoplasm, less differentiated than that of any other cells in the body, and under the microscope appear as granular, colourless, transparent bodies, spherical in form when at rest, and containing a nucleus, often tri- or multi-lobed. Many of the leucocytes of frog's blood at the ordinary temperature, and of mammalian blood when artificially heated on the warm stage, may be seen to undergo slow changes of form. Processes called pseudopodia are pushed out at one portion of the surface, retracted at another, and thus the corpuscle gradually moves or 'flows' from place to place, 
and envelopes or eats up substances, such as grains of carminc, which come in its waty. This kind of motion was furst observed in the anoba, and is therefore called amoeboid. The lencocytes of human blood are not all of the same size, and differ also in other respects. They may be classified according to the presence or absence of granules in their protoplasm, and the fineness or coarseness of the gramules; according to the chemical nature of the dyes with which the granules most readily stain, and according to the form of the nucleus. Five varieties of leucocytes may thus be distinguished in normal blood (Plate I.) :

I. Polymorphomuclear Nentrophile Cells.-The nucleus assumes a great variety of forms, often contorted or deeply lobel. the lobes being united by finc strands of chromatin. The protoplasin contains numerous fine refractive granules, which stain best neither with simple acid dyes like eosin nor with simple basic dyes like methylene bluc, but with mixtures which must be assumed to contain 'ncutral ' stains, like Ehrlich's so-called triacid stain.* These cells make up

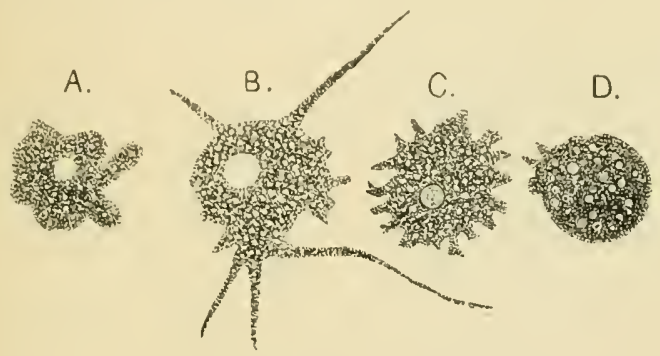

Fig. 2.-Ameboid Movenent.

$A, B, C, D$, successive changes in the form of an amøeba.

$6_{5}$ to 75 per cent. of the total number of leucocytes. Their diameter is 10 to $12 \mu$.

2. Eosinophile Cells ( 12 to I $_{5} \mu$ in diameter), much less numerous in normal blood than the neutrophiles (less than 5 per cent. of the whole), but found in considerable numbers in the serous cavities, the connective tissue, and the bone-marrow. The granules in the protoplasm are coarser than the neutrophile granules, and stain much more deeply with cosin. The nucleus may be simple, lobed, or even divided into fragments between which no connection can be traced. It is less rich in chromatin, and stains less easily with basic dyes, like methylene blue, than the nuclens of the first variety.

3. Hyaline Cells, or Large Mononuclear Lencocytes, with a diameter of 12 to $15 \mu$. They possess a large simple nucleus, poor in chromatin, surrounded by a relatively great amount of protoplasm, with no evident granules. They constitute 3 to 5 per cent. of the total number of leucocytes.

4. Lymphocytes. - Smaller cells than any of the preceding (diameter $6 \mu$ ), possessing a single large nucleus, surrounded by a comparatively small amount of protoplasm; 20 to 25 per cent. of the leucocytes of the blood belong to this group.

* A mixture of orange G., acid fuchsin, and methyl green. 
5. 'Mast Cells,' or 'Basophiles,' the least numerous variety $\left(0^{\circ} 5\right.$ per cent. of the total number). Very few are to be found in the normal blood of achults, but more in children. They are somewhat smaller than the neutrophiles (average dianeter about no $\mu$ ). The nucleus is irregularly trilobed. The protoplasm shows coarse granules, which (lo not glitter like the granules of the eosinophile cells, and are therefore less conspicuous in the unstained conclition. Unlike the eosinophile granules, they stain with basic dyes, such as methylene blue.

Blood-plates.-When blood is examined immediately after being shed, small colourless bodies ( $\mathrm{I}$ to $3 \mu$ in dianeter) of various shapes, but usually round or oval, may be seen. These are the blood-plates or platelets. They can be collected by placing a drop of blood on a smooth and clean piece of paraffin, and keeping it in a moist chamber. Clotting is long delayed, and the white and coloured corpuscles sink to the bottom, while the platelets rise to the top of the drop, from which they can be removed by a cover-slip. They can be best studied when the blood is mixed directly with some fixing solution, such as Hayem's solution (sodium chloride, I grm. ; sodium sulphate, 5 grm. ; mercuric chloride, 0.5 grm. ; water, 200 grm.), or osmic acid. They can even, like leucocytes, be kept alive on the warm stage in an appropriate medium (agar, to which certain salts have been added), and then show lively amoboid morements (Deetjen). While some observers believe that they represent the remains of the nuclei of the erythroblasts, it is more probable that they are independent elements. They have even been described as nucleated cells, although the nucleus is not easy to stain. They are not produced by the breaking up of other elements of the shed blood, for they have been observed within the freshly-excised, and therefore still living, capillaries - in the mesentery of the guinea-pig and rat (Osler).

Enumeration of the Blood-corpuscles.-This is done by taking a measured quantity of blood, diluting it to a known extent with a liquid which does not destroy the corpuscles, and counting the number in a given volume of the diluted blood (p. 58$)$.

The average number of red corpuscles in a cubic millimetre of blood is about $5,000,000$ in a healthy man, and about $4,500,000$ in a healthy woman, but a variation of $1,000,000$ up or down can hardly be considered abnormal. In persons suffering from profound anemia the number may sink to I,000,000 per cubic millimetre, or even less, while in new-born children and in the inhabitants of high plateaus or mountains it may rise to $7,000,000$, or even more. In the latter instance a residence of a fortnight in the rarefied air is sufficient to bring about the increase, 
and a subsequent residence of a fortnight in the lowlands to annul it.*

The number of white blood-corpuscles is on the average about Io,000 per cubic millimetre of blood, or one leucocyte for every 500 red blood-corpuscles. But if the count is made when digestion is relatively inactive, four to five hours after a meal, it gives no more than 7,000 to the cubic millimetre. In newborn children the average number is over 18,000 per cubic millimetre. In leukemia the number of white corpuscles is enormously increased - on the average to about 300,000 , but in extreme cases to 600,000 per cubic millimetre-while at the same time the number of the red corpuscles is diminished; and the ratio of white to red may approach I: 4. As the anæmia rapidly advances towards the fatal termination of an acute case, and the erythrocyte count falls to $1,000,000$, or even less, the ratio may come still nearer to unity. An increase in the number of leucocytes has also been observed in certain infective diseases as part of the inflammatory reaction. There are also physiological variations, even within short periods of time; for example, the number of lymphocytes is increased when digestion is going on. The normal number of blood-plates varies from a quarter to half a million to the cubic millimetre, but may be greater in disease and at high levels (Kemp).

Life-history of the Corpuscles.

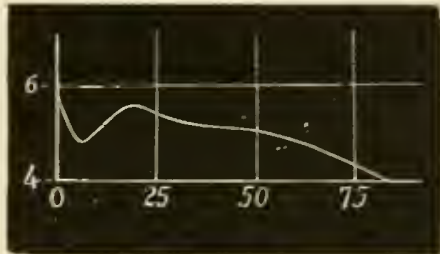

Fig. 3.- ClRve ShOWisg THE Nituber of Red Corptiscles AT DIFFERENT AGES (AFTER Sörexsey's Estimatioss).

The figures along the horizontal axis are years of age, those along the vertical axis millions of cor. puscles per cubic millimetre of blood. -The corpuscles of the blood, like the body itself, fulfil the allotted round of life, and then die. They arise, perform their functions for a time, and disappear. But although the place and mode of their origin, the seat of their destruction or decay, and the average length of their life, have been the subject of active research and still more active discussion for many years, much yet remains unsettled.

* In I 3 apparently healthy students (male) the average number of red corpuscles was $5,190,000$ per cubic millimetre. In 104 of these, the number ranged from $4,000,000$ to $6,400,000$; in 7 I (or 63 per cent. of the whole), from $4,400,000$ to $5,500,000$; in 3 , from $3,500,000$ to $3,900,000$; in 5 , from $6,500,000$ to $7,000,000$. In one observation the number reached $7,300,000$. In the new-born child the average is over $6,000,000$. In one case of pernicious anæmia, only i 43,000 corpuscles per cubic millimetre were present, the lowest number recorded. Over i 3,000,000 have been counted in a case of cyanosis (imperfect oxygenation of the bloorl, with blueness of the lips, etc.) due to congenital disease of the heart. 
In the embryo the red corpuscles, even of those forms (mammals) which have non-nucleated corpuscles in adult life, are at first possessed of nuclei, and approximately spherical in form. In the human foetus, at the fourth week all the red corpuscles are nucleated. Later on the nucleated corpuscles gradually diminish in number, and at birth they have almost or altogether disappeared, some of them, at least, having been converted by a shrivelling of the nucleus into the ordinary non-nucleated form. In the newly-born rat, which comes into the world in a comparatively immature state, many of the red corpuscles may be seen to be still nucleated. The first corpuscles formed in embryonic life are developed outside of the embryo altogether.

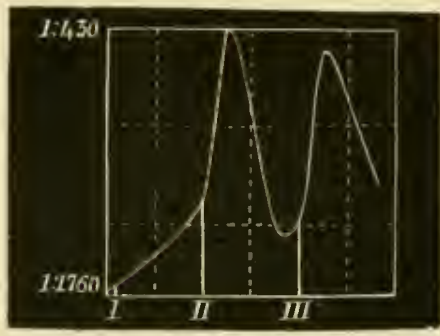

F1G. 4.-CURVE SHOWIXg PROPORTION OF WiITE CORPUSCles tO RED AT DIFFERENT TIMES OF THE Day (aFter the Results of HIRT).

At I the morning meal was taken : at II the mid-day meal; at III the evening meal. During active diges. tion the number of lymphocytes in the blood is greatly increased, both absolutely and relatively to the number of the other leucocytes.
Even before the heart has as yet begun to beat, certain cells of the mesoderm (see Chap. XII.) in a zone ('vascular area ) around the growing embryo begin to sprout into long, anastomosing processes, which afterwards become hollowed out to form capillary bloodvessels. At the same time clumps of nuclei, formed by division of the original nuclei of the cells, gather at the nodes of the network. Around each nucleus clings a little lump of protoplasm, which soon develops hæmoglobin in its substance; and the new-made corpuscles float away within the new-made vessels, where they rapidly multiply by mitosis. In later embryonic life the nucleated corpuscles continue in part to be developed within the bloodvessels in the liver, allantois, spleen, and red bone-marrow, and in certain localities in the connective tissue, by mitotic division of previously existing nucleated corpuscles, in part to be formed endogenously within special cells in the liver and perhaps other organs. Still later the nucleated corpuscles give place in the blood of the mammal to non-nucleated erythrocytes. Many of these are doubtless derived from the nucleated corpuscles, but some appear to be produced in the interior of certain cells of the connective tissue, and are non-nucleated from the start.

In the mammal in extra-uterine life the chief seat of formation of the red blood-corpuscles is the red marrow of the bones of the skull and trunk, and of the ends of the long bones of the 
linbs. Special mucleated cells in the marrow, originally colourless, multiply by karyokinesis, take up hemoglobin or form it within their protoplasm, and are transformed by varions stages into the ordinary non-nucleated red corpuscles, which then pass into the blood-stream. These blood-forming cells have received the name of erythroblasts or hamatoblasts. According to their size, erythroblasts have been distinguished as normoblasts, megaloblasts, and microblasts. The normoblasts are most numerous, and have about the same diameter as the full-formed erythrocrtes, into which they are believed to develop. The megaloblasts are larger, and the microblasts smaller, and they are thought to be the precursors of those aberrant forms of erythrocytes sometimes found in the blood in certain diseases. After hemorrhage rapid regeneration of the blood takes place, so that in a few weeks the loss of even as much as a third of the total blood is made good. The plasma is much sooner restored to its normal amount than the corpuscles. Microscopical examination shows in the red marrow the tokens of increased production of coloured corpuscles. Other organs also, particularly the spleen, may, in such emergencies, take on a bloodforming function.

A constant destruction of red blood-corpuscles must go on, for the bile-pigment and the pigments of the urine are derived from blood-pigment. The bile-pigment is formed in the liver. It contains no iron; but the liver-cells are rich in iron, and on treatment with hydrochloric acid and potassium ferrocyanide, a section of liver is coloured by Prussian blue. Iron must, therefore, be removed by the liver from the blood-pigment or from one of its derivatives; and there is other evidence that the liver is either one of the places in which red corpuscles are actually destroyed, or receives blood charged with the products of their destruction. Although it cannot be doubted that in all animals whose blood contains hæmoglobin the iron found in the liver bears an important relation to the building up or breaking down of the blood-pigment, the injection of hæmoglobin or hæmin, indeed, increasing markedly the amount of iron in the liver, as well as in the spleen, bone-marrow and other tissues, this does not seem to be the only function of the hepatic iron, for the liver of the crayfish and the lobster, which have no hæmoglobin in their blood, is rich in iron. Destruction of erythrocytes may also take place in the spleen and bone-marrow. Although the statement that free blood-pigment exists in demonstrable amount in the plasma of the splenic rein is incorrect, red corpuscles have been seen in various stages of decomposition within large amœboid cells in the splenic pulp; and deposits containing iron have been found there and in the red bone-marrow in certain pathological 
conditions. But there is no good foundation for the statement sometimes rather fancifully made that the spleen is in any special sense the 'graverard of the red corpuscles.' Some of the coloured corpuscles may break up in the blood itself, forming granules of pigment, whicl may then be taken up by the liver, spleen, and lymph glands. Indeed, it is probable that a large proportion of the worn-out erythrocytes are finally destroyed in the bloodstream. The portal circulation may be more than other vascular tracts a seat of this natural decay, perhaps in virtue of the presence of substances with a hemolytic action (p. 27) absorbed from the alimentary canal.

The lymphocytes are undoubtedly derived from the lymph. They are identical with the small lymph-corpuscles, and have little, if any, power of amceboid movement. They are formed largely in the lymphatic glands, for the lymply coming to the glands is much poorer in corpuscles than that which leaves them. The lymplatic glands, however, are not the only seat of formation of lymplocytes, for lymph contains some corpuscles before it has passed through any gland ; and although a certain number of these may have found their way by diapedesis from the blood, others are formed in the diffuse adenoid tissue, or in special collections of it, such as the thymus, the tonsils, the Peyer's patches and solitary follicles of the intestine, and the splenic corpuscles. The hyaline cells are possibly dereloped by the enlargement of lymphocytes. It is probable that the eosinophile cells, the polymorphonuclear neutrophiles, and the 'mast ' cells are formed in the bone-marrow. To a very small extent white blood-corpuscles may multiply by karyokinesis or indirect division in the blood.

The fate of the leucocytes is even less known than that of the red corpuscles, for they contain no characteristic substance, like the blood-pigment, by which their destruction may be traced. That they are constantly disappearing is certain, for they are constantly being produced. Not a few of them actually escape from the mucous membranes of the respiratory, digestive, and urinary tracts. The remnants of broken-down leucocytes have been found in the spleen and lymph glands. It must be assumed that many break up in the blood-plasma itself.

\section{Physical and Chemical Properties of the Blood.}

Fresh blood varies in colour, from scarlet in the arteries to purple-red in the veins. It is a somewhat viscid liquid, with a saline taste, and a peculiar odour.

Viscosity of Blood.--The viscosity of normal dog's blood is about six times greater than that of distilled water at body temperature. It can be determined by allowing the blood to 
flow through a capillary tube of known dimensions under a definite pressure, and measuring the amount which escapes in a given time. In general the viscosity and specific gravity of the blood vary in the same direction, although there is not an exact proportionality between them. Thus, sweating, which causes a diminution of the water of the blood, causes also an increase in its viscosity. With increasing temperature the viscosity of the blood diminishes, as is the case with other liquids (Opitz).

In polycythænia, where the number of erythrocytes in proportion to plasma is greatly increased, the viscosity of the blood increases in an equal degree. In one case of polycythemia, with a blood-count of $8,300,000$, the viscosity was 9.4 times that of water; in a case of marked chlorosis it was only $2 \cdot 14$. But the importance of this factor in causing an abnormal bloodpressure by increasing or diminishing the resistance to the bloodflow has been exaggerated. Although it has been shown that in the living vessels, so long as their calibre remains constant, the flow is affected by changes in the riscosity of the blood, just as in glass tubes, compensation by adjustment of the vascular calibre is so ample and so easy that even the greatest alterations of viscosity produce little effect on the mean blood-pressure.

Reaction of Blood.-In the sense in which the term is used in physical chemistry, the reaction of a solution depends on the proportion between its content of hydrogen $(\mathrm{H}+)$ and hydroxyl $(\mathrm{OH}-)$ ions, an excess of hydrogen ions corresponding to an acid and an excess of hydroxyl ions to an alkaline reaction. It has been shown by a physical method (the determination of the electromotive force of a cell containing blood or serum as one liquid) that hydroxyl ions are present only in small excess, and that blood is really but a little more alkaline than distilled water. Practically, it may be regarded as a neutral liquid. Under a great variety of conditions, physiological and pathological, its reaction remains almost unchanged. The administration of large quantities of acid or alkali causes a surprisingly small effect. In diabetes, even when it can be shown that an abnormal production of acid substances is taking place, the blood shows little. if any, diminution in the proportion of hydroxyl ions; it remains to all intents and purposes a neutral liquid. In diabetic coma, where the blood may in extreme cases turn blue litmus red, the true reaction is only slightly altered.

The manner in which the reaction of the blood, the tissue liquids, and probably the protoplasm itself, is regulated within such narrow limits is a subject of great interest. Although it cannot be said that all the details of the process have been satisfactorily explained, two factors have been shown to be of importance: (I) The power of the proteins to combine either with 
acids or with bases, so that, when excess of base is adcled to blood, the proteins act as acids, and neutralize the base; when excess of acid is added, the proteins act as hases, and nentralize the acid. (2) The equilibrimm of certain of the inorganic constituents of the blood (carbon dioxide, the carbonates, and the phospliates) is such that even great variations in the concentration of any of these, such as may nomally occur, produce scarcely any effect upon the coucentrations of the liydrogen and liyclroxyl ions.

The so-called 'titratable' alkalinity of blood or sermm, measured by the amomint of standard acid which must be adled before the colour of the inclicator used clianges from alkaline to aci 1 , bears no necessary or fixed proportion to the actual alkalinity. When blood, for instance, is titrated with hydrochloric aciol, with methyl orange as inclicator, at the point where the red colour appears all the disodium plospluate and sodium bicarbonate will lave been changed into monosolimm phosphate and carbon dioxide, all the alkali removed from combination with proteins, a certain amomnt of acirl-protein compounds formed, and other minor reactions produced (Henderson). It is difficult to correlate the quantity deduced from such a titration with any pliysiological condition, although undoubterlly it bears some relation to the acid-nentralizing power of the blool, and some relation to its real reaction.

Wlat is estimated here is the quantity of acirl required to satisfy the proteins and to react with the carbonates and plosphates before that concentration of luydrogen and hydroxyl ions just necessary to cause the change of colour is establisherl. This is not the same for clifferent indicators. since there is a certain mininum ratio in the concentration of these ions at which each indicator turns in one or the other direction, none turning preciscly at the neutral point. Thus serum appears to be acid when tested with plienolphthalein. and alkali must be added to the serum before the pink colour inclicating alkalinity is produced. On the otler hand, with litmus or methyl orange it gives the alkaline reaction, and a considerable amount of acis must be added before the colour of the indicator which denotes acility appears. The true reaction of the serum is not, of course, at one and the same time both alkaline and acid ; but it is so near neutrality that it falls just below the degree of alkalinity necessary to give the pink colour with phenolphthalein. and just below the degree of acidity which gives the pink colour correspondling to an acid reaction witl methyl orange. Certain inclicators-for example, rosolic acid - turn so as to give sharp colour reactions at about the concentration of hyclrogen and hydroxyl ions in the blood, and these may possibly be of use in tetermining the changes in the true reaction for clinical purposes (Aller).

Much more closely related to the true alkalinity of the blood than the titratable alkalinity is the carbon dioxide content. This follows from the facts that much the greatest part of the carbon dioxide is mited with bases, chiefly with sodium, and that the quantity in simple solution is approximately constant. The estimation of the total carbon dioxide in a sample of blood throws light upon the capacity of the blood to perform one of its most important functions-the transportation of carbon 
dioxide and to preserve one of its essential properties an almost neutral reaction in the presence of an excessive intake or production of acid substances. In herbivorous animals the carbon dioxide content of the blood is easily lessened by the administration of acids, but in carnivora and in man it is much more dificult to bring about such a decided effect, the acid being neutralized hy ammonia, which is split off from the proteins. In many discases, however, and particularly in those accompanied by fever, this protective mechanism breaks down, and the alkalinity of the blood, as measured by its content of carbon dioxicke, becomes seriously reduced.

Specific Gravity of Blood. - The average specific gravity of hlood is about I066 at birth. It falls during infancy to about ro $5^{\circ}$ in the third year, then rises till puberty is reached to about ro 58 in males (at the seventeenth year), and ro5.5 in females (at the fourteenth year). It remains at this level during middle life in males, but falls somewhat in females. In chlorotic anæmia of young women it may be as low as I030 or I035. It rises in starvation. Sleep and regular exercise increase it (Lloyd Jones).* The specific gravity of the serum or plasma varies from 1026 to 1032 .

The Electrical Conductivity of Blood.-The liquid portion of the blood conducts the current entirely by means of the electrolytes dissolved in it, the most important of these being the inorganic salts; and the conductivity of the serum varies, in different specimens of blood, within a comparatively narrow range. The conductivity of entire (defibrinated) blood, on the contrary, varies within wide limits. For instance, in a case of pernicious anæmia the conductivity of the blood was found to be almost double that of normal hmman blood, while the conductivity of the serum was normal. The most influential factor which governs this variation is the relative volume of the corpuscles and sermm. When the blood is relatively rich in corpuscles and poor in serum, its conductivity is low; when it is poor in corpuscles and rich in sermm, its conductivity is high. The explanation is that the corpuscle refuses passage to the ions of the dissociated molecules, which, in virtue of their electrical charges, render a liquid like blood a conductor (p. 400), or permits them only to pass very slowly, so that the intact red corpuscles have an electrical conductivity so many times less than that of serum, that they may, in comparison, be looked upon as non-conductors (Practical Exercises, p. 60).

* In I65 students (male) the average specific gravity of the blood, as determined in the writer's laboratory by Hammerschlag's method (p. 5t) was $1054^{\circ} 4$. In 149 of these the variation was from 1050 to 1065 ; in 94 (or 57 per cent. of the whole), from $105+$ to 1060 ; in 4 , from 1046 to 1049 ; in 9 , from 1066 to 1070 . In 3 the specific gravity was only 1040 to $10+2$. 


\section{The Relative Volume of Corpuscles and Plasma in Unclotted} Blood, or, what can be converted into this by a small correction, the relative volume of corpuscles and serum in defibrinated blood, can be easily determined, with approximate accuracy, by comparing the electrical conductivity of entire blood with that of its serum.* Another method, more suitable for clinical work, though not so accurate, is the so-called hematocrite method. A small quantity of blood is centrifugalized in a graduated glass tube of narrow bore until the corpuscles have been collected into a solid 'thread' at the outer extremity of the tube. Their volume and that of the clear plasma which has been separated from them are then read off on the scale. The hxmatocrite must rotate at such a high speed (10,000 turns a minute) that separation of the corpuscles from the plasma is accomplished before clotting has occurred. Dilution of the blood with liquids which prevent clotting is not permissible for exact work (Practical Exercises, p. 59). By these and other methods too elaborate for description here, it has been shown that the plasma or serum usually makes up rather less than two-thirds, and the corpuscles rather more than one-third, of the blood. But this proportion is, of course, liable to the same variations as the number of corpuscles in a cubic millimetre of blood. It depends, further, the number of corpuscles being given, on the average volume of each corpuscle. For instance, when the molecular concentration, and therefore the osmotic pressure (p. 398), of the plasma is reduced, as by the addition of water or the abstraction of salts, water passes into the corpuscles and they swell; when the molecular concentration of the plasma is increased, by the abstraction of water or the addition of salts, water passes out of the corpuscles, and they shrink. In luman serum the average depression of the freezing-point below that of distilled water, which is a measure of the molecular concentration and of the osmotic pressure, is about $0^{\circ} 56^{\circ} \mathrm{C}$. (Practical Exercises, p. 64). For clinical purposes, the determination of the relative volume of corpuscles and plasma is most useful in cases where the average size of the erythrocytes departs from the normal, and where, accordingly, the enumeration of the corpuscles would give an erroneous idea of their total mass.

Laking of Blood, or Hæmolysis.-Even in thin layers blood is opaque, owing to reflection of the light by the red corpuscles.

* The formula $p=\begin{aligned} & \lambda(b) \\ & \lambda(s)\end{aligned}(174-\lambda(b))$, where $p$ is the number of c.c. of serum in 100 c.c. of blood: $\lambda(b), \lambda(s)$, the conductivity respectively of the blood and serum (both measured at or reduced to $5^{\circ} \mathrm{C}$, and expressed in reciprocal ohms multiplied by $\left.10^{8}\right)$, may be used in the calculation. A reciprocal ohm is the conductivity of a mercury column I 063 metres long and $\mathrm{I}$ square millimetre in section. 
It becomes transparent or 'laky' when by any means the pigment is brought out of the corpuscles and goes into true solution. Repeated freezing and thawing of the blood, the addition of water, the passage of electrical currents, constant and induced,* putrefaction, heating the blood to $60^{\circ} \mathrm{C}$., and many chemical agents (as bile-salts, ether, saponin), cause this cliange. Certain complex poisons of animal origin. such as snake-venoms, beepoison, spider - poison or arachmolysin, and certain toxins produced by pathogenic bacteria - for instance, tetanolysin, formed by the tetanus bacillus-also possess decided hamolytic power. The blood-serum of certain animals acts on the coloured corpuscles of others, and sets free their pigment-for example, the serum of the dog or ox causes hemolysis of rabbit's corpuscles; the serum of the ox, goat, dog, or rabbit lakes guinea-pig's corpuscles. But rabbit's serum does not lake dog's corpuscles, and guineapig's serum is inactive towards the corpuscles both of the rabbit and the dog. It has been shown that in hæmolysis by foreign serum two bodies are concerned: one, which is easily destroyed by heating to about $56^{\circ} \mathrm{C}$., the so-called complement, and another, the intermediary body or amboceptor, which is not affected by being heated to this temperature. Thus, if dog's serum be heated to $56^{\circ} \mathrm{C}$. for twenty minutes, no amount of it will lake rabbit's washed corpuscles - that is, rabbit's corpuscles freed from their own serum by repeated washing with salt solution and centrifugalization. If, however, serum which is not itself hæmolytic for rabbit's blood (e.g. , rabbit's or guinea-pig's serum) be added to the washed rabbit's corpuscles, they will be laked by the heated dog's serum. Unheated dog's serum will lake rabbit's corpuscles, whether they have been washed free from their own serum or not (Practical Exercises, p. 63).

The hypothesis which best explains these facts and many similar ones is that dog's serum contains both of the bodies necessary for hæmolysis of rabbit's corpuscles. When the complement has been rendered inactive by heating, the amboceptor cannot cause laking by itself. Rabbit's serum contains complement, but not the specific amboceptor necessary for the laking of rabbit's corpuscles. Accordingly, the addition of fresh rabbit's serum to heated dog's serum restores complement to the latter, and thus it is again rendered active for rabbit's corpuscles. The amboceptor is supposed to unite on the one hand with certain groups in the corpuscle and on the other with the complement, which is thus enabled to develop its hæmolytic action upon the envelope or the stroma. The complement is

* The laking action of induced currents is due simply to the heating of the blood. Condenser discharges, which cause liberation of the hæmoglobin without raising the temperature of the blood as a whole to the point at which heat-laking occurs, possibly act in the same way by causing local heating of the corpuscles owing to their high resistance. 
incapable of acting, even in the presence of ambuceptor, if the temperature is reduced to $0^{\circ} \mathrm{C}$. Nevertlueless the corpuscles take up amboceptor at this temperature, and on this fact is based a method of freeing sermm from amboreptor. For example, if rlog's serum and excess of rabbit's wasled corpuscles, both previously cooled to $0^{\circ} \mathrm{C} .$, be mixed and placed at o ${ }^{\circ} \mathrm{C}$. for some hours, and the sermm then removed, it will be found that it has lost the power of laking rabbit's corpuscles, waslied or unwashed, at air or body temperature, althougl it will still do so on the addition of dog's serum in which the complement has been destroyed by lieating it to $50^{\circ} \mathrm{C}$.

As to the mamer in which hamolytic agents cause the liberation of the bloo(l-pigment, the fact that in so many forms of laking the corpuscles swell wp before the hamoglobin escapes indicates that the entrance of water is an important step. The entrance of water is favoured by changes produced in the clienical and plysical condition of certain constituents of the superficial layer (cnvelope) of the corpuscle, as well as by changes in its interior. Sajonin and ether, for example, are known to be solvents of cholesterin and lecithin, and cholesterin and lecithin are important constituents of the stroma and envelope of the erythrocyte. It is easy to understand that if a portion of one or both of these substances is dissolved, or altered without being actually dissolved, profound changes may be produced in the permeability of the corpuscle to water and to the salts dissolved in the liquid in which the erythrocytes are suspended. In adclition to this change of permeability, many laking agents, perhaps all, exert also a more clirect influence on the normal relations of the native blood-pigment to the stroma. Fither and saponin, for instance, secm to act in two ways-by disorganizing the envelope through solution of its lipoids, and thus increasing its permeability to water; and by helping to dissociate the blookpigment-stroma complex by exerting a pull on the lipoirls of the stroma, while the water simultaneously exerts a pull on the pigment.

The conclusion follows from this view of hamolysis that the erythrocytes, normally so perfectly adapted to the plasma in which they float, may, when the conditions on which their cquilibrium with it depends are altered. be rapidly and inevitably destroyed by that very plasma itself. It is, indeed, the very fact of the exquisite adaptation of liquicl and cell for a strictly regulated cxchange of material which constitutes the langer when the regulation is upset. A liquid like mercury, which is not alapted either to give anything to erythrocytes in contact with it or to take anything from them, would not cause hamolysis, even if the permeability of the corpuscles for water or solimm chloricle were increased to any cxtent. The continued survival of the erythrocytes in an arpuenus solution of salts and proteins like the plasma-nay. more. the protection of the corpuscles up to a certain point by the plasma against the attack of extraneous hemolytic agents-are facts we are prone to take so much for granted as to forget that they depend entirely njon a most delicate adjustment of the permeability of the corpuscles for essential constituents of the plasmal. Disturb these relations to a sufficient (legree, and the plasma becomes a poison to the erythrocytes not much less deadly than distillerl water.

When we add to blood a hrmolytic substance, and sec that 
presently the blood-pigment hats left the corpuscles, we are apt on first infulse to attribute the whole effect to the foreign material adcled. We are apt to say that the saponin, the ether, the alien serum, las laked the blook. In a certan sense this is truc, but it is not the whole truth. In reality the hamolytic agent has acterl in an essential degree, although not exchusively, by overthrowing the equilibrim between the corpuscles and the aqueous solution of certain substances in which they are suspended. To say that the forcign substance alone causes the laxmolysis is no more accurate than it would be to say that a man swimming strongly in a rough sea, who sinks when hit and stunned by a piece of wreckige, was cliowned by the blow, and not by the sea. No loubt it is true that, but for the blow, he would latve continued to swim; yet, in reality. he loses his life because he is environed by a medium deadly to him as soon as his power of adjustument to it has been too much diminished. On land, the blow would have stumned, but would not have killed him. In like manner, to glance at one phase of the natural decay of the corpuscles within the body, an erythrocyte may float socure in its watery environment through many rounds of the circulation. But its security is not static, like that of a $\log$ floating on the water. It is dymamic, a trimph of perfect physico-chemical poise, as the security of the swimmer, still more of the tight-rope clancer, is dynamic, a trimmph of perfect neuromuscular poise. The time, however, arrives when, either through changes in the corpuscle itself (the changes of cellular senility, as we may call them), or through changes in the environing medium, or through a combination of the two, the adjustment is upset, and the erythrocyte is now destroyed by the plasma in which it has so long lived.

In general luxmolysis by foreign serum is preceded by agglutination or aggregation of the corpuscles into groups. Agglutination may be obtained witlout hamolysis by heating the hæmolytic serum to the temperature at which the complement is destroyed, since the agglutinating agents, or agglutinins, are relatively resistant to heat. When the corpuscles of one animal are injected intraperitoneally or subcutaneously into an animal of a different kind, the serum of the latter acquires the property of agglutinating and laking the corpuscles of an animal of the same kind as that whose corpuscles have been injected. This is especially marked if the injection is several times repeated at intervals of a few days. If, for instance, dog's corpuscles are injected into a rabbit, the rabbit's serum after a time becomes strongly liamolytic for dog's corpuscles. It also agglutinates them. This is due to the appearance in the rabbit's serum of an amboceptor and an agglutinin which have a specific action on dog's corpuscles. Many other cells besides the coloured bloodcorpuscles give rise, when injected, to similar specific substances (cytolysins), which cause destruction of cells of the same kinde.g., leucocytes and spermatozoa. The process of hamolysis is more easily followed than the cytolysis of ordinary cells. Yet in its main features it is essentially similar. Hence it has been studied not only for its own interest, but even more for the light 
it throws upon that peculiar and specific response which the body makes to the presence of foreign cells or juices, and which constitutes an attempt to render itself 'immune' to them.

In each case the specific antibody scems to be produced in response to the presence of some particular constituent of the foreign cell. The substances which on injection give rise to antibodics are spoken of as antigens. In the case of the erythrocytes there is evidence that the antigens (both the hæmolysinogen, which causes the production of specific amboceptor, and the agglutininogen, the substance which gives rise to specific agglutinin) are lipoids, or are so closely associated with the lipoids of the corpuscles that they are extracted by the same solvents. Thus ethereal extracts of erythrocytes cause the production of hæmolysin and agglutinin, just as the entire corpuscles clo. Indeed, the response of the animal body to the presence of foreign substances is so catholic, and at the same time so exquisitely specific, that many artificially isolated proteins, even those of vegetable origin, after as careful purification as possible, occasion, when injected, the production of antibodies which will precipitate from a solution only the variety of protein injected.

Precipitins.-When the serum of one animal is injected into another of a different group, the serum of the latter acquires the property of causing a precipitate in the normal serum of animals of the same group as that whose serum was injected, but not in the serum of any other kind of animal. Thus, if human blood or serum is repeatedly injected into a rabbit, the serum of the rabbit will cause a precipitate in diluted human blood or serum, but not in the blood or serum of other animals, except that of monkeys, where a slight reaction may be obtained. The specific bodies which cause the precipitation are termed precipitins. The phenomenon has been made the basis of a method of distinguishing human blood for forensic purposes.

Since changes begin in the blood as soon as it is shed, having for their outceme clotting or coagulation, we have to gather from the composition of the stable factors of clotted blood, or of blood which has been artificially prevented from clotting, some notion of the composition of the unaltered fluid as it circulates within the ressels. The first step, therefore, in the study of the chemistry of blood is the study of coagulation.

Coagulation of the Blood.-When blood is shed, its viscidity soon begins to increase, and after an interval, varying with the kind of blood, the temperature of the air, and other conditions, but in man seldom exceeding ten, or falling below three, minutes, it sets into a firm jelly. This jelly gradually shrinks and squeezes out a straw-coloured liquid, the serum. Lnder the microscope the serum is seen to contain few or no red corpuscles ; these are nearly all in the clot, entangled in the meshes of a kind of network of fine fibrils composed of fibrin. In uncoagulated blood no such fibrils are present; they have accordingly been 
formed by a change in some constituent or constituents of the normal blood. Now, it has been shown that there exists in the plasma-the liquid portion of unclotted blood-a substance from which fibrin can be derived, while no such substance is present in the corpuscles. In various ways coagulation can be prevented or delayed, and the plasma separated from the corpuscles. For example, the blood of the horse clots very slowly, and a low temperature lessens the rapidity of coagulation of every kind of blood. If horse's blood is run into a vessel surrounded by ice and allowed to stand, the corpuscles, being of greater specific gravity than the plasma, gradually sink to the bottom, and the clear straw-yellow plasma can be pipetted off. Or the addition of neutral salts to blood may be used to delay coagulation, the blood being run direct from the animal into, say, a third of its volume of saturated magnesium sulphate solution. The plasma may then be conveniently separated from the corpuscles by means of a centrifugal machine. Again, two ligatures may be placed on a large bloodvessel, so that a portion of it can be excised full of blood and suspended vertically (the so-called experiment of the 'living test-tube'); coagulation is long delayed, and the corpuscles sink to the lower end. In these and many other ways plasma free from corpuscles can be got; and it is found that when the conditions which restrain coagulation are removed-when, for instance, the temperature of the horse's plasma is allowed to rise or the magnesium sulphate plasma is diluted with several times its bulk of water-clotting takes place, with formation of fibrin in all respects similar to that of ordinary blood-clot. The corpuscles themselves cannot form a clot.* From this we conclude that the essential process in coagulation of the blood is the formation of fibrin from some constituent of the plasma, and that the presence of corpuscles in ordinary blood-clot is accidental. In accordance with this conclusion, we find that lymph entirely free from red corpuscles clots spontaneously, with formation of fibrin; and when fibrin is removed from newly-shed blood by whipping it with a bundle of twigs or a piece of wood, it will no longer coagulate, although all the corpuscles are still there.

What, now, is the substance in the plasma which is changed into fibrin when blood coagulates? If plasma, obtained in any of the ways described, be saturated with sodium chloride, a precipitate is thrown down. The filtrate separated from this precipitate does not coagulate on dilution with water; but the precipitate itself-the so-called plasmine of Denis-on being

* Bird's corpuscles, however, washed free from plasma, will form a clot when laked in various ways, as by addition of water or by freezing and thawing. 
dissolved in a little water, does form a clot. Fibrin is therefore derived from something in this precipitate. Now, "plasmine' contains two protein hoclies fibrinogen, which coagulates by heat at about $5^{\circ}{ }^{\circ} \mathrm{C}$. , and serum-globulin, which coagulates at about $75^{\circ} \mathrm{C}$., and it was at one time believerl that both of these entered into the formation of fibrin (Schmidt). Hammersten, however, has shown that fibrinogen alone is a precursor of fibrin; pure serum-globulin neither helps nor hinders its formation. This observer isolated fibrinogen from bloodplasma by adding sodium chloride till about I 3 per cent. Was juesent. With this amount the fibrinogen is precipitated, while serum-globulin is not precipitated till 20 per cent. of salt is reached. After precipitation of the fibrinogen the plasma no longer coagulates; and a solution of pure fibrinogen can be made to clot and to form fibrin, while a solution of sermmglobulin cannot. Blood-serum, too, which contains abundance of serum-globulin, but no fibrinogen, will not coagulate.

So far, then, we have reached the conclusion that fibrin is formed by a change in a substance, fibrinogen, which can be obtained by certain methods from blood-plasma. It may be added that there is evidence that fibrinogen exists as such in the circulating blood ; for if unclotted blood be suddenly heated to about $56^{\circ} \mathrm{C}$, the temperature of heat-coagulation of fibrinogen, the blood for ever loses its power of clotting. The liver seems to be an important place of origin of fibrinogen, which may also be formed in the bone-marrow. Since fibrinogen is readily soluble in dilute saline solutions, and fibrin only soluble with great difficulty, we may say that in coagulation of the blood a substance soluble in the plasma passes into an insoluble form.

How is tlis cliange detemined when blood is slied? We have said that a solution of pure fibrinogen can be made to coagulate, but it does not coagulate of itself. The addition of another substance in minute quantity is necessary. This other substance does not itself seem to be used up in the process, nor to enter bodily into the fibrin formed; a small puantity of it can cause an indefinitely large amount of fibrinogen to clot;* its power is abolished by boiling. For these reasons it is considered to be a ferment (p). 3It), and is spoken of as fibrinferment or thrombin.

Two forms of fibrin-ferment have been distinguished by some writers : $\boldsymbol{a}$-thrombin, whicl exists in serum hefore anything has been added to it, and $\beta$-thrombin, or Schmidt's fibrin-ferment, in

* According to Rettger's recent work this is erroneous. He state's that the quantity of fibrin formed is proportional to the amount of thrombin present, and that thrombin does not act like a ferment. The incuiry is complicated by the fact that fibrin, once formed, tends to adsorb the remaining thrombin and so to interfere with its further action. 
a solution which can be obtained by precipitating blood-serum, or defibrinated blood, with fifteen to twenty times its bulk of alcohol, letting the whole stand for a month or more, and then extracting the precipitate with water. All the ordinary proteins of the blood having been rendered insoluble by the alcohol, the fibrin-ferment passes into solution in the water, and the addition of a trace of the extract to a solution of fibrinogen causes coagulation. The supposed distinction between this form of thrombin and the other is not so well estalblished that we need take account of it here.

The action of fibrin-ferment on fibrinogen helps to explain many experiments in coagulation. Thus, transudations like hydrocele fluid do not clot spontaneously, although they contain fibrinogen, which can be precipitated from them by a stream of carbon dioxide or by sodium chloride. But the addition of a little fibrin-ferment causes hydrocele fluid to coagulate. So does the addition of serum, not because of the serum-globulin which it contains, as was once believed, but because of the fibrinferment in it. The addition of blood-clot, either before or after the corpuscles have been washed away, or of serum-globulin obtained from serum, also causes coagulation of hydrocele fluid, and for a similar reason, the fibrin-ferment having a tendency to cling to everything derived from a liquid containing it. On the other hand, serum, which, although fibrin-ferment is present in it, does not of itself clot, because the fibrinogen has all been changed into fibrin during coagulation of the blood, can be made to coagulate by the addition of hydrocele fluid, which contains fibrinogen. We have thus arrived a step farther in our attempt to explain the coagulation of the blood: it is essentially due to the formation of fibrin from the fibrinogen of the plasma under the infuence of fibrin-ferment.

The Formation of Fibrin-ferment from its Precursors.There is good reason to believe that thrombin is formed by the interaction of three factors: (I) A substance which, since it is a precursor of thrombin, is called thrombogen, or prothrombin. It is already present in the circulating plasma. (2) A substance liberated from the formed elements of the shed blood, but which can be obtained also from the cells of all tissues. Since it has been supposed to act upon thrombogen, changing it into fully-formed thrombin, much in the same way as enterokinase (p. 33I) acts upon trypsinogen, changing it into fullyformed trypsin, it is called thrombokinase (Morawitz).* (3) Cal-

* Others believe, however, that the substances in tissue extracts which favour coagulation do so not by activating prothrombin, but by a direct action upon fibrinogen similar to, but not necessarily identical with, that exerted by thrombin, and speak of them as coagulins (L. Loeb). It is possible that the tissues yield both activating substances (kinases) and coagulins. 
cium ions. The following experiments illustrate the rôle of these three factors:

The plasma obtained by drawing off bird's blood-c.g., the blood of a fowl or goose - through a perfectly clean cannula into a perfectly clean vessel, without contact with the tissues, and then rapidly centrifugalizing off the formed elements, can be kept unclotted for days and even weeks. The addition of a small amount of tissue extract (procured by rubbing up bloodfree liver, thymus, muscle, or other organs with sand, and extracting for several hours with salt solution) to the bird's plasma causes rapid coagulation. The plasma contains thrombogen and calcium salts, but is lacking in thrombokinase, which is supplied by the tissue extract. A solution of fibrinogen containing calcium will clot if serum, in which fibrin-ferment is always present, be added. It will not clot on addition of tissue extract alone, nor on addition of bird's plasma alone (obtained as above), but will readily coagulate if both tissue extract and bird's plasma be added. Therefore, something in the bird's plasma (thrombogen), plus something in the tissue extract (thrombokinase), produce in the presence of calcium the same effect as the thrombin of serum. It can be shown that calciun is only necessary for the formation of the fibrin-ferment, but not for its action on fibrinogen. For instance, a calcium-free solution of fibrinogen can be made to clot by serum from which the calcium has been removed.

If a soluble oxalate (potassium or ammonium oxalate) is mixed with freshly-drawn dog's blood to the amount of 0.2 or o.3 per cent., the blood remains unclotted. The plasma separated from this oxalated blood contains both thrombogen and thrombokinase, but it does not coagulate, because the calcium has been precipitated out in the form of insoluble calcium oxalate. In the absence of calcium the reaction of the thrombogen and thrombokinase which leads to the formation of thrombin does not take place. All that is necessary to bring about coagulation is to add calcium chloride in somewhat greater quantity than is required to combine with any excess of oxalate present. If more than a certain amount of calcium be added clotting is hindered instead of being helped, so that it is only within certain limits of concentration that calcium favours coagulation. From oxalate plasma a nucleo-protein or a mixture of nucleo-proteins can be separated which contains thrombogen and thrombokinase, but little or no calcium, and does not cause clotting, but which on treatment with a calcium salt acquires the properties of fibrinferment. In the curious hereditary disease known as hamophilia, a deficiency of calcium seems occasionally to be responsible for the diminished coagulability of the blood; and the internal 
administration of a solution of calcimm chloride has sometimes been thought to lessen the tendency to hemorrhage, or its local application to cut short an actual attack. It is possible that in some cases there may be a want of thrombokinase in the blood or in the cut tissues, and that the application of normal tissue extract (extract of thymus, for example) or of a solution containing fibrin-ferment may be of benefit. The injection of normal sermm into the circulation, or the transfusion of normal blood, have also been used with temporary advantage.

When sodium fluoride is added to freshly-drawn blood to the amount of $\mathrm{O}_{3}^{3}$ per cent., coagulation is also prevented. But there is this difference between oxalate and fluoride plasmathat, although the calcium has been precipitated in both, the addition of calcium chloride to fluoride plasma is not sufficient to induce clotting. Tissue extract containing thrombokinase must be supplied as well. In some way or other sodium fluoride interferes with the liberation of thrombokinase from the formed elements of the blood, although in the concentration mentioned it does not hinder the action of fully-formed thrombin, as is shown by the fact that fluoride plasma coagulates on the addition of a little serum, which supplies fibrin-ferment. The fluoride blood clots readily if it is diluted with water, and at the same time mixed with calcium chloride solution, for the water damages the formed elements, and thus favours the liberation of thrombol:inase.

When proteoses (or peptones) are injected into the circulation of a dog or goose, the blood is deprived of the power of coagulation. The peptone plasma must be assumed to contain both thrombogen and thrombokinase, since it can be made to clot in various ways (e.g., by dilution with water or by slight acidulation with acetic acid) without the addition of anything which could supply either of these factors. Yet a little tissue extract causes it to clot much more rapidly than simple dilution or acidulation, and more rapidly than the addition of serum. So that either the thrombokinase already present in peptone plasma is present in an unavailable form, or in some way the formation of thrombin from its precursors is hindered. But this is not the only cause of the incoagulability of peptone plasma. It may be shown to contain an antithrombin, a body which antagonizes the action of fully-formed thrombin, and which does not seem to be a ferment, since it acts quantitatively in proportion to the amount present. This is the reason why, although peptone plasma can always be made to clot by the addition of fibrinferment, in serum, for instance, relatively large quantities of it must be supplied (Practical Exercises, pp. 56, 57).

An extract of the head of the medicinal leech in salt solution 
prevents the clotting of blood both in the test-tube and when injected into the circulation. The plasma ohtained differs from peptone plasma in refusing to coagulate unless tissue extract is added. It is therefore deficient in thrombokinase, or, rather, as has been shown, the kinase present is mable to act, because neutralized by antikinase present in the leech-extract. Leechextract also contains an antitlumbin, which can be nentralized by a sufficient amount of thrombin. In the small wound from which the leech sucks blood this sufficient amount is not present, and the blood remains unclotted, as it also does in the alimentary canal of the leech. The anticoagulant substance, hirudin, has been isolated, and gives the reactions of an albumose.

Sources of Thrombogen and Thrombokinase.-It has already been stated that thrombogen exists in the circulating plasma. This is showm by the fact that fluoride plasma obtained from blood drawn directly throngh a wide cannula into sodium fluoride solution, with all precautions to prevent alteration of the blood, and immediately separated from the formed elements by the centrifuge, will clot on the addition of tissue extract. The source of the thrombogen has been thought to be the blood-plates, but this has not been proved. Thrombokinase is not present in the circulating plasma. In shed and clotting blood wlich has not been allowed to come into contact with cut tissues the only possible sources of thrombokinase, so far as we know, are the corpuscles and the blood-plates. The red corpuscles we may at once dismiss, for although the stromata, especially of nucleated corpuscles, contain thrombokinase, or can under artificial conditions be made to develop that action on coagulation by which we recognize its presence, not only do they remain intact under ordinary circumstances during coagulation, but there is strong evidence, as has already been pointed out, that they do not make any essential contribution to the process. We liave left over the lencocytes and the platelets, and it is highly probable that from both thrombokinase is liberated in the first moments after blood is drawn, and, acting on the thrombogen already present in the plasma, changes it into actual fibrin-fement. This surmise is strengthened by the fact that in freshly-shed mammalian blood extensive destruction of blood-plates takes place. It has also been shown that the blood of the crayfish, which coagulates with extreme rapidity, contains certain colourless corpuscles which, immediately it is withdrawn, break up with explosive suddenness, and that substances which hinder the breaking " In the blood of another crustacean Limulus, the kingcrab, coágulation is preceded by an agglutination of the leucocytes 
which exhibit amolooid movements. They become entangled by the interlacing of the psendopodia which they protrucle (i. Loeb). In the blood of some mammals (rabbit) many lencocytes, especially the polymorphomuclear lencocytes, disitppear, but in man, the pig, and the ox this is not the case. It must be remembered, however, that the lencocytes in shed blood, without actually being destroyed, may, under the influence of what is for them a foreign environment, undergo changes which permit the escape of thrombokinase and other substances. Further, the white layer or 'buffy coat' which tops the tardily-formed clot of horse's blood, and consists of the lighter, and therefore more slowly sinking, platelets and white corpuscles, causes clotting in otherwise incoagulable liquids like hydrocele fluid much more readily than the red portion of the clot, and vields far more of Schmidt's fibrin-ferment on treatment with alcohol.

In blood collected in paraffined vessels, so as to delay clotting, and immediately centrifugalized, coagulation is seen to begin in and around the layer of white elements, and then to spread upwards in the stratum of plasma and downwards in the stratum of erythrocytes.

Thrombokinase has not only been shown to exist in the leicocytes, the platelets, and the stromata of the coloured corpuscles, but, as already stated, in all tissues hitherto examined. Under ordinary circumstances it appears that a larger amount of thrombogen is liberated or is already present in shed blood than can be changed into thrombin by the thrombokinase set free, since serum contains a surplus of thrombogen in addition to the fully-formed ferment. This is shown by the fact that the activity of a given quantity of serum in causing the coagulation of a plasma not spontaneously coagulable or of a fibrinogen solution is increased by the addition of tissue extract (containing thrombokinase).

The thrombin of any particular kind of vertebrate blood has no marked specific action-that is, will cause coagulation in solutions of fibrinogen or plasma of very different origin. For example, the sera of all vertebrates hitherto investigated induce clotting in goose's plasma. On the other liand, it appears that a greater degree of specificity exists in the case of the thrombokinase and thrombogen, the specificity being absolute in some cases, relative in others. That is to say, the thrombokinase of one animal may activate the thrombogen of an animal of another group, while it may fail to activate the thrombogen of an animal belonging to a third group. But it will always activate the thrombogen of an animal of the same kind.

To sum up, we may suy that when blood is shed fibrin-ferment. 
(thrombin) is rapidly formed by the action of thrombokinase, liberated from the lencocytes, the blood-plates, and possibly to some extent from the erythrocytes, upon thrombogen, already present in the circulating plasma. Further and this is of great practical importance-since no vessel is opened under ordinary circumstances except through a wound in the overlying structures, the cut tissues supply a store of thrombokinase at the point where it is required to aid in the stanching of the wound. Calcium is essential to the reaction by which thrombogen and thrombokinase form fibrin-ferment, lint is not necessary for that action of fibrin-ferment on fibrinogen by which fibrin is produced (Practical Exercises, pp. 55-57).

Both thrombogen and thrombokinase have close relations with a substance or substances belonging to the group of nucleoproteins. If they are not actually nucleo-proteins, they cling to them with such tenacity that it is not easy by ordinary means to separate them. Thus nucleo-protein can be obtained from solutions of fibrin-ferment, and, by appropriate treatment and in the presence of proper conditions, solutions of nucleo-protein can be made to influence coagulation in the way characteristic of fibrin-ferment. Nucleo-proteins are contained in the nuclei and protoplasm of cells, and have been prepared from the thymus, testis, kidney, lymphatic glands, and other organs, by precipitating their watery extracts with dilute acetic acid (Wooldridge), or by extracting with sodium chloride and then precipitating with excess of water (Halliburton). The precipitated nucleo-protein can be dissolved in dilute sodium carbonate solution. When it is injected slowly or in small amount into the veins of an animal, it abolishes for a time the power of coagulation of the blood; and when this 'negative phase,' as it is called, has been once established, even a very large and rapid injection produces no further effect, possibly because an antibody which neutralizes the action of thrombokinase has been produced. If, however, a considerable quantity of the solution has been injected at the first, the result is very different: extensive intravascular clotting instantly ensues; the animal dies in a few minutes; and the right side of the heart, the vence cave, the portal vein, and perhaps the pulmonary arteries, may be found choked with thrombi. Here the injected thrombokinase is responsible for the clotting, thrombogen and calcium being already present. Curiously enough, intravascular coagulation fails to be produced in a certain proportion of cases when albino animals are injected with nucleo-protein from pigmented animals, while there is no absolute failure of coagulation when albinos are injected with nucleo-protein from albinos, and no failure when pigmented animals are injected with material either from other pigmented animals or from alhinos. Intravascular 
coagulation is especially striking in birds on injection of tissue extracts.

To a certain extent the action of nucleo-protein in coagulation can be imitated by other substances of animal origin, such as the venoms of some vipers (Martin), and even by certain artificial products of the laboratory, the synthesized colloids of Grimaux, which, when injected into the blood, produce the same phenomena of intravascular coagulation down to the finest detail and including the negative phase. It is not known whether these substances act on the blood-plates, lencocytes, or other cells, and thus cause an increased production or an increased liberation of one or more of the precursors of fibrin-ferment, or whether they take part directly in its formation. But there is some evidence that the venoms which favour coagulation do so in virtue of their containing a kinase. On the other hand, cobravenom prevents coagulation by means of an antikinase-that is, a substance which antagonizes the action of kinase, and so hinders the formation of fibrin-ferment. It does not contain an antithrombin-that is, a body which will prevent the action of fibrin-ferment already formed (Mellanby).

So far we have been considering the problen of coagulation as if all the data for its solution could be obtained by a study of the blood itself. In other words, our main business up to this point has been the explanation of coagulation in the shed blood; it has been only incidentally, and with the object of casting light on the question of extravascular clotting, that we have touched on the coagulation of the blood within the living vessels. It is not possible here to adequately discuss, nor even to define, the differences between the two problems. All we can do is to warn the student, and to emphasize our warning by one or two illustrations, that valuable as is the knowledge derived from experiments on extravascular coagulation, it would be totally misleading if applied without modification to the circulating blood. For instance, we have recognized in the leucocytes and bloodplates an important source of the thrombokinase which plays so great a part in the clotting of shed blood; but we may be sure that leucocytes and blood-plates are constantly breaking down in the lymph and the blood, and we have to inquire how it is that coagulation does not occur, except in disease, within the vessels. Calcium is not wanting to the circulating plasma, fibrinogen is not wanting, and it has already been mentioned that thrombogen exists in perfectly fresh and, as we may say, still living blood. Why, then, does it not coagulate? Some have said that coagulation is 'restrained' by the contact of the living walls of the bloodvessels; but although it is certain that the contact of foreign matter-and all dead matter is foreign to 
living cells-does hasten the destruction of leucocytes and bloodplates or that alteration in thrm on which the liberation of the precursors of the ferment depends, it is evident that it is just this 'restraining' influence of the vessels, if it is due to anything more than the mere smoothness of their endothelial lining, which has to be explained. The best answer which can be given to the question is: First, that the quantity of thrombokinase free in the plasma at any given time must be small, since no evidence of its presence in fluoride plasma can be obtained. If thrombokinase is liberated in the circulating blood, we may assume that it is clanged into some inactive substance or quickly eliminated. And it appears that, unlike the true ferments, thrombokinase acts quantitatively-i.e., in proportion to its amount-upon thrombogen. Second, an antithrombin exists in the circulating plasma, and even if fully formed fibrin-ferment were present, it could not cause coagulation until the antithrombin had been neutralized. This antithrombin is probably not manufacturerl in the blood, or at least not exclusively in the blood, but in the tissues, and there is no reason to deny the ressels themselves a share in its production, even if its presence has not hitherto been demonstrated in the internal coat (L. J,oeb). So that living blood within the living vessels may be said to be acted upon by two sets of influences, one tending to favour coagulation, the other to oppose it. Under normal conditions, the processes that make for coagulation never obtain the upper hand. But anything which interrupts the circulation, and consequently the free interchange between blood and tissues, interferes with the elimination or neutralization of the precursors of fibrin-ferment, and with the entrance of the substances that render the fully-formed ferment inactive. In the clotting of extravascular plasma, free from corpuscles, we may indeed see the continuation, under modified conditions, of a normal process always going on within the bloodvessels. In the lungs it would seem that the forces which favour coagulation are feeble, or the forces that resist it strong, for blood, after passing many times through the pulmonary circulation without being allowed to enter the systemic vessels, loses its power of clotting.

The liver is another organ whose relations to the coagulation of the blood are peculiar. We have already mentioned that the injection of commercial peptone, which consists chiefly of proteoses, into the blood of dogs causes it to lose its coagulability. The effect gradually passes away, till after some hours the original power of coagulation is restored (p. 55). The liver is known to be intimately concerned in the production of this remarkable result, for if the circulation through it be interrupted, the injection of proteose is ineffective. Further, if a solution of proteose 
is artificially circulated through an excised liver, a substance (perhaps an antithrombin) is formed which is capable of suspending the coagulation of blood outsicle of the horly, a property which proteoses themselves do not possess, or possess only in slight degree. It is not believed that the proteose is actually changed into this anticoagulant substance, but rather that the liver cells produce it as a reaction' to the presence of the foreign substance, being perhaps stimulated in some way by the circulating proteose. In part the abnormally great alkalinity of the peptone blood, due to the excess of alkali secreted by the liver, is responsible for its slow coagulation. Under certain conditions, some of which are known and others not, the injection even of one or other of the purified proteoses causes not retardation, but hastening, of coagulation; and if this has been the result of a first injection, a second is equally unsuccessful. It is possible that by an effort of the organism to restore the normal coagulability of the blood, on which its very existence depends, substances which favour coagulation are produced, and that the result of an injection of proteose is determined by the relative amount of coagulant and anticoagulant secreted in a given time. Protamins (products obtained from the ripe milt of certain fishes, and believed to be the simplest proteins) exert, when injected intravenously, a retarding influence on coagulation, and lower the blood-pressure, just as albumoses do (Thompson). Even serum - albumin and serum - globulin possess this property in some degree. All these substances also cause a diminution in the number of leucocytes in the blood owing, in the case of albumose at any rate, to their accumulation in the abdominal vessels, and not to any actual destruction of them.

\section{The Chemical Composition of Blood.}

The serum of coagulated blood represents the plasma minus fibrinogen; the clot represents the corpuscles plus fibrin. Thus:

$$
\begin{aligned}
& \text { Plasma }- \text { Fibrin (ogen) = Serum. } \\
& \text { Corpuscles }+ \text { Fibrin=Clot } \\
& \text { Plasma }+ \text { Corpuscles }=\text { Serum }+ \text { Clot }=\text { Blood. }
\end{aligned}
$$

Bulky as the clot is, the quantity of fibrin is trifling $(0.2$ to 0.4 per cent. in human blood). The plasma contains about ro per cent. of solids, the red corpuscles about 40 per cent., the entire blood about 20 per cent.

Serum contains 8 to 9 per cent. of proteins, about o. 8 per cent. of inorganic salts, and small quantities of neutral fats, soaps, cholesterin esters, lecithin, urea, kreatin, dextrose, lactic acid, and other substances. The chief proteins are serum-albumin and sermm-globulin. In the rabbit the former, in the horse the latter, 
is the more abundant; in man they exist in not far from equal amount. A small quantity of mucleo-prolein (which is eitler tle fibrin-ferment or is closely united with it) and of fibrino-globulin (which some consider a soluble product formed from fibrinogen in clotting) is also present. Ferments which cause hyclrolysis of proteins and carbohydrates, possibly a ferment (lipase) which acts upon fats, and certain oxidizing ferments (oxvdases), liare also been demonstrated. The chemical nature of the hories

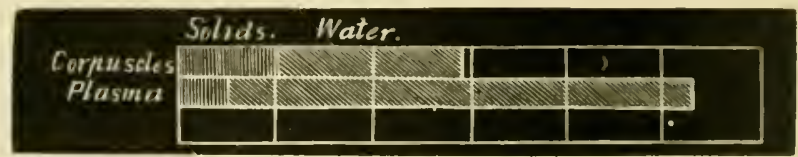

Fig. 5.-Diagrair showing Relative QtaANtity of Solids and Water IN Red CORPLSCles AND PLASMA.

which confer on serum or plasma its specific hamolytic, agglutinating, precipitating, and bactericidal properties has not been definitely determined.

The quantitative composition of serum, especially as regards the inorganic salts, is remarkably constant in animals of the same species, and even in animals of different species belonging to the same. or to not very widely-separated, natural groups. In colel. blooded animals the serum-albumin is scantier than in mammals. the globulin relatively more plentiful.

Serum-albumin belongs to the class of native albumins. It has been obtained in a crystalline form from the serum of horse's blood. It is soluble in listilled water, and is not precipitated by saturating its solutions with certain neutral salts. Heatel in neutral or slightly acid solution, it coagulates first at $73^{\circ}$, then at $77^{\circ}$, then at $84^{\circ} \mathrm{C}$. Although this is not of itself sufficient proof. there is other evidence that it consists of a mixture of proteins.

Serum-globulin belongs to the globulin group of proteins. When heated, it coagulates at about $75^{\circ} \mathrm{C}$. (p. 8). It is insoluble in listilled water. and is precipitated by saturation with such neutral salts as magnesium sulphate or by half-saturation with ammonium sulphate. It has been shown that. as thus obtained, it is not a single substance, but a mixture of at least two proteins-eu-globulin. which can be precipitated from its saline solution by dialyzing off the salts, and pseudo-globulin. which cannot be so precipitated.

Of the inorganic salts of ssrum. the most important are sodium chloride and sodium carbonate. Small amounts of potassium. calcium, and magnesium, united with phosphoric acid or chlorine. and a trace of a fluoride. are also present. A portion of the silts is loosely combined with the proteins.

The Red Corpuscles consist of rather less than bo per cent. of water and rather more than 40 per cent. of solids. (of the solids the pigment hamoglobin makes up about go per cent. the proteins and nucleo-protein of the stroma about 7 per cent. ; lecithin and cloolesterin 2 to 3 per cent. ; inorganic salts (which vary greatly in their relative proportions in different 
animals, but in man consist chiefly of phosphates and chloride of potassium, with a much smaller amount of sodium chloride) about I per cent. There is evidence that a portion of the salts is more firmly combined than the rest, so that, even after the action of the most energetic laking agents, this fraction remains attached to the stroma.

Hemoglobin.-Of all the solid constituents of the blook, hamoglobin is present in greatest amount, constituting, as it does, no less than 13 per cent., by weight, of that licpuicl. It is an cxcecelingly complex boly, containing carbon, hydrogen, nitrogen, and oxygen in much the same proportions in which they exist in ordinary proteins (p. 1). Iron is also present to tine extent of almost exactly one-thircl of I per cent., and there is also a little sulphur, the amount of which stands in a very simple relation to the quantity of iron ( 1 atom of iron to 3 of sulphur in (log's hæmoglobin, and $x$ atom of iron to 2 of sulphur in the hemoglobin of the horse, ox, and pig). Hæmoglobin is marle up of a protein element which contains all the sulphur and a pigment which contains all the iron, the protein constituting by far the larger portion of the gigantic molecule, whose weight has

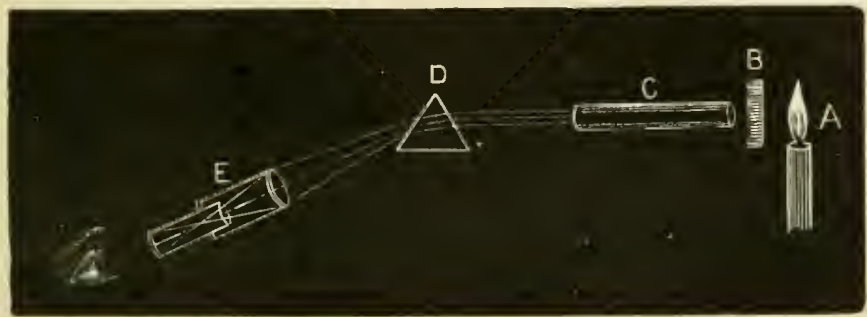

Fig. 6.-Diagrail of Spectroscope.

A, source of light ; B, laver of blood ; C, collimator for rendering rays parallel ; D, prism ; E, telescope.

been estimated at more than 16,000 times that of a molecule of hydrogen. Since its percentage composition is still undetermined with absolute precision, it is impossible to give an empirical formula that is more than approximately correct. For dog's hæmoglobin Jaquet gives $\mathrm{C}_{75 \times} \mathrm{H}_{1203} \mathrm{~N}_{195} \mathrm{~S}_{3} \mathrm{FeO} \mathrm{O}_{21 \times}$, which would make the molecular weight 16.669 .

The most remarkable property of hæmoglobin is its power of combining loosely with oxygen when exposed to an atmosplere containing it, and of again giving it up in the presence of oxidizable substances or in an atmosphere in which the partial pressure of oxygen (pp. 248,254 ), has been reduced below a certain limit. It is this property that enables liæmoglobin to perform the part of an oxygen-carrier to the tissues, a function of the first importance, which will be more minutely considered when we come to deal with respiration.

The bright red colour of blood drawn from an artery or of venous blood after free exposure to air is due to the fact that the 
hamoglobin is in the oxidized state-in the state of oxyluemoglobin, as it is called. If the oxygen is removed by means of reducing agents, such as anmonium sulphide, or by exposure to the racuum of an air-pump, the colour darkens, the bloodpigment being now in the form of reduced hemoglobin. In ordinary venous blood a large proportion of the pigment is in this condition, but there is always oxylatmoglobin present as well. In asphyxia (p. 23I), however, nearly the whole of the oxylizmoglobin may disappear.

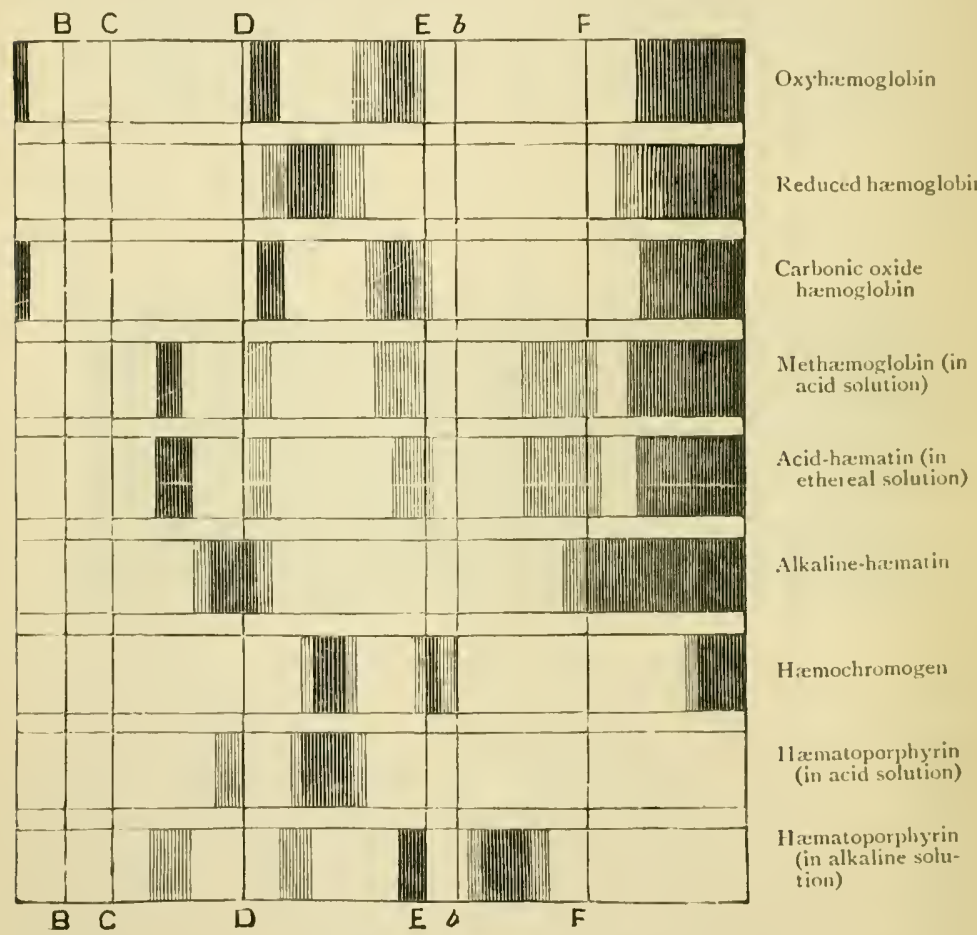

Fig. 7.-Table of Spectra of Henoglobin and its Derivatives.

Crystallization of Hamoglobin. - In the circulating blood the hæmoglobin is related in such a way to the stroma of the corpuscles that, although the latter are suspencled in a liquicl readily capable of dissolving the pigment, it yet remains under ordinary circumstances strictly within them. In a few invertebrates, however, it is normally in solution in the circulating liquid. As a rare occurrence hamoglobin may form crystals inside the corpuscles (p. 63). When it is in any way brought into solution outside the body, it shows in many animals, but not in the same degree in all, a tendency to crystallization; and the ease with which crystallization can be incluced is in inverse proportion to the solubility of the hamoglobin. Thus, it is 
far more difficult to obtain crystals of oxyhamoglobin from human blood than from the blood of the rat, guinea-pig, or dog. whose bloodpigment is less soluble than that of man, and for a like reason the oxyhamoglobin of the bird, the rabbit, or the frog crystallizes still less readily than that of human blood.

As to the form of the crystals, in the vast majority of animals they are rhombic prisms or needles, but in the guinea-pig they are tetrahcdra belonging to the rhombic system, and in the squirrel six-sided plates of the hexagonal system (Fig. 8).

Reduced hamoglobin can also be cansed to crystallize, though with more difficulty than oxyluemoglobin, since it is more soluble. Crystals of re. duced liamoglobin were first prepared from liuman blood by Hüfner, who allowed it to putrefy in sealed tubes for several weeks.

When a solution of oxyhæmoglobin of moderate strength is examined with the spectroscope, two wellmarked absorption bands are seen, one a little to the right of Fraunhofer's line $\mathrm{D}$, and the other a little to the left of E. A third band exists in the extreme violet between $G$ and $H$. It cannot be detected with an ordinary spectroscope, but has been studied by the aid of a fluorescent eyepiece, by projecting the spectrum on a fluorescent screen, and by photographing the spectrum. The addition of a reducing agent, such as ammonium sul-

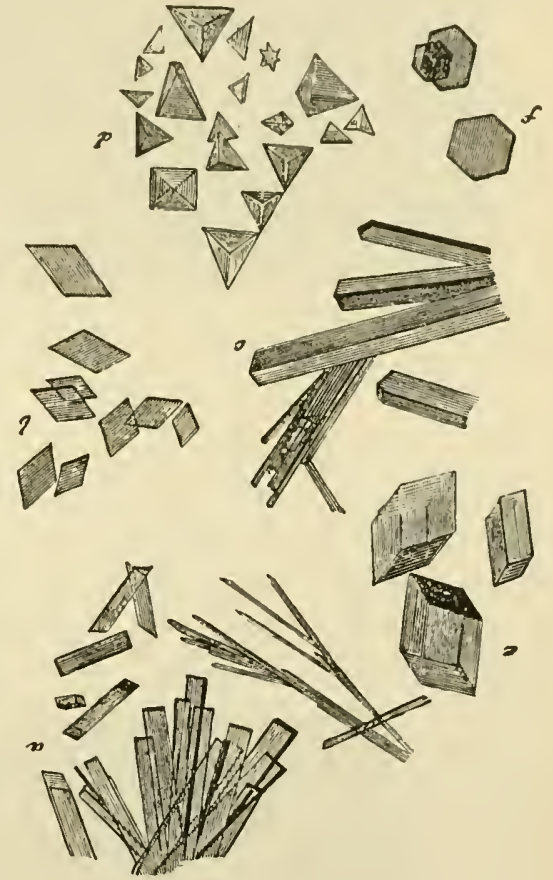

Fig. 8.-Oxyhemoglobin CRystals. $a, b$, from man $: c$, from cat; $d$, from guinea pig $c$, from hamster; $f$, trom squirrel (Frey). phide, causes the bands in the visible spectrum to disappear, and they are replaced by a less sharply-defined band, of which the centre is about equidistant from $\mathrm{D}$ and $\mathrm{E}$. This is the characteristic band of reduced hæmoglobin. The spectrum of ordinary venous blood shows the bands of oxyhæmoglobin.

Carbonic oxide hamoglobon is a representative of a class of hæmoglobin compounds analogous to oxyhæmoglobin, in which the looselycombined oxygen has been replaced by other gases (carbon monoxide, nitric oxide) in firmer union. Its spectrum shows two bands very like those of oxyhæmoglobin, but a little nearer the violet cnd. Carbonic oxide hæmoglobin is formed in poisoning with coal-gas. 
Owing to the great stability of the conpound, the hemoglobin can no longer be oxidized in the lungs. and cleath may take place from isphyxia. It is, however, gralually broken up, and therefore artificial respiration way be of use in such cases. Inhalation of oxygen and especially transfusion of blood are also of great value.

Methrmoglobin is a derivative of oxyliamoglobin which can be formed from it in various ways, e.g., by the adclition of forricyanile

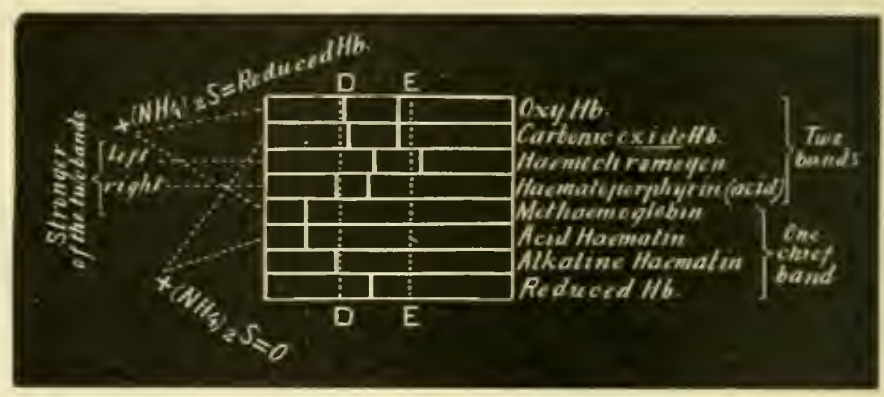

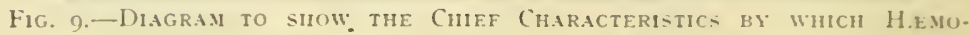
GLOBIX AXD SOME OF ITS DERINATIVES MAY BF RECORNIZED SPECTRONCOII.

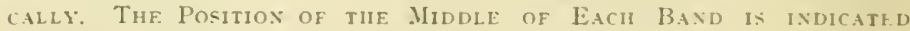
ROTGHLY BY A V'FRTICAL LINE.

of potassium or nitrite of anyl (Gamgec). by electrolysis (in the neighbourhood of the anode), or by the action of the oxidizing ferment 'echidnase' in the poison of the viper (Phisalix). It very' often appears in an oxyhremoglobin solution which is cxposed to the air. It has been found in the urine in cases of hemoglobinuria. in the fluid of ovarian cysts, and in hematoceles. The strongest band in its spectrum is in the red, between $C$ and $D$. but nearer C. nearly in the same position as the band of acid-hæmatin. Reducing

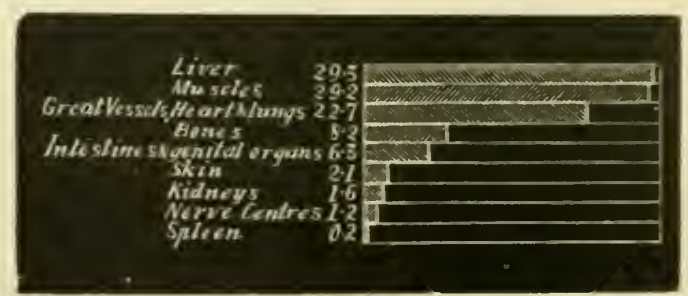

Fig. io, -Dlagram to illestrate the Distribetion of the Blool) IN the VARIOL'S OrgaNs of a RabBit (AFTER RaNkE'S MEAstremeNis).

The numbers are percentages of the total blood.

agents. such as ammonium sulphide, change methamoglobin first into oxyhamoglobin and then into reduced hamoglobin. It has by some been regarded as a more highly oxidized hemoglobin than oxyhamoglobin. Rebutting evidence has, however, been offered to the effect that the same quantity of oxygen is required to saturate both pigments. and this evidence appears to be sound. The difference lies rather in the manner in which the oxygen is united to the 
hamoglobin in the methamoglobin molecule than in the quantity of oxygen which it contains. For methemoglobin, unlike oxyhemoglobin, parts with no oxygen to the vacum, while, on the other hand, in the presence of reducing agents it yiclds up its oxygen even more readily than oxyliamoglobin rloes (Haldane) (p. 25I).

By the action of acils or alkalies oxylzemoglobin is split into a pigment, laxmatin and protein borlies, of which much the most important is globin, a substance belonging to the histon group. It is casily precipitated from solution by ammonia. As to the pigment moicty, when hamoglobin is acted on by acids in the absence of oxygen. hamochromogen is first formed, which then gralually loses its iron and is changed into hematoporphyrin. If oxygen be present, hamatin is the final product. By the action of alkalies reduced hamoglobin yiekls hamochromogen, which is stable in alkaline solution, and gives a beautiful spectrum with two bands, bearing some resemblance to those of oxyhemoglobin, but placed nearer the violet encl. The band next the red end is much sharper than the other (p. 68).

Hematin, the most frequent result of the splitting up of hæmoglobin, is generally obtained as an amorphous substance with a bluish-black colour and a metallic lustre, insoluble in water, but soluble in dilute alkalies and acids, or in alcohol containing them. In addition to the iron of the hæmoglobin, hæmatin contains the four chicf elements of proteins-carbon, hydrogen, nitrogen, and oxygen (Practical Exercises, pp. 67, 68).

Hematoporphyrin, or iron-free hæematin, may be obtained from blood or hæmoglobin by the action of strong sulphuric acid. Its spectrum in acid solution shows two bands, one just to the left of $D$, the other about midway between D and E. Like oxyhæmoglobin, reduced hæmoglobin, carbonic oxide hæmoglobin, methæmoglobin and other derivatives of hæmoglobin, it also has a band in the ultra-violet.

Hemin is a compound of hæmatin and hydrochloric acid, which crystallizes in the form of small rhombic plates, of a brownish or brownish-black colour (Fig. I6, p. 67). They are insoluble in water, but readily soluble in dilute alkalies (Practical Exercises, p. 7I).

Chemistry of the White Blood-corpuscles.-The composition of pus-cells and the leucocytes of lymphatic glands has alone been investigated. The chief constituents of the latter are a globulin coagulating by heat at $48^{\circ}$ to $50^{\circ} \mathrm{C}$. ; a nucleo-protein coagulating in 5 per cent. magnesium sulphate solution at $75^{\circ} \mathrm{C}$, and causing coagulation of the blood on injection into the veins of rabbits; an albumin coagulating at $73^{\circ} \mathrm{C}$.; and a ferment with powers like the pepsin of the gastric juice. In pus-cells glycogen has been found, and it can be demonstrated microchemically in the leucocytes of blood by the iodine reaction in various conditions. Fats, cholesterin, and lecithin are also present, as well as the so-called protagon. The ordinary inorganic constituents have been demonstrated-namely. potassium, sodium, calcium, magnesium, and iron, united with chlorine and phosphoric acid. The total solids amount to II to I 2 per cent.

The Quantity of Blood.-The quantity of blood in an animal is most accurately determined by the method of Welcker. The animal is bled from the carotid into a weighed flask. When blood has ceased to flow the vessels are washed out with water or physiological saline solution, and the last traces of blood are removed by chopping up the body, after the intestinal contents have been 
cleared away, and extracting it with water. The extract and washings are mixed and weighed ; a given quantitv of the mixture is placed in a hematinometer (a glass trough with parallel sides, e.g.), and a weighed quantity of the unmised blood cliluted in a similar vessel till the tint is the same in both. From the amomnt of dilution required, the quantity of blood in the watery solution can be calculated. This is added to the amount of ummixed blood directly determined. Since hamorrhage is immediately followed by the entrance of liquid into the bloodvessels from the lymph and tissue fluids, somewhat too high a result will be obtained if the bleeding is at all prolonged. It is well, therefore, to take only a moderate amount of blood for direct estimation, and to compute the balance by the colorimetric method.

Many other methods have been devised on the principle of injecting a known guantity of some substance into the circulating blood, and then, after an interval has been allowed for mixture, determining the change produced in a sample. Thus, the specific gravity of a drop of blood having been measured, a certain quantity of a solution of sodium chloride isotonic with the plasma may be injected into a vein, and the specific gravity again determined. Or the electrical resistance of a small sample of blood may be measured before and after injection of a given quantity of isotonic salt solution.

The quantity of blood in the body was greatly overestimated by the ancient physicians. Avicenna put it at $25 \mathrm{lb}$., and many loose statements are on record of as much as $201 \mathrm{~b}$. being lost by a patient without causing death. The proportion of blood to body-weight has been found to be in the dog I : I3. new-born child I : I9, cat I : I4, horse I : I5, frog I : I7, rabbit I : I9, fowl I : 20. The total mass of the blood in a living man has been estimated by causing the person to inhale a known volume of carbon monoxicle mixed with oxygen or air, and then determining in a sample of blood taken from the finger the percentage anount to which the hemoglobin has become saturated with carbon monoxide. All that remains is to estimate the volume of carbon monoxide (or, what is precisely the same thing, the volume of oxygen) which roo c.c. of blood will take up. This latter quantity is called the percentage oxygen capacity. From these data the total volume of the blood can be calculated. If the volume is multiplied by the specific gravity the mass is obtained.

Thus, if the hemoglobin was found to be 25 per cent. saturated witl carbon monoxide after the person had absorbed i 50 c.c. of that gas, the whole of the blood would require 600 c.c. of carbon monoxide to saturate it completely. If the percentage oxygen capacity was 20,20 c.c. of oxygen or carbon monoxide would be needed to saturate 
1ou c.c. of blood. Therefore the tutial volume of the blood would be $000 \times{ }_{20}^{100}=3.000$ c.c. And the mass, if the specific gravity wats 1.055. Would be $3,000 \times 1 \cdot 055=3,165$ grammes. According to this method the blood on the average in man constitutes only f'9 per cent., or $: 0^{1} ;$ of the body-weight (say, $3 \frac{b}{2}$ kilogrammes in a 70 kilu 111212). varying in fourteen persons between is and is. Probably these results are somewhat too small. The amount has recently been estimated at $1: 1$ of the body-weight (Plesch). But, upon the whole. the method is sufficiently accurate, as shown by control experiments with Welcker's method on animals. In chlorosis and pernicious ancmia the puantity of blood is markedly increased. In one case of pernicious anamia it amounted to $\frac{I}{7^{\prime} 2}$ of the body-weight.

This is clue solely to increase in the plasma.

Fig. Io (p. 46) illustrates the distribution of the blood in the various organs of a rabbit. The liver and skeletal muscles each contain rather more than one-fourth; the heart, lungs, and great vessels rather less than one-fourth; and the rest of the body about one-fifth, of the total blood. The kidney and spleen of the rabbit each contain one-eighth of their own weight of blood, the liver between one-third and one-fourth of its weight, the muscles only one-twentieth of their weight.

\section{Lymph and Chyle.}

Lymph has been defined as blood without its red corpuscles (Johannes Müller) ; it resembles, in fact, a dilute blood-plasma, containing leucocytes, some of which (lymphocytes) are common to lymph and blood, others (coarsely granular basophile cells) are absent from the blood. The reason of this similarity appears when it is recognised that the plasma of tissue-lymph (p. 407) is derived, in large part at any rate, from the plasma of blood by a process of physiological filtration (or secretion) through the walls of the capillaries into the lymph-spaces that everywhere occupy the interstices of areolar tissue, while the lymph of the lymphatic vessels is in turn derived from the tissue fluid. But in addition to the constituents of the plasma, lymph appears to contain certain substances produced in the metabolism of the tissues which pass into it directly. Lympl, as collected from one of the large lymphatic vessels of the limbs, or from the thoracic duct of a fasting animal, is a colourless or sometimes yellowish or slightly reddish liquid of alkaline reaction. Its specific gravity is much less than that of the blood (Ior5 to I030). It coagulates spontaneously, but the clot is always less firm and less bulky than that of blood. The plasma contains fibrinogen, from which the fibrin of the clot is derived. Serum-albumin and serum-globulin are present in much the same relative proportion as in blood, although in smaller absolute amount. Neutral fats, urea, and 
sugar are also found in small quantities. The inorganic salts are the same as those of the blood-serum, and exist in about the same amount, sodimn preponderating among the hases, as it does in serum. The following table show's the results of analyses of lympli from man and the horse (Munk) :

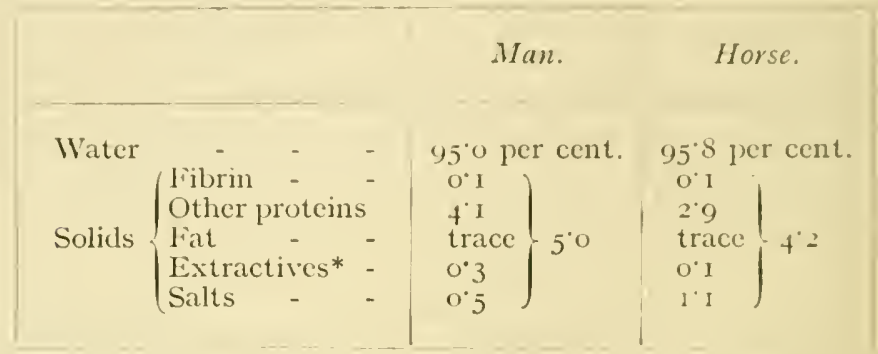

Chyle is merely the name given to the Iymph coming from the alimentary canal. The fat of the food is absorbed by the lymphatics, and during digestion the chyle is crowded with fine fatty globules, which give it a milky appearance. There may also be in chyle a few red blood-corpuscles, carried into the thoracic duct by a back-flow from the veins into which it opens. Clyyle clots like ordinary lymph, the size of the clot varying according to the quantity of fat present and enmeshed by the fibrin. Wounds of the thoracic duct or of lymphatics opening into it are occasionally procluced in operations on the neck, and when these remain open chyle may be readily collected. In samples obtained from a patient only a week after the section of a branch of the duct during an operation for the removal of tubercular glands, water constituted 928.90 parts in 1,000 , total solids $7 \mathrm{I} \cdot \mathrm{I} 0$, inorganic solids 6.04 , organic solids $65^{\circ} .00$, proteins I8.52, ether extract (fatty substances) I9:30 (Sollmann). The following is the composition of a sample analyzed by Paton, and obtained from a fistula of the thoracic duct in a man :

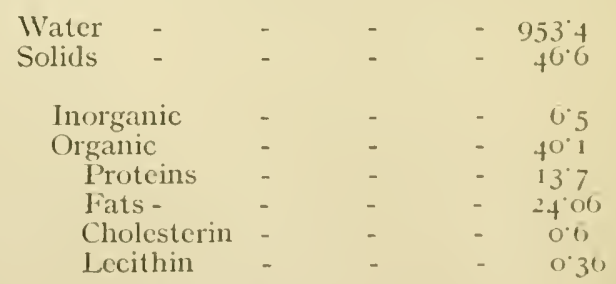

The quantity of chyle flowing from the fistula was estimated at as much as 3 to + kilos per twenty-four hours, or nearly as

* The term 'extractives' is somewhat loosely applied to organic substances which exist in so small an anount, or have such indefinite chemical characters that they cannot be separately estimated. 
numch as the whole of the blood. The flow has been calculated in various animals at one-eighteenth to one-seventh of the borlyweight in the twenty-four hours. The quantity of lymph in the hody is unknown, but it must be very great-perhaps two or three times that of the blood.

Allied to tissue-lymph, but not identical with it, are the fluids present in health in very small amount in such serous cavities as the pericardium. The synovial fluid of the joints differs from lymph especially in containing a small amount of a mucin-like substance.

The gases of the blood and lymph will be treated of in Chapter III., the formation of lymph in Chapter V.

\section{The Functions of Blood and Lymph.}

We have already said that these liquids provide the tissues with the materials they require, and carry away from them materials which have served their turn and are done with. These materials are gaseous, liquid, and solid. Oxygen is brought to the tissues in the red corpuscles; carbon dioxide is carried away from them partly in the erythrocytes, but chiefly in the plasma of the blood and lymph. The water and solids which the cells of the body take in and give out are also, at one time or another, constituents of the plasnra. The heat produced in the tissues, too, is, to a large extent, conducted into the blood and distributed by it throughout the body. It is not known whether the leucocytes play any part in the normal nutrition of other cells, although it is probable that they exercise an influence on the plasma in which they live; but they have important functions of another kind, to which it is necessary to refer briefly here.

Phagocytosis.-Certain of the amœboid cells of blood and lymph, and the cells of the splenic pulp, are able to include or ' eat up ' foreign bodies with which they come in contact, in the same way as the amcba takes in its food. Such cells are called phagocytes; and it is to be remarked that this term neither comprises all leucocytes nor excludes all other cells, for some fixed cells, such as those of the endothelial lining of bloodvessels, are phagocytes in virtue of their power of sending out protoplasmic processes, while the small, immobile, uninuclear leucocyte, or lymphocyte, is not a phagocyte.

Although it is not at present possible to assign a physiological value to all the phenomena of phagocytosis, either as regards the phagocytes themselves or as regards the organism of which they form a part, there seems little doubt that under certain circumstances the process is connected with the removal of 
structures which in the course of development have becone obsolete, or with the neutralization or elinination of harmful substances introduced from without, or formed by the activity of bacteria within the tissucs. During the metamorphosis of some larve, groups of cilia and muscle-fibres may be absorbed and eaten up by the leucocytes. In the metamorphosis of maggots, for example, the muscular fibres of the abdominal wall, which are used in creeping, and are therefore not required in the adult, degenerate, and are devoured by swarms of leucocytes which migrate into them. In the human subject an example of absorption of tissue by the aid of leucocytes is the removal of the necrosed decidua reflexa, the fold of uterine mucous membrane which envelops the ovum (Minot).

The behaviour of phagocytes towards pathogenic microorganisms is of even greater interest and importance. Metschnikoff laid the foundation of our knowledge of this subject by his researches on Daphnia, a small crustacean witl transparent tissues, which can be observed under the microscope. When this creature is fed with a fungus, Monospora, the spores of the latter find their way into the body-cavity. Here they are at once attached by the leucocytes, ingested, and destroyed. But after a time so many spores get through that the leucocytes are unable to deal with them all; some of them develop into the first or ' conidium' stage of the fungus; the conidia joison the leucocytes, instead of being destroyed by them, and the animal generally dies. Occasionally, however, the leucocytes are able to destroy all the spores, and the life of the Daplmia is preserved. This battle, ending sometimes in victory, sometimes in defeat, is believed by Metsclınikoff to be typical of the struggle which the phagocytes of higher animals and of man seem to engage in when the germs of disease are introduced into the organism. He supposes that the immunity to certain diseases possessed naturally by some animals, and which may be conferred on others by vaccination with various protective substances, is, to a large extent, due to the early and complete success of the phagocytes in the fight with the bacteria; and that in rapidlyfatal diseases-such as chicken-cholera in birds and rabbits, and anthrax in mice-the absence of any effective plagocytosis is the factor which determines the result. Others have laid stress on the action of protective substances supposed to exist in the living plasma itself, altlough only as yet demonstrated in the serum. It is possible that such substances are manufactured by the leucocytes, and either given off by them to the plasma by a process of 'excretion,' or liberated by their complete solution.

The most recent investigations go to show that Metschnikoff's phagocytic theory of immunity requires modification, at any 
rate in the case of the higher animals and man, although the hrilliant hiological ohservations on which it was originally built retain all their value. He supposed that in the immunizing process the lencocytes underwent certain changes, acquired, so to speak, a sort of 'education' that enabled them to cope with hacteria against which they were previously powerless. It seems more probable that in the presence of the substances that confer immunity, not only the leucocytes, but other cells, are stimulated to produce bodies which cut short the life, or inhibit the growth, of the bacteria (alexins), or prepare them for being taken up by the phagocytes (opsonins). It lias been shown that bacteria which have been in contact with serum containing the appro. priatc opsonins are taken up readily by leucocytes washed free from serum constituents by physiological salt solution, whereas the washed lencocytes either lo not ingest bacteria which have not been acted on by serum, or take them up in much smaller numbers. There is some evidence that in certain bacterial infections-for example, chronic furunculosis, a condition in which crops of boils continue to appear-the grip of the bacteria on the body is perpetuated by a deficiency in the amount or in the activity of opsonins capable of acting specifically upon the micro-organisms in question. A numerical expression, which in certain cases, perhaps, gives a measure of the patient's resistance to the infection, has been worked out by Wright under the name 'opsonic index.' This index is the ratio between the average number of bacteria taken up, under certain fixed conditions, by each polymorphonuclear leucocyte in an emulsion made with the patient's serum, and the average number taken up by similar leucocytes in an emulsion made with normal serum. The significance of this index, and even the practicability of the methods used to ascertain it, are still the subject of lively discussion.

Diapedesis.-The fact that leucocytes can pass out of the bloodvessels into the tissues has a very important bearing on the subject of phagocytosis. The phenomenon is called diape-desis, and is best seen when a transparent part, such as the mesentery of the frog, is irritated. The first effect of initation is an increase in the flow of blood through the affected region. If the irritation continues, or if it was originally severe, the current soon begins to slacken, the corpuscles stagnate in the vessels, and inflammatory stasis is produced. The leucocytes adhere in large numbers to the walls of the capillaries, and particularly of the small veins, and then begin to pass slowly through them by amœboil movements, the passage taking place at the junctions between, or it may be through the substance of, the endothelial cells. Plasma is also ponred out into the tissues, 
the whole forming an inflammatory exudation. Even red bloodcorpuscies may pass out of the vessels in small numbers. The exudation may he gradually reabsorbed, or destruction of tissue may ensue, and a collection of pus be formed. The cells of pus are emigrated leucocytes (Practical Exercises, Chap. II., p. I77).

Their emigration is connected with the defence of the organism against the entrance of certain forms of bacteria at the seat of injury, and with the repair of the injured tissue, but the nature of the summons which gathers them there is not yet clearly understood. It is probably some sort of chemical attraction (chemiotaxis) between constituents of the bacteria or decomposition products of the injured tissue on the one hand, and constituents of the leucocytes on the other.

\section{PRACTICAL EXERCISES ON CHAPTER I.}

N.B.-In the following exercises all experiments on animals which would cause the slightest pain are to be done under complete ancesthesia.

1. Reaction of Blood.--(1) Put a drop of fresh dog's or ox blood on a piece of glazed neutral litmus paper (the litmus paper can be glazed by dipping it into a neutral solution of gelatin and allowing it to dry). Wash the blood off in ro to 30 seconds with distilled water. A bluish stain will be left, showing that fresh blood is alkaline. (2) Repeat with dog's or ox serum. It is not necessary to wash the serum off, as it does not obscure the change of colour. (3) Repeat (I) with human blood. With a clean suture-needle or a good-sized sewing-needle which has been sterilized in the flame of a Bunsen burner, prick one of the fingers behind the nail. Bandaging the finger with a handkerchief from above down wards, so as to render its tip congested, will often facilitate the getting of a good-sized drop, but for quantitative experiments, like 2, 7, and 14 (4), this should not be done.

2 Specific Gravity of Blood-Hammerschlag's Method.-(I) Put a mixture of chloroform and benzol of specific gravity 1.060 into a small glass cylinder. Put a drop of dog's or ox defibrinated blood into the mixture by means of a small pipette. If the drop sinks add chloroform, if it rises add benzol, till it just remains suspended when the liquid has been well stirred. Then with a small hydrometer mensure the specific gravity of the mixture, which is now equal to that of the blood. Fiilter the liquid to free it from blood, and put it back into the stock-bottle. (2) Obtain a drop of human blood as in $\mathrm{I}$, and repeat the measurement of the specific gravity.

3. Coagulation of Blood.*-(1) Take three tumblers or beakers, label them $\alpha, \beta$, and $\gamma$, and measure into each 100 c.c. of water. Mark the level of the water by strips of gummed paper, and pour it out. (If a sufficient number of graduated cylinders is available, they may of course be used, and this measurement avoided.). Into a prut 25 c.c. of a saturated solution of magnesimm sulphate. into $\beta 25$ c.c. of a 1 per cent. solution of potassium or ammonium oxalate in 0.9 per cent. solution of sodium chloridle, and into $\gamma 25$ c.c. of a

* This experiment requires two laboratory periods, the various blood mixtures being obtained during the first and worked up during the second. 
1.2 per cent. solution of solium fluorile in $0^{\prime} 9$ per cent. salt solution. If the log proviled is a large one, these quantities may be all dombled; for a small dog they may be all halved.

(2) Insert a cannula into the central end of the carotill artery of a log anastlietized with morphine* and ether, or A.C.E. mixture.†

To put a Cammula into an Avtery. - Select a glass cannula of suitable size, feel for the artery, make an incision in its course through the skin, then isolate about an inch of it with forceps or a blunt needle, carefully clearing away the fascia or connective tissue. Next pass a small pair of forceps under the artery, and draw two ligatures through below it. If the cannula is to be inserted into the central end of the artery, tie the ligature which is farthest from the heart, and cut one end short. Then between the heart and the other ligature compress the artery with a small clamp (often spoken of as 'bull(log ' forceps). Now lift the artery by the distal ligature, make a transverse slit in it with a pair of fine scissors, insert the cannula, and tic the ligature over its neck. Cut the ends of the ligature short. If the cannula is to be put into the distal end of the artery, both ligatures must be between the clamp and the heart, and the bulldog must be put on before the first ligature (the one nearest the heart) is tied, so that the piece of bloodvessel between it and the ligature may be full of blood, as this facilitates the opening of the artery.

(3) Run into $\alpha, \beta$, and $\gamma$ enough blood to fill them to the mark. Shake the vesscls, or stir up once or twice with a glass rod, to mix the blood and solution.

(4) Take a small thin copper or brass vessel, and place it in a freczing mixture of ice and salt. Run into it some of the blood from the artery. It soon freezes to a hard mass. Now take the vessel out of the freezing mixture and allow the blood to thaw. It will be scen that it remains liquid for a short time and then clots.

(5) Run some of the blood into a porcelain capsule, stirring it vigorously with a glass rod. The fibrin collects on the rod; the blood is defibrinated and will no longer clot.

(6) Now let some blood run into a small beaker or jar. Notice that the blood begins to clot in a few minutes, and that soon the vessel can be tilted without spilling it. Note the time required for clotting to occur. Set the coagulated blood aside in a cool place, and observe next day that some clear yellow serum has separated from the clot.

(7) Weigh out a quantity of Witte's 'peptone' equivalent to 0.5 gramme for every kilo of body-iveight of the dog. Dissolve the peptone in about twenty times its weight of $0^{\circ} 9$ per cent. salt solution. Put a cannula into the centra'- end of a crural vein (p. 200). Fill the cannula with the peptone solution and connect it with a burctte. Put I 5 drops of the peptone solution into a test-tube labelled ' Peptone A.' Put the rest into the burette and see that the connecting tube is filled with the solution and free from air. Run into the test-tube about 5 c.c. of blood from the cannula in the carotid. Now let the peptone solution flow from the burette into the vein. Feel the pulse over the heart as the solution is running in. If the heart becomes very weak, stop the injection; otherwise the animal may dic from the great lowering of blood-pressure (p. 20I). As soon as the

* One to 2 centigrammes of morphine hydrochlorate per kilogramme of body-weight should be injected subcutaneously about half an hour before the operation. Ten c.c. of a 2 per cent. solution is sufficient for a dog of good size. Note that diarrhœa and salivation are caused by such a close. For directions for fastening the dog on the holder, see footnote on p. I $\$ 6$.

$t$ A mixture of I part of alcohol, 2 of ether, and 3 of chloroform. 
injection is finished, draw off a sample of 5 c.c. of blood into a testtube labelled 'Peptone B,' and let it stand. In ten minutes collect five further samples of 5 c.c. ('Peptone C, D, E, Ji, (r)'), and a large one, $1 \mathrm{H}$; in half an hour another set of five small samples, and at as long an interval as possible thereafter five more. Now letting the dog bleed to death, observe that the flow of blood is temporarily increased by pressure on the abdominal walls, which squeczes it towards the heart, by passive movements of the hindlegs, and also during the convulsions of asphyxia, which soon

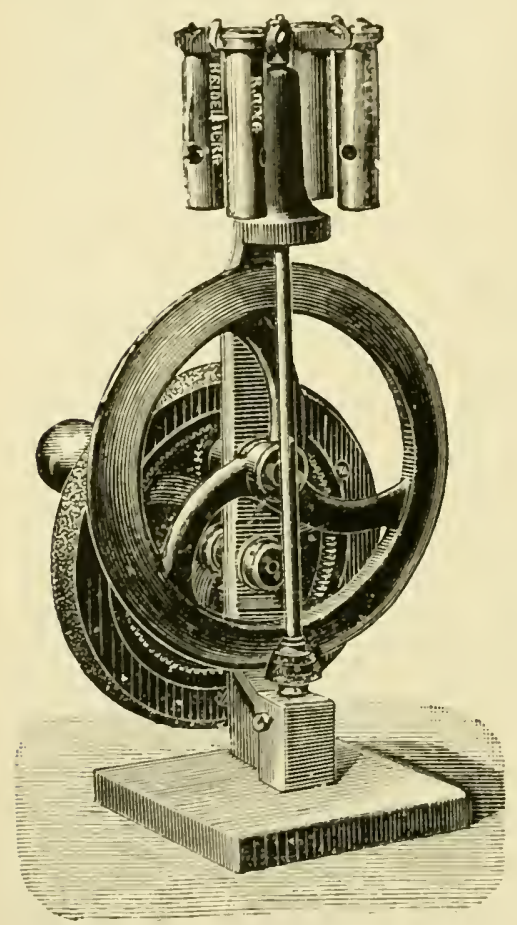

The four cylinders shown at the top of the figure are soswung that they become
Fig. II.-CENTRIFTGE (JUNG). horizontal as soon as speed is got up.

appear. Adil to the peptone blood D 5 c.c. of serum, to J a little sodiun chloride extract of liver, to $\mathrm{I}^{*}$ a little extract of muscle, and to G I 5 drops of a 2 per cent. Solution of calcium chloride, and put C, D. F, I; and $C_{x}$ into a water-bath at $40^{\circ} \mathrm{C}$. Treat the other sets of small samples in the same way. Note how long each specimen takes to clot, and report your results. *

(8) Observe that the blood in $\alpha, \beta$, and $\gamma$ has not coagulated. Iabcl four test-tubes '()xalate A, B, C, D,' and put into each about 5 c.c. of the oxalater blood. Add to $A$ and $B$ or 6 drops of a 2 per cent. solution of calcium chloricle, to $\mathrm{C}$ I2 drops, and to D as much as there is of the blood. Leave $A$ at the ordinary temperature, put the other test-tubes in a water-batls at $40^{\circ} \mathrm{C}$, and note when clotting occurs.

(9) To Io c.c. of the fluoride blood add a little more $\mathrm{CaCl}_{2}$ than is required to combine with the excess of fluoride present. Jabel four test-tubes 'Fluoride A, B, C, D,' and into each put about 2 c.c. of this 'recalcifica' fluoride blood. To $\mathrm{I}$ adrl I c.c. liver extract ; to C I c.c. muscle cxtract, and to D 4 c.c. water. Jabel two more test-tubes " Fluoride $\mathrm{E}$ and $\mathrm{F}$ : Into each put 2 c.c. of the fluoride blood without $\mathrm{CaCl}_{2}$. Add also to $\mathrm{I}$ I c.c. liver cxtract and to F I c.c. serum. Put all the tubes in a bath at about $40^{\circ} \mathrm{C}$., and observe in which and in what time coagulation takes place.

(so) By means of a centrifuge (Fig. II) separate the plasma

* Sometimes the injection of peptone liastens coagulation instead of hindering it. It has been asserted that this is only the case when small doses are used (less than $0^{\circ} \mathrm{O} 2$ gramme per kilo of body-weight). But in 2 dogs out of I I a dose of $0^{\circ} 5$ gramme per kilo has been seeu to hasten coagulation, and in 1 out of 12 to leave it unaffecterl; in the other a coagulation was markedly retarded. 
from the corpuscles in $\alpha, \beta$, and $\gamma$, and also from the peptone bloorl.

With the oxalate plasma from $\beta$, and the fluoricle plasma from $\gamma$. repeat the observations in (8) and (9), using smaller quantities of the plasma, if necessary, in small test-tubes. With the plasma from a perform the following experinents : Put a small quantity of the plasma ( $\mathrm{I}$ c.c.) into four test-tubes, labelling them 'Magnesium Sulpliate A, B, C, D.' Dilute B with four times, C witli eight times. and $\mathrm{D}$ with twenty times as much distilled water as was taken of the plasma. Observe in which, if any, coagulation occurs, and the time of its occurrence, and report the resnlt.

(Ix) With peptone plasma from $H$ and from the peptone blood obtained later repeat the experiments done in (7). In arldition dilute I c.c. of the plasma with three volumes of water and I c.c. of it with ten volumes of water, and put in the bath at $40^{\circ} \mathrm{C}$. Observe whether clotting occurs.

If no centrifuge is available, the various blood-mixtures must be left standing in a cool place for 12 to 24 hours till the corpuseles settle. The plasma can then be siphoned or pipetted off. Instead of clog's blood, the blood of an ox or pig may be obtained at the slaughter-house.

4. Preparation of Schmidt's Fibrin-ferment.-Precipitate bloodserum with ten times its volume of alcoliol. Let it stand for several weeks, then extract the precipitate with water. The water clissolves out the fibrin-ferment, but not the coagulated serum proteins.

5. Preparation of Tissue Extracts containing Thrombokinase.In a dog or rabbit lilled by bleeding insert a cannula in to the lower end of the thoracic aorta. Fill the cannula with $0^{\prime} 9$ per cent. salt solution or water, and connect it with a bottle also containing salt solution or water. Wash out the vessels of the lower portion of the body, maling an opening in the inferior vena cava above the diaphragm to allow the liquid to escape. For the sake of cleanliness, a cannula armed with a piece of rubber tubing should be inserted for this purpose into the inferior vena cava. Continue the injection till the liquid issues colourless. Then remove portions of liver and muscle. Mince each separately. Rub up with sand in a mortar. Add $0^{\circ} 9$ per cent. sodium chloride solution and rub up again. Put into bottles and keep in the ice-chest. For use take off some of the liquid from the top with a pipette, or strain through cheese-cloth.

6. Serum.-Test the reaction, and determine, both by the hydrometer and the pycnometer, or specific gravity bottle, the specific gravity of the serum provided, or of the serum obtained in experiment 3 .

Serum Proteins.-(I) Saturate serum with magnesium sulphate crystals at $30^{\circ} \mathrm{C}$. The serum-globulin is precipitated. Filter off. Wash the precipitate on the filter with a saturated solution of magnesium sulphate. Dissolve the precipitate by the addition of a little distilled water, and perform the following tests for globulins: (a) Saturate with magnesium sulphate. A precipitate is obtained. (b) Drop into a large quantity of water, and a flocculent precipitate falls down. (c) Heat. Coagulation occurs. Determine the temperature of coagulation (p. S).

(2) To a portion of the filtrate from (I) add sodium sulphate to saturation. The serum-albumin is precipitated. (Neither magnesium sulphate nor soclium sulphate precipitates serum-albumin 
alone, but the double salt solio-magnesium sulphate precipitates it, and this is formed when sodimm sulpluate is added to magnesimm sulphate.)

(3) Dilute another portion of the filtrate from (1) with its own bulk of water. Very slightly acidulate with dilute acctic acirl, and determine the temperature of heat coagulation.

(4) Precipitate the serum-globulin from another portion of serum by adding to it an equal volume of saturated solution of ammonium sulpliate. Filter. Precipitate the serum-albumin from the filtrate by saturating with ammonium sulphate crystals.

(5) Dilute serum with ten to twenty times its volume of distilled water, and pass through it a stream of carbon dioxide. The serumglobulin is partially precipitated. This is the starting-point of a method said to be the best for obtaining pure serum-globulin.

(6) Acidulate some serum with dilute acetic acid and boil. Filter off the coagulum, and to the filtrate add silver nitrate. A non-

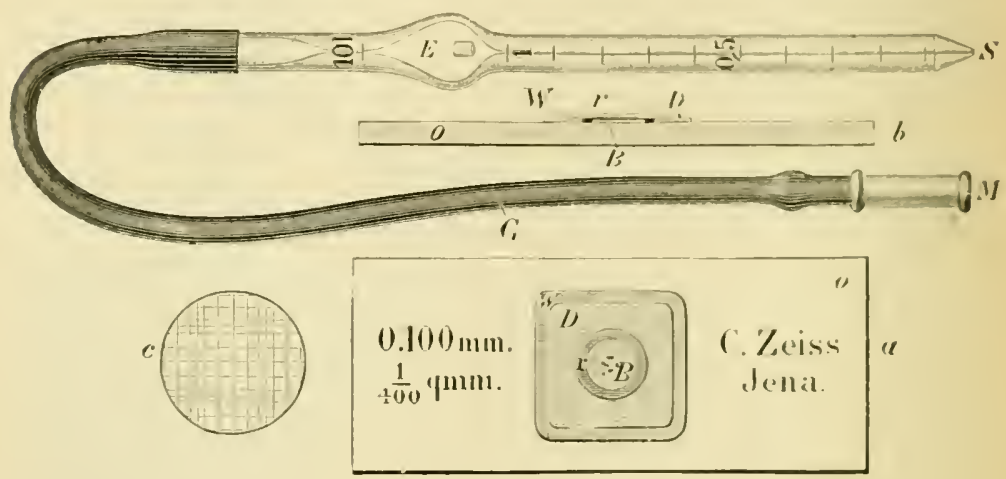

FIG. I2.-THOMA-ZEISS H.EMOCYTOMETER.

$M$, mouth-piece of tube $G$, by which blood is sucked into $S ; E$, bead for mixing ; $a$, view of slide from above $b$, in section; $c$, squares in middle of $B$, as seen under microscope.

protein precipitate insoluble in nitric acid but soluble in ammonia indicates the presence of chloricles.

7. Enumeration of the Blood-corpuscles.-Use the Thoma-Zeiss apparatus (Fig. 12). (I) Suck a (lrop) of ox or (log's blood up into the capillary tube $S$ to the mark $I$. Wipe off any blood which may adhere to the end of the tube. Then fill it with IIayem's solution (p. IS) or 3 per cent. sodium chloride to the mark 101 . This represents a dilution of Ioo times. Mix the blood and solution thoroughly, then blow out a drop or two of the liquid to remove all the solution which remains in the capillary tube. Now fill the shallow cell $B$ with the blool mixture. Put the cover-glass on, taking care that it does not float on the liquid, but that the cell is exactly filled. Put the slide muler the microseope (say Leitz's oc. III., obj. 5), and count the number of red corpuscles in not less than ten to twenty squares. Sixteen squares is a good rontine number. The greater the number of squares counted, the nearer will be the approximation to the truth. Now take the average number in a square. The depth of the cell is $l_{1}^{\prime}, 1 m 11$, the area of 
each square $\frac{1}{60}$ sq. $m$ m. The volume of the colimn of liquicl standing upon a square is fúcin cub. $111 \mathrm{~m}$. ()ne cub. mus. of the diluted blood would therefore contain 4 ,ooo times as many corpuscles as one square. But the blood has becn diluted roo times, therefore 1 cub. mm. of the undiluted bloor would contain foo,ooo times the number of corpuscles in one square. Suppose the average for a squarc is found to be i3. This would correspond to $5,200,000$ corpuscles in I cub. $13 m$. of blood. Compare your result with the true number supplied by the demonstrator. (2) Prick the finger to obtain a drop of blood, and repeat the count as in (1).*

To Count the IVhite Corpuscles. - Add to I part of blood 9 parts of $\frac{1}{3}$ per cent. acetic acid, in order to lake the coloured corpuscles and render it casy to sec the leucocytes.

8. Relative Volume of Corpuscles and Plasma by Hæmatocrite. (I) For practice, fill the two graduated glass tubes with the defibrinated blood of an animal. The rubber tube with mouthpiece supplied with the apparatus is to be attached to the glass tube, and the blood sucked up. Press the tip of the index-finger against the pointed end, and carefully remove the rubber tube. Place the tube

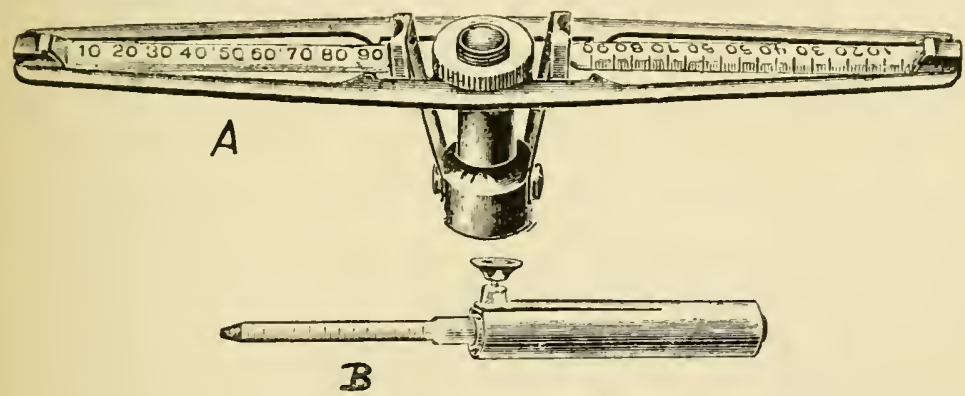

Fig. I3.-Hamatocrite.

$A$, hæmotocrite attachment with graduated tubes; $B$, automatic pipette for filling the tubes (Daland).

in the hæmatocrite frame, blunt end outwards - that is, farthest from the axis of rotation-and then slip the pointed end down into position against the spring. Instead of the rubber tubc, a special suction pipette for automatically filling the graduated tubes may be employed (Daland). Attach the hæmatocrite frame to the centrifugc, and rotate till the volume of sediment (corpuscles) ceascs to diminish. The graduations are best read with a hand lens. The leucocytes will be seen to form a thin whitish line proximal to the column of red corpuscles.

(2) Prick the finger or the lobe of the car, fill the tubes as in (I), and centrifugalize. Everything must be done as rapilly as possible, so that the blood may not clot till the separation of plasma and corpuscles is completed. The centrifuge must rotate very rapiclly (about 10,000 revolutions a minute) for two or threc minutes. For clinical purposes it is best to rotate the centrifuge always at the same speed for the same length of time rather than to aim at reaching a constant length of the column of corpuscles. In this way useful

* If the tube has not been properly filled, blow the blood out immediately. On no account permit it to clot in the capillary tube 
comparative results can be obtained. It is well, to avoicl the risk of acciclent, to rotate the centrifuge under a guard.

9. Electrical Conductivity of Blood.-(I) Fill a small $\mathbf{U}$-tube with blood up to a mark. In each limb insert a platimum electrode* connected with a holer, which insures that the electrode shall always dip to the same depth into the tube. Arrange the $U$-tube so that it is inmersed at least to the mark in water of comstant temperature. Water runuing freely from the coll-water tap) into and out of a large vessel will have a sufficiently constant temperature for the purpose. A thermoneter must be fixed in the water with its bulb in contact with the $\mathbf{U}$-tube. Connect the electrocles with a resistance-box in the Wheatstone's bridge arrangement (Fig. 204 , p. 617). so that the $U$-tube occupies the position of the unknown resistance CD. Instead of the battery fir, connect the poles of the secondary of a small induction-coil, arranged for an interrupted current, with $A$ and $C$, and insteal of the galvanometer ( $;$ insert a telephone. The resistances $\Lambda B$ and $A D$ (the arms of the bridge) will be obtaincel by taking out two plugs from the appropriate part of the resistance-box. Whether the arms should be equal (say, ro : 10, roo : 100, or 1,000 : r, o00 ohms) or uncqual (say, Io : 100, or $100:$ I, ooo, or Io : I, O00 ohms) will depend upon the resistance of the tube of liquid to be measured. Take out from the part of the box corresponding to $\mathrm{BC}$ a plug representing a resistance something like that which the tube of blood is expected to have. Close the primary circuit of the induction-coil, and apply the telephone to the ear. A buzzing sound will be heard, which will be louder the farther from the true resistance of the tube the resistance taken out of the box is. Go on altering the resistance in the box by taking out or putting in plugs till the sound disappears, or is reduced to a minimum. The temperature of the water should now be reat off. The resistance of the tube of blood for this temperature can casily be calculated from the formula on p. 6r7. It increases about 2 per cent. for each degree Centigrade of diminution of temperature. The conductivity is the reciprocal of the resistance. By determining once for all the resistance of the tube when filled with a standard solution of a salt whose conductivity is known, the specific conductivity of the blood can be expressed in lefinite mits, but this is not necessary for the purposes of the student. Compare the resistances of defibrinated blood, serum, $0^{*} 9$ per cent. sodium chloride solution, and a sediment of blood-corpuseles separated by centrifugalization.

(2) Instead of the resistance-box a wire momnted on a scale may be used for the bridge arms AB, AD, the ends of the wire being connected at $B$ and $D$. A slider with an insulated handle moving along the gradluated wire is joined by a flexible wire with one pole of the sceondary coil, the other pole being connected at C. The resistance $\mathrm{BC}$ is constituter by a rheostat from which a fixed resistance can be taken out. Instead of obtaining the minimum somnd in the telephone hy varying the resistance $B C$ in the box, the measurement is mate by varying the position of the slider ; in other worels. by changing the ratio $A B: \Lambda D$.

(3) If no rheostat is arailable instructive comparative measurements may still be made with the gradnated wire by substituting for the resistance $\mathrm{BC}$ a $\mathbf{U}$-tube of another licpuicl.

* If the platinum electroles are of good size and the resistance of the tube of liquid considerable, it is not necessary to platinize them-i.e., to cover them by electrolysis of a solution of platinic chloricle with a layer of platinum-black. 
If the tubes are of the same dimensions, and the licpuids with which they are filled are approximately at the same initial temperature, it is not necessary to immerse them in water at constant temperature. It is sufficient to place them side by side in the air. I'erform the following experiments in this way:

(a) Label the tubes $\Lambda$ and B. Fill then both to the mark with oo per cent. NaCl solution. Comnect as in the figure, and move the slider along the wire till the sound is a minimum. Probably the two tubes are not exactly of the same dimensions, and therefore the slider will not be exactly in the midalle of the wire. Suppose it is at $49^{\circ} \mathrm{o}$, the total length of the wire being soo. Then resistance of $\mathrm{A}:$ resistance of $\mathrm{B}:: 49^{\circ} \mathrm{O}: 51^{\circ} \mathrm{O}$, i.e., resistance of $\mathrm{A}=\frac{49}{5^{\mathrm{I}}}$ resistance
of $\mathrm{B}$.

(b) Fill A with defibrinated blood, keeping $\mathrm{B}$ filled with $\mathrm{NaCl}$ solution, and repeat the measurement. The slider must now be moved much farther away from the zero of the scale. Suppose the minimum sound is obtained with the slider at $70^{\circ} \mathrm{O}$. Then resistance of blood $=\frac{7}{3} \times \frac{5^{\mathrm{T}}}{49}$ resistance of the $\mathrm{NaCl}$ solution.

(c) Compare in the same way the resistance of serum with that of the NaCl solution. It will be found much less than that of the blood.

(d) Centrifugalize some of the blood for as long as is convenient, and compare the resistance of the blood from the top of the tubes and from the bottom of the tubes with that of the $\mathrm{NaCl}$ solution. The resistance of the blood from the bottom of the tubes will bo found much greater than that of the blood from the top.

IO. Opacity of Blood.-Smear a little fresh blood on a glass slide, and hold the slide above some printed matter. It will not be possible to read it, because the light is reflected from the corpuscles in all directions, and little of it passes through.

I 1. Laking of Blood by Chemical and Physical Agents.--(I) Put a little fresh blood into three test-tubes, A, B and C. Dilute A with an equal volume, $B$ with two volumes, and $C$ with three volumes, of distilled water, and repeat experiment 9 . The print can now be read probably through a layer of $A$, but certainly through $B$ and $C$, since the hremoglobin is dissolved out of the corpuscles by the water and goes into solution, the blood becoming transparent or laked. That the clifference is not due merely to dihtion can be shown by putting an equal quantity of blood in two test-tubes, and gradually diluting one with distilled water and the other with a $0^{\circ} 9$ per cent. solution of sodium chloride, which does not dissolve out the hamoglobin. Print can be read through the first with a smaller degree of dilution than through the second. Examine the laked blood with the microscope for the 'ghosts,' or shadows of the red corpuscles. The addition of a drop or two of methylene blue will render them somewhat more distinct.

(2) Heat a little dog's or ox blood in a test-tube immersed in a water-bath. Put a thermometer in the test-tube, taking care that there is enough blood to cover the bulb. Keep the temperature about $60^{\circ} \mathrm{C}$. In a few minutes the blood becomes dark and laking occurs.

(3) (a) Put a little blood into each of four test-tubes. To one add a little ether, to another a little chloroform, to the thircl dilute acetic acid in $0^{\circ} 9$ per cent. $\mathrm{NaCl}$, and to the fourth a dilute solution 
of bile salts (or of solium taurocholate) in $0^{\circ} \mathrm{g}$ per cent. $\mathrm{NaCl}$ solution. Laking occurs in all.

(b) To 5 c.c. of blood auld o 5 c.c. of a 3 per cent. solution of sisponin in $0^{\circ} 9$ per cent. $\mathrm{NaCl}$ solution, and put the mixture at $f 0^{\circ} \mathrm{C}$. Laking soon occurs.

(c) Using a ro per cent. dilution of blood (blood to which nine volumes of $\mathrm{NaCl}$ solution have been added) or a 5 per cent. suspension of washed corpuseles in $\mathrm{NaCl}$ solution (i.e., a suspension of corpuscles which have been washed free from serum by being repeatedly mixed with $\mathrm{NaCl}$ solution and centrifugalized), determine the minimum dose of $\mathrm{O}^{\prime} 3$ per cent. saponin solution which will just cause complete laking. Keep the tubes at about $40^{\circ} \mathrm{C}$, and observe them from time to time. Now add to some of the ro per cent. dilution or the 5 per cent. suspension of blood an equal volume of serum from the same kind of blood, and repeat the determination of the minimum dose of saponin necessary for laking. It will be found that more is now required. The cholesterin in the serum neutralizes the action of some of the saponin.

(4) (a) Put I c.c. of blood into each of two test-tubes. To one add I c.c. of 2 per cent. aqueous solution of urea, and to the other 3 c.c. Laking will take place in the second, whether this has been the case in the first or not.

(b) Repeat the experiment with a 2 per cent. solution of urea in 0.9 per cent. $\mathrm{NaCl}$ solution. Laking does not occur. This shows that the urea in the first experiment did not act as a hremolytic agent. Laking occurred because urea penetrates the corpuscles easily, and therefore, although the freczing-point of the urea solution is not very different from that of the $\mathrm{NaCl}$ solution, its actual osmotic pressure, in relation to the envelopes of the corpuscles, is very much less, and the laking is really water-laking.

(5) Put some blood into a flask or test-tube, cork up, and let it stand till it begins to putrefy. It becomes laked. The same occurs when the blood is collected aseptically in a sterile tube and sealed up, although it takes a longer time for the laking to become complete.

(6) With blood containing nucleated corpuscles (necturus, frog or chicken) diluted with isotonic salt solution, perform the following experiments under the microscope :

(a) With a glass-rod drawn to a fine point put a small drop of blood on a slide, and near it a drop of distilled water. Carefully lower the cover-slip and observe the interface with the microscope, first with the low and then with the high power. Then mix and see complete laking. Add a little methylene bluc. Note that the nuclei still stain.

(b) Place a small cIrop of a 3 per cent. solution of saponin in isotonic salt solution on a slide, and near it a small drop of blood. Observe as in $(a)$. Repeat with a 2 per cent. solution of sodium taurocholate in salt solution. If necturus corpuscles, which are splendid objects for such experiments on account of their great size, have been used, intracorpuscular crystallization of the hremoglobin may be observed.

(c) Repeat $(a)$ and $(b)$ with mammalian blood. Note that the corpuscles swell before being laked by the saponin. If any of the corpuscles are crenated, it may be secn that before being laked by the saponin the crenations disappear, the corpuscles becoming round, while in the taurocholate solution they may remain crenated till laking has occurred. This indicates that the permeability of the envelopes is not affected in the same way by the two laking agents. 
12. Hæmolysis and Agglutination by Foreign Serum.-(1) 'To a small quantity of rabbit's blood adel an equal volume of dorg's serum. Mix and let stand at to $^{\circ} \mathrm{C}$. The colonr of the blood is soon darker than before, and it can be seen to be laked. Examine microscopically.

(2) Place a small drop of rabbit's blood and a somewhat larger (lrop of the dog's scrum on a slicle, near, but not quite in contact with, cacli other. Now put on a cover-slip, so that the drops just come together, and cxamine at once with the microscope with a moderately high power. Where the two drops mingle, the red corpuscles will be secn first to become agglutinated into groups, and then to fade out, leaving only their ' ghosts.' A few of the corpuscles which come into contact with the, as yet, undiluted scrum may be cntirely dissolved.

(3) Heat some of the dog's scrum to $60^{\circ} \mathrm{C}$. for ten minutes, and repeat ( 1 ) and (2). No laking will now be produced in the rabbit's corpuscles, but agglutination may be observed as before.

(4) Repeat (1) and (2) with (log's blood and rabbit's serum. The blood will not be laked, although sometimes the dog's corpuscles may become crenated. There will be no agglutination.

(5) With a 5 per cent. suspension of rabbit's washed corpuscles perform the following experinents :*

Put into each of six small test-tubes I c.c. of the suspension. Label the tubes $\mathrm{A}, \mathrm{A}^{\prime}, \mathrm{B}, \mathrm{B}^{\prime}, \mathrm{C}, \mathrm{C}^{\prime}$.

(a) To $\mathrm{A}$ and $\mathrm{A}^{\prime}$ add respectively $0^{\circ} \mathrm{I}$ c.c. and $0^{\circ} 5$ c.c. ox serum.

(b) To $\mathrm{B}$ and $\mathrm{B}^{\prime}$ add respectively $O^{\circ} \mathrm{I}$ c.c. and $0^{\circ} 5$ c.c. dog's serum.

(c) To $\mathrm{C}$ and $\mathrm{C}^{\prime}$ add respectively $\mathrm{O}^{\circ} \mathrm{I}$ c.c. and $0^{\circ} 5$ c.c. of $0^{\circ} 9$ per cent. soclium chloride solution.

Put all the tubes in a bath at $40^{\circ} \mathrm{C}$. Compare the amount of laking and agglutination in the various tubes at intervals of two minutes or less. Repeat $(a),(b)$, and $(c)$ with guinea-pig's washed corpuscles and scrum of ox and dog. Determine which of these sera has the strongest hamolytic power. $\dagger$

(6) Heat I c.c. of ox and dog's serum respectively to $56^{\circ} \mathrm{C}$., keeping it at that temperature, or not more than a couple of degrees above it, for ten $\ddagger$ minutes, and repeat experiment (5), labelling the tubes

* The material obtained from one medium-sized dog, two rabbits, and one guinea-pig is enough for fifty or sixty students, working together in sets of two, to perform experiments (5) to (8). In order to obtain a serum more strongly hamolytic for rabbit's corpuscles than normal dog's serum, a dog may be 'immunized' by previous injection of all the washed corpuscles obtainable from a rabbit. The injection should be made under the skin or, better, in to the peritoncal cavity-of course, with aseptic precautions. It should be repeated not less than twice, with an interval of ten days between the successive injections, and the dog's blood should be drawn off about ten days after the last injection.

$\dagger$ To determine the amount of laking at any given moment, drop the small test-tubes into the metallic centrifuge cups after shaking them up, and centrifugalize. A very short time is sufficient to separate a clear supernatant liquicl, from the tint of which the extent of the hæmolysis can be deduced. Before replacing the tubes in the thermostat, they should, of course, be shaken up. Small test-tubes of about $8 \mathrm{~mm}$. internal diameter and short enough to go conveniently into the centrifuge cups are the most serviceable.

$\ddagger$ For exact work a longer time is recommended. But for the student the time is made as short as possible, and it is only in exceptional cases that ten minutes is not enough. 
$D, D^{\prime}, E, E^{\prime}, I^{\prime}, I^{\prime}$. Satve the rest of the heated serat for (\$). There is no laking in any of the tubes, but probably agglutination in D, D', and E, E'. (The complement is destroyed, but not the intermediary bolly or amboceptor, or the agglutinin-p. 27.)

(7) Put half of the contents of tubes D, D', E, E', into four separate test-tubes, and add to each $\mathrm{O}^{\circ} 2$ c.c. of rabbit's serum. If there is laking now it is because the rabbit's serum contains complement. Save the balance of $\mathrm{D}, \mathrm{D}^{\prime}$, E and $\mathrm{E}^{\prime}$ for (8).

(S) Allow $0^{\circ} 5$ c.c. of ox serum to act at $0^{\circ}$ C. on the rabbit's washed corpuscles contained in 5 c.c. of the 5 per cent. suspension after removal of the sodium chloride solution. The ox serum and rabbit's corpuscles are separately cooled to $\mathrm{O}^{\circ} \mathrm{C}$. before being mixed, and the mixture is then kept at $0^{\circ} \mathrm{C}$. for one hour. Centrifugalize the scrum off rapidly. Label it 'Serum S.' To $0^{\circ} 2$ c.c. of the original 5 per cent. suspension of rabbit's washed corpuscles add $O^{\prime}$ I c.c. of this serum (labelling the tube G), and put at $f 0^{\circ} \mathrm{C}$. with a control-tube containing the same amount of suspension plus salt solution instead of serum. Add the rest of the serum $\mathrm{S}$, cooled to $0^{\circ} \mathrm{C}$., to the same cooled rabbit's corpuscles, and leave for a further period at $0^{\circ} \mathrm{C}$. Then centrifugalize rapidly, and to $0^{\circ} 2$ c.c. of the original suspension of washed rabbit's corpuscles add O I c.c. of serum S (labelling the tube $\mathrm{H}$ ), and put at $\mathrm{O}^{\circ} \mathrm{C}$. with a sodium chloride tube as control. There may be no laking in either $\mathrm{G}$ or $\mathrm{H}$, or if there is laking it may be greater in $\mathrm{G}$ than in $\mathrm{H}$. The amboceptor has been removed from serum $\mathrm{S}$ by the rabbit's corpuscles. Add o. I c.c. of this 'inactivated ' serum to the balance of $\mathrm{D}, \mathrm{D}^{\prime}$, and $\mathrm{E}, \mathrm{E}^{\prime}$ (left from 6). Laking will occur because the serum $\mathrm{S}$ contains complement, and the heated serum added in (6) to these tubes contains amboceptor. Wash the rabbit's corpuscles which have been treated with ox serum at $\mathrm{o}^{\circ} \mathrm{C}$. with cooled sodium chloride solution. Adel to them some of serum $\mathrm{S}$ (that from the top of tube $\mathrm{H}$ will (lo if no more is left), and put at $40^{\circ} \mathrm{C}$. Laking will occur, showing that the amboceptor was fixed by the rabbit's corpuscles at $0^{\circ} \mathrm{C}$. To a further portion of the washed rabbit's corpuscles which were treated with ox serum at $0^{\circ} \mathrm{C}$. add normal rabbit's serum, and put at $40^{\circ} \mathrm{C}$. If laking occurs it is because the rabbit's serum contains complement.

Dog's serum may be used instead of ox serum for experiment ( 8 ).

13. Osmotic Resistance of the Coloured Corpuscles. - irill a burette with a I per cent. solution of sodium chloride and another with distilled water. Talie a series of ten test-tubes and run into the first 6 c.c. of the $\mathrm{NaCl}$ solution, into the sccond $5^{\circ} \mathrm{S}$ c.c.. into the thirel $5^{\circ} 6$ c.c., and so on, always making a difference of $0^{\circ} z$ c.c. between each two successive test-tubes. From the other burette run in enough clistilled water to make up ro c.c. of solution in each tubethat is, $f$ c.c. of clistilled water for the first tube, $f^{\prime} z$ c.c. for the second, and so on. Shake up. The tubes now contain a series of solutions of salt differing in strength by $0^{\circ} \mathrm{O} 2$ per cent. in successive tubes, the strongest being $0^{\circ} 6$ per cent., and the weakest $0^{\circ} 42$ per cent. Number the tubes I to 10, beginning with the strongest solution. Put into each tube one drop of perfectly fresh blood. Shake moderately so as to mix the blood and salt solution, and allow the tubes to stand for ten to thirty minutes. Observe the colour of the clear liquid above the sediment of corpuscles. Determine in which tube the first tinge of hamoglobin appears. The next higher concentration of the salt solution is that in which all the corpuscles are just able to retain their hamoglobin, and is a measure of the minimum osmotic resistance 
of the corpuscles, or the resistance of the weakest corpuscles. Repeat with blood which has stood at room tenperature for twelve to twenty-four hours. For clinical purposes tubes, each containing 5 c.c. of salt solution, maly be used. $\Lambda$ single drop of blood can then be clistributed between the tubes witl a fine pipette or a glass rorl, beginning witl the most concentrated solution, and passing lown to the less concentrated. The blood must be distributed rapidly before coagulation occurs. Only such concentrations of the salt solution as are known to correspond to the possible variations of the osmotic resistance for any particular cliscase or for any particular varicty of blood neck be enployed.

14. Blood-pigment-(I) Preparation of Hæmoglobin Crystals.--(a) To a little dog's blood in a narrow test-tube add its own volume or twice its volume of chloroform. Invert the tube ten or twelve times so as to allow the chloroforn to act on the blood, but avoid violent shaking. When the tube is now allowed to stand for a few minutes the laked blood all rises to the top. Remove a little of the layer of blood without taking with it any of the chloroform layer, and cxamine the oxyhæmoglobin crystals with the microscope. Tliey form long rhombic prisms and needles (Fig. 8, p. 45).

(b) Add a little crude saponin to dog's blood in a test-tube. Shake

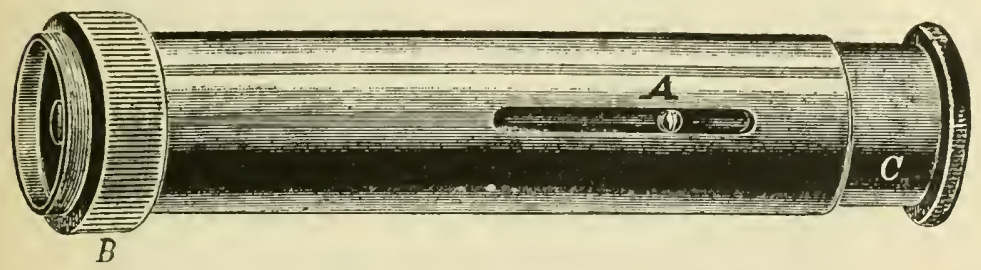

Fig. 14.-Direct Vision Spectroscope of Simple Type.

$A$, slot in which a pin on the eyepiece $C$ slides in focussing the spectrum; $B$, milled head, by the rotation of which the slit is narrowed or widened.

up well, and allow it to stand till the colour becomes dark. Then shake vigorously, and a mass of hremoglobin crystals will be formed.

(c) Put a small drop of guinea-pig's blood on a slide. Mix with a drop of Canada balsam and cover. Tetrahedral crystals of oxyhæmoglobin will form after a time. The slide may be kept.

(2) Spectroscopic Examination of Hæmoglobin and its Derivatives. - (a) With a small, dircct-vision spectroscope look at a bright part of the sky or a white cloud. Focus by pulling out or pushing in the eycpiece until the numerous fine dark lines (Fraunhofer's lines), running vertically across the spectrum, are seen. Narrow the slit by moving the milled edge till the lines are as sharp as they can be made. Note especially the line $\mathrm{D}$ in the orange, the lines $\mathrm{E}$ and $b$ in the green, and $F$ in the blue. Always hold the spectroscope so that the red is at the left of the field. Now dip an iron or platinum wire with a loop on the end of it into water, and then into some common salt or sodium carbonate, and fasten or hold it in the flame of a fishtail burner. On examining the flame with the spectroscope, a bright yellow line will be seen occupying the position of the dark line $D$ in the solar spectrum. This is a convenient line of reference in the spectrum, and in studying the spectra of hæmoglobin and its derivatives, the position of the absorption bands with regard to the $D$ line should always be noted. The dark lines in the solar spectrum. 
are lue to the absorption of light of a definite range of wave-lengths by metals in a state of vapour in the sun's atmosphere, and of course no lark lines are seen in the spectrum of a gas-flame. Put some defibrinated blood into a test-tube. Fasten it vertically in a clamp in front of the flame and examine it with the spectroscope. holding the latter in one hand with the slit close to the test-tube, and focussing the eyepice with the other. Or arrange the spectroscope, test tube and gas-flame on a stand as in Fig. 15. Nothing can be seen till the blood is diluted. Pour a little of the blood into another testtube, and go on diluting till, on focussing, two bands of oxyhemoglolin are seen in the position indicated in Fig. 7. Draw the spectrum; then dilute still more, and observe which of the bands first disappears. Now put 5 c.c. of the blood into another test-tube, and

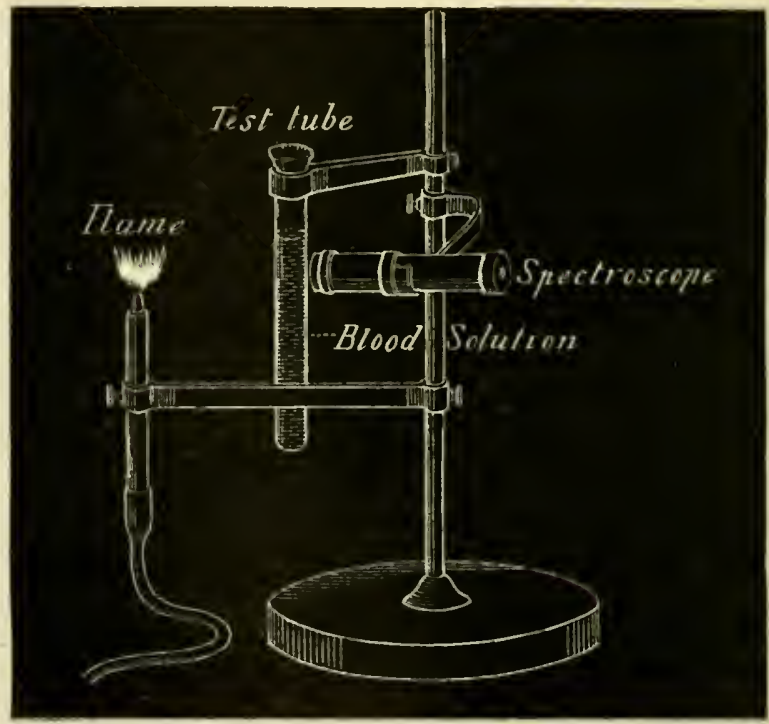

Fig. I5.-Spectroscopic Examination of Blood.pigment.

dilute it with four times its volume of water. Take 5 c.c. of this dilution, and again add four times as much water, and so on till the solution is only faintly coloured. Note with what degree of dilution the bands disappear. Then examine each of the solutions with the spectroscope and draw its spectrum.

(b) Make a solution of blood which shows the oxyhamoglobin bands sharply. Add some ammonium sulphide solution to reduce the oxyhamoglobin. Heat gently to about body temperature. A single, ill-clefined band now appears, occupying a position midway between the oxyhamoglobin bands, and the latter disappear. This is the band of reduced hamoglobin (Fig. 7).

(c) Carbonic Oxide Hamoglobin.--Pass coal-gas through blood for a considerable time. Examine some of the blool (after dilution) with the spectroscope. Two bands, almost in the position of the oxyhrmoglobin bands, are seen; but no cliange is caused by the 
addition of ammonium sulphide, since carbonic oxicle hamoglobin is a more stable compound than oxyhremoglobin.

(d) Methmeoglobin. - l'ut some blood into a test-tube, add a few clrops of a solution of ferricyanide of potassium, and heat gently. On diluting a well-marked band will be seen in the red. On addition of ammonimm sulphicle this band disappears; the oxyhemoglobin bands are secn for a moment, and then give place to the band of reduced hamoglobin (Iig. 7).

(e) Acid Hamatin.- To a little diluted blood add strong acetic acid and heat gently. The colour becomes brownish. The spectrum shows a band in the red between $\mathrm{C}$ and $\mathrm{D}$, not far from the position of the band of methamoglobin. The addition of a llopl or two of ammonium sulphicle causes no change in the spectrum, and this is a means of distinguishing acid-haematin from methamoglobin. If more ammonium sulphide be alded, hamatin will be precipitated when the acid solution has been rendered neutral, and a further adclition of ammonium sulphicle or sodium hydroxide will cause the hrmatin to be again dissolver, a solution of alkaline haematin being formed. This in its turn may be reduced by an excess of ammonium sulphide, and the spectrum of hæmochromogen may be obtained (Fig. 7).

Since the watery solution of acid hæmatin obtained as above is usually somewhat turbid, a solution in acid ether is sometimes employed for spectroscopic examination. Add to a little undiluted defibrinated blood about half its volume of glacial acetic acid, and then not less than an equal volume of ether. Mix well, pour off the ethereal extract and examine it with the spectroscope, diluting, if necessary, with ether and glacial acetic acid. It shows a strong band in the red somewhat farther from the $D$ line than the

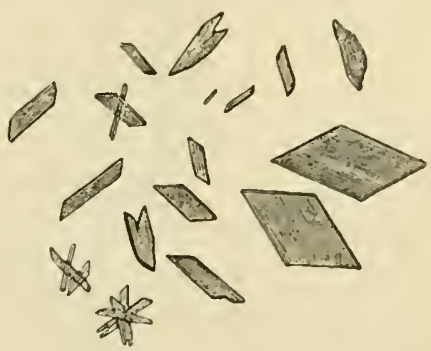

Fig, I6.-CRYSTALS OF HA:MiN (FREY). methremnglobin band. On dilution, three additional fainter bands may be seen.

(f) Alkaline Hamatin.-To diluted blood add strong acetic acid and warm gently for a few minutes. Then, when the spectroscopic examination of a sample shows that acid-hæmatin has been formed, neutralize with sodium hydroxide. A brownish precipitate of hæmatin is thrown down. which dissolves in an excess of sodium hyclroxide, giving a solution of alkaline hæmatin (or alkali-hamatin).

Or add sodium hydroxide to blood directly, and warm for a couple of minutes after the colour has changed decidedly to brownish-black. The spectrum of alkaline hæmatin is a broad but ill-defined band just overlapping the $\mathrm{D}$ line, and situated chiefly to the red side of it (Fig. 7). The solution should be shaken up with air before being examined, as some of the alkali-hrematin is changed into hæmochromogen by reducing substances formed by the action of the alkali on the blood.

(g) Hamochromogen.-To a solution of alkaline hæmatin add a drop or two of ammonium sulphide. The band near D disappears, and two bands make their appearance in the green (Fig. 7).

(h) Hamatoporphyrin.-Put some strong sulphuric acid in a testtube. Add a few drops of blood, agitate the test-tube till the blood 
dissolves, and examine the purple liquid. cliluting it, if necessary. with sulphuric aciol. Its spectrum sliows two well-markerl bands, one just to the left of D. and the other midway between D and E (Fig. 7).

(3) Guaiacum Test for Blood. - 1 test for bloor - much $11 \mathrm{sed}$ in hospitals, and, inclecel, a delicate one, but not always trustworthy unless certain precautions be taken - is the guaiacum test. A (lrop) of freshly-prepared tincture of guaiacum is adeled to the liquisl to be tested, and then peroxide of hyolrogen. If blood be present. the guaiacum strikes a blue colour. The decomposition of the peroxide by the blood is due mainly to the hatmoglobin of the corpuscles. Any derivative of hamoglobin which still contains the iron will act. and boiling does not abolish this power. ()n the other hancl. oxydases or oxidlizing ferments present not only in the formed elements

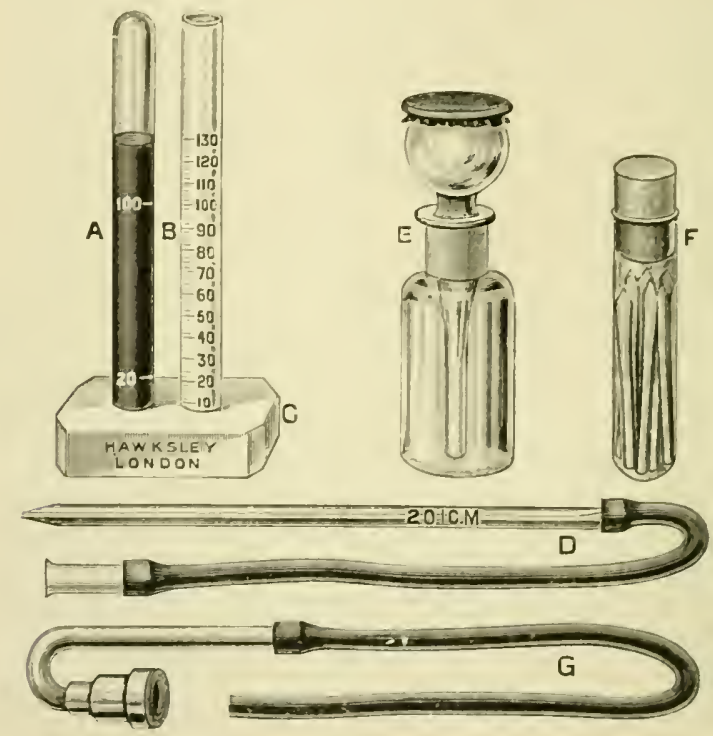

Fig, r7.-Haldane's Monhication of Gowers' Hamoglobinometer.

of blood but elsewhere, e.g.. in fresli vegetable protoplasm, milk, seminal fluicl. and pus, will cause the same colonr (p. 26f), but not if they have been previously boiled.* The test has been

* The formed elements of blood rably contain no less than three ferments of interest in this connection: (1) I catalase which decomposes peroxide of hydrogen into water and molecular oxygen (i.e., oxygen not in the atomic or nascent state). This reaction is given by both bloor! and pus. (2) An oxydase (also spelled oxiclase), which oxilises gua iacum and similar substances without the presence of hydrogen peroxide. This reaction is obtainable eren from acfucous extracts of lencocytes. (3) A peroxydase falso spelled peroxirlase) which canses the oxirlation of these substances only in the presence of hyclrogen peroxide, a reaction also given by leucocytes. These ferments are all inactivated by boiling (Kiastle). 
considered chiefly of value as a negative test. When the blue colour is not obtained, we have good cridence that blool is absent. But, according to Buckmaster, if the precantion of first boiling the lippinel suspected to contain blood be adopterl, it is also a good positive test.

(1) Quantitative Estimation of Hamoglobin - (a) By Haldane's Molificalion of Cozers' Hemoglobinomeler. - Place in the graduated tube 13 (Fig. I7) an amomnt of water less than will nltimately be repuired to dilute the blool to the required tint. Puncture the finger or lobe of the ear with one of the small lancets in $\mathrm{F}$, and fill the capillary pipette D to a little beyond the mark 20. Wipe the point of the pipette and dab it on a piece of filter-paper till the blood stands exactly at the mark. Blow the blood into the water in [3, and rinse the jipette with the water. Attach the cap of tube (; to a gas-burner. Introrluce the rubber tube into 13 nearly to the level of the water, and allow gas to pass for a few seconds. Witl-

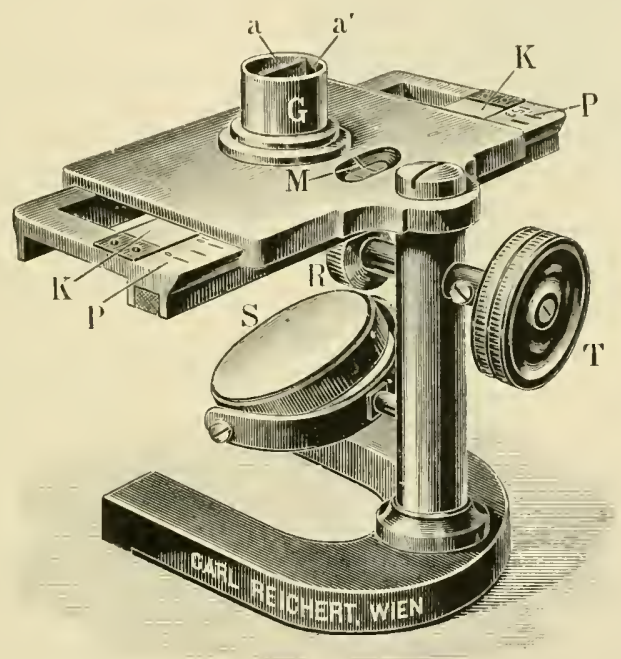

Fig. I8.-FLeischl's Hziometer.

draw the tube while the gas is still passing. Immediatcly close the end of $B$ with the finger, and move the tube so that the liquid passes from end to end of it at least a dozen times, to saturate the hremoglobin with carbonic oxide. While this is being done, the tube should be held in a cloth, otherwise it will become heated, and liquid will spurt out when the finger is removed. Water is now added drop by drop with the pipette stopper of the bottle E, which is used for holding the water, the tube being inverted after each addition, till the tint in $B$ is the same as that in $A$. In comparing the tubes, they should be held against the light from the sky or from an opal glass lamp-sharle. It is necessary to transpose the tubes repeatedly. The level at which the tints are equal is read off on $B$ half a minute after the addition of the last drop of water. Water is now again added by drops till the tint in $B$ is just noticeably weaker than in $A$, and the mean of the two readings is taken. 
The result is the percentage actually present of the average proportion of hicmoglobin in the bloos of healthy arlult males. Healthy women give an averige of only 89 per cent., and healthy cliblecn an average of only $\$ 7$ per cent., of the proportion in men. Tlic liquicl in $\Lambda$ is a 1 per cent. solution of blood containing the average percentage of hamoglobin found in the hlood of healthy arlult males, and lusing an oxygen citpacity of 18.5 per cent.-i.e., roo c.c. of the blood with which the standard wats made would take up) in combination from air 18.5 c.c. of oxygen. The solution in $A$ has

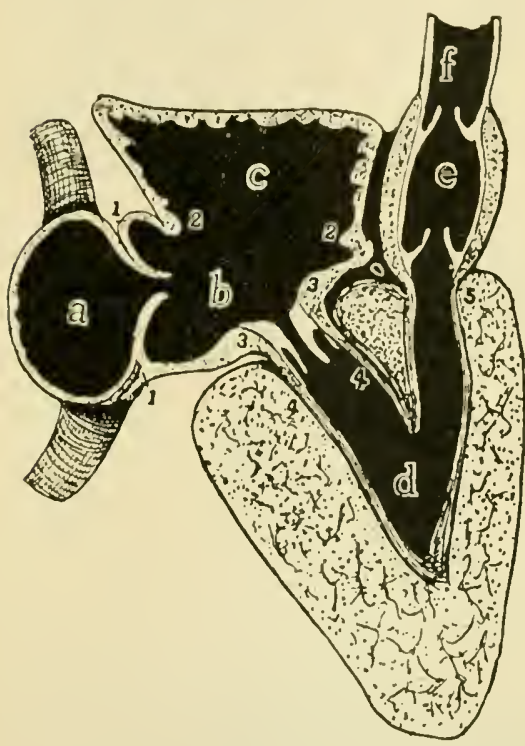

Fici. 19.-J)IAGRAM OF I'RIMITIVE VERTE. BRATE HEART, COMHINING FEATULES FOUN1) IN THE EEL, DOGFISH, AND FROG (FLACK, AFTER KEEITH).

$a$, Sinus venosus; $b$, auricular canal ; $c$, auricle; $d$, ventricle; $c$, bulbus curdis; f. aorta; I-I, sino-auricular junction and venous valves; $2-2$, junction of canal and auricle ; $3-3$, annular part of auricle; 5 , bulboventricular junction. the white surface $S$, aranged below the compartments a and $a$. being illuminated by a lamp. (or the instrument may be placed in a small box, lighted by a candle. It is best that each result should be the mean of two readings. one just too large and the otlicr just too small. In any case the instrmment does not give readings accurate to less than 5 per cent.

(c) Hoppe-Seyler's Method.-Two parallel-sided glass trougls are used. In one is put a standarel solution of oxyliemoglobin of known strength, in the other a measured quintity of the blood to be tested. The latter is diluted with water until its tint appears the 
same as that of the standard solution, when the troughs are placed side by side on white paper. From the quantity of water adcled it is easy to calculate the proportion of hamoglobin in the undiluted blood. (ireater accuracy is obtained if the hemoglobin in the standard solution and that of the blood are converted into carbonic oxide hamoglobin by passing a stream of coal-gas through them.

(d) Tallquist's Method.-In this method the tint prodnced by a (Irop of blood on a piece of white filter-paper is compared with a scale representing 10 percentages of hamoglobin (from io to Ioo per cent.). The standard filter-paper is supplied in the form of a book with the scale. To make an estimation, all that is necessary is to touch a (lrop) of blood with a picce of the filter-paper, and allow the blood to diffuse slowly through the paper, so as to give an even stain. The position of the stain is then determined by the scalc; e.g., it may be deeper than 9o, but fainter than 100 , in which case the percentage of hemoglobin lies between 90 and Ioo. The method is by no means a very accurate one, but more accurate than it appears at first siglit.

(5) Microscopic Test for Blood-pigment.-Put a drop of blood on a slicle. Allow the blood to dry, or heat it gently over a flame, so as to evaporate the water. Add a drop of glacial acetic acid; put on a cover-glass. and again heat slowly till the liquid just begins to boil. Take the slide away from the flame for a few seconds, then heat it again for a moment; and repeat this process two or thrce times. Now let the slicle cool, and examine with the microscope (high power). The small black, or brownish-black, crystals of hæmin will be seen (Fig. I6, p. 67). This is an important test where only a minute trace of blood is to be examined, as in some medico-legal cases. If a bloodstain is old, a minute crystal of sodium chloride should be added along with the glacial acetic acid. Fresh blood contains enough sodium chloride.

A blood-stain on a piece of cloth may first of all be soaked in a small quantity of distilled water, and the liquid examined with the spectroscope or the micro-spectroscope (a microscope in which a small spectroscope is substituted for the eyepiece). Then evaporate the liquid to dryness on a water-bath, and apply the hæmin test. Or perform the hæmin test directly on the piece of cloth. In a fresh stain the blood-corpuscles might be recognized under the microscope. Very few liquids, however, are available for washing out the blood, as all ordinary solutions, and even serum itself, cause laking of dried corpuscles (Guthrie). Absolute alcohol, or 35 per cent. potassium hydroxide, may be used to soak and rub up the cloth in. 


\section{CHAITER II}

\section{THE CIRCULATION OF THE BLOOD AND LYMPH}

THE blood can only fulfil its functions by continual movement. This movement implies a constant transformation of energy; and in the animal body the transformation of energy into mechanical work is almost entirely allotted to a special form of tissue, muscle. In most animals there exist one or more rhythmically contractile muscular organs, or hearts, upon which the chicf share of the work of keeping up the circulation falls.

Comparative.-In Echinus a contractile tube connects the two vascular rings that surround the beginning and end of the alimentary canal, and plays the part of a heart. In the fower crustacea and in insects the heart is simply the contractile and gencrally sacculated dorsal bloodvessel ; in the higher crustacea, such as the lobster, it is a well-clefined muscular sac situated dorsally. A closerl vascular system is the exception among invertebrates. In most of them the blood passes from the arteries into irregular spaces or lacunce in the tissues, and thence finds its way back to the heart. In the primitive vertebrate heart five parts can be distinguished as we procecel from the venous to the arterial end : (1) The sinus venosus, into which the great veins open; (2) the auricular canal, from the clorsal wall of which is developed-(3) the auricle; (4) the ventricle; (5) the bulbus arteriosus, from which the chief artery starts (Fig. 19, p. 70). Amplnioxus, the lowest vertebrate, has a primitive lacunar vascular system ; a contractile clorsal bloodvessel serves as arterial or systemic heart, a contractile ventral vessel as venous or respiratory heart. From the latter. vessels go to the gills. Fishes possess only a respiratory heart, consisting of a venous sinus, auricle, ventricle, and bulbus arteriosus. This drives the blood to the gills, from which it is gathered into the aorta; it has thence to find its way without further propulsion through the systemic vessels. Amphibians have a venous sinus, two auricles. a single ventricle, and an arterial bulb; reptiles, two auricles and two incompletely-separated ventricles. In birds and mammals the respiratory and systemic hearts are completely separatel. The former. consisting of the right auricle and ventricle. propels the blood through the lungs; the latter, consisting of the left auricle and ventricle, receives it from the pulmonary veins, and sends it through the systemic vessels.

The sinus renosus seems 10 be represented in the mammalian heart by certain small portions of tissue, especially the so-called sinoauricular node, a little knot of primitive fibres at the mouth of the 
superior vena cava. The auricular canal is probably repressented by the auriculo-ventricular bundle, which will again be referred to in relation to the conduction of the heart-beat from anricles to ventricles (p. 135). This bumdle starts from a clump of primitive tissue, the anriculo-ventricular nole at the base of the interaricular saptum on the right side. bolow and to the right of the coronary sinus, and runs down the interventricular septum. The sino-auricular and the auriculo-ventricular nodes are connected by fibres which run in the interauricular septum, so that it may be considered that the primitive carliac tube is still represanted from base to apex of the aclult mammalian heart, although only by very slencler threats of tissue, amidist the massive secondary developments of auricular and ventricular muscle (Keith and Flack).

General View of the Circulation in Man.--The whole circuit of the blood is divided into two portions, very distinct from each other, both anatomically and functionally-. the respiratory or lesser circulation, and the systemic or greater circulation. Starting from the left ventricle, the blood passes along the systemic ressels - arteries, capillaries, reins-and, on returning to the heart, is poured into the right auricle, and thence into the right ventricle. From the latter it is driven through the pulmonary artery to the lungs, passes through the capillaries of these organs, and returns through the pulmonary veins to the left auricle and ventricle. The portal system, which gathers up the blood from the intestines, forms a kind of loop on the systemic circulation. The lymph-current is also in a sense a slow and stagnant side-stream of the bloodcirculation; for substances are

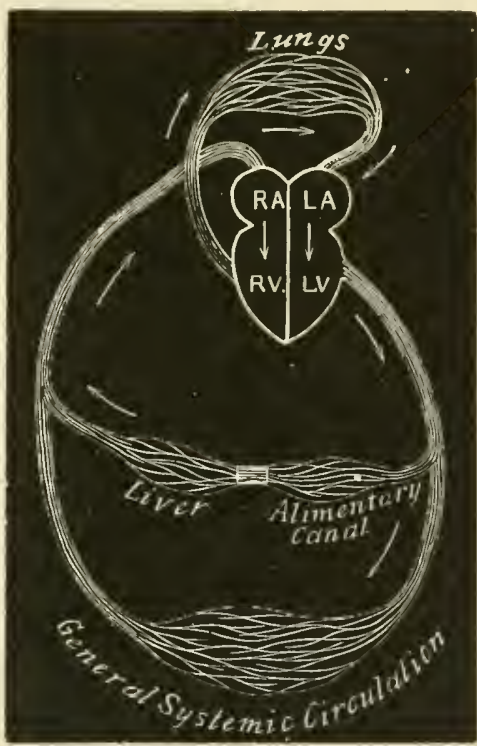

Fig. 20. Diagram òf THE General Course of the Circulation.

RA, LA, right and left auricles; RV LV, right and left ventricles. constantly passing from the bloodvessels into the lymph-spaces, and returning, although after a comparatively long interval, into the blood by the great lymphatic trunks.

Physiological Anatomy of the Vascular System. - The heart is to be looked upon as a portion of a bloodvessel which has been modified to act as a pump for driving the blood in a definite direction. Morphologically it is a bloodvessel ; and the physiological property of automatic rhythmical contraction which belongs to the heart in so eminent a degree is, as has been mentioned (p. 72), an endowment of bloodvessels in many animals that possess no localizerl heart. Even in some mammals contractile bloodvessels occur; the veins of the bat's wing, for example, beat with a regular rhythm, and perform the function of accessory hearts.

The whole vascular system is lined with a single layer of endo- 
thelial cells. In the capillaries nothing else is present; the endothelial layer forms the whole thickness of the wall. In young animals, at any rate, the endothelial cells of the capillaries are capable of contracting when stimnlated; and changes in the calibre of these vessels can be brought about in this way. The walls of the arteries and veins are chiefly made $u p$ of two kinds of tissue, which render them distensible and elastic : non-striped muscular fibres ansl yellow elastic fibres. The muscular fibres are mainly arranged as a circular midelle coat, which, especially in the smaller arteries, is relatively thick. One conspicuous layer of elastic fibres marks the boundary between the middle and inner coats. In the larger arteries elastic lamina are also scattered freely among the muscular fibres of the middle coat. The onter coat is composed chicfly of ordinary comnective tissue. The veins differ from the arteries in lawing thinner walls, with the layers less distinctly markel, anil containing a smaller proportion of non-striped muscle and elastic tissue; although in some veins, those of the pregnant uterus, for instance, and the cardiac ends of the large thoracic veins, there is a greater development of muscular tissue. Further, and this is of prime physiological importance, valves are present in many veins. These are semilunar folds of the internal coat projecting into the lumen in such a direction as to favour the flow of blool towards the heart, but to check its return. In some veins, as the venæ cavæ, the pulmonary veins, the veins of nost internal organs, and of bone, there are no valves; in the portal system they are rudimentary in man and the great majority of mammals. The valves are especially well marked in the lower limbs, where the venous circulation is uphilt. When a valve ceases to perform its function of supporting the column of blood between it and the valve next above, the foundation of varicose veins is laid; the valve immediately below the incompetent one, having to bear up too great a weight of blood, tends to yield in its turn, and so the condition spreads. The smallest veins, or venules, are very like the smallest arteries, or arterioles, but somewhat wider and less muscular. The transition from the capillaries to the arterioles and venules is not abrupt, but may be consiclered as marked by the appearance of the non-striped muscular fibres, at first scattered singly, but gradually becoming closer and more numerous as we pass away from the capillaries, until at length they form a complete layer.

In the heart the muscular element is greatly developed and differentiated. Both histologically and physiologically the fibres scem to stand between the striated skeletal muscle and the smooth muscle. In the mammal the cardiac muscular fibres are generally described as made 1 p of short oblong cells, devoid of a sarcolemma, often branched, and arranged in anastomosing rows, each cell having a single nucleus in the midlle of it. But it has recently been shown that the muscle fibrils run right through the apparent cell boundaries, and form a continuous sheet of tissue anastomosing in every dircction. The fibres are transversely striated, but the stria are not so distinct as in skeletal muscle. A sarcolemmat is not absent, althongh it is more delicate than in skeletal muscle, and perhaps of a different nature. Many fibres pass from one auricle to the other, and from one ventricle to the other.

In the frog's heart the muscular fibres are spindle-shaped, like those of smooth muscle, but transversely striated, like those of slicletal muscle. From the sinus to the apex of the ventricle there is a continnous sheet of nuscular tissue. 
The problems of the circulation are partly physical, partly vital. Some of the phenomena observed in the blood-stream of a living animal can be reproduced on an artificial model; and they may justly be called the physical plienomena of the circulation. Others are essentially bound up with the properties of living tissues; and these may be classified as the vital or physiological phenomena of the circulation. The distinction, although by no means sharp and absolute, is a convenient one-at least, for purposes of description; and as such we sliall use it. But it must not be forgotten that the physiological factors play into the sphere of the physical, and the physical factors modify the physiological. Considered in its physical relations, the circulation of the blood is the flow of a liquid along a system of elastic tubes, the bloodvessels, under the influence of an intermittent pressure produced by the action of a central pump, the heart. But the branch of dynamics which treats of the movement of liquids, or hydrodynamics, is one of the most difficult parts of physics, and, in spite of the labours of many eminent men, is as yet so little advanced that even in the physical portion of our subject we are forced to rely chiefly on empirical methods. It would, therefore, not be profitable to enter here into mathematical theory, but it may be well to recall to the mind of the reader one or two of the simplest data connected with the flow of liquids through tubes:

Torricelli's Theorem.- Suppose a vessel filled with water, the level of which is kept constant; the velocity with which the water will escape from a hole in the side of the vessel at a vertical depth $h$ below the surface will be $v=\sqrt{2 g h}$, where $g$ is the acceleration produced by gravity.* In other words, the velocity is that which the water would have acquired in falling in vacuo through the distance $h$. This formula was deduced experimentally by Torricelli, and holds only when the resistance to the outflow is so small as to be negligible. The reason of this restriction will be easily seen, if we consider that when a mass $m$ of water has flowed out of the opening, and an equal mass $m$ has flowed in at the top to maintain the old level, everything is the same as before, except that energy of position equal to that possessed by a mass $m$ at a height $h$ has disappeared. If this has all been changed into kinetic energy $\mathrm{E}$. in the form of visible motion of the escaping water, then $\mathrm{E}=\frac{1}{2} m v^{2}=m g h, i . e, v=\sqrt{2 g} \hbar$. If, however, there has been any sensible resistance to the outflow, any sensible friction, some of the potential energy (energy of position), will have been spent in overcoming this, and will have ultimately been transformed into the kinetic energy of molecular motion, or heat.

Flow of a Liquid through Tubes. - Next let a horizontal tube of uniform cross-section be fitted on to the orifice. The velocity of outflow will be diminished, for resistances now come into play. When the liquidl flowing through a tube wets it, the layer next the wall of the tube is prevented by adhesion from moving on. The

* I.e., the amount added per second to the velocity of a falling body $(g=32$ feet $)$. 
particles next this stationary layer rub on it, so to speak, and are retarded, although not stopped altogether. The next latyer rubs on the comparatively slowly moving particles outsicle it, and is also delayed, although not so much as that in contact with the immovable layer on the walls of the tube. In this way it comes about that every particle of the liquicl is hindered by its friction against otluers - those in the axis of the tube least, those near the periphery most - and part of the energy of position of the water in the reservoir is used up in overcoming this resistance, only the remainder being transformed into the visible kinctic energy of the liquid escaping from the open encl of the 1 ube.

If vertical tubes be inserted at different points of the horizontal tube, it will be found that the water stands at continually decreasing licights as we pass away from the reservoir towards the (ip)en cuel of the tube. The height of the liguid in any of the vertical tubes indicates the lateral pressure at the point at which it is inserted; in other worls, the excess of potential energy, or encrgy of position, which at that point the liquid possesses as compared with the water at the frec end, where the pressure is zero. If the centre of the cross-

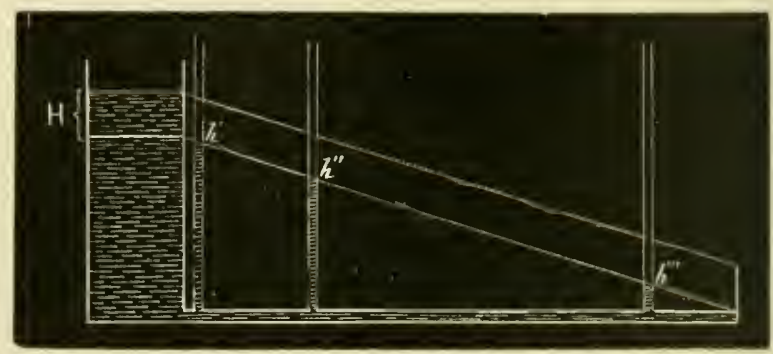

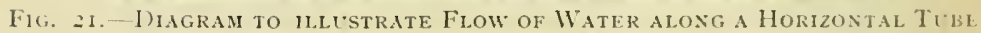
CONNECTED WITH A RESERVOIR.

section of the free curl of the tube be joined to the centres of all the menisci, it will be fomel that the line is at straght line. The litteral pressure at any point of the tube is therefore proportional to its clistance from the free end. Since the sime yuantity of walter must pass through each cross-section of the horizontal tube in a given time as flows out at the open ent, the kinetic encrgy of the lipuid at every cross-section must be constant and equal to $\varliminf_{-2}^{1} v^{2}$. where $r$ is the mean velocity (the quantity which escapes in unit of time dividerl by the cross-section) of the water at the free encl.

Just inside the orifice the total energy of a mass $m$ of water is $m g h$; just beyond it at the lirst vertical tube. mgh' $h^{\prime} m w^{2}$. where $h^{\prime}$ is the lateral pressure. On the assumption that between the inside of the orifice and the first tube, no energy has been transtormed into heat (an assmmption the more nearly correct the smaller the clistance between it and the insicle of the orifice is madc). we have $m g h=m g h^{\prime}$ $+2 m v^{2}$. i.e., $3 m v^{2}=m g\left(h-h^{\prime}\right)$. In other worels. the portion of the energy of position of the water in the reservoir which is transformed into the kinctic energy of the water flowing along the horizontal tube is measured by the difference between the height of the level of the reservoir and the lateral pressure at the beginning of the horizontal tube-that is, the height at which the straight line joining the menisci of the vertical tubes intersects the column of water in the reservoir. Let $H$ represent the height corresponding to that part of 
the energy of position which is transformed into the kinetic energy of the flowing water. H is easily calculated when the mean velocity of efflux is known. For $v=\sqrt{2 g l l}$ by Torricelli's theorem (since none of the cnergy corresponding to $\mathrm{H}$ is supposed to be used up in orercoming friction), or $\mathrm{H}=\frac{v^{2}}{2 g}$. At the sceond tube the lateral pressure is only $h^{\prime \prime}$. The sum of the visible kinetic and potential energy here is therefore $m v^{2}+m g h^{\prime \prime}$. A quantity of energy $m g\left(h^{\prime}-h^{\prime \prime}\right)$ must have been transformed into heat owing to the resistance caused by fluid friction in the portion of the horizontal tube between the first two vertical tubes. In general the energy of position represented by the lateral pressure at any point is equal to the energy used up in overeming the resistance of the portion of the path beyond this point.

Velocity of Outflow. - It has been found by experiment that $v$, the mean velocity of outflow, when the tube is not of very snall calibre, varies directly as the diameter, and therefore the volume of outflow as the cube of the cliameter. In fine capillary tubes the mean velocity is proportional to the square, and the volume of outflow to the fourth power of the diameter (Poiseuille). If, for example, the linear velocity of the blood in a capillary of $10 \mu$ in diameter is ${ }_{2}^{1} \mathrm{~mm}$. per sec., it will be four times as great (or $2 \mathrm{~mm}$. per sec.) in a capillary of $20 \mu$ diameter, and one-fourth as great (or $\frac{1}{8} \mathrm{~mm}$. per sec.) in a capillary of $5 \mu$ diameter, the pressure being supposed equal in all. The volume of outflow per sccond is obtained by multiplying the cross-section by the linear velocity. The cross-section of a circular capillary, $10 \mu$ in diameter, is $\pi\left(5 \times \frac{1}{1000}\right)^{2}=$, say, $1 \pm \frac{1}{50 \pi} \mathrm{sq} . \mathrm{mm}$. The outflow will be,$\frac{1}{2} 50 \times \frac{1}{2}=\frac{1}{2} 500 \mathrm{cub}$. mm. per sec. The outflow from the capillary of $20 \mu$ diameter would be sixteen times as much, from the $5 \mu$ capillary only one-sixteenth as much. Some idea of the extremely minute scale on which the blood-flow through a single capillary takes place, may be obtained if we consicler that for the capillary of ro $\mu$ diameter a flow of $\overline{2} \frac{1}{500}$ cub. mm. per sec. would scarcely amount to $\mathrm{I}$ cub. $\mathrm{mm}$. in six hours, or to $\mathrm{I}$ c.c. in $25^{\circ}$ days.

When the initial energy is obtained in any other way than by means of a 'head' of water in a reservoir-say, by the descent of a piston which keeps up a constant pressure in a cylincler filled with liquidthe results are exactly the same. Even when the horizontal tube is distensible and elastic, there is no difference when once the tube has taken up its position of equilibriım for any given pressure, and that pressure loes not vary.

Flow with Intermittent Pressure.-When this acts on a rigid tube, everything is the same as before. When the pressure alters, the flow at once comes to correspond with the new pressure. Water thrown by a force-pump into a system of rigid tubes escapes at every stroke of the pump in exactly the quantity in which it enters, for water is practically incompressible, and the total quantity present at one time in the system cannot be sensibly altered. In the intervals between the strokes the flow ceases; in other words, it is intermittent. It is very clifferent with a system of clistensible and elastic tubes. During each stroke the tubes expand, and make room for a portion of the extra liquid thrown into them, so that a smaller quantity flows out than passes in. In the intervals between the strokes the distended tubes, in virtue of their elasticity, tend to regain their original calibre. Pressure is thus exerted upon the liquid, and it continues to be forced out, so that when the strokes of the pump succeed each other with sufficient rapidity, the outflow 
becomes continuous. This is the state of affairs in the vascular system. The intermittent action of the heart is toned lown in the elastic ressels to a continuous stealy flow.

The Beat of the Heart.-In the frog's heart the contraction can be seen to begin about the months of the great veins which open into the sinus venosus. Thence it spreads in succession over the sinus and auricles, hesitates for a moment at the auriculo-ventricular junction, and then with a certain sud. denness invades the ventricle. In the mammalian heart the starting-point of the contraction is likewise the mouths of the veins opening into the auricles (especially the superior vena cava). which are richly provided with muscular filores akin to those of the heart. But the wave advances so rapidly that it is difficult to trace in its course a regular progress from base to apex, although the ventricular beat undoubtedly follows that of the auricle, and the capillary electrometer indicates that, in a heart beating normally, the electrical change associated with contraction begins at the base, then reaches the apex (p. 730), and finally passes towards the orifices of the great arteries.

The most conspicuous events in the beat of the heart, in their normal sequence, are: (I) the auricular contraction or systole, (2) the ventricular contraction or systole, each followed by relaxation, (3) the pause. The auricles, into which, and beyond which in to the ventricles, blood has been flowing cluring the pause from the great thoracic veins, contract sharply, the right, perhaps, a little before the left. The contraction begins in the muscular tissue that surrounds the orifices of the veins, so that these, destitute of valves as they are, are functionally, at least, if not anatomically, sealed up for an instant, and regurgitation of blood into them is to a great extent, if not entirely, prevented. Apparently, complete closure of the inferior cava is unnecessary, the pressure of the blood in it being sufficiently high to hinder any important back flow. The action of the circular fibres of the veins in closing their orifices is reinforced by the contraction of a band of muscle (the tania terminalis) in the roof of the right auricle. This band compresses especially the mouth of the superior vena cava. The filling of the ventricles is thus completed; their contraction begins either simultaneously with the relaxation of the auricles or a little later.* The mitral and tricuspid valves, whose strong but delicate curtains liave during the diastole been hanging down into the ventricles and swinging

* It has often been lebated whether any appreciable interval exists between the end of the auricular and the beginning of the ventricular systole of the warm-blooded heart. According to Chauveau, not only is this period (the intersystole) well marked and sharply delimited (in the horse), but it is occupied by a definite series of events, including the contraction of the papillary muscles. 
freely in the entering current of blood, are floated up as the intraventricular pressure begins to rise, so that, in the first moment of the sudclen and powerful ventricular systole, the free elges of their segments come together, and the auriculo-ventricular orifices are completely closed (Fig. 85, p. I90). In the measure in which the pressure in the contracting ventricles increases, the contact of the valvular segments becomes closer and more extensive ; and their tendency to belly into the auricles is opposed by the pull of the chordie tendiner, whose slender cords, inserted into the valves from border to base, are kept taut, in spite of the shortening of the ventricles by the contraction of the papillary muscles. The arrangement and connections of the muscular fibres of the heart are such that during the auricular systole the auriculo-ventricular groove moves towards the base of the heart, while during the systole of the ventricles it moves towards the apex, which constitutes a relatively fixed point on account of the mutual action of the numerous fibres which converge here and constitute the " whorl." The line joining the apex and the origin of the aorta does not shorten when the ventricles contract, but all parts of the heart are drawn towards this line. The apex is, therefore, pushed forwards, while the rest of the ventricular surface is being drawn inwards. During the systole, the ventricles change their shape in such a way that their combined cross-section-which in the relaxed state is a rough ellipse with the major axis from right to left-becomes approximately circular, and they then form a right circular cone. As soon as the pressure of the blood within the contracting ventricles exceeds that in the aorta and pulmonary artery respectively, the semilunar valves, which at the beginning of the ventricular systole are closed, yield to the pressure, and blood is driven from the ventricles into these arteries.

The ventricles are more or less completely emptied during the contraction, which seems still to be maintained for a short time after the blood has ceased to pass out. The contraction is followed by sudden relaxation. The intraventricular pressure falls. The lunules of the semilunar valves slap together under the weight of the blood as it attempts to regurgitate, the corpora Arantii seal up the central chink, and the aorta and pulmonary artery are thus cut off from the heart. Then follows an interval during which the whole heart is at rest, namely, the interval between the end of the relaxation of the ventricles and the beginning of the systole of the auricles. This constitutes the pause. The whole series of events is called a cardiac cycle or revolution (see Practical Exercises, p. I86).

It will be easily understood that the time occupied by any one of the events of the cardiac cycle is not constant, for the 
rate of the heart is variable. If we take about 70 beats a minute as the average normal rate in a man, the ventricular systole will occupy about 0.3 second ; the diastole, ${ }^{*}$ including the ventricular relaxation, about 0.5 second. The systole of the auricle is onethird as long as that of the rentricle.

This rhythmical beat of the heart is the ground phenomenon of the circulation. It reveals itself by certain tokens-sounds, surface-movements or pulsations, alterations of the pressure and velocity of the blood, changes of volume in parts-all periodic phenomena, continually recurring with the same period as the heart-beat, and all fundamentally connected together. And if we hold fast the idea that when we take a pulse-tracing, or a blood-pressure curve, or a plethysmographic record, we are really investigating the same fact from different sides, we shall be able, by following the cardiac rhythm and its consequences as far as we can trace them, to hang upon a single thread many of the most important of the physical phenomena of the circulation.

The Sounds of the Heart.- When the ear is applied to the chest, or to a stethoscope placed over the cardiac region, two sounds are heard with every beat of the heart; they follow each other closely, and are succeeded by a period of silence. The dull booming 'first sound' is heard loudest in a region which we shall afterwards have to speak of as that of the "cardiac impulse' (p. 82) ; the short, sharp, 'second sound' over the junction of the second right costal cartilage with the sternum.

There has been much discussion as to the cause of the first sound. That a sound corresponding with it in time is heard in an excised bloodless heart when it contracts, is certain; and therefore the first sound cannot be exclusively due, as some have asserted, to vibrations of the auriculo-ventricular valves when they are suddenly rendered tense by the contraction of the ventricles, for in a bloodless heart the valves are not stretched. Part of the sound must accordingly be associated with the muscular contraction as such.

Again, the fact that the first sound is heard during the whole, or nearly the whole, of the rentricular sistole is against the idea that it is exclusively due to the vibrations of membranes like the valves, which would speedily be damped by the blood and rendered inaudible. But while there is good reason to believe that the vibration of the suddenly-contracted rentricles is the fundamental factor, the shock sets up ribrations also in the blood, the chest-wall, and perhaps the resonant tissue of the lungs. Further, as we shall see later on (p. 660), the sound caused by a contracting muscle readily calls forth sympathetic resonance in

* The term 'diastole' is variously used, as meaning the pause, the pause plus the period during which relaxation is occurring, or the period of relaxation alone. W'e shall employ it in the second sense. 
the ear, and the peculiar booming character of the first sound may be due to the superposition of these various resonance tones upon the muscular note. But, in addition, the vibration of the auriculo-ventricular valves undoubtedly contributes to the production of the sound, and some observers have been able to distinguish in the first sound the valvular and the muscular elements, the former being higher in pitch than the latter, but a minor third below the second sound. In the excised empty heart the deeper tone of the first sound is alone heard, while the higher note is elicited when in a dead heart the auriculoventricular valves are suddenly put under tension (Haycraft) When the initral valve is prevented from closing by experimental division of the chordæe tendinex, or by pathological lesions, the first sound of the heart is altered or replaced by a ' murmur.' This evidence is not only important as regards the physiological question, but of great practical interest from its bearing on the diagnosis of cardiac disease. It may be added that the point of the chest-wall at which the first sound is most easily recognised is also the point at which a changed sound or murmur connected with disease of the mitral valve is most distinctly heard. The sound is, therefore, best conducted from the mitral valve along the heart to the point at which it comes in contact with the wall of the chest. Changes in the first sound connected with disease of the tricuspid valve are heard best, in the comparatively rare cases where they can be distinctly recognised, in the third to the fifth interspace, a little to the right of the sternum.

The second sound is caused by the vibrations of the semilunar valves when suddenly closed, 'the recoiling blood forcing them back, as one unfurls an umbrella, and with an audible check as they tighten' (Watson). The sharpness of its note is lost, and nothing but a rushing noise or bruit can be heard, when the valves are hooked back and prevented from closing. It is altered, or replaced by a murmur when the valves are diseased. As there is a mitral and a tricuspid factor in the first sound, so there is an aortic and a pulmonary factor in the second. The place where the second sound is best heard (over the junction of the second right costal cartilage and sternum) is that at which any change produced by disease of the aortic valves is most easily recognised. The sound is conducted up from the valves along the aorta, which comes nearest to the surface at this point. Changes connected with disease of the pulmonary valves are most readily detected over the second left intercostal space near the edge of the sternum, for here the pulmonary artery most nearly approaches the chest-wall. The first sound is 'systolic '-that is, it occurs during the ventricular systole; the 
second is 'diastolic,' loeginning at the commencement of the diastole.

The Cardiac Impulse.- $A$ surface-movement is seen, or an impulse felt, at every carliac contraction in various situations where the heart or arteries approach the surface. The pulsation, or impulse, of the heart, often styled the apex-beat, is usually most distinct to sight and touch in a small area lying in the fifth left intercostal space, between the mammary and the parasternal line, ${ }^{*}$ and generally, in an adult, about an inch and a half to the sternal side of the former. It is due to the systolic hardening of the ventricles, which are here in contact with the chest-wall, the contact being at the same time renclered closer by their change of shape, and by a slight movement of rotation of the heart from left to right during the contraction (Practical Exer-

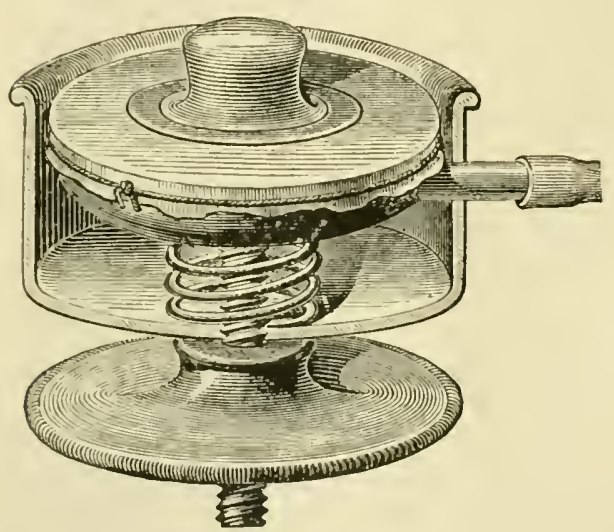

Fig. 22.-Diagram of Marey's Cardiograpit. cises, p. I9I). When the left ventricle is in contact with the chest at the position of the apex-beat, as is usually. the case, an important element in the impulse is the actual forward thrust of the apex. When the apex-beat corresponds in position with the right ventricle, there is no actual forward movement, although the hardening of the ventricle may be felt as a thrust by the finger. Even in health the position of the impulse varies somewhat with the position of the body and the respiratory movements. In children it is usually situated in the fourth intercostal space. In disease its displacement is an important cliagnostic sign, and may be very marked, especially in cases of effusion of fluid into the pleural cavity. It is sometimes, though not invariably, a little lower in the standing than in the sitting position. and shifts an inch or two to the left or right when the person lies on the corresponding side.

Various instruments, called cardiographs, have becn devised for magnifying and recording the movements produced by the cardiac

* The mammary line is an imaginary vertical line supposed to be drawn on the chest through the middle point of the clavicle. It usually, but not necessarily, passes through the nipple. The parasternal line is the vertical line lying midway between the mammery line and the corresponding border of the sternum. 
impulse. Marey's carcliograph (Fig. 22) consists essen tially of a small chamber, or tambour, filled with air, and closed at one enil by a flexible membrane carrying a button, which can be arljusted to the wall of the chest. This receiving tambour is connected by a tube with a recording tambour, the flexible plate of which acts upon a lever writing on a travelling surface-a miformly-rotating clrum, for example-covered with smoled paper. Any movement communicated to the button forces in the end of the tambour to which it is attached, and thus raises the pressure of the air in it and in the recording tambour; the flexible plate of the latter moves in response, and the lever transfers the movement to the paper. The tracing, or carliogram, obtained in this way shows a small elevation corresponding to the auricular systole, succeeded by a large abrupt rise corresponding to the beginning of the first sound, and caused by the ventricular systole. This ventricular elevation is the essential portion of the curve; it is alone felt by the palpating hand, and the auricular clevation is often absent from the carcliogram in man. The rise is maintained, with small secondary oscillations, for about 0.3 of a second in a tracing from a normal man, then gives way to a sucken clescent, that marks the relaxation of the ventricles, the beginning of the second sound, and the closure of the semilunar valves. An interval of about 0.5 sccond elapses before the curve begins again to rise at the next auricular contraction.

Such was the interpretation which Chauveau and Marey put upon their tracings. Although neither their results nor their deductions from them have escaped the criticism of succeeding investiga-

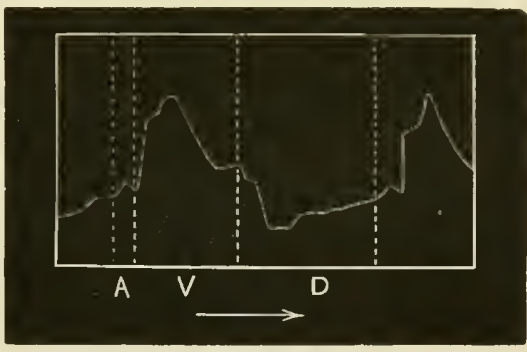

FIG. 23.-CARDIOGRAM TAKEN WITH MAREY'S CARDIOGRAPH.

$A$, auricular systole; $V$, ventricular systole; D, diastole. The arrow shows the direction in which the tracing is to be read. tors, it is doubtful whether any adequate reason has been brought forward for discarding them, and Chauveau has recently furnished fresh proofs of their accuracy. The difficulties that beset the subject are great, for the cardiogram is a record of a complex series of events. The very rapid variation of pressure within the ventricles, the change of volume and of shape of the heart, the slight change of position of its apex, must all leave their mark upon the curve, which is besides distorted by the resistance of the elastic chest-ivall, the inertia of the recording lever, and the compression of the air in the connecting tubes. It is only by comparing in animals the cardiographic record with the changes of blood-pressure in the heart and arteries that our present degree of knowledge of the human cardiogram has becn attained. Could we register directly the fluctuations of pressure in the interior of the human heart, the cardiographic method would be rarely employed. For clinical purposes the receiving tambour can be advantageously replaced by a small glass funnel or a small metal cup, the open end of which is applied without a membrane over the cardiac impulse, the stem being connected with the recording tambour. In 
cases in which the right ventricle is in contact with the chest-wall at the position of the apex-beat the carliogram is 'inverted' - that is to say. the chest-wall is drawn in cluring systole and protruded cluring rliastole of the ventricles. Inversion of the cardiogram is, therefore, not an infallible sign of the pathological condition known as adherent pericardium (Mackenzic).

Endocardiac Pressure. - The function of the heart is to maintain an excess of pressure in the aorta and pulmonary artery sufficient to overcome the friction of the whole vascular channel, and to keep up the flow of blood. So long as the semilunar valves are closed, most of the work of the contracting ventricles is expended in raising the pressure of the blood within them. At the moment when blood begins to pass into the arteries, nearly all the energy of this blood is potential; it is

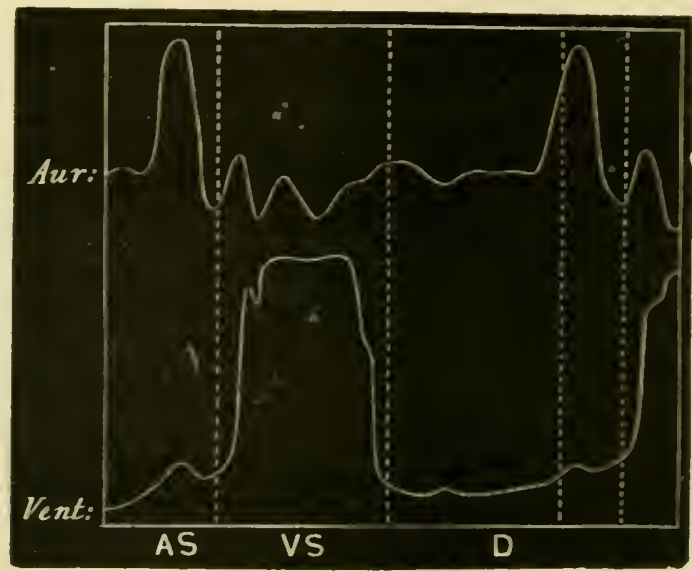

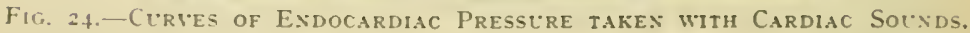

A ur., auricular curve ; I'ent., ventricular curve ; AS, period of auricular systole, including relaxation : VS, of ventricular systole, including relaxation ; D, pause.

the energy of a liquid under pressure. During a cardiac cycle the pressure in the cavities of the heart, or the endocardiac pressure, varies from moment to moment, and its variations afford important data for the study of the mechanics of the circulation.

For the study of the endocardiac pressure, the ordinary mercurial manometer (p. 101) is unsuitable, since, owing to the relatively great "tmount of work required to produce a given displacement of the mercury, it does not readily follow rapid changes of pressure, and the mercurial column, once displaced, continues for a time to execute vibrations of its own, which are compounded with the true oscillations of blood-pressurc. But by introducing in the connection between the manometer and the heart a valve so arranged as to oppose the passage of blood towards the heart, while it favours its 
passage towards the manometer, the maximm pressure attained in the carliac cavities during the cycle may be measured with considerable accuracy. When the valve is reversed the apparatus becomes a minimum manometer. In this way it has been found that in large dogs the pressure in the left ventricle may rise as high as 230 to 240 mml of mercury, and sink as low as -30 to $-50 \mathrm{~mm}$. ; while in the right ventricle it may be as mucl as $70 \mathrm{~mm}$, and as little as $-25 \mathrm{~mm}$. In the riglit auricle a maximum pressure of $20 \mathrm{~mm}$. of mercury has been recorded, and a minimum pressure of - Io $\mathrm{mm}$. or even less. But these results were obtained under somewhat exceptional experimental conditions, and the normal maximum pressures in the heart cavitics in man are prob. ably not so high, especially in the right auricle and ventricle.

Our knowledge of the maximum and minimum pressure attained in the cavities of the heart, even if it were far more precise than it actually is, would only carry us a little way in the study of the endocardiac pressure-curve, for it would merely tell us how far above the baseline of atmospheric pressure the curve ascends, and how far below the base-line it sinks. To exhaust the problem, we require to have tracings of the exact form of the curve for each of the cavities of the heart, and to know the time-relations of the curves so as to be able to compare them with each other, and with the pressure-curves of the great arteries and great veins. To obtain satis-

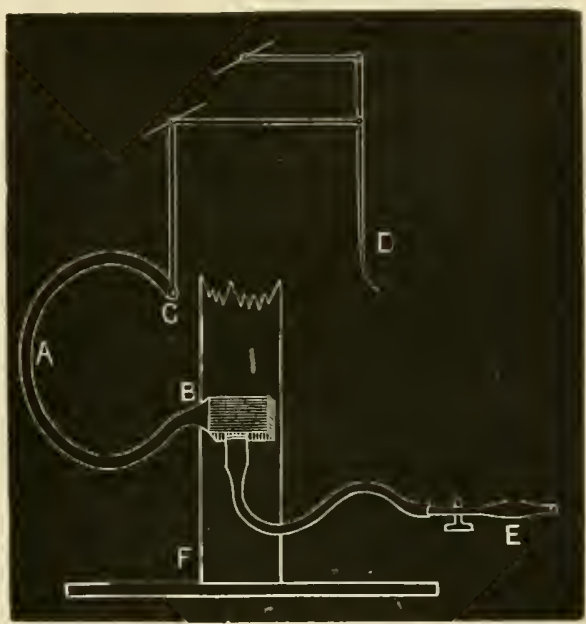

Fig. 25.-Diagran OF Fick's C-spring MANOMETER.

A, hollow spring filled with alcohol. Its open end $\mathrm{B}$ is covered with a membrane and is fixed to the upright $\mathrm{F}$; the other end $\mathrm{C}$ is free to move, and is connected with a sy'stem of levers, which move the writing point $\mathrm{D}$; $\mathrm{E}$ is the cannula, which is connected with the bloodvessel. When the pressure in the spring is increased it tends to straighten itself.

factory tracings of the

swiftly-changing endocardiac pressure is a task of the highest technical difficulty, and it is only in very recent years that it has been accomplished with any approach to accuracy by the use of elastic manometers, in which the blood-pressure is counterbalanced, not by the weight of a column of liquid, as in the mercurial manometer, but by the resistance to compression of a small column of air or the tension of an elastic disc or of a spring. One of the earliest of these was the now somewhat obsolete C-spring manometer of Fick, of which a diagram is given in Fig. 25. Examples of the most perfect elastic manometers of the modern type are the improved instrument of Fick (Fig. 26), with the various modifications it has undergone, and especially the manometers of Hürthle. 
Iïrthle's spring manometer consists of a small drum covered with an indiarubber membrane, loosely arranged so as not to vibrate with a period of its own. The drum is connected with the heart or with a vessel, and the blool-pressure is transmitted to a stecl spring by means of a light metal disc fastened on the membrane. The spring acts on a writing lever. The instrument is so constructed that for a given change of pressure the quantity of liquid displaced is as small as possible, and it is on this that its capacity to follow sudien variations of pressure chicfly depends. The manometer is connected with the cavity of the heart by an appropriately curved camnula of metal or glass, which, after being filled with some liquid that prevents coagulation (Practical Exercises, p. 195), is puslied through the jugular vein into the right auricle or ventricle, or through the carotid artery and aorta into the left ventricle. Some observers fill only the cannula with fluid, and leave the capsule of the elastic manometer and as much of the connections as possible full of air. Others fil the whole system with liquid. And around the question of the relative merits of 'transmission' by liquid and by air has raged a

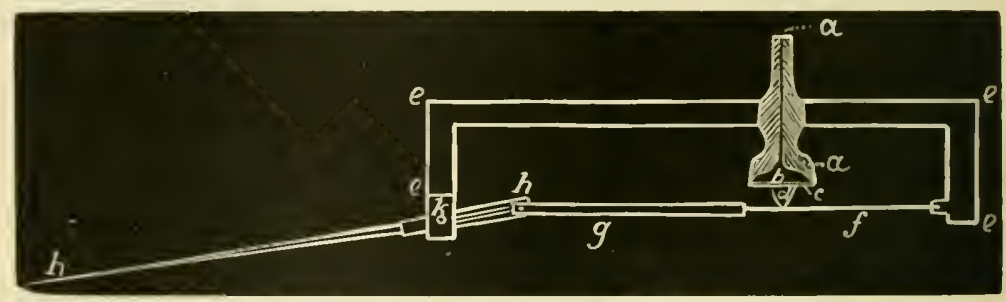

Fig. 26.-Fick's Elastic MANOMEter.

$a, a$ is a metal piece tunnelled by a narrow canal of about $\mathrm{I} \mathrm{mm}$. in diameter which enlarges below to a shallow saucer-shaped space $b$. The wide opening of $b$ is covered by a thin piece of indiarubber $c$, to the centre of which an ivory button $d$ is at tached. The button presses on a strong steel spring $f$, which is attached at one end to the brass frame $e, c$, and at the other, by means of an intermediatc piece $g$, to the lever $h ; b$ is filled with a few drops of water, but the canal $a$ contains only air. When $a$ is connected with the interior of the heart or of an artery, the changes of pressure are transmitted to the spring, and recorded by the writing-point of the lever.

controversy which, however, now shows signs of coming to an end. For there is reason to suppose that the character of the curves obtained is modified among other circumstances by the manner in which the pressure is transmitted.

Thus, the pressure-curve of the ventricle, according to most of those who lave employed manometers with liquid transmission and small inertia of the moving parts (Fig. 27), remains after the first abrupt rise, which undoubtedly corresponds to the ventricular systole, almost parallel to the abscissa line for a considerable time, and then descends somewhat less suddenly than it rose. This systolic 'plateau, although usually broken by minor heights and hollows, which may be partly due to inertia oscillations of the liquid or the recording apparatus, would indicate 
that the ventricular pressure, after reaching its maximum, maintained itself there thronghont the greater part of the systole. The tracings yielded by most of the manometers with air transmission (Fig. 2S) show the same suddenness in the first part of the upstroke and the last part of the descent-that is, the same abmutness in the beginning of the contraction and the end of the relaxation. But they differ totally in the intermediate portion of the curve, which, climbing ever more grachally as it nears its apex, remains but a moment at the maximum, then immediately descending forms a 'peak,' and not a plateau.

Without entering further into a technical discussion, we may say the bulk of the evidence goes to show that the plateau is

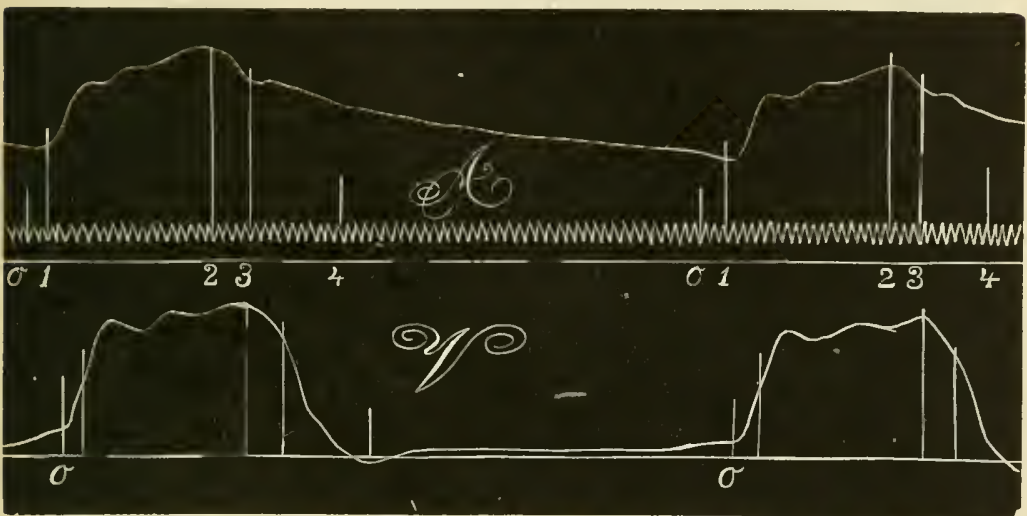

Fig. 27.-Sinultaneous Record of Pressure in Left Ventricle (V) and Aorta (A). (Ḧ̈RThle.)

The traciugs were taken with elastic manometers ; $o$ indicates a point just before the closure of the mitral valve; $I$, the opening of the semilunar valve; 2 , begin. ning of the relaxation of the ventricle; 3 , the closure of the semilunar valve; 4, the opening of the mitral valve. The ventricular curve shows a 'plateau.'

not, as the advocates of the peak have claimed, an artificial phenomenon, but does in reality correspond to that continuation of the systole of the ventricle, that dogged grip, if we may so phrase it, which it seems to maintain upon the blood after the greater portion of it has been expelled.

This conclusion is essentially in accordance with the results of Chauveau and Marey, obtained long ago by means of their "cardiac sound,' which was in principle an elastic manometer (Fig. 29).

It consisted of an ampulla of indiarubber, supported on a framework, and communicating with a long tube, which was connected with a recording tambour. The ampulla was introduced into the heart through the jugular vein or carotid artery in the way already described. Sometimes a double sound was employed, armed with two ampullæ, placed at such a distance from each other that when 
one was in the right ventricle the other was in the auricle of the same side. Each ampulla communicated by a separate tube in the common stem of the instrument with a recorcling tambour, and the writing points of the two tambours were arranged in the same vertical line. When any change in the blood-pressure takes place, the degree of compression of the ampulla is altered, and the change is transmitted along the air-tight connections to the recorling tambours. Simultancous records of the changes in the blood-pressure in the right auricle and ventricle obtained in this way indicate a sudden rise of the auricular pressure corresponding with the auricular systole, followed by a sudden fall (Fig. 24). This is represented on the ven-

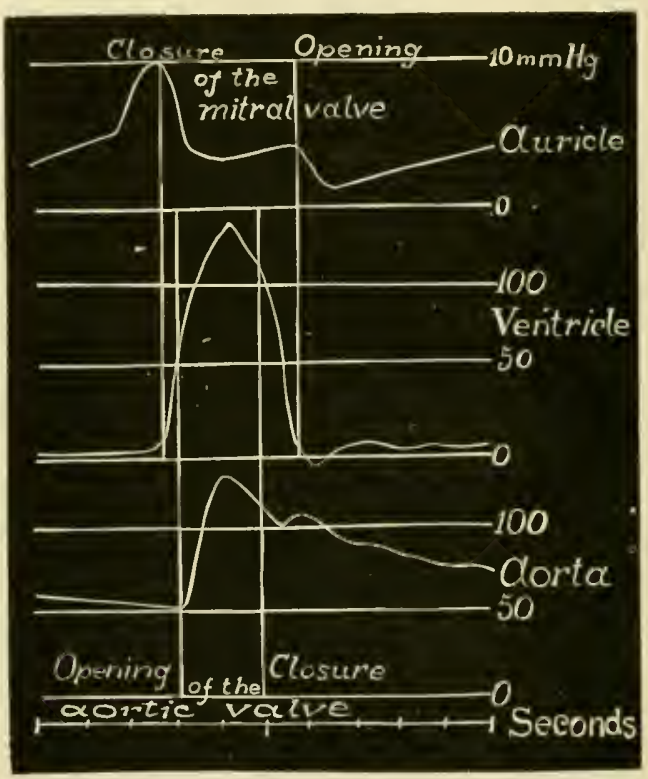

Fig. 28.-COMPARISON OF PRESSURE - CURVES OF Left Auricle, Left Ventricle, AND AOrta (v. FREY).

Recorded by elastic manometers with air trans. mission. The ventricular curve shows a 'peak.' iricular curve by a smaller elevation. which shows that the pressure in the ventricle lias been raised somewhat by the blood driven into it from the auricle. Then follows imme. dialcly a great and abrupt increase of ven tricular pressure, the result of the systole of the ventricle. The beginning of this clcvation is synchronous with the begimning of the first sound ; it remains for some time at the maximum, and then the curve sud. aenly sinks as the ventricle relaxes. On the descending limb there is a slight elevation, lue, as Marcy siI)posed, to the closure of the semilunar valves, which causes a better-marked and simultancous clevation in the curve of aortic pressure when
passed into the aorta this is registered by means of a sound passed into the aorta through the carotid artery. Both the auricular and ventricular curves now begin again to rise slowly, showing a gradual increase of pressure as the blood flows from the great veins into the auricle, and through the tricuspid orifice into the ventricle. This slow rise continues till the next auricular systole.

It is probable that some of the smaller elevations on the curves of Chauveau and Marey, and particularly that which they associated with the closure of the semilunar valves, were due to the oscillations of their apparatus. For it is a remarkable 
fact that on most of the endocardiac pressure tracings of the best modern manometers, whether the curves belong to the type of the peak or of the plateau, no sudden change of curvature, no nick, or crease, or undulation reveals the moment of opening or closure of any valve. But by experimentally graduating a pair of elastic manometers, and obtaining with them simultaneous records of the pressure in auricle and ventricle, or by using a ' differential' manometer, in which the pressures in two cavities are opposed to each other, so that the movement of the membrane corresponds to their difference, we can calculate at what points of the ventricular curve the pressure is just greater than and just less than the pressure in the auricle. The first point, it is evident, will correspond to the instant at which the mitral or tricuspid valve, as the case may be, is closed, and the second to the instant at which it is opened. And in like manner, by comparing the pressure-curve of the aorta with that of the left ventricle, the moment of opening and closure of the semilunar valves may be determined (Figs. 27 and 28 ). According to the best observations, the closure of the semilunar valves takes place at a time corresponding to a point on the upper

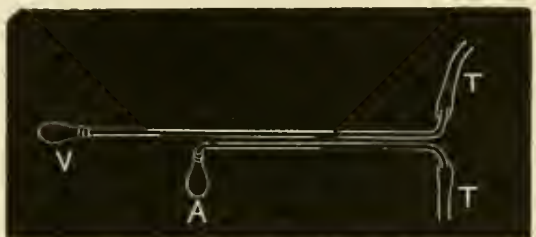

FIG. 29.-DIAGRAM OF CARDIAC SOUND FOR Simlltaneols Registration of ENDoCARDiaC Pressure ix AURICle aNd VEN. TRICLE.

A, elastic ampulla for auricle: $V$, for verltricle; $\mathrm{T}$, tubes connected with recording tambours.

portion of the descending limb of the intraventricular curve.

On the blood-pressure curve of the aorta, simultaneously registered. the corresponding point is near the bottom of the so-called 'aortic' notch (p. 96) which precedes the dicrotic elevation. For clinical purposes, in man the moment of closure of the semilunar valves (denoted by the abbreviation S.C. point) may be taken as $0^{\circ} 03$ second before the bottom of the aortic notch in sphygmographic tracings from the carotid, this being approximately the average time taken by the pulse-wave in travelling from the aorta to the carotid. The S.C. point, the A.O. point, or moment of opening of the auriculoventricular valves, and the beginning of the ventricular systole, are three important points of reference in the measurement and interpretation of pulse-tracings in clinical work. The A.O. point in man may be taken as a point ' $\mathrm{O}^{\circ} \mathrm{O} 3$ second in advance of the summit of the dicrotic wave' on the carotid pulse-tracing (Lewis). But this is the most difficult of the three standard points to determine clinically with anything like accuracy.

The study of the curves of endocardiac pressure enables us to add precision in certain points to the description of the events 
of the cardiac cycle which we have already given, and, as regards the ventricles, to divide the cycle into four periods :

(I) A period during which the pressure is lower in the ventricles than either in the auricles or the arteries, and the auriculo-ventricular valves are consequently open, and the semilunar valves closed. This is the period of 'filling' of the heart, or the pause.

(2) A period, beginning with the ventricular systole, during which the pressure is increasing abruptly in the ventricles, while they are as yet completely cut off from the auricles on the one hand and the arteries on the other by the closure of both sets of valves. This is the period of 'rising pressure,' during which the ventricles are, so to say, 'getting up steam.' The interial between the beginning of the rentricular systole and the opening of the semilunar valves is termed the 'presphygmic' interval.

(3) A period during which the pressure in the ventricles or'ertops that in the arteries, and the semilunar valves are open, while the auriculo-ventricular valves remain shut. This is the period of 'discharge' or 'sphygmic' period.

(t) A period during which the pressure in the ventricles is again less than the arterial, while it still exceeds the auricular pressure, and both sets of valves are closed. This is the period of rapid relaxation. The interval between the closure of the semilumar and the opening of the auriculo-ventricular valves is sometimes called the "postsphygmic' interval.

Of the four periods, the second and fourtl are exceedingly brief. The third is relatively long and constant, being but slightly dependent on either the pulse-rate or the pressure in the arteries. The duration of the first period varies inversely as the frequency of the heart; with the ordinary pulse-rate it is the longest of all.

From records taken in a person with a defect in the chest-wall which renclered the heart accessible the following results were obtained as to the duration of the various events of the cardiac cycle : First and fourth periods together, $0^{*} 445$; third period, $0^{*} 254$; second period (presphygmic interval), $0^{\circ} \mathrm{O} 5 \mathrm{I}$ seconcl, the pulse-rate being so a minute (Tigerstedt). In another case with a similar defect the first period lasted 0.32 , the fourth period (post-sphygmic interval) $0^{\circ} \circ 6$, the second and third periods together $0_{4}$, and the auricular systole 0.1 second, the pulse-rate being 66 .

The fluctuations of pressure in the auricles, although confined within narrower limits than in the ventricles, are of equal interest. They have been studied of late years in considerable detail both in animals and by indirect methods in man. No fewer than three distinct elevations or ' positive waves,' separated or followed by three depressions or 'negative waves,' have been described on the curve of intra-auricular pressure (Fig. 24). The 
furst elevation corresponds to the systole of the auricle. The second coincides with the onset of the ventricular systole, and is, perhaps, due to the sudden bulging of the auriculo-ventricular valve into the auricle, or even to a slight regurgitation of blood from the ventricle througl the valve before it has completely closed. The cause of the third elevation, which occurs during the period occupied in the ventricular pressure-curve by the plateau, is less clearly made out. In man, the events taking place in the right auricle during its systole can be followed to some extent by recording the venous pulse in the jugular veins, especially the internal jugular, at the root of the neck (Fig. 30). Successful tracings can be obtained, not onlyin certain pathological conditions, but in many nomal individuals, and it is probably

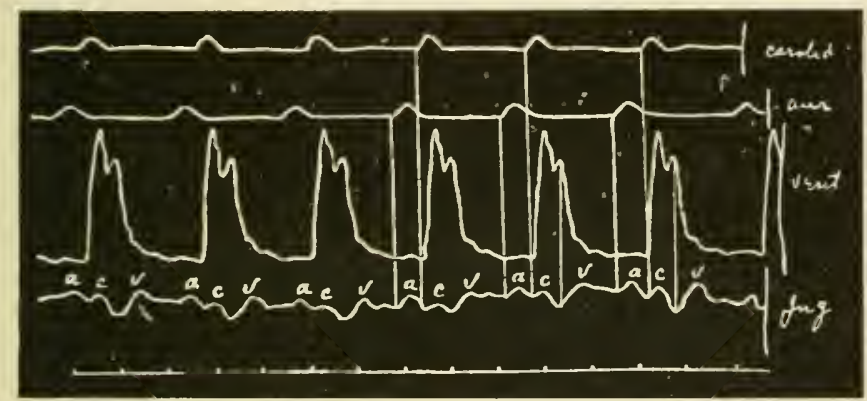

Fig. 30.-Simtltaneots Record of Jugtlar Pulse, Ventrictlar Contrac. tion, Alricllar Contraction, and Carotid Plise ix Dog (Cushay and GROSH).

$a, c, v$, the three elevations of the jugular pulse. Time-trace, fifths of a second.

only a matter of improved technique to obtain them in all. The jugular venous pulse-tracing, like the intra-auricular pressurecurve, shows in general three well-marked elevations and three depressions, and there is good evidence that, broadly speaking, these features of the jugular curve correspond as regards their origin with the changes of pressure in the auricle. Some difference of opinion exists as to how the changes of pressure in the auricle are propagated into the reins, although there seems to be little reason to suppose that in normal persons any actual regurgitation of blood takes place. It is also a debatable question how far pulsation transmitted from the great arteries of the thorax and neck may affect the jugular tracing. But be this as it may, the jugular curve, when properly interpreted, affords valuable information as to the action of the auricle, information of the same kind as that afforded by the arterial pulse-tracing and the cardiogram as to the action of the ventricle. The 
student must, however, be warned that the proper interpretation of such tracings in the study of cardiac disease requires special knowledge and training.*

We have already said that a negative pressure may be detected in the carliac cavities by means of a special form of mercurial manometer. This is confirmed by an examination of the tracings written by good elastic manometers, for the curves of both ventricles may often kescend below the line of atmospheric pressure. The cause of this negative pressure has been much discussed. In part it may be ascribed to the aspiration of the thoracic cage when it expands during inspiration (1). 210). But since the pressure in a vigorously-beating heart may still become negative, when the thorax has been openerl, and the influence of the respiratory movements eliminated, we must conclude that the recoil of the somewhat narrowed, or at least distorted, auriculo-ventricular rings, and of elastic structures in the walls of the ventricles, exerts of itself a certain suction upon the blood. This, however, is not an important factor in the maintenance of the circulation.

The Arterial Pulse.-At each contraction of the heart a quantity of blood, probably varying within rather wide limits (p. I 27), is forced into the already full aorta. If the walls of the bloodvessels were rigid, it is evident (j). 77) that exactly the same quantity would pass at once from the veins into the right auricle. The work of the ventricle would all be spent within the time of the systole, and only while blood was being pumped out of the heart would any enter it. Since, however, the vessels are extensible, some of the blood forced into the aorta during the systole is heaped up in the arteries, beyond which, in the narrow arterioles and in the capillary tract, with its relatively great surface, the chief resistance lies. The arteries are accordingly distended to a greater extent than before the systole, and, being elastic, they keep contracting upon their contents until the next systole over-distends them again. In this way, during the pause the walls of the arteries are executing a kind of elastic systole, and driving the blood on into the capillaries. The work done by the ventricle is, in fact, partly stored up as potential energy in the tense arterial wall, and this energy is being continually transformed into work upon the blood during the pause, the heart continuing, as it were, to contract by proxy during its diastole. Thus, the blood progresses along the arteries in a series of waves, to which the name of 'blood-waves' or 'pulsewaves' may be given. Wherever the pulse-wave spreads it manifests itself in various ways-by an increase of blood-pressure, an increase in the mean velocity of the blood-flow, an increase in the volume of organs, and by the visible and palpable signs to whicl the name of pulse is commonly given in a restricted

* The necessary details must be sought in such works as Mackenzie's 'Diseases of the Heart.' 
sense. The intermittence in the flow with which the pulsewave is necessarily associated is at its height at the beginning of the aorta. In middle-sized arteries, such as the radial, it is still well marked, but it dies away as the capillaries are reached, and only under special conditions passes on into the veins, where, however, as has just been mentioned, pulsatory phenomena of a different origin may be detected.

The pulse was well known to the Greek physicians, and used by them to a certain extent as an indication in practical medicine. Harvey demonstrated with some clearness the relation of the pulse to the contraction of the heart, but Thomas Young was the first to form a proper conception of it as the outward token of a wave propagated from heart to periphery.

When the finger is placed over a superficial artery like the carotid, the radial or the temporal, a throb or beat is felt, which, without measurement, seems to be exactly coincident with the cardiac impulse. In certain situations the pulse can be seen as a distinct rhythmical rise and fall of the skin over the vessel. The throbbing of the carotid, especially after exertion, is familiar to everyone, and the beat of the

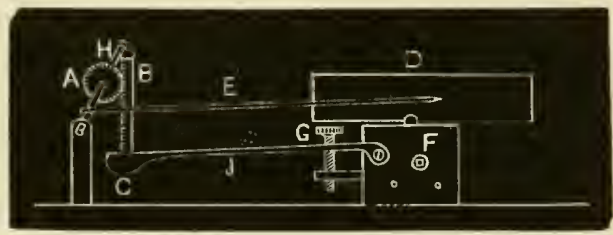

Fig. 3i.-Scheme of Marey's Sphygmograph.

$A$, toothed wheel connected with axle $\mathrm{H}$, and gearing into toothed upright B; C, ivory pad which rests over bloodvessel and is pressed on it by moving $\mathrm{G}$, a screw passing through the spring $\mathrm{J}$; $\mathrm{E}$, writing-lever attached to axle $\mathrm{H}$, and noved by its rotation. E writes on D, a travelling surface moved by clockwork $\mathrm{F}$. ulnar artery can be easily rendered visible by extending the hand sharply on the wrist. When the pulse is felt by the finger, it is not the expansion, but the hardening of the wall of the vessel, due to the increase of arterial pressure, that is perceived ; and even a superficial artery, when embedded in soft tissues so that it cannot be compressed, gives no token of its presence to the sense of touch. Sometimes an artery is longitudinally extended by the pulse-wave, and this extension may be far more conspicuous than the lateral dilatation. This is particularly seen when one point of the vessel is fixed and a more distal point offers some obstruction to the bloodflow, as at a bifurcation or in an artery which has been ligatured and divided.

By means of the sphygmograph, the lateral movements of the arterial wall, or, rather, in man, the movements of the skin and other tissues lying over the bloodvessel, can be magnified and recorded. 
It would be very unprofitable to enumerate all the sphygmographs which ingenuity has invented and found names for. The first rude attempt to magnify the movements of the pulse was marle by loosely attaching a thin fibre of glass or wax to the skin with a little lard, in order to demonstrate the venous pulse which appears under certain conditions. Vierordt inproved on this by using a counterpoised lever writing on a blackened surface. But the inertia of the lever was so great that the fincr features of the pulse were obscured. In all modern sphygmographs there is a part. usually button-shaped, which is pressed over the artery by means of a spring, as in Marey's and Dudgeon's sphygmographs, or by a weight, or by a column of liquid. In Marey's instrument, the button acts upon a toothed rod gearing into a toothed wheel, to which a lever, or a system of levers,

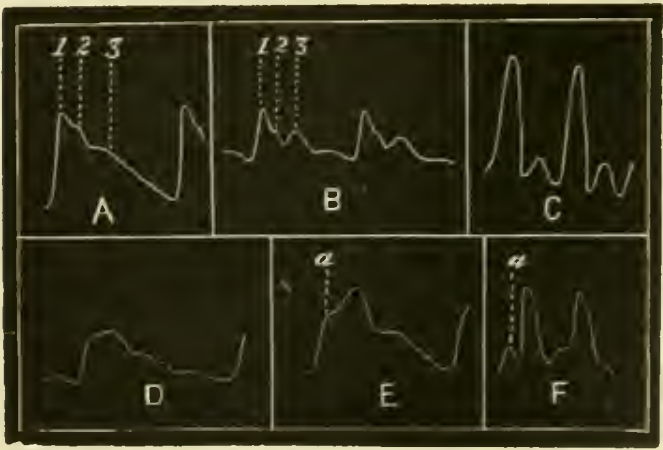

Fig. 32.-PULSE-traciNGs.

I, primary elevation; 2, predicrotic or first tidal wave; 3 , dicrotic wave. The depression between 2 and 3 is the dicrotic or aortic notch: 3 is better marked in $\mathrm{B}$ than in $\mathrm{A}$. C, dicrotic pulse with low arterial pressure; D, pulse with high arterial pressure-summit of primary elevation in the form of an ascending plateau. E, systolic anacrotic pulse; the secondary wavelet $a$ occurs during the upstroke corresponding to the rentricular systole. F, presystolic anacrotic pulse; $a$ occurs just before the systole of the ventricle. In this rarer form of anacrotism, a may sometimes be due to the auricular systole when the aortic valves are incompetent. is attached. The lever has a writingpoint which recoris the movement on a smokerl plate, or a plate covered with smaked paper. drawn uniformly along by clockwork (Figs. 31, S7). For many clinical purposes, especially in the study of rlis. ordered cardiac function, isolated records of the ar. terial pulsc are of comparatively little value. Special forms of sphygmo. graphs (polygraphs) have, therefore, becn devised, which, by the ad. dition of one or more recording tambours, permit the simultaneous record of move. ments from two or more points of the vascular system-for example, the radial artery and the jugular vein, or the radial or carotid artery, jugular vein, and the apex of the heart. In rare cases, with defect of the chest wall, a tracing may be obtained even from the aorta (Fig. 33).

In a normal arterial pulse-tracing (Fig. 32) the ascent is abrupt and unbroken; the descent is more gradual. and is interrupted by one, two, or even three or more, secondary warelets. The most important and constant of these is the one marked 3, which has received the name of the dicrotic wave. L'sually less marked, and sometimes absent, is the wavelet 2 between the dicrotic elevation and the apex of the curve. It is generally termed the 
predicrotic wave. Oscillations, clue to vibrations of the recording apparatus, appear on many pulse-tracings, and it is important to recognise their cause, so that no weight may be given to then.

In the explanation of the pulse-tracing, a fundamental fact to be borne in mind is the elasticity of the vessels. When an incompressible fluid like water is injected by an intermittent pump into one end of an elastic tube a wave is set up, which is transmitted to the other end of the tube. It is a positive wave-that is, it causes an increase of pressure and an expansion of the tube wherever it arrives; and if a series of levers be placed in contact with the tube, they will rise and sink in succession as the wave passes them. After the passage of this primary wave the walls of the tube, instead of coming instantly to rest in their original position, regain it by a series of oscillations, first shrinking too much, then expanding too much, but at each novement coming ncarer to the position of equilibrium. Each vibration of the clastic wall is of course accompanied by a change of pressure in the contents of the tube. This change of pressure runs along the tube as a wave; and such waves, succeeding the primary one. may be called secondary waves of oscillation. These secondary waves will be set up in an elastic system whether the distal end of the system be closed or open. But if it is closed, or sufficiently obstructed without being actually closed, secondary waves of another kind may also be generated, the primary wave on arriving at the distal end being reflected there. The reflected wave running back towards the central end may there again undergo reflexion, and pass out once more towards the distal end as a centrifugal, twice-reflected wave. When the liquid ceases to enter the tube at the end of the stroke, a wave of diminished pressure-a negative wave-is generated at the central end, and is propagated

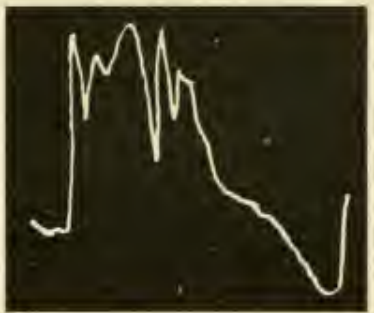

FIG. 33.-PULSE-CURVE FROM HUMAN AORTA (AFTER TIGERSTEDT). to the distal end, where it may be reflected just like the positive wave.

Although under certain conditions the dicrotic wave is so marked that the double beat of the pulse was discovered and llamed by physicians long before the invention of any spliygmograph, perhaps no physiological question has been more discussed or is less understood than the mechanism of its production. Two points, however, seem to be clear: (I) That it is a centrifugal, and not a centripetal, wave-that is to say, it travels away from, and not towards, the heart; (2) that the aortic semilunar valves have something to do with its origin.

It is not a centripetal wave, for in tracings taken at all parts of the arterial path, no matter what the distance from the heart and the capillaries (e.g., the origin of the carotid and the radial at the wrist), the dicrotic wave is separated by the same interval from the beginning of the primary elevation. This can only be explained by supposing that it has the same point of origin, and 
travels with the same velocity and in the same direction as the primary wave. It is not, then, a wave reflected directly from the peripheral distribution of the artery from which the pulse-tracing is taken.

Some writers have contended that it is a centrifugal twice-reflected wave, and, indeed, traces of such waves may be detected in the vessels of newly-killed animals when changes of pressure of the same order of magnitude as the arterial pulse are artificially produced by a pump and recorded by elastic manometers connected with the interior of an artery. It has been supposed that these seconclary waves are reflected first from peripheral points at which the bloodflow is particularly obstructed (the bifurcations of the larger arteries, and the small arteries and capillaries in general), and that running towarls the heart, they are again reflected outwards from the semifunar valves. It has been urged in support of this view that in very small animals (guinea-pigs) no dicrotic elevation occurs on the pulsetracing, since the path which the reflected wave has to follow is so short that it arrives at the root of the aorta before the primary elevation is over. But this argument is by no means conclusive. and, indeed, the great difference in the distance from the heart of the numerous points at which reflection must take place is one of the chief difficulties of the hypothesis. For it is not easy to understand how the reflected fragments of the primary wave, arriving at lifferent intervals at the heart, can be integrated into the single considerable dicrotic elevation.

The explanation that best takes account of the facts and renders most clear the rôle of the semilunar valves is somewhat as follows: When the systole abruptly comes to an end and the outflow from the ventricle ceases, the column of blood in the aorta tends still to move on in virtue of its inertia, and a diminution of pressure, accompanied by a corresponding contraction of the aorta, takes place behind it, just as a negative wave is set up in the central end of the elastic tube when the stroke of the pump is over. At the same moment, and while the semilunar valves are still for an instant incompletely closed, the diminution of pressure in the beginning of the aorta is intensified by the aspiration of the relaxing ventricle, which sucks the blood back against the valves, and draws them a little way into its cavity. A negative wave, therefore-a wave of diminished pressure, represented in the pulse-curve by the ' aortic notch' - travels out towards the periphery. The diminution of pressure is quickly followed by a rebound, as always happens in an elastic system. The recoiling blood meets the closed semilunar valves. The aorta expands again, and the expansion is propagated once more along the arteries as the dicrotic elevation. It is possible that this elevation may be reinforced by a reflected wave produced in the manner described.

When the semilunar valve becomes incompetent in disease, or is rendered insufficient in animals by the artificial rupture of one or 
more of its segments, the licrotic wave, as will be readily understood from the manner in which it is produced, either disappears altogether or is markedly enfeebled. But apart fiom any anatomical lesion or functional defect in the aortic valves, the prominence of the wave varies with a great number of circumstances, some of which are in a measure understood, while others remain obscure. It varies in particular with the abruptness of (lischarge of the ventricle and the extensibility of the arteries. The conditions are usually favourable when the arterial pressure is low, for the blood then passes quickly from the ventricle into the arteries, which, already only moderately tense, are easily dilated by the primary wave, then sharply collapse, and are again abruptly distended when the dicrotic wave arrives. And, in fact, an exaggeration of the dicrotic wavelet may be artificially produced by nitrite of amyl (Fig. 89, p. I93), a drug which lessens the blood-pressure by dilating the small arteries. Iuscular exercise (Fig. 88, p. I93), running or bicycling, for instance, has a similar effect on the sphygmogram, although the explanation can scarcely be the same, since the blood-pressure mounts rapidly when moderate excrcise begins and only gradually falls during its continuance, with an abrupt decline to normal or below it on cessation of work (Bowen). The increase in the pulse-rate may have something to do in this case with the exaggeration of the dicrotism, which is very frequently, although by no means invariably, associated with a rapidly-beating heart, and therefore is often seen in fever. On the other hand, in certain diseases associated with a high arterial pressure the dicrotic elevation almost disappears. Atheromatous arteries, being very inextensible, do not allow a dicrotic pulse.

Since the pulse represents a periodical increase and diminution in the amount of distension of an artery at any point, the line joining all the minima of the pulse-curve will vary in its height above the base-line, or line of no pressure, according to the amount of permanent distension, i.e., permanent blood-pressure, which the heart in any given circumstances is able to maintain. Any circumstance that tends to lessen the permanent distension will cause a fall of the line of minima, and any circumstance tending to increase the distension will cause that line to rise. If, for example, the arm be raised while a pulse-tracing is being taken from the wrist, the line of minima falls because the pernanent pressure in the radial artery is diminished.

The form of the pulse-curve varies in the different arteries, and therefore in making comparisons the same artery should be used. When the wave of blood only enters an artery slowly, the ascending part of the curve will be oblique. This is normally the case in a pulse-curve of a distant artery, such as the posterior tibial. The height of the wave is also less than in an artery nearer the heart, such as the carotid, or even the axillary, where the primary elevation is relatively abrupt (Fig. 90, p. I93).

Anacrotic Pulse.-As a rule, the ascent of the tracing is unbroken by secondary waves, but in certain circumstances these may appear on it. This condition, which, when well marked at any rate, may be considered pathological, is called anacrotism (Fig. 32). It is seen when the discharge of the left ventricle into the aorta is slow and difficult-e.g., in old people 
whose arteries have been rendered less extensible by the deposit of lime-salts in their walls (atheroma), and in cases where the orifice of the aorta has been narrowed from disease of the semilunar valves (aortic stenosis). Since these conditions are in general associated with hypertrophy and dilatation of the left ventricle, the slow emptying of the ventricle is partly due to the greater quantity of blood which it contains. In whaterer way the delay in the emptying of the ventricle is brought about, the most probable explanation of the anacrotic pulse is that the delay affords time for one or more secondary waves to be dereloped in the arterial ststem before the summit of the curve has been reached, and that these are superposed upon the longdrawn primary elevation. In aortic insufficiency, where the left side of the heart is never cut off entirely from the aorta, the auricular impulse is sometimes marked on the pulse-curve as a distinct elevation; and this gives rise to a peculiar kind of anacrotic pulse, especially in the arteries nearest the heart (Fig. 32, F).

Frequency of the Pulse.-In health, the working of the cardiac pump is so smooth and apparently so self-directed, that it needs a certain degree of attention to perceive that the rate of the stroke is not absolutely constant. It is, in reality, affected by many internal conditions and external influences.

At the end of fotal life the rate is given as I 44 to I33; from birth till the end of the first year, I 40 to I 23 ; from Io to I 5 years, 9 I to 76 ; from 20 to 25 years, 73 to 69 . It remains at this till 60 years, and increases again somewhat in old age.* At all ages the pulse is somewhat quicker in the female than in the male, the excess amounting to about $S$ beats a minute. So that if we take the average rate for a man (in the sitting position) as 72 , the average for a woman will be So. The difference is partly due to the fact that the average man is taller than the average woman; and it is known that in persons of the same sex and age the pulse-rate has an inverse relation to the stature. But there may be, in addition, a real sexual difference. It must not be forgotten that a small number of perfectly healthy persons have an habitually slow pulse, not above 50 in the minute. The position of the body exercises a slight, but relatively constant,

* It must be remembered that these numbers are merely averages. Some healthy individuals have a much lower pulse-rate than 72 per minute, and some a rate considerably greater. Thus, while the average pulse-rate (taken in the sitting position) of $\delta$; healthy (male) students (in the writer's laboratory), whose ages ranged from is to 36 years, was 73 , the extreme variation was from if to 93 . In the standing position the average was So, and the variations 64 to 105 . In the supine position, average 69 , and variations $q 5$ to 99 . After a short spell of muscular exercise (generally running up and down some flights of stairs) the average in the standing position was 119 , the variations $; 5$ to 164 , and the average increase 32 . 
influence on the rate, which is greater in the standing than in the sitting posture, and greater in the latter than in the recumbent position. And this is true even when muscular action is as far as possible eliminated by fastening the person to a board. The pulse is further affected by the respiratory movements, especially when they are exaggerated in forced breathing, being accelerated during each inspiration (p. 269). It is also increased by the taking of food, and especially of alcoholic stimulants, by muscular exercise, in fever and many other pathological conditions, and by a high external temperature. A warm bath, for example, causes a very distinct acceleration of the heart; and Delaroche found that in air at the temperature of $65^{\circ} \mathrm{C}$. his pulse went up to I6o. A cold bath may depress the pulse-rate to 60 , or even less. During sleep it may fall to 50 . It is greatly influenced by psychical events, and that in the direction either of an increase or a decrease. Finally, it ought to be remembered as of some practical importance that the pulse-rate in women and children, but particularly in the latter, is less steady than in men, and more apt to be affected by trivial causes. And it is a good general rule to let a short interval elapse after the finger is laid on the artery before beginning to count the pulse, so that the acceleration due to the agitation of the patient may have time to subside.

Rate of Propagation of the Pulse-wave. - When pulsetracings are taken simultaneously at two points of the arterial system unequally distant from the heart, by two sphygmographs whose writing-points move in the same vertical straight line, it is found that the ascent of the curve begins later at the more distant than at the nearer point. Since waves like the pulsewave travel with approximately the same velocity in different parts of an elastic system like the arterial 'tree,' this ' delay' must be due to the difference in the length of the two paths. The difference in length can be measured; the time-value of the 'delay' can be deduced from the rate of movement of the recording surface; dividing the length by the time, we arrive at the rate of propagation of the pulse-wave. The average rate has been found to be about 7 metres per second in man in the arteries of the upper limb, and 8 metres in those of the lower limb, the difference being due to the smaller distensibility of the latter. In sleep the velocity diminishes almost a metre a second. It increases in arterio-sclerosis, where the distensibility of the arteries is diminished, and in chronic nephritis with hypertrophy of the heart, in which the blood-pressure is increased. The mean velocity of the pulse-wave is about the same as the speed of a moderately fast steamship (say, I7 miles an hour), but less than that of a wave of the sea in a strong gale. The velocity of the pulse-wave must not be confounded with that 
of the blood-stream itself, which is not one-thirtietls as great. A ripple passes over the surface of a river at its own rate-a rate that is independent of the velocity of the current. The passage of the ripple is not a bodily transference of the particles of water of which at any given moment the wave is composed, but the propagation of a change of relative position of the particles. The mere fact that the ripple can pass upstream as well as down is sufficient to illustrate this. The pulse-wave does not, however, correspond in every respect to a ripple on a stream, for the bodily transfer of the blood depends upon the series of blood-waves which the heart sets travelling along the arteries. Every particle of blood is advanced, on the whole, by a certain distance with every pulse-wave in which for the time it takes its place. But no particle continues in the front of the pulse-wave from beginning to end of the arterial system. The 'delay' or 'retardation' of the pulse (the interval, say', between the beginning of the ascent of the carotid and radial curves) is practically constant in the same individual, not only in health, but also in most diseases. But the retardation is markedly increased when the pulse-wave has to pass through a portion of an artery whose lumen is either greatly widened (in aneurism), or greatly constricted (in endarteritis obliterans).

The Blood-pressure Pulse. - In man it is only possible to trace the pulse-wave along the arteries by movements of the walls of the vessels transmitted through the overlying tissues. In animals the changes of pressure that occur in the blood itself can be directly registered, and these changes may be spoken of as the blood-pressure pulse. At bottom, as already pointed out, the phenomenon is exactly the same as that we have been dealing with in our study of the external pulse. We are only now to follow, by a more direct, and in some respects a more perfect method, the same wave of blood along the same cliannel.

Measurement of the Blood-pressure.-Hales was the first to measure the blood-pressure. This he did by connecting a tall glass tube with the crural artery of a horse. The height to which the blood rose in the tube indicated the pressure in the vessel. Poiseuille, nearly half a century later, applied the mercury manometer, which had already been used in physics, to the measurement of bloodpressure. Ludwig and others improved this method by making the manometer self-registering by means of a float in the open limb, supporting a style which writes on a revolving drum, or kymograph. (For the method of taking a blood-pressure tracing, see p. 195.)

For reasons already mentioned the mercurial manometer is better suited for measuring the mean blood-pressure, or for recorling changes in the pressure which last for some time, than for following the rapid variations of the pulse-wave. For the latter purpose, one of the class of elastic manometers is required (p. 86).

A blood-pressure tracing taken from an artery with a manometer of this sort yields the truest picture of the pulse-wave which it is 
possible to obtain, because the reproduction of it is the most direct. The fact that such a tracing shows a close agreement with the trace of a good spliygmograph properly applied to the corresponding artery on the other side, is a striking proof of the general accuracy of the sphygmographic method for physiological purposes, and enables us to gnide ourselves in transferring to man, in whom, of comse, the sphysmograpl can alone be used, the information derived from direct manometric observations in animals.

For the same reason it is unnecessary to discuss the manometric tracings, as regards the pulsatory phenomena, in all their

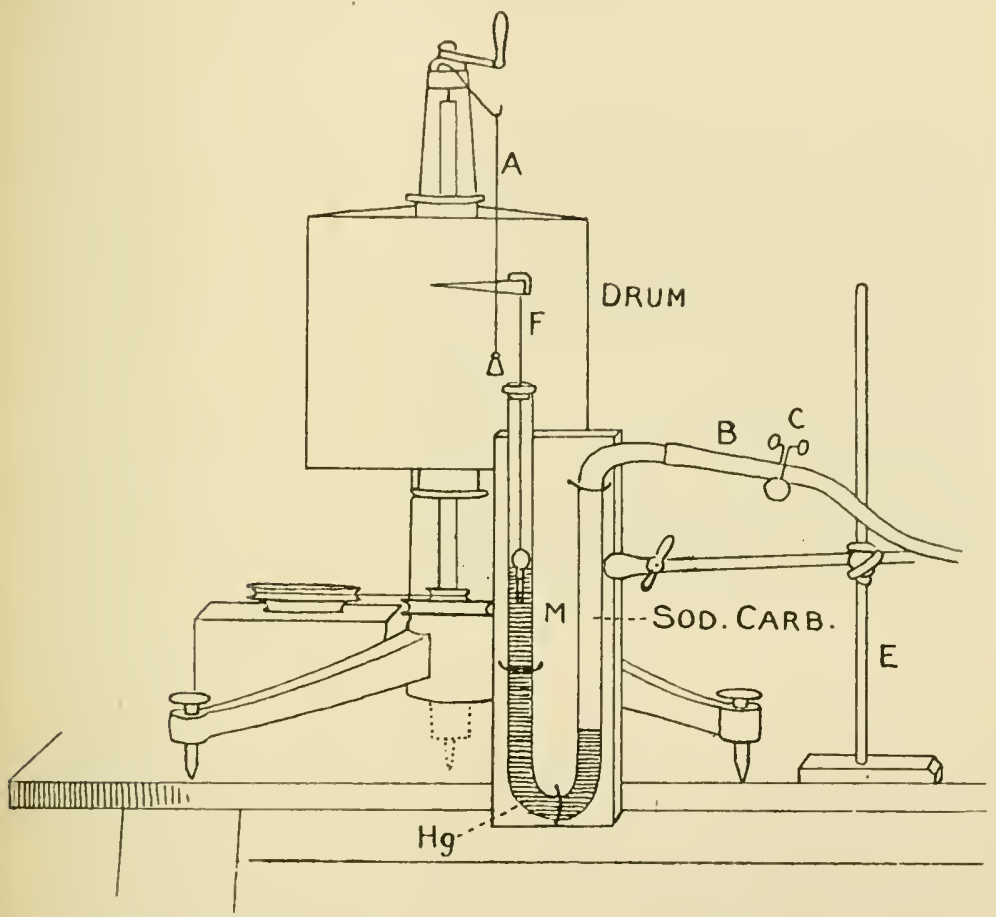

Fig. 34.-Arrangement for taking a Blood-pressure Tracixg.

II. manometer: $\mathrm{Hg}$, mercury ; F, float armed with writing-point; $\mathrm{A}$, thread attached to a wire projecting from the drum and supporting a small weight. The thread keeps the writing-point in contact with the smoked paper on the drum. $\mathrm{B}$ is a strong rubber tube connecting the manometer with the artery ; $\mathrm{C}$, a pinchcock on the rubber tube, which is taken off when a tracing is to be obtained.

details. It will be sufficient to say that while the form of the blood-pressure pulse-curve varies with the mean blood-pressure, the dicrotic wave is always marked on it, preceded by one or more oscillations falling within the period of the systole, and followed by one or more within the period of the diastole. When the blood-pressure is low, the first or primary elevation is the 
highest of the whole curve (Fig. 35). When the blood-pressure is high, the maximum falls later, coinciding with one of the secondary systolic waves, but always preceding the dicrotic wave; and the curve assumes an anacrotic character.

That all the secondary oscillations, including the dicrotic wavelet, are centrifugal, and not centripetal, may be shown, just as in the sphygmographic method, by recording the blood-pressure simultaneously at two points of the arterial systen at different distances from the heart-e.g., in the crural ancl carotid arteries. The secondary waves are found, by measuring the tracings, to reach the more distal point later than the more central.

The increase of pressure during the systolc, as inclicated by the

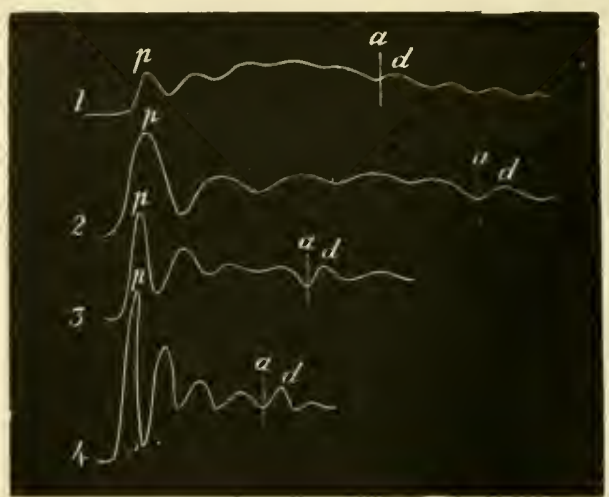

Fig. 35.-Curves of Blood-pressure taken WITH A SPRING MANOMETER FROM TIIE Carotid ARtery of a Dóg (Hürtille).

When I was taken the blood-pressure was high; 2 corresponds to a medium, 3 to a low, and + to a very low, blood-pressure : $p$ is the primary elevation-this and the succeching elevations between $p$ and $a$ are called systolic waves; the systolic waves are followed by a marked elevation $d$, which corresponds $t o$ the dierotic wave. height of the prinary clevation, is always very large, much larger than it appears in a tracing taken with a mercury manometer. In the rab. bit this pulsatory varia. tion is one-third to onefourth of the minimum pressure. In the dog it is still greater, owing to the slower rate of the heart, and often amounts to $50 \mathrm{~mm}$. of mercury, while under favourable conditions(low minimum pressure and slowly beat. ing heart) the systolic increase of pressure may be actually more than double the minimum (Hürthle). Fick found also, by means of his spring manometer, that the pulsatory variations of bloor-pressure were greater than the respiratory variations (p. 103 ), although in the records of the mercury manometer the reverse appears often to be the case. Landois, too, in the course of experiments in which a divided artery was allowed to spout against a moving surface, and to trace on it a sort of pulsc-curve painted in blood (a hamautogram as it is called), observed that the rate of escape of the blood was nearly 50 per cent. greater lluring the systole than luring the pause of the heart. The existence of the dicrotic wave on this tracing was long looked on as the best proof that it was not an artificial phenomenon.

The wave of increased pressure, as it runs along the arterial system, carries with it wherever it arrives an increase of potential energy. But this excess of potential energy is continually being 
worn down, owing to the friction of the vascular bed; and although in the comparatively large arteries the loss of energy is not great, it rapidly increases as the arteries approach their termination, and begin to break up into the narrow arterioles which feed the capillary network. For not only is the total surface, and therefore the friction, increased with every bifurcation, but the mere change of direction and division of the wave cannot take place without loss of energy. For this reason the fluctuations of blood-pressure are greater in the large arteries near the heart than in arteries smaller and more remote. In the wide and muchbranched capillary bed the pulse-wave disappears altogether, and the blood-pressure becomes relatively constant or permanent. And it is for some purposes convenient to look upon the bloodpressure in the arteries as made up of a permanent element, with pulsatory oscillations superposed on it. Since no portion of the arterial system is more than partially emptied in the interval between two blood-waves, the minimum or permanent pressure is always positive-i.e., always above that of the atmosphere, the beats of the heart succeeding each other so rapidly that the successive waves overlap or 'interfere,' and are only separated at their crests.

If the heart is stopped while a blood-pressure tracing is being taken-and we shall see later on how this can be done (p. I43)the minimum line of the tracing goes on falling towards the zeroline. When the heart begins

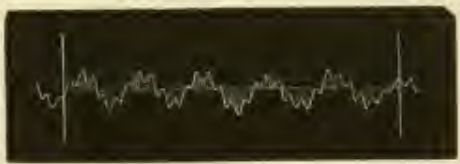

Fig. 36.-BLOod-PRESSURE TRACING.

The horizontal straight line intersecting the curves is the line of mean pressure.

beating again, the pressure-curve rises, not by a continuous ascent, but by successive leaps, each corresponding to a beat of the heart, and each overtopping its predecessor, till the old line of minimum or of mean pressure is again reached.

The mean arterial blood-pressure is the permanent pressure plus one-half of the average pulsatory oscillation. In a bloodpressure tracing the line of permanent pressure joins all the minima; the line of maximum pressure joins all the maxima; the line of mean pressure is drawn between them in such a way that of the area included between it and the blood-pressure curve as much lies above as below it (Fig. 36). As has been said, a tracing taken with a mercury manometer gives approximately the mean blood-pressure. Each beat of the heart is represented on it by a single elevation of variable size, sometimes not amounting to more than one-twentieth of the height of the curve above the line of zero or atmospheric pressure, but sometimes much larger. The oscillations due to the heart-beat are 
superposed upon much longer, and often, as registered in this way, larger waves, caused by the movements of respiration. So much having been said by way of definition, we have now to consider the amount of the mean arterial pressure, the variations which it undergoes, and the factors on which its maintenance depends.

As to its amount, it will be sufficiently accurate to say that in the systemic arteries of warm-blooded animals in general (including birds), and of man in particular, the mean pressure does not, under ordinary conditions, descend much below $100 \mathrm{~mm}$. of mercury, nor rise much above $200 \mathrm{~mm}$. ; while in cold-blooded animals it seldom exceeds $50 \mathrm{~mm}$., and may fall as low as $20 \mathrm{~mm}$.

It does not seem possible, at least with our present data, to further subdivide these two great groups; nor do we know preciscly whether the distinction depends mainly on morphological or mainly on physiological differences, whether, that is to say, the warm-blooded animal has a higher blood-pressure than the cold-blooded chiefly because its vascular system (and especially its heart) is anatomically more perfect, or because its heart beats faster and works harder. It may be that it is for both of these reasons that the birds, which in certain other respects are more nearly related to the reptiles than to the mammals, mount, as regards the pressure of the blood, into the mammalian class, and that a manometer in the carotid of a goose will rise as high, or almost as high, as in the carotid of a horse, a sheep, or a clog. while the pressure in the aorta of a tortoise is no higher than in the aorta of a frog. But we know that the mere average rate of the heart has of itsclf comparatively little influence on the blood-pressure within either group, for the heart of a rabbit beats, on the average, very much faster than the heart of a dog, ancl yet the arterial pressure in the dog is certainly at least as great as in the rabbit. Nor does the size of the body seem to have any definite relation to the mean pressure, even in animals of the same species; and there is no reason to suppose that the pressure is materially less in the radial artery of a dwarf than in the radial artery of a giant.

Measurement of the Blood-pressure in Man.-In man the bloodpressure has been estimated by adjusting over an artery an instrument known as a sphygmomanometer or sphygmometer, which, in its most modern form, consists essentially of a hollow rubber pad or bag containing liquid or air, and connected with a metallic pressure gauge or a mercurial manometer.

The sphygmomanometer of Erlanger (Fig. 37) is arranged to obtain graphic records of the pulse, from which both the maximum and the miminum blood-pressures may be deduced. The mean pressure cannot be directly measured, but must lie much nearer to the minimum than to the maximum, since the line of mean pressure bisects the area enclosed by the pulse-curve, and this area is broad at the base and narrow at the apex. The rubber bag is applied in the form of a cuff or armlet to the arm above the elbow over the brachial artery. It communicates with a mercury manometer. which gives the pressure exerted upon the arm. It is also connected with a 
rubber bulb, B, enclosed in a glass bulb, G, and through a stopcock with a syringe bulb. V, provided with a valve. The space between $B$ and $G$ conmunicates (I) with the tambour; (2) with the exterior through the stopcock by the tube E, and also through a pin-point opening in the membrane of the tambour. While the armlet is being adjusted the stopcock is turned so that the rubber bag is in communication with the cxternal air through $F$. The same is true of the space TS in the glass bulb. The tambour is thus protected against undue strain during adjustment. The stopcock is now rotated so as to cut off the armlet from the exterior and to permit the entrance of air through $\mathrm{F}$ from $\mathrm{V}$, which is used as a pump to raisc the pressure, the space TS and the tam. bour being still in communication with the exterior. When the desired pressure has been reached, the stopcock is turned into an intermediate position, which cuts off both the armlet and the space TS from the exterior, and the pulse is then transmitted to the tambour and recorded on the drum. By certain adjustments of the stopcock air can be allowed to escape more or less rapidly from the armlet.

To determine the maximum or systolic blood-pressure, the air-pressure in the armlet is raised considerably (about 50 $\mathrm{mm} . \mathrm{Hg}$ ) above what it is expected to be.

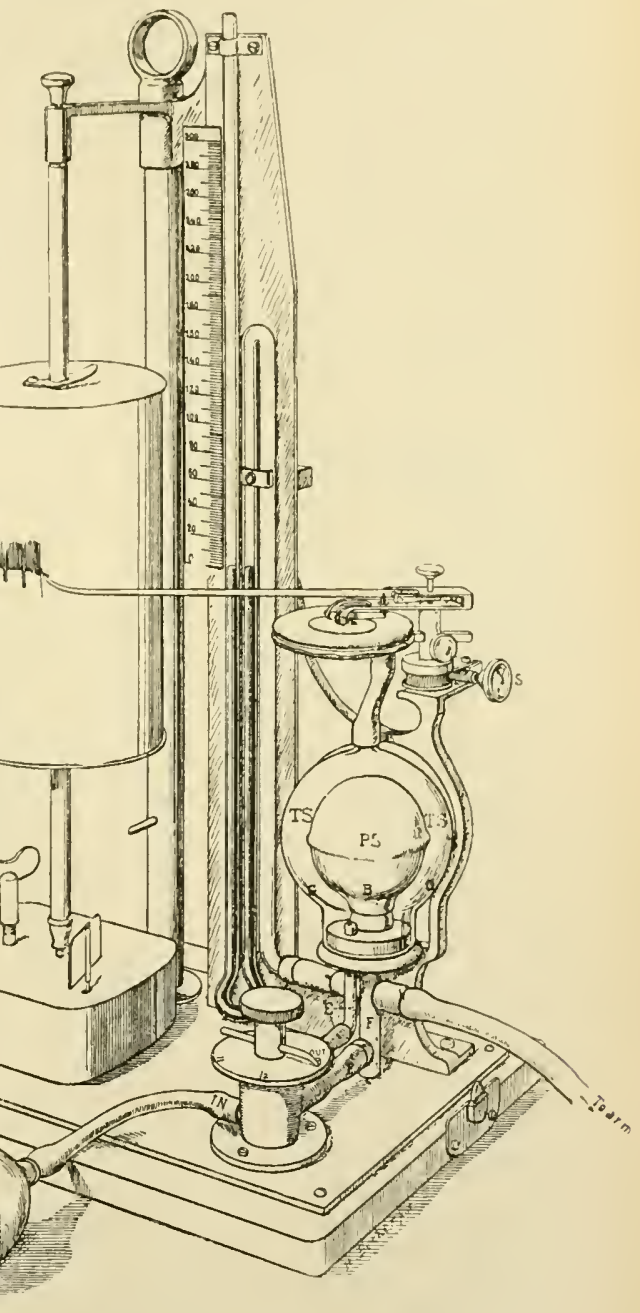

Fig. 37.-Sphygmomanometer of ErLanger.

While the lever is writing on the drum the small oscillations due to the impact on the bag of the pulse-ivaves in the central portion of the obliterated artery, the pressure is gradually diminished by allowing air to escape. At the moment when the pressure upon the 
arm falls below the maximum blool-pressure, and the pulse-wave is first able to break through the brachial artery, the oscillations of the lever will more or less abruptly increase in amplitude. The pressure shown by the manometer at this point is the systolic blood-pressure. To obtain the minimum or diastolic pressure, the air-pressure in the armlet is raised somewhat ( 1 o to $15 \mathrm{~mm}$. $\mathrm{Hg}$ ) above the pressure expected. The pressure is diminished by $5 \mathrm{~mm}$. Hg at a time, records of the oscillations being taken on the drum. The manometer reading at the point at which the oscillations, after reaching the maximum, begin abruptly to diminish, corresponds to the minimum blood-pressure.

In using the splyygmometer of Hill and Barnard (Fig. 38), the bag is inflated with air till the pulsation inclicated by the inclex of the pressure gauge reaches a maximum. The mean pressure shown by the gauge at this point is approximately equal to, or somewhat greater than, the minimum arterial pressure. With this instrmment it has been found that in the brachial artery the normal arterial pressure in most healthy young men is 110 to $130 \mathrm{~mm}$. of mercury in the sitting posture. When the person is

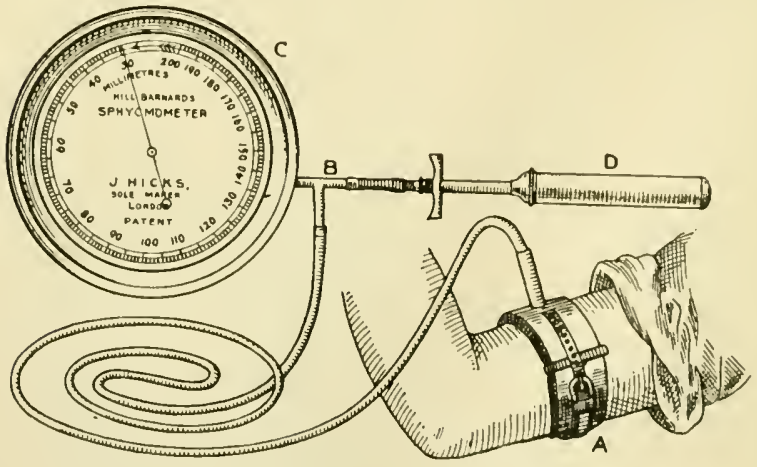

Fig. 38.-Sphygaometer of Hill and Barnard.

It consists of a broad armlet, $A$, which is strapped round the upper arn. On the inside of the armlet is a thin rubber bag containing air, and connected by a $T$-tube, $B$, with a pressure gauge, $C$, and a small compressing air-pump, $D$, fit ted with a valve. resting in the recum bent posture, the pressure may be as low as $95 \mathrm{~mm}$.ofmer. cury. Harl work and nervous strain may raise the pressure to $I_{4}$ or 145 min. of nercury.

The effect of muscular exercise upon the pressure is influenced by the nature of the work. Such an effort as the lifting of a heavy weight causes a sudden and great increase, which is very transient. Thus, the average arterial pressure in a number of men was II I before, 180 during, and i 10 two to three minutes after the lift (McCurdy). The rise of pressure in this case is due largely to the marked diminution of the calibre of the bloodvessels mechanically produced by the strong and sustained contraction of the muscles. This increases the resistance to the passage of the blood along the arteries, while the veins are emptied by the pressure, and more blood thus reaches the right side of the heart. It is obvious that the heart and vessels may easily be exposed to an injurious strain during such efforts. In such an excrcise as running, while the pressure mounts to some cxtent at first, as already mentioned, the rise is not maintained, owing to the dilatation of the cutancous vesscls. In the anterior tibial artery of a 
boy whose leg was to be amputated, the blood-pressure, measured by means of a manometer connected directly with the artery, was found to vary from 100 to $160 \mathrm{~mm}$., according to the position of the bocly and other circumstances. In a woman sixty years old, in good health, the following readings were obtained with a sphygmomanometer :

$\begin{array}{crc}\text { June } & 2 S & - \\ \text {," } & 29 & - \\ \text { Aug. } & 3 & - \\ ,, & 7 & - \\ , & 12\end{array}$

$$
\begin{array}{ll}
126-130 \mathrm{~mm} \text {. of mercury. } \\
126-136 & , \\
132-144 & , \\
134-140 & , \\
136-144 & ,
\end{array}
$$

Such measurements on man show that the mean blood-pressure under similar conditions in one and the same artery, and in one and the same individual, may vary for a considerable time only within comparatively narrow limits.

This relative constancy of the general arterial pressure is the result of a delicate adjustment between the work of the heart, the resistance of the ressels, and the volume of the circulating liquid. The quantity of the blood is tolerably steady in health, and considerable changes may be artificially produced in it (p. I74) without affecting the pressure in any great degree. On the other hand, the work of the heart and the peripheral resistance are highly variable and vastly influential. A narrowing of the arterioles throughout the body or in some extensive vascular tract increases the peripheral resistance; and if the heart continues to beat as before, the pressure must rise. If the arterioles are widened, while the heart's action remains unchanged, the pressure must fall. In like manner an increase or a decrease in the activity of the heart, in the absence of any change in the peripheral resistance, will cause a rise or a fall in the blood-pressure. But if a slowing of the heart is accompanied by an increase in the peripheral resistance, or a dilatation of the arterioles by an increase in the activity of the heart, the one change may be partially or completely balanced by the other. and the pressure may vary within narrow limits or not at all.

Not only is the mean pressure, as measured in a large artery, tolerably constant, but if recorded simultaneously in two arteries at different distances from the heart, it is seen to decrease very gradually so long as the arteries remain large enough to hold a cannula. It is nearly as high, for instance, in the crural artery of a dog as in the carotid. It is easy to see that this must be so, for the resistance of the arteries between the point where the arterioles are given off and the heart is only a small fraction of the total resistance of the vascular path; and we have said (p. 76) that the lateral pressure at any cross-section of a system of tubes through which liquid is flowing is proportional to the resistance still to be overcome. This is also the reason why the pressure is always much lower in the pulmonary artery and 
right ventricle than in the aorta and left ventricle (only onethird to one-sixth as great), for the total resistance of the vascular path through the lungs is much less than that of the systemic circuit. In dogs with natural respiration the pressure in the pulmonary artery was found to vary between If and $26 \mathrm{~mm}$. of mercury, averaging about $20 \mathrm{~mm}$.

The Velocity-pulse.- We have seen that the blond is propelled through the arteries in a series of waves that travel from the heart towards the periphery. The particles in the front of the pulse-wave are constantly changing, but since every section of the arterial tree is successively distended, every section contains more blood while the pulse-wave is passing over it than it contained immediately before. And since there is always a fairly free passage for this blood towards the periphery, there is a bodily transfer on the whole of a certain quantity with every wave.

The translation of the blood, instead of being entirely intermittent, as it would be in a rigid tube or in an elastic system with a slow action of the central pump, is to some extent constantly going on ; for a portion of a blood-wave is always passing through every section of the arterial channel. Thus, we arrive at the same distinction as to the onward movement of the blood itself as we previously reached in regard to the blood-pressure. the distinction between the constant or permanent factor of the velocity and the periodic factor, which we may call the velocity-pulse.

The Velocity of the Blood. - By the velocity or rate of flow of a river we should mean, if the flow were uniform throughout the whole cross-section. the rate of movement of any given portion or particle of the water. If we could identify a portion of the water, we could determine the velocity by measuring the distance travelled over by that portion in a given time. If the velocity was uniform over the channel, we could predict the actual time which would be requirer to traverse any fractional part of the measured distance. If. however. the velocity of the current changed from point to point, then we could only rleduce from our observation the mean rate of the river for the measured distance. To determine the actual rate for any given portion of this distance over which the rate was uniform. we should have to make a separate observation for this portion alone.

But as soon as we pass from an ideal frictionless river to an actual stream, in which the water at the bottom and near the banks flows more slowly than that in the middle and on the surface. we are in every case restricted to the notion of mean velocity. We may clistinguish between the velocity of different parts of the current, between that of the mid-stream and the side current, the bottom and the surface layers; but when we consider the river as a whole, we take cognizance only of the mean or average velocity. And at any cross-section this may be defined as the volume of water passing per hour. or whatever the unit of time may be. divided by the crosssection of the current. It is evident that this does not enable us to 
letermine the actual velocity of any given particle of the water at any given moment within a measured interval ; nor does it tell us whether or not the average velocity of the current has itself undergone variations within the period of observation.

We have dwelt upon this point because the measurement of the velocity of the blood, to which we must now turn, involves the same considerations. Within the smaller arteries, as the microscope shows us, and as we should in any case expect from what we know of fluid motion, the blood-current, apart from the periodical variations in its velocity, due to the action of the heart, varies in speed from point to point of the same crosssection. The layer next the periphery of the ressel, the socalled peripheral plasma-layer or Poiseuille's space, moves more slowly than the central portion, the axial stream. In fact, we must suppose that in the large as well as in the small vessels the layer just in contact with the vessel-wall is at rest, while the stratum internal to this slides on it and has its velocity diminished by the friction. The next layer again slides on the last, but since this is already in motion, its velocity is not so much diminished, and so on. The velocity must therefore increase as we pass towards the axis of the bloodvessel, and reach its maximum there ( $p$. I 77 ).

Again, the relocity must be altered wherever an alteration occurs in the width of the bed, that is, in the total cross-section of the vascular system; for since as much blood comes back in a given time to the right side of the heart as leaves the left side, the same quantity must pass in a given time through every cross-section of the circulation. Wherever the total section of the vascular tree increases, the blood-current must slacken; wherever it diminishes, the current must become more rapid. Now, the total section, increasing somewhat as we pass from the heart along the branching arteries, undergoes an abrupt augmentation, and reaches its maximum in the capillary region. It suddenly diminishes again at the venous end of the capillary tract, and then more gradually as we pass heartwards along the veins, but never becomes so small as in the arterial tract. We must, therefore, expect the mean velocity to be greatest in the large arteries, less in the veins, and least in the arterioles, capillaries, and venules. It must, of course, be remembered that the total section varies from time to time at any given distance from the heart. The capillary tract is especially variable in its area, and capillaries full of blood at one moment may be collapsed and empty at another, according to the changes of calibre and pressure in the arteries which feed and the veins which drain them.

Although in strictness we are only at present concerned with the arteries, it will be well to consider here what a change of velocity at 
any part of the vascular channel really implies. To say that when the channel widens the velocity diminishes, is not to explain the meaning of this diminution. A climinution of velocity implies a diminution of kinetic energy, and it is necessary to know what becomes of the energy that disappears. The stock of energy imparted by the contraction of the heart to a given mass of blood constantly diminishes as it passes round from the aorta to the right sicle of the heart, for friction is constantly being overcome and heat generated. This energy, as we have seen, exists in a moving liquid in two forms, potential and kinetic, the former being measured by the lateral pressure, the latter varying directly as the square of the velocity. Whenever the velocity, and therefore the kinetic energy, of a given mass of the blood is diminished without a corresponding increase in the potential energy, some of the total stock of energy must have been used up to overcome resistance (p. 76 ).

In a uniform, rigid, horizontal tube, as has been already remarked, the velocity (and consequently the kinetic energy) is the same at every cross-section of the tube, while the potential energy, represented by the lateral pressure, diminishes regularly along the tube. When the calibre of the tube varies, it is different. Suppose, for instance, that the liquid passes from a narrower to a wider part, the velocity must diminish in the latter. The kinetic energy of visible motion which has disappeared must have left something in its room. Here there are three possibilities: (1) The kinetic energy that has disappeared may be just enough to overcome the extra friction in the wider part of the tube due to eddies and consequent change of direction of the lines of flow; in this case the potential energy of a given mass of the liquid will be the same at the beginning of the wider part as in the narrower part. The lost kinetic energy will have been transformed into heat. (2) The kinetic energy which has disappeared may be greater than is enough to overcome the extra resistance ; a portion of it must, therefore, have gone to increase the potential energy, and the lateral pressure will be greater in the wide than in the narrow part. (3) The lost kinetic energy may be less than enough to overcome the extra resistance; in this case both the lateral pressure and the velocity will be less in the wide than in the narrow part. It has been experimentally shown that when a narrow portion of a tube is succeeded by a considerably wider portion, and this again by a narrow part, case (2) holds ; and the liquid may, under these conditions, actually flow from a place of lower to a place of higher lateral pressure.

In the vascular system the conditions are not the same. The widening of the bed which takes place as we proceed in the direction of the arterial current is not due to the widening of a single trunk, but to the branching of the channel into smaller and smaller tubes. In the larger arteries the increase of resistance is so gradual that both the potential and the kinetic energy diminish only slowly, and the lateral pressure and velocity are not much less in the femoral artery than in the aorta or carotid. But in the artericles the friction increases so greatly that although the velocity, and therefore the kinetic energy, in the capillary region is much less than in the arteries, the amount of kinetic energy lost is not upon the whole equivalent to the energy consumed in over- 
coming the extra resistance; the potential energy of the blood is also drawn upon, and the lateral pressure falls sharply in the capillary region, as well as the velocity. Where the capillaries open into the veins, the lateral pressure again sinks abruptly, while the velocity begins to increase, till in the largest veins it is probably about half as great as in the aorta.

Where does the extra kinetic energy of the blood in the veins come from? To say that the vascular channel again contracts as the blood passes from the capillaries into the veins, and that, since the same quantity must flow through every cross-section of the channel, the velocity must necessarily be greater in the narrower than in the wider part, does not answer the question. The greater portion of the kinetic energy of the arterial blood is, as we have seen, destroyed, or, rather, changed into an unavailable form, into heat, in the capillary region. The mean velocity of the blood in the capillaries is not more than $\frac{1}{300}$ to एकo of the velocity in the aorta; the kinetic energy of a given mass of blood in the capillaries cannot therefore be more than $\left(\frac{1}{200}\right)^{2}$, or $\frac{1}{400 \overline{0}}$ of its kinetic energy in the aorta. In the veins, taking the velocity at half the arterial velocity, the kinetic energy of the mass would be one-fourth of that in the aorta, or at least I0,000 times as great as in the capillary region. This extra kinetic energy comes partly from the transformation of some of the potential energy of the blood. The resistance in the veins is very small compared with that in the capillaries; less of the potential energy represented by the lateral pressure at the end of the capillary tract is required to overcome this resistance, and some of it is converted into the kinetic energy of visible motion, the lateral pressure at the same time falling somewhat abruptly. Contributory sources of kinetic energy in the veins are the aspiration caused by the respiratory movements and the pressure caused by muscular contraction in general, which, thanks to the valves, always aids the flow towards the heart. From these two sources new energy is supplied, to reinforce the remnant due to the cardiac systole (p. I2I).

Measurement of the Velocity of the Blood.-1. Direct Observation. - (a) This method can be applied to transparent parts by observing the rate of flow of the corpuscles under the microscope. But it is only where the blood moves slowly, as in the capillaries, that the method is of use. (b) Part of the path of the blood through a large vessel may be artificially rendered transparent by the introduction of a glass tube, of approximately the same bore as the vessel i'Volkmann). The tube is filled with salt solution, and the blood admitted by means of a stopcozk at the moment of observation. The time which the blood takes to pass from one end of the tube to the other is noted, and the length divided by the time gives the velocity of the blood in the tube. If the calibre of the tube is the same as that of the artery, this is also the velocity in the vessel; but if the calibre is 
different, a correction would lave to be made. The method is not a good one, for the reason, among others, that the long tube introduces an extra resistance.

2. Ludwig's Stromuhr.-This instrument measures the quantity

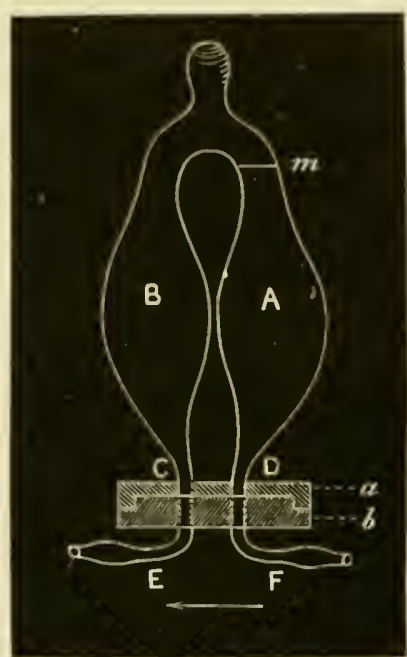

Fig. 39,-Stronulir of LUdWiG AND DOGIEL.

$\mathrm{A}, \mathrm{B}$, glass bulbs; $a$, a metal dise, to which $A$ and $G$ are at. tached, and which can be rotated on the disc $b ; \mathrm{E}, \mathrm{F}$, cannule attached $t \leftrightarrow b$, and connected with the peripheral and central end; of a divided bloodvessel. At the beginning of the experiment, A and the junction between $\mathrm{A}$ and $\mathrm{B}$ are filled with oil ; $B$ is filled with physiolngical solt solution or defibrinated blood: $a$ being turned into the position sh $\mathrm{Wn}$ in the figure, the blood passes through $\mathrm{F}$ and 1 ) into $A$, and the oil is forced into B. As soon as the blood has reachel the mark $m$, the dise $a$, with the bulbs, is rapidly rotated. so that $\mathrm{C}$ is now opposite $\mathrm{F}$. The blood now passes into $\mathrm{B}$, and the oil is again driven into $A$. When the oil has re ched 1 , reversal is again made, and so on.

is $\pi \times\left(\begin{array}{l}3 \\ 2\end{array}\right)^{2}=3_{4}^{3.14 \times 0}=7.06$ sq. mm. The velocity is $\frac{1000}{7.00}=141 \mathrm{~mm}$. per second.

Various improvements in this method have been made, such as a graphic registration of the reversals of the stromuhr. of blood which passes in a given time through the vessel at the cross-section where it is inserted. It consists of a $U$-shaped tube, with the limbs widened in to bulbs, but narrow at the free ends, which are connected with a metal disc. By rotating the instrument, these ends can be placed alternately in communication with a cannula in the central, and another in the peripheral portion of a divided artery; or they can be placed so that none of the blood passes through the bulbs, but all goes by a short-cut. One limb of the instrument is filled with oil, and the other with defibrinated blood. The limb containing the oil is first put into communication with the central end, and that containing the blood with the peripheral end of the artery. The blood from the artery rushes in and displaces the oil into the other limb, the defibrinated blood passing on into the circulation. As soon as the blood has reached a certain height, indicated by a mark, the instrument is reversed, and the oil is again displaced into the limb it originally occupicd. This process is repeated again and again, the time from beginning to end of an experiment being carefully noted. The number of times the blood has filled a bulb in that period, the capacity of the bulb and the cross-section of the vessel being known, all the data required for calculating the velocity of the blood in the ressel have been obtained.

Suppose, for example, that the capacity of the bulb up to the mark is 5 c.c., and that it is filled twelve times in a minute, the quantity flowing through the cross-section of the artery is I c.c., or 1.000 cub. $\mathrm{mm}$. per second. l.et the diameter of the vessel be $3 \mathrm{~mm}$, then its sectional area 
3. I tube or box, in which swings a small pendulum, is inverterl in the course of the vessel. The pendulum is deflected by the blemel. and the anrount of the deflection bears a relation to the velocity of the stream ( Iierordt's hermilachometer: Chatreau and lorict:s much more perfect dromogiaph) ( Fig. +1$)$.

4. Pitot's 'Tubes. - If two vertical tubes, " and $b$. of the form shoin in lig. fo, be inserted into is horizontial tube in which licpuid is llowing in the direction of the arrow. the level will be higher in a than would be the case in an ordinary sicle-tube

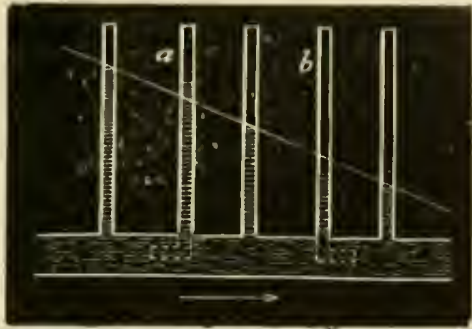

Fig. 40.-Pitot's Tubes. without an elbow: in $b$ it will be lower. For the moving liquid will exert a push on the columm in $a$, and a pull on that in $b$. The amount of this push and pull will vary with the velocity, so that a change in the latter will correspond to an alteration in the difference of level in the two tubes. Instruments on this principle have been constructed by Marey and Cybulski, the former registering the movements of the two columns of blood by comnecting the tubes to tambours provided with writing levers, the latter by photography (Fig. +4).

5. The electrical method, described on 1 ). 123 , for the measurement of the circulation time, can also be applied to the estimation of the mean velocity of the blood between two cross-sections of the arterial path which are separated by a sufficient distance. For example, salt solution can be injected into the left ventricle or the beginning of the aorta, and the interval which it takes to reach a pair of electrodes in contact with, say, the femoral artery determined. Knowing the distance between the point of injection and the electrodes, we can then calculate the mean velocity.

Of these methods, 3 and 4 are alone suited for the study of the velocity-pulse, that is, the change of velocity occurring with every beat of the heart. The curves obtained by Chauveau's dromograph show a general agreement with blood-pressure tracings taken

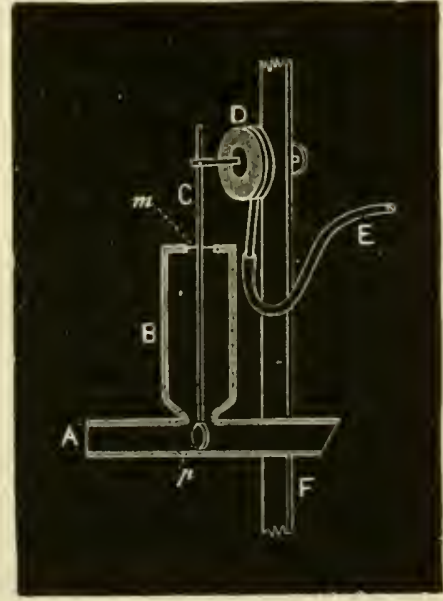

Fig. +1.-Chauveau's DromoGRAPH.

A, tube connected with blood. vessel; $B$, metal cylinder in communication with $A$. The upper end of $\mathrm{B}$ has a hole in the centre, which is covered by a membrane, $m$, through which a lever, $C$, passes; $C$ has a small disc, $p$, at its end, which projects in:o the lumen of $A$, and is $d e$ flected in the direction of the bloodstream through A. The deflection is registered by a recording tambour in communicition by the tube $E$ with $A$ tambour $D$, the flexible membrane of which is connected with the lever or pendulum $\mathrm{C}$. 
by a spring manometer, and with records of the external pulse obtained hy a spliygmograph. There is a primary increase of velocity corresponding with the ventricular systole, and a

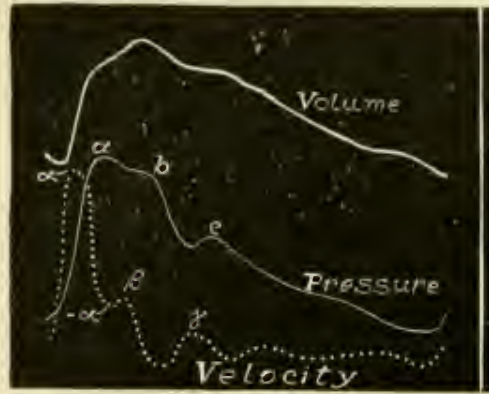

FIG. 42.

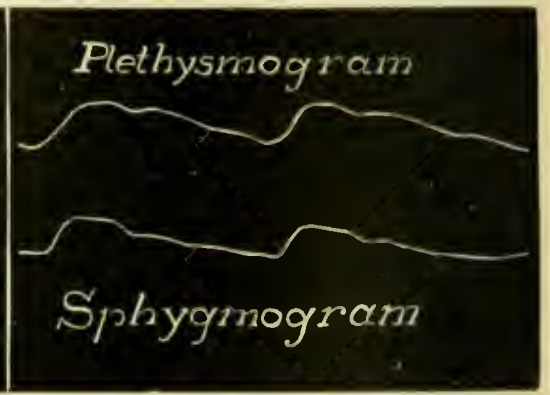

FIG. 13.

Fis. 42.-The highest of the three curves is a plethysmograplic record taken from the hand; the second curve is a sphygmogram taken simultaneously from the corresponding radial artery; the lowest (interrupted) eurve is the curve of velority deduced from a comparison of the first two (Fick).

FIG. 43.-Simultaneous plethysmographic and sphygmographic tracings.

secondary increase corresponding with the dicrotic wave (Fig. 45). Like all the other pulsatory phenomena, the velocity-pulse disap-

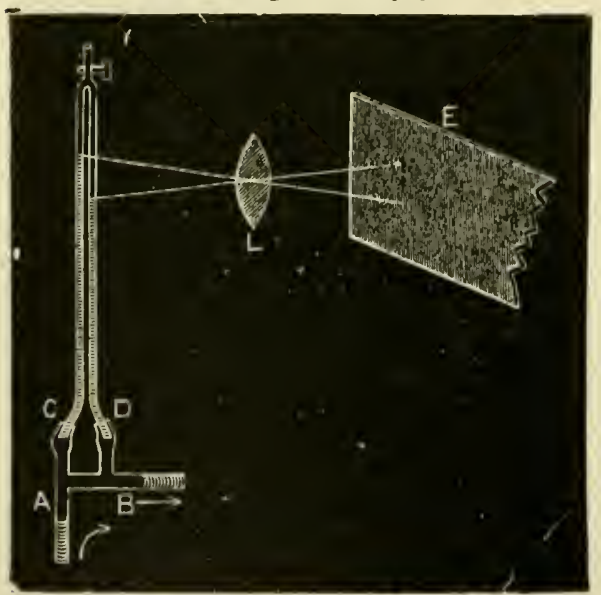

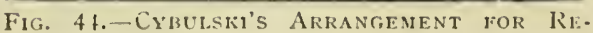
CORDING VARIATIONS IN TIIE VElOCITY OF TIIE BLOOD.

A, tube comecled with central, B with peri plieral end of divided bloodvessel. The blood stand: higher in the tube $C$ than in D. A beam of light passing through the meniscus in both tubes is focussed by the leus I. on the: travelling photographic plate $\mathrm{E}$. The velocity at any moment is deduced from the height of the meniseus in the two tubes $C$ and $D$. pears in the capillaries, and is only present under exceptional circumstances in the veins.

Fick, from a comparison of sphygmographic and plethysmographic tracings (p. II7), taken simultaneously from the radial artery and the hand, lias demon. strated that in mar. the velocity-pulse exhibits the same general cliaracters as in animals (Figs. 42 and 43). And v. Kries has confirmed Fich's conclusions by actual records of the velocitypulse obtained by means of an arrangement called a gas tachograph (Fig. 45 ). 
This consists of a plethysmograph connected with the tube of a gas-burner. When the part enclosed in the plethysmograph expands, air issues from the connecting tube, and canses an increase in the height of the flame. When the part shrinks, air is drawn in from the flame, which is depressed. Since the speed

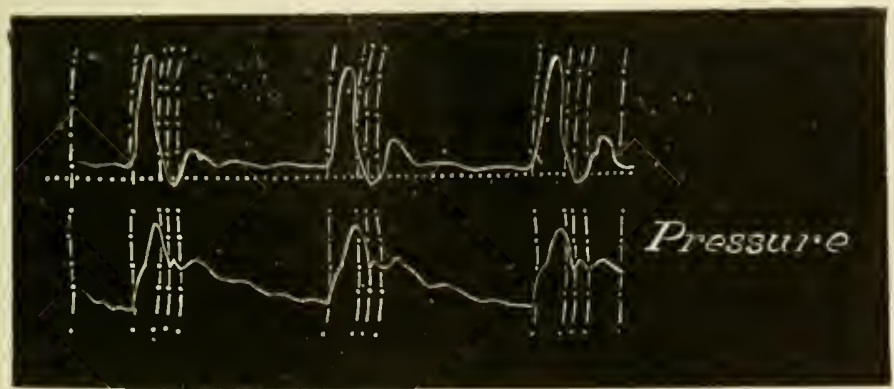

Fig. 45.-Sinultaneol's Tracisgs of tie Velocity (Upper Curve) a.je Pressitre (Lower Curve) (Lortet).

The tracings were taken from the carotid artery of a horse. The curve of velocity was obtained by the dromograph. The dicrotic wave is marked on it. The slightly curved ordinates drawn through the curves indicate corresponding points.

of the blood in the veins may be considered constant during the time of an experiment, the rate at which the volume of the part alters at any moment is a messure of the pulsatory change of velocity in the arteries of the part. And by photographing the movements of the tlame on a travelling sensitive surface, the velocity-pulse is directly recorded.

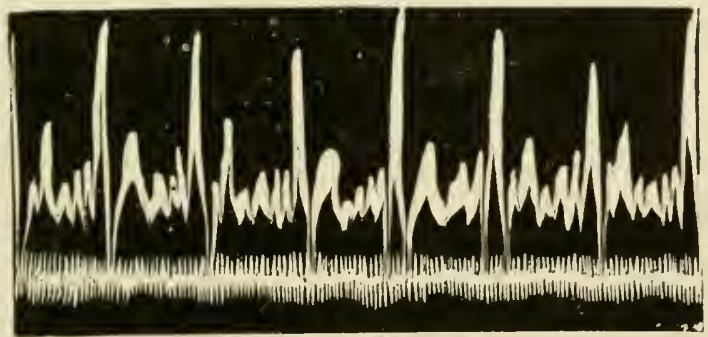

Fig. 4U.-Photographic Record of the Velocity-pulse obtained by rhe Gas Tachograph (v. Kries).

The upper curve is the photographic representation of the movements of the flame, and corresponds to the curve of velocity.

The mean velocity, like the mean blood-pressure, is more variable in the large arteries near the heart than in the smaller and more distant arteries. Dogiel found in measurements takeu with the stromuhr (a good instrument for the estimation of mean speed), within a period of two minutes, velocities ranging from 
over $200 \mathrm{~mm}$. to under $\mathrm{IOO} \mathrm{mm}$. per second in the carotid of the rabhit, and from over $500 \mathrm{~mm}$. to less than $250 \mathrm{~mm}$. in the carotid of the dog. Chauveau, with the dromograph, found the velocity in the carotid of a horse to be $520 \mathrm{~mm}$. per second during systole, I50 $\mathrm{mm}$. during the pause, $220 \mathrm{~mm}$. during the period of the dicrotic wave.

It is probable, however, that if these numbers are at all accurate for bloodvessels in the immediate neighbourluood of the heart, there must be a rapid diminution in the velocity even while the arteries are still of considerable calibre. For it has been found by the electrical method that, in anrsthetized dogs at any rate, as is shown in the following table, the mean velocity between the origin of the aorta and the crural artery in the middle of the thigh is usually less than roo $\mathrm{mm}$. per second.

\begin{tabular}{|c|c|c|c|c|c|c|}
\hline $\begin{array}{l}\text { No. of } \\
\text { Experi- } \\
\text { ment. }\end{array}$ & $\begin{array}{l}\text { Body. } \\
\text { weight } \\
\text { in Kilos. }\end{array}$ & $\begin{array}{c}\text { Distance between } \\
\text { Point of Injection } \\
\text { and Electrodes, } \\
\text { in Millinietres. }\end{array}$ & $\begin{array}{l}\text { Average Time be } \\
\text { tween Injection } \\
\text { and Arrival of the } \\
\text { Salt Solution, in } \\
\text { Seconds. }\end{array}$ & $\begin{array}{c}\text { Average } \\
\text { Pulse-rate } \\
\text { per Mlinute. }\end{array}$ & $\begin{array}{l}\text { Average } \\
\text { Velocity } \\
\text { per ieennd. } \\
\text { in Mlilli- } \\
\text { metres. }\end{array}$ & $\begin{array}{l}\text { Av: rage } \\
\text { Distance } \\
\text { traversed per } \\
\text { Heart beat, in } \\
\text { Nlillimetres. }\end{array}$ \\
\hline $\begin{array}{l}\text { I. } \\
\text { II. } \\
\text { III. } \\
\text { IV. } \\
\text { V. }\end{array}$ & $\begin{array}{l}34 \cdot 55 \\
17 \cdot 5 \\
14 \cdot 99 \\
10 \cdot 32 \\
7 \cdot 165\end{array}$ & $\begin{array}{l}420 \\
495 \\
400 \\
470 \\
330\end{array}$ & $\begin{array}{l}4 \cdot 62 \\
5 \cdot 7 \\
5 \cdot 0 \\
7 \cdot 12 \\
7 \cdot 83\end{array}$ & $\begin{array}{c}105 \\
69 \\
102 \\
74.5 \\
46.3 \\
\text { (weak beat) }\end{array}$ & $\begin{array}{l}90.9 \\
86 \cdot 8 \\
80 \\
72 \cdot 9 \\
42 \cdot 1\end{array}$ & $\begin{array}{l}51^{\circ} 9 \\
75^{\circ}+4 \\
47^{\circ} 0 \\
58^{\circ} 7 \\
54^{\circ} 5\end{array}$ \\
\hline
\end{tabular}

In I. the injecting cannula was in the descending part of the thoracic aorta, in V. at the very origin of the aorta, and in II., III., and $I V$. in the left ventricle.

As to the speed of the blood in the arteries of man, our data are insufficient for more than a loose estimate. But it does not seem likely that the mean velocity of a particle of blood in moving from the heart to the femoral artery can exceed $150 \mathrm{~mm}$. per second for the whole of its path. This would correspond to rather more than a third of a mile per hour. In the arch of the aorta the average speed may be twice as great. "The rivers of the blood' are, even at their fastest. no more rapid than a sluggish stream. A red corpuscle, even if it continued to move with the velocity with which it set out through the aorta, would only cover about 5 miles in twenty-four hours, and would require five years to go round the world.

The Volume-pulse.-When the pulse-wave reaches a part it distends its arteries, increases its volume, and gives rise to what may be called the volume-pulse.

This may be readily recorded by means of a plethysmngraph, an instrument consisting essentially of a chamber with rigid walls which 
enclose the organ, the intervening space being filled np with liquicl (Fig. 47). The movements of the liquid are transmitted citler. through a tube filled with air to a recording tambour, or directly to) a piston or float acting npon a writing lever. Special names lave been given to plethysmographs adapted to particular organs; for example, Roy's oncometer for the kidney. The method has been successfully applied to the investigation of circulatory changes in man, a finger, a hand or an entire limb being enclosed in the plethysmograph. With a fairly sensitive arrangement, every beat of the heart is represented on the tracing by a primary elevation and a dicrotic wave (Fig. $\left.f^{8}\right)$.

The general appearance of the curve is very similar to that of an ordinary pulse-tracing, though there are some differences of detail, especially in the time relations. A volume-pulse has been actually observed not only in limbs and portions of limbs, but also (in animals) in the spleen, kidney and brain, and other organs, and in the orbit.

The so-called cardio-pneumatic movements also constitute

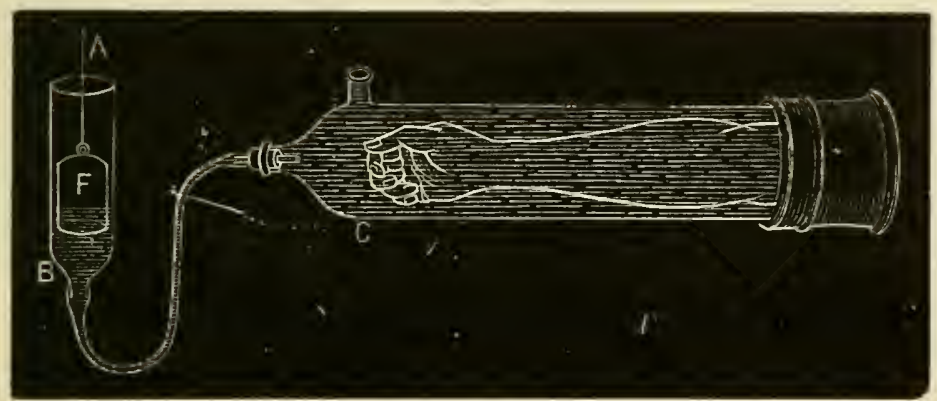

Fig. 47.-PlethysMograpiI FOR ARM.

$F$, float attached by $A$ to a lever which records variations of level of the water in $B$, and therefore variations in the volume of the arm in the glass vessel $C$. Or the plethysmograph may be connected to a recording tambour. The tubulure at the upper part of $\mathrm{C}$ is closed when the tracing is being taken.

a volume-pulse, although of complex origin. This name is given to the rhythmical changes of pressure accompanying the beat of the heart, which can be detected in the air of the respiratory passages when one nostril is connected with a recording tambour, or water manometer, the other nostril and the mouth being closed, and the respiration suspended in inspiration, with the glottis open. Or the mouth may be connected with the recording apparatus, the nostrils being closed. One factor in the production of these movements may be the change of blood-volume in the soft tissues of the mouth, naso-pharynx, and perhaps also in the lower respiratory passages accompanying the heart-beat. Another factor, and a more influential one, is the rhythmical alteration of pressure caused directly by the alternate systole and diastole of 
the heart in the air contained in the lung-tissue surrounding it, which acts as a kind of air plethysmograph. One interesting way in which the cardio-pneumatic movements may reveal themselves is by a variation with each beat of the heart in the intensity of a note prolonged in singing, especially after fatigne has set in. Upon the whole, the air-pressure falls during systole, owing to the expulsion of blood from the chest, and rises during diastole. The main cardio-pneumatic movement is, therefore, a systolic inspiration and a diastolic expiration (Practical Exercises, p. 29I).

Doubtless the weight of an organ would also show a pulse corresponding to the beat of the heart, and so would the temperatureat least, of the superficial parts. For the amount of heat given off by the blood to the skin increases with its mean velocity, and, therefore, although the difference may not in general be measurable, more heat is presumably given off luring the systolic increase of velocity than during the diastolic slackening. And this, along with other

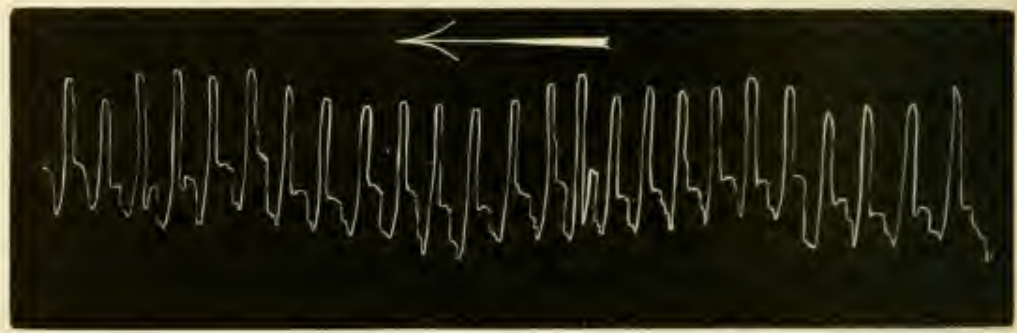

Fig. 48.-PletuYsmograph Tracixg From Arm.

The tracing was taken by means of a tambour connected with the plethysmograph. The dicrotic wave is distinctly marked.

considerations, suggests that, at any rate in certain situations and under certain conditions, there may even be a pulse of chemical change ; that is, a slight and as yet cloubtless inapprecialble ebb and flow of metabolism corresponding to the rhythm of the heart.

The Circulation in the Capillaries.-From the arteries the blood passes into a network of narrow and thin-walled vessels, the capillaries, which in their turn are comnected with the finest rootlets of the veins. Physiologically, the arterioles and venules must for many purposes be included in the capillary tract, but the great anatomical difference-the presence of circularlyarranged muscular fibres in the arterioles, their absence in the capillaries-has its physiological correlative. The calibre of the arterioles can be altered by contraction of these fibres under nervous influences; the calibre of the capillaries, although it varies passively with the blood-pressure, and is possibly to some extent affected by active contraction of the endothelial cells, cannot be under the control of vaso-motor nerves acting on muscular fibres (but see p. I57). 
Harver had deduced from his observations the existence of channels between the arteries and the veins. Malpighi was the first to observe the capillary bloorl-stream with the microscope, and thus to give ocular demonstration of the truth of Harvey's brilliant reasoning. He used the lungs, mesentery and bladder of the frog. The web of the frog, the tail of the tadpole, the wing of the bat, the mesentery of the rabbit and rat, and other transparent parts, have also been frequently employed for such investigations. From the apparent velocity of the corpuscles and the degree of magnification, it is easy to calculate the velocity of the capillary blood-stream. It has been estimated at from 0.2 to $0.8 \mathrm{~mm}$. per second in different parts and different animals. .

The comparative slowness of the current and the disappearance of the pulse are the chief characteristics of the capillary

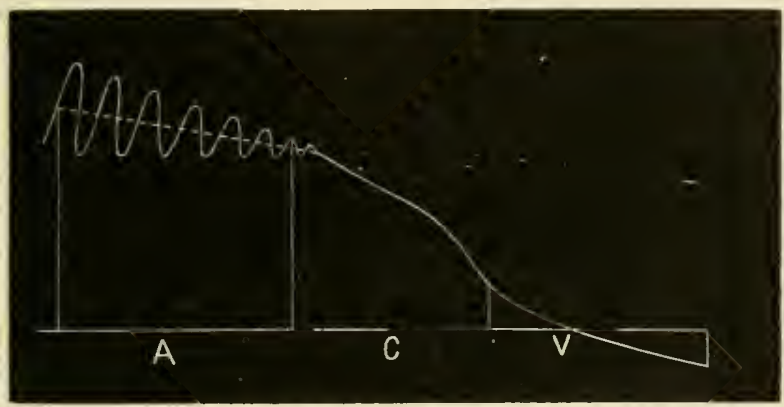

Fig. 49.-Diagrau to illéstrate the Slope of Pressure along the Vasculai: SYSTEM.

A, arterial ; C, capillary ; V, venous tract. The interrupted line represents the line of mean pressure in the arteries, the wavy line indicating that the pressure varies with each heart-beat. The line passes below the abscissa axis (line of zero or atmospheric pressure) in the veins, indicating that at the end of the venous system the pressure becomes negative.

circulation. The explanation we have already found in the great resistance of the narrow arterioles and the much-branched capillary ressels. Although the average diameter of a capillary is only about Io $\mu$ ( 5 to $20 \mu$ in clifferent parts of the body), the number of branches is so prodigious that the total cross-section of the systemic capillary tract has been estimated at 500 to 700 times that of the aorta. Such estimates are, of course, by no means exact.

The total cross-section of the vascular channel gradually widens as it passes away from the left ventricle. In the cap:1lary region it undergoes a great and sudden increase. At the venous end of this region the cross-section is again somewhat abruptly contracted, and then gradually lessens as the right side of the 
heart is approached; but the united sectional area of the large thoracic veins is greater than tlat of the aorta.

Attempts liave been made to measure the blood-pressure in the capillaries by weigliting a small plate of glass laid on the back of one of the fingers behind the nail, until the capillaries are just emptied, as shown by the paling of the skin (v. Kries), or by olserving the height of a column of liquid that just stops the circulation in a transparent part (Roy and Graham Brown). The last-named observers found that a pressure of 100 to $150 \mathrm{~mm}$. of water fabout 7 to $11 \mathrm{~mm}$. of $\mathrm{Hg}$ ) was needed to bring the blood to a standstill in the capillaries and veins of the frog's web; that is, about a third of the blooklpressure in the frog's aorta. The pressure in the capillaries at the root of the nail in man varies from 30 to $50 \mathrm{~mm}$. of mercury, as estimated by the methol of $v$. Kries. But the method is exposed to serious errors.

Under certain conditions the pulse-wave may pass into the capillaries and appear beyond them as a venous pulse. Thus,

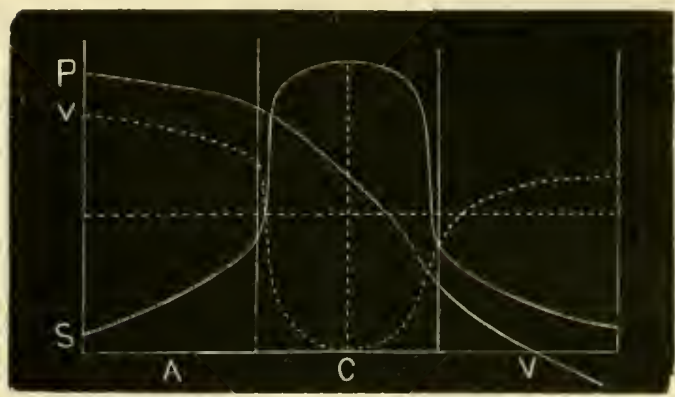

Fici. 50, - Relation of BloOd-PREsst"Re, Velocity. ANI CROSS-SECTION.

The curves $P, V$, and $S$ represent the blond-pressiure, velocity of the blood, and total cross-section respectively in the arteries $\mathrm{A}$, capillaries $\mathrm{C}$, and veins $\mathrm{V}$. ally when they are partially empticd by pressure-as a flicker of pink that comes and goes with every beat of the heart.

We have seen that the lateral pressure at any point of a uniform rigid tube through which water is flowing is proportional to the amount of resistance in the portion of the tube between this point and the outlet. In any system of tubes the sum of the potential and kinetic energy must diminish in the direction of the flow; and although the problem is complicated in the vascular system by the branching of the channel and the variation in the total cross-section, yet theory and experiment agree that in the larger arteries the lateral pressure diminishes but slowly from the heart to the periphery, the resistance being small compared with the resistance of the whole circuit. In the capillary region the vascular resistance abruptly increases; the velocity (and therefore the kinetic energy) abruptly diminishes, and the lateral pressure falls much more stecply between the begimning and the end of this region than between the heart and 
its commencement. In the veins only a small remnant of resistance remains to bz overcome, and the lateral pressure must sink again rather suddenly about the end of the capillary tract. Fig. 5o shows by a rough diagram the manner in which the pressure, velocity and cross-section probably change from part to part of the vascular system.

The Circulation in the Veins.-The slope of pressure, as we have just explained, must fall rather suddenly near the beginning and near the end of the capillary tract. It continues falling as we pass along the veins, till the heart is again reached. In the right heart, and in the thoracic portions of the great veins which enter it, the pressure may be negative-that is, less than the atmospheric pressure. And since nowhere in the venous system is the pressure more than a small fraction of that in the arteries, its measurement in the veins is correspondingly difficult, because any obstruction to the normal flow is apt to artificially raise the pressure. A manometer containing some lighter liquid than mercury, such as water or a solution of sodium citrate or magnesium sulphate, is usually employed, so that the difference of level may he as great as possible. In the sheep the pressure was found to be $3 \mathrm{~mm}$. of mercury in the brachial, and about $\mathrm{I} \mathrm{mm}$. in the crural vein. Opitz obtained the following pressures in dogs (of about I5 kilos): left facial vein, 5.I; right external jugular, $-0.1 \mathrm{I}$; central end of superior vena cava, -2.8 ; femoral vein, 5.4 ; renal vein, 10.9; portal vein, $8.9 \mathrm{~mm}$. of mercury.

The venous pressure being so low, or, in other words, the potential energy which the systole of the heart imparts to the blood being so greatly exhausted before it reaches the veins, other influences begin here appreciably to affect the bloodstream :

I. Contraction of the Muscles.-This compresses the neighbouring veins, and since the blood is compelled by the valves, if it moves at all, to move towards the heart, the venous circulation is in this way helped.

2. Aspiration of the Thorax.-In inspiration the intrathoracic pressure, and therefore the pressure in the great thoracic veins, is diminished, and blood is drawn from the more peripheral parts of the venous system into the right heart (p. 21o).

3. Aspiration of the Heart.-When the heart, after its contraction, suddenly relaxes, the endocardiac pressure becomes negative, and blood is sucked into it, just as when the indiarubber hall of a syringe is compressed and then allowed to expand. But we cannot attribute any great importance to this; and, of course, it is only the relaxation of the right ventricle which could directly affect the venous circulation.

4. Every change of position of the limbs, as in walking, aids the vellous circulation (Braune), and this independently of the 
muscular contraction. When the thigh of a dead body is rotated outwards, and at the same time extended, a manometer connected with the femoral vein shows a negative pressure of 5 to Io $\mathrm{mm}$. of water. When the opposite movements are made, the pressure becomes positive.

It follows from the number of casually-acting influences which affect the blood-flow in the veins that it cannot be very regular or constant. We liave seen that in the great arteries there is a considerable variation of velocity and of pressure witl every discharge of the ventricle, and although this variation is absent from the veins, since normally the pulse, due to the ventricular discharge, does not penetrate into them, the venous flow is, nevertheless, as a matter of fact, more irregular than the arterial. So that if it is difficult to give a useful definition of the term 'velocity of the blood' in the case of the arteries, it is still more difficult to do so in the case of the veins. Where voluntary movement is prevented, one potent cause of variation in the venous flow is eliminated; and in curarized animals certain observers have found but little difference between the mean velocity in the veins and in the corresponding arteries. Others have found the velocity in the reins considerably less, which is indeed what we slould expect from the fact that the average cross-section of the venous system is greater than that of the arterial system. Opitz, by means of a stromuhr, obtained a mean velocity of $\mathrm{I}+7 \mathrm{~mm}$. per second in the external jugular vein of a I 3-kilo dog.

To sum up, we may conclude that, upon the whole, the blood passes with gradually-diminishiug velocity from the left ventricle along the arteries; it is greatly and somewhat suddenly slowed in the broad and branching capillary bed; but the stream gathers force again as it becomes more and more narrowed in the venous chanuel, although it never acquires the speed which it has in the aorta.

Venous Pulse.--To complete the account of the circulation in the veins, it may be recalled that, in addition to the venous pulse described on p. I20, which, as an occasional phenomenon, may travel through widened arterioles and capillaries from the arteries into the veins, and therefore in the direction of the bloodstream, a so-called venous pulse, travelling from the heart against the blood-stream and depending on variations of pressure in the right auricle, may be detected in the jugular vein in healtly persons, and far more distinctly in certain disorders of the circulation. In animals a venous pulse of this nature has been demonstrated in the vena cavie, the jugular vein, and with a delicate manometer even in the large veins of the limbs. It moves with a speed of $\mathrm{I}$ to 3 metres a second (.Norrow). It is most 
easily observed in the jugular veins in man, because of their proximity to the leart. IVe have already pointed out the significance of the study of this venous pulse for the analysis of cardiac events (p. qI). A jugular venous pulse of a perfectly different origin is seen in cases of incompetence of the tricuspid valve. Here the chief elevation is synchronous with the ventricular systole, and is caused by the regurgitation of blood from the right ventricle through the auricle into the veins. The so-called 'communicated renous pulse' is simply due to the proximity of some large artery, especially when enclosed in a common sheath, whose pulsations are directly transmitted to the rein. The changes of pressure in the great veins accompanying the respiratory morements (p. 266) are also sometimes spoken of as a venous pulse, but they are produced in an entirely different way-namely, by the rhythmical alteration in the intrathoracic pressure, which alternately favours and hinders the venous return to the heart.

The Circulation-time.-Hering was the first who attempted to measure the time required by the blood, or by a blool-corpuscle, to complete the circuit of the vascular system. He injected a solution of potassium ferrocyanide into a rein (generally the jugular), and collected blood at intervals from the corresponding vein of the opposite side. After the blood had clotted, he tested for the ferrocyanide by addition of ferric chloride to the serum. The first of the samples that gave the Prussian blue reaction corresponded to the time when the injected salt had just completed the circulation. This method was improved by Vierordt, who arranged a number of cups on a revolving disc below the vein from which the blood was to be taken. In these cups samples of the blood were received, and the rate of rotation of the disc being known, it was possible to measure the interval between the injection and appearance of the salt with considerable accuracy. Hermann made a further advance by allowing the blood to play upon a revolving drum covered with paper soaked in ferric chloride, and by using the less poisonous sodium ferrocyanide for injection.

Even as thus modified, the method laboured under serious defects. It was not possible to make more than a single observation on onc animal, at least without allowing a considerable interval for the elimination of the ferrocyanide, and, further, the method was unsuited for the estimation of the circulation-time in individual organs. In both of these respects the more recently introduced electrical method presents considerable advantages; for by its aid we can not only obtain satisfactory measurements of the circulation-time in such organs as the lungs, liver, kidney, etc., but we can repeat them an indefinite number of times on the same animal.

A cannula, connected with a burette (or a Mariotte's bottle, or a syringe), containing a solution of sodium chloride (usually a $\mathrm{I}^{*} 5$ to 2 per cent. solution), is tied into a ressel-say, the jugular vein. Suppose that the time of the circulation from the jugular to the carotid is required-that is, practically the time of the lesser or pulmonary circulation. A small portion of one carotid artery is 
isnlated, and laid on a pair of hook-shapwl plitinum electrodes.* covered, except on the concate side of the hook, with at layer of insulating varnish. To further secure insulation, at bit of very thin sheet-indiarubber is slipped between the artery and the tissues. By means of the electroles the piece of artery lying between them, with the blond that flows in it, is connected 11 p as onc of the resistances in a Wheatstone's briclge $(p .617)$. The secondary coil of at small inductorium, arranged for giving an intermpted current, and with a single Daniell or iry cell in its primary, is substituted for the battery, and a telephone for the galvanometer, according to Kohlrausch's well-known methor for the measurement of the resistance of electrolytes. It is well to have the induction nachine set $1 p$ in a separate room and connecterl to the resistance-box by long wires,

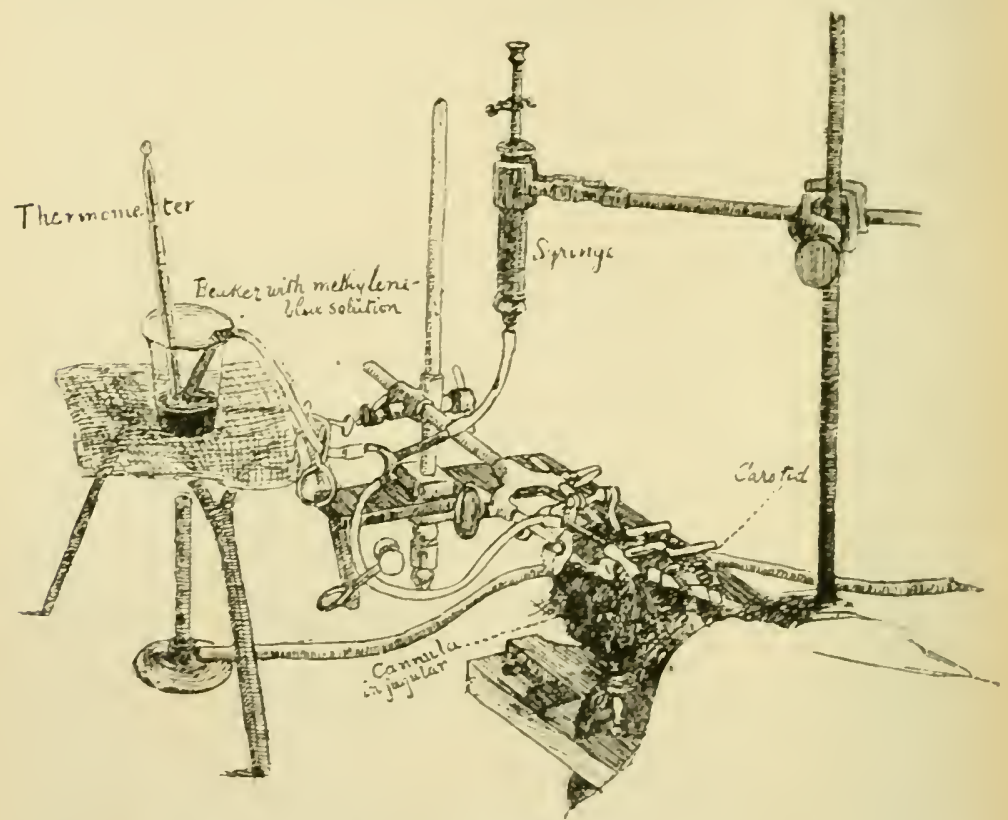

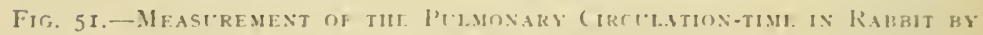
INJECTION OF MITITHA.NF BH.F.

so that the noise of the Neef's hamner may be inaulible. The bridge is balancel by adjusting the resistances until the sound heard in the teleplone is at its minimum intensity, the secondary coil being placed at such a distance from the primary that there is no sign of stimulation of muscles or nerves in the neighbourhnol of the electrodes when the current is closed. A definite, small guantity of the salt solution is now allowed to run into the vein by turning the stop-cock of the burette. It moves on with the velocity of the blood, and reaching the artery on the clectroles causes a diminution of its

* The electrodes can easily be malle hy beating out one end of a piece of thick platinum wire to a brearlth of 5 or $6 \mathrm{~mm}$., and then bencling the flattened part into a hook, or by bencling pieces of stout platinum foil. 
electrical resistance (j. 25). This disturbs the balance of the brialge. and the somel in the telephone becomes lourler. The time from the beniming of the injection to the alteration in the somel is the circula. tion-time between jugular and carotid. It can be reale off by al stop-watch, or more accurately by an clectric time-maker writing on a revolving drum (lig. 52). Instead of the telephone a galvanometer may be uscel, the crual aud oppositely directed induction shocks being replaced by a weak voltaic current and the platinum by unpolarizable clectrodes (1). 025). But this is less comveniont.

The circulation-time of an organ like the kidncy can be measured by adjusting a pair of electroles maler the renal artery and another under the renal vein, and reading off the interval required by the salt solution to pass from the point of injection first to the artery and then to the vein. The difference is the circulation-time through the kidney.

lior certain purposes, and particularly for measurements on small animals like the rabbit, or on organs whose ressels are too delicate to be placel on electroles without the risk of serious interference with the circulation, another method may be employed with advantage. It depends on the injection of a pigment, like methylene blue, which at first overpowers the colour of the blood and shows through the walls of the bloodvesscls, but is soon relucel to a colourless substance (Fig. 51). The details of the method are given in the Practical Exercises (p. 203).

It may be said in general terms that in one and the same animal the time of the lesser circulation is short as compared aith the total circulation-time, relatively constant, and but little affected by changes of temperature. In animals of the same species it increases with the size, but nore slowly. and rather in proportion to the increase of surface than to the increase of weight.

Thus a dog weighing 2 kilogrammes had an average puimonary circulation-time of $4^{\circ} \mathrm{O}$ seconds, while that of a $\log$ weighing II'S lilos was 8.7 seconds, and that of a log with a body-weight of 18.2 kilos only $\mathrm{IO}^{\circ}+$ scconds. It is probable that in a man the pulmonary circulation-time is not usually much less than 12 seconds, nor much more than is scconds.

The circulation-time in the kidney, spleen and liver is relatively long and much more variable than that of the lungs, being easily affected by exposure and changes of temperature (increased by cold, diminished by warmth).

In a dog of $13^{\circ} 3$ kilos weight the average circulation-time of the spleen was I0.95 seconds; kidney, I3.3 seconds; lungs, S 4 seconds. The circulation-time of the stomach and intestines is (in the rabbit) comparatively short, not exceeding very greatly that of the lungs, but it is lengthened by exposure. The circulation-time of the retina and that of the heart (coronary circulation) are the shortest of all.

The total circulation-time is properly the time required for the whole of the blood to complete the round of the pulmonary and systemic circulation. But there are many routes open to any given particle of blood in making its systemic circuit. If it passes from the aorta through the coronary circulation it takes an exceedingly short 
route. If it patsses through the intestines and liver, or through the kiclney, or through the lower limbs, it takes a long route. So that to iletermine the total circulation-time by direct measurement we must know (1) the quantity of blood that passes on the average by each path in a given time, and (2) the average circulation-time of each path. If the average weight of blood in each organ be representel by $w_{1}, w_{2}, w_{3}$. etc. ; and the average circulation-times by $t_{1}, t_{2}, t_{3}$. etc.; and $t$ be the total systemic circulation-time; then $w_{1 t_{1}}^{t}, w_{2} \frac{t}{t_{2}}, w^{\prime} t_{3}, \quad$ ctc. will represent the quantity of blood passing through each organ in $t$ seconds, since in the average circulationtime of an organ the whole of the blosel in it at the beginning of the period of observation will have been exchangel for fresh blood.

1
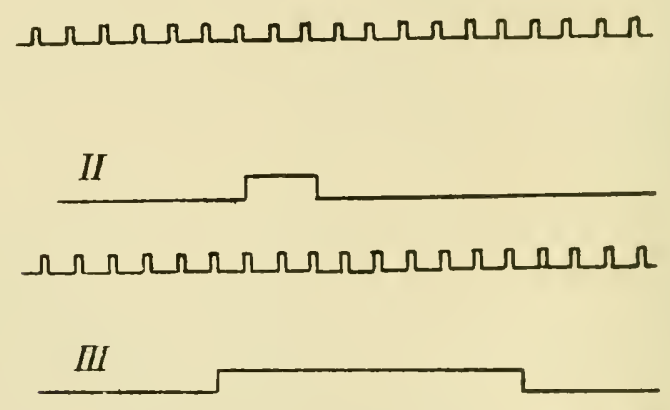

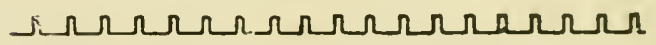

Fig. 52.-Time of tite Lesser Circulation. C.it añestietizen
witu Eibler.

Tine-trace, seconds. The line above the tiune-trace was writen liy an eleutro. magnetic signal, the circuit of which was clused at the mument when injection of methylene blue into the jugular vein was hegun, and opened at the moment when the change of colour in the carotid was whserved. I. mormal circulation-time : 1I, circulation-time after section of both vagi (muth (liminislsed): III, circulation-time during stimulation of the periplieral end of one vagus (much increased).

But the whole of the blood in the bolly. which we may call W: passes once round the systemic circulation in $t$ seconds. Therefore, $w_{1 t_{1}}+w_{2 t_{2}}^{t}+w_{3 t_{3}}^{t}$, etc., $=W$. In this equation everytling can be determined by experiment except $t$, and therefore $t$ can be calculated. Adding $t$ to the pulmonary circulation-time, we arrive at the total circulation-time.

Although our experimental data are as yet too meagre to make the calculation more than a rough approxination, it appears probable that in certain animals the total circulation-time is five or six times as great as the pulmonary circulation-time. If the same ratio holds good in man, the total circulation-time is unlikely to be much less than a minute or much greater than a minute and a quarter. We 
shall see dircetly that this estimate is confirmed by data derived from a different source. In the meantime, we may usc it jrovisionally to calculate the work done by the heart. l.ct us take for simplicity the total circulation-time as 1 minute in a 70 -kilo man, the quantity of blood as $f^{\frac{1}{2}}$ kilos, * and the mean pressure in the aorta as $150 \mathrm{~mm}$. of mercury. Up to the time when the semilunar valves are opened, the work lone by the left ventricle is spent in raising the intraventricular pressure tilt it is sufficient to overcome the pressure in the aorta. If a vertical tube were connected with the left ventricle. the blool would rise till the column was of the same weight as it column of mercury of equal section and $150 \mathrm{~mm}$. high. This column of blood would be about 1.92 metres in tieight. If a reservoir were placed in communication with the tube at this height, a quantity of blool equal to that ejected from the ventricle would at each systole pass into the reservoir; and the work which the blood thus collected would be capable of doing. if it were allowed to fall to the level of the heart. would be equal to the work expeniled by the heart in forcing it up. 'Thus, in 1 minute the work of the left ventricle would be cqual to that done in raising $+\frac{1}{2}$ kilos of blood to a height of $1 \cdot 92$ metres - that is, about $8.6+$ kilogramme-metres; in $2+$ hours it would be, say, I 2.450 kilogramme-metres. Taking the mean pressure in the pulmonary artery at one-third of the aortic pressure, we get for the daily work of the right ventricle about 4 , I 50 kilogrammemetres. The work of the two ventricles is thus about 16,600 kilogramme-metres. $f$ which is enough to raise a weight of nearly 4 pounds from the bottom of the decpest mine in the world to the top of its highest mountain, or to raise the man himself to $\left[\frac{1}{2}\right.$ times the height of the spire of Strasburg Cathedral, or twice the height of the loftiest 'skyscraper' in New York. By friction in the bloodvessels this work is almost all changed into its equiralent of heat, nearly to calories (p. 584). Further, since the contraction of the heart is always maximal (p. 14 I), and there is reason to believe that the quantity of blood ejected at a single systole by the left rentricle (being dependent upen the inflow from the pulmonary veins, and therefore upon the inflow into the right sicle of the heart from the systemic veins) varies widely, some of the mechanical effect of the contraction must be wasted when the quantity is less than the ventricle is capable of expelling.

Output of the Heart. - If $4^{\frac{1}{2}}$ kilos of blood pass through the heart in I minute with the average pulse-rate of 72 per minute, the quantity ejected by cither ventricle with crery systole will be $\begin{gathered}45^{\circ} \\ 72\end{gathered}=62^{\circ} 5 \mathrm{grm}$, or a little less than 60 c.c. This is much less than the amount assigned by Vicrordt, which at one time gained great vogue in physiological text-books, but all recent observers who have directly measured the output are agreed that Vicrordt's estimate is too high. Thus, in a series of experiments on more than twenty dogs, ranging in weight from 5 to nearly 35 kilos, it has been shown that the output. or contraction volume. as it is sometimes called, of the left ventricle per kilo of body-weight diminishes as the size of the animal increases; and the relation between body-weight and output is such that in a man weighing 70 kilos we can hardly suppose that the

* The mean of the $5 \frac{1}{2}$ kilos given by most writers, and of the $3 \frac{1}{2}$ kilos obtained by Haldane and Smith (p. 49).

+ Since the blood on expulsion is moving with a certain velocity, an addition might be made for its kinetic energy. But this would only increase the total work by a small fraction (about 1 per cent.). 
ventricle discharges more than 105 grm. of blood per second, or $\$ 7$ grm. (So c.c.) per heatt-beat with a pulse-rate of 72 . Putting this result aloug with that defuced from the circulation-time, we can pretty sifely conclude that the aterage anomut of blond thown out by each veritricle at each beat is not more than 70 or So c.c. \%untz. from the quantity of oxygen absorbed by the blood in the lungs, hats cstimated the output at 60 c.c. But according to him this maty be Ioubled during severe muscular work, when, ats al matter of fact, by the airl of the Röntgen-rays or by percussion of the chest. the volume of the heart nity be shown to be considerably increatsed. In the midllle of the eighteenth century, Passanant calculated the output at fo. 5 grm., which is certanly too low. Tigerstedt puts it at 50 to roo c.c.. Plesch at 59 c.c.

\section{The Relation of the Nervous System to the Circulation.}

So far we have been considering the circulation as a purely physical problem. We have spoken of the action of the heart as that of a force-pump, and perhaps to a small extent that of a suction-pump too. We have spoken of the bloodvessels as a system of more or less elastic tubes through which the blood is propelled. We have spoken of the resistance which the blood experiences and the pressure which it exerts in this system of tubes, and we have considered the causes of this resistance, the interpretation of this pressure, and the physical changes in the vascular system that may lead to variations of botlı. But so far we have not at all, or only incidentally and very briefly, dealt with the physiological mechanism through which the plyssical changes are brought about. We hase now to see that although the heart is a pump), it is a living pump ; that although the vascular system is an arrangement of tubes, these tubes are alive; and that both heart and ressels are kept constantly in the most delicate poise and balance by impulses passing from the central nervous system along the nerves.

In many respects, and notably as regards the influence of nerves on it, we may look upon the heart as an expanded, thickened and rhythmically-contractile bloodvessel, so that an account of its imnervation may fitly precede the description of vaso-motor action in general.

The Relation of the Heart to the Nervous System. A very simple experiment is sufficient to prove that the beat of the heart does not depend on its comnection with the central nervous system, for an excised frog's lieurt may, under faromable conditions, of which the most important are a moderately low temperature, the presence of oxygen and the prevention of evaporation, continue to beat for days. The mammalian heart also, after removal from the body, beats for a time, and indeed, if delibrinated blood be artificially circulated through the coronary vessels, for several or even many hours. But although this 
proves that the heart can beat when separated from the central nervous system, it does not prove that nervous influence is not essential to its action, for in the cardiac substance nervous elements, bot hi cells and libres, are to be found.

The Intrinsic Nerves of the Heart. - In the heart of the frog numerous nerve-cells occur in the sinus venosus, especially near its junction with the right auricle (Remak's ganglion). A branch from each vagus, or rather from each vago-sympathetic nerve (for in the frog the vagus is joined a little below its exit from the skull by the sympathetic), enters the heart along the superior vena cava (pp. I43, I82).

Running through the sinus, with whose ganglion-cells the true vagus fibres, or some of them, are believed to make physiological junction (p. 149), the nerves pursuc their course to the auricular septum. Here they form an intricate plexus, studded with ganglioncells. From the plexus nerve-fibres issue in two main bundles, which pass down the anterior and posterior borders of the septum to end in two clumps of nerve-cells (Bidder's ganglia), situated at the auriculo-ventricular groove. These ganglia in turn give off fine nerve-bundles to the ventricle, which form three plexuses-one inder the pericardium, another under the endocardium, and a third in the muscular wall itself, or myocardium. From the last of these plexuses numerous non-medullated fibres run in among the muscular fibres and end in close relation with them. Similar plexuses of nerve-fibres exist in the mammalian ventricle. But while scattered ganglion-cells are found in the upper part of the ventricular wall. most observers have been unable to demonstrate any either in the mammal or the frog in the apical half. In the rat's heart, according to the careful observations of Schwartz, truc ganglion-cells are confined to an area on the posterior surface of the auricles, lying always under the visceral pericardium. Other writers, however, have stated that ganglion-cells do exist in the apex both of the cat's and of the frog's heart. In connection with the whole question it must be borne in mind that in other organs improved histological methols have brought typical nerve-cells to light in situations where they were not suspected or were clenied to exist, and, further, that all investigators are not agreed upon the histological criteria by which ganglion-cells are to be distinguished.

Cause of the Rhythmical Beat of the Heart.-Scarcely any physiological question has excited greater interest for many years than the mechanism of the heart-beat. Several properties of the cardiac tissue ought to be distinguished in discussing this question: (I) Its automatism-i.e., its power of beating in the absence of external stimuli ; (2) its rhythmicity-i.e., its power of responding to continuous stimulation by a series of rhythmically repeated contractions; (3) its conductivity-i.e., its power of conducting the contraction wave or the impulse to contraction once it has been set up; and (4) the power of co-ordination, in virtue of which the various parts of the heart beat in a regular sequence. 
The excitability of the cardiac tissue-that is, its power of appropriate response (namely, by contraction) to a suitable stimulus - does not particularly concern us here, since it is in no wise a property special to the heart. Only, as we shall see in the sequel, the time-relations of this excitability are of interest, for the existence of a refractory period-that is, an interval during which the cardiac muscle refuses to respond to excitation -throws light upon the rhythmicity of the leart-beat. The tonicity of the heart-i.e., its power of remaining contracted to a certain extent in the intervals between successive beats-is another property of great importance in certain aspects, but which only needs to be mentioned at present.

That the heart-beat is automatic, is sufficiently shown by the fact that, as already mentioned, an excised and empty heart will go on beating for a time, for many hours or even for days in the case of cold-blooded animals. When blood, or even a suitable solution of such inorganic salts as exist in serum, is caused to circulate through the coronary vessels of the excised heart of a warm-blooded animal, it also continues to contract for a long time. But where the cause of the automatism resides, in the muscular tissue or in the intrinsic nervous apparatus, cannot be decided offhand, because in nearly all animals litherto investigated the muscular tissue, ganglion-cells, and nerve-fibres are inseparably intermingled. In Limulus, however, the horseshoe or king crab, the cardiac ganglion-cells are collected in a nerve-cord running longitudinally in the median line along the dorsal surface of the segmented heart, and sending off at intervals branches to two lateral cords, and also branches which enter the heart muscle directly (Fig. 53). When the median nerve-cord is removed, as can be done without injuring the muscle, the heart ceases for ever to beat spontaneously. It still contracts when directly stimulated, mechanically or electrically, but the contraction never outlasts the stimulation. The antomatic power therefore resides in the nerve-cord alone, and not in the muscle. The same is true of the rhythmical power, for excitation of the nerves that pass from the median cord to the muscle produces, ' not a rhytlimical series of beats in the resting, and an acceleration of the rhythm in the pulsating heart, but a tetanus closely resembling that produced in skeletal muscle on stimulation of a motor nerve' (Carlson). Conduction and co-ordination are also effected in this heart through the nervous mechanism, and essentially through the median nerve-cord; for section of the longitudinal nerves in any segment of the heart abolishes the co-ordination of the two ends of the heart on either side of the lesion, while division of the muscle in any segment does not 
affect the co-ordination. It is not permissible to transfer these results wholesale to higher hearts, and especially the conclusions as to rhythm, conduction, and co-ordination. But in the case of the higher animals also facts may be adduced in favour of the neurogenic origin of the beat. The isolated auricular appendices of the mammalian heart, in which no ganglion-cells have been found, refuse to beat spontaneously. If in the frog we divide the sinus, which is conspicuously rich in ganglion-cells, from the lower portion of the heart, it continues to pulsate. A fragment from the base of the ventricle will go on contracting if it includes Bidder's ganglion, but not otherwise. We cut off the lower two-thirds of the frog's ventricle, the so-called apex preparation, which either contains no ganglion-cells or is relatively poor in them, and it remains obstinately at rest. Further, if, without actually cutting off the apcx, we dissever it physiologically from the heart by crushing a narrow zone of tissue midway

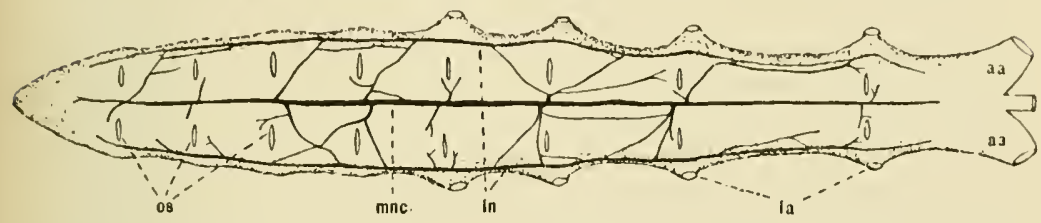

Fig. 53. - Tile Heart and the Heart Nerves of limulus : Dorsal View (CARLSON).

(The heart is figured one-half the natural size of a large specimen.)

$a a$, Anterior artery : $l a$, lateral arteries; $l n$, lateral nerves; mnc, median zerve. cord ; os, ostia.

between it and the auriculo-ventricular groove, we abolish for ever its power of spontaneous rhythmical contraction. The frog may live for many weeks, but in general the apex remains in permanent diastole. It can be caused to contract by an artificial stimulus, but it neither takes part in the spontaneous contraction of the rest of the heart, nor does it start an independent beat of its own.

What can be simpler than to assume that the sinus beats because it has numerous ganglion-cells in its walls, and that the apex refuses to beat because it has comparatively few or none? Could we pick out the nerve-cells from the sinus, without injuring the muscular tissue, as easily as we can extirpate the median nerve-cord in Limulus we may well suppose that it would lose its power of automatic contraction. And although, if we pursue our investigations a little farther, facts may emerge which seem to contradict the neurogennic hypothesis, the contradiction is usually only apparent. Let us inquire, for instance, what 
happens to the auricles and ventricle of the frog's heart when the sinus is cut off. The answer is that, as a rule, while the sinus goes on beating, the rest of the heart comes to a standstill, in spite of the numerous ganglion-cells in the auricular septum and the auriculo-ventricular groove. Not only so, but if the ventricle be now severed from the auricles by a section carried through the groove, it is the former, poor in nerve-cells though it be, which will usually first begin to beat. We shall again have to discuss this experiment (P. I5I). It, at any rate, cannot be interpreted as proving that the automaticity of the heart does not depend upon the presence of ganglion-cells. For although a portion of the heart rich in ganglion-cells may, under the circumstances mentioned, refuse for a time to beat, there is good evidence that this is due either to a peculiar condition called inhibition into which the muscular tissue or the nerve-cells of the lower portions of the heart have been thrown by the first section, or more probably to the loss of the accustomed impulses from the sinus which normally give the signal for the auricular contraction. A stronger argument in favour of the myogenic theory is the fact that the embryonic heart beats with a regular rhythm at a time when as yet no ganglion-cells have settled in its walls. But it may well be that this primitive automatic power of the cardiac muscle, absolutely necessary at first, since the carly establishment of the circulation is essential for the development of the tissues in general and of the nervous system in particular, falls into abeyance when the intrinsic cardiac nervous mechanism is completed, or at least becomes subordinated to the latter. The advocates of the myogenic theory further state that the isolated bulbus aorte of the frog, and even tiny fragments of it, will pulsate spontaneously, and that the same is true of small portions of the great veins which open into the sinus. The rhythmical contraction of the veins of the bat's wing has also been considered an argument in favour of myogenic automatism. In none of these cases, however, can the complete absence of ganglion-cells be considered satisfactorily demonstrated. The statement that a portion of the apex of the dog's ventricle continues for a considerable time to beat with a rhythm of its own when connected with the rest of the heart by nothing but its bloodvessels and the narrow isthmus of visceral pericardimm and connective tissue in which they lie has not been confirmerl by all observers. But even if it be accepted, it can hardly be used as a decisive argument against the neurogenic theory so long as the absence of ganglion-cells from such a ventricular strip has not been demonstrated.

The fact that under the influence of a constant stimulus portions of the heart can le marke to beat rhythmically has 
been sometimes, though erroneously, hromght forward as evidence of myogenic automatism. 'Thus the supposedly ganglion-free apex of the frog's heart, lifeless as it secms when left to itself, can be caused to execute a long and faultless scries of pulsations when its cavity is distended with defibrinated blood or serum, or certain artificial nutritive fluids, or even physiological salt solution. The passage of a constant current through the preparation may also start a regular rhythm. But apart from the question whether nervous elements would not be subjected to the constant stimulus impartially with the muscular elements (and nerve-fibres, at any rate, are acknowledged to be present), the beat here produced ought not to be considered as an automatic beat, but as a contraction evoked by an external stimulus. Sucli experiments, in fact, throw no light upon the automatism of the leart, but prove clearly its rhythmicity-i.e., its power of responding to a continuous stimulus by regularly recurring contractions. While we are hardly at present in a position to discriminate sharply between the influence of constant stimulation upon the nervous and upon the muscular elements of the heart, and certainly not in a position to deny to the nervous elements the power of responding to such stimulation by rhythmical discharges, it can hardly be doubted that the cardiac muscle itself possesses rhythmical power. This is a property which also belongs to the smootli muscle of such tubes as the ureter, whose rhythmical contraction is affected by distension much as that of the heart is, and in a smaller degree even to ordinary skeletal muscle, which can contract with a kind of rhythm under the stimulus of a certain tension and in certain saline solutions. But just as the primitive automatism of the cardiac muscle nuay have become subordinated in the course of development to the automatism of the nervous elements, so the primitive rhythmical power of the muscle may under ordinary conditions remain in abeyance and yet be capable of asserting itself in favourable circumstances, and when the normal rhythmical impulses from the nervous apparatus are withdrawn. In any case, in the normally beating heart the opportunity for the cxercise of the rhythmical power of the muscle does not arise, at least in the case of the lower portions of the heart. For the impulses which. (in the frog's heart), descending from the sinus, liberate the contraction of the auricles, and the impulses which, descending from the auricles, liberate the contraction of the ventricle appear to be discrete, and not continuous; in other words, the lower portions of the heart do not receive from the upper portions a continuous stream of stimuli to which they respond by rhytlinical contractions, but a series of rhythmically repeated impulses, each of which evokes a single contraction. One of the best 
proofs of this is that, if the sinus is heated the ventricle beats much more rapidly in unison with the rapidly beating sinus and auricles, while if the ventricle itself is heated no change takes place in its rhythm. Now, if the ventricle responds to a constant stimulus by rliythmical beats, the condition of the ventricular tissue ought to affect the rate of its beat. In the mammalian heart, too, an alteration in the temperature of a definite area of the wall of the right auricle lying between the mouths of the venæ cavæ, produces a change in the rate of the whole heart, while no effect is caused by altering the temperature of any other portion of the heart. It has already been stated that the impulses from the nerve-cord which maintain the rhythm in the Limulus heart are also discontinuous.

Conduction and Co-ordination.-The question of the conduction of the excitation over the heart and the co-ordination of its parts is in the same position as the question of the automatism and rhythmicity. In the horseshoe crab, as already remarked, the mechanism appears to be a nervous one. In higher hearts, on the other hand, facts have been discovered which favour each of the rival hypotheses. In the frog's heart the probability that the contraction wave is propagated from fibre to fibre of the muscle without the intervention of nerves has been much insisted upon, since the muscular tissue, although presenting certain variations in its character in the different divisions of the heart and at their junctions, forms a practically continuous sheet over the whole organ from base to apex. In support of this view has been brought forward the observation that the delay of the wave at the auriculoventricular groove is much greater than it ought to be if the excitation were transmitted by nerves, since the velocity of the nerve-impulse is exceedingly great (p. 689); and the further observation that, when the ventricle is caused to contract by artificial stimulation of the auricle, this delay is appreciably greater when the stimulus is applied as far from the ventricle as possible than when it is applied as near to it as possible. The delay has been attributed to the 'embryonic' character of the muscular tissue at the junction of the sinus with the auricles and of the auricles with the ventricles. But it has never been demonstrated that muscular fibres with the histological characters described do, as a matter of fact, conduct the contraction wave so much more slowly than the other cardiac muscular fibres. It is just as probable, and indeed more so, that whether the contraction travels in any particular division of the heart directly from muscle-fibre to muscle-fibre or not, the impulse to contraction is transferred from each division of the heart to the next by a nervous mechanism whose action is timed with the very object 
of securing a certain interval between the systoles of successive divisions. In any case, since we know that the velocity of the nerve-impulse is very different in different varieties of nerves, the question cannot be decided by general arguments of this kind. In Limulus, as a matter of fact, the velocity in the intrinsic heart nerves is only one-tenth as great as in the ordinary motor (limb) nerves of the animal (Carlson).

In the mammalian heart the alleged absence of muscular connection between the auricles and ventricles was long the foundation of the general belief that the link was a nervous one. Certainly there is no dearth of nerves which might serve as such a

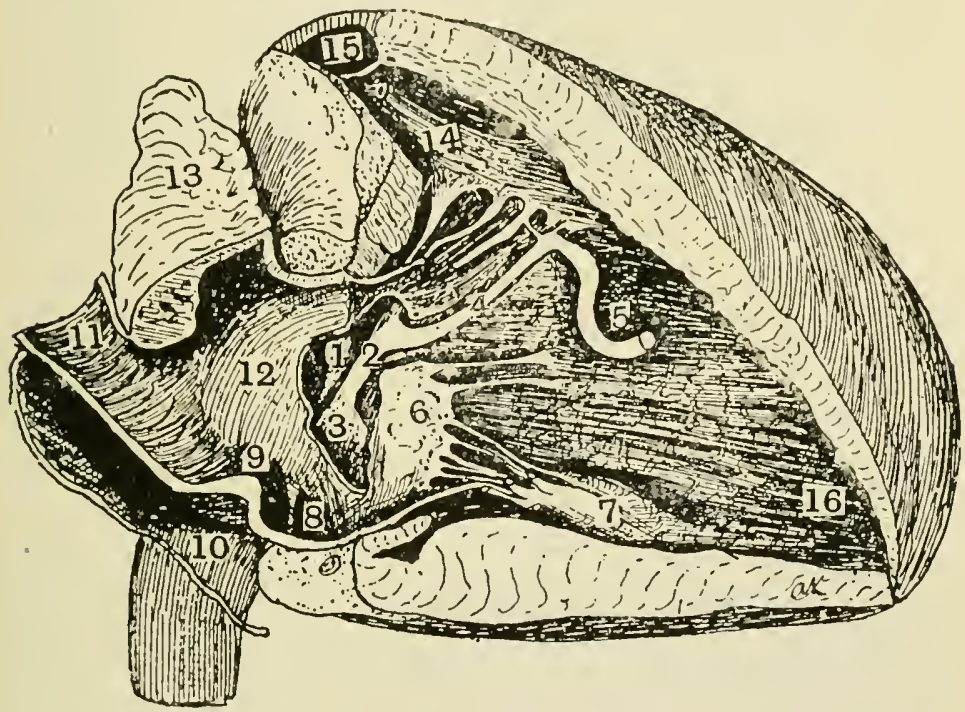

Fig. 54-Rigit Auricle and Ventricle of Calf, to show Auriculoventricular Band (Keith).

I, Central cartilage ; 2, main auriculo-ventricular bundle ; 3 , auriculo-ventricular (A-V) node; 4 , right septal division of the bundle; 5 , moderat or band; 6 , medial or septal cusp of tricuspid valve; 8 , coronary sinus.

bridge. But it has been shown (Kent, His, etc.) that in the mammalian heart, too, a slender band of muscular fibres, arising at a definite point near the coronary sinus on the right side of the interauricular septum below the fossa ovalis, passes forwards and downwards through the fibrous ring between the auricles and ventricles under the septal cusp of the tricuspid valve. It then divides into two branches, one for each ventricle, which run down the interventricular septum towards the apex, spreading out as the Purkinje fibres or their equivalent, to blend at last, with the ordinary muscle of the ventricles, and particularly of the 
interventricular septum. The fibres of the bundle are narrower than the other fibres of the auricles, very rich in nuclei, and only slightly differentiated into fibrillie. They seem to represent the remains of the primitive cardiac tube, which by the development of certain pouches and twists becomes transformed into a multichambered heart. Their resemblance to embryonic fibres suggests that they may have retained the primitive capacity of the mesodermic tissue of the embryonic heart to conduct, and even to originate, the rhythmical contraction. But while there is no decisive evidence that they constitute an automatic cardio-motor centre, as some anthors have supposed, they, or at least the narrow bridge of tissue in which they lie, do play an important part in the conduction of the contraction from the auricles to the ventricles. For compression of the band produces a block, just as the pressure of a clamp in the auriculo-rentricular groove does in the frog's heart (Kent). With a certain degree of pressure the ventricle beats only once for two beats of the auricle, witls greater pressure only once for three or more auricular beats. W'ith a still greater pressure or after crushing or section of the bundle conduction is abolished, and the ventricle either renains at rest for a time, as in the frog's heart, or, what is much more common, immediately starts beating with an independent rhythm, which is slower than that of the auricles (Erlanger). It can be considered certain that in these observations nerves may have been involved in the block as well as the muscle of the auriculo-rentricular band, since this band is richly provided with nerve-fibres as well as ganglion-cells (Wilson). Yet it is unlikely that all the nerves capable of conducting the impulses to contraction should be gathered into such a narrow compass, and therefore the experiment supports the view that the conduction is carried ont in the muscular tissue. And if the conduction of the excitation from auricles to ventricles is accomplished by a muscular connection, it is natural to suppose that the co-ordination of symmetrical portions of the heart on either side of the longitudinal axis, the co-ordination in virtue of which the two auricles contract together and the two rentricles together, is also achieved by the passage of impulses through the muscular tissue. In accordance with this, it has been shown that the ventricles in the dog and cat continue to beat in unison, after the attempt has been made to sever any nerves connecting them by extensive zigzag incisions, so long as they are united by a narrow bridge of muscle (Porter).

In disease, interference with the conduction of the stimulus from auricles to ventricles along the atrio-ventricular bundle is a not uncommon phenomenom. Accorling to the legree of interference. the ventricular contraction may be simply delayed. or only a certain proportion of the auricular contractions (every second. every third, or every fourtli) may be conducted to the ventricle, or. finally. the block may be complete. and the ventricle then contracts quite incle- 
penclently of the aturicle, the stimulus to contraction roriginating. perlatss, in the minjured portion of the bunclle below the seat of the block. These conclitions are most easily recogniserl by comparing tracings simultaneously obtaincel from the jugular vein and the raclial artery or apex-beat ( 1 . 82). When the block is complete the rate of the ventricle is very slow (about 30 in the minute, or less),

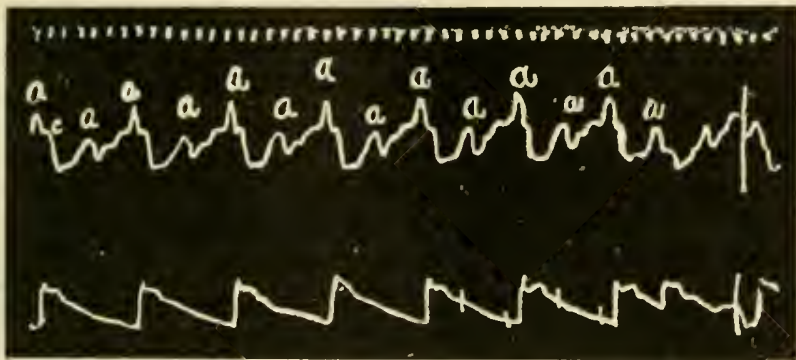

Fig. 55.-JUGULAR (UPPER) AND CAROTID (LOWER) I'ULSE TRACING FROM I

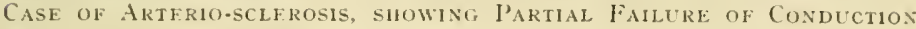
IN TIE AURICUlO-VENTRICULAR BENDLE (CUSINY AND GRUSII).

The rentricle only beats once to two beats of the auricle. Tine-trace, fifths of a second.

the time of the ventricular beat is clearly unrelated to that of the auricular, and the stability of the ventricular rhythm is abnormally great. such circumstances as usually cause a marked increase in the pulsc-rate-mental excitement, for instance-affecting it little or not at all. This is the condition in the so-called Stokes-Aclans

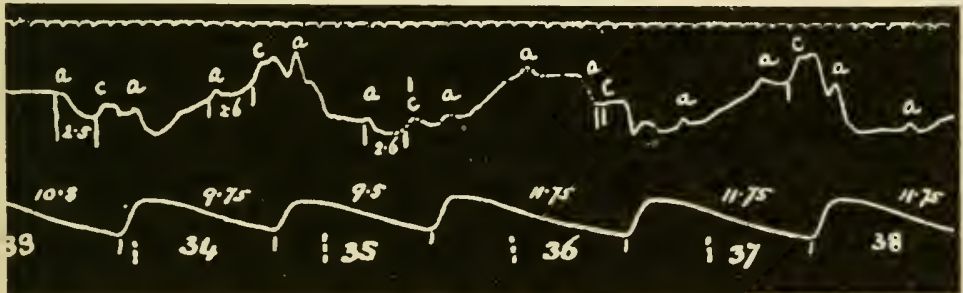

Fig. 56. -Tracing of Jugular (LPPER) AND RAdial (Lower) PUlse From a MAN WITH HEART-BLOCK (LEWIS AND MACNALTY).

In the cycles inarked $3.4,35$, and 36 the ventricular contraction, although less frequent than the auricular, was initiated from the auricle. In the last two cycles ( 37 and $3^{8}$ ) and the pause of 36 complete heart-block was present. On the jugular trace the $a-c$ interval (representing the interval between the onset of the auricular and ventricular contractions) is given, and on the radial trace the duration of a cardiac cycle, both in fifths of a second.

discase. In some of these cases pathological (syphilitic) changes in the atrio-ventricular bundle have actually becn discovered at autopsy. In others there is some reason to believe that abnormal cxcitation of the cardio-inhibitory nerves may be responsible even for longcontinued block, especially when the conductivity of the bundle has been already permanently diminished. 
In the case of the warm-blooded heart a complete breakdown of co-ordination occurs under certain circumstances, producing the phenomenon known as fibrillary contraction, or delirium cordis, a condition in which each minute portion, perhaps each fibre, of the whole heart, or of a portion of it, goes on contracting in a disorderly manner, quite independently of the rest. The condition is often seen in a heart that has been exposed for some time, particularly in the ventricle, and can be induced by stimulating it with strong induction shocks or by ligation of the coronary arteries. There is no reason to believe that fibrillary contraction is connected with the loss of impulses from any special co-ordinating centre, for it is not peculiar to the heart, but is typically seen in the tongue when the circulation after a long interruption is restored. The peculiar 'boiling' movement is exactly similar to that observed in the heart, probably because the tongue also contains fibres running in several directions.

Without entering further into a discussion of the rival hypotheses, we may sum up by saying that for one heart (that of Limulus) the automatism and the rhythmical power have been clearly shown to reside in the local nervous apparatus; for the hearts of other animals full and formal proof of the neurogenic theory, so far as those two properties of the cardiac tissue are concerned, has not been given. It is probable, but not proven. As regards the conduction and co-ordination of the contraction, the bulk of the cvidence (leaving the Limulus heart out of account) points to the muscular tissue as the channel through which the effective impulses pass. The normal order or sequence in which the different parts of the heart contract depends upon the fact that the automatism of the upper portions is more pronounced than that of the lower, so that under strictly physiological conditions the contraction is only propagated, and not originated, by the lower parts of the heart. When, however, the signal to contraction normally given by the basal region is prevented from reaching the lower parts, an independent automatic rhythm of the latter may be developed, as in the case of the mammalian ventricle mentioned above. Here we may suppose that the automatic mechanism of the lower portions of the heart discharges itself as soon as a sufficient accumulation of energy has taken place in it, although it requires a longer time to reach the point of discharge than the automatic mechanism of higher parts, and therefore is normally discharged from above. Under certain conditions the normal sequence can be reversed. In the heart of the skate it is easy, by stimulating the bulbus arteriosus, to cause a contraction passing from bulbus to sinus. The power of propagating the contraction may also be artificially altered. As already mentioned, it may be diminished or abolished by 
pressure. The same effect maly be produced by fatigue or cold, while heating a portion of the heart in general increases its power of conducting the contraction.

Chemical Conditions of the Beat.-When we liave localized the essential mechanisu of the rhythmical beat in the nervous or in the muscular elements, the question may still be asked what the chemical and physical conditions are which are necessary to its maintenance. While it is known that a supply of arterial blood at or near body-temperature, and under a sufficient pressure, is required for permanent cardiac contraction, much simpler solutions will suffice to maintain the activity even of the isolated mammalian heart for a considerable time. One of the best of these is a solution containing sodium chloride, potassium chloride, calcium chloride, and sodium bicarbonate in the proportions in which they exist in blood-serum, with the addition of a small quantity of dextrose (Locke). When this solution, properly oxygenated and warmed, is circulated through the coronary vessels of an excised rabbit's or cat's heart, strong and regular beats may be observed for many hours. Some investigators have claimed for sodium chloride, and even for sodium ions, others for calcium salts or calcium ions, a special role in the origination or maintenance of the rhythmical beat. There is no doubt that strips from the ventricle of the tortoise or turtle, which after isolation have ceased beating, and if left to themselves in a moist chamber do not develop rhythmical contractions, begin after a while to beat when immersed in or irrigated with a solution of sodium chloride or a solution of canesugar containing a little of that salt. They refuse to beat in any solution which does not contain sodium chloride (Lingle). The addition of calcium chloride to the sodium chloride solution, or prelininary treatment of the strip with a solution of a calcium salt before its immersion in the sodium chloride solution hastens the onset of the contractions, and increases the length of time for which they are kept up (Erlanger). It is unquestionable that for the normal beat of the heart the presence of both salts is one of the necessary conditions, but there is at present no sufficient foundation for the view that either the one or the other acts as a special chemical excitant of the automatic contraction.

Resuscitation of the Heart.-Not only can the beat of the freshly-excised mammalian heart be long maintained by artificial circulation, but many hours or even days after somatic death pulsation may be restored by the perfusion of such a solution of inorganic salts as Locke's through the coronary vessels. Kuliabko in this way was able to restore a rabbit's heart which had been kept forty-four hours in the ice-chest. Even after an interval of three to five days from the death of the animal, in other 
experiments, pulsation returned in certain parts of the heart, while twenty hours after death from double pneumonia the lieart of a boy three months old was restored, and went on beating for over an hour. He obtained also more or less complete restoration of the beat in the hearts of persons dead from bronclitis combined with peritonitis or meningitis, and from cholera infantum, but was unsuccessful in cases of diphtheria complicated with septicemia or erysipelas, and in cases of pleurisy with effusion. It is to be remarked, however, that although beats of a kind can be obtained a long time after death, they are either confined to the auricles or to portions of them, or, if they involve the ventricles too, they are only shallow and local contractions, especially seen in the neighbourhood of the larger coronary vessels, and are utterly inadequate to the maintenance of an efficient circulation. The heart can also be resuscitated in situ for some time after complete stoppage without the injection of any solution by clamping the aorta in the thorax and practising direct cardiac massage, the lower end of the animal at the same time being elevated to allow blood to pass out of the engorged abdominal veins to the right auricle. The clamping of the aorta permits a sufficient pressure to be attained for the filling of the coronary arteries. Tle injection of adrenalin into the blood has also been recommended as a means of raising the blood-pressure by constricting the small arteries, and stimulating the action of the cardiac muscle. The possibility of restoration of the mammalian heart many hours after somatic deatl has been considered by some a strong argument for the myogenic theory of cardiac automatism, since, they say, it is improbable that ganglion-cells, elsewhere such physiologically fragile structures, should in the heart retain their vitality for so long a time. But it is easy to overdo this argument, and we must not assume without proof that ganglion-cells in all parts of the body have an egual capacity of survival. Indeed, we know that there are gleat differences, the nervous mechanism concerned in respiration, $c . g$. being capable of restoration when the circulation is renewed after total animia of the brain and cervical cord lasting for as much as an hour (in cats), while the nervous mechanism concerned in volumtary movements cannot be completely restored even after a much shorter interval. It is very probable that the cardiac ganglia, if the all-important automatic function of the heart depends upon them, are, like the cardiac muscle, endowed with exceptional powers of resistance to those changes which constitute death. The possibility also must not be overlooked that the contractions obtained after such long intervals are not truly automatic, but similar rather to the rhythmical beats developed under the influence of pressure in the frog's ajex preparation or by immersion in salt solutions of tortoise ventricle strips. 
In addition to its marked power of rhythmical contraction, the cardiac muscle is distinguished from ordinary skeletal muscle by other peculiarities. It used to be considered the most striking of these peculiarities that "it is everything or nothing with the heart '; in other words, that the heart muscle, when it contracts, makes the best effort of which it is capable at the time; a weak stimulus, if it can just produce a beat, causing as great a contraction as a strong stimulus. Recent work, however, has indicated that this property is also possessed by the skeletal musclefibre. When a whole sheletal muscle is excited either directly or through its motor nerre, it is true that throughout a considerable range increase of stimulus is accompanied by an apparent increase in the strength of contraction. But there is reason to believe that this is because a larger and larger number of fibres become involved in the excitation as the stimulus is increased, and not because each fibre responds more and more strongly (Lucas). In skeletal muscle the fibres are completely isolated from each other and the excitation does not spread from fibre to fibre, as happens in the heart.

A more characteristic property of the cardiac muscle than the 'all or nothing' law is that a true tetanus of the heart cannot be obtained at all, or only under very special conditions. When the ventricle of a normally beating frog's heart is stimulated by a rapid series of induction shocks, its rate is generally increased, but there is no definite relation between the number of stimuli and the number of beats. Many of the stimuli are ineffective. In the same way a portion of the heart, such as the apex of the ventricle, when stimulated in the quiescent condition by an interrupted current, responds by a rhythmical series of beats, and not by a tetanus. It is evident that the cardiac muscle, like ordinary striped muscle, is for some time after excitation incapable of responding to a fresh stimulus - i.e., there is a refractory period. But this is immensely longer in cardiac than in skeletal muscle. When the phenomenon is analyzed, it is found that a stinuulus falling into the heart muscle between the moment at which the contraction begins and the moment at which it reaches its maximum, produces no effect-is, so to speak, ignored. When the stimulus is thrown in at any point between the maximum of the systole and the beginning of the next contraction, it causes what is called an extra contraction. The extra contraction is followed by a longer pause than usual-a so-called compensatory pause-which just restores the rhythm, so that the succeeding systole falls in the curve where it would have fallen had tliere been no extra contraction (Fig. 57).

In man, extra systoles followed by compensatory pauses may occur under pathological conditions, giving rise to an important 
gromp of cardiac irregularities. These extra systoles may be either auricular or ventricular, the auricle or the ventricle contracting prematurely without waiting for the signal of the sinus rhythm normally originating at the mouths of the great veins. The analysis of pulsetracings showing these irregularities has led to results of great physiological and clinical interest (Cushny, Mackenzic, etc.), but cannot be dwelt on here. When every second beat is an extra systole, generally weaker than the preceding and the succeeding normal beat, the condition is called pulsus bigeminus. The weaker beat is always followed by a compensatory pause of greater luration than that prececling it. From the pulsus bigeminus must be distinguished that form of alternating pulse termed pulsus alternans. in which every second beat is diminished in size, but the intervals
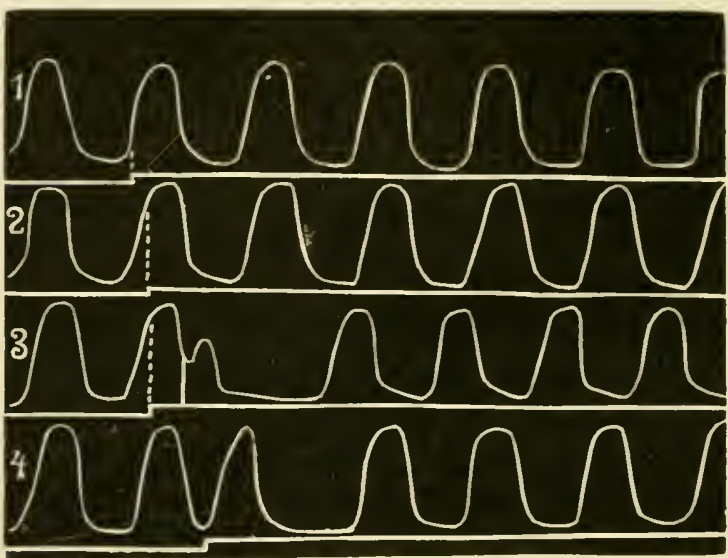

F19. 57.-Refractory Period and Compexsatory Panse (MAREY).

A frog's heart was stimulated at a point corresponding to the nick in the horizontal line below each curve. In I and 2 there was no response; in 3 and + there was an extra cuntraction, succeeded by a compensatory pause. separating the beats are of uniform length. This form of irregnlarity inlicates that the power of the heart-muscle to contract is fail. ing.

The refractory period is shorter for stronger than for weak stimuli, and is markedly diminished by raising the temperature of the heart. So that stimulation of the heated heart with a

series of strong induction shocks may cause a tetaniform condition, if not a typical tetanus. The contraction of the normally beating heart is really a simple contraction, and not a tetanus. The capillary electrometer shows only the electrical changes corresponding to a single contraction ( $p$. 720); and when the nerve of a nerve-muscle preparation is laid on the heart, the muscle responds to each beat by a simple twitch, and not by tetanus (p. ISS). That the cardiac muscle itself, apart from the intrinsic nervous mechanism, shows the phenomenon of 'refractory state' lias been shown in the Limulus heart after extirpation of the ganglion (Carlson).

Like ordinary skeletal muscle, the cardiac muscle is at first benefited by contraction, perliaps by an 'augmenting' action of 
fatigue-products such as carbon dioxide (Lee), so that when the apex is stimulated at regular intervals, each contraction is somewhat stronger than the preceding one. To this phenomenon the name of the staircase or 'treppe' has been given from the appearance of the tracings (p. 648 ).

The Extrinsic Nervous Mechanism of the Heart.- While, as we have seen. the essential cause of the rhythmical beat of the heart resides in the tissue of the heart itself, it is constantly affected by impulses that reach it from the central nervous system. These impulses are of two kinds, or, rather, produce two distinct effects: inhibition, or diminution in the rate or force of the heart-beat, and augmentation, or increase in the rate or force. Both the inhihitory and the augmentor impulses arise in the medulla oblongata, and perhaps a narrow zone of the neighbouring portion of the cord ; and they can be artificially excited by stimulation in this region. They pursue their course to the heart by fibres which may in certain animals be mingled together, but are anatomically distinct. We may, therefore, divide the extrinsic or external nervous mechanism of the heart into a cardio-inhibitory centre with its efferent inhibitory nerve-fibres and a cardio-augmentor centre with its efferent accelerator or augmentor fibres. Both of those centres, as we shall see, have also extensive relations with afferent nerve-fibres from all parts of the body, including the heart itself.

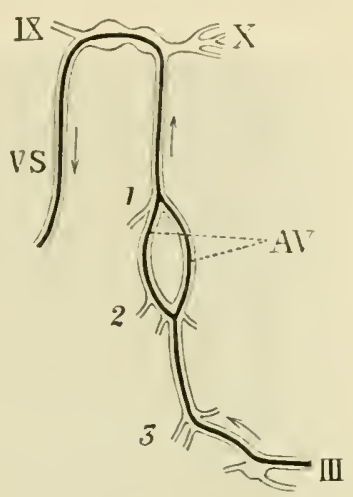

Fig. 58. - Diagrail of ExTRINSIC NERVES OF Frog's Heart (AFter Foster).

III, 3rd spinal nerve; AV, annulus of Vieussens; $\mathbf{X}$, roots of vagus; IX, glosso-pharyngeal nerve; VS, combined vagus and sympathetic ; 1,2 , and 3 , the Ist, 2nd, and 3 rd sympathetic gang. lia. The dark line indicates the course of thesympathetic fibres. The arrows show the direction of the augmentor impulses.

It was in the vagus of the frog that inhibitory nerves were first discovered by the brothers Theber more than sixty years ago, and even now our knowledge of the cardiac nervous mechanism is more complete in this animal than in any other. We shall, therefore, first describe the phenomena of inhibition and augmentation as we see them in the heart of the frog, and then pass on to the mammal.

In the frog the inhibitory fibres leave the medulla oblongata in the vagus nerve. The augmentor fibres come off from the upper part of the spinal cord by a branch from the third nerve to the third sympathetic ganglion, and thence find their way along the 
sympathetic corl to its junction with the vagus, in which they run, mingled with the inhibitory fibres, down to the heart.

When the vago-sympathetic in the frog or toad is cut, and its peripheral end stimulated, the heart in the rast majority of cases is stopped or slowed, or its beat is distinctly weakened without, it mav be, anv marked slowing. In other words, the rate at which the heart was working before the stimulation is greatly diminished, or reduced to zero. Such an effect, a diminution of the rate of working, we call Inhitition. IIhat precise form the inhibition shall take, whether the stoppage shall be complete or partial, appears to depend partly upon the strength of the

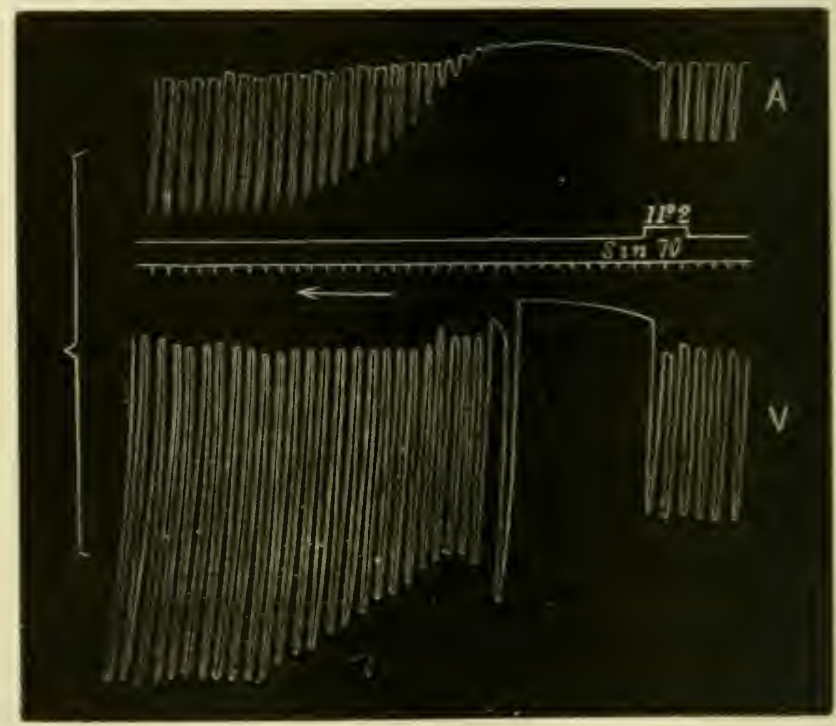

F1G. 59.-TRACING FrOM Frog's HEART.

A, auricular, V, ventricular tracing. Sinus stimulated (primary coil jo mm. from secondary). Heart at temperature $I^{\prime} \geq^{\circ} \mathrm{C}$. Complete standstill. The time-tracing between the curves marks intervals of two seconds.

stimulus used and partly upon the state of the heart itself. Some hearts it mar be impossible to stop with weak stimulation, although other signs of inhibition may be distinct, while they are readily stopped by stronger stimulation. In other cases the strongest stimulation may not produce complete standstill. Again, a heated heart may be more readily brought to standstill by stimulation of the vagus than a heart at the ordinary temperature or a cooled heart.

But there are other points of importance to be noted in regard to this inhibition: (I) It does not begin for a little time after 
stimulation las begin. In other words, there is a distinct latent period; and the length of this latent period is related to the phase of the heart's contraction at which the stimulus is thrown in, and to the rate at which the heart is beating. As a general rule, the heart makes at least one beat before it stops.

(2) The inhibition does not continue indefinitely, even if stimulation of the nerve is kept up. Sooner or later, and usually, in fact, after an interval of a few seconds, the heart begins again to beat if it has been completely stopped, or to quicken its beat if it has only been slowed, or to strengthen it if the inhibition has only weakened the contraction, and it

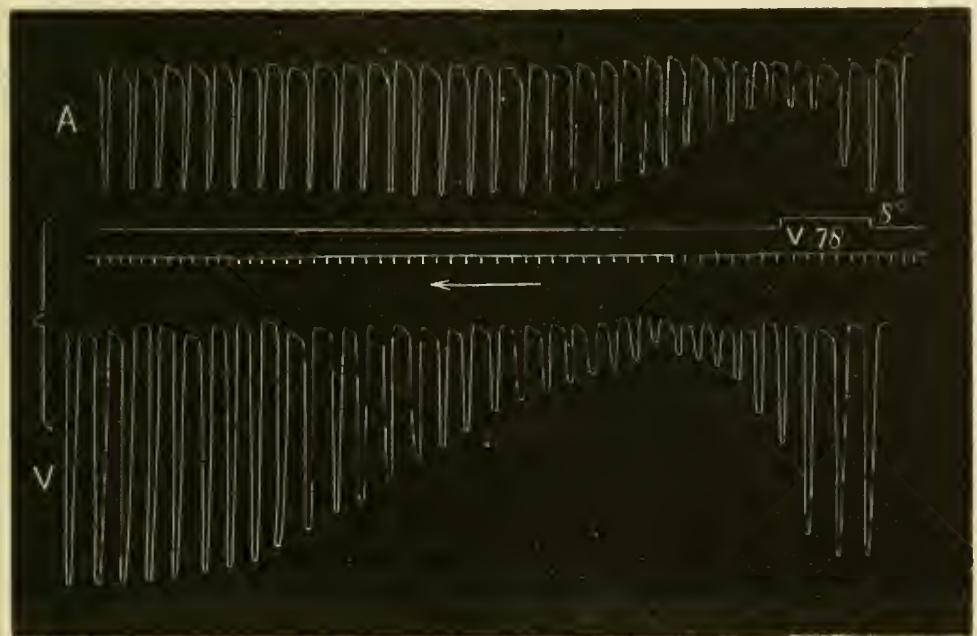

Fig, 6o.-Frog's Heart : Vagus Stimulated.

Temperature of heart $8^{\circ} \mathrm{C}, ; 78 \mathrm{~mm}$. between the coils. Diminution in force of auricle and ventricle, but not complete standstill. Time-tracing shows two-second intervals.

soon regains its old rate of working. Not only so, but very often there follows a longer or shorter period during which the heart works at a greater rate than it did before the inhibition, and this greater rate of working may be manifested by increased frequency of beat, or increased strength of beat, or by both. When the temperature of the heart is low, increased frequency; when it is high, increased strength, is generally seen during this period of secondary augmentation.* The cause of this secondary augmentation, and of the primary augmentation sometimes seen in fresh preparations and often in hearts that have been long

* Augmentation is termed 'secondary' when it is preceded by inhibition, 'primary' when it is not so preceded. 
exposed (Fig. 6I), excited much speculation before it was known that sympathetic fibres existed in the vagus. There is no longer any doubt that it is due to the stimulation of these accelerator or, as it is better to call them (since mere acceleration is not the only consequence of their stimulation), augmentor fibres in the mixed nerve. For (I) excitation of the roots of the vagus proper within the skull, and therefore above the junction of the simpathetic fibres, causes no secondary augmentation, or very little. and the inhibition lasts far longer than when the mixed trunk is stimulated. (2) Fxcitation of the upper or ceplalic end of the sympathetic cord before it has joined the vagus causes, after a relatively long latent period, marked angmentation. And if the contractions of the leart are registered, the tracing bears a close resemblance to the curve of secondary augmentation following excitation of the mixed nerve on the other sicle with an equally strong stimulus and for an equal time. (3) When the vago-sympathetic is stimulated weakly there is little or no secondary augmentation. Now, it is known that the augmentor fibres require a comparatively strong stimulus to cause any effect when they are separately excited. whereas a weak stimulus will excite the inlibitory fibres.

The question arises at this point, why it is that, when the inhibitory and augmentor fibres are stimulated together in the mixed nerve (and the same is true when the sympathetic on one side and the vagus on the other are stimulated at the same time). the inhibitory effect always comes first, when there is any inhibitory effect, while the augmentation always has to follow. The answer has sometimes been given, that the latent period of the augmentor fibres is longer than that of the inhibitory fibres. But although this is certainly the case, the answer is insufficient. For the period of postponement may be much greater than the latent period of the sympathetic fibres when stimulated by themselves. The inhibition apparently runs its course without being affected by the simultaneous augmentor effect, which, lying latent until the end of the inhibition, then bursts out and completes its own curve. It is not like the passing of two waves through each other, but rather like the stopping of one wave until the other has passed by. It seems as if augmentation cannot develop itself in the presence of inhibition-at least. until the latter is nearly spent. Like a musical-box devised to play a series of melodies in a fixed order, and from which a particular tune cannot be obtained till those preceding it have been run through, the heart, in some way or other, is arranged, in the presence of competing impulses from its extrinsic nerves, to play the tune of inhibition before the tune of augmentation. In the frog, at any rate, the two processes can hardly be considered as antagonistic, in the sense that a definite amount of augmentor excitation can overcome a lefinite amount of inhibitory excitation. Nor is it the case that when the heart is played upon at the same time by impulses of both kinds, it pits them against each other and strikes the balance accurately between them. It is possible, however, that when the inhibitory fibres are very weakly. and the augmentor fibres very'strongly stimulated, the amonnt of inhibition may be some what diminished. In 
mammals, on the other hand, a true antagonism scems to exist ; and stimulation of the inhibitory nerves is less effective when the augmentors are excited at the same time. The carliac nerves affect not only the rate and force of the contraction, but also the conductivity of the heart. Thus in the frog's heart cluring stimulation of the vagus, the contraction passes more slowly, and during stimulation of the sympathetic more quickly from auricles to ventricle.

In mammals (and in what follows we shall restrict ourselves chicfly to the dog, cat, and rabbit, as it is in these animals that the subject has been most carefully studie(l) the inhibitory fibres run down the vagus in the neck and reach the heart by its cardiac branches. They are derived from the bulbar roots of the spinal accessory, whose inner

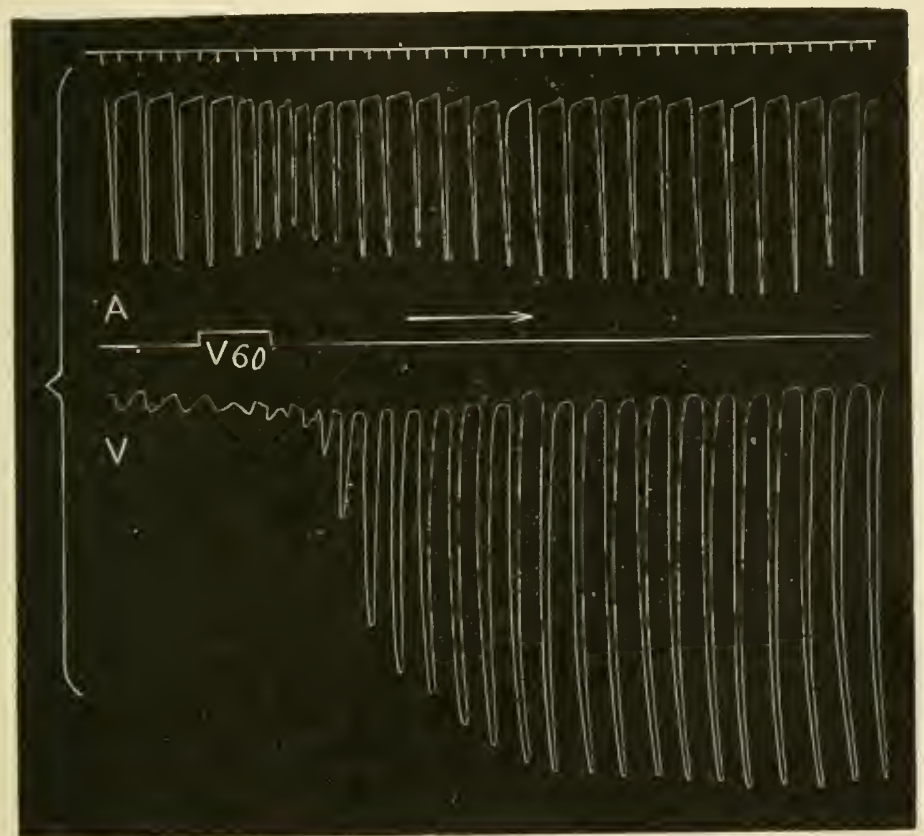

Fig. 6I.-FroG's HEART.

A. auricular, V, ventricular tracing. Ventricle beating very feebly. Vagus stimulated ( $60 \mathrm{~mm}$, between coils). Marked augmentation of ventricular beat.

branch joins the vagus. The augmentor fibres leave the spinal cord in the anterior roots of the second and third thoracic nerves, and possibly to some extent by the fourth and fifth. Through the corresponding white rami communicantes they reach the sympathetic cord, and running up through the stellate ganglion (first thoracic), and the annulus of Vieussens, which surrounds the subclavian artery, to the inferior, cervical ganglion, they pass off to the heart by separate 'accelerator' branches, taking origin either from the annulus or from the inferior cervical ganglion. Some augmentor fibres are often, if not always, present in the dog's vago-sympathetic in the neck. It is especially easy to demonstrate their presence five or six days after section of the nerve, when the excitability of the inhibitory fibres has disappeared. 
In the log the vagus and cervical sympathetic are, in the great majority of cases, contained in a strong common slieath, and pass together througl the inferior cervical ganglion. Epon opening this sheatli they may with care be separated, the fibres ruming in distinct strands, and not mixed together as in the vago-sympathetic of the frog. For some distance below the superior cervical ganglion the cervical sympathetic is not connected with the vagus, and here the nerves may be separately stimulated without any artificial

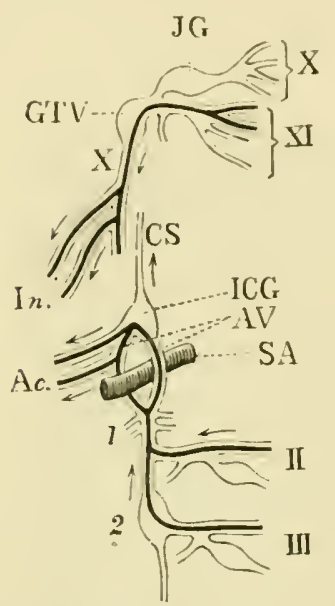

Fig. 62.-Diagrail of Cardiac NERVES IX THE D)OG (AFTER Foster).

II, III, second and third dorsal nerves: SA. subelavian ar. terv: A $\mathrm{Y}$, annulus of V'jeussens ; ICG, inferior cervical ganglion : CS, cervical sympathetic : $\mathbf{I}$, first thoracic or stellate ganglion of the sympathetic: 2 , second thoracic ganglion ; Ac., acrelerator or augmentor fibres passing off towards the heart : $\mathbb{X}$, routs of vagus: $\mathrm{XI}$, roots of spiual arressory: $\mathrm{J}(\vec{r}$, jugular ganglion : GlV, ganglion trunci vagi ; In., juhil,itory hibres passung off towards the heart. isolation, but the electrodes must be very well insulaterl. as the available length of nerve is small.

In the rabbit and some other manmals, including man, the vagus and sympathetic run a scparate course in the neck.

The effects of stimulation of the vagus or vago-sympathetic in the mammal are very much the same as in the frog, except that secondary augmentation is in general less marked, though often present in some degree, and that in the mammal the inhibitory fibres have a smaller direct action on the ventricle. It indeed beats more slowly when the auricle is slowed, but this is only because in the normally beating heart the ventricle takes the time from the auricle. The strength of the ventricular contractions may be not at all diminished, even when the auricle is beating very feebly during inhibition. When the auricle is completely stopped, which does not occur so readily as in the frog, the ventricle also stops for a short time, but soon begins to beat again with an independent rhvthm of its nwn. In the frog the ventricle is directly affected by stimulation of the vagus, and the force of its beats is diminisled independently of the inhibitory effects in the auricles (Practical Exercises, Pp. IS2, IS7).

The inhibitory fibres, then, influence the heart particularly through the auricles; they are par excellence auricular nerves. On the other hand, the accelerantes in all mammals which have been investigated not only extend to the ventricles, but are even mainly distributed to them. They are empluatically ven- 
tricular fibres, and in accordinee with its greater mass the left ventriche receives more libres than the right.

stimulation of the accelerator nerves in the dog causes an increase in the force of both the arricular and ventricular contraction, and, as a rule, in addition, some increase in the rate of Lle: leat.

As to the nature of the physiological linkage between the cardiac nerves and the muscular tissue of the heart we know but little. It has been supposed that within the heart itself there may exist peripheral nervous mechanisms which mediate between the nerves and the muscle. We have already given reasons for assigning to the intrinsic cardiac nervous mechanism an important share in the maintenance of the rhythmical beat. If this be the case, it is natural to assume. that the extrinsic nerves act on the heart-muscle not directly, but through the ganglion-cells. Some of these cells lie on the comse of the vagus fibres, and although the view has been adrocated that they are simply stations where the inhibitory impulses pass from nedullated to non-medullated filores, and where possibly other

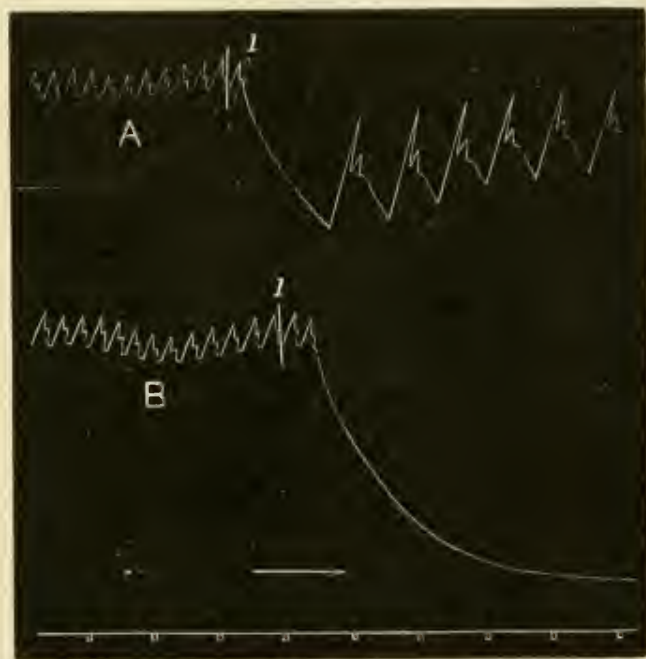

Fig, 63.-Blood-pressure Tracings: Rabit.

Vagus stimulated at $I$. Stimulus stronger in B than in $\mathrm{A}$ (Hiirthle's spring manometcr). anatomical clianges

and rearrangements occur, they may just as well be important intermediate mechanisms which essentially modify the physiological impulses falling into them and shape the visible results that follow those impulses. The nervi accelerantes are already non-medullated before they reach the heart, and it is not known whether they make comnection with intracardiac ganglion-cells. The fact that the action of the accelerantes can be restored by perfusing the heart with a nutrient solution at a much longer interval after somatic death than the action of the vagus strengthens the siggestion that ganglion-cells are interposed on the inhibitory though not on 
the augmentor path, without, however, proving of itself that such a difference exists. In one experiment the leart of an anthropoid ape was revived when three successive perinds-viz., four and a half, twenty-eight and a half, and fifty-three hours respectively - had elapsed after the death of the animal, although during the last period the heart had been twice frozen liard. The vagus was shown to be still capable of causing some inlibition six hours after death, and the accelerans some augmentation as late as fifty-three hours after death (Hering).

In the cliscussions that have arisen over the relation of the cxtrinsic to the intrinsic cardiac nervous apparatus appeal has frequently been marle to the action of certain poisons on the heart.

Thus, after nicotine has been injecterl subcutanconsly, or painterl directly on the lieart of a frog. stimulation of the vago-sympathetic causes no inhibition ; it may caus aug:nntation. But stimulation of the junction of the sinus and auricle sill catises inhibiti n als in the normal heart.

Atropine and its allies, such as dalurine, not only abolish the inhibitory effect of stimulation of the vagus trunk, but also that of stimulation of the junction of sinus and auricle.

Muscarine, a poison containcel in cortain muslirosins (p. is 3 ), catuses diastolic arrest of the heart, which whon the circulation is intact. becomes swollen and engo:"gad with bloxl. This action talkes place in a hart already poisoned with nicotine or one of its congeners, but not in a heart uncler the influenc? of atropin" or its allies. And a heart bronght to a standstill by muscarine can be marle to beat again by the application of atropin ', although not by nicotine.

These facts may be explained as follow's: Nicotine paralyos not the very ends of the vagus, but the gangliat thrsughts which its fibres pass. Stimulation of the sinus, which is practically stimulation of the vagus fibres betwen the granglion-cells and the muscular fibres. is therefore effective, although stimulation of the nerve-trunk is not (Langley). On the other hand, the atropine groul paralyzes the nerve-endings thenselves, or interferes with the reception of the inhibitory impulses by acting on a so-callerl receptive sulsstance in the muscle $(p, 166)$, so that neither stimulation of the sinus nor of the nerve-trunk can cause inlabition. Nuscarine, on the contrary, stimulates the vagus fibres between the nerve-eclls and the muscle. or the actual nerve-endings, or exerts an inhibitory action on the muscle itself through the appropriate receptive substance, and thus keeps the heart in a state of permanent inhibition, which is removed when atropine cuts out the nerve-enelings. or combines with the receptive substance. It is quite in accordance witl this that muscarine has no effect on a heart whose ragus nerves, as occasionally happens, have no inhibitory power. Pilocarpine has very much the same action as muscarine.

The view that muscarine and atropine can directly affect the cardiac muscle gains a certain amount of smppert from the facts that these drugs act very much in the same way on the heart of the manmalian cumbryo (rat, rabbit, etc.) before and after the development of its intrinsic nervous system, and that the passige of an interrupterl current through the heart of very young conbryos causes distinct inlibition. But, as has already been pointed out, it is not 
lenitimate to transfer without guestion to the muscie of the fully developed leart the propertics of the enbryonic cardiac tissuc. And, on the other liand, muscarine fails to affect the heart in many invertebrate animals - for instance, in the Daphnia (Pickering). let it is probable that, while the various tissues in the heart possess a different susceptibility to one and the same (lrug, if the dlose is large enouglı it maly affect them all. In the Limulus heart, where the question can be nuost casily tested, it has been found that the sclective action of alkaloids, aniestletics, and various other substances on the three heart-tissues (ganglion, motor nerve plexus, and inuscle) is one of (legree only (Meek).

Stannius' Experiment.-Another series of phenomena, intimately relaterl to our present subject, have excited, since they were first made known by Stannius, an cnormous amount of discussion. The clicf facts of this classical experiment we have already mentioned (p. 132), and they are also described in the Practical Exercises (p. $\left.1 \&_{3}\right)$. They are easy to verify. but difficult to interpret. The nost probabl zexplanation of the standstill caused by the first ligature is that the lower portion of the heart, when cut off from the sinus in which the beat normally originates, needs some time for the development of its automatic power to the point at which an independent rhythm can be maintained. The effects following the sccond Stannius ligature are supposed by some to be clue to

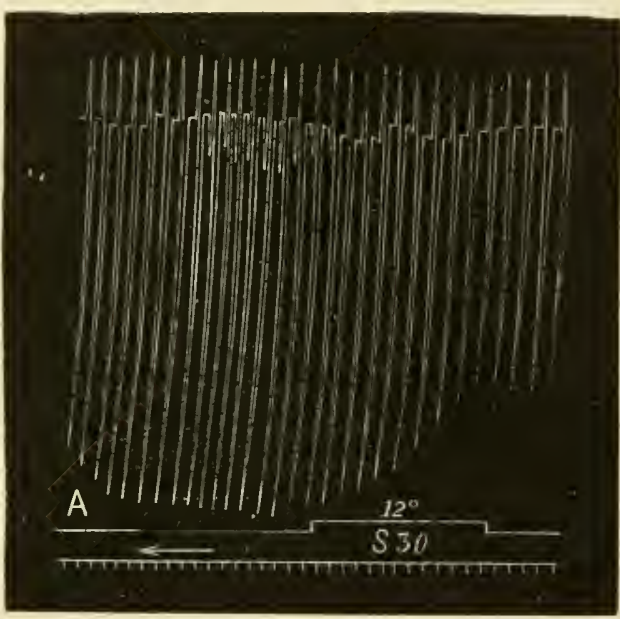

FIG. 64.-FROG'S HEART.

Sympathetic stimulated $(30 \mathrm{~mm}$. between the coils). Temperature $12^{\circ}$. Marked increase in force. Only auricular tracing reproduced. Time-trace, two-second intervals. stimulation of the muscular tissue in the auriculo-ventricular groove by the ligature.

Another view is that the first ligature stimulates the inhibitory mechanism (vagus fibres) at the junction of the sinus and right auricle, a position in which it is specially sensitive to stimuli. This causes inhibition of the whole of the heart bclow the ligature. The sccond ligature cuts off the ventricle from the inhibitory impulses, while leaving the auricle still under their influence. The Stannius experiment does not succeed in the mammalian heart-or, at any rate, only imperfectly.

Nature of Inhibition and Augmentation.--So far we have been discussing the phenomena of inhibition and augmentation as ultimate facts. We have not attempted to go behind them, nor to ask what it is that really happens when inhibitory impulses fall into a heart, which from the first days of embryonic life has gone on beating with a regular rhythm, and in the space of a second or two bring it to a 
standstill. The question camnot fail to press itself upon the mind of anyone who hats ever witnesserl this most beantiful of physiological experiments ; but as yet there is mo answer except ingenious speculations. The most plausible of these is the trophic theory of (iatskell, who sees in the vagus a nerve which so acts npon the chemical changes going on in the heart as to give them a trophic, or anabolic, or constructive turn, and thus to lessen for the time the destructive changes underlying the muscular contraction. The augmentor nerves. on the other hanel, are supposed to exert a katabolic influence, and to favour these elestructive changes. And while, according to (jaskell, the natural consequence of inhibition is a stage of increased efficiency and working power when the inhibition has passed away, the natural
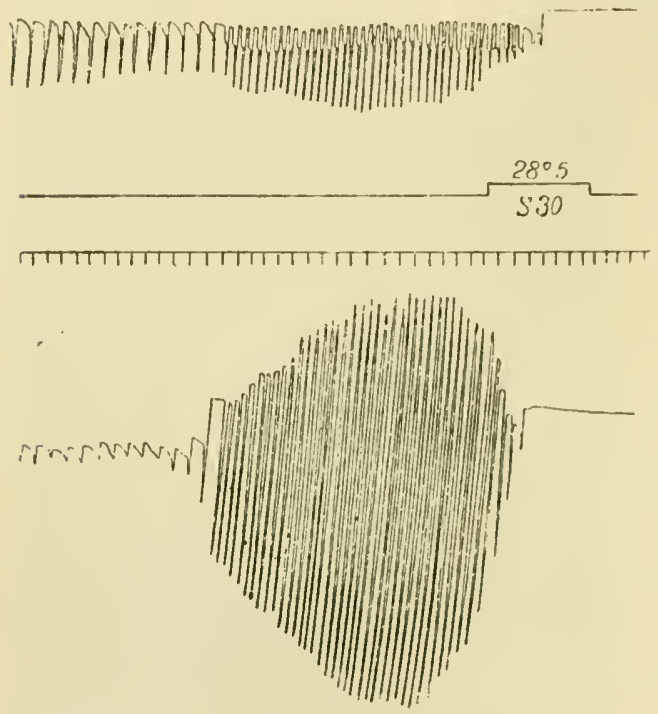

Fig. 65.-Effect of Stimulation of Firug's Cardiac Sympathetic during Complete Standsilll or tile HEART AT $28.5^{\circ} \mathrm{C}$.

Upper tracing, auricle; lower, ventricle. To le read fron right to left. Time-trace, two-second intervals. complement of augmentation is a temporary exhaustion. But it must be remembered that this clistinction is not as yet basecl upon any very solicl founclation of actually observed and easily interpreted facts, while to some of the facts brought forwarl in its favour unclue importance has been given.

Whatever the exact mechanism of augmentation may be, there is no founclation for the statement that the card i o-augmentor nerves have an ac. tion on the heart so fundamentally different from the action of motor nerves (u) slicletal muscle that they camnot originate contractions in a heart entirely at rest. Excitation of the cardio-augmentor nerves can cause rhythmical contractions in the perfectly quiescent heart of molluses, and a suelden and prolonged outburst of beats of great force in the frog's heart, which has been brought to a standstill by cautiously heating it to $40^{\circ} 10+3^{\circ} \mathrm{C}$. (Practical Exercises, p. 178) for a minute or two, or to a considerably lower temperature, for a longer time (1ijg. 65). A similar effect can be obtained on the quiescent mammalian heart by stimulation of the nervi accelerantes.

The Normal Excitation of the Cardiac Nervous Mechanism. -We have now to inquire how this elaborate nervous mechanism is normally set into action. And we may say at once that, 
striking ats are the cffects of experimental stimulation of the: vagus trunk or the nervi accelerantes in theje course, it is only muder exceptional circumstances that the efferent nerve-fibres, at auy rate before they have entered the heart, can be directly excited in the intact body. In certain cases the pressure of a tumour of an aneurism on the nerve-trunks, or, in the case of the accelerators, the progress of a pathological change in the sympathetic ganglia through which the fibres pass, has been thought to bring about by direct stimulation a slowing or a quickening of the pulse. In some individuals the vagus may be excited by compressing it against the vertebral column or against a bony tumour in the neck. But it is from the cardio-inlibitory and cardio-augmentor centres in the medulla oblongata that the impulses which regulate the activity of the heart are normally discharged. Inhibitory impulses are constantly passing out from the medulla, for section of both vagi causes almost invariably an increase in the rate of the heart, at least in mammals, although the increase is less conspicuous in animals like the rabbit, whose normal pulse-rate is high, than in animals like the dog, whose pulse-rate is comparatively low. Section of one vagus usually causes only a comparatively slight increase, for the other is able of itself to control the heart. It is not certainly known whether the augmentor centre in like manner discharges a continuous stream of impulses, or is only roused to occasional activity by special stimuli. For the results of section of the nervi accelerantes, or the extirpation of the inferior cervical and stellate ganglia, are dubious and conflicting. But if it does exert a tonic influence on the heart, this is feebler than the tone of the inhibitory centre. As to the nature of this inhibitory tone, and the manner in which it is maintained, we know but little. It may be that the chemical changes in the nerve-cells of the inhibitory centre lead of themselves to the discharge of impulses along the inhibitory nerves. But there is some evidence that, in the complete absence of stimulation from without, the activity of the centre would languish, and perhaps be ultimately extinguished. For when the greater number of the afferent impulses have been cut off from the medulla oblongata by a transverse section carried through its lower border, division of the vagi produces little effect on the rate of the heart. Also, when the upper cervical cord and the brain are resuscitated after a period of anzmia, the return of cardio-inhibitory tone is tardy in comparison with the return of the truly automatic function of respiration, and does not seem to precede the opening up of the afferent paths to the cardio-inhibitory centre. Indeed, reflex inhibition may be produced at a time when the inlibitory centre has regained none of its tone. 
The suggestion is that the normal tone of the centre is largely dependent upon reflex impulses. Be this as it may, we know that the actirity of the inlibitory centre is profomally influenced -and that both in the direction of an increase and of a diminution-by impulses that fall into it through afferent nerves and by stimuli directly applied to it. And we may assume that the same is true of the augmentor centre. The common statement that stimulation of the central end of one vagus, the other being intact, produces distinct inhibition does not hold for all mammals. In dogs this is sometimes the case, but often (under anesthesia, at any rate) there is little or no inhibition, or even augmentation. In etherized cats, on the other hand, some inhibition is always seen. Of all the afferent fibres of the vagus, the pulmonary fibres produce the most marked reflex inhibition. The cardiac fibres are much less effective.

These pulmonary nerves also influence the respiratory and vaso-motor centres. The respiration is temporarily arrested, and the blood-pressure falls through the dilatation of the small arteries when they are excited. As will be again pointed out in connection with the subject of deat le during the administration of anesthetics, the afferent vagus fibres coming from the alveoli of the lungs can be chemically stimulated when irritant vapours, such as chloroform or ammonia, are inhaled (p. 235).

The depressor nerve, a branch of the vagus, which is easily found in the rabbit as a slender nerve running close to the sympathetic in the neck, and a little to its inner side, but in the dog is usually blended with the vago-sympathetic, falls into the same category with the vagus itself as regards its rellex action on the heart, to which it bears an importint relation. In all mammals some of its fibres end in the wall of the aorta, but some of them may run down over the heart to the ventricle. Stimulation of its peripheral end lias no effect, for the fibres in it which influence the circulation are afferent, not efferent. But excitation of its central end causes a marked fall of bloodpressure (p. I69), accompanied by, but not essentially due to, a distinct slowing of the heart. If the animal is not anesthetized, there may be signs of pain, and for this reason the depressor has sometimes been spoken of, somerhat loosely, as the sensory nerve of the heart. The ahdominal sympathetic (of the frog) also contains afferent fibres, through which reflex inhibition of the heart can be produced when they are excited mechanically by a rapid succession of light strokes on the abdomen with the handle of a scalpel.

On the other liand, when the central end of an ordinary peripheral nerve like the sciatic or hrachial is excited the common effect is pure angmentation (Fig. 66), which sometimes develops 
itself with even greater suddenness than when the accelerator nerves are directly stimulated. Occasionally, howerer, the augmentation is abruptly followed by a typical vagus action. Here the reflex inhibitory effect seems to break in upon and cut short the reflex angmentor effect.

These examples show that certain afferent nerves are especially related to the cardio-inhibitory, and others to the cardio-augmentor, centre, or at least that the central connections of some nerves are such that inhibition is the usual effect of their reflex excitation. while the opposite is the case with other nerves. But it is improbable that the effect of a stream of afferent impulses reaching the cardiac centres hy any given nerve is cletermined solely by anatomical relations. The intensity and the

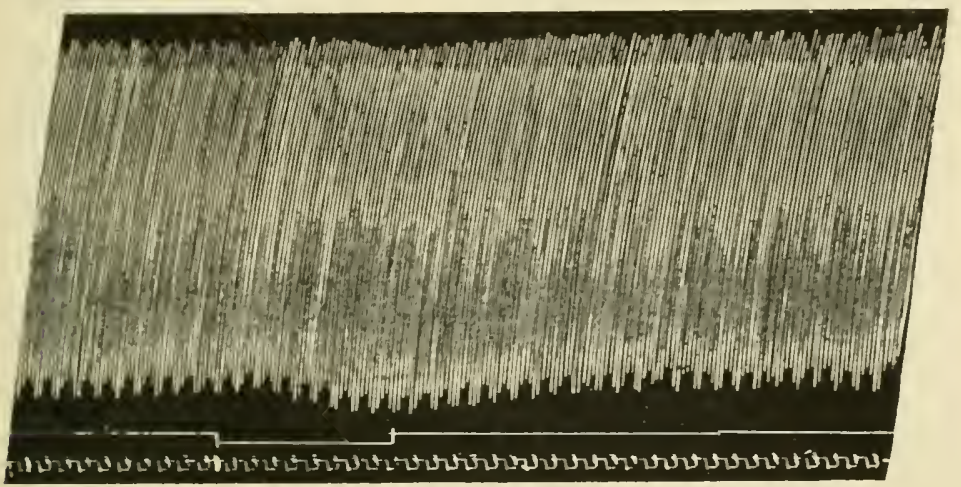

F1G. 66.-Mracardiographic Tracing of Cat's Ventricle.

The signal line shows the point at which the central end of the brachial nerve was stimulated during resuscitation of the animal after a period of cerebral anania. Some angmentation of the ventricular beat is seen. The notches in the ventricular tracing are due to the artificial respiration. Time-trace, seconds.

nature of the stimulus seem also to have something to do with the result. For when ordinary sensory nerves are weakly stimulated, augmentation is said to be more common than inhibition, and the opposite when they are strongly stimulated. And while a chemical stimulus, like the inhaled vapour of chloroform or ammonia, causes in the rabbit reflex inhibition of the heart through the fibres of the trigeminus that confer common sensation on the mucous membrane of the nose, the mechanical excitation of the sensory nerves of the pharynx and nesophagus when water is slowly sipped causes acceleration. ${ }^{*}$ The stimulation of the nerves of special sense is followed sometimes by the one effect and sometimes by the other. To complete the cata-

* In 75 healthy students the average pulse-rate (in the sitting position) was increased from 73 to $S_{5}$ per minute by sipping water. 
logue of the nervous channels liy which impulses may reach the cardiac centres in the medulla, we may ardel that there must be an extensive connection between them and the cerebral cortex, since erery passing emotion leaves its trace npon the curve of carliac action. The so-called 'reflex cardiac deatla,' which is an occasional conserpuence of intense psychical influences (anxiety, fright, etc.), may be due to the prolonged excitation of the cardio-inhibitory centre, as well as to the disturbance of other centres in the bulb by the cortical storm. It is a remarkahle fact, too, and one that can only be explained hy such a connection, that although in the vast majority of individuals the will has no influence whatever on the rate or force of the heart, except, perhaps, indirectly through the respiration, some persons have the power, by a voluntary effort, of markedly accelerating the pulse. In one case of this kind it was noticed that perspiration broke out on the hands and other parts of the body when the heart was voluntarily accelerated. A rise of bloodpressure due to constriction of the ressels has also been oloserved. The effort cannot be kept up for more than a short time, and the pulse-rate quickly goes back to normal. It has been recently shown that this peculiar power is more common than has been supposed, and that where it is present in rudiment, it can he cultivated, although it is a dangerous acquisition.

As an example of the direct action of a chemical stimulus on a cardiac centre, we may cite the inhibition produced hy injection of adrenalin into a vein (p. 20I), and ats an instance of the direct action of a physical change, the slowing of the heart in asphyxia as the blood-pressure rises (p. I72). Jle variation in the pulse-rate associated with changes in the position of the body, to which we have already referred (p. (g) , is brought about by direct stimulation of the inlibitory centre by the increase of blood-pressure in the medulla oblongata when a person who has been standing assunes the supine, or even the sitting, posture. But it is also due in part to changes in the amount of numscular contraction, since muscular exercise causes acceleration of the heart either reflexly, through afferent muscular nerves, or by a direct effect of waste products of the metabolism of the muscles on the cardiac centres in the bulb or on the heart itself (p). 220).

Theoretically, quickening of the heart might be caused either by a diminution in the inhibitory tone or by an increase in the activity of the augmentor centre; and slowing of the heart might be due either to a climinution in the angmentor tone, if such exists, or to an increase in the activity of the inhibitory centre. So that it is not always easy to interpret such results as we have quoted above. But it would appear that under ordinary conditions the rate of the heart is mainly regulated 
by the inhibitory centre, which, within a considerable range, can profuce variations in either clirection. The angmentor mechanism is perhaps merely anxiliary to the inlibitory, being called into action only in emergencies.

Vaso-motor Nerves. - Just as the muscular walls of the heart are governed by two sets of nerve-filores, a set which keeps down the rate of working and a set which may increase it, the muscular walls of the ressels are under the control of nerves which have the power of diminishing their calibre (vaso-constrictor), and of nerves which have the power of increasing it (a'aso-dilator). All nerves that affect the calihre of the vessels, whether vaso-constrictor or vaso-dilator, are included under the general name vaso-motor. These vaso-motor nerves, like the angmentor and inhibitory fibres of the heart, are connected with a centre or centres, which in turn are in relation with numerous afferent nerves. It is convenient to distinguish the afferent nerves which cause on the whole a vaso-constriction and a consequent increase of arterial pressure as pressor nerves, and those which cause on the whole vaso-dilatation, with fall of pressure, as depressor nerves, reserving the terms vaso-constrictor and vaso-(ilator for the efferent portions of the reflex arcs. It is through this reflex mechanism that the bloodvessels are mainly influenced, although the endings of the vaso-motor nerves in the smooth muscular fibres or the muscular fibres themselves are sometimes directly affected by substances circulating in the blood. Albumose, for instance, causes by peripheral action dilatation of the vessels and a fall of bloodpressure (p. 2oI); suprarenal extract, or its active principle, adrenalin, constriction, with a rise of pressure (p. 20I). Apocodeine paralyzes the raso-motor nerve-endings after a preliminary stimulation, and now adrenalin causes no constriction. Chrysotoxin, an active principle of ergot, causes a marked rise of bloodpressure by stimulating the sympathetic ganglion-cells or the pre-ganglionic filres of the vaso-constrictor patl. Vaso-motor nerves control chiefly the small arteries. They have no direct influence on the capillaries.* Nor has the existence of an effective vaso-motor regulation of the calibre of the veins, except in the portal system, been proved up to this time by any clear and

* It is usually taught that the capillaries, being devoid of muscular fibres in their walls, are not supplied with vaso-motor fibres, and that the only kind of active contraction of which they are capable is due to a process analogous to the turgescence of vegetable cells, the thickness of the wall being increased at the expense of the lumen, while the total cross-section of the vessel remains unchanged. It has recently been asserted, however, that a true contraction, in which both the total section and the lumen are diminished, may be caused in the capillaries of the nictitating membrane of the frog either by direct stimulation or by excitation of vaso-motor fibres in the sympathetic (Steinach and Kahn). 
unambiguous experiment, although there are grounds on which it has been surmiserl that the nervous system does influence the 'tone' of the whole venous tract. These grounds will be mentioned in the proper place. Meanwhile, before describing the distribution of the best-known tracts of vaso-motor fibres and defining the position of the vaso-notor centres, we must glance at the methods by which our knowledge has been attained.

(1) In translucent parts inspection is sufficient. Paling of the part indicates constriction; flushing. (Jilatition of the small ressels. This methor has been mucls used, sometimes in conjunction with (2), in such parts as the balls of the toes of logs or cats, the ear of the rabbit, the conjunctiva, the mucous membrane of the mouth ancl gums, the web of the frog, the wing of the bat, the intestines, etc.

(2) Obscrvation of changes in the temperature of parts. This method has been chicfly employed in investigating the vaso-motor nerves of the limbs, the thermometer bulb being fixed between the toes. In such peripheral parts the temperature of the blond is normally less than that of the blood in the internal organs, beciuse the opportunities of cooling are greater. The effect of a freer circulation of bloos (dilatation of the arteries) is to raise the temperature; of a more restricted circulation (constriction of the arteries), to lower it.

(3) Measurcment of the blookl-pressure. If we measure the arterial blood-pressure at one point, and find that stimulation of certain nerves increases it without affecting the action of the heart, we can conclude that upon the whole the tone of the small ressels has been increased. But we cannot tell in what region or regions the increase has taken place; nor can we tell whether it has not been accompanied by diminution of tone in other tracts.

But if we measure simultancously the bloot-pressure in the chice artery and chief vein of a part such as a limb, we can tell from the changes caused by section or stimulation of nerves whether, and in what sense, the tone of the small vessels within this areat has been altered. For example, if we foumd that the lateral pressure in the artery was diminished, while at the same time it was increased in the vein, we should know that the 'resistance' between artery and vein had been lessened, and that the blood now found its way more readily from the artery into the vein. If, on the other liand, the venous pressurc was diminished, and the arterial pressure simnltaneously increased, we should have to conchule that the vascular resistance in the part was greater than before. If the pressure both in artery and vein was increased, we could not come to any conclusion as to local changes of resistance without knowing how the general blood-pressure hat varied.

(4) The measurement of the velocity of the blood in the ressels of the part. This may be done by the stromulhe or dromograph. or by allowing the blood to escape from a small vein and measuring the ont flow in a given time, or, without opening the vesscls, by estimiting the circulation time (p. I23). When changes in the general arterial pressure are eliminated, slowing of the blood-stream through a pirt corresponds to increase of vascular resistance in it: increase in the rate of flow implies diminished vascular resistance. Sometimes the red colour of the blood issuing from a cut vein, and the visible pulse in the stream, indicate with eertainty that the vessels of the organ have bcen dilated. 
(5) Alterations in the volume of an organ or limb are often taken as indications of clianges in the calibre of the small vessels in it. We have atrealy scen how these alterations are recorded by means of a plethysmograph (p. 1 I7). The brain is encloserl in the skull as in a natural pletlyssmograph, anel changes in its volume maly be registered by connecting a recording apparatus with a trephine hole.

(6) for the separation of the effects of stimulation of vasoconstrictor and vaso-dilator fibres when they are mingled together, as is the case in many nerves, advantage is taken of certain differences between them. For example, the vaso-constrictors lose their excitability sooncr than the vaso-dilators when cut off from the nerve-cells to which they belong. So that if a nerve is divided, and some days allowed to clapse before stimulation, only the dilators will be excited. The vaso-clilators are more sensitive to weak stimuli repeated at long intervals than to strong and frequent stimuli, and the opposite is truc

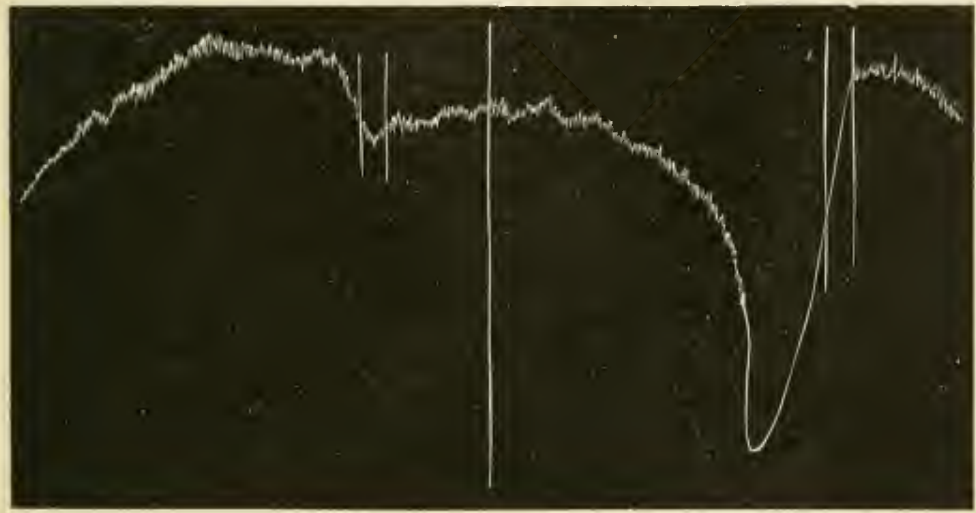

Fig. 67.-Plethyshograns: HiNd-Limb of CAT (AFter Bowditch ANd WARREN).

To be read from right to left. On the left hand is shown the effect of slow stimu. lation of the seiatic ( $I$ per second) : on the right hand the effect of rapid stimulation (6+ per second). In the first case the limb swelled owing to excitation of the vaso-dilators; in the second, it shrank through excitation of the vaso-con. strictors.

of the constrictors. When a nerve containing both kinds of fibres is heated, the excitability of the vaso-constrictors is increased in a greater degrec than that of the dilators; when the nerve is coolerl, the dilators preserve their excitability at a tempcrature at which the constrictors have ceased to respond to stimulation (Fig 67).

The Chief Vaso-motor Nerves.-The first discovery of vasomotor nerves was made in the corvical sympathetic. When this nerve is cut, the corresponding side of the head, and especially the ear, become greatly injected owing to the dilatation of the vessels. This experiment can be very readily performed on the rabbit, and the changes are most easily followed in an albino. The ear on the side of the cut nerve is redder and hotter than the other; the main arteries and veins are swollen with blood, 
and many vessels formerly invisible come into view. The slow rlyythmical changes of calibre, which in the normal rablit are very characteristically' seen in the middle artery of the ear, clisappear for a time after suction of the sympatletic, although they 11timately again become visible (Practical Exercises, p. 202).

Stimulation of the ceplalic end of the cut sympathetic causes a marked constriction of the vessels and a fall of temperature on the same side of the head. From these facts we know that the cervical sympathetic in mammals contains vaso-constrictor fibres for the side of the head and ear, and that these fibres are constantly in action. Certain parts of the eye, and the salivary glands, larynx, resophagus, and thyroid gland, are also supplied witl vaso-motor (constrictor) nerves from the cervical sympathetic.

It has been asserted that the cervical sympathetic contains some of the vaso-constrictor fibres that supply the corresponding half of the brain and its membranes, but this has been disputecl, and some observers ileny that the vessels of the brain have any vaso-motor nerves. Non-melullated nerve-fibres, however. may be seen in and around the walls of the cercbral and spinal bloodvessels, and it is difficult to believe that these have not a vaso-motor function, although this has not as yet been clearly demonstrated by experimental methods.

It has sometimes been argued that we ought not to expect the brain to be supplierl with vaso-motors. For it is enclosed in a rigid box, and the quantity of blood in it can be increased or diminished only to the slight extent to which the cerebro-spinal liquicl can be displacerl into the vertebral canal. Important changes in the cerebral blood-supply are therefore brought about, it is said, not by a widening or narrowing of the cerebral vessels, but by an alteration in the velocity of the blood in them as a result of a rise or fall of the systemic arterial pressure. This argument, however, leaves out of account the consideration that in general the brain does not function as a whole, but that certain parts of it may often become active and relatively hyperemic, while other parts are inactive and relatively ancmic, and that important changes in the clistribution of the blond in the encephalon may be effecterl, although the total mass of blood in the organ undergoes little or no alteration. It is, of course, true that it is not the absolute quantity of bloorl in an organ which is a function of its activity, but the rate at which it is rencwerl. And it is theoretically possible that an organ at rest shoulel contain as much blood as when it is active, or even more. But such cases. if they cxist, are certainly rare. The retina, which from the standpoint of elevelopment is a portion of the brain. is undoubtedly supplied with vaso-constrictor fibres which run in the cervical sympathetic.

That the cervical sympathetic contains some dilator fibres is proved liy the fact that stimulation of the ceplatic end in the clog causes flusling of the mucous memhrane of the mouth on the same sicle. Further, after clivision of the nerve on one side in the rabbit it may be olserved that when the animal is excited 
the vessels of the ear whose nerve is intact maty become still more dilated than those whose constrictor libres have been paralyzed. The only explanation is that vaso-dilators are being excited from the central nervous system. In the cat the cervical sympathetic contains vaso-dilators for the submaxillary gland (p. 367 ).

The vaso-motor fibres of the head rum up in the cervical sympathetic, and then pass into various cerebral nerves, of which the lifth or trigenimus is the most important.

The trigemimes nerve contains vaso-constrictor nerves for various parts of the eye (conjunctiva, sclerotic, iris), and for the mucous nuembrane of the nose and gums, and section of it is followed hy dilatation of the vessels of these regions. The lingual branch of the trigeminus supplies vaso-motor fibres to the tongue, and apparently both vaso-constrictor and vaso-dilator.

In some animals - the ral)bit, for instance-the ear derives part of its vaso-motor supply through the great auricular nerve, a branch of the third cervical nerve, which they reach as grey rami from the stellate ganglion.

Another great vaso-motor tract, the most influential in the body, is contained in the splanchnic nerves, which govern the vessels of many of the abdominal organs. Section of these nerves causes an immediate and sharp fall of arterial pressure. The intestinal vessels are dilated and overfilled with blood. As a necessary consequence of their immense capacity, the rest of the vascular system is underfilled, and the blood-pressure falls accordingly. Stimulation of the peripheral end of the splanchnic nerves causes a great rise of blood-pressure, owing to the constriction of vessels in the intestinal area. We therefore conclude that in the splanchnics there are vaso-motor fibres of the constrictor type, and that impulses are constantly passing down them to maintain the normal tone of the vascular tract which they command. The presence of dilator fibres (for the intestines and the kidney, for example) has also been demonstrated in the splanchnic nerves, although the constrictors predominate, and special methods have to be employed for the detection of the dilators.

The same is true of the nerves of the extremities, which certainly contain vaso-dilator fibres in addition to vaso-constrictors, although the difficulty of demonstrating the presence of the former is fully as great as it is in the splanchnics. For the investigation is complicated by the fact that such nerves as the sciatic supply with raso-motor fibres two leading tissuesskin and muscle; and these are not necessarily affected in the same direction or to the same extent by stimulation of their vaso-motor fibres. The vaso-constrictors under ordinary conditions preponderate, so that section of the sciatic or the braclial is generally followed by flushing of the balls of the toes and rise 
of temperature of the feet stimulation loy paling and fall of temperature. lisy taking advantage, lowever, of the menelual excitability of dilators and constrictors in a degenerating nerve, and of the differences between the two kinds of filores in their reaction to electrical stimuli (p. 159), it has been slown that vaso-dilators are also present, and come to the front when the conditions are rendered favourable for them and unfavourable for the constrictors.

Vaso-motor fibres for the fore-limb ( $(\log )$ issue from the cord in the anterior roots of the third to the cleventh dorsal nerves, and for the linel-linb in the anterior roots of the eleventh dorsil to the third lumbar. Stimulation of most of these roots caluse's constriction of the vessels, but stimulation of the eleventh dorsal maty cause dilatation (Bayliss and Bradford).

The Vaso-motor Nerves of Muscle.-When the motor nerve of the thin mylo-hyoid muscle of the frog, which can be observed under the microscope, is cut, and the peripheral end stimulated, the vessels are seen to dilate distinctly, and this effect is not abolished when contraction of the muscle is prevented by a dose of curara insufficient to paralyze the vaso-motor nerves. This indicates the existence in the nerse of vaso-dilator fibres. But we must be calutious in transferring this result to ordinary skelctal muscle, for the mylo-liyoid is more closely allied to the muscles of the tongue than, for example, to the muscles of the limbs, and in the mammal the tongue muscles are known to be better supplied with vaso-dilator fibres than the limb muscles. The average flow of bloos through a mammalian muscle is inclecel increased during voluntary contraction, and during rhythmically repeated artificial tetanization of its motor-nerve. The out flow of blood from the main vein of the levator labii superioris, one of the muscles used in feeding in the horse, was found to be in one experiment nearly eight times, in another about seven times, and in a third three and a half times as great during voluntary work with it (in chewing) as in rest. But as no increase in the blook-1tow through the skcletal muscles of a completely curarized mammal during excitation of their nerves has ever been satisfactorily demonstrated, we must conchude that they are very scantily provieled with vaso-clilator fibres or not at all. It is uncertain whether they are suppliech with vaso-constrictors. The undoubted incrcase in the bloodflow in contraction may therefore be connected in some way with the mechanical or chemical changes in the muscular fibres themselves.

It has been suggested that the muscular vessels are widlened by the direct action of the acid products of the active muscle. since very dilute acids (Lactic acil, e.g.) cause general dilatition of the small vessels. A similar explanation has been extended to the dilatation of the vessels of the brain churing cerebral activity by some of those who deny the existence of vaso-motor nerves for that organ, but here the evielence is by no means satisfactory. The vagus has been stated to contain vaso-constrictor. and the annulus of Vieussens vaso-dilator, fibres for the cormary arteries of the lieart. But this question is far from being settled. There is some reason to believe that the metabolic products liberated in the heart-muscle, and especially carbon dioxide, govern the changes in the calibre of the coronary arterioles. A close relationship exists between the output of carbon dioxide and the rate of flow through the coronary circulation (Barcroft and Dixon). 
Vaso-motor Neres of the Langs.- There hats been much discussion ats to the course, and even ats to the cxistence, of valso-motor fibres for the lungs. The problem is perliats the most difficult in the whole range of vaso-motor topography, for the pulumonary circulation is so related to other vasculat tracts, that clatuges produced in the vessels of distant organs by the stimulation or section of nerves maty affect the quantity of blood received by the right side of the heart, and therefore the quantity propelled through the lungs and the pressure in the pulmonary artery. And changes in the systemic arterial pressure may favour or hinder the discharge of the left ventricle, and therefore affect the pressure in the left auricle and the pulmonary veins. All that we can really say is that the lungs are probably supplied with vaso-constrictor fibres, altlough less richly than most other organs. In mammals these fibres seem to pass ont from the upper half of the dorsal spinal cord, and some of them can be detected nearer their destination in the annulus of Vicussens.* The vago-sympatlietic of the tortoise contains vaso-constrictors for the lung of the sanne side (Irogh).

Vaso-dilator Fibres.-In most of the peripheral nerves these are mingled with vaso-constrictors; but in certain situations, for an anatomical reason that will be mentioned presently, nerves exist in which the only vaso-motor fibres are of the dilator type. Of these, the most conspicuous examples are the chorda tympani and the nervi erigentes or pelvic nerves; and, indeed, it was in the chorda that vaso-dilators were first discovered by Bernard. The chorda tympani contains vaso-dilator and secretory fibres for the submaxillary and sublingual salivary glands. With the secretory fibres we have at present nothing to do; and the whole subject will have to be returned to, and more fully discussed in Chapter IV. But a most marked vascular change is produced by stimulation of the peripheral end of the divided chorda tympani nerve. The glands flush red; more blood is evidently passing through their vessels. Allowed to escape from a divided vein, the blood is seen to be of bright arterial colour and shows a distinct pulse. The small arteries have been dilated by the action of the vaso-motor fibres in the nerve. The resistance being thus reduced, the blood passes in a fuller and more rapid stream through the capillaries into the veins, and on the way there is not time for it to become completely venous. These vaso-dilator fibres are not in constant action, for section of the nerve, as a rule, produces little or 10 change. Vaso-constrictor fibres pass to the salivary glands from

* Brodie and Dixon, perfusing isolated 'surviving' lungs with blood under constant pressure, and measuring the outflow, came to the conclusion that no pulmonary vaso-motor fibres exist, since adrenalin causes no vaso-constriction. They assume that adrenalin acts upon vaso-motor nerve-endings (but see p. 564), and that in organs in which this drug does not produce vaso-constriction no vaso-constrictor fibres are present. But Plumier, working independently with the same method, saw strong constriction of the pulmonary vessels under the influence of adrenalin, and also on stimulation of the annulus of Vieussens. Wiggers also obtained constriction with adrenalin. 
the cervical sympatlutic, along the arteries, and stimulation of that nerve causes narrowing of the vessels and rliminution of the blood-flow, sometimes almost to complete stopy)age.

"The neri erigenles are the nerves through which erection of the penis is causerl. When they are divirlerl there is no effect, lut stimulation of the peripheral end caluses dilatation of the vessels of the erectile tissuc of the organ, which becomes overfilled with blood. During stimulation of these nerves, the quantity of lolood flowing from the cut dorsal vein of the penis nua be fifteen times greater than in the absence of stimulation. It spurts out in a strong stream, and is brigliter than ordinary venous blood (Eckhard). Stimulation of the perijpleral end of the nerius pudendus causes constriction of the ressels of the penis, so that it contains vaso-constrictor fibres which are the antagonists of the nervi erigentes.

$V$ aso-motor Nerves of Veins.-Like arteries, veins have plexuses of nerve-fibres in their walls, and contract in response to various stimuli. In some cases-e.g., in the wing of the bat-rhythmical contractions of the veins are strikingly displayed, but they do not depend on the central nervous system, as they persist after section of the brachial nerves. The first clear proof of the existence of vaso-motor nerves for veins was furnished by Mall, who showed that vaso-constrictor fibres for the portal vein exist in the splanchnic nerves. When these were stimulated, after the disturbing effect of changes in the circulation through the intestines had been eliminated by compression of the aorta in the thorax, an actual shrinking of the vein could be olsserved. The fibres issue from the spinal cord by the anterior roots of the third to the eleventh dorsal nerves, but chiefly in the fiftl to the ninth dorsal. When the liver is enclosed in a plethysmogralph, and the central end of an ordinary sensory nerve, like the sciatic, excited, reflex vaso-constriction tahes place in the portal area, the volume of the organ diminishes, and the blood-pressure rises in the portal vein (François-Franck).

The vena portæ and its branches are in the physiological sense arteries rather than veins, since they break $11 p$ into capillaries, and it was to be expected that the regulation of the bloudflow in them would be carried out in the same way as in ordinary arteries, namely, by means of vaso-motor nerves. But we must not, without special proof, extend the results obtained in the portal system to ordinary veins. A certain amount of evidence, lowever, exists that even such reins as those of the extremities are supplied, though scantily, with vaso-constrictor (veno-motor) fibres. After ligation of the crural artery or atorta, stimulation of the peripheral end of the sciatic has been seen to cause contraction of short portions of the superficial reins of the leg. 
Course of the Vaso-motor Nerves. - In the log the vaso-constrictors pass ont as fine medullated fibres $(1.8$ to $3.6 \mu$ in diameter) in the anterior roots of the second dorsal to about the second lumbar nerves. They proceed by the white rami communicantes to the lateral sympatletic ganglia, where, or in more rlistal ganglia such as the jnferior mesenteric, they lose their medulla, and their axiscylinder processes (Chap. N1l.) break up into fibrils that come into close relation with the nerve-cells of the ganglia. These ganglioncells in their turn send off axis-cylinder processes, which, enveloped by a nenrilemma, pass as non-medullated fibres by various routes to their final destination, the unstriped muscular fibres of the bloodvessels. Tlieir course to the head has been already described. To the limbs they are distributed in the great nerves (brachial plexus, sciatic, etc.), which they reach from the sympathetic ganglia by the grey rami communicantes.

The outflow of vaso-clilator fibres is not restricted to the same portion of the corl from which the outflow of constrictor fibres takes place. Their existence is inclecel most easily demonstrated in nerves springing from those regions of the cerebro-spinal axis from which vaso-constrictor fibres do not arise, and where, therefore, wo have not to contend with the difficulty of interpreting mixed effects. Vaso-dilators for the external generative organs and the mucons membrane of the lower end of the rectum pass out as small medullated fibres of the anterior roots of certain of the sacral nerves (mainly the second and third in the cat) into the pelvic nerve (nervus erigens). They cnd in relation with ganglion-cells in the neighbourhood of the organs which they supply. The serenth and ninth cranial nerves carry vaso-dilator fibres which are listributed by way of the lingual and other branches of the fifth to the salivary glands, the tongue, the mucous membrane of the floor of the mouth, and part of the soft palate. Those in the lingual, passing througls the chorda tympani.end in ganglion-cells near the submaxillary and sublingual glands, and the axons of these cells continue the path to the vessels of the glands. It is supposed that the vaso-dilators distributed in other branches of the fifth also have ganglion-cells on their course. In fact, there is gool evidence that every efferent vaso-motor fibre is interrupted by one, and only by one, ganglion-cell between the cord and the bloodressels. The remarkable statement has been recently made that for certain regions of the body, especially the skin of the limbs. the vaso-clilator nerves are contained, not in the anter:or, but in the posterior roots. And these, it is claimed, are not aberrant efferent fibres which have strayed in the course of development into the wrong roots, but true posterior root-fibres whose cells of origin lie in the spinal ganglia, and which conduct efferent vaso-dilator im pulses in the wrong direction, so to speak, from the cord to the periphery-- antidromic " impulses (Bayliss). But the question is still under discussion.

Effect of Nicotine on Nerve-cells.-A method which has been found most fruitful in studying the relations of sympathetic ganglioncells to the vaso-motor fibres. as well as to the pilo-motor* and secretory fibres which in certain situations are so intricately mingled with them. must here be mentioned. It depends upon the fact that when a suitable close of nicotine (Io milligrammes in a cat) is injecterl into a vein, or a solution is painted on a ganglion with a brush, the passage of nerve-impulses tlurough the ganglion is blocked for a time (Langley). The nerve-fibres peripheral to the ganglion are not

* Pilo-motor nerves supply the smooth arrector pili muscles, whose contraction causes the hair to 'stand on eni.' 
affected. The question whether efferent fibres are connected with nerve-cells between a given point and their peripheral distribution can. therefore, be answered by observing whether any effect of stimulation is abolished by nicotine. If, for instance, the excitation of a nerve caused constriction of certain bloodvessels before, and has no effect after, the application of nicotine to a ganglion, its raso-constrictor fibres, or some of them, must be connected with nerve-cells in that ganglion. Langley has recently brought forwarl evidence that many of the bolies which are cominonly supposed to act upon nerve-cndings (as nicotine, curara, atropine, pilocarpine, adrenalin, etc.) really act upon 'receptive' substances of the cells in connection with which the nerve-fibres end. These receptive substances are conceived to be capable of being specifically affected by chemical bodies and by nervous stimuli. and in their turn to be capable of influencing the metabolism of the main cell substance on which its function depends. The receptive substances thus form beyond the histological link of the nerve-ending a kind of chemical link between the nerve-fibre and the cell which it supplies.

We have thus traced the vaso-motor nerves from the cerebrospinal axis to the bloodvessels which they control ; it still remains to define the portion of the central nervous system to which these scattered threads are related, which holds them in its hand and acts upon them as the needs of the organism may require.

Vaso-motor Centres.-Now, experiment has shown that there is one very definite region of the spinal bulb which has a most intimate relation to the vaso-motor nerves. If while the bloodpressure in the carotid is being registered, sav, in a curarized rabbit, the central end of a peripheral nerve like the sciatic is stimulated, the pressure rises so long as the bull is intact, this rise being largely due to the reflex constriction of the vessels in the splanchnic area. If a series of transverse sections be made through the brain, the rise of pressure caused by stimulation of the sciatic is not affected till the upper limit of the hulb is almost reached. If the slicing is still carried dowmwards, the bloodpressure sinks, and the rise following stimulation of the sciatic becomes less and less. When the medulla has been cut away to a certain level, only an insignificant rise or none at all can be obtained. The portion of the medulla the removal of which exerts an influence on the blood-pressure, and its increase by reflex stimulation, extends from a level t to $5 \mathrm{~mm}$. above the point of the calamus scriptorius to within 1 to $2 \mathrm{~mm}$. of the corpora quadrigemina. Stimulation of the medulla causes a 1ise, destruction of this portion of it a severe fall, of general blood-pressure. There is evidently in this region a nervous ' centre' so intimately related, if not to all the vaso-motor nerves, at least to such very important tracts as to deserve the name of a vaso-motor centre. Experiment has shown that this is much the most influential centre, and it is usually called the chief or general vaso-motor centre. Some writers prefer to 
speak of it as the vasn-constrictor centre, since it is undoubtedly connected with most or all of the vaso-constrictor paths, and lias not been shown to be similarly connected with the vasodilator paths. There is, indeed, not the same solid evidence for the existence of a general vaso-dilator centre in the bull as for the existence of the general vaso-constrictor centre. Yet there are facts which indicate that the bulbar vaso-motor centre or centres, when reflexly stimulated, can, and often do, respond not merely by an increase or a renission of vaso-constrictor tone, but by a simultaneous inhibition of vaso-constrictor fibres and excitation of vaso-dilators leading to a fall of pressure, or by a simultaneous inhibition of vaso-dilators and excitation of vasoconstrictors leading to a rise of pressure.

The spinal cells of origin of the pre-ganglionic segments of the vaso-constrictor paths constitute subordinate centres which either normally support a certain degree of vascular tone, or come to do so after the chief vaso-motor centre has been cut off.

Thus, in the frog it is possible to go on destroying more and more of the cord from above downwards, and still to obtain reflex vaso-motor effects, as seen in the vessels of the web, by stimulating the central end of the sciatic nerve. Although these effects indeed diminish in amount as the destruction of the cord proceeds, yet a distinct change can be caused when only a small portion of the cord remains intact.

Similarly, in the mammal evidence has been obtained of the existence of 'centres' at various levels of the cord, capable of acting eventually, if not at once, as vaso-constrictor centres after the loss of the controlling influence of the bulb. The best example of a vaso-dilator centre is that situated in the lumbar cord, which controls the erection of the penis. After total section of the cord at the upper limit of the lumbar region, erection, which is known to be due to a reflex dilatation of the arteries of the organ through the nervi erigentes, can still be caused (in dogs) by mechanical stimulation of the glans penis, so long as the afferent fibres of the reflex arc contained in the nervus pudendus are intact. Destruction of the lumbar cord abolishes the effect. It is impossible to aroid the conclusion that a vaso-dilator or erection centre, which is in relation on the one hand with the nerri erigentes, and on the other with the nervus pudendus, exists in the lower portion of the spinal cord. Vaso-motor centres for the hindlimbs have also been located in the same region. When the brain, the bulb, and the upper portion of the cord have been eliminated by ligation of all the arteries from which blood can possibly reach them, a sufficient vascular pressure persists to permit the circulation to go on in the lower portion of the body for hours. And while section-or freezing (Fig. 6S) - of the cord in the lower 
cervical region causes a marked fall of pressure, this is not permanent if the animal is allowed to survive. Forty-ene dity after total section of the cord at the seventh cervical segment in a rlog an arterial pressure of $130 \mathrm{~mm}$. of mercury was found. A mechanism for the maintenance of vascular tone exists even beyond the limits of the central nervous system. For when the lower portion of the cord is completely destroyed, the dilatation of the vessels of the hind-limbs, which is at first so com-jicu(sus, passes away after a time, the functions of vas()-motor ceutres having perhaps been assumed by the sympatletic ganglia (Goltz and Ewald). When the lumbo-sacral sympathetic chain is extirpated, there is a further loss of vascular tone in the affected region. But even this is not irremediable. After a time recovery again occurs, although it may be more partial and tardy than before. This may take place either through the intervention of still more

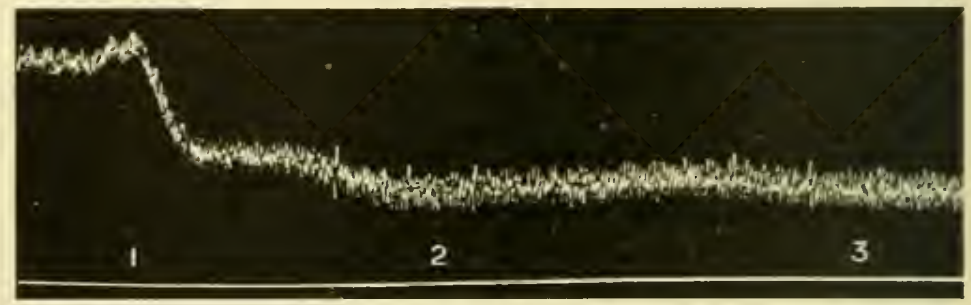

Fig. 68.-EfFect ON Biond-presstre in Dog OF Fritezing, Spinal Cord (PIKF).

At I the first or second dorsal segment of the cord was frozen with liquicl air : at 2 and 3 central end of sciatic stimulated withrut effect on pressure (respertively one and $a$ half and three minutes after freezing of cord). (Four-tifthsof original size.)

peripheral ganglia, or through the development of a certain tonus by the muscular fibres of the vessels when abandoned to themselves.

As to the nature of the tone of the general vaso-motor centre, the same question may be asked which has leen alreacly discussed for the cardio-inhibitory centre. Is it reflex, or does it depend upon direct excitation of the centre by some constituent of the blood or lymph, or some substance produced in the centre itself? The best answer which can at present be made is that a constant central excitation by the carbon dioxide formed in the centre or circulating in the blood is a not unimportant factor in the maintenance of the vaso-motor tone. A marked diminution in the carbon dioxide tension of the blood, a condition which is termed ' acapnia,' may indeed contribute to the severe fall of bloodpressure associated with surgical shock (p. 175) (Henderson). In addition to the direct influence of carbon dioxide, and possibly of other substances, the arrival of afferent impulses at the centre seems to play a part in maintaining that continual discharge 
of efferent impulses along the vaso-motor nerves which constitutes its tone. In this regard, the vaso-motor centre occupies an intermediate position between the respiratory centre, the most purely antomatic, and the cardio-inhibitory centre, the most purely reflex of the three great bulbar mechanisms.

of the anatomical relations of the nerve-cells that make up the bulloar and spinal vaso-motor centres, little more is known than may be (leluced from the physiological facts we have been reciting. It has been sumised on histological grounds that certain cells of small size scattered 1t]) and (lown the thoracic and npper lumbar regions of the cord in the lateral horn (intermedio-lateral tract), and perinaps cropping out also in the bulb, are vaso-motor cells. There is good eviclence that the pre-ganglionic sympathetic fibres, including the vaso-motor fibres which we have alrealy discovered emerging from the cord in the spinal roots, are connected with these cells. Ancl, indeel, there is reason to believe that the connection is made without the intervention of any other nerve-cells, and that the axiscylinclers of these vaso-motor fibres are the axis-cylincler processes of the vaso-motor cells. So that the simplest efferent path along which vaso-motor impulses can pass may be considered as built up of two neurons, one with its cell-body in the corcl, and the other in a sympathetic ganglion. Less is known of the elements which constitute the bulbar centre and of their connections. But since it would appear that the spinal vaso-motor centres are uncler the control of the chicf centre in the bulb, it is necessary to suppose that the axis-cylinder processes of some of the cells of the bulbar centre come into relation with the spinal vaso-motor cells. and that impulses passing, let us say. from the bulb to the ressels of the leg. woull have to traverse three neurons (see Chap. XII.).

Vaso-motor Reflexes. - Ire have already seen that the cardiac centres are constantly influenced by afferent impulses, and that in the direction either of augmentation or inhibition. The vasomotor centre in the bulb is equally sensitive to such impulses. They reach it for the most part along the same nerves, and by increasing or diminishing its tone cause sometimes constriction and sometimes dilatation of the vessels, the result depending partly upon the anatomical connection of the afferent fibres, but apparently in part also upon the state of the centre.

Of the afferent nerves that cause vaso-dilatation, the most important is the depressor, whose reflex inhibitory action on the heart lias been already described. The fall in the arterial pressure is due chiefly, not to the inhibition of the heart, but to inhibition of the vaso-constrictor tone of the bulbar vaso-motor centre, combined with stimulation of vaso-dilator nerves, and consequent general dilatation of the arterioles throughout the body. That the depressor action involves excitation of vasodilators follows from the fact that vaso-dilatation occurs in the limbs on stimulation of the depressor after their vaso-constrictor nerves have been cut. Stimulation of the depressor produces its usual result after section of the vagi. It has been suggested that 
the function of the nerve is to act as an automatic check upon the hlood-pressure in the interest both of the heart and the ressels, its terminations in the aorta or the ventricular wall being mechanically stimulated when the pressure tends to rise towards the danger limit. In rare cases, eflerent inlibitory libres for the heart have been found in the depressor of the rabbit.

Many of the peripheral nerves contain fibres whose stimulation is followed by dilatation of the bloodvessels in special regions, usually the areas to which they are themselves distributed, accompanied by constriction of distant and, it may
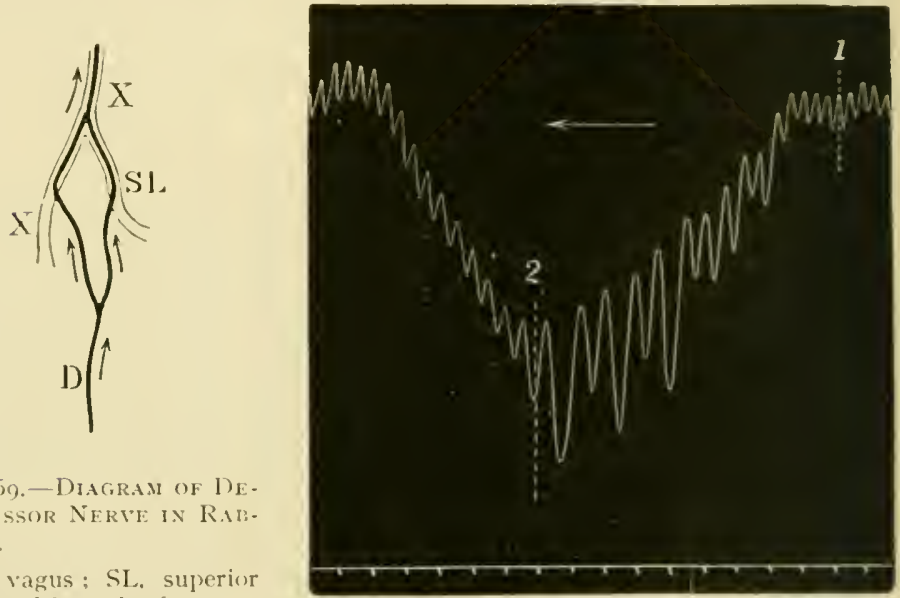

Fig. 69.-DIAGRAM OF DE. PRESSOR NERTE IN R.IIBIT.

$X$, vagus: SL. superior laryngeal branch of vagus ; D. depressor fibres. The arrows slow the course of the impulses that affect the blond-pressure.

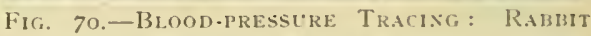
(MERCURY MANOMITER).

Central end of depressor stimulated at I : stimulation stopped at 2. Time-trace, seonds.

be, more extensive vascular tracts. Thus, the usual local effect of stimulating the afferent fibres of the lowest three thoracic nerves, in whose anterior roots run the vaso-motor fibres for the kidney, is a dilatation of the renal vessels (Bradford), and the usual local effect of stimulating tle infra-orbital or supraorbital nerve a dilatation of the external maxillary artery. But the general effect in both cases is vaso-constriction in otlier regions of the body, which more than compensates the local dilatation, so that the arterial hlood-pressure rises. It is not difficult to see that both of these clianges render it easier for the part to obtain an increased supply of blood.

Sometimes the reciprocal relation between vaso-clilatation in one part of the body and vaso-constriction in another is only apparent. For cxample, stimulation of the cut end of the sciatic causes. as we have already secn, cxtensive vaso-constriction and a notable rise in the blood-pressurc. The constriction certainly involves the splanch- 
nic area; but superficial parts, as the lips, may be secn to be fluslied with blood In asplyxia. When the vaso-motor centres are directly stimulated by the venons bloms, this apparent antagonism is still better marked: the cutaneons ressels are widely rlilated and engorged, the face is livid. but the abdominal organs are pale and blondless (Heidenhain). The blool-pressure rises rapidly, reaches a maximum, and then gradually falls as the vaso-motor centre becomes paralyzed (Figs. 72 and 73). It has been shown that in both cases vaso-constriction of the skin is really produced as well as vasoconstriction of the intemal organs, but the increased blood-pressure mechanically overcomes the constriction of the cutancous vessels.

The kind of stimulus seems to have something to do with the direction of the reflex vaso-motor change. For while electrical stimulation of every muscular nerve, even of the very finest twigs that can be isolated and laid on electrodes, provokes

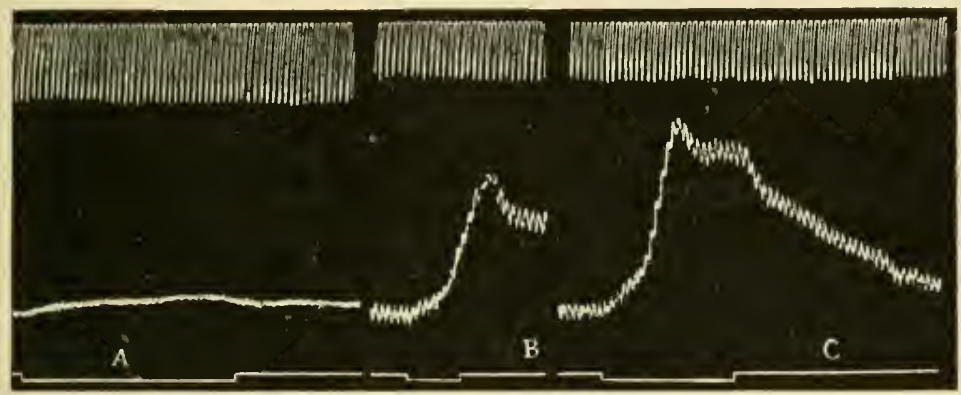

Fig. 71.-PRessor Effect of Stimulation of Central ENd of Vagís in a CAT DURING RESUSCITATION AFter CEREBRAL ANæMia.

The depressions in the signal line $\mathrm{ABC}$ indicate the duration of three suecessive excitations of equal strength, sixty-five, seventy-three, and seventy-nine minutes respectively after restoration of the circulation. The pressor effect increases as resuscitation proceeds. Later on the original depressor effect was again obtained. The upper tracing is that of the artificial respiration. (Two-thirds original size.)

always, whether the shocks follow each other rapidly or slowly, a rise of general blood-pressure, mechanical stimulation of a muscle, as by kneading or massage, causes a fall. The condition of the afferent fibres also exerts an influence. For example, excitation of the central end of a sciatic nerve that has been cooled is followed by vaso-dilatation and fall of pressure, the opposite of the ordinary result. These and similar facts have led to the idea that most afferent nerves contain two kinds of fibres, whose stimulation can affect the activity of the vasomotor centres - ' reflex vaso-constrictor,' or ' pressor' fibres, and 'reflex vaso-dilator,' or 'depressor' fibres. The branch of the vagus, however, to which the name ' depressor' has been specially given, is usually described as the only peripheral nerve the excitation of which is in all circumstances followed by a general 
diminution of arterial pressure. But this is not strictly correct, for at an early period in the resuscitation of the brain after anemia excitation of the rabbit's 'depressor' causes a slight rise of pressure not followed by any fall. This, perhaps, indicates the presence in the 'depressor' of a small number of preasor nibres, which are resuscitated sooner than the depressor fibres proper. The same phenomenon, only mrre marked, may le seen when the central end of the cat's ragus, containing the depressor fibres, is excited at intervals during resuscitation (Fig. 7I). Or the result may depend upon a change in the response of the altered vaso-motor centres to impulses reaching them along the depressor fibres. If specific 'depressor' fibres exist in wther nerves, they are so mingled with 'pressor ' fibres that their action is masked when both are stimulated together. The state of the

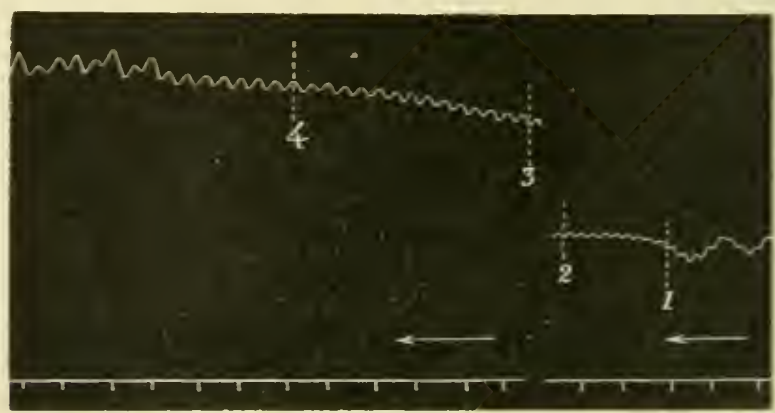

FIG. 72.-RISE OF BLOOD-PRESSLRE IX ASPHYXIA: RABBIT.

Respiration stopped at $I$. Interval between 2 and 3 (not ref riduced) $4+$ siorinds. during which the blood-pressure steadily rose. At 4 . respiration resumed. Time trace, seconds.

vaso-motor centre is unquestionably a factor which has some importance in determining the result of reflex vaso-motor stimulation. For instance, in an animal deeply ana-thetized with chloroform or chloral, excitation of pressor fibres (in an orilinary sensory nerve) causes, not a rise, but a fall of blond-pressure; while in an animal fully under the influence of strvelunine stimulation of the depressor nerve causes not a fall, but a rise.

These facts enable us to some extent to understand the manner in which the distribution of the blood is adjusted to the requirements of the different parts of the hodr, so that to a certain degree of approximation no organ has too much, and none too little. The blood-supply of the organs is always shifting with the calls mpon them. Now, it is the actively-digesting stomacl and the actively-secreting glands of the alimentary tract which must be fed with a full stream of blood, to supply waste and to 
carry away absorbed nutriment. Again, it is the working muscles of the legs or of the anms that need the clief blowelsupply. But wherever the call may be, the vaso-motor mechanism is able, in health, to answer it by bringing about a widening of the small arteries of the part which needs more blood, and a compensatory narrowing of the vessels of other parts whose needs are not so great.

The amount of blood flowing through an organ in a given time is not, of course, proportional to the quantity contained in it at any moment. For it elepends also upon tlie velocity of the blool-stream, and the blood flows at a rlifferent rate in different organs and in the same organ at clifferent times. The flow for Ioo grammes of organsubstance per minute under certain conditions has been determined by observations with the stromuhr in logs as follows: Posterior extremity, 5 c.c. ; skeletal muscles, I2 c.c. ; heacl, 2o c.c. ; intestine, 3 I c.c. ; spleen, 58 c.c. ; brain, I36 c.c. ; kidney, I5o c.c. ; thyroid gland, 560 c.c. (Opitz, etc.).

It is also through the vaso-motor system, and especially by the action of that portion of it which governs the abdominal vessels, and of the nerves that regulate the work of the heart, that in animals to which the upright position is normal (monkey) and in man the influence of changes of posture on the circulation is almost completely compensated.* The pressure in the upper part of the human brachial artery has been measured with a sphygmomanometer, first in the horizontal and then immediately afterwards in the standing posture, and in health it has been found to remain practically unchanged (Hill). But if the person was overworked or out of sorts, the compensation was less complete. It is well known that in debilitated persons, especially if long confined to bed, the sudden assumption of the upright position may cause vertigo, and even syncope, the normal compensatory mechanism being deranged. In such animals as the rabbit this compensation is totally inefficient. When a domesticated rabbit, which has been kept in a hutch, is sus-

* Two factors may be distinguished in the blood-pressure, the hydrostatic and the hydrodynamic elements. The hydrostatic portion of the pressure is due to the weight of the column of blood acting on the vessel ; the hydrodynamic portion of the pressure is due to the work of the heart. If a dog be securely fastened to a holder arranged in such a way that the animal can be placed vertically, with the head up or down, and the mean blood-pressure in the crural artery be measured in the two positions, there will be a considerable difference. For when the legs are uppermost the heart has to overcome the weight of the column of blood rising above it to the crural artery; when the head is uppermost the action of the heart is reinforced by the weight of the blood. And if no change were produced in the action of the heart, or in the general resistance of the vascular path, by the change of position, this difierence would be equal to the pressure of a column of blood twice as high as the straiglt-line distance between the cannula and the point of the arterial system at which the pressure is the same with head up as with head down (indifferent point). 
pended vertically with the feet down, the blood drains into the abdominal ressels, syncope speedily ensues, and in a period that ranges from less than a puarter to three-quarters of an hour the aninnal dies in the convulsions of acute cerebral aniemia (Salathé, Hill). The head-down position has no ill effects. In wild rabbits, whose abdominal wall is more tense and elastic, these fatal symptoms are not easily produced, and the same is true of cats and dogs. But in all animals, when the compensation is destroyed, as in paralysis of the vaso-motor centre by chloroform, the circulation may be profoundly influenced by the position of the body: elevation of the head may lead to cerebral anxmia, syncope, and even death ; elevation of the legs, and particularly the abdomen, may restore the sinking pulse by filling the heart and the ressels of the brain. If a chloralized dog be fastened on a board which can be rotated about a lorizontal axis passing under the neck, the blood-pressure in the carotid artery falls greatly when the animal is made to assume the vertical position with the head up, and either rises a little or remains practically unchanged when the head is made to hang down. So great may the fall of pressure be in the former position that death may occur if it be long maintained (Practical Exercises, p. I99).

Finally, it is in virtue of the amazing power of accommodation possessed by the vascular system, as controlled by the vaso-motor and cardiac nerves, that so long as these are not disabled the total quantity of blood may be greatly diminished or greatly increased, without endangering life, or even causing more than a transient alteration in the arterial pressure. It is not until at least a quarter of the blood has been withdrawn that there is any notable effect on the pressure, for the loss is quickly compensated by an increase in the activity of the heart and a constriction of the small arteries. An animal may recuver after losing considerably more than half its blood.* Conversely, the volume of the circulating liquid may be (loubled by the injection of blood or physiological salt solution without causing death, and increased by 50 per cent. without any marked increase in the pressure. The excess is promptly stowed away in the dilated vessels, especially those of the splanchnic area; the water passes rapidly into the lymple, and is then more gradually eliminated by the kidneys.

From these facts we can deduce the practical lesson, that bloodletting, unless fairly copious, is useless as al means of lowering

* It is not usually possible to obtain quite two-thircls of the total blood by bleeding a dog from a large artery. In seven dogs bled from the carotid in the laboratory of the writer, the ratio of the weight of the blood obtained to the body-weight was $1: 247,1: 217,1: 20^{\circ} 7,1: 20^{\circ}(7,1: 15 \%, 1: 16$, $1: 13 \%$. In the last case, the blood clotted with abnormal slowness, and the animal died in a few minutes. 
the general arterial pressure, while it need not be feared that transfusion of a considerable quantity of blood, or of salt solution, in cases of severe himorrhage will clangerously increase the pressure. And from the physiological point of view the term 'hemorrhage' includes more than it does in its ordinary sense. For as dirt to the sanitarian is "matter in the wrong place,' latmorrhage to the pliysiologist is blood in the wrong place. Not a drop of blood may be lost from the body, and yet death may occur from hiemorrhage into the pleural or the abdominal cavity, into the stomach or intestines. Not only so, but a man may bleed to death into his own bloodvessels; in surgical shock, as well as in ordinary fainting or syncope, the blood which ought to be circulating through the brain, heart and lungs may stagnate in the dilated vessels of the splanchnic and

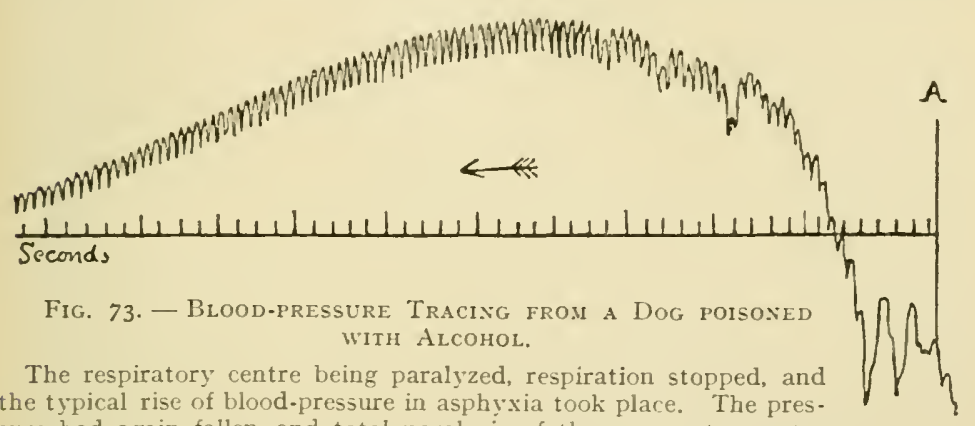
the typical rise of blood-pressure in asphyxia took place. The pres. sure had again fallen, and total paralysis of the vaso-motor centre was near at hand, when at $A$ the animal made a single respiratory movement. The quantity of oxygen thus taken in was enough to restore the vaso-motor centre, and the blood-pressure again rose. This was repeated five or six times. (Threefourths original size.)

other areas. The rapid feeble pulse in shock is due to a similar loss of activity of the cardio-inhibitory centre. The cause of the vascular symptoms of surgical shock is by no means clear, and is probably complex. Some observers have laid stress upon the supposed effect of excessive stimulation of afferent nerves in producing long-continued inhibition or fatigue of the vaso-motor centres. In farour of this hypothesis is the fact that in experimental shock in animals the rise of blood-pressure which can be obtained by stimulation of a nerve like the sciatic is not so great as under normal conditions (Crile, Howell). As already mentioned (p. I69), a diminution of the carbon dioxide pressure in the blood occasioned by increased pulmonary ventilation due to the excitation of afferent nerves, or in the case of abdominal operations or wounds by the direct escape of carbon dioxide through the peritoneum, has also been suggested as an important factor. 
The Lymphatic Circulation. - Is has alreisly becen stated. some of the constitnents of the blood, insteatel of passing back to the heart from the citpillatries along the veins. find their way by a much more tedious route along the lymplatics. The biosicappillaries are everywhere in very intimate relation with lymphcapillatries, which, completely lined with epithelioirl cells, lie in irregular spaces in the connective-tissue that everywhere aceompanies and supports the bloodvessels. The constituents of the bloox-plasma are filtered through, or secreted by the citpillary walls into these lympl spaces, and mingling there with wiste products discharged by the cells of the tissues, ferm the lipuid known ats tissuc licpuid or tissue lymph. From the tissue liquid the lympls capillarics take up the constituents of the 'Iymplatic' lympli. which then passes into larger lymphatic vessels, with lymphatic glands at intervals on their course. These fall into still larger trunks, and finally the greater part of the lymph reaches the bloud again by the thoracic duct, which opens into the venous system at the junction of the left subclavian and internal jugular veins. The lymph from the right side of the head and neck. the right cxtremity. and the right side of the thorax, with its viscera. is collected by the right lymphatic duct, which opens at the junction of the right subclavian and internal jugular veins. The openings of botlı clucts are guarded by scmilunar valves, which prevent the reflux of blood from the veins. Scrous cavities like the pleural sacs, although differing from ordinary lymph spaces, are connected through small openings, called stomata, with lympliatic ressels.

The rate of flow of the lympl in the thoracic cluct is very small compared with that of the blood in the arterics-only about + mm. per second, according to one observer. Nevertheless, a substance injected into the blood can be detecter in the lymph of the duct in four to seven minutes (Tschirwinsky). The factors which contribute to the maintenance of the lymph flow are :

(I) The pressure under which it passes from the blood capillarics into the lymph spaces and from the lymph spaces into the lymph capillaries. The pressure in the thoracic duct of a lorse may be as high as $12 \mathrm{~mm}$. of mercury; in the dog it may be less than I $\mathrm{mm}$. The difference is probably duc, in part at lcast, to a difference in the experimental conclitions, dogs being usually anasthetized for such measurements, horses not. The pressure in the lymple capillaries must, of course, be higher than in the thoracic duct-how much higher we do not know.

(2) The contraction of muscles increases the pressure of the lymph by compressing the channcls in which it is contained, anel the valves, with which the lymplatics are even more richly provided than the veins, hinder a backward and favour an onward llow. The contractions of the intestines, and especially of the villi, are an important aid to the novement of the chyle. By the contraction of the diaphragm, substances may be sucked from the peritoneal cavity into the lymphatics of its central tendon, through the stomata in the scrous layer with which its lower surface is clad. It is even possible by passive movements of the diaphragm in a lead rabbit to inject its lymphatics with a coloured liquid placed on its peritoneal surface. Passive movements of the limbs and massage of the muscles are also known to hasten the sluggish current of the lympl, and are sometimes employed with this object in the treatment of disease.

(3) The movements of respiration aid the flow. At every iuspira- 
tion the pressure in the great veins near the heart becomes negative, and lymph is sucked into them (p. 2 in).

(4) In some animals rhythmically-contracting muscular sacs or hearts exist on the course of the lymphatic circulation. The frog laas two pairs, an anterior and a posterior, of these lymph hearts, which pulsate, although not with any great regularity, at an average rate of sixty to seventy beats a minute, and are governed by motor and inhibitory centres situated in the spinal cord. The beat is not directly initiated from the cord, but the tonic influence of the cord is necessary in orler that the lvmph heart may continuc to beat (Tschermak). Such hearts are also found in reptiles. It is possible that in animals without localized lymph hearts the smooth muscle, which is so conspicuous an clement in the walls of the lymphatic vessels. may aid the flow by rhythmical contractions.

\section{PRACTICAL EXERCISES ON CHAPTER II.}

I. Microscopic Examination of the Circulating Blood.--(I)' Take a tadpole and lay it on a glass slide. Cover the tail with a large cover-slip, and examine it with the low power (Leitz, oc. III., obj. 3). Gencrally the tail will stick so closely to the slide, and the animal will move so little, that a sufficiently good view of the circulation can be obtained. If there is any trouble, destroy the brain with a needlc. Obscrve the current of the blood in the arteries, capillarics and veins. An artery may be casily distinguished from a vein by looking for a place at which the vessel bifurcates. In veins the blood flows in the two branches of the fork towards the point of bifurcation, in arteries away from it. Skctch a part of a field.

To Pith a Frog. - Wrap the animal in a towel, bend the head forwards with the index-finger of one hand, feel with the other for the depression at the junction of the head and backbonc, and push a narrow-bladed knife right down in the middle line. The spinal cord will thus be divided with little bleeding. Now push into the cavity of the skull a piece of pointed lucifer match. The brain will thus be destroyed. The spinal cord can be destroyed by passing a blunt needle down inside the vertebral canal.

(2) Take a frog and pith its brain only, inserting a match to prevent bleeding. Pin the frog on a plate of cork into one end of which a glass slide has been fastened with sealing-wax. Lay the web of one of the hind-legs on the glass and gently separate two of the toes, if necessary by threads attached to them and secured to the cork plate. Put the plate on the microscope-stage and fasten by the clips (see pp. I5, Io9).

(3) After the normal circulation has been studied thoroughly put a very small drop of tincture of cantharides on the portion of the web which is in the field of the microscope, using a finc pipette. Observe the process of inflammation, including stasis and diapedesis (p. 53).

2. Anatomy of the Frog's Heart.-Expose the heart of a pitherl frog by pinching up the skin over the abdomen in the middle line, dividing it with scissors up to the lower jaw, and then cutting through the abdominal muscles and the bony pectoral girdle. The external abdominal vein, which will be observed on reflecting the skin, can bc easily avoided. The heart will now be seen encloserl in a thin membrane, the pericardium, which should be grasped with finc- 
pointed forceps and freely divided. Connecting the posterior surface of the heart and the pericardium is a slender band of connective tissue, the fraenum. A silk ligature may be passel around this with a threaded curved needle, or curved finc-pointed forceps, and ticl, and then the framum may be divided posterior to the ligature. The anatomical arrangement of the various parts of the heart should now be studied. Note the single ventricle with the bulbus arteriosus, the two auricles, and the sinus venosus, turning the heart over to see the latter by means of the ligature. Cobserve the whitish crescent at the junction of the sinus venosus and the right auricle (Fig. 7.1).

3. The Beat of the Heart.--Note that the auricles beat first, and then the ventricle. The ventricle becomes smaller and paler during its systole, and blushes red during diastole. Comnt the number of beats of the heart in a minute. Now excise the heart, lifting it by
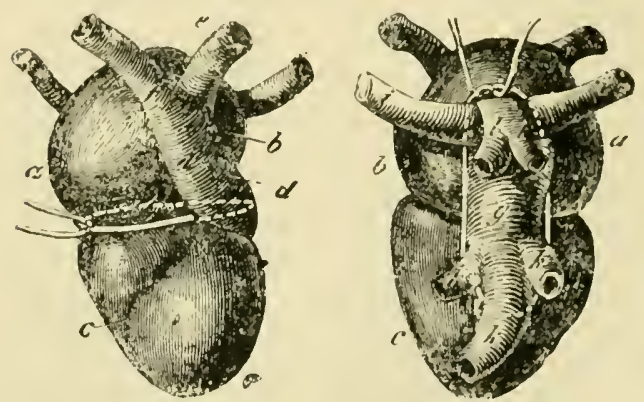

Fig. 74.-Frog's HeART WITH STANNIUS' LIGATCRES in Position (Cyon).

Anterior surface of heart shown on the left, posterior surface on the right. $a$, right auricle; $b$, left auricle; $c$, ventricle : $d$, bulbus arterinsus : $\ell, f$, aort $x ; g$, sinus venosus. means" of the ligature. and taking care to cut wicle of the sinus venosus. Place the heart in a small porcelain capsulc on a little blotting-patper moistened witl physiological salt solution.* ()b. serve that it goes on beating. Put a little ice or snow in contact with the heart, and count the number of beats in a minute. The rate is greatly diminished. Now remove the ice and blottingpaper, cover the heart with the salt solution. and heat, noting the temperature with a thermometer. Observe that the heart beats faster and faster as the temperature rises. $A t+0^{\circ} \mathrm{C} . \mathrm{to}^{\circ}+3^{\circ} \mathrm{C}$. it stops beating in liastole (heat standstill). Now at once pour off the heaterl liquicl, and run in some cold salt solution. The heart will begin to beat again.

4. Cut off the apex of the ventricle a little below the aturiculoventricular groove. The auricies, with the attached portions of the ventricle, go on beating. The apex does not contract spontaneously, but can be made to beat by stimulating it mechanically (by pricking it with a needle) or electrically. Divide the still contracting portion of the heart by a longitudinal incision. The two halves go on beating.

5. Heart Tracings.-(I) Fasten a myograph-plate (Fig. 75) on a stand. Take a long light lever consisting of a straw or a piece of thin chip, armed at one end with a witing-point of parchment-pajer, supported near the other end by a horizontal axis, and pierced not far from the axis by a needle carrying on its point a small piece of cork or a ball of sealing-wax.

* For frog's tissues this should be 0.7 to 0.75 per cent. sodium chloride solution, for mammalian tissues a little stronger (about 0.9 per cent.). 
A counterpoise is adjusted on the short arm of the lever in the form of a small leaden weight. Cover a drum with glazed paper and smoke it. The paper must be put on so tightly that it will not slip. To smoke the drum. liold it by the spindle in both hands over a fish-tail burner, depress the drum in the flame, and rotate rapidly. Avoid putting on a heavy coating of smoke, as a niore lelicate tracing is obtained when the paper is lightly smoked. The speed of the drum can be varied by putting in or taking out a small vane. Arrange an clectro-magnetic time-marker for writing seconds (Fig. $\left.7^{6}\right)$. I'ith a frog

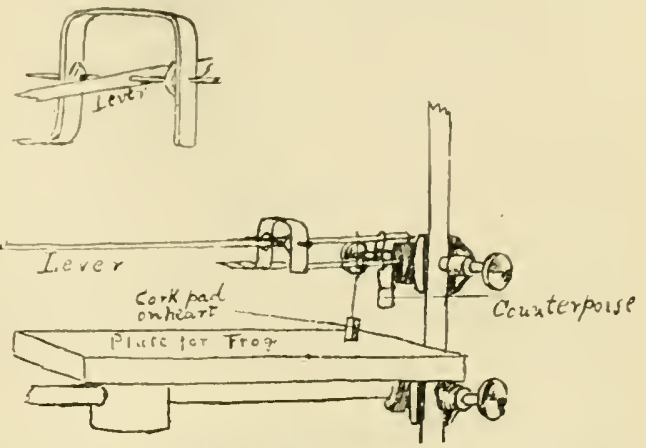

Fig. 75.-ARRaNgemext For obtaising a Heart TRACING FRON A FROG.

(brain only), expose

the heart, and put under it a cover-slip to give it support. Pin the frog on the myograph-plate. and adjust the foot of the lever so that it rests on the ventricle or the auriculo-ventricular junction. Bring the writing-point of the lever and that of the time-marker vertically under each other on the surface of the drum. Set off the drum at the slow speed (say, a centimetre a second). When the lever rests on the auriculo-ventricular junction. the part of the tracing corresponding to the contraction of the heart will be broken into two portions, representing the systole of the auricles and ventricle respectively. Cut the paper off the drum with a knife (keeping the back of the knife to the drum to avoid scoring it) and carry it to the

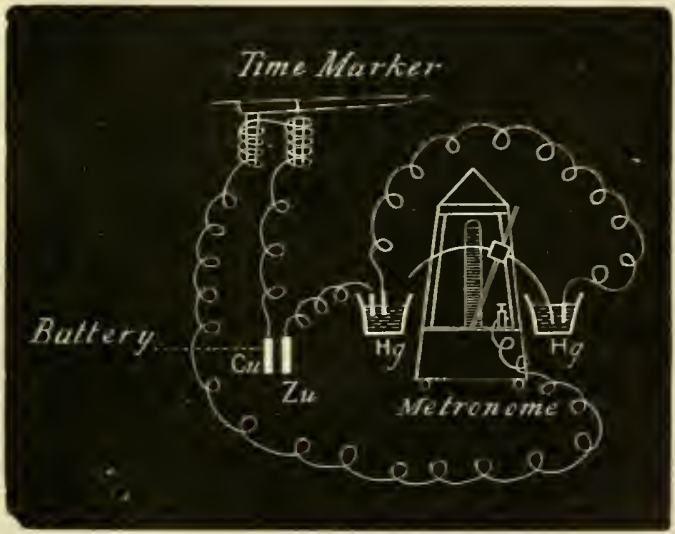

Fig. 76.-ELECT'RO-MAGNETIC TIME-MARKER CONNECTED WITH METRONOME.

The pendulum of the metronome carries a wire which closes the circuit when it dips into either of the mercury cups, $\mathrm{Hg}$.

varnishing-trough, holding the tracing by the ends with both hands, smoked side up. Immerse the middle of it in the varnish, draw first one end and then the other through the varnish, let it drip for a minute into the trough, and fasten it $\mathrm{up}$ with a pin to dry. 
(2) Heart Tracing, with Simultaneous Record of Auricular and Ventricular Contractions.-(a) For this purpose two levers may be arranged, one resting on the auricle, the other on the ventricle, the writing points being placed in the same vertical straight line on the flum. $\Lambda$ convenient form of apparatus is shown in Fig. 77.

(b) Gaskell's Method (a modification of).-Attach a silk ligature to the very apex of the ventricle. Divicle the fraenum, cut the aorta across close to the bulbus, pinch up a tiny portion of the auricle and ligature it. Remove the intestines, liver, lungs, etc., care being taken in cutting away the liver not to injure the sinus. Then remove the lower jaw, and cut away the whole of the body except the head, part of the osophagus, and the tissue connecting it with the heart. Fix the head in a clamp sliding on an ordinary stand. The heart is held at the auriculo-ventricular junction in a Ciaskell's clamp supported on a separate stand. The thread connected with the ventricle is brought round a pulley and attached to a lever above the heart. The auricle is connected with another lever. The

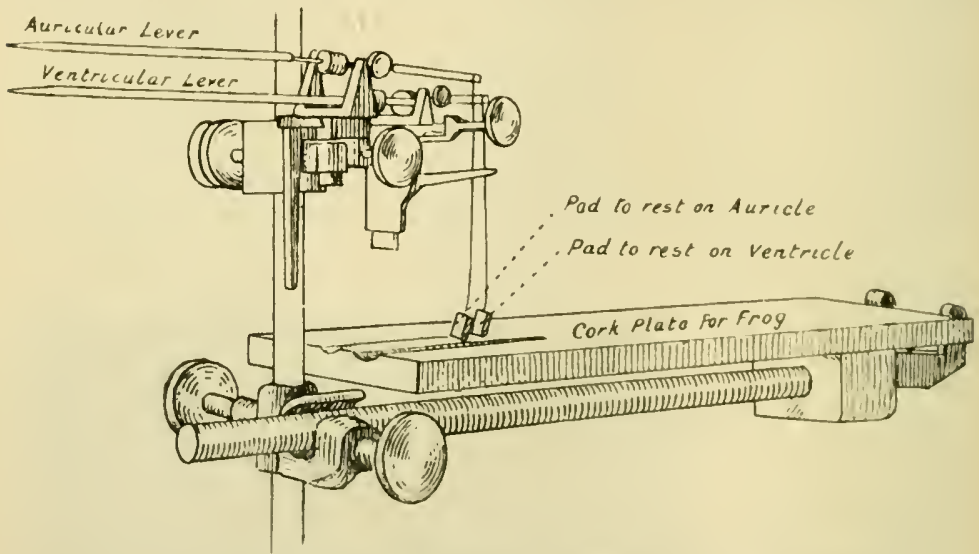

Fig, 77. - Apparatus for obtaining a Simeltaneot's Tracing of Aurictlar and Ventricular Contractions.

writing-points of the two levers are arranged in a vertical line on the drum. The small pulley must be oiled from time to time to lessen the friction (Fig. 78 ).

If tortoises or turtles are available, the much larger heart of these animals may be used for Experiments $5(2)(a)$ and $(b)$. The animal having been killed by cutting off its head, the ventral portion of the carapace is detached by the saw. The pericardium can now be slit open, and the pads of the levers arranged on auricles and ventricle respectively, as in Experiment 5 (2) (a), without further disturbing the heart. Or the heart may be removed, together with the upper portion of the body, the pericardium openerl, and the liver cut away. The aortic trunk is then divided, and the portion of it attached to the heart grasjed by a small forceps clamp. Fine silk ligatures are attached to the apex of the ventricle and the top of the right auricle. The vagus nerves are exposed in the neck, ligated, and divided. The upper portion of the body is supported on a stand. The forceps grasping the aorta is fixed in an ordinary holder, and the threads are attached to the levers, as in Experiment 5 (2) (b). 
With the vagi, Experiment 7 may be performel. It must be remembered that the activity of the two vagi is unequal in the tortoise, the right being the niore active.

6. Dissection of the Vagus and Cardiac Sympathetic Nerves in the Frog.-(1) Put the tissues in the region of tho neck on the stretch by passing into the gullet a narrow testtube or a thick glass rod moistened with water, and by pinning apart the anterior limbs. Expose the heart by cutting through the pectoral girdle in the way described in 2 (p. 177 ). On clearing away a little connective tissue and muscle with a secker, three large nerves will come into view. The upper is the glossopharyngeal, the lower the loypoglossal; the vagus crosses diagonally between them (Fig. 79). Above the vagus trunk, running parallel to it, and separated from it by a thin muscle and a bloodvessel (the carotid artery), lies its laryngeal branch. The vagus should be traced up to the ganglion situated on it near its exit from the skull.

(2) Then cut away the lower jaw, dividing and reflecting the membrane covering the roof of the mouth. At the junction of the skull and the backbone will be seen on each side the levator anguli scapulie muscle (Fig. 8o). Remove this muscle carefully with fine forceps. Clear away a little connective tissue lying just over the upper cervical vertebra,

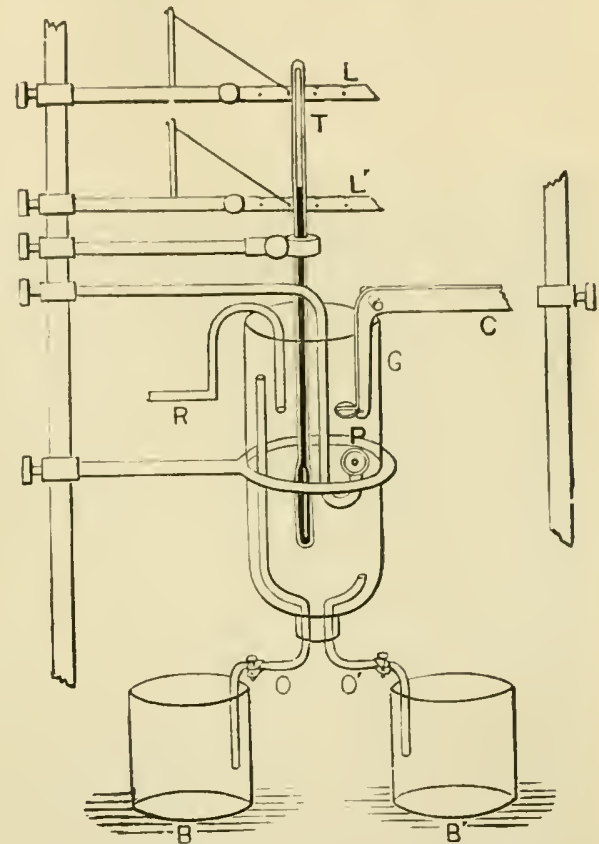

Fig. 70.-Arrangement for recording Auricu LAR AND Ventricular Contractions (AND Studying the Influence of Temperature ON THE HEART).

C, clamp holding the heart at the auriculo. ventricular groove; $P$, pulley round which a thread attached to the apex of the ventricle passes to the lever $L^{\prime} ; \mathrm{L}$, lever connected with auricle. (The rest of the arrangement is for studying the influence of temperature on the heart and its nerves, $G$ being a vessel filled with physiological salt solution in which the heart is immersed; $R$, an inflow tube from a reservoir containing salt solution at the temperature required; $\mathrm{O}^{\prime}$, an outflow tube by which $G$ may be emptied into the beaker $B^{\prime} ; O$, a tube passing to the beaker $B$ to prevent overflow from $G ; T$, a thermometer.) and the sympathetic chain, with its ganglia, will be seen. Pass a fine silk thread beneath the sympathetic about the level of the large brachial nerve, by means of a sewing-needle which has been slightly bent in a flame and fastened in a handle. Tie the ligature, divide the sympathetic 
below it, and isolate it carcfully with fine scissors up to its junction with the vagus ganglion.

Batteries-To set up a Daniell Cell.-Fill the porous pot (Fig. 203, p. 615) previously well soaled in water, with dilute sulphuric acid (1 part of commercial acid to 10 or 15 parts of water) to within $1 \frac{1}{2}$ inches of the brim, and place in it the piece of amalgamated zinc. If the zine is not properly amalgamated, leave it in the pot for a minute or two to clean its surface. Then lift it out, pour over it a little mercury, and rub the mercury thoroughly over it with a cloth. Put the pot into the outer vessel, which contains the copper plate, and is filled with a saturated solution of sulphate of copper,

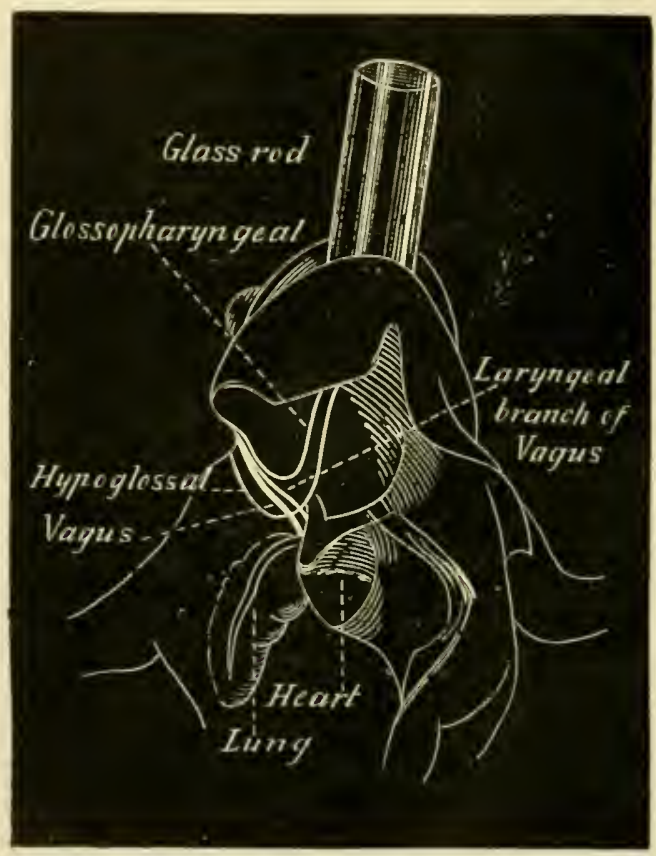

Fig. 79.-The Relations of the VAgus 1N THE FROG. with some undissolved crystals to keep it saturated. After using the Danicll, it must always be taken down. The outer pot is left with the copper plate and the sulphate solution in it. The zinc is washed and brushed bright. The sulphuric acid is poured into the stock bottle, and the porous pot put into a large jar of water to soak.

The Bichromate Cell contains only one liquid-a mixture of I part of sulphuric acid with + parts of a 10 per cent. solution of potassium bichromate. In this is placed one, or in some forms two, carbon plates and a plate of amalgamated zinc. After using the battery, take the zinc out of the liquid.

The Leclanché battery consists of a porous pot filled with a mixture of manganese dioxide and carbon packed around a carbon plate, which forms the positive pole. The pot stands in an outer jar of glass filled with a saturated solution of ammonium chloride, into which dips an amalgamated zinc rod, which constitutes the negative pole. Various ferms of $d r y$ batteries can be conveniently used for running induction-coils or time-markers, but are not adapted for yielding constant currents of long duration.

7. Stimulation of the Vagus in the Frog.-Make the same arrangements as in 5 (1) (p. 178), but, in addition, set up an induction machine arranged for an interrupted current (Fig. SI), with a Daniell, a bichromate, a Leclanclie, or a dry cell in the primary 
circuit. which should also include a simple key. Insert a shortcircuiting key in the secondary circuit. Attach the electrocles to the short-circuiting key, pusl the secondary coil up towards the primary until the shocks are clistinctly felt on the tongue when the Neef's hammer is set going and the short-circuiting key opened. Pith the brain of a frog, expose the lieart, clissect out the vagus on one side, ligature it as high up as possible, and divide above the ligature. Fasten the electrodes on the cork plate by means of an indiarubber band, and lay the vagus on them. Set the drum off (at slow speed). After a dozen leart-beats liave been recorded, stimulate the vagus for two or three seconds by opening the short-circuiting key. If the nerve is active, the heart will be slowed, weakened, or stopped. In the last case the lever will trace an unbroken straight line; but even if the stimulation is continued the beats will again begin.

S. Stimulation of the Junction of the Sinus and Auricles.-After a sufficient number of the observations described in 7 have been taken with varying time and strengtl of stimulation, take the writingpoints off the drum, apply the electrodes directly to the crescent at the junction of the sinus venosus with the right auricle, and stimulate. The heart will be affected very much in the same way as by stimulation of the vagus, except that during the actual stimulation its beats may be quickened and the inhibition may only begin after the electrodes havc been removed (Fig. 59, p. I44).

9. Effect of Muscarine (or Pilocarpine) and Atropine.

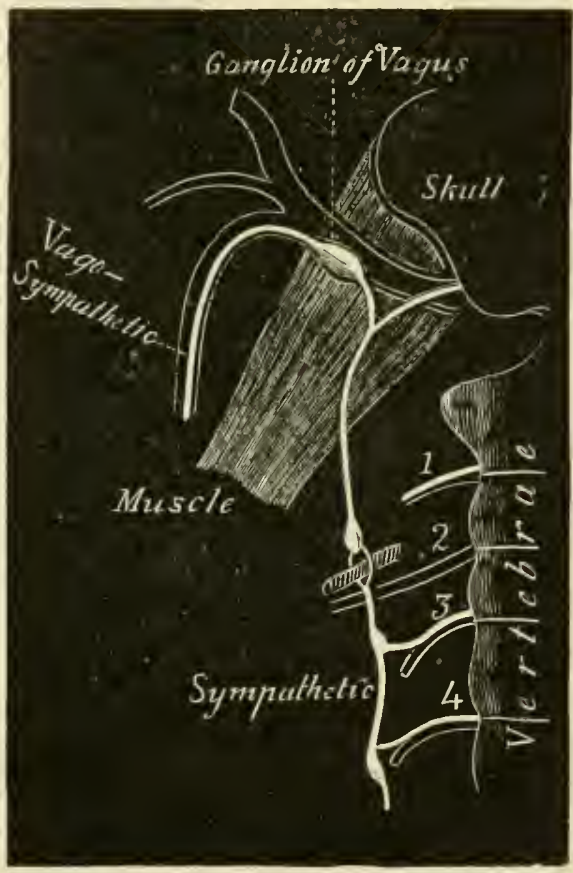

Fig. 8o.-Relation cF the Sympatiletic TO THE VAGUS IN THE Frog.

I, 2, 3, 4 are spinal nerves.

- Paint on the sinus veno-

sus with a small camel's-hair brush a very dilute solution of muscarine (or of pilocarpine). The heart will soon be scen to beat more slowly, and will ultimately stop in diastole. Now apply a dilute solution of sulphate of atropine to the sinus. The heart will again begin to beat. Stimulation of the vagus will now cause no inhibition of the heart, because its endings have been paralyzed by atropine. (Muscarine or pilocarpine has also been applied to the heart, but it could be shown by a separate experiment that atropine by itself has the same effect on the vagus endings-p. I 50 ).

Io. Stannius' Experiment.-Pitl a frog. Expose the heart in the 
way described under $2(p, 177)$. Ligature the franum with a fine silk thread, and use the thread to manipulate the heart. With a curved needle pass a moistened silk thread between the aorta and the superior vena cava, and tic it round the junction of the sinus and right auricle (Fig. 74 ). The auricles and ventricle stop beating as soon as the ligature is tightened. The sinus venosus goes on beating. Now separate the ventricle from the rest of the heart by an incision through the anriculo-ventricular groove, or tie a second ligature in the groove. The ventricle begins to beat again, the auricle remaining quiescent in diastole (p. I5I). Occasionally both auricle and ventricle, or only tho auricle, may begin to beat.

11. Stimulation of Cardiac Sympathetic Fibres in the Frog.(1) In the vago-sympathetic after the inhibitory fibres have been cut out by atropine.-Arrange everything as in 7 (p. 182). Assure yourself. by stimulating the vagus, that it inhibits the heart, and take a

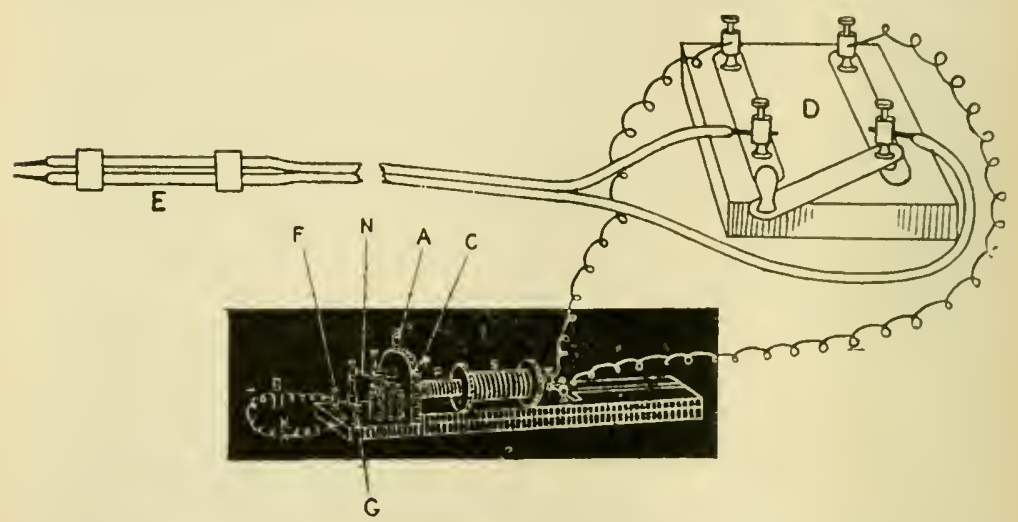

Fig. 8i.-ArRangement of Induction Machine for Tetanus.

$\mathrm{B}$, battery ; $\mathrm{K}$, simple key ; $\mathrm{P}$, primary coil ; $\mathrm{S}$, secondary coil ; $\mathrm{A}$, $\mathrm{C}$, binding screws to be connected with battery for single shocks; $F, G$, binding screws for tetanizing current; $N$, Neef's hammer; $D$, short-eireuiting key in secondary; $\mathrm{E}$, electrodes. $\mathrm{D}$ and $\mathrm{E}$ are drawn to a much larger scale than the rest of the figure.

tracing cluring stimulation. Then paint a dilute solution of atropine on the sinus. Stimulation of the vagus, which is really the vagosympathetic (sce Fig. 8o), will now cause, not inhibition, but augmentation (increase in rate or force, or both), since the cndings of the inhibitory fibres have been paralyzed by atropine. The strength of the stimulating current required to bring out a typical augmentor effect is greater than that needed to stimulate the inhibitory fibres. Take a tracing to show augnentation produced by stimulating the nerve.

(2) By direct stimulation of the cervical sympathetic.-Make the same arrangements as in I (I), but, instead of isolating the vagus, dissect out the sympathetic on one side in the manner described in 6 (2) (p. I 8 I), and do not apply atropine to the heart. Lay the upper (cephalic) end of the sympathetic on very fine and well-insulated electrodes, and stimulate (Fig. 64, p. 151). (To insulate clectrodes the points may be covered witl melted paraffin. When the paraffin 
has cooled, a narrow groove, just sufficient to lay bare the wires on the upper side, is made in it, and the nerve is laid in this groove.)

Experiments 7, 1 I (I) and II (2) will be rendered more cxact by connecting a second electro-magnetic signal with a Pohl's commutator without cross-wires (Fig. S2) $_{2}$, in such a way that the circuit is interrupted at the instant when stimulation begins.

12. The Action of Inorganic Salts on Heart-muscle.-Exposc and remove the heart of a tortoise or turtle (p. I8o). Cut off the apical twothirds of the ventricle by an incision parallel to the auriculo-ventricular groove. By a second parallel cut remove a ring of tissue 2 or 3 millimetres wide from the upper end of this portion of the ventricle. Divide the ring at opposite ends of a diameter, so as to form two strips. Tic a fine silk thread to cach end of one strip. Attach one of the threads to the short limb of a glass rod bent at right angles, so that it can be immersed at will in a beaker. The other end of the rod is fixed in a holder sliding on a stand. Attach the second thread to the short arm of a counterpoised lever arranged to write on a

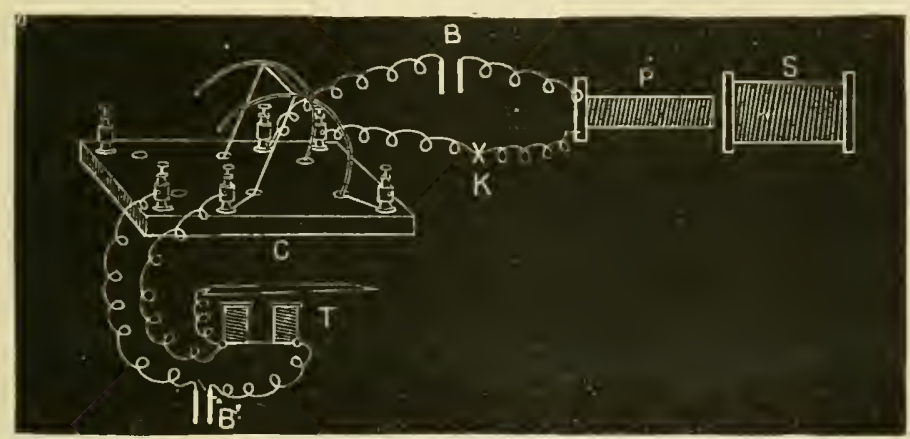

Fig. 82.-ARRANGEMENT FOR RECORDING THE BEGINNING AND END OF StimLlation.

C, Pohl's commutator without cross-wires; B, battery in circuit of primary coil $\mathrm{P}: \mathrm{B}^{\prime}$, battery in circuit of electro-magnetic signal $\mathrm{T}: \mathrm{K}$, simple key in primary circuit; $\mathbf{S}$, secondary coil. When the bridge of the commutator is tilted into the position slown in the figure, the primary circuit is closed and the circuit of the sigual broken.

slowly-moving drum. If the strip is still beating, wait till the contractions have ceased; then

(I) Immerse the strip in a beaker filled with 0.7 per cent. solution of sodium chloride. After a time it begins to beat rhythmically. The contractions become rapidly stronger, and then after a while diminish, and gradually cease. The tone or tonus of the strip is diminished by the solution.

(2) Arrange the other strip in the same way, and immerse it in a solution of calcium chloride (about I per cent.) isotonic with the sodium chloricle solution used in (I). If the strip is contracting, the contractions will cease. Rhythmical contractions will not appear as in the solium chloride solution. The tone of the strip may be increased.

(3) Remove most of the calcium chloride solution from the beaker, and fill it up with 0.7 per cent. sodium chloride solution. The rhythmical contractions will appear after a longer or shorter latent 
period, and will be stronger and last for a longer time than in the sodintn chloricle solution alone.

(4) Immerse a fresh strip in a solution containing sodium chloride $\left(0^{\circ} 7\right.$ per cent.). calcium chloride ( $0^{\circ} 025$ per cent.), and potassium chloride (0.03 per cent.) (a modified Ringer's solution). A longer series of rhythmical contractions will be obtained than in either ( $I$ ) or (3). That this is not due to the potassium chloride acting alone can be shown by immersing a strip in a solution of potassium chloride (about $0^{\circ} 9$ per cent.) isotonic with the solium chloride solution used in (I). No contractions will be caused.

13. The Action of the Mammalian Heart.-Inject under the skin of a (log (preferably a small one) I c.c. of a 2 per cent. solution of morphine hydrochlorate for every kilo of body-weight. As soon as the morphine has taken effect (in 15 to 30 minutes, but, better, after an hour), fasten the animal back (lown on a holder (as in Fig. I 29, 1). 288), pushing the mouth-pin behind the canine teeth and screwing the nut home. ${ }^{*}$ In the meantime select a tracheal cannulat of suitable size, and get ready instruments for dissection-one or two pairs of artery-forceps, a pair of artery-clamps (bulldog pattern), two or three glass cannulae of various sizes for bloodvessels, ten strong waxed ligatures, sponges, hot water, a towel or two, and a pair of bellows to be connected with the tracheal cannula when the chest is opened. Arrange an incluction-coil and electrodes for a tetanizing current (Fig. 8 I, p. 184). With scissors curved on the flat clip away the hair from the front of the neck. Put the hair carefully away, ancl remove all the loose hairs with a wet sponge so that they may not get into the wounds. Give ether, or pour into the stomach by a tube 5 c.c. of a 0.5 per cent. solution of chloroform in ro per cent. alcohol per kilo of bodyweight, diluted before administration with 3 or + volumes of water (Gréhant's method).

To put a Canmula in the Trachea.-The ladir having been clipped in the middle line of the neck and the skin shaved, a mesial incision is to be made, beginning a little below the cricoid cartilage, which can be felt with the finger. The trachea is then cleared from its attachments by forceps or a blunt needle, and tivo strong ligatures are passed beneath it. A single loop is placed on each of these but is not drawn tight. Raising the trachea by means of the upper ligature, the student makes a longitudinal incision through two or three of the cartilaginous rings, inserts the cannula, and ties the

* A simple but efficient and convenient holder for a dog may be easily constructed as follows. Take a board of the length required ( $2 \frac{1}{2}$ to 5 feet. according to the size of the (log). At one end fasten two short upright wooden pins, with a clear space of 4 to 6 inches between them. These are pierced from side to side with four or live holes at different heights. An iron pin passes behind the eanine teeth of the animal through two corresponding holes in the uprights, and the muzzle is tied over this by a cord which secures the head. For a large dog an upper pair of holes is used, for a small dog a lower pair. The feet are fastened by cords to staples inserted into the sides of the board, the fore-legs being drawn tailwards for all operations on the neck or head, headwards for operations on the thorax. A rabbit-holder can be made in exactly the same way.

$\dagger \Lambda$ tracheal cannula is easily made by heating a piece of glass tubing, about 6 inches long, a short distance from one end, and clrawing it out slightly so as to form a 'neck.' The tubing is then bent about its middle to an obtuse angle, and the end next the neck is ground obliquely on a stone. The diameter of the cannula should be about the sanic as that of the trachea, in to which it is to be inserted by its oblique end. 
lower ligature firmly around its neck. The upper ligature can now be withdrawn.

Clip off the hair on each side of the sternum. Make an incision on each side through the skin and down to the costal cartilages about 2 inches from the edge of the breast-bone, and long enough to expose about four costal cartilages (say, 3 rd to 6 th). With a curved needle pass waxed ligatures round the cartilages, and tie firmly to compress the intercostal vessels. The bellows should now, or carlier if any symptoms of impeled respiration have appeared, be connecter with one end of the horizontal limb of a glass $T$-piece, the other end of which is similarly connected with the tracheal cannula. The stem of the $T$-piece is provided with a short piece of rubber tubing, which, when artificial respiration is being carried on, is to be alternately closed and opened-closed during inflation of the lungs, and opened when the air is to be allowed to escape from them. Or a screw-clamp may be adjusted on the piece of tubing so that the opening is sufficiently narrow to permit the lungs to be properly inflated when the bellows are compresserl, and yet sufficiently wide to permit easy escape of the air and collapse of the lungs at the end of each inflation. Ether may, when necessary, be administered, by inserting between the $\mathbf{T}$-piece and the tube from the bellows an ether bottle with two tubes passing through the cork to within an inch or two of the ether. If the camnula has a side-opening, as is usually the case with metal cannulæ, the $T$-piece may be dispensed with. One student should take sole charge of the artificial respiration, which ought to be begun as soon as the chest has been opened, and continued at the rate of about twenty inflations per minute. The costal cartilages are rapidly cut through with strong scissors just on the sternal side of the ligatures, the artificial respiration being suspended for an instant, as each cut is made, to avoid wounding the lungs. The sternum is clivided at its lower end and turned up. If there is much bleeding a ligature should be tied round its upper end. With a curved needle a ligature is passed below the internal mammary arteries as they approach the sternum. That bone may now be removed, and the heart, enclosed in the pericardium, comes into view. A thread is passed with a suture-needle through each side of the pericardium, which is then stitchecl to the chest-wall and opened.

(a) Note the various portions of the heart, right and left ventricles, right and left auricles, with the auricular appendices. Feel the heart with the hand, and observe that the right ventricle is softer and has thinner walls than the left, and that the auricles are softer than the ventricles. Note how all the parts of the heart harden in the hand during systole and soften during diastole (pp. 78, 82).

(b) Dissect out the vago-sympathetic on one side in the neck of the dog. The guide to the nerve is the carotid artery. These two structures and the internal jugular vein lie side by side in a common sheath. Feel for the artery a little external to the trachea, cut down on it, open the sheath, isolate the vago-sympathetic for about an inch, pass two ligatures under it, tie them, and divide between the ligatures. The peripheral and central ends of the nerve may now be successively stimulated. Stimulation of the peripheral end causes slowing of the heart, or stoppage in diastole. Feel that it softens when it stops. It soon begins to beat again. Stimulation of the central end of the vago-sympathetic may or may not cause inhibition. If it cloes, expose the other vago-sympathetic, clivide it, and repeat the stimulation of the central end. There will 


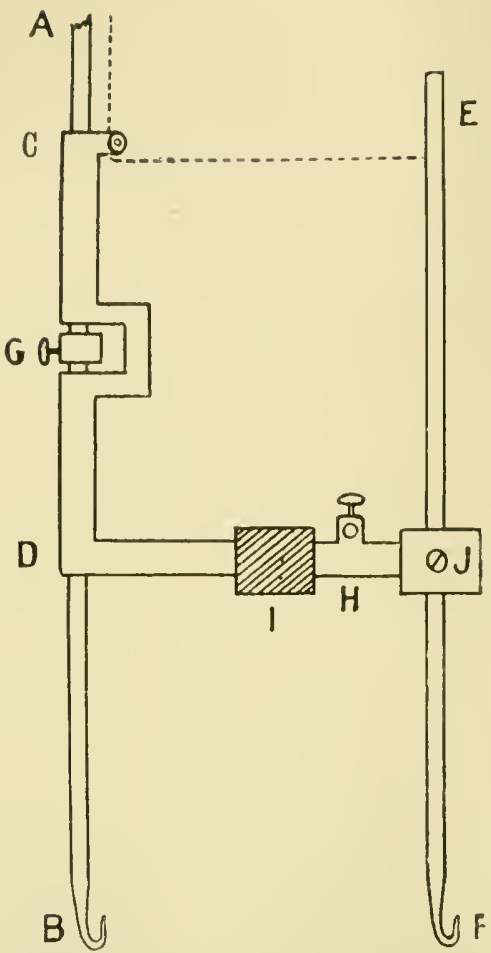

F1g. 83. MYOCARDICIRATH OF ADAM AvD Ros (MODIFED By CUSHNY AND MATtIEWS).

$A B$, a perpendicular rod descending f:o n a universal joint. which is not shown in the figure: (1), a brass shath, moving easily on the o.l, an! hearing o: its upper end an ivory puilev, and at its lower end a horizontal har, which is inter. rupted by a plate of hard rubber, I. The per. pendicular rod EF moves on the horizontal $i \mathrm{a}$. by the hinge-joint, J. E: is hooked at o:ne cud for attachment to the heart, and bored at the other for a thread which, passing over the pulley at $C$, passes through the universal joint a di moves a writing lever not shown in the figure. $C D$ is prevented from moving up $A B$ bv a ring of brass, $\mathrm{F}$, which is screwed to $\mathrm{AB}$, b.1t is not attached to $\mathrm{CD}$; the hook $F^{*}$ can therefore inove to and from $A B$, and can rotate round $\mathrm{i}$ :. whil. it c nno: move up or down. The hooks Fand is are insulated from each other by the hard rublser, 1 . $\mathrm{II}$ is a binding post thro:igh which, and Ihro glt ano her connected with $A$, induction siocks may be sent at will through the portion of the beart lying between the hooks. now be no inhibition of the heart. Incidentally it may be seen that stimulation of the central cnd of the vago-sympathetic causes strong, though, of course, with opened chest, abortice, respiratory movements.

(c) Pith a frog (brain and cor(l), dissect out the sciatic nerve on one side up to the sitcral plexus. Cint off the whole leg. Drop the cut end of the nerve on the heart, and holel the preparation so that the nerve touches the heart also by its longituclinal suriace. It each cardiac beat the nerve is stimulated by the atction current (Chap. XI.), and the muscles of the $\operatorname{leg}$ contract.

(d) Raise the boird so that the hearl of the animal is down and the hinclfeet np, and note whether there is any effect on the action and filling of the heart. Repeat the observation with hcald up and fect lown.

(e) Compress the arta with the fingers, and observe the effect on the regrec of dilatation of the various cavities of the heart. Repeat the experiment with the inferior vena caval, and compare the results.

(f) Smloke a drum. Insert the liooks of the myocaroliograph ( into the rentricle, taking care not (o) penctrate decply jutotlo wall. Ariange the lever to write on the drum. While a tracing is being taken stimulate the peripheral end of the vagus. Unlook the cardiogräpl.

(g) Stop the artificial 
respiration, and observe the changes which take place in the auricles and ventricles, comparing particularly the riglit side of the heart with the left. Before the leart has stopped beating, recommence the artificial respiration

(h) Connect a cylinuler of oxygen with a good-sized rubber catheter,

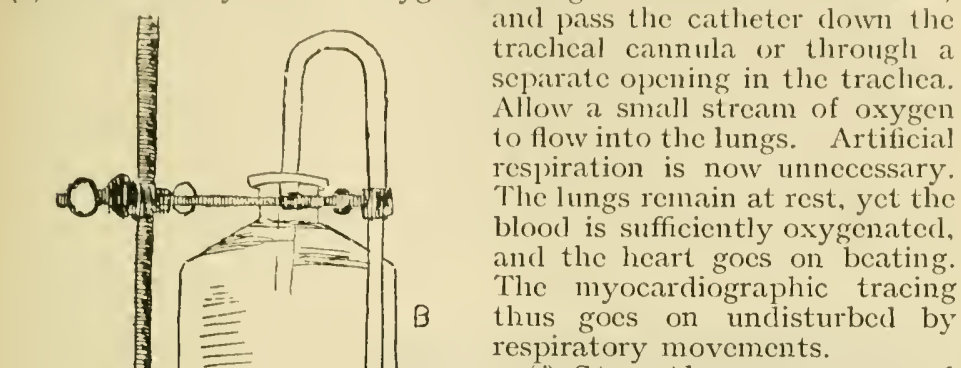
and pass the catheter town the separate opening in the trachea. Allow a small stream of oxygen to flow into the lungs. Artificial respiration is now unnecessary. The lungs remain at rest, yet the blood is sufficiently oxygenated, and the heart goes on beating. thes gocs on respiratory movements.

(i) Stop the oxygen, and resume the artificial respiration. Make a small penetrating wound with a scalpel in the left ventricle. Obscrie the

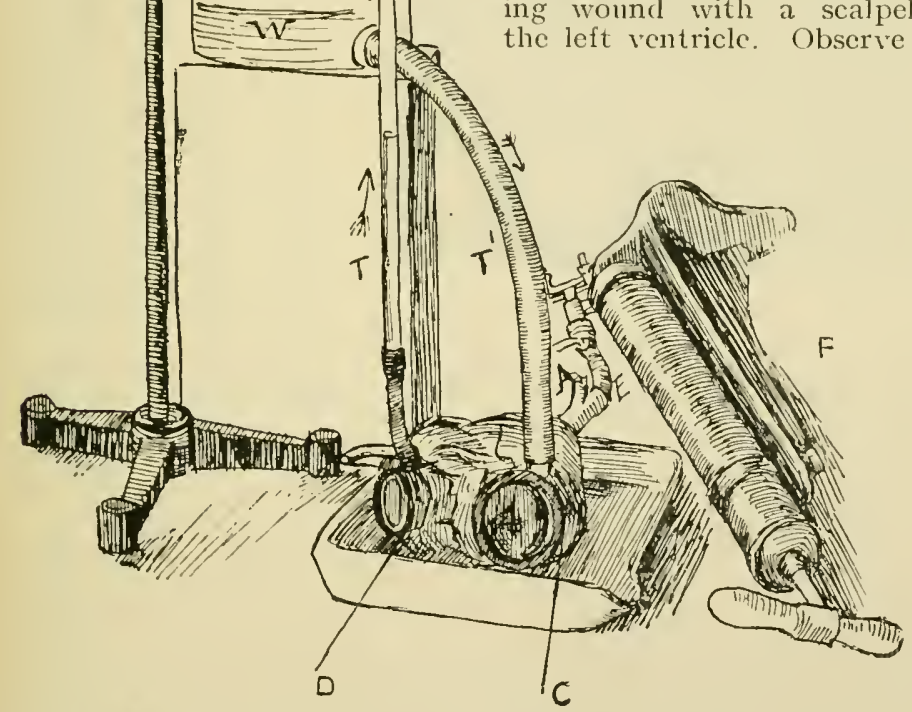

Fig. 84.-Arrangement to illustrate Action of Cardac Valves in the HEART OF AN OX (GAD).

$\mathrm{C}$, glass window in left auricle: $\mathrm{D}$, window in aorta; $\mathrm{E}$, tube inserted through apex of heart into left ventricle and connected with pump P; A, side tube on $\mathrm{E}$, through which wires are connected with a tiny incandescent lamp in the ventricle; $I V$, water in bottle $B$; T, 'T', tubes.

course of the hæmorrhage, and note cspecially the difference in systole and diastole.

(j) Lay the electrodes on the heart, and stimulate it with a strong interrupterl current. The character of the contraction soon becomes profoundly altered. Shallow, irregular contractions flicker over the 
surface, with a kind of simmering movement suggestive of a boiling pot (clclirium cordis, fibrillar contraction). Now kill the animal by stopping the artificial respiration. Obscrve how long the heart continues to beat, and which of its divisions stops last.

(k) Make a dissection of the cervical sympathetic up to the superior cervical ganglion, and down through the inferior cervical ganglion to the stcllate or first thoracic ganglion. Make out the annulus of Vicussens and the cardiac sympathetic (accelerator) branches going off from the annulus or the inferior cervical ganglion to the cardiac plexus (Fig. 62, p. I. 8 ).

I. Action of the Valves of the Heart.-(r) Study the action of the valves of the ox-heart, connected with the pump $P$ and bottle $\mathrm{B}$ in the artificial scheme, as shown in Fig. 84 . The cavity of the heart is illuminated by means of a small electric lamp. the wires of which pass in at $\Lambda$. When the piston of the pump is pushed down,

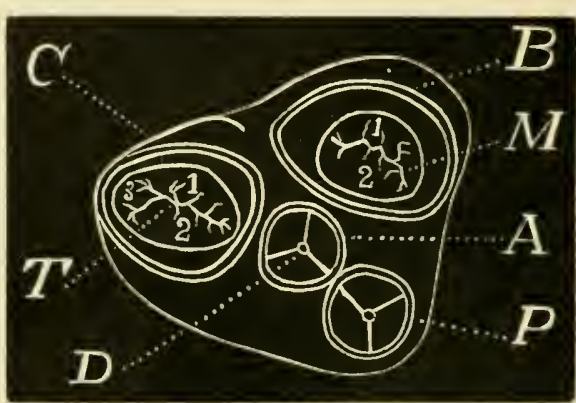

F1G. 85.-Diagrain of Valves of the Heart.

The valves are supposed to be viewed from above, the auricles having been partially removed. $A$, aorta with semilunar valve : $I$, posi. tion of corpora Arantii ; $P$, pulmonary artery ; $B$, wall of left auricle; $M$, mitral valve, with $I$ and 2 , its posterior and anterior segments; $C$, wall of right auricle : $T$, tricuspid valve, with $r$, its posterior ; 2 , its anterior ; and 3 . its external segment. reflected around the great vessels at the base of the heart. Distinguish the pulmonary artery, the aorta. the superior and inferior venæe cavæ, and the pulmonary veins. The trachea and portions of the lungs may also be attached. If so, remore them carefully without injuring the heart.

(b) Take two wide glass tubes, drawn slightly into a neck at one end. One of the tubes should be about Io $\mathrm{cm}$. long, and the other about $50 \mathrm{~cm}$. Tic the short tube A firmly by its neck into the superior vena cava, the long tube $B$ into the pulmonary artery. Ligature the inferior vena cava. Connect A by a small piece of rubber tubing with a funnel supported in a ring on a stand. Pour water into the funnel till the right side of the heart is full. It will escape from the left azygos vein, which must be ticd. Put on any additional ligatures that may be needed to render the heart watertight. Support B in the vertical position by a clamp. Fill the 
funnel with water, and it will rise in B to the same level as in the funnel. Now compress the riglt ventricle with the hand, and the water will rise ligher in B. Relax the pressure, and notice that the water remains at the higher level in $B$, being prevented by the semilunar valves from flowing back into the ventricle. By alternately compressing the ventricle and allowing it to relax, water can be pumped into $B$ till it escapes from its upper end, and if this is so curvel that the water falls into the funnel, a 'circulation' which imitates that of the blood can be established. Note that during the pumping the sinuses of Valsalva, behind the semilunar valves at the origin of the pulmonary artery, become prominent.

(c) Take out B and tear ont one of the segments of the semilunar valve. Replace $B$, and notice that while compression of the ventricle has the same effect as before, the water no longer keeps its level on relaxation, but regurgitates into the ventricle. This illustrates the condition known as insuffciency or incompetence of the valves. But if the injury is not too extensive, it is still possible, by more vigorously and more rapidly compressing the heart, to pump water into the funnel. This illustrates the establishment of compensation in cases of salvular lesion.

(d) Now remove both tubes. Tie the pulmonary artery. Cut away the greater part of the right auricle. Pour water into the auriculo-ventricular orifice, and notice that the segments of the tricuspicl valve are floated up so as to close the orifice. Invert the heart, and the ventricle will remain full of water. Open the right ventricle carefully, and study the papillary muscles, and the chordæe tendinex. noting that the latter are inserted into the lower surface of the segments of the tricuspid valve, as well as into their free edges.

(e) Repeat $(b),(c)$, and $(d)$ on the left side of the heart, tying tube $B$ into the aorta as far from the heart as possible, and $A$ into the left auricle.

(f) Separate the aorta from the left ventricle, cutting wide of its origin so as not to injure the semilunar valves, and tic a short wide tube into its distal end. Fill the tube with water, and notice that the valves support it. Cut open the aorta just between two adjacent segments of the valve, and notice the pockets behind the segments. and how they are related to each other, and connected to the wall of the ressel.

I5. Sounds of the Heart.-(a) In a fellow-student notice the position of the cardiac impulse, the chest being well exposed. Use both a binaural and a single-tube stethoscope. Place the chest-piece of the stethoscope over the impulse, and make out the two sounds and the pause. (b) With the hand over the radial or brachial artery. try to determine whether the beat of the pulse is felt in the period of the sounds or of the pause. (c) Listen with the stethoscope over the junction of the second right costal cartilage with the sternum, and compare the relative intensity of the two sounds as heard here with their relative intensity as heard over the cardiac impulse.

I6. Cardiogram.- Smoke a drum, and arrange a recording tambour and a time-marker beating half or quarter seconds to write on it (Fig. 76, p. I79). Apply the button of a cardiograph (Fig. 22, p. 82) over your own cardiac impulse, and fasten it round the body by the bands attached to the instrument. Connect the cardiograph by an indiarubber tube with a recording tambour (Fig. 86). Set the drum off at a fast speed, take a tracing, and varnish it. Compare with Fig. 23 (p. 83), and if the tracing is sufficiently typical, as is 
often not the case witl luman cardiograms, measure out the time. value of the varinus events in the carcliac revolution.

For the cardiograph, a small glass funnel, or thistle tube, the stem of which is connected with the recording tambour. may be substituted, the broad end of the funnel being pressed over the apex-beat.

17. Sphygmographic Tracings.-Attach a .Marcy's sphygmograph (Fig. 31, p. 93) to the arm. Fasten a smokerl paper on the plate D. Apply the pad $C$ of the sphygmograph to the wrist over the point

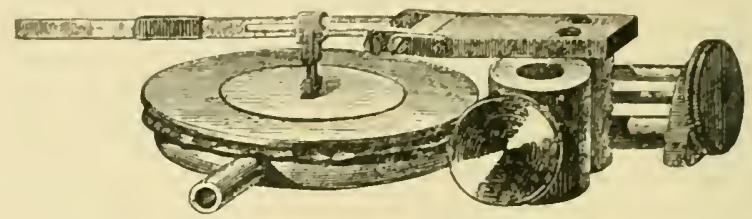

Fig. 86.-.Marey's Tambour.

where the pulse of the radial artery can be most distinctly felt. Adjust the pressure by moving the screw G. The writing-point of the lever $E$ will rise and fall with every pulse-beat. When cverything is satisfactorily arranged, set off the clockwork which moves the plate D. and a pulse tracing will be obtained. Study the changes which can be produced in the pulse curve- $(a)$ by altering the position of the body (sitting, standing, and lying down) : (b) by

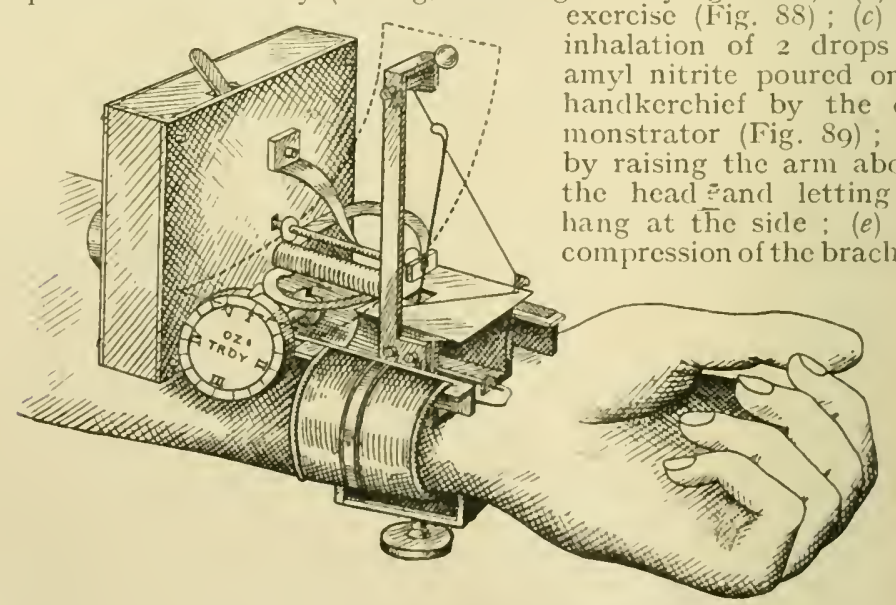

Fig. 87.-Dudgen's SPHYgirograpir.

artery at the bend of the elbow; $(f)$ by altering the pressure of the pad. Varnish the tracings after marking on them the conditions under which they were obtained.

A Duclgeon's splyygmograph (Fig. 87) may also be employed. Or a small glass funnel or thistle tube connected with a recording tambour may be pressel over the carotid artery. The lever of the tambour writes on a drum, on which at the same time half or quarter scconds are markel by an electro-magnetic signal. 
18. Venous Pulse Tracing from the Jugular Vein.-Arrange a recording tambour to write on a drum. Connect the tambour with the stem of a small glass thistle-tube (or with a small metal cup) by a picce of narrow rubber tubing, and apply the cup-shaped end of the thistle-tube over the right jugular bulb of a fellow-student. This lies about 1 inch external to the riglut sterno-clavicular articulation, and a little above it. The receiver may have to be moved about a little until the best pulsation is obtained. 'The 'patient' should be lying down, the shoulders slightly raised, the head on a pillow and turned slightly to the right, in order to relax the right sterno-mastoid unuscle (Mackenzic).

19. Polygraph Tracings.Arrange the polygraph over the radial artery, as with an ordinary sphygmograph, so that the lever will record the radial pulse when the strip of paper is set moving. If the

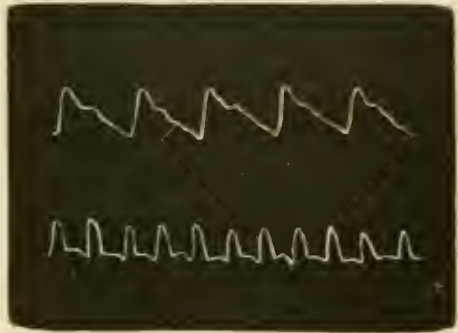

Fig. 88. - EFFECT OF EXERCISE ON the Pulse (Marey).

Upper tracing, normal; lower, after running. instrument has only one tambour, connect the tambour to a receiver or thistle-tube over the jugular bulb, and arrange the writing-point of the tambour immediately below the writing-point connected with the radial. If the polygraph is provided with clockwork to record time, set off the time-marker writing fifths of a second. When it is

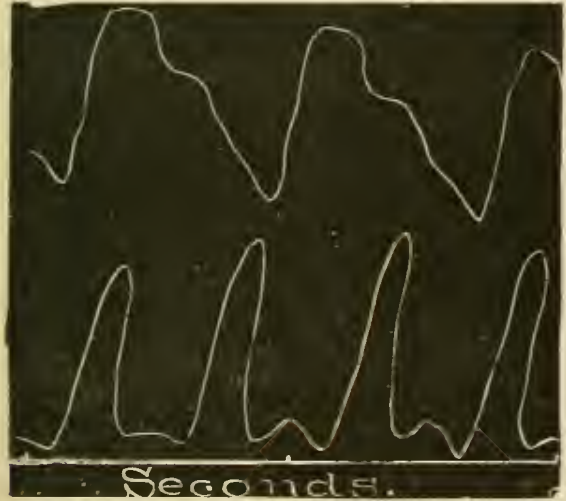

Fig. 89.-Effect of AMrl Nitrite oN the PULSE (MAREY).

Upper tracing, normal ; lower, after inhala. tion of amyl nitrite.

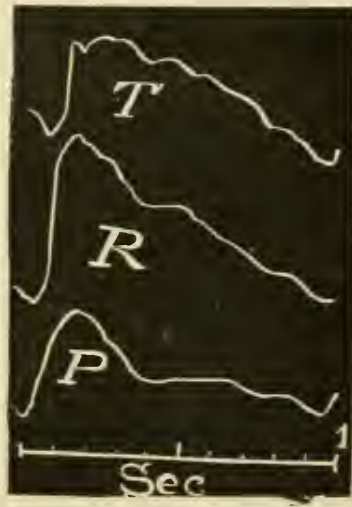

Fig. 90. - Pulse Tracixgs FROM DIFFERENT ARTERIES.

$T$, temporal ; $R$, radial ; $F$, artery of foot (v. Frey).

seen that the writing-points are marking properly, start the clockwork which moves the strip of smoked paper. Repeat the observation with the tambour connected with the apex-beat. Letter the curves as far as possible as in Figs. 55 and: $: 56$ (p. 137) jwithout at present attempting their exact analysis.

If the polygraph has two tambours, simultaneous tracings of the 
rarlial pulse, the jugular pulse, and the cardiac impulse, or of the carotid pulse, the jugnlil pulse, and the apex-beat, may be taken, and other combinations as well. If no polygraph is avilable. a drum may be employed, the tracings being all taken with thistletubes connected with recorfing tambours. The levers of the tambours must be arranged to write on the drum in the same vertical straight line, or, without making the adjustment quite exact, vertical lines of reference may be drawn through each curve, with the drum at rest, indicating the relative positions of the writing-points.

20. Plethysmographic Tracings.-Connect the vessel C (Fig. 47 p. 117 ) with $B$, place the arm in $\mathrm{jt}$, and adjust the indiarubber band to make a watertight connection. Support $C$ so that the arm rests easily within it, and fill it with water at body temperature. Adjust a

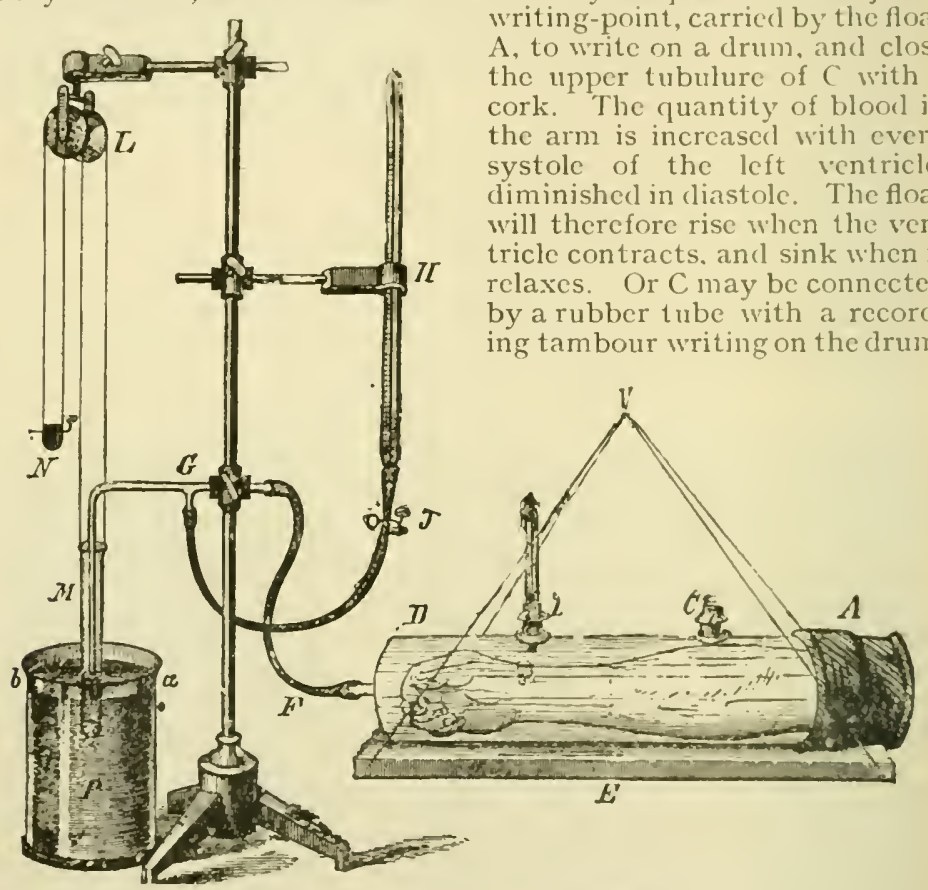

Fig. 91.-Plethysmograph (Mosso).

$M$, balanced test-tube, in communication with $D$. When water passes from vessel $D$ to $M$, or from $M$ to $D, M$ moves down or up, and its movements are re. corded by the writing point $X . M$ is steadied by the liquid in $I$, into which it dips.

No water must get into the tambour, and it is well to insert a piece of glass tubing in the connection between it and the plethysmograph, so that it may be seen when the water is rising too high. A T-picce with a short piece of rubber tubing on the stem should be inserted in the course of the tube learling to the tambour. All adjustments are made with the $T$-piece open, and when a tracing is to be taken the short rubber tube is closed by a clip. Arrange a time-marker to write half or quarter seconds (liig. 76, p. 179). Mosso's arm plethysmograph (Fig. 91) may also be used. 
(I) Take tracings witl the arm (a) horizontal, (b) hanging down.

(2) With the arm lorizontal, take tracings to show the effect (a) of closing and opening the fist inside the plethysmograph ;* (b) of applying a tight bandage round the arm a little way above the indiarubber band $1(c)$ of inhaling 2 drops of amyl nitrite.

Instead of the arm plethysmograph a small plethysmograph to loold a finger may be employed. It consists of a glass tube clrawn ont at one end. The wide end is provided with a rubber collar. The narrow end is connected by a small rubber tube with a very small and sensitive recording tambour, a $\mathbf{T}$-piece being inserted on the connection as before. With the $\mathbf{T}$-piece closed fill the tube with water. Then, holding up the wide end of the tube, the tip of the finger is put in so as just to close the tube. The $\mathbf{T}$-piece is then raised and opened, and the finger pushed in as far as it will go. The collar must fit the finger so as to form a watertight joint. Now get the proper pressure in the tambour by blowing into the $\mathrm{T}$-piece, and close the clamp. A time-tracing can be taken as before.

21. Pulse-rate.-(1) Count the radial pulse for a minute in the sitting, supine, and standing positions. Use a stop-watch, setting it off on a pulse-beat and counting the next beat as one. Make three observations in each position.

(2) Count the pulse in a person sitting at rest, and then again in the sitting position immediately after active muscular exertion. Note how long it takes before the pulse-rate comes back to normal.

(3) Count the pulse in a person sitting at rest. Repeat the observation while water is being slowly sipped, and note any change.

(4) With one hand over the thorax of a rabbit, count its pulse. Then notice the effect (a) of suddenly closing its nostrils, $(b)$ of bringing a small piece of cotton-wool sprinkled with ammonia or chloroform in front of the nose (reflex inhibition of the heart).

22. Blood-pressure Tracing.- (a) Put a dog under morphine (p. 55). Set up an incluction machine arranged for an interrupted current (Fig. 8 I, p. I 84 ). Fill the $U$-shaped manometer tube (if this has not already been done) with clean mercury to the height of Io to $12 \mathrm{~cm}$. in each limb. If the float tends to stick, half an inch of oil may be put above the mercury in the distal (straight) limb before putting in the float. But where the mercury is clean and dry, and the size of the float properly adjusted to that of the tube, this is not necessary, and is to be avoided. Then, tilting the tube carefully, fill the proximal limb (i.e., the limb which is to be connected with the bloodvessel) with a saturated solution of sodium carbonate or a half-saturated solution of magnesium sulphate, or what is better for most purposes, a 2 per cent. solution of sodium citrate. This is easily done by means of a pipette furnished with a long point. Now attach a strong rubber tube to the proximal end of the manometer. and fill it also with the solution. All air must be got out of the manometer and its connecting-tube. Raise the end of the rubber tube and blow into it, so as to cause a difference of about $10 \mathrm{~cm}$. in the height of the mercury in the two limbs of the manometer, and, without releasing the pressure, clamp the tube with a pinchcock or screw clamp (Fig. 34, p. IO I).

Now smoke a drum, and arrange the vrriting-point of the manometer-float so that it will write on it. Suspend a small weight by a piece of silk thread from a support attached to the stand of the

* Closing the fist causes a fall in the curve, i.e., a diminution in the volume of the arm. On opening the hand, the curve regains its level. 
drum, so that it hangs down ontsicle of the writing-point of the manometer-float and always kecps it in contact with the smoked surface without unduc friction. Or a piece of glass rol drawn out to a fine threal in the blowpipe flame answers very well. Below the writing-point of the float, and in the same vertical line with it, adjust the writing-point of a time-marker beating seconds (Fig. 76 , p. 179).

Next, fasten the animal on a holder. back down. Give ether and insert a tracheal cannula (p. 186). (The tracheal cannula is not absolutely required for the experiment, but it is convenient, as the animal is more under control, and artificial respiration can be begun at any moment, sliould this be necessary.) Insert a glass cannula, armed with a short piece of rubber tubing, into the

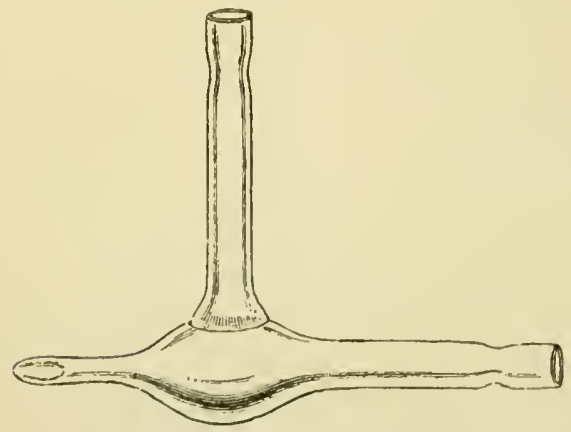

Fig. 92. -THREl-IVAY CaNidul.

central (cardiac) end of the caroticl artery (p. 55). Leaving the bulldog forceps on the artery, fill the cannulit and tube with the sodium citrate or one of the other solutions. Slip the rubber tube over a short glass connecting-tube. Fill this also with the solntion, and connect it with the manometer-tube, sceing that both are quite full of liquid, so that no air may be enclosed.

Where a permanent working place is provided for blood-pressure experiments it is convenient to connect the cannula and manometer with a pressure-bottle containing the sodium citrate solution, and to use a three-way cannula for the bloodvessel (Fig. 92). The cannula

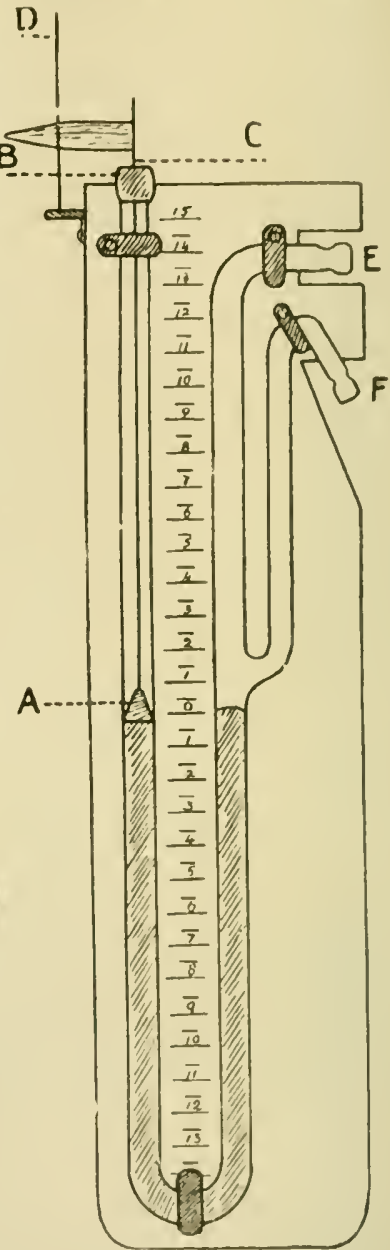

F1(;. 93.-MANOMETIR WIT Side-tube (GUTIIRIE).

$A$, float ; B, collar through which the wire $C$ of the fluat moves; D, vertical wire fixed to manometer - holder, which keeps the writing-point on the drum; E, limb of manometer connected with cannula, with its side-piece, F. has a bulbous enlargenent. which hinders clotting. The end of the cannula is connected with the tube from the pressure-bottle, which 
is closed by a clip, and the side-tube is connected with one limb, E, of the manometer slown in Fig. 93. E is itself provided with a side-tube, F, armed with a short piece of rubber tubing. The cammula does not require to be filted with liquid before being inserted into the artery. By opening $F$ and releasing the clip on the tube from the pressure-bottle the canmula and the tube connecting it with the manometer can be filled, and any blood-clots can be easily washed out in the course of an experiment. Before the bullklog forceps is taken off the artery to obtain a blood-pressure tracing, I: must be closed, and the clip on the tube from the pressure-bottle opened. The bottle is attached to a strong cord passing over a pullcy, by which it is raised to a height sufficient to balance approximately the pressure in the artery. The tube to the pressure-bottle

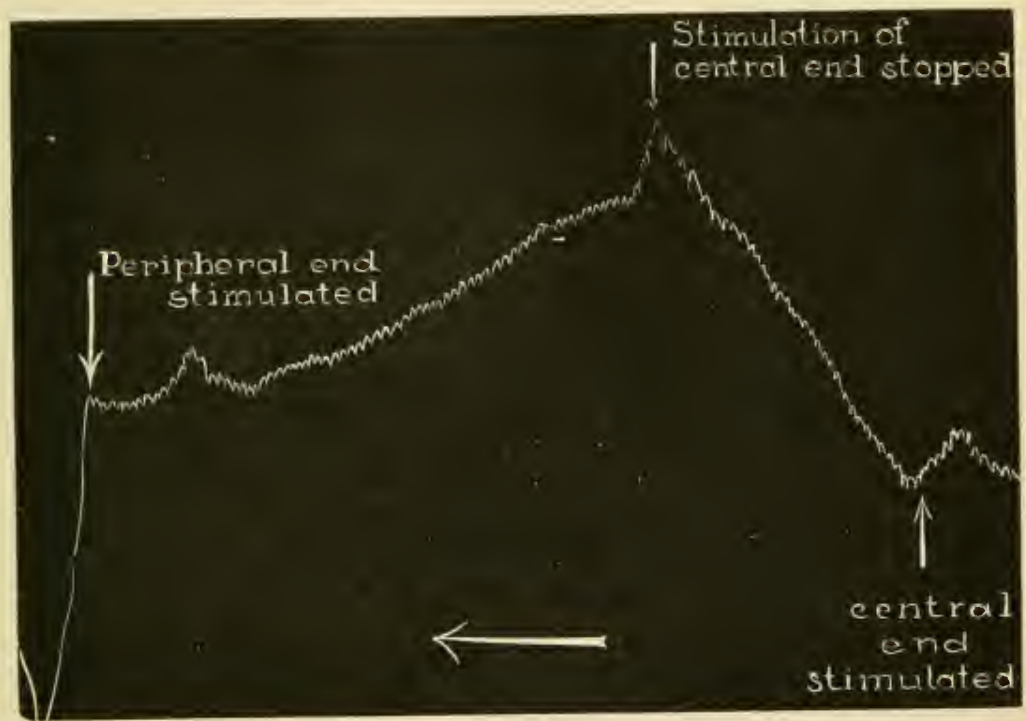

Fig. 94.-Blood-pressure Tracing fron a Dog: Stimulation of Central AND PERIPHERAL Ends of VAGUS.

The other vagus was intact. Stimulation of the peripheral end caused stoppage of the heart and a marked fall of pressure. Stimulation of the central end produced a great rise of pressure, with, perhaps, a slight acceleration of the heart.

is then clipped. If no manometer with side-tube is available, a $T$-picce can be inserted in the connection between the cannula and the manometer, and the cannula can be washed out through this.

Now take the bulldog forceps off the artery, and allow the drum to revolve at slow speed. The writing-point of the manometer-float will trace a curve showing an elevation for each heart-beat, and longer waves due to the movements of respiration.

(b) Isolate the vago-sympathetic nerve in the neck. Ligature doubly, and cut between the ligatures. Stimulate the peripheral (lower) end; the heart will be slowed or stopped, and the bloodpressure will fall. Stimulate the central (upper) end; there may be 
inhibition of the lieart or acceleration, and the pressure may fall or rise (p. 15f).

(c) Expose and divide the other vago-sympathetic while a tracing is being taken. Again stimulate the central end of the nerve and observe whether there is any effect.

(d) Expose the sciatic nerve in one leg, as follows: The leg having been loosened from the holder, the foot is seized by one liand and lifted straight $u p$, so as to put the skin of the thigh on the stretcli. An incision is now made in the middle line on the posterior aspect of the thigh, through the skin and subcutaneous tissne. The muscles are separated in the line of the incision with the fingers, and the sciatic nerve comes into view lying decply between them. Place a double ligature on it, and divide between the ligatures. Stimulate the upper (central end); the blood-pressure probably rises, and the heart may be accelerated. Stimulate the periplieral end of the nerve; there is little change in the blood-pressure and none in the rate of the heart.

(e) Note, incidentally, that stimulation of the central end of the sciatic or the upper (cephalic) end of the vago-sympathetic may cause increase in the rate and depth of the respiratory movements. Dilatation of the pupil is also causecl by stimulation of the upper cnd of the vago-sympathetic through the sympathetic (pupillo-dilator) fibres that supply the iris.

(f) Again stimulate the peripheral end of one vagus, or of both at the same time, while a tracing is being taken, and see how long it is possible to keep the heart from beating. Sometimes, but rarely, in the dog inhibition can be kept up so long that the animal dics.

(g) Close the tracheal cannula so that air can no longer enter the lungs. In a very short time the blood-pressure curve begins to rise (rise of asphyxia). After some minutes the pressure falls, and finally. when the circulation has stopped completely and the pressure lias become equalized throughout the whole vascular system, a residual pressure of only a few $\mathrm{mm}$. (usually about $10 \mathrm{~mm}$. $\mathrm{Hg}$ ) is inclicated. In order to get the true zero pressure, disconnect the arterial cannula from the manometer, and allow the writing-point to trace a horizontal straight line (line of zero pressure) on the drum (Figs. 72 and 73).

23. Estimation of the Arterial Blood-pressure in Man.-IVith the Erlanger sphygmomanometer estimate the systolic and diastolic pressures in the brachial artery of a fellow-student as clescribed on p. I05. Begin with the observed person in the sitting position.

Compare the results with those obtained on the same artery with any other sphygmomanometer which may be available, especially with one like the Riva-Rocci, with which the systolic pressure is obtained by observing the height of the mercurial manometer at the moment when the pressure in the cuff over the brachial has fallen to the point at which the pulse at the wrist is just obliterated. By compressing rapidly the rubber bulb the mercury is first raised to a height somewhat greater than that necessary to completely obliterate the radial pulse. Then the bulb is kept compressed, and the mercury allowed to fall steadily, the point being noted at which the fingers over the radial just perceive the returning pulse. The observer's left hand may be used for palpating the pulse, and the right for working the bulb. Repeat the observations with the person standing up and lying down. Investigate the effect of inuscular exercise on the blood-pressure. 
24. The Influence of the Position of the Body on the Bloodpressure. - Inject into the rectum of a $\log 3$ to + grm. of cliloral hyclate clissolved in a little water. See that it does not run ont again immediately after injection. In ten minutes anesthetize the animal fully with a mixture of equal parts of alcohol, chloroform, and ether (one of the so-called A.C.E. mixtures), or with chloroform, and tic it very securely, back downward, on a board, which can be rotated around a horizontal axis, corresponding in position to the point at which the cannula is to be inserted.* Set up a drum and manometer as in 22 (p. 195), but with a rubber connecting-tube of such length as will allow fiec rotation of the board. Put a cannula in the trachea. Insert a cannula into the central end of the carotid artery at a point immediately above the axis of rotation of the board, and connect it with the manometer.

(a) Take a blood-pressure tracing with the board horizontal.

(b) Whilst the tracing is being taken, rotate the board so that the position of the animal becomes vertical, with the feet clown. Mark on the tracing the moment when the change of position takes place. The pressure falls. Replace the dog in the horizontal position. The manometer regains its former level. Now rotate the board, till the animal is again vertical, but with feet 11) and head down, and observe the effect on the blood-pressure. The respiratory variations in the pressure are usually greater with fect down than with head down. Notice in both cases whether there is any change in the rate of the heart.

(c) Taks the board off the stands, lay it on a table, expose the femoral artery, and insert a cannula into it. Shift the axis so that it now lies below this cannula. Replace the board on the stands, and repeat $(a)$ and $(b)$. The fall of pressure will now take place in the head-down position. $\dagger$ In the feet-down position (with the cannula in the femoral artery) a rise of pressure in general takes place. But sometimes this is very small, and lasts only a few seconds, being succeeded by a fall, during which the heart-beats on the tracing are much weaker than before, since enough blood is not reaching the heart to enable it to maintain the pressure. In the feet-down position see whether the corneal reflex can be got. If not, as is likely, turn the animal into the head-down position. The reflex may now soon be obtained, and it may again disappear on putting the animal in the feet-down position. If the chloroform anæsthesia is light the reflex may not be abolished in the feet-down position, although strong respiratory

* A simple arrangement for this purpose is a board with a number of staples fastened in pairs into its lower surface, so that an iron rod can be pushed through any pair, and form a horizontal axis at right angles to the length of the board. The dog having been tied down, the rod is pushed through the pair of staples corresponding to the position of the cannula in the artery that is to be connected with the manometer. The projecting ends of the rod rest in two ordinary clamp-holders, fastened at a convenient height on two strong stands, whose bases are clamped to the end of a table. The other end of the board is supported by a piece of wood that rests on the floor, and can be removed when the board is to be rotated.

$\dagger$ In 16 dogs the fall of pressure in the carotid in the feet-down position varied from 12 to $100 \mathrm{~mm}$. of mercury; average fall, $44^{\circ} 4 \mathrm{~mm}$. In 12 out of the 16 animals the rise of pressure in the head-down position varied from 2 to $36 \mathrm{~mm}$. ; in I there was no change; in 3 there was a fall of 5 to $24 \mathrm{~mm}$. 
movements may occur, owing to ancmia of the meilulla oblongata.

25. Effects of Hæmorrhage and Transfusion on the Bloodpressure.-Anaesthetize a clog with morphine and ether, and insert a cannula into the trachea. Put a cannula into the central end of the carotid artery and another into the central end of the femoral artery. Then insert a canuula, which should have a piece of incliarubber tubing 2 to 3 inches in lengtl on its wide end, into the central end of the femoral vein on the opposite side. In doing this more care is necessary than in putting a cannula into an artery. Feel for the femoral artery, cut down over it, and with forceps or a blunt neclle separate the femoral vein from it for abont an inch. Fass two ligatures under the vein, and tie a loose loop on eacli. Put at pair of bulldog forceps on the vein between the ligatures and the lieart. Now tie the lower (distal) ligature, and cut one end short. The piece of vein between it and the bullilog forceps is thus clistended with blood, and this facilitates the next step. With fincpointerl scissors make a snip in the wall of the vein. The cannula is now pushed through the slit in the vein, and the upper ligature tied firmly round its neck. By the aid of a pipette, made by drawing a piece of glass tubing out to a long point, the cannulat and rubber tube are then completely filled with $0^{\circ} 9$ per cent. salt solution. Be sure to pass the point of the pipette right clown to the point of the cannula, so as to dislodge any bubble of air that may tend to cling there. Then, holding $1 \mathrm{p}$ the open end of the rubber tube, close it, without allowing any air to enter, by means of a screw clamp or bulldog forceps, or a small piece of glass rod. Connect the cannula in the carotid with a manometer, arranged to write on a lrum as in experiment 22 (p. 195). Take the bulldog off the carotid, and measure the difference in the level of the mercury in the two limbs of the manometer with a millimetre scale.

(I) (a) While a tracing is being taken, draw off about io c.c. of blood from the femoral artery, and observe whether there is any effect on the tracing. Mark on the tracing the moment when the removal of the blood begins and ends.

(b) Repeat $(a)$, but run off abont ioo c.c.* of blood, and let this be immediately defibrinated. Then draw off portions of Ioo c.c.* at short intervals until a distinct fall of blood-pressure has been produced. All the samples of blool should be defibrinated and strained through cheese-cloth.

(2) (a) Now, while a tracing is being taken, inject the whole of the defibrinated blood slowly through the cannula in the femoral vein by means of a funnel supported by a stand at such a height that the blood runs in easily. A pincheock should be put on the tube connecting the funnel and the cannula, and this should be closed before the funnel is quite empty, so as to obviate any risk of air getting into the vein. Of course, the cannula and connecting-tubes must all be freed from air before injection is begun. Again measure the difference in the level of the mercury and compare the pressure with that observed before the first hamorrhage.

(b) Inject into the vein, while a tracing is being obtained, about Ioo c.c. ${ }^{*}$ of $0^{\circ} 9$ per cent. Salt solution heated to $40^{\circ} \mathrm{C}$.. and go on injecting portions of 100 c.c. until a distinct rise of pressure has taken place, kecping a record of the total amount injected, and warking the time of each injection on the curve. 
(c) After an interval of thirty minutes, again measure the heiglit of the meremy in the manometer. Then bleed the dog to eleath white a tracing is being recordecl.

26. The Influence of Albumoses (and Peptones) on the Bloodpressure. - Set up the apparatus for taking a blood-pressure tracing as in cxperiment 22 (1). [95), but omit the induction-coil. Weigh a dog. Weigh out a quantity of WVitte's peptone equivalent to 0.5 grm. for every kilo of body-weight. Dissolve the peptone in abont/ten times its weight of $0^{\circ} 9$ per cent. Salt solution. Anasthetize the $\log$ with morphine and ether or A.C.E. mixture. Insert a cannulit into the trachea. Put cannula into the central end of onc carticl and of one femoral vein (p. 200). Comnect the carotid with the manometer, and the femoral vein with a burette or large syringe containing the peptone solution. Take care that the connecting-tube and canmula are free from air. Now commence to take a blood-pressure tracing, and while it is going on inject the peptone solntion. The pressure falls owing largely to a dilatation of the small arteries through the direct action of the peptone

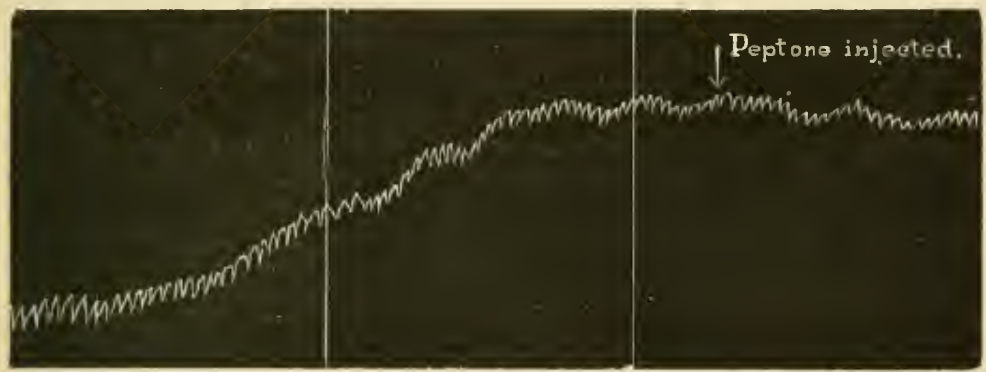

Fig. 95.--Effect of Injection of Peptone on the Blood-pressure IN A Dog.

(To be read from right to left.)

on their muscular tissue or on the endings of the vaso-motor nerves. *

27. Effect of Suprarenal Extract on the Blood-pressure.-Nake the arrangements for a blood-pressme tracing from a $l o g$ as in 22 , p. 195. Put a cannula in the carotid and another in the femoral vein or one of its branches (p. 20o). Expose both vagi in the neck, and pass threads loosely under them. Connect the carotid with the manometer and take a tracing. Then, while the tracing is continued, inject slowly into the femoral vein an amount of watery extract corresponding to about $0^{*} 2 \mathrm{grm}$. of suprarenal, or, what is more convenient, a few c.c. of a solution of adrenalin chloride of the strength of I to 50,000 in 0.9 per cent. sodium chloride solution, the dose depending, of course, on the size of the animal. The blood-

* In 12 logs the blood-pressure always fell, the amount of the fall varying from 81 to $2 \mathrm{Imm}$. of mercury (average, $60 \mathrm{~mm}$.). It sometimes returned to normal in twenty to thirty minutes, but usually required a longer time. In some logs, after the injection of the whole of this amount of peptone, death occurs before there has been any considerable recovery of the pressure. 
pressure rises* owing to constriction of the arterioles by direct excitation of the junction between their vaso-constrictor nerves and their muscular tissue. The heart is slowed, but its beat is strengthenel. At once cut both vagi while a tracing is being taken ; the blood-pressure rises still more (p. $\left.15^{6}\right)$. The rise of pressure is sometimes so great that to prevent the mercury from being forced out of the manometer the tube must be clipper. The rise is not long maintained, but a second injection causes a renewerl increase of pressure.

25. Section and Stimulation of the Cervical Sympathetic in the Rabbit. Set up an incluction-coil arranged for an interrupted current (lig. si, p. IS 4 ). and connect it through a short-circuiting key with electrodes. The preparations necessary for an operation with antiseptic precautions are supposed to have been previously made-the instruments, sponges, and ligatures boiled in water; the instruments then immersed in a 5 per cent. solution of carbolic acid, the sponges and ligat ures in corrosive sublimate solution ( $0^{*}$ i per cent.). Instead of sponges swabs of sterile gauze or cotton may be used, and until the observations on the nerve have been made it is better to use sterile $0^{\circ} 9$ per cent. salt solution for such slight sponging as the wound may require rather than the antiseptic solutions. The hands are to be thoroughly washed, with diligent us? of the nailbrush, in soap and water before the cutting operation begins, and then soaked successively in alcohol and in the corrosive sublimate solution.

Fasten the rabbit on a holder, back downwards, as in Fig. $5 \mathrm{I}$. licep the animal warm by covering it with a cloth. and do not handle or wet its ears. Clip off the hair on the anterior surface of the neck. Remove loose hairs with a wet sponge, shave the neck, and wash it thorouglily, first with soap and water and then with corrosive sublimate. Give ether. Make a longitudinal incision in the middle line over the trachea, beginning a little balow the thyroid cartilage and extending down wards for an inch and a half. Feel for the carotid artery. cxpose, and raise it up. Two nerves will now ba seen coursing beside the artery. The larger is the vagus, the smaller the sympathetic. A third and much finer nerve (the depressor. or superior cardiac branch of the vagus) may also be seen in the same position, but the student should neglect this for the present. Pass a ligature under the sympathetic, and tie it, the ear being held up to the light while this is being (lone. so that its vessels may be clearly seen. A transient constriction of the arteries may be seen at the moment when the nerve is ligatured. This is due to stimulation of the vaso-constrictor fibres. Then follows a marked dilatation of the bloodvessels, due to paralysis of these fibres. The ear is flushed and hot. Note also that the pupil is probably narrower on the side on which the nerve has been tied. On stimulation of the upper (cephalic) end of the sympathetic with the electrodes, the vessels are markedly constricted, the car becomes pale and cold. and the pupil dilates. Cut the nerve above and below the ligature

* The amount of the initial rise of pressure is very variable, since the slowing of the heart tends to diminish the pressure, while the constriction of the arterioles tends to increase it. Thus, in one experiment the increase of pressure on injection of the extract was only $6 \mathrm{~mm}$. of mercury, while in another it was $56 \mathrm{~mm}$. On section of the vagi in this second experiment, there was an additional rise of $6+\mathrm{mm}$., and after a second injection a further rise of $70 \mathrm{~mm}$., making an increase of $190 \mathrm{~mm}$. in all above the original pressure. 
and take out the ligature. Wash the wound thoroughly with corrosive sublimate, then with sterile (boilerl) water, and close it, the muscles being first brought together by a row of interrupted sutures and then the skin by another row. Since it is rlifficult to thoroughly disinfect the hair-follicles, ancl a suture passed through a septic follicle is apt to give rise to suppuration, subcutancous stitchesi.e., stitches passed by a curverl needle through the deep layer of the skin witlout coming through to the surface-may be employed. The wound is to be protected by a coating of collodion. No other dressing is required. The animal is now removed from the holeler and put back to its hutch. The stulent must examine it at least

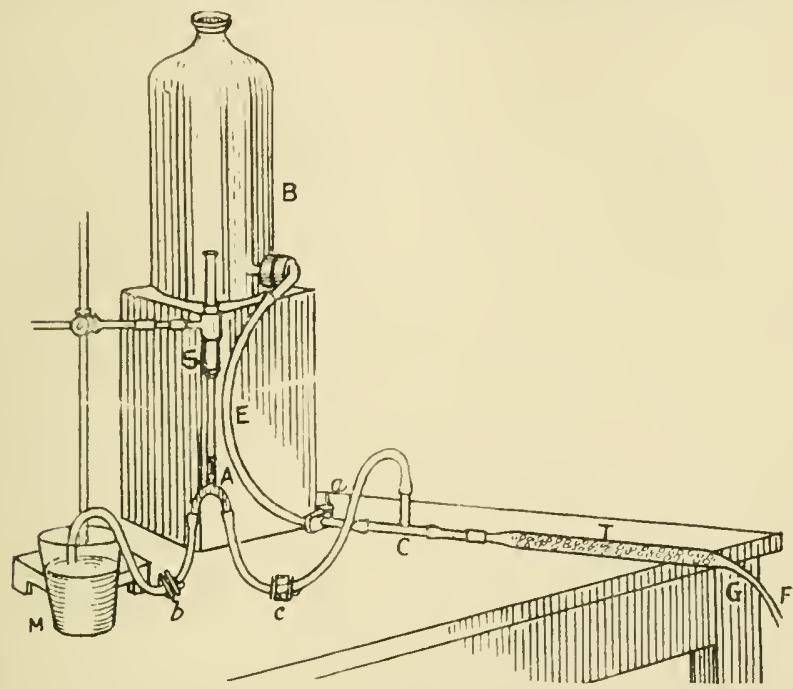

Fig. 96.-Artificial Schene to illustrate a Method of Measuring tie Circulation-time.

$\mathrm{B}$, bottle containing water, the rate of outflow of which is regulated by screw clamp $a ; \mathrm{S}$, syringe filled with methylene-blue solution, connected with $\mathrm{T}$-piece A ; II, beaker containing methylene-blue solution; $b, c$, screw-clamps; C, T. piece, inserted in the course of the flexible tube $\mathrm{E}$, and connected with the glass tube $\mathrm{T}$, which is filled with beads; $\mathrm{F}$, outflow tube. The clamp $c$ having been closed and $b$ opened, the syringe is filled with the methylene-blue solution $: b$ is then closed, $c$ opened, and a definite quantity of the solution injected into the system. The time from the beginning of injection till the appearance of the blue at $\mathrm{G}$ is measured with the stop-watch.

once a day for the next week, and study the differences between the two cars (p. I 59) and the two pupils.

29. Determination of the Circulation-time.-(a) Begin with an artificial scheme (Fig. 96). Fill the syringe with a $0^{\circ} 2$ per cent. solution of methylene blue. Allow the water to flow from the bottle by loosening the clamp. Inject a definite quantity of the methylenebluc solution, and with a stop-watch observe how long it takes to pass from the point of injection to the end of the glass tube filled with beads. Make ten readings of this kind and take the mean. Then raise the bottle so as to increase the rate of flow of the water, 
and repeat the observations. The 'circulation-time' will be found to be diminished. This corresponds to an increase of blood-pressure due to increased activity of the heart, without change in the calibre of the bloodvessels. Next, leaving the bottle in its present position, diminish the outflow by tightening the clamp; the circulation-time will be increased. This corresponds to an increase of blool-pressure due to diminution in the calibre of the small arteries.

(b) Fill the syringe* with methylene-blue solution $\left(0^{\circ} 2\right.$ per cent. in 0.9 per cent. Salt solution), as in $(a)$. Keep the solution warmed to $40^{\circ} \mathrm{C}$. by immersing the small beaker containing it in a water-bath, or heating it over a bunsen with a small flame. Weigh a rabbit or cat. In the case of the rabbit, inject $\frac{1}{2} \mathrm{grm}$. chloral hydrate into the rectum, and later on give ether if necessary. If a cat, give ether alone. Fasten it on a holder, back downwards (Fig. 5 I, p. 124). Cover it with a towel to keep it warm. Clip off the hair on the front of the neck, and make an incision $I \frac{1}{2}$ inches long in the middle line, beginning a little way below the cricoid cartilage. Reflect the skin and isolate the external jugular vein, which is quite superficial. Carcfully separate about 3 inch of the vein from the surrounding tissue, and pass two ligatures under it, but do not tie them. Compress the vein with a pair of bulldog forceps between the heart and the ligatures. Now tie the uppermost of the two ligatures (that next the hearl). but only put a single loose loop on the other. The piece of vein between the upper ligature and the bulldog is now distended with blood. With fine-pointed scissors make a small slit in the rein, taking great care not to divide it completely, insert the cannula, and tie the loose ligature firmly over its neck. Fill the cannula and the small piece of rubber tubing attached to it with $0^{\circ} 9$ per cent. salt solution by means of a pipette with a long point. Expose the caroticl on the other sicle, isolate it for $\frac{3}{4}$ inch, clear it carefully from its sheath, slip under it a strip of thin sheet indiarubber, and between this and the artery a little piece of white glazed paper. Connect the cannula in the jugular with the $\mathbf{T}$-piece attached to the syringe. Care must be taken that no air remains in the cannula or its connecting-tube. as a rabbit not unfrequently dies instantaneously when a bubble of air is injected into the right heart, although a considerable quantity of air can generally be injected into the jugular of a clog without killing it.

Now take off the bulldog from the vein, and make a series of observations on the pulmonary circulation-time. The animal must be so placed that a good light falls on the carotid. If necessary, the light of a gas-flame may be concentrated on it by a lens. The student holds the stop-watch in one hand, and injects a measured quantity of the methylene-bluc solution with the other. Uniformity in the quantity injected is secured by fastening on the piston of the syringe a screw-clamp, which stops the piston at the clesired point. The observation consists in setting off the watch at the moment when injection begins and stopping it when the blue appears in the carotid. After each injection the screw-clamp or pinchcock on the

* A burette, sloped so as to make a small angle with the horizontal, may be substituted for the syringe. The burette is supported on a stand at such a height that the methylene-blue solution runs without great force into the jugular (say $10-15 \mathrm{~cm}$. above the level of the camnula). The (langer of producing an abnormal result by suddenly raising the pressure in the right side of the lieart is thus avoided. 
tube comnected with the cannula must be tightened, the other opened, and the syringe refilled. (ireat eare must be taken never to open the two clamps at the same time, as in that case blood may regurgitate through the jugular and fill the syringe, or methylene blue may be sucked into the circulation. As many observations as possible should be taken, and the mean determined. The circulation-time observed is approximately that of the lesser circulation, the time taken by the blood to pass from the left ventricle to the carotid being negligible for the purposes of the student.

The specific gravity of the blood may also be tested at the beginning and end of the experiment by Hammerschlag's methoul (p. 5t). If a large number of injections have been made in quick succession, the specific gravity will be lass than normal; but if a consiclerable interval has been allowed to elapse after the last injection, little or no difference may be found, as the surplus liquid readily passes out of the bloodvessels.

Autopsy.-Observe particularly the state of the lungs, whether the bladler is distended or not, and whether any of the serous cavities or the intestines contain much liquid; so as to determine, if possible, by what channel the water injected into the blood may have been climinated. Study the distribution of the methylene blue in such organs as the kidneys and the muscles immediately after death, and notice that the blue colour becomes more pronounced after exposure for a time to the air. Make a longitudinal section through a kidney, and observe that the pigment is found especially in the cortex and around the pelvis at the apices of the pyramids, or it may be only in the cortex. The urine is greenish. If some methylene blue has been injected after the heart ceased to beat, the bloodvessels, particularly in the mesentery, may be beautifully mapped out by the pigment. This is not the case if the last injection took place before deatl, since the methylene blue is rapidly reduced by living tissues to a colourless substance, leuco-methylene blue. 


\section{CHAPTER III}

\section{RES P IR A T I O N}

Respiration in its widest sense is the sum total of the processes by which the ultimate elements of the body gain the oxygen they require, and get rid of the carbon dioxide they produce.

Comparative.--In a uniccllular organism no special mechanism of respiration is needed; the oxygen diffuses in, and the carbon clioxide diffuses ont, through the general surface. The simple wants of such multicellular animals as the cœlenterates, the group to which the sea-anemone belongs, are also supplied by diffusion through the ectoderm from and into the surrounding water, and through the endoderm from and into the contents of the body-cavity and its ranifications.

But in animals of more complex structure special arrangements become necessary, and respiration is divided into two stages: (1) External respiration, an interchange between the air or water and a circulating medium or blood as it passes through richly vascular skin, gills, tracheæ, or lungs : and (2) internal respiration, an interchange between the blood, or lymph, and the cells.

In the lower kinds of worms respiration goes on solely through the skin, under which plexuses of bloodvessels often exist, but in some higher worms there are special vascular appendages that play the part of gills. The crustacea also possess gills, while in the other arthropola respiration is carried on either by the general surface of the body (in some low forms), or more commonly by means of trachex, or branched tubes surrounded by blood spaccs and communicating externally with the air and internally by their finest twigs with the individual cells. Most of the mollusca breathe by gills, but a few only by the skin.

Among vertebrates the fishes and larval amphibians breathe by gills, but most adult amphibians have lungs. The skin, too, in such animals as the frog has a very important respiratory function. more of the gaseous exchange taking place through it in some conditions than through the lungs.

One small group of fishes, the dipnoi, has the peculiarity of posscssing both gills and a kind of lungs, the swim-bladder being surrounded with a plexus of bloodvessels and taking on a respiratory function.

In all the higher vertebrates the respiration is carried on by lungs ; the trifling amount of gascous interchange which can possibly take place throngh the skin is not worth taking into account. The lungs are to be regardecl as developed from outgrowths of the alimentary canal, beginning near the mouth. 
The object of all special respiritory arrangements being, in the first instance, to facilitate the gaseous exchange between the surrounding medium (air or water) and the blood, a prime necessity of a respiratory orgitn, be it skin, gill, trachea, or lung. is a free supply of blood, in vessels so fine and thin that diffusion readily takes place into them and out of them. But a free supply of blood would be of no avail if the medium to which the blood gave up its carbon clioxirle and from which it drew its oxygen was not being constantly and sufficiently renewed.

Sometimes the natural currents of the water or the air are of themselves sufficient to secure this renewal ; in other cases, artificial currents are set up by cilia, or special bailing organs, like the scaphognathites of the lobster. In all the higher animals, active movements, by which air or water is brought into contact with the respiratory surfaces, are necessary; and it is possible that such movements take place eren in the trachea of insects and other air-breathing arthropoda. Fishes, by rhythmical swallowing movements, take in water through the mouth and pass it over the gills and out by the gill-slits, while the frog distends its lungs by swallowing air.

Physiological Anatomy of the Respiratory Apparatus.-In man the respiratory apparatus consists of a tube (the trachea) widened at its upper part into the larynx, which contains the special mechanism of voicc, and communicates through the nose or mouth with the external air. Below, the trachea divides dendritically into innumerablc branches, the ultimate divisions of which are called bronchioles. Each bronchiole breaks up into several wider passages, or infundibula, the walls of which are everywhere pitted with recesses or alcoves, called alvcoli. The infundibula constitute the essential distensible clements of the lung, by the alternate stretching and relaxation of which the respiratory changes in the volume of the organ are mainly brought about. The trachea and larger bronchi are strengthened by hyaline cartilage in the form of incomplete rings, connected behind by non-striped muscular fibres, which also exist in the intervals between the rings. The middle-sized bronchi within the Iungs have the cartilage in the form of detached pieces in the outer portion of the wall, while nearer the lumen lies a complete ring of non-striped muscle.

In the bronchioles, no cartilage is present, but the circularlyarranged muscular fibres still persist, and also form a thin layer in the infundibula. In the air-cells, or alveoli, however, there are no muscular fibres. Their walls consist essentially of a network of elastic fibres, continuous with a similar layer in the infundibula and bronchioles, and covered on the side next the lumen by a single layer of large, clear epithelial scales, with here and there a few smaller and more granular polyhedral cells.

From the larynx to the bronchioles the mucous membrane is ciliated on its free surface, the cilia lashing upwards so as to move the secretion towards the larynx and mouth. In the infundibula the ciliated epithelium begins to disappear, and is absent from the alveoli. Part of the nasal cavity and the upper part of the pharynx are also lined with ciliated epithelium. IIucous glands are present in abundance in the upper portions of the respiratory passages, but disappear in the smaller bronchi.

Blood-supply of the Lungs. - The quantity of blood traversing the lungs bears no proportion to the amount required for their actual nourishment. Small, however, as this latter quantity is, it cannot 
apparently be clerived from the vitiated blood of the right ventricle. but is obtained directly from the aortic system by the bronchial arteries. These are distributed with the bronchi. which they supply as well as the connective-tissue of the interlobular septa running through the substance of the lung, the pleura lining it and the walls of the large bloodvessels. Most of the blood from the bronchial arteries is returned by the bronchial veins into the systemic venous system, but some of it finds its way by anastomoses into the pulmonary veins.

The brancles of the pulmonary artery are also distributed with the bronchi, and b:eak up into a dense capillary network around the alveoli. From the capillaries veins arise which, gradually uniting. form the large pulmonary veins that pour their blood into the left auricle.

The same quantity of blood must, on the whole, pas.s per unit of time through the lesser as through the greater circulation, otherwise equilibrium could not exist. and blood would accumulate either in the lungs or in the systemic vessels. But it does not follow that at each heart-beat the output of the two ventricles is exactly cqual. If, indeed, the capacity of the lesser circulation were constant, the quantity driven out at one systole by the right ventricle would be the same as that ejected at the next by the left ventricle. But it is known that the capacity of the pulmonary vessels is altered by the movements of respiration and probably in other ways, so that it is only on the average of a number of beats that the output of the two ventricles can be supposed equal.

The time required by a given small portion of blood, e.g. by a single corpuscle, to complete the round of the lesser circulation. is, as we have seen (p. I25), much less than the average time needed to complete the systemic circulation. In the rabbit the ratio is probably about $\mathrm{I}: 5$. Since all the blood in a vascular tract must pass out of it in a period equal to the circulation time. the average quantity of blood in the lungs and right heart of a rabbit must be about onefifth of that in the srstemic ressels. On the assumption that the same proportion holds for a man, not less than 700 grm. out of the $4^{\frac{1}{2}}$ kilos* of blood in a 70 kilo mitn must be contained in the lesser circulation, and about $33^{3}$ kilos in the greater. This corresponds sufficiently well with calculations from other clata.

For example, the average weight of the lungs in three persons, executed by beheading. was $45 \mathrm{j}$ grm. (Gluge). The average weight r) the lungs in a great number of persons who had died a natural death was $102+$ grm. (Juncker). The weight of the pulmonary tissue alone in the first set of cases must be less th $21,7 \mathrm{grm}$., for the lungs of a person who has bled to death are never bloodless. In a dog killed by bleeding from the carotid. one-quarter of the weight of the lungs consisted of blond. Assuming the same proportion for the decapitated individuals, we get $3+3$ grm. as the net weight of the blood-free lungs. Deducting this from $1024 \mathrm{grm}$. we arrive at $68 \mathrm{I}$ grm. as the average quantity of blood in the lungs. Adding to this the quantity in the right side of the heart (p. I2S), we get, in round numbers, $750 \mathrm{grm}$. as the amount in the lesser circulation. It is true that in the living body the conditious are not the same as after death: but it is probable that in a large number of cases taken at random the rifferences would be approximately equalized.

It has been further calculated that the total area of the alveolar

* See footnote on p. 12\%. 
surface of the lungs of a man is about 100 square metres (sixty times greater than the area of the skin), of which, perhaps, 75 square metres are occupied by capillarics. The average thickness of this immense sheet of blood has been reckoned to be equal to the diameter of a red blood-corpuscle, or. say, $8 \mu$. This would give 600 c.c. (630 grm.) as the quantity of blood in the lungs, which is probably somewhat too low an estimate.

If we take the pulmonary circulation-time as 13 seconds (p. 125), and the quantity of blood in the lungs as 700 grm., then $\frac{0.7 \times 60 \times 60}{13}$ $=194$ kilos of blood will pass through the lungs in an hour, or 4.656 kilos (say, 4,400 litres) in twenty-four hours. This would fill a cubical tank in which the man could almost stand upright with the lic closed.

\section{Mechanical Phenomena of Respiration.}

The lungs are enclosed in an air-tight box, the thorax; or it may be said with equal truth that they form part of the wall of the thoracic cavity, and the part which has by far the greatest capacity of adjustment. The alreolar surface of the lungs is in contact with the air. The pleura, which covers their internal surface, is reflected over the chest-walls and diaphragm, so as to form two lateral sacs, the pleural cavities. In health these are almost obliterated, and the visceral and parietal pleuræ, separated and lubricated by a few drops of lymph, glide on each other with every movement of respiration. But in disease the pleural cavities may be filled and their walls widely separated by exudation, as in pleurisy, or by blood, as in rupture of an aneurism, or by air in the condition known as pneumo-thorax. Between the two pleural sacs lies a mesial space, the mediastinum, commonly divided into an anterior mediastinum in front of the heart, and a posterior mediastinum behind it. The pleural and pericardial sacs and the mediastinum constitute together the thoracic cavity. The external surface of the chest-wall and the alveolar surface of the lungs are subjected to the pressure of the atmosphere, to which the pressure in the thoracic cavity (intrathoracic pressure) would be exactly equal if its boundaries were perfectly yielding. But in reality the intra-thoracic pressure is always normally something less than this. For even the lungs, the least rigid part of the boundary, oppose a certain resistance to distension, and so hold off, as it were, from the thoracic cavity a portion of the alveolar pressure; and in any given position of the chest the intra-thoracic pressure is equal to the atmospheric pressure minus this elastic tension of the lungs.

The object of the respiratory movements is the renewal of the air in contact with the alveolar membrane-in other words, the ventilation of the lungs. Two main methods are followed by sanitary engineers in the ventilation of buildings: they force air 
in, or they draw it in. In both cases the movement of the air depends on the establishment of a slope of pressure from the inlet to the interior. In the first method, this is done by increasing the pressure at the inlet; in the second. by diminishing the pressure at the outlet. In certain animals Nature, in solving its problem of ventilation, has made use of the first principle. Thus, the frog forces air into its lungs by a swallowing morement. In artificial respiration, as practised in physiological

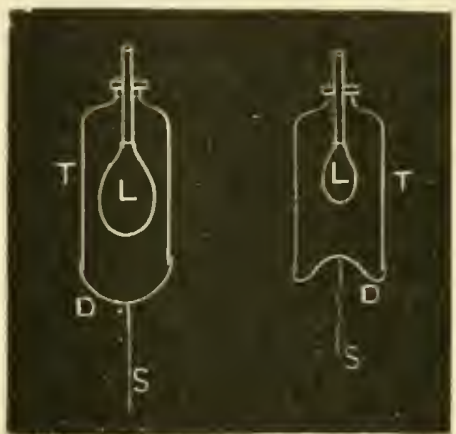

FIG. 97.-SCHEME TO ILLESTRATE THE MOVEMENTS OF THE LUNGS IN THE Chest.

$T$ is a bottle from which the bottom has been removed; I), a flexible and elastic membrane tied on the botıle, and capable of being pulled out by the string $\mathrm{S}$ so as to increase the eapacity of the bottle. L is a thin elastic bag representing the lungs. It communicates with the external air by a glass tube fitted airtight through a cork in the neek of the bottle. When $D$ is drawn down, the pressure of the external air calises $\mathrm{L}$ to expand. When the string is let go, $\mathrm{L}$ contracts again, in virtue of its elasticity. in contact with the vertebral column, the nediastinal surface in contact with the pericardium and the contents of the mediastinum, and the surface of the apex, move but little. The surfaces in contact with the diaphragm, ribs, and sternum have the greatest range of movement. Intermediate portions of the parenclivma of the lungs expand in a degree determined by their listance from the relatively stationary and mobile surfaces. The pressure of the air in the alveoli rluring the rapid expansion of the lungs necessarily sinks below that of the atmosphere, and air rushes in through the trachea and bronchi till the difference is equalized. Then commences the novement of expiration. The expanded 
chest falls back to its origrinal limits; the pressure in the thoracic carity increases; the distended lungs, in virtue of their elasticity, shrink to their former volume; the pressure of the air in the alreoli rises ahove that of the atmosphere, and with this reversal of the slope of pressure air streams out of the bronchi and trachea.

In inspiration the chest dilates in all its diameters. Its vertical diameter is increased by the contraction of the diaphragm, which, composed of a central tendon, a peripheral ring of muscular tissuc, and the two muscular crura, bulges up into the thorax in the form of two flattener clomes, one on each side, and thus closes its lower aperture. When the diaphragm contracts, even in ordinary quiet breathing, the central tendon descends distinctly (about half an inch) after the manner of a piston. The acute angle which the muscular ring makes during relaxation with the thoracic wall opens out around its whole circumference, so as to form a groove of triangular section. But the most peripheral portion of the ring is always kept in close apposition to the chest-wall by the negative intrathoracic pressure. The lungs follow the descending diaphragm, their lower borders keeping accurately in contact with it. The descent of the diaphragm is not directly downwards, but downwards and forwards. For it is compounded of two morements, the spinal segment of the muscle (the crura) causing a vertical elongation of the thorax, while the sterno-costal part (the muscular ring) pushes the abdominal viscera downwards and forwards (Keith). Since the diaphragm is attached to the lower ribs, there is a tendency during its contraction for these to be drawn inwards and upwards ; but this is opposed by the pressure of the abdominal viscera, and by the action of the quadratus lumborum, which fixes the twelfth rib, and of the scrratus posticus inferior, which draws the lower four ribs backward. When these and the other inspiratory muscles that act especially upon the ribs are paralyzed by injury to the spinal cord, and respiration is carried on by the diaphragm alone, the line of its attachment to the ribs is distinctly marked during inspiration by a shallow circular groove.

The thorax is also enlarged by the action of certain muscles that act upon the ribs. Among the elevators of the ribs, as their name indicates, are usually reckoned, although erroneously, the levatores costarum-twelve in number on each side. They arise from the transverse processes of the last cervical and first eleven dorsal vertebra, and passing obliquely downwards and outwards, are inserted between the tubercle and the angle into the first or second rib below their origin. They do not elevate the ribs, but take part in lateral movements of the spinal column. The scalene muscles, which may in a lean person be felt to be 
tense during inspiration, fix the first and second ribs (scalenus anticus and medius, the first; scalenus posticus, the second rib), and so afford a fixed line for the intercostal muscles to work from on the lower ribs.

The most important elevators of the ribs are the external intercostals. The intercartilaginous portions of the internal intercostals (the intercartilaginei muscles, as they are sometimes called) also contract simultaneously with the diaphragm, and may therefore be included in the list of inspiratory muscles; but instead of elevating the ribs they depress the costal cartilages, and thus help to widen the angles between them and the ribs. In addition to increasing the capacity of the chest, the contraction of the external intercostals and the intercartilaginous muscles aids in inspiration by augmenting the rigidity of the intercostal spaces, and so preventing them from being drawn in as easily as would otherwise be the case when the thorax is expanded by the action of the diaphragm and the other inspiratory muscles.

Leaving out of account the floating ribs, which functionally form a part of the abdominal wall, the ribs in relation to their respiratory functions may be divided into the following groups: (I) The first rib, which, moving itself very little, provides a fixed line towards which the next set of ribs may be raised.

(2) An upper costal series consisting of the ribs from the second to the fifth. These are raised in inspiration towards the fixed first rib by the contraction of the intercostal muscles. The movement of these ribs is, mainly at any rate, a rotation around a transverse axis, the axes on which they move corresponding to their necks. The manner in which they are articulated to the vertebre prevents any sensible rotation around an antero-posterior axis or 'bucket-handle' movement. Since these ribs slant downwards and forwards to their sternal attachments, the sternum is raised when they are elevated; or, rather, since the manubrium is practically immovable in ordinary breathing, the body of that bone is lient on the manubrium at the manubrio-sternal joint. This causes an increase in the antero-posterior diameter of the thorax. Further, since the arches formed by the ribs widen in regular progression from above downwards in the upper portion of the thoracic cage, so that the second rib is a segment of a larger circle than the first, and the third than the second, it is clear that a general elevation of the chest will tend to increase the transverse diameter at any given level. Such an increase is also favoured by the opening out of the angles between the bony ribs and the costal cartilages under the influence of the couple (or pair of oppositely directed forces) that acts on them-viz., the upward pull of the external intercostals exerted on the ribs, and the downward pull of the 
intercartilaginei and the resistance of the stermum to further displacement exerted on the cartilages. The whole arrangement is perfectly adapted to permit the expansion of the roughly conical upper lobes of the lungs.

(3) The lower costal series, consisting of the ribs from the sixth to the tenth. These ribs, with their muscles, form a mechanism which normally acts along with the diaphragm (Keith). They are so arranged that in inspiration the lateral and anterior part of each moves outwards to a greater extent than the one above it. There is not only a rotation around a transverse axis, by which the lower end of the sternum, connected to these rils by the combined cartilages of the sixth to the ninth, is elcvated, lut also a rotation around an anteroposterior axis. The movement of the lower ribs results, therefore, in increasing both the back-to-front diametcr and the transverse diameter of the lower portion of the thorax. The widening of the thorax from side to side may also be in a slight degree ascribed to a twisting morement of the ribs, which tends to evert their lower borders. With the diaphragm, these lower ribs arranged in a vertical series of not very different curvature constitute a mechanism for the inspiratory cxpansion of the roughly cylindrical lower lobes of the lungs.

Expiration in perfectly tranquil breathing is brought about with less aid from active muscular contraction. The sense of effort disappears as soon as the chest ceases to expand. The diaphragm and the elevators of the ribs relax. The structures that have been stretched or twisted recoil into their original positions; the structures that have been raised against the force of gravity fall back by their weight, and in the measure in which the pressure increases in the thoracic cavity the elasticity of the lungs causes them to shrink. The pressure in the alveoli, which at the end of inspiration was just equal to that of the atmosphere, is thus increased, and the air expelled. It is probable that, even in man and in quiet respiration, the interosscous portions of the internal intercostals help by their contraction in depressing the ribs, and that a slight contraction of the abdominal muscles hastens the return of the diaphragm to its position of rest. In reptiles and birds, expiration is normally effected by an active muscular contraction. This is also true in some mammals - the rabbit, for instance, in which the external oblique muscles of the abdominal wall take an important share in the expiratory act.

Types of Respiration.-Differences exist also, not only between different groups of animals, but even between women and men, in the relative importance in inspiration of the diaphragm and the muscles that raise the lower ribs on the one hand, and the muscles that elevate the upper ribs on the other. When the 
morements of the diapliragm predominate, the respiration is saicl to be of the abdominal or diaphragmatic type: when the movements of tle upper ribs and sternum are most conspicuous, of the costal or thoracic type. In abdominal respiration, the inspiratory movement commences at the diaphragm, and then involves the lower ribs and the tip of the stermum. In costal respiration, the upper ribs initiate the movement, and are followed by the abdomen. In the rabbit, cluring quiet breathing. the respiration is purely diapliragmatic, the ribs remain motionless; and herbivorous animals in general conform more or less closely to this type. In the carnivora, on the contrary, the costal type prevails. Man allies himself as regards his respiration with the rabbit and the sheep; he uses his liaphragm more than his wpper ribs. Civilized woman falls into the class of the wolf and the tiger; she uses her upper ribs more than her diaphragm. The cause of the difference between men and women has been much discussed. It is not a primitive sexual difference, for it is far from being universal; in the uncivilized and semi-civilized races that have been investigated, the women breatlue like the men. It is therefore probable that the predominance of the costal type among women of European race is a peculiarity developed by a mode of dressing which hampers the movements of the diaphragm while permitting the elevation of the ribs. This conclusion is strengthened by the fact that in children no difference exists ; bot h boys and girls show the abdominal type of respiration.

All this refers to ordinary breatling. In forced respiration, when the need for air becomes urgent, costal breathing alwars becomes prominent alike in men, in women, and in animals, for by elevation of the ribs the capacity of the chest can be increased to a greater degree than by any contraction of the diapliragm.

In forced inspiration, indeed, all the muscles that can elevate the ribs may be thrown into contraction, as well as other muscles which give these fixed points to act from. During a paroxysm of asthma, for example, the patient may grasp the lack of a chair with his hands, so as to fix the arms and shoulders and allow the pectorals and serratus magnus to raise the ribs. Similarly in forced expiration all the muscles are used which can depress the ribs, or increase the intra-abdominal pressure and push up the diaplıragm.

Artificial Respiration.-An efficient pulmonary ventilation can be obtained by various methods when the natural breatling is in abeyance. In animals the method most commonly employed for experimental purposes is the rlythmical inflation of the lungs by a pump or bellows, or by a stream of compressed air which is regularly interrupter, the cliest heing allowed to collapse after each inflation. When the animal is to be kept alive after the 
experiment the inflation is protuced througle at tube introluced thromgh the glottis. If the anmal is not to be kept alive, the apparatus is generally connecter with a cammla in the trachea. lis man the exchange of air between the atmosphere and the lungs may be most readily accomplished by strong rhythmical compression of the lower part of the chest. "This forces out some of the air from the lungs; on relaxing the pressure the chest expands again and air is drawn in. Schäfer has shown that this is the most efficient method of respiration in resuscitation of the apparently drowned. "The patient is placed face downwards on the ground, with a folded coat under the lower part of the chest. The operator puts himself athwart or at the side of the patient, facing his head and kneeling wpon one or both knees (Fig. 9S), and places his liands on each side over the lower part of the back (lowest ribs). He then slowly throws the weight

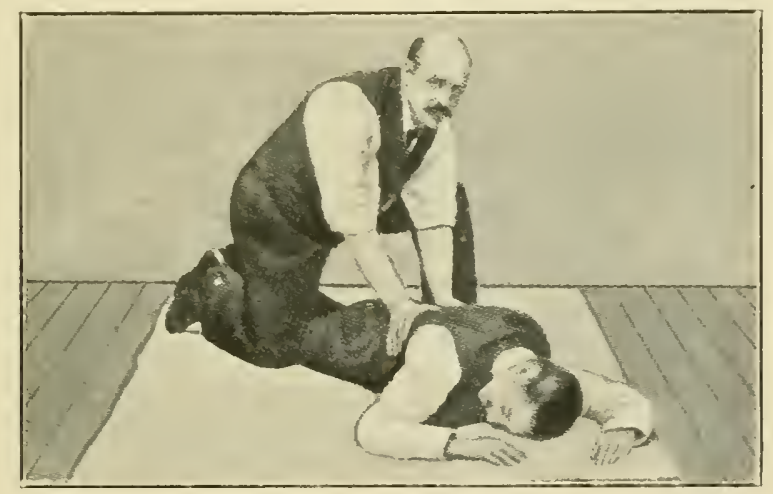

Fig. 98.-Artificial Respiration in Cases of Drowing (after Schäfer).

of his body forward to bear upon his own arms, and thus presses upon the thorax and forces air out of the lungs. He then gradually relaxes the pressure by bringing his own body up again to a more erect position, but without moring the hands.' Air is thus drawn into the lungs. The process is repeated twelve to fifteen times a minute.

Certain accessory phenomena (movements and sounds) are associated with the proper movements of respiration. The larynx rises in expiration, and sinks in inspiration. The glottis (and particularly its posterior portion, the glottis respiratoria) is widened during deep inspiration and narrowed during deep expiration. The same is the case with the nostrils, ancl, indeed, in some persons the ala nasi move even in ordinary breathing. It has long been known that in deep respiration changes in the calibre of the bronchi synchronous with the respiratory move- 
ments may nccur. In young persons it may be directly observed with the bronchoscope, an instrument used by laryngologists for exploring the larger bronchi, that these dilate in inspiration and constrict in expiration (Ingalls). In part at least these movements are passively produced by the changes of intrathoracic pressure, but it has not been definitely determined whether they are not in part caused by alternate contraction and relaxation of the circular bronchial muscles. To these muscles has sometimes been attributed the function of regulating the flow of air into and out of the infundibula, as the muscle of the arterioles regulates the distribution of the blood in the organs.

As regards the respiratory sounds, all that is necessary to be said here is that when we listen over the greater portion of the lungs with the ear, or, much better. with a stethoscope. a soft breezy murmur, that has been compared to the rustling of the wind through distant trees, is heard. This has been called the iesicular murmur. It is only heard in health during inspiration and the very beginning of expiration, and is louder in children than in adults. Around the larger bronchi and the trachea a blowing sound is heard, which certainly nriginates at the glottis. and is strengthened by the resonance of the air-tubes. In health this is not recognised over the greater portion of the lung. But in certain diseases in which the alveoli are filled up with exudation, this bronchial or iubular breathing may be heard over a large area, the resicular sound being now suppressed and the bronchial sound being better conducterl through the smaller bronchi towards the surface of the lungs when their walls have been rendered more rigid by the solidification of the parenchyma. in spite of the fact that the consolidated tissue as such does not conduct the sound so well as the air-containing alveoli.

It has been much debated whether the vesicular murmur also arises at the glottis, and is modified by transmission through the pulmonary tissue. or whether it arises somewhere in the terminal bronchi, the infundibula or the alveoli. Both views mav be supported by certain arguments, and to both some objections may be raised. The fact appears to be that there are two elements in the inspiratory murmur-a true vesicular sound, produced about the place where the terminal bronchioles give off the infundibula, and a resonance sound set up in the trachea and bronchi by the glottic murmur. This resonance sound as heard over portions of the lung containing only small bronchi has a different character from that heard over large bronchi. inasmuch as the fundamental note, and to a still greater extent the overtones (p. 2So), are much weakened in those small and easilydistensible tubes. The true vesicular element is heard all over the lungs, but the resonant laryngeal element in large animals, 
like the horse and ox, dies out as an audible murmur before it reiches the remotest lobules, and can only be distinguished over a portion of the pulmonary area. When the glottic sound is eliminated by causing an animal to breathe through a tracheal fistula, the vesicular murmur is still heard, and in the horse is even somewhat sharper than normal, although in the dog it is softer and weaker. The expiratory murmur does not seem to contain a true vesicular element, but is exclusively due to the resonance of the expiratory glottic sound (Marek). It is generally admitted, and this is of great importance in practical medicine, that when the normal vesicular sound is heard over any portion of the lung tissue, it may be inferred that this portion is being properly distended, and that air is freely entering its alveoli.

$\mathrm{Up}$ to this point we have contented ourselves with a purely qualitative description of the mechanical phenomena of respiration. We have now to consider their quantitative relations, and the methods by which these have been studied.

The expansion of the lungs in inspiration may be easily demonstrated in man, and even a rough estimate of its amount obtained, by the clinical method of percussion. For example, the resonant note that is elicited when a finger laid on the chest at a part where it overlies the right lung is smartly struck can be followed down until it is lost in the "liver dulness.' If the lower limit of the resonant area be marked on the chest-wall first in full inspiration and then in full expiration, the mark will be lower

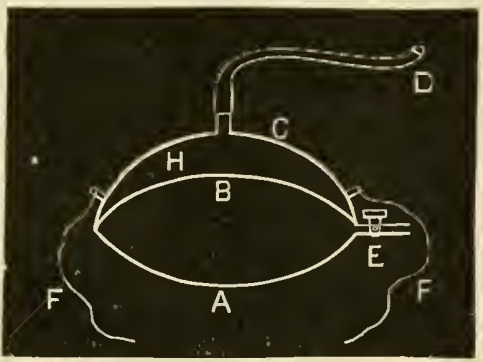

Fig. 99.-SCheMe of TAMGOUR For Recording Respiratory Move. MENTS.

C, a metal capsule connected airtight with $\mathrm{B}, \mathrm{A}$, two caoutchouc membranes, the chamber formed by which can be inflated by means of the tube and stopcock $\mathrm{E}$. The tube D connects the space $\mathrm{H}$ with a registering tambour provided with a lever. The membrane $A$ is applied to the chest, round which the inextensible strings $F$ are tied. At every expansion of the chest the pressure in $\mathrm{H}$ is increased, and the increase of pres. sure is transmitted to the registering tambour. in the former than in the latter, and the difference will represent the difference in the vertical length of the shrunken and distended lung. A similar enlargement in the transverse direction may be demonstrated in the same way, the inner borders of the lungs coming nearer to the middle line in inspiration, and receding from it in expiration. The examination of the chest by the Röntgen rays has also yielded results of importance in the study of normal respiratory conditions, and still more important results in pulmonary disease.

For most physiological purposes, however, a faithful graphic record of the respiratory movements is indispensable. This may be obtained-

(I) By registering the movements of a single point, or the varia- 
tions in a single circumference, of the boundary of the thoracic cavity. In man changes in the circumference of the thorax at any level can be recordel by means of a tambour adjusted to the chest (lïgs. on and 128), and in communication with another. which is provicled with a writing lever (Figs. S6 and 131). Or an clastic tube, with a spiral spring in its lumen. may be fastened around the thorax or abdomen and connected with a piston-recorler (a small cylinder in which works a piston carrying a writing-point) (Fitz).

(2) By recorling the changes of pressure produced in the airpassages by the respiratory movements. This can be lone by connecting a cannula in the trachea of an animal with a recorling tambour in the manner described in the Practical Exercisss, p. 28s, The variations of pressure may be measured by connecting a manometer with the trachea, or in man with the nostril.

(3) By writing off the changes of pressure which occur in the thoracic cavity luring respiration. For this purpose a trocil is introduced through an intercostal space into one of the pleural sacs. without the admission of air, or into the pericardium. and then connected with a manometer or other recorling apparatus. Or a tube. similar in construction to a cardiac sound (p. \&9), may be pusherl

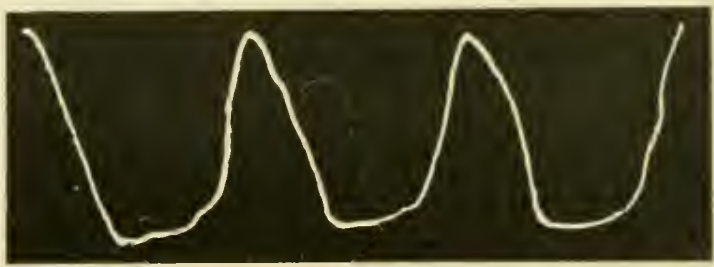

Fig. ioo.-Respiratory TraciNg from Ma: (MAREy).

1)own stroke, inspiration; up stroke, expiration.

down the oesophagus. The variations in the intrathoracic pressure are transmitted to the air in the elastic bag, and thence to a tambour.

(4) In the rabbit the part of the cliaphragm attached to the ensiform cartilage may be isolated from the rest and its contractions recorderl by a lever (Head). For some purposes this is the best method.

When the respiratory movenents are studied in any of these ways, it is found that there is practically no pause between the end of inspiration and the beginning of expiration. Nor, although the chest collapses more gradually than it expands, is there any distinct interval in ordinary breathing between the end of expiration and the beginning of the succeeding inspiration. When, however, the respiration is unusually slow, an actual pause (expiratory pause) may occur at this point. Fxpiration takes somewhat longer time than inspiration, the ratio varying from $7: 6$ to $3: 2$, according to age, sex, and ot her circumstances.

The frequency of respiration is by 110 means constant even in health. All kinds of influences affect it. It is dificult even to direct the attention to the respiratory act without bringing 
about a modification in its rhythm. In the adult i5 to 20 respirations per minute may be taken as about the normal. In young children the frequency may be twice as great (newboin child, 50 to 70 ; child from I to 5 years old, 20 to 30 per minute). It is greater in a female than in a male of the same age. A rise of temperature increases it; $\mathbf{5} 50$ respirations per minute have been seen in a dog with a high temperature. sudilen cooling of the skin, exercise, and various emotional states. increase the rate, and slecp diminishes it. The will can alter the frequency and deptl of respiration for a time, and even stop it altogether, but in less than a minute, in ordinary individuals, the desire to breathe becomes imperative. Cato's assertion that he could kill himself at any time 'merely by holding his breath' is only a proof that le was a better philosopher than physiologist. After a period of forced respiration the breath can be held for a much longer time. This is due to the "washing out ' of the carbon dioxide, the normal stimulus to the respiratory centre (p. 23I). After six minutes of forced breathing the interval of roluntary inhibition can be extended beyond four minutes. A professional diver has remained under water in a tank for about four and three-quarter minutes. When oxygen is inhaled instead of air during the last few breaths of the forced respiration, the interval during which the breath can be held may be much increased (up to nine or ten minutes). In animals the rate of respiration can be greatly affected by drugs and by the section and stimulation of certain nerves; but to this we shall return when we come to consider the nervous mechanism of respiration.

It cannot fail to be observed that to a great extent the rate of respiration is affected by the same circumstances as the frequency of the heart (p. q8), and in the same direction. And, indeed, in health, these two physiological quantities, amid all their absolute variations, maintain to each other a fairly constant ratio ( $I$ to 4 or $I$ to 5 in man). Even in many diseases this proportion remains tolerably stable, although in others it is disturbed.

The total quantity of air expired, or, what comes to the same thing, the alteration in the capacity of the chest during expiration, can be measured by means of a gas-meter or of a spirometer (Fig. IOI), which consists of an inverted graduated glass cylinder dipping by its open mouth into water and balanced by weights. The vessel is sunk till it is full of water, the air being allorred to escape by a cock. The expired air is now permitted to enter it through a tube, and displaces some of the water. The spirometer is adjusted so that the level of the water inside and outside is the same, and then the volume of air contained 
in it is read off. This gives the volume of the expired air at atmospheric pressure. Similarly, by breathing air from the spirometer the amount inspired can be measured (p. 29I).

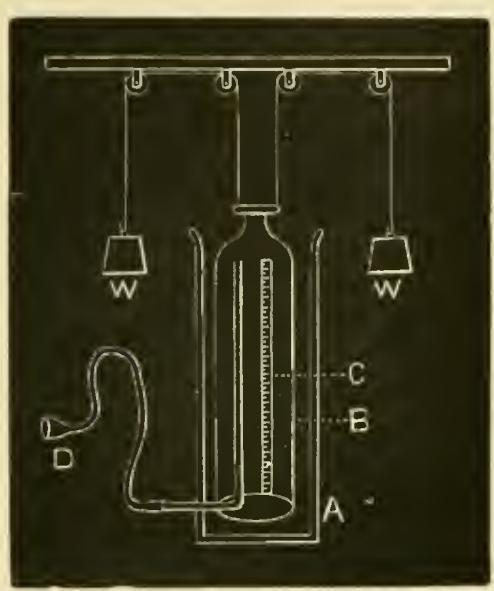

Fig. iot.-Diagram of Spirometer.

$A$, vessel filled with water. $B$, glass cylinder with scale $\mathrm{C}$, swung on pulleys and counterpoised by weights W. D, tube for breathing through.

From 400 to 500 c.c. of air* are taken in and given out at each respiration in quiet breathing. This is called tidal air. It amounts to 35 pounds by weight in twenty-four hours, or enough to fill, at atmospheric pressure, a cubical box with a side of 8 feet. With the decpest possible inspiration room can be made for 2,000 c.c. more; this is called complemental air. By a forced expiration I,500 c.c. can be expelled besides the tidal air ; and to this quantity the name of supplemental or reserve air has been given. After the deepest expiration there always remains $I, 000$ to $I, 200$ C.C. of air in the lungs (Durig), and this is called the residual air. After a normal expiration following a normal inspiration the lungs still contain stationary air to the amount of about 2,500 c.c.

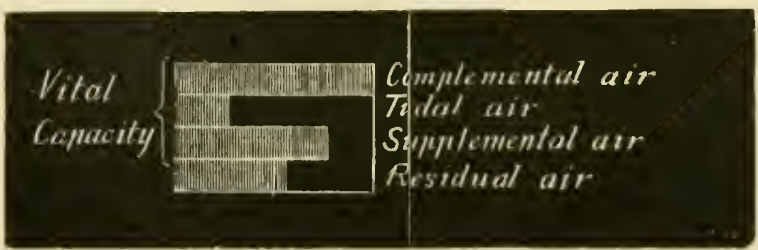

Fig. I02.-Diagram to llzustrate tile Relative Amount of Complemental, Tidal, Supplemental, and Residual Air.

The term vital or respir a tor y capacity is applied to the quantity of air which can be expelled by the dcepest expiration following the deepest inspiration, and amounts in an adult of average height to 3,500 or 4,000 c.c. The maximum quantity of air which the lungs can contain is evidently equal to vital capacity plus residual air. At one time the vital

* The average obtained by the writer for $S$ I healthy students, with an average body-weight of 66 kilos, was 460 c.c., or 7 c.c. per kilo. In 4 newborn children the tidal air varied from 20 to $30 \mathrm{c.c}$, and from $7 \cdot 6$ to $7 \cdot 3 \mathrm{c.c}$. per kilo, which is not very different from the amount in the adult. The pulmonary ventilation must therefore be far more rapid in the child, since its respiratory frequency is so much greater. 
capacity was thought to be capable of affording valuable information in the diagnosis of chest discases; but little stress is now laid upon it, as it varies from so many causes. For instance, it can be increased by practice with the spirometer. It is greater in mountaineers than in the inhabitants of lowland plains.

It is clear from the figures we have given that in ordinary breathing only a small proportion of the air in the lungs comes in direct at each inspiration from the atmosphere, and only a small proportion escapes into the atmosphere at each expiration. The greater part of the air in the lungs is simply moved a little farther from the upper respiratory passages, or a little nearer them; and fresh oxygen reaches the alveoli, as carbon dioxide leaves them, mainly by diffusion, aided by convection currents due to inequalities of temperature, and to the churning which the alternate expansion and shrinking of the lungs, and the pulsations of their arteries, must produce. But that some of the tidal air strikes right down to the alveoli is evident enough. For the respiratory ' dead space'-that is, the capacity of the upper air-passages and the bronchial tree down to the infundibula -is only I40 c.c., or one-third of the amount of the tidal air (Zuntz, Loewy). There is no direct way of determining whether any respiratory exchange goes on through the walls of the upper air-passages. But by indirect methods it has been estimated that about 30 per cent. of the rolume of the tidal air is pure air (Haldane and Priestley). This, of course, corresponds to the ' effective' dead space. Taking the average tidal air at 460 c.c. (p. 220), it is clear that the effective corresponds very closely with the anatomical dead space-that is to say, the respiratory function of the air-passages above the point where the infundibula are given off is negligible. Although such calculations can only be approximately correct, the agreement is of interest. The immense extent of the pulmonary surface, and the extreme thinness of the layer of blood in the capillaries of the lungs, facilitate the interchange between the gases of the blood and the gases of the alveoli.

The Amount and Variations of the Intrathoracic Pressure.In the deepest expiration the lungs are never completely collapsed; their elastic fibres are still stretched; and the tension of these acts in the opposite direction to the external atmospheric pressure, and diminishes by its amount the pressure inside the thoracic cavity. In the dead body Donders measured the value of this tension, and therefore of the negative pressure of the thorax, by tying a manometer into the trachea, and then causing the lungs to collapse by opening the chest. It varied from $7.5 \mathrm{~mm}$. of mercury in the expiratory position to $9 \mathrm{~mm}$. in the inspiratory. So far as can be judged from observations made 
on persons suffering from various diseases of the respiratory organs, the alterations during ordinary breathing do not amount to more than 3 or $4 \mathrm{~mm}$. of mercury. But when an attempt is made in the dead body to imitate a deep inspiration by making traction on the chest-walls so as to expand the lungs, the intrathoracic pressure may fall to $-30 \mathrm{~mm}$. of mercury; and in a living rabbit during a deep natural inspiration, a pressure of $-20 \mathrm{~mm}$. has been seen.

The reason why the lungs collapse when the chest is opened is that the pressure is now equal on the pleural and alveolar surfaces, being in both cases that of the atmosphere. There is therefore nothing to oppose the elasticity of the lungs, which tends to contract them. So long as the chest is unopened, the pressure on the pleural surface of the lungs is less than that on the alveolar surface, and the elastic tension can only cause them to shrink until it just balances this difference.

In intra-uterine life, and in stillborn children who have never breathed, the lungs are completely collapsed (atelectatic), and there is no negative intrathoracic pressure. They are kept in this condition by adhesion of the walls of the bronchioles and alveoli. If the lungs have been once inflated, this adhesion ceases to act, and they never completely collapse again.

Amount and Variations of the Respiratory or Intrapulmonary Pressure. - As we have already remarked, the pressure in the alveoli and air-passages is less than that of the atmosphere while the inspiratory movement is going on, greater than that of the atmosphere during the expiratory movement, and equal to that of the atmosphere when the chest-walls are at rest. When the external air-passages are closed, e.g., by connecting a manometer with the mouth and pinching the nostrils, the greatest possible variations of pressure are produced. In the deepest inspiration under these conditions a negative pressure of about $75 \mathrm{~mm}$. of mercury (i.e., a pressure less than that of the atmosphere by this amount) has been found, and in deep expiration a somewhat greater positive pressure* (Practical Exercises, p. 292).

But with ordinary breathing, the variations of pressure as measured by this method do not exceed 5 to Io mm. of mercury above or below the pressure of the atmosphere.

When the external openings are not obstructed, as, for example. when the lateral pressure is taken in tlie trachea of an animal by means of a cannula with a side-tube connected with a manometer, still smaller, and doubtless truer, values have been found (2-3 $\mathrm{mm}$. of mercury, as the positive expiratory pressure and

* The maximum negative pressure in deepest inspiration averaged for 49 students, $-73 \mathrm{~mm}$. (highest observation - $137 \mathrm{~mm}$.) of mercury; the maximum jositive pressure in deepest expiration, too $\mathrm{mm}$. (highest observation +1 to $\mathrm{mm}$.). 
I mum. as the negative inspiratory pressure in (logs). But since the respiratory passages are abruptly narrowed at the glottis, the variations of pressure must he greater below than above it, and in generil they must increase with the distance from that orifice, being greater, for instance, in the alveoli than in the bronchi.

Relation of Respiration to the Nervous System.-- Unlike the beat of the lieart, the respiratory movements are entirely dependent on the central nervous system. The 'centre' which presides over then is situated in the spinal bulb. It is a bilateral centre-that is, it has two functionally symmetrical halves, one on each side of the middle line. Fach of these halves has to do more particularly with the respiratory muscles of its own side, for destruction of one-half of the spinal bulb causes paralysis of respiration only on that side. Anatomically the respiratory centre has not been sharply localized; but it lies lower than the vaso-motor centre, not far from the point of the calamus scriptorius. Stimulation of this region during apnoea (p. 23I) is stated to cause co-ordinated inspiratory movements and widening of the opening of the glottis through abduction of the rocal cords. The centre is brought into relation with the muscles of respiration by efferent nerves. The phrenic nerves to the diaphragm, and the intercostal nerves to the muscles which elevate the ribs, are the most important of those concerned in ordinary breathing. The respiratory centre is further related to afferent nerves, of which the most influential is the vagus, particularly its pulmonary fibres and its superior laryngeal branch. But almost any afferent nerve may powerfully affect the centre; and it is also influenced by fibres passing to it from the higher parts of the central nervous system.

Section of the spinal cord in animals above the origin of the phrenic nerves causes complete paralysis of respiration, and consequent death. The phrenics arise from the third and fourth cervical nerves, and are joined by a branch from the fifth; and in man fracture of any of the four upper cervical vertebra is, as a rule, instantly fatal. But in one case respiration was carried on, and life maintained for thirty minutes, merely by the contraction of the muscles of the neck and shoulders in a man entirely paralyzed below this level (Bell). Section of the cord just below the origin of the phrenics leaves the diaphragm working, although the other respiratory muscles are paralyzed. A case has been recorded of a man in whom, from disease of the spine in the lower cervical region, all the ribs became completely immovable. He was able to lead an active life, and to carry on his business, although he breathed entirely by his diaphragm and abdominal muscles. 
Section of one phrenic is followed by paralysis of the corresponding laalf of the diaphragm, section of both phrenics by complete paralysis of that muscle, and although respiration still goes on by means of the muscles which act upon the ribs, it is usually inadequate to the prolonged maintenance of life. In the horse, however, not only has survival been seen after this operation, but the animal, after the first temporary increase in the frequency of the breathing had disappeared, could be driven in a light vehicle without any marked dyspnœa. The phrenic nuclei in the two halves of the cord are connected across the middle line. For when a semisection of the cord is made between this level and the respiratory centre in the medulla, respiratory impulses are still able to reach both phrenic nerves. In some animals both halves of the diaphragm go on contracting. But when, as usually happens, this is not the case, and the diaphragm on the side of the semisection has ceased to act, it at once begins to contract again when the opposite plirenic nerve is cut, and the respiratory impulse, descending from the bulb, is blocked out from the direct, and forced to follow the crossed path. It has been shown that the crossing takes place at the level of the phrenic nuclei, and nowhere else (Porter).

When one vagus is divided, there is little or no change in the respiratory movements. Half an inch of one vagus nerve has been excised in removing a tumour, and the patient showed no symptoms whatever. But section of both vagi in such animals as the dog, cat and rabbit causes respiration to become much deeper and slower, the one change for a time compensating the other, so that the total amount of air taken in and given out, the amount of carbon dioxide eliminated, and the partial pressure of that gas in the pulmonary alveoli are not greatly altered. The relative duration of the two respiratory phases is completely changed, inspiration being much more prolonged than expiration. It has been shown that the effect is really due to the loss of impulses that normally ascend the vagi, not to any irritation of the cut ends. For a nerve can be frozen without exciting it ; and when a portion of each vagus is frozen, the respiration is affected in precisely the same way as when the nerves are divided.

After section of both ragi certain fibres coming from the brain above the respiratory centre appear to take a share in the regulation of the respiratory movements. The bloodvessels supplying these fibres, or the centres from which they come, can be blocked by injection of paraffin wax into the common or internal carotid, or the bulb can be severed with the knife above the level of the respiratory centre, without any"effect being produced upon the breathing, except that the rate is, as a rule, somewhat lessened 
But when both the vagi and these upper patlis are cut the character of the respiration is changed, exceedingly prolonged inspiratory spasms alternating with long periods of complete relaxation of the diaphragn till the animal clies.

From these facts it appears that the periodic antomatic discharges of the respiratory centre are being continually controlled and modified by impulses passing up the vagus, and that in the absence of these impulses a certain degree of control is exercised by the higher paths, which, however, do not appear to be normally in action, at any rate to the full measure of their capacity. When the vagi are severed, the control of the higher paths comes into play, and is sufficient still to keep the breathing regular, although it is slowed. When the higher paths are cut off, the vagus of itself is able to regulate the discharge. But when both are gone, the respiratory centre, freed from control, passes into a condition of alternate spasm and exhaustion. Of the central connections of these upper paths but little is surely known. The corpora quadrigemina, however, seem to contain centres which can affect the respiration. Certain areas on the cerebral cortex have also been described, the excitation of which modifies the respiratory movements. There is no question that the cortex is connected, and extensively connected, with the respiratory centre, since the rate and depth of the co-ordinated respiratory movements, which are universally acknowledged to involve the activity of the centre, can be altered not only by the will, but by the most varied psychical events.

The rhythmical excitation of the regulating vagus fibres must be brought about either by mechanical stimulation of the nerveendings in the lungs, due to the alternate stretcling and shrinking, or by chemical stimulation depending on the changes that occur with each respiration in the content of oxygen and carbon dioxide in the alveolar air, and therefore in their pressure (p. 248 ) in the blood. Both views have found advocates, but whatever influence the chemical changes in the blood may exert, there is no doubt that the mechanical factors are the more important. That the vagus is really excited is shown by the fact that a negative variation (Chap. XI.) is set up in the nerve when the lungs are inflated. An electrical change, although not so pronounced, is also observed when air is sucked out of the lungs (Alcock and Seemann).

When the normal excitation of the vagus fibres by expansion of the lungs is exaggerated by closing the trachea at the end of inspiration, the diaphragm inmediately relaxes, and a long expiratory pause ensues, broken at last by a series of inspirations much deeper and more prolonged than those which were taking place before occlusion. When the trachea is occluded 
at the end of expiration, a series of deep and long-drawn inspirations occurs, the first of which begins at the monent when the next nomal inspiration ought to have taken place had the windpipe been left free. The most obvious explanation of these results is that the expansion of the lungs sets up impulses in the vagi which cut short the inspiratory activity of the respiratory centre (inspiration-inhibiting fibres), while in collapse inpuises are set up which excite it to renewed inspiratory discharge (inspiration-exciting fibres). Since ordinary cxpiration is in the main not associated with active muscular contraction, the inspiration-inhibiting fibres would be at the same time expiration-exciting. Clearly this would constitute a so-called 'selfsteering' arrangenent, each inspiration leading inevitably to the succeeding expiration, and each expiration providing the neces-

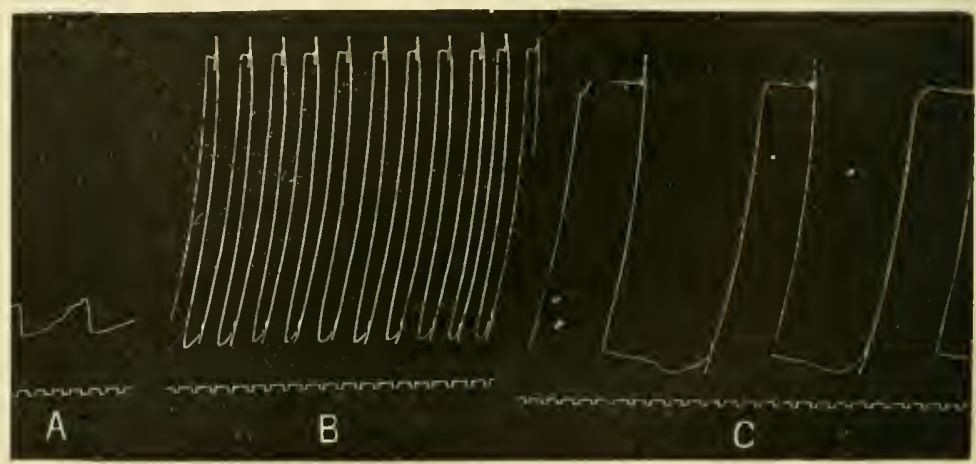

Fig. IO3.-RESPIRATORY TRACINGS: 1)OG.

A, normal : 13, effect of stimulation of the central end of vagus ; $C$, effect of section of both vagi. (Tracing taken as in Fig. 129, p. 285.) Time-tracing, seconds.

sary stimulus for the succeeding inspiration. On this hypothesis section of the vagi must necessarily be followed by slowing of the respiratory movements, and we have seen that this is the case.

A rival liypothesis is that the antomatic activity of the respiratory centre leads normally to the discharge of motor impulses to the inspiratory muscles, which are cut short at each expansion of the lungs by the inhibitory action of the ragus, the nerve not boing excited during pulmonary collapse, and therefore carrying no inspiratory impulses to the centre. On this assumption, we may think of the centre as being ' wound up ' like a clock, the periodic arrival of regulating impulses acting like an escapenent movement, and allowing a certain amount of clischarge. When the vagi are cut the inspirations are greatly prolonged and deepened, because the check on the discharge of the centre has been remored. 
Attempts have been made by experimental stimulation of the vigrus trunk to determine whether, as a matter of fact, it contains both inspiratory and expiratory fibres. But the results are neither so clear nor so coustant that we can confidently ilppeal to them in making a decision, and even some of the investigators who maintain the existence of but one anatomical set of fibres believe that these are affected differently by different kinds of stimulation momentary stimuli, for example, setting up in them impulses which we may call inspiratory, and longlasting stimuli impulses which we may call expiratory.

Excitation of the central end of the cut vagus below the origin of its superior laryngeal branch, with induction shocks of moderate strength, certainly causes quickening of respiration. If the
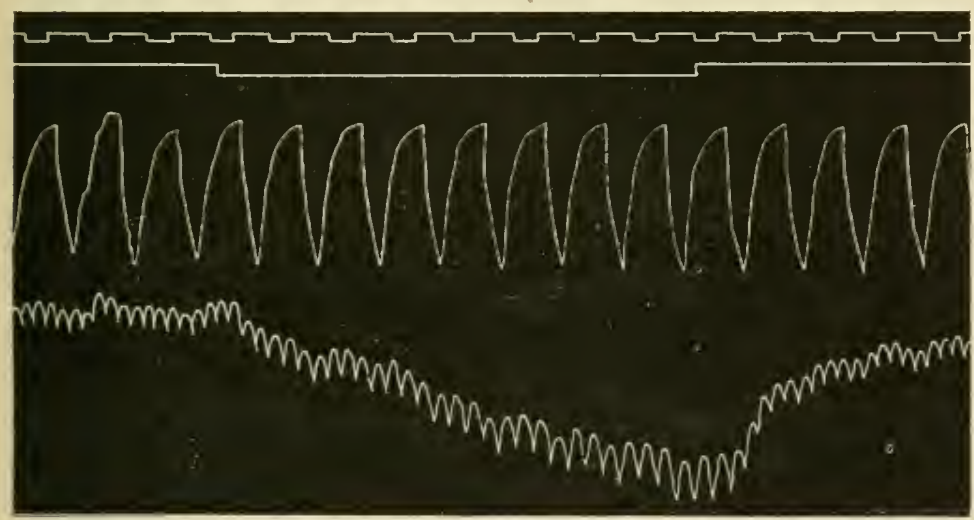

Fic. IO4.-EFfect of Stintlation of CeNtral Exd of VAgUs ix a Cat. Liper Trace, Respiration; LOWER Trace, Blood-presstre.

At the top are the time trace (seconds), and below it the signal line, the depres. sion in which indicates the duration of the excitation. Practically no effect was produced on the respiration, but a fall of blood-pressure with slowing of the heart.

cxcitation be strong, there is arrest in the inspiratory phase. A brief mechanical stimulus, or a series of such, has a similar effect. But chemical stimulation (e.g., with a strong solution of potassium chloride) or long-continued mechanical excitation like that produced by stretching or compression of the nerve, or certain kinds of electrical stimulation-for instance, the very weakest induction shocks, or the closure of an ascending roltaic current*_cause slowing of the respiratory movements or expiratory standstill. This is also the usual, though not the invariable, result of stimulating the superior laryngeal, even when weak induction shocks are employed. With stronger stimulation energetic contractions of the expiratory muscles

* I.e., a current passing towards the head in the nerve. 
11aty occul. These fatcts undoubtedly suggest the existence in the vagus of two kinds of afferent nerve-filses that affect the respiratory centre in opposite ways-inspiratory fibres, which stimulate it to greater activity of discharge, and expiratory fibres, which inlibit its action. The latter varicty we may suppose to be more numerous in the superior laryngeal, the former in the pulmonary branches of the vagus. And there is nothing forced in the hypothesis that cortain kinds of stimuli act particularly on the one set of fibres, and certain kinds on the other, for we have already seen an instance of this in studying the differences between the vaso-constrictor and the vaso-dilator nerves (p. I59).

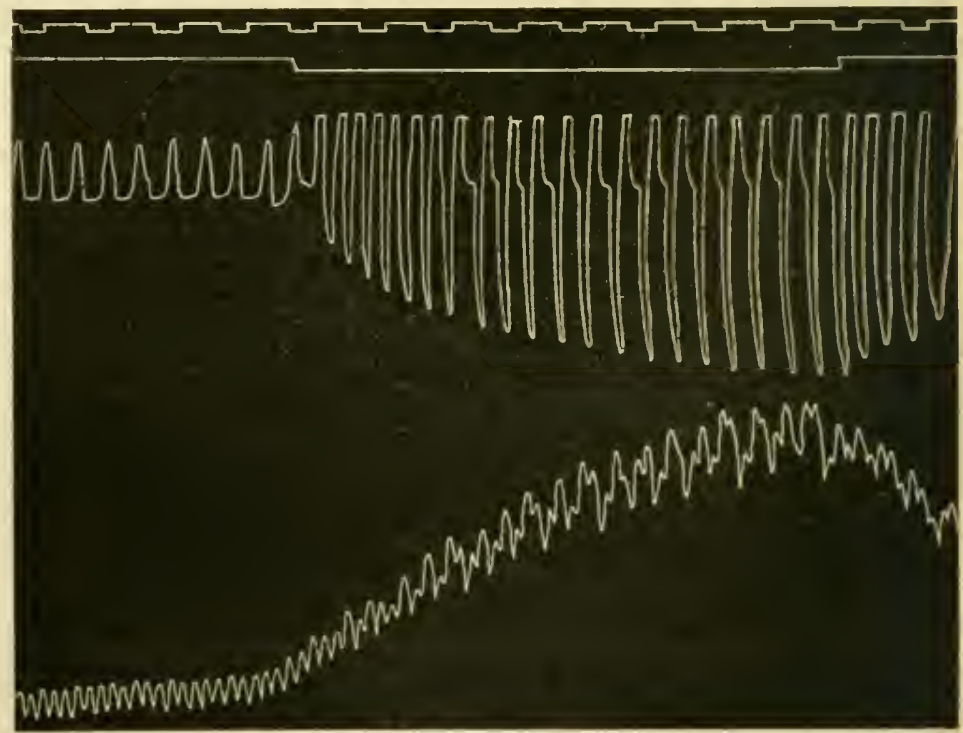

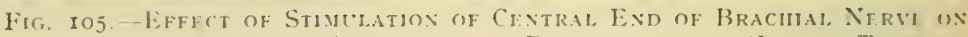
RESPRATION (IPPIR TKACING) ANI) BLORD-PRLSSLRE (LOWLR TRACISG) IX THE CAT.

At the top of the figur. are the lime trace (seeronds) and the signal line, shrwing beginning and end of stimulation.

The most probable conclusion, and the one which best reconciles the conflicting hypotheses, is that two sets of fibres are present: (I) Fibres which inhibit inspiration (and cause expiration), and are excited in ordinary inspiration by the cxpansion of the lungs. (2) Fibres which cause inspiration (and inhibit expiration), and are excited in strong expiration, as in dyspnœa, by the collapse of the lungs, but are not active in ordinary expiration. 
However this mav be, the facts we have been discussing have an importance of their own, apart from any hypothetical explanations of them. Some of them have been more than once unintentionally illustrated on man. In one case the left ragus trunk was included in a ligature with the common carotid. The respiratory movements immediately stopped, the pulse was slorred, and death occurred in thirty minutes (Rouse). The superior laryngeal fibres, unlike those of the vagus proper, are not constantly in action, as section of both nerves lias no effect on respiration. Any source of irritation in the larynx may stimulate these fibres and produce a cough, which may also be cansed by irritation of the puhnonary fibres of the vagus.

The cutaneous nerves, and especially those of the face (fifth nerve), abdomen and chest, have a marked influence on respiration. They can be easily excited in the intact body by thermal and mechanical stimulation. A cold bath, for instance, usually causes acceleration and deepening of the respiratory movements; and the efficacy of mechanical stimulation of sensory nerves in stirring up a sluggish respiratory centre is well known to midwives, who sometimes slap the buttocks of a newborn child to start its breathing. The reflex expiratory standstill caused in rabbits by inhalation of such sharp-smelling substances as ammonia, acetic acid, and tobacco-smoke is due to afferent impulses passing up the trigeminus fibres from the mucous membrane of the nose, and is still obtained after section of the olfactory nerves.

Another set of afferent nerves which have been supposed by some to bear an important relation to the respiratory centre are those which supply the muscles. We have already noticed that the frequency of respiration is greatly augmented by muscular exercise. The simplest explanation would seem to be that afferent muscular nerves are stimulated either by mechanical compression of their terminal 'spindles,' or by the chemical action on them of certain waste products produced in contraction. It is quite likely that this is one way in which the adjustment is achieved. But this is not the only, and perhaps not the most important, way. For an increase in the respiratory movements is caused by tetanizing the muscles of a limb whose nerves have been completely severed, and which is indeed connected with the rest of the body by no other structures than its hloodvessels. This can only be due to two things : a direct action on the respiratory centre by the blood that has passed through, and been altered in, the contracting muscles, or an action exerted by the blood indirectly on the centre through the excitation of afferent respiratory nerves whose connection with it is still intact-for example, the other muscular nerves or the pul- 
monary branches of the vagus. That the sction is direct is shown by the fact that after section of the vagi, the sympathetic, and the spinal cord below the origin of the phrenics, an increase in the respiratory movements is still produced by tetanizing a $\lim b$.

It is generally acknowledged that the respiratory centre may be excited botli by blood that is ricli in carbon dioxide and by blood that is poor in oxvgen, the actual stimulating substance in the latter case being, perliaps, an easily oxidizable bodypossibly lactic acid-which rapidly disapjears from properly oxygenated bloorl.

But it luas been the subject of long-continued discussion whether excess of carbon dioxide or deficiency of oxrgen is the more potent stimulus. The best evidence points to the conclusion that comparatively small alterations in the amount of carbon lioxide in the inspired air cause a relatively great increase in the respiration, while in the case of the oxygen the cleparture from the normal proportion must be much more decided to bring about any notable effect. Nor is it at all ont of harmony with this that, when very large quantities of carbon dioxide (30 per cent. and upwards in rabbits) are inllaled, a condition of narcosis comes on without any previous respiratory clistress. For many substances act differently in large and in small doses. Haldane has pointed out how exquisitely sensitive the respiratory centre is to even small changes in the partial pressure of carbon dioxide in the alveolar air, and therefore in the blood and the centre itself, and luas demonstrated that this is the way in which the amount of the pulmonary ventilation (the volume of air breathed per unit of time) is chiefly regulated in ordinary breathing.

For instance, an increase of as little as 0.2 per cent. of carbon dioxide in the alreolar air, corresponding to an increase of $\mathrm{I} .4 \mathrm{~mm}$. of mercury in the partial pressure (p. $2+8)$ of the gas, caused an increase in the pulmonary ventilation of roo per cent. The alveolar oxygen pressure had to be diminished to I3 per cent. of an atmosphere before any decided increase in the respiration occurred. 1)uring moderate muscular work the percentage of "arbon dioxide in the alveolar air, and therefore in the blood, increases slightly, causing an increase in the rentilation, and this is one of the ways in which the hyperpmoea associated with muscular exercise is brought about. In serere work lack of oxygen, with accummlation of lactic acid and ot leer metabolic products, which stimulate the respiratory centre or render it excitable by smaller pressures of carhon dioxide, also plays a part.

To sum up, the regulation of normal breathing is trofold-a 
chemical regulation (through the carbon dioxide) of the amount of air moved into and out of the lungs per unit of time; and a nervous regulation (chicfly through the vagi) of the rate and depth of the movements necessary to effect the given amount of ventilation.

When the vagi have been divided, an increase in the carbon dioxide pressure within certain limits is responded to by an increase in the total ventilation, just as in the normal animal, but the form of the response is different. Whereas in the normal animal both the rate and the depth of respiration are increased, in the ragotomized animal there is a marked increase in depth, with little or no increase in rate (Scott).

When the gaseous exchange in the lungs from any cause liecomes insufficient, the respiratory movements are exaggerated, and ultimately every muscle which can directly or indirectly act upon the chest-wall is called into play in the struggle to pass more air into and out of the lungs. To a lesser and greater degree of this exaggeration of breathing the terms Hyperpncea and Dyspncen have been respectively applied. If the gaseous interchange remains insufficient, or is altogether prevented, asphyxia sets in. Sometimes in man impending asphyxia from loss of function by a part of the lungs (with crippling of the lesser circulation), as in pneumonia, may be warded off by inhalations of oxygen. Increase in the temperature of the blood circulating through the spinal bulb, as when the carotid arteries of a dog are laid on metal boxes through which hot water is kept flowing, also causes dyspnœa (heat-dyspncea) (p. 29o). But if the temperature be too high, the respiratory movements may be slowed, perhaps by a partial paralysis or inhibition of the respiratory centre. When the blood is cooled the respiration becomes deeper and slower, but if the temperature is greatly and suddenly lowered, the centre may be stimulated and the breathing quickened. In man the increased temperature of the blood in fever is a cause, though not the only one, of the increase in the rate of respiration.

Apnœa.-The physiological opposite of dyspnœa is apncea. This condition may be produced in an animal by rapid or prolonged artificial respiration. It is especially easy to obtain in an animal in which the circulation through the brain and bulb is interrupted for a time and then restored, while artificial respiration is being kept up. Spontaneous respiration returns after a longer or shorter interval, but if the artificial respiration be still maintained, it again ceases. In a successful experiment the animal remains without breathing for many seconds after the artificial respiration is stopped. In apnœa the chest remains at rest in the expiratory phase if the lungs lave been inflated by the artificial respiration and then allowed to collapse of themselves (expiratory apnœa), but in the inspiratory phase if they 
luave been emptied by suction and then permitted of themselves to expand (inspiratory apnoea). The apnoea is not produced, as some have thought, by the accumulation of an excess of oxygen in the blood, for rapid and repeated inflation of the lungs with hydrogen may cause the condition. Indeed, towards the enci of the apnocic period the venous blood may be very distinctly poorer in oxygen than normal venous blood. Apnoxa is easily caused in man by a period of deep and rapid breathing and in other ways. The essential thing in this chemical or true apncea (apncea vera) is the lowering of the partial pressure of carbon dioxide in the alveolar air, and therefore in the arterial blood and the respiratory centre. The carbon dioxide is washed out of the body, so to say, by the excessive pulmonary ventilation.

In addition to chemical apnoea, which is obtainable whether the ragi are intact or not, a so-called mechanical apnoea, or apnoea vagi, exists - that is to say, a stoppage of the respiration due to an inhibitory effect produced through the vagi on the respiratory centre when the vagus endings in the lungs are excited mechanically by inflation. Some observers state that this vagus apnoea does not outlast the inflation. Others believe that the results of successive inflations can be 'summated' in the centre, giving rise to an apnœea which persists after stoppage of the artificial respiration. That a 'memory' of a prolonged rhythmical inflation of the lungs can impress itself in some way on the respiratory centre is shown by the curious phenomenon that in resuscitation of the bulb after a period of ancenia the natural respiration, when it returns, may have for a short time exactly the same rhythm as the artificial respiration which has just been stopped.

That the blood when the gaseous exchange in the lungs is interfered with produces dyspnœea by acting on some portion of the brain may be shown in an interesting manner by establishing what is called a cross-circulation in two rabbits or dogs. The vertebral arteries and one carotid are tied in both animals; the remaining carotids are divided and connected crosswise by glass tubes, or, what is better, as it avoids the risk of clotting, they are crossed by suturing the cut ends, so that the brain of each is supplied by blood from the other. When the respiration is artificially hindered or stopped in one of the animals, it shows no dysproea ; it is in the other, whose brain is being fed with improperly ventilated blood, that the respiratory movements become exaggerated. The point of attack of the 'venous' blood has been further localized in the spinal bulb by the observation that when the brain has been cut away above it, the cord severed below the origin of the phrenics, and all otler nerves connected with the region between the two planes of section divided, any interference 
with the gaseous exchange in the lungs is at once followed by dyspncea.*

Automaticity of the Respiratory Centre.-The question has been raised whether, in the absence of this 'natural' stimulation by the bloor, and of the impulses that constantly reach the centre along its afferent nerves, it would continue to discharge itself, or whether it would sink into inaction. $\mathrm{We}$ have already discussed a similar question in regard to the cardiac and vaso-motor centres, and the subject must again present itself when we come to examine the functions of the central nervous system. In the meantime it is only necessary to say that there is evidence that it is not the mere presence of carbon dioxide (or other substances) in the blood circulating through the respiratory centre which determines the constant excitation of the centre, but rather the accumulation of carbon dioxide in the centre itself when the partial pressure of that gas in the blood is raised. The idea that the continuous excitation of the centre is ' autochthonous ' - in other words, that it is due to an internal stimulating substance or substances manufactured in the centre itself, as well as carried to it in the blood-renders it easy to understand that the discharge of the respiratory centre, although modified by the quality of the blood which circulates in it, is not essentially dependent on it. Indeed, in cold-blooded animals whose blood has been replaced by physiological salt solution, and (in frogs) even after the circulation has been stopped altogether by excision of the heart, quiet, regular breathing may be seen for a considerable time. Of course, blood is essential for the continued nutrition of the centre and its connections, and it eventually breaks down and ceases to discharge. The respiratory discharge is still less dependent for its initiation upon the arrival of afferent impulses. For after section of the bulb above the centre, of the cord below the origin of the phrenics, of the vagi and of the posterior roots of all the upper cervical nerves, the spasmodic respiration which we have already described as occurring when the vagi and the higher paths have been severed continues without essential modification. It has also been observed that during resuscitation of the bulb and upper cervical cord after a period of anæmia, stimulation of afferent nerves, including the vagi, is entirely without influence on the respiratory movements for some time after respiration has returned, presumably because the synapses (p. 749) on the afferent paths lying within the previously anæmic area are as yet unable to conduct the nerve impulses. Nevertheless, the respiratory centre con-

* The conclusion is doubtless correct, but this experiment is not decisive. For the phrenic nerves themselves contain afferent fibres, the stimulation of which can influence the respiration after section of the vagi. 
tinues steadily to discharge itself along the efferent paths, whose srnapses are situated berond the anxmic region. Section of the bull above the level of the respiratory centre, and of the cord below the origin of the phrenic nerves, in addition to the anamia, makes no essential difference in the result. The initial rate of discharge of the centre thus isolated from afferent impulses is apjhroximately constant in different experiments (about four a minute in cats).

Action of Drugs on the Respiratory Centre.-The respiratory centre is directly affected by numerous drugs. Pituri and nicotine, for instance, cause in various animals a quickening and deepening of the respiration, followed, if the dose has been large, by slowing and ultimate cessation. The action of the great majority of such substances, however, possesses only a pharmacological interest, and it would be out of place even to enumerate them in a text-book of physiology. But there are one or two points in the action on the respiratory centre of chloroform and alcohol-substances so greatly employed in practical medicine and in physiological research-which may properly be touched on here.

Chloroform.--The cause of the deaths from chloroform which, at rare intervals, startle the operating theatre of every great hospital where this anæsthetic is nsed, has been, on account of its extreme practical interest, the subject of prolonged discussion and experiment. Is it the heart that fails? Or is it the respiration? The answer of what is known as the 'Edinburgh School' was that the respiration (in physiological terms, the respiratory centre) is always first paralyzed. Their golden rule of doctrine in chloroform administration was. "Watch the respiration; the heart will take care of itself'-a rule which, however, in 'Edinburgh' practice did not exclude careful observation of the pulse. This view, having the merit of simplicity, was widely adopted. It was upheld by a scientific commission appointed by the Nizam of Hyderabad to investigate the question with the aid of modern physiological methods. But the conclusions of the Hyderabad Commission seem to have been too absolutely drawn. For it has been shown by a number of observers that chloroform may paralyze the heart without primarily affecting the respiration; and, further, that paralysis of the vaso-motor centre, and the consequent withdrawal of blood from the heart and brain to the dilated splanchnic area, may be an important factor in bringing about a fatal result (p. I74). In nomal chloroform anæsthesia in man it is easy to demonstrate by the sphygmomanometer a fall of blood-pressure in the brachial artery of 20 to $f 0 \mathrm{~mm}$. of mercury (Hill). It would seem that death from chloroform may take place either from 
primary failure of the respiratory centre followed by failure of the heart, or from primary paralysis of the heart or of the whole rascular mechanism (including the muscular tissue of the heart and bloodvessels and the vaso-motor centre), followed by paralysis of the respiratory centre. Sometimes the respiratory failure and the vascular paralysis may be simultaneous; often one may follow so hard on the heels of the other that it is difficult to decide which is primary and which secondary. Much depends upon the concentration of the chloroform vapour. Where it is dilute (at any rate, up) to 5 per cent. of the inspired air in cats), and is administered by means of an apparatus which insures a definite and uniform concentration, the respiration invariably stops before the heart. The chloroform is mainly taken up by the coloured corpuscles. The percentage of the drug in the blood increases very rapidly in the first minutes of administration, and then rapidly declines, to increase again to a maximum, which is now maintained during the remainder of the anæsthesia. In those first few minutes occurs a definite danger point as regards the respiratory centre (Buckmaster). The stronger the chloroform mixture inhaled the greater is the damage to the vascular mechanism relative to that inflicted upon the respiratory apparatus, and the more rapidly does it become irreparable. It has been shown that the concentration of chloroform necessary to produce serious effects upon the isolated mammalian heart when added to the liquid used for perfusion is practically identical with that found in the blood of animals fully narcotized with the drug by inhalation. In ether narcosis the quantity of the anæsthetic in the blood is not sufficient to seriously affect the heart, and this is the reason why ether is so much safer than chloroform. The practical lesson is that in administering chloroform both the respiration and the pulse must be watched. The drug should be given by a method which allows exact control of its concentration in the air. The primitive drop-bottle and towel method should be abandoned. In addition to the dangers connected with the direct action of chloroform on bulbar centres, the possibility of untoward reflex effects must be borne in mind. At a certain stage in chloroform anæsthesia, before it has become very deep, comparatively trifling causes may bring about great and sudden changes in the pulserate, owing to the abnomal mobility of the vagus centre (MlacWilliam). It is further of interest in connection with the causation of death during the administration of anæsthetics that the afferent nerves of the alveoli can be chemically stimulated when irritant vapours, such as chloroform, hydrochloric acid, ammonia, bromine, or formaldehyde are inhaled through a tracheal cannula, causing reflex arrest of the heart and of the respiratory 
movements and a fall of blood-pressure through vaso-clilatation (Brodie).

Alcohol in small doses, when given by the stomach or (in animals) injected into the blond, causes stimulation of the respiratory centre and increase in the pulmonary ventilation. In man, this increase usually amounts to $S$ to 15 per cent., but is occasionally much greater. But the limit which separates the favourable action of the small dose from the hurtful action of the large, is easily overstepped. When this is done, and the dose is continually increased, the activity of the respiratory centre is first diminished and finally abolished. In dogs, for instance, after the injection of considerable quantities of alcohol into the stomach, death takes place from respiratory failure, and the breathing stops while the heart is still unweakened (Fig. 73, p. I75). This is the final outcome of a progressive impairment in the activity of the centre, of which the slow and heavy breathing of the drunken man represents an earlier stage.

Spinal Respiratory Centres.-Although the chicf respiratory centre lies in the medulla oblongata, under certain conditions impulses to the respiratory muscles may originate in the spinal cord. Thus, in young mammals (kittens, puppies), especially when the excitability of the cord has been increased by strychnine, in birds and in alligators, movements, apparently respiratory, have been seen after destruction of the brain and spinal bulb. In adult cats, when the functions of the brain, medulla, and cervical cord have been abolished by occlusion of their vessels, similar movements of the thoracic and abdominal muscles may be seen, but they are not sufficient for effective respiration. No proof has ever been given that in the intact organism the spinal cord below the level of the bulb takes any other part in respiration than that of a mere conductor of nerve impulses; and it is not justifiable to assume the existence of automatic spinal respiratory centres on the strength of such experiments as these.

Death after Double Vagotomy.-Alterations in the rhythm of respiration are not the only effects that follow division of both vagi (or vago-sympathetics) in the neck. In certain animals, at least, this operation is incompatible with life. In the rabbit, as a rule, death takes place in twent $y$-four hours. A sheep may live three days, and a horse five or six. I)ogs often live a week, occasionally a month or even two, and in rare instances they survive indefinitely: The most prominent symptoms (in the $\log$ ), in addition to the marked and permanent slowing of respiration, quickening of the pulse and contraction of the pupils, are difficult deglutition, accompanied by frequent romiting and progressive emaciation. The appetite is sometimes ravenous, but no sonner is the food swallowed than it is rejected; and this is particularly true of water or liquid 
food. Sometimes the rejected food is simply regurgitated alter having reached the lower end of the asophagus, without entering the stomach. The fatal result is usually caused, or at least preceded, by changes of a pnemunnic nature in the lungs. The precise significance of the puhnonary lesion is obscure. But it would seem that paralysis of the laryngeal and cesophageal muscles, with the conseguent entrance of saliva, food, or foreign bodies, carrying bacteria into the lungs, is responsible to a great extent. And when only a partial palsy of the glottis is produced, by dividing the right vagus below the origin of the recurrent laryngeal, and the left as usual in the neck, pnemmonia either does not occur or is long delayed. It may be that the tissue of the lungs is rendered particularly susceptible to such insults in consequence of trophic or vascular changes induced by section of the pulmonary and cardiac fibres in the vagi. It may be quite clearly demonstrated, however, in animals which live for some weeks, that, notwithstanding the paralysis of the glottis associated with aphonia, no pulmonary symptoms may be present till a day or two before death. The picture presented in these cases is that of an animal suffering, above all, from alimentary disturbances. The respiration is, to be sure, very different from the normal in frequency. depth, and type, but there is nothing to suggest that the lungs are the seat of any pathological process. Suddenly the picture changes. Pulmonary symptoms obtrude themselves. The physical signs of consolidation of the lungs may be detected, and in a short time the animal is inevitably dead. Occasionally the determining cause of the pulmonary lesion seems to be some external circumstance, as a sudden fall of the air temperature. The idea is exceedingly apt to present itself to the observer that the pneumonia is an accident, an acute intercurrent affection breaking the course of a chronic malnutrition, which in any case must lave ended in death. Of course, the ragotomized animal is predisposed to this accident, but there is no definite time after section of the nerves at which it must take place. The romiting is certainly connected with the paralysis and consequent dilatation of the cesophagus; and by previously making an artificial opening into the stomach, or by a surgical prophylaxis still more heroic, the establishment of a double gastric and cosophageal fistula (p. 374), death may be prevented for many months. Elimination of all the pulmonary fibres of the ragi, by extirpation of one lung, followed after an interval by section of the opposite vagus in the neck, is not fatal in rabbits. This is also in farour of the view that in double ragotomy the stress falls mainly on the digestive system.

Innervation of the Bronchial Muscles.-Both constrictor and dilator fibres for the bronchi are contained in the vagus. 
They are not constantly in action, but can be reflexly excited, most easily (in the dog and cat) by stimulating the nasil mucous membrane, and particularly at small area well back upon the nasal septum. Cauterization of the corresponding area in man is said to give permanent relicf in certain cases of spasmodic astluna, a condition in which the recurrent atlacks of dyspnoxa seem, according to the most generally accepted view; to be associated with spasm of the bronchial muscles.

Special Modifications of the Respiratory Movements. Cheyne-Stokes Respiration is the name given to at peculiar tyje of breathing, marked by pauses of many seconds alternating with groups of respirations. In each group the nowements grarlually increase to a maximum amplitude, and then becone gradually shallower again, till they cease for the next pause. The phenomenon often occurs in certain discases of the brain and of the circulation, and pressure on the spinal bulb may produce it. In cats in which the circulation in the brain and medulla ololongata has been interrmpted for a time and then restored it is often noticed at a certain stage of resuscitation of the respiratory centre. In frogs, Cheyne-Stokes breathing has been observed as the result of interference with the circulation in the spinal bulb, 'drowning,' or ligature of the aorta, and also as a consequence of removal of the brain, or parts of it (hemispheres and optic thalami). But it is not peculiar to pathological conditions, heing also scen, more or less perfectly, in normal sleep, especially in children, in healthy men at ligh altitudes, in libernating animals, and in morphine and chloral poisoning.

Well-marked Cheyne-Stokes brcathing can be obtained experimentally in normal persons in a variety of ways. If, for example, the subject is caused to breathe deeply and frequently for about two minutes, so as to produce a prolonged apncea, the respiration, when it is resumed spontaneously, is of the Cherne-stokes type (Haldane). The explanation given by Haldane is that the fall in the partial pressure of the oxygen in the pulmonary alveoli (p. 232) during the primary apncea, with the conseguent fall of oxygen pressure in the arterial blood and the respiratory centre, leads to the production of lactic acid in the respiratory centre and elsewhere, which stimulates the centre in the same way as carbon dioxide, and thus permits it to be excited by a smaller partial pressure of carhon dioxide than that nomally necessary: As soon as the pressure of carbon dioxide, which is increasing during the period of apnoea, has reached the exciting value breathing is resumed. The respirations, beginning as very feeble movements, rapidly increase in strength till the breatling becomes quite decp) or actually dyspneic. The store of oxygen is replenished by this thorough ventilation of the lungs, the changes 
in the excitability of the respiratory centre due to lack of oxygen (lisappear (perlapes by oxidation of the lactic acid), and the centre relapses into a period of repose. During this periond of apnocil the oxygen pressure sinks once more to the point at which the cliange in the excitability of the respiratory centre by carbon dioxide occurs, and the breathing again starts. In pathological cases the want of oxygen may be associated either with deficient circulation through the bulb-centre or with deficient intake by the lungs. The administration of oxygen through at mask has been shown in suclı cases to abolish the priodicity in the respiration, and to render it more nomal.

Peculiarly modified, but more or less normal, respiratory acts are coughing, sneezing, yawning, sighing, and hiccup).

A cough is an abrupt expiration with open mouth, which forces open the previously closed glotis. It may be excited reflexly from the mucous membrane of the respiratory tract or stomach through the afferent fibres of the vagus, from the back of the tongue or mouth, and (by cold) from the skin.

Sneezing is a violent expiration in which the air is chiefly expelled through the nose. It is usually excited reflexly from the nasal mucous membrane through the branch of the fifth nerve which supplies it. Pressure on the course of the nasal nerve will often stop a sneeze. A bright light sometimes causes a sneeze, and so in some individuals does pressure on the supraorbital nerve, when the skin over it is slightly inflamed.

Yawning is a prolonged and very deep inspiration, sometimes accompanied with stretching of the arms and the whole body. It is a sign of mental or physical weariness.

A sigh is a long-drawn inspiration, followed by a deep expiration.

Hiccup, or hiccough, is due to a spasmodic contraction of the diaphragm, which causes a sudden inspiration. The abrupt closure of the glottis cuts this short and gives rise to the characteristic sound. The following readings of the intervals between successive spasms were obtained in one attack: I3 secs., I 2 secs., I5 secs., 9 secs., I4 secs., etc.-i.e., one-fourth or one-fifth of the frequency of the ordinary respiratory movements. The mere fixing of the attention on the observations soon stopped the hiccup.

Hiccup is generally considered to be a reflex movement, brought about through the respiratory centre by afferent impulses originating in the stomach. The irritation may be merely due to some slight digestive disturbance set up by overfilling of the stomach, perhaps. This is exceedinglycommon in infants. But persistent hiccup may also be a distressing symptom of very formidable diseases-for example, carcinoma of the pylorus. Experimentally, reflex contractions of the diaphragn can sometimes be elicited by stimulation of the central end of the vagus at 
a time when no spontaneous respiratory movenents are going on. This has been observed, for instance, in cats during resuscitation of the brain after a period of ancmia. In man also, in a case of Clieyne-Stokes respiration accompanied by hiccup, it was seen that the hiccup persisted during the periods of apucea. If the respilatory centre is the centre for the hiccup reflex, it can therefore be excited by afferent nervous injpulses at a time when it is not excited by the normal chenical stimulus (Machicnzie and Cushny).

\section{Chemistry of Respiration.}

Our knowledge of this subject las been entirely acquired in the last 200 years, and cliefly in the last century.

Boyle slowed by means of the air-pump that animals dic in a vacumm, and Bernouilli that fish cannot live in water from which the air has been driven out by boiling.

Mayow, of Oxford, seems to a considerable extent to have anticipated Black, who in 1757 demonstrated the presence of carbonic acid (carbon dioxide) in expired air by the turbidity which it causes in line-water.

A most fundamental step was the discovery of oxygen by Priestley in I77 I, and his proof that the venous blood could be made crimson, like arterial, by being slaken up with oxygen.

Lavoisier discovered the composition of carbonic acid, and applied his discovery to the explanation of the respiratory processes in animals, the heat of which he showed to be generated like that of a candle by the mion of carbon and oxvgen. He made many further important experinents on respiration, publishing some of lis results in 1789 , when the French Revolution, in which he was to be one of the most distinguisled victins, was breaking out. He made the mistake, however, of supposing that the oxidation of the carbon takes place in the blood as it passes through the lesser circulation.

That some carbon dioxide is formed in the lungs there is 110 reason to doubt, and the quantity may even he considerable. But that they are not the chief seat of oxidation was sufficiently proved as soon as it was known that the blood which comes to them from the right lieart is rich in carbon dioxide, while the blood which leaves them through the pulmonary veins is comparatively poor.

There are two main lines on which research has gone in trying to solve the chemical problems of respiration: (I) The analysis and comparison of the inspired and expired air, or, in general, the investigation of the gaseous exchange between the blood and the air in the lungs. (2) The analysis and comparison of the gases of arterial and venous blood, of the other liquids, and of the solid tissues of the body, with a view to the determination 
of the gaseous excliange between the tissues and the blond. IVe shall take these up as far as possible in their order.

The methods which liave been used fol comparing the composition of inspired and expired air are very various.

(1) Breathing into one spirometer and out of another, the inspired and expired air being directed by valves. The contents of the spirometers are analyzed at the end of the experiment (Speck). In the arrangement of $\%$ ant\% and Creppert, insteat of the whole of the expired air, a sample is collected for analysis during the entire duration of the experiment, while the total volume expired is measured by a gas-meter. This is a very convenient method for observations on man, especially in clisease, but each experiment can only be carried on at most for fifteen to twenty minutes.

(2) A small apparatus, much on the same principle, was used for rabbits by Pfluger and his pupils. A cannula in the trachea was connected with a balanced and self-arljusting spirometer containing oxygen, and the inspired and expired air separated by potassium hvilroxide valves, which absorbed the carbon dioxide. The amount of oxygen used could be read off on the spirometer, and the amount of carbon dioxide produced estimated in the liquid of the valves.

(3) Elaborate arrangements, such as Pettenkofer's great respiration apparatus, and the still larger and more efficient modifications of it constructed since his time, in which a man, or even several men, can remain for an inclefinite period, working, eating, and sleeping. Air is drawn ont of the chamber by an engine, its volume being measured by a gas-meter. But as it would be far too troublesome to analyze the whole of the air, a sample stream of it is constantly drawn off, which also passes through a gas-meter, through drying-tubes containing sulphuric acid, and through tubes filled with baryta water. The baryta solution is titrated to determine the quantity of carbon dioxide; the increase in weight of the drying tubes gives the quantity of aqueous vapour. A similar sample stream of the air before it passes into the chamber is treated exactly in the same way, and from the data thns got the quantity of carbon dioxide and aqucous vapour given off can readily be ascertained. The oxygen can be calculated, as the difference between the final body-weight and the original body-weight plus the weight of the carbon dioxide and water eliminated, but may also be directly estimated by special methods.

(4) Haldane and Pembrey have elaborated a gravimetric method, which is very suitable for small animals. It depends upon the absorption of carbon dioxicle by soda lime. (See Practical Exercises, p. 293). In Atwater's so-called respiration calorimeter, which will be referred to again under 'Animal Heat,' and by which, not only the gaseous metabolism, but the heat production can be measured in man, the carbon dioxicle is estimated in the same way.

The expired air is at or near the body temperature, and is saturated with watery vapour. In ordinary breathing it contains about 4 per cent. of carbon dioxide, while the inspired air only contains a trace. The expired air contains I 6 or 17 per cent. of oxygen, the inspired air about 2I per cent. The percentage of carbon dioxide in the alveolar air is, of course, greater than in the ordinary expired air, since the relatively pure air of the dead space 
constitutes a substantial fraction of the ticlal air. The carbon cloxide percentage in the alveolar air at the end of expiration, witl the body at rest, is remarkably constant in one and the sane individual at comstant atmospheric pressure (j). 257). There are in addition in expired air small quantities of hydrogen and marshgas derived from the alimentary canal, either directly from eructation or after absorption into the blood. Sometimes a trace of ammonia can be detected in the air of expiration, but tlis is clue to decomposition of proteins taking place in the mouth, especially in carious teeth, or in the air-passages and lungs in disease of these organs. It has indeed been sliown that the lungs are practically impermeable for ammonia. Expired air is entirely free from floating matter (chust), which is always present in the inspired air. The volume of the expired air, owing to its higher temperature and excess of watery vapour, is somewhat greater than that of the inspired air, but if it be measured at the temperature and degree of saturation of the latter, the volume is somewliat less. Since the oxygen of a given quantity of carbon dioxide would have exactly the same volume as the carbon dioxide itself at a given temperature and pressure, it is clear that the deficiency is due to the fact that all the oxygen which is taken up in the lungs is not given off as carbon dioxide. Some of it, going to oxidize hy diogen, reapjears as water. A small amount of it mites witl the sulphur of the proteins (1).444). The yutient of the volume of oxigen given ont as carbon clioxicle by the volume of oxygen taken in is the respiratory quotient. It shows what proportion of the oxigen is used to oxidize carbon. It may ajploach nuity on a carbohydrate diet, which contains enough oxygen to oxidize all its own liydrogen to water. With a rich diet $j$ fat it is least of all ; with a diet of lean meat it is internediate in amomnt. For ordinary fat contains no more than one-sixtl, and proteins not one-half, of the oxygen needed to oxidize their liydrogen. Inman on a mixed diet the respiratory quotient may be taken as 0.8 or 0.9. So long as the type of respiration is not clianged, the respiratory quotient may remain constant for a wide range of metabolism. In hibernating animals, however, the respiratory quotient may become very small during winter sleep (as low as 0.25 ), both the ontput of carbon dioxide and the consumption of oxygen falling cnomously, but the forner in general more than the latter. This has been explained on the assumption that oxygen is stored away in winter sleep in the form of inconpletely oxidized substances. On the other hand, in dyspnara accompanying muscular exertion the respiratory quotient has been found as ligh as I.2. It must be remembered that even a voluntary increase in the respiratory movements causes an immediate temporary increase in the respiratory quotient, owing to the 'washing out' of carbon dioxide 
from the blood and tissues. This change has no metabolic significance. Indeed, the determination of the respiratory (juotient for short periods has only a linited value, and such observations nust be interpreted with great care. In starvation the respiratory quotient diminishes, the production of carbon dioxide falling off at a greater rate than the consumption of oxygen, for the starving organism lives on its own fat and proteins, and has only a trifling carbo-hydrate stock to draw upon. In a diabetic patient, fed on a diet of fat and protein alone, the respiratory quotient was only 0.6 to 0.7 , just as in a starving man.

The amount of oxygen absorbed in a man at rest has been determined under certain conditions as about 0.29 gramme per hour, and the discharge of carbon dioxide as about 0.33 gramme per hour per kilogramme of body-weight. In an average man weighing 70 kilos the mean prodnction of carbon dioxide is about Soo grammes (400 litres) in twenty-four hours, and the mean consumption of oxygen about 700 grammes (490 litres). But there are very great variations depending upon the state of the body as regards rest or muscular activity and on other circumstances. In hard work the production of carbon dioxide was found to rise to nearly I,300 grammes, and in rest to sink to less than joo grammes, the consumption of oxygen in the same circumstances increasing to nearly I, Ioo grammes and diminishing to 600 grammes. In rest, in moderate exertion, and in hard work, the production of carbon dioxide was found to be nearly proportionate to the numbers 2,3 , and 6 respectively. When unaccustomed work is performed, the increase in the carbon dioxide output (and oxygen intake) may be much greater. With training it diminishes. In a case of diabetes the consumption of oxygen was 50 per cent. greater than in a healthy man, corresponding to the higher heat-equivalent of the food of the diabetic patient.

Ventilation.-Taking 400 litres per twenty-four hours, or I7 litres per hour, as the mean production of carbon dioxide by an average male adult at rest or doing only light work, we can calculate the quantity of fresh air which must be supplied to a room in order to keep it properly ventilated.

It has been found that when the carbon dioxide given off in respiration amounts to no more than 2 parts in I0,000 in the air of an ordinary room, the air remains sweet. When the carbon dioxide given off reaches + parts in 10,000 , the room feels distinctly, and at 6 in 10,000 disagreeably, close. while at 9 parts in I0,000 it is oppressive and almost intolerable. This has been supposed by some to be due to a volatile poison exhaled from the lungs, for pure carbon dioxide added alone in similar proportions to the air of a room has not the same bad effect. Certain observers, indeed, alleged that the condensed vapour of the breath, when injected into rabbits, produced fatal symptoms. But this 
has heen shown to be aroneous; and the most careful experiments latve failed to detect in the air expired hy lealt ly persons any trace of such a poison. It has therefore been suggested that the odour and other ill-eflects of a close room are due to substances given off in the sweat and the sebum, and allowed by persons of uncleanly liabits to accumulate on the skin, and also to the products of slow putrefactive processes constantly going on, under farourable conditions, on the walls, flonr, or furniture, but only becoming perceptible to the sense of smell when rentilation is insufficient. In a small, newly-painted chanlacr. presumably free from sucli impurities, it was not until the carbom dioxide reacled ; $10+$ per cent. that marked discomfort, with dyspunat, hegan to be felt. No close odour could be detected.

Nevertheless, experience has slown that it is a good working rule for ventilation to take the limit of permissible respiratory impurity at 2 parts of carbon dioxide per Io,000; and the I 7 litres of carbon clioxide given off in the hour will require 85,000 litres (or 3,000 cubic feet) of air to dilute it to this extent. This is the average guantity reguired for the male adult per hour. For men engaged in active labour, as in factories or mines, twice this amount may not he $t(x)$ much. For women and children less is required than for men. If a room smells close, it needs ventilation, whatever be the proportion of earbon dioxide in the air. It must be remembered that in permanenty-occupied romms mere increase in the size will not compensate for incomplete renewal of the atr, although it mat he easier to ventilate a large room than a small one without causing draughts and other inconveniences. But as few apartments are occupied during the whole twenty-four hours, a large room whicl can be thorouglily ventilated in the absence of its immates lias a distinct adrantage over a small one in its great initial stock of fresh air. The cubic space per head in an ordinary dwelling-lıouse sliould be not less than 28 cubic metres or I, ooo cubic feet.

The quantity of carbon dioxide given off (and of oxygen consumed) is not only affected by muscular work, but also by everything which influences the general metabolism. In males it is greater on the average than in females (in the latter there is a temporary increase cluring pregnancy), but for the same bodyweight and under similar external conditions there is 110 difference between the sexes. Tlie gaseous exchange is greater in probortion to the body-ıveight in the child than in the adult. This depends largely on the fact that, other things being equal, the metabolism is relatively to the body-neight more active in a small than in a large organism, since the surface (and therefore the heat loss) is relatively greater in the former. But it las been shown that even in proportion to the surface the metabolism is 
greater in youth than in adult life, and greater in the vigorous whlt than in the old man. So that the age of the organism has an influence apart from the extent of surface. The taking of food increases the gaseous exchange, partly from the increased nechanical and chemical work performed by the atimentary canal and the digestive glands. But that this is not the sole cause of the increase is shown by the fact that it varies with different kinds of food to a greater extent than can be explained by differences in the ease with which they are digested. For instance, maize produces a much greater increase than oats when given in equal amount, and a protein dict a greater increase than a diet of carbo-hydrate or fat. Sleep diminishes the production of carbon dioxide partly because the muscles are at rest, but also to some extent because the external stimuli that in waking life excite the nerves of special sense are absent or ineffective. Even a bright light is said to cause an increase in the amount of carbon dioxide produced and of oxygen consumed; but probably only by increasing muscular movements, including the movements of respiration. The external temperature also has an influence. In poikilothermal animals (such as the frog), the temperature of which varies with that of the surrounding medium, the production of carbon dioxide, on the whole, diminishes as the external temperature falls, and increases as it rises. In homoiothermal animals, that is, animals with constant blood temperature, external cold increases the production of carbon dioxide and the consumption of oxygen. But if the connection of the nervous system with the striated muscles has been cut out by curara, the warm-blooded animal behaves like the cold-blooded (Pflüger and his pupils in guinea-pig and rabbit). These interesting facts will be returned to under 'Animal Heat.'

Cold-blooded animals produce far less carbon dioxide, and consume far less oxygen, per kilo of body-weight than warm-blooded.

The following table shows the relation between the bodyweight and the excretion of carbon dioxide in man :

\begin{tabular}{|c|c|c|c|c|}
\hline \multicolumn{2}{|c|}{ Age. } & Weight in Kilos. & \multicolumn{2}{|c|}{$\begin{array}{l}\mathrm{CO}_{g} \text { excretel per } \\
\text { Killo per Hour. }\end{array}$} \\
\hline \multirow{6}{*}{ Male } & 158 & $8_{+} \cdot 6$ & \multicolumn{2}{|c|}{ O 41 gramme } \\
\hline & 44 & $76 \cdot 5$ & $0: 48$ & , \\
\hline & 35 & 65 & $0^{\circ} 5 \mathrm{I}$ & , \\
\hline & 28 & 82 & 0.49 & ," \\
\hline & 16 & $57^{\circ} 7$ & 0.59 & ," \\
\hline & $\left(9^{\circ} 6\right.$ & 22 & 0.92 & , , \\
\hline \multirow{4}{*}{ Female } & $(66$ & $66^{\circ} 9$ & $0 \cdot 39$ & \\
\hline & 30 & $53^{\circ} 9$ & 0.54 &, \\
\hline & 19 & $55^{\circ} 7$ & 0.45 & ", \\
\hline & 110 & 23 & 0.83 & , \\
\hline
\end{tabular}


The next table illustrates the difference in the intensity of metabolism in different kinds of animals, a difference, however, largely dependent upon relative size :

\begin{tabular}{|c|c|c|c|c|c|}
\hline \multirow[t]{2}{*}{ Animal. } & \multicolumn{2}{|c|}{$\begin{array}{l}\text { Oxyigen absorbed } \\
\text { per liilo per Hour. }\end{array}$} & \multicolumn{2}{|c|}{$\begin{array}{l}\text { (arbon 1)inxide given } \\
\text { off per Kilo per Hour }\end{array}$} & $\begin{array}{l}\text { Respiratory () uotient } \\
\text { (CO. or } \mathrm{O}_{2 .}\left(\mathrm{In} \mathrm{CO}_{2}\right) \text {. }\end{array}$ \\
\hline & in $\mathrm{grm}$. & in c.c. & in $\mathrm{g} \mathrm{cm}$. & in r.r. & $\mathrm{O}_{2}$ \\
\hline Greenfinch & 13000 & 9001 & $13^{\circ} 500$ & 6909 & $3 \cdot 76$ \\
\hline Hen & 1.058 & $7+0$ & 1.327 & 675 & 0.91 \\
\hline Dog & $1 \cdot 303$ & 911 & $1 \cdot 325$ & 674 & $0 \cdot 74$ \\
\hline Rabbit & 0.087 & 690 & $1 \cdot 244$ & 632 & O० ?I \\
\hline Sheep - & $0: 490$ & $3+3$ & 0.671 & $3+1$ & 0.29 \\
\hline Boar - & 0.391 & 273 & $00+43$ & 225 & $0 \cdot 82$ \\
\hline Frog - & 0.105 & $73^{\circ} 4$ & 0.113 & $57 \%$ & 0.78 \\
\hline Crayfish - & 0.054 & 38 & 0.064 & $32 \cdot 7$ & $0 \cdot 86$ \\
\hline
\end{tabular}

Forced respiration, although it will temporarily increase the quantity of carbon dioxide given off by the lungs, and thus raise for a short time the respiratory quotient, does not sensibly affect the production; it is only the store of already formed carbon dioxide in the body which is drawn upon. The amount of oxygen taken up is little altered by changes in the movements of respiration. Within wide limits the oxygen consumption of the organism is independent of the supply of oxygen offered to it.

How it is that the depth of the respiration may affect the rate at which carbon dioxide is eliminated, we can only understand when we have examined the process by which the gaseous interchange between the blood and the air of the alveoli is accomplished; and before doing this it is necessary to consider the condition of the oxygen and carbon dioxide in the blood.

\section{The Gases of the Blood.}

Physical Introduction.-Watter may be assumed to be made up of molecules beyond which it cannot be divided without altering its essential character. A molecule may consist of two or more particles of matter (atoms) bound to each other by chemical links. The kinetic theory of matter supposes the molecules of a substance to be in constant motion. frequently colliding with each other, and thus having the direction of their motion changed.

In a gas the mean free path, that is, the average distance which a molecule travels without striking another, is comparatively long, and far more time is passed by any molecule without an encounter than is taken up with collisions. Although the average velocity of the molecules is very great, these collisions will produce all sorts of differences in the actual velocity of different molecules at any given time. Some will be moving at a greater, some at a slower rate, than the average; while some may be for a moment at rest. If the gas is in a closed vessel, the nolecules will be constantly striking its 
sides and rebounding from them. If a very small opening is mado in the vessel, some molecules will occasionally hit on tho opening and escape altogether. If the opening is made larger, or the experiment continued for a louger time witl the small opening, all the molecules will in course of time have passed out of the vessel into the air, while molecules of the oxygen, nitrogen, and argon of the air will have passed in. In a gas, then, not enclosed by impenetrable boundaries, there is no restriction on the path which a molecule may take, no tendency for it to keep within any limits.

When two chemically indifferent gases are placed in contact with each other, diffusion will go on till they are uniformly mixed. The diffusion of gases may be illustrated thus. Suppose we have a perfectly level and in every way uniform field divided into two equal parts by a visible but intangible line, the well-known whitewash line, for instance. On one side of the line place 500 blind men in green, and on the other 500 blind men in red. At a given signal let them begin to move about in the field. Some of the men in green will pass over the line to the 'red' side; some of the men in red will wander to the 'green' side. Some of the men may pass over the line and again come back to the side they started from. But, upon the whole, after a given interval has elapsed, as many green coats will be seen on the red side as red coats on the green. And if the interval is long enough there will be at length about 250 men in red and 250 in green on each side of the boundary-line. When this state of equilibrium has once been reached, it will henceforth be maintained, for, upon the whole, as many red uniforms will pass across the line in one direction, as will recross it in the other.

In a liquid it is very different; the molecule has no free path. In the depth of the liquid no molecule ever gets out of the reach of other molecules, although after an encounter there is no tendency to return on the old path rather than to choose any other ; so that any molecule may wander through the whole liquid. Although the average velocity of the molecules is much less in the liquid state than it would be for the same substance in the state of gas or vapour (gas in presence of its liquid), some of them may have velocities much above the average. If any of these happen to be moving near the surface and towards it, they may overcome the attraction of the neighbouring molecules and escape as vapour. But if in their further wanderings they strike the liquid again, they may again become bound down as liquid molecules. And so a constant interchange may take place between a liquid and its vapour, or between a liquid and any other gas, until the state of equilibrium is reached, in which on the average as many molecules leave the liquid to become vapour as are restored by the vapour to the liquid, or as many molecules of the dissolved gas escape from solution as enter into it.

For the sake of a simple illustration, let us take the case of a shallow vessel of water originally gas-free, standing exposed to the air. It will be found after a time that the water contains the atmospheric gases in certain proportions-in round numbers, about $\frac{1}{100}$ of its volume of oxygen and $\frac{1}{50}$ of its volume of nitrogen (measured at $760 \mathrm{~mm}$. mercury and $0^{\circ} \mathrm{C}$.).

Now, let a similar vessel of gas-free water be placed in a large airtight box filled with air at atmospheric pressure, and let the oxygen be all absorbed before the water is exposed to the atmosphere of the box. The latter now consists practically only of the nitrogen of the air, and its pressure will be only about four-fifths that of the external 
atmosphere. Nevertheless, the quantity of nitrogen absorbed by the water will be exactly the same as was absorbed from the air. If the box was completely exhausted, and then a quantity of oxygen, equal to that in it at first, introduced before the water was exposed to it, the pressure would be found to be only about one-fifth that of the external atmospliere; but the quantity of oxygen taken up by the water would be exactly equal to that taken up in the first experiment.

Two well-known physical laws are illustrated by our supposed experiments : (I) In a mixture of gases which do not act chemically on cach other the pressure excricd by each gas (called the partial pressure of the gas) is the sume as it would exert if the others were absent. (2) The quantity (mass) of a gas absorbed by a liquid which does not act chcmically upon it is proportional to the partial pressure of the gas. It also clepends upon the nature of the gas and of the liquid, and on the temperature, increase of temperature in general diminishing the quantity of gas absorbed. It is to be noted that when the volume of the absorbed gas is measured at a pressure equal to the partial pressure under which is was absorbed, the same volume of gas is taken up at every pressure.

Suppose, now, that a vessel of water, saturated with oxygen and nitrogen for the partial pressures under which these gases exist in the air, is placed in a box filled with pure nitrogen at full atmospheric pressure. As we have seen, there is a constant interchange going on between a liquid which contains gas in solution and the atmosphere to which it is exposed. Oxygen and nitrogen molecules will therefore continue to leave the water; but if the box is large, few oxygen molecules will find their way back to the water, and ultimately little oxygen will remain in it. In other words, the quantity of oxygen. absorbed by the water will become again proportional to the partial pressure of oxygen, which is not now much above zero. On the other hand, molecules of nitrogen will at first enter the water in larger number than they escape from it, for the pressure of the nitrogen is now that of the external atmosphere, of which its partial pressure was formerly only four-fifths. In unit volume of the gas above the water there will be 5 molecules of nitrogen for every 4 molecules in the same volume of atmospheric air. Therefore, on the average 5 nitrogen molecules will in a given time get entangled by liquid molecules for every 4 which came within their sphere of attraction before. On the whole, then, the water will lose oxygen and gain nitrogen, while the atmosphere of the air-tight box will gain oxygen and lose nitrogen.

If, now, the partial pressures of oxygen and nitrogen under which the water had been originally saturated were unknown, it is evident that by exposing it to an atmosphere of known composition, and afterwards detcrmining the changes produced in the composition of that atmospherc by loss to, or gain from, the gases of the water, we could find out something about the original partial pressures. If, for example, the quantity of oxygen in the atmosphere of the chamber was increased, we could conclude that the partial pressure of oxygen under which the water had been saturated was greater than that in the chamber at the beginning of the experiment. And if we found that with a certain partial pressure of oxygen in the atmosphere of the chamber there was neitlier gain nor loss of this gas, we might be sure that the partial pressure (the temperature being supposed not to vary) was the same when the water was saturated. We shall see 
later on how this principle lass heen applied to determine the partial pressure of oxygen or carbon dioxide which just suffices to prevent blood. or any other of the liyuids of the body, from losing or gaining these gases. This pressure is evidently equal to that exerted by the gitses of the liquid at its surface, which is sometimes called their 'tension'; for if it were greater, gas would, upon the whole, pass into the blood: and if it were less, gas would escape from the blood. Thus, the tension of a gas in solution in a liquid is equal to the partial pressure of that gas in an atmosphere to which the liquid is exposed, which is just sufficient to prevent gain or loss of the gas by the liquid (p. 256 ).

The following imaginary experiment may further illustrate the meaning of the term 'tension' of a gas in a liquid in this connection :

Suppose a cylinder filled with a liquid containing a gas in solution, and closed above by a piston moving air-tight and without friction, in contact with the surface of the liguid (Fig. IO6). Let the weight of the piston be balanced by a counterpoise. The pressure at the surface of the liquid is evidently that of the atmosphere. Now, let the whole be put into the receiver of an air-pump, and the air gradually exhausted. Let exhaustion proceed until gas begins to escape from the liquid and lies in a thin layer between its surface and the piston, the quantity of gas which has become free being very small in proportion to that still in solution. At this point the piston is acted upon by two forces which balance each other, the pressure of the air in the receiver acting downwards, and the pressure of the gas escaping from the liquid acting upwards. If the pressure in the

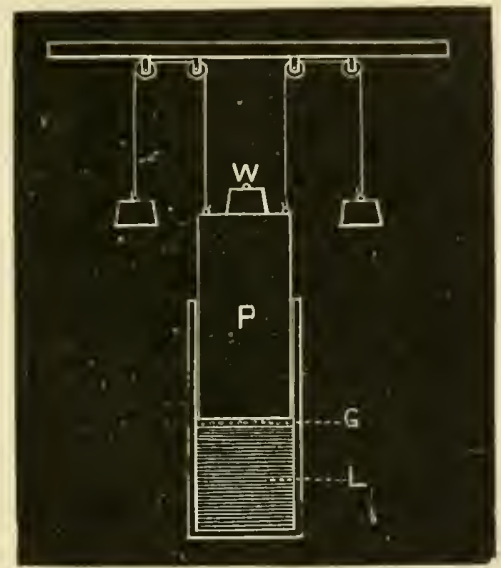

Fig. I06.-IMaginary Experiment to illustrate 'Tension' of a Gas in A Liguid.

$P$, frictionlcss piston; $L$, liquid in cylinder: $G$, gas beginning to escape from liquid. P is exactly counterpoised. In addition to the manner described in the text, the experiment may be supposed to be performed thus: Let the weight, W, be determined which. when the receiver is completely exhausted, suffices just to keep the piston in con. tact with the liquid. The pressure of the gas is then just counterbalanced by $\mathrm{IV}$; and if $\mathrm{S}$ is the area of the cross. section of the piston the pressure of the gas per unit of area is $\frac{\mathrm{W}}{\mathrm{S}}$. Or if the piston is hollow. and mercury is poured into it so as just to keep it in contact with the liquid. the height of the column of mercury required is also equal to the pressure or tension of the gas.

receiver is now slightly increased, the gas is again absorbed. The pressure at which this just happens, and against which the piston is still supported by the impacts of gaseous molecules flying out of the liquid, while no pressure is as yet exerted directly between the liquid and the piston, is obviously equal to the pressure or tension of the gas in the liquid. 
From the above principles it follows that a gas held in solution may be cxtracted by exposure to an atmosphere in which the partial pressure of the gas is mate as small as possible. Thus, oxygen can be obtained from liquids in which it is simply dissolved by putting them in an atmosphere of hydrogen or nitrogen, in which the partial pressure of oxygen is zero, or in the vacum of an air-pump, in which it is extremely small. Heat also aids the expulsion of dissolved gases. Some gases held in weak chemical union, like the looscly-

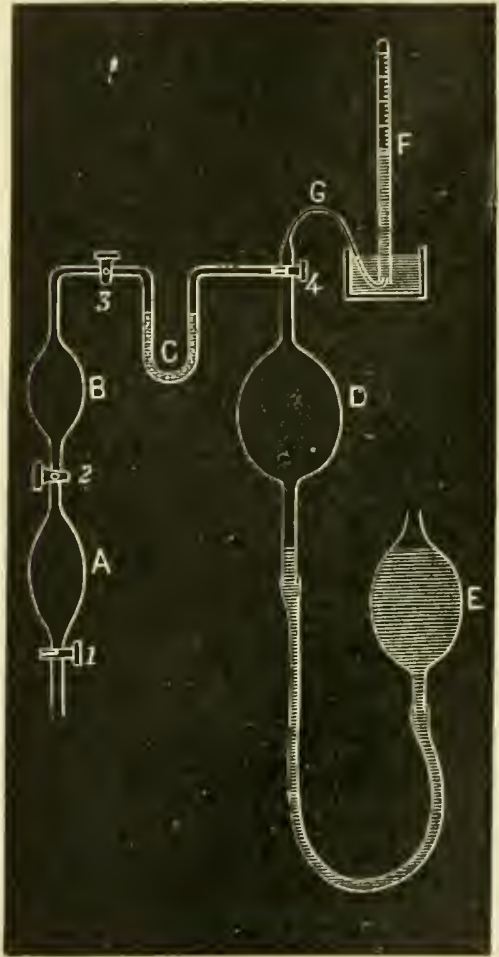

Fig. I07.-SChEME OF Gas-PUMP.

A, the blood bulb; $B$, the froth rhamber: $C$, the drying tube: 1), fixed mercury tube; $E$, movable mercury bulb connected by a llexitle tube with D ; F, eudiometer ; (i, a narrow delivery tube: $I, 2,3,4$, taps, + being a three-way tap. $\mathbf{A}$ is filled with blood by connecting the tap I by means of a tube with a bloodvessel. Taps I and 2 are then closed. The rest of the apparatus from $B$ to $D$ is now exhausted by raising $E$, with tap 4 turned so as to place D only in communication with G, till the mercury fills D. Tap 4 is now turned so as to connect C with $\mathrm{D}$, and cut off $\mathrm{G}$ from $\mathrm{D}$, and $\mathrm{E}$ is lowered. The mercury passes out of D, and air passes into it from $B$ and C. Tap $t$ is again turned so as to cut off C from D and connect $G$ and D. E is raised and the mercury passes into $D$ and forces the air out through $G$, the end of which has not hitherto been placed under $F$. This alternate raising and lowering of $\mathrm{E}$ is continued till a manometer connected between $\mathrm{C}$ and $f$ indicates that the pressure has been sufficiently reduced. The tap 2 is now spened; the gases of the blood buhble up into the froth chamber, pass through the drying-tube $C$, which is filled with pumice-stone and sulphuric acid, and enter D. The end of $G$ is placed under the eudiometer $F$, and by raising $\mathrm{E}$., with tap 4 turned so as to cut off $\mathrm{C}$, the gases are forced out through $\mathrm{G}$ and collected in $\mathrm{F}$. The movements required for exhaustion can be repeated several times till no more gas cornes off. The escape of gas from the blood is facilitated by im. mersing the bull, $\mathrm{A}$ in water at $40^{\circ}$ to $50^{\circ} \mathrm{C}$.

combined oxygen of oxyhamoglobin, can be obtained by dissociation of their compounds when the partial pressure is reduced. Nore stable combinations may require to be broken up by chemical agents - carbonates, for instance, by acids.

Extraction of the Blood-gases. - This is best accomplished by exposing biood to a nearly perfect vacumn. The gas-pumps which have been most largety used in blood analysis are constructed on the principle of the Torricellian vacuum. A diagram of a simple form of 
Pfïger's gas-pump is given in Fig. 107. The gases otained are ultimately dried and collected in a cudiometer, which is a graduated glass tube with its mouth dipping in to mercury. The carbon dioxide is estimatel by introducing a little potassimm hycl roxide to absorb it. Th" diminution in the volume of the gas contained in the endiometer gives the volume of the carbon dioxide. The oxygen may be estimated by putting into the cucliometer more than enough hydrogen to unite with all the oxygen so as to form water, and then, after reading off the volume, exploding the mixture by means of an electric spark passed through two platinum wires fused into the glass. (Inethird of the diminution of volume represents the quantity of oxygen present. It can also be estimated by absorption with a solution of prrogallic acid and potassinm hydroxide.* The remainder of the original mixture of blood-gases, after deduction of the carbon dioxide and oxygen, is put down as nitrogen (with, no doubt, a small proportion of argon). For the sake of easy comparison, the obscrved volume of gas is always stated in terms of its equivalent at a standard pressure and temperature $(760 \mathrm{~mm}$., or sometimes on the Continent 1 metre of mercury, and $\mathrm{O}^{\circ} \mathrm{C}$.).

It is also possible in various ways to estimate the amount of oxygen in blood without the use of the pump. Thus, since a definite volume of oxygen ( ${ }^{\circ} 338$ c.c. at $0^{\circ} \mathrm{C}$. and $760 \mathrm{~mm}$. pressurc) combines with a gramme of hæmoglobin, we can calculate the total volume of oxgyen present if we know how much of the blood-pigment is in the form of oxyhæmoglobin; and this can be determined by means of the spectrophotometer. Or potassium ferricyanide may be added to the blood. This expels the oxygen from its combination with the hrmoglobin, which then unites with an exactly equal amount of oxygen obtained from the ferricyanide to form methrmoglobin (Haldane) (p. 67).

In dog's blood, which has been up to this time chiefly investigated, there are considerable variations in the quantity of oxygen and carbon dioxide which can be extracted. This is particularly true of the venous blood, as might naturally be expected, since even to the eye it varies greatly according to the vein it is obtained from, the rapidity of the circulation, and the activity of the tissues which it has just left. On the average,

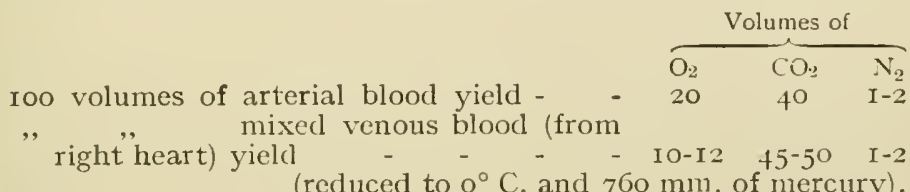

Average venous blood contains 7 or 8 per cent. by volume less oxygen, and 7 or 8 per cent. more carbon dioxide, than arterial blood. Thus, in the lungs the blood gains about twice as many volumes of oxygen per cent. as the air loses, and the air gains about half as many volumes of carbon dioxide per cent. as the blood loses. It is easy to see that this must be so, for

* Or an alkaline solution of sodium hydrosulphite, which is more cleanly. 
the volume of the air inspiaed in a given time is about twice as great as that of the blood which passes through the pulmonary circulation (pl). 209, 220, 242). Even arterial blood is not cuite saturated with oxygen; it can generally still take up one-tenth to one-fifteenth of the quantity contained in it. Nor is venous blood nearly saturated with carbon dioxide; when shaken with the gas it can take 11p abont $5_{50}$ volumes per cent.

When the gases are not removed from blood immediately after it is drawn, its colour becomes darker, and it yields more carbon dioxide and less oxvgen than if it is evacuated at once (Ptliiger). lirom this it is concluded that oxidation goes on in the blood for some time after it is shed. The oxidizable substances are, however, confined to the corpuscles, which suggests that ordinary metabolism simply continues for some time in the formed elements of the shed blood, and that the disappearance of oxygen is not due to the oxidation of substances which have reached the blood from the tissues.

The Distribution of the Gases in the Blood.-The oxygen is nearly all contained in the corpuscles. A little oxygen can be pumped out of serum ( $0^{\circ} \mathrm{I}$ to $0^{\circ} 2$ per cent. by rolume), but this follows the Henry-Dalton law of pressures; that is, it comes off in proportion to the reduction of the partial pressure of the oxygen in the pump, and is simply in solution.

When blood is being pumped out, very little oxygen comes off till the pressure has been reduced to about half an atmosphere. At abont a thircl of an atmosphere, if the blood is nearly at body temperature, the oxygen begins to escape a little more freely; and when the pressure has fallen to about one-sixth of an atmosphere (corresponding to a partial pressure of oxygen of 25 to $30 \mathrm{~mm}$. of mercury), it is disengaged with a burst. This shows that it is not simply absorbed, but is united by chemical bonds to some constituent of the blood. The same thing is seen when defibrinated blood is saturated at body temperature with oxygen at different pressures. The quantity taken up lessens but slowly as the pressure is reduced, till at about 25 to $30 \mathrm{~mm}$. of mercury an abrupt diminution takes place. It is found that a solution of pure hitmoglobin crystals behaves towards oxygen somewhat differently from blood containing the same proportion of blood-pigment; and although there is no doubt that the body in blood with which the oxygen is loosely united is intimately related to the hæmoglobin, which can be artificially prepared from it, there are good reasons for believing that they are not identical. Some writers for this reason prefer to give the special name hæmochrome to the native blood-pigment as it exists within the unaltered corpuscles, reserving the term hemoglobin for the more or less artificial though, perhaps, only slightly altered product. 
We mat suppose that at the ordinary temperature and pressure* some oxygen is continually escaping from the bonds by which it is tied to the hemoglobin; but, on the whole, an equal number of free molecules of oxygen, coming within the range of the hemoglobin molecules, are entangled by them, and thus equilibrium is kept up. If now the atmospheric pressure, and therefore the partial pressure of oxygen, is reduced, the tendency of the oxygen to break off from the hamoglobin will be unchanged, and as many molecules on the whole will escalpe as before; but even after a considerable reduction of pressure the lirmoglobin, such is its avidity for oxygen, will still be able to seize as many atomis as it loses. The nore, hovever, the partial pressure of the oxygen is diminished-that is to say, the fewer oxyen molecules there are in a given space above the liamoglobin-the smaller will be the chance of the loss being made 11) by accidental captures. $\Lambda$ t a certain pressure the escapes will become conspicuously more numerous than the captures; and the gas-pump will give evidence of this, although it could give no information as to mere molecular interchange, so long as equilibrium was maintained. The higher the temperature of the hemoglobin is, the greater will be the average velocity of the molecules, and the greater the chance of escape of molecules of oxygen. The " dissociation tension' of oxyhamoglobin, or the partial pressure of oxygen at which the oxylhamoglobin begins to lose more oxygen than it gains, is increased by raising the temperature. Curves of dissociation of oxyhamoglobin and blood are shown in Figs. 108 and 109. According to Bohr, Fig. 109 represents the curves for blood and a hæmoglobin solution of equal strength. It will be observed that the two curves are not identical, the blood-pigment in the corpuscles not behaving just as the artificially-produced oxyhæmoglobin.

The Carbon Dioxide of the Blood.-Blood freed from gas absorbs carbon dioxide partly in proportion to the pressure, and in part independently of it. Some of the carbon dioxide must therefore be simply dissolved; some, and this the greater portion, is chemically combined. The serum contains a larger percentage of carbon dioxide than the clot, but this percentage is not great enough to allow us to assume that the whole of the carbon dioxide is confined to the serum. About a third of it belongs to the corpuscles.

In the serum the combined carbon dioxide exists chiefly as carbonate and bicarbonate of sodium, the relative amount of each depending on the quantity of carbon dioxide and of other acids, such as phosphoric acid, which dispute with it the possession of the bases. That its relations are peculiar, however, is shown by the fact that from defibrinated blood the whole of the carbon dioxide can in time be pumped out without the addition of an acid to displace it from the bases with which it is combined. It is hardly necessary to say that this could not be done with a solution of sodium carbonate. Yet when sodium carbonate is

* The partial pressure of oxygen in air at $760 \mathrm{~mm}$. atmospheric pressure is $\frac{21}{100} \times 760$, or $159^{\circ} 6 \mathrm{~mm}$. 
added to blood, even in considerable ansount, all the carbon dioxide in it can be obtained by the pump. From serum a great deal, but not the whole, of the carbon dioxicle can be likewise pumped out. The residue (from io to IS per cent. of the whole) is set free on the addition of an acid, e.g., phosphoric acid.

The most satisfactory explanation is that in the serum there exist substances which can act as weak acicls in gradually driving out the carbon dioxide, when its escape is rendered easier by the vacuum. The quantity of these. however, is so small that a

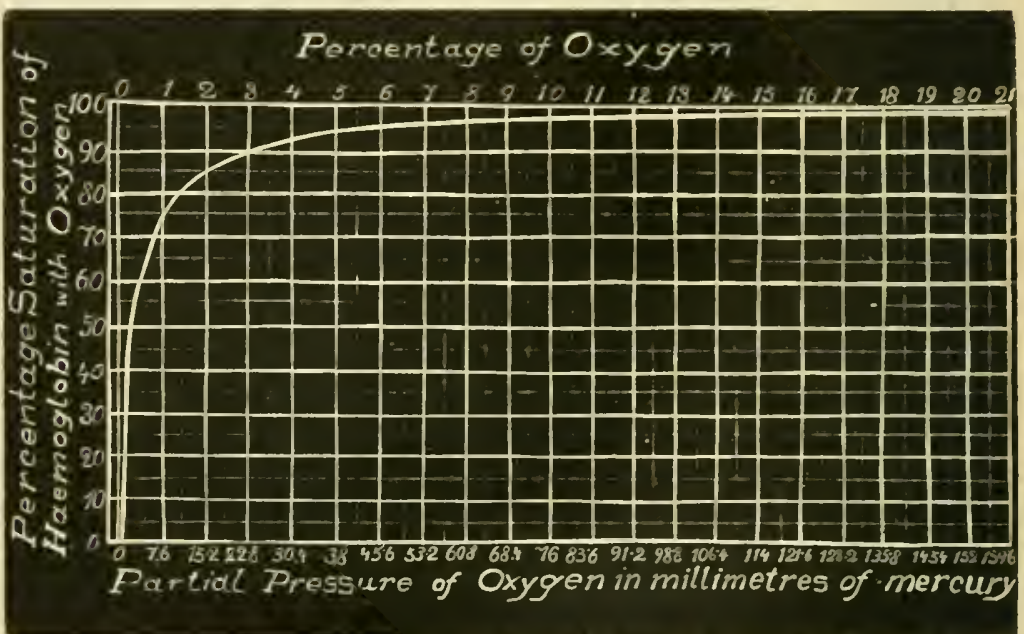

Fig. ZO8.-CURTE OF DISSOCIATION OF OXYHAMOGLOBIX AT $35^{\circ}$ C. (AFTER H('FNER'S RESLLTS).

Along the horizontal axis are plotted the partial pressures (numbers below the curve) of oxygen in air, to which a solution of hatnoglobin was exposed. The corresponding percentages of oxygen are given above the rurve. Along the vertical axis is plotted the percentage saturation of the hitnoglobin with oxygen. Thus, on exposure to an atmosphere in which oxygen existed to the exent of I per cent., corresponding to a partial pressure of $7.6 \mathrm{~mm}$. of 1nercury, the hatuo. globin took up about 75 per cent. of the amount of oxygen required to saturate it. When the oxygen was present in the atmosphere to the amount of about 10 per cent., corresponding to a partial pressure of $; 6 \mathrm{~mm}$. of mercury, the quantity taken up by the hæmoglobin was about g6 per cent. of that required for saturation.

portion of the carbon dioxide remains in the serum. The proteins of the serum, such as serum-globulin, behave in certain respects like weak acids, and maly contribute to the driving out of the carbon clioxide. When defibrinated blood is pumped out, the whole of the carbon dioxide can be removel. apparently because substances of acid nature pass from the corpuscles into the serum and help to break up the carbonates. and because the hamoglobin in the corpuscles acts as a weak acid.

In the red corpuscles a portion of the carbon clioxide is in combination with alkalies. We know that the corpuscles contain 
alkalics, for potassimm lats been demonstrated microchemically in frog's erythrocytes (Micallum) (Froutispiece), and the titratable alkalinity of 'laked' blood (1)p. 24,27 ) is greater than that of unlaked blood, unless a long time is allowed in the case of the latter for the alkalies of the corpuscles to reach the acid used in titration. Some observers believe that a weak compound of carbon dioxide can be formed with liewsoglobin; for a solution of lramoglobin absorbs more of this gas than water, and the quantity absorbed is not proportional to the pressure. The hamoglobin of the corpuscles may therefore hold a portion of the carbon dioxide in combination.

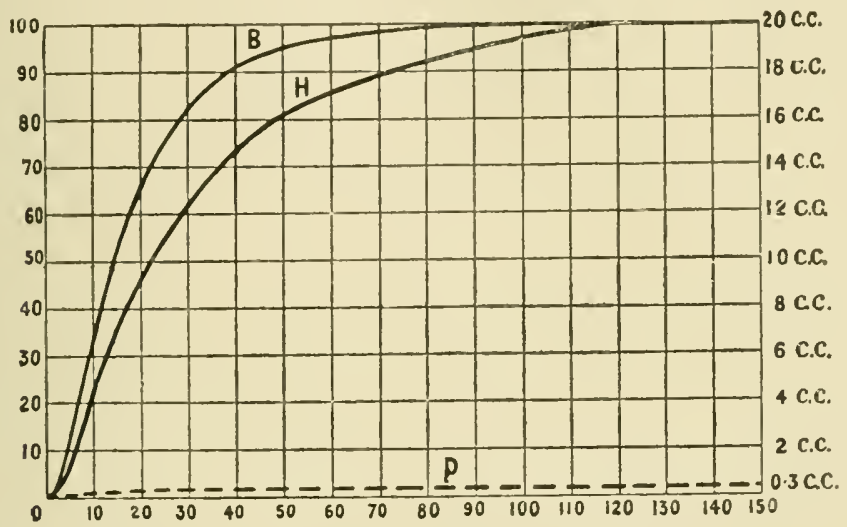

Fig. IO9. - CuRves of DISSOCIATION OF OXIGEN fOR HORSE's BlOOD (B) AND Dog's H.woglobix Solution (H) At $38^{\circ} \mathrm{C}$. (Bohr).

The figures along the base-line and the vertical axis at the left have the same signification as in Fig. 108. The figures along the vertical at the right give the actual number of c.c. of oxygen chemically combined by roo c.c. of the blood for each pressure of oxygen. Thus, with pressure $10 \mathrm{~mm}$. 6 c.c. of oxygen were taken up by the blood-pigment in Ioo c.c. of blood. The interrupted line $\mathrm{P}^{\prime}$ indicates the amount of oxygen dissolved in the plasma of the blood at each partial pressure on the assumption that the plasma is two-thirds of the volume of the blood. Thus, at $150 \mathrm{~mm}$. oxygen pressure the plasma of $100 \mathrm{c} . \mathrm{c}$. of blood took up 0.3 c.c. oxygen.

When blood is saturated with carbon dioxide and then separated into serum and clot, the serum is found to yield more gas than the clot; but if the serum and clot are separately saturated, the latter takes up morc carbon dioxide than the former. From this it is argued that a substance combined with carbon dioxide must in blood saturated with the gas pass out of the corpuscles into the serum. This cannot be hæmoglobin. for it remains in the corpuscles, but it may very well be an alkali, combined with the carbon clioxide and thus set free fron its connection with the hæmoglobin And, as a matter of fact, under the circumstances described. it has been found that alkalies do pass from the clot into the serum, and chlorine from the serum into the corpuscles, which at the same time gain water and become larger. The molecular concentration (p. 398) of the serum of defibrinated blood, as measured by the lowering of the freezing-point, increases when it is saturated with carbon dioxide. On the other hand, when blood is saturated with 
oxygen, alkalies patss out of the serum into the corpuseles, which at the sante time lose wates and shrink in volume, while the molecular concentration of the serum is diminished. Hamburger luas extended these observations to the circulating blool, and has shown that the plasma of venous blood has a higher pereentatge of alkali, protein, sugar, and fat, than the plasma of arterial blood, and that the corpuscles have a greater volume, though not a greatci diameter. We maty therefore suppose that in the pulmonary capillarics. under the influence of oxygen, water passes into the plasma from the corpuscles. In the systemic capillaries the blood becomes loaded with carbon dioxide, and therefore the corpuseles take up water from the plasma, which accorlingly has a more concentrated supply of food-substances to offer to the tissues than the plasma of arterial blood itsclf. Some writers see in this interclange an automatic arrangement by which oxidation is favoured. Whatever may be thought of this riew - and objections to it are not wanting-the current theory, that the corpuscles are simply passive carrices of oxygen, and exercise no further influence on the plasma, breaks down in face of the facts. We must admit that an active and many-sided commerce exists between them and the liquid in which they float.

The nitrogen of the blood is simply absorbed.

The Tension of the Blood-gases.-If the gases of the blocel existed in simple solution, their tension or partial pressure could be deduced from the amount dissolved and the co-efficient of absorption. Since they are chemically combined, it is necessary to determine it directly.

This has been lone by means of an apparatus called the acrotonometer. The blood is male to pass directly from the vessel to glass tubes, which it traverses at the same time, the stream being divided between them; it then passes out again. The tubes are warmed by means of a water-jacket to the body temperature. Some of them are filled with gascous mixtures having a greater, and the others with mixtures having a smaller, partial pressure, say of carbon dioxide, than is expected to be found in the blood. As the latter runs in a thin sheet over the walls of the tubes, it loses carbon dioxide to some of them and takes up carbon dioxide from others. From the alteration in the proportion of the carbon choxide in the tubes, it is casy to calculate the partial pressure of that gas in the blool; that is, the partial pressure which it would be necessary to have in the tubes in order that the blood miglit pass through then without losing or gaining carbon dioxide (p. 248).

The pressure of oxygen in arterial blood was given by Strassburg as about $30 \mathrm{~mm}$. of mercury in the dog (corresponding to the partial pressure of oxygen in a gaseous mixture at atmospheric pressure when + per cent. of it is present), and in venous blood as something like $20 \mathrm{~mm}$. If we were to accept the experiments of Bohr, made by means of a special form of aerotonometer constructed and worked much in the same way as Ludwig's stromuhr (p. II2), and inserted into the course of a bloodvessel, it would be necessary to treble or quadruple these numbers. 
The pressure of carbon dioxide in arterial blood we mat take at 10 to fo mm., in venous blood at 30 to $50 \mathrm{~mm}$, according to the results of different observers.

Whenever the venous blood has to pass through a region in which the pressure of carbon dioxide is higher than its own, carbon dioxide will enter it. When it enters a region in which the carbon dioxide pressure is kept lower than in itself, the carbon dioxide compounds formed in its passage through the tissues will be dissociated, and it will begin to lose carbon dioxide by diffusion. If the pressure of oxygen in this region is at the same time higher than in the renous blood, some of it will be taken up. And to bring about these results no peculiar 'vital' force need be inroked; ordinary physical processes will, under the assumed conditions, be alone required.

Now, we know that in the lungs carbon dioxide is given off from the blood, and oxygen taken up by it. We have, therefore, to inquire what the partial pressures of these gases are in the alveoli, and whether they are so related to the corresponding partial pressures in the blood that a simple process of dissociation and diffusion will be sufficient to explain pulmonary respiration.

The percentage of carbon dioxide in expired air cannot tell us the pressure of that gas in the alveoli, for the air in the upper part of the respiratory tract is necessarily expelled along with the alveolar air, and dilutes the carbon dioxide in it. But the mean of the carbon dioxide percentages in samples taken from the last portions of the air of two deep expirations, one following an ordinary inspiration and the other following an ordinary expiration, is themean percentage in the alveoli. This quantity, while, as already remarked (p. 242), very constant in a given individual, varies in different men from $4^{.6}$ to 6.2 (mean 5.5$)$ per cent. of the dry alveolar air. In women and in children of both sexes it is less than in men. From this we conclude that in men the partial pressure of carbon dioxide in the alveoli may be at least one-eighteenth of an atmosphere, or $42 \mathrm{~mm}$. of mercury (Fitzgerald and Haldane).

In animals, samples of the alveolar air have been drawn off directly by means of a pulmonary catheter. This consists of two tubes, one within the other. The inner tube, which is a fine elastic catheter, projects free from the other for a little distance at its lower end. The outer tube terminates in an indiarubber ball, which can be inflated so as to block the bronchus into which it is passed, and cut off the corresponding portion of the lung from communication with the outer air. A sample of the air below the block can be drawn off through the inner tube. In this way the proportion of carbon dioxide in the alveoli of the dog was found to be only about 3.8 per cent., corresponding to a partial pressure of about $29 \mathrm{~mm}$. of mercury. 
In liohr's experiments, in some of which the animals were made to freathe air containing carbon dioxide in various proportions, the tension of that gas in the air of the lungs varied from 5.8 to $34^{6} \mathrm{~mm}$. of mercury, while in arterial blond, taken at the same time, it usually ranged from 10 to 38 mm., and was often less than in the alveolar air.

If we accept these results, we seem shut up to the conclusion that carbon dioxide does not pass through the walls of the alveoli solely by diffusion. And although Bohr's experiments have been severely criticized, it does not seem improbable in itself that the physical process of diffusion. which undoubtedly plays a great part, is aided by some other process, which may provisionally be termed secretion. It is possible, too, that when the conditions are especially unfavourable to diffusion-when, for instance, the partial pressure of carbon dioxide is artificially. increased in the alveoli-the cells which line them are stimulated to increased activity:

As to the oxigen, we are in the same position. Its partial pressure does not appear to he alwars higher, even under normal conditions, in the alveoli than in the arterial blood as it leaves the lungs. Indeed. Bohr found that in the majority of his observations on dogs, the oxygen tension was distinctly greater in the blood than in the pulmonary air. And Haldane and smith, using a new method,* have obtained a value for the oxygen tension in human blond $(26.2$ per cent., equal to $200 \mathrm{~mm}$. of mercury) that even exceeds the partial pressure of oxygen in the external air, and is about twice as great as that of the air of the alveoli. This remarkable result cannot be reconciled with any purely physical explanation of the absorption of oxygen. But the method by which it was obtained, although correct in principle, has not escaped criticism as to its details (Osborne).

Additional evidence in favour of the view that there is, besides diffusion, an element of selective secretion in the exchange of gases through the pulmonary membrane is afforded by a study of the gases of the swim-bladder in fishes. These

* The subject of the experiment breathes air containing a definitely. known very small percentage of carbon monoxide until the hxmoglobin has united with as much of that gas as it will take up for the given concentration of it in the air. Then the percentage amount to which the hxmoglobin has become saturated with carbon monoxide is determined in a sample of blood taken, say, from the finger. Now, the final saturation with carbon monoxide of a hxmoglobin solution brought into contact with a gascous mxture containıng carbon monoxide and oxigen, depends on the relative tensions of the two gases in the liquid. But the tension of carbon monoxide in the blood leaving the lungs will (after absorption has ceasc d) be the same as that in the mspred air. hinowing this tension and the degree of saturation of the hiemoglohin with carbon monoxide, the oxigen tension in the hlood leaving the lungs-i.e, in the arterial bloodis known. 
consist of oxygen, nitrogen, and ustlally a small quantity of carbon dioxide, but in very different proportions from those in which they exist in the air or the water. Thus, as much as 87 per cent. of oxygen has been found in the bladder of fishes taken at a considerable depth, but a smaller amomnt in those captured near the surface. When the gits is withdrawn by puncturing the bladder with a trocar, the organ rapidly refills, and tle percentage of oxygen increases. Further, this process of gaseous secretion is under the influence of nerves, for gas ceases to accumulate in the organ when the branches of the vagi that supply it are cut. In the tortoise stimulation of the peripheral end of the vagus causes a fall of gaseous exchange in the corresponding lung, with an accompanying rise in the other lung. That this is not the consequence of an alteration in the pulmonary circulation is indicated by the fact that the change is greater in the intake of oxygen than in the output of carbon dioxide. In the mammal, however, no such effect has been clearly demonstrated, and the decisive proof that the lungs are gas-secreting glands which would be afforded by the discovery of secretory nerves is still wanting.

We have now completed the description of the phenomena of external respiration, with the discussion of its central fact, the exchange of gases between the blood and the air at the surface of the lungs. It remains to trace the fate of the absorbed oxygen, and to determine where and how the carbon dioxide arises.

Internal Respiration-Seats of Oxidation.-The suggestion which lies nearest at hand, and whicl, as a matter of fact, was first put forward, is that the oxygen does not leave the blood at all, but that it meets with oxidizable substances in it, and unites with their carbon to form carbon dioxide. While there is a certain amount of truth in this riew, oxygen, as already mentioned, being to some extent taken up by freshly-shed blood, and also by blood under other conditions, to oxidize bodies, other than hæmoglobin, either naturally contained in it or artificially added, there is no doubt that the cells of the body are the busiest seats of oxidation. This is shown by the presence of carbon dioxide in large amount in lymph and other liquids which are, or have been, in intimate relation with tissue elements ; by its presence, also in considerable amount, in the tissues themselves-in muscle, for instance; by its continued and scarcely lessened production not only in a frog whose blood has been replaced by physiological salt solution, and which continues to live in an atmosphere of pure oxygen, but in excised muscles; and by the remarkable connection between the amount of this production and the functional state of those tissues. In insects the finest $t$ wigs of the trachea, through which oxygen passes to the tissues, actually 
end in the cells; and in luminous insects, like the glow-worm, it lias been noticed that the pliosphorescence, which is certainly dependent on oxidation, begins and is most brilliant in those parts of the cells of the light-producing organ that surround the ends of the trachere. Microscopic evidence has been obtained that the nucleus plays a predominant part in intracellular oxidation ; e.g., in the indophenol (p. 265) and similar reactions the coloured oxidation products are deposited chicfly in and around the nuclei of such cells as liver and kidney cells and frog's red corpuscles (Lillie).

The fact observed by Bohr that an increase in the carbon dioxide tension of blood diminishes its combining power for oxygen, and therefore favours the giving up of oxygen to the lymph and tissues, may liave an important influence on internal respiration. The effect is much more marked where the oxygentension is low than where it is high, so that in the lungs the taking up of oxygen is scarcely interfercd with even by a ligh carbon dioxide tension. Lympl, bile, urine, and the serous fluids contain very little oxygen, but so much carbon dioxide that the pressure of that gas in all of them is greater than in arterial blood, while in lympl alone (taken from the large tloracic duct) has it been found less than that of venous blood. And it is probable that lympli gathered nearer the primary seats of its production (the spaces of areolar tissue) would show a higher proportion of carbon dioxide. Strassburg found tliat with a pressure of carbon dioxide in the arterial blood of $21 \mathrm{~mm}$. of mercury, the pressure in bile was $50 \mathrm{~mm}$., in peritoneal fluid $58 \mathrm{~mm}$., in urine 68 mm. in the surface of the empty intestinc $58 \mathrm{~mm}$. Saliva, pancreatic juice, and milk, also contain much carbon dioxide, and only a little, if any, oxygen. From muscle (to facilitate pumping, the muscle is minced and warmed) no free oxygen at all can be pumped out, but as much as I 5 volumes per Ioo of carbon dioxide, some of which is free, that is, is given up to the vacuum alone, while some of it is fixed, and only comes off after the addition of an acid.

Muscle may be safely taken as a type of the other tissues in regard to the problems of internal respiration. It is instructive, therefore, to observe that the great scarcity of oxygen in the parenchymatous liquids which bathe the tissues, liere in the tissues themselves deepens into actual famine. The inference is plain. The active tissues are greedy of oxygen; as soon as it enters the muscle it is seized and 'fixed' in some way or other. The traces of oxygen in the lymph cannot therefore be journeying away from the tissue elements; they must have come from another source, and this can only be the blood. Could we gather tissue lymph for analysis directly from the thin sheets that lie between the blood capillaries and the tissues, we might find more oxygen present as well as more carbon dioxide. But if we did find more 
oxygen, it would still be oxygen in transit from the capillaries towards places where the partial pressure of oxygen is less. In the lymph, the pressure is kept low by the avidity of the tissues with which it is in contact, and possibly by the existence in it of oxidizable substances which lave cone from the tissues. In the tissues there is no partial pressure at all, because the oxygen that reaches them is at once stowed away in some compound, in which it has lost the properties of free oxygen.

Assuming, then, that at least a great part of the oxidation and consequent production of carbon dioxide goes on in the tissues, let us follow the steps of the process, as far as we can, in the light of our knowledge of the respiration of muscle.

Respiration of Muscle. - It is a remarkable fact that an excised frog's muscle is capable of going on producing carbon

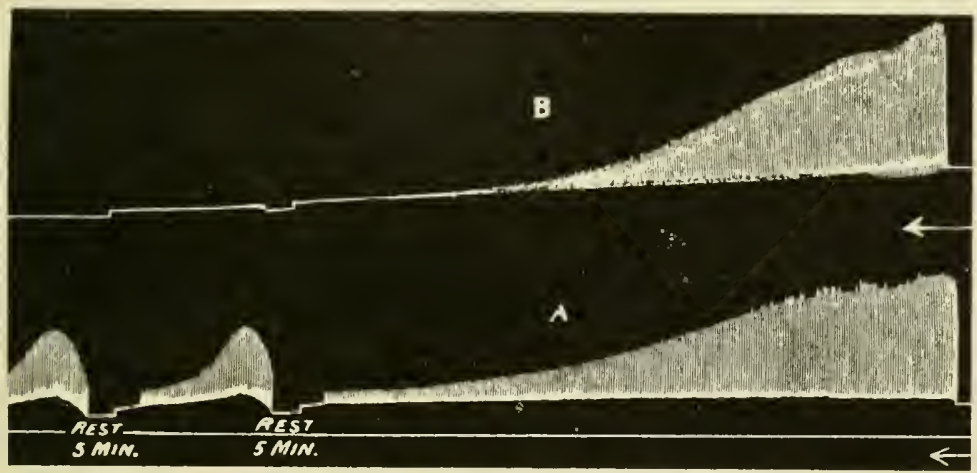

Fig. iro.-Fatigue of a Pair of Sartorius Muscles (Fletcher).

$A$. in an atmosphere of oxygen : B, in an atmosphere of nitrogen. A is partially restored by a rest of five minutes.

dioxide for a long time, in the entire absence of oxygen, in a chamber, for instance, filled with nitrogen or other indifferent gas. Not only so, but it can be made to contract many times in this oxygen-free atmosphere, although it loses its power of contraction sooner than in oxygen, and does not show the same capacity for recuperation during an interval of rest. In mammals the muscles can also be made to contract repeatedly when the dissociable oxygen has, as far as possible, been got rid of from the blood by asphyxiating the animal, and to produce a correspondingly large quantity of carbon dioxide, although they lose their contractility much more rapidly than the muscles of the frog. This leads us to the important conclusion that the carbon dioxide does not arise, so to speak, on the spot, from the immediate union of carbon and oxygen. Oxygen is essential to muscular life and action. But a stock of it is apparently 
taken up by the muscle, and stored in some compound or compounds, probably essential constituents of the living muscular substance, which are broken down during muscular contraction,
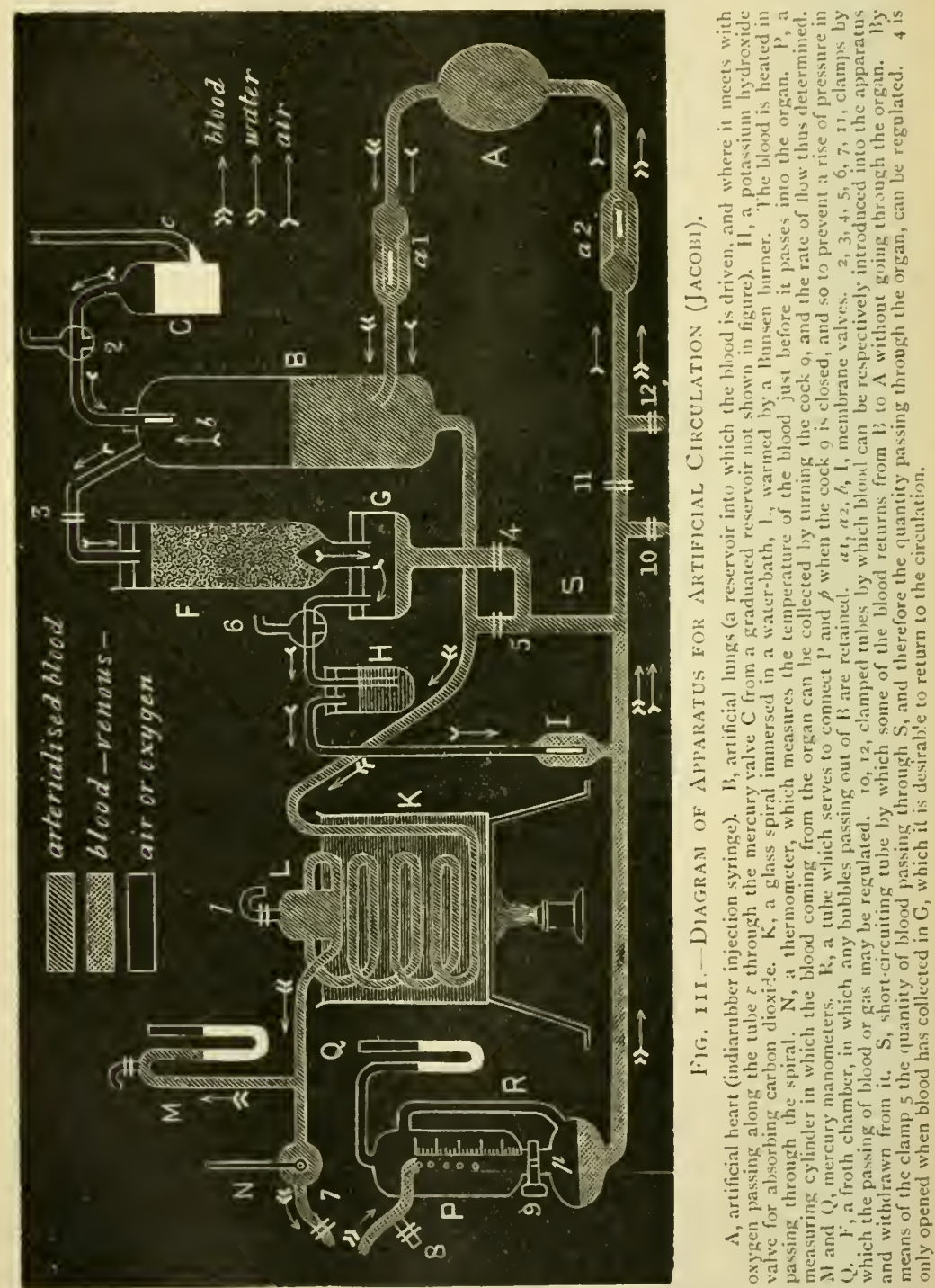

and more slowly during rest, carbon dioxide in both cases being one of the end products. It is possible that there is an ascending series of bodies through which oxygen passes up, and a descend- 
ing series through which it passes down, hefore the fina! stage is reached. In a nommal inuscle with intact circulation, while carbon dioxide is given off, certain of the other decomposition products appear, in conjunction with oxygen and some substance ich in carbon, like sugar, to be regenerated into the material which breaks down in contraction. When oxygen is not available, as in an atmosphere of nitrogen, carbon dioxide is still given off, but the other decomposition products are not regenerated to contractile substance, but accummlate in the muscle, producing the plenomena of fatigue, and eventually of rigor.

When muscle goes into rigor (Chap. IX.) - and this is most strikingly seen when the rigor is caused by raising the temperature of frog's muscle to about $40^{\circ}$ or $4 \mathrm{I}^{\circ} \mathrm{C}$.- there is a sudden increase in the quantity of carbon dioxide given off. Moreover, in an isolated muscle the total quantity of carbon dioxide obtainable during rigor is markedly less if the muscle has been previously tetanized. From this it has been argued that the hypothetical substance, the decomposition of which yields carbon dioxide in contraction, is also the substance which decomposes so rapidly in rigor; that a given amount of it exists in the muscle at the time it is removed from the influence of the blood; and that this can all explode either in contraction or in rigor, or partly in the one and partly in the other. According to Fletcher, there is no increase in the amount of carbon dioxide given off during tetanus by an excised frog's muscle unless the stimulation is so severe and prolonged as to hasten the onset of rigor. He therefore supposes that in the contraction the decomposition does not proceed quite to the formation of carbon dioxide, which in the intact body is afterwards liberated from some more complex carbon-containing waste-product.

The respiration of muscles in situ can be studied by collecting samples of the blood coming to and leaving them and analyzing the gases. The mere difference of colour between the venous and arterial blood of a muscle, or other active organ, is sufficient to show that oxygen is taken up and carbon dioxide given out by it to the blood. This is the case in muscles at rest, and even in muscles with artificial circulation after they have become inexcitable. In active muscles more oxygen is used up and more carbon dioxide produced than in the resting state. Chauveau and Kaufmann, in their experiments on the levator labii superioris muscle of the horse in feeding, found that the consumption of oxygen and the production of carbon dioxide might be many times as great in activity as in rest.

Thus in one experiment the amount of oxygen taken in, expressed in c.c. per gramme of muscle per minute, was 0.0079 during rest, and O.I4 during work; the corresponding quantities 
for the carbon dioxide given off were $0.005 S$ and 0.18 . The respiratory quotient rose to $\mathrm{I}^{\cdot} 3$ in two experiments, and even to $I^{\prime} 7$ in a third, showing that the increase in the production of carbon dioxide was relatively greater than the increase in the intake of oxygen. These experiments were performed under conditions so normal that the animal continued to eat its hay with seeming unconcern throughout the observations, although these involved the exposure of the main bloodvessels of the muscle, and the collection of samples of blood from them.

In the heart of a small dog through which blood was pumped by a larger dog the oxygen intake when the heart was beating feebly was, on the average, about o.or c.c. per gramme of heartmuscle per minute. When the heart was caused to beat very strongly under the influence of adrenalin, the oxygen intake rose in one case to 0.08 , and in two others to 0.04 . In the resting pancreas the oxygen intake has been found to be 0.03 to 0.05 c.c. per gramme per minute; in the active pancreas, O.I c.c. The corresponding number for the submaxillary gland at rest is $0^{\circ} 03$, and in activity $0^{\circ} 09$; for the kidney, $0^{\circ} 03$ at rest or during scanty secretion, and 0.07 during active secretion (Barcroft).

Nature of the Oxidative Process. - When we have recognised the cells as the seat of oxidation, the question immediately presents itself. How do they accomplish the feat of burning such masses of food substances as can only be rapidly oxidized in the laboratory at the temperature of the body by the most energetic chemical reagents? The researches of late years have furnished a key to the solution of this long-standing puzzle by demonstrating the existence in the tissues of oxidizing ferments or oxydases. Of these, one of the most widely distributed is a ferment which splits off oxygen from hydrogen peroxide. Since any oxidation produced is only secondary to this decomposition. ferments which decompose hydrogen peroxide are often spoken of as catalases, to distinguish them from the oxydases proper. A catalase is found in practically all the tissues of the body, as well as in vegetable cells, and we have already mentioned instances of its action in connection with the oxidation of the guaiaconic acid in tincture of guaiacum in the presence of the peroxide (p. 69). As regards the activity of this ferment, blood comes first; then follow spleen, liver, pancreas, thymus, brain, muscle, and ovary. It is present in the blood-free organs as well as in the blood. Some tissues, both animal and regetable, contain a ferment, an oxydase, which causes the oxidation of guaiaconic acid in the presence of atmospheric oxygen, and these do not need peroxide of hydrogen in order to render guaiacum blue. An allied ferment which also induces the blue colour in tincture of guaiacum is the so-called laccase found in the most 
active form in the latex of the tree from which Japanese lacquer is obtained, but also in many other plants. Many fungi contain a ferment, tyrosinase, which oxidizes tyrosin, and in certain animals tyrosinases have also been demonstrated. Another well-known oxidizing ferment in fresh animal tissues is characterized by the property of forming indophenol by oxidation in an alkaline solution of paraphenylenediamin and $a$-naphthol, and may therefore be termed indophenyloxydase. The colourless solution becomes reddish or violet. This ferment is contained in pancreas, salivary glands, spleen, thymus, and bonemarrow, but has not been detected in muscle, lungs, brain, kidneys, and other organs. Finally, we may mention a ferment which favours the oxidation of aldehydes to the corresponding acids, and is appropriately named aldehydase. Evidence of its presence in most organs lias been obtained, but it seems to be absent from muscle, pancreas, bone-marrow, and mammary glands. It is to be expected that other oxydases capable of favouring oxidation of specific kinds of food substances or their decomposition products will be discovered, but it would be rash to conclude that this is the only way in which living protoplasm can bring about the rapid oxidation which is so characteristic a feature of its activity.

The Influence of Respiration on the Blood-pressure.- We have already stated, in treating of arterial blood-pressure (p. ro4), that a normal tracing shows a series of waves corresponding with the respiratory movements.

The relationship between the respiratory phases and the rise and fall of the blood-pressure is not by any means a simple and invariable one. It depends upon a number of factors, which need not be equally influential under different conditions or in different animals (Lewvis). Something depends upon the rate, something upon the relative preponderance of costal and abdominal respiration, and something probably upon the size of the animal. For instance, an inspiratory rise of blood-pressure occurs in man with pure diaphragmatic, and a fall with pure thoracic, breathing (Fig. II2). In cats with fairly fast and not very deep respiration the blood-pressure rises in expiration and sinks in inspiration. With deep and slow respiration the opposite effect may, upon the whole, be seen. In dogs, according to Einbrodt, although the mean blood-pressure is falling for a short time at the beginning of inspiration, it soon reaches its minimum, then begins to rise, and continues rising during the rest of this period. At the commencement of expiration it is still mounting, but soon reaches its maximum, begins to fall, and continues falling through the remainder of the expiratory phase.

A partial explanation is afforded by a consideration of the 
mechanical changes produced in the thorax by the respiratory movements. (of these, the influence of variations in the intrathoracic pressure on the filling of the heart is of special importance. IVith deep ablominal breathing the changes of intra-ahdominal
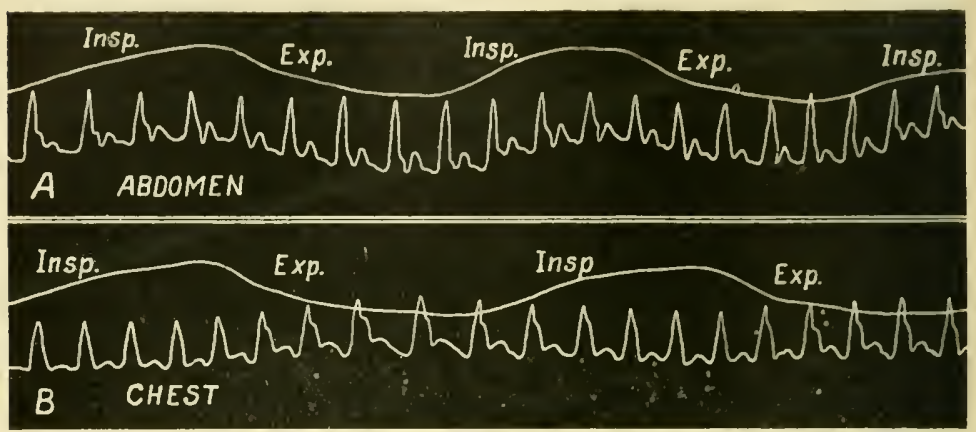

Fig. II 2.-Respiratory Waves in the Blood-pressure : Simultaneous Tracings of Movements of Respiration and of Radial Pulge in Human SUBJIECT (LEWIS).

In A the respiration was diaphragmatic; in 13, costal. In A the respinatury traring was taken from the abdominal wall : in 13, from the chest.

pressure also affect the filling of the heart, an increase of pressure (in inspiration) tending to cause more blood to be squeezed from the ablominal veins fowards the chest. The changes of vascular resistance in the lungs, due to the alteration in the calibre of the pulmonary ressels, also contribute, but, for such rariations

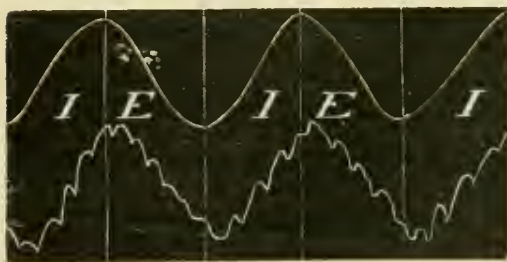

Fic. II3.

The upper tracing shows the respiratory movements in a rabbit with rather deep and slow diaphragmatic breathing: the lower tracing is the blood-pressurt: curve; I, inspiration ; li, expiration, including the pause. atmosphere, unless during a forced expiration when the free escape of air from the lungs is obstructed, diminishes in inspiration and increases in expiration. The great reins outside the chest, the jugular reins in the neck, for example, are uncler 
the atmospheric pressure, which is readily transmitted through their thin walls, while the heart and thoracic veins are under a smaller pressure. The venous blood both in inspiration and expiration will, accordingly, tend to be drawn into the right auricle. In inspiration the venous flow will be increased, since the pressure in the thorax, and therefore in the pericardial carity, is diminished; and upon the whole more venous blood will pass into the right heart cluring inspiration than during expiration. Now, the right ventricle is not in general working as hard as it can work. Ilence, the excess of blood which reaches it during an inspiration is at once sent into the lungs, although not even the first of it can have passed through to the left side of the heart at the end of the inspiration, since the pulmonary circulation-time (four to five seconds in a small dog, two to three seconds in a rabbit) is longer than the time of a conplete inspiration at any ordinary rate. The increase in the quantity of blood pumped into the pulmonary artery will, if not counteracted by other circumstances, tend to raise the bloodpressure in the artery and its branches, and therefore at once to accelerate the outflow through the pulmonary veins. This will be aided if at the same time the vascular resistance in the lungs is reduced, as is known to be the case. The left ventricle, like the right, is capable of discharging more blood than it ordinarily receives. The excess of blood coming to it is easily and promptly ejected. The systemic arteries are better filled and the arterial pressure rises.

In expiration the contrary will happen. The return of blood to the thorax will be checked. This is well shown by the swelling of the veins at the root of the neck in expiration, their shrinking in inspiration, the so-called respiratory venous pulse. Less blood being drawn into the right heart, less will be pumped into the pulmonary artery, in which the pressure will, of course, fall. The outflow into the left auricle will thus be diminished-all the more as in the expiratory phase the vascular resistance in the lungs is increased-and the systemic arterial pressure will be lowered. In both cases, however, the change seen in the blood-pressure curve will be belated, and will not coincide exactly with the commencement of the inspiration or the expiration. If it is delayed for a period about equal to the length of an inspiration or an expiration, the blood-pressure will be seen to sink in inspiration and to rise in expiration. If the period of delay is less than this, the pressure will be mounting during a part of each respiratory phase and falling during the rest. As to the explanation of the delay, several factors may be concerned.

The negative pressure of the thorax acts on the aorta, as well as on the thoracic veins, although, on account of the greater 
thickness of its walls, to a smaller extent than on the reins. The diminution of pressure in inspiration tends to expand the thoracic aorta, and to draw blood back out of the systemic arteries, while expiration has the opposite effect. And although the hindrance caused in this way to the flow of blowel into the arteries during inspiration, and the acceleration of the thw during expiration may not be great, they will, of conrse, be better marked in small animals with comparatively vielding arteries than in large animals. Yet, whether great or small, the tendency will be to diminish the pressure in the ont phase and increase it in the other. As soon as the changes of pressure produced by alterations in the flow of venous blood into the chest and through the lungs are thoroughly established, the arterial effect will be overborne; but before this happens, that is, at the beginning of inspiration and expiration, it will be in evidence, and will help to delay the main change.

Another factor in this delay is found in the changes of vascular capacity which take place in the lungs when they pass from the expanded to the collapsed condition. The expansion of the lungs in natural respiration causes a widening of the pulmonary capillaries, with a consequent increase of their capacity and diminution of their resistance. When the vessels at the base of the heart are ligatured either at the height of inspiration or the end of expiration, so as to obtain the whole of the blood in the lungs, it is found that they invariably contain more blood in inspiration than in expiration. During inspiration, as we have seen, the right ventricle is sending an increased supply of blood into the pulmonary artery; but before any increase in the outflow through the pulmonary veins can take place, the vessels of the lung must be filled to their new capacity. The first effect, then, of the lessened vascular resistance of the lungs in inspiration is a temporary falling off in the outflow through the aorta, and therefore a fall of arterial pressure. As soon as a more copious stream begins to flow through the lungs, this is succeeded by a rise. In like manner the first effect of expiration, which increases the resistance and diminishes the capacity of the pulmonary vessels, is to force out of the lungs into the left auricle the blood for which there is no room. This causes a rise of arterial blood-pressure, succeeded by a fall as soon as the lessened blood-flow through the lungs is established.

The changes in the diastolic capacity of the chambers of the heart jtself, with the clianges of pericardial pr-ssure, must also act in the same direction. It is obvious, then, how greatly the rate and depth of respiration in relation to the size of the animal and the other circumstances already mentioned may influence the time relations of the respiratory oscillations in the arterial 
pressure curve, so that we ought not to expect them to be absolutely constant.

In artificial respiration oscillations of blool-pressure, synchronous with the movements of the lungs, are also scen. During inflation (inspiration) the arterial pressure rises ; during deflation (expiration) it falls. The wares are not entirely abolisherl even when the thorax is opened. In the latter case there are, of course, no variations of intrathoracic pressure, and the oscillations must be connected with the changes in the pulmonary circulation. the inflation squeezing blood from the lungs into the left side of the heart, while deflation permits the pulmonary vessels to become filled to their new capacity at the expense of the stream flowing into the left auricle. When artificial respiration is stopped at the height of inflation (Fig. 114), the arterial blood-pressure falls rapidly, and continues low until the rise of asphyxia begins. The fall of pressure when the chest has been previously opened is due to the increased vascular resistance

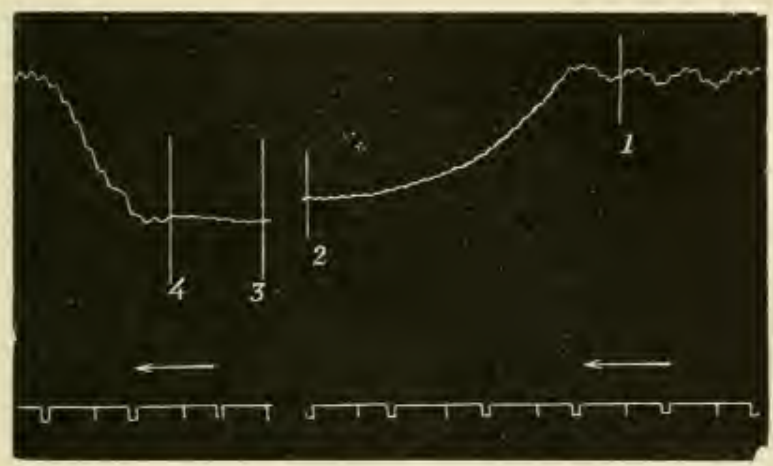

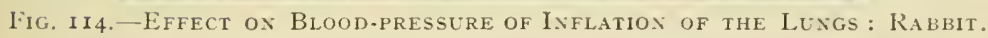

Artificial respiration stopped in inflation at $\mathrm{r}$. Interval between 2 and 3 (not reproduced) 5 I seconds, during which the curve was alınost a straight line. Time tracing shows seconds.

in the lings, due to the narrowing of the capillaries by the increased alveolar pressure. With intact chest the increased intrathoracic pressure due to the inflation is also an important factor. When the respiration is stopped in collapse, instead of a fall a steady rise of pressure occurs (as in Fig. 72, p. 172). This ultimately merges in the elevation due to asphyxia. which shows itself sooner than in inflation. When the tracheal cannula is closed in natural respiration, no initial fall of pressure takes place (Fig. II5).

Besides the mechanical effects of the respiratory movements on the circulation, it may be influenced by changes in the cardioinhibitory and vaso-motor centres synchionous with the rhythm of the respiratory centre. In many animals (the dog, for instance) and in man, it can be very easily made out that the rate of the heart is greater during inspiration, especially towards its end, than in expiration. The phenomenon is especially distinct in 
deep and slow respiration. It is catused hy at rhythnical rise and fall in the activity of the carlio-inhibitory centre, synchronous with the respiratory movements, for the difference disappears after division of both vagi. The normal respiratory oscillations of blood-pressure are not due in any great degree to such changes in the rate of the heart, for they persist after section of the vagi, and they are seen in animals like the rabbit, in which in ordinary breathing little or no variation in the rate of the heart is connected with the phases of respiration. The most probable explanation of the respiratory variations in the pulse-rate is that the respiratory centre, when it is discharging itself in inspiration, sends out impulses as a sort of overflow along fibres connecting it with the cardio-inhibitory centre. These increase the tone of that centre, but, owing to the long latent period of the cardioinhibitory apparatus, the inhibition does not reveal itself till the

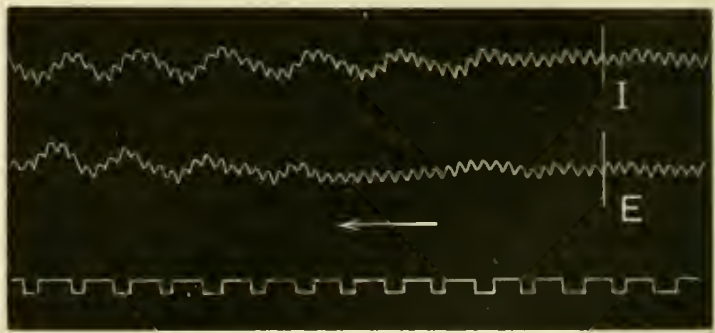

Fic. II 5. - BLOOD-PRESSURE TRACING: RABBIT, LNDER CHLORAL.

Natural respiration stopped at I in inspiration, at li in expiration. The mean blond-pressure is scarcely altered; but the respiratory waves become nuch larger owing to the abortive efforts at breathing. Time tracing shows seconds.

succeeding expiration. It may be, however, that the impulses discharged from the respiratory centre in inspiration diminish the tone of the cardio-inhibitory centre, and thus lead to acceleration of the heart towards the end of the inspiratory plase. In certain pathological conditions the influence of the respiration on the pulse-rate is exaggerated (so-called respiratory arhythmia).

Traube-Hering Curves.--Rhythmical changes in the activity of the vaso-motor centre, also associated witl periodic discharges from the respiratory centre, may be observed under certain conditions-e.g., when in an animal paralyzed by curara, and therefore unable to breathe spontaneously, the artificial respiration is stopped for a time. If such a dose of curara he given as will still permit slight spontaneous respiration to go on, and both vagi be cut, it can be scen on stopping the artificial respiration that the waves on the blood-pressure curve are exactly synchronous with the slow respiratory movements. The 
Tranle-Hering watres sink in insporation and rise in exponat tion.

The fact that they have invarialdy a lomger period than the matural respiratory mosements indicates that they are not concerned in the production of the normal respiratory oscillations of arterial pressure. Probably the reason why the Tranbe waves ilplear after section of the vagi is the increased vigour of the slow respiratory discharges, compled with a hyperexcitability of the vaso-motor centre, due to the long pauses in the acration of the blood. In the aspleyial rise of pressure in a curarized dog they are constantly seen, and are often observed when the circulation in the medulla oblongata is in any way interfered with (Fig. I I6). In addition to the true Traube-Hering waves, other and much longer periodic variations in the blood-pressure are sometimes noticed.

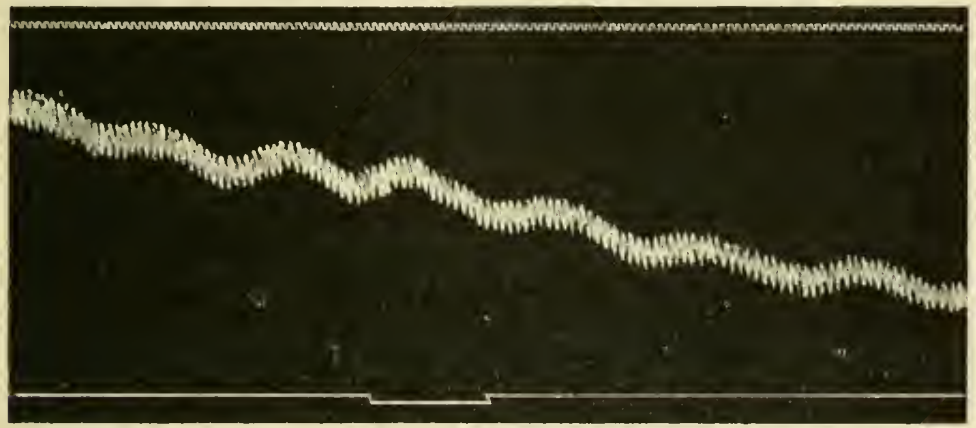

Fig. II6.-Traube-Hering Waves as the Blood-pressure is FAlling during Occlusion of the Cerebral Arteries in a Cat.

If spontaneous respiration is going on their long sweeping curves then show the ordinary respiratory waves superposed on them.

The normal respiratory oscillations in the reins, as might be expected, run precisely in the opposite direction to those in the arteries, and so do the Traube-Hering curves. The increased flow from the veins to the thorax during inspiration lowers the pressure in the jugular vein, while it increases the pressure in the carotid. The constriction of the small bloodvessels to which the Traube-Hering curves are due increases the blood-pressure in the arteries, because it increases the peripheral resistance to the blood-flow; in the veins it lowers the pressure, because less blood gets through to them. Accordingly, when the Traube-Hering curve is ascending in the carotid, it is descending in the jugulas.

The respiratory variations in the volume of the brain, which are so striking a phenomenon when a trephine hole is made in the skull, but which can also take place, thanks to the 
displacement of cerebro-spinal fluid (p. I $(00)$, when the cranimm is intact, have by some been attributed to interference with the venous outflow from the cranial cavity cluring expiration, and by others to those changes in the arterial pressure whose causes we have just been discussing. The truth is that neither factor is exclusively concerned. The question turns largely upon the time-relations of the movements. The swelling of the brain is sometimes synchronous with expiration, and the shrinking with inspiration. Here the damming back of the blood in the sinuses when the outflow is checked by the expiratory rise of pressure in the thoracic veins either conspires with an expiratory rise of arterial pressure or is more than enough to counterbalance an expiratory fall of pressure in the cerebral arteries if the respiratory conditions are such as to lead to an expiratory fall. But sometimes the dura mater bulges into the trephine hole in inspiration and sinks down in expiration. Here the increase in the volume of the brain produced by the increased pressure in the arteries and capillaries in inspiration is more than sufficient to counterbalance the quickened escape of blood from the cerebral reins.

The effects of breathing condensed and rarefied air are(I) mechanical, shown chiefly by changes in the circulation, in the blood-pressure, for instance; (2) chemical.

The mechanical effects differ according to whether the whole body, or only the respiratory tract, is exposed to the altered pressure. When the trachea of an animal is connected with a chamber in which the pressure can be raised or lowered, it is found that at first the arterial blood-pressure rises as the pressure of the air of respiration is increased above that of the atmosphere. But a maximum is soon reached; and when respiration begins to be impeded, the pressure falls in the arteries and increases in the veins. When the pressure of the air in the chamber is diminished a little below that of the atmosphere, there is a slight sinking of the arterial blood-pressure, which rises if the air-pressure is further diminished.

It is clear that any change of the air-pressure which tends to diminish the intrathoracic pressure will favour the venous return to the heart, and therefore, if the exit of blood from the thorax is not proportionally impeded, the filling of the arteries. An increase in the intra-alveolar pressure must tend on the whole to increase, and a diminution in it to lessen, the pressure inside the thorax, which always remains equal to the intra-alvcolar pressure, minus the clastic tension of the lungs. Breathing compressed air should, therefore, under the conditions described, be upon the whole unfavourable to the venous return to the heart and to the filling of the arteries, and the arterial pressure should fall; while breathing rarefied air should have the opposite effect. But a very great diminution of the intrathoracic pressure is not necessarily favourable to the circulation, since the auricles are then unable to contract perfectly. 
Certain chest (liseases have been treated by the use of apparatus by which the patient is made to breathe either compressed or rarefied air ; or to inspire air at one pressure and to expire into air at another pressure. And it has, upon the whole, been found, in agreement with theory, that condensed air cannot help the circulation however it is applied, but always hinders it; while rarefied air aids the circulation both in inspiration and in expiration. But the increased work of the inspiratory muscles may counterbalance the advantage.

Valsalva's experiment, which is performed by closing the month and nostrils after a previous inspiration, and then forcibly trying to expire, is an imitation of breathing into compressed air. The intrathoracic pressure is raised, it may be, to considerably more than that of the atmosphere; the venous return to the heart is

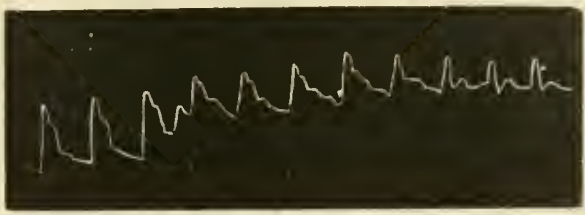

Fig. il 7.--Pulle Tracing in Valsalya's Experiment (Rollett). impeded, and may be stopped; and the pulse curve is altered in such a way as to indicate first an increase and then a decrease of the arterial blood-pressure (Fig. I I 7 ).

Mïller's experiment, which should be bracketed with Valsalva's, consists in making, after a previous expiration, a strong inspiratory effort with mouth and nostrils closed. Here the intrathoracic pressure is greatly diminished, more blood is drawn into the chest, and upon the whole effects opposite to those of Valsalva's experiment are produced (Fig. I I 8 ). Neither experiment is quite free from langer. In both the dicrotism of the pulse becomes more marked.

When the whole body is subjected to the changed pressure, as in a balloon or on a

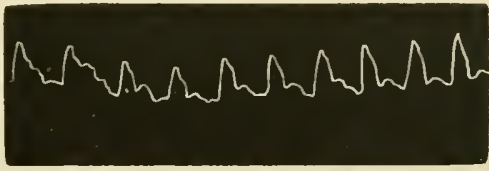

Fig. Ir 8.-PULSE Tracing IN MÜLleR'S EXPERIMENT (ROLLETT). mountain, in a diving-bell or a caisson used in building the piers of a bridge, the conditions are very different. For the blood-pressure, the intrathoracic pressure, and the intra-alveolar pressure, all fall together when the pressure of the atmosphere is diminished, and all rise together when it is increased. It is possible not only to live, but to do hard manual labour, at very different atmospheric pressures.

As regards the chemical effects of condensed and rarefied air, Loewy found that the quantity of oxygen absorbed by a man breathing air in the pneumatic cabinet remained constant at all pressures between about two atmospheres and half an atmosphere. At $440 \mathrm{~mm}$. of mercury dyspnœea became evident; but if the person was now made to work, the dyspnœa passed away, and did not again manifest itself till the pressure was reduced to $410 \mathrm{~mm}$. There are towns on the high tablelands 
of the Andes, and in the Himalayas, where the barometric pressure is not more than 16 to 20 inches, ret the inhabitants feel no ill effects. And in the caissons of the Forth Bridge the workmen were engaged in severe toil under a maximum pressure of over three atmospheres, while in the caissons of the St. Louis Bridge in America a maximum pressure of over four atmospheres (i.e., more than three atmospheres in addition to the ordinary air-pressure) was reached.

Inside the caissons the men sometimes suffer from pain and noise in the ears, due to excessive pressure on the external surface of the tympanic membrane. If the pressure in the tympanum is raised by a swallowing movement, which opens the Eustachian tube and permits air to enter it, the symptoms generally disappear. The suldenness of the change of pressure has much to do with its effects, and it is found that the men are most liable to dangerous symptoms while passing through the air-lock from the caissons to the external air. It may be concluded from experiments on animals, that some of the most serious of these-the localized paralysis usually affecting the legs (paraplegia) and the circulatory disturbances - are clue to the formation of gaseous emboli, by the liberation of nitrogen in the blood and other body-fluids when the pressure is abruptly reduced. And, indeed. it is found that the symptoms can often be caused to disappear, both in animals and men, by promptly subjecting them again to compressed air. To avoid gas embolism on decompression. the shift should be so short that the body-fluids do not become fully saturated with nitrogen, and the decompression should be slow. Even with a rate of decompression of twenty minutes for each atmosphere of excess pressure the equilibrium between the dissolved and the atmospheric nitrogen is not entirely established fifteen minutes after decompression.

But that the action of air under a high pressure is not merely mechanical follows from the singular fact that in pure oxygen at a pressure of + to 5 atmospheres, which corresponds to air at 20 to 25 atmospheres. convulsions are often produced in vertebrate animals, while exposure to 6 to 25 atmospheres of oxygen causes dyspnœa and coma, usually without convulsions. All animals, so far as investigated, are instantly convulsed and killed under a pressure of 50 atmospheres of oxygen (Hill and Macleorl). Even seeds and regetable organisms in general are killed in a short time in oxygen at 3 to 5 atmospheres: and an atmosphere of pure oxygen, equal to five atmospheres of air. hinders the development of eggs. Lorrain Smith has shown that in small birds and mice exposure for many hours to a pressure of between I and 2 atmospheres of pure oxygen causes pneumonia. He confirms Bert's observations on the acute toxic effects produced by higher pressures. and supposes that in the production of caisson clisease the special action of the oxygen at high pressure may play a part as well as the rapid decompression.

When the air-pressure is diminished below a certain limit, death takes place from asphyxia, more or less gradual according to the rate at which the pressure is reduced. The hrmoglobin cannot get or retain enough oxygen to enable it to perform its respira- 
tory function; its dissociation tension is no longer balanced by an equal or greater partial pressure of oxygen in the air. The tension of carbon dioxide in the blood is also lessened, owing to the dyspncea and the consequent increase of pulmonary ventilation.

To such changes, as well as to the cold, some of the deaths in high balloon ascents must be attributed. Messrs. Glaisher and Coxwell supposel that they reached the height of 37,000 feet; the former bccame unconscious at 29,000 fect ( 8,800 metres), at which height the amount of oxygen in the arterial blood would probably not excecl ro volumes per cent., but recovered during the descent. The symptoms of the 'mountain sickness' so familiar to Alpine climbers (nausca. headache, and marked depression), the undue hyperpnoa proluced by muscular excrtion, and the slecp disturbed by irregular breathing, are also mainly due to deficiency of oxygen in the blood. The most rational prophylaxis is to leave the high peaks severely alone. But for the enthusiasts who cannot do this a portable apparatus for generating oxygen has been devised. Experiments in the preumatic cabinet indicate that the hyperpnoea is due to the indirect action of want of oxygen already referred to in cliscussing the normal regulation of respiration (p. 230) - that is, to the formation, in consequence of the insufficient oxygen supply, of lactic acid or other substances which have the same influence as carbon dioxide on the respiratory centre-so that less carbon dioxide is required to excite the centre. Although the hyperpnoea leads to a diminution in the partial pressure of carbon dioxide in the pulmonary alveoli, there is no evidence that lack of carbon dioxide ('acapnia') is the primary cause of mountain sickness (Haldane). It must be remembered, however, that here the influence of the low barometric pressure is complicated by other conditions. For example, while in the pneumatic cabinet, as already stated, diminution of the pressure does not affect the oxygen consumption, it is relatively much greater on the high mountain levels both during rest and during work than on the plains. This is not the case in balloon ascents. And evidence has been brought forward that changes in the mechanics as well as in the chemistry of respiration are concerned (the breathing, for instance, taking on a periodic character, with some approach to the Cheyne-Stokes type [p. 238$]$ ), and that there is something not connected with the want of oxygen which diminishes the capacity for muscular work. This

'something' is perhaps a peculiar excitation of the nervous system in the fierce light of those high levels, which acts not only on the retina, but on the skin, and may even affect the distribution of the blood. It is said that a so-called light bath, as used in the treatment of certain diseases, may increase the quantity of blood in rabbits by 25 per cent. in four hours. The shorter wave-lengths which are relatively more intense in the mountain light are most effective.

Cutaneous Respiration.- - It has already been remarked that a frog survives the loss of its lungs for some time, respiration going on through the skin. Indeed, it has been calculated that in the intact frog, under ordinary conditions, as much as three-quarters of the total gaseous exchange may be cutaneous. Two frogs were seen to live thirty-three days, and one even forty days, after excision of the lungs. The effect of exclusion of the pulmonary respiration on the gaseous exchange depends on the previous intensity of the metabolism. If this is high the gaseous exchange sinks markedly; if it is low there is scarcely any alteration. At their naximum 
efficiency the frog's lungs are capable of sustaining a much greater exchange than the skin. Besides this quantitative, there is a qualitative difference, the carbon dioxide passing more easily through the slin than the oxygen, so that the respiratory quotient is increased by clinination of the lungs. In mammats the structure of the skin is different, and respiration can only go on through it to a very slight extent. The amount of carbon dioxide excreted in man, although only about $4 \mathrm{grm}$. or 2 litres in twenty-four hours, is much greater than corresponds to the guantity of oxygen absorbed through the skin. It has been asserted. and no doubt with justice, that some at least of the carbon clioxide given off is due to putrefactive processes taking place on the surface of the body. Such proceseses, as has already been pointed out, secm also responsible in part for the heavy odlowr of a 'close' room. For no harmful products appear to be exhaled from the slin when it is properly cleansed. In spite of the romantic statements to the contrary in ancient and modern books (for instance, the story of the child that was gilded to play the part of an angel at the coronation of a medieval pope, but died before the ceremony began), the whole of the human skin may be coated with an impermeable varnish without any ill effects. The entire surface of the body of a patient with cutaneous disease was covered with tar, and kept covered for ten days. There was not the least disturbance of any normal function. Thic serious effects of varnishing the skin in animals are due, not to retention of poisonous substances, but to increased heat loss. Varnishing is not so rapidly harmful in large animals like dogs as in rabbits, which have a relatively great surface and a delicate skin. The danger of widespread superficial burns is well known. But it is not due to diminished cxcretion by the skin, for death occurs when large cutancous arcas remain uninjured. The patient nearly always dies when a quarter of the whole skin is burnt: yet the remaining threequarters may surely be considered capable, from all analogy. of making up the loss by increased activity. One kidney is enough to eliminate the products of the nitrogenous metabolism of the whole body. It is difficult to see why the excretion of the trifling amount of solid matter in the perspiration should be interfered with by the loss of 25 per cent. of the sweat-glands. The real explanation of the serious effects of extensive sujerficial burns is perhaps the excessive irritation of the sensory nerves, which may lead to changes in the nervous centres, or reflexly in other organs, or the chenical changes in the damaged tissue, for example, in the blood-corpuscles, or the transudation of lymph at the injured part, and consequent increase in the concentration of the blood.

\section{Voice and Speech.}

Voice.-Sounds of various kinds are frequently produced by the movements of animals as a whole, or of individual organs. The muscular sound, the sounds of the heart and of respiration, we have already had to speak of. Such sounds may be considered as purely accidental as the footfall of a man or the buzzing of a fly. The wings of an insect beat the air, not to cause sound, but to produce rnotion; the respiratory murmur is a mere indication that air is funding its way into the lungs, it is in no way related 
to the oxidation of the blood in the pulmonary cappillaries. But in many of the higher animals mechanisms exist which are specially devoted to the utterance of sounds as their prime and proper end. In man the voice-producing mechanism consists of a triple series of tubes and chambers: (I) The trachea, through which a blast of air is blown; (2) the larynx, with the vocal cords, by the vilorations of which sound-waves are set up; and (3) the upper resonance chambers, the pharynx, mouth, and nasal cavities, in which the sounds produced in the larynx are modified and intensified, and in which indepenclent notes and noises arise.

The larynx is a cartilaginots box, across which are stretched, from front to back, two thin and sharp-edged membranes, the (true) vocal cords. In front the cords are attached to the thyroid cartilage. one a little to each side of the middle line; behind they are connected to the vocal or anterior processes of the pyramidal arytenoid cartilages. The thyroid and the two arytenoids are mounted upon a cartilaginous ring, the cricoid. The arytenoids can rotate on the cricoid about a vertical axis, while the cricoid can rotate on the thyroid cartilage around a transverse horizontal axis. The cricoid can thus be raised by the contraction of the crico-thyroid muscle, and the vocal cords stretched. By the pull of the posterior crico-arytenoid muscles, attarhed to the external or muscular processes of the arytenoid cartilages, the vocal processes are rotated outwards, the cords separated from cach other or abducted, and the chink between them, the rima glottidis, widened. When the vocal processes are approximated by contraction of the lateral crico-arytenoid inuscles and the consequent forward movement of the muscular processes, the vocal cords are brought closer together, or adducted, and the rima is narrowed. The transverse or posterior arytenoid muscle, which connects the two arytenoid cartilages behind, also helps, by its contraction, to narrow the glottis by shifting the cartilages on their articular surfaces somewhat nearer the middle line. Running in each vocal cord, and, in fact, incorporated with its clastic tissue, is a muscle, the thyro-arytenoid. the external portion of which may to some extent cause inward rotation of the vocal processes and adduction of the cords; but the main function, at least of its inner part, is to alter the tension of the cords. The diagrams in Figs. IIg and 120 illustrate the action of the abductors and adductors of the vocal cords.

The crico-thyroid muscle and the deflectors of the epiglottis are supplied by the superior laryngeal branch of the vagus, which also contains the sensory fibres for the mucous membrane of the larynx above the vocal cords. In the dog and rabbit motor fibres also reach the crico-thyroid by the so-called middle iaryngeal nerve which arises from the superior pharyngeai branch 
of the vagus. All the other intrinsic muscles are supplied by the recurrent laryngeal branch of the vagus. It receives these motor fibres from the spinal accessory, and supplies sensory fibres to the mucous membrane of the larynx below the vocal cords and to the trachea.

The voice is produced, like the sounds of a reer instrument, by the rhythmical interruption of an expiratory blast of air by the vibrating vocal cords. When a bell is struck, vibrations are set up in the metal, which are communicated to the air. It is not the same with the vibrations of the vocal cords; if they were plucked or struck, they would only produce a feeble note. The air in the mouth, pharyux, larynx, trachea, and lungs is

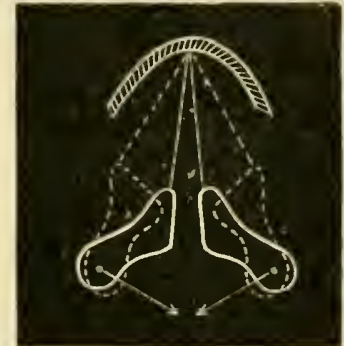

Fig. ITy.-DIAGRAMAATIC HORIZONTAL SECTION OF LARYNX TO SHOW THE II. RECTION OF PULL OF THE I'OSTERIOR CRICO-ARYTENOID MUSCLES, WHICII ABDUCT the Vocal, Cords.

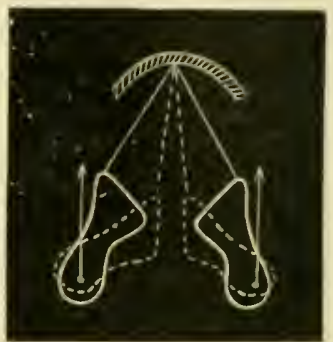

Fig. 120.-DiRection of PUll OF the Lateral Crico. ARYTENOIDS, WHICH ADDUCT IIIE VOCAI, CORDS.

Dotted lines show position in adduction.

Dotted lines show position in abduction.

the real sounding body; a pulse of alternate rarefaction and condensation is set up in it by the interference, at regular intervals, of the vocal cords with the expiratory blast. Forced abruptly' from their position of equilibrium as the blast begins, they almost immediately regain and pass below it, in virtue of their elasticity, and continue to vibrate as long as the stream of air continues to issue in sufficient strength. Not only do they vibrate up and down, but also towards and away from the middle line, so that, at least in the chest voice, they come into contact with each other at each swing. The sound-waves thus set up spread out on every side, impinge on the tympanic membrane, set it quivering in response, and give rise to the sensation of sound.

We may say, in a word, that the whole exquisite mechanism of cartilages, ligaments, and muscles, has for its object the production of a sufficient pressure in the blast of air driven through the windpipe by an expiratory act, and of a suitable tension in 
the vibrating cords. An approximation of the cords, a narrowing of the glottis, is essential to the production of voice; with a widely-opened glottis the air escapes too easily, and the necessary pressure cannot be attained. The pressure in the windpipe was found in a woman with a tracheal fistula to be about $12 \mathrm{~mm}$. of mercury for a note of medium lieight, about $15 \mathrm{~mm}$. for a high note, and about $72 \mathrm{~mm}$. for the lighest possible note. The period of vibration of structures like the vocal cords depends on their length, thickness, density and tension; the shorter, thinner, morc tense and less dense a stretched string is, the greater is the vibration frequency, the higher the note. In the child the cords are short ( 6 to 8 mm.), in woman longer (Io to I $2 \mathrm{~mm}$. when slack, I3 to $I_{5} \mathrm{~mm}$. when stretched), in man longest of all ( $\mathrm{I}_{4}$ to $\mathrm{I} S \mathrm{~mm}$. in the relaxed, and $\mathrm{I} 8$ to $22 \mathrm{~mm}$. in the stretched position); and the lower limit of the voice is fixed by the maximum lengtl of the relaxed cords. A boy or a woman cannot utter a dec]) bass note, hecause their vocal cords are relatively short, and do not vibrate with sufficient slowness. It is true that by the action of the crico-thyroid muscle the cords can be lengthened, and that the maximum length in a woman approaches or excecds the minimum length in a man. But the lengthening of the vocal cords in one and the same individual is always accompanied by other changes-increase of tension, decrease of breadth and thickness-which tell upon the vibration frequency in the opposite way, and more than compensate the effect of the increase of length, so that for high notes the cords are longer than for low. The contraction of the thyro-arytenoid muscle is a more influential factor in altering the tension of the cords than the contraction of the crico-thyroid. It is probable that when the lighest notes are uttered, only the anterior portions of the cords are free to vibrate, their posterior portions being damped by the approximation of the vocal processes of the arytenoid cartilages by the contraction of the lateral crico-arytenoid and transverse arytenoid muscles. The range of an ordinary voice is 2 octaves; by training $2 \frac{1}{2}$ octaves can be reached: but in exceptional cases a range of 3 , and even $3 \frac{1}{2}$, octaves (as in the celebrated singer Catalani) has been known.

The development of the voice in children is of great interest. At the age of six years the boy's voice has a rather narrower range than the girl's in both dircctions. The boy's voice reaches its full height in the twelfth and its full depth in the thirteenth year, when the range is almost 3 octaves, its upper limit being a semitone higher than the girl's, but its lower limit a whole tone deeper. When the voice 'brealss' in boys at the age of puberty it falls about an octave. The control of the vocal organs becomes so incomplete that only in one-fourth of the cases can notes of sufficient steadiness to be used in music be produced. The vocal cords, as may be seen with the laryngoscope, are frequently, though not alwıys, congested. 
The pitch of a note, while it depends chiefly, as has been said, on the tension of the vocal cords, rises and falls somewhat with the strength of the expiratory blast; the highest notes are only reached with a strong expiratory effort. The intensity of all rocal sounds is determined by the strength of the blast, for the amplitude of vibration of the cords is proportional to this. Besides pitch and intensity, the ear can still distinguish the quality or timbre of sounds; and the explanation is as follows: Two simple tones of the same pitch and intensity, that is, the sounds caused by two series of air-waves of the same period and amplitude-of the same frequency and height, to use less technical terms-would appear absolutely identical to the sense of hearing; just as the aerial disturbances on which they depend would be absolutely alike to any physical test that could be applied. But no musical instrument ever produces sound-wares of one definite period, and one only; and the same is true of the voice. When a stretched string is displaced in any way from its position of rest, it is set into vibration : and not only does the string vibrate as a whole, but portions of it vibrate inclependently and give out separate tones. The tone corresponding to the vibration period of the whole string is the lowest of all. It is also the loudest, for it is more difficult to set up quick than slow vibrations. The ear therefore picks it out from all the rest : and the pitch of the compound note is taken to be the pitch of this, its fundamental tone. The others are called partial or overtones, or harmonics of the fundamental tone, their vibration frequency being twice, three times, four times, etc., that of the latter. Now, the fundamental tone of a compound note or clang produced by two musical instruments may be the same, while the number, period, and intensity of the harmonics are different; and this difference the ear recognises as a difference of timbre or quality. The timbre of the voice depends for the most part on partial tones produced or intensified in the upper resonance chambers.

A great deal of our knowledge as to the mode and mechanism of the production of voice has been acquired by means of the laryngoscope (Fig. I2I). This consists of a small plane mirror mounted on a handle, which is held at the back of the mouth in such a position that a beam of light, reflected from a larger concave mirror fastened on the forehead of the observer, is thrown into the larynx of the patient. The ohserver looks through a hole in the centre of the large mirror: and an image of the interior of the larynx is seen in the small mirror, in which the parts that are anterior appear as posterior. the arytenoid cartilages in front, the thyroid behind, and the vocal cords stretching between. The small mirror is warmed to body. 
temperature before being introduced, so as to prevent the condensation of moisture on it. The tendency to retch which is caused by contact of the instrument with the soft palate may be removed or lessened by the application of a solution of cocaine.

Examined with the laryngoscope during quiet respiration, the glottis is seen to be moderately, though not widely, open, and the rocal cords almost motionless. Although the portion between the arytenoid cartilages has received the name of glottis respiratoria, in contradistinction to the glottis vocalis between the vocal cords, the rima in its whole extent from front to back is really concerned in the respiratory act. In deep expiration the vocal cords come nearer to the middle line, and the glottis

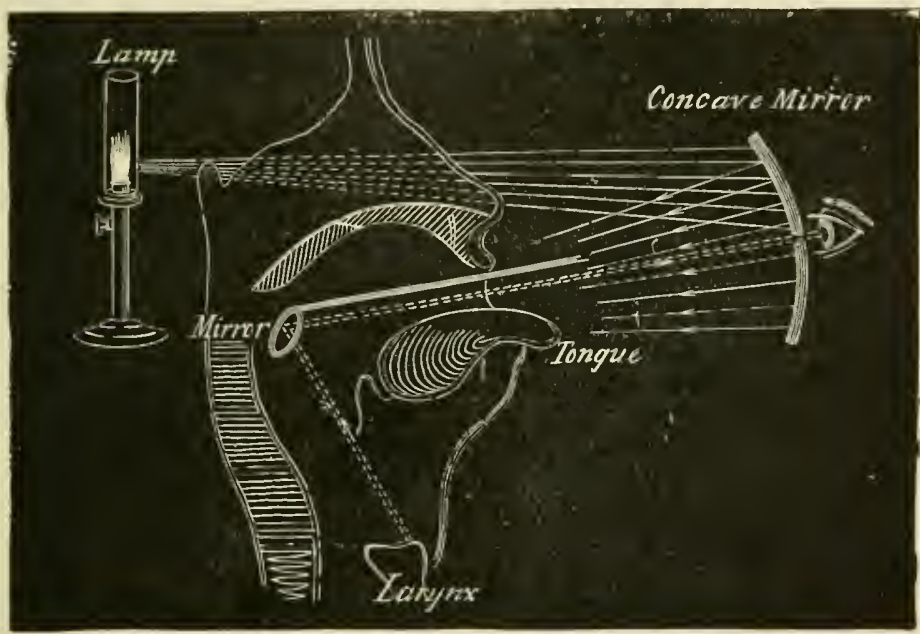

Fig. I2I.-DIAGRAM OF I.ARYNGOSCOPE.

is narrowed; in deep inspiration they are widely separated, and the rings of the trachea, and even its bifurcation. may be disclosed to view. When a sound is produced-a note sung, for example - the cords are approximated (Figs. I22 and I23) ; and with a high note more than with a low.

The essential difference between the production of notes in the lower register, or chest voice, and in the higher register, or falsetto. has been much debated. The lowest notes which can be uttcred by any given voice are chest notes, the highest are falsetto notes : but there is a debatable land common to both registers, and medium notes can be sung either from the chest or from the head. Chest notes impart a vibration or fremitus to the thoracic walls, from the resonance of the lower air-chambers, the trachea and bronchi ; and this can be distinctly felt by the hand. In head notes or falsetto the resonance is chiefly in the upper cavities, the pharynx, mouth, 
and nose. As to the nechanical conditions in the larvix, there is a pretty general agreement that during the production of falsetto notes the vocal cords are less closely approximated thaz in the sounding of cliest notes. The escape of air is consequently more rapid in the head voice, and a falsetto note cannot be maintained sc long as a note sung from the cliest. But it is only the anterior part of the rima glottidis that is wider in the falsetto voice; the whole of the glottis respiratoria. and even the posterior portion of the glottis vocalis, are closed during the emission of falsetto notes.

Oertel has stated, and the statement has been confirmed by others, that the free edge of the vocal cord alone vibrates in the falsetto voice, one or more nodes or motionless lines parallel to the edge being formed by the contraction of the internal part of the thyroarytenoid muscle, which thus acts like a stop upon the cord.

Approximation of the rocal cords may take place in certain acts unconnected with the production of voice. Thus, a congh,

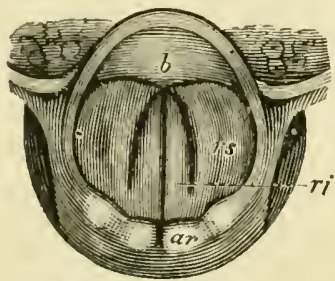

FIG. I 22 . POSITION OF THE GLOTTIS PRELIMINARY TO THE UTTERANCE OF SOUND.

$r$, false vocal cord; $r$, true vocal rord; ar, arytenoid carti. lage; $b$, pad of the epiglottis.

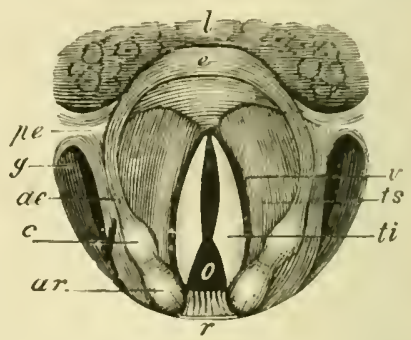

Fig. 123.-Position OF OPEN Glottis.

l. tongue: $e$, epiglot tis; $a e$, ary. eniglottidean fold; $c$, cartilaze of Wrisberg; ar, arytenoid cartilage; 0 , glottis; $v$, ventricle of Morgagni ; $t i$, true vocal cord; $t s$, false vocal cord.

as has already been mentioned, is initiated by closure of the glottis. During a strong muscular effort, too, the chink of the glottis is obliterated, and respiration and phonation both arrested. The object of this is to fix the thorax, and so afford points of support for the action of the muscles of the limbs and abdomen. But considerable efforts can be made even by persons with a tracheal fistula.

Speech.-Ordinary speech is articulated voice-roice shaped and fashioned by the resonance of the upper air-cavities, and jointed together by the sounds or noises to which the varying form of these cavitics gives rise. Here we come upon the fundamental distinction between vowels and consonants. Vowels are musical sounds; consonants are not musical sounds, but noises - that is to say, they are due to irsegular vibrations, not to regularly recurring waves, the frequency of which the ear can 
apmreciate as a defunite pitch. This difference of character corresponds to a difference of origin : the vowels are produced by the ribrations of the rocal cords; the consonants are due to the rushing of the expiratory blast through certain constricted yortions of the buccal chamber, where a kind of temporary glottis is established by the approximation of its walls. One of these 'positions of articulation' is the orifice of the lips; the consonants formed there, such as $p$ and $b$, are called labials. A second articulation position is between the anterior part of the tongue and the teeth and hard palate. Here are formed the dentals, $t$, $d$, etc. The ordinary English $r$, and the $r$ of the Berwickshire and East Prussian 'burr,' also arise in this position through a vibratory motion of the point of the tongue. The third position of articulation is the narrow strait formed between the posterior portion of the arched tongue and the soft palate. To the consonants arising here the name of gutturals has been given. They include $k, g$, the Scottish $c h$. and the uvular German $r$. The latter is produced by a vibration of the uvula. The aspirated $h$ is a noise set up by the air rushing through a moderately wicke glottis, and some have therefore included the glottis as a fourth articulation position for consonants. Certain sounds like $n, m$, and $n g$, when fina! (as in pen, dam, ring), although produced at the glottis, are intensified by the resonance of the air in the nose and pharynx, and are sometimes spoken of as nasal consonants.

As we have said, the vowels are produced by vibrations of the vocal cords, but to what they owe their special timbre or quality has been much discussed. According to the view with which Helmholtz's name is particularly connected this is due to the reinforcement of certain overtones by the resonating cavities, the shape and fundamental tone of which are different for each vowel.

When a vowel is whispered, the mouth assumes a characteristic shape, and emits the fundamental tone proper to the form and size of the particular 'vowel-cavity,' not as a reinforcement of a tone set up by the vibrations of the vocal cords, but in response to the rush of air through the cavity; just as a bottle of given shape and size gives out a definite note when the air which it contains is set in vibration, by blowing across its mouth. A whisper, in fact, is speech without voice ; the larynx takes scarcely any part in the production of the sound; the vocal cords remain apart and comparatively slack; and the expiratory blast rushes through without setting tham in vibration.

The fundamental tone of the 'vowel-cavity' may be found for each vowel by placing the mouth in the position necessary for uttering it, then bringing tuning-forks of different period in front of it, and noting which of them sets up sympathetic resonance in the air of the mouth, and so causes its sound to be intensified. The fundamental tone is lowest for $u$ (as in lute). Next comes $o$; 
then $a$ (as in path): then $a$ (as in fane): then $i$ : whle $e$ is highest of all. A simple illustration of this maty be found in the fact that when the vowels are whispered in the order griven, the piteln rises. When 4 or $o$ is sounded, the buccal cavity has the form of a wirlebellied flask, with a short and narrow neck for $u$, a still shorter but wider neck for $o$. For $e$ the tongue is raised and almost in contact with the palate, and the cavity of the mouth is shaped like a flask with a long narrow neck and a very short belly. For $i$ the shape is similar, but the neck is not so narrow. For $a$ (as in palh) the vowelcavity is intermediate in form between that of $u$ and $e$, being roughly funnel-shaped, and the mouth is ratl er widely opened. For $u(00)$ the resonating cavity is made as long as posible, the larynx being depressed and the lips protruded : for $e$ the resonating cavity is at its shortest. the larynix being raised as much as possible and the lips retracted (Figs. 124 to 126 ).

According to Helmholtz, all that the resonating cavity does is to strengthen certain of the partials or overtones of the laryngeal note. If this is true, the partials which give a vowel-sound the timbre by

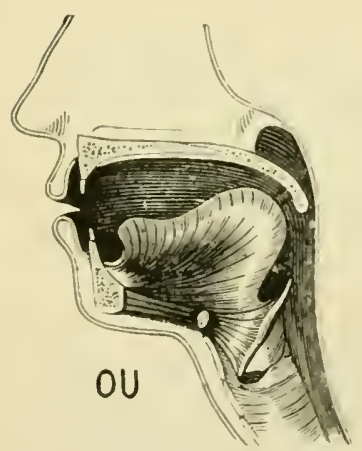

F1G. I24.

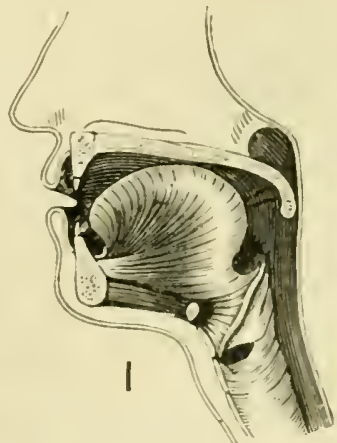

FIG. 125 .

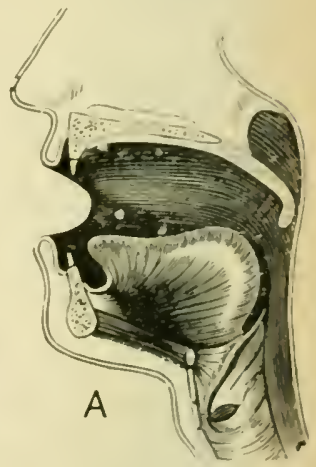

FIG. 126 .

which we recognise it as different from other vowel-sounds cannot preserve the same numerical relation to the fundamental tone when the pitch of the latter is altered. Suppose, for example, that a given vowel is souncled with a pitch corresponding to 100 vibrations a second, and that the partial which is particularly strengthened by the resonance of the mouth cavity is the fifth overtone, corresponding to 600 vibrations. Then when the same rowel is sounded with a pitch of 200 vibrations the reinfored partial which will now give the quality to the sound will still correspond to 600 vibrations a second, since this is the rate which most easily elicits the resonance, but it will not now be the fifth but the second overtone.

Universally accepted for a time. the Helmholtz theory has been in recent years assailed, especially by Hermann, who bases his criticism on microscopic examination of curves obtained by the Edison phonograph, and on reproductions of such records obtained by photographing on a moving drum covered with sensitive paper a beam of light reflected from a small mirror attached to a system of levers whose movements fellow the curves faithfully and greatly magnify them. Hermann has come to the conclusion that the mouth does 
not act as a mere resonator, but that for each vowel, in addition to the funclamental note dine to the vibration of the vocal cords, the pitch of which is, of course, viriable, one or, it may be, two other notes (formants, as he calls them), not necessarily harmonics of the laryngeal note, but separated from it by a constant or nearly constant musical interval, are directly produced by the passage of the regularly interrupted cxpiratory blast through the mouth, the air contained in that cavity being for an instant set into vibration at each interruption. On this view it is the musical effect produced by the oscillation or continual recurrence, in short series, of these vibrations which gives the vowels their quality. The fact that it is by no means difficult to sing (with the larynx) and whistle (with the mouth) at the same time, shows the possibility of Hermann's view, that a fixed tone can be generated in the mouth by the intermittent stream of air issuing from between the vibrating vocal cords, just as a tone is generated in a pipe by blowing into or over it, and his records do show continually recurring groups of vibrations as his theory requires. McKenlrick takes up a middle position, belicving that both theories are partially true, and this seems to be the best conclusion which can at present be arrived at. It seenis clcar, at any rate, that more than one factor is concerned in the timbre of the vowel sounds.

When the vowels are being uttered, the soft palate closes the entrance to the nasal chambers completely, as may be shown by holding a candle in front of the nose, or trying to inject water through the nares. If the cavities of the nose are not completely blocked off, the voice assumes a nasal character in pronouncing certain of the vowels; and in some languages this is the ordinary and correct pronunciation.

Many animals have the power of emitting articulated sounds; a few have risen, like man, to the dignity of sentences, but these only by imitation of the human voice. Both vowels and consonants can be distinguished in the notes of birds, the vocal powers of which are in general higher than those of mammalian animals. The latter, as a rule, produce only vowels, though some are able to form consonants too.

The nervous mechanism of voice and speech will have to be again considered when we come to study the physiology of the brain and spinal cord. But the curious physiological antithesis between the functions of abduction and of adduction of the rocal cords may be mentioned here. The abductor muscles are not employed in the production of voice; they are associated with the less specialized, the less skilled and purposive function of respiration. The adductor muscles are not brought into action in respiration; they are associated with the highlyspecialized function of speech. Corresponding to this difference of function, we find that adduction is preponderatingly represented in the cortex of the brain, abduction in the medulla oblongata. Stimulation of an area in the lower part of the 
ascending frontal convolution, near the fissure of Rolando, in the macaque monkey, causes adduction of the vocal cords, never abduction. In the cat, however, abduction of the cords may also be obtained by stimulation of the cortex. The same is true of the dog, but only when the peripheral adductor nerves have been divided. Stimulation of the medulla oblongata (accessory nucleus) causes abdnction, never adduction. The skilled adductor function is, therefore, placed under control of the cortex. The vitally important, but more meclanical, abductor function is governed by the medulla. The abductor movements are more likely to be affected by organic discase, the adductor movements by functional changes. But the distinction between the two groups of muscles is not entirely due to a difference of central connections, since by altering the strength of the stimulus and the external conditions the one or the other may be separately

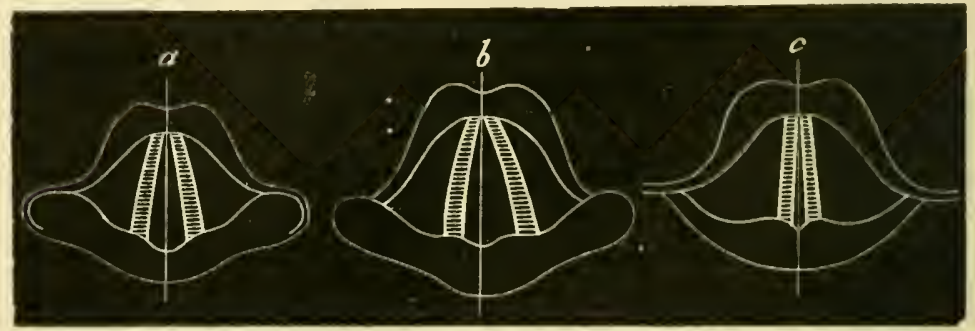

Fig. I27. - Diagram of Vocal Cords ix Paralyses of the Laryx.

$a$, Paralysis of both inferior laryngeal nerves. The vocal cords have taken up the 'mean' position. $b$. Paralysis of right inferior laryngeal nerve. An attempt is being made to narrow the glottis for the utterance of sound. The right cord remains in its 'mean' position. $c$, Paralysis of the abductor muscles only, on both sides. The cords are approximated beyond the "mean' position by the action of the adductors.

excited through the inferior laryngeal nerve. Thus, strong stimulation of the inferior laryngeal causes closure of the glottis, for although it supplies both abductors and adductors, the latter, as the stronger muscles, prevail. With weak stimulation, and in young animals, the abductors, owing to the greater excitability. of the neuro-muscular apparatus, carry off the victory, and the glottis is opened (Russell).

When the nerve is cooled the abductors give way before the adductors. The same is true when it is allowed to become dry. And after death in a cholera patient it was observed that the posterior crico-arytenoid, an abductor muscle, was the first of the intrinsic laryngeal muscles to lose its excitability. I-esions of the medulla oblongata are often accompanied by marked changes in the character of the voice and the power of articulation.

Section or paralysis of the superior laryngeal nerve causes the 
voice to hecome hoarsa, and renders the sounding of high notes an inpossibility, owing to the want of power to make the vocal cords tense. Stimulation of the vagus within the skull causes contration of the crico-thyroid muscle and increased tension of the cords. Section or paralysis of the inferior laryngeal nerves leads to loss of voice or aphonia, and dyspnoea (Fig. 1 27). Both adductor and abductor muscles are paralyzed; the vocal cords assmme their mean position - the position they have in the dead body-and the glottis can neither be narrowed to allow of the production of a note, nor widened during inspiration. It is said, however, that young animals, in which the structures around the glottis are more vielding than in adults, can still utter shrill cries after section of the inferior laryngeals, the contraction of the crico-thyroid muscle alone being able, while increasing the tension of the cords, to draw them together.

Interference with the connections on one side between the higher cerebral centres and the medulla oblongata, as by rupture of an artery and effusion of blood into the posterior portion of the internal capsule (giving rise to hemiplegia, or paralysis of the opposite side of the body), is not followed by loss of voice; the laryngeal muscles on both sides are still able to act.

\section{PRACTICAL EXERCISES ON CHAPTER III.}

I. Tracing of the Respiratory Movements in Man.-Pass a tape through the rings $B$ of the stethograph shown in Fig. I28, and then around the neck or over the shoulders, so as to support the instrument on the chest at a convenient height. Fasten tapes to the books and tie them by a slip - knot round the chest. The tube $\mathrm{E}$ is connected to a recording tambour, writing on a drum. Or use the belt stethograph or spirograph of Fitz (p. 218), fasten-

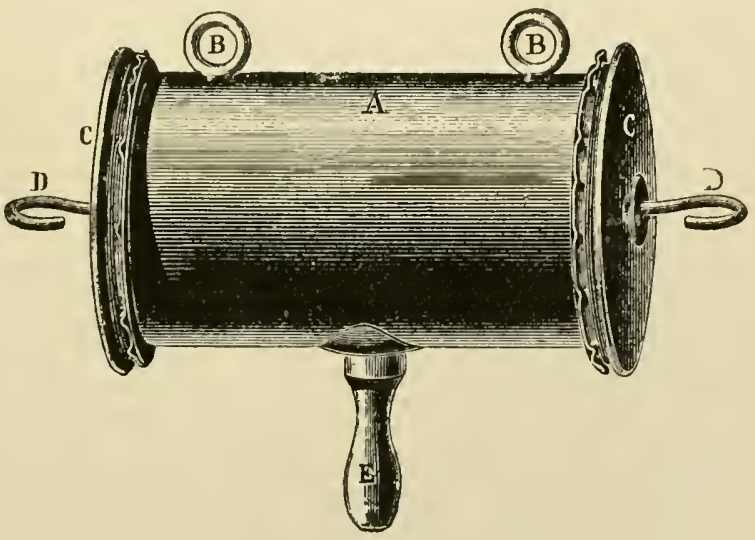

Fig. I28.-STEthograpH.

ing the elastic tube round the chest with the chain, and connecting it with a tambour or the bellows recorder shown in Fig. I3I. Compare the extent of the excursion when the tube is adjusted at different levels over the thorax and abdomen. 
2.* Production of Apnœa and Periodic Breathing in Man.-Arrange for taking tracings of the respiratory movements from a fellowstuclent as in 1. 1,et the subject of the experiment reclinc in a perfectly easy position in an armehair. Let him then breathe deeply and frequently for about two minutes, so as to produce a prolonged apnœa of about two minutes' duration. Whenever any desire to breathe returns, the breathing is to be allowed to take its own course. It may be expected at first to be of the periodic (Cheync-Stokes) type.

3. Tracing of the Respiratory Movements in Animals. - (a) Set up the arrangement shown in Fig. 129. and test whether it is air-

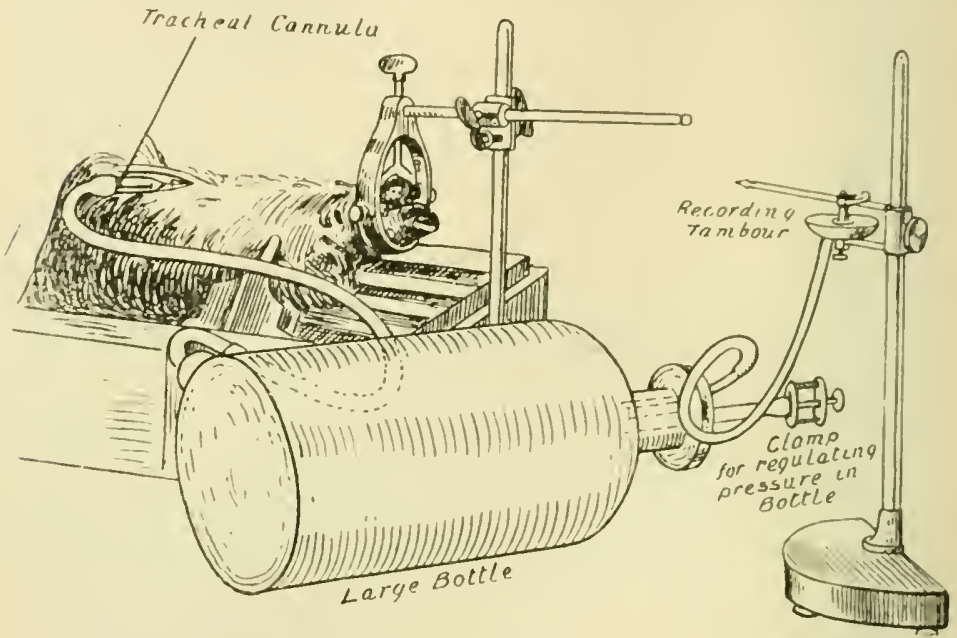

F1G. 129.-ARRANGEMENT FOR REspIRATORY TRACING.

Two glass tubes are inscrted through a cork in the mouth of the large bottle. One of them has a small piece of indiarubber tubing on it, which is closed or opened, as may be required, by a screw-clamp. The other is connected by a rubber tube with a recording tambour. The tubulure at the bottom of the bottle is closed by a cork, through which passes a glass tube, connected by a rubber tube with the tracheal cannula. If no bottle with tubulure is available, it is only necessary to pass through the cork, down to the bottom of the bottle, a third glass tube, which is connerted with the tracheal cannula. While a tracing is being taken the animal breathes the air contained in the bottle. When this becomes vitiated the respiratory movements are exaggerated and a norınal tracing is no longer obtained. For this reason the tracheal cannula must be connected with the bottle only at the moment when a tracing is to be taken. The arrangement is most suitable for a sinall animal.

tight. Have also in readiness an induction machine and electrodes arranged for an interrupted current. Anxsthetize a rabbit with chloral or ether (p. 204), or a small dog† with morphine and ether,

* This experiment is only to be attempted under the direct supervision of the demonstrator.

t If a large dog is used the bottle should be omitted, the tracheal cannula being connected with the stem of a $\mathrm{T}$-tube. One end of the horizontal limb of the T-tube is connected with the tambour; the other is provided with a rubber tube, which can be partially closed by a screw-clamp to regulate the excursion. Ether may be given when required by connecting the horizontal limb of the T-tube with a bottle with two glass tubes in the cork (p. is6). 
or A.C.E. mixture. Insert a cannula into the trachea (p. r86), and connect it with the large bottle by a tube. Connect the bottle with a recording tambour adjusted to write on a drum, and regulate the amount of the excursion of the lever by slackening or tightening the screw-clamp. Set the drum off at slow speed, and take a tracing.

(b) Then disconnect the cannula from its tube. Dissect out the vagus in the lower part of the neck, pass a ligature uncler it, but clo not tie it. Commect the cannula again with the bottle, and while a tracing is being taken ligature the vagus. Cut below the ligature and stimulate its central end with weak shocks, marking the time of stimulation on the clrum. Repeat the stimulation with strong shocks, and observe the results.

(c) Apply a strong solution of potassium chloride with a canzel'shair brush to the central end of the vagus while a tracing is being taken, and observe the effect.

(d) Isolate the sciatic nerve (p. I98), ligature it, and cut below the ligature. Stimulate its central end while a tracing is being taken. The respiratory movements will be increased.

(e) Disconnect the cannula, and isolate the vagus on the other sicle. While a tracing is being taken, divide it. The respiratory movements will probably at once become deeper and less frequent.

(f) Again disconnect the cannula. Isolate the superior laryngeal branch of the vagus. This will be found entering the larynx at the point where the laryngeal horn of the hyoid bone is connected with the thyroid cartilage. If the finger is passed back along the upper border of the thyroid cartilage, this point will easily be felt. Ligature the nerve, and divide it between the

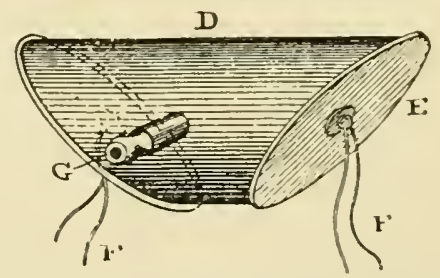

Fig. i3o.-Stethograph (Crile). larynx and the ligature. Reconnect the cannula. Take a tracing first with weak, and then with strong stimulation of the central end of the superior laryngeal.

$(g)$ Make an incision through the abdominal wall in the linea alba, and study the movements of the diaphragm. Find the nerves from which the phrenics take origin in the neck. In the dog they arise from the fifth, sixth, and seventh cervical nerves. Divide the phrenic fibres on one side, and observe that the diaphragm on the corresponding side is now paralyzed.

(h) Insert a cannula into the carotid artery. While a respiratory tracing is being taken, allow blood to flow from the artery. Dyspnœea and exaggeration of the respiratory movements will be seen when a considerable quantity of blood has been lost. Mark and varnish the tracings.

In the whole of this experiment the tracheal cannula is to be disconnected, except when the lever is actually writing on the drum, in order that the period during which the animal must breathe into the confined space of the bottle may be diminished as much as possible. Instead of the method described, the stethograph shown in Fig. I30 may be used to obtain respiratory tracings from animals, a broad canvas band being put round the animal's chest. To each end of this band is clamped with sufficient tension a strong threarl (F). fastened to a small metal disc on the inside of the rubber dam closing 
the obliquely-cut ends of the metal cylinder D. The tube $\mathrm{r}$ is connected with a tambour or with a bellows recorder (Fig. 13I).

4. The Effect of Temperature on the Respiratory Centre-Heat

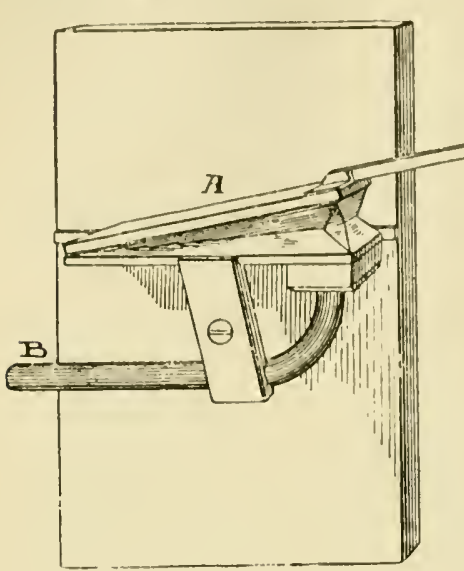

FIG. I3I.-BELLOWS RECORDER (CRILE).

$B$, a lead tube connected with the small bellows $A$, which consists of a light wooden base and top, to which is cemented very flexible (organ key) leather, properly creased for expansion and contraction; $C$, writing lever.

Dyspnœa.-Set up an arrangement for taking a respiratory tracing as in 2 (footmote, p. 288). Anirsthetize a rlog, and fasten it, back lownward, on a holeler. Make an incision in the middle line of the neck. commencing a little below the cricoid cartilage, and extending down for + or 5 inches. Insert a cannula into the trachea. Isolate both carotid arteries for as great a distance as possible, and arrange them on the brass tubes shown in lig. 132. Connect two adjacent ends of the tubes by a short rubber tube. Connect one of the remaining encls to a funnel, supported on a stand, and the other to a rubber tube lianging over the table above a large jar. Slip two or three folds of paper between the tubes and the vagus nerves. Heat two or three litres of water to about $65^{\circ} \mathrm{C}$. (a) Now connect the tracheal cannula with the tambour. As soon as the tracing is under way. let the lot water run through the funnel and tubes into the jar. Mark on the tracing the point at which the flow of the hot water was begun, and go on passing it until it has produced an effect. Then stop the clrum, and circulate water at the ordinary temperature

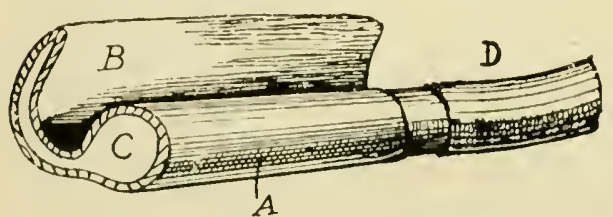

Fig. I32.-ARRANGFMENT FOR HEATING OR COOI.ING THE BLOOD IN THE CAROTID ARTERIES.

A, cylindrical portion of tube: 13 , flattened portion in the groove between which and $A$, the artery, lies; C, cross-section, showing the lumen extending into $\mathrm{B} ; \mathrm{D}$, rubber tube attached to a brass tube soldered into $A$. The other end of $A$ has a similar lorass tube soldered into it not shown in the figure). This is connected by a rubber tube with a similar apparatus, on which the other carotid lies. $D$ is connected with a funnel containing hot or cold water or with the outflow tube, as the case may be. passed through the tubes, and compare the results. Always allow
the water to pass for a time before making an observation. normal. Then, while a tracing is being taken, pass ice-cold water through the tubes, and again notice the effect.

(b) Expose the sciatic. Pass ice-water through the tubes, and while a respiratory tracing is being taken stimulate its central end witl inluction shocks so weak as just to cause an effect. Pass water at air temperature through the tubes, and repeat the stimulation with the coils at the same listance. Do the same while hot water is being till the breathing is again 
5. Measurement of Volume of Air inspired or expired - Vital Capacity. - A spirometer (Fig. IoI, p. 220) of sufficient accuracy for this experiment can be malle by removing the bottom of a large bottle with a capacity of not less than 4 litres. A good cork, through which passes a glass tube connected with a rubber tube, is fitted into the neck. The bottle is fixed vertically, mouth downwards, the glass tube being closed for the time, and graduated, by pouring in measured quantities of water, say roo c.c. at a time, and marking the level. The divisions are then etched in. If the cork loes not fit air-tight, it is covered with wax. The bottle is swng on two pulleys, counterpoised and immersed, bottom down, in a large glass jar or a small cask nearly full of water. A smaller bottle may be used for the determination of the tidal air, so as to reduce the error of reading.

(I) Submerge the bottle to the stopper, after opening the pincheock on the rubber tube. Breathe into the bottle, close the cock, adjust the bottle so that the level of the water is the same insicle and outside, and then read off the level. Determine the volume of air expired in :

(a) A normal expiration after a normal inspiration (tidal air) ;

(b) The greatcst possible expiration after a normal inspiration (supplemental air plus tidal air) ;

(c) The greatest possible expiration after the greatest possible inspiration (vital capacity).

(2) Open the cock and raise the bottle till it is nearly full of air. Determine the volume of air inspired in :

(a) A normal inspiration after a normal expiration (tidal air) ;

(b) The greatest possible inspiration after a normal expiration (complemental air plus tidal air) ;

(c) The greatest possible inspiration after the greatest possible expiration (vital capacity).

Iake several observations of each quantity, and take the mean.

(3) Count the rate of respiration for three minutes, keeping the brcathing as nearly normal as possible ; repeat the observation ; and from the mean result and the amount of the tidal air calculate the quantity of air taken into the lungs in twenty-four hours (pulmonary ventilation).

6. Cardio-Pneumatic Movements.-Fill a $\mathbf{U}$-tube with tobaccosmoke. One end of the tube is placed in the nostril of a fellowstudent, and made tight with a little cotton-wool. The other nostril and mouth are closed, and respiration suspended. The column of smoke moves in and out at each beat of the heart. By feeling the apex-beat, try to verify the fact that during systole the cardiopneumatic movement is inspiratory, and in diastole expiratory.

7. Auscultation of the Lungs.-Make the following observations on a fellow-student, who should strip to the waist, and should be seated on a stool, so that the back can be easily examined as well as the front of the chest:

(a) Vesicular Breathing.-Place the stethoscope 2 or 3 inches below the axilla. The rustling vesicular sound will be heard. It is more intense in inspiration than in expiration, and much more prolonged, being heard during the whole of the inspiratory act, but in health only at the beginning of expiration. Another position in which to listen for the typical vesicular sound is below the angle of the scapula. Study the sound in deep and in ordinary breathing. Now go over the whole chest systematically, noting the positions where the typical vesicular sound can be clearly heard and the positions where it, is not heard or is obscured by- 
(b) Bronchial Breathing.--Place the stethoscope over the trachea, where the breath-sounds are of the bronchial type. although lourler than the bronchial breathing heard over a consoliclaterl area of lung in pneumonia. The expiratory sound is generally lourler than the inspiratory, and at least as long as the inspiratory sound, extending throughout the greater part of expiration. Both sounds are higlier in pitch than the vesicular murmur. and have a blowing character. Go over the chest. and note the points at which bronchial breatling can be hearcl.

(c) Repeat $(a)$ and $(b)$. using the ear applied to a towel lairl over the various regions of the chest instead of the stethoscope.

(d) Perform the following experiment on a dog used for some other purpose: Open the trachea as described on P. IS6. Insert into it the cross-piece of a glass $\mathbf{T}$-tube of as large a bore as possible. tying the trachea over it on each side of the stem. The stem projecting from the wound is armed with a short piece of rubber tubing. which can be closed at will with a clip. When the tube is thus closed the animal breathes through the glottis in the ordinary way. When the tube is open, and the mouth and nose covered tightly with a cloth. no air goes through the glottis. The tube being closed, listen with the stethoscope or the ear alone over a part of the chest where the vesicular murmur is well heard. If the rubbing of the hairs below the stethoscope causes disturbing sounds, shave a portion of the skin. Continue listening while an assistant closes the tube and covers up the animal's muzzle. Determine whether any change takes place in the resicular sound.

(e) Repeat (d) while listening over the lower part of the trachea, and determine whether any change takes place in the bronchial breathing sound.

8. Respiratory Pressure.-Connect a strong rubber tube with a glass bulb, and the bulb with a mercurial manoneter provided with a scale. (I) Fasten the tube with a little cotton-wool in one nostril. breathe through the other with closed mouth, and observe the amount by which the level of the mercury is altered in ordinary inspiration and expiration.

(2) Repeat the observation with forced breathing. pinching the tube at the height of inspiration and expiration, and reading off the maximum inspiratory and expiratory pressure.

(3) Repeat (1) with the tube connected to the mouth by a glass tube held between the lips, and the nostrils open.

(4) Repeat (2) with the tube in the mouth and the nostrils closed.

9. Determination of Carbon Dioxide and Oxygen in inspired and expired Air-(J) Estimation of Carbon Dioxide. lill a burette with water. and close the pinchcock on the rubber tube. Immerse the wide end of the burette in a large vessel of water. and fill it with carbon dioxide by putting into it below the water a tube connected with a bottle in which carbon dioxide is being evolved by the action of hydrochloric acid on marble chips. See that gas has been coming off freely from the bottle for a little time before the tube is put under the burette. Do not fill the burette with gas beyond the graduated part. To prevent warming the burette by the hand, hold it. by means of a clamp or test-tube holder. in the vertical position. its mouth being still immersed. Make the level of the water the same inside and outside, and read off the meniscus. Then introduce a piece of stick sodium hylroxide. close the burette with a finger or the palm of the hand, lift it out of the water. and by a sort of see-saw 
movenent shake the solim hydrate repeatedly from end to end of it. Igain immerse the burette, and reacl the level of the meniscus. Ilost of the gas will be absorbed. Repeat the shaking. If the realing is still the same, absorption is now complete.

(2) Estimation of Oxygen (Analysis of inspired Air).- litl the burette with the air of the litboratory. Open the pincheock, and immerse the wide end of the burette till the water reaches the graduation. Then close the cock, and read off the meniscus. Introduce a picce of sodium hydroxicle, and proceed as in (I). Notice that there is no appreciable absorption. (This methorl is not suitable for the measurement of the small quatity of carbon dioxicle in ordinary air.) Now introduce. uncler water, some pyrogallic acicl. This can be done conveniently by wrapping up some of the crystals in thin paper so as to form a kind of small cigarette, which is pushed up into the burctte. A little more sodium hydroxide may also be adderl. if the piece first introduced is entirely dissolved. Shake as described in (1),

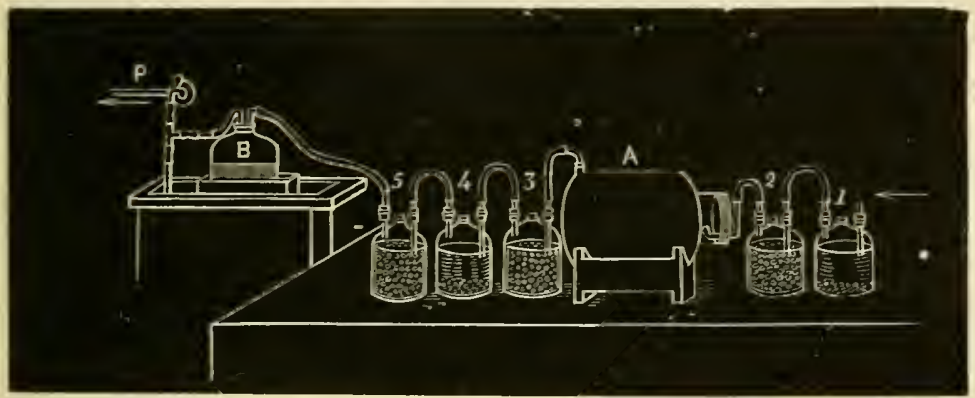

Fis. I33.-Haldane's Apparatus for Measuring the Quantity of CO. and AnUEOUS VApour gIVEN OFF bir an ANIMAL.

$A$, chamher into which the animal is put; I and 4 , Woulff's bottles filled with soda-lime to absorb carbon dioxide; 2,3 , and 5 , Woulff's bottles filled with numice-stone soaked in sulpluuric acid to absorb watery vapour ; $B$, glass bell-jar suspended in watcr, by means of which the negative pressure is known; $\mathrm{P}$, water. pump which sucks air through the apparatus; I and 2 are simply for absorbing the rarbon slioxide and water of the ingoing air.

till no more absorption takes place. Then read off the meniscus again (always making the level the same inside and outside the burette). The difference in the two readings gives the amount of oxygen present. What remains in the burette is nitrogen (and a little argon). Its amount is, of course, equal to the reading of the burette, plus the capacity of the ungraduated part at the narrow end of the burette, which must be determined once for all by a separate measurement.

(3) Analysis of expired Air.-(a) Fill the spirometer with water. Breathe into it several times in your ordinary way, but be careful not to inspire any air from the spirometer; then fill the burette with the expired air from it. Or simply expire several times through the burette, seeing that none of the inspired air comes through it. Determine, as in (I) and (2), the percentage amount of carbon dioxide, oxygen, and nitrogen. (b) Repeat $(a)$ with air expired after the lungs have been thoroughly ventilated by taking a number of deep breaths in succession, and determine whether there is any difference in the percentage amounts.

IO. Estimation of the Quantity of Water and of Carbon Dioxide 
given off by an Animal (Haldane's Method).-(I) Connect the apparatus slown in Fig. 133 with the water-pump. Allow a negative pressure of 5 or 6 inches of water to be established in it, as shown by the rise of water in the belt-jar $B$. Then close the open tube of carbon dioxide bottle 1 , and clamp the tube between the water-pump and the bell-jar. If the negative pressure is maintained, the arrangement is air-tight. Now weigh bottle 3 and bottles 4 and 5 , the last two together. Place a cat in the respiratory chamber $\mathrm{A}$, connect the chamber directly with the water-pump, and test whether it is tight. Then take the stopper out of bottle 1 , and adjust the rate at which air is drawn through the apparatus. Let the ventilation go on for a few minutes, then insert bottles 3,4 , and 5 again. Note the time

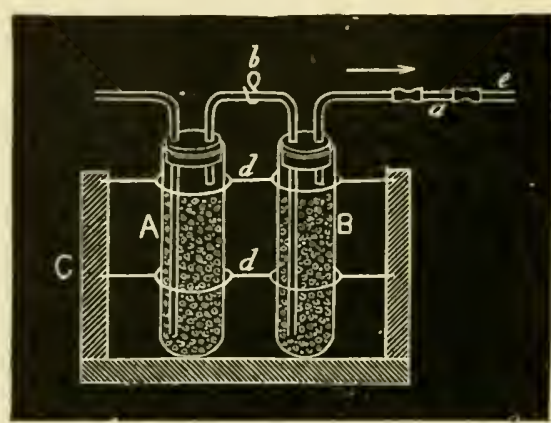

FIG. 134. ABSORITIO: TUBES FOR CO, AND MOISTURE.

A, soda-lime tube ; $B$, sulphuric acid tube: $C$, wooden frame, in which $A$ and $B$ are supported by wires $d: b$, wirc hook, which grips the glass tube firmiy, and by means of which the tubes are lifted out of the frame in order to be weighed; $a$, short piece of glass tubing, by taking out which the absorption tubes are disconnected from the rest of the apparatus ; $\varepsilon$, glass tube going off to animal chamber. cxactly at this point, and after an hour disconnect 3, 1 , and 5. and again weigh. The difference of the two weighings of 3 shows the quantity of water given off by the animal in an hour; the difference in the combined weight of 4 and 5 , the quantity of carbon dioxide. Weigh the cat. and calculate the amount of water and of carbon dioxide given off per kilo per hour.

(2) For the student it is more convenient to use smaller animals. The mouse nay be taken as an example of a warm-blooded animal. and the frog of a cold-blooded. Instead of the Woulff's bottles use wide test-tukes connected as in Fig. 134, and for the animal chamber a small beaker, closed with a very carefully-fitted cork which has been boiled in paraffin. The inlet and outlet tubes of the chamber are to be introduced through this cork. The holes for these are to be bored with the greatest carc, and the tubes to be put in while the cork is still hot from boiling in paraffin. Also insert a thermoneter about 6 inches long registering from $\mathrm{o}^{\circ} \mathrm{C}$. to $45^{\circ} \mathrm{C}$. Modeller's wax is to be used finally to render all the junctions air-tight.

Add to the series of tubes clescribed in the apparatus a single tube containing baryta-water. This is placed to the left of tube 5, and sn arranged that the air-current bubbles through the water. As long as the absorption of carbon dioxide is complete, the baryta-water remains clear. Bcyond this a water-bottle should be placed to act as a valve and to indicate the negative pressure in the apparatus. It can be most simply constructed by using a cylinder of stout glass tubing in a wide-mouthed bottle containing some water, the inlet and outlet tubes passing through a paraffined cork which seals the upper end of the cylinder.

Before making an observation, test whether the apparatus is airtight, as explaincd above, after introducing the animal into the 
chamber, sealing the latter with wax, and connecting it with the absorption-subes. But a negative pressure of 2 or 3 inches of water is a sufficient test for the small apparatus.

To make an observation. set the air-current going at the desired rate. Allow it to run for a few minutes till the carbon dioxide, which hos accumulated during the testing, has been swept out. At a time which has been decided on ind noted, stop the current by disconnc:ting the water-pump. Discnnnect and stopper up the animal chimber. and weigh it as quickly as possible. Connect up again, using only recently-weighed absorption-tubes, and finally connect with the water-pump and allow the current to pass for a definite period. say an bour.

The soda-lime should not be too dry, or absorption is not sufficiently rapid. The following facts are made out in the observatien :

(a) The loss of weight by the animal chamber (chiefly loss by the animal). (b) The gain of the sulphuric acid tube in water. (c) The gain of the soda-lime tubes in carbon dioxide.

If we compare total loss and total gain. we find that they do not correspond, the gain being always greater then the loss. The surplus can only be oxygen which has Leen absorbed by the animal and added to the hydrogen and carbon of its substance to form water and carbon dioxide. Calculate the respiratory quotient (p. 242).

I. Muscular Contraction in the absence of Free Oxygen (sce p. 26I).-(I) Pith a frog (brain and cord). Cut off one hind-leg at the middle of the thigh. ard strip the skin from it. Pass a thread under the tendo Achillis, tie it, and divide the tendon below it. Free the tendon and the gastrocnemius muscle from the loose connective tissuc lying between them and the bones of the leg, and divide the latter just below the knee. Remove superfluous thigh muscles, and fasten the gastrocnemius in a moist chamber by means of the femur. Attach the thread on the tendon to a lever. Connect the poles of the secondary coil of an induction machine by fine copper wires to the femur and the tendon. Put a battery and simple key in the primary, and arrange it for single shocks. Stimulate the muscle and observe the height of the contraction. Now pass into the chamber a current of washed hydrogen gas from a bottle containing granulated zinc, upon which a little dilute sulphuric acid is poured from time to time. The air in the moist chamber will soon be entirely displaced Jy the hydrogen. Nevertheless, the muscle will contract on being stimulated as before, and the stimulation can be repeated many times.

12. Oxidising Ferments.-Wash ont the bloodvessels of a dog or rabbit (Practical Exercises, p. 57). Chop up finely portions of pancreas, spleen, muscle, lungs, and kidney, keeping each separate, and avoiding any contamination of one by another. Grind up half of each portion with sand in a small mortar, and extract with a small quantity of water, keeping all the extracts separate. Into each of clcven test-tubes put Io c.c. of a colourless dilute alkaline solution of paraphenyleneriamin and $\alpha$-naphthol (freshly made by mixing solutions of the two substances in equimolecular proportions* and adding a little sodium carbonate). To five of the tubes add the chopped organs, to five the watery extracts of the organs, and enough water to make the volume equal in all the tubes. To the remaining tube add the same amount of water. Observe in which tube a change of colour takes place (p, 265).

* 1.e., the weight of each of the two substances in the mixture shou'd be proportional to its molezular weight. 


\section{CHAPTER I V}

\section{DIGESTION}

In the last chapter we have described the manner in which the interchange of gases between the tissucs and the air is carried out. We have now to consider the digestion and absorption of the solid and liquid food, its further fate in relation to the chemical changes or metabolism of the tissues, and finally the excretion of the waste products by other channels than the lungs.

Logically, we ought to take metabolism after absorption and before excretion, tracing the food through all its vicissitudes from the moment when it enters the blood or lymph till it is cast out as useless matter by the various excretory organs. Unfortunately, however, the steps of the process are as yet almost entirely hidden from us; we know only the beginning and the end. We can follow the food from the time it enters the alimentary canal till it is taken up by the tissues of absorption; and we have really a fair knowledge of this part of its course. We can collect the end products as they escape in the urine, or in the breath, or in the sweat; and our knowledge of them and of the manner in which they are excreted is considerable. But of the wonderful pathway by which the dead molecules of the food mount up into life, and then descend again into death, we catch only a glimpse here and there. Only the introduction and the conclusion of the story of metabolism are at present in our possession in fairly continuous and legible form. We will read these before we try to decipher the handful of torn leaves which represents the rest.

Comparative.-In the lowest kinds of animals, such as the Amøba, there is neither mouth, nor alimentary canal, nor anus: the food, wrapped round by pseudopodia, is taken in at any part of the animal with which it happens to come in contact. A vacuole is formed around it. Acid is secreted into the vacuole, the food is dirested within the cell-substance, and the part of it which is useless for nutrition is cast out again at any part of the surface.

Coming a little higher, we find in the Coclenterates a inouth and $29^{3}$ 
alimentary tube, which opens into the body-cavty, where cortin amomt of digestion seems to take place, and from which the food is absorbed either through the cells of the endoderm, or, as in Meclusa, hy means of fine canals, which radiate from the body-cavity into its walls, and form part of the so-called gastro-vascular system. In the Echinodermata we have a further development, a complete alimentary canal with month and anus, and entirely shut off from the bodycavity. In many Arthropods it is possible already to distinguish parts corresponding to the stomach, and the small and lisge intestines of higher forms, the digestive glinds being represented by organs which in some groups seem to be homologous with the liver, and in others with the salivary glands of the higher Vertebrates. A few Molluscs seem in addition to possess a pancreas.

Among Vertebrates fishes have the simplest, and birds and mammals the most complicated, alimentary system. In the lowest fishes the stomach is only indicated by a slight widening of the anterior part of the digestive tube. In water-living Vertebrates there are $n$ salivary glands. In birds the osophagus is gencrally dilated to form a crop, from which the food passes into a stomach consisting of two parts, one pre-cminently glandular (proventriculus), the other pre-eminently muscular (ventriculus). Among mammais a twofold division of the stomach is distinctly indicated in rodents and cetacea, but this organ reaches its greatest complexity in ruminants, which possess no fewer than four gastric pouches. "The differentiation of the intestine into small and large intestine and rectum is more distinct, both anatomically and functionally, in mammals than in lower forms ; but there are marked differences between the various mammalian goups both in the relative size of the several parts of the digestive tube, and in the proportion between the total length of the alimentary canal and the length of the body. In general, the canal is longest in herbivora, shortest in carnivora. Thus, the ratio between length of body and length of intestine is in the cat $1: 4$. $\operatorname{dog} 1: 6$, man $1: 5$ or 6 , horse $1:$ I 2 , cow $1: 20$, sneep $1: 27$. The relative capacity of the stomach, small intestine, and large intestine, is in the $\operatorname{dog} 6: 2: 1{ }^{\circ}$, in the horse $1: 3{ }^{\circ}: 7$, in the cow $7: 2: 1$ The area of the mucous surface of the alimentary canal is very considerable, in the dog more than half that of the skin, the surface of the small intestine being three times that of the stomach and four times that of the large intestine. In the horse the mucous surface has twice the area of the skin.

Anatomy of the Alimentary Canal in Man.-The alimentary canal is a nuscular tube, which, beginning at the mouth, runs under the various names of pharynx, osophagus, stomach, small intestine, large intestine, and rectum, till it ends at the anus. Its walls are largely composed of muscular fibres ; its lumen is clad with epithelium, and into it open the ducts of glands, which, morphologically speaking, are involutions or diverticula formed in its course. In virtue of its muscular fibres it is a contractile tube : in virtue of its epithelial lining and its special glands it is a secreting tube ; in virtue of both it is fitted to perform those mechanical and chemical actions upon the food which are necessary for digestion. Its inner surface is in most parts richly supplied with bloodvessels, and in special regions beset with peculiarly-arranged lymphatics; by both of these channels the alimentary tube performs its function of absorption. From the beginning of the cesophagus to the end of the rectum the muscular vall consists, broadly speaking. of an outer coat of longitudinally- 
arranged fibres, and a thicker inner coat of fibres running circularly or transversely around the tube. Between the layers lies a plexus of non-medullated nerves and nerve-cells (Aucrbach's plexus). In the stomach the longitudinal fibres are found only on the two curvatures, and a third incomplete coat of oblique fibres makes its appearance internal to the circular layer. In the large intestine, again, the longitudinal fibres are chicfly collected into three isolated strands. In the pharynx the typical arrangement is cleparterl from, inasmuch as there is no regular longitudinal layer; but the three constrictor muscles represent to a certain extent the great circular coat. The muscles of the mouth and of the pharynx are of the striperl variety. So is the muscle of the upper half of the resophagus in man and the cat, and of the whole oesophagus in the dog and the rabbit. In the rest of the alimentary canal the muscle is smooth, except at the very end. where the external sphincter of the anus is striped. In certain situations the circular coat is leveloped into a regular anatomical sphincter, a definite muscular ring, whose function it is to shut one part of the tube off from another (sphineter pylori, ileocolic sphincter), or to help to close the external opening of the tube (internal sphincter of anus). Elsewhere a tonic contraction of a portion of the circular coat, not anatomically developed beyond the rest, creates a functional sphincter (cardiac sphincter of stomach).

Throughout the greater part of the digestive tract the peritoneum. forms a thin serous layer, external to the muscular coat. Internally the muscular coat is separated from the mucous membrane, the lining of the canal, by some loose areolar tissue containing blondressels, lymphatics, and nerves (Meissner's plexus), and callerl the submucous coat. Between the inucous and submucous layers, but belonging to the former, in the whole canal below the beginning of the oesophagus, is a thin coat of smooth muscular fibres, the muscularis mucos $x^{\text {, con- }}$ sisting in some parts, e.g., in the stomach, of two. or cven three, layers. Between this and the lumen of the canal lie the ducts and alveoli of glands, surrounded by bloodvessels and embedded in adenoid or lymphoid tissue, which in particular regions is collecterl into well-defined masses (solitary follicles, Peyer's patches, tonsils), extending, it may be, into the submucous tissue. In the mouth, pharynx, and osophagus, the glands lie in the submucosa, as do the glands of Brunner in the rluodenum ; everywhere else they are confined to the mucous membrane proper. Between the openings of the glands the mucous membrane is linerl with a single layer of columnar epithelial cells, sometimes (in the small intestine) arranged along the sides of tiny projections or villi. At the ends of the alimentary canal, $v i z$. , in the mouth, pharynx, and oesophagus, and at the anus. the epithelium is stratified squamous, and not columnar.

The purpose of ford is to supply the waste of the tissues, to replenish the stores of material from the oxiclation of which the energy required for the running of the bodily machine is derived, and thus to maintain the normal composition of the body. In the body we find a multitude of substances marked off from eacl other, some by the sharpest chemical differences, others by characters much less distinct, but falling upon the whole into the few fairly definite groups already described (p). I). Thus, there are bodies like serum-albumin, serum-globulin, myosinogen. and so on, which are so much alike that they can all be placed 
in one great class, the proteins. Then we have substances like glycogen and dextrose, vastly simpler in their composition, and belonging to the group of carbo-hydrates. Then, again, fats of virious kinds are widely distributed in normal animal bodies; and inorganic materials (water and salts) are never absent.

Now, although it is by no means necessary that a substance in the body belonging to one of these great groups should be clerived from a substance of the same group in the food, it has been found that no diet is sufficient for man unless it contains representatives of all ; a proper diet must include proteins, carboliydrates, fats, inorganic salts, and water. These proximate principles have to be obtained from the raw material of the foodstuffs-that is, as regards the first three groups, which can alone yield energy in the body, from the tissues and juices of other living things, plants or animals ; it is the business of digestion to sift them out and to prepare them for absorption. This preparation is partly mechanical, partly chemical.

The water and salts and some carbo-hydrates, such as dextrose, are ready for absorption without change. Fats are split into glycerin and fatty acids before absorption. Indiffusible colloidal carbo-hydrates, like starch and dextrin, are changed into diffusible and readily soluble sugars, and the natural proteins into diffusible peptones, and eventually - in great part, at leastinto much simpler decomposition products. These changes are obviously favourable to absorption. But this is not their whole significance. For disaccharides, such as cane-sugar, maltose, or lactose, although easily soluble in the contents of the gut, and in themselves perfectly capable of being absorbed without change, are, unless present in unusually large amount, all converted into monosaccharides, such as dextrose, levulose, or galactose, either in the lumen or in the wall of the alimentary tube. The reason is that the disaccharides are unsuitable as pabulum for the cells. Digestion is not only a preparation of the food for absorption by the gut, but for assimilation by the tissues after absorption. An equally important instance of this double function is seen in the digestion of proteins. The complete shattering of the protein molecule into amino-acids and the other groups yielded by its decomposition (p. 332) is required, in the case of that portion of the protein which goes to build up the tissues, because of the high degree of specificity of the tissue proteins. The myosinogen of beef cannot be cobbled into the myosinogen of human muscle, still less we may suppose into the serum albumin of human blood. It is necessary that the food protein should be completely ' wrecked' in digestion so that protein which is to take its place in protoplasm may be built exactly to order from the bricks. A satisfactory 'fit' cannot be obtained with ready- 
marle protein. Mechanical division of the ford is an important aid to the chemical action of the digestive juices. We shall sec that this mechanical division forms a great part of the work of the stomach, but it is normally begun in the mouth, and it is of consequence that this preliminary stage should be properly performed.

\section{The Mechanical Phenomena of Digestion.}

Mastication.- It is among the mammalia that regular mastication of the food first makes its appearance as an important aid to digestion. The amphibian bolts its fly, the hirl its grain, and the fish its brother, without the ceremony of chewing. In ruminating mammals we see mastication carried to its highest point; the teeth work all day long, and most of them are specially adlapted for grinding the food. The carnivora spend but a short time in mastication ; their teeth are in general adapted rather for tearing and cutting than for grinding. Where the dict is partly animal and partly vegetable, as in man, the teeth are fitted for all kinds of work; and the process of mastication is in general neither so long as in the purely vegetable feechers, nor short as in the carnivora.

In man there are two sets of teeth: the temporary or milk teeth, and the permanent teeth. The milk teeth are twenty in number, and consist on each side of four incisors or cuttingteeth, two canines or tearing-teeth, and four molars or grindingteeth. The central incisors emerge at the seventh month from birtl, the other incisors at the ninth month, the canines at the eighteenth, and the molars at the twelfth and twenty-fourtli month respectively. Each tooth in the lower jaw appears a little before the corresponding one in the upper jaw. Each of the milk-teeth is in course of time replaced ly a permanent tooth, and in addition the vacant portion of the gums behind the milk set is now filled up by twelve teeth, six on each side, three above and three below. These twelve are the permanent molars; they raise the number of the permanent teeth to thirtytwo. The permanent teeth which occupy the position of the milk molars now receive the name of premolars. 'The first tooth of the permanent set (the first true molar) appears at the age of $6 \frac{1}{2}$ years; the last molar, or wisdom-tooth, does not emerge till the seventeenth to the twenty-fifth year.

In mastication the lower jaw is moved up and down, so as to alternately separate and approximate the two rows of teetl. It has also a certain amount of movement from side to side, and from front to back. The masseter, temporal and internal pterygoid muscles raise, and the digastric, with the assistance of the 
mylo- and genio-hyoid, depresses, the lower jaw, but its downward movement is mainly a passive one. The extemal pterygoids pull it forward when both contract, forward and to one side when only one contracts. The lower fibres of the temporal muscle retract the jaw. The huccinator and orbicularis oris muscles prevent the food from passing between the teeth and the cheeks and lips. The tongue keeps the food in motion, works it up with the saliva, and finally gathers it into a bolus ready for deglutition.

Deglutition. - This act consists of a voluntary and an involuntary stage. Just before the beginning of the voluntary stage mastication is suspended, and a slight contraction of the diaphragm generally takes place. The anterior part of the tongue is suddenly elevated and pressed against the hard palate, and the elevation travels back from the tip towards the root, as the mylohyoid muscles in the floor of the mouth contract sharply, so as to thrust the bolus through the isthmus of the fauces. As soon as this has happened and the food has reached the posterior portion of the tongue, it has passed beyond the control of the will, and the second or involuntary stage of the process begins.

This stage may be divided into two parts: (I) Pharyngeal, (2) osophageal-both being reflex acts. During the first the food has to pass through the pharynx, the upper portion of which forms a part of the respiratory tract, and is in free communication with the larynx during ordinary breathing. It is therefore necessary that respiration should be interrupted and the larynx closed while the food is being moved through the pharynx. But that the interruption may be short, the food must be rapidly passed over this perilous portion of its descent. The main propelling force under which the bolus is shot through the back of the pharynx is derived from the contraction of the mylo-hyoid muscles already mentioned, assisted to some extent by the styloand palato-glossi ; and that none of the purchase may be lost, the pharyngeal cavity is cut off from the nose and mouth as soon as the bolus has entered it. The soft palate is raised by the levator palati and palato-pharyngei muscles; at the same time the upper part of the pharynx, narrowed by the contraction of the superior constrictor, comes forward to meet the soft palate, closes in upon it, and so prevents the food from passing into the nasal cavities. The pharynx is cut off from the mouth by the closure of the fauces through the contraction of the palatopharyngeal muscles which lie in their posterior pillars. The upper free end of the epiglottis (the so-called pharyngeal part) aids the back of the tongue in completing a movable partition across the pharynx, which keeps close to the bolus as it passes down between the posterior surface of the epiglottis and the posterior wall of the pharynx. Almost immediately after the 
contraction of the mylo-hyoids the larynx is pulled upwards and forwards by the contraction of the thyro-hyoid muscle, and the elevation of the hyoid bone by the muscles which connect it to the lower jaw. The base of the tongue is simultaneously drawn backwards by the stylo- and palato-glossus. The lower or laryngeal portion of the epiglottis is thus caused to come into contact with the upper orifice of the larynx, occluding it completely, but the pharyngeal portion projects beyond the larynx. and takes no share in its closure (Eykman). The glottis is closed by the approximation of the rocal cords and the arytenoid cartilages. The epiglottis. however, is not absolutely indispensable for closing the larynx. since swallowing proceeds in the ordinary way when it is absent. The morsel of food, grasped by the middle and lower constrictors as it leaves the back of the tongue. passes rapidly and safely over the closed larynx, the process heing accelerated by the pulling up of the lower portion of the pharyn over the bolus by the action of the palatn- and stylo-pharyngei.

The second or aesophageal portion of the involuntary stage is a more leisurely performance. The bolus is carried along by a peculiar 'peristaltic' contraction of the muscular wall of the cesophagus, which travels down as a wave, constricting the tube and pushing the food before it. In front of the constricting wave moves a wave of inhibition, so that the part of the cesophagus in to which the bolus is about to pass is always relaxed, while the part behind it is contracted. This exact co-ordination of inhibition and contraction is the essential thing in peristalsis. When the food reaches the lower end of the gullet the tonic contraction of that part of the tube is for a moment relaxed by reflex inhibition, and the morsel passes into the stomach. Beaumont saw, in the case of St. Martin, that the esophageal orifice of the stomach contracted firmly after each morsel was swallowed, and so did the gastric walls in the neighbourhood of the fistula when food was introduced by this opening. In the dog the whole process of swallowing from mouth to stomach has been shown to occupy four to five seconds. but the time is by no means constant. In man the peristaltic wave requires about five to six seconds to travel from the level of the glottis to the cardiac orifice. The rate of movement is greater in the upper than in the lower portion of the resophagus.

Such is the mechanism of deglutition when the bolus is of such consistence and size that it actually distends the oesophagus. But it has been shown that liquid food is swallowed in a different way. The food lying on the dorsum of the tongue, suddenly put under pressure by the sharp contraction of the mylo-hyoid muscles, is shot rapidly down to the lower part of the lax cesophagus, or, occasionally, some of it even into the stomach. So 
far the process has only occupjed one-tenth of a second. After sereral seconds, the food, or the portion which still remains in the (esoplagus, is forced through the cardiac sphincter into the stomach by the arrival of the tardy peristaltic contraction of the resophageal wall (Kronecker and Meltzer). Two sounds may be heard in man on listening in the region of the stomach or usophagus during deglutition of liquids, especially when, as generally happens, they are mixed with air. The first sound occurs at once, and is due to the sudden squirt of the liquid along the gullet; the second, which is heard after a distinct interval (about six seconds), is caused by the forcing of the fluid through the cardiac orifice of the stomach by the contraction of the cesophagus.

There are certain peculiarities which distinguish this peristaltic movement of the cesophagus from that of other parts of the alimentary canal. It is far more closely related to the central nervous system, and, unlike the peristaltic contraction of the intestine, can pass over any muscular block caused by ligature, section, or crushing, so long as the nervous connections are intact. But division of the cesophageal nerves causes, as a rule, stoppage of cesophageal movements; although an excised portion of the tube retains its vitality for a long time, and may, under certain circumstances, go on contracting in the characteristic way after removal from the bdoy. Stimulation of the mucous membrane of the pharynx will cause reflex movements of the cesophagus, while stimulation of its own mucous membrane is ineffective. From these facts we learn that although the osophageal wall may possess a feeble power of spontaneous peristaltic contraction, yet this is usually in abeyance, or at least overmastered by central nervous control; so that impulses discharged as a "fusillade' from successive portions of the vagus centre, and travelling down the cesophageal nerves, excite the muscular fibres in regular order from the upper to the lower end of the tube.

Nervous Mechanism of Deglutition.-The centre for the whole involuntary stage (both pharyngeal and oesophageal) lies in the upper part of the medulla oblongata. When the brain is sliced away above the medulla, deglutition is not affected; but it the upper part of the medulla is removed, the power of swallowing is abolished. In man, disease of the spinal bulb interferes far more with deglutition than disease of the brain proper.

Normally, the afferent impulses to the centre are set up by the contact of food or saliva with the mucous membrane of the posterior part of the tongue, the soft palate and the fauces, the nerve-channels being the superior laryngeal, the pharyngeal branches of the vagus, and the palatal branches of the fifth 
nerve.* A feather has sometimes heen swallowed involuntarily by a reflex movement of deglutition set up while the soft palate or pharynx was being tickled to produce vomiting. Artificial stimulation of the central end of the superior laryngeal will cause the movements of deglutition independently of the presence of food or liquid ; but if the central end of the glosso-pharyngeal nerve be stimulated at the same time, the movements do not occur. The glosso-pharyngeal is therefore able to inhibit the deglutition centre, and it is owing to the action of this nerve that in a series of efforts at swallowing, repeated within less than a certain short interval (about a second), only the last is successful. It is also through the glosso-pharyngeal nerve that the respiratory movements are inlibited during deglutition. When the central end of this nerve is stimulated, respiration is stopped for four or five seconds, and this cessation is distinguished from that produced by any other afferent nerve by the circumstance that it occurs not in expiration exclusively or in inspiration exclusively, but with the respiratory muscles in the precise degree of contraction in whicl they happened to be at the moment of stimulation. The efferent nerves of the reflex act of deglutition are the hypoglossal to the tongue and the thyro-hyoid and other muscles concerned in raising the larynx; the glosso-pharyngeal, vagus, facial and fifth to the muscles of the palate, fauces, and pharynx; the fifth to the mylo-hyoid; and the vagus to the larynx and cesophagus. Section of the vagus interferes with the passage of food along the nesophagus; stimulation of its peripheral end causes cesophageal movements.

Movements of the Stomach and Intestines.-The whole of the stomach does not take part equally in the movements associated with digestion. We may divide the organ, both anatomically and functionally, into two portions - a pyloric portion, or antrum pylori, comprising about a fifth of the stomach, and a larger cardiac portion, or fundus.t At the junction of the antrum and the fundus the circular muscular coat is slightly thickened into a ring called the 'transverse band,' or 'sphincter of the antrum.' In the living stomach the region of the transverse

* It appears that the most influential reflex paths may differ in different animals. In the rabbit, e.g., the reflex is set up by excitation of the trigeminal fibres which supply the mucous membrane anterior to the tonsils, in the dog and cat by excitation of the glosso-pharyngeal fibres in the posterior wall of the pharynx, and in monkeys by excitation of the trigeminal branches distributed to the mucous membrane over the tonsils (Kalnn).

$\dagger$ Here 'fundus' is used in the sense in which it is generally employed in speaking of the stomach of the dog or cat as signifying the whole of the organ with the exception of the antrum pylori. By the fundus of the human stomach most writers mean only the cul-cle-sac at the cardiac encl ; the portion intervening between it and the antrum pylori is often termed the body of the stomach. 
band is usually contracted so strongly and continuously that at distinct groove is seen to separate the tubular antrum from the bag-like cardiac end. The suggestion of a massive constricting ring of muscle is belied by an examination of the dead viscus. The transverse band is really little more than a physiolngical sphincter. The empty stomach is contracted and at rest. A few minutes after food is taken contractions begin in the antrum, and nun on in constricting nudulations (in the cat at the rate of six in the minute) towards the pyloric sphincter. Each wave takes about twenty seconds (in the cat) to pass from the middle of the stomach to the pylorus. Feeble at first, they become stronger and stronger as digestion proceeds, and gradually come to involve the portion of the fundus next the sphincter of the antrum, but their direction is always towards the pylorus, never, in normal digestion, away from it. The food is thus subjected to energetic churning movements in the pyloric end of the stomach, and worked up thoroughly with the gastric juice. Kept in constant circulation, it gradually becomes reduced to a semi-liquid mass, the chyme, which is at intervals driven against the pylorus by strong and regular peristaltic contractions of the lower end of the stomach, the sphincter relaxing from time to time by a reflex inhibition to admit the betterdigested portions into the duodenum, but tightening more stubbornly at the impact of a hard and undigested morsel. The nature, as well as the consistence of the food, influences the length of its sojourn in the stomach. Carbo-hydrate food passes more rapidly through the pylorus than fatty food, and fat more rapidly than protein. The reason is that the acidity of the gastric juice varies with the different kinds of food, hydrochloric acid being secreted in abundance in the presence of proteins, and to a much smaller extent in the presence of fats and carbohydrates. Now, dilute hydrochloric acid when introduced into the stomach remains there for a much longer time than water. This depends upon the fact that such portions of the acid as get into the duodenum stimulate afferent fibres in its mucous membrane, and so cause reflex spasm of the pyloric sphincter. When the acid chyme becomes neutralized to a certain point by the bile and pancreatic juice, inhibitory impulses pass up from the duodenum and cause the sphincter to relax. The cardiac division of the stomach, with the exception of the portion that borders the transverse band, takes no share in the peristaltic movements. And, indeed, it is far more difficult to cause such contractions by artificial stimulation in the fundus than in the pylorus. The two portions of the stomach are partially, or in certain animals from time to time completely, cut off from each other by the contraction of the sphincter of the antrum. The 
fundus. so far as its mechanical functions are concerned, acts chiefly as a reservoir for the food, which, like a hop'per, it gradually passes into the pyloric mill as digestion goes on by a tonic contraction of its walls. The existence of this reservoir enables larger quantities of food to be taken at one meal, which can then be digested gradually. These facts

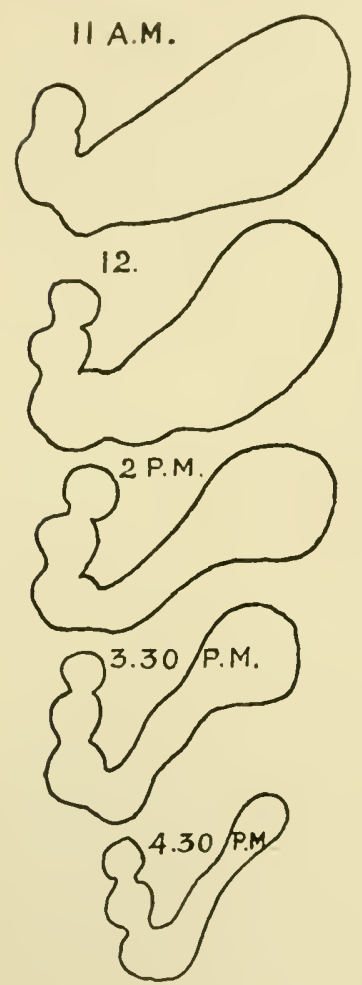

Fig. 135.-Cat's Stonacu SeEN B?' RöNtgex Ray's (CANion).

The outlines of the stomach containing food mixed with bis. suth subnitrate were drawn at intervals from in a.m. to 4.30 P.m.

ahnormal conditions, as ahnormal conditions, as in the exposed 'surviving' intestines of the rabbit, contractions, probably similar to the pendulum movements, but running indifferently in both directions, can be set up by local stimulation. The function of these pendulum morements seems to be the thorough mixing of the food with the digestive juices in the intestine. 
When an animal is forl with food containing bismuth subnitrate and observed with the liöntgen rays, it is seen that the foor in a coil is oftern divided into small segments, which then join together to form longer masses, these being in turn again divided. This segmentation is rliythuically repeated (in the cat at the rate of thirty times a minute). Althougl of itself it insures only the mixing of the contents of the gut, and not their onward progress, it is usually accompanied by peristalsis, so that while the food is undergoing segmentation, it is also slowly passing down the intestine. Often, however, a column of food remains for a considerable time, dividing, uniting, and dividing again, without sensibly shifting its position. In addition to the relatively rapid pendulum movements, much slower periodic variations of tone of the whole musculature may be normally observed.

(2) True peristaltic movements, in which a ring of constriction, obliterating the lumen, moves slowly down the tube, with a speed, it may be, no greater than I $\mathrm{mm}$. per second. The portion of the intestine immediately below the adrancing constriction is relaxed and motionless, so that we may say that a wave of inhibition precedes the wave of contraction. The peristaltic movements of the small intestine, the most typical of their kind, are most easily excited by mechanical stimulation of the mucous membrane, as by the contact of a morsel of food or an artificial bolus of cotton-wool. Travelling, under normal conditions, always downwards, the constriction squeezes the contents of the tube before it, and the wave usually ends at the ileo-cæcal valve, which separates the small intestine from the large. The cause of the definite direction of the peristaltic wave is grounded in the anatomical relations of the intestinal wall. For when a portion of the intestine is resected, turned round in its place and sutured, so that what was before its upper is now its lower end, the contraction wave is unable to pass, and the obstruction to the onward flow of the intestinal contents causes marked dilatation of the gut, and sometimes serious disturbance of nutrition. The most probable explanation is that the peristalsis is governed by a local reflex nervous mechanism (Auerbach's plexus), the stimulation of which by the contact of the food with the mucous membrane or by the distension of the gut causes excitation of the circular muscular fibres above the point of stimulation and inhibition of them below it. The automatic pendulum movements and also the slow, rhythmical variations of tone, have a different relation to the local nervous mechanism, for they behave differently to poisons like cocaine and nicotine, which act on that mechanism. The pendulum movements are, if anything, increased in intensity and made more regular. But the peristaltic waves, although they can 
be locally excited ly direct stimulation of the muscular fibres, are no longer propagated, and a bolus introduced into the intestine remains at rest where it is placed. Some have interpreted these facts as indicating that the pendulum movements are myogenic in origin. But evidence has lately been obtained that, although they are not reflex movements elicited by afferent impulses from the mucous membrane, since they continue in unaltered intensity, in isolated loops of intestine immersed in Locke's solution (p. I39) after removal of both mucosa and submucosa, they are nevertheless dependent upon Auerbach's plexus. For when the circular muscular coat is separated from this plexus, the automatic movements of this coat are abolished. although the excitability of the musculature to direct stimulation is not affected. The longitudinal coat, which is still in connection with Auerbach's plexus, goes on contracting spontancously (Magnus). Under certain conditions a movement of food or secretions in the reverse of the normal direction can be set up in the small intestine in the intact body, e.g., in the case of obstruction of the intestine leading to vomiting of its contents. But this does not necessarily indicate a reversal of the normal direction of the peristalsis. Such a reversal, if it occurs at all, is not easy to realize by artificial stimulation, and even when an antiperistaltic wave is apparently started it travels up the intestinc only for a short distance and then dies out. A third variety of intestinal movement has sometimes been described, the socalled 'peristaltic rush ' (Meltzer, etc.). It consists of a rapidly moving peristaltic contraction, preceded by relaxation of a long portion of the tube. Such a contraction may even sweep down without pause from the duodenum to the end of the ileum.

The movements of the large intestine differ from those of the small mainly in the great frequency of antiperistalsis. This, indeed, seems to be the usual movement of the transverse and ascending colon. The antiperistalsis recurs in periods about every fifteen minutes, and each period generally lasts about five minutes. The constrictions, running towards the crecum, thoroughly churn and mix the remnants of the food, a considerable absorption of which may take place in the upper part of the large intestine. Regurgitation into the ileum in man is prevented partly by the oblique entry of the ileum through the wall of the colon (so-called ileo-cæcal valve), but essentially by the tonic contraction of the ileo-colic sphincter. The sphincter usually permits the passage of material only in the direction from small to large intestine. But as an occasional phenomenon, a reverse movement may occur. Thus food may actually pass back through the ileo-colic sphincter into the small intestine under the action of a long-continued and vigorous antiperistalsis, and a this way a considerable portion of a bulky enema may be 
erentually disposed of (Cammon). This so-called antiperistalsis is not precisely the same kind of movement, except for its direction, as the peristalsis already described in the small intestine, since it is not preceded lyy a wave of inhibition. True peristaltic contractions preceled by relaxation of the gut may also be observed to start in the crecum, and to travel down the large intestine. They are not very frepuent in comparison with those of the small intestine, and they die away before reaching the end of the colon, allowing the food to be driven back again towards the caecum by the antiperistalsis. A true downward peristalsis is more commonly seen in the descending colon, and is here associated with the propulsion and collection of the frece, which are mainly stored in the sigmoid flexure. 'These peristaltic contractions do not normally reach the rectum, which, except during defaccation, remains at rest.

Influence of the Central Nervous System on the Gastrointestinal Movements.-As we have already said, these movements are much less closely dependent on the central nerrous system than are those of the cesophagus. They can not only go on, but are in general better marked when the extrinsic nervous connections are cut; they cannot spread when the continuity of the tube is destroyed, and the mere presence of food will excite them when other than local reflex action has been excluded by section of the nerves. Nevertheless, the central nervous system does exercise some influence in the way of regulation and control, if not in the way of direct initiation of the movements, and the swallowing or even the smell of food has been observed to strengthen the contractions of a loop of intestine severed from the rest, but with its nerves still intact. The vagus is the efferent channel of this reflex action : stimulation of its peripheral end may cause movements of all parts of the alimentary canal from resophagus to large intestine, and may strengthen movements already going on ; but section of it does not stop them, nor hinder the food from causing peristalsis wherever it comes. The vagus also contains inhibitory fibres for the lower end of the resophagus and the whole of the stomach. Stimulation of it is followed first by inhibition, and then, after an interval, by an increase of tone and augmentation of the contraction of the whole stomach, including the cardiac and pyloric sphincters. The splanchnic nerves contain fibres by which the intestinal movements can be inhibited, and they appear to be always in action, for after section of these nerves the movements are strengthened. On the other hand, stimulation of the peripheral end of the cut splanchnic causes arrest of the movements. Occasionally, however, it has the opposite effect. Contractions of the small intestine are more easily caused by excitation of the vagus after the inhibitory splanchnic nerves have been cut. The splanchnics also contain 
inhibitory fibres for the stomach, and it is only when these are intact that complete reflex inhibition of the organ can be obtained in the rabbit (Aner). The gastric movements are not permanently affected by section of these nerves alone, or even by simultaneous section of the splanchnics and the gastric branches of the vagi. But if the vagi are cut while the splanchnics remain intact, the peristalsis of the stomach is weakened, its onset delayed, and the proper emptying of the viscus through the pylorus interfered with. In all probability these results are due to the uncontrolled action of the inhibitory fibres. The splanchnics have a special relation to the ileo-colic splincter, which closes when they are stimulated, and becomes insufficient when they are cut. The vagus does not affect it.

The lower part of the large intestine is influenced by the sacral nerves (second, third, and fourth sacral in the rabbit), and by certain lumbar nerves, in the same way as the higher parts of the alimentary. canal, and particularly the small intestine, are inflnenced by the vagus and the splanchnics. Stimulation of these sacral nerves within the spinal canal, or of the pelvic nerves (nervi erigentes) into which they pass, causes contraction of the parts of the large intestine concerned in defæcation--that is, in the dog, of the whole colon, with the exception of the cæcum ; in the cat, of the distal two-thirds of the colon. The colon first undergoes rapid shortening duc to the contraction of the longitudinal fibres and the recto-coccygeus muscle. After a few seconds this is followed by contraction of the circular fibres, beginning at the lower limit of the region in which antiperistalsis can occur, and spreading downwards, so as to cmpty the portion of the bowel involved in the contraction. This is a very close imitation of what occurs in natural defæcation. In man the parts involved in these movements are probably the sigmoid flexure and rectum. In addition to these characteristic motor effects on the lower part of the large intestine, stimulation of the pelvic nerves causes an increase in the antiperistalsis of its upper portions. Stimulation of the lumbar nerves or of the portions of the sympathetic into which their visceral fibres pass (lumbar sympathetic chain from second to sixth ganglia, or the rami from it to the inferior mesenteric ganglia) causes inhibition of the movements of the crecum and the whole colon, including the antiperistaltic movements.

Excitation of the sacral nerves initiates or increases the contraction of both coats of the portions of the large intestine on which they act. excitation of the lumbar nerves inhibits botli. And in the small intestine the same law holds good; the two coats are contracted together by the action of the vagus or inlibited together by that of the splanchnics. With the establishment of these facts, the theory that the same nerve which causes contraction of the circular coat in all tubes whose walls are made up of two layers of miscle also contains fibres that bring about inhibition of the longitudinal coat, and vice versâ, falls to the ground. It was suggested that in this way antagonism between the two coats was prevented.

Defæcation is partly a voluntary and partly a reflex act. But in the infant the voluntary control has not yet been developed; in the adult it may be lost ly disease; in an animal 
it may be abolished hy operation, and in eacll case the action hecomes wholly reflex. In the normal course of events, the pressure of the freces accumulating in the sigmoid flexure at last begins to elicit the discharge of that reflex contraction of the lower portion of the bowel already described (p. 3I0), of which the pelvic nerves constitute the efferent path. At the same time, the sensations set up by the presence of freces in the rectum, the lower part of which, at any rate, is empty and quiescent in the intervals of clefrecation, give rise to the characteristic desire to empty the bowels. This desire may for a time be resisted by the will, or it may be yielded to. In the latter case the abdominal muscles are forcibly contracted, and the glottis being closed, the whole effect of their contraction is expended in raising the pressure within the abdomen and pelvis, and so aiding the muscular wall of the bowel itself in driving the freces from the sigmoid flexure to the rectum. The two sphincters which close the anus-the internal sphincter of smooth muscle, and the external of striated--are now relaxed by the inhibition of a centre in the lumbar portion of the spinal cord, through the activity of which the tonic contraction of the sphincters is normally maintained. This relaxation is partly voluntary, the impulses that come from the brain acting probably through the medium of the lumbar centre. But in the dog, after section of the cord in the dorsal region, the whole act of defæcation, including contraction of the abdominal muscles and relaxation of the sphincters, still takes place, and here the process must be purely reflex. Even after complete destruction of the lumbar and sacral portions of the spinal cord the tone of the sphincters returns after a time, and defæcation is carried on as in a normal animal, the control of the sphincters being due either to a property of the muscular tissue itself or to local ganglia. The contraction of the levatores ani helps to resist overdistension of the pelvic floor and to pull the anus up over the fæces as they escape. The nervi erigentes carry efferent constrictor fibres, and the hypogastrics, as a rule, efferent dilator fibres, to the sphincters. While the internal sphincter is by itself capable of maintaining a tonus of considerable strength, the external sphincter contributes an important share (30 to 60 per cent.) to the closure of the rectum.

The time of passage of substances through the alimentary canal has been studied by administering collodion capsules filled with subnitrate of bismuth to human beings, and observing their progress by taking shadow pictures of them at intervals with the Röntgen rays. During the first twenty minutes two such capsules swallowed at the same time by a healthy young man were clearly seen in the greater curvature of the stomach, but in the interval between the first half-hour and the seventh or 
eighth hour no further trace of them was detected. Ahout the eighth hour they reappeared in the catum. Where they remainer with little or no onward movement till the fourteentle hour. From the fourteenth to the sixteenth hour ther travelled along the ascending colon, and tarried a long time at the left angle of the colon. From the nineteenth to the twenty-second or twentyfourth hour they slowly passed downward in the descending colon and stopped at the sigmoid flexure, till their expulsion in clefetcation. In some subjects the entire passage of the capsules was complete in sixteen hours, in others not until after thirty hours.

Vomiting.-We have seen that under normal conditions the movements of the alimentary canal always tend to carry the fond in one definite direction, along the tube from the mouth to the rectum. The peristaltic waves generally run only in this direction, and, further, regurgitation is prevented at three points by the cardiac and pyloric sphincters of the stomach and the ileocolic sphincter and valve. But in certain circumstances the peristalsis may be reversed, one or more of the guarded orifices forced, and the onward stream of the intestinal contents turned back. In obstruction of the howel, the freal contents of the large intestine may pass up beyond the ileo-crecal valve, and. reaching the stomach, be driven by an act of romiting through the cardiac orifice ; in what is called a 'bilious attack,' the contents of the duodenum may pass back through the pylorus and be ejected in a similar way; or, what is by far the most common case, the contents of the stomach alone may be expelled.

Vomiting is usually preceded by a feeling of nausea and a rapid secretion of saliva, which perhaps serves, by means of the air carried down with it when swallowed, to dilate the carcliac orifice of the stomach, but may be a mere by-play of the reflex stimulation bringing about the act. The diaphragm is now forced down upon the abdominal viscera, first with open and then with closed glottis. The thoracic portion of the resophagus is thus placed under diminished pressure, and therefore widened, while saliva and air are aspirated into it out of the mouth. The abdominal muscles strongly contract. At the same time the stomach itself, and particularly the antrum pylori, contracts, the cardiac orifice relaxes, and the gastric contents are shot up into the lax (esophagus, and through it into the pharynx, and issue by the mouth or nose. The movements of the stomach during romiting induced by apomorphine have been studied in the cat by the Röntgen ray method. There is first observed extreme relaxation of the cardiac end ; then a deep constriction appears a little below the cardiac orifice, and runs towards the pylorus, increasing in depth as it goes. When the transverse band is reached, this contracts firmly and remains contracted, and the constriction passes on 
()ere the antrum pylori. Ten or twelve similar waves follow, at the end of which time the constriction in the region of the transverse band divirles the stomach into the firmly-contracted antrum and the relaxed fundus. Now follows a sudden contraction of the diaphragm and abdominal muscles accompanied by the opening of the cardiac orifice. Either the diapluragm and alulominal muscles alone, without the stomach, or the riaphragm and stomach together, withont the abdominal muscles, can carry ont the act of vomiting. For an animal whose stomach has been replaced by a bladder filled with water can be made to vomit by the administration of an emetic (Magendie) ; and Hilton saw that a man who lived fourteen years after an injury to the spinal cord at the height of the sixth cervical nerve, which caused complete paralysis below that level, conld romit, though with great difficulty. In a young child in which very slight causes will induce romiting, the stomach alone contracts during the act. But in the adult such a contraction is ineffectual, and the same is the case in animals, for a dog under the influence of a moderate dose of curara, which paralyzes the voluntary muscles but not the stomach, cannot romit.

The nerve-centre is in the medulla oblongata. It may be excited by many afferent channels: the sensory nerves of the fauces or pharynx, of the stomach or intestines (as in strangulated hernia), of the liver or kidney (as in cases of gall-stone or renal calculi), of the ntenus or ovary, and of the brain (as in cerebral tumour), are all capable, when irritated, of causing vomiting by impulses passing along them to the romiting centre.

The vagus nerve in man certainly contains afferent fibres by the stimulation of which this centre can be excited, for it has been noticed that when the vagus was exposed in the neck in the course of an operation, the patient vomited whenever the nerve was touched (Boinet, quoted by Gowers). In meningitis, vomiting is often a prominent symptom, and is sometimes due to irritation of the vagus nerve by the inflammatory process.

Some drugs act as emetics by irritating surfaces in which efficient afferent impulses may be set up, the gastric mucous membrane, for example; sulphate of zinc and sulphate of copper act mainly in this way. Apomorphine, on the other hand, stimulates the centre directly, and this is also the mode in which romiting is produced in certain diseases of the medulla oblongata. The efferent nerves for the diaphragm are the phrenics, for the abdominal muscles the intercostals. The impulses which cause contraction of the stomach pass along the vagi. Dilatation of the cardiac orifice is brought about by the inhibitory fibres in the ragus alrearly mentioned. 


\section{The Chemical Phenomena of Digestion.}

Ferments. - The chemical changes wrought in the food as it passes along the alimentary canal are due to the secretions of various glands which line its cavities or pour their juices into it through special ducts. These secretions owe their power for the most part to substances present in them in very small amount, but which, nevertheless, act with extraordinary energy upon the various constituents of the food, causing profound changes without, upon the whole, being themselves used up, or their digestive power affected. The active agents are the enzymes, sometimes spoken of as unformed or unorganized ferments- unorganized because their action does not depend upon the growth of living cells, which was long supposed to be the case for some other ferments, such as yeast. Since it has been shown that specific enzymes can be separated from cells which were formerly believed to act by their mere growth, the distinction between formed and unformed ferments has lost its significance, and has to a great extent been superseded by the distinction between intra- and extra-cellular enzymes-i.e., between ferments which normally act in the interior of the cells where they are produced and ferments which act outside of the cells that secrete them. From yeast cultures, for instance, by crushing the cells, a substance can be obtained which in the complete absence of living yeastcells, and, indeed, of any living micro-organism, forms alcohol and carbon dioxide from sugar, just as living yeast does. There is every reason to believe that it is by the intracellular action of this endoenzyme that the yeast-cell normally causes alcoholic fermentation. The digestive ferments are typical extracellular enzymes. Their chemical nature has not been exactly made out; some of them at least do not appear to be proteins, or to contain a protein group. Some of them apparently exist in the colloidal condition, although this has not been shown for all. In certain cases the more or less stable union of a definite inorganic substance with the ferment, or its actual inclusion in the ferment molecule, seems to be a condition of its action. Thus there is reason to believe that in gastric digestion hydrochloric acid is loosely combined with the pepsin. In the plant oxydase, laccase (p. 264), manganese is present. And the fact that manganese salts oxidize certain substances as laccase does suggests that it is the manganese in combination with some protein or other organic compound in the ferment molecule which confers upon laccase its oxidizing power. A similar relation between iron and some animal oxydases is possible, though not definitely proved. But none of the ferments of the digestive juices has as yet been satisfactorily isolated, and at present it is only by their effects that we recognise 
them. Some of then act best in an alkaline, some in an acicl medium. They all agree in having an 'optimum' temperature, which is more favourable to their action than any other; a low temperature suspends their activity, and boiling abolishes it for "ver. The optimum temperatures of the majority of enzymes lie between $37^{\circ}$ and $53^{\circ} \mathrm{C}$. ; the ' killing' temperatures between $60^{\circ}$ and $75^{\circ} \mathrm{C}$. when they are heated in solutions, but considerably higher when they are heated clry. The action of all of them is hydrolytic-i.e., it is accompanied with the taking up of the clements of water by the substance acted upon. The accumulation of the products of the action first checks and then arrests it. It has been demonstrated in the case of some enzymes that this is clue to their action being reversible. For example, lipase (p. 334) not only decomposes ethyl butyrate or glycerin butyrate, but also builds them up again from the decomposition productse.g., glycerin butyrate from glycerin and butyric acid (Hanriot, Kastle and Ioevenhart). Sometimes the action is not strictly reversible in the sense that precisely the original material is reconstructed, but from the products of the hydrolysis substances are synthesized or condensed, which are then incapable of being split by the ferment. When a concentrated solution of dextrose is acted on for a long time by yeast maltase, a ferment obtained from yeast which changes maltose into dextrose, some of the dextrose is reconverted into isomaltose and dextrin-like bodies. Isomaltose is not again hydrolysed by maltase. The ferment emulsin contained in almonds behaves in the converse way. It hydrolyses isomaltose so as to form dextrose, and then condenses dextrose to maltose (Armstrong).

Many of the ordinary substances of the laboratory will accelerate a reaction which goes on slowly in their absence. These are called catalysers. Some writers also speak of catalysers which retard a reaction progressing quickly in their absence. The process by which the reaction is accelerated (or retarded) is termed catalysis. A typical catalyser can exert its action when it is present in exceedingly small amount in comparison with the substance acted upon. However it may enter into the reaction, it does not take part in the formation of the final products nor contribute to the energy changes, and for this reason is often apparently unaltered at the end of the process. A classical instance of catalysis is the inversion of cane-sugar by weak acids, i.e., the change of the cane-sugar into a mixture of equal quantities of dextrose and levulose - a reaction which may be represented by the equation :

$$
\begin{aligned}
& \mathrm{C}_{12} \mathrm{H}_{22} \mathrm{O}_{11}+\mathrm{H}_{2} \mathrm{O}=\mathrm{C}_{66} \mathrm{H}_{12} \mathrm{O}_{6}+\mathrm{C}_{6} \mathrm{H}_{12} \mathrm{O}_{6} \text {. } \\
& \text { Cane-sugar. Water. Dextrose. Levulose. }
\end{aligned}
$$

This is a reaction which occurs also when the sugar is simply dissolved in water, but with extreme slowness at the ordinary 
temperature, although more rapidly at $100^{\circ} \mathrm{C}$. The effect of the acid is to catalyse the reaction, to markedly accelerate it. The hyclrogen jons of the free acid appear to be responsible for the catalysis. The same action upon cane-sugar is exerted by an enzyme, invertase, found in intestinal juice, although the laws governing the reaction are somewhat different. And it is probable that there is no fundamental difference between the action of the digestive enzymes and that of the inorganic catalysers.

Not even the markedly specific action of the digestive ferments can be considered an essential distinction. It is true that invertase will act upon dextrose, and not at all upon maltose or lactase. But there are other sugars, e.g., raffinose, a trisaccharicle with the formula $\mathrm{C}_{1 \times} \mathrm{H}_{32} \mathrm{O}_{1 \mathrm{f}}$, obtained from bect-sugar residues, which it will hydrolyse. Raffinose is made up of one molecule each of dextrose, levulose, and galactose. On heating with dilute acids, it is decomposed into these substances. Invertase, however, only splits off the levulose molecule, leaving a disaccharide isomeric, but not identical with lactose. Similarly lactase, which is without action upon cane-sugar or maltose, will hydrolyse the $\beta$-galactosides, and maltase, inert as regards cane-sugar or lactose, will hydrolyse the $\alpha$-glucosides. On the other hand, emulsin decomposes the $\beta$-glucosilles, to which group most of the natural glucosides belong, as well as the $\beta$-galactosides and lactose. From raffinose emulsin splits off galactose. leaving cane-sugar. Since the $\alpha$ and $\beta$ compounds are isomeric, and cliffer not in their composition but in their structure, it has been concluded that the structure of the molecule of a substance must be related to the structure of the enzyme which can act on it, in some such way as a lock is related to its proper key. Thus the key lactase fits in the lock lactose, but not in the lock dextrose or the lock maltose.

As to the manner in which an enzyme increases the velocity of its appropriate reaction, it is not easy to make any very positive statement. Several possibilities are recognised, of which two have been especially discussed. (I) The existence of the enzyme in colloidal solution may be important. It is characteristic of colloidal solutions, in which the dissolved substance is present in the form of extremely fine particles, that the total surface of the particles is very great in proportion to the mass of the substance in solution. Thus, a sphere of about the same volume as the eycball, with a diameter of. say, 2 centimetres, would have a surface of 12.5 square centimetres. If this material were subdivided into spheres of about the same volume as a leucocyte, with a liameter of, say, $10 \mu$, it would form eight thousand million of these spheres, with a total surface of over $2 \frac{1}{2}$ square metres. If the small spheres were further subdivided into spherical particles, with a diameter only the thousandth part of that of a leucocyte, say ${ }_{\text {Ioo }}^{\mu}$, cach would form a thousand million of these particles, and the total surface of all the particles would be about 2,500 square metres.

Now, it is known that the intensity of action of some of the inorganic catalysers is proportional to the surface exposed. For example, hydrogen peroxide, if left to itself, is slowly decomposed in to water and oxygen. The addition of fincly divided platinum, in the 
forn of platinum black, greatly hastens the lecomposition, and the oxygen bubbles off. A colloidal solution of platinum, prepared by passing electric sparks between two platinum electrodes immersed in distilled water, and containing the metal in the form of ultramicroscopic particles, is still more effective. The precise nature of the surface effect is not entirely clear. One factor appears to be an increase in the concentration of dissolved substances at the surface, and the better opportunity for mutual action thus afforded to the ferment and the substrate, as the substance acted on by the ferment is termed. The great extension of the surface cannot be the only factor in the catalysis; otherwise any fine powder or suspension would have a catalytic action. But kaolin, or fine sand, or colloidal solutions of ordinary proteins or gelatin, have little, if any, effect on the decomposition of hydrogen peroxide.

(2) Enzymes may produce their effects by contributing to the formation of bodies intermediate between the substrate and the endproducts. If the time required for the formation of a given quantity of the intermediate compound and the time required for the decomposition of this compound into the final products of the ferment action are in sum less than the time required for the direct change of the substrate into the end-products, the enzyme will clearly act as a catalyser of the reaction. It has been shown that in the case of certain inorganic catalysers this does occur. There is some evidence that the ferment actually combines with the substrate, the combination then breaking up to form the end-products.

The Quantitative Estimation of Ferment Action.--Since we have as yet no certain method of freeing the digestive ferments from impurities, our only quantitative test is their digestive activity. And since a very small quantity of ferment can act upon an indefinite amount of material if allowed sufficient time, we can only make comparisons when the time of digestion and all other conditions are the same. If we find that a given quantity of one gastric extract, acting on a given weight of fibrin, dissolves it in half the time required by an equal amount of another gastric extract, or dissolves twice as much of it in a given time, we conclude that the digestive activity of the pepsin is twice as great in the first extract as in the second. But this does not permit us to say that the one contains twice as much pepsin as the other. For it has been found that the amount of digestion in a given time is not directly proportional to the quantity of ferment present, but to the square root of the quantity of ferment (Schütz's law). This law was deduced by Schütz for pepsin, but is said to hold also for trypsin, steapsin, and ptyalin (Pawlow, Vernon). To determine the amount of proteolysis the nitrogen of the protein which has gone into solution may be estimated (p. ${ }_{4} 82$ ). The following table shows the results of one experiment:

\begin{tabular}{|c|c|c|}
\hline Pepsin Solution used \\
in c.c.
\end{tabular}


() r a piece of a glass capillary-tube filled with heat-coargulated eggwhite may be cut off and placel in the digestive mixture (Mett's tubes). At the end of the period of digestion the length of the piece of tube and that of the undigested remnant of the column of coagulated protein are measured with a millimetre scale under a low-power microscope. The difference gives the length of the column rligested. If I c.c. of gastric juice caused in a given time digestion of $2 \mathrm{~mm}$. of the egg-white, $\&$ c.c. of the same juice would digest in the same time and under identical conditions about $+\mathrm{mm}$., and 9 c.c. about $6 \mathrm{~mm}$.

Besides the ferments of the digestive juices which act extracellularly in the lumen of the alimentary canal, and those which do their work intracellularly in its walls, micro-organisms are present in the gut, and even in normal digestion contribute to the changes brought about in the food; while under abnormal conditions they may awaken into troublesome, and even dangerous, activity. It is now known that many of these act by producing intracellular enzymes.

It may be noted here, although the subject must be again referred to (p. 36I), that specific substances capable of inhibiting the action of ferments exist. Some of these antiferments are normally present in the body-an antitrypsin, for instance, in normal blood-serum. Numerous antiferments may be artificially obtained by immunising animals with the original ferments. Thus an antilipase is found in the serum of rabbits after injection of pancreatic lipase, and an antiemulsin after injection of emulsin. Injection of rennin causes the formation of antirennin, which can be demonstrated in the blood-serum and milk of the immunized animal.

It is now necessary to consider in detail the nature of the various juices yielded by the digestive glands, and the mechanism of their secretion. Since it is along the digestive tract that glandular action is seen on the greatest scale, this discussion will practically embrace the nature of secretion in general. And here it may be well to say that, although in describing digestion it is necessary to break it up into sections, a true view is only got when we look upon it as a single, though complex, process, one part of which fits into the other from beginning to end. It is, indeed, the business of the physiologist, wherever it is possible to insert a cannula into a duct and to drain off an unmixed secretion, to investigate the properties of each juice upon its own basis; but it must not be forgotten that in the body digestion is the joint result of the chemical work of five or six secretions, the greater number of which are actually mixed together in the alimentary canal, and of the mechanical work of the gastro-intestinal walls. 


\section{The Chemistry of the Digestive Juices.}

Saliva.-The saliva of the mouth is a mixture of the secretions of three large glands on each side, and of many small ones. The large glands are the parotid, which opens by Stenson's luct opposite the second upper molar tooth; the submaxillary, which opens by Wharton's duct under the tongue; and the sublingual, opening by a number of ducts near and into Wharton's. The small glands are scattered over the sides, floor, and roof of the mouth, and over the tongue.

Two types of salivary glands, the serous or albuminous and the mucous, are distinguished by structural characters and by the nature of their secretion; and the distinction has been extended to other glands. The parotid of many, if not all, mammals is a purely serous gland; it secretes a watery juice with a general resemblance in composition to dilute blood-serum. The submaxillary of the dog and cat is a typical mucous gland; its secretion is riscid, and contains mucin. The submaxillary gland of man is a mixed gland; mucous and serous alveoli, and even mucous and serous cells, are intermingled in it. The submaxillary of the rabbit is purely serous. The sublingual is, in general, a mixed gland, but with far more mucous than serous alveoli. Some of the small glands are serous, others mucous in type.

The mixed saliva of man is a somewhat viscous, colourless liquid of low specific gravity (I002 to I008, average about I005), alkaline to litmus, acid to phenolphthalein, but when tested by the electrical method (p. 23) almost neutral. Besides water and salts, it contains mucin (entirely from the submaxillary, the sublingual and the small mucous glands of the mouth), to which its viscidity is due, traces of serum-albumin and serum-globulin (chiefly from the parotid), and a ferment called ptyalin, which hydrolyses starch, and therefore belongs to the group of amylases or diastases. An oxydase or oxidizing ferment is also present. The salts are calcium carbonate and phosphate (often deposited as 'tartar' around the teeth, occasionally as salivary calculi in the glands and ducts), sodium bicarbonate, sodium and potassium chloride, and almost always a trace of sulphocyanide of potassium, detected by the red colour which it strikes with ferric chloride.* The total solids amount only to five or six parts in the thousand. A great deal of carbon dioxide can be pumped out from saliva, as much as 60 to 70 c.c. from Ioo c.c. of the

* In 100 students investigated by the writer the saliva only once failed to give the reaction, and in this individual a trace of sulphocyanide was present 3 days later. It is absent from the saliva of many animals. In 25 dogs submaxillary saliva obtained by stimulation of the chorda tympani only once gave the ferric chloride reaction, and then faintly. 
secretion-i.c., more than can be obtained from renous blood. Only a small proportion of this is in solution, the rest existing as carbonates. ()xygen is also present even in saliva which has not come into contact with the air, and, incleed, in somewhat greater guantity than in serum (about o.6 volume per cent. in (log's saliva). Uneler the microscope epitlelial scales, dead and swollen leucocytes (the so-called salivary corpuscles), bacteria, and portions of food, may be found. All these things are as accidental as the last - they are mere flotsam and jetsam, washed by the saliva from the insicle of the mouth. But greater significance attaches to certain peculiar bodies, either splierical or of irregular shape, that are seen in the viscid submaxillary saliva of the dog or cat. They appear to be masses of secreted material. The quantity of saliva secreted in the twenty-four hours varies a good deal. On an average it is from I to 2 litres (Practical Exercises, p. 422).

Besides its functions of dissolving sapid substances, and so allowing them to excite sensations of taste, of moistening the food for deglutition and the mouth for speech, and of cleansing the teeth after a meal, saliva, in virtue of its ferment, ptyalin, has the power of digesting starch and converting it into maltose, a reducing sugar. In man the secretion of any of the three great salivary glands has this power, although that of the parotid is most active. In the log, on the other hand, parotid saliva has little action on starch, and submaxillary none at all ; while in animals like the rat and the rabbit the parotid secretion is highly active. In the horse, sheep, and ox, the saliva secreted by all the glands seems equally inert.

When starch is boiled, the granules are ruptured, and the starch passes into imperfect solution, yielding an opalescent liquid. If a little saliva be added to some boiled starch solution which is free from sugar, and the mixture be set to digest at a suitable temperature (say $40^{\circ} \mathrm{C}$.), the solution in a very short time loses its opalescence and becomes clear. It still, however, gives the blue reaction with iodine; and Trommer's test ( $p$. Io) shows that no sugar has as yet been formed. The cliange is so far purely a physical one ; the substance in solution is soluble starch. Later on the iodine reaction passes gradually through violet into red; and finally iodine causes no colour change at all, while maltose is found in large amount, along with some isomaltose, a sugar having the same formula as maltose, but differing from it in the melting-point of the crystalline compound formed by it with phenyl-hydrazine (p. 48S). Traces of dextrose, a sugar which rotates the plane of polarization less than maltose, but has greater reducing power, may be found among the end-products when the digestion is conducted in 
vilro. It is possible that this is produced from the maltose by maltase, which some writers assert to be present in small amount in saliva. But the observation has also been made that the saliva itself (in the cat) may contain a trace of dextrose (Carlson).

The red colour indicates the presence of a kind of dextrin called erythrodextrin; the violet colour shows that at first this is still mixed with some unchanged starch. Soon the erythrodextrin disappears, and is succeeded by another dextrin, which gives no colour with iorline, and is therefore called achroodextrin. This is partly, but in artificial digestion never completely, converted into maltose, and can always at the end be precipitated in greater or less amount by the addition of alcohol to the liquid. It is probable that a whole series of dextrins is formed during the digestion of starch. Some of these may appear as forerunners of the sugar, others merely as concomitants of its production. The latter may never pass into sugar; and it is certain that sugar may appear before all the starch has been converted into achroodextrin. When the sugar is removed as it is formed, as is approximately the case when the digestion is performed in a dialyser, the residue of unchanged dextrin is less than when the sugar is allowed to accumulate (Lea). In ordinary artificial digestion, for instance, under the most favourable circumstances at least $\mathrm{I}_{2}$ to $\mathrm{I}_{5}$ per cent. of the starch is left as dextrin; in dialyser digestions the residue of dextrin may be little more than 4 per cent. This goes far to explain the complete digestion of starch which takes place in the alimentary canal, a digestion so exhaustive that, although soluble starch and dextrin may be lound in the stomach after a starchy meal, they do not occur in the intestine, or only in minute traces. Here the amylolytic ferment of the pancreatic juice, which is essentially the same in its action as ptyalin, only more powerful, must effect a very complete conversion of the starch molecules accessible to its attack. It is not inconsistent with this, that unchanged starch granules may sometimes be excreted in the fæces, especially when imbedded in raw vegetable structures.

It is a notable fact that amylolytic or starch-splitting ferments, also called diastases or amylases, are not confined to the animal body, but are widely distributed in plants. A diastase, which is present in all sprouting seeds, and may be readily extracted by water from malt, forms dextrin and maltose from starch. The optimum temperature of malt diastase, however, is about $55^{\circ} \mathrm{C}$., while that of pytalin is about $40^{\circ} \mathrm{C}$.

While a neutral or weakly alkaline reaction is not unfavourable to salivary digestion, it goes on best in a slightly acid medium. It has been shown that the activity of ptyalin on starch, both having been previously dialysed to get rid as far as possible 
of salts, is increased by the addition of very sulall amounts of aciels and of the neutral salts of strong monobasic acids. The action is decreased by larger amounts of acid $(0.0007$ to $0.00 \mathrm{r} 2$ per cent. of hydrochloric acid) and by neutral salts of weak acids. An acidity equal to that of a $O^{\circ} I$ per cent. solution of hyclrochloric acid stops salivary digestion completely, although the ferment is still for a time able to act when the acidity is sufficiently reduced. Strong acids or alkalies permanently destroy it. These fácts indicate that in the mouth, where the reaction is weakly alkaline, the conditions are comparatively favourable to the action of the ptyalin. They are still more favourable in the stomach for some time after the beginning of a meal, while the reaction is yet weakly acid. It has been observed that (in cats) salivary digestion may go on for an hour or more in the cardiac end of the stomach, since free hydrochloric acid does not appear here before that time. Since the contents of the cardiac end are not frecly intermixed with those of the pyloric end, a greater proportion of sugar is found in the former, and the difference is more marked with solid than with liquid food (Cannon and Day). But during the greater part of gastric digestion the degree of acidity is such that the ptyalin must be hindered. Although the food stays but a short time in the mouth, therc is no cloubt that, in man at least, some of the starch is there changed into sugar (1). $\left.4^{2}+\right)$. But this is not the case in all animals. Something depends on the amylolytic activity of the saliva, and something upon the form in which the starchy food is taken, whether it is cooked or raw, enclosed in vegetable fibres or exposed to free admixture with the secretions of the mouth.

The fact already mentioned that hydrolytic changes of the same nature as those produced by enzymes can be brought about in other ways holds good for ptyalin. If starch is heated for a time with dilute hydrochloric or sulphuric acid, it is changed first into dextrin, and then into a form of reducing sugar, which, however, is not maltose, but dextrose. If maltose is treated witl acid in the same way, it is also changed into dextrose. When glycogen (j) I) is boiled with dilute oxalic acid at a pressure of three atmospheres, isomaltose and dextrose are formed (Cremer). Facts will be cited later on which show that the action of the other digestive ferments, as already mentioned, can also be imitated by purely artificial means. Indeed, we may say that the ferments accomplish at a comparatively low temperature what can be done in the laboratory at a higher temperature, and by the aid of what may be called more violent methods.

Gastric Juice.-The Abbé Spallanzani, although not, perhaps, the first to recognise, was the first to study systematically, the chemical powers of the gastric juice, but it was by the careful 
and convincing experiments of Beammont that the fomndation of our exact knowledge of its composition and action was laid.

It is difficult to speak without enthusiasm of the work of Beaumont, if we consider the difficulties under which it was carried on. In army surgeon stationed in a lonely post in the wilderness that was then called the territory of Michigan, a thousand miles from a University, and four thousand from anything like a physiological liboratory, he was accidentally called upon to treat a gun-shot wound of the stomach in a Canadian voyageur, Alexis St. Martin. When the wound healed a permanent fistulous opening was left, by means of which food could be introduced into the stomach and gastric juice obtained from it. Beaumont at once perceived the possibilities of such a case for physiological rescarch, and began a series of experiments on digestion. After a while, St. Martin, with the wandering spirit of the voyageur, returned to Canada without Dr. Beammont's consent and in his absence. Beaumont traced him, with great difficulty, by the help of the agents of a fur-trading company, incluced him to come back, provided for his family as well ats for hinself, and procecded with his investigations. A second time St. Martin went back to his native country, and a sccond time the zealous investigator of the gastric juice, at heavy expense, sccured his return. And although his experiments were necessarily less cxact than would be permissible in a modern research, the modest book in which he published his results is still counted among the classics of physiology. The production of artificial fistula in animals, a method that has since proved so fruitful, was first suggested by his work.

Gastric juice when obtained pure, as it can be from an accidental fistula in man, or, better, by giving a dog with an cesophageal as well as a gastric fistula a 'sham-meal' (p. 374), is a clear, thin, colourless liquid of low specific gravity (in the dog I003 to I006) and distinctly acid reaction. The total solids average about 5 parts per thousand, of which the ash (chiefly sodium and potassium chloride, with small quantities of calcium and magnesium phosphate) represents about a fourth, and heatcoagulable substances (proteins, nucleoprotein) about a third. None of these has any special importance in digestion. Of quite a. different significance are the three ferments present: pepsin, which changes proteins into peptones; rennin, which curdles milk (but see p. 327) ; and a fat-splitting ferment which, under certain conditions at least, splits up emulsified neutral fats-e.g., the fat of milk - into glycerin and fatty acids, but has 110 action upon non-emulsified fat. The acidity is due to free hydrochloric acid, the other important constituent of the juice. In the dog the proportion of this acid varies from 0.46 to 0.58 per cent. In such analyses as have been made of approximately pure human gastric juice a smaller percentage of hydrochloric acid has usually been obtained (at most 0.35 to 0.4 per cent.). But there is some reason to believe that if the human juice could be collected in a faultless manner, and especially free 
from any achixture with saliva or with a patholngical secretion of mucus, it would show as high a percentage of acid as the dog's juice.

In cases of cancer, whether the growtl is situated in the stomach or not, the free hydrochloric acid of the gastric juice is usually mucl reduced, and often absent. Under sucl conditions some lactic acid may be present in the stomach, being produced from the carbo-hydrates by the action of bacteria (Bacillus acidi lactici), which are normally held in check by the hydrochloric acid, although not rendered incapable of growth when they have passed on into the intestine. Even in the strength of 0.07 to $0^{\circ} 08$ per cent. hydrochloric acid prevents the formation of lactic acid from dextrose. Indeed, when all the hydrochloric acid of the gastric juice is combined with proteins, the protein-acid compound still inhibits the growth of bacteria in the stomach, although not so efficiently as the same amount of free acid. That in normal gastric juice the acidity is not due to lactic acid can be shown by shaking the juice with ether, which takes up lactic acid, and then applying Uffelmann's test to the ethereal extract (Practical Excrcises, 1). 428 ).

More than this, it is not due to an organic, but to an inorganic acid, for healthy gastric juice causes such an alteration in the colour of aniline dyes like congo-red and methyl violet as would be produced by dilute mineral acids, and not by organic acids, even when present in much greater strength.* Finally, when the bases and acid radicles of the juice are quantitatively compared, it is found that there is more chlorine than is required to combine with the bases; the excess must be present as free hydrochloric acid. In the pure gastric juice of fishes like the dogfish and skate, however, the acid is said not to be hydrochloric but an organic acid. The quantity of gastric juice secreted depends upon the nature and amount of the food. It has been estimated at as much as 5 litres in twenty-four hours, or several times the quantity of saliva secreted in the same time. With sham fecding a dog may yield 200-300 c.c. in an hour.

The great action of gastric juice is upon proteins. In this two of its constituents have a share, the pepsin and the free acid. One member of this chemical copartnery cannot act without the other; peptic digestion requires the presence both of pepsin and of acid ; and, indeed, an active artificial juice can be obtained by cligesting the gastric mucous membrane witl dilute $(0.2$ to $0^{*}+$ per cent.) hydrochloric acid. A glycerin extract of a stomach

* A dilute solution of congo-red is turned violet by organic and blue by inorganic acids; the gastric juice turns it blue. Nethyl violet is rendered blue by an inorganic acid like hydrochloric acid, and green if more of the acid be added. It is not altered by organic acids. Gastric juice turns it blue. 
which is not too fresh also possesses peptic powers: but it requires the addition of a sufficient quantity of acid to render then available.

Well-washed fibrin obtained from blood is a convenient proteiu for use in experiments on digestion. Since the blood contains traces of pepsin, the fibrin should be boiled to destroy any which may be present (see also p. 422 ).

If we place a little fibrin in a beaker, cover it with gastric juice obtained from a dog or with $\mathrm{O}^{\circ}+$ per cent. hydrochloric acid, to which a small quantity of pepsin or of a gastric extract has been adcled, and put the beaker in a water-bath at $40^{\circ} \mathrm{C}$, the fibrin soon swells up and becomes translucent, then begins to be dissolved, and in a short time has disappeared (see Practical Exercises, p. 426). If we examine the liquid before digestion has proceeded very far, we shall find chiefly acid-albumin in solution; later on, chiefly albumoses; and of these the priniary albumoses (proto-albumose and hetero-albumose) are the first to appear in quantity, followed by secondary or deutero-albumose (p. Io). Still later, peptones in large and always relatively increasing amounts will be present along with the albumoses. From this we conclude that acid-albumin is a stage in the conversion of fibrin into albumose, and albumose a half-way house between acid-albumin and peptone. It must not be supposed, however, that all the protein is first changed into acid-albumin before any of the acid-albumin is changed into albumose, or that all the protein has already reached the albumose stage before peptone begins to appear. On the contrary, a certain amount of albumoses and of peptones are present very early in peptic digestion, while the greater part of the original protein is still unaltered. Similar, but not identical, intermediate substances occur in the digestion of the other proteins, including that of bodies like gelatin, which are not ordinary proteins, but which pepsin can digest. The generic name of proteose properly includes all bodies of the albumose type, the term 'albumose' itself being sometimes reserved for such intermediate products of the digestion of albumin; while those of fibrin are called fibrinoses; of globulin, globuloses; of casein, caseoses ; and so on. The peptones produced from different proteins are also not absolutely identical. If the digestion is prolonged, the peptones are in turn further hydrolysed, so that eventually a considerable proportion of the original protein is converted into amino-acids and other substances (p. 332).

In the stomach, however, during the four or five hours for which gastric digestion ordinarily lasts, little, if any, of the protein passes beyond the stage of proteose and peptone, and it is in these forms that the bulk, at any rate, of the protein food enters the duodenum. The pancreatic juice, as we shall see later on, not only effects a more complete conversion into peptone, but can split up the whole or a very large proportion of the peptone itself into substances which are no longer protein. Since the subject of protein digestion must come up again, it will be well to postpone any closer discussion of the process till we can view it as a whole. In the meantime it is 
only necessary to repeat that pepsin alone cannot rligest proteins at all. Its action requires the presence of an acid; in a neutral or alkaline medium peptic digestion stops. As in the case of other ferments, there is a certain temperature at which pepsin acts best, an 'optimum' temperature $\left(35^{\circ}\right.$ to $40^{\circ} \mathrm{C}$, or about that of the body). At $0^{\circ} \mathrm{C}$. it is inactive, except in coldblooded animals (frog). Boiling destroys it.

Dilute acid alone does not dissolve coagulated protems like boiled fibrin, or does so only with extreme slowness. Uncoagulated proteins, howerer, are readily changed by it into acidalbumin ; and by the prolonged action of acids, especially at a high temperature, further changes of much the same nature as those produced in peptic digestion may be caused in all proteins. But under the ordinary conditions of natural gastric digestion, it may be said that the acid alone does little until it is aided ly the ferment, just as the ferment alone does nothing without the aid of the acid. The acid enters into a temporary combination with the protein, the more highly hyclrolysed proteins, such as peptone, combining with a greater proportion of acid than such proteins as fibrin or albumin. These compounds so easily undergo liydrolytic dissociation that, in spite of its union with the proteins, the hydrochloric acid is able to act along with the pepsin, so that peptic digestion goes on even when enough protein is present to combine with all the acid. There is evidence that in the gastric juice the pepsin exists in the form of an unstable compound with hydrochloric acid, and it is probably this pepsin-liydrochloric acid compound which is actual catalytic agent in peptic digestion. Althougl hyclrochloric acid acts most powerfully, other acids, such as nitric, phosplioric, sulplunric, or lactic (arranged in the order of their efficacy), can replace it.

The milk-curdling ferment, rennin, or chymosin, is contained in large amount in an extract of the fourth stomach of the calf. which, as rennet, has long heen used in the manufacture of cheese. It exists in the healtly gastric juice of man, but disappears in cancer of the stomach and in chronic gastric catarrh. It is doubtful whether the properties of rennin are ever found in gastric juice or any preparation obtained from it or from the gastric mucous membrane unless pepsin is present. This has suggested that there is no separate milk-curdling ferment, but that the clotting of caseinogen is merely an associated action of the pepsin. Proteolytic ferments of the most varied origin will curdle milk. Pawlow has recently shown that it is highly probable that the milk-curdling property not only of the gastric juice, but also of the pancreatic juice and of the secretion of Brunner's glands, is associated with the proteolytic ferment. 
He states that when the comparison is instituted under proper conclitions there is an exact parallelism between the proteolytic and the milk-culdling power of these secretions, no matter what the circumstances may be in which they are collected, or the influences to which they are exposed after collection. He has fomnd it impossible to separate from any one of them a fraction which has milk-curlling power without proteolytic power.

The curdling of milk by the gastric ferment includes two processes: (I) An action on caseinogen in the course of which a substance, whey-protein, not previously present in the milk, is proluced. This substance is not capable of being converted into casein, and remains in solution in the whey. (2) The altered caseinogen is precipitated in the presence of calcium salts, but not otherwise, as casein, which is insoluble, and forms the curd. Dilute acid will of itself precipitate caseinogen, and the presence of acid, and particularly hydrochloric acid, in the gastric juice helps its milk-curdling action. But that a ferment is really concerned is indicated by the fact that the juice, after being made neutral or alkaline, still curdles milk, and that this power is destroyed by boiling. The optimum temperature is the same as that of the other ferments of the digestive tract, about $40^{\circ} \mathrm{C}$. (p. 3I5).

As to the exact function of the milk-curdling ferment of the gastric juice in digestion, we have no precise knowledge. It seems superfluous if we suppose that the free acid is able of itself to do all that the ferment does along with it. But there is evidence that the curd produced by the ferment is more profoundly changed than the precipitate caused by dilute acids; for the latter may be redissolved, and then again curdled by rennin in the presence of calcium salts, while this cannot be done with the former. We may suppose, then, that the ferment is capable of effecting changes more favourable to the subsequent action of the pepsin upon the casein than those which the acid alone would effect. Or it may be that the ferment acts in the early stages of digestion before much acid has been secreted. We do not know whether the curdling of milk renders it easier for the watery portion to be absorbed by the walls of the stomach, or insures that it shall be more rapidly passed on into the duodenum. If this were the case, it would be a raison d'etre for early curdling, since milk is a very dilute food, and the immense proportion of water in it might weaken the gastric juice too much for rapid digestion of the proteins. But caution should be exercised in giving a physiological value to all the details of the milkcurdling action of the gastric juice. Milk-curdling ferments, or, at any rate, ferments with a milk-curdling influence, have an 
extremely wide distribution, both in secretions which in norma] circumstances can never come into contact witl milk, and in the tissues of animals and plants. Many bacteria produce them. And it appears that in the suchling, where it might be expected, if anywhere, to have a definite and important office. the rennet action of the gastric juice is distinctly less than in the adult. It is worthy of note that the curcl formed by rennet from human milk is more finely divided than that formed from cow's milk, and therefore is more easily digested. The addition of lime-water or barley-water to cow's milk keeps the curd from adhering in large masses, and thus aids its digestion - a fact which is sometimes usefully applied in the artificial feeding of infants.

On fats gastric juice has usually been supposed to have no action, although everybody admits that it will dissolve the protein constituents of fat-cells and the protein substances which heep the fat-globules of milk apart from each other. It has, however, been recently shown that both in the stomach and in vitro (with glycerin extracts of the gastric mucous membrane) a considerable amount of well-emulsified fat may be split up. Gastric juice splits up fat, both in neutral and in weakly acid solutions. The slightest excess of alkali checks the action. The glycerin extract is much more resistant to alkali, while very sensitive to hrdrochloric acid. This indicates that the fat-splitting ferment exists in the mucous membrane in a different form from that in which it exists in the juicenamely. as a zymogen or mother-substance. As regards the carbo-hydrates the swallowed saliva will continue to act on starch in the stomach, so long as the acidity is not too great; while the hydrochloric acid of the gastric juice is able to invert cane-sugar, changing it into a mixture of dextrose and levulose. ${ }^{*}$ and also. doubtless, to hydrolyse to dextrose a portion of the maltose formed by the saliva. Altogether, there is no doubt that the proportion of the carbo-hydrates of the food digested in the stomach is far from insignificant.

The Antiseptic Function of the Gastric Juice.-The stomach, with its acid contents, forms during the greater part of gastric digestion a valve or trap to cut off the upper end of

* These are both reducing sugars, but, as their names indicate, they rotate the plane of polarization in opposite directions. The specific rotatory power of levulose is greater than that of dextrose, so that when cane-sugar is completely inverted, although equal quantities of dextrose and levulose are produced, the plane of polarization is rotated to the left. Cane-sugar itself rotates it to the right. The term 'inversion ' has been extended to include the similar hydrolysis of other sugars of the disaccharride group-e.g., maltose to dextrose, and lactose to a mixture of dextrose and galactose, even although the products are not levo-rotatory. 
the intestine from the hacteria-infested regions of the mouth and pharymx, and to destroy or inhibit the micro-organisms swallowed with the food and saliva. The occasional presence in romited matter of sarcinae or regularly arranged groups of micrococci, generally four to a group, shows that under abnormal conditions the gastric contents are not perfectly aseptic; and even from a normal stomach active micro-organisms of various kinds can be obtained. But upon the whole there is no doubt that the acidity of the gastric juice is an important check on bacterial activity during the first part of digestion, and in the 11)per portion of the alimentary canal. Koch has shown that the acidity of the gastric juice of a guinea-pig is sufficient to kill the comma bacillus of cholera. Normal guinea-pigs fed with cholera bacilli were unaffected. But if the gastric juice was neutralized by an alkali before the administration of the bacilli the guinea-pigs died. Charrin found, too, that digestion with pepsin and hydrochloric acid causes an appreciable destruction or attenuation of diphtheria toxin. Bacteria, like the lactic acid bacillus, which form acid products, may be less profoundly affected by the acid gastric juice than the putrefactive bacteria, which, on the whole, form alkalies, and are therefore accustomed to an alkaline medium. Yet we have seen that the growth of even the lactic acid bacillus is very strictly controlled when the gastric juice contains the normal amount of bydrochloric acid.

It has been supposed by some that this bactericidal action is the chief function of the stomach, and the question has been asked why we should attribute any digestive importance to the secretion of that viscus, since the pancreatic juice can do all that the gastric juice does, and some things which it cannot do. Further, it has been shown that a dog may live five years after complete excision of the stomach, comport himself in all respects like a normal dog, and when killed for autopsy show every organ in perfect health (Czerny). In man, too, the stomach has been excised with a successful result. But if this is to be admitted as evidence against the digestive function of the stomach, it is just as good evidence against the bactericidal function, particularly as it has in addition been shown that even putrid flesh has no harmful effect on a dog after excision of the stomach, any more than on a normal dog. And, indeed, the reasoning is fallacious which assumes that what may happen under abnormal conditions must happen when the conditions are normal. For nothing is impressed more often on the physiological observer than the extraordinary power of adaptation, of making the best of everything, which the animal organism 
possesses. Dumbtless, a dog withrut a stomach will use to the best arlvantage the digestive fluids that remain to him ; and the pancreatic juice, with the aid of the bile and the succus entericus, may be arlecpuate to the complete task of digestion. So, tor, a man from whom the surgeon has removed a kirlney, or a testicle, or a lobe of the thyroid gland, may be in no respect worse off than the man who possesses a pair of these organs. But what (l) We derluce from this? Not, surely, that the excised thyroirl, or testicle, or kidney was useless, or the gastric juice inactive, but that the organism has been able to compensate itself for their loss. Further, it would seem that the fate of the protein or of part of the protein digested and absorbed by the stomach is different from that digested and absorbed by the intestine. For after the operation of gastro-enterostomy (the establishment of an artificial opening between the stomach and the small intestine through which the food passes rapidly witlout having to submit to the challenge of the pyloric sphincter), the ingested nitrogen is more quickly eliminated than when the protein is frrst subjected to full gastric digestion. So that when the quantity of protein in the food is increased above that necessary for nitrogen equilibrium (p. 529) none of the excess is assimilated and stored up, as is the case in a normal animal (Levin, etc.).

Pancreatic Juice.-Pancreatic juice, bile, and intestinal juice are all mingled together in the small intestine, and act upon the food, not in succession, but simultaneously. But by artificial fistulie in animals they can be obtained separately; and occasionally some of them can be procured through accidental fistulae in man. It is said that under certain conditions, especially when fat or oil is introduced into the stomach, the pylorus may remain open long enough to permit the passage of pancreatic juice or bile from the duodenum into the stomach, and this has been recommencled as a practical method of obtaining these secretions in man.

Human pancreatic juice, as obtained from a fistula, is a clear. only slightly viscirl liquid of distinctly alkaline reaction to litmus. Its specific gravity is about I007 to IoIo. The total solicls constitute about $1 \cdot 5$ or 2 per cent., of which a little less than I per cent. is made up of inorganic salts, chiefly solium carbonate, with small quantities of chloricles. The balance of the solids consists mainly of proteins. The alkaline reaction is due to the sodium carbonate, and it is worthy of remark, as showing the important part taken by this secretion in the neutralization of the cliyme, that when titrated against standard acid the alkalinity of the pancreatic juice is not much less than the 
acility of the gastric juice when titrated against stanclard alkali. The quantity of pancreatic juice secreted during the twenty-four hours in an average man has been estimated at 500 to soo c.c. from obscrvations on cases of fistula. Probably under perfectly normal conditions it is greater. A so-called artificial pancreatic juice can be made by extracting the pancreas with water or glycerin. Since better methods of obtaining the natural juice have been leveloped, these extracts have lost some of their importance.

Fresl pancreatic juice contains four ferments: (I) The mothersubstance, trypsinogen. of a proteolytic or protein-digesting ferment, trypsin; (2) an anylolytic ferment, amylopsin; (3) a fat-splitting or lipolytic ferment, steapsin: (4) a milk-curdling ferment. It is doubtful whether the last is a different body from the trypsin (sce p. 326). In any case, it cannot be considered as taking any practical share in digestion, since it can harlly ever happen that milk passes through the stomach without being curdled.

Trypsinogen has no action upon proteins, but in normal digestion it is changed into active trypsin by the enterokinase of the intestinal juice (p. 343). Pancreatic juice collected without contact with intestinal contents or with the mucous membrane of the intestine does not digest proteins. The same is truc of extracts of perfectly fresh pancreas, but if the pancreas is allowed to stand for a time, the extracts contain active trypsin. perhaps because some decomposition product has activated the trypsinogen. Some writers, however, state that when contamination of the gland with intestinal contents or contact with the mucosa lias been avoided in its removal from the body, such extracts will remain inactive for months, although the trypsinogen can at once he activated to trypsin by the addition of enterokinase.

Trypsin, to a certain extent, corresponds with pepsin in its action on proteins. But it acts energetically in an alkaline as well as in a not too acid medium (a very slight amount of digestion may go on in distilled water); and its action, unlike that of pepsin-at least in digestions of moderate duration-does not stop mainly at the peptone stage, but goes on rapidly to the production of the amino-acids, the basic substances arginin, lysin, and histidin, known as the hexone bases, and most of the other decomposition products obtained by boiling proteins with dilute acids. The most important of these products, so far as they have been isolated and identified. are enumerated in the following table (see also pp. I-3) : 


\section{CEITEF DECOMPOSITION PROIDCTS OJ: TROTEINS.}

WONOAMINO-ACIDS AND THITR COMPOUNIUS.

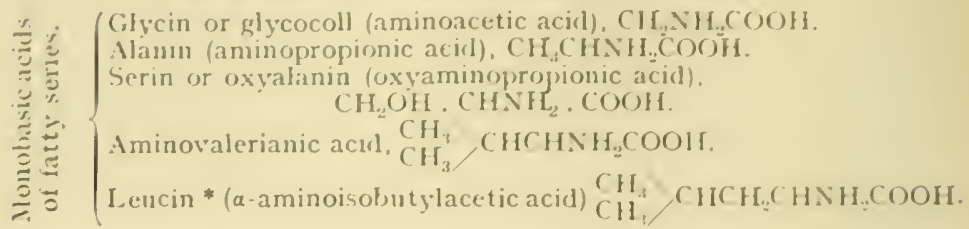

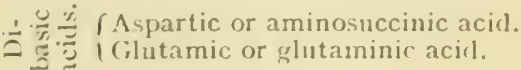

Tyrosin* (para-oxyphenylaminopropionic acirl).
$\mathrm{C}_{6} \mathrm{H}_{4} \mathrm{OH}$. CH., CHNH, COOH.

Phenylalanin (phenylaminopropionic acid), $\mathrm{C}_{6} \mathrm{H}_{5} \mathrm{CH}_{2} \mathrm{CHNH}_{2} \mathrm{COOH}$.

(Prolin (pyrroliclin carboxylic acid).

Oxyprolin (oxypyrrolidin carboxylic acid).

ITryptophane (indol-aminopropionic acil).

DIAMNO-ACIDS AND THEIR COMPOUNDS.

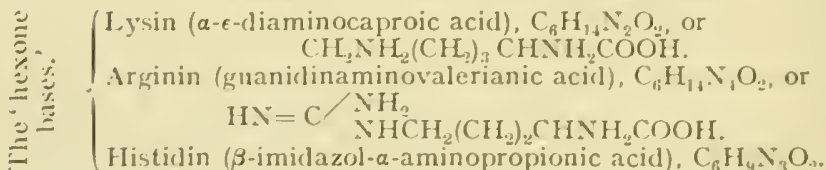

Cystin, $\mathrm{C}_{6} \mathrm{H}_{12} \mathrm{~N}_{2} \mathrm{~S}_{2} \mathrm{O}_{4}$ (derived from a complex amino-acirl, aminothiolactic acid, containing the greater part of the sulphur of the protein molecule).

Ammonia (representing the so-called 'amide-nitrogen," and liberated from the products of acjd hydrolysis of proteins by heating the mixture after addition of alkali).

After the most prolonged artificial digestion with trypsin, a residue of the protein remains unconverted into these relatively simple substances. But even this small portion of the original protein has undergone a great change, for it no longer gives the biuret reaction. It can be split into amino-acids, etc., by heating with acid. When trypsin acts upon protein already digested by pepsin, this partially hydrolysed residue is smaller than when the trypsin acts alone, no matter for how long a time. This illustrates the co-operative relation of these two ferments-a

* Leucin is formed from a-isobutyl acetic acid by the replacement of one $\mathrm{H}$ by $\mathrm{NH}_{2}$. Tyrosin is related to propionic acid $\left(\mathrm{C}_{3} \mathrm{H}_{6} \mathrm{O}_{2}\right)$. If one $\mathrm{H}$ in propionic acid is replacel by $\mathrm{NH}_{2}$, we get aminopropionic acid, $\mathrm{C}_{3} \mathrm{H}_{3}\left(\mathrm{NH}_{2}\right) \mathrm{O}_{2}$. If another $\mathrm{H}$ is replaced by oxyphenyl $\left(\mathrm{C}_{\mathrm{n}} \mathrm{H}_{4} \mathrm{OH}\right)$, an aromatic radicle, we get tyrosin. 
a clation still more clearly implied in the fact that, althougl trypsin readily forms allumoses and peptones from native protein when such is offered to it, yet in natural digestion the great allumoseand peptone-forming ferment is pepsin. In the lumen of the intestine the trypsin is confronted mainly with protein already hydrolysed to the albumose and peptone stage in the stomach.

There is no reason to believe that there is any fundamental difference between the action of trypsin and pepsin on the protein molecule - at any rate, up to the point of peptone formation.

The order in which they appear and their relative amount at different stages of the digestion show that the alkali-albumin and the albumoses produced when trypsin acts in an alkaline medium, such as a I per cent. solution of sodium carbonate, are, like the acid-albumin and albumoses of peptic digestion, mainly, at any rate, intermediate substances through which protein passes on its way to peptone. In both cases the action consists in a splitting up of the complex protein with assumption of water, so that each successive product is further hydrated than the last. Nor is it possible to point out any radical difference between the peptone of gastric and the peptone of pancreatic digestion. The further rapid hydrolysis of the peptone by trypsin into decomposition products of low molecular weight is a distinction merely of degree. For pepsin can also, as we have seen, produce a certain amount of these after prolonged digestion. Trypsin is a more powerful ferment than pepsin, and naturally carries the decomposition farther, and accomplishes it with greater ease.

In all that we have hitherto said regarding tryptic digestion we have supposed that putrefaction has been entirely prevented. If no antiseptic is added to a tryptic digest, it rapidly becomes filled with micro-organisms, and emits a very disagreeable frecal odour; and now various bodies which are not found in the absence of putrefaction make their appearance, such as indol, skatol, and other substances, to which the frecal odour is due. They are not true products of tryptic digestion, but are formed by the putrefactive micro-organisms, which can themselves split off from proteins numerous decomposition products, including tyrosin, and change tyrosin into indol.

Amylopsin, or pancreatic ptyalin, the diastatic or sugarforming ferment of pancreatic juice, changes starch into dextrin and maltose, just as the ptyalin of saliva does. The two ferments are possibly identical, but under the conditions of action of the pancreatic juice its diastatic power is greater than that of saliva, and it readily acts on raw starch as well as boiled. Amylopsin is mainly, perhaps entirely, present in the juice in the form of active ferment. If a zymogen stage exists, the mother-sub- 
stance is less stable or less easily extracted from the gland than is trypsinogen. In this respect amylopsin also resembles ptyalin. I small amount of maltase is contained in pancreatic juice, and further hydrolyses to dextrose a portion of the maltose formed by the amylopsin.

Steapsin splits up neutral fats into glycerin and the corresponding fatty acids. The latter unite with the alkalies of the pancreatic juice and the bile to form soaps. In this important process bile acts as the helpmate of pancreatic juice; together they effect much more than either or both can accomplish by separate action. Many tissues contain fat-splitting ferments or lipases, which are probably not identical with steapsin. Steapsin exists as active ferment in the pancreatic juice, but there is some reason to believe that a portion of it may be present as a mother-substance, steapsinogen, in the gland, and probably in the secretion as well. Active steapsin can also be extracted from the pancreas by glycerin or water. It is to be noted that it is only the proteolytic enzyme which is totally inactive till it reaches the intestine. The significance of this will be discussed later on.

Bile.-Bile is a liquid the colour of which varies in different groups of animals, and even in the same species is not constant, depend ng on the length of time the fluid has remained in the gall-bladder and other circumstances. When it is recognised that the colour is due to a series of pigments, which are by no means stable, and of which one can be caused to pass into another by oxidation or reduction, this want of uniformity will be easily intelligible. The fresh bile of carnivora is goldenred. The bile of herbivorous animals is in general of a green tint, but, when it has been retained long in the gall-bladder, may incline to reddish-brown. Fresh human bile, as it flows from a fistula just established, is of a reddish-brown, golden-yellow or yellow colour. Beaumont speaks of the yellowish bile which he could press into the stomach of St. Martin by manipulating the abdomen. In a case observed by the writer, it was seen that when the bile flowing from a fistula was allowed to sjoread out in a dressing, it became greenish, because of oxidation of a part of the bilirubin to biliverdin, although as it actually escaped from the fistula it was yellow. The bile of a monkey taken from the gall-bladder immediately after death is dark green, but if left a few hours in the gall-bladder it is brown, the green pigment having been reduced. It should be remembered that human bile from the post-mortem room may alter its colour in the interval which must elapse before it can usually be procured after death. Bile, as obtained from fistula in otherwise healthy persons, has a specific gravity of about roos to Ioro. In the 
gall-hladder water is absorbed from the bile and mucin added to it, so that the specific gravity of bladder bile is as high as I030 to rofo. The reaction is feelbly alkaline to litnus.

The composition of two specimens of human bile-one from a fistula, the other from the gall-bladder-is shown in the following table:

Bladder Bile.

Fistula Bile.

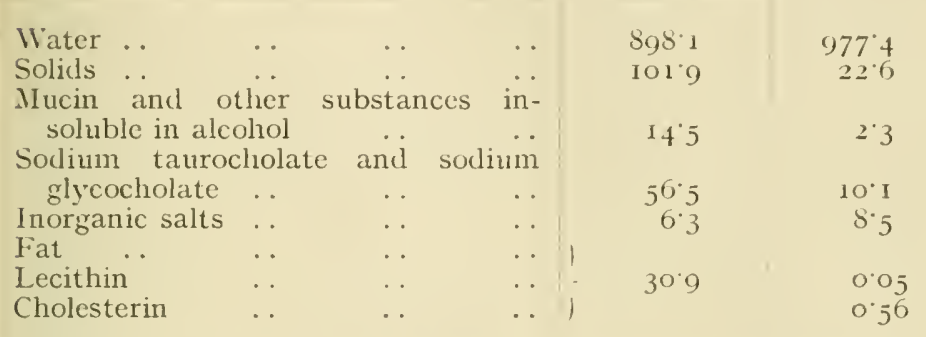

The substance which renders bladder bile viscid, but which is present in much smaller amount in bile from a fistula, and is probably entirely absent from the fluid as it is secreted by the liver-cells. is commonly termed 'mucin.' It has been shown, however, that in many animals-for example, the ox, dog, sheep, etc.-the substance is not a true mucin. It does not yield, like mucin, reducing substances on boiling with dilute acid, or only very small amounts of these. It is relatively rich in phosphorus, and consists-mainly, at any rate-of a phospho-protein (p. 2). The mucilaginous substance of human bile consists largely of true mucin.

Mucin is scarcely to be looked upon as an essential constituent of bile; it is not formed by the actual bile-secreting cells, but by mucous glands in the walls and goblet-cells in the epithelial lining of the larger bile-ducts, and especially of the gall-bladder.

Bile-pigments.- It has been said that these form a series, but only two of the pigments of that series are present in normal bile, bilirubin, and biliverdin. In human bile, the former, in herbivorous bile and that of some cold-blooded animals, such as the frog, the latter is the chief pigment. But bilirubin can be extracted in large amount from the gall-stones of cattle; while the placenta of the bitch contains biliverdin in quantity, although, as in all carnivora, it is either absent from the bile or exists in it in comparatively small amount. These facts show that the two pigments are readily interchangeable.

Bilivubin is best prepared from powdered red gall-stones by dissolving the chalk with hydrochloric acid, and extracting the residue with chloroform, which takes up the pigment. From this solution, on evaporation, beautiful rhombic tables or prisms of bilirubin separate out; and the crystals are finer when the solution also contains cholesterin than when it is pure.

Biliverdin can be obtained from the placenta of the bitch by extraction with alcohol. It is insoluble in chloroform, and by means of this property it may be separated from bilirubin when the two happen to be present together in bile. Biliverdin can also be formed 
from bilirubin by oxidation. By the aid of active oxidlizing agents, such as yellow nitric acid (which contains some nitrous acid), a whole series of oxiclation products of bilirubin is obtained, beginning with biliverdin. and passing through bilicyanin, a bluc pigment, to bili-purpurin, which is purple, and finally to cholctelin, a yellow substance. It is possible that there are other intermecliate bodies. This is the foundation of Gmelin's test for bile-pigments (sec Practical Excrcises, p. 43I). The same substances are produced, and in the same order, when a solution of bilirubin in chloroform is treated with a dilute alcoholic solution of iodine.

The positive pole of a galvanic current catuses the same oxiclative changes, the same play of colours, while the reducing action of the negative pole reverses the effect, if the action of the positive electrode has not gone too far. Starting from biliverdin, the negative pole causes the green to pass through yellowish-green into golden-yellow, and ultinuately into pale yellow, indicating a series of bodies formed by reduction of the biliverdin. These reactions can also be used for the detection of bile-pigments.

By the reducing action of sodium amalgam, or of tin and hydrochloric acid, on bilirubin, hydrobilirubin is obtained. This is sinilar to but not identical with the urobilin of urine, or with the urobilin (stercobilin) found in the faces (partly in the form of its mothersubstance or chromogen, urobilinogen) from birth onwards, although not in the meconium (p. 396). Urobilin is derived from the normal bilepigment by reduction in the intestine itsclf, where reducing substances due to the action of micro-organisms are never absent in extra-uterine life. The changes occurring in oxidation and reduction of the bile-pigment may be partially represented as follows:

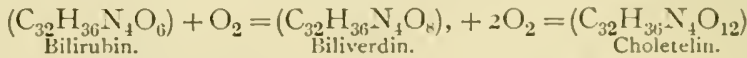

$$
\begin{aligned}
& \left.2 \underset{\text { Bilirubin. }}{2\left(\mathrm{C}_{32} \mathrm{H}_{36} \mathrm{~N}_{4} \mathrm{O}_{6}\right)}-\mathrm{O}_{2}+{ }_{4} \mathrm{H}_{2} \mathrm{O}=\underset{\text { Hydrobilirubin. }}{2} \underset{\text { Het }}{\left(\mathrm{C}_{32}\right.} \mathrm{H}_{40} \mathrm{~N}_{4} \mathrm{O}_{-}\right) .
\end{aligned}
$$

The bile of most animals shows no characteristic absorption spectrum. But the fresh bile of certain animals, the ox, for instance, does show bands - a strong one over $\mathrm{C}$, and two weaker bands, one of which is just to the left of $\mathrm{D}$, and the other to the right of it, but nearer $\mathrm{D}$ than $\mathrm{E}$. The two last bands grow stronger when the bile is allowed to stand for twenty-four hours, and in about three days, in warm weather, a fourth sharp band may appear between $\mathrm{C}$ and $\mathrm{B}$. But none of these bands is due to the normal bile-pigment, and they are not essentially changed when this is oxidized or reduced by electrolysis. Mac.Iunn attributes the spectrum of the bile of the ox and sheep to a body which he calls cholohæmatin, and which does not belong to the bile-pigments proper. Of the derivatives of the bilirubin set, only the lowest and the highest members, hydrobilirubin and choletelin, are clescribed as giving absorption spectra.

The Bile-salts. - These are the sodium salts of two acids, glycocholic and taurocholic. In the bile of omnirora, including man, both are in general present, and in various proportions; in human bile there is more glycocholic than taurocholic acid; sometimes taurocholic acid is entirely absent. In the bile of many carnivorae.g., the dog and cat-only taurocholic acid is found; in that of the carnivora generally it is by far the more important of the two acids. In the bile of most herbivora there is much more glycocholic than taurocholic acid. 
Both acids are made up of a mon-nitrogenous body, cholic or cholalic acid, and a nitrogenous borly, glycin or glycocoll in glyencholic, and taurin in tanrocholic acidl.

The decomposition of the bile-acids into these substances is cffected by boiling them with dilute acid or alkali, a molecule of water being taken up); thus-

$$
\begin{aligned}
& \mathrm{C}_{24} \mathrm{H}_{43} \mathrm{NO}_{4 \mathrm{i}}+\mathrm{H}_{2} \mathrm{O}=\mathrm{C}_{2} \mathrm{H}_{5} \mathrm{NO}_{2}+\underset{\text { Chlycin. }}{\mathrm{C}_{21} \mathrm{H}_{411} \mathrm{O}_{5}} \text {; }
\end{aligned}
$$

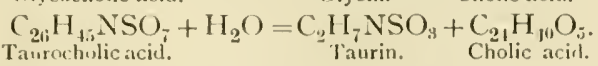

Taurocholic acid is much more easily split up than glycocholic; even boiling with water is sufficient.

Clycin, as already stated, is amino-acctic acid, taurin is aminoethyl-sulphonic acid, an atom of hydrogen bcing in each case replaced by $\mathrm{NH}_{2}$. A notable difference between glycocholic and taurocholic acid is that the latter contains sulphur. The whole of this belongs to the taurin. Botli glycin and taurin are derived from the clisintegration of proteins, and the sulphur of the taurin represents a portion of the sulphur of the proteins. We have already seen that among the products of protcin hydrolysis a sulphurcontaining body, cystin, is found, and there is good evidence that taurin is derived from cystin.

Traces of cholic acid, formed by hydrolysis from the bile-acids by the action of putrefactive bacteria, are found in the intestines, espccially in the lower portion.

Pettenkofer's test for bile-acids (Practical Exercises, p. 430), accidentally discovered in examining the action of bile upon sugar, depends upon three facts: (I) That cholic acid and furfuraldehyde give a purple colour when brought together; (2) that the bile-salts yield cholic acid when acted upon by sulphuric acid; (3) that when cane-sugar is lecomposed by strong sulphuric acid, furfuraldehyde is formed.

Since a similar colour is given when the same reagents are added to a solution containing albumin, it is necessary to remove this, if present, from any liquid which is to be tested for bile-acids.

Lecithin and cholesterin are by no means peculiar to bile (p. 4). They are very widely distributed in the body. Lecithin belongs to the group of phosphatides, fat-like phosphorus-containing substances present in all cells. It is a compound of glycerin with two molecules of fatty acid and one of phosphoric acid. The phosphoric acid is at the same time united with a base cholin. The fatty acid (stearic, palmitic, oleic, etc.) varies in different varieties of lecithin. Heated with baryta-water, lecithin yields the corresponding fatty acid in the form of a soap, along with cholin and glycero-phosphoric acid.

Cholesterin is an alcohol with the empirical formula $\mathrm{C}_{27} \mathrm{H}_{40} \mathrm{O}$. It is best obtained from white gall-stones, of which it is the chicf, and sometimes almost the sole constituent (see Practical Exercises, p. 43I).

The chief inorganic salts of bile are sodium chloride, sodium carbonate, and alkaline sodium phosphate. The phosphoric acid of the ash comes partly from the phosphorus of organic compounds (lecithin and bile-mucin), the sulphuric acid from the sulphur of taurocholic acid, the sodium largely from the bile-salts. Iron is a notable inorganic constituent of bile, although it exists only in traces, in the form of phosphate of iron. Manganese is also present in minute amount. Ioo c.c. of fresh bile yields 50 to roo c.c. of carbon dioxide, part of which is in solution and part combined with alkalies. 
The quantity of bile secreted in twenty-four hours in an average man is probably from 750 c.c. to a litre. In nine cases of fistula of the gall-blarder in patients operated on for gall-stones or cchinococcus the daily quantity varied from 500 to I, IOO c.c. (Brand).

The great action of the bile in digestion is undoubtedly the preparation of the fats for absorption. In this preparation four processes are important: two chemical actions, hydrolysis of nentral fats to glycerin and fatty acids, and suponificution, or the formation of soaps by the union of fatty acids with bases, especially sodium; and two pliysical processes, emulsification, or the formation of a mechanical suspension of such fine globules of unaltered neutral fat as exist in milk, and solution of soaps and fatty acids. While there has been much discussion as to the relative share taken by these processes, and especially by saponification and emulsification in the absorption of fat (p. +12). there is no doubt that they are all concerned in the digestion of fat or the preparation of it for absorption. In this, indeed, the processes are complementary to each other, for an essential preliminary to emulsification in the intestine seems to be the formation of a certain amount of soaps, soluble in the intestinal contents, while the formation of an emulsion at least increases the surface of contact between the unaltered fat and the digestive juices, and so favours more rapid saponification and solution. In the whole series of changes the bile plays a part, though not an independent one; it acts always in conjunction with the pancreatic juicc.

While no complete explanation has been given of the precise nature of this partnership, it is certain that the fat-splitting ferment of the pancreatic juice, on the one hand, and the bilesalts on the other, contribute largely to the total action. An alkaline solution, a solution of sodium carbonate, e.g., is unable of itself to emulsify a perfectly neutral oil ; but if some free fatty acid be added, emulsification is rapid and complete (p. 12). Now, there is no doubt that here a soap is formed by the action of the alkali on the fatty acid, and there is equally little doubt that the formation of the soap is an essential part of the emulsification. But it is not clear in what manner the soap acts, whether by forming a coating round the oil-globules, or by so altering the surface-tension, or other physical properties of the solution in which it is dissolved, that they no longer tend to run together. However this may be, in pancreatic juice we have the two factors present which this simple experiment shows to be necessary and sufficient for emulsification; we have a ferment which can split up neutral fats and set free fatty acids, and an alkali which can combine with those acids to form soaps. Accordingly, pancreatic 
juice is able of itself to form emulsions with perfectly neutral oils. It is possible that the protein constituents of pancreatic juice may have a share in emulsification, since the addition of protein -e.g., egg-white-to a soap solution increases the stability of the emulsions formed by the soap. In bile, on the contrary, although the alkali is present, there is no fat-splitting ferment, and according to the best experiments, bile alone has no emulsifying power on perfectly neutral fat. But we now come to a remarkable fact: this inert bile when added to pancreatic juice greatly intensifies its emulsifying action, and a solution of bilesalts has much the same effect as bile itself. The fact is unloubted, but the explanation is obscure. What it is that the bile or bile-salts can add to the pancreatic juice which so increases its power of emulsification, we do not know. It has been surmised that a characteristic physical property of bile, the diminution of the surface-tension of watery liquids to which it is added, may play an important part, perhaps, in enabling the fatsplitting ferments or the emulsifying soaps to get into closer contact with the unaltered fat. It is also true that bile by itself, presumably in virtue of the chemical action of its alkaline salts, can, in presence of a free fatty acid, rapidly form an emulsion. But the pancreatic juice itself contains so considerable a quantity of sodium carbonate that it would scarcely seem to require the relatively feeble reinforcement of the alkaline salts of the bile.

A part of the effect of the bile is certainly due to its favouring the fat-splitting action of the pancreatic juice. By the addition of bile, the quantity of fat split up by a definite amount of dog's pancreatic juice may be increased two to threefold. It has been shown that this is an action of the bile-salts. The sodium salts of synthetically-obtained glycocholic and taurocholic acids produce the same effect. The capacity of dissolving soaps, which is a property of the bile-salts, is also of great importance in supplementing the solvent power of the intestinal liquids for the products formed by the pancreatic juice. The solution of soaps in the bile-salts has the power in its turn of dissolving free fatty acids. The significance of this in fat absorption will be referred to again. Although our knowledge of the mutual action of the two juices on the digestion of fats is still incomplete, there is no doubt that they are equally necessary. For in some diseases of the pancreas fat or fatty acid often appears in the stools, and this token of imperfect digestion of the fatty food may be confirmed by the wasting of the patient. The same may occur when the bile is prevented by obstruction of the duct or by a biliary fistula from entering the intestine. Yet in some cases of fistula, where there is every reason to believe that all the bile is escaping externally, the nutrition of the patient-at 
any rate, on a diet not abnormally rich in fat-is unaffected. The mere defieiency of bile in the intestine is, of comse, complicated in obstructive jaundice by the harmful effects of the biliary constituents circulating in the lolood.

The white stools of jaundice owe their colour, not merely to the absence of bile-pigment, but also to the presence of fat. Their highly offensive odlour used to be adduced as evidence that bile is the 'natural antiseptic' of the intestine. It seems rather to be due to the coating of the particles of food with mndigested fat, which shields the proteins from the action of the digestive juices while permitting the putrefactive bacteria to revel in them uncheckerl. As a matter of fact, the bile itself has little, if any, power of hindering the growth of micro-organisms, although the free bile-acicls are tolerably active antiseptics. In suckling clildren it is not uncommon to see the feeces white with fat. This is a less serious symptom than in adults, and perhaps betokens merely that the milk in the feeding-bottle is undiluted cow's milk, which is richer in fat than human milk, and ought to be mixed with water.

Bidder and Schmidt found that the chyle in the thoracic duct of a normal dog contained 3.2 per cent. of fat. In a dog with the bile-duct ligatured the proportion fell to 0.2 per cent. It is an instance of the extraordinarily exact adaptation of the digestive juices to the nature of the food, the mechanism of which will present itself for discussion later on, that the reinforcing action of the bile upon the fat-splitting ferment of the pancreatic juice is said to be greater when the food is rich in fat (p. 38I).

Bile has been credited with a physical power of aiding the passage of fat through membranes moistened with it by diminishing the surface tension, and it has been inferred that this has an important bearing on the absorption of fat from the intestine. But the inference does not follow from the statement, and the statement has been itself denied. There is at present no evidence that the digestive function of the bile extends beyond the preparation of the food for absorption to the preparation of the mucosa for absorbing it.

On proteins bile has either no cligestive action, or only a feeble one. Fibrin is slightly digested by the bile of the dog and of man. But the addition of it to fresh pancreatic juice considerably increases the proteolytic power of that secretion (Rachford), although not so decidedly as in the case of the fat-splitting action. The amylolytic action of the pancreatic juice is also favoured by the bile, and in about the same degree as its proteolytic effect. Although bile sometimes exerts by itself a feebly amylolytic action, this is not to be included among its specific powers, for a diastatic ferment in small quantities is widely diffused in the body.

The addition of bile or bile-salts to a gastric digest causes the 
precipitation of any nnaltered native protein, acid-allumin, albunose, and pepsin. The precipitate, which is a salt-like componnd of protein with tamocholic acid, is reclissolved when the liquid is renclered alkaline, and therefore in excess of hile, or of a solution of bile-salts, but the pepsin las no longer any power of digesting proteins. Part of the bile-acids and bile-mucin is also thrown down by the acid of the digest. It has been suggesterl that by thus precipitating the constituents of the chyme which lave not been carried to the peptone stage bile prepares them for the action of the pancreatic juice. But it is difficult to sec how the precipitation of a substance can prepare the way for its (ligestion, and it is more probable that if any physiological value is to be given to this reaction, it has the function of preventing the absorption of proteins which have not been sufficiently split up. There is little cloubt, however, that the rendering of the pepsin inactive has physiological significance, for pepsin exerts an injurious influence upon the ferments of the pancreatic juice. In digestion, then, the bile has a troofold function, farouring greatly the activity of the pancreatic ferments, especially the fat-splitting ferment, and aiding in establishing the conditions necessary for the transition of gastric into intestinal digestion.

Succus entericus.-This is the name given to the special secretion of the small intestine, which is supposed to be a product of the Lieberkühn's crypts. In order to obtain it pure, it is of course necessary to prevent admixture with the bile, the pancreatic juice, and the food. This can be done by dividing a loop of intestine from the rest by two transverse cuts, the abdomen having been opened in the linea alba. The continuity of the digestive tube is restored by stitching the portion below the isolated loop to the part above it. One end of the loop is sewed in to the lips of the wound in the linea alba, and the other being closed by sutures, the whole forms a sort of test-tube opening externally (Thiry's fistula). Or both ends are made to open through the abdominal wound (Vella's fistula). Another method is to make a single opening in the intestine, and by means of two indiarubber balls, one of which is pushed down, and the other up through the opening, and which are afterwards inflated, to block off a piece of gut from communication with the rest. Or several openings may be made at different levels in the intestine, each being allowed to heal into a wound in the abdominal wall. When pure juice is required it is collected from the lower fistulæ, while the upper fistula are opened to permit the escape of the secretions which enter the higher portions of the alimentary canal (gastric juice, pancreatic juice, and bile). The intestinal juice so obtained is a thin yellowish liquid of alkaline reaction, generally somewhat 
turbid from the presence of a certain number of leucocytes and cpithelial cells. Its specific gravity is about roro, the total solids about $\mathrm{I}_{5} \mathrm{y}$ per cent. It contains a small amount of proteins, including serum albumin and serum globulin, and about the same proportion of inorganic salts as most of the liquids and solids of the body, namely, 0.7 or 0.8 per cent., chiefly sodium carbonate and sodium chloride; but, like the other digestive liquids, it is adapted to the nature of the food, and therefore its composition is not quite constant. Like bile, intestinal juice acts but feebly on the food substances by itself, and if we contented ourselves with examining the pure and isolated secretion, we should greatly underestimate its importance. The sodium carbonate, in which it is relatively rich, will, to be sure, form soaps with fatty acids produced by the action of the pancreatic juice or of the fat-splitting bacteria in which the intestine abounds, and thus aid in the digestion of fats. A lipase, feebler than that of the pancreatic juice, or present in smaller concentration, is also a constituent of the succus entericus. That a great deal of fat may be split up in the alimentary canal in the absence both of bile and pancreatic juice is well ascertained. The alkali of the succus entericus must at the same time aid in neutralizing the original acidity of the chyme, and in preserving the proper reaction of the intestinal contents. A ferment called invertase, or sucrase -which is not introduced with the food or formed by bacterial action as has been suggested, since it occurs in the aseptic intestine of the new-born child-will invert cane-sugar. The ferments maltase and lactase will cause a corresponding change in maltose and lactose (see footnote, p. 328). It is worthy of remark that these inverting enzymes are present in the intestinal mucosa as well as in the intestinal juice, and extracts of the mucosa are usually distinctly more active than the juice itself. So that there is reason to believe that hydrolysis of the disaccharides may take place both in the lumen of the gut before absorption and in the wall of the gut during absorption. Inverting enzymes appear in the intestine early in embryonic life. Maltase is the most generally distributed of all these enzymes, and it is found along with lactase in the intestine of the embryo pig, while invertase is missing till after birth (Mendel). On native proteins and starch the isolated succus entericus has little or no action. But it contains a ferment, erepsin, which, although it does not affect native proteins like serum- and eggalbumin (fibrin and caseinogen may be slightly digested), exerts a powerful action on the first products of protein hydrolysis, albumoses, and peptones, breaking them up into bodies which no longer give the biuret reaction (ammonia, mono-amino acids, hexone bases, etc.). It destroys the diphtheria toxin, which 
is also rendered imnomous by trypsin. Erepsin, however, is not specific to the secreted intestinal juice, for it occurs also, not only in the mucous membrane of the intestine, which, indeed, contains a greater quantity of it than the succus entericus, but in all animal tissues hitherto investigated. The kidney in mammals is even richer in erepsin than the intestinal mucous membrane. Next to these come the pancreas, spleen, and liver, then at a long interval the heart muscle, while skeletal muscle and brain-tissue are poorest of all in the ferment. The intestinal mucosa varies in its erepsin content at different levels and on different diets. In cats on a meat or a mixed diet the duodenum is about five times richer in the ferment than the stomach. The ileum is about half as rich as the duodenum, and the jejunum occupies an intermediate position between the duodenum and ileum (Vernon). The secretion of Brunner's glands in the duodenum, which resemble in structure the pyloric glands of the stomach, digests coagulated albumin, although its proteolytic powers are feebler than those of the pancreatic juice.

The most characteristic constituent of succus entericus is a ferment, enterokinase, which differs from all the ferments we have hitherto described in acting not directly upon the foodstuffs, but upon the trypsinogen of the pancreatic juice, changing it into the active enzyme trypsin. It may therefore be spoken of as a ferment of ferments. It has been previously stated that freshly secreted pancreatic juice is without action upon proteins. The addition of succus entericus immediately confers upon it a high degree of proteolytic power. In one experiment pancreatic juice, obtained by a temporary fistula, required four to six hours to dissolve fibrin, and did not attack coagulated albumin even in ten hours. On addition of succus entericus, the same pancreatic juice dissolved fibrin in three to seven minutes, and rapidly digested coagulated albumin (Pawlow). In like manner a glycerin extract of a fresh pancreas has hardly any effect on proteins; a similar extract of a stale pancreas is active. The fresh pancreas contains trypsinogen, which is soluble in glycerin, for the inert extract becomes active when it is treated with dilute acetic acid, or even when it is diluted with water and kept at the body-temperature. If the fresh pancreas be first treated with dilute acetic acid, and then with glycerin, the extract is at once active. The trypsinogen can therefore be activated within the pancreatic cells, gradually when the pancreas is simply allowed to stand after excision, more rapidly in presence of the dilute acid. The ordinary tests for ferment action (destruction by boiling, activity in very small amounts, etc.) have shown that this property of the intestinal juice is due to a ferment, although it differs in certain respects from most ferments-for instance, in 
requiring a relatively high temperature to inactivate it. The smallest trace of enterokinase will convert a large guantity of trypsinogen into trypsin if time be given. At the same time, althougl to a much smaller extent, the fat-splitting and starchdigesting activity of the pancreatic juice is increased. The secretion of the duodenum causes a greater increase in the proteolytic power than that of the other portions of the small intestine, while no such difference lias been made out in the case of the anylolytic and lipolytic functions. It is probable that the enterokinase, which is secreted mainly in the upper part of the small intestine, and solely by the intestinal epithelium, acts only on the trypsinogen, and that the amylopsin and steapsin are aided in some other way. Enterokinase is only found in the intestinal juice when pancreatic juice is jresent in the gut. It is therefore secreted in response to the presence of trypsinogen or of some other constituent of the pancreatic juice.

Delczenne has attempted to explain the interaction of enterokinase and trypsinogen as an adaptive phenomenon of the same kind as the formation of antitoxins and hamolysins (p. 27). According to him, enterokinase acts like a complement in hamolysis, while trypsinogen plays the part of an intermediary body or amboceptor which enables the enterokinase to attack the protein molecule. He asserts that enterokinase, or a substance which produces a similar effect on trypsinogen, is contained not only in the mucous membrane of the intestine, but also in leucocytes, in fibrin (one of whose properties it is to pick out ferments from liquids containing them), in lymph-glands, in snake venom, and even in cortain anaerobic bacteria. On this view trypsin would not be a definite substance produced by the interaction of enterokinase and trypsinogen, but only an expression for these two bodies acting together. Strong evidence against this view, and in favour of the independent existence of trypsin, has been brought forward by Bayliss and Starling, and it does not seem to merit further consideration.

According to Pawlow, the reason why the trypsin is not secreted in the active form is that active trypsin readily destroys the amylolytic and lipolytic ferments. In the intestine, where trypsin is rendered active by enterokinase, these ferments are protected from its attack by the proteins of the food and by the bile.

Having now finished our review of the chemistry of the digestive juices, our next task is to describe wlat is known as to their secretion-the nature of the cells by which it is effected and their histological appearance in activity and repose, and the manner in which it is called forth and controlled.

\section{The Secretion of the Digestive Juices.}

The digestive glands are formed originally from involutions of the mucous membrane of the alinentary canal, the salivary glands from the ectoderm, the others from the endolerm (Chap. Xl $V^{\top}$.) Some are simple unbranched tubes, in which there is either no clis- 
tunction into body and duct, as in licberkïln's crypts in the intestines, or in which one or more of the tubes open into aluct, as in the glands of the fundus of the stomach. Some are branched tubes, several of which may end in a common (luct; such are the glands of the pyloric end of the stomach, and the Brunner's glands in the luodenum. In others the main aluct ramifies into a more or less complex system of small channels, into each of the ultimate branches of whicl one or more (usually several) of the secreting tubules or alveoli open. The salivary glands and the pancreas belong to this class of compound tubular or racemose glands, and so does the liver of such animals as the frog. But in the latter organ the typical arrangement is obscured in the higher vertebtates by the predominance of the portal bloodvessels over the system of bile-channels as a groundwork for the grouping of the cells.

In every secreting gland there is a vascular plexus outside the cells of the gland-tubes, and a system of collecting channels on their inner surface; and in a certain sense the cells of every gland are arranged with reference to the bloodvessels on the one hand, and the ducts on the other. But in the ordinary racemose gland the blood-supply is mainly required to feed the secretion; the cells of the alveoli have either no other function than to secrete, or if they have other functions, they are not such as to entail a great disproportion between the size of the cells and the lumen of the channels into which they pour their products. For both reasons the relation of the grouping of the cells to the duct-system is very obvious, to the blood-system very obscure. In the liver the conditions are precisely reversed. We cannot suppose that the manufacture of a quantity of bile less in volume than the secretion of the salivary glands, though doubtless containing far more solids, requires an immense organ like the liver, and a tide of blood like that which passes through the portal vein. And, as we shall see, the liver has other functions, some of them certainly of at least equal importance with the secretion of bile, and one of them evidently requiring from its very nature a bulky organ. Accordingly, both the richness of the blood-supply and the size of the secreting cells are out of proportion to the calibre of the ultimate channels that carry the secretion away. The so-called bile-capillaries, which represent the lumen of the sccreting tubules, are mere grooves in the surface of adjoining cells ; and the architectural lines on which the liver lobule is built are: (1) the interlobular veins which carry blood to it; (2) the rich capillary network which separates its cells and feeds them; and (3) the central intra-lobular vein which drains it. Thus a network of cclls lying in the meshes of a network of blood-capillaries takes the place of a regular dendritic arrangement of ducts and tubules; and in accordance with this the bile-capillaries, instead of opening separately into the ducts, form a plexus with each other within the hepatic lobule (see also footnote, p. I4).

The ducts and secreting tubules of all glands are lined by cells of columnar epithelial type, but the type is most closely preserved in the ducts. In none of the digestive glands is there more than a single complete layer of secreting cells. But the alveoli of the mucous salivary glands show here and there a crescent-shaped group of small deeply-staining cells (crescents of Gianuzzi) outside the columnar layer (Fig. I 4 I, $\mathrm{A}^{\prime \prime}, \mathrm{B}^{\prime \prime}$ ), and between it and the basement membrane, while the gland-tubes of the fundus of the stomach have in the same situation a discontinuous layer of large ovoid cells, 
termed parictal from their position, oxyntic (or acid-sacreting) from their supposed function (Figs. I38-1 40). Access to the lumen of the glands is proviled for these decply-placed parictal cells and for the cells of the crescents by fine branching channels which enter and surround the cells. The serous salivary glands, the pyloric glands of the stomach, and the Lieberkïhn's crypts, have but a single layer of cpithelium; and since there is no hepatic cell which is not in contact with at least one bile-capillary, the liver may be regarcled as having no more. The same is true of the pancreatic alveoli, except that in the centre of many of the acini a few spindle-shapel cells (centroacinar cells), apparently continued from the lining of the smallest ducts, may be seen. Remarkable histological changes, evilently connected with changes in functional activity, have been noticed in most of the digestive glands. In discussing these, it will be best to omit for the present any detailed reference to the liver. since, although there are histological marks of secretive activity in this gland as well as in others, and of the same general character, they are accompanied, and to some extent overlaid, by the microscopic
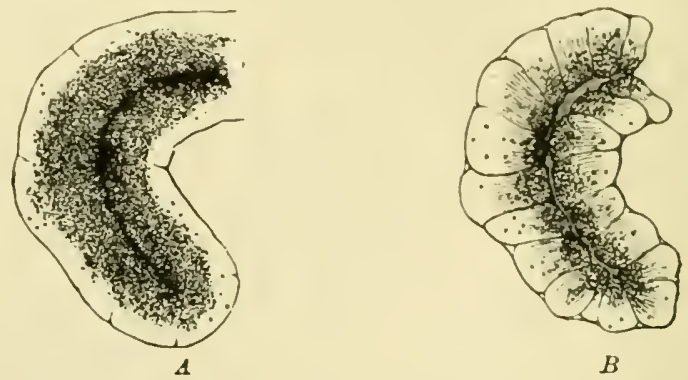

Fig. I36.-Pancreas in 'Loaded' and 'Discharged' State.

$A$, alveolus of rabbit's pancreas, 'loaded' (resting); $B$, ' discharged' (active), observed in the living animal (Kühne and Lea).

evidences of other functions (p. 5 II). The serous salivary glands and the pancreas can be taken together; so can the glands of the various regions of the stomach; the mucous salivary glands must be considered separately.

Changes in the Pancreas and Parotid during Secretion.The cells of the alveoli of the pancreas or parotid cluring rest, as can be seen by examining thin lobules of the former between the folds of the mesentery in the living rabbit, or fresh teased preparations of the latter, are filled with fine granules to such an extent as to obscure the nucleus. In the parotid the whole cell is granular, in the pancreas there is still a narrow clear zone at the outer edge of the cell which contains few granules or none ; in both, the divisions between the cells are very indistinct, and the lumen of the alveolus cannot be made out. During activity the granules seem to be carried from the outer portion of the cell towards the lumen, and there discharged; the clear outer zone 
of the pancreatic cell grows broader and broader at the expense of the inner granular zone, until at last the granular zone may in its turn be reduced to a narrow contour line around the lumen (Fig. I36). In the uniformly clouded parotid cell a similar change takes place; a transparent outer zone arises ; and, after prolonged secretion, only a thin edging of granules may remain at the inner portion of the cell (Fig. I37). In both glands the outlines of the cells become more clearly indicated, and a distinct lumen can now be recognised. The cells are smaller than they are during rest.

The disappearance of granules from without inwards cluring activity suggests that these are manufactured proclucts eliminated in the secretion.

In one respect the changes in the pancreas differ remarkably from those in the salivary glands. The 'islets of Langerhans,' those characteristic groups of small polygonal cells, richly supplied with bloodvessels, but not arranged in the form of alveoli
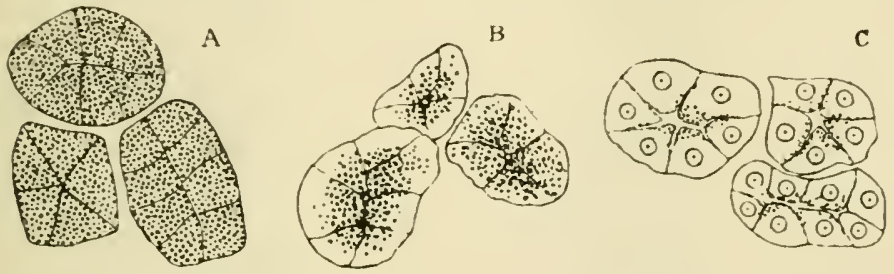

Fig. I37.-Alveoli of Parotid Gland : A, at Rest ; B, after a Short Period of Activity; C, after a Prolonged Period of Activity (Fresh Pre. PARATIONS) (LANGLEY).

In $\mathrm{A}$ and $\mathrm{B}$ the nuclei are obscured by the granules of zymogen.

and unprovicleck with ducts, which are scattered here and there among the alveoli, are markedly increased in size and, it is said, in number when the pancreas is caused to secrete actively by repeated injections of secretin, and also in starvation. Some observers consider that they are derived from the ordinary secreting cells, which when exhausted undergo rearrangement, and that they can, in turn, give rise to new alveoli by a process of proliferation. Others look upon them as independent structures, with a different function from the pancreatic alveoli (p. 554). The discussion of this question is assuredly not yet closed.

Changes in the Glands of the Stomach during Secretion.-The mucous membrane of the stomach is covered with a single layer of columnar epithelium, largely consisting of mucigenous goblet-cells. It is studded with minute pits, into which open the ducts of the peptic and pyloric glands, the ducts being lined with cells just like those of the general gastric surface. Three varieties of gastric 
glands have been distinguisherl: (I) The glands of the cardia. In man these occupy a small portion of the mucous membrane at the cardiar end, near the orifice of the eesoplagens. Some of the glands are single tulunles, but others have two or more tulules opening into a common duct. Both are lined by a single layer of slort columnar epithelium, which contaims granules. (2) The glands of the pyloric canal or antrum. These consist of short,

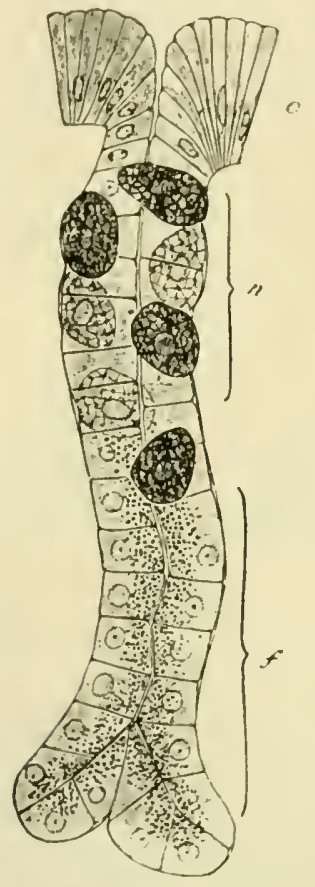

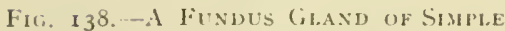
Form from tile Bat's Stomacil (Osmic ACID PREPARATION) (LANGLEY).

c. colummar epithelium of the surface : $n$, neck of the gland with chief or centra! and parietal cells : $f$, hase, occupied only by chief crlls, which show the granules accumulated lowards the lumen of the gland.

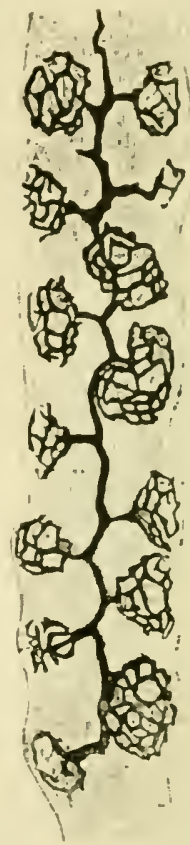

Fici. 139. - A Fundis Glaxn PREPARED BY GOLGI'S METIODD. SHOWING THE MODE OF COM. MUNICATION OF THE P'ARIETAL CELLS WITU THE (ILAND-LIME.N (Schafer, AFter $i$. Wi Ller).

branched tubules, which open by twos and threes into long ducts. (3) The glands of the fundus or oxyntic glands, occupying the intermediate and greater portion of the organ. The gland tubules are long and seldom branched, and the ducts, into each of which open from one to three tubules, are relatively short. The secreting parts of both kinds of glands are lined hy short columnar granular cells; and in the pyloric tubules no others 
are present. But, as we lave said, in the glands of the fundus there are besides large ovoicl cells scattered at intervals like beads between the losement membrane and the lining of chiof cells. The cells of the pyloric glands have a general resemblance to the chief cells of the fundus glands, but they are not quite the sime. For example, the gramules are less distinct in the pyloric grands. In the hmman stomach it is only quitc near the pylorus that the parietal cells disapuear altogether. The parietal cells also contain granules, hut they are smaller and less mumeroms than those of the chief cells, so that the reeper portioms of the fundus glands are mucl: larker in appearance than the more superficial portions, since the oxyntic cells are more numerous in the neighbourliood of the lucts (Bensley).

The histological changes connected with gastric secretion do not liffer essentially from those described in the pancreas and the parotid, but there is mucl greater difficulty in making observations on the living, or at least but slightly altered, cells. For the mammal the best method is to use animals with a permanent gastric fistula, and to remove from time to time small portions of the mucous membrane for examination in the fresh condition. During digestion the granules disappear from the outer part of the chief cells of the fundus glands, leaving a clear zone, the lumen being bordered by a granular layer. Or, more rarely, there may be a uniform decrease in the number of granules throughout the cell. The total volume of the cell is less than in the fasting condition. The ovoid cells, which are small in the fasting animal, swell up, so as to bulge out the membrana propria. They reach their maximum size (in the dog) very late in digestion (the thirteenth to the fifteenth hour). No such definite changes in their contents as those observed in the other cells have been made out. The granules in the ovoid cells during and after activity seem to be as large and as numerous as in the resting cell, or even larger. After sham feeding in dogs the histological changes in the gastric glands are very slight, even when considerable amounts of gastric juice have been secreted (Noll and Sokoloff).

The chief cells of the oxyntic, and the similar if not identical cells of the pyloric glands, are believed to manufacture the pepsinforming substance. The ovoid cells of the tormer are supposed to secrete the hydrochloric acid. The evidence on which this belief is based is as follows :

The glands of the antrum pylori in the dog, in which in most situations no ovoid cells are to be seen, secrete pepsin, but no acid. The pyloric end of the stomach or a portion of it has been isolated, the continuity of the alimentary canal restored by sutures, and the secretion of the pyloric pocket collected. It was found to be 
alkaline, and contained pepsin. The glands of the frog's aesophagus, which contain only chief cells, secrete pepsin, but no acid. It seems fair to conclude that the chief cells of the funclus glands in the mammal secrete none of the free hydrochloric acid, but certainly some of pepsin. But it does not follow that all the pepsin is formed by these cells, although it would seem that all the hydrochloric acid must be secreted by the only other glandular
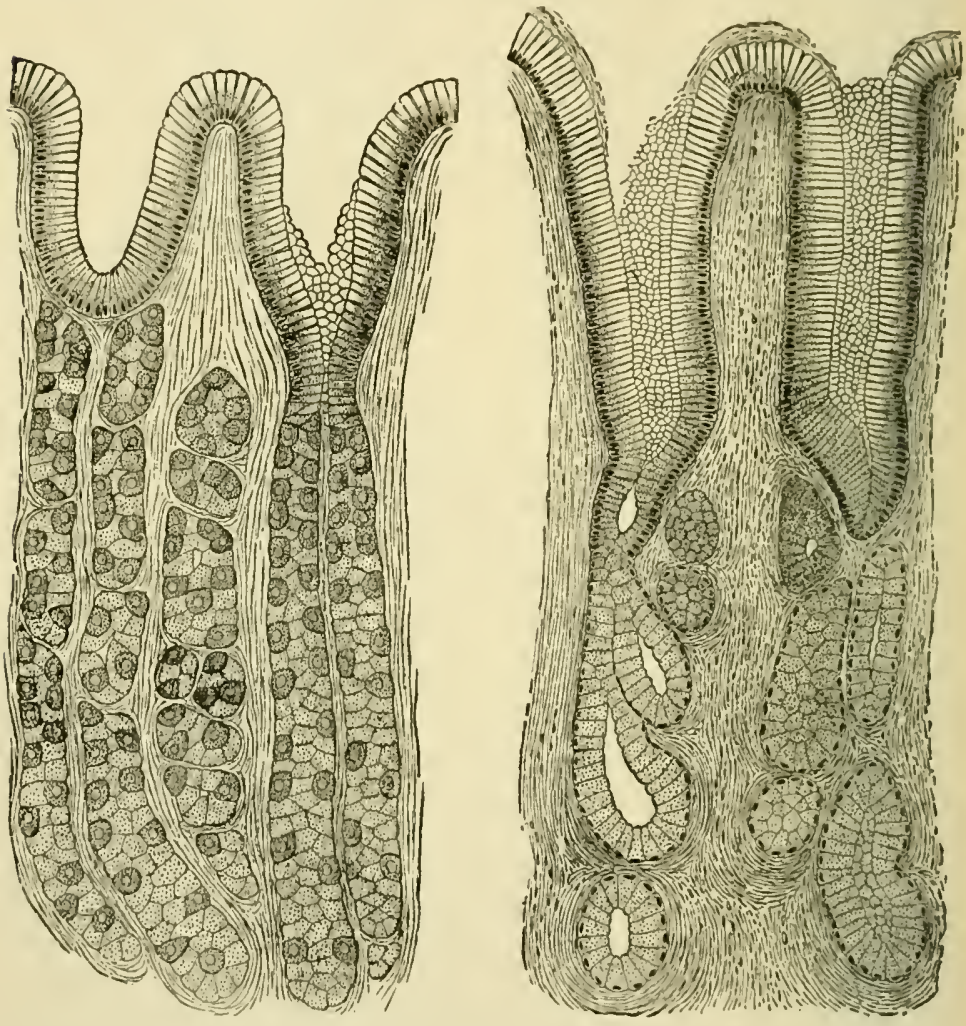

Fig, r40,-The Gastric Glands (Eibtein).

On the left, oxyntic; right, pyloric.

elements present, the ovoid or 'border' cells. And, indeed, the glands in the fundus of the frog's stomach, which are composed only of ovoid cells, whilst secreting much acid, also form some pepsin, although far less than the asophageal glands. During winter sleep (in the marmot) the production of hydrochloric acid in the parietal cells stops altogether, while the chief cells continue to accumulate granules of pepsinogen. 
That some pepsin is secreted by the pyloric end of the stomach is not difficult to prove. The secretion collected from the isolated pyloric portion is, indeed, like the secretion of the Brunner's glands in the duodenum, quite unable to digest protein until dilute hydrochloric acid is added. But this is because in both cases the juice as it flows from the glands is slightly alkaline, and, as we have alrearly seen, pepsin only acts in the presence of an acid. The milk-curdling action of these two juices also unfolds itself only when the secretions are first acidulated, and later on again neutralized ; in other words, the ferments must be activated by the addition of an acid. In normal digestion the pepsin of the (in itself) alkaline secretion of the pyloric end of the stomach becomes a constituent of the acid gastric juice; and it may, perhaps, be considered a morphological accident, so to speak, that the oxyntic cells of the fundus should mingle their acid products with the (presumedly) alkaline secretion of the chief cells in the lumen of each gland-tube, instead of being massed as a separate organ with a special duct.

We are not without other examples of digestive juices fitted or destined to act in a medium with an opposite reaction to their own. The 'saliva ' of the cephalopod Octopus macropus, strongly acid though it is, contains a proteolytic ferment which in vitro acts, like trypsin, better in an alkaline than in an acid solution. And trypsin, whose precursor is a constituent of the alkaline pancreatic juice and the enterokinase which activates it, a constituent of the alkaline succus entericus, performs a part at least of its work in an acid medium.

Attempts made to demonstrate an acid reaction in the border cells have hitherto failed, perhaps because the acid is poured into the ducts as fast as it is formed. But it may be mentioned, although only as a matter of historical interest, that some observers have denied that the acid is secreted in the depths of any cell from the chlorides of the blood, and have asserted that it is formed at the surface of contact of the stomach-wall with the gastric contents from the sodium chloride of the food by an exchange of sodium ions (p. 4oo) for hydrogen ions from the blood or lymph. It was pointed out in favour of this view that when, instead of sodium chloride, sodium bromide is given in the food, the hydrochloric acid in the stomach is to a large extent replaced by hydrobromic acid. And it was argued that this cannot be due to the decomposition of the bromide by hydrochloric acid, since it occurs in animals deprived for a considerable time of salts, and in 'salt-hunger' the stomach contains no acid (Koeppe). It may be, however, that even in "salthunger ' the presence of sodium bromide in the stomach stimulates the secretion of hydrochloric acid, which then decomposes the bromide, with the formation of hydrobromic acid. The sodium chloride formed in the double decomposition might be re-absorbed, and the stock of chlorides in the blood remain undiminished. It is in any case a decisive objection to this now defunct theory that a copious secretion of gastric juice, containing hydrochloric acid in 
abundance, can be obtained, without the introrluction of any food into the stomach, either by the process of sham feeding (p. 37f) or by psychical stimulation of the gastric glands when food is shown to an animal.

Changes in Mucous Glands during Secretion.-In the mucous salivary and sther mucous glands similar, lut apparently more complex, changes occur. During rest the cells which line the lumen may be seen in fresh, teased preparations to be filled with granules or 'spherules.' After active secretion there is a great diminution in the number of the granules. Those that remain are chiefly collected around the lumen, although some may also be seen in the peripheral portion of the cell; and there is no very distinct differentiation into two zones. That a discharge of material takes place from these cells is shown by their smaller size in the active gland. That the material thus cliscliarged is not
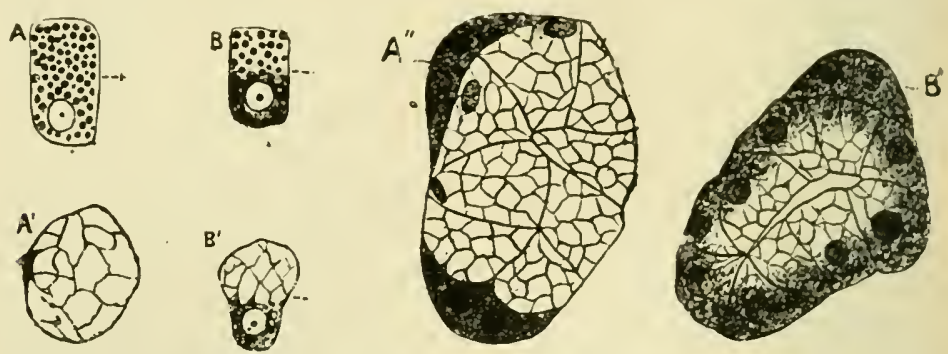

Fig, i4t.-Mucous Cells (from Submaxillary of Dog) in Rest and Activity (La.igley).

$A, B$, fresh ; $A^{\prime}, B^{\prime}$, after treatment with dilute acetic acid; $A^{\prime \prime}, B^{\prime \prime}$, alvcoli hardened in alcohol and stained with carmine. A, $A^{\prime}$, and $A^{\prime \prime}$ represent the loaded; B, B', and B", the discharged condition.

protoplasmic is indicated by the behaviour of the cells to protoplasmic stains such as carmine. The resting cells around the lumen stain but feebly, in contrast to the deep stain of the demilunes, while the discharged cells take on the carmine stain much more readily. Further, when a resting gland is treated with various reagents (water, dilute acids, or alkalies), the granules swell up into a transparent substance identical with mucin, which fills the meshes of a fine protoplasmic network.

In ordinary alcohol-carmine preparations only the network and nucleus are stained; the nucleus, small and shrivelled, is situated close to the outer horder of the cell. When a discharged gland is treated in the same way there is proportionally more "protoplasm' (or 'bioplasm ') and less of the clear material, what remains of the latter being chiefly in the inner portion of the cell, while the nucleus is now large and spherical, and not so near the basement membrane (Fig. I4I). 
$$
\text { . }
$$ 


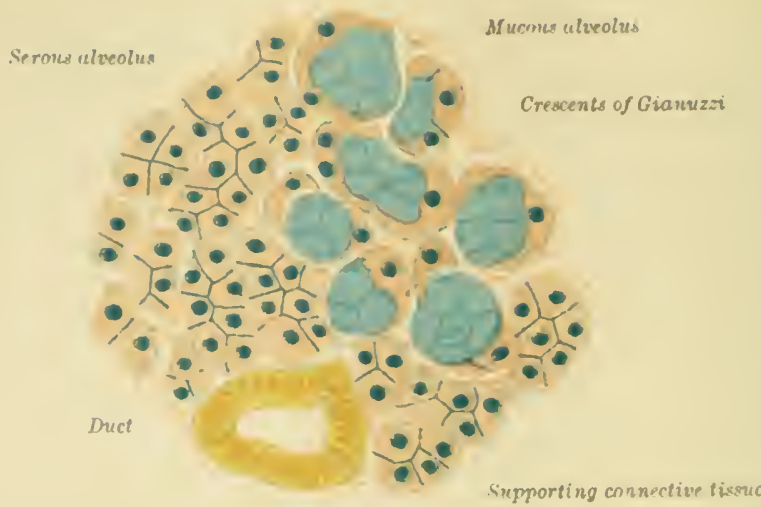

1. Section of submaxillary gland showing both mucous and serous alveoli, $\times 250$. (Stained with humatoxylin.)

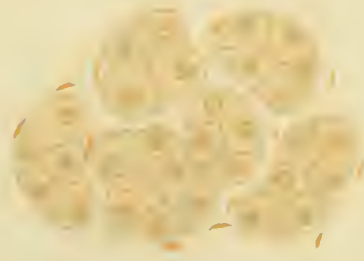

Before sccrction (resting)

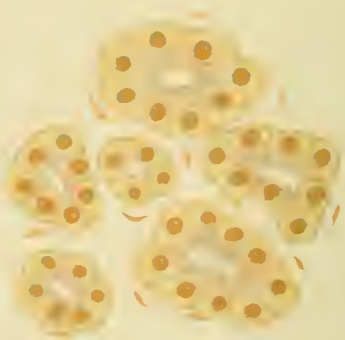

Afler secretion (active)

2. Section of serous gland, $\times 300$. (Sluined with borax carmine.)

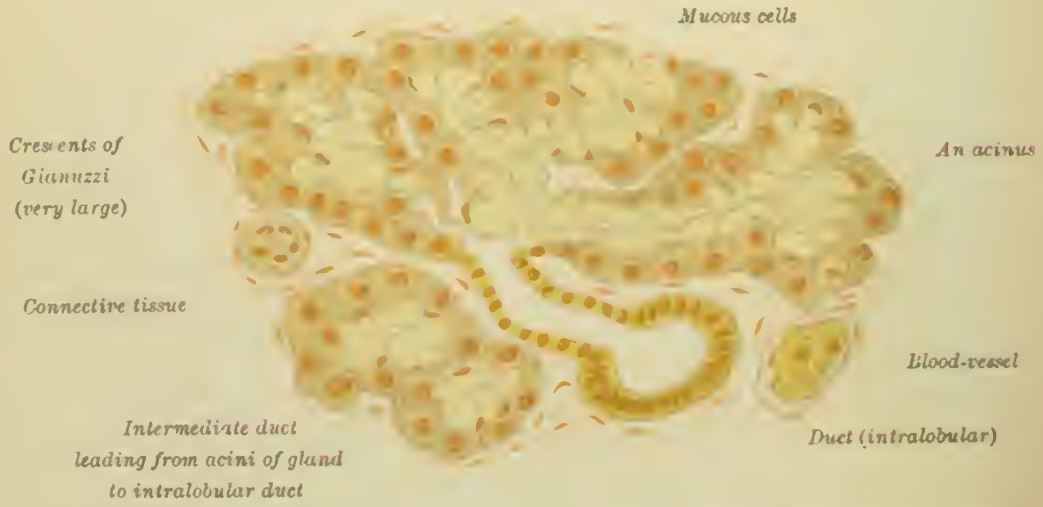

3. Section of mucous gland (after secretion), $\times 300$. (Stained with picrocarmine.) 
Everything, therefore, points to the granules in what we may now call the mucin-forming cells as being in some way or other precursors of the fully-formed mucin; manufactured during 'rest' by the protoplasm and partly at its expense, moved towards the lumen in activity, discharged as mucin in the secretion. It has been asserted that not only is the protoplasm lessened in the loaded cell and renewed after activity, but that many of the mucigenous cells may be altogether broken down and discharged, their place being supplied by proliferation of the small cells of the demilunes. This conclusion, however, is not supported by sufficient evidence. The cells of the crescents contain fine granules, but none which can be changed into mucin. They are of serous and not of mucous type. But the fact on which we would specially insist is that the granules of the resting mucigenous cell may be looked upon as a mother-substance from whicli the mucin of the secretion is derived; they are not actual, but potential, mucin.

So in the pancreas, the serous or albuminous salivary glands, and the glands of the stomach, there is every reason to believe that the granules which appear in the intervals of rest, and are moved towards the lumen and discharged during activity; are the precursors, the mother-substances, of important constituents of the secretion. These granules are sharply marked off from the protoplasm in which they lie and by which they are built up. By every mark, by their reaction to stains, for instance, they are non-living substance, formed in the bosom of the living cell from the raw material which it culls from the blood, or, what is more likely, formed from its own protoplasm, then shed out in granular form and secluded from further change. The granules in the ferment-forming glands are not in general composed of the actual ferments. and, indeed, in several instances it has been shown that the actual ferments are not present in the secreting cells at all.

We have already seen that the pancreas and even the fresh pancreatic juice are deroid of active trypsin. Similarly, a glycerin extract of a fresh gastric mucous membrane is inert as regards proteins, or nearly so. But if the mucous membrane has been previously treated with dilute hydrochloric acid, the glycerin extract is active, as is an extract made with acidulated glycerin. Here we must assume the existence in the gastric glands of a mother-substance, pepsinogen, from which pepsin is formed. The rennin of the gastric juice, which is formed in the chief cells, also has a precursor, which, if the ferment is identical with pepsin (p. 326), must be pepsinogen. The proteolytic power of an extract of the pancreas, when the trypsinogen has been activated into trypsin, or of the gastric mucous membrane, when the pepsinogen has been changed into pepsin, seems to be, roughly 
speaking, in proportion to the quantity of granules present in the cells. Therefore it is concluded that the granules represent mother-substances of the ferments or zymogens. Some observers believe they have obtained evidence of stages in the elaboration of the terments still further back than the mother-substances, grandmother-substances so to speak, or prozymogens. Benslev: e.g., concludes that the nuclei of the chief cells in the fundus glands of the stomach talie part in the formation of a prozymogen, the precursor of the zymogen or pepsinogen, as jepsinogen is the precursor of the enzyme pepsin.

A glycerin or watery extract of the salivary glands always contains active anylolytic ferment, if the natural secretion is active. So that if ptyalin is preceded by a zymogen in the cells, it must be very easily changed into the actual ferment.

But we should greatly deceive ourselves if we supposed that granules of this nature in gland-cells are necessarily related to the production of ferments. The mucigenous granules have no such significance. Mlost digestive secretions contain protein constituents, with which the granules may have to do, as well as with ferments. And bile, a secretion which contains no mucin, no proteins, and either no ferments or mere traces, as essential and original constituents, is formed in cells with granules so disposed and so affected by the activity of the gland as to suggest some relation between them and the process of secretion. In the liver-cells of the frog, in addition to glycogen, and oil-globules, small granules may be seen, especially near the lumen of the gland tubules; they diminish in number during digestion, when the secretion of bile is active, and increase when food is witheld and secretion slow. And in fasting dogs the secreting cells of Brunner's glands, the pyloric glands and the pancreas, as well as the lining epithelium of the bile-ducts, have been found to contain many fatty granules. Possibly some of these represent the fat which is known to be excreted into the alimentary canal (pp. 4I5, 4I6).

The Nature of the Process by which the Digestive Secretions are Formed.-We have spoken more than once of the gland-cells as manufacturing their secretions. It is an idea that rises naturally in the mind as we follow with the microscope the traces of their functional activity. And when we compare the composition of the digestive juices with that of the blood-plasma and lymph, the suggestion that the glands which produce them are not merely passive filters, but living laboratories, acquires additional strength. It is evident that everything in the secretion must, in some form or other, exist in the blood which conses to the gland, and in the lympl which bathes its cells. No glandular cell, if we except the leucocytes, which in some respects are to he considered as unicellular glands, dips directly into the 
blool ; everything a gland-cell receives must pass through the walls of the bloodvessels. (But see footnote on p. I4.) So that anything which we find in the secretion and do not find in the blood must have been elaborated by the gland epithelium (or by the capillary endothelium) from raw material brought to it by the blood.

Take, for example, the saliva or gastric juice. These liquids both contain certain things that also exist in the blood, but in addition they contain certain things specific to themselves: mucin in saliva, hydrochloric acid in gastric juice, ferments in both. It is true that a trace of pejsin and a trace of a diastatic ferment may be discovered in blood; but there is no reason whatever to believe that this is the source of the pepsin of the gastric juice, or the ptyalin of the salivary glands, except, perhaps, in animals like the cat, whose saliva contains a diastase in still smaller concentration than the serum (Carlson). On the contrary, it is possible that the ferments of the blood may be in part absorbed from the digestive glands, the rest being formed by the leucocytes and liberated when they break down. The liver affords an even better example of this 'manufacturing' activity of gland-cells, and many facts may be brought forward to prove that the characteristic constituents of the bile, the bilepigments and bile-acids, are formed in the liver, and not mercly separated from the blood. Bile-pigment has indeed been recognized in the normal serum of the horse, and bile-acids in the chyle of the dog, but only in such minute traces as are easily accounted for by absorption from the intestine. Frogs live for some time after excision of the liver, but no bile-acids are found in the blood or tissues. But if the bile-duct be ligatured, bile-acids and pigments accumulate in the body, being absorbed by the lymphatics of the liver (Ludwig and Fleischl). If the thoracic duct and the bile-duct are both ligatured in the dog, no bileacids or pigments appear in the blood or tissues. Wertheimer and Lepage state that bile or bilirubin injected into a bile-duct appears sooner in the urine than in the lymph of the thoracic luct, and therefore conclude that the bloodvessels are the most important channel of absorption. This conclusion, however, cannot be accepted until it is shown that in these experiments the injection did not cause rupture of some of the hepatic capillaries and direct entrance of the bile-pigment into the blood. It is not improbable that the pressure attained by the bile in the bile-capillaries is a factor in determining the path by which it is absorbed, and that when the pressure rises beyond a certain limit it may pass both into the bloodvessels and into the lymphatics. In mammals life cannot be maintained for any length of time after ligature of the portal vein, since this throws 
the whole intestinal tract out of gear. But after an artificial communication has been made between the portal and the left renal vein or the inferior cava, the portal may be tied and the animal live for months (Eck). The liver can now be completely removed, but deatll follows in a few hours. A good method of establishing an Eck's fistula is to make a longitudinal incision in the inferior vena cava and the portal or superior mesenteric vein, and to suture the edges of the two openings together with a very fine sewing-needle and thread (Carrel and Guthrie).* In birds there exists a communicating branch between the portal vein and a rein (the renal-portal) which passes from the posterior portion of the body to the kidney, and there breaks up into capillaries; and not only may the portal be tied, but the liver may be completely destroyed without immediately killing the animal. In the hours of life that still remain to it no accumulation of biliary substances takes place in the blood or tissues. A further indication that bile-pigment is produced in the liver is the

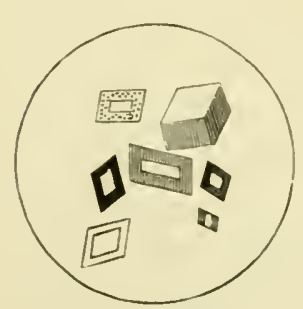

FIG. I42.-HENATOIDIX, fact that the liver contains iron in relative abundance in its cells (p. 2I), and eliminates small quantities of iron in its secretion. Now, bile-pigment, which contains no iron, is certainly formed from blood-pigment, which is rich in iron, for hæmatoidin (Fig. I42), a crystalline derivative of hæmoglobin found in old extravasations of blood, especially in the brain and in the corpus luteum, is identical with bilirubin. The seat of formation of bile-pigment must therefore be an organ peculiarly rich in iron. The existence of hæmatoidin, however, shows that bile-pigment may, under certain conditions, be formed outside of the hepatic cells. The occurrence of biliverdin in the placenta of the bitch points in the same direction. But the pathological evidence in favour of the pre-formation of the biliary constituents tends rather to shrink than to increase. For many cases of what used to be considered 'idiopathic' or 'hæmatogenic' jaundice, i.e., an accumulation of bile-pigments and bile-acids in the tissues, due to defective elimination by the liver, are now known to be caused by obstruction of the bile-ducts and consequent reabsorption of bile ('obstructive' or 'hepatogenic' jaundice).

But if substances such as the ferments, mucin, hydrochloric acid, the bile-salts and bile-pigments, are undoubtedly manufactured in the gland-cells, it is different with the water and inorganic salts which form so large a part of every secretion.

* By means of a small clamp, the jaws of which are shaped so as to isolate a longitudinal strip at one side of a bloodvessel while the circulation goes on through the rest of the lumen, the veins may be opened and sutured without interrupting the circulation. 
No tissue lacks them ; no physiological process goes on without them; they are not high and special products. As we breathe nitrogen which we do not need because it is mixed with the oxygen we require, the secreting cell passes through its substance water and salts as a sort of by-play or adjunct to its specific work. But this is not the whole truth. The gland-cell is not a mere filter through which water and salts pass in the same proportions in which they exist in the liquids that the cell draws them from. When, e.g., the salivary glands secrete against the resistance of an abnormally high pressure in the ducts, the percentage of salts in the saliva increases. The secretions of different glands differ in the nature, and especially in the relative proportions, of their inorganic constituents. They differ also in their osmotic pressure and electrical conductivity, which depend so largely upon those constituents, notwithstanding the fact that the osmotic pressure and conductivity of the bloodserum (p. 25) vary only within narrow limits. Even the secretion of one and the same gland is by no means constant in this respect, as we shall have to note more especially when we come to deal with the influence of the nervous system on secretion (p. 367). The following tables illustrate this point:

\begin{tabular}{|c|c|c|c|c|}
\hline \multirow[t]{2}{*}{ Dog. } & \multicolumn{2}{|c|}{ Blood-serum.* } & \multicolumn{2}{|c|}{ Filtrate of Gastric Contents. } \\
\hline & $\Delta t$ & $\mathrm{~K} \div\left(5^{\circ} \mathrm{C}.\right) \times 10^{+}$. & $د$ & $\mathrm{~K} \div\left(5^{3} \mathrm{C}.\right) \times 10^{4}$ \\
\hline $\begin{array}{l}\text { I. } \\
\text { II. } \\
\text { III. }\end{array}$ & $\begin{array}{l}0.643^{\circ} \\
0.628^{\circ} \\
0.602^{\circ}\end{array}$ & $\begin{array}{l}92^{\circ} 0 \\
87^{\circ} 6 \\
87^{*} 7\end{array}$ & $\begin{array}{l}0.585^{\circ} \\
0.585^{\circ} \\
0.642^{\circ}\end{array}$ & $\begin{array}{l}3 \text { I }: 5 \\
179: 4 \\
35 \div \cdot 7\end{array}$ \\
\hline \multicolumn{3}{|c|}{$\begin{array}{l}\text { Vomit of man with complete intes- } \\
\text { tinal obstruction } \ldots\end{array}$} & $0^{*}+33^{\circ}$ & $8_{4} \cdot 7$ \\
\hline
\end{tabular}

Pancreatic Juice of Dog (Pincussohn).

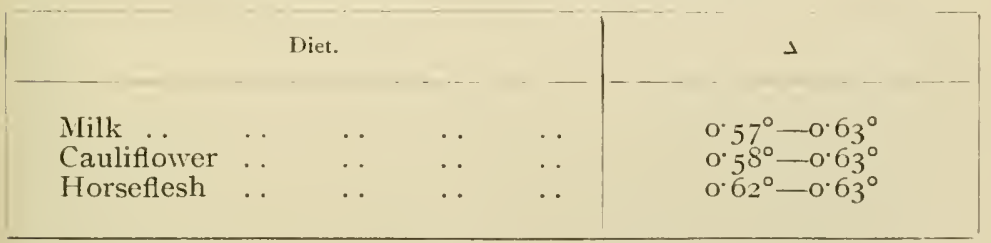

* The blood and gastric contents were obtained from the animals in the writer's laboratory twenty-four hours after the last meal.

$\uparrow$ The depression of the freezing-point below that of distilled water.

$\ddagger K$ is the specific conductivity of a cube of the liquid of I centimetre side, the conductivity of a similar cube of mercury being taken as unity. The number in brackets is the temperature at which the measurement was made. To obtain expressions for $\mathrm{K}$ in whole numbers, it is multiplied by $10^{4}$. 
Gastric Juice from Miniature Stomach in a Dog in Different Experiments (Bickel).

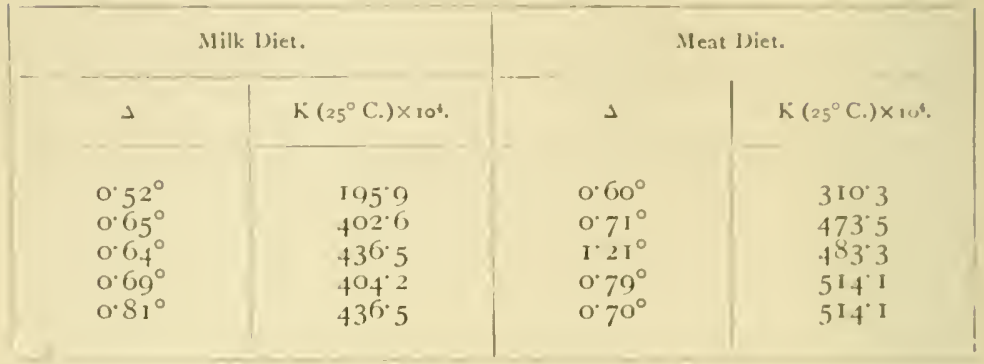

$\Delta$ of Blood and Saliva Compared (Jappelli).

\begin{tabular}{|c|c|}
\hline د of Ylood. & $د$ of Submaxillary Saliva of Dog. \\
\hline $\begin{array}{l}0.570^{\circ} \\
0.610^{\circ} \\
0.600^{\circ} \\
0^{\circ} 590^{\circ} \\
0.580^{\circ} \\
0.605^{\circ} \\
0.650^{\circ} \\
0.610^{\circ}\end{array}$ & $\begin{array}{l}0^{\circ}+10^{\circ} \\
0^{\circ} 350^{\circ} \\
0^{\circ}+30^{\circ} \\
0^{\circ} 410^{\circ} \\
0^{\circ} 450^{\circ} \\
0^{\circ} 425^{\circ} \\
0^{\circ} 380^{\circ} \\
0^{\circ} 475^{\circ}\end{array}$ \\
\hline$د$ of Human Fistula Bile. & $د$ of Human Bladder Bile. \\
\hline $\begin{array}{l}0^{\circ} 56^{\circ} \\
0.547^{\circ} \\
0.615^{\circ} \\
0.60^{\circ} \\
0.545^{\circ}\end{array}$ & $\begin{array}{l}0.65^{\circ} \\
0.865^{\circ} \\
0.78^{\circ} \\
0.92^{\circ} \\
-\end{array}$ \\
\hline
\end{tabular}

$\triangle$ of Dog's Submaxillary Suliva.

Chorda stimulated :

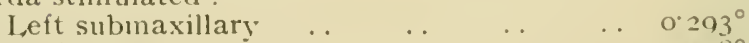

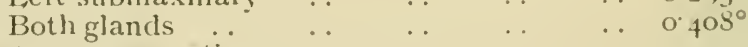

$\begin{array}{cccccc}\text { Spontancous secretion : } & & & & & \\ \text { Right submaxillary } & \ldots & \ldots & \ldots & \ldots & 0^{\circ} 195^{\circ} \\ \text { دof dog's serum } & \ldots & \ldots & \ldots & \ldots & 0^{\circ} 590^{\circ}\end{array}$

The protein substances, such as serum-albumin and globulin. common to blood and to some of the digestive secretions, take a middle place between the constituents that are undoubtedly 
manufactured in the cell and those which seem by a less special and lahorious, though a selective, process to be passed through it from the hlood. Their practical absence from bile, and, as we shall see, from urine, their relative abundance in pancreatic aucl scantiness in gastric juice, point to a closer dependence upon the special activity of the gland-cell than we can suppose necessary in the case of the salts.

Although it is in the cells of the digestive glands that the power of forming ferments is most conspicuous, it is by no means confinerl to them. It seems to be a primitive, a native power of protoplasm. Lowly animals, like the amoba. lowly plants. like bacteria, form ferments within the single cell which serves for all the purposes of their life. The ferment-secreting gland-cells of higher forms are perhaps only lop-sided amnebre, not so much endowed with new properties as disproportionately developed in one direction. The contractility has been lost or lessened, the digestive power has been retained or increased ; just as in muscle the power of contraction has been developed, and that of digestion has fallen behind. The muscle-cell and the cartilage-cell are parasites, if we look to the function of digestion alone. They live on food already more or less prepared by the labours of other cells; and it is a universal law that in the measure in which a power becomes useless it clisappears. But the presence of pepsin in the white blood-corpuscles, the parasites as well as the scavengers of the blood, and of amylolytic, proteolytic and lipolytic ferments in many tissues, should warn us not to conclude that the power of forming ferments belongs exclusively to any class of cells. There is good and growing evidence that food-substances absorbed from the blood are further decomposed and, in turn, elaborated by ferment action within the tissues thenselves; while many facts show that the power of contraction is widely diffused among structures whose special function is very different, and a few point to its possession in some degree even by glandular epithelium. On the other hand, it must be remembered that none of the digestive glands absorb food directly from the alimentary canal to be then digested within their own cell-substance ; the ferments which they form do their work outside of them ; their cells feed also upon the blood.

\section{Why are the Tissues of Digestion not affected by the Digestive} Ferments?-This is the place to mention a point which has been very much debated. Why is it that the stomach or the small intestine does not digest itself ? This is really a part of a wider question: Why is it that living tissues resist all kinds of influences, which attack dead tissues with success? And we have to inquire whether the immunity of the alimentary canal to the digestive juices is an example of a general resistance of all living tissues to destructive agencies, or a specific resistance of certain tissues to certain influences.

That all living tissues cannot withstand the action of the gastric juice has been shown by putting the leg of a living frog inside the stomach of a $\log$ : the leg is gradually eaten away (Bernard). 
It is true that it has first been killed and then cligesterl, but the question is, why the stomach-wall is not first killed and then digested? Wlien the wall luas been injured by caustics or by an embolus, the gastric juice acts on it. But the living epithelium that covers it is able to resist the action of the acid and prejosin, which clestroys the tissues of the frog's leg. The explanation is not to be found in the alkalinity of the blook, for the frog's blood is also alkaline, and the cells that line the intestine are preserved from the pancreatic juice, which is intensely active in an alkaline medium, while the living frog's leg is not larmed by a weakly alkaline pancreatic extract, which does not digest the epitlelium because it cannot kill it. A certain amount of protection may be afforded to the walls of the stomacls by the thin layer of mucus which covers the whole cavity, for mucin is not affected by peptic digestion. And a mucous secretion seems in some other cases to act as a protective covering to the walls of hollow viscera, whose contents are such as would certainly be harmful to more delicate membranes, e.g., in the urinary bladder. large intestine, and gall-bladder. Still, however important such a mechanical protection may lie, it does not explain the whole matter, and it is necessary to suppose that the gastric ejoithelium has some special power of resisting the gastric juice, either by turning any of the ferment which may invade it into an inert substance and neutralizing any intrusive acid, or by opposing their entrance as the epithelium of the bladder opposes the absorption of urea. There is reason to believe that, as a matter of fact, free hydrochloric acid cannot penetrate the living cells, and it is to be noted that both active pejosin and free acid must be present at the same point within the cells before digestion of them can take place. In the glancl-cells of the pancreas the protoplasm is, no doubt, shiclded from digestion by the existence of the ferment in an inert form as zymogen; and it is possible that this is one of the reasons for the existence of the mothersubstance. But no such explanation is, of course, available for the intestinal epithelium. Trypsin when injected below the skin causes the tissue to break clown and ulcerate. And while an active solution of trypsin can be allowed to remain a long time in an isolated loop of small intestine without producing any ill effect, damage is soon cansed not only to the intestinal wall, but also to the liver, when the mucous membrane of the loop las been injured hefore the introduction of the trypsin. We must suppose, then, that the normal mucous membrane of the intestine prevents the absorption of trypsin, or, if it absorbs any of it, renders it harmless. Indeed, it is impossible to escape the conclusion that eacl membrane becomes accustomed, and, so to speak. 'immune,' to the secretion normally in contact with 
it, althongh not necessitrily to other secretions. It is easy to multiply illustrations of this principle.

Few tissues but the lining of the urinary tract or of the large intestine could bear the constant contact of urine or freces. When urine is extravasated under the skin, or the contents of the alimentary canal burst into the peritoneal cavity, they come into contact with tissues which, although alive, are much less fitted to resist them than the surfaces by which they are normally enclosed ; and the consequences are often disastrons. I encocytes thrive in the blood, but perish in urine. Blood does not harm the enclothelial cells of the ressels, but kills a muscle whose crosssection is dipped into it. The defensive, or in some cases, offensive liquids secreted by many animals are harmless to the tissues which produce and enclose them. A caterpillar inrestigated by Poulton secretes a liquid so rich in formic acid that the mere contact of it would kill most cells. The so-called saliva of Octopus macropus contains a substance fatal to the crabs and other animals on which it preys. The blood of the viper contains an active principle similar to that secreted by its poison-glands, but its tissues are not affected by this substance, so deadly to other animals.

A step in the solution of our problem has lately been taken by Weinland. Starting with the idea that if special protective mechanisms against the digestive juices were anywhere to be found it would be in the intestinal parasites whose whole existence is passed among them, he has made the important discovery that in these parasitic worms specific antiferments exist-i.e., substances which inhibit the action either of pepsin or of trypsin or of both. These substances can be precipitated from the expressed juice of the worms by alcohol, without completely losing their activity. Fibrin can be impregnated with them, and it is then, just like the 'living tissue,' rendered for a longer or shorter time unassailable by the proteolytic ferments. While the supposed proof that similar antiferments are contained in the cells of the mucous membrane of the stomacl and intestines of the higher animals appears to have broken down, these facts are full of suggestion for future work. As already mentioned. it is known that an antitrypsin exists in the blood, with the same properties as the antitrypsin in the intestinal worms (Hamill). This explains the resistance of blood-serum to the digestive action of trypsin. In addition to this body, which hinders the action of fully-formed trypsin. and has . o effect upon enterokinase, the blood of some animals contains an antikinase-i.e., a substance which hinders the action not of trypsin, but of enterokinase, preventing it from activating the trypsinogen into trypsin. 


\section{The Influence of the Nervous System on the Digestive Glands.}

(I) The Influence of Nerves on the Salivary Glands.-All the salivary glauds have a double nerve-supply, from the medulla oblongata through some of the cranial nerves, and

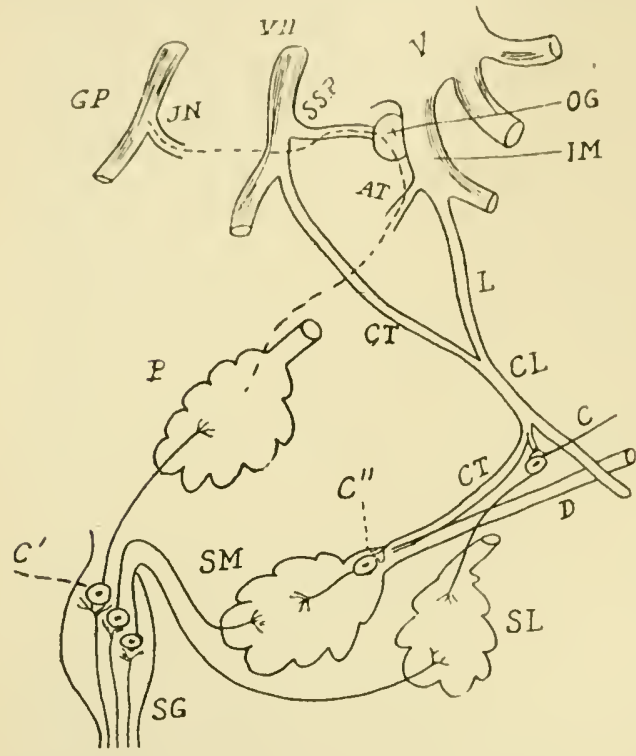

Fic. I43.-Nerves of tHe Salivary Cilands.

S.I and SL, submaxillary and sublingual glands : P. parotid : V, fiftl nerve: VII, facial : GP, glossopharyngeal : L, lingual : CT, chorda tympani : CL, chordo-lisgual; D, submaxillary (Wharton's) duct : C, ganglion cell of so-called submaxillary ganglion in the chordo-lingual triangle, connected with a nerve filre going to sublingual gland; $\mathrm{C}^{\prime \prime}$, ganglion cell in hilus of submixillary gland: SSP, small superficial petrosal branch of the facial: $\mathrm{OC}_{\mathrm{r}}$, otic ganglion; IM, inferior maxillary division of fifth nerve: AT, auriculo-temporal branch of fifth: $\mathrm{JN}$. Jacobson's nerve: C'. ganglion cells in superior cervical ganglion (SC) connected with sympathetic fibres going to parotid, submaxillary and sublingual glands. The figure is schematic.

end in a plexus on the basement membrane of the alveoli. From the plexus fibrils run in among the gland-cells, but do not secm to penetrate them. The lingual, the chorda tympani proper, and Wharton's duct form the sides of what is called the chorclolingual triangle. Within this triangle are situated many ganglion cells, a special accumulation of which has received the name of the submaxillary ganglion. 'This, however, should rather be called the 
sublingual ganglion, since its cells, as well as the others in the chordo-lingual triangle, are the cells of origin of axons which proceed as non-medullated fibres to the sublingual gland. The sublingual gland receives its cerebral fibres partly from branches given off from the lingual in the chordo-lingual triangle after the chorla tympani proper has separated from it, and ending around the nerve-cells within that triangle, partly from the chorda itself in the terminal portion of its course. These statements rest on anatomical and physiological evidence. The latter we shall return to.

The cerebral fibres for the parotid (in the dog) pass from the tympanic branch of the glosso-pharyngeal (Jacobson's nerve) through connecting filaments to the small superficial petrosal branch of the facial, with this nerve to the otic ganglion, and thence by the auriculo-temporal brancli of the fifth to the gland.

The sympathetic fibres for all the salivary glands appear to arise from nerve-cells in the upper dorsal portion of the spinal cord. Issuing from the cord in the anterior roots of the upper thoracic nerves (first to fifth, but mainly second thoracic for the submaxillary), they enter the sympathetic chain, in which they run up to the superior cervical ganglion. Here they break up into terminal twigs, and thus come into relation with ganglion cells, whose axons pass out as non-medullated fibres, and, surrounding the cxternal carotid, reach the salivary glands along its branches. Langley has shown, by means of nicotine (p. I65), that the sympathetic fibres for the submaxillary and sublingual, and, indeed, for the head in general in the dog and cat, are connected with nervecells in this ganglion, but not between it and their termination, or between it and their origin from the spinal cord.

Stimulation of the Cranial Fibres.-When in a dog a cannula is placed in Wharton's duct, and the saliva collected (p. 424), it is found that stimulation of the peripheral end of the divided chorda causes a brisk flow of watery saliva, and at the same time a dilatation of the vessels of the gland, which we have already described in dealing with vaso-motor nerves (p. I63). Notwithstanding the vaso-dilatation, the volume of the gland is in general diminished, owing to the rapid passage of water into the duct (Bunch). The blood has been shown to lose water in making the circuit of the submaxillary gland during excitation of the chorda, but doubtless some of the water of the saliva comes directly from the cells or from the lymph. That the increased secretion is not due merely to the greater blood-supply, and the consequent increase of capillary pressure, is shown by the injection of atropine, after which stimulation of the nerve, although it still causes dilatation of the vessels, is not followed by a flow of saliva. Mere increase of pressure could not in any case of itself account for the secretion, since it has been found that the maximum pressure in the salivary duct when the outflow of saliva from the duct is prevented may, during stimulation of the chorda, much exceed the arterial blood-pressure (Ludwig). In one experiment, for example, the pressure in the carotid of a dog was $125 \mathrm{~mm}$., in Wharton's duct I95 mm. of mercury. 
Even in the head of a decapitaterl animal a certain amount of saliva may be causect to flow by stimulation of the chorda, but too much may easily be made of this. And since the blond is the ultimate source of the secretion, we could not expect a permanent or copious flow in the absence of the circulation, even if the glandcells could contimue to live. In fact, when the circulation is almost stopped by strong stimulation of the sympathetic, the flow of saliva caused by excitation of the chordat is at the same time greatly lessened or arrested, even though the sympathetic itself possesses secretory fibres. So that, while there is no doubt that the chorda tympani contains fibres whose function is to increase the activity of the gland-cells, its vaso-dilator action is, under normal conditions, closely connected with, and, indeed, anxiliary to, its secretory action, although the dilation of the vessels does not directly produce the secretion. This is only a particular case of a physiological law of wicle application, that an organ in action in general receives more blood than the same organ in repose, or, in other words, that the tissues are fed according to their needs. The contracting muscle, the secreting gland, is flusherl with blood, not because an increased blood-flow can of itself cause contraction or secretion, but because these high efforts require for their continuance a rich supply of what blood hrings to an organ, and a ready removal of what it takes away.

The quantity of blood passing through the parotid of a horse when it is actively secreting during mastication may be quadrupled (Chaureau). The parallel between the muscle and the gland is drawn closer when it is stated that electrical changes accompany secretion (p. 734), and that the rate of production of carbon dioxide and consumption of oxygen (in the submaxillary gland) is three or four times greater during activity than during rest. The temperature of the saliva flowing from the dog's submaxillary during stimulation of the chorda has been found to be as much as $1.5^{\circ} \mathrm{C}$. above that of the blood of the carotid, althongh with the gland at rest no constant difference could be detected between the arterial blood and the interior of Wharton's duct. But such measurements are open to many fallacies; and while there is no doubt that more heat is produced in the active than in the passive gland, it will not be surprising, when the vastly-increased blood-flow is remembered, that no difference of temperature between the incoming and outgoing blood has been satisfactorily demonstrated.

It has already been mentioned that most of the fibres of the chorda tympani proper become connected with ganglion-cells, and lose their medulla inside the submaxillary gland. only a few having already lost it by a similar connection with ganglion-cells in the chordo-lingnal triangle. These facts have becn made nut by means of the nicotine 
method previonsly described (p. 165). Thus, it is found that, after the injection of nicotine ( 5 to $10 \mathrm{mg}$. in a rabbit or cat, fo or $50 \mathrm{mg}$. in a $(\mathrm{log})$, stimulation of the chorda tympani proper or of the chordolingual nerve catuses 110 secretion from the submaxillary gland; but stimulation of the hilus of the gland is followed by a copions secretion -as mucl,, if the stimulation is fairly strong, as was causer by excitation of the nerve before injection of nicotine. That this is due neither to any direct action on the gland-cells, nor to stimulation of the sympathetic plexus on the submaxillary artery, but to stimulation of chorda fibres beyond the hilus, is shown by the fact that after atropine has been injected in sufficient amount to paralyze the nerve endings of the chorda, but not of the sympathetic, stimulation of the hilus causes little or no flow of saliva. 'The application of nicotine solution to the chordo-lingual triangle does not affect the submaxillary secretion caused by stimulation of the chordo-lingual nerve, even in cases where a few secretory fibres for the submaxillary do not leave the chordo-lingual nerve in the chorda tympani proper, but are given off to the chorlo-lingual triangle. This shows that none of the ganglion-cells in the triangle are connected with the secretory fibres of the submaxillary gland. By observations of the same kind they are known to be connected with fibres going to the sublingual. In a similar way, by observing the effects of stimulation of the chorda on the bloodvessels before and after the application of nicotine, it has been found that the vaso-dilator fibres are connected with ganglion-cells in the same positions as the secretory fibres (Langley).

Stimulation of the Sympathetic Fibres.-The sympathetic, as has been already indicated, contains both vaso-constrictor and secretory fibres for the salivary glands. If the cervical sympathetic in the dog is divided, and the cephalic end moderately stimulated, a few drops of a thick, viscid and scanty saliva flow from the submaxillary and sublingual ducts, while the current of blood through the glands is diminished. As a rule, no visible secretion escapes from the parotid, but microscopic exan1ination shows that many of the ductules are filled with fluid, which is apparently so thick as to plug them up (Langley); while the cells show signs of 'activity' (p. 347).

\section{Simultaneous Stimulation of Cranial and Sympathetic Fibres.-} When the chorda and sympathetic are stimulated together, the former prevails so far, with moderate stimulation of the latter, that the submaxillary saliva is secreted in considerable quantity, and is not particularly viscid. It is, however, richer in organic matter than is the chorda saliva itself. When the chorda is weakly, and the sympathetic strongly, excited, the scanty secretion (if there is any) is of sympathetic type, thick and rich in organic matter. With strong stimulation of both nerves, the secretion, at first plentiful and watery, soon diminishes, even below the amount obtained by stimulation of the chorda alone, because of the diminution in the blood-flow, and therefore in the oxygen supply, produced by the vaso-constrictors 
of the sympathetic (Heidenhain). With stimulation just strong enough to cause secretion when applied separately to either nerve, there is no secretion when it is applied simultaneously to both.

All this refers to the dog. In this animal, then, there seems to be a certain amount of physiological antagonism between the secretory action of the two nerves. But it differs in one respect from the antagonism between their vaso-motor fibres; for with strong stimulation the constrictors of the sympathetic always swamp the dilators of the chorda, while the secretory fibres of the chorda appear upon the whole to prevail over those of the sympathetic. And in all probability this apparent secretory antagonism is very superficial, and is due largely to the difference in the rasomotor effects of the two nerves. For it has been shown that when the blood-flow through the submaxillary gland is artificially dininished by graduated compression of its artery, stimulation of the chorda gives rise to a thick viscid and scanty saliva, relatively rich in organic solids (Heidenhain). When the amount of blood passing through the gland is made approximately the same as during stimulation of the sympathetic, the chorda saliva becomes practically identical in composition with the sympathetic saliva. This is one reason, perhaps the chief one, why the sympathetic, when both nerves are stimulated together, without artificial interference with the blond-supply, always appears to add something to the common secretion when there is a secretion at all, this something being represented by an increase in the percentage of organic matter. The observation that the sympathetic effect persists after stimulation has been stopped, and that excitation of the chorda after previous stimulation of the sympathetic causes a flow of saliva richer in organic matter than would have been the case if the sympathetic had not been stimulated, has long been considered a proof that the secretory fibres of the two nerves are widely different in function. To explain this result, Heidenhain assumed the existence in the sympathetic of a preponderance of fibres concerned in the building up in the cells of the organic constituents of the saliva (so-called "trophic," or, better, since the word trophic is usually. associated with the building up of the bioplasm itself, " trophicsecretory " fibres). It would seem, however, that the increase in organic constituents is only realized when a sufficient time has not been allowed, after stimulation of the sympathetic, for the normal circulation to become re-established in the gland. The saliva obtained by stinulation of the chorda immediately. after a period of artificially diminished blood-flow, without any previous excitation of the sympathetic, also contains a surplus of organic matter (Carlson). 
Indeed, the distinction between chorda and sympathetic saliva, which, by taking account of the parotid as well as the submaxillary and sublingual glands, has been generalized into a distinction between ccrebral and sympathetic saliva, and which, when the vasomotor conditions are left out of account, aplears t) hoil good in the $\log$ and the rabbit, breaks down before a wider induction. For in the cat the sympathetic saliva of the submaxillary gland, although much more scanty, is more watery than the chorda saliva (Langley), which, however is by no means riscid; and the two secretions differ far less than in the dog. The discovery of Carlson that the cat's cervical sympathetic contains so many vaso-dilator fibres for the submaxillary gland that the usual effect of its stimulation with a weak interrupted current is a marked augmentation in the blood-flow affords an explanation. In accordance with this functional similarity, there is a much smaller difference in the action of atropine on the two sets of fibres in the cat than in the dog, although even in the cat the sympathetic is less readily paralyzed than the chorda.

In their secretory action there is not even an apparent antagonism in the cat, with minimal stimulation of both nerves, which causes as much secretion as would be produced if both were separately excited. Further, even in the dog, after prolonged stimulation of the sympathetic, the submaxillary saliva is no longer viscid, but watery, the proportion of solids, and especially of organic solids, being much lessened, as it is also in chorda saliva after long excitation. When the cerebral nerve of the resting gland is strongly excited, it is found that up to a certain limit the percentage of organic matter in a small sample of saliva subsequently collected during a brief weak excitation increases with the strength of the previous stimulation; this is also true of the inorganic solids. But there is a striking difference when the experiment is made on a gland after a long period of activity; here increase of stimulation causes no increase in the percentage of organic material, while the inorganic solids are stil] increased. In both cases the absolute quantity of water, and therefore the rate of flow of the secretion, is augmented.

All this points to the same conclusion as the microscopic appearances in the gland-cells, that the cells during rest manufacture the organic constituents of the secretion, or some of them, and store them up, to be discharged during activity. The water and the inorganic salts, on the other hand, seem rather to be secreted on the spur of the moment, so to speak, and not to require such elaborate preparation. And it has been stated that when the chorda tympani is stimulated with currents of varying strength. the quantity of organic substances in small samples of 
Sativa collected from a fresh gland is more nearly proportional to the rate of secretion than is the quantity of water and salts, which varies also with the blood-supply.

Lest the apparently insignificant result of artificial stimulation of the svmpathetic in such animals as the dog shoulel cause its secretory action to be appraised at too low a value, it should be remembered that in the intact boly the svmpatletic secretror fibres, when they are excited, are, it may he assumed, excited inclependently of the vaso-constrictors, and eren in conjunetion with the vaso-dilators of the sillivary glands.

It is conceivable that such differences between chorda and sympathetic saliva as are not accomnted for by the differences in the blood-flow during their stimulation are due, not to the nerve-fibres, but to the end organs with which they are connected: that is, the two nerves may supply, not the same, but different gland-cells. And it is well known that even after prilonged stimulation of the chorda or chordo-lingual alone. some alreoli of the dog's submaxillary gland remain in the 'resting 'state; after stimulation of the sympathetic alone, the number of unaffected alveoli is much greater: while after stimulation of both nerves, few alveoli seem to have escaped change. If there is no essential difference between the cranial and sympathetic secretory fibres, it is casy to understand that they will be distributed to different secreting elements. The supposed proof that there must be some overlapping in the nerve-supply-i.e., that some cells must be supplied from both sources, since excitation of the sympathetic influences the amount of organic material in the saliva obtained by subsequent stimulation of the chorda-is, as we have just seen, by no means so cogent as has been assumed. And, indeed. we know nothing of a division of labour between the cells of a gland, except when there are obvious anatomical distinctions. Thus, the submaxillary gland in man contains both serous and mucous acini. and mucin-making cells are scattered over the ducts of most glands, and, indeed, on nearly every surface which is clad witl columnar epithelium. In these cases we cannot doubt that one constituent-mucin-of the entire secretion is manufactured hy a portion only of the cells. In the cardiac glands of the stomach. too, the ovoid cells, in all probahility, yield the whole of the acid of the gastric juice. But, so far as we know. every hepatic cell is a liver in little. Every cell secretes fully-formed bile; every cell stores up, or may store up, glycogen. So it is with the secretory alvecli of the pancreas, if we consider the islands of I-angerhans as having no connection with the alveoli; one cell is just like another; all apparently perform the same work : each is.a unicellular pancreas. (But see p. 55t). 
Paralytic Secretion.-When the chorda tympani is divided, a slow 'paralytic" secretion from the submaxillary gland begins in a few hours, and continues for a long time accompanied by atrophy of the gland. There is also a secretion of the same kind from the submaxilary on the opposite side, but it is less copious. This is called the 'antilytic ' secretion, which is most pronouncel in the first few days after the operation, and seems to be a transient phenomenon. It can be at once abolished by section both of the chorda and the sympathetic on the corresponding side, and is therefore the to impulses arising in the central nervous system. The canse of the paralytic secretion has not been fully marle out. If within two or three dlays of clivision of the chorda the sympathetic on the same side is cut, the secretion is greatly climinished or stops altogether; and it is conclurled that up to this time it is maintained by impulses passing along the sympathetic to the gland from the salivary centre, the excitability of which has been in some way increased by (livision of the chorda, possibly by some such degenerative process in the cells as the clianges seen in cerebro-spinal motor cells whose axons have been divided (p. 756). This may also account for the antilytic secretion. But if section of the sympathetic is not performed for several days, it has no effect on the paralytic secretion, which at this stage seems to depend on local changes in or near the gland itsclf, leacling to a mild continuous cxcitation of those nervecells on the course of the fibres of the chorda to which reference has already been made. Section of the sympathetic alone causes neither secretion nor atrophy, nor does removal of the superior cervical ganglion. The histological characters of the gland-cells cluring paralytic secretion are those of 'rest.'

Reflex Secretion of Saliva.-The reflex mechanism of salivary secretion is very mobile, and easily set in action by physical and mental influences. It is excited normally by impulses which arise in the mouth, especially by the contact of food with the buccal mucous membrane and the gustatory nerve-endings. The mere mechanical movement of the jaws, even when there is nothing between the teeth, or only a bit of a non-sapid substance like indiarubber, causes some secretion. The vapour of ether gives rise to a rush of saliva, as does gargling the mouth with distilled water. The smell, sight, or thought of food, and even the thought of saliva itself, may act on the salivary centre through its connections with the cerebrum, and make " the teeth water.' A copious flow of saliva, reflexly excited through the gastric branches of the vagus, is a common precursor of vomiting. The introduction of food into the stomach also excites salivary secretion.

The researches of Pawlow and his pupils have shown that the salivary glands are not excited indifferently by everything which comes into contact with the buccal mucous membrane. A remarkable adaptation exists between the properties of food or foreign bodies introduced into the mouth and their effects upon the secretion of saliva. When solid dry food is given to a dog saliva is copiously poured out; much less is secreted when the 
food is moist. Acids or salts induce an abundant flow, in order that they may be neutralized, cliluted or washed ont of the mouth. In this case a watery liquid, poor in mucin, flows from the mucous glands. Mucin is a lubricant to facilitate the swallowing of solid food, and here it could be of no use. When clean pebbles are put in the dog's mouth the animal may try to chew them, but eventually ejects them. Either no saliva or very little is secreted, since it could not aid in their expulsion. If, however, the very same stones are reduced to sand and again introduced into the animal's mouth, saliva is plentifully secreted to wash it out.

The serous and inncous salivary glands are not necessarily excited by the same food materials, and here again we can trace an astonishingly exact adaptation. A permanent parotid or submaxillary fistula can easily be made in a dog by freeing Stenson's or Wharton's duct from the surrounding mucous membrane for a little distance, bringing the natural orifice of the duct out through a small wound in the cheek, and stitching it in position there. When it is desired to collect saliva, the wide end of a funnel-shaped tube, whose stem is bent so as to hang vertically, can be attached by a little shellac of low melting-point to the skin around the orifice of the duct and at some distance from it, and on the narrow end can be hung a small graduated tube, into which the saliva drops. When fresh meat is given to the animal little or no parotid saliva is secreted, while a copions flow takes place from the submaxillary gland, mucin being required to lubricate it for deglntition, while water is not specially needed. But if the meat is in the form of a dry powder the parotid pours out a plentiful secretion, while the submaxillary also secretes a fluid relatively rich in mucin. The same difference is seen between fresh moist bread and dry bread. The afferent nerveendings from which impulses are carried to the reflex centres (or the portions of the salivary centre) which presicle over the various salivary glands must possess the power of very delicate selection as regards the kinds of stimulation by which they are affected. The mere relish of the animal for the different kinds of food plays but a small part. Most dogs clisplay a much livelier interest in a piece of meat than in a piece of dry biscnit, yet it is the biscuit which excites the parotid to activity.

The sight of dry food causes an abundant flow of watery saliva from the paroticl, and a flow of fluid rich in mucin from the submaxillary. Various uneatable substances, including substances which in contact with the mucous membrane of the mouth produce strong and disagreeable stimulation of it, and excite disgust, cause also, when viewed from a distance, secretion by all the salivary glands; but the submaxillary saliva, as ought to be 
the case for substances unfit for food, and therefore not destined to be swallowed, is poor in mucin. When the animal is shown pebbles and sand the phenomena are qualitatively the same as when they are put into its mouth-the glands remaining inactive in presence of the pebbles, but secreting plentifully at sight of the sand. In short, the same adlaptation is observer in the case of the so-called psychical secretion as when the stimulating substances act directly upon the endings of the afferent salivary nerves in the buccal mucons membrane. It is further worthy of note that when the animal is hungry the psychical secretion is most copjous and most easily obtained. After a full meal it cannot be elicited at all. When food (or other exciting substance) is repeatedly shown to a fasting animal the reaction becomes each time weaker, and finally the glands cease to respond. All that is then necessary to restore the reaction is to put into the animal's mouth a little of the food (or other object). When it is now shown it at a distance the ordinary effect follows promptly. This indicates that the condition of the salivary centre exercises an important influence upon the psychical secretion, its excitability to the weaker stimulus set up by the sight of the object being increased by the stronger reflex stimulation coming directly from the mouth. In the conclition of satiety the inexcitability of the centre may be due to the action of food-products in the blood.

In most animals and in man the activity of the large salivary glands is strictly intermittent. But the smaller glands that stud the mucous membrane of the mouth never entirely ccase to secrete, and the same is the case with the parotid in ruminant animals.

The centre is situated in the medulla oblongata, stimulation of which causes a flow of saliva. The chief afferent paths to the salivary centre are the lingual branch of the fifth and the glossopharyngeal; but stimulation of many other nerves may cause reflex secretion of saliva. In experimental reflex stimulation, the sole efferent channel seems to be the cerebral nerve-supply of the glands. After section of the chorda, no reflex secretion by the submaxillary gland can be caused, although the sympathetic remains intact.

It was alleged by Bernard that, after division of the chordolingual, a reflex secretion could be obtained from the submaxillary gland by stimulating the central end of the cut lingual nerve between the so-called submaxillary ganglion and the tongue, the ganglion being supposed to act as ' centre.' It has been shown, however, that this is not a true reflex effect, but is due to the excitation of certain (recurrent) secretory fibres of the chorda that run for some distance in the lingual, then bend back on their course and pass to the gland. It may be in part a pseudo- 
or axon-reflex (p. 8o9), elicited by excitation of efferent fibres, which send branches to some of the ganglion cells.

The salivary centre can also be inhibited, especially by emotions of a painful kind - for instance, the nervousness which often dries up the saliva, as well as the eloquence, of a beginner in public speaking, and the fear which sometimes made the medieval ordeal of the consecrated bread pick ont the guilty.

In rare cases the reflex nervous mechanism that governs the salivary glands appears to completely break down; and then two riposite conclitions may be seen-xerostomia, or 'dry mouth,' in which no saliva at all is secreted, and chronic ptyalism, or hydrostomia, where, in the absence of any discorerable cause, the amount of secretion is permanently increased. Both conditions

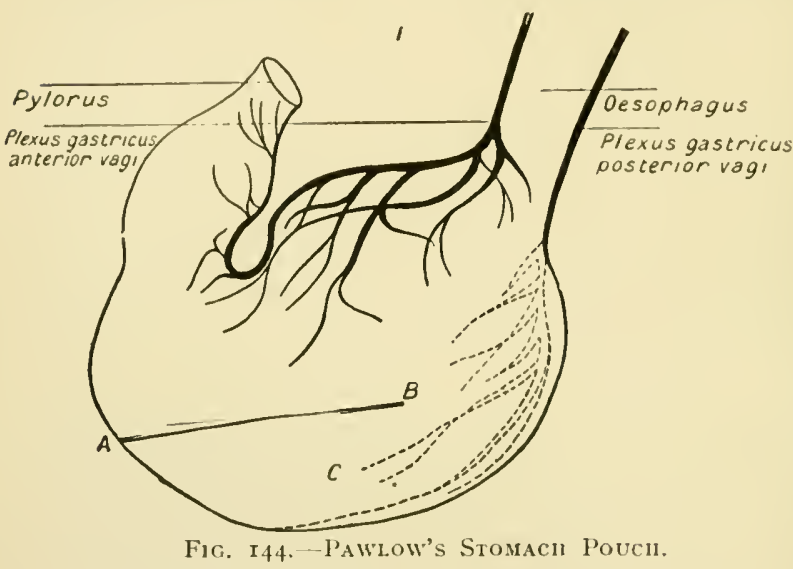

AB, line of incision ; $C$, flap for forming the stomach pouch. At the base of the flap the serous and miscular coats are preserved, and only the mucous membrane divided, so that the branches of the vagus going to the pouch are not severed.

are said to be more common in women than in men.

The Influence of Nerves on the Gastric Glands.-Like saliva, gastric juice is not secreted contintously, except in animals such as the rabbit, whose stomachs are never empty. The normal and most efficient stimulus is the eating of food and its presence in the stomach. Mechanical stimulation of the gastric mucous membrane with a non-digestible substance, such as a feather or a glass rod, causes secretion of mucus, but not of gastric juice. But the observations mentioned above on the difference of response of the salivary glands to different substances suggest that the local mechanical stimulation of the food on the gastric glands may be more effective. There is also at first thought much to indicate that the gastric glands are stimulated chemically in a more direct manner than the salivary glands by the local action of food substances reaching the cells by a shortcut from the cavity of the stomach, or in a more roundalout way by the blood. And it might be very plausibly argued that the 
gastric glands are favourably situated for direct stimulation, while the large salivary glands are not; and that the great function of saliva being to aid deglutition, an almost momentary, and at the same time a perilous act, it is necessary to provide by a nervous mechanism for an inmediate rush of secretion at any instant, while it is not important whether the gastric juice is poured out a little sooner or a little later, and therefore it is left to be called forth by the more tardy and haphazard method of local action. Nevertheless, on looking a little closer, we find that this does not exhaust the subject, and that the gastric secretion can be influenced by events taking place in distant parts of the body, just as the salivary secretion can. In a boy whose cesophagus was completely closed by a cicatrix, the result of swallowing a strong alkali, and who had to be fed by a gastric fistula, it was found that the presence of food in the

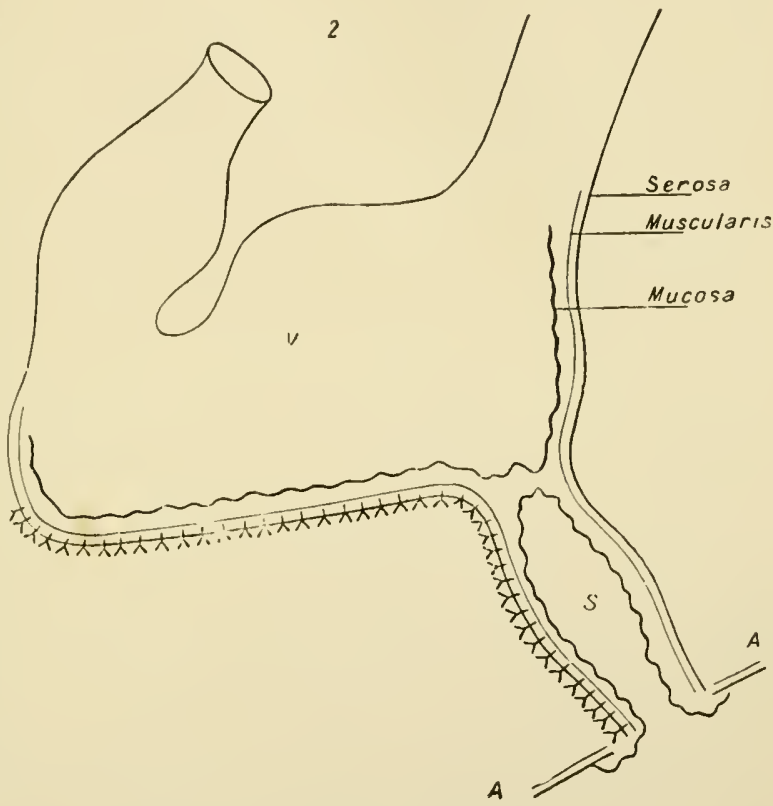
Fig, 145.-PAwlow's Stomach Pouch. month, and even the sight or smell of food, caused secretion of gastric juice (Richet).

Here there must have been some nervous mechanism at work. The secretion cannot have been excited by the direct action of absorbed food-products circulating in the blood-an explanation which might be given, though an insufficient one, of the secretion seen in an isolated portion of the cardiac end of the stomach during the digestion of food in the rest. The efferent nervous channels through which these effects are produced have been defined by Pawlow's experiments on dogs. He first made 
a gastric fistula, then a few days afterwards divided the nesophagus through a wound in the neck, and stitched the two cut ends to the edges of the wound. After the animals had recovered, it was observed that when meat was given to them by the mouth, a copious secretion of gastric juice followed in five or six minutes, notwithstanding the fact that in this 'sham feeding' the food immediately escaped from the opening in the upper portion of the divided œesophagus. Mnch the same result was seen when the food was simply shown to the animal. Indeed, when a hungry animal is tempted with the sight of meat, the flow of gastric juice, always occurring after a latent period of five or six minutes, may be even greater than with sham feeding. Division of the splanchnic nerves had no effect on this reflex secretion, while it could not be obtained after division of both vagi below the origin of their cardiac and pulmonary branches, by which disturbance of the heart and respiration are avoided. Further, stimulation of the peripheral end of the vagus in the neck* caused secretion. These experiments show that secretory fibres for the gastric glands run in the vagi. It is probable that the vagi also contain efferent filores which inhibit the gastric secretion. The excitation of the secretory fibres is not produced reflexly by the processes of mastication and deglutition as such. Dilute acid is the most powerful chemical stimulus for the buccal mucous membrane, and when it is introduced into the mouth of a dog with a double œsophageal and gastric fistula, an abundant secretion of saliva at once ensues. But no matter how long the animal continues to swallow the mixture of saliva and acid, no gastric juice is formed. The same is the case in sham feeding with salt, pepper, mustard, smooth stones, and even extract of meat. It is the desire for food-the appetite, as we call it-and the feeling of satisfaction associated with eating food that the animal relishes, which is the efficient cause of the gastric secretion in sham feeding. The more eagerly the dog eats, the greater is the flow of gastric juice.

Pawlow also performed the converse experiment. In dogs in which a pouch had been isolated from the stomach and made to open to the exterior by the surgical procedure illustrated in Figs. I 44 and I 45 , he introduced into the large stomach, without the animal's knowledge, food of various kinds. This is best done in a sleeping dog. The secretion of gastric juice, both in the main stomach and in the ponch or miniature stomach, which is known in a great variety of conditions to present an exact picture of the process of secretion in

* The nerve was not stimulated till a few days after the section, so as to allow the cardio-inhibitory fibres to degenerate. Otherwise the heart would have been stopped by the stimulation. 
the large, is markedly delayed and scanty when it does appear Bread and coagulated egg-white did not yield a single drop chring the first hour or more. Raw flesh excited a secretion, but after an interval of fifteen to forty-five minutes, instead of live or six to ten, as in sham feeding. It was very scanty during the first hour (only one-third the normal amount), and possessed a very low digestive power. The importance of the psychical element is slown by the fact that in one dog, which, after a weighed amount of meat had been introduced into its stomach (without its knowledge) received a sliam meal of meat, the amount of protein digested after one and a half hours was five times greater than in another animal treated exactly in the same way, except that the sham meal was omitted. But even after division of the vagi, gastric secretion is still caused by the introduction of various substances into the stomach, especially water and meat extract. The active substances in the meat extract are, for the most part, insoluble in alcohol. Kreatin is inactive. It is in virtue of these substances that raw meat placed directly in the stomach causes some secretion after a time. Milk and gelatin solution are also clirect excitants of gastric secretion apart from the water in them. Starch, fat, and egg-white are totally inert. After section of both vagi in dogs, no marked qualitative or quantitative changes have been observed in the gastric juice. The secretion caused by the presence of food in the stomach is still obtained when, in addition to the vagi, all other nerves which can possibly connect the central nervous system with the organ have been severed and the sympathetic abdominal plexuses have been destroyed (Popielski). We must therefore suppose that the gastric glands, while normally under the control of a nervous mechanism in the upper portion of the cerebro-spinal axis whose efferent fibres run in the vagi, are also capable of being locally stimulated through the peripheral ganglia in the stomach walls or the chemical action of the products of digestion absorbed into the blood. Edkins showed that the injection of food substances or the products of their digestion (broth, dextrin, peptone) or of acid into the blood caused no secretion of gastric juice, while the injection of an extract of the pyloric mucous membrane, made by boiling it with water, acid, or peptone, excited a certain amount of secretion. $\mathrm{He}$ therefore concluded that the secondary secretion of gastric juice is determined, not by local stimulation of a reflex mechanism in the gastric wall, but by the production in the mucous membrane of the pyloric end of a chemical substance, the gastric secretin or gastric hormone, * which is absorbed by the blood, and acts as

* 'Hormone' (from uppaw, I arouse or excite) is the name given to a substance which, carried by the blood from the place where it is formed 
an excitant $t^{\prime}$ all the gastric glands. The carcliac mucosa was found incapable of forming this substance.

It is not to be imagined that the 'psychical' secretion and the secretion called forth by the direct action of the food or foodproducts in the stomach perform independent offices. They can. in various instances, be shown to supplement each other. For example, not more than one-lialf or one-third of the gastric juice secreted during the digestion of bread or boiled egg-albumin can be ascribed to the psychic effect. Yet these substances, when introduced directly into the stomach, cause practically no secretion. We must suppose that during the digestion of the bread nd albumin by the psychically secreted juice certain products analogous to those in the meat extract are formed, which act as chenical excitants of the local secretory apparatus. The psychic juice is indispensable in this case to start the process, "to set the stove ablaze,' as Pawlow puts it. In the case of meat it is not indispensable, since the meat can chemically excite the gastric glands; but it greatly hastens the process of digestion. These facts emplasize the importance of appetite in digestion, a truism in treatment which thus receives for the first time a rational explanation. The influence of good-humour upon nutrition, which experience has crystallized into the proverb ' Laugh and grow fat,' lias also been shown to depend-in great part, at least-1ipon a beneficial action on the digestive functions, both motor and chemical. The movements of the cat's stomach and intestines have been observed to cease when the animal became angry or excited by unpleasant emotions; and in a dog whose gastric glands were pouring out a copious psychical secretion in response to a sliam meal, secretion stopped abruptly when the animal's wrath was awakened by what is probably to the normal dog the most specifically 'adequate' stimulus for the emotion of anger-the sight of a cat which he was restrained from chasing.

By means of experiments with the miniature stomach it has been further shown that each kind of food has its own cliaracteristic curve of gastric secretion. With flesh diet the maximum rate of secretion occurs during the furst or second hour, and in each of the first two lours the quantity of juice furnished is approximately the same. With bread diet we have always a sharply-indicated maximum in the first hour, and with milk a similar one during the second or the third hour (Fig. If6). The juice secreted on different diets also differs in digestive power-i.e., in the amount of protein which a given guantity of

acts as a chemical messenger in exciting the activity of some more or less listant orgau. The classical example is the pancreatic secretin which, manufactured in the intestinal mucosa, excites the secretion of the pancreatic juice. 
it will digest in a given time. 'Bread juice' is much stronger in ferment than 'meat juice, and 'meat juice' somewhat stronger than 'milk juice' (Fig. I 47 ). But 'meat juice' has a higher acidity than 'bread juice,' 'milk jujce' being intermediate. These differences do not necessarily indicate that the gastric 111ucous menbranc responds in a specific way to each kind of food substance, as suggested by Pawlow. Tiley may depend on several circumstances, and particu-

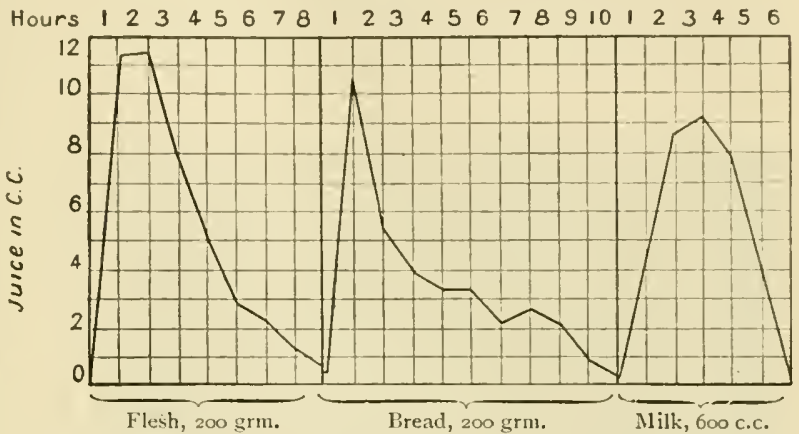

Fig. I46.-RATE OF SECRETION OF GASTRIC JIICE With Diets of Meat, Bread, AND Milk (PAwlow). larly on this - that the quantity, though not the quality, of the psychical or 'appetite' juice is related to the relish with which the animal eats the food. The products formed in the digestion of the different foods by the psychical juice may therefore be different in nature and amount, and thus the quantity of the gastric hormone which determines the secondary secretion may val'y with the food.

The young $\mathrm{mam} \mathrm{mal}$, like the a dult, secretes gastric juice before the food reaches the st o m a ch.

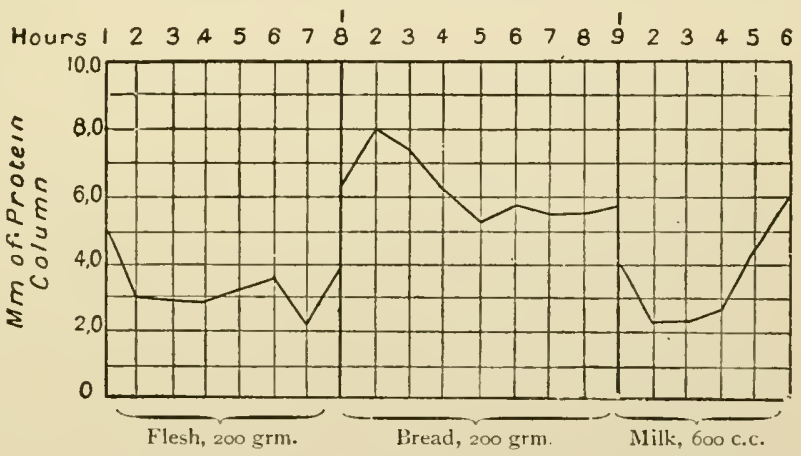

Fig. 147.-Digestive Power of Gastric Juice (PaWlow).

The digestive power of the juice, as measured by the length of the protein column digested in Mett's tubes, is represented hour by hour, with diets of flesh, bread, and milk.

In puppies from one to eighteen days old sham feeding (sucking the teats of the mother after an œesophageal fistula has been made in the younger animals and a double œsophageal and 
gastric fistula in the older) causes a liquid with the properties of gastric juice to gather in the stomach. This power, then, is a congenital one. The individual does not gain it by experience; it comes into the world with him (Cohnheim).

The Influence of Nerves on the Pancreas.-Like the stomach, the pancreas receives secretory fibres through the vagus. These are probably connected with a reflex centre in the medulla oblongata. It has long been known that when the medulla is stimulated a flow of pancreatic juice is occasionally set up,

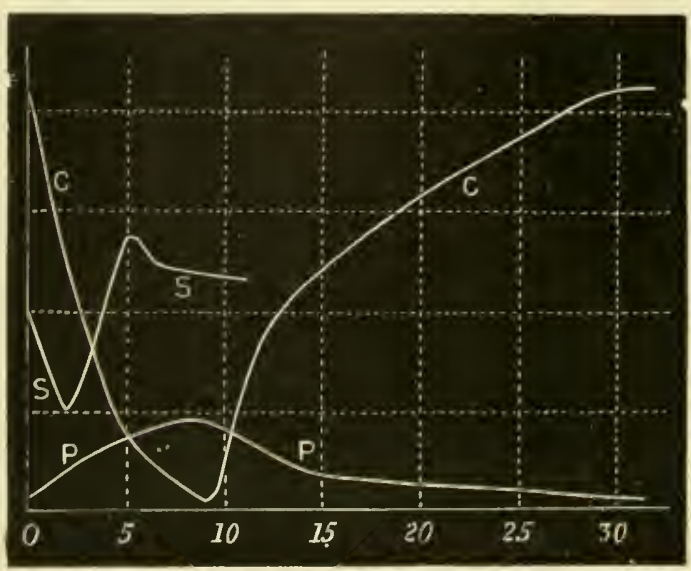

IFIG. I.48.-SECRETION OF PEPSIN.

$C$ shows the quantity of pepsin(ogen) in the mucous membrane of the cardiac end of the stomach at different times during digestion ; $P$, the quantity of pepsin(ogen) in the inucous membrane of the pyloric end; $S$, the quantity of pepsin in the secretion of the cardiac glands. The numbers marked along the horizontal axis are hours since the last nieal. Alonut five hours after the meal, $S$ reaches its inaximun. From the very beginning of the meal $\mathrm{C}$ falls steadily down to the tenth hour, and then begins to rise -i.e., the gland-cells of the cardiac end of the stomach becone poorer in pepsin(ogen) as secretion proceeds.

or is increased if already going on. The same is true when the vagus is stimulated in the ordinary way in the neck. But the experiment often failed, for the pancreas is peculiarly susceptible to circulatory (isturbances, and stimulation of the bulb or the vagus may interfere with the blood-flow through the gland by exciting its vaso-constrictor fibres or causing inhibition of the heart. These disturbing influences may be avoided, as Pawlow has show1, by stimulating the vagus, three or four clays after dividing it, with slowly-recurring stimuli (induction shocks or light blows from a small hammer worked by an electro-magnet at the rate of about one in the second). The secretory fibres are still susceptible of excitation, while the cardio-inhibitory fibres, which degenerate more rapidly, are almost or altogether inexcitable, and the vaso-constrictors are but little affected by these slow rhythmical stimuli, which excite the secretory neries (p. I59). A pancreatic fistula has previously been established by excising a small portion of the duodenal wall containing the 
opening of the pancreatic duct, closing the intestine by sutures, and stitching the orifice of the duct into the ablominal womnd. On stimulation of the ragus the juice will begin in two to three minntes to drop from a cannula in the duct, and will continue to How for several minutes after cessation of the stimulus. The sympathetic also contains secretory fibres for the pancreas. Efferent fibres which inhibit the secretion have also been discovered in the ragus. Their presence may be most clearly demonstrated when that nerve is stimulated during the flow of pancreatic juice excited by the introluction of dilute acid into the duodenum. Stinulation of the central end of the vagus and of the other nerves is capable of reflexly inhibiting the pancreatic secretion. Painful impressions have a strong inhibitory influence. This is one of the reasons why many observers failed to detect the secretory nerves. The inhibition cansed by vomiting is probably due to impulses ascenting the vagus. It is possible that through these nervous channels the pancreatic secretion is affectad by the psychical conditions comnected with rating and the elesire for tood, just as in the case of the gastric secretion; but our information on this subject is scantier and less precise. A flow of juice may undoubtedly take place within three or four minutes after food is taken, but it is not quite certain whether this is noi determined by the passage of some of the acid gastric contents into the duodenum.

Secretin.- IVe have already referred to the fact that pancreatic secretion is excited by the presence of acid in the duodenum. The mechanism of this action is of great interest. Two or three minutes after the introduction of 0.4 per cent. hydrochloric acid into the duodenum pancreatic juice begins to flow. A similar effect is seen when the acid is placed in the jejunum, but not when it is injected into the lower part of the ileum. It is obtained as strongly and as promptly from an isolated loop of intestine when all the nerves passing to it have been cut, and the solar plexus extirpated, and also after the administration of atropine, which paralyzes the endings of secretory nerves elsewhere. The secretion accordingly does not depend upon a local reflex mechanism with its afferent endings in the intestinal mucous membrane, but upon some substance which is carried to the pancreas by tlie blood, and acts directly upon its cells. This substance is not the acid, for the injection of 0.4 per cent. hydrochloric acid into the blood produces no effect upon the pancreas. It has been shown by Bayliss and Starling that the exciting substance is a diffusible body of low molecular weight, probably of organic nature, but not a protein, which they call secretin. It is soluble in alcohol or alcohol and ether, and is not destroyed by boiling. It is produced in the mucous membrane of the jejunum or duo- 
denum on exposure to dilute hydrochloric acid. Extracts of mucous membrane so treated cause a copious pancreatic secretion, and a smaller secretion of bile, when injected in small quantities into the blood of animals in which no such secretion is taking place, but have no influence on any other gland. At the same time the arterial blood-pressure falls somewhat. The substance which produces the fall of blood-pressure is different from secretin, since acid extracts of the lower end of the ileum, which lave no effect on the flow of pancreatic juice, diminish the blool-pressure. A precursor of secretin, called pro-secretin, exists in the intestinal mucous membrane, and can be extracted from it by physiological salt solution. It does not affect the pancreatic secretion. By boiling or by the action of acid secretin is split off from it. Pro-secretin is most abundant in the

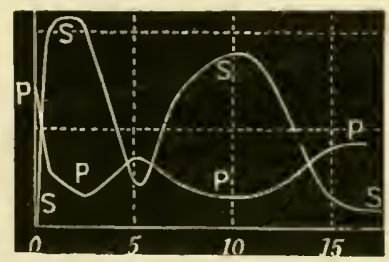

Fig. I49.-RATE OF SECRETION OF PaNcreatic Juice.

$S$ shows the variation in the rate of secretion of the pancreatic juice in a log: $P$, the variation in the percentage of solids in the juice. It will be seen that the maxima of $S$ fall at the same time as the maxima of P. The numbers along the horizontal axis are hours since the last meal. duolenum. and diminishes as we pass down the intestine.

Secretin is very widespread in the animal kingdom. In the monkey, log, cat, rabbit, man, ox, sheep, pig, squirrel. goose, tortoise, salmon, dog-fish, and skate evidence of its presence has been obtained. The secretin of one animal will excite a flow of pancreatic juice in an animal of a different kind as well as in one of the same kind. In normal digestion secretin is formed under the influence of the acid chyme, not in the stomach, but after it las passed into the duodenum. The passage of the chyme through the pylorus, as previously mentioned ( $p .305)$, is regulated by the reaction of the duodenal contents, as well as by the consistence of the gastric contents. So long as the liquid in the duodenum is acid. the pylorts remains closed. As soon as the first small portion of acid chyme ejected from the stomach has been neutralized by the increased secretion of the pancreatic juice and the outpouring of bile from the gall-bladder in response to the stimulus of the acid, the pylorus opens again.

According to Pawlow, certain food substances, notably fat, and water stimulate the pancreatic secretion, and with great promptness, even before any acid lias been prodnced in the stomach, and therefore before any can liave passed into the duodenum. Possibly this effect is elicited through the long reflex paths already described as running in the vagi or through a local ner- 
vous mechanism, which, although it does not take part in the excitation of the pancreatic secretion by acid, may yet exist for the performance of other offices. It is more probable, however, that it is due to the passage of some of the gastric contents through the pylorus; for when oil is introduced into the small intestine, it causes the production of secretin, although, unlike dilute acid, it is quite ineffective in forming secretin when rubbed up with the scrapedoff mucous menbranc.

The pancreatic, like the gastric, juice is said to vary as regards its digestive properties with the nature of the food. On a dict of bread the juice is very poor in fat-splitting ferment, while on a 兰 24 diet of flesh it is richer, and on a diet of milk richest of all. With bread the juice is relatively rich in amylolytic ferment. When we take the quantity of the juice as well as its strength in ferments into consideration, it is stated that bread 111111 iv V 1 II III IV V VI VII VIIII 11 III I8 V VI

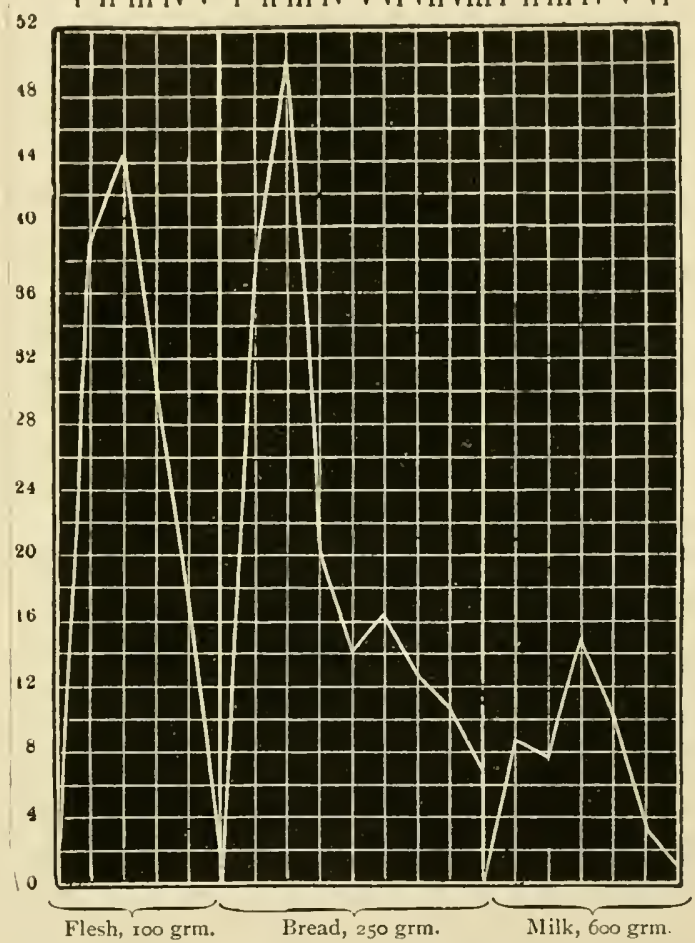

Fig. I50.-Secretion of Pancreatic Juice with Different Diets (Pawlow).

The hours are in roman numerals. occasions the secretion of a juice with a greater quantity of proteolytic ferment than either milk or meat, although it is relatively dilute (Fig. I50). The vegetable proteins require more ferment to digest them than proteins of animal origin. There is no more evidence that the adaptation of the pancreatic juice to the nature of the food is due to a specific sensibility of the duodenal mucosa to the various food-stuffs than there is in the case of the adaptation of the gastric juice. If the volume of the chyme and its acidity 
are related to the nature of the food, then the amount of secretin formed, and therefore the intensity of secretion in the pancreas, will be similarly related. The one apparently proved example of specific adlaptation of the pancreatic juice has not stood the test of a critical examination. It was asserted that in dogs ferl for some days with food containing lactose (milk) the ferment, lactase, is present in that secretion, while the pancreatic juice of dogs whose food is frce from lactose does not contain lactase. The adaptation of the pancreas to lactose was supposed to be achieved through some substance produced by the action of lactose on the intestinal mucous membrane, which plays the part of a specific chemical stimulus to the pancreatic cells or their secretory nervous mechanism, causing them to form lactase. But it has been conclusively shown that when tegs are fed with lactose for weeks no lactase appears in the pancreatic juice (Plimmer).

The natural secretion of pancreatic juice is by no means so intermittent as that of saliva. In the rabbit the pancreatic, like the gastric, juice flows continuously. In the dog it legins almost as soon as food is taken, rises in two or three hours to a maximum, then falls till the fifth or sixtl hour, after which it may mount again somewhat, and then, gradually diminishing, ultimately stops (Figs. I 49, I50). During normal activity the bloodvessels of the gland are dilated. But under experimental conditions the increased secretion caused by secretin is accompanied sometimes by an increase and sometimes by a diminution in the blood-flow, and secretion may continue for some time after comple te cessation of the circulation, while the increased consumption of oxygen which goes hand in hand with the increased secretion is also independent of the blook-supply (May, Barcroft and Starling). This shows how far the secretory process is from a mere mechanical filtration, although it does not follow that, under normal conditions, a decreased blood-flow ever does accompany an increased secretion. There is one difference between the normal secretion of pancreatic juice and of saliva which may still be mentioned: the pressure of the latter in the submaxillary duct may, as we have seen, greatly exceed the arterial blood-pressure, without reabsorption and consequent celema of the gland occurring; but the secretory pressure of the pancreatic cells is very low, not more than a tenth of that of the salivary gands. Cilema begins before a manometer in the duct shows a pressure of $20 \mathrm{~mm}$. of mercury, the secreted fluid passing very easily in to the lympli spaces.

The mutual relations of the spleen and pancreas have formed the subject of numerous inquiries. Some authors maintain that the spleen plays an important rôle in the elaboration of the proteolytic ferment of the pancreas, forming a sub- 
stance which we may call pro-trypsinggen, since it is supposed to be carried in the blood to the pancreatic cells, and changerl by them into trypsinogen. There is some evirlence that extracts of the spleen prepared from it when congested during digestion exert a farourable influence on the proteolytic power of the pancreas (Menclel). And there is no doubt that the spleen, like other organs, contains an intracellular enzyme which can ais in the digestion of protein. The products of the action in an acid medium of this enzyme are the same as those formed by trypsin in an alkaline medium (Leathes). But this is not enough to prove that the spleen has any special relation to pancreatic rligestion.

The Influence of Nerves on the Secretion of Bile.-Nthough bile is secreted constantly, it only passes at intervals into the intestine. For the liver in many animals, unlike every other gland except the kidney, has in connection with it a reservoir, the gall-bladder, in which its secretion accumulates, and from which it is only expelled occasionally. We have therefore to distinguish the bile-secreting from the bile-expelling mechanism, To study the rate of secretion of bile (Fig. I5I), a fistula of the gall-bladder can be established. But to learn the function of bile in digestion it is more important to know when and at what rate it enters the intestine. For this purpose a fistula is made by cutting the natural

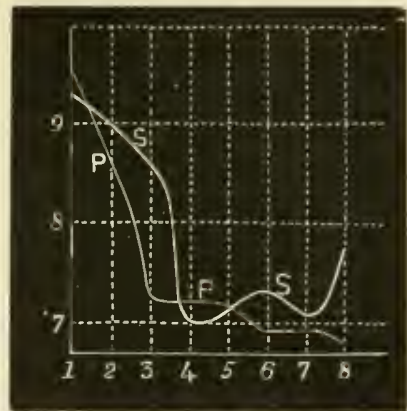

Fig. I5I.- Rate of Secretion OF B1LE.

$\mathrm{S}$ shows how the rate of secre. tion of bile falls in a dog when a biliary fistula is first made, and the bile thus prevented from entering the intestine; $\mathrm{P}$ shows the fall of the percentage of solids. The numbers along the horizontal axis are quarters of an hour since bile began to escape through the fistula. The num. bers along the vertical axis refer only to curve $S$, and represent the rate of secretion in arbitrary units. orifice of the common bile-duct with a piece of the surrounding mucous membrane out of the intestine and transplanting it upon the serous coat, where it is sutured. The loop of intestine, with the orifice of the duct facing outwards, is then stitched into the abdominal wound, where it is allowed to heal. Of course, since a circulation of the bile-acids takes place -i.e., an absorption from and re-excretion into the intestinethe formation of that juice cannot proceed upon absolutely normal lines when the bile no longer enters the duodenum. The only condition under which fistula bile could have the same composition as nomal bile would be that in which as great an amount of bile-acils is introduced into the gut as escapes through 
the fistula. A circulation of a smaller proportion of the bilepigments is also probable, but there is no circulation of the biliary cliolesterin (Stadelmann).

Of the direct influence of nerves, either on the secretion of bile or on its expulsion, we have scarcely any knowledge, scarcely even any guess which is worth mentioning here. It is true the secretion of bile may be distinctly affected by the section and stimulation of nerves which control the blood-supply of the stomach, intestines. and splecn, for the quantity of blood passing by the portal vein to the liver depends upon the quantity passing through these organs, and the rate of secretion is diminished when the blond-supply is greatly lessened. In this way stimulation of the medulla oblongata. the spinal cord, or the splanchnic nerves stops or slows the secretion of bile by constricting the abdominal vessels; and the same effect can be reflexly produced by the excitation of afferent nerves.

The right splanchnic nerve contains inhibitory with some motor fibres, and the vagi (especially the left) contain motor fibres for the gall-bladder. Probably its contraction takes place naturally in response to reflex impulses from the mucous membrane of the duodenum, for the application of dilute acid to the mouth of the bile-duct causes a sudden flow of bile, ${ }^{*}$ and the acid contents of the stomach, when projected through the pylorus into the intestine, have a similar effect. But, in addition, as we have seen, the secretin formed will cause an increase in the rate of secretion of the bile. In studying the effect of secretin it is necessary to obtain it free from bile-salts, since these cause of themselves an increased secretion of bile. When this is done by dissolving out with alcohol any bile-salts which may be present in the extract of intestinal mucous membrane, a solution of the residue containing the secretin still erokes a rapid secretion of bile. The fact that the same hormone excites the formation both of pancreatic juice and bile is obviously related to that common action of the two juices in digestion on which we have already dwelt.

When food passes into the stomach, there is at once a sharp rise in the rate of secretion of bile. A maximum is reached from the fourth to the eighth hour-that is, while the food is in the intestine. There is then a fall, succeeded by a second smaller rise about the fifteenth or sixteenth hour, from which the secretion gradually declines to its minimum. Upon the whole, the curves of secretion of pancreatic juice and bile show a fairly close correspondence, except that the latter is more nearly continuous. But when we compare the curves representing the rate at which the bile actually enters the intestine with the curve of pancreatic secretion (Fig. I52), we are struck by their almost absolute parallelism. This lends additional support to the con-

* This result seems to be difficult to realise experimentally. Bainbridge and Dale could not elicit reflex contraction of the gall-bladder (in anesthetized animals) in this way. 
clusion deduced from their chemical and physical properties, that in digestion they are partners in a common work.

While the rate at which bile passes into the intestine seems to be influenced by digestion much in the same way as the rate of pancreatic secretion, the details are as ret less exactly known. In the fasting animal no bile enters the gut. When food is taken the flow begins after a definite interval, which varies for the different kinds of food. As long as digestion lasts bile continues to escape, but both the quantity and quality depend upon the nature of the food. Water, raw egg-white, and starch paste, whether given by the mouth or introduced direstly into the stomach of a dog, cause 110 flow of bile. But fat, the extractives of meat, and the products of digestion of egg-white produce a copious discharge. This discharge may be determined by the relatively large amount of acid chyme passed through the pylorus when proteins are digested in the stomach and the stimulus to the formation of secretin occasioned by the
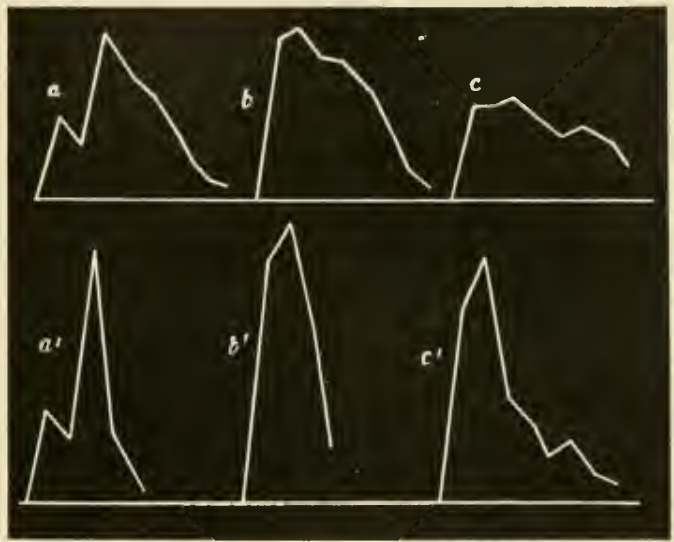

Fig. i52.-Pancreatic Juice and Bile (Pawlow).

The upper curves represent the hourly rate of pan. creatic secretion, and the lower the rate at which the bile enters the intestine; $a, a^{\prime}$, milk diet $; b, b^{\prime}$, meat : $c, c^{\prime}$, bread. Only the general form of the curves is to be compared. The scale of the ordinates of the various curves was not the same.

presence of this

chyme or of fatty material in the duodenum. In the case of fat a further favourable influence on the secretion of bile is the absorption of bile-salts which accompanies the absorption of the fatty acids and soaps produced in fat digestion. Bilesalts stimulate the secretion of bile, including bile-salts themselves. An increased flow of bile-salts into the intestine accelerates the splitting of fats by the pancreatic juice, and therefore the absorption of bile-salts acting as solvents for, or chemically united to, the fatty acids and soaps. A circle analogous to the 'vicious circle' of the logicians, but constituting a physiological adaptation of most potent virtue in the digestion of fats, is thus established. Not only is the quantity of bile poured into the 
intestine increased on a dict rich in fat, hut it is said that a given amount of it aids the fat-splitting action of the pancreatic juice more powerfully than if the diet were poor in fat. This may depend upon an increase in the concentration of the hile-salts in bile secreted when a large amount of fat is ingested. But it is well to recognise that we do not at present know with any great exactness the mechanism by which the rate of secretion and expusion of bile and the properties of that juice are influenced hy digestion. It has been conjectured that the first alrupt rise may be started by reflex nervous action, and that later on secretin and, in the case of fat digestion, bile-salts may directly. excite the hepatic cells.

The pressure under which the bile is secreted is higher than the pressure of the portal blood, and therefore the liver ranges itself with the high-pressure salivary glands rather than with the low-pressure pancreas. But although the biliary pressure is high relatively to that of the blood with which the secreting cells are supplice, it is absolutely low, the maximum heing no more than $25 \mathrm{~mm}$. of mercury.* This is a point of practical importance, for a comparatively slight obstruction to the outflow, even such as is offered by a congested or inflamed condition of the duodenal wall about the mouth of the duct, may be sufficient to cause reabsorption of the bile through the lymphatics, and conseguent jaundice. Of course, complete plugging of the duct by a biliary calculus is a much more formiclable barrier, and inevitably leads to jaundice, just as ligature of a salivary duct, in spite of the great secretory pressure, inevitably causes cedema of the gland.

The Influence of Nerves on the Secretion of Intestinal Juice.As to the influence of nerves on the secretion of the succus entericus, our knowledge is almost limited to a single experiment, and that an inconclusive one. Moreau placed four ligatures on a portion of the small intestine, so as to form three compartments separated from each other and from the rest of the gut. The mesenteric nerves going to the middle loop were divided, and the intestine returner to the abdomen. After some time a watery secretion was found in the middle compartment, little or none in the others. This is a truc 'paralytic' secretion, and not a mere transudation depending simply on the vascular dilatation caused by section of the vaso-constrictor nerves. for it has the same composition and digestive action as normal succus entericus obtained from a fistula. The secretion begins about four hours after section of the nerves, goes on increasing

* In the $\log$, cat, and monkey the average maximum pressure at which as much bile is secreted as is taken up from the bile-paths ly the portal lymphatics is about $300 \mathrm{~mm}$. of bile. The highest pressme recorded was $373 \mathrm{~mm}$. of bile in a cat (Herring and Simpson). 
for about twelve hours, and then rapidly diminishes, so that after about two days the middle loop, as well as the other two, will be found empty. The interpretation usually put upon the experiment is that nerves which normally inhibit the local secretory mechanism have been divided. But there is no real proof of the existence of such nerves.

The same adaptation is seen in the secretion of the succus entericus as in the secretion of the other digestive juices, and the adaptation is naturally most striking in regard to those points in which the intestinal juice is peculiar. While mechanical stimulation of the stomach is ineffective as regards the secretion of gastric juice, mechanical stimulation of the intestine, as by the contact of a camnula, produces a free flow of succus entericus. The reaction is a localized one, the secretion only taking place from the portion of the mucous membrane stimulated. This fact acquires significance when we reflect that the food moves very slowly in the intestine, and a secretion could be of use only at the points where the food happened to be. The juice secreted in response to mechanical stimulation is poor in enterokinase But if a little pancreatic juice be put into the intestine, and left there for some time, the juice afterwards secreted is rich in enterokinase.

Effect of Certain Drugs on the Digestive Secretions.-A small dose of atropine, as has been said, abolishes the secretory action of the chorda tympani. This it does by paralyzing the nerve-endings or 'receptive' substances in the gland-cells through which the nerveimpulses excite secretion. The gland-cells are not completely paralyzed, for the sympathetic can still cause secretion. The nerve-fibres are not paralyzed, because the direct application of atropine does not affect them ; nor is the seat of the paralysis the ganglion-cells on the course of the fibres, for stimulation between those cells and the gland-cells is ineffective. Pilocarpine is the physiological antagonist of atropine, ancl restores the secretion which atropine has abolished. In small d'oses it causes a rapid flow of saliva. its action being certainly a peripheral action, and probably an action on the nerve-endings (or receptive substances), for it persists after all the nerves going to the salivary glands have been divided, and after the ganglion-cells have been paralyzed by nicotine. Atropine and pilocarpine act similarly on some of the other digestive glands, atropine paralyzing the pancreatic secretion elicited by stimulation of the vagus, although not that obtained by the introduction of acid into the intestine. Pilocarpine seems only to cause a secretion of pancreatic juice when other stimuli are already acting, especially the stimulus determined by the presence of acid in the duodenum. Pilocarpine increases the secretion of gastric, and probably of intestinal juice, but atropine does not stop the secretion caused by division of the intestinal nerves. Physostigmine and muscarine act on the whole like pilocarpine, but physostigmine in small doses gives rise to an abundant flow of pancreatic juice, even when the intestine is empty, probably by stimulating the endings of the secretory fibres in the vagus. 
The action of alcohol on the secretion of gastric juice has been studied in a dog with a double gastric and oesoplageal fistula. Before or during a sham meal of meat, alcohol dilnted with water was given as an enema. After the enema the quantity of hydrochloric acid secreted increased in about the same proportion as the quantity of juice, but the pepsin was diminished, reaching a miniinum after three-quarters to one arid a quarter hours. The increase in the total quantity of the juice and in the acil over-compensated the moderate diminution in the digestive power, so that the net result was beneficial (Pekelharing). But it must be remembered that strong alcoholic beverages, when mixed with the gastric juice, and therefore when taken by the mouth, retard the proteolytic action, so that any favourable effect on the secretion of the juice may casily be lost in the subsequent digestion, unless the alcohol is dilute (Chittenden and Mendel). The action of alcolnol introduced into the rectum on the gastric secretion is both reflex and direct.

Cholagogues. - The action of a host of clrugs on the secretion of bile has been investigated by various observers, but till something like unanimity has been reached it would not be profitable to go into details here. The only real cholagogues at present positively known appear to be the salts of the bile-acids, which, given by themselves or in the bile, cause not only an increase in the volume of the biliary secretion. but also an increase in its solids. Certain compounds of salicylic acid, as salol (phenyl salicylate) and sodium salicylate, also appear to slightly increase the flow, while usually diminishing the concentration of the bile. The injection of hremoglobin into the blood-stream, or its liberation there by substances, such as toluylenediamin and arseniuretted hydrogen, which cause destruction of the corpuscles, leads to an increased secretion of bile-pigment as well as a more rapid flow of bile.

Summary.-Here let us sum up the most important points relating to the secretion of the digestive juices. They are all formed by the activity of gland-cells originally derived from the epithelial lining of the alimentary canal. The organic constituents or their precursors (including the mother-substances of the ferments) are prepared in the intervals of rest-absolute in some glands, relative in others-and stored up in the form of gramules, which during activity are moved towards the lumen of the gland tubules, and there discharged.

The nerves of the salivary glands are, as regards their origin, (a) cerebral, (b) sympathetic; the former group is vaso-dilator, the latter (usually) vaso-constrictor; both are secretory. Secretion of saliva depends strictly on the nervous system. That nerves influence the gastric and pancreatic secretions is also made out. The psychical secretion is of greater importance for the saliva and gastric juice than for the pancreatic juice. The direct action of secretin (produced in the intestinal mucous membranc by the influence of the chyme) is the most characteristic factor in pancreatic secretion. As regards the intestinal glands and the liver, it has not been proved that their secretive activity is under the control of the nervous system, except in so far as the latter may indirectly govern it through 
the blood-supply, although various circumstances suggest the probability of a more direct action. All the digestive juices show a certain adaptation to the nature of the food, although it has not been demonstrated that this is due to a specific sensibility of the mucous membranes for each kind of food-stuff. The action of one juice on the secretion of another is also of great significance. Thus, the water of the saliva directly excites a flow of gastric juice when it reaches the stomach; the acid of the gastric juice excites a flow of pancreatic juice when it reaches the duodenum; and the pancreatic juice excites the intestinal mucous membrane to the production of enterokinase, the most characteristic constituent of the succus entcricus. In all the glands the blood-flow is increased during activity; in some (salivary giands) this is known to be caused through vaso-motor nerves. In the salivary glands clectro. motive changes accompany the active state, and more heat is produced. Both in the salivary glands and the pancreas it has been showen that much more carbon dioxide is given off, and much more oxygen used up, during secretion than during rest. In the other glands we may assume that the same occurs. This is one proof that work is done in the separation or manufacture of the constituents of the various secretions.

\section{Digestion as a Whole.}

Having discussed in detail the separate action of the digestive secretions, it is now time to consider the act of digestion as a whole, the various stages in which are co-ordinated for a common end. The solid food is more or less broken up in the mouth and mixed with the saliva, which its presence causes to be secreted in considerable quantity. Liquids and small solid morsels are shot down the open gullet without contraction of the constrictors of the pharynx, and reach the lower portion of the œsophagus in a comparatively short time ( $\frac{1}{10}$ second) ; while a good-sized bolus is grasped by the constrictors, then by the œsophageal walls, and passed along by a more deliberate peristaltic contraction.

Chemical digestion in man begins already in the mouth, a part of the starch being there converted into dextrins and sugar (maltose), as has been shown by examining a mass of food containing starch just as it is ready for swallowing (p. 424). This process is no doubt continued during the passage of the food along the œsophagus.

The first morsels of a meal which reach the stomach find it free from gastric juice, or nearly so. They are alkaline from the admixture of saliva; and the juice which is now beginning to be secreted, in response to the psychical excitement, and 
reflexly through the presence of the food and the water of the saliva in the stomach, is for a time neutralized, and anylolytic digestion still permitted to go on. For 20 to fo minntes after digestion has begun there is no free hydrochloric acid in the stomach, although some is combined with proteins, and during this period the ptyalin of the swallowed saliva will be able to act even better than in the mouth, being favoured by a weakly acid reaction. Indeed, for a time, as the meal goes on, the successive portions of food which arrive in the stomach will find the conditions more and more favourable for amylolytic digestion. But as the acidity continues to increase, the activity of the ptyalin will first be lessened, and ultimately abolished; and, upon the whole, a considerable proportion of the starches must usually escape complete conversion into sugar until they are acted upon by the pancreatic juice. This is particularly the case with unboiled starch, as contained in vegetables which are eaten raw; and, indeed, we know that sometimes a certain amount of starch may escape even pancreatic digestion, and appear in the freces. Meanwhile, pepsin and hydrochloric acid are being poured forth; the latter is entering into combination with the proteins of the food; and before the end of an ordinary meal peptic digestion is in full swing. The movements of the pyloric end of the stomach increase, and eddies are set up in its contents, which carry the morsels of food with them, and throw them against its walls. In this way not only are the contents thoroughly mixed, and fresh portions of food constantly brought into contact with the gastric juice secreted mainly in the more passive cardiac end, but a certain amount of mechanical disintegration is brought about. This is aided by the digestion of the gelatin-yielding connective tissue which holds together the fibres of muscle and the cells of fat, and the digestible structures in vegetable tissue which enclose starch granules. If milk has formed a portion of the meal, the cascinogen will have been curdled soon after its entrance into the stomach, by the action of the rennet ferment alone (see p. 326) when the milk has been taken at the beginning of digestion before the gastric contents have become distinctly acid, by the acid and ferment together when it has been taken later. The caseinogen and other proteins of milk, like the myosinogen and other proteins of meat, and the globulins, albumins, and other proteins of bread and of vegetable food in general, are acted upon by the pepsin and hydrochloric acid, yielding ultimately peptones; while variable quantities of these proteins and of the acid-albumin and proteoses derived from them may escape this final change, and pass on as such into the duodenum. In the dog, indeed, a very large proportion of a meal of flesh has been found to be digested to the 
peptome stage while still in the stomach, leaving for the juices that act on it in the intestine only its further hydrolysis to amino-acids, etc. But we may safely assume that, in the case of a man living on an ordinary mixed diet, a good deal of the foor proteins passes through the pylorus chemically unchanged, or having mudergone only the first steps of hydration. For, even a few minutes after food has been swallowed, especially liquid food or water, the pyloric sphincter may relax and allow the stomach to propel a portion of its contents into the intestine; and such relaxations occur at intervals as digestion goes on, althongh it is not for several hours (three to five) that the greater portion of the food reaches the duodenum. During this period the acidity has at first been constantly increasing, although for a time the hydrochloric acid has combined, as it is formed, with the proteins of the food. Then comes a stage where the hydrochloric acid has so much increased that, after combining with all the proteins, some of it remains over as free acid. After a time the total acidity begins to fall, the partially digested proteins continually passing on through the pylorus, while a considerable proportion is so fully digested as to he absorbed by the gastric mucous membrane itself. Thus, in one experiment on the digestion of meat in a dog. it was found that 30 per cent. was absorbed in the stomach.

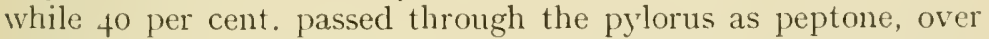
20 per cent. as undissolved or soluble protein (acid-albumin), and a little more than 8 per cent. as proteose (Tobler). The large proportion of peptone is noteworthy, as indicating some kind of selective passage of the different digestive products from the stomach into the duodenum. "For the gastric contents contain plenty of proteose, although only traces of peptone. The total ' titratable acidity' goes on diminishing till the third or fourth hour, the proportion of free to combined acid continuing, nevertheless, to rise, since nearly all that is now secreted remains free. In addition to a certain amount of protein, small quantities of soluble and easily diffusible substances, like sugars and some of the organic crystalline constituents of meat-c.g., kreatin-may also be absorbed into the blood by the gastric mucous membrane.

The substances which reach the duodenum are: (I) The greater part of the fats. The partial digestion in the stomach of the envelopes and protoplasm of the cells of adipose tissue, and of the protein which keeps the fat of milk in emulsion, prepares the fats which are not split up by the gastric juice for what is to follow in the intestine. (2) All the proteins which have not been carried to the stage of peptone, and much peptone. (3) All the starch and dextrins - and glycogen, if any be presentwhich have not been converted into sugars, and probably a 
portion of the sugars. (4) Elastin, nucleins, collulose, and other substances not digestible, or digestible only with difficulty, in gistric juice. (5) The constituents of the gastric juice itself, including pepsin. The ptyalin of the saliva has been already destroyed.

It must be remembered that all this time, even from the beginuing of digestion, a certain amount of pancreatic juice has been finding its way into the duodenum in response first perhaps to the psychical excitation, and later to that action of the acid chyme on the intestinal mucous membrane which has been described. In the duodenum its trypsinogen is becoming activated to trypsin by the enterokinase of the intestinal juice. The secretion of bile, too, has quickened its pace, the gall-bladder is getting more and more full as the meal proceeds and gastric digestion begins, and some of the bile may very soon escaje into the intestine. The pylorus opens occasionally for a moment whenever the small portions of clyme which at this stage are beginning to pass through have been sufficiently neutralized by the pancreatic juice and bile, although it is not necessary that the reaction should become actually neutral. When the acid chyme, a greyish liquid, turbid with the débris of animal and vegetable tissues-with muscular fibres, fat globules, starch granules, and dotted ducts-gushes through the pylorus and strikes the duodenal wall, the muscular fibres of the gall-bladder contract, and sudden rushes of bile take place from the common duct. By-and-by, as bile and pancreatic juice continue to be poured out, the reaction in the duodenum, as tested by litmus, becomes less acid and even weakly alkaline for a time. But it soon becomes acid again, and the acidity at first increases as the food passes down the gut. In the lower portion of the small intestine the acidity diminishes, and the contents may be neutral or actually alkaline for some distance above the ileocrecal valve. To phenolphthalein the reaction is acid throughout the whole intestine. But methyl orange shows an alkaline reaction, all the way from the lower end of the duodemum to the crecum (Noore and Rockwood). In the upper part of the duodenum the reaction with this indicator is sometimes found acid, but sometimes neutral or alkaline. All this refers to the conditions during full digestion ( 3 or 4 to $S$ or 9 lours after the taking of food). When digestion is over (20 to 24 hours after a meal) the reaction becomes acid to methyl orange, litmus, and phenolphthalein throughout the whole intestine.* But it must be remembered that the differences in true reaction at different

* In 18 dogs fed with meat zo to 24 hours before death this was found to be the case. In + of the dogs the gastric contents were almost neutral to litmus and methyl orange, lut slightly alkaline to pluenolplithalein ; in the rest acid to all three indicators. 
stages of intestinal digestion and at different levels of the gut are always slight. There is never a great preponderance either of hydroxyl or of liydrogen ions between the point at which the pancreatic juice and hile are mingled with the gastric chyme and the lower part of the ileum.

Reaction of Intestinal Contents. $-\Lambda$ consideration of the properties of the indicators mentioned cnables us to interpret in some measure these results, which at first sight appear so confusing. Methyl orange, the nost stable of the scries, is not affected by weak organic acids, but reacts acid to inorganic, and the stronger organic acids like lactic, acetic and butyric acids, and alkaline to salts of the weaker acicls, such as sodium carbonate and bicarbonate. I'henolphthalein is very sensitive to acids, even to weak organic acicls such as the fatty aciels derived from the fat of meat, and to carbonic acid. Litmins is intermediate between methyl orange and phenolphthalein. The chyne, as it passes throwgh the pylorus, contains free hydrochloric acid. it mingles immediately with the alkaline contents of the duodenum. If these contain a sufficient quantity of bases to combine with the whole of the acids which would affect methyl orange, that indicator will show a neutral or alkaline reaction. Phenolphthalein may at the same time react acid on account of the presence of weaker acisls, including carbonic acid, either originally dissolved in the intestinal fluid or liberated by the action of the acids of the chyme on the carbonates. If there is not enough alkali to combine with the whole of the stronger acids, the reaction will be at first acid to all the indicators, but may soon become alkaline to methyl orange or even to litmus, as pancreatic juice and bile continue to enter the duodenum. As the food progresses along the intestine a certain amount of lactic acicl is produced by the action of micro-organisms on the carbo-hydrates. The alkalies of the intestinal secretions are being continually used up, both to neutralize this acid, and to form soaps with the fatty acids set free from the fats by the steapsin and the fat-splitting bacteria. The point may easily be reached, and as a rule is reached, at which enough of the weak acids or of acid salts is present to give an acid reaction with phenolphthalein or litmus, while the reaction is still alkaline to methyl orange. By the time the food has arrived at the lower end of the small intestine the greater part of the fat-splitting may be supposed to be over, and the greater part of the fatty acids absorbed. The acids that renain may be easily neutralized by the alkaline succus entericus, reinforced by the alkalies, especially ammonia, produced by the ordinary putrefactive bacteria from proteins ; and the reaction, previously alkaline to methyl orange only, may thus become alkaline to litmus as well. Dissolved carbonic acid will still account for the acid reaction to phenolphthalein. Towards the end of intestinal digestion the discharge of pancreatic juice, bile and succus entericus having almost or entirely ceased, the acid-forming bacteria appear again to get the upper hand; and since the reaction is acid to methyl orange as well as to the other indicators, we must assume that strong organic acids, like lactic acid, are present. Very early in the meal the inflow of alkaline pancreatic juice, and perhaps of succus entericus, into the intestine begins ; and for a considerable time this is not counteracted by the escape of any large quantity of acid chyme through the pylorus. We nust accordingly suppose that the conditions for the establishment of an alkaline reaction of the intestinal contents are 
unfavourable at the cnd of intestinal cligestion, and favourable at the beginning of gastric agestion.

Trypsin, like pepsin, performs its work in part in an acid medimm : and although the canse of the acidity and the character of the medimm are far from being the same as in the gastric juice, it is obviously an advantage that the chief proteolytic ferment should be able to act upon the proteins in all parts of the intestine and at every stage of intestinal digestion whether the reaction is acid or alkaline. The proteins of the chrme are all carried by the trypsin to the stage of peptone, and the peptene, or a great part of it, even in perfectly normal digestion, is further split up into amino- and diamino-acids by the trypsin and by the erepsin of the succus entericus.

In the lower portions of the small intestine hacteria of various kinds are present and active; and it is not unlikely that even throughout its whole length a certain range of action is permitted to them, checked by the acidity of the chyme, though scarcely by the feeble antiseptic properties of the bile.

The lower end of the small intestine is not cut off by any bacteria-poof harrier from the large intestine, in which putrefaction is constantly going on. It has been actually shown that small particles, such as lycopodium spores, suspended in water, soon reach the stomach when injected into the rectum. So that micro-organisms, aided by the antiperistalsis of the colon, may be able to work their way above the ileo-colic splineter and valve, even against the downward peristaltic movement of the small intestine. But even if this were not the case, a few bacteria or their spores, passing through the stomach with the food, would be enough to set "1p extensive changes as soon as they reached a part of the alimentary canal where the conditions were farourable to their derelopment. Indeed, from the time when the first micro-organism enters the digestive tmbe soon after birth, it is never free from bacteria ; and their multiplication in one part of it rather than another depends not so much on the number originally present to start the process, as on the conditions which enconrage or restrain their increasc.

A certain amount of already emulsified fats is broken up into their fatty acids and glycerin in the stomach, memulsified fats entirely by the fat-splitting ferment of the pancreatic juice. The acirls will form soaps with alkalies wherever they meet them in the intestinal contents, or even in the mucous membrane. A portion of those soluble soaps may be immediately absorbed; the rest will aid in the emulsitication of the fats not yet chemically: decomposed, and thus greatly hasten the fat-spliting action of the pancreatic juice. The starch and dextrin which have escaped the action of the saliva are changed into maltose by the amylopsin. 
The succus entericus, in addition to its important functions alleady mentioned, aticls as an alkaline liguide in lessening the acidity of the chyme and establishing the reaction farourable to intestinal chigestion. It will invert any cane-sugar, maltose, or lactose, which nay reach the intestine; but it cannot he doubted that some cane-sugar may be absorbed by the stomach, after being inverted by the hydrochloric acid of the gastric juice or by inverting ferments taken in with the food, or on its way through the gastric walls.

Epon the whole no great amount of water is absorbed in the small intestine, or at least the loss is balanced by the gain, for the intestinal contents are as concentrated in the cluodenum as in the ileum. But as soon as they pass beyond the ileo-crecal valve water is rapidly absorbed, and the contents thicken into normal fieces, to which the chief contribution of the large intestine is mucin, secreted by the vast number of goblet cells in its Lieberkïhn's crypts.

Bacterial Digestion.-So far we have paid no special attention to other than the soluble ferments of the digestive tract, althougl we have incidentally mentioned the action of the lactic acid hacilli on carbo-hydrates and of the fat-splitting bacteria - on fats. It is now necessary to recognise that the presence of bacteria is an absolutely constant feature of digestion; and although their action must in part be looked upon as a necessary evil which the organism has to endure, and against the consequences of which it has to struggle, it is not mnlikely that in part it may be ancillary to the processes of aseptic digestion. But bacteria are not essential (in mammals, at any rate, living on milk), as some have supposed. For it has been shown that a young guinea-pig, taken by Caesarean section from its mother's uterus with elaborate aseptic precautions, and fed in an aseptic space on sterile milk, grew apparently as fast as one of its sisters brought up in the orthodox microbic way. The alimentary canal remained free from bacteria (Nuttall and Thierfelder). On the other hand, chickens hatched from sterile eggs and kept in a sterile enclosure lived, indeed, for a time, but did not thrive in comparison with the control animals, and died at latest after eighteen days (Schottelius). It is probable that the difference in the results is to be attributed to the difference in the food, purely regetable food requiring the aid of bacteria for its proper digestion, while an easily-digestible food like milk does not.

Among the more important actions of bacteria on the protein food-products in the intestines may be mentioned the formation of indol, plenol, and skatol, the first having tyrosin for its precursor, and being itself after absorption the precursor of the 
'indican' in the urine; the second being to a small extent thrown out with the faces, but chiefly absorbed and eliminater by the kidneys as an aromatic compound of sulphuric acid; the third passing ont mainly in the faces.

The large intestine is the chosen haunt of the bacteria of the alimentary canal; they swarm in the faeces, and by their influence, especially in the cecum of herbivora, but also to a small extent in man, even cellulose is broken up, the final products being carbon dioxide and marsh gas. A cellulose-dissolving enzyme of great activity is present in the liepatic secretion of the snail, which rapidly produces sugar from cellulose. But in herlivorous mammals no such ferment has been found; and although cellulose can be split up by bacteria in their intestines, sugar is not among the products. In this case the cellulose makes only an insignificant contribution to the metabolism of the animal. The contents of the large bowel are generally acid from the products of bacterial action, although the wall itself is alkaline.

Fæces.-In addition to mucin, secreted mainly by the large intestine, the frecs consist of indigestible remnants of the food, such as clastic fibres, spiral vessels of plants, and in general all vegetable structures chiefly composed of cellulose. They are coloured with a pigment, stercobilin, derived from the bile-pigments. Stcrcobilin is identical with urobilin, which forms a common, though not an invariable, constituent of bile itself. A portion of it is absorbed by the intestine and then excreted in the urine, the urobilin in which is often mucl increased in fever ('febrile' urobilin). No bilirubin or biliverdin occurs in normal freces, although pathologically they may be present. The dark colour of the freces with a meat dict is due to hamatin and sulphide of iron, the latter being formed by the action of the sulphuretted hydrogen which is constantly present in the large intestine on the organic compounds of iron contained in the food or in the secretions of the alimentary canal. A small amount of altered bilcacids and their products is also found; and in respect to these, and to the altered pigments, bile is an excretion. And althougl its entrance into the upper instead of the lower end of the intestine, the ascertained importance of its function in digestion, and the fact that the greater part of the bile-salts is reabsorbed, show that in the adult it is very far from being solely a waste product, the equally cogent fact, that the intestine of the newborn child is filled with what is practically concentrated bile (meconium), proves that it is just as far from being purely a digestive juice. Skatol and otler bodies, formed by putrefactive clianges in the proteins of the food, are also present in the treces, and are responsible for the freal odour. Masses of bacteria are 
invariably present, and often make up a very considerable proportion of the total fecal solids. Of the inorganic substances in faces the numerous crystals of triple phosphate are the most characteristic. When the diet is too large, or contains too much of a particular kind of food, a considerable quantity of digestible material may be found in the fxecs-e.g., muscular fibres and fat. But it should be remembered that under all circumstances the composition of the fieces differs from that of the food. The intestinal contribution is always an important one, although relatively more important with a flesh than with a vegetable diet. The xanthin or purin bases normally found in human freces come both from the food directly and from the metabolism of the tissues. They are increased in amount on a diet rich in purin bodies (such as meat extract or thymus), but are also formed on a cliet like milk, from which xanthin bases cannot be obtained. 


\section{CHAPTER $V^{r}$}

\section{A B S O R P T I O N}

Physical Introduction.-Imbibition, or molecular imbibition, is the term applied to the entrance of liquid into a colloid, without the loss of its properties as a solid, when no preformed capillary spaces are present. The entrance of water into a piece of gelatin, or an epidermic scale, is an example of molecular imbibition. Nost animal and regetable tissues possess this property, which is believed to be of importance in such physiological processes as absorption, secretion, and the excretion of water from the lungs and skin. The process by which liquid passes into a solid with preformed capillary spaces-e.g., a sponge-is sometimes spoken of as capillary imbibition.

Diffusion.--When a solution of a substance is placed in a vessel. and a layer of water carefully run in on the top of it, it is found after a time that the dissolved substance has spread itself through the water, and that the composition of the mixture is uniform throughout. The result is the same when two solutions containing different proportions of the same substance, or containing different substances, are plared in contact. The phenomenon is called difusion. The time required for completc diffusion is conparatively short in the case of a substance like hydrochloric acid or sodium chloride, exceedingly long in the case of albumin or gum. In both it is more rapid at a high temperature than at a low.

Osmosis.- If the solution be separated from water by a membrane absolutely or relatively impermeable to the dissolved substance, but permeable to water, water passes through the membrane into the solution. This phenomenon is called osmosis. E.g., a membrane of ferrocyanide of copper, nearly impermeable to cane-sugar, can be formed in the pores of an unglazed porcelain pot by allowing potassium ferrocyanide and cupric sulphate to come in contact there. If the pot is filled with, say, a I per cent. solution of cane-sugar, closed by a suitable stopper, connected to a manometer, and then placed in a vessel of water, water passes into it till the pressure indicated by the manometer rises to a certain height. With a 2 per cent. solution it reaches twice this height, and in general the osmotic pressure, as it is called, is in any solution proportional to the molecular concentration* of the solution, or, in other words, to the number of molecules of the dissolved substance in a given volume of the solution. If in this sentence we substitute ' gaseous pressure' for 'os-

* The molecular concentration is strictly defined as the number of grammes of the dissolved substance in a litre of the solution divided by the gramme-molecular weight. The gramme-molecular weight, or gramme-molecule, is the number of grammes corresponding to the molecular weight. Thus, the gramme-molecular weight of sodium chloride $(\mathrm{NaCl})$ is $5 \mathrm{~S} .36$ grammes, and of cane-sugar $\left(\mathrm{C}_{22} \mathrm{H}_{22} \mathrm{O}_{11}\right), 342$ grammes. 
motic pressure,' and 'gas ' for ' solution,' we have a statement of Boyle's law, which asserts that the pressure of a gas is proportional to its density. Indeed, it has heen slown that the osmotic pressure of the clissolved substance is the same as the pressure that would be exerted by a gils, say hydrogen, if all the water were removed, and a molecule of ly yllogen substituted for each molecule of the sulsstance, or as would be exerted by the substance itself if, after removal of the solvent, it conld be left as a gils filling the same volmme. And the osmotic pressure of a solntion of one substance is the same as that of a solution of any other substance which contains in a given volmme the same number of molecules of the dissolved substance. In other words, the osmotic pressure is not dependent on the nature, but on the molecular concentration, of the substance. The analogy of the laws of osmotic to those of gaseous pressure becomes still more obvious when it is added that the osmotic pressure of a substance with any given molecular concentration is proportional to the absolute temperature; and that when al solution contains more than one dissolved substance the total osmotic pressure is the sum of the partial osmotic pressures which each substance would exert if it were present alone in the same volume of the solution.

The osmotic pressure of a solution may reach an enormous amount. Thus, a I per cent. solution of cane-sugar has a pressure at $0^{\circ} \mathrm{C}$. of $493 \mathrm{~mm}$. of mercury. A ro per cent. solution of cane-sugar would have an osmotic pressure of more than six atmospheres, and a i 7 per cent. solution of ammonia a pressure of no less than 22.t atmospheres. The manner in which the phenomenon known as osmotic pressure is developed is not definitely known. One theory attributes it to the attraction between the dissolved molecules and the molecules of the solvent on the other side of the membrane. The most commonly accepted view is that the osmotic pressure is due to the kinetic energy of the moving molecules. Where the molecules are hindered from passing a bounding membrane, the pressure exerted by their impacts on the boundary is greater than where the membrane is easily perncable, because in the latter case many of the molecules pass through, carrying with them their kinetic energy. The pressure must be still less when

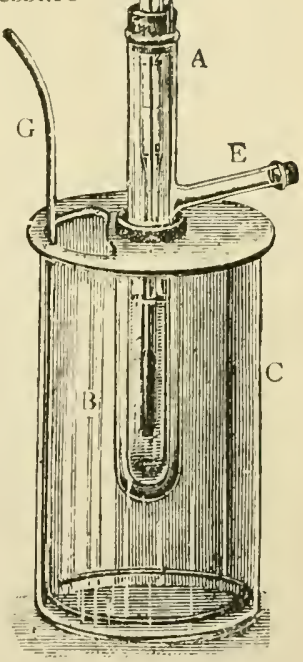

FIG. 153.-BЕСКмANN's Apparatus.

For description, see p. 492 . a dissolved substance diffuses freely into water; but however small it may become, it is in the same force which gives rise to the osmotic pressure of the molecules of the dissolver substance that the cause of diffusion must be sought. Recently interest in the nature of the nrembrane itself as an important factor in osmosis has been revived (Kahlenberg, Armstrong, etc.). There are many facts which indicate that in physiological processes the affinity of the dissolved substances for, or their solubility in, the cell envelopes or the cytoplasm plays an important rôle.

It is as yet impossible to directly measure the osmotic pressure with accuracy by means of a semi-permeable membrane like ferrocyanide of 
copper. Recourse is therefore had to indirect methods, especially one which depends on the fact that the freezing-point of a solution is lower than that of the solvent, salt water, e.g., freezing at a lower temperature than fresh water. The amount by which the freezing-point is lowered depends on the molecular concentration of the dissolved substance, to which, as we have seen, the osmotic pressure is also proportional. When a gramme-molecule of a substance is dissolved in water, and the volume made up to a litre, the freezing-point is lowered by $1.86^{\circ} \mathrm{C}$.; the osmotic pressure is $22^{\circ} 35$ atmospheres (16,986 mm. of mercury). It is therefore easy to calculate the osmotic pressure of any solution if we know the amount by which its freezing-point is lowered. A I per cent. solution of cane-sugar, for example, would freeze at about $-0^{\circ} 054^{\circ} \mathrm{C}$. Its osmotic pressure $=\frac{0.054}{I^{\circ} 68} \times 16,986=493 \mathrm{~mm}$. of mercury.

A convenient apparatus for making freezing-point measurements is shown in Fig. 153. The details of the metlod are given in the Practical Exercises. p. 492.

The osmotic pressure of different solutions may also be compared by observing the effect produced on certain vegetable and animal cells. When a solution with a greater osmotic pressure than the cell-sap (a hyperisotonic solution) is left for a time in contact with certain cells in the leaf of Tradescantia discolor, plasmolysis occursthat is, the protoplasm loses water and shrinks away from the cellwall. If the osmotic pressure of the solution is lower than that of the coloured cell-sap (hypoisotonic solution), no shrinking of the protoplasm takes place. By using a number of solutions of the same substance but of different strength, two can be found, the stronger of which causes plasmolysis, and the weaker not. Between these lies the solution which is isotonic with the cell-sap-that is, has the same molecular concentration and osmotic pressure. The strength of an isotonic solution of some other substance can then be determined in the same way with sections from the same leaf.

Animal cells (red blood-corpuscles) may also be employed, the liberation of hrmoglobin or the swelling of the corpuscles, as measured by the hæmatocrite (p. 59), being taken as evidence that the solution in contact with them is hypoisotonic to the contents of the corpuscles. Here we may suppose that the impacts of the molecules of the salts of the corpuscle on the inside of its envelope, not being balanced by similar impacts on the outside, tend to distend it, and thus to create a potential vacuum for the surrounding water, which accordingly enters. If the corpuscles shrink, the solution is hyperisotonic to their contents. But since the cells are much more permeable to certain substances than to others, this method does not always yield trustworthy results.

Electrolytes.-We have said that the osmotic pressure is proportional to the concentration of the solution, but this statement must now be qualified. For certain compounds, including all inorganic salts and many organic substances, the osmotic pressure decreases less rapidly than the theoretical molecular concentration as the solution is diluted. The explanation is that in solution some of the molecules of these bodies are broken up into simpler groups or single atoms, called ions. Each ion exerts the same osmotic pressure as the molecule did before. The proportion between the average number of these dissociated molecules and of ordinary molecules is constant for a given concentration of the solution and a given temperature. But as the solution is diluted, the proportion of 
dissociated molecules becomes greater. The bodies which behave in this waly are electrolytes - that is, their solutions conduct a current of clectricity: bodies which do not exhibit this behaviour do not conduct in solution. And there are many reasons for believing that the dissociation of the electrolytes is the essential thing in electrolytic conduction. WV may suppose that in a solution of an electrolytesodium chloride, for instance-a certain number of the molecules fall asunder into a kation ( $\mathrm{Na})$, * carrying a cluarge of positive electricity, and an anion $(\mathrm{Cl})$, carrying an equal negative charge. These electrical charges, it must be remembered, are not created by the passage of a current through the solution. We do not know how they arise, but the ions must be supposed to be electrically charged at the moment when the molecule is broken up. And the ions of different substances must each be supposed to carry the same quantity of electricity. But since they are all wandering frecly in the solution, no excess of negative or of positive electricity can accumulate at any part of it-in other words, no difference of potential can exist. When clectrodes connected with a voltaic batterv are dipped into a solution of an electrolyte, a difference of potential, an electrical slope, is established in the liquid, and the positively charged kations are compelled to wander towards the negative pole, the negatively charged anions towards the positive pole. In this way that movement of electricity which is called a current is maintained in the solution. It is clear that the greater the number of ions, and the faster they move in the solution, the greater will be the quantity of electricity carried to the electrodes in a given time, when the difference of potential between the electrodes, or the steepness of the electric slope, remains constant. In other words, the specific conductivity of a solution of an electrolyte varies as the number of dissociated molecules in a given volume and the speed of the ions. It increases up to a certain point with the concentration, because the absolute number of dissociated molecules in a given volume increases. The molecular conductivity - that is, the conductivity per molecule, or, strictly, the ratio of the specific conductivity to the molecular concentration, increases with the dilution, because the relative number of dissociated molecules, as compared with undissociated, increases. At a certain degrec of dilution the molecular conductivity reaches its maximum, for all the molecules are dissociated. The ratio of the molecular conductivity of any given solution to this maximum or limiting value is therefore a measure of the proportion between the number of dissociated, and the total number of molecules. The molecular conductivity of the salts dissolved in the liquids of the animal body, for the degree of dilution in whicin they exist there, is such that we must assume them to be for the most part dissociated.

Absorption of the Food.-In the preceding chapter we have traced the food in its progress along the alimentary canal, and sketched the changes wrought in it by digestion. IVe have next to consider the manner in which it is absorbed. Then, for a reason which has already been explained, instead of following its fate within the tissues, until it is once more cast out of the

* J. J. Thomson has shown that the chemical atoms must be assumed to consist of smaller corpuscles or particles of electricity called electrons. 
body in the form of waste products, it will be best to drop the logical order and pick up the other end of the clue-in other words, to pass from alssorption to excretion, from the first step in metabolism to the closing act, and afterwards to return and fill in the interval as best we can.

Comparative.-And here, first of all, it should be remembered that the epitlielial surfaces, through which the substances needed by the organism enter it, and waste products leave it, are, physiologicallv consiclered, outside the body. The mucous membranes of the alimentary. respiratory, and urinary tracts are in a sense as much external as the fourth great division of the physiological surface, the skin. The two latter surfaces are in the mammal purely excretory. Absorption is the dominant function of the alimentary mucous membrane, but a certain amount of excretion also goes on through it. The pulmonary surface both excretes and absorbs, and that in an equal measurc. But it is by no means necessary that the surface through which

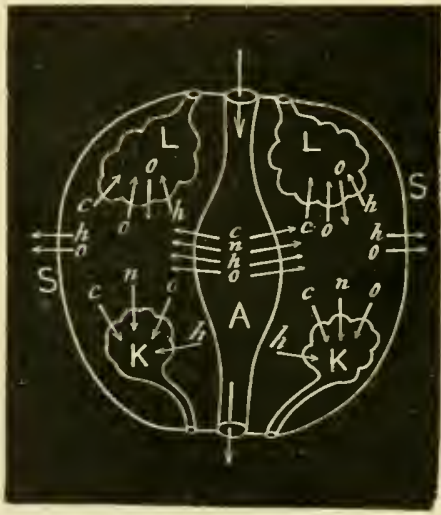

Fig. Ij1.-DiAgRAM OF ABSORP. TION AXD EXcRETION.

Carbon $c$, nitrogen $n$, hydrogen $h$, and oxygen $o$. L represents the pulmonary surface ; $K$, the surface of the renal epithelium; $A$, the alimentary canal; S, the skin. oxgyen is taken in and gaseous waste products given off should be buried deep in the body, and communicate only by a narrow channel with the exterior. In the frog the skin is largely an absorbing as well as an excreting surface; oxygen passes freely in through it, just as carbon dioxide passes freely out. In most fishes, and many other gillbearing animals, the whole gaseous interchange takes place through surfaces immersed in the surrounding water, and therefore distinctly external. In certain forms it has even been shown that the alimenttary canal may serve conspicuously for absorption and excretion of gaseous, as well as liquid and solid substances. Still lower down in the animal scale, the surface of a single tube may perform all the functions of digestion, absorption and excretion. Lower still, and even this tube is wanting, and everything passes in and out through an external surface pierced by no permanent openings.

Indeed, even in man the functions of the various anatomical divisions of the physiological surface are not quite sharply marked off from each other. Though gaseous interchange goes on far more readily through the pulmonary membrane than anywhere else, swallowed oxygen is easily enough absorbed from the alimentary canal and carbon dioxide given off into it ; and to a small extent these gases can also pass through the slin. Though water is excreted chicfly by the skin, the pulmonary and the urinary surfaces, and on the whole absorbed chiefly from the digestive tract, there is no surface which in the twenty-four hours pours out so much water as the mucous membrane of the stomach. Under normal conditions, it is true, by far the greater part of this is reabsorbed in the intestine, 
vet in diarrhea, whether natural or caused by purgatives, the intestines thenselves maly, insteal of absorbing, contribute largely to the excretion of water. Again, althougl the solicls of the excreta are normally given off in far the greatest quantity in the urine and faeces (only part of the latter is truly an excretion, since much of the feeces of a mixed diet hats never been physiologically inside the body at all), yet salts and traces of urea are constantly found in the sweat, and salts and nuncin in the excretions of the respiratory tract. Further, although the solicls and liquids of the food are usually taken in by the alimentary nutcons surface, it is possible to cause substances of both kincls to pass in through the skin; and a certain amount of absorption may also take place through the urinary bladeler. So that really it may be considered, from a physiological point of view, as more or less an accident that a man should absorb his food by (lipping the villi of lis intestine into a digested mass, rather than by dipping his fingers into properly prepared solutions, as a plant dips its roots among the liquids and solids of the soil; or that he should draw air into organs lying well in the interior of his thorax, instead of letting it play over special thin and highly vascular portions of his skin; or that the surface by which he excretes urea should be buried in his loins, instead of lying frce upon his back.

It has been already explained that, although digestion is a necessary preliminary to the absorption of most of the solids of the food, we are not to suppose that all the food must be digested before any of it begins to be absorbed. On the contrary, the two processes go on together. As soon as any peptone, or, at least, any amino-acids, have been formed from the proteins, or any dextrose from the starch, they begin to pass out of the alimentary canal ; and by the time digestion is over, absorption is well advanced.

Even in the month it has already begun, although the amount of absorption here is quite insignificant, and it is continued with greater rapidity in the stomach. Here a not inconsiderable part of the proteins - at least, in the easily digested form of animal food - a certain amount of the sugar representing the carbo-hydrates and diffusible substances like alcohol, and the extractives of meat, which form an important part of most thin soups and of beef-tea, are undoubtedly absorbed. Water is very sparingly taken up by the stomach. It is in the small intestine that absorption reaches its height. The mucous membrane of this tube offers an immense surface, multiplied as it is by the valvulæ conniventes, and studded with innumerable villi. Here the whole of the fat, much sugar, proteose and peptone, or rather the products of the further action of the ferments of the intestine on these derivatives of the native proteins, and certain constituents of the bile are taken in. In the large intestine, as has been already said, water and soluble salts are chiefly absorbed.

What now is the mechanism by which these various products 
are taken up from the digestive tube, and what patlis do they follow on their way to the tissues?

Theories of Absorption. - Not so very long ago it was supposed by many that the processes of diffusion, osmosis and filtration offered a tolerably complete explanation of physiological absorption. At that time: the dominant note of physiology was an eager appeal to chemistry and physics to 'come over and help it '; and as new facts were discovered in these sciences they were applied, with a confidence that was almost naive, to the problems of the animal organism. The phenomena of the passage of liquids and lissolved solicls through animal membranes, upon which the work of Gralnam had cast so much light, seemed to find their parallel in the absorptive processes of the alimentary canal. And when digestion was more decply studied, facts appeared which seemed to show that its whole drift was to increase the solubility and diffusibility of the constituents of the food. But as time went on, and more was learnt of the phenomena of absorption and the powers of cells, these crude physical theories broke down, and discarled 'vitalistic' hypotheses began once more to arouse attention. Then came the investigations of De Vries, Van 'T Hoff, and others in the domain of molecular physics, which gave to our notions of osmosis the precision that was wanted before its relation to many physiological processes could be profitably discussed. At the present time it must be arlmitted that we possess no explanation of absorption which is more than a confession of ignorance, and does not itself need to be explained. Some physiologists, impressed with the vast progress of physics and chemistry, believe that it will eventually become possible to explain on mechanical and chemical principles all the peculiar phenomena which we observe in the passage of substances through the walls of the alimentary canal. Others, taking account of the number and nature of these peculiarities, oppressed with the perennial parados of vital action, incline to the less sanguine view, that after all physical explanations have been exhausted, the real secret of the cell will still lurk in some ultimate 'vital' property of structure or of function, and still elude our search. Both the optimist and the pessimist, the adherent of the physical ari the adherent of the vitalistic hypothesis, admit that the phenomena of absorption are essentially connected with the cells that line the alimentary canal. But the one must confess what the other proclaims, that while the processes carried on in these cells are definite, well ordered, and evidently guided by laws, these laws have as yet denied themselves to the modern physiologist, with chemistry in one hand and physics in the other, as they denied themselves to his prealecessor, equipped only with his scalpel, his sharp eyes, and his mother-wit. So that in the present state of our knowledge all we can really say is that, while absorption is certainly aided by physical processes, like osmosis and cliffusion, possibly by physical processes like imbibition, it is at bottom the work of cells with a selective power which we do not understand. Thus, dissolved substances pass with equal case in either direction through an ordinary diffusion membrane, but in general they pass, with the water in which they are dissolved, more readily out of the intestine than into it. This normal clirection of the stream is still maintained for a considerable time after stoppage of the circulation, provided that the intestine is kept in good condition by being suspended in well-oxyzenated blood. IVater or 
solutions of sodium chloricle or sugar disappear from the lumen. And this is not wue to mere imbibition by the intestinal wall, but the liquid is actually transported across it. The theory that liquirls might be taken up from the gut by imbibition, and the water then mechanically removed by the blood flowing on the other side of the imbibing cells, is incompatible with this experiment (Cohnheim). When the cells that line the intestine are injured or clestroyed, or subjected to the action of certain poisons, absorption from it is diminished or abolished. And in their normal state they (lo not take 11p indiscriminately all kinds of cliffusible substances, or absorb those which they do take 11 in the direct ratio of their liffusibility. Nor do they reject everything which does not diffuse. Albumin, for example, which does not pass through dead animal membranes, is to a certain extent taken up from a loop of intestine without change. Cane-sugar (after inversion) and dextrose are absorbed more rapidly than their velocity of diffusion would indicate, when compared with inorganic salts. Glauber's salt diffuses in water fiftecn times as fast as cane-sugar, but cane-sugar is absorbed from the intestines ten times faster than Glauber's salt. The velocity of absorption is (lifferent even for simple stereoisomeric sugars-i.e., sugars whose molecule, with the same number of atoms combined in the same way, has a different form (Nagano). Nor is there any clear relation between the rate of absorption of the various sugars and their osmotic pressure. Dextrose and cane-sugar are always absorbed in greater amount than lactose from solutions of the same osmotic pressure. Indeed, as we shall sec, lactose is practically not taken up at all as such (p. $4^{16}$ ), and in concentrated solutions may even cause a reversal of the normal movement of water, and act as a purgative. Even the water, organic and inorganic solids of the serum of an animal, are absorbed from a loop of its intestine when the bloodpressure in the capillaries of the intestinal wall is considerably greater than the pressure in the cavity of the gut. Since the serum in the intestine and the plasma in the capillaries must be isotonic, and practically identical in chemical composition, the absorption cannot be clue to ordinary osmosis or diffusion. Nor can it be due to filtration, since the slope of pressure is from the capillaries to the lumen of the gut (Reid). It is therefore extremely difficult to reconcile this experiment with any purely physical theory of absorption. The same investigator, summing up the result of careful experiments on the absorption of weak solutions of glucose, concludes that "with the intestinal membrane as normal as the experimental procedure will permit, phenomena present themselves which are as distinctly opposed to a simple physical explanation as those previously studied in the absorption of serum.'

But if it be true that the action of the columnar epithelium of the intestinal mucous membrane is secretory and selective, making use of purcly physical processes, but not mastered by them, the possibility must be admitted that in the cells of endothelial type which line the serous cavities, the lymphatics, the bloodvessels, the alveoli of the lungs, and the Bowman's capsules of the kidney (p. 452$)$, the clement of secretion may be less marked, and more overshadowed by the physical factors. And it may very plausibly be urged that changes of considerable physiological complexity can only be wrought on substances that have to pass through a cell of considerable depth, while a mere film of protoplasm suffices for, and indeed favours, mechanical filtration and diffusion. IVe have already seen (p. $25^{8}$ ), in the case of the lungs, that whatever the 
complete explanation may be of the gascous exchange which takes place through the alveolar membrane, physical diffusion undoubtedly plays an important part. IVe shall see, too (p. 466$)$, that in the case of the kidney the endothelium of the Bowman's capsule. althongh by no means devoid of selective power, does seem to have allotted to it a simpler task than falls to the share of the 'rorlded ' epithelium.

Absorption from the Peritoneal Cavity.--Furtler, it has been stated that interchange between blood-serum, circulated artificially in the vessels of dogs and rabbits which have been dead for hours, and liquids introduced into the peritoneal cavity, is essentially the same as in the living animal, and can be explained on purcly physical principles (Hamburger). But there is one experiment, at any rate, which is certainly difficult so to explain - ri\%, the absorption from the peritoneal cavity of sodium chloride solution isotonic with the bloodserum, an absorption which goes on with considerable rapidity. Starling has supposed that this is due to the circumstance that the proteins of the serum exert osmotic pressure, the peritoneal membrane being almost or altogether impermeable for them in comparison to its permeability for the salt solutions. In consequence, water passes into the bloodvessels from the peritoneal cavity. The solution thus becomes more concentrated as regards sodium chloride, some of which accordingly enters the blood by diffusion, and so on. But even isotonic serum is absorbed from the peritoneal cavity, and it seems to savour of special pleading to suggest, as has been done. that this takes place through the lymphatics, and not at all through the bloodvessels.

Up to a certain point an increase in the intraperitoneal pressure favours absorption, but beyond this it hinders it by interfering with the circulation. The removal of a portion of the fluid in this condition facilitates the absorption of the rest-a fact which has long been applied in the operation of tapping. Ligation of the thoracic duct has little effect on the fate of liquids injected into serous cavities. since the bloodvessels play the chief part in their absorption, just as strychnine, when injected under the skin-i.e., into the lympl spaces of areolar tissue-is taken up by the blood and does not appear in the lymph.

But eren if we admit that substances can pass, by physical processes alone, from serous cavities in to the blood, and from the blood into serous cavities, this has little bearing upon the question of intestinal absorption. For we can liardly put anything into the peritoneal cavity which is not foreign to it. It was never intencled to come into contact with the hundred and one solutions, extracts. suspensions, and what not, which the industrious experimenter has offered to its unsoplisticated endothelium. It cannot possibly liave developed any high degree of 'selective' power. In the intestine everything is different. The mucosa is adapted to come into contact with an inmense varicty of materials, all kinds of footsubstances iningled witl many kinds of refuse, the products of the action of numerous digestive ferments, and of a vigorous and varied bacterial flora. All these it has to sift and try. It cannot fail to have properties which suggest a severe and searching sclection.

Formation of Lymph.-Closely connected with the question of absorption from and secretion (or transudation) into the serous 
cavities is the question of the factors concerned in the formation of the lymph, even although recent researches throw grave doubt on the common view that these sacs are merely expanded lymph spaces, and indicate that the liquid found in them has a different origin from lymph. We ought to distinguish the lymph as we collect it from the great lymphatic trunks, not only from the liquids of the serous cavities, but still more sharply from the liquid which fills the multitudinous clefts and spaces of the tissues. It is now pretty definitely established that the tissue spaces do not communicate by actual passages with the lymphatic vessels, but that the latter form everywhere a closed system like the blood-vascular system, the lymph capillaries merely lying in the tissue spaces (WV. G. MIcCallum, etc.). This conception entails a radical change in the current views of lymph production. If the lymphatics form a closed system, the lymph cannot be actual tissue fluid, but only tissue fluid modified by its passage through the walls of the lymph capillaries, just as tissue fluid is not actual blood-serum, but serum modified by its passage through the walls of the blood capillaries.

Although it is customary to speak of the lymph obtained from the lymphatic vessels as if it were perfectly homogeneous, is no experimental ground for supposing that the lymph from different tracts, or the tissue liquid in contact with the cells of different organs, or even the tissue liquid in contact with one and the same cell at different parts of its periphery, has a uniform composition, or even a uniform molecular concentration. There are indeed certain general considerations which show that this cannot be so.

The teaching of Ludwig, that lymph is formed by the filtration, and in a minor degree, diffusion, of the constituents of bloodplasma through the walls of the capillaries into the tissue spaces, was based on such facts as the increase in the tissue liquid of a limb or organ which occurs when the exit of blood from it by the veins is hindered, or when the quantity of the circulating liquid is increased by the injection of blood or salt solution. It was first seriously called in question by Heidenhain, who advanced the theory that lymph is secreted by the endothelium of the blood capillaries. One of Heidenhain's strongest arguments in favour of his secretion theory was the existence of substances which, when injected into the blood, increased the flow of lymph from the thoracic duct of the dog without affecting appreciably the arterial pressure. He divided these so-called lymphagogues into two classes: (I) substances like peptone, extracts of the head and liver of the leech, extract of crayfish muscle, egg-albumin, etc., which cause not only an increase in the rate of flow, but an increase in the specific gravity and total solids of the lymph; 
(2) crystalloid sulustances, like sugar, salt, etc., which cause an increased flow of lymph more watery than normal.

Starling has shown that although the lymphagogues of the second class do not raise the arterial pressure, they 10 , hy attracting water from the tissues and thus causing hydramic plethora (an excess of blood of low specific gravity), bring about a marked rise of venous, and therefore, what is the important thing for lympli filtration, of capillary pressure. But it can be demonstrated that vaso-dilatation with increase of capillary pressure is not in itself sufficient to increase the formation of lymph. We have seen, e.g. (p. I 63 ), that when the chorda 1 ympani nerve is stimulated in the dog the arterioles of the submaxillary gland

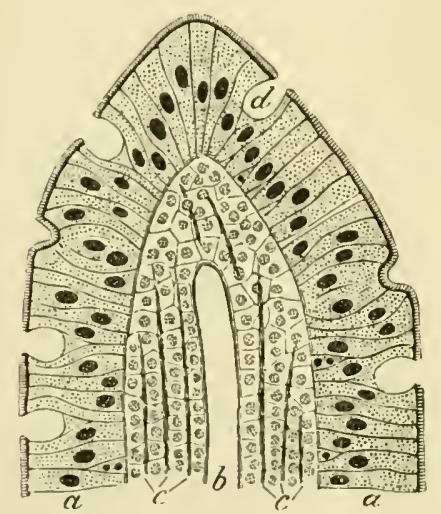

Fic. I55.-VERTICAL SEction of a VillLe: CAT. $\times 300$.

a, laver of columnar epithelium covering the villus - the outer edge of the rells is striated; $b$, central lacteal of villus ; $c$, unstriped mus. cular fibres; $d$, mucin - forming goblet-cell.

stabstances necessary to sustain the increased secretion. But a balance is maintained between the production of tissue liquid and its removal by the gland-cells. When the gland is quiescent. the small amount of tissue liquid normally formed from the blood capillaries for the nutrition of the cells is balanced by, upon the whole, an equal amount of lymph secreted from the tissue spaces into the Jymph capillaries.

We may say, indeed, that the closed lymphatic system has for its great function the regulation of the quantity and quality of the tissue liquid. In glands with an external secretion increased irrigation of the tissue spaces from the blood does 110 , as a rule, lead to increased flow of lympl, hecause the surplus 
fluid is required to form the secretion. In other organs, lowrerer such as the muscles and the ductless glands, it is probable that the angmented irrigation rendered necessary by functional activity is always associated with an accelerated flow of lymph, which carries off the surplus liquid, including a portion of the waste products. It is possible that an important factor in the production of nedema may be the derangement of the mechanism. whatever it is, through which the adjustment of the rate of formation of tissue liquid to that of lymphatic lymph is achiered. But it must be remembered that in all the organs the blood capillaries not only supply materials to the tissue spaces. but take up) materials from them. So that, while the lymphatics constitute an important drainage system, the bloodressels irrigate the tissnes and drain them as well.

A mere increase in the capillary blood-pressure does not of itself accelerate the formation of tissue liquid from the blood any more than that of lymph from the tissme liquid, as is shown by the fact that, when the chorda tympani is stimulated after injection of a dose of atropine sufficient to prevent all salivary secretion, there is neither cedema of the gland nor increase in the flow of lymph from it, although the arterioles are as widely dilated as before. Further, after division or embolism of the medulla oblongata, and consequent paralysis of the vaso-motor centre and general rascular dilatation, it is stated that the injection of sodium chloride produces an increase in the lymph-flow as great and as dirable as in the normal animal, and which can continue even after death (Pugliese). The action of the first class of lymphagogues, which cannot be explained as the consequence of an increase of capillary pressure, because the pressure in the capillaries is not consistently increased, and may even in the case of some of these lymplragogues be diminished, Starling attributes to an injurious effect on the capillary endothelium (and especially on the endothelium of the capillaries of the liver, since nearly the whole of the increased lymph-flow comes from that organ), which increases its permeability. But it is not easy to distinguish an increase of permeability produced by lymplagogues from an increase of secretory activity of the endothelial cells.

Hamburger, too, has brought forward results which it is difficult to reconcile with a theory of filtration even for the second class of lymphagogues. Further, Heidenhain has shown that some time after injection of a crystalloid substance, like sugar, into the blood, a greater percentage of the substance may be found in the lymph than in the blood. Now, when a mixture of crystalloids and colloids is filtered through a thin membrane. the percentage of crystalloids in the filtrate is never, at most, greater than in the original liquid. And although Cohnstein 
states that if time enough be allowed, the maximum concentration of sodiun chloride in the lymph, after intravenous injection, becomes approximately the same as the maximum in the blood, this fact loses its weight as an argument in favour of the filtration hypothesis when we remember that, according to Asher. all the solids of the lymph are markedly increased when even small quantities of crystalloids are injected into the veins. Nor is it at all easier to explain lymph formation as a matter of osmosis or diffusion combined with filtration. Lazarus-Barlow found, for example, that in the great majority of his experiments the injection of a concentrated solution of sodium chloride, dextrose or urea into a vein was followed, not by an initial diminution in the outflow of lymph (as might have been expected if the exchange of water between the blood and the tissue spaces. and between the tissue spaces and the lymph capillaries, was regulated solely by differences in osmotic pressure), but by an immediate increase. And Carlson has shown that the osmotic pressure of lymph coming from the active salivary glands, as measured by the freezing-point method, may, under chloroform or ether anæsthesia, be distinctly less than that of the bloodserum. Water must therefore be passing from a liquid of higher to one of lower osmotic concentration.

So far we have considered the passage of the lymph constituents, on the one hand through the endothelium of the blood capillaries into the tissue spaces; on the other, from the tissuc spaces through the endothelium of the lymph capillaries. But it is not to be supposed that the liquid lying in clefts, partly bounded by blood capillaries, partly by lymph capillaries, partly by tissue-cells, should be affected solely by the first two. The third anatomical element must contribute something to, or withdraw something from, the tissue liquid, and may thus play a part in the formation of lymph from the latter. The recent researches of Asher and his pupils have raised the question of the relation between the physiological activity of the organs, and especially of the glands, and the formation of the lympli. They conclude that the common doctrine that lymph is simply a diluted blood-plasma is erroneous. Lymph, they say, far from being a mere filtrate or even a secretion from the blood, is formed by the activity of the organs, and may actually be absorbed by the blood from the tissue spaces. In fact, according to their view, the intravenous injection of lymphagogues, both crystalloid and colloid, only causes an increased flow of lymph in so far as it leads to increased glandular secretion. But this generalization has had only a short-lived vogue, and one by one the results which seemed to support it have been disproved. For example, it was stated that secretin causes a flow of lymph from 
the lymphatics of the pancreas, as well as a flow of pancreatic juice. But it has been showr that the increased production of lymph is not due to the secretin at all, but to lymphagogue substances, including albumose, extracted with the secretin from the intestinal mucous membrane. A solution of secretin can be prepared which causes a considerable increase in the secretion of pancreatic juice and bile, but no augmentation whatever in the flow of lymply from the thoracic duct. Again, it was asserted that peptone, a noted lymphagogue, produces a great increase in the biliary secretion. It has been demonstrated, however, that the action of the peptone is merely to cause expulsion of the contents of the gall-bladder by the mechanical effect of the swelling of the liver, and not at all to stimulate the liver-cells to form more bile. For it produces no effect on the flow of bile if the gall-bladder be emptied or the cystic duct tied before the injection (Ellinger). That active salivary secretion is not accompanied by increased lymph-flow from the lymphatics of the salivary glands has been mentioned abore. Nevertheless, we may safely assume that the activity of the organs does make a contribution to the lymph-to its solids. if not in any important degree to its water-content, although to say that they alone are concerned in its formation, to the exclusion of the capillaries, is altogether an over-statement. The waste-products of the tissues pass into the lymph, and possibly, as Koranyi suggests, may, by increasing its molecular concentration, cause the passage of some water into it from the blood. Or the decomposition of the large protein molecules. which in tissue metabolism are breaking down into numerous smaller molecules, may entail an increase of osmotic pressure in the cells themselves, which in turn may lead to withdrawal of water by the cells from the tissue liquid. The osmotic pressure of the liquid may thus rise, and water may pass into the tissue spaces from the blood. The molecular concentration of lymph (except in anæesthetized animals as mentioned above) is in general somewhat greater than that of blood-serum-e.g., in one observation $\Delta$ of serum was $0.605^{\circ} \mathrm{C}$., and of lymph $0.610^{\circ} \mathrm{C}$. For the solid tissues, the freezing-point of which, however, cannot be as satisfactorily determined as that of liquids, the following values of $\Delta$ were obtained: Brain, $0.65^{\circ}$; muscle, $0.68^{\circ}$; kidney, $0.94^{\circ}$; liver, $0.97^{\circ}$; while for blood it was $0.57^{\circ}$ (Sabbatani).

To sum up, we may say that while the physical processes of filtration, osmosis and diffusion may play a part in the passage of water and solids through the walls of the blood capillaries, as well as from the tissue-cells into the tissue spaces, and from these spaces into the lymph capillaries, there is much which they leave mex- 
plained, and athich at present, for the a'ant of a more precise term a'c must altribute to secretory activity.

Although no definite lymph-secretory nerve-fibres have as yet been discovered, it is possible that they exist (Sihler). As already pointed out, the same volume of liquid as escapes into the ducts of the active submaxillary gland must, upon the whole, pass out of the blood capillaries. On what principle shall we distinguish one only of these processes as physiological secretion? They begin together when the chorda tympani is stimulated. A drug which paralyzes secretory nerve-endings abolishes both effects. The simplest explanation is that the chorda contains secretory fibres which influence the formation both of saliva and of the tissue liquid from which it is recruited; and, so far as this consideration goes, it is just as logical to consider the increase in the supply of tissue liquid as the cause of the increase in the flow of saliva as to consider the increased salivary secretion as the cause of the increased flow of liquid into the tissue spaces. The increased flow of liquid may be brought about either by an action of the nerve on the gland-cells, causing them to produce a hormone, which then affects the blond capillaries (Carlson), or by a direct action on the capillary endothelium. The advantage to cells engaged in the active secretion of saliva of being immersed in an abundant bath of tissue liquid is obvious. The postmortem flow of lymph, which may continue in some cases long after complete cessation of the circulation-for an hour after injection of dextrose to produce hydræmic plethora; for as much as four hours after injection of extract of the strawherry, which is a lymphagogue of Heidenhain's first group (Mendel and Honker) - is a phenomenon whose relation to normal lympls formation has not been definitely settled.

It ought to be remembered in this whole discussion that the epithelium of ordinary glands derives its supplies of material from the tissue lymph. The vicissitudes of blood-pressure affect it only in a secondary and indirect manner. On the other hand, the endothelial cells of the capillaries are in direct contact with the blood. And it is interesting to observe that in this respect the glomeruli of the kidney and the alreoli of the lungs (if the endothelial lining of Bowman's capsule and the alveolar membrane are assumed to be complete) take a middle place between the glands proper and the quasi-glandular capillaries.

Absorption of Fat.- - It has been already mentioned that fat is split up in the intestine into glycerin and fatty acids, but it has been a subject of discussion whether it all undergoes this change or only a portion of it. The common view has long been that the greater part of the fat escapes decomposition, and, after emulsification by the soaps formed from the liberated fatty 
acids, is absorbed as neutral fat by the eputhelial cells covering the villi. If an animal is killed during digestion of a fatty meal, these cells are found to contain globules of different sizes, which stain black with osmic acid, are dissolved ont by ether leaving vacuoles in the cell substance, and are therefore fat (Fig. I 56). It has always been difficult to explain how droplets of emulsified fat could get into the interior of the epithelial cells, although, perhaps, no more difficult than to explain the passage of living tubercle bacilli from the contents of the intestine into the chyle of the thoracic duct - a fact which has been clearly demonstrated (Ravenel). The fat certainly passes into the cells, and not between then. When fat is found in the cement substance between the cells, it has been mechanically squeezed out of them by the shrinking of the villi in preparation. This difficulty is obviated if we suppose that the whole of the fat is split up in the intestine, the products being absorbed in solution, the glycerin as such, and the fatty acids either as soaps or in the free state, or partly free and partly saponified. If this is the true theory-and the evidence of its truth has of late years been continually growing - neutral fat must again be built up in the epithelial cells from the absorbed glycerin and the fatty acids or soaps. Now, it has been shown that when an animal border; $C$, lymph corpuscles; $l$, lacis fed with fatty acids they are teal.

not only absorbed, but appear as

neutral fats in the chyle of the thoracic duct, having combined with glycerin in the intestinal wall; and the epithelial cells contain globules of fat, just as they do when the animal is fed with neutral fat. Further, it is known that fat-splitting goes on in the alimentary canal to a much greater extent than would be necessary merely for the formation of a quantity of soap sufficient to emulsify the whole of the fat in the food. Indeed, at certain stages of digestion most of the fatty material, both in the small and large intestine, has been found to consist of fatty acids. To clinch the matter, it has been proved that when mixtures of paraffin and fat, which can be emulsified in a watery solution of sodium carbonate, are eaten, the paraffin is completely excreted with the freces, while the greater part of the fat is absorbed. And fatty substances which are not easily split up and saponified (for example, lanolin, the fat of sheep's wool, 
a mixture of compounds of fatty acids with cholesterin and allied bodies) are not absorbed even when they are easily emulsified. Even fats with a melting-point lar above the temperature of the hody can be absorbed after being split up. The palnitate of cetyl alcohol, the chief constituent of spermaceti, melting at $53^{\circ} \mathrm{C} .$, was absorbed to the extent of I 5 per cent., 85 per cent. being excreted in the freces. It appeared as palmitin in the chyle of a human being flowing from a fistula, the palmitic acid having been absorbed as such, or as a sodium soap, and having then united with glycerin to form the neutral fat, palmitin.

Some observers have endeavoured to show that the fat is absorhed without change by introducing into the intestine fat stained with dyes, such as alkanna red or Sudan 111 ., which are insoluble in water. The stained fat was found in the epithelial cells of the villi, in the lacteals, and, in the case of a patient suffering from chyluria, in the urine. But this evidence is not conclusive, for it has been shown that the pigments might easily have been absorbed after decomposition of the fat, since, although insoluble in water, they are soluble in fatty acids, and therefore to some extent in the intestinal contents, and readily pass into the lymph.

As already pointed out, the bile plays an important part in the solution of the fatty acids, which may form loose compounds with the anide group of the bile-acids. In these loose combinations, soluble in water, the fatty acids can be absorbed from the intestinal contents (Plliger).

Leucocytes have been asserted to be the active agents in the absorption of fat. They have been described as pushing their way between the epithelial cells, fishing, as it were, for fatty particles in the juices of the intestine, and then travelling back to discharge their cargo into the lymph. This view, however, is erroneous. But, although the leucocytes do not aid in the absorption of fat from the intestine, they appear to take it ujs from the epithelial cells, conveying it through the spaces of the network of adenoid tissue that occupies the interior of the villus, to discharge it into the central lacteal, where it mingles with the lymph and forms the so-called molecular basis of the chyle. A part of the fat reaches the lacteal in another way. The contraction of the smooth muscular fibres of the villus and the peristaltic movements of the intestinal walls alter the capacity of the lacteal chamber, and so alternately fill it from the lymph of the adenoid reticulum, and empty it into the lymphatic vessel with which it is connected. By this kind of pumping action the passage of fat and other substances into the lymphatics is aided. In the dog most of the fat goes into the lacteals, and thence by the general lymph-stream through the tharacic duct into the blood. And in man the chyle collected from a 
lymplatic fistula contained a large proportion of the fat given in the food (Munk). But this bare statement woukl be misleading if we clicl not add that the fat taken in can never be entirely recovered in the chyle collected from the thoracic duct. A small fraction of the deficit might be accounted for as fat directly used up for the nutrition of the intestinal wall itself. But even after ligation of the thoracic and right lympliatic ducts a large proportion of a meal of fat (32 to $4^{8}$ per cent.) is absorbed from the intestine, obviously by the channel of the bloodvessels, since the fat-content of the lolood increases up to, it may be, six times the highest amount present in the blood of fasting animals. The statement that only fatty acids can be absorbed under these conditions is erroneous (Munk and Friedenthal).

A dog normally absorbs 9-2I per cent. of the fat in a meal in three to four hours; $2 \mathrm{I}-46$ per cent. in seven hours; and 86 per cent. in eighteen hours (Harley). After excision of the pancreas the absorption of fat is hindered, though not abolished. Nore fat, indeed, can be recovered from the intestine than is given in the food. This at first sight paradoxical result is explained by the well-established fact that a certain amount of fat is normally excreted into the intestine.

As to the manner in which the synthesis of the fat in the intestinal epithelium is accomplished, the most fascinating theory is that which attributes it to the reversed action of a fatsplitting ferment or lipase, possibly the very same steapsin as originally split it up in the intestine. The reversibility of the action of various enzymes under changed conditions has been well made out, and it has been stated that even outside of the body the pancreas, intestinal mucous membrane, lymph glands, etc., and even cell-free extracts of these organs have the power of synthesizing the ester, ethyl butyrate from butyric acid, and ethyl alcohol (p. 3I5). Moore, however, finds that in the case of ordinary fats the synthesis takes place in the intestinal wall only in situ and while the circulation is going on. In the intestinal mucosa the greater part of the fatty acid is already combined with glycerin as neutral fat, although considerable quantities of free fatty acid are also present. In the lymph coming directly from the mesenteric glands practically the whole of the fatty acids are in the form of neutral fat.

An additional, and in some respects even more remarkable, illustration of the synthesizing powers of the intestinal wall is the discovery of Munk, already referred to (p.4I3), that fatty acids given by the mouth appear in the lymph of the thoracic duct as neutral fats, having somewhere or other, in all probability on their way through the epithelium of the gut, been combined with glycerin. 
Since, however, the amount of nemtral fat recosered from the thoracic duct is not equivalent to more than one-third of the fatty acids given, it has been suggested that this synthesis of fat is only apparent, and that the whole of the fat which appears in the chyle after a meal of fatty acids comes from the fat excreted into the intestine (Frank), which is increased when fatty acids are given by the mouth. But the suggestion is more ingenious than the evidence advanced in its support is convincing. And, as we have seen (p. fI5), a part of the deficit may be accounted for hy absorption directly into the bloodvessels.

Absorption of Carbo-hydrates. - Carbo-hyclrates are normally absorbed from the alimentary canal only in the form of monosaccharides, such as dextrose, levulose, and galactose, but especially dextrose. These monosaccharides are readily formed from polysaccharides like starch and dextrin, and the disaccharide maltose, which they yield, as well as from disaccharides like cane-sugar and lactose, by the ferments alrearly studied. That, as a matter of fact, the hydrolysis in the intestine must convert practically all the carbo-hydrate into monosaccharides before absorption can be shown in various ways. The ferment lactase, while present in the small intestine of all young mammals, is regularly absent in some mammalian groups in the adult. In other species, including man, it is found in some adults, but not in all. In birds and other animals below the mammals, it has not hitherto been found at any age. It has been surmised that these differences lepend upon the presence or absence of lactose (milk) as a regular constituent of the food. (But see p. 382.) If, now, lactose is introrluced into a loop of intestine in an animal which does not possess lactase - e.g., an adult rabbit - it is not absorbed, but remains in the lumen till it is at last decomposed by bacterial action. In animals in which lactase is present the lactose is rapidly absorbed. Maltose is easily taken up from the intestine because of the action of the ferment maltase, which is the most widely spread of all the inverting ferments. The dextrose formed by the maltase is so rapidly absorbed that none, or only traces, of it can be detected in the contents of the intestinal loop. But if absorption be interfered with by injuring the intestine, maltose disappears, and dextrose accumulates in the lumen. The reason for the discrimination exercised by the intestinal mucosa in favour of the monosaccharides becomes apparent when an attempt is made to circumvent it by injecting the sugars subcutaneously. Cane-sugar and lactose so introduced are excreted unchanged in the urine. Dextrose, levulose. and galactose are used up in the body, and maltose likewise, thanks to the presence of lactase in the blood and tissues. The cells of the body in general will burn only monosaccharides, and 
not di- or poly-saccharides. Therefore the intestine admits the simple, but rejects the more complex sugars. It is only in the presence of abnormally great quantities or abnormally great concentrations of the sugars which are not directly utilizable that they are to a certain extent taken up unaltered, to be quickly excreted as such (p. 5i6). The sugar absorbed from the intestine passes normally into the rootlets of the portal vein, not into the chyle, for no increase in the quantity of that substance in the contents of the thoracic duct takes place during digestion, while the sugar in the portal blood is increased after a starchy meal. The blood of the portal vein of a dog in the fasting condition contained $0^{\circ} 2$ per cent. of dextrose. During absorption of a meal rich in carbo-hydrates it contained as much as 0.4 per cent. In the lymph issuing from the thoracic duct the amount was the same in both conditions-viz., 0.I6 per cent. In a case of lymph (chyle) fistula in a human being, where almost all the lymph from the digestive tract escaped through the fistula, out of Ioo grammes of carbo-hydrate taken ( 50 grammes starch and 50 grammes sugar), only $\frac{1}{2}$ gramme, or not I per cent. of the sugar corresponding to the carbo-hydrates of the food, could be recovered in the chyle. But when a large amount of a dilute solution of sugar is introduced into the intestine, some of it is taken up by the lacteals.

Absorption of Water and Salts.-The main channel for absorption of these is the bloodvessels of the intestine. As much as 3 to 5 litres of water can be absorbed in a day in the intestine of a healthy man, exceptionally even 6 to Io litres, without the fres altering their normal consistence. Absorption of the water and dissolved salts may theoretically take place either through the epithelial cells (intra-epithelial absorption), or between the cells (interepithelial absorption). According to Höber, most metallic salts (silver, mercury, lead, bismuth, copper, manganese, etc.) are absorbed interepithelially, while iron salts form an exception, and pass into the epithelial cells. The distinction between interepithelial and intra-epithelial absorption does not rest upon an absolutely sure foundation. Yet it is probable that everything which is useful in the nutrition of the body is taken up by the cells, while such substances as metallic salts which are foreign to the organism, and are denied entrance into the cells, may pass in small amount between them, their passage being perhaps associated with more or less injury to the interstitial substance. The vigilant selection exercised by the mucosa is well illustrated by the facts that, although manganese and iron are chemically so closely related, iron, which is necessary for the formation of the blood-pigment, is absorbed in immensely greater amount than manganese; and that chlorides, especially 
sodimn chloricle, ate readily taken $u p$, sulphates with difficulty. Jron is absorbed by the bloodvessels, but also to some extent by the lacteals. From the blood it is carried to various organs, especially the spleen and liver. There is reason to believe that the eosinophile lencocytes take some share in its transportation.

It was supposed by Bumge that only organic compounds of iron conld be absorbed, and that the undoubted benefit derived from the administration of inorganic iron compounds, such as ferric chloride, in chlorosis, was due not to their direct absorption, but to their shielding the organic compounds from the attack of the sulphuretted hydrogen in the intestine (1).396). But this theory has been shown to be inconsistent with the facts. For instance, after the administration of salts of iron, the iron in the blood, liver, spleen, and other organs increases, but there is no accumulation of iron in the liver of an animal to which salts of manganese have been given, although these are equally decomposed by sulphuretted hydrogen.

Absorption of Proteins. - The proteins of the food or their digested products also pass directly into the blood-capillaries which feed the portal system. For it has been shown that after ligature of the thoracic duct protein substances are still absorbed from the intestine, and the urea corresponding to their nitrogen appears in the mrine. And the total nitrogen in the chyle flowing from a fistula of the thoracic duct in a man was not found to be increased during the digestion of protein food. The yuantity of chyle escaping in a given time was also unaffected, whereas during the digestion of fats it was greatly augmented (Munk).

Although a certain amomnt of egg-albumin, serum-albumin, alkali-albumin, and other native or slightly altered protein substances can be absorbed as such by the small, and even by the large, intestine, there is no evidence that, under ordinary conditions, this mode of absorption is of any practical importance. For when native proteins, with the possible exception of the serum proteins from an animal of the same species, are introdnced 'parenterally '-that is, injected directly into the blood, tle peritoneal cavity, or the muscles, or under the skin, so that they do not reach the tissues by way of the alimentary canal-they behare in a very different mammer from the same proteins when given by the nouth. One notable difference is that the parenterally administered proteins give rise in general to the formation of specific precipitins (p. 30). This is not the case when they are administered per os, muless, like raw egg-white, which, as already mentioned (p. 375), evokes no secretion of gastric juice, they remain long undigested in the alimentary canal, when an amount sufficient to cause the production of precipitins may 
eventually be absorbed maltered. Secondly; they are not, as at rule, utilized in the metabolism of the body, or are utilized very incompletely. Eggrallumin, for instance, when injected into the blood, is excreted in the urine. It has been previously pointed out that the various proteins differ remarkably in the kind and quantity of the amino- and diamino-acids which can be obtained from them (p. 2). This is unquestionably one important reason why the food proteins are-for the most part, at any rate-so thoroughly hydrolysed before absorption. Another may be that it is easier for the intestine to take up the small molecules of the decomposition products than the large colloid aggregates of the original protein solutions.

So far as the first reason is concerned, the degree of decomposition need not be the same for all the food proteins. A new house has to be built from the materials of an old one. How far the work of demolition must be carried will depend upon the difference between the plans of the two houses. Sometimes the main part of the old building may be saved, and only the wings require reconstruction. In like manner it is conceivable that the central group or nucleus of the molecule of a given food protein may be identical with that of a given body protein, and that only the side chains may be so different that they must be broken up and reconstructed. Or, again, the whole architectural plan of the new house may be so distinct from that of the old that the only feasible method is to completely demolish the latter, and then to use the individual bricks in the new construction; just as a protein in the food may differ so radically from a tissue protein into which it is to be transformed that it must be decomposed into the simplest products of proteolysis before the reconstruction of the molecule can begin. It is not known what the minimum degree of hydrolysis is which will permit of effective absorption and utilization. There can be no doubt that by far the greater part of the proteins of the food is first changed into proteoses and peptones. But proteose and peptone are absent from the blood, and, indeed, when injected into the blood, they are excreted in the urine. When injected in larger amount, they pass also into the lymph, from which they gradually reach the blood again, and are eventually, as before, eliminated by the kidneys. The clear inference is that if they are absorbed as such from the alimentary canal, they must be changed in their passage through its walls. The fact that a portion of the peptone and albumose is decomposed into aminoacids, etc., in the lumen of the intestine has been already alluded to. It would seem that a further portion of the remaining peptone and albumose-probably the whole-is similarly decomposed by the action of erepsin in the intestinal wall. It 
has actually been shown that during the digestion of a protein meal the mucosa of the stomach and intestine contains proteose and peptone, while none is present in the muscular coat or in any other organ. They rapidly disappear from a portion of the mucous membrane kept at a temperature of about $40^{\circ} \mathrm{C}$. outside of the body, and their disappearance is clue, not to their regeneration into serum proteins, as was once supposed, but to their decomposition by the erepsin. We must suppose that the serum and organ proteins are built up from the products of this decomposition. But whether the mucosa of the alimentary tract is especially a seat of the synthesis is unknow (p. 497). It is at least equally probable that it occurs in all the cells of the body, each one building up for itself the particular kind of protein which it needs. The direct way of testing the question would be to examine the blood coming from the intestine during the absorption of proteins, and to determine quantitatively any changes which might have occurred in the nitrogenous constituents. But the flow of blood through the intestine is so great, the absorption of the digestive products so gradual, and their removal from the blood by the tissues, in all probability, so rapid, that there is no reason for surprise that hitherto the results of such determinations have been ambiguous. It has, however, been shown that when peptone, albumose, or the final products of tryptic digestion are introduced into a ligated segment of a dog's small intestine, there is always, when absorption occurs, an increase in the nitrogenous substances in the blood, in the form of compounds which are not precipitated by tannic acid, and therefore are neither native proteins nor proteose. Urea accounts for about one-half of the increase; the rest probably represents amino-acids and similar substances (Leathes). A much more conclusive experiment has been made on the intestines of certain octopods, which, when excised and suspended in the oxygenated blood, will live for many hours. A solution of peptone was introduced into the isolated intestine, and after twenty hours the crystalline products, leucin, tyrosin, ly.sin, and arginin, were found in the blood. In the intact animal none of these bodies could be detected in the blood (Cohnheim). The inference is that protein in these animals is absorbed in the form of amino-acids, etc., which are then carried to the tissues and utilized there. It unay be that some of the proteose and peptone are regenerated by a shorter process, and without having been further split up, but of this, too, there is no definite proof. The regeneration, wherever it occurs, must presumably take place in cells, and the only available cells in the digestive mucous membrane are those which line the tube, or the leucocytes which wander between them. Accordingly, both have been credited 
with the power of absorling (and perhaps transforming) these substances, but the balance of evidence is in favour of the epithelial cells. We cannot, however, as in the case of the fat single out any particular tract of epithelium as alone engaged in the absorption (and possibly in the resynthesis) of the products of the digestion of the proteins. In all likelilıood the cells covering the villi are actively concerned, but there is no valid reason for denying a share to the general lining of the stomach and small intestine, even including the Lieberkiihn's crypts, which morphologically form a kind of inverted villi. It is, incleed, true that the crypts do not take part in the absorption of fat, for no grantles blackened by osmic acid occur in them during digestion of a fatty meal. But this is a ground for attributing to them other absorptive functions rather than for altogether denying to them a share in absorption, unless, indeed, we assume that the secretion of the succus entericus engrosses the whole activity of this extensive sheet of cells.

The extraordinary efficiency of the small intestine in digestion and absorption is shown by the fact that after removal of even 70 to 83 per cent. of the combined jejunum and ileum in dogs, the metabolism is not necessarily much affected. On a diet poor in fat the animals absorb as much of the fat as a normal dog, although a smaller proportion when the diet is rich in fat. It has been generally stated that it is never permissible to remove more than one-third of the small intestine in man. But in one case $2 \frac{3}{4}$ metres was resected, or quite one-half, and the patient recovered. Even the large intestine, which possesses Lieberkïhn's crypts, but no villi, is able to absorb not only peptones and sugar, especially monosaccharides like dextrose, but also fats and undigested native proteins. And, although these are powers which can be rarely exercised to any great extent in normal digestion, they form the physiological basis of the important method of treatment by nutrient enemata. The observation already nentioned (p. 309), that considerable quantities of food administered by the rectum can pass through the ileo-colic sphincter and valve into the lower part of the ileum, thanks to the antiperistaltic movements of the large intestine, indicates that an important part of the preliminary digestion and of the absorption of enemata may occur in the small intestine. But remnants of the proteolytic, amylolytic, fat-splitting, and inverting ferments which have done their work in the small intestine are passed on into the large, and may be demonstrated in its contents. Doubtless these are able to act upon food substances which may have escaped complete digestion and absorption in the higher parts of the alimentary canal, as well as upon food substances injected into the rectum. 


\section{PRACTICAL EXERCISES ON CHAPTERS IV. AND V.}

Quantitative Estimation of Ferment Action. - For pepsin an easy methosl, although not a very accurate one, of estimating the rate at which the fibrin disappears is to use fibrin stained with carmine. As solution goes on, the dye colours the liquid more and more leeply, and by comparing the depth of colour at any time with standard solutions of carmine. we get the quantity of dye set free, and therefore of filorin aligested. This method cannot be used for trypsin. A much better method is that of Mett (p. 318). Fluicl egg-white is sucked up into fine glass tubes (of 1 to 2 mun. bore). The tubes are then heated in a bath at $95^{\circ} \mathrm{C}$. For use short pieces $(1$ or $2 \mathrm{~cm}$. long) are cut off and placed in I or 2 c.c. of the liquid to be tested. the whole being kept at $38^{\circ}$ to $40^{\circ} \mathrm{C}$. At the encl of a certain time the length of the colmmn of unclissolved protein is measured with a scale and low-power microscope. Deducting this from the length of the tube, we get the length of the column digested. As a test of the activity of a diastatic ferment, we take the amount of sugar formed in a giren time in a given quantity of a standard starch solution. Where rapid work is required, glass tubes filled with tinted starch paste may be used in the same way as the Mett's tubes. A more accurate inethod, and yet a rapid and convenient one, is baserl upon the time which is necessary in order that the iodine reaction with starch may just disappear when a standard starch solution is cligested with a dilution of the ferment solution at $40^{\circ} \mathrm{C}$. To letermine the activity of pancreatic juice as regards fat-splitting ferment. the acidity of the emulsion formed by the juice and fat after standing for a definite time at a given temperature (witl occasional shaking) can be estimated by titration with baryta solution.

I. Saliva.-Collection and Microscopic Examination of Saliva.Chew a piece of paraffin-wax, or inhale ether or the vapour of strong acetic acid. The flow of saliva is increased. Collect it in a porcelain capsule. Examine a drop under the microscope. It may contain a few flat epithelial scales from the mouth and a few round granular bodlies, the salivary corpuscles, the granules in which often show a lively, dancing movement (Brownian motion). Filter the saliva to free it from air-bubbles, and perform the following experiments :

(a) Test the reaction with litmus paper. It is usually alkaline. An acid reaction may indicate that bacterial processes are abnormally active in the month.

(b) Add dilute acetic acid. A precipitate indicates the presence of uncin (p. 3I9). Filter.

(c) Add a drop or two of silver nitrate solution to the filtrate from (b). A precipitate insoluble in nitric acicl, soluble in ammonia. proves that chloricles are present.

(d) Adcl to another portion a few drops of clilute ferric chloride slightly aciclulated with clilute hyclrochloric acid. and the same quantity to as much clistilled water in a control test-tube. A reddish coloration is obtained, due to the presence of sulphocyanic acicl. which is combined with potassium and other bases in the saliva. The colour is discharged by mercuric chloride. Or, a drop of saliva may be allowed to fall from the mouth on a test-paper (prepared by soaking filter-paper with a dilute starch solntion, containing a littlo iodic acid, and allowing it to (try in the air). The paper is coloured 
blue by the union of the starcli with iocline set frec from the iolic acisl by the action of the sulphocyanic acid.

(e) Take some boiled starch mucilage, and test it for reclucing sugar by 'Trommer's test (p. ro). If no sugar is found, take three test-tubes, label them $\mathrm{A},[3, \mathrm{C}$, and nearly half fill each with the boiled starch. To $A$ aclil a little saliva,* to $B$ some saliva which has been boiled, to $\mathrm{C}$ a little saliva which has been neutralized, and as much $0^{\circ}+$ per cent. hydrochloric acid as lias been taken of the mucilage, so as to make the strength of the acid in the mixture $0^{\prime} 2$ per cent., a proportion well below that of the gastric juice. Put the testtubes into a water-bath at $40^{\circ} \mathrm{C}$. In a few minutes test the contents for reducing sugar. Abundance will be found in $\mathrm{A}$, none in $\mathrm{B}$ or $\mathrm{C}$. In $B$ the ferment ptyalin has been destroyed by boiling; in $C$ its action has been inhibited by the acicl. If the test-tubes have been left long enough in the bath, no blue colour will be given by $A$ on the aclitition of iodine, but a strong blue colour by $\mathrm{B}$ and $\mathrm{C}-$ i.e., the starch will have completely disappeared from A.

(f) Put some starch in a test-tube, add a little saliva, and loold in the hand or place in a bath at $40^{\circ} \mathrm{C}$. On a porcelain slab place several separate drops of dilute iodine solution. With a glass rod add a clrop of the mixture in the test-tube to one of the drops of iodine at intervals as digestion goes on. At first only the blue colour given by starch will be scen ; a little later a violet colour, due to the presence of erythrodextrin in adilition to some unaltered starch. A little later the colour will be reddish, the starch having disappeared and the erythrodextrin reaction being no longer obscured. Later still no colour reaction will be obtained, the erythrodextrin having undergone further changes, and only sugar (maltose, isomaltose, and perhaps a trace of dextrose) and achroodextrin - a kind of dextrin which gives no colour with iodine-being present.

(g) Put two pieces of glass tube filled with tinted starch paste (p. +22 ) in separate test-tubes. Cover one with 3 c.c. and the other with 6 c.c. of saliva. The saliva must all be taken from the same stock, and must not be collected separately. Put in a bath at $38^{\circ} \mathrm{C}$., and when a fair amount of cligestion has taken place in each, measure the length of the column digested, and determine the relation between the amount digested in the two tubes (p. $3 I_{7}$ ).

(h) Dilute 2 c.c. of saliva with distilled water up to 20 c.c., and filter. Take six test-tubes of the same width, and label them $A, B$, C, etc. Measure into A 3 c.c. of the diluted saliva, into B 2 c.c., into $\mathrm{C} \mathrm{I}_{3} 3$ c.c., into $\mathrm{D} 0.9$ c.c., into $\mathrm{E} 0^{\circ} 6$ c.c., and into $\mathrm{F} \mathrm{O}^{\circ}+$ c.c. Thus a series is obtained in which each tube contains (approximately) two-thirls as much ferment as the one it follows. Add distilled water to tubes B to $F$, sufficient to make up the volume in each to 3 c.c. Place the tubes in a beaker of iced water ; add to each ro c.c. of a I per cent. solution of boiled starch previously coolecl in iced water, and shake so as to mix the contents. Each tube now contains starch in uniform concentration, and ferment in varying concentration. The low temperature prevents digestion till all the tubes are ready. Now put the tubes simultaneously into a waterbath at $40^{\circ} \mathrm{C}$. for half an hour, and then back again into iced water to prevent further digestion. Move them about in the icel water to cool rapidly. Fill up the tubes with distilled water nearly to the top, add a drop or two of iodine solution to cach, and mix uniformly.

* As it filters slowly, unfiltered saliva may be used for Experiments $(e)$. ( ) , and $(i)$. 
The tubes to which the smallest amounts of saliva were arlded will probably still sliow a clistinct bluc colour, while those at the other end of the series will be brown or yellow, and the intermediate tubes bluish-violet. Suppose D is the last tube still showing a bluish tint, then in the next higher tube. C, all the starch lias been liyelrolysed at least to dextrin-that is, $1 \cdot 3$ c.c. of the ten-times diluted saliva, or $0^{*} 13$ of the original saliva, has been sufficient to change all the starch in 10 c.c. of the I per cent. solution. With another specimen of saliva the same result might be reached in tube E, containing an amount of ferment equal to that in o. of c.c. of the original saliva. We could then conclude that the diastatic power of the second saliva was abont twice as great as that of the first. A closer approximation can now be made by setting up two fresh tubes (C and E respectively for the two salivas) and determining the time required for the bluc reaction with jodine to disappcar, taking out a drop from time to time and testing on a porcelain slab.

(i) Put a little distilled water into a porcelain capsulc, and bring the water to the boil. Now put into the month some boiled starch paste, and move it about as in mastication. After half a minute spit the starch ont into the boiling water. Divide the water into two portions. Test one for sugar, and the other for starch. Repeat the experiment, but keep the starch two minutes in the mouth. Teport the result.

(j) Starch solution to which saliva has been added is placed in a dialyser tubc of parchment-paper for twenty-four hours. At the end of that time the dialysate (the surrounding water) should be tested for sugar and for starch. Sugar will probably be found, but no starch. If no reaction for sugar is obtained, the dialysate should be concentrated on the water-bath, and again tested.

2. Stimulation of the Chorda Tympani.-(I) Having previously studied the anatomy of the mouth and submaxillary region in the dog by dissecting a dead animal (Fig. I57), put a good-sized dog under morphine. Set up an induction-machinc for a tetanizing current (p. I8.4). and conncet it with finc electrodes. Fasten the dog on the holder, give cther if necessary, and insert a cannula in the trachea (p. I 86). Then make an incision 3 or 4 inches long throngh skin and platysma muscle, along the inner border of the lower jaw beginning about the angle of the mouth, and continuing backwards towards the angle of the jaw. Such branches of the jugular vein as come in the way may be generally pushed aside, but if necessary they may be doubly ligated and divided. Feel for the facial artery, so as to be able to avoid it. Divide the digastric muscle about its anterior third, and clear it carefully from its attachments ; or, without dividing it, pull it outwards with a hook. The broad. thin mylo-hyoid muscle will now be seen with its motor nerve lying on it. Divide the muscle about its middle at right angles to its fibres, and raise it carcfully. The lingual nerve will be seen energing from under the ramus of the jaw. It runs transversely towards the middle line, and then, bending on itself, passes forwards parallel to the larger hypoglossal nerve. In its transverse course the lingual will be seen to cross the ducts of the submaxillary and sublingual glands. These structures having been identified, the lingual nerve is to be ligatured before it enters the tongue and cut peripherally to the ligature. Then a glass cannula of suitable size is to be inserted into the submaxillary luct (the larger of the two), just as if it were a bloodressel (p. 55). A short piece of narrow rubber tubing is care- 
fully slipped on the end of the cannula. The lingual is now to be lifted by means of the ligature, and traced back towards the juw till its chorda tympani branch is seen coming off and running backwards along the liuct. The chordo-lingual nerve (Fig. I $+3,1$, 362) is then to be cut centrally to the origin of the chorla tympani, which can now be easily laid on electrodes by means of the ligature on the lingual. On stimulating the chorda, the flow of saliva through the canmula will be increased. The current need not be very strong. If the flow stops after a short time, it can be again caused by renewerl stimmlation after a brief rest. A quantity of saliva may thus be collected, and the experiments alrealy made with human saliva repeated.

(2) Expose the vago-sympathetic nerve in the neck on the same side; ligature it; divide below the ligature; and note the effect produced by stimulation of the upper end on the flow of saliva.

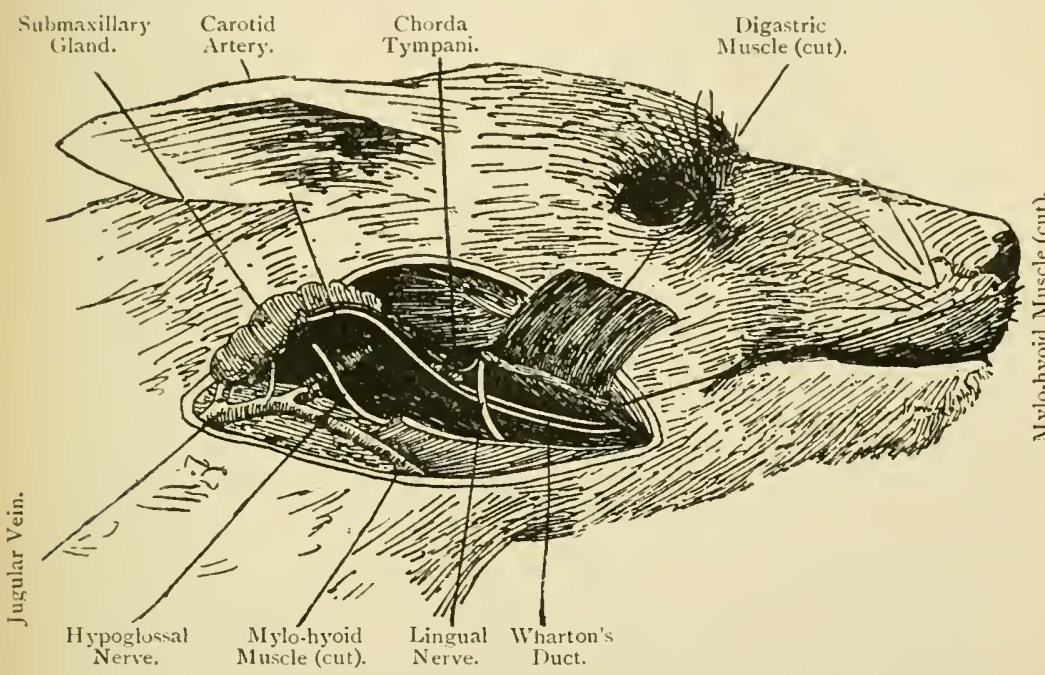

Fic. r57.- Dissection for Stimelation of Chorda TyMpani (After

BERNARD).

(3) Set up another induction-machine, and connect it with electrodes. Stimulate the chorda, and note the rate of flow of the saliva. Then, while the chorda is still being excited, stimulate the vago-sympathetic, and observe the effect. If the experiment is successful, finish by stimulating the chorda for a long time. Then harden both submaxillary glands in absolute alcohol, make sections, stain with carmine, and compare them.

3. Effect of Drugs on the Secretion of Saliva.-(I) Proceed as in 2 (I), but, in addition, insert a cannula in to the femoral rein (p. 200). On the cannula put a short piece of rubber tubing, filled with o' 9 per cent. salt solution and closed by a small clamp, or a small piece of glass rod, or a pair of bulldog forceps. While the chorda is being stimulated inject into the vein ro to I 5 milligrammes of sulphate of atropine by pushing the needle of a hypodermic syringe through the rubber tube. This will stop the flow of saliva. and abolish the 
effect of stimulation of the chorda. See whether the sympathetic is also inactive, and report the result.

(2) Now empty the cannula in the submaxillary duct by means of a feather, and fill it with a 2 per cent. solution of pilocarpine nitrate by means of a fine pipette. Fill also the short rubber tube attached to the cannula, and close it again. Compress the tube, and so force into the luet a small quantity of the solution. Open the tube. Secretion of saliva will again begin, and stimulation of the chorela will again cause an increase in the flow. But after a few ninutes the action of the atropine will reassert itself, and the flow will stop. Renewed secretion may be caused by a fresh injection of pilocarpine.

4. Gastric Juice.--(a) Preparation of Antificial Gastric Juice.'Take a portion of the pig's stomach provided, strip off the mucous membrane (except that of the pyloric end, which is relatively poor in pepsin), cut it into small picces with scissors, and put it in a bottle with Ioo times its weight of $\mathrm{O}^{*}+$ per cent. hyclrochloric acid. Label and put in a bath at fo $^{\circ} \mathrm{C}$. for three hours, and then in the cold for twelve hours. Then filter.

(b) Take another portion of the mucous membranc, cut it into pieces, and rub up with clean sand in a mortar. Then put it in a small bottle, cover it with glycerin, label, and set asille for two or three days. The glycerin extracts the pepsin.

(c) Take five test-tubes, A, B, C, D, E, and in each put a little washed and boiled fibrin or a small cube of coagulated egg-white. To A add a few clrops of glycerin extract of pig's stomach, and fill $11 \mathrm{p}$ the test-tube with $\mathrm{O}_{4}$ per cent. hydrochloric acid. To $\mathrm{B}$ add glycerin extract and distilled water; to $\mathrm{C}$ glycerin extract and I per cent. sodium carbonate ; to $\mathrm{D} \mathrm{o}_{+}+$per cent. hyclrochloric acid alone; to $\mathrm{E}$ glycerin extract which has been boiled, and $\mathrm{O}_{+}+$per cent. hydrochloric acid.

Put up another set of five test-tubes in the same way, except that a few clrops of a watery solution of a commercial pepsin are substituted for the glycerin extract. Label the test-tubes $\Lambda^{\prime}, B^{\prime}, C^{\prime}, D^{\prime}, E^{\prime}$.

Into another test-tube put a little fibrin (or an egg-white cube), and fill up with the filtered acid extract from $(a)$. Label it $l^{\circ}$. Place all the test-tubes in a tumbler, and set them in a water-bath at $+0^{\circ} \mathrm{C}$. Put a piece of a Mett's tube $(p,+22)$ into each of two testtubes, and add I 5 c.c. of $\mathrm{O}_{4}$ per cent. hydrochloric acid. To one tube add 5 clrops and to the other ro drops of the same filtered glycerin extract of gastric mucous membrane. Put the tubes in the bath, and when digestion is clistinct at the ends of both tubes measure the length of the column digested in each. What is the relation between the two (1).317) ? The experiment can be repeated with the hydrochloric acid extract of the mucous membrane.

After a time the fibrin (or egg-white) will have almost completely disappeared in $\mathrm{A}, \mathrm{A}^{\prime}$, and $\mathrm{F}$, but not in the other test-tubes. Filter the contents of $\mathrm{A}, \mathrm{A}^{\prime}$, and $\mathrm{F}$ into one dish.

(d) Test the filtrate for the products of gastric digestion :

(a) Neutralize a portion carefully with clilute sodium hyclroxide. A precipitate of acid-albumin may be thrown down. Filter.

$(\beta)$ To a portion of the filtrate from (a) adel excess of sodium hydroxide and a drop or two of very dilute copper sulpliate. A rose colour indicates the presence of proteoses or peptones. The cupric sulphate must be very cautiously adled, because an excess gives a violet 
colour, and thus obscures the rose reaction. If still more cupric sulphate be added, blue cupric hydroxicle is thrown down, and nothing can be inferred as to the presence or the nature of proteins in the liquicl.

$(\gamma)$ Heat another portion of the filtrate from $(a)$ to $30^{\circ} \mathrm{C}$. and adel crystals of ammonium sulphate to saturation. A precipitate of proteoses (albumoses) may be obtained. [iilter off.

(s) All to the filtrate from $(\gamma)$ a trace of cupric sulphate and excess of sodium hydroxide. A rose colour inlicates that peptones are present. Nore sodium hydroxide must be adled than is sufficient to break 11) all the ammonimm sulphate, for the biuret reaction requires the presence of free fixed alkali. A strong solution of the sodium hydroxide should therefore be used, or the stick caustic soda. The addition of ammonium sulphate will cause the red colour to disappear; so will the addition of an acid. Sodium hydroxide will bring it back. Ammonia does not affect the colour.

$(\epsilon)$ Use another portion of the filtrate from $(a)$ to separate the primary and secondary albmmoses as in experiment $(2)(\gamma)$ (p. Io).

(e) To some milk in a test-tube add a lrop or two of rennet extract, and place in a bath at $40^{\circ} \mathrm{C}$. In a short time the milk is curclled by the rennin. (See p. 326.)

5. (I) To obtain Normal Chyme.--Inject subcutaneously into a dog, one and a half hours after a meal of minced meat and water, $2 \mathrm{mg}$. of apomorphine. Half of one of the ordinary tabloids is enough. Collect the romit.

(2) To obtain Pure Gastric Juice. - If the laboratory possesses a dog with Pawlow's double oesophageal and gastric fistula, the juice may be obtained in large amount by sham feeding with meat (p. 374). If not, proceed as follows: Put a fasting dog under ether, and fasten on the holder. Clip the hair and shave the skin in the middle line below the sternum. Make a longitudinal incision through the skin and subcutaneous tissue from the xiphoid cartilage downwards for 3 or 4 inches. The linea alba will now be seen as a white mesial streak. Open the abdomen by an incision through it. Pull over the stomach towards the right, and stitch it to the abdominal

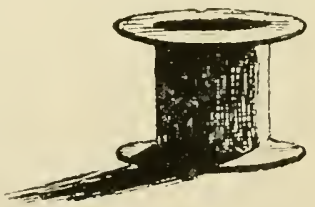

Fig. I58.-STOMACH CaNNula. wall, open it, and insert a stomach cannula (Fig. ${ }_{5} 8$ ). Make an incision through the serosa and muscularis. Doubly ligate and divide any vessels exposed in the submucosa. Then make an opening in the mucosa of sufficient size to just admit the gastric cannula. This will go into a smaller opening if it is provided with a nick in the flange which enters the stomach. Be careful to prevent blood from getting into the stomach. Immediately stitch the wound in the stomach over the flange of the cannula, but do not pass the stitches through to the internal surface of the mucosa. Suture the muscles and skin separately. Then stitch up the wound in the abdomen. Wash out any stomach contents with warm water. Put a cork in the 
cannula, and coicr the animal with a cloth. The following cxperiments may now be performed. Expose both vagi in the neck. Connect two pairs of electrodes with the scconclary coil of an in(luctorium arranged for single shocks. By means of a key in the primary stimulate the nerves with slow rhythmical induction shocks at the rate of about one a sccond. Continue the stimulation for fifteen minutes, collect any juice that may have been secreterl, and apply the tests in (3). If secretion is slow, a little distilled water may be put into the stomach, and the vagus stimulation repeated. Mechanical stimulation of the mucous membrane with a feather causes no secretion of acid gastric juice. but may cause a secretion of alkaline mucus.

(3) (a) Test the reaction to litmus of the chyme obtainel in (I). and of the pure juice obtained in (2).

(b) Test their proteolytic powers by putting in a bath at $40^{\circ} \mathrm{C}$. for two lours two test-tubes containing respectively filtered chyme and fibrin, and gastric juice and fibrin. The fibrin will be digested in both. Estimate the proteolytic power quantitatively by Mett's tubes (p. +22$)$.

(c) Add a few clrops of the chyme and gastric juice to milk in two test-tubes, and place them in a bath at $40^{\circ} \mathrm{C}$. Repeat $(c)$ after neutralizing the liquids.

(d) Examine a drop of the unfiltered chyme under the microscope. Partially digested fragments of the food will be secn-muscular fibres, or fat cells. Filter, and proceed as in $4(d)($ p. 426$)$.

(4) Test the filtrate from the chyme and the gastric juice for lactic acid by Uffelmann's test or Hopkins's test (p. 7 I 6 ), and for hydrochloric acid by Günzburg's reagent.

Uffelmann's Test for Lactic Acid.- The reagent is a dilute solution of carbolic acid to which dilute ferric chloride has been added till the colour is bluish (say a drop of a I per cent. ferric chloride solution to 5 c.c. of a I per cent. carbolic acid solution). The blue colour of the mixture is turned yellow by lactic acid, but not by dilute hydrochloric acid. Since Iffelmann's test is given also by phosphates, alcohol. and sugar, which may sometimes be present in the stomach contents, it is best to sliake the gastric contents with ether, dissolve the ethereal extract in water, and then make the test on the watery solution.

Gunzburg's Reagent for Free Hydrochloric Acid in Gastric Juice is made by dissolving 2 parts of phloroglucinol and I part of vanillin in 30 parts by weight of absolute alcolıol. A few drops of the reagent are added to a few (lrops of the filterel gastric juice in a small porcelain capsule. and the whole evaporated to dryness over a small bunsen flame. If free hydrochloric acid is present, a carmine-red residue is left. If all the hydrochloric acid is united to proteins in the stomach contents, the reaction does not succeed. It is also hindered by the presence of leucin.

6. Pancreatic Juice.-(a) Take a piece of the pancreas of an ox or dog which has been kept twenty-four hours at the temperature of the laboratory, and make a glycerin extract in the same way as in the case of the pig's stomach in $+(b)$. Put in a small bottle. and set aside for a day or two.

(b) Put a little boiled fibrin into each of six test-tubes, A, B, C, D. E. F. To $A$ add a few lrops of glycerin extract of pancreas. and fill up with a I per cent. sollium carbonate solution : to B add glycerin extract and distilled water; to $C$ glycerin extract and 
excess of $u_{5}$ per cent. Iydrochloric acid ; to D I per cent. soulium carbonate alone; to li I per cent. sodium carbonate in which a few drops of glycerin extract of pancreas have been previously boiled ; to $F^{\prime}$ glycerin extract and excess of $0^{*} 2$ per cent. hydrochloric acid.*

Set up six test-tubes. $A^{\prime}, 1^{\prime} . C^{\prime}, 1^{\prime}$. $E^{\prime}$, $I^{\prime \prime}$, in the same way, but substitute a few drops of a solution of conmercial pancreatin for the glycerin extract. Set up two test-tubes as in experiment $+(p .+26)$ witl Mett's tubes. Put all the test-tubes in a tumbler, and place in a batll at $40^{\circ} \mathrm{C}$. The fibrin will be gradually eaten away in $\mathrm{A}$ and $\mathrm{A}^{\prime}$, by the action of the trypsin, but will not swell up or become clear before disappearing, as it does in dilute hydrochloric acid with glycerin extract of stomacl. Filter the contents of these test-tubes. Neutralize the filtrate with dilute acid; a precipitate will consist of alkali-albumin. If such a precipitate is obtained, filter it off and test the filtrate for proteoses and peptones as in $4(d)($ p. 426$)$. Some digestion, and perhaps a considerable amount, may also hare taken place in $\mathrm{F}$ and $\mathrm{F}^{\prime}$; less or none at all in $\mathrm{C}$ and $\mathrm{C}^{\prime}$; and none in the other test-tubes (pp. 331, 39.4).

(c) Add a few drops of the glycerin extract to a test-tube containing starch mucilage, which has bcen previously found free from reducing sugar. Put in a bath at $+0^{\circ} \mathrm{C}$. After a short time abundance of reducing sugar will be found, owing to the action of the ferment, amylopsin.

(d) Mince thoroughly a good-sized piece of fresh pancreas, and shake up well with three or four times its bulk of water. Put 5 c.c. of fresh cream into a test-tube, then Io c.c. of the extract, a few drops of chloroform to prevent the growth of bacteria, a few drops of litmus solution. and if necessary enough of very dilute sodium hydroxide to just render the colour distinctly blue. Shake up, and divide the mixture into two portions, $A$ and $\dot{B}$. Boil one portion (B), and place the test-tubes at $4 \mathrm{O}^{\circ} \mathrm{C}$. Examine from time to time. The blue colour will disappear in $\mathrm{A}$. owing to the formation of fatty acids from the neutral fats, and sodium hydroxide must be added to it to restore the colour. In $\mathrm{B}$ the fat-splitting ferment has been destroyed by boiling, and fat-splitting will not occur. Probably a distinct result will not be obtained for several hours, and it will be best to leave the tubes in the incubator over-night.

(e) If the laboratory possesses an animal with a pancreatic fistula, the following experiment may be done by a limited number of students with fresh pancreatic juice + collected from the fistula. Take five test-tubes, A, B, C, D, E. Add 5 c.c. of pancreatic juice to each tube. Boil E, and then cool it. Put into $\mathrm{A}$ and $\mathrm{B}$ small pieces of heat-coagulated egg-white, into $\mathrm{C}$ a little starch mucilage, and into D and E 5 c.c. of fresh cream. Add further to B a scraping of the mucous membrane of the upper part of the small intestine which has first been washed free of contents. To D and E add a

* With hydrocliloric acid of different strengths the rapidity of digestion of boiled fibrin by glycerin extract of dog's pancreas (I volume of extract to 25 of acid) was found about the same for 0.3 and 0.17 per cent. acid ; much less for $0^{\circ} \mathrm{OS}$ per cent., while in $0^{\circ} \mathrm{O}_{4}$ per cent. acid there was practically no digestion at all. In $0^{\circ}+$ per cent. acid digestion took place more rapidly than in $0^{\circ} \mathrm{OS}$ per cent., but much less rapidly than in $0^{-1} 17$ per cent. In acid of all strengths digestion was markedly slower than in I per cent. sodium carbonate.

t A considerable flow of pancreatic juice can be obtained from a dog with a pancreatic fistula by injecting intravenously an extract of intestinal mucous membrane containing secretin (p. 379). 
drop or two of litmus solution, and, if necessary, enough of dilute sodium liyclroxicle to just establish a blue colour. Then put the test-tubes at $40^{\circ} \mathrm{C}$., and examine after a time. No digestion will have taken place in $\mathrm{A}$, because the pancreatic juice, as secreted, does not contain active trypsin. In $B$ cligestion may take place, because the enterokinase in the intestinal mucous membrane will activate the trypsinogen to trypsin. In $\mathrm{C}$ and $\mathrm{D}$ there will be evidence of the production of reducing sugar and fatty acids respectively, since the pancreatic juice, as secreted, contains active amylopsin and steapsin. E will be unchanged unless by bacterial action.

(f) Leucin and Tyrosin.- As examples of amino-acids formed when pancreatic digestion of proteins (fibrin or casein, e.g.) is allowed to go on for some days,* leucin and tyrosin may be isolated. Add bromine water by drops to 5 c.c. of the digest; a pink colour indicates tryptophane. If the " digest' be neutralized, then filtered, and the filtrate concentrated and allowed to stand, a crop of tyrosin crystals will separate out, since tyrosin is only slightly soluble in watery solutions of neutral salts. These crystals having been filtered off, the proteoses (albumoses) and peptones can be precipitated together by alcohol, and afterwards separated. if that is rlesired, by reclissolving the precipitate in water and throwing down the proteoses by saturation with ammonium sulphate. The alcoholic filtrate will contain any leucin that may be present, since that boly is moderately soluble in alcohol, as well as traces of tyrosin, which, however. is much less soluble in this medium. On concentration, crystals of botl substances will be obtained. Tyrosin crystallizes characteristically from animal liquids in beautiful silky necdles united into sheaves, leucin in the form of indistinct fatty-looking balls, often marked with radial strize and coloured with pigment (Figs. I69 and I $70, \mathrm{p} \cdot+52)$.

7. Bile.-(a) Test the reaction of ox bilc. It is alkaline to litmus.

(b) Add dilute acetic acid. A precipitate of bile-mucin (really nucleo-albumin) falls down. Some of the bile-pigment is also precipitated. Filter. (Pig's bile contains more of the mucin-like substance than ox bile.)

(c) Put a little of the filtrate from $(b)$ or of the original bile into a porcelain capsule, add a drop or two of a dilute solution of canesugar, and mix with the bile. Then add a few drops of strong sulpliuric acid, and stir. Then a few lrops more of the sulphuric acid, stirring all the time. A purple colour appearing at once, or after gentle heating, shows the presence of bile-acids (Pettenkofer's reaction). The bile may be diluted before the adclition of the sulphuric acid. In this case a greater amount of the acicl must be added. Examine the purple liquid in a test-tube witl a spectroscope (p. 65 ). Dilute tlie liquid with water. adlling some sulphuric acicl to partially clear up the precipitate caused by the water. Two absorption bands are seen. one to the red side of $\mathrm{D}$, and the other, a stronger and broader band, over and to the right of $E$. When only a very small amount of bile-salts is present, the reaction is made more sensitive if a solution of furfuraldehycle (1 to 1,000) is userl instead of cane-sugar.

(d) Hay's Sulphur Test.-Sprinkle a little sulphur (in the form of the fine powder known as flowers of sulpliur) on the surface of some bile in a small beaker or deep watch-glass. The sulphur will soon sink to the bottom. Repeat with water; the sulphur will float. The reaction is due to the diminution of the surface tension produced by

* A little chloroform is adcled to prevent bacterial growth. 
the bile-acids, and succecds also in a solution of bile-salts. The test is very sensitive. But in stomach contents, vomit, or stools, it rarely gives good results, since alcohol or acetic acil is often present in the gastric liquid, and phenol and its clerivatives in intestinal contents, and all of these cause such an alteration in the surface tension that the sulphur sinks. Ether, chloroform, turpentine, benzine and its derivatives, anilin and soaps, also vitiate the test in the same way.

(c) Adcl yellow nitric acid (containing nitrous acid) to a little bile on a white porcelain slab. $\$ play of colous, beginning with green and running through blue to ycllow and yellowish-brown, indicates the presence of bile-pignent (Gmelin's reaction). The reaction may also be obtained by putting some yellow nitric acid into a test-tube, and then rumning a little bile from a pipette on to the surface of the acicl. The play of colours is seen at the surface of contact. Where the bile-pigment is present only in traces, some of the liquid may be filtered througl, white filter-paper, and the test applied by putting a drop of the nitric acid on the paper.

(f) Cholesterin (Fig. 159) - Preparation. - Extract a powdered gall-stone (preferably a white one) with hot alcohol and ether in a test-tube. Heat the test-tube in warm water, not in the free flame. Put a drop of the extract on a slide. Flat crystals of cholesterin, often chipped at the corncrs, separate out. (a) Carefully allow a drop of strong sulphuric acid and a drop of dilute iodine to run under the cover-glass. A play of colours-violet, bluc, green, red-is seen.

( $\beta$ ) Evaporate a drop of the solution of cholesterin in a small porcelain capsule, add a drop of strong nitric acid, and heat gently over a flanıe. A ycllow stain is left, which becomes red when a drop of ammonia

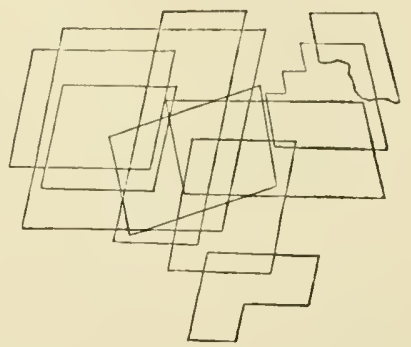

Fig. I59. -- CRY'stals of ChOlesTERIN (FREY). is poured on it while it is still warm.

$(\gamma)$ Dissolve a little cholesterin in chloroform. Add an equal bulk of strong sulphuric acid, and shake gently. The solution turns red and the subjacent acid shows a green fluorescence.

$(\delta)$ Put a drop or two of water in a watch-glass, and add a drop of an ethereal solution of cholesterin. The cholesterin is precipitated, and will not dissolve in the water even on heating. Repeat the observation with bile instead of water. The cholesterin dissolves in the bile.

(g) To a little of the filtrate from a peptic digest (e.g., fibrin which has been digested for twenty-four hours with pepsin and hydrochloric acid) add some bile. A precipitate is thrown down, which is redissolved in excess of the bile (p. $34 \mathrm{I}$ ).

(h) Shake up a little bile with some rancid olive-oil in a test-tube. An cmulsion is formed. Repeat the experiment with the same quantities of bile and oil, but use perfectly fresh oil. Compare the stability of the two emulsions, allowing the tubes to stand together for a while.

(i) To some starch mucilage, shown to be free from sugar, add a little bile, and place in a bath at $4^{\circ} \mathrm{C}$. After a time test for reducing sugar. Report the result. Bile often has a slight diastatic power. 
(j) To demonstrute the Presence of Iron in the Liver Cells.--Steep, sections of liver in a solution of potassimm ferrocyanide, and then in lilute hydrochloric acid. Or a $\mathrm{r}^{*} 5$ per cent. solution of potassium ferrocyanide in $0^{\circ} 5$ per cent. hydrochloric acid may be used. (The iron may previously be fixed in the tissue by hardening it in a mixture of alcohol and ammonium sulphide.) The sections become bluish from the formation of Prussian blue. A fine-pointed glass rod or a platinum lifter should be usecl in manipulating them. A stecl neculle cannot be cmployed. Mount in glycerin or Farrant's solution, or (after dehydrating with alcohol and clearing in xylol) in xylol-balsam. Blue granules may be seen under the microscope in some of the hepatic cells. Sections of spleen may also be exanined for this reaction.

8. Microscopical Examination of Fæces.--Examinc under the microscope the slides provided. Draw, and as far as possible deterinine the nature of, the objects seen (p. 396).

9. Absorption of Fat.--(a) Feed a rat or frog with fatty food ; kill the rat in three or four hours, the frog in two or three clays. Immediately after killing the rat open the abdomen, carefully draw out a loop of intestine, and look through the thin mesentery. The white lacteals will probably be seen ramifying in the mesentery. They appear white on account of the presence of globules of fat in the chyle with which they are filled. Strip off tiny picces of the mucous membrane of the small intestine, and steep them in $\frac{1}{2}$ per cent. solution of osmic acid for forty-eight hours. Then tease fragments of the mucous membrane in glycerin and examine under the microscope. To preserve the specimens take off the glycerin with blotting-paper and mount in Farrant's medium, which is a preservative glycerin mixture. Other portions of the mucous membrane may be hardened for a fortnight in a mixture of 2 parts of IIüler's fluid and I part of a I per cent. Solution of osmic acid. Sections are then made with a freezing microtome after embeclding in gum. No process must be used by which the fat would be clissolved out (Schäfer). (See Fig. I 56 , p. 4 I 3 .)

(b) Feed a cat with 30 grammes of butter stainecl a deep red with the dye Sudan III. After five hours ansesthetize the animal with ether, insert a camnula in the carotid artery, and obtain a sample of blood. Defibrinate the blood, and separate the serum by the centrifuge. If digestion and absorption of the fat have procecded normally, the serum will contain numerous fat droplets, and will be tinged pink by the dye, which can be dissolved out of it by shaking up with ether. On opening the abdomen it will be seen that the mucous membrane of the small intestine, as far down as the fat has reached, is stained pink, and that the lacteals in the mesentery are also pink. Observe whether any of the pigment has passed into the urine.

ro.* Time required for Digestion and Absorption of Various Food Substances. - Feed three dogs, A, B, and C, which have previonsly fasted for twenty-four hours, with a meal containing starch (proved to be free from sugar), lard, and meat.

(1) After fifteen minutes inject subcutaneously into A 2 c.c. of a o. I per cent. solution of apomorphine. Note the time which clapses before the animal vomits. Collect the vomit.

* Experiments 10 and 11 are conveniently lone in a class by assigning each of the three animals to a separate set of students. The contents of the stomach and intestine are clivided into three portions, so that each set has a sample from each dog. 
(a) Examine a little of it muler the microscope, and make ont fat globules, muscular fibres and starch granules. The latter can be recognised by their being coloured bue by a drop or two of iotline solution.

(b) Filter the chyne, mixing it, if necessary, with a little water, and test it as in $+(d)(p)+2())$ for the products of digestion of proteins. In addition, test for starch, dextrin, and reducing sugar

(2) One and a (puarter hours after the meal inject apomorphine into $\log B$, and proceed as in (I).

(3) Two and a half hours after the meal inject apomorphine into dog $C$, and procecl as in (I). Compare the results from the three specimens of chyme.

11.* Quantity of Cane-sugar inverted and absorbed in a Given Time.-Take three $\operatorname{logs}, \mathrm{A}, 13$, and C, which have fasted for twentyfour hours. The animals should be about the same size. Feed A and $B$ with roo c.c. of a stanclard solution of cane-sugar (about a 20 per cent. solution) or as much more as they will take. If the logs have been liept without water for a day they will more readily take the sugar solution. Or it may be given through a tube passed into the stomach, and in this case a larger quantity of sugar can be given. A gag consisting of a piece of wood with a hole in the middle of it, through which the tube is passed, must first be secured in the dog's mouth. Feed $\mathrm{C}$ with 50 grammes of powdered canc-sugar mixed with lard, the mixture being rolled into little balls.

(I) After a quarter of an hour put A under chloroform or the A.C.E. mixture, and fasten it on a holder. Kill the animal with chloroform, open the abdomen, tic the osophagus, place double ligatures on the pyloric end of the stomach and the lower end of the small intestine, and livide between them. Cut out the stomach and intestine: wash their contents into separate vessels, and test the reaction with litmus paper. Add water and rub up thoroughly. Filter. Wash the residue repeatedly with small quantities of water, and pass all the washings through the filter. Make up each of the two filtrates to 200 c.c.

(a) Test the filtrates from the contents of the stomach and intestines qualitatively for dextrose by Trommer's (p. Io) and the phenylhydrazine test (p. 488).

(b) If no reducing sugar is present, add to 20 c.c. of each filtrate I c.c. of hydrochloric acid, boil for half an hour, and again test for reducing sugar. If it is now found, some cane-sugar is present.

(c) If reducing sugar is found, estimate its amount as dextrose by Fehling's solution (p. 489 ) in a measured quantity of the original filtrate of the gastric or intestinal contents before and after boiling with one-twentieth of its volume of hydrochloric acid.

(d) Estimate in the same way the amount (as dextrose) of the invert sugar in the standard solution of cane-sugar after inversion, and before inversion if it gives the qualitative test for reducing sugar before it has been boiled with acid.

From the data obtained (and taking 95 parts of cane-sugar as equal to oo parts of dextrose) calculate the amount of cane-sugar absorbed, left unchanged, and inverted, though not absorbed.

(2) One and a half hours after the meal anzesthetize B, and proceed as in $(1)$.

(3) Two hours after the meal proceed in the same way with $\mathrm{C}$. But in addition observe the lacteals in the mesentery, by gently 
lifting up a loop of intestine immediately after opening the abdomen. If the absorption of the fat lias begun, they will be easily visible, as a network of fine milk-white vessels. Also examine the gastric and intestinal contents with the microscope for fat globules. Compare your results on the amount of sugar obtained from the three animals. Probably much more unabsorbed sugar will be found in (' than in $B$, as the lard hinders it from being dissolved.

I2. Auto-digestion of the Stomach.-In some of the previous experiments the stomach of an animal killed during ligestion should be removed from the body after double ligation of cesophagus and duoderum, and placed in a water-bath at $40^{\circ} \mathrm{C}$. After several hours the contents should be washed out, and the mucous membrane examined. It may be entirely eaten away in parts. 


\section{CHAPTER VI}

\section{EXCRETION}

WE have now followed the ingoing tide of gaseous, liquid, and solid substances within the physiological surface of the body. There we leave them for the present, and turn to the consideration of the channels of outflow, and the waste products which pass along them. In a body which is neither increasing nor diminishing in mass the outflow must exactly balance the inflow ; all that enters the body must sooner or later, in however changed a form, escape from it again. In the expired air, the urine, the secretions of the skin, and the fæces, by far the greater part of the waste products is eliminated. Thus the carbon of the absorbed solids of the food is chiefly given off as carbon dioxide by the lungs ; the hydrogen, as water by the kidneys, lungs and skin, along with the unchanged water of the food; the nitrogen, as urea by the kidneys. The freces in part represent unabsorbed portions of the food. A small and variable contribution to the total excretion is the expectorated matter, and the secretions of the nasal mucous membrane and lachrymal glands. Still smaller and still more variable is the loss in the form of dead epidermic scales, hairs, and nails. The discharges from the generative organs are to be considered as excretions with reference to the parent organism, and so is the milk, and even the fœetus itself, with respect to the mother.

Excretion by the lungs and in the fæces has been already dealt with. All that is necessary to be said of the expectoration and the nasal and lachrymal discharges is that the first two generally contain a good deal of mucin, and are produced in small mucous and serous glands, the cells of which are of the same general type as those of the mucous and seruus salivary glands. The lachrymal glands are serous like the parotid; and the tears secreted by them contain some albumin and salts, but little or no mucin. The sexual secretions and milk will be best considered under reproduction (Chap. XIV.), so that there remain only the urine and the secretions of the skin to be treated here 


\section{Excretion by the Kidneys.}

The Chemistry of the Urine.-Normal urine is a clear yellow liquid acid to litmus and similar indicators, but nearly neutral or very weakly acid in the physico-chemical sense (p. 23). The average specific gravity is about I020, the usual limits being IOI 5 and I025, although when water is taken in large quantities, or long withheld, the specific gravity may fall to I005, or even less, or rise to 1035, or eren more. The quantity passed in twentyfour hours is very variable, and is especially dependent on the activity of the sweat-glands, being, as a rule, smaller in summer when the skin sweats much, than in winter when it sweats little. The average quantity for an adult male is 1200 to Ifoo c.c. (say, to to $50 \mathrm{oz}$.).*

Composition of Urine.-This is very closely related to the quantity and quality of the food. Hence it is impossible to speak of a definite normal composition of the urine. It is essentially a solution of urea and inorganic salts, the proportion of the latter being generally about I' 5 per cent., or double the usual amount in physiological liquids. Besides urea, there are other nitrogenous bodies in mich smaller quantity, such as ammonia, uric acid, and the allied xanthin bases, hippuric acid, and kreatinin. Some of these at least are products of the metabolism of proteins within the tissues. And besides the inorganic salts there are certain organic bodies-indoxyl, phenyl, pyrokatechin, skatoxyl-united with sulphuric acid, which are primarily derived from the products of the putrefaction of proteins within the digestive tube.

Folin has published analyses of 'normal' urines from six persons, weighing from $5^{6} \cdot 6$ to $70^{\circ} 9$ kilos (average $63^{\circ} 4$ kilos), who were kept for seven days on one standard uniform diet. The diet consisted of 500 c.c. of milk, 300 c.c. of eream (containing I 8 to 22 per cent. of fat), 450 grammes of eggs, 200 grammes of Horlick's malted milk, 20 grammes of sugar, 6 grammes of sodium chloride, water enough to make the whole up to two litres, and 900 c.c. of additional water. The ingredients contained il 9 grammes of protein, about 148 grammes of fat, and 225 grammes of carbo-hydrates. The average results of all the determinations are given in the following table:

* The average quantity of urine varies not only with the season, but also with the habits of the person, especially as regards the amount of liquid taken. The average for seventeen healthy (American) students, whose urine was collected for six to eight snccessive days in winter, was 1166 c.c. The highest average in any one individual for the observation period was 1487 c.c. (for seven days), and the lowest $7+3$ c.c. (for eight rlays). The greatest quantity passed in any one period of twenty-four hours was 2286 c.c. (by the individual whose average was the highest). The smallest quantity passed in twenty-four hours was 6;0 c.c. (by the individual whose average was the lowest). 


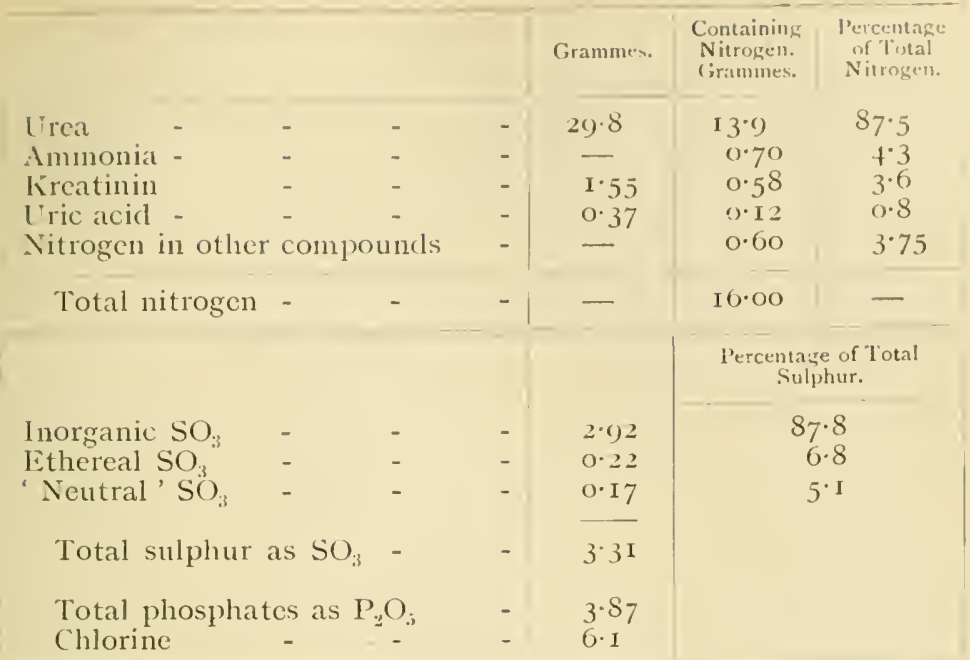

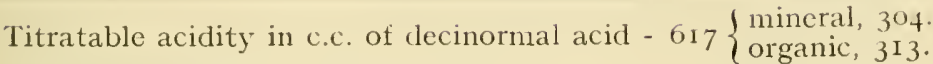

Indican (Fehling's solution $=\mathbf{1 0 0} *$ ) -

- 77

- I 430 C.c.

The great influence of diet on the composition of the urine is illustrated in the following table. Urine I. was obtained from a man weighing $S_{7}$ kilos on the standard protein-rich diet described above. Urine II. was obtained from the same person on a diet very poor in protein (40o grammes of starch and 300 c.c. of cream), containing only about I gramme of nitrogen, as against I g grammes in the first diet.

\begin{tabular}{|c|c|c|c|c|c|c|}
\hline & & & \multicolumn{2}{|r|}{ I. } & \multicolumn{2}{|c|}{ II. } \\
\hline Volume of urine & - & & I I 7 & o c.c. & $3^{8} 5_{5}$ & 5 c.c. \\
\hline Total nitrogen & - & - & $\begin{array}{l}\text { Grammes } \\
\text { I } 6.8\end{array}$ & Per Cent. & $\begin{array}{c}\text { Grammes. } \\
3.60\end{array}$ & Per Cent. \\
\hline Urea-nitrogen & - & & 14.70 & $=87.5$ & $2 \cdot 20$ & $=6 \mathrm{I} \cdot 7$ \\
\hline Ammonia-nitrogen & & 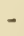 & $0 \cdot 49$ & $=3 \cdot 0$ & 0.42 & $=I \mathbf{I} \cdot 3$ \\
\hline Uric acid-nitrogen & - & - & $0 \cdot 18$ & $I \cdot I$ & 0.09 & 2.5 \\
\hline Kreatinin-nitrogen & & - & 0.58 & $3 \cdot 6$ & 0.60 & $=17 \cdot 2$ \\
\hline Nitrogen in other ce & mpoun & & 0.85 & $4 \cdot 9$ & $0 \cdot 27$ & $7 \cdot 3$ \\
\hline Total $\mathrm{SO}_{3}-$ & - & - & $3 \cdot 64$ & & & \\
\hline Inorganic $\mathrm{SO}_{3}$ & - & - & $3 \cdot 27$ & $=90.0$ & $0 \cdot 46$ & $=60.5$ \\
\hline Ethereal $\mathrm{SO}_{3}$ & - & - & $0 \cdot 19$ & $5 \cdot 2$ & $0 \cdot 10$ & $=1.3 \cdot 2$ \\
\hline Neutral $\mathrm{SO}_{3}$ & - & - & 0.18 & $4 \cdot 8$ & 0.20 & $=26 \cdot 3$ \\
\hline Total phosphates a & $\mathrm{P}_{2} \mathrm{O}_{3}$ & - & $4 \cdot I$ & & $\mathrm{I} \cdot \mathrm{O}$ & \\
\hline Chlorine & - & - & $6 \cdot I$ & & $1 \cdot 6$ & \\
\hline
\end{tabular}

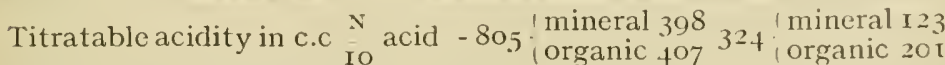
Indican (Fehling's solution = IOo) - $120 \quad-\quad-\quad 0$

* The indican is given in arbitrary units, the indigo-blue being obtained from the urine and then estimated colorimetrically, using Fehling's solu- 
The titratable acidity of urine (see p. 2.1) is chicfly clue to the acid (monobasic) plosphates, such as acid sodium phospliate $\left(\mathrm{NaIH}_{2} \mathrm{l}^{\prime} \mathrm{O}_{4}\right)$, but in an important degree also to organic acids. According to Folin, indeed, the organic acidity may be more than half the total acidity. Normally the acidity diminishes distinctly, or even gives place to alkalinity, during digestion, when the acicl of the gastric juice is being secreted. This is sometimes fancifully denominated the alkaline tide. After a fast, as before breakfast, the opposite condition, the acid tide. occurs.

The acidity varies with the quantity of vegetable food in the diet. The: urine of herbivora and vegetarians is alkaline. and is either turbid when passed, or on standing soon becomes turbid from precipitated carbonates and phosphates of earthy bases, while that of carnivora and of fasting herbivora, which are living on their own tissues, is strongly acid and clear. Normal human urine may deposit urates soom after discharge, as they are more soluble in warm than in cold water. They carry down some of the pigment, and therefore usually appear as a pink or brick-red sediment. When urine is allowed to stand after being voicled, what is generally described as ' acid fermentation' occurs. The acidity grarlually increases : acid sodium urate is produced from the neutral urate, and comes down in the form of amorphous granules, while the liberated uric acicl is deposited

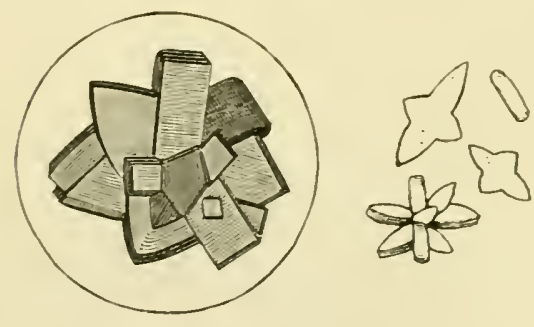

FiG. I60.-URIC ACID.

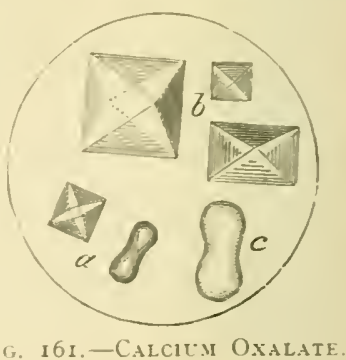

often in 'whetstone' crystals, coloured yellow by the pigment (Fig. 16o). Calcium oxalate may also be thrown down as 'envelope,' $a, b$, or. less frequently, 'sand-glass' crystals, c (Fig. 16I). If the urine is allowed to stand for a few days. especially in a warm place. or in a place where urine is decomposing, the reaction becomes ultimately strongly alkaline, owing to the formation of ammonium carbonate from urea by the action of micro-organisms (Micrococcus urea, Bacterium urea, and others) which reach it from the air, and produce a soluble ferment, in whose presence the urea is split up with assumption of water. Thus :

$$
\mathrm{CON}_{2} \mathrm{H}_{4}+2 \mathrm{H}_{2} \mathrm{O}=\underset{\text { Immonium carbonate. }}{\left(\mathrm{NH}_{4}\right)_{2} \mathrm{CO}_{3} .}
$$

The substanies insoluble in alkaline urine are thrown down, the deposit containing ammonio-magnesic or triple phosphate. formed by the union of ammonia with the magnesium phosphate present in fresh urine. and precipitated as clear crystals of "knife-rest " or 'coffin-lid ' shape (Fig. I62), along with amorphous earthy phosphates, and often

tion as a standard. Fehling's solution is employerl because it is a blue liquid of definite depth of tint already prepared in cvery physiological laboratory. 
acid ammonium urate in the form of dark balls occasionally covered witls spines (l.ig. I65). Calcinm phosphate $\left(\mathrm{CaHH}_{\mathrm{PO}}\right)$ is another plosphate found in serliments deposited from alkaline or faintly acirl urine. It is usually amorphous, but sometimes in the form of long prismatic crystals arranged in star fashion, and hence spoken of as stellar plosphate (Fig. 164). It is not pigmented.

It is only in pathological conditions that the alkaline fermentation takes place within the bladcler. The reaction of the urine can readily be marle alkaline by the arlministration of alkalies, alkaline carbonates, or the salts of vegetable acids like malic, citric, and tartaric acid, which are broken up in the body and form alkaline carbonates with the alkalies of the blood and lymph. It is not so

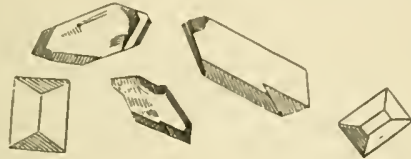

Fig. i62.-Triple Pilosphate.

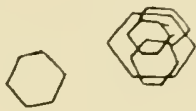

Fig. I63.-Cystin.

easy to increase the acidity of the urine, although mineral acids do so up to a certain limit. If the administration of acid be pushed farther, ammonia is split off from the proteins, and is excreted in the urine as the ammonium salt of the acid.

Determination of the Acidity.-A titration method is described in the Practical Exercises (p. 477). In speaking of the reaction of blood, it has already been mentioned (p. 24) that we cannot determine by titration the true acidity or alkalinity of a liquid in the physico-chemical sense-i.e.. the concentration of the dissociated hydrogen and hydroxyl ions respectively. E.g., when we titrate equal quantities of decinormal* acetic acid and decinormal hydrochloric acid with decinormal potassium hydroxide, using, say, phenol-

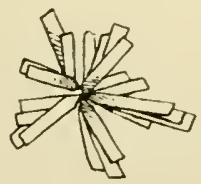

Fig. I64.-Stellar Phosphate CRYSTALS.

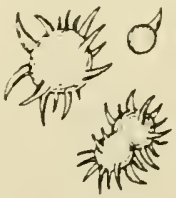

Fig. I65.-AMMONIUM URATE (AFter MilRoy).

phthalein as the inclicator, nearly the same volume of the potassium hydroxide solution will be needed to neutralize each acirl. Yet it can be shown by physico-chemical methods that the acetic acid in the strength used is only dissociated to the extent of a little more than I per cent., while about 80 per cent. of the hydrochloric acid is dissociated. The concentration of the hydrogen ions is there-

* A normal solution of a substance contains in a litre a number of grammes of the substance equal to the number which expresses its equivalent weight-a decinormal (usually written No) solution one-tenth of this amount, a centinormal one-hundredth, etc. Thus, a normal solution of potassium hydroxide contains $; 6$ grammes of $\mathrm{KOH}$, and a decinormal solution $5^{*} 6$ grammes in 1000 c.c. 
fore eighty times as great in the hydrochloric as in the acetic acid solution. What we determine by the titration is not the true acidity, but the total amount of hydrogen which can be replaced by metal. The concentration of the hydrogen ions in normal urine is very small, on the average only about 0.003 milligrammes in the litre, or about thirty times as much as is present in the purest clistilled water. Urine departs about as much from neutrality in the one direction as blood docs in the other.

Urea, $\mathrm{CO}\left(\mathrm{NH}_{2}\right)_{2}$, is the form in which by far the greater part of the nitrogen is under ordinary conditions discharged from the body. Its amount is as important a measure of protein metabolism as the quantity of carbon dioxide given out by the lungs is of the oxidation of carbonaceous material. Yet a glance at the table on p. 437 shows that, when the total protein metabolism is greatly reduced by diminishing the protein in the food, the relative as well as the absolute amount of nitrogen eliminated as urea suffers a great diminution. The relative amount of the other nitrogenous urinary constituents, espccially of the kreatinin, is markedly increased. The significance of this difference is alluded to in speaking of the kreatinin content of urine, and will have to be again considererl under Protein Metabolism. Urea is soluble in water and in alcohol, and crystallizes from its solutions in the form of long colourless needles, or four-sided prisms with pyramidal ends. It can be easily prepared from urinc. Urea can also be obtained artificially by heating its isomer, ammonium cyanate $\left(\mathrm{NH}_{4}-\mathrm{O}-\mathrm{CN}\right)$, to $100^{\circ} \mathrm{C}$. This reaction is of great historical interest, as it forms the final step in Wöhler's famous synthesis of urea, the first example of a complex product of the activity of living matter being formed from the ordinary materials of the laboratory. Heated in watery solution in a sealed tube to $180^{\circ} \mathrm{C}$, urea is entirely split up into carbon dioxide and ammonia, a cliange which can also be brought about, as already mentioned, by the action of micro-organisms. Nitrous acid, hypochlorous acid, and salts of hypobromous acid carry the decomposition still further, carbon dioxide, nitrogen, and water being the products of their oxidizing action on urea. Thus: $\mathrm{CO} .2\left(\mathrm{NH}_{2}\right)+3 \mathrm{NaBrO}=$ $3 \mathrm{NaBr}+2 \mathrm{H}_{2} \mathrm{O}+\mathrm{CO}_{2}+\mathrm{N}_{2}$. This reaction is the basis of the hypobromite method of estimating the quantity of urea in urine (Practical Exercises, p. 480).

Ammonia. - The ammonia in urine is united with acids in the form of salts. Its formation from proteins is determined, as we shall see later on, by the necessity of neutralizing certain acids produced in metabolism-e.g., those derived from the sulphur and phosphorus of the proteins, or acids administered experimentally. According to some observers, the percentage amount of the total nitrogen in the urine in the form of ammonia remains the same whether the food be rich or poor in protein (Schittenhelm, etc.). but others state that when the protein is reduced, there is a relative increase in tlie ammonia-nitrogen (see table on $\mathrm{p} .+37$ ) (Folin).

Uric acid $\left(\mathrm{C}_{5} \mathrm{H}_{3} \mathrm{~N}_{4} \mathrm{O}_{3}\right)$ exists in large amount in the urine of birds. The excrement of serpents consists almost entirely of uric acid. In both cases it is mainly in the form of acid ammonium urate. In contrast to urea, uric acid is very insoluble, requiring 1,900 parts of hot, and 15,000 parts of cold, water to dissolve it. In man and mammals the quantity is comparatively small in liealth, but is increased after a meal, particularly one containing substances rich 
in nucleins - e.g., the thymus-or substances containing purin bases-e.g., hypoxanthin in meat. When the amount of protein in the food is greatly reduced, the absolute quantity of uric acid is diminished, but the proportion of the total nitrogen of the urine climinated as uric acid is increased, since the 'cndogenous uric acid (p. 507) still continues to be formed and excreted.

The purin bases (sometimes called the nuclein bases, the alloxuric bases, or the xanthin bases) are a group of substances allied to uric acid, and including, besides xanthin itself, hypoxanthin, guanin, adenin, and other bodies. They exist in very small amount in urine, but. like uric acid, are increased in amount by the ingestion of nuclein-containing substances. The greater part of the purin bases produced in the body is transformed into uric acid; it is only the untransformed residue which appears in the urine. An interesting fraction of the purin bases in the urine which is not related to the nuclein metabolism is composed of the so-called heteroxanthin. derived from caffeine in the coffee and tea, $l$-methylxanthin, derived from theobromine in the cocoa. and paraxanthin, derived from theophyllin in the tea. consumed as beverages.

The purin bodies are so called because they, and uric acid also. are all to be considered chemically as derivatives of a substance called purin $\left(\mathrm{C}_{5} \mathrm{H}_{4} \mathrm{~N}_{4}\right)$, which, howcver, has itself never been discovered in the body. Their empirical formulæe are as follows:

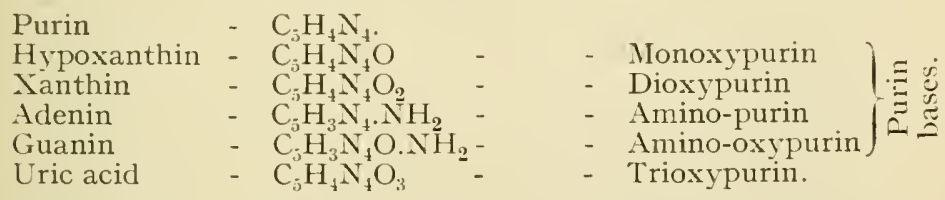

Hippuric acid $\left(\mathrm{C}_{9} \mathrm{H}_{3} \mathrm{NO}_{3}\right)$ occurs in considerable quantity in the urine of herbivora (Practical Exercises, p. 486 ); in the urine of carnivora and of man only in traces; in that of birds not at all. Its amount is much more dependent on the presence of particular substances in the food than that of the other organic constituents of urine. Anything which contains benzoic acid, or substances which can be readily changed into it (such as cinnamic and quinic acids), causes an increase of the hippuric acid in urine. In fact, one of the best ways of obtaiming the latter is from the urine of a person to whom benzoic acid is given by the mouth; the sweat may also in this case contain a trace of hippuric acid. Chemically, it is a conjugated acid formed by the union of benzoic acid and glycin. Thus :

$$
\underset{\text { Benzoic acid. }}{\mathrm{C}_{7} \mathrm{H}_{6} \mathrm{O}_{2}}+\underset{\text { Glycin. }}{\mathrm{C}_{2} \mathrm{H}_{5} \mathrm{NO}_{2}}=\underset{\text { Hippuric acid. }}{\mathrm{C}_{3} \mathrm{H}_{4} \mathrm{NO}_{3,3}}+\underset{\text { Water. }}{\mathrm{H}_{2} \mathrm{O}}
$$

Benzoic acid, therefore, mects glycin in the body, and combines with it. as fatty acids meet glycerin and combine with it. But while a minute amount of free glycerin has been found in the plasma, no free glycin has been detected in the normal blood or tissues. Glycin. however, has been demonstrated in the blood and ascitic fluid in cases of nephritis.

Amino-acids. - The only amino-acid hitherto detected with certainty in normal urine is glycin.

Oxalic acid is always present, although in very small amount. Some of it comes from the oxalates of the food, but a portion of it 
arises in the metabolism of the tissues probably from the decomposition of uric acid. It is known that outside of the body uric acid may be made to yicld oxalic acid. Calcium oxalate crystals are often seen in urinary secliments.

Kreatinin $\left(\mathrm{C}_{4} \mathrm{H}_{7} \mathrm{~N}_{3} \mathrm{O}\right)$.-- Kreatinin is the anhydride of kreatin (lig. 16(i). Its formula differs from that of kreatin only in possessing the elements of one molecule of water less; and kreatinin can be obtained by boiling kreatin with dilute sulphuric acid, then neutralizing with barium carbonate, filtering, evaporating the filtrate to dryness on the water-bath, and extracting the residue withalcolool. From its alcoholic solution it crystallizes in colourless prisms Kreatinin forms crystalline compounds with various acids and salts. One of the best known of these is kreatinin-zinc-chloride, formed on the addition of zinc chloride to an alcoholic or watery solution of lireatinin, often in the shape of beautiful thick-set rosettes of neeriles (Fig. I67). A portion of the urinary kreatinin is derived from the kreatin of the meat taken as fool. But this is not its only source, for on a meat-free dict and in starvation kreatinin is still excreted. The absolute quantity in the urine on a meat-free list is constant for one and the same inclividual, although different

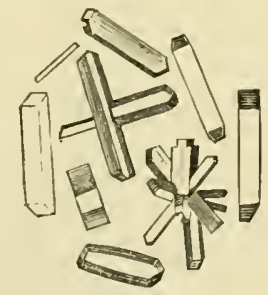

Fig, i66.-KREATIN.

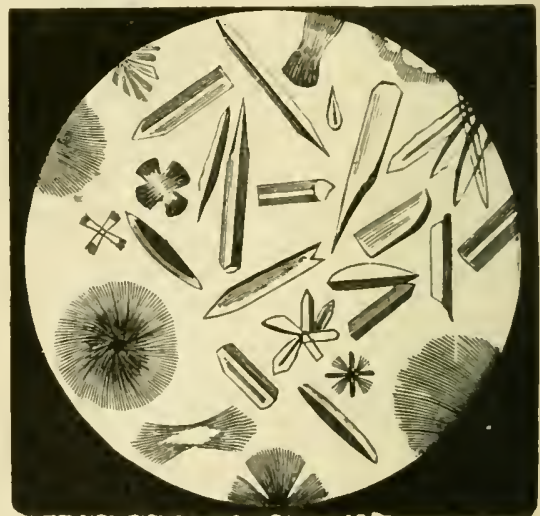

FIG. I67.-KREATININ-ZINC-CHLORIDE.

in different persons, and independent of the total amount of nitrogen eliminated. Hence on a dict poor in protein the percentage of the total nitrogen excreted as kreatinin is nuch greater than on a protein-

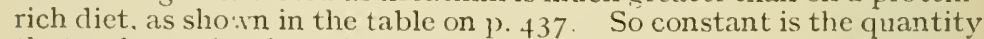
that a determination of the kreatinin may be used as a check upon the complete collection of the urine.

Carbo-hydrates are normally present in human urine, but only in very small amount. Three are known with certainty-dextrose. isomaltose, and the so-called animal gum or urire dextrin. Glycuronic acid $\left(\mathrm{C}_{10} \mathrm{H}_{10} \mathrm{O}_{7}\right)$, a body which can be derived from dextrose, and which occurs in the urine in increased amount after the administration of chloroform, chloral, nitrobenzol. camplior, and other drugs, seems also to be constantly, or at least frequently present in small amount, probably paired with phenol, indoxyl or sliatoxyl, as a potassium salt. It gives Fehling's test, and thus may easily be mistaken for sugar. The total quantity of carbo-hydrates, including glycuronic acid, excreted in the urine of the twenty-four hours has been estimated at 2 to 3 grammes. The quantity of lextrose in 
normal human urine is about $0^{\circ} \mathrm{O} 2$ per cent., or about one-fifth of the proportion in blood.

Proteins, mainly serum-allomin, are also foumd in normal urinc in minute quantities, on the average about $0^{\circ} 0036$ per cent. (Mörner).

Pigments of Urine.-The pigments of urine have not hitherto been exhanstively studied; but we already know that normal urine contains several, and pathological urines probably additional, pigmentary substances. The best-known pigments in normal urine are urochrome, the vellow substance which gives the liquid its ordinary colour; uroerythrin, the pink pignent which often colours the deposits of urates that separate even from healthy urine; and urobilin, sometimes termed normal urobilin, to distinguish it from the so-called febrile urobilin, which, as has been already stated, is identical with the faca'. pigment stercobilin, and occurs not only in many febrile conditions, but also in cases with no fever, such as functional derangements of the liver, dyspepsia, chronic bronchitis, and valvular diseases of the heart. Normal and febrile urobilin are said to show certain spectroscopic differences, but are nevertheless one and the same substance, and represent, mainly at least, the

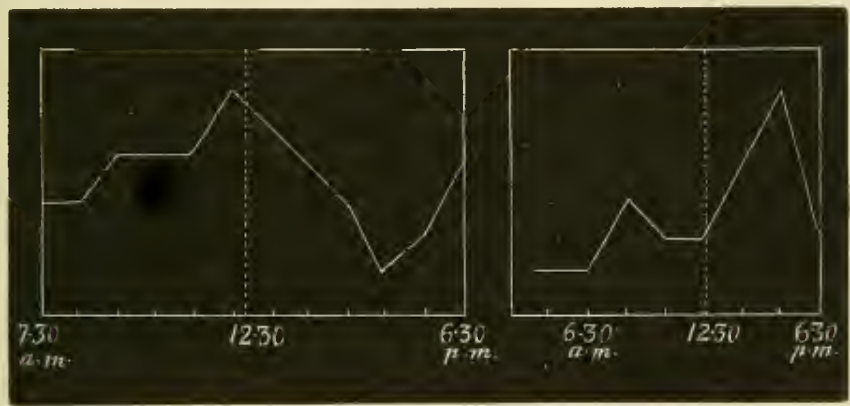

Fig. i68.-Pepsin in Urine. Diastatic Ferment in Urine. At Different Times of the Day (Hoffuann).

portion of the stcrcobilin which is not excreted with the fæces, but absorbed from the intestine into the blood. The urobilin in normal urine only exists in small amount in the fully-formed condition, most of it being present as a chromogen or mother-substance (urobilinogen), which by oxidation, as on standing exposed to the air, is converted into urobilin. On the addition of ammonia and zinc chloride to a solution of urobilin a beautiful green fluorescence is obtained, and the solution now shows an absorption balld between $b$ and F. Urobilin and urochrome are related substances, but the exact nature of the relation has not been settled. Therc is some evidence that a portion of the urobilin of urine is not derived from the intestine, but manufactured probably in the liver. In hunger urobilin is still excreted in the urine, although in greatly reduced amount. During menstruation it is markedly increased, both in fasting and in normally fed individuals. Urorosein is a red pigment which is produced from its chromogen by the action of mineral acids-e.g., strong hydrochloric acid-especially in the presence of an oxidizing agent.

The pigments of the blood and bile and some of their derivatives are of common occurrence in the urine in disease. Hamatoporphyrin has not only been found in some pathological conditions, but is 
constantly present in minute traces in normal urine. Certain drugs - e.g.. sulphonal canse an increase in its amount. It can be separated from urine by the addition of sodium or potassium liydroxide, which precipitates the earthy phosphates. The hamatoporphyrin is carried down with the precipitate, and may be dissolved out with chloroform. The chloroform is then evaporated and the residuce dissolved in alcohol acidified with hydrochloric acid. The alcololic solution is filtered, and examined with the spectroscope. In paroxysmal hamoglobinuria, methamoglobin, mixed with some oxyhamoglobin, is found in the urine in large amount; and it is wortlyy of note that it is not formed in the urine after secretion, but is already present as such when it reaches the bladder.

In the rare condition termed alkaptonuria a bocly, alkapton. now known tobeidentical with homogentisinic acid $\left(\mathrm{C}_{61} \mathrm{H}_{3} .(\mathrm{OH})_{2} \mathrm{CH}_{2} . \mathrm{COOH}\right)$, a dioxyphenylacetic acid, is present. The uriue becomes dark brown on the addition of an alkali, or simply on exposure to air. It gives Felnling's test for sugar. The substance has relations to the aromatic amino-acids tyrosin and phenyl-alanin, and when either of these is given to a person suffering from alkaptonuria, the amount of alkapton excreted is increased. IVe may suppose, therefore, that in this condition the normal decomposition of these products of proteolysis is interfered with.

Ferments. - The urine contains traces of protcolytic and anlylolytic ferments (Fig. I68). These may be easily separated from it by putting a little fibrin, which has the power of fixing (adsorbing) enzymes, into the urine.

Of the inorganic constituents of urine the most important and most easily estimated are the chlorine, phosphoric acid, and sulphuric acid.

Chlorine.-Much the greater part of the chlorine is united with sodium, a smaller amount with potassium. The chloricles of the urine are undoubtedly to a great extent derived directly from the chlorides of the food, and have not the same metabolic significance as the organic, and even as some of the other inorganic constituents. But it is noteworthy that in certain discased conditions the clulorine may clisappear entirely from the urine, or be greatly liminishede.g., in pnemmonia, and in general in cases in which much material tends to pass out from the blood in the form of effusions (1). 477).

Phosphoric Acid. - The phosphoric acid of the urine is chicfly derived from the phosphates of the food, but must partly come from the waste products of tissues rich in phosphorus-containing substances, such as lecithin and nuclein. The phosplooric acil is united partly with alkalies, especially as acid sodium phosphate, and partly with earthy bases, as phosphates of calcium and magnesium. Thic earthy phosphates are precipitated by the aldition of an alkali to urine, or in the alkaline fermentation. In some pathological urines they come down when the carbon clioxide is clriven off by heating; a precipitate of this sort cliffers from heat-coagulated albumin in being readily soluble in acids (P'ractical Exercises, p. ${ }_{4} 86$ ). A small anount of phosphorus may appear in the urine in a less oxidized form than phosphoric acicl.

Sulphuric Acid.-This is only to a slight extent derived from ready-formed sulphates in the food. The greater part of it is formed by oxidation of the sulphur of proteins. About nine-tenths of the 
sulphur in normal nrine is present as inorganic sulphates, mainly those of potassinm and sodium. Of the other tenth, a portion is represented by ethereal sulphates, and the remainder by the socalled 'neutral' sulphur, including the sulphur associated with the pigment uroclurouse, and the small amount of sulplur occurring in less oxidized forms than sulphates in sucli compounds as the sulplocyanicle, which is probably, in part but not entirely, clerived from that of the saliva; and ethyl sulphide, a substance with a penetrating odour, which appears to be a constant constituent of log's nrine $(A \mathrm{bel})$.

Thiosulpluric acicl $\left(\mathrm{H}_{2} \mathrm{~S}_{2} \mathrm{O}_{3}\right)$ occurs almost constantly in cat's urine, often in dog's. It is not free, but combined with bases.

The ethereal sulphates are compounds in which the sulphuric acid is 111 ited with aromatic bodies (indol, phenol, etc.). Silch are potassium-phenyl-sulphate $\left(\mathrm{C}_{\mathrm{i}} \mathrm{H}_{5} \mathrm{KSO}_{4}\right)$, potassium-kresyl-sulphate $\left(\mathrm{C}_{7} \mathrm{H}_{7} \mathrm{KSO}_{4}\right)$, potassium-indoxyl-sulphate $\left(\mathrm{C}_{8} \mathrm{H}_{1 ;} \mathrm{NKSO}_{1}\right)$, potassiumskatoxyl-sulphate $\left(\mathrm{C}_{9} \mathrm{H}_{4} \mathrm{NKSO}_{1}\right)$, and two double sulphates of jotassium and pyrocatechin. The formation of potassium incloxyl sulphate may be thus represented : Indol, $\mathrm{C}_{6} \mathrm{H}_{4}<\underset{\mathrm{NH}}{\mathrm{CH}} \mathrm{CH}$ on absorption from the intestine is changed into indoxyl, $\mathrm{C}_{6} \mathrm{H}_{4} / \mathrm{NH} . \mathrm{CH} . \mathrm{CH}$, which $+\mathrm{SO}_{2} \mathrm{OH}$ (potassium hydrogen sulphate) yields $\mathrm{SO}_{2} / \mathrm{OCK}_{8} \mathrm{H}_{6} \mathrm{~N}$ (potassium indoxyl sulphate) $+\mathrm{H}_{2} \mathrm{O}$. The 'pairing ' of these aromatic bodies with sulphuric acid renclers them innocuous to the organism. Most of the compounds are present in greater amount in the urine of the horse than in the normal urine of man. But in disease the quantity of 'indican' in the latter may be much increased ; and to a certain extent it must be looked upon as an index of the intensity of putrefactive processes in the intestine and of absorption from it. Munk made the observation that in the urine of a starving dog the phenol-forming substances are absent, while in the urine of a starving man they are present in abnormally large amount. The indigo-forming substances ('indican '), on the other hand, are in hunger excreted in considerable quantity by the dog, and not at all by man (Practical Exercises, p. 479).

Phenol and kresol can casily be obtained from horse's urine by mixing it with strong hydrochloric acid and distilling. These aromatic bodies pass over in the distillate. Pyrocatechin remains behind, and can be extracted by ether. It gives a green colour with ferric chloride, which becomes violet on the addition of sodium carbonate.

The sulphur of the inorganic sulphates is the fraction of the total sulphur which fluctuates in proportion to the total protein metabolism. In this regard it follows the variations in the urea. It represents 'exogenous' metabolism. The neutral sulphur occupies a position analogous to that of the kreatinin : the smaller the amount of protein in the food, and the smaller therefore the total protein decomposed, the larger is the fraction which the neutral sulphur forms of the total sulphur. The neutral sulphur accordingly represents endogenous metabolism. The ethereal sulphur takes an intermediate position in this regard, but upon the whole it also becomes a more prominent fraction of the total sulphur when the food contains little or no protein. The ethereal sulphates are therefore not entirely derived from the putrefaction of protein. 
Carbonates of solium, ammonium, calcium, and magnesium occur in allialine urine. Their source is the carbonates and the vegetable organic acids of the food. In acid urine a certain amount of carbon (lioxide is present, although not firmly united with bases, so that most of it can be pumped out.

Physico-chemical Analysis of Urine.-The freczing-point of urinc is often (leterminel to obtain a measure of the molecular concentration. which with the total quantity of urine secreted in a given time is an index of the work of the kidney. The greater the volume of urine secreter per unit of time, and the greater the number of molecules dissolved in unit volume of it, the greater is the work of the sccrctory apparatus in scparating it from the blood ( $\left.p . f^{6}\right)_{5}$ ). Normally, $\lrcorner$ has a higher value for urine than for blood i.e., the molecular concentration of the urine is higher than that of the serim. But when large draughts of water are taken $\perp$ may be lower for wrine than for blool, and in general it varies within far wider limits (from $0^{\circ}$ I I $5^{\circ}$ to $2.546^{\circ} \mathrm{C}$. according to Köppe). The following table from Kövesi and Roth-Schulz sliows the changes in $\perp$ uncler the influence of water :

\begin{tabular}{|c|c|c|}
\hline Time. & Urine in c.c. & $د$ \\
\hline Io to 2 & 240 & $\mathrm{I} \cdot 8 \mathrm{O}$ \\
\hline 2 to 6 & 255 & $\mathrm{I} \cdot 72$ \\
\hline 6 to 10 & 161 & $I \cdot 93$ \\
\hline Io to 2 & I3I & $2 \cdot 18$ \\
\hline 2 to 6 & 160 & $2 \cdot 23$ \\
\hline 6 to $\mathrm{IO}$ & 120 & $I \cdot 9 \mathrm{I}$ \\
\hline I I to I 2 & I.8 litres 'Salvator' water taken & - \\
\hline I 2 to $\mathrm{I} 2.30$ & $5^{00}$ & $0 \cdot 12$ \\
\hline I 2.30 to I & $4+4$ & O. I I \\
\hline I to $\mathrm{I} .30$ & $4+2$ & $0 \cdot 10$ \\
\hline I. 30 to 2 & 46 & $0 \cdot 78$ \\
\hline 2 to 2.30 & +5 & $\mathrm{I} \cdot 30$ \\
\hline
\end{tabular}

If the electrical conductivity is determined, we obtain an approximate measure of the number of dissociated ions in unit volume, mainly the inorganic salts. Deducting this from the total number of molecules per unit volume (measured by $\lrcorner$ ), we arrive at the concentration of the urine in non-clissociated molecules, mainly urea and other organic constituents. Precision is added to such calculations by estimating also in the orlinary way (by titration, e.g.) one or more of the inorganic constituents, especially the chlorine, since sodium chloricle is quantitatively the most important of the salts. Various formulxe have been leduced from such determinations connecting the freczing-point and conductivity with other physical constants of the urine. E.g., $s-\mathrm{I}=\mathrm{K}=75$, where $s$ is the specific gravity and $K$ a constant with the value $75 ; \begin{gathered}\lambda \times 10^{6} \\ h\end{gathered}=K=145$, where $\lambda$ is the specific conductivity, $h$ the percentage of ash, and $K$ a constant $=$ $I^{*} 45$. The quotient $\frac{\perp}{\mathrm{NaCl}}$, representing the ratio of the total concentration to the sodium cliloride concentration varies within rela- 
tively narrow limits in health, according to Koranyi, the diet exercising no influence upon it whatever. Thus, in a large number of healthy individuals $\mathrm{NaCl}$ fluctuated only between $1 \cdot 23$ and 1.69 , while $s$ varied from $1.26^{\circ}$ to $2 \cdot 35^{\circ}$, and the percentage of sodium chloride from 0.85 to 1.54 . This is illustrated in the table:

\begin{tabular}{|c|c|c|c|}
\hline $\begin{array}{l}\text { Urine in c.c. in } \\
\text { Twenty-four Hours. }\end{array}$ & $د$ & $\begin{array}{c}\text { Percentage of } \\
\text { NaCl. }\end{array}$ & $\underset{\mathrm{NaCl}}{\mathrm{S}}$ \\
\hline I. 365 & I. $+13^{\circ}$ & I. 08 & $I \cdot 32$ \\
\hline $1,7+5$ & $1 \cdot 60^{\circ}$ & $I \cdot 24$ & $I \cdot 29$ \\
\hline I, 680 & $\mathrm{I} \cdot 68^{\circ}$ & $\mathrm{I} \cdot 28$ & $I \cdot 3 I$ \\
\hline 1,015 & $\mathrm{I} \cdot 8_{f^{\circ}}$ & $\mathrm{I} \cdot \mathrm{I} 5$ & $I \cdot 60$ \\
\hline 865 & $I \cdot 8 I^{\circ}$ & $I \cdot 26$ & I. 44 \\
\hline 1,360 & $I \cdot 62^{\circ}$ & I.OG & I.49 \\
\hline 840 & $2 \cdot 26^{\circ}$ & $I \cdot 50$ & $I \cdot 5 I$ \\
\hline$I, 600$ & $\mathrm{I} \cdot 46^{\circ}$ & $I \cdot I_{4}$ & $I \cdot 28$ \\
\hline 2,080 & $I \cdot 33^{\circ}$ & 0.85 & $I \cdot 68$ \\
\hline
\end{tabular}

The Urine in Disease.-Although, strictly speaking, a truly pathological urine has no place in physiology, the line which separates the urine of health from that of disease is often narrow, sometimes invisible; while the study of abnormal constituents is not only of great importance in practical medicine, but throws light upon the physiological processes taking place in the kidney, and upon the general problems of metabolism. Even in health the quantity of the urine, its specific gravity, its acidity, may vary within wide limits. A hot day may increase the secretion of sweat, and correspondingly diminish the secretion of urine, and the deficiency of water may lead to a deposit of brick-red urates. A meal rich in fruit or vegetables may render the urine alkaline, and its alkalinity may determine a precipitate of earthy phosphates. But neither the scanty acid urine with its sediment of urates, nor the alkaline urine with its sediment of phosphates, comes into the category of pathological urines; the deviation from the normal does not amount to disease. The maximum deviation from the line of health is the total suppression of the urine. If this lasts long, a train of symptoms, of which convulsions may be one of the most prominent, and which are grouped under the name of uræmia, appears. At length the patient becomes comatose, and death closes the scene. Suppression of urine may be the consequence of many pathological conditions, but there is one case on record in the human subject which, in effect, though not in intention, belongs to experimental physiology. A surgeon diagnosed a floating kidney in a woman. With a natural impatience of loose odds and ends of this sort, he offered to remove it, and in an evil 
hour the patient consented. The surgeon, a perfectly skilful man, who acted for the best, and to whom no blame whatever attached, carried the kidney to a well-known pathologist for examination. The latter, to the horror of the operator, suggested, from the appearance of the organ, that it was the only kidney the woman possessed. This turned out to be the fact. Not a drop of urine was passed. Apart from this ominous symptom, all went well for seven or eight days; but then uræmic troubles came on, and the patient died on the eleventh or thirteenth clay after the operation. The autopsy showed that her only kidney had been taken away.

In disease the urine may contain abnormal constituents, or ordinary constituents in abnormal amounts. Of the normal constituents which may be altered in quantity, the most inlportant are the water, the inorganic salts, the urea, the uric acid, and the aromatic substances.

Water.-A marked and persistent diminution in the quantity of urine-that is to say, practically in the water, with or without an increase in the specific gravity-is suggestive of disorganization of the renal epithelium. In some infective diseases the kidney is liable to be secondarily involved, its secreting cells being perhaps crippled in the attempt to eliminate the bacterial poisons. In the form of parenchymatous or tubal nephritis which so frequently complicates scarlet fever, the quantity of urine has in some cases fallen to 50 or 60 c.c. in the twenty-four hours.

In chronic interstitial nephritis ('granular kidney'), on the other hand, where the structural changes in the tubules are, for a long time at least, comparatively circumscribed, the quantity of urine is often increased and of low specific gravity. In these cases the increase in the blood-pressure, associated with hypertrophy of the heart, may be a factor in the exaggerated renal secretion. In diabetes mellitus the quantity of urine is greatly increased, perhaps in some cases because more urea is excreted than normal, and urea acts as a diuretic, perhaps also because the elimination of sugar draws with it an increased excretion of water to hold it in solution. Although a specific gravity as low as 1002 has been seen in healthy persons (after copious potations), the persistence of a density below IOIO should suggest hydruria. Watson mentions the case of a boy with diabetes insipidus, who voided in twenty-four hours 9 or Io pints ( 5 to 6 litres) of urine with a specific gravity of rooz. On the other hand, while the specific gravity has been occasionally observed to mount in health to at least 1036, its persistence at 1025 or I030 or anything above this, especially if the urine is pale and apparently dilute, should suggest diabetes mellitus. 
Inorganic Salts. - The changes in the quantity of the inorganic constituents of the urine in disease are not, in the present state of our knowledge, of as much importance as the changes in the organic constituents. The chlorides are clininished in most acute febrile diseases and may even totally disappear from the urine, and their reappearance after the crisis is, so far as it goes, a farourable symptom. In most cases in which the quantity of the urine is markedly lessened, all the inorganic substances are diminished in amount.

Urea.-The quantity of urea is, as a rule, increased in fever, cither absolutely or in proportion to the amount of nitrogen in the food. In the interstitial varieties of kidney disease the urea is usually not diminished, but when the stress of the change falls on the tubules (parenchymatous nephritis), it is distinctly decreased-it may be even to one-twentieth of the normal.

Uric acid is diminished in the urine in gout (perhaps to oneninth of the normal), not only during the paroxysms, but in the intervals. It accumulates in the blood and tissues, and, as sodium urate, may form concretions in the joints, the cartilage of the ear, and other situations. Watson relates the case of a gentleman who used to avail himself of his resources in this respect by scoring the points at cards on the table with his chalky knuckles. In leukæmia the quantity of uric acid and purin bases in the urine is greatly increased, not only absolutely, but also in proportion to the urea. As much as $4 \frac{1}{2}$ grammes of free uric acid, in addition to about $\mathrm{I} \frac{1}{2}$ grammes of ammonium urate, has been found in a urinary sediment in a case of leukæmia.

The aromatic bodies, of which indoxyl may be taken as the type, are increased when the conditions of disease favour the growth of bacteria in the intestine-e.g., in cholera, acute peritonitis, and carcinoma of the stomach. A marked increase in the amount of the 'indican' in the urine may, as far as it goes, be taken as an indication that the bacteria are gaining the upper hand in the intestinal tract; a marked diminution is usually a sign that the battle has begun to turn in favour of the organism (Practical Exercises, p. 479). Tryptophane, a substance which we have already recognised among the products of the tryptic digestion of proteins, has been shown to be a precursor of indol, which is formed from it under the influence of bacteria. When tryptophane is injected into the cæcum of rabbits, the indican in the urine is markedly increased. Putrefactive processes in other parts of the body than the intestine may also increase the indican in the urine-e.g., a collection of putrid pus in the pleural cavity.

Abnormal Substances in Urine.-Sugar, proteins, the pigments of bile and blood, or their derivatives, are the most 
important abmornal substances found in solution in the urine. Normal wrine, as has been stated, contains a trace of dextrose, but so little that it camnot be detected by ordinary tests, and for practical purposes it may be considered absent. Dextrose is the sugar found in the urine in diabetes. In the mrine of nursing mothers lactose may be present. Pentoses, sugars with five carbon atoms in the molecule (instead of six, as in the liexoses, of which group dextrose is a member), may also occasionally occur in urine. Pentoses give the ordinary reduction tests for sugar, and yicld osazones, but do not ferment with yeast. Various plants contain pentoses, and when these are eaten the pentoses are excreted in the urine, but in cases of true pentosuria they originate in the body, possibly from nucleo-proteins. The condition has not the same sinister significance as diabetes. Specific toxic substances produced by bacterial action have been demonstrated in the urine in certain diseases. Red blood-corpuscles and leucocytes (pus corpuscles, white blood-corpuscles, mucus corpuscles) are the chief organized deposits; but spermatozoa may occasionally be found, as well as pathogenic bacteria-e.g., the typhoicl bacillus; and in disease of the lidney casts of the renal tubules are not uncommon. These tube-casts may be composed chiefly of red blood-corpuscles, or of leucocytes, or of the epitlelimm of the tubules, sometimes fattily degenerated, or of structurcless protein, or of amyloid substance. Abnormal crystalline substances, such as the amino-acids, leucin (Fig. IOg), and tyrosin (Fig. I70), and cystin (Fig. I63) may be on rare occasions found in urinary sediments; but generally the unorganized deposits of pathological urine consist of bodies actually contained in, or obtainable from, the nomal secretion, but present in excess, either absolutely, or relatively to the solvent power of the urine. Cystin is of interest because of its relations to the sulphur of the protein molecule (1).332). It is not found in the nomnal organism. It very occasionally forns calculi in the bladeler. There are individuals who constantly pass as much as one-fourth of all the sulphur in the form of cystin, without any otlier symptoms.

Various anino-acids are present in solution in the urine in many pathological conditions. Of these the least soluble are leucin and tyrosin, and this is the reason why they are most easily detected. A gencral reaction for animo-acids is their precipitation as sparingly soluble compounds ( $\beta$-naplitlalinsulphones) by $\beta$-naplithalinsulphochloride in the presence of an alkali (sodium liydroxide). In acute yellow atroply of the liver leucin and tyrosin have been found in large amounts in the liver itself, ats well as in the urine. In phosphorus poisoning these amino-acids, as well as glycocoll, have been detected in 
the urine, and there is no doubt that other amino-acids, arising from the decomposition of proteins, are also present in such conditions.

Sugar.-In diabetes mellitus, although the quantity of urine is usually much increased, its specific gravity is above the normal ; and this is due chicfly to the presence of sugar (dextrose), which generally amounts to 1 to 5 per cent., but may in extreme cases reach io or even ${ }_{5}$ per cent., more than half a kilogramme being sometimes given off in twenty-four hours.

The name of the tests for dextrose is legion. They are mostly founded on its reducing action in alkaline solution. Hydrated oxide of bismuth (Bocttcher), salts of gold, platinum and silver, indigo (Mulder), and a host of other substances, are reduced by dextrose, and may be used to slow its presence. The reduction of cupric salts (Trommer), fermentation by yeast, and the formation of crystals of phenyl-glucosazone are the best established tests. (See Practical Exercises, p. 488 .)

Proteins.-Serum-albumin and serum-globulin are the proteins most commonly found in pathological urine. Both are coagulated by heating the urine, slightly acidulated if it is not already acid, or by the addition of strong nitric acid in the cold. Proteoses (albumoses) are also occasionally present, e.g., in the disease called 'osteomalacia' and in conditions associated with the formation and especially with the decomposition of pus. They may be recognised by the tests given in the Practical Exercises (p. +26$)$. It is doubtful whether the presence of true peptone has as yet been satisfactorily made out.

The presence of bile-salts may be shown by Hay's test, or Pettenkofer's test (p. +30).

The pigments of blood and bile may be detected by the characters described in treating of these substances; the spectrum of oxylæmoglobin, or methæmoglobin, or any of the other derivatives of hæmoglobin, with the formation of hæmin crystals, would afford praof of the presence of the former, and Gmelin's test of the latter. The red blood-corpuscles, seen with the microscope, are the most decisive evidence of the presence of blood, as leucocytes in abundance are of the presence of pus. It should be remembered that pus in the urine of women has sometimes no significance except as showing that there has been an admixture of leucorrheal discharge from the vagina. (Sec Practical Exercises, pp. 65, 494.)

The Secretion of the Urine.-We have now to consider the mechanism by which the urine is formed in the kidney from the materials brought to it by the blood. And here the same questions arise as have already been discussed in the case of the salivary and other digestive glands: (I) Are the urinary constituents, or any of them, present as such in the blood? (2) If they do exist in the blood, can they be shown to be separated from it by processes mainly physical or mainly 'vital '-in other words, by ordinary filtration, diffusion and osmosis, or by the selective action of living cells? In the case of the digestive juices it has been seen that some constituents are already present in the blood, but that physical laws alone, so far as we at present 
understand them, cannot explain the proportions in which they occur in the secretions, or the conditions under which they are separated; while other constituents-and these the more specific and important-are manufactured in the gland-cells.

In the kidneys the conditions seem at first sight favourable to physical separation, as opposed to physiological secretion. [rine las been described as essentially a solution of urea and salts, and botli are ready formed in the blood. The arrangement of the bloodvessels, too, suggests an apparatus for filtering under pressure.

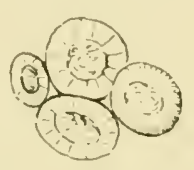

Fig. 169.-LeuciN Cristals.

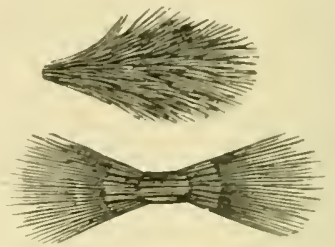

Fig. I70.-TYROSIN CRYSTALS.

Bloodvessels and Secreting Tubules of Kidney.-The renal artery splits up at the hilus into several branches, which pass in between the Malpighian pyramids, and form at the boundary of the cortex and medulla vascular arches, from which spring, on the one side, interlobular arteries running up into the cortex between the pyramids of Ferrein, and, on the other, vasa recta running down into the bounclary layer of the medulla (Fig. I 7 I). The interlobular arteries give off at intervals afferent vessels; each of these soon breaks up into a glomerulus or tuft of vascular loops, which gather themselves up again into a single efferent ressel of somewhat smaller calibre than the afferent. The glomerulus is fitted into a cup or capsule (of Bowman), which is closely reflected over it, except where the affent and efferent vessels pass through, and forms the beginning of a urinary tubule. If we suppose the tuft pushed into the blind end of the tubule so as to indent it, it will be easily understood that the single layer of flattened epithelium reflected on the glomerulus is continuous with that lining the capsule, which in its turn is continuous with the epithelial layer of the rest of the urinary tubule. This has been divided by histologists into a number of parts which it is unnecessary to enumerate here, further than to say that the urinary tubule proper begins in the cortex in Bowman's capsule and the proximal convoluted tubule (with its continuation, the spiral tubule), and ends in the cortex with the clistal convoluted tubule, the connection between the two being made by a long loop (Henle's) with a descending and an ascending limb (1 ig. 172). Between the ascending limb and the distal convoluted tube is interposed the zigzag tubule. The tubule throughout its length is bounded by a basement membrane lined by a single layer of epitlelium, which cliffers in its character in clifferent parts of the tubule.

The distal convoluted tube joins by means of the short connecting tubule one of the straight tubules which form the pyramids of Ferrein or medullary rays in the cortex, and which run down into the medulla, always uniting into larger and larger tubes as they 


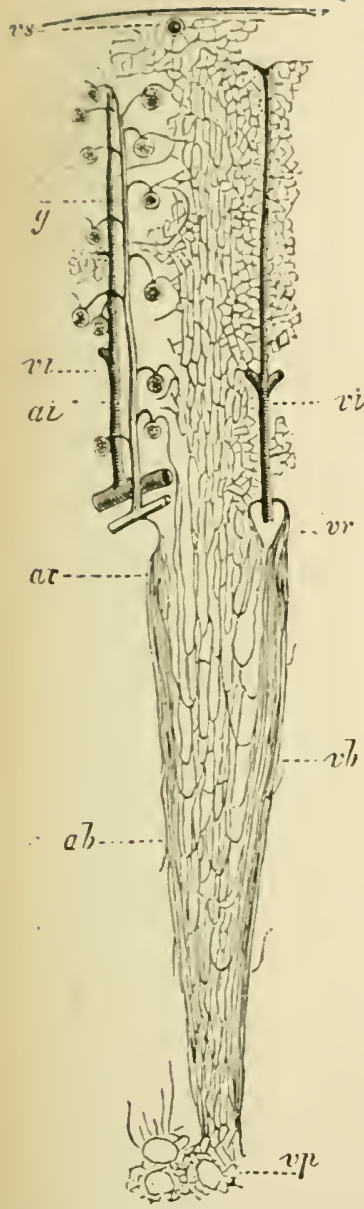

FIG. I7I.—DIAGRA II OF Bloodvessels OF KidNey (KLEIN, AFTER LUDWIG).

$a i$, interlobular artery ; $v i$, interlobular vein : $g$, glomerulus, to which an afferent artery is seen coning from the interlobular artery, and from which an efferent artery proceeds to break up into a capillary network surrounding the renal tubules: $v s$, vena stellata; $a r$, arterice rectic; $v b$, leash of venæ rectæ; vp, vas. cular network round ducts at apex of a papilla.

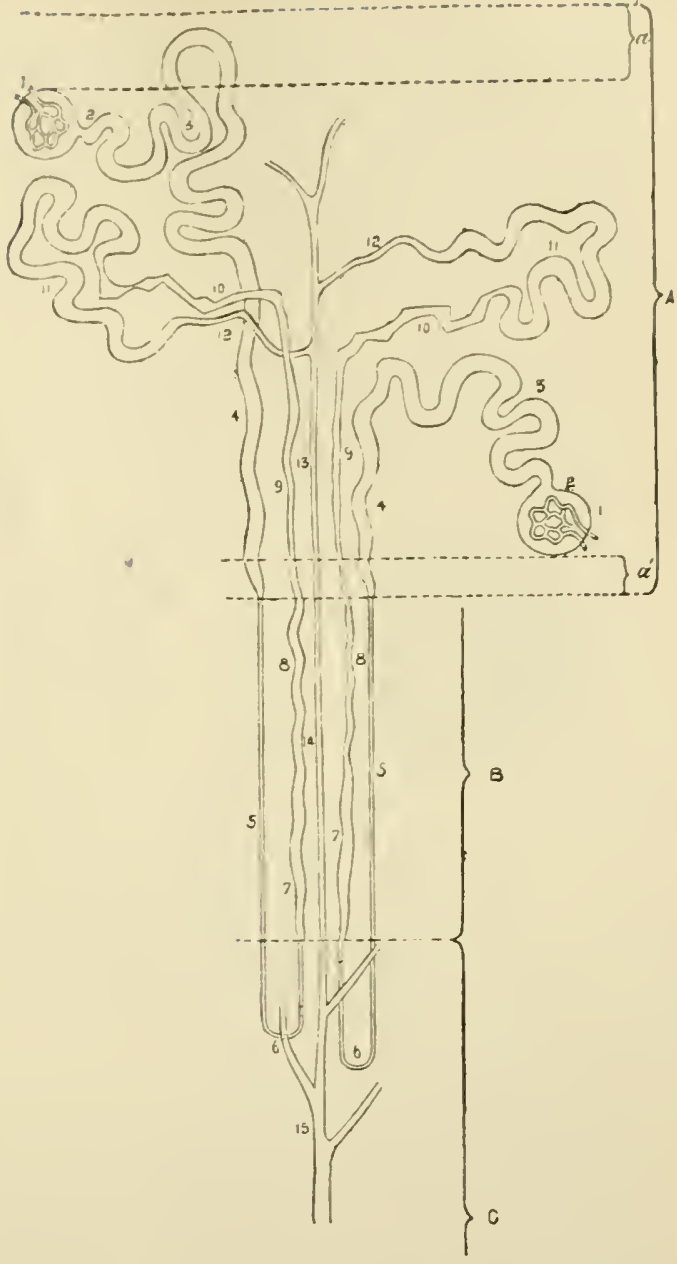

Fig. I72.-Diagray OF RENAL TUBULE (KLEIN).

A, cortex ; $a$, layer of cortex immediately under capsule containing no Malpighian corpuscles; $a^{\prime}$. inner layer of cortex devoid of Malpighian corpuscles ; B, boundary laver ; C, papillary zone of medulla; I, Bowman's capsule ; 2, neck of capsule ; 3, proximal convoluted tubule : 4, spiral tubule; 5, descending part of Henle's looptubule; 6 , the loop ; 7,8 , and 9 , ascending limb of loop-tubule; Io, irregular tubuie ; I 1 , distal convoluted tubule; I2, junctional tubule; I3, collecting tubule, in a medullary ray or pyra. mid of Ferrein I4, collecting tubule in the boundary layer; I5, large collecting tubule ending in a duct of Bellini. 
go, until at length they open as dluets of Bellini on the apex of a papilla. The two convoluted tubules (with the spiral and zigzag tubules) are lined by similar epithelial cells with granular contents, and the tendency of the granules to be arranged in rows perpendicular to the basement membrane gives them a striated or "rodeled " appearance (Fig. I73). The granules are cosinophile (p. I7), which is also a character of the granules of other secreting cclls. Towards the lumen the cells may show a brush of processes, looking like cilia, but in mammals these are not motile. The ascending part of Henle's loop also has cells of the salne general character. with

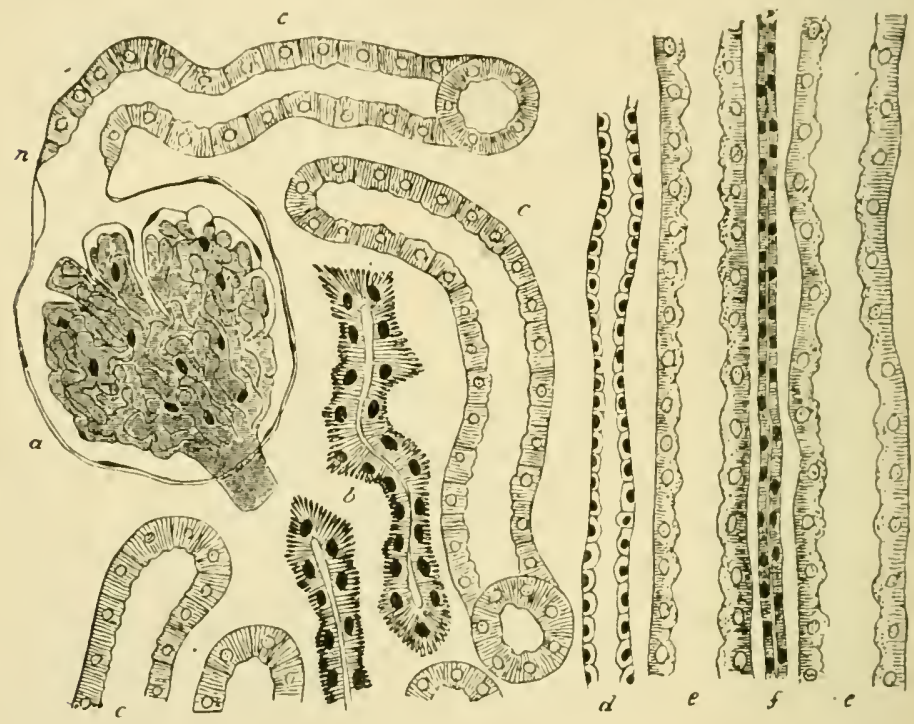

Fig. i73.-From a Vertical Section of Dog's kinney to show tile Struc. tURE of DifFerent portions of the ReNal TUbUle (KLlein).

a, Bowman's capsule enclosing glomerulus, the capillaries of which are arranged in lobules separated by a little connective tissue. The capsule and glomerulus

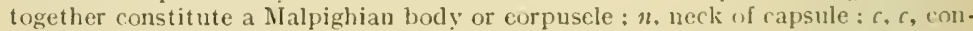
voluted tubules, eut in various directions ; $b$, irregular or zigzag tubule : $d, e^{2}$ and $f$ are straight tubules, which take part in the formation of a medullary ray or pyramid of Ferrein ; $l$, collecting tubule : $c, c$, spiral tubule : $\%$, narrow part of ascending limb of Henle's lonp-tubule; $b . c$, and $e^{\prime}$ are lined with rudded epithelium.

numerous granules, although the 'rodlling' may not he so distinct. We shall see directly that the morphological resemblance is the index of a functional likeness. The blood-supply of the tubules. especially of the convoluted portions, is excecdingly rich, the efferent vessels of the glomeruli breaking up around them into a closemeshed network of capillaries, from which the blood is collected into interlobular veins rumning parallel to the interlobular arteries between the pyramids of Ferrein. The straight tubules of the medulla are also surrounded by capillaries given off from straight arteries (arterixe recta) running down into it partly from the arterial arches and partly from efferent vessels of the glomeruli nearest the 
boundary layer, the blood passing away by straight veins (vence recta) which join the larger veins accompanying the arterial arches. The greater part of the blood going through the kidney has to pass through two sets of capillaries, one in the glomeruli, the other around the tubules. Even the portion of it which does not go through the glomeruli has for the most part a long route to traverse in narrow arterioles and venules to and from its capillary distribution. And the mean circulation-time through the kidney has been found to be longer than that through most other organs (p. I25).

Theories of Renal Secretion.--To come back to our problem of the nature of renal secretion, the anatomical structure of the kidney might be expected to throw light upon the question. And, indeed, it was on a purely histological foundation that Bowman established his famous 'vital' theory of renal secretion. Impressed with the resemblance between the renal epithelium and the epithelial cells of other glands, and with the distribution of the bloodvessels in the kidney, he came to the conclusion that the characteristic constituents of urine, including urea, were secreted from the blood by the tubules. To the Malpighian bodies he assigned what he doubtless considered the humbler office of separating water from the blood for the solution of the all-important solids. To Ludwig, on the other hand, with his whole attention fastened on the mechanical factors by which the flow of urine could be influenced, the tubules seemed of secondary importance, while the glomeruli appeared a complete apparatus for filtering urine from the blood into Bowman's capsule. He saw that the efferent vessel was smaller than the afferent; that it was therefore easier for blood to come to the glomerulus than to get away from it, and that the pressure in the capillaries of the tuft must be higher than in ordinary capillaries, because the resistance beyond them in the comparatively narrow efferent vessel, and especially in the second plexus, is greater than the resistance beyond a single capillary network. And experimental investigation soon showed him that the rate at which urine was formed could be greatly influenced by changes in the blood-pressure.

On such considerations, Ludwig founded the 'mechanical' theory of urinary secretion, which, although in a much modified form, still divides with the 'vital' theory the allegiance of physiologists. It is impossible here to enter in detail into a controversy that has extended over more than half a century and produced an extensive literature. The result of the discussion has been, in our opinion, to establish in its essential principles the 'vital' theory of Bowman, or at least to show that no purely physicochemical theory as yet constructed will account for all the facts.

Indwig supposed that the mine, qualitatively complete in all its constituents, was simply filtered throngh the glomeruli, the 
work done in this filtration being performed entircly at the expense of the energy of the lieart-beat represented as lateral pressure in the vessels of the tufts. But as the proportion of salts, and especially of urea, is very far from being the same in mrine as in blood, it had further to be assumed that the liquid which passes into Bowman's capsule is exceedingly dilnte, and that absorption of water, and perhaps of other constituents, takes place in its passage along the renal tubules. This process of reabsorption he pictured as a purely physical diffusion between the dilnte urine in contact with the free ends of the epithelial cells lining the tubules and the much more concentrated lymph with which their deep ends are bathed. The great length of these tubules, as compared with those of most other glands, might indeed seem to indicate a long sojourn of the urine in them, and the probability of important changes being caused in its passage along them. But if we consider the immense length $(60$ to $70 \mathrm{~cm}$.) of the seminal tubules and of their gigantic dincts (epididymis 6 metres), where, of course, absorption of water on a large scale is out of the question, it will be granted that little can be built upon the mere length of the renal tubules. On the other hand, the salivary glands, where there are no glomeruli, secrete as much water as the kidneys are supposed to filter; and the pancreas, whose capillaries form the first of a donble set, and therefore in this respect correspond to the renal glomeruli, secretes less water than the liver, whose capillaries correspond to the low-pressure plexus around the convoluted tubules of the kidney. So that deductions drawn from the anatomical relations of the bloodvessels are not in this case of much value, unless supported by physiological results.

It is somewhat unfortunate that systematic witers have fallen into the habit of discussing the mechanism of urinary secretion as if the Ludwig theory and the Bowman theory presented an exact antithesis, as if the one offered a complete 'mechanical ' explanation of a process, which the other viewed as entirely 'vital,' and therefore withdrawn from physical explanation.

We need not concern ourselves here with the historical development of this discussion. Three main questions require our attention :

I. Is there any evidence that reabsorption actually occurs in the tubules? If reabsorption on an important scale does take place, it follows at once that there must be a difference of function between the two parts of the renal apparatus, through which urinary constituents pass in opposite directions.

2. But if there is no reabsorption, or none of importance, it may still be asked whether, the direction of movement of the urinary constituents through the glomeruli and the tubular 
cpithelimm being the same, some quantitative or qualitative difference in their activity may not exist, certain constituents, c.g., passing mainly or exclusively through the one or the other.

3. When these questions lave been settled, we are in a position to consider the nature of the process by which the urinary constitnents find their way from the blood into the lumen of the capsules and the tubules, or, if there is reabsorption, out of the tubules into the lymph and blood again, and to see whether or no it can be entircly explained on mechanical and physicochemical principles.

That some absorption can take place from the kidney when the pressure in the ureter is abnormally raised need not be doubted, and when substances like potassium iodide or strychnine are introduced into the ureter or the pelvis of the kidney under these circumstances, they can speedily be detected in the blood. When the ureter pressure (in dogs) is only slightly increased, instead of evidence of reabsorption, we obtain evidence of increased secretion. The volume of urine, the total quantity of sulpliate in the urine when sodium sulphate is injected into the blood as a diuretic, and the total amount of reducing sugar when phloridzin is injected, are all greater on the obstructed than on the normal side. These facts are quite opposed to the idea that filtration and reabsorption are important factors in the preparation of normal urine (Brodie and Cullis). Cluanges in the blood-flow through the kidney have nothing to do with the results, since the small increase in pressure in the ureter was shown not to affect the rate of flow of the blood. The attempt has been made to decide whether absorption normally occurs by removing as much of the tubules as possible, and seeing whether the character of the urine is altered. In rabbits the whole or a large portion of the medulla has been excised from one kidney and the other then extirpated. From the mutilated kidney two or three times as much urine was said to flow as was secreted by a control rabbit operated on in the same way, except for the removal of the renal medulla (Ribbert). The conclusion was drawn that the greater quantity of urine escaping was due to the smaller opportunity for reabsorption of the water. But experiments mentioned on p. 569 suggest a different interpretation of these observations. And Boyd, who repeated Ribbert's work, obtained quite different results after partial removal of the medulla. He found it impossible to remove the whole. So that hitherto the direct method of eliminating the tubules has left the matter where it was.

Some light has been thrown on this question, by taking advantage of the anatomical fact that the kidney of batrachians, and, indeed, that of fishes and ophidia as well, has a double 
blood-supply: The renal artery gives off afferent vessels to the glomeruli; the venu advehens, or renal portal vein, breaks up, like the portal vein in the liver, into a plexus of capillaries surrounding the tubules, and there seems to be no communication between the two vascular systems.

By tying all the arteries going to the kidneys in frogs the circulation through the glomeruli can be completely cut off, while ligation of the renal portal vein does not affect the blood-supply of the glomernli, though markedly interfering with that of the tubules. Gurwitsch has found that after ligation of the renal portal rein of one kidney in (male) frogs, the flow of urine from that kidney is much diminished as compared with the other.

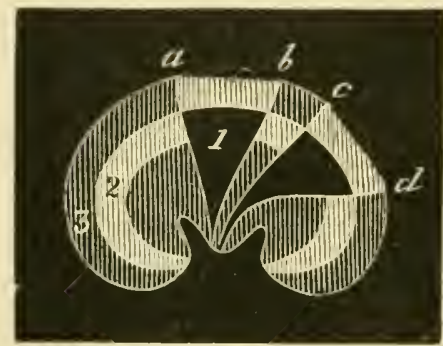

FIG. I74.-DIAGRAM OF DISTRIBU. TION OF PIGMENT IN KIDNFE' AFTER INJECTION INTO BLOOD.

The cortex between $a$ and $b$ and hetween $c$ and $d$ was cauterized be. fore the injection. In the black wedge-shaped portions, I, there was no pigment. In the zones shaded like 2 there was some pigment, but not so much as in the areas shaded like 3 . pigment is insoluble. His results were as follows: (I) When the spinal cord was cut before the injection in order to reduce the blood-pressure, the blue granules were found in the 'rodded' epithelimm of the convoluted tubules and the ascending limb of Henle's loop, and in the lumen of the tubules, but nowhere else. Bowman's capsules contained no pigment. The renal cortex was coloured bhe. (2) When the spinal cord was not cut, the pigment was found in the medullat and pelvis of the kidney, ats well as in the cortex, but alwars in the lumen of the tubules, and not in the epithelium, except in the situations mentioned. (3) If a portion of the cortex of the kidney had been canterized with nitrate of silver before injection of the pigment, the spinal cord being left intact, a wedge of the renal substance, corresponding to this area, remained coloured only in the cortex, although the rest 
was blue in the medulla also. The 'rodded' epithelium was filled with blue granules as hefore (Fig. I74).

(I) Slows that the epithelium is capable of excreting some substances at least. (2) Appears to show that when the bloodpressure is normal water is poured out from some part of the tubule, and washes the pigment separated by the "rodded" epithelium down towards the papillae. (3) Suggests that it is throngh the glomernli that most of the water passes. For canterization lias not destroyed the powver of the epithelinm to excrete pigment, and therefore, presumably, would not have destroyed its power to excrete water if it possessed this power in any great degree; and the glomeruli and their capsules are the only other part of the renal meclianism which can have been affected. The fact that in birds and serpents, whose urine is solid or semi-solid, the glomernli are smaller than in mammals is corroborative evidence that the glomeruli have to do with the excretion of water.

When pigments are injected into the dorsal lymph-sac of a frog without interference with the renal circulation, they are found plentifully in the limen of the convoluted tubules, and also in the epithelial cells lining them. The suggestion has been made that the pigments have been absorbed by the cells from the lumen and not excreted by them into it. And certainly pigments soluble in the cytoplasm or in the substances that form the envelopes of cells, and therefore capable, like methylene blue, of staining them during life, might be taken up by the renal epithelium if excreted into the tubules by the glomeruli, and might cause staining of them, particularly, of course, of the free ends of the cells next the lumen. But this suggestion is inadmissible since on injection of the same pigments after ligation of the renal portal vein the convoluted tubules contain little or no pigment in their lumen. And when the urinary flow is stopped on one side in mammals by temporary compression of the renal artery the corresponding kidney takes up fully as much carmine as its fellow (Carter). There is no doubt that not only pigments capable of 'vital staining,' like methylene blue, but also pigments which do not stain living cells, are taken $11 p$ from the blood (or lymph) by the epithelial cells, and, lying in vacuoles in their cytoplasm, are transported towards the lumen, and there extruded. It is not the solubility of the pigments in lipoids, and therefore their solubility in the supposed lipoid envelope of the cells, which determines whether they shall be excreted. The degree in which they are capable of heing presented to the cells in non-colloid solution appears to some extent to be a determining factor. The pigments not taken up are highly colloidal (Gurwitsch, Höber). Shafer has recently confirmed Heidenhain's state- 
ments as to the place of excretion of indigo-carmine. When lenco-indigo-carmine (a colourless reduction-product of indigocarmine) was injected, the blue oxidized substance was found in the lumen of the convoluted tubules and in the collecting tubules, but not at all in the Bowman's capsule. The cells of the convoluted tubules were colourless, because they kept the pigment in its reduced condition, and it only became oxidized in the lumina of those parts of the tubules whose contents, according to Dreser, show an acid reaction. On oxidation by peroxide of hydrogen the cells of the convoluted tubules became faintly green, but the Bowman's capsule remained colourless. This can only be explained on the assumption that the leuco-product of the pigment was excreted by the cells of the convoluted tubules.

But these cells are far from taking up all pigments indifferently. Some pigments are extruded mainly by one part, others mainly by another part of the renal tubule, and some even by the glomeruli, as shown long ago for ammonium carminate. The glomeruli, however, are in general far less active in this regard than the epithelial cells, and the fact that the latter pick out from the blood such substances as these foreign pigments which pass through the Malpighian tufts unchallenged, renders it likely that the tubules also exercise a special function in the secretion of the normal constituents of urine. More direct evidence of this is not wanting, for Bowman saw crystals of uric acid in the epithelium of the convoluted tubules of birds. Heidenhain found that urate of soda injected into the blood of a rabbit is excreted by the epithelium of the convoluted tubules and the ascending part of Henle's loop, just as is the case with indigo-carmine. And Nussbaum's experiments, although not quite conclusive, have made it probable tluat in the frog urea is actually separated by the epithelium of the tubules. They were founded on the anatomical peculiarity in the renal circulation of the frog already mentioned. By tying the renal arteries in that animal, he thought he could at will stop the circulation in the glomeruli. and he found that after this was done there was no further spontaneous secretion of urine. But when urea was injected intravenously the secretion of urine again began, urea being eliminated by the kidneys, and water along with it. Sugar, peptone, and egg-albumin, injected into the blood, no longer passed into the urine, even when the secrotion was excited by simultaneous injection of urea, although they readily did so when the arteries were not tied. He concluded that the Malpighian corpuscles have the power of excreting water, sugar, peptone, and albumin, while the epithelium of the tubules excretes urea as well as water.

Beddard has confirmed Nussbaum's statement that when all the arteries going to the kidney are tied the glomeruli are completely and permanently deprived of blood. 'The spontaneous 
secretion of urine is totally stopped, as Nussbaum found, but only in three experinents ont of eighteen was it possible to start the secretion by injection of nrea. The epithelium of the tubules degencrated and desquamated after complete ligation of all the renal arteries, showing that it repuires some arterial blood as well as the venous blood from the renal portal to maintain its vitality. The degeneration of the epithelium can be prevented by keeping the frogs in an atmosphere of oxygen after ligation of the arteries. In six such frogs, in which the complete elinination of the glomeruli was controlled by subsequent injection, secretion of urine followed the injection of urea, alone or in combination with dextrose, phloridzin, or di-sodinm hydrogen phosphate $\left(\mathrm{Na}_{2} \mathrm{HPO}_{4}\right)$. In all the cases the urine contained urea, chlorides, and sulpliates, and was acid to phenolphthalein. In one case after injection of urea and dextrose, and in another after urea and phloridzin, the urine reduced Fehling's solution, and therefore presumably contained dextrose (Beddard and Bainbridge). When the frog's kidney is perfused in situ with oxygenated salt solution a certain flow of urine takes place. Substitution of nonoxygenated saline markedly slows the flow (Cullis).

Apparently, then, the tubules have the capacity to secrete practically all the constituents of urine, and when the flow of urine is small, probably most of it comes from the tubules. When, as in the diuresis produced by salt solutions, large quantities of water and salts have to be rapidly excreted, the bulk of the liquid comes from the glomeruli, but also by a process of secretion.

Lindemann has endeavoured to exclude the glomeruli in mammals by injecting oil through the renal artery. After a short time, according to him, the oil emboli clear away from practically all parts of the kidney except the glomeruli, which remain plugged. If indigo-carmine be subsequently injected into the blood, it is not only taken up from it by the embolized kidney as well as by a normal one, but is excreted. The quantity of urine is much diminished and its specific gravity increased, but its composition is not essentially altered. He infers that the tubules are in a high degree independent of the glomeruli as an apparatus for the secretion of urine.

As regards our first two questions we may conclude that there is no good evidence that reabsorption of water or other constituents of the urine in the renal tubules plays an important part in the preparation of that secretion. Many facts favour the conclusion that the glomeruli and the renal epithelium act as separate although, of course, mutually supplementary mechanisms, the glomeruli separating the larger portion of the water and salts, the epithelium the larger portion, if not the whole, of the characteristic organic constituents.

As regards the third question, it is now generally admitted, 
even hy those who mphold a modified 'mechanical 'theory, that even if the urine is originally separated from the blood by liltration at the expense of the energy of the heart-beat represented by the pressure of the blood in the glomeruli, the reabsorption in the tubules camnot be attributed to simple diffusion, but must be a selective process analogous to absorption in the intestine and entailing the expenditure of a large amount of work at the expense of the food materials or the protoplasm of the epithelial cells. Every attempt at a strictly mechanical explanation breaks down for the kidney, as for other glands.

The practical absence from urine of the proteins and sugar of the blood under normal circumstances, and the elimination by the kidney of egg-albumin, peptone, and other bodies when injected into the veins, show a selective power inexplicable except by reference to the vital activity of cells. Urea and dextrose, both highly diffusible substances, circulate side by side in the bloodvessels of the kidney. The one is taken and the other left. The nrea is a waste-product of no further use in the economy. The sugar is a valuable food-substance. The kidney selects with nnerring certainty the urea, of which only + parts in $\mathrm{I} 0,000$ are present in the blood, but rejects the sugar, of which there is three times as much.

Egg-albumin injected into the blood passes through the renal circulation side by side with the serum-albumin of the plasma. Both are indiffusible through membranes, and to the physical chemist the differences between them may appear superficial and minute. But the kidney does not hesitate for an instant. A large part of the egg-albumin is promptly excreted as a foreigil substance; the serum-albumin passes on untouched.

Not only does the kidney exercise a power of qualitative selection; it also takes cognizance of the quantitative composition of the blood. So long as there is less sugar in the plasma than about 2 to 3 parts in $\mathbf{I}, 000$, it is refused passage into the renal tubules. But when this limit is passed, and the proportion of sugar in the blood becomes excessive, the kidney begins to excrete sugar, and continues to do so till the balance is redressed.

The advocates of the theory of filtration through the glomeruli have made their firmest stand on the excretion of the inorganic constituents of the urine, and have laid stress particularly on the fact that the hydriemic plethora caused by intravenous injection of salts is accompanied by dimesis. It is true that the direct introduction of water into the blood, or its attraction from the lymphspaces when the osmotic pressure of the blood is increased by the injection of substances like urea, sugar, and sodium chloride, may cause a condition of hydremio plethora, and that this plethora may sometimes be associated with an increase of pressure in the capillaries in general, and therefore in the vessels of the Mal- 
pightian tuft. It maty also be admitted that such ath intreatse of pressure might be accompanied hy an increased filtration of water and salts into Bowman's capsule. Even in the exciscel kidney, after the vital activity of its cells may be presumed to have ceased, filtration of the inost varied solutions occurs when the organ is perfused with them through the renal artery. The liquid which escapes from the weter always has the same composition as the perfusion fluid (Sollmann). It would certainly appear unlikely that the glomerular epithelium should make no use whatever for the furtherance of its task of the difference of hydrostatic pressure on its two surfaces. It is in taking advantage of such circumstances for the promotion of its specific work 11] to the point at which they cease to favour it that a great part of the true secretory activity of cells may be supposed to consist. When we see a barge passing through a lock, and being gradually lifted to the proper level by the inrush of water, we never dream of saying that the whole thing is an affair of the laws of hydrostatics. We know that the part played by the lock-keeper, the opening and closing of the gates and sluices at the proper time, is all-inportant, although he does not lighten by one ounce the weight which the water must lift. He uses the head of water for a specific purpose-namely, to lift the barge. In like manner it is to be expected that the glomerular epithelimm, when the difference of pressure on its two surfaces is increased by hydremic plethora, will use the increased facility of filtration to rapidly excrete a portion of the water. But who will believe that the addition of a tumbler of water, absorbed from the alimentary canal, to + or 5 litres of blood circulating in a system of ressels whose capacity can and does vary within wide limits, should cause in the capillaries of the kidney an increase of pressure exactly proportional to the increase in the elimination of water in the urine, lasting for the same time and disappearing at the moment when the normal composition of the blood is restored ? Nor is it easier to explain on any mechanical hypothesis how it is that in a starving animal, the quantity of inorganic substances eliminated in the urine drops almost to zero, while the proportional amount in the blood and tissues is little, if at all, affected. In a rabbit rendered poor in sodium chloride by feeding it with salt-free food the injection of a solution of sodim chloride isotonic with the blood produces no diuresis for a considerable time, but, on the contrary, a diminished flow of urine, while a similar solution injected into the reins of a rabbit previously fed with salted food causes an immediate and considerable diuresis. When small quantities of isotonic solutions of various salts are injected, those not normally present in the blood produce a greater diuresis than'normal constituents. Sodium chloride, which is present in normal plasma in greater 
amount than any other salt, canses the smallest diuresis of all (Haake and Spiro). Such facts suggest that the secreting rells of the kidney are stimulated $\mathrm{ol}^{\circ}$ inhibited by the contact of blood or lymph in which the normal colstituents are present in too great of in too small amount, and that the intensity of the action is proportional to the degrec of deficiency or excess. The greater the velocity of the circulation in the kidney, the more effective will be the stimulation produced by any given substance present in excess, and therefore the greater the total amount of it climinated in a given time. For in making the round of the renal circulation the concentration of the substance in any given portion of blood will fall less, and therefore the average stimulation exerted by it during the round will be greater the faster the blood flows. It is quite in agreement with this that when plethora is occasioned by transfusion of blood there is little or no diuresis, although the increase of arterial, capillary, and venous pressure, and the dilatation of the kidney, are evident. For the rapid passage of liquid out of the vessels would lead to a great increase in the relative proportion of corpuscles to plasma-that is to say, to an abnormal condition of the blood. On the other hand, when plethora is produced by injection of serum diuresis occurs (Cushny). This, again, is what we should expect, since the elimination of the superfluous liquid will restore the normal proportion. The diminished viscosity of the blood (1).22) produced by the excess of serum will aid the flow through the kidney and therefore increase the diuresis, while in the case of the plethora produced by injection of blood the elimination of liquid will at once increase the viscosity, diminish the velocity of the renal flow, and tend to lessen diuresis.

There is, then. little more reason to assume that the copious flow of urine which follows the absorption of a large quantity of water is due to a mere process of filtration than there is to believe that filtration, and not selective secretion, is the cause of the gush of saliva which precedes vomiting: or the sudden outburst of sweat on sudden and severe exertion. In addition, there are the positive proofs already mentioned that the 'rodded ' epithelium of the tubules, which no one supposes to he abandoned more to mere physical influences than the epithelium of the salivary glands, plays a part in the secretion of some of the urinary constituents.

As to the nature of the mechanism set in motion, and the series of events that take place as the constituents of the urine journey from the interior of the bloodressels to the lumen of the tubules, we know no more than in the case of other glands. This alone is clear, that the separation of the urine from the blood impiies the performance of a large amount of work by 
the kirlney. For the osmotic pressure of urine is several times as great as that of the plasma of the blood. Bloorl-plasma freezes at $-0.55^{\circ}$ to $-0.05^{\circ} \mathrm{C}$. (on the average, say, $-0.0^{\circ} \mathrm{C}$.). The osmotic pressure corresponding to $-0.6^{\circ} \mathrm{C}$. is $5,662 \mathrm{mill}$ metres of mercury (p. 4no), or, in round numbers, 75 metres of water. Human urine has been found to freeze at $-\mathrm{I} \cdot 3 \mathrm{~S}^{\circ}$ to $-2 \cdot I^{\circ} \mathrm{C}$. (say, on the average, $-\mathrm{I} \cdot \mathrm{S}^{\circ} \mathrm{C}$.), and for highly concentrated urines the depression of the freezing-point may be considerably greater. The osmotic pressure corresponding to $-\mathrm{I} \cdot \mathrm{S}^{\circ} \mathrm{C}$. is $\mathrm{I} 6,0 \mathrm{~S} 6$ millimetres of mercury or 225 metres of water. This exceeds the osmotic pressure of the plasma by I50 metres of water. In separating a kilogramme of urine from the blood the kidney accordingly does work approximately equivalent to raising a weight of a kilogramme to the height of I50 metres-i.e., I50 kilogramme-metres. It is evident that the excess of the blood-pressure in the glomeruli over the pressure of the urine in the tubules, which, even if we neglect the latter altogether-since there is only slight resistance to the flow of urine towards the bladder-cannot at most be greater than Ioo millimetres of mercury, or I·35 metres of water, will account for only an insignificant part of this work. The rest must be done at the expense of the energy of the food materials taken up by, and transformed in, the cells concerned with the secretion of the urine. But we do not know in what way these cells, by applying this energy, perform the remarkable feat of permanently maintaining a difference of fifteen atmospheres in the osmotic pressure of the liquids in contact with their attached and free surfaces. A token of the intensity of the metabolic effort required is the marked increase in the absorption of oxygen (as much as 0.28 c.c. per gramme) which occurs during diuresis, although it is not in proportion to the amount of the diuresis. In one experiment the oxygen absorbed by a dog's kidneys was I I per cent. of what would have been used up by the entire animal under normal conditions. There is no definite relation between the oxygen taken in and the carbon dioxide given out at any moment.

What is the significance of the peculiar arrangement of the glomerular bloodvessels, if the epithelium of the capsules has secretive powers like that of ordinary glands? It is difficult to believe that these unique vascular tufts have not a near and important relation to the renal function; but it is by no means clear what that relation is. The secretion of water, and even its rapid secretion, is not at all bound up with any set arrangement of bloodvessels. Gland-cells all over the body secrete water under the most varied conditions of blood-pressure, although a comparatively high pressure is upon the whole favourable to a copious outflow.

But the kidney has other functions than mere excretion (Pp. 508 , 569). And it may be that the simplest part of the latter process, 
the elimination of water and salts, is largely thrown upon the Malpighian corpuscles, as a physiologically cheaper machine than the epithelium of the tubules, which is left free for more complex labours. These may include not only the separation of nitrogenous metabolites, but also synthetic processes possibly concerned in the regulation of protein metabolism. (One characteristic synthesis, the union of benzoic acid and glycin to hippuric acis, has already been referred to. As will be shown later (p. 50S), it takes place mainly, in some animals perhaps cxclusively, in the kidncy. The epithelium of the glomerulus, being a less highly organized and less delicately selective mechanism than that of the convoluter tubules. may more easily respond to increasz of blood-pressure by increased secretion. At the sime time, placed as it is at the last flond-gate of the circulation, where the escape of anything valuable means its total loss, the glomerular epithelium may be endowed with a general power of resistance to transulation, which renders a com? paratively high blood-pressure a necessary condition of its acting at all. And as a matter of fact water ceases to be secreted by the kilney long before the blood-pressure in the glomeruli can have fallen below that which suffices for the highest activity of the liver. Perlaps. however, the high minimum pressure required (30 to fo mm. of mercury in the $(\mathrm{log})$ is merely the necessary consequence of the long and difficult path which most of the blood going through the kidney has to take, and that a sufficient blood-flow cannot be kept up with less. It may be, too, that the comparatively small surface of the glomeruli, restricted in order to leave room for the more highly organized parts of the renal mechanism, entails the more intense and concentrated activity, which the high blood-pressure renders possible, and the simplicity of work and organization renders harmless.

An obvious result, and perhaps an important one, of the peculiar arrangement of the bloodvessols of the kidney is that the renal tubules proper are shiclded from an excessive blood-pressure by the interposition of the glomeruli as a block. This may be cither because the epithelium of the tubules would not perform its work so well under a high blood-pressure, or because there would be a danger of substances which ought to be retained being cast out into the urine. In this connection it is interesting to note that the specific constituents of wrine are separated by epithelium surrouncled by capillaries of the s?cond order, and therefore witl a smaller bloodpressure than exists in the capillaries of most glands, while the same is true of bile, another (practically) protein-free secretion.

The maximum secretory pressure in the kidney, as shown by a manometer tied into the divided ureter, is about $60 \mathrm{~mm}$. of mercury in the dog, or less than half that of saliva. If the escape of the urine is opposed by a greater pressure than this, or if the ureter is tied, the kidney becomes cedematous. Whether the oedema is due to reabsorption of urine or to the pouring out of lymph owing to the pressure of the dilated tubules on the veins has not been definitely settled. It has been already pointed out that there is no necessary relation between the blood-pressure in the capillaries of a gland and its secretory pressure; and, so far as this goes, water might just as well be 
secreted at a pressure of $60 \mathrm{~mm}$. of mercury from the lowpressure blood of the second set of renal capillaries as from the high-pressure blood of the glomeruli. By obstruction the molecular concentration of the urine is diminished to half or threequarters of the normal.

The Influence of the Circulation on the Secretion of Urine.-Although the activity of no organ in the body is governed more by the indirect effects of nervous action than that of the kidney, no proof has been given of the existence of secretory fibres for it comparable to those of the salivary ylands. All the changes in the rate of renal secretion which follow the section or stimulation of nerves can be explained as the consequences of the rise or fall of local or general blood-pressure, and of the corresponding variations in the velocity of the blood in the renal vessels.

The best way to study variations in the calibre of the renal vessels is the plethysmographic method, and the oncometer of Roy is a plet hysmogra ph adapted to the kidney. It consists of a metal capsule lined with loose membrane. between which and the metal there is a space filled with oil. The two halves of the capsule open and shut on a hinge ; and the kidney, when intro-

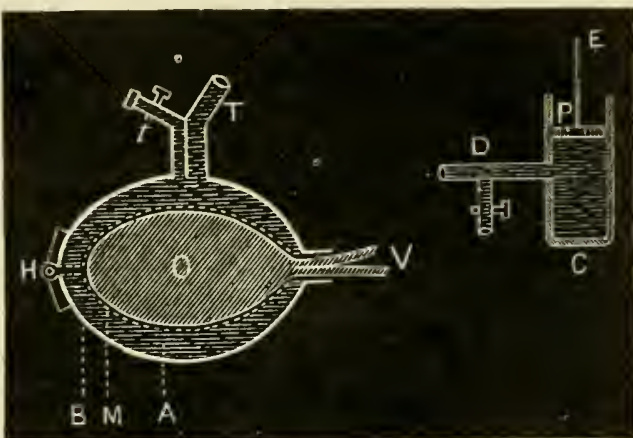

Fig, I75.-Diagray OF ORgan-PletHysmograph OR OYCOMETER.

$B$, metal box in two halves opening on the hinge $\mathrm{H}$; II, thin membrane: A, space filled with oil ; $\mathrm{O}$, organ enclosed in oneometer; $\mathrm{V}$, vessels of organ; $t$, tube for filling insirument with oil ; $\mathrm{T}$, tube con. nected with $\mathrm{D}$, which opens into cylinder $\mathrm{C} ; \mathrm{C}$ is also filled with oil; $\mathrm{P}$, piston attached by $\mathrm{E}$ to a writing lever. duced into it, is sur. rounded on all sides by the membrane, the vessels and ureter passing out through an opening. The oil-space is connected with a cylinder also filled with oil, above which a piston, attached to a lever, moves. The lever registers on a drum the changes in the volume of the kidney-i.e., practically the changes in the quantity of blood in it, and therefore in the calibre of its vessels. A still better oncometer is that of Schäfer, in which air is employed instead of oil.

Nerves of the Kidney.-Both vaso-constrictor and vaso-dilator fibres for the renal vessels, but most clearly the former, have been shown to leave the cord (in the dog) by the anterior roots of the sixth thoracic to second lumbar nerves, and especially of the last three thoracic. They run in the splanchnics, and then through the renal plexus-around the renal artery-into the kidney. The vasoconstrictors predominate, so that the general effect of stimulation of the nerve-rnots, the splanchnics, or the renal nerves is shrinking of 
the kidney, with diminution or cessation of the secretion of urine. But slow rhythmical stimulation of the roots causes increase of volume, the scanty dilators being by this methorl excited in preference to the coustrictors.

The remal nerves, entering at the hilum, branch repeatedly, so as to form a wide-meshed plexus aromel the arteries, and accompany them coen to their fincst ramifications in the cortex. Coming off from the nerves surroumling the arteries are fune fibres which are distributed to the convoluted tubules. Some of them terminate in globular ends, others in fine threads that pass through the membrana propria (Berkely).

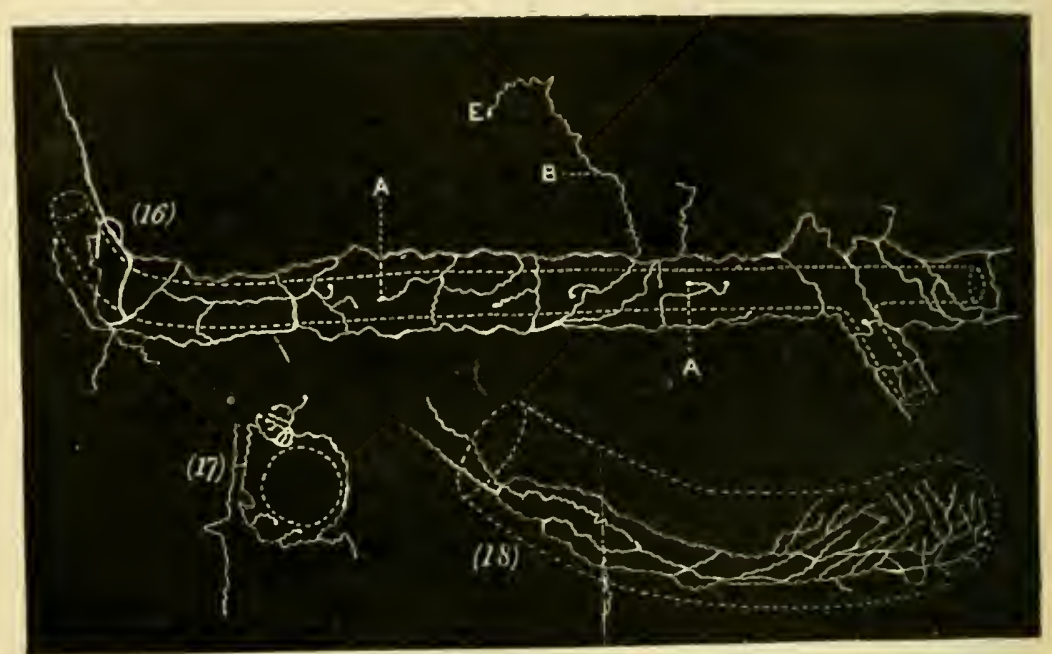

FIG. I76.-NERVES OF KIDNEY (BERKELY).

(16) medium - sized artery with its nerve-plexus ; A, terminal knobs; $B$, aberrant branch ending in terminal knob E; the dotted lines outline the artery: (17) nerve-fibres surrounding a Bowman's capsule, which is indicated by a dotted line; some of the endings are close to the membrane; ( 18 ) convoluted tubule shown in outline with fine nerve-fibres on $\mathrm{it}$, which seem to enter the basement membrane.

Section of the renal nerves is followed by relaxation of the small arteries in the kidney, and consequent swelling of the organ. The flow of urine is greatly increased, and sometimes albumin appears in it, the excessive pressure in the capillaries (particularly in those of the glomeruli) being supposed to favour the escape of substances to which a passage is refused under normal conditions.

- An experiment which is sometimes quoted as a decisive test of the relative importance of changes in the rate of flow, and in the pressure of the blood within the glomeruli, is that of tying the renal vein. This undoubtedly does not lower the 
intra-glomerular pressure-on the contrary, it must increase it but the secretion of urine stops. If the venous outhow from the kidney is only partially interfered with, the flow of urine is immediately diminisled, but the administration of a diuretic like potassimm nitrate causes an increase. It is more than likely that in these experiments the secretion stops or slackens not because a high blood-pressime, but because an active circulation is its necessary condition. When the blood stagnates in the kidney the natural stimulus to the renal apparatus speedily disappears owing to the elimination of the urinary constituents to the neutral or indifferent point (p. 46.4). The experiment, however, is not perfectly conclusive. For few glands can go on performing their function after the circulation has ceased. The kidney must be able to feed itself in order to continue its work. Above all it needs oxygen; and it might be urged that if the blood in the glomeruli could be kept at the normal standard of arterial blood, secretion might still go on after ligation of the renal vein.

According to Ludwig, indeed, the flow of urine stops, in spite of continued filtration through the glomeruli, because the swelling of the reins in the boundary layer compresses the tubules, and may even obliterate their lumen. There is no conclusive experimental evidence, however, and no it priori probability, that the obstruction so produced is sufficiently sudden or sufficiently complete to cause instant and total cessation of the flow. It is even less justifiable to conclude from the experinent that the liquid part of the urine is, at any rate, not separated by the epithelium of the tubules, since the bloodpressure in the capillaries around the tubules must rise very greatly after ligature of the vein, and yet secretion is stopped. It might equally well be argued that the renal epithelium normally secretes water under a low blood-pressure, but is disorganized under the excessive and entirely unaccustomed pressure which follows the closure of the vein.

It is not only through nerves directly governing the calibre of the vessels of the kidney that the rate of urinary secretion can be affected. Any change in the general blood-pressure, if not counteracted by, still more if conspiring with, simultaneous local changes in the renal ressels, may be followed by an increased or diminished flow of urine; and the law which explains all such variations, or at least serves to sum them up, is that in general an increase in the rate of the blood-flow through the kidney is followed by an increase in the rate of secretion. It will be remarked that this is the converse of the great law, of which we have already seen so many illustrations, that functional activity increases blood-flow. It is probable that this law holds for the kidney as well as for other organs, but that the influ- 
ence of actuvity on blood-supply is subordinatecl to that of blood-supply on activity, while in most tissues, as in the muscles, the opposite is the case. It is evident that an increase in the blood-flow would favour the secretory activity of the renal cells, since the average concentration of the blood presenter to them as regards those constituents which they select would remain relatively ligh in its circuit through the kiclney. The 'stimulus' to secretion would, therefore, be relatively intense.

Destruction of the medulla oblongata (i.e., of the vaso-motor centre), or section of the cord below it, diminishes the secretion of urine, because the arterial pressure is lowered so much as to over-compensate the dilatation of the renal vessels, which the operation also brings about. If the blood-pressure falls below $40 \mathrm{~mm}$. of mercury, the secretion is abolished. Stimulation of the medulla or cord also lessens the flow of urine by constricting the arterioles of the kidney so much as to over-compensate the rise of general blood-pressure, caused by the constriction of small ressels throughout the body.

If the renal nerves have been cut, stimulation of the medulla oblongata increases the urinary secretion, because now the rise of general blood-pressure is no longer counterbalanced by constriction of the renal vessels. An increase in the urinary flow can be produced in the rabbit by a lesion in a part of the funiculi teretes, which can be reached in the floor of the fourth ventricle (Eckhard), perhaps by destroying the portion of the vaso-motor centre governing the renal nerves, while the rest remains uninjured, or is even stimulated, and thus keeps up or even increases the general blood-pressure. There is either no glycosuria, or it is very slight.

Section of the splanchnic nerves causes a fall of arterial pressure, which is, however (in animals like the dog, in which compensation soon takes place), more than balanced by the simultaneous dilatation of the renal vessels, and therefore for some time the flow of urine is increased, but not so much as when the renal nerves alone are cut. In the rabbit there is no increase. On the other hand, stimulation of the splanchnics stops the urinary secretion, because the general rise of pressure is not enough to make up) for the constriction of the renal vessels.

Diuretics are substances that increase the flow of urine. Some of them act mainly on the circulation, as by increasing the general blood-pressure, others mainly by a direct influcnce on the secreting mechanism. Digitalis is a representative of the first class; urea and caffem belong to the sccond. The action of digitalis is to strengthen the beat of the heart, which is at the same time somewhat slowed, and to constrict the arterioles. Both effects contribute to the increase of pressure. But the accompanying diuresis is clue to the cardiac factor, the vasi-constriction which involves the renal vessals also, being overcompensated. The diuretic effect of digitalis 
is much greater in carrliac disease with dropsical effusions than in liealth. Calfein, when injected into the blood, affects the pressure but little. It causes dilatation of the renal vessels after a passing constriction, and an increase in the flow of urine after a temporary climinution. The vascular dilatation is not the chicf reason for the diuretic effect, for the latter is still obtained when the vaso-motor mechanism has been paralyzed by chloral hydrate, and even after the secretion of urine has been stopped by the fall of pressure consequent on section of the spinal cord. Caffein, therefore, acts directly on the renal epithelium. The action of urea, potassium nitrate, and the saline diuretics is probably also a direct action on the secreting structures, although some have supposed that their primary effect is to cause vaso-dilatation in the kidney, and in consequent local increase in the capillary pressure. The influence of ancesthetics on diuresis is of practical importance. Ether generally increases, while chloroform generally diminishes the flow of urine in dogs. A.C.E. mixture has a variable effect, but there is always a marked after-increase. A mixture of ether and chloroform constitutes the ideal anzestletic for experiments on the kidney, since it alters the diuresis only slightly (Thompson).

Summary.-Our knowledge of renal secretion may be thus summed 11p: The reater and sults of the urine are chiefly separated by the glomernli; the process is not a mere physical filtration, but a true secretion. Substances like sugar, peptone, egg-albumin, and hamoglobin when injected into the blood are probably excreted mainly by the glomeruli; and so is the sugar of diabetes. Urea, uric acid, and presumably the other organic constituents of normal urine, with a portion of the water and salts, are excreted by the physiological activity of the 'rodded' epithelimm of the renal tubules. The rate of secretion of urine rises and falls with the pressure, and still more with the velocity, of the blood in the renal vessels. No secretory nerves for the kidney have been found; the effects of section or stimulation of nerves on the secretion can all be explained by the changes produced in the renal blood-flow. Some diuretics act by increasing the blood-flow, others directly on the epithelium of the tubules or the glomeruli.

Micturition.-The urine, like the bile, is being constantly formed; although secretion varies in its rate from time to time, it never ceases. Trickling along the collecting tubules, the urine reaches the pelvis of the kidney, from which it is propelled along the ureters by peristaltic contractions of their walls, and drops from their valve-like orifices into the bladder. When this becomes distended, rhythmical peristaltic contractions are set $\mathrm{up}$ in it, and notice is given of its condition by a characteristic sensation, which is perhaps aided by the squeezing of a few drops of urine past the tonically contracted circular fibres that form a sphincter round the neck of the bladder, and into the first part of the urethra. The desire to empty the bladder can be resisted for a time, as can the desire to empty the bowel. If it is yielded to, the smooth muscular fibres in the wall of the viscus are thrown 
into contraction. This is aicled by an expulsive effort of the ablominal muscles. The sphincter vesice is relaxed; and the urine is forcel along the urethra, its passage being facilitated by cliscontinuous contractions of the ejaculator urinc muscle, which also serve to squeeze the last drops of urine from the urethral camal at the completion of the act.

liegurgitation into the ureters is to a great extent preventer by their compression between the mucous and muscular coats of the blarkler, where they run for more than half an inch before opening at the posterior angle of the trigone. But it has been shown that a certain amount of back flow can take place. Small bodies like diatoms suspended in water and pigments dissolved in it have been found in the pelvis of the kidney, the renal tubules, and even the circulation after being injected in to the bladder.

The pressure in the bladder of a man may be made as high as Io cm. of mercury during the act of micturition : about half this amount is clue to the contraction of the resical walls alone, the rest to the contraction of the abdominal muscles. A pressure of $\mathrm{It}$ to $26 \mathrm{~mm}$. Of mercury is required to open the sphincter of a rabbit's bladkler in life.

Although the whole performance seems to us to be completely voluntary, there are facts which show that it is at bottom a reflex series of co-ordinated movements. that can be started by impulses passing to a centre in the spinal cord from above or from below-from the brain or from the bladder. In dogs, with the spinal cord divided at the upper level of the lumbar region, micturition takes place regularly when the bladder is full, and can be excited by such slight stimuli as sponging of the skin around the anus (Goltz). Here, of course, the act is entirely reflex ; and the centre is situated at the level of the fifth lumbar nerves. The efferent nerves of the bladder, like those of the rectum, come partly from the cord directly through the sacral nerves, and partly througl the lumbar sympathetic chain (second to sixth ganglia). The sacral fibres are comnected with nerve cells in the hypogastric plexus, and the sympathetic, partly at least, in the inferior mesenteric ganglia. This anatomical coincidence acquires interest in view of the striking physiological similarity between the processes of micturition and defaecation, a similarity which is emphasized by the fact that the latier is almost invariably accompanied by the former. An important difierence, however, is that the will can far more readily snt in motion the machinery of micturition than that of derecation; a man can generally empty his bladeler when he likes, but he cannot empty his bowels when he likes

Sometimes in disease, and especially in diseatse of the spinal cord, the mechanism of micturition breaks domm; the bladder 
i* no longer emptied; it remains distended with urine, which dribbles away through the urethra as fast as it escapes from the ureters. To this condition the term incontinence of urine is properly applied.

Reflex emptying of the bladder, without an act of will or during unconsciousness, is not true incontinence. The involuntary micturition of children during sleep, for example, is a perfectly normal reflex act, although more easily excited and less easily controlled than in adults. Section either of both nervi erigentes, or of looth liypogastrics, is never followed by more than quite temporary disturbance of function of the bladder in dogs, both male and female. In a few days the urine is normally passed. In bitches the same is true when botlı pairs of nerves are divided. But in male dogs true incontinence of urine follows section of the four nerves, as well as intense tenesmus due to paralysis of the lower part of the large intestine.

\section{Excretion by the Skin.}

Besides permitting of the trifling gaseous interchange already referred to (p. 275), the skin plays an important part in the elimination of water by the sweat-glands.

Sweat is a clear colourless liquid of low specific gravity ( $\mathrm{IOO}_{3}$ to ro06), consisting chiefly of water with small quantities of salts, neutral fats, volatile fatty acids, and the merest traces of proteins and urea. It is acid to litmus except in profuse sweating, when it may become neutral or even alkaline. It is secreted by simple gland-tubes, which form coils lined with a single layer of columnar epithelium, in the subcutaneous tissue, with long ducts running up to the surface through the true skin and epidermis. Unless collected from the parts of the skin on which there are no hairs, such as the palm, it is apt to be mixed with sebum, a secretion formed by the breaking down of the cells of the sebaceous glands, which open into the hair follicles, and consisting chiefly of glycerin and cholesterin fats, soaps, and salts. Sebum is probably of consilerable importance for maintaining the normal condition of the hair and skin.

Although it is only occasionally that sweat collects in visible amount on the skin, water is always being given off in the form of vapour. This invisible perspiration leaves behind it on the skin, or in the glands, the whole of the non-volatile constituents, which may be to some extent reabsorbed; and since even the visible perspiration is in large part evapolated from the very mouths of the glands in which it is formed, the sweat can hardly be considered a vehicle of solid excretion, even to the small extent indicated by its chemical composition.

The total quantity of water excreted by the shin, and the 
relative proportions of visible and invisible perspiration, vary greatly. A dry and warm atmosphere increases, and a moist and cold atmosphere diminishes the total, and, within limits, the invisible perspiration. Visible sweat-given the condition of rapid heat-production in the body as in muscular labouris more readily deposited on freely exposed surfaces when the air is moist than when it is dry. The air in contact with surfaces covered by clothing is never far from being saturated with watcry vapour. Here, accordingly, a comparatively slight increase in the activity of the sweat-glands suffices to produce more water than can be at once evaporated; and the excess appears as sweat on the skin, to be absorbed by the clothing without evaporation, or to be evaporated slowly, as the pressure of the aqueous vapour gradually diminishes in consequence of diffusion. The power of imbibition (p. 398) of water by the various layers of the skin diminishes as we pass outwards, and the cells of the epidermis are characterized by the rapidity with which they return from a condition of excessive imbibition to their normal state. This constitntes a protective mechanism against excessive loss of water. When the skin is thoroughly moistened its degree of imbibition is three times the normal.

The quantity of sweat given off by a man in twenty-four hours varies so much that it would not be profitable to quote here the numerical results obtained under different conditions of temperature and humidity of the air (but sec p. 588). It is enough to say that the excretion of water from the skin is of the same order of magnitude as that from the kidneys: a man loses upon the whole as much water in sweat as in urine. But it is to be carefully noted that these two channels of outflow are complementary to each other ; when the loss of water by the skin is increased, the loss by the kidneys is diminished, and vice versâ.

The Influence of Nerves on the Secretion of Sweat.- The sweat-glands are governed directly by the nervous system; and though an actively perspiring skin is, in health, a flushed skin, the vascular dilatation is a condition, and not the chief cause of the secretion. Stimulation of the peripheral end of the sciatic nerve causes a copious secretion of sweat on the pad and toes of the corresponding foot of a young cat, and this although the ressels are generally constricted by excitation of the vasomotor nerves. Not only so, but when the circulation in the foot is entirely cut off by compression of the crural artery or by amputation of the limb, stimulation of the sciatic still calls forth some secretion. As in the case of the salivary glands, injection of atropine abolishes the secretory power of the sciatic, while leaving its vaso-motor infuence untouched; and pilocarpine 
increases the flow of sweat by direct stimulation of the cndings of the secretory nerves in the glands.

That the sweating caused by a ligh external temperature is normally brought about by nervous influence, and not by direct action on the sccreting cells, is shown by the following experiments. One sciatic nerve is divided in a cat, and the animal put into a hot-air chamber. No sweat appears on the foot whose nerve has been cut, but the other feet are bathed in perspiration. Similarly, a venous condition of the blood (in asplyyia) causes sweating in the feet whose nerves have not been divided, but none in the other foot; and stimulation of the central end of the cut sciatic has the same effect. All this points to the existence of a reflex mechanism; and it is certain that aspliyxia acts by direct stimulation of the centre or centres. The vaso-motor centre is at the same time stimulated, and the bloodressels constricted, as in the cold sweat of the death agony. Fear may also cause a cold sweat, impulses passing from the cerebral cortex to the vaso-motor and sweat centres.

It is probable that a general sweat-centre exists in the medulla oblongata. but its position has not been exactly determined nor ever its existence definitely proved. On the other hand, it is known that in the cat there are at least two spinal centres, one for the fore-limbs in the lower part of the cervical cord, and another for the hind-limbs where the dorsal portion of the cord passes into the lumbar. That this latter centre does not exist or is comparatively inactive in man is indicated by the following case: A man fell from a window and fractured his backbone at the fifth dorsal vertebra. The lower half of the body was paralyzed for a time, but recorered. Ultimately, however, the paralysis returned; and shortly before his death (twenty-one years after the accident) it was noticed that a copious perspiration broke out several times on the upper part of the body, while the lower portion remained perfectly dry. If there is any functional spinal centre in man, it appears to lie above the fifth spinal segment. For it was seen in a professional diver who fractured his neck at that level, and lived three months after the accident, that sweat frequently appeared on parts of the body above the lesion, but never below. At the autopsy the whole thickness of the cord, except perhaps a small portion of the anterior columns, was found destroyed. Of course, it may be that in man the spinal centres, although normally active, lose their function for a long time after such severe injuries to the cord owing to the condition known as shock.

The secretory fibres for the fore-limbs (in the cat) leave the cord in the anterior roots of the fourth to ninth thoracic nerves. They pass by white rami communicantes to the sympathetic chain, in which they reach the ganglion stellatum, where they are all connected with nerve-cells. Then, as non-medullated fibres, they gain the brachial plexus by the grey rami, and run in the median and ulnar to the pads of the feet. The fibres for the hind-limbs leave the cord in the anterior roots of the twelfth thoracic to the third or fourth lumbar nerves; pass by the white rami to the sympathetic ganglia, in which 
they form comnections with ganglion cells ; then, as non-medullated fibres, run along the grey rami, and are distributed to the foot in the sciatic.

The evidence of the direct secretory action of nerves on the sweat-glands is singularly striking and complete, in contrast to what we know of the kidney. In the latter, blond-flow is the inportant factor; increased blood-flow entails increased secretion. In the former, the nervous impulse to secretion is the spring which sets the machinery in motion; vascular dilatation aids secretion, but does not generally cause it. It would, however, be easy to lay ton much stress on this distinction, for in the horse the mere dilatation of the bloodvessels of the head after section of the cervical sympathetic has been found to be accompanied by increased secretion of sweat, and urinary secretion can certainly be affected by the direct action of various substances on the secretory mechanism, independently of vascular changes. But the broad difference stands out clearly enough, and the reason of it lies in the essentially different purpose of the two secretions. The water of the urine is in the main a vehicle for the removal of its solids; the solids of the sweat are accidental impurities, so to speak, in the water. The kidney eliminates substances which it is vital to the organism to get rid of ; the sweat-glands pour out water, not because it is in itself hurtful, not because it cannot pass out by other channels. but because the evaporation of water is one of the most important means by which the temperature of the body is controlled. In short, urine is a true excretion, sweat a heatregulating secretion. No hurtful effects are produced when elimination by the skin is entirely prevented by varnishing it, provided that the increased loss of heat is compensated. A rabbit with a varnished skin dies of cold, as a rabbit with a closely-clipped or shaven skin does; suppression of the secretory function of the skin has nothing to do with death in the first case any more than in the second (p. 276).

\section{PIRACTICAL EXERCISES ON CHAPTER VI.}

\section{Urine.}

For most of the experiments human urine is employed-in the quantitative work the mixed urine of the twenty-four lours. Urime may also be obtained from animals. In rabbits pressure on the abdomen will usually empty the bladder. Dogs may be taught to micturate at a set time or place, or kept in a cage arranged for the collection of urine. Or a catheter may be used (p. 609). 
I. Specific Gravity.-Pour the wrine into a glass cylincler, and remove froth, if necessary, with filter-paper. Place a urinomet(r ( 1 ig. 177 ) in the urine, and see that it loes not come in contact with the side of the ressel. Rearl off on the gradnaterl stem the division which corresponds with the bottom of the meniscus. This gives the specific gravity.

2. Reaction. - (a) Test with litmus-paper. Fencrally the litmus is redilened. but nccasionally in healtl the urine first passed in the morning is alkaline. Somelimes urinc lias an amphicroic reactioni.e., affects both red and blue litmus-paper. This is the case when there is such a relation between the bases and acids that both acid and 'neutral' (dibasic) phosphates are present in certain proportions. The acid phosphate reddens blue litmus, and the 'neutral phosphate turns red litmus blue.

(b) Titratable Acidity. - To 25 c.c. of urine add 15 to 20 grammes of powdered potassinm oxalate. and onc or two drops of a $I$ per cent. solution of phenolphthalein. Shake the mixture rapidly for a minute or two. and then titrate with decinormal sorlium hydroxide at once (while still cold from the solution of the oxalate) till a faint pink colour remains permanent on shaking. The potassium oxalate is adderl to counteract the tendency of the calcium present in urine to form basic phosplnates, which would be precipitated, and the acidity of the urine thus increased (Folin).

3. Chlorides-(a) Qualitative Test.-Add a drop of nitric acid and a clrop or two of silver nitrate solution. The nitric acid is added to prevent precipitation of silver phosphate. A white precipitate soluble in ammonia shows the presence of chlorides. The precipitate appears to be incompletely soluble in ammonia, since the ammonia brings down a small precipitate of earthy phosphates.

(b) Quantitative Estimation.-The quantitative estimation of the chlorine in urine without previous evaporation and incineration is best marle by one of the modifications of Volhard's method. It depends upon the com-

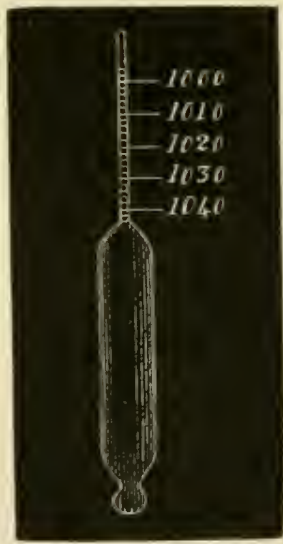

FIG. I77.-URINOMETFR. plete precipitation of the chlorine combined with the alkaline metals, and also of sulphocyanic acid, by silver from a solution containing nitric acid in excess; and avoids the error introduced into simpler methods, like Mohr's, by the union of some of the silver with other substances than chlorine. A given quantity of a standard solution of silver nitrate (more than sufficient to combine with all the chlorine) is added to a given volume of urine. The excess of silver is now estimated by means of a standard solution of ammonium sulphocyanide, which precipitates the silver as insoluble silver sulphocyanide. A fairly strong solution of the double sulphate of iron and ammonium (known as iron-ammoniaalum) is taken as the indicator, since a ferric salt does not give the usual red colour with a sulphocyanide so long as any silver in the solution is uncombined with sulphocyanic acid. The ironammonia-alum forms the red salt, ferric sulphocyanide, when any excess of ammonium sulphocyanide is present, but it does not react with silver sulphocyanide. 
The standard solution of silver nitrate can be made by dissolving $29^{\circ} 063$ grammes of pure fused silver nitrate in distilled water and making up the volume of the solution accurately to I litre. The solution should be kept in the dark. One c.c. of this solution corresponds to $0^{\circ}$ I gramme $\mathrm{NaCl}$ or $0^{\circ} 00607$ gramme $\mathrm{Cl}$.

The standard solution of ammonimm sulphocyanide is prepared as follows: Dissolve 13 grammes pure ammonium sulphncyanide $\left(\mathrm{NH}_{4} \mathrm{CNS}\right)$ in a litre of distilled water. Measure with a pipetie into a beaker 20 c.c. of the standard silver nitrate solution, and add 5 c.c. of the iron alum solution and + c.c. of pure nitric acid (specific gravity 12 ). Fill a burette with the sulphocyanide solution, and run it into the silver nitrate solution until a faint permanent red tinge is obtamed. Note the number of c.c. of the sulphocyanide solution required, and then dilute the solution till 2 c.c. of the sulphocyanide solution correspond exactly to I c.c. of the silver solution, so as just to allow of the end reaction witl the iron solution being seen. and no more.

To carry out the metlool, put in c.c. of urine, which must be free from albumin. in a stoppered flask, with a mark corresponding to 100 c.c.. or a graduated cyliniler. Aile 50 c.c. of water, 4 c.c. of pure nitric acid (specific gravity $1 \cdot 2$ ), and is c.c. of the standarl silver solution; shake well, fill with water to the mark, and again shake. After the precipitate has settled, filter it off. Take 50 c.c. of the filtrate. add 5 c.c. of the solntion of iron-ammonia-alum, and run in from a burette the standard solution of ammonium sulpliocyanidc until a weak but permanent recl coloration appears.

Suppose $x$ c.c. of the sulpliocyanicle solution are required, then the chlorine in Io c.c. of wrine evidently corresponds to $(15-x)$, o.ol gramme $\mathrm{NaCl}$.

4. Phosphates-(I) Qualitative Tests.-(a) Render the urine alkaline with ammonia. A precipitate of earthy phosphates (calcium and magnesium phosphates) falls down. Filter. The filtrate contains the alkaline phosphates. To the filtrate add magnesia mixture.* The alkaline plosphates (sodium, potassium, or ammonium phosphates) are precipitated as ammonio-magnesic or triple phosphate. (b) Add to urine half its volume of nitric acid and a little molybdate of ammonium, and heat. A yellow precipitate of ammonium phospho-molybdate shows that plosphates are present. This test is given both by alkaline and eartliy phosphates.

(2) Quantitative Estimation. - The quantitative estimation of phosphoric acid in urine is best done volumetrically, by titration with a standard solution of uranium nitrate, using ferrocyanide of potassium as the indicator. Iranium nitrate gives with phosphates, in a solution containing free acetic acid. a precipitate with a constant proportion of phosphoric acid. As soon as there is more uranium in the solution than is required to combine with all the phosphoric acid. a brown colour is given with potassium ferrocyanide, due to the formation of uranium ferrocyanide. In carrying out the method. 5 c.c. of a mixture of acetic acid and sodium acetate (there are Io grammes of sodium acetate and Io grammes of glacial acetic acid in 100 c.c. of the mixture) are added to 50 c.c. of urine, which is then heated in a beaker on the water-bath almost to boiling. The standard uranium solution (which contains $35^{\circ} 5$ grammes of uranium nitrate in the litre, and I c.c. of which corresponds to 0.005 gramme

* Magnesium chloride 110 grammes, ammonium chloride i 40 grammes, ammonia (specific gravity $0^{\circ} 9$ I) 250 c.c., and water 1,750 c.c. 
$\left.\mathrm{P}_{\mathrm{y}} \mathrm{O}_{\mathrm{i}}\right)$ is now run in from a burette, until a drop of the urine gives, with a drop of potassium ferrocyanide solution, on a porcelain slab. a brown colour. Uranium acetate may be used instead of uranium nitrate, but the latter keeps best. When uranium acetate is employel it is not necessary to acld the solinm acetate mixture.

5. Sulphates - (I) Qualitative Test.-Add to urine a drop of hydrochloric acid and then a few trops of barium chloricle. A white precipitate comes down, showing that inorganic sulphates are present. The hychrochloric acid prevents precipitation of the phosphates.

(2) Qunntitative Estimation of the Sulphates (Inorganic and Ethereal). Adld to 50 c.c. of albumin-frec urine in a $200-c . c$. Erlenmeyer llask 5 c.c. of a + per cent. potassimm chlorate solution and 5 c.c. of strong hydrocliloric acid, and boil the mixture to break up the ethereal sulphates. In five to ten minutes it becomes perfectly colourless. While it continues to boil, 25 c.c. of a 10 per cent. solution of barium chloride are added by (lrops, at such a rate that it takes about five minutes to add this quantity. The flask is now put on the water-bath for one-half to one hour, till the precipitate has settled. Then filter through an asli-free filter. Wash the precipitate on the filter for half an hour with loot water. During the first twenty minutes of the washing, at intervals of a few minutes, substitute hot 5 per cent. ammonium chloride solution for the water. At the end of the half-hour's washing, as soon as the water has run through the filter, fold up the latter and press it gently betwcen dry filter-papers to remove a portion of the water. Then place the filter in a weighed porcelain crucible. Pour into the crucible 3 or + c.c. of alcohol, and ignite it, to dry and partially burn the filter-paper. Then incinerate till all the carbon is burned off, cool, and weigh. From the weight of the barium sulphate, the sulphuric acid in 50 c.c. of urine is easily calculated $\left(\mathrm{SO}_{4}\right.$ in I gramme of barium sulphate, $0^{\circ}$ I I 87 gramme) (Folin).

(3) Quantitative Estimation of the Sulphuric Acid united with Aromatic Bodies (Aromatic or Ethereal Sulphates).- Put 200 c.c. of the same urine as used in (2) into a beaker. Add Ioo c.c. of Io per cent. barium chloride solution in the cold. Let stand for twenty-four hours. Then decant off the clear supernatant liquid, and filter it. Measure 150 c.c. of the clear filtratc, corresponding to 100 c.c. of the urine, into a foo-c.c. Erlenmeyer flask. Add 10 or 15 c.c. of concentrated hydrochloric acid, and io to i 5 c.c. of 4 per cent. potassium chlorate. Heat the mixture to boiling. and proceed as in (2). From the weight of the barium sulphate, the ethereal sulphuric acid in 100 c.c. of urine can be calculated. Deducting this from the quantity per 100 c.c. of urine obtained in (2), we get the amount of inorganic sulphuric acid per roo c.c. (Folin).

6. Indoxyl (contained in the urine as indican, the potassium salt of indoxyl-sulphuric acid) can be oxidized into indigo, and so detected and estimated.

A qualitative test is the following: Ten c.c. of horse's urine is mixed with ro c.c. of Obermayer's reagent (pure concentrated hydrochloric acid containing 2 to 4 parts of ferric chloride in 1,000$)$, and shaken well for a minute or two; a bluish colour appears if, as is generally the case, indoxyl is present, indigo $\left(\mathrm{C}_{16} \mathrm{H}_{10} \mathrm{~N}_{2} \mathrm{O}_{2}\right)$ being formed by the oxidizing action of the ferric chloride on the indoxyl, the compound of which with sulphuric acid has been broken up by the hydrochloric acid. The urine must be free from albumin. In performing the test in human urine, which contains a smaller quantity 
of the indigo-forming substance, the faint blue liquil should be sluaken up with a few (lrops of chloroform. The latter tikes up the colour, which is thus renlered more eviclent. If there is difficulty in obtaining the reaction, the urine may furst be decolonrized by precipitating it with acctate of lear, alvoiding excess. The precipitate is filtered off, and the test then applies to the clear filtrate. The skatoxyl of urine can also be oxidized to indigo, but it is present in far smaller anount. The average quantity of indigo obtained from a litre of lorse's urine is about 150 milligrammes; from a litre of human urine, not a twentieth of that amount.

lior comparative (1uantitative determinations the method of Fotin may be used. One-hundredth of the twenty-four hours' urine is taken. In this the indigo is developed by the addition of an equal volume of Obermayer's reagent $(p-479)$, and the indigo-blue (lissolved by means of 5 c.c. of chloroform. The chloroform solution is then compared colorimetrically with Fehling's solution. This can be done by putting the indigo solution and 5 c.c. of the Fehling's solution respectively into small test-tubes of equal calibre, and comparing the depth of tint. If the Feluling's solution is stronger than the indigo solution, run water into the former from a pipette. graduated in tenths of a c.c., shaking up after each addition, till equality of tint has been reached. If the incligo solution has a stronger blue colour than the Fehling's solution, clilute a measured amount of it first of all with such a quantity of chloroform (say an equal volume) as will make its tint distinctly weaker than that of the Fehling's solution. Then dilute the Fehling's solution with water, as before, till the tint is the same. From the amount of lilution the quantity of indigo can be expressed in arbitrary units, taking Fehling's solution as I00. Thus, if I c.c. of water must be added to the 5 c.c. ot Fehling's solution, the indican can be expressed as $\underset{5+\mathrm{I}}{\mathrm{IO0}}=\underset{6}{500}=83$.

5

The comparison can be made more accurately by a colorimeter, if one is available. To determine the absolute amount of indigo obtained, comparison must be marle with a standard solution of incligo.

7. Urea-(1) Decomposition of Urea.-Heated dry in a test-tube, it gives off ammonia. The resiclue contains biuret, which, when (lissolved in water, gives a rose colour with a trace of cupric sulphate and excess of sodium hydroxide (or of the hydroxides of certain other metals of the alkalies and alkaline earths, p. 7). Some proteinspeptones and albumoses-in the presence of the same reagents, give a similar colour, the so-called biuret reaction.

(2) Quantitative Estimation - The Hypobromite Method.-The urea is split up by sodium hypobromite $(p .+40)$, and the carbon dioxide being absorbed by the excess of sodium hydroxide used in preparing the hypobromite, the nitrogen is collected over water in an inverted burctte. It is easy to calculate the weight of urea corresponding to a given volume of nitrogen measured at a given temperature and pressure. The nitrogen of urea is $\ddot{30}$, or ${ }_{1}^{i} ;$ of the whole molecular weight. Now, I c.c. of $\mathrm{N}$ weighs, at 760 millimetres of mercury and $0^{\circ} \mathrm{C}$. . o oor 25 gramme. Therefore, I c.c. of $N^{\top}$ corresponds to $0.00125 \times 0^{5}=0.00268$ gramme urea. Suppose, now, that I c.c. of urine was found to yield Io c.c. of $\mathrm{N}$ measured at $\mathrm{I} 7^{\circ} \mathrm{C}$. and $75^{\circ} \mathrm{milli}$ metres barometric pressure. Since a gas expands $z: 3$ part of its volume at $0^{\circ}$ for every degree above $0^{\circ}$, we must correct the apparent 
volume of nitrogen by multiplying by a gas is inversely proportional to the pressure, we must further

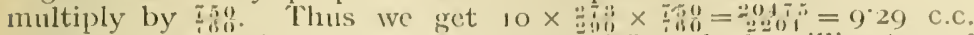
as the volume of the nitrogen reduced to 1$)^{\circ} \mathrm{C}$. and 760 millimetres of mercury. Multiplying this by 0.00268 , we get 0.0249 grimme urea for I c.c. urine, which for a diaily yichl of 1,200 c.c. would correspond to $29^{\circ} 88$ grammes urea.

As a matter of fact, however, it has been found that there is always a deficiency of nitrogen - that is, a given quantity of urea yielels less than the estimated amount of gas. $\Lambda$ gramme of urea in urine, insteat of giving off 373 c.c. of nitrogen. gives only $35+$ c.c. at $0^{\circ} \mathrm{C}$. and 760 millimetres pressure. We must therefore take I c.c. of $\mathrm{N}$ as corresponding to 0.00282 gramme, instead of $0^{\circ} 00268$ gramme urea. But it is affectation to make this correction if, as is constantly done in hospitals, tlic temperature is not taken into account.

A convenient apparatus is shown in Fig. I 78 . In $B$, place Io c.c. of a solution made by adding bromine to ten times its volume of 4o per cent. sodium hydroxide solution. Mix 5 c.c. of urine with 5 c.c. of water. Put 5 c.c. of the mixture into the thimble $A$, which is then set in the small bottle $B$. The cork is now carefully fixed in $B$, and the tube $F$ being open, the level of the water in the burette is read off. The pinchcock having been closed, the bottle B is now tilted so that the urine in the thimble is gradually mixed with the hypobromite solution, and the nitrogen given off is added to the air in the burette and its connections. The level of the water in the burette is therefore depressed. When gas ceases to be given off, and a short time has been allowed for the whole to cool, the tube is raised till the

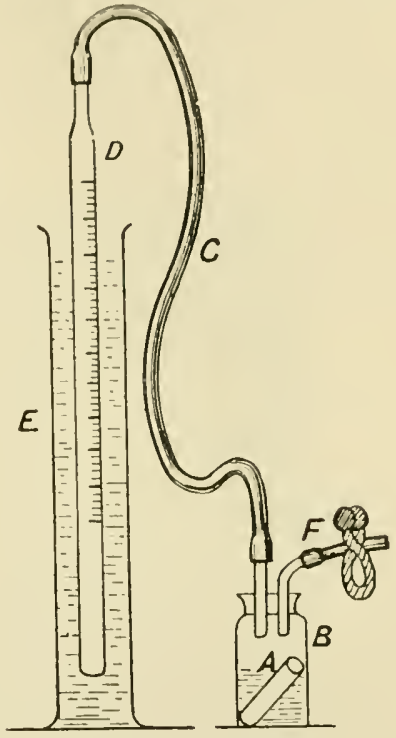

Fig. I78.-HYPoвRoMite MethoD OF ESTIMATING UREA.

A, glass thimble; $B$, bottle, through the rubber cork of which pass two short glass tubes, one con. nected by the rubber tube $C$ with a burette D, and the other armed with a short piece of rubber tube $\mathrm{F}$. $\mathrm{F}$ is provided with a pinchcock. The burette is supported on a stand, and immersed in water contained in the glass cylinder $\mathrm{E}$.

level of the water is once more the same inside and out. The level is again read off; the difference of the two readings gives the volume of nitrogen at the temperature of the air and the barometric pressure. In order that the temperature of the water may be the same as that of the air, the cylinder should be filled a considerable time before the observations are begun.

For clinical purposes sufficiently accurate results may be very easily obtained with the so-called ureometer of Doremus (Fig. I 79). A little urine is poured into the side-tube A, the stopcock C being closed. The stopcock is then opened for an instant, so as to fill 
its bore, and then closed again. Any urine which lias passed into the tube $B$ is washed out with water, and $B$ is then filled with hypobromite solution. $A$ is now filled up with urine to the top of the graduation. By opening the stopeock, I c.c. of urine for less if the urine is concentrated) is permitted to pass into $B$ and to mix with the hypobromite solution. The nitrogen collects in 13. and when it lias ceased to come off, the meniscus of the liquicl is reacl off. The corresponding degree on the scale gives the amount of urea in grammes contained in the quantity of urine cmployerl.

8. Estimation of the Total Nitrogen.-1t is sometimes more important to determine the total nitrogen of the urine than the urea alone : and this is conveniently done by Kjeldahl's method (or some modification of it). which can also be applied to the estimation of the nitrogen in the faces, or in any of the solids or liquids of the body. It depends on the oxidation of the nitrogenous matter (or, rather. in the case of urine, mainly its hyclrolysis) in such a way that the nitrogen is all represented as ammonia. The ammonia is then distilled over, collected and estimated, and from its amount the

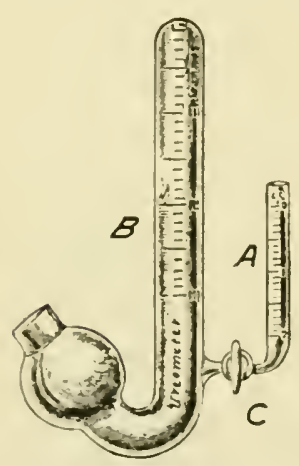

F1G. I79.-DOREMUS UREOMETER. nitrogen is easily calculated. In urine the methorl can be carried out by adding to a measured quantity of it (say 5 c.c.) four times its volume of strong sulphuric acid, and boiling in a long-necked flask (capacity 200 c.c.), after the addition of a globule of mercury (about o.I c.c.), which hastens oxidation and obviates bumping. A part of the mercuric sulphate formed remains in solution; the rest forms a crystalline deposit. The heating should con. tinue for half an hour, or until the liquid is decolourized. It should be kept gently boiling. This completes the process of oxidation; and the next step is to liberate the ammonia from the substances with which it is united in the solution, and to distil it over. Dilute the liquid with water, after cooling, up to about I 50 c.c., and pour into a larger long-necked flask. Add enough of a solution of sodium hydroxide (specific gravity about $\mathrm{I}^{\cdot 25}$ ) to render the liquid alkaline, avoiding excess, as this favours bumping. The proper quantity can be found by determining beforchand how much of the alkali is needed to neutralize the acid used for oxidation, and a little more than this amount should be added. Twenty c.c. of strong sulphuric acid needs about 75 c.c. of 40 per cent. sodium hydroxide to neutralize it. Bumping may further be prevented by the addition of a little granulated zinc. Sinake the flask two or three times. Add also about 12 c.c. of a concentrated solution of potassium sulphicle (I part to I t parts water). which favours the setting free of the ammonia from the amino-compounds of mercury that have been formed during oxidation. Commercial 'liver of sulphur' will do quite well. Immediately connect the distilling-flask with the worm, as shown in Fig. I So, and distil the ammonia over into 5o c.c. of standard (decinormal) sulphuric acid (sec footnote, p. 439) contained in a flask into which a glass tube connected with the lower end of the worm dips. Heat the distilling flask at first gently, then strongly, and boil for three-quarters of an hour, or juntil about two-thirds of the liquid has passed over. Then lift the tube out of the standard acid, and 
continue the distillation for two or three minutes longer. The ammonia is now all united with the standard acid, a certain amount of which is left over. By determining this amount we arrive at the quantity combined with ammonia, and therefore at the quantity of ammonia. Fill a burette with a lecinormal solution of potassium or sodium hydroxide. Add a little methyl-orange solution to the standard sulphuric acid, to serve as indicator. Then run in the potassium or sodium hychoxicle till the pink tinge gives place to a permanent but just recognisable yellow. Let $x$ be the number of c.c. run in. Since r c.c. of any decinormal solution is equivalent to I c.c. of any other, $x$ represents also the number of c.c. of the standard sulphuric acid left uncombined with ammonia; and $50-x$, the quantity combined with ammonia. Then, l c.c. of decinormal solium or potassium hydroxide being equivalent to I c.c. of deci-

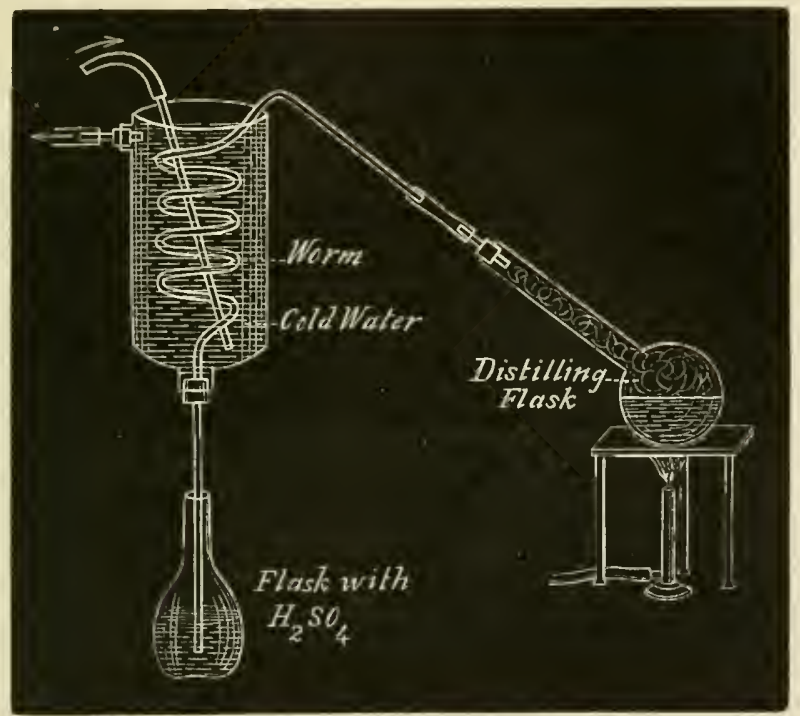

Fig. i8o.-Arrangenext for Distillation in Estimation of Total VITROGEN.

normal ammoniumi hydroxicle, and I c.c. of decinormal ammonium hydroxide containing $0^{\circ}$ oo I 4 gramme nitrogen, we get $\left(5^{\circ}-x\right) \times 0^{\circ} 00$ I 4 as the quantity of nitrogen in 5 c.c. of urine.

Instead of mercury, potassium sulphate and copper sulphate may be added to the sulphuric acid in order to aid the decomposition in the first stage of the estimation. About 3 grammes of potassium sulphate and I gramme of copper sulphate are added to 5 c.c. of urine, and then 5 c.c. of sulphuric acid. The liquid is gently boiled for an hour, or until it is quite clear. The neutralization and distillation are conducted as before, the proper quantity of sodium hydroxide being determined in advance. No potassium sulphide is added, but a small quantity of talc may be put in to prevent bumping. Instead of methyl orange. 'alizarin red,' which is bright rea in thel presence of the slightest trace of alkali, may be used. 
9. Uric Acid-(I) Qualitative Test for Uric Acid-Murexide Test.A small quantity of uric acid or onc of its salts is heated with a little dilute nitric acid. The colour of the residue left by evaporation becomes yellow, and then red, and on the addition of ammonia changes to deep purple-red. Potassium or sodium hydroxide changes the yellow to violet. The purple-red substance is murexide or ammonium purpurate. It is also formed by the action of nitric acid and ammonia on theobromine (dimethylxanthin), an alkaloid in cocoa, and theine or caffeine (trimethylxanthin), an alkaloid in tea and coffee, which are also purin derivatives (p. $44 \mathrm{I})$.

(2) Onantitative Estimation--(a) Hopkins's Method of Estimation of Uric Acid.-Add about 25 grammes of ammonium chloride to Ioo c.c. of urine in a beaker. Stir well to dissolve all the salt. Then add 2 c.c. of strong ammonia. Let the mixture stand till the precipitate of ammonium urate which is formed has entirely settled to the bottom and the liquid above it is clear. Then filter through a small filter-paper. Wash the precipitate on the filter twice with saturated solution of ammonium chloride from a washbottle. Then wash the precipitate into a porcelain capsule with hot water from a wash-bottle with a fine jet, unfolcling the filterpaper over the capsule so as to get it all off. For this purpose not more than 20 to 30 c.c. of water should generally be necessary : but if much more has been used the excess should be got rid of by evaporation on a water-bath. Pour about I c.c. of strong hychrochloric acid into the capsule, lieat to boiling, and then allow the capsule to stand in the cold till the uric acill crystallizes out. The time required for this is 2 to 12 homs, being shorter the lower the temperature. The crystals are filtered off through a small weighed filter, the filtrate being received in a graduated cylinder and measured. The crystals are then washed on the filter with cold distilled water. till the washings come through the filter free from chlorides, as tested by silver nitrate (1. 477). The filter with the crystals is dried in the oven and weighed. To the weight of the uric acid thus obtained i milligramme must be added for each I5 c.c. of the filtrate (the mother-liquor) collected in the gradluated cylincler.

$O r$, instead of being estimated by weighing, the uric acicl crystals may, after laving been washed with the cold distilled water as (lescribed, till free from chlorides, be washed again with hot water off the filter (which need not in this case be a weighed one) into a capsule. The capsule is filled up with distilled water, 1 c.c. of ro per cent. sodium carbonate solution added, and the contents heated to boiling to dissolve the uric acid. The solution is allowed to cool, and then emptied into an Erlenmeyer's flask with a mark roughly corresponding to roo c.c. The solution is made up to roo c.c. with distilled water. A burette is filled with standard potassium permanganate solution (a twentieth-normal solution made by dissolving $\mathrm{I}^{\circ}{ }_{5} 8 \mathrm{I}$ grammes of the permanganate in a litre of water). Twenty c.c. of strong sulphuric acid are poured into the flask, which is then shaken. The permanganate is now run in with constant shaking of the flask. At first the pink colour produced where the drops of permanganate fall into the liquid disappears at once without spreading through the liquid. When a certain amount has been run in, however, the whole liquid becomes pink, although the colour soon disappears. This indicates that enough of the permanganate has been added. Each c.c. of the permanganate used is equivalent to 0.00375 gramme uric 
acicl. One milligramne must be added, as before, to the result for each 15 c.c. of the mother-liquor from which the uric acid erystals were deposited.

(b) Folin's Modification of Hopkins's Method.-The chicf reagent is a solution of 500 grammes ammonium sulphate, 5 grammes uranium acetate, and bo c.c. 10 per cent. acetic acid, in $65^{\circ}$ c.c. of water.

One hundred and fifty c.c. of urine is measured into a tall, narrow beaker or a cylinder, and $37^{\frac{1}{2}}$ c.c. of the reagent added. If enough urine is available, $200 \mathrm{c.c}$. of urine and $50 \mathrm{c.c}$. of reagent are to be used. Allow the mixture to stand without stirring for about half an hour. The uraniunn precipitate has then settled, and the clear supernatant liquicl is removed by siphoning or decantation. One hundred and twenty-five c.c. of this liquid is measured into another beaker, 5 c.c. of strong ammonia added, and the mixture set aside till next day. The precipitate is then filtered off, and washed with Io per cent. ammonium sulphate solution until the filtrate is quite or nearly free from chlorides. The filter is then removed from the funnel, opened, and the precipitate rinsed back into the beaker. Enough water to make about 100 c.c. is added, and the precipitate is then dissolved by means of 15 c.c. concentrated sulphuric acid, and at once titrated with permanganate solution, each c.c. of which corresponds to 3.75 milligrammes of uric acid. The very first pink coloration, extending through the entire liquid through the addition of two drops of permanganate solution, marks the end point. A correction of 3 milligrammes, owing to the solubility of ammonium urate, is added to the result.

Io. Kreatinin.-Qualitatively, kreatinin may be recognised in very small amounts by IVeyl's test. A few drops of a dilute solution of sodium nitro-prusside are added to urine, and then dilute sodium hydroxide drop by drop. A ruby-red colour appears, which soon turns yellow. If the urine is now strongly acidified with acetic acid and heated, it becomes first greenish and then blue. Enough acid must be added to more than neutralize the alkali.

Neubauer has made the reaction of kreatinin with zinc chloride the basis of a method for its quantitative estimation (Fig. I67, p. 442), but the results seem to be somewhat uncertain. A more convenient method is that of Folin. It depends upon the comparison of the colour which kreatinin gives with picric acid in an alkaline solution with that of a standard solution of potassium bichromate. Ten c.c. of urine is measured into a 500 c.c. measuring-flask; I 5 c.c. of a saturated picric acid solution (containing about I2 grammes per litre), and 5 c.c. of a ro per cent. solution of sodium hydroxide are added. The mixture is allowed to stand for five minutes. Then water is added up to the 500 c.c. mark, and the flask shaken to mix uniformly. Samples of the liquid are then at once compared colorimetrically with a half-normal solution of potassium bichromate containing 2455 grammes per litre. The colour of the urine does not introduce a sensible error on account of the great dilution. For exact work the comparison must be made with a good colorimeter. It has been found experimentally that, when ro milligrammes of kreatinin are present in 500 c.c. of a solution made as described, a layer of the solution $8^{\circ}$. millimetres in thickness has the same depth of tint as 8 millimetres of the bichromate solution. Suppose it takes 9 millimetres of the 
urine-picrate solution to equal 8 millimetres of the bichromate, then the Io c.c. of urine contains Io $\times \frac{8 \cdot 1}{9}=9.0$ milligrammes of kreatinin.

I I. Hippuric Acid.-From horse's or cow's urine hippuric acicl is prepared by evaporating to a small bulk, and adcling strong hydrochloric acid. The crystalline precipitate is washerl with cold water, then dissolved in hot water, and filtered hot. Hippuric acid separates out from the filtrate in the cold in the form of long four-sicled prisms with pyramidal ends. Heated dry in a test-tube, the crystals melt, and benzoic acid and oily drops of benzonitrilc, a substance witl a smell like that of oil of bitter almonds, are formed.

ABNORMAL SUBSTANCES IN URINE.

12. Proteins - (I) Qualitative Tests.-(a) Boil and arld a few drops of nitric acid. A precipitate on boiling, increased or not affected by the acid, shows the presence of coagulable proteins (scrumalbumin or globulin). A precipitate of eartily phosphates sometimes forms on boiling. It is distinguished from a precipitate of proteins by dissolving on the addition of acid.

(b) Heller's Test.-Put some nitric acid in a test-tube. Pour carefully on to the surface of the acid a little urine. A white ring at the junction of the liquids indicates the presence of albumin or globulin. If much albumose is present, a white precipitate, which disappears on heating, may be formed. When this test is performed with undiluted urine, uric acid may be precipitated and cause a brown colour at the junction. A similar ring may be found in the absence of proteins when the test is made on the urine of a patient who has been taking copaiba. In very concentrated urine a white ring of nitratc of urea may be formed. A coloured ring is frequently seen, owing to the oxidation of certain chromogens of urine.

(c) Filter some urine, and add to the filtrate its own volume of acetic acid. A precipitate may indicate mucin or nucleo-albumin. If any is formed, filter it off, and add to the filtrate a few drops of potassium ferrocyanide. A white precipitate shows the presence of proteins.

(d) Test for Globulin in Urine.--Serum-globulin probably never occurs in urine apart from serum-albumin. It may be detected thus: Make the urine alkaline with ammonia, let it stand for an hour and filter. Half saturate the filtrate with ammonium sulphate-i.e., add to it an equal volume of a saturated solution of ammonium sulphate. Serum-globulin is precipitated, scrum-albumin is not.

(e) Test for Albumose in Urine (Albumosuria).-Coagulable proteins are removed by boiling the urine (acidulated if necessary), and filtering off the precipitate if any. The filtrate is neutralized. If a further precipitate falls clown it is filtered off. the clear filtrate is heated in a beaker placed in a boiling water-bath, and there saturated with crystals of ammonium sulpliate. A precipitate indicates that albumoses (proteoses) are present. A slight precipitate might possibly be due to the formation of ammonium urate. A further test may be performed on the original nrine if it is free from coagulable proteins, or on the filtrate after their removal. Adci a clrop or two of pure nitric acid. If albumoses are present, a precipitate is thrown down which disappears on heating, and reappears on cooling the testtube at the cold-water tap. 
(n Test for Peptone in Crine (Peptonuria).-Place some of the urine in a beaker on a boiling water-bath for thirty minutes, and saturate with ammonium sulphate crystals. Then boil over a small flane or in an air-bath for half an hour. All the proteins, including peptones, are precipitated. But the peptones can still be redissolved by water, the others not. Filter hot. Wash the precipitate on the filter with a boiling saturated solution of ammonium sulphate. Then extract the resiclue with cold water, filter, and test the filtrate by the biurct test (addition of very dilute cupric sulphate and excess of sodium liydroxide). A rose colour indicates the presence of peptone (1). 7), but if the reaction is only a faint one, it may be due to urobilin (Stokvis). True peptone is rarely found in urine.

(2) Quantitative Estimation of Coagulable Proteins (Serum-Albumin and Globulin)-(a) Gravimetric Method.--Heat 50 to Ioo c.c. of the urine to boiling, adding a dilute solution ( 2 per cent.) of acetic acid by drops as long as the precipitate seems to be increased. Filter through a weighed filter. Wash the precipitate on the filter with hot water, then with hot alcohol, and finally with ether. Dry in an airbath at $\mathrm{I}^{\circ} \mathrm{C}$, and weigh between watch-glasses of known weight.

(b) Method of Roberts and Stolnikow (modified by Brandberg).This method is founded on the fact that the time taken for the white ring to appear in Heller's test depends on the proportion of coagulable protein present. It has been found that when I part of albumin is contained in 30,00o parts of an albuminous solution (0.0033 per cent.), the ring appears in two and a half to three minutes. The amount of dilution of the urine which is necessary to delay the formation of the ring for this length of time is what has to be determined. To do this, proceed as follows : Dilute a portion of the urine (say 5 c.c.) ten times-that is, add to it nine times its volume of distilled water from a burette. Place some pure nitric acid in a test-tube with a pipette, taking care not to wet the sides of the test-tube with the acid. Now run on to the surface of the nitric acid some of the diluted urine. Hold the test-tube up against the light from a windows Between the test-tube and the window hold obliquely a dull black surface a few inches from the test-tube, moving it up and down a little below the junction of the urine and acid. Note the interval that elapses before formation of the white ring. If it is more than three minutes, the diluted urine contains less than I part in 30,000, and the undiluted urine less than I part in 3,000 (i.e., less than $0^{\circ} 033$ per cent.) of coagulable protein, and the experiment must be repeated with urine diluted to a smaller extent. If the ring appears after a shorter interval than three minutes, the diluted urine contains more than I part in 30,000 (the original urine more than $0^{\circ} 033$ per cent.) and must be further diluted. Fill a burette with the diluted urine. Run I c.c. of it into a test-tube and add 9 c.c. of distilled water. Repeat the test with this second dilution. If the ring appears at it longer interval than three minutes, the twice-diluted urine contains less than I part of albumin in 30,000 , and the original undiluted urine less than I part in $300-i . e .$, less than 0.33 per cent. So far, then, we have found, let us suppose, that the proportion of albumin in the original urine lies between $0^{\circ} 033$ and $0^{\circ} 33$ per cent. Now rum I c.c. of the urine of the first dilution (the urine diluted ten times) into a test-tube, and add + c.c. of distilled water-i.e., dilute again five times. If this gives the white ring in Heller's test in three minutes, the original urine will contain 1 part of albumin in $\frac{30,000}{10 \times 5}-i . e$., in 
600 parts, or $0^{\prime} 1{ }^{\prime}$ per cent. If the interval is longer or shorter than three minutes. the urine of the first clilution ( 1 to 10) must be diluted less or more than five times until the interval amounts to about three minutes. The total dilution corresponcling to a percentage of 0.0033 of albumin is thus known. and the percentage in the undiluted urine can be easily calculated.

(c) Esbach's . Method.-Esbach's reagent is made by dissolving Io grammes of picric acid and 20 grammes of citric acid in boiling water (8oo or 900 c.c.), and then making up the volume to a litre. The so-called albuminimeter is simply a strong glass tube graduated and marked in a certain way. Fill the tube up to the mark $\mathrm{I}^{*}$ with the urine. Then add the reagent up to the mark R. Close the tube with the rubber cork, and invert it a dozen times without shaking.
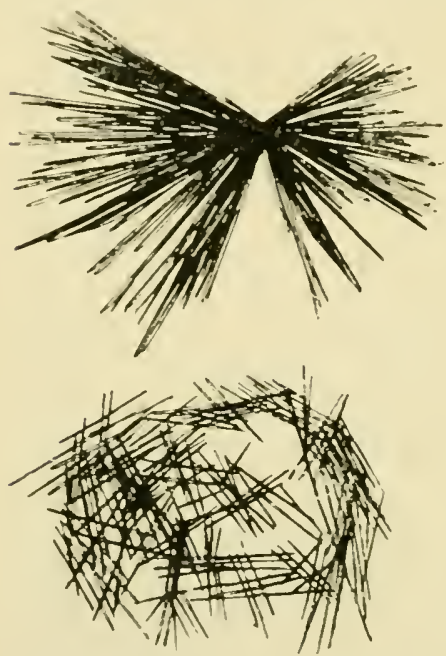

Fig. I8I.-PhExyl-glucosazoNe Axd

PheNYl-MALTOSAZONE CRYSTALS (MACLEOD).

The phenyl-glucosazone crystals are in the upper part of the figure, the phenyl-maltosazone in the lower. found in rare cases in disease (p. 444$)$. If less than $0^{\prime} 5$ per cent. of sugar is present in the urine, no precipitate of cuprous oxide will be formed till the urine is cooled. The test may also be performed with IFehling's solution.

(b) Phenyl-hydrazine \%est.-This test depends upon the fact that phenyl-hydrazine forms with sugars such as glucose (dextrose), maltose, isomaltose. etc.. but not with cane-sugar, characteristic crystalline substances (phenyl-glucosazone. phenyl-maltosazone, etc.) which can be recognised under the microscope, and are distinguished from each other by melting at different temperatures. Phenylglucosazone $\left(\mathrm{C}_{18} \mathrm{H}_{22} \mathrm{~N}_{4} \mathrm{O}_{4}\right)$ melts at $205^{\circ} \mathrm{C}$. To perform the test for dextrose in the urine. proceed thus: Put 5 c.c. of urine in a testtube, add I decigramme of hydrochlorate of phenyl-hylrazine and 
2 ilecigrammes of sodium acetate. It is sufficiently accurate to auld as nucl plenyl-hydrazine as will lie on a sixpence (or a rlime) and twice as mucli sodium acetate. Heat the test-tube in a boiling waterbath for half an hour. Then cool at the tap and examine the deposit under the microscope for the yellow plienyl-glucosazone crystals (Fig. I 8I). Sometimes the osazone precipitate is amorphous. lest this should be the case, the precipitate, if no crystals can be seen. must be dissolved in hot alcohol. The solution is then diluterl with water and the alcoliol boiled off, when the osazone, if any be present, will crystallize out. Very minute traces of sugar can be detected in this way (as little as $0^{\circ} \mathrm{I}$ per cent. in urine). Often in normal urine yellow crystals are deposited during the first fifteen minutes' heating. They must not be mistaken for glucosazone. They probably consist of a compound of glycuronic acid and phenylhydrazine. They are changed as the heating goes on into an amorphous brownish-yellow precipitate (Abel).

(c) The Yeast Test is an important confirmatory test for distinguishing the fermentable sugars from other reducing substances, but it is not very delicate, and will with difficulty detect sugar when less than 0.5 per cent. is present. It can be performed thus : A little yeast (the tablets of compressed yeast do very well) is added to a test-tıbe half filled with urine. The test-tube is then filled up with mercury. closed with the thumb, and inverted over a dish containing mercury. The dish may be placed on the top of a waterbath whose temperature is about $40^{\circ} \mathrm{C}$. After twenty-four hours the sugar will have been broken up into alcohol and carbon dioxide. The latter will have collected above the mercury in the test-tube, and the former will be present in the urine. The tests for sugar will either be negative or will be less distinct than before. A control test-tube containing water and yeast should also be set up, as impurities in the yeast sometimes yield a small amount of carbon dioxide. Specially constructed tubes are also often used for performing the test.

(2) Quantitative Estimation of Sugar in Urine.--(a) V'olumetrically, the sugar can be estimated by titration with Fehling's solution. As this does not keep well, two solutions containing its ingredients should be kept separately and mixed when required. Solution $I$. : Dissolve $34^{\circ} 64$ grammes pure cupric sulphate in distilled water, and make up the volume to 500 c.c. Solution II. : Dissolve 173 grammes Rochelle salt in 400 c.c. of water, add to this $5^{-6}$ grammes sodium hydroxide, and make up the volume with water to 500 c.c. Keep in well-stoppered bottles in the dark. For use, mix together equal volumes of the two solutions. Ten c.c. of this mixture is reduced by 0.05 gramme dextrose. To estimate the sugar in urine, put Io c.c. of the mixture into a porcelain capsule or glass flask, and dilute it four or five times with listilled water. Dilute some of the urine, say ten or twenty times, according to the quantity of sugar indicated by a rough determination. Run the diluted urine from a burette in to the Fehling's solution, bringing it to the boil each time urine is added, until, on allowing the precipitate to settle, the blue colour is seen to have entirely disappeared from the supernatant liquid. The observation of the colour must be made while the liquid is still hot.

Suppose that 10 c.c. of Fehling's solution is decolourized by 20 c.c. of the ten-times diluted urine. Then 2 c.c. of the original urine contains $0^{\circ} 5$ gramme dextrose. If the urine of the twenty-four hours 
(from which this sample is assumed to have been taken) amounts to 4.000 c.c. the patient will have passel $0.05 \times 2.000=100$ grammes sugar, in twenty-four hours. Various modifications of Fehling's solution, which have for their object the prevention of the precipitate of cuprous oxide. with its clisturbing effect upon the reading of the end-point. liave been devised. 'Thus, in Pavy's solution ammonia holds the cuprous oxide in solution, and the end-point is the disappearance of the blue colour. Ten c.c. of Pary's solution =1 c.c. of Fehling's solution $=0.005$ gramme of dextrose.

(b) The polarimeter affords a rapid and, with practice. a delicate means of estimating the quantity of sugar in pure and colourless solutions, but diabetic urine must in general be first decolourizerl by adding lead acetate and filtering off the precipitate. What is measured is the amount by which the plane of polarization of a ray of polarized light of given wave-length (say sodium light) is rotated when it passes through a layer of the urine or other optically active solution of known thickness. Let $a$ be the observed angle of rotation, $l$ the length in decimetres of the tube containing the solution, $w$ the number of grammes of the optically active substance per c.c. of solution, and $(a)_{1}$ the specific rotation of the substance for light of the wave-length of the part of the spectrum corresponding to the $\mathrm{D}$ line (i.e.. the amount of rotation expressed in degrecs which is produced by a layer of the substance I decimetre thick, when the solution contains I gramme of it per c.c.). Then $(a)_{1}= \pm \frac{a}{w} \cdot$. In this equation $a$ and $l$ are known from direct measurement; $(a)_{1}$, has been determined once for all for most of the important active substances, and therefore $w$ is easily calculated. For dextrose $(a)_{w}$ may be taken as $52.6^{\circ}$. It varies somewhat with the concentration, but for most investigations on the urine these variations may be neglected.

It is not possible to describe here the numerous forms of polarimeter that are in use. Those constructed on what is called the "halfshadow' system (Fig. IS2) gire sufficiently satisfactory results. A half-shadow polarimeter consists, like other polarimeters. of a fixed Nicol's prism (the polarizer), and a nicol capable of rotation (the analyzer). In addition, there is an arrangement which rotates by a definite angle the plane of polarization in one-half of the field, but not in the other-e.g., a small nicol occupying only half of the field. In the zero position of the analyzer, both halves of the field are equally dark. The solution to be investigated is placed in a tube of known length. the ends of which are closed by glass discs secured by brass screw caps. The glass cliscs must be slid on, so as to exclude all air. The tube having been introduced between the polarizer and analyzer. the sharp vertical line which indicates the division between the two half-fields is focussed with the eye-piece, and then the analyzer is rotated till the two halves are again equally shadowed. The angle of rotation, $a$, is read off on the graduated arc, which is provicied with a vernier.

Pentoses reduce Fchling's solution, but do not give the yeast test. They give the following characteristic tests, which may be performed with gum arabic. which contains arabinose, one of the pentoses:

(I) Phloroglucin Reaction.-Warm in a test-tube some pure concentrated hydrochloric acid to which an equal volume of distilled water has been added. Add phloroglucin until a little remains undissolved. Add a small quantity of gum arabic, and keep the test- 
tube in a water-bath at $100 \mathrm{C}$. The solution becomes clierry-red, and a precipitate gradually scparates, which maty be dissolved in amyl alcohol. The solution sliows with the spectroscope a band between $\mathrm{D}$ and $\mathrm{E}$.

(2) Orcin Reaction. - I'se orcin instead of phloroglucin in (I). The solution becomes redelish-blue on warming, and shows a band between $C$ and D, near D. The colour quickly changes from violet to blue. red, and finally green. A bluish-green precipitate separates, which is soluble in amyl-alcohol. Glycuronic acid gives all the above reactions of pentoses.

Bile-Salts-(Hay's Test).-Put a little fincly-divided sulphur, in the form of flowers of sulpliur, on the top of a glass of urine. If bilc-

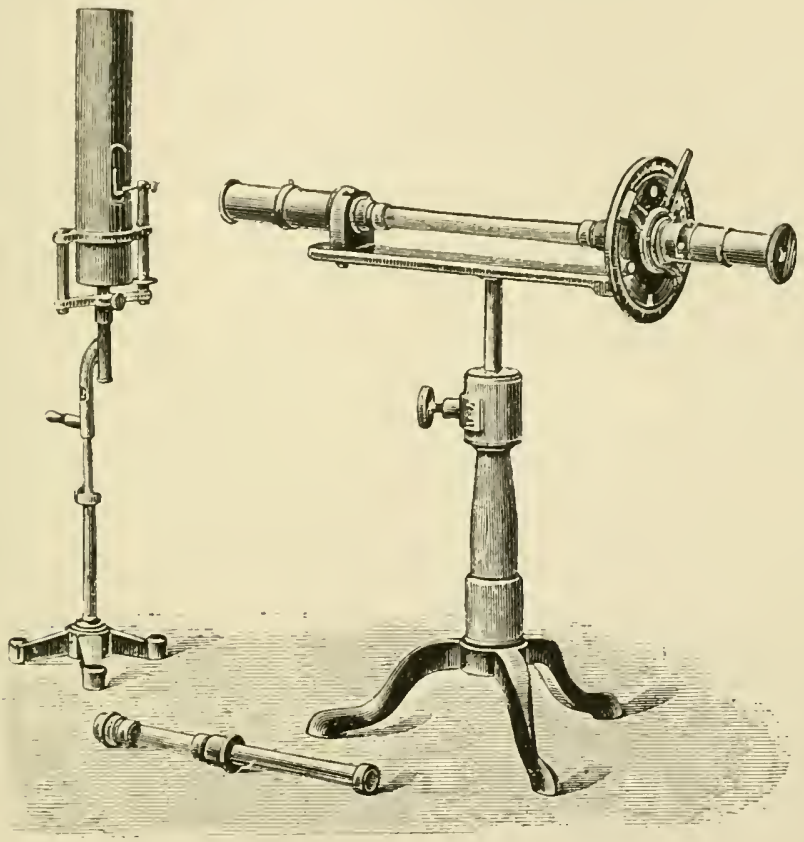

Fig. I82.-Mitscherlich's Polarimeter.

(Half-Shadow Instrument.) (Simple Form.)

salts are present the sulphur will sink to the bottom. If there are no bile-salts it will float on the top. The difference is duc to an alteration in the surface tension of the urine produced by the bile-salts. We must exclude the presence of acetic acid, alcohol, ether, chloroform, turpentine, benzine and its derivatives, phenol and its derivatives, anilin and soaps, all of which also cause such an alteration in the surface tension of urine that the sulphur sinks to the bottom. The urine should be fresh, and if it has to be kept it should be preserved from decomposition by cyanide of mercury, which does not alter the surface tension. The reaction has the great advantage over other tests of being easily carried out at the bedside. 
Acetone.-(1) Legul's Test (Rothera's modification).-To 5 to 10 c.c. of the acetone-containing urine add enough ammonium sulphate crystals to form a layer at the bottom of the test-tube, then 2 or 3 clrops of a fresh 5 per cent. solution of sodium nitro-prusside and i to 2 c.c. of strong ammonia. The development of a colour like that of permanganate of potassium, often in the form of a ring a little above the undissolved salt, indicates the presence of acetone. 'The reaction must not be declared negative till half an hour has clapsed. The colour slowly fades.

(2) Where there is doubt as to the presence of acetone, it is best first to clistil it over. Put 250 to 500 c.c. of the urine suspecterl to contain acetone into a litre flask. Arld a few c.c. of phosphoric acid ; connect the flask with a worm (see Fig. $180, \mathrm{p} .4^{8} 3$ ), and distil over the urine into a small flask. For qualitative tests it is best to collect only the first 20 to 30 c.c., as most of the acetone is contained in this. Test the distillate for acetone by (I) or by

Lieben's Test.-To a few c.c. of the distillate in a test-tube add a few drops of solution of iodine in potassium iodide and then sodium or potassium hydroxide. A precipitate of yellow iodoform crystals (sixsided tables) is thrown down if acetone be present. Examine them under the microscope. On heating, the odour of iolloform may be recognised. If the precipitate is amorphous it may be dissolved in ether (free from alcohol), which is allowed to evaporate on a slide. when crystals may be obtained.

Determination of the Freezing-point of Urine.-Study Beckmann's apparatus shown in Fig. I 53, p. 399. Note the large thermometer D graduated in hundredths of a degree centigrade. It is inserted through a rubber cork into the inner thick test-tube $\Lambda$. A platinum wire F, bent at the lower end into a circle or a spiral, which passes easily up and down between the bulb of the thermometer and the tube, serves to stir the urine. The thermometer must be so supported by the rubber cork that the bulb is in the axis of the tube and a centimetre or two from the bottom of it. The side-piece $E$ on the tube $\mathrm{A}$ is not absolutely necessary, but it is convenient for 'inoculating ' the urine with a crystal of ice at the proper time. A passes through a rubber cork into a shorter and wider outer glass tube $B$. The space between $A$ and $B$ serves as a badly conducting mantle, which prevents too rapid cooling of the contents of $\mathrm{A}$. B passes through a hole in the metal or wooden cover of a strong glass jar C, which contains the freezing mixture. B should fit the hole so tightly that it does not bob up out of the mixture when $A$ is removed. In $\mathrm{C}$ is a stirrer, $\mathrm{G}$, of strong copper wire, the end of which passes through the lid. This serves to stir up the freezing mixture from time to time.

Pulverize some ice by pounding it in a strong woolen box with a heavy piece of wook. Take the inner tube with the thermometer out of the apparatus. It is convenient to take the thermometer out of the tube, and to hang it up carefully on a stand by means of a fine flexible copper wire passing through the eye. The rubber cork can be taken out with the thermometer, and the platinum wire also, the bent free end of the latter supporting it in the cork, or it may be fastened temporarily to the thermometer stem by a small rubber band, which is slid up over the cork when the thermometer is reinserted. Tube $\mathrm{A}$ can be set temporarily in a specially heavy testtube rack. Remove the lid of $C$, and with it tube B. Now put ice and salt alternately into $\mathrm{C}$ until it is nearly full, mixing them up well. Add some cold water from the tap till the stirrer $G$ can move freely 
up and down in the mixture. For very exact work the temperature of the freezing mixtme must not be more than a few (legrees below the freczing-point of the liguid which is being examined. Put on the lirl, and inmerse tube B. Into A, which must be perfectly clean, put enough pure distilled water to fully cover the bulb of the thermometer, and introduce the latter. For ordinary purposes distilled water previously boiled to expel the carbon dioxide, and then cooler in a stoppered flask, is sufficiently pure. Immerse A directly in the freezing mixture through the hole by which $\mathrm{G}$ comes out, or through a separate hole (not shown in the figure) till some ice has formed in the water. Take A out of the mixture, wipe it with a cloth, and hold the lower part of it in the hand till nearly the whole of the ice has melted. If there is a cake of ice at the bottom, see that it is displaced by the platinum stirrer. A trace of ice being still left floating in the water, place $A$ in $B$, and allow the temperature to fall to a few tenths of a degree below the freezing-point you expect to get, as detemined by a previous rough experiment. The freezing mixt ure is stirred up occasionally. The meniscus of the thermometer is to be carefully followed, as it goes on falling, by means of a weak land lens. Now stir the water in A briskly. Suddenly it will be seen that the mercury begins to rise. Keep stirring with the platinum wire, and read oft the maximum height of the mercury, at which it is stationary for some time. The temperature can be estimated between the gradiations to thousandths of a degree. Take out $A$, and observe the fine ice crystals in the water. Heat $A$ in the hand as before till nearly all the ice has disappeared ; then replace $A$ in $B$, ancl make another freezing-point determination. A third one may also be made, and the mean of the three readings taken.

Take out the thermometer, and dry it and the platinum wire with clean filter-paper, or dip them in some of the urine, which is then thrown away. Dry A or rinse it with urine. Then make a determination of the freezing-point of the urine in the same way as was done with the water. The freezing-point of the urine will lie much lower on the scale.

Instead of freezing the liquid first and then leaving a little ice in it when $A$ is placed in $B, A$ may be put into $B$ before any ice has formerl. Cooling is then allowed to go on with gentle stirring to a few tenths of a degree below the anticipated freezing-point. A small crystal of clean dry ice is then introduced through the side-piece on a clean splinter of wood or the loop of a cooled platinum wire, the end of which passes through a piece of cork, by which it is held to prevent conduction of heat. The platinum stirrer can be raised to receive the crystal. The liquid is now vigorously stirred; freezing occurs, and the observation is made as before.

Instead of the above method, the liquid may first be cooled directly in the freezing mixture, but not so much that ice forms. A is then put in B, and cooling allowed to go on while it is being stirred. When it has been undercooled to a certain extent-i.e, cooled below its freezing-point-the vigour of the stirring is increased. Ice forms suddenly, as before, and the temperature rises to the freezing-point. With urine this method is sufficiently satisfactory, but it is not usually easy to get freezing of the distilled water till the undercooling is considerable, and it has been shown that this introduces some error.

Suppose the freezing-point of the distilled water on the scale of the thermometer was $5^{\circ} 245^{\circ}$ and that of the urine $3^{\circ} 625^{\circ}$, the value 
of $\lambda$ for the urine is $1 \cdot 620^{\circ}$. Since for most purposes it is sufficient to fix the second decimal point, much smaller and less expensive thermometers than the ordinary Beckmann may be employed.

In the same way the freezing-point of blood-serum (or blood), bile, and other physiological liquids can be determined.

Systematic Examination of Urine.-In examining urine, it is convenient to allopt a regular plan, so as to avoid the risk of overlooking anything of importance. The following simple scheme may serve as an example; but no routine should be slavishly followerl, the object being to get at the important facts with the minimum of labour. Iore extensive information must be sought in the treatises on examination of the urine for clinical purposes.

I. Anything peculiar in colour or smell ? If the colour suggests blood, examine with spectroscope, hremin test. guaiacum test (pp. $6_{5}$. 68,7 I) ; if it suggests bile. test for bile-pigments by Gmelin's test (p. $43 \mathrm{I})$, and for bile-salts by Pettenkofer's test (p. $\left.43^{\circ}\right)$ and by Hay's test (pp. 430, 49I).

2. Reaction.

3. Sediment or not? Sediment may be procured by letting the urine stand in a conical glass. or in a few minutes by the centrifuge. If the appearance of the sediment suggests anything more than a little mucus, examine with the microscope. "The sediment may contain organized or unorganized deposits.

Organized Sediments.- (a) Red blood-corpuscles (considerably altered if they have come from the upper part of the urinary tract).

(b) Leucocytes. A few are present in health. A large number indicates pus. When pus is present the sediment may be white to the naked eye.

(c) Epithelium from the bladder. ureters, pelvis of the kidney or the renal tubules. A few squamous epithelial cells from the urethra are always present in normal urine.

(d) Tube casts.

(e) Spermatozoa (occasional).

(f) Bacteria.

(g) Parasites (rare).

(h) Portions of tumours (rare).

\section{Unorganized Sediments.}

IN ACID URINE.

Uric Acid.-Crystals coloured brownish - yellow with urinary pigment. Various shapes, especially' oval 'whetstones,' rhombic tables, and elongated crystals, often in bundles (Fig. I60).

Urates.-Usually amorphous, in the form of fine granules, often tinged with urinary pigment, sometimes brick-red. Soluble on heating. On addition of acids (including acetic acid) they dissolve and uric acid crystals appear in their placc. Acid urate of sodium and of
IN ALKALINE URINE.

Triple Phosphate - Clear. colourless, coffin-lid or knife-rest crystals. Also deposited in the form of feathery stars (Fig. I62).

Calcium Hydrogen Phosphate ('stellar' phosphate), $\mathrm{CaHPO}_{4}$. -Crystals often wedge-shaped and arranged in rosettes. Iay also occur in a dumb-bell form. (A phosphate of calcium is alsoe occasionally seen in weakly acid urine.) (Fig. I6 . p. 439.)

Calcium Phosphate, $\mathrm{Ca}_{3}\left(\mathrm{PO}_{4}\right)_{2}$. -Amorphous.

Magnesium Phosphate.-Long 


\section{Unorganized Sediments (continued) -}

IN ACID URINE.

ammonium occasionally found in the crystalline form (rosettes of neelles).

Calcium Oxalate.-Octahedral, 'cnvelope' crystals, not coloured. Insoluble in acetic acid. Soluble in hydrochloric acicl (Fig. I6I, p. +38 ).

Cystin. - Hexagonal plates. Rare (Fig. 163, p. +39).

Iencin and Tyrosin (Figs. I 69, I 7o, p. 452).-Rare. Also found in alkaline urine.but rarely.

Triple Phosphate.-Sometimes found in weakly acid urine.
IN ALKALINE URINE.

rhombic tablets, which are dissolved at the elges by ammonium carbonate solution, unlike triple phosphate. All the abovo are soluble in acetic acidwithont effervescence.

Calcium Carbonate. - Small spherical or dumb-bell-shaped borlies soluble in acctic acid with effervescence.

Ammonium Urale. - Dark balls, often covered with spines. Soluble in acetic or hyclrochloric acid, with formation of uric acid crystals (Fig. I65, p. 439).

4. Specific gravity.

5. Quantity of mine in twenty-four hours. If the quantity is abnormally large and the specific gravity high, test for sugar.

6. Inorganic constituents not generally of clinical importance, but in special diseases they should be examined-e.g., chlorides in pileumonia.

7. Normal organic constituents. Sometimes quantitative estimation of urea or total nitrogen in fever, and in rliabetes and Bright's disease.

8. Chemical examination for abnormal organic constituents, especially albumin and sugar.

Albumin.--(1) Heat some of the urine in a test-tube to boiling. A precipitate insoluble on addition of a few drops of acetic acid consists of coagulable protein. A precipitate soluble in acetic acid consists of carthy phosphates.

(2) Heller's test. Put some strong nitric acid in a test-tube and run on to it some urine. A white ring indicates protein.

A quantitative estimation may be made by the method of Roberts and Stolnikow or Esbach (p. 487 ).

Sugar.--(I) Trommer's test. (Fehling's solution may be used.) If the result is indecisive :

(2) Phenyl-hydrazine test (p. 488).

(3) In case of doubt confirm by yeast test.

A quantitative estimation may be made with Fehling's solution or the polarimeter. 


\section{CHAPTER VII}

\section{METABOLISM, NUTRITION AND DIETETICS}

WE return now to the products of digestion as they are absorbed from the alimentary canal, and, still assuming a typical diet containing proteins, carbo-hydrates and fats, we have to ask, What is the fate of each of these classes of proximate principles in the body? what does each contribute to the ensemble of vital activity? It will be best, first of all, to give to these questions what roughly qualitative answer is possible, then to look at metabolism in its quantitative relations, and lastly to focus our information upon some of the practical problems of dietetics.

I. Metabolism of Proteins. - The two chief proteins of the blood-plasma, serum-globulin and serum-albumin, must, as has been already pointed out, be recruited from proteins absorbed from the intestine and for the most part, at any rate, profoundly altered in its lumen and in their passage through the epithelium which lines it. The physiological reasons for this alteration are in a measure known, and have already been alluded to in connection with the digestion of proteins. No doubt the far-reaching decomposition of the protein molecule may to some extent facilitate the absorption of protein food. No doubt also it is imperative that such slightly hydrolysed products as peptone, and particularly proteose, should not appear in quantity in the blood, for when injected they cause profound changes in that liquid, one expression of which is the loss of its power of coagulation, and are rapidly excreted by the kidneys, or separated out into the lymph. But the passage of the food from the stomach is so gradual an affair, the quantity of digesting protein present at one time in any loop of intestine is so small, and the rush of blood which irrigates the active mucosa is so large, that the concentration of peptone or proteose necessary to produce injurious effects could hardly in any case be realized. Again, there is no evidence that the simpler decomposition products of further hydrolysis are not in equal concentration as poisonous as proteose and peptone. 
Apart from any influence which it may have in favouring absorption, the complete shattering of the protein molecule has a clouble significance. In the first place, as already pointed out, the food-proteins cannot be used dircctly in the upbuilding and repair of the protoplasm ( 1 . $\left.f^{\mathrm{I}} 9\right)$ ), since the tissue-proteins differ from them and from each other in the amount and nature of the amino-acids and other gromps in their molecule (p. 2). Secondly, nnder ordinary dictetic conditions a surplus of nitrogen in the protein food has to be got rid of by being converted into urea without being built up into the tissue substance. Here we come upon the fundamental fact that the protein katabolism is not a single uniform process. Two forms may be distinguished which are essentially independent in course and character. One kind varies extremely in its quantitative relations, according to the amount of protein in the food. Its chief end-products are urea, representing the nitrogen, and inorganic sulphates, representing the sulphur of the proteins. Since this form of katabolism, as we shall see directly, is not essentially connected with the life and nutrition of the living substance, it is termed exogenous. The other variety is practically constant in amount for one and the same individual, and independent of the quantity of protein in the food. Its characteristic end-products are kreatinin and neutral sulphur. This form of protein katabolism is essentially an expression of the waste of the living substance itself, and is therefore spoken of as endogenous.

Some have supposed that the intestinal mucosa has as one of its special functions the resynthesis of a great part of the digestive decomposition products into the proteins of the blood-plasma. If this is the case, these proteins must be again decomposed in the cells of the various tissues in order that the " buildingstones " may be recombined to form the tissue-proteins. For the proteins of the organs are not the same as those of the blood, and the proteins of different organs differ characteristically from each other. The significance of the synthetic function of the intestinal wall would then lie in this: that from the varying mixture of amino-acids, etc., derived from the food-proteins an alway's uniform and suitable protein mixture (the blood-proteins) is fabricated for the feeding of the tissues. An alternative assumption, and superficially at least a simpler one, is that no more extensive synthesis of proteins occurs in the wall of the alimentary canal than is necessary for the needs of the tissues composing it, and that the decomposition products of the proteins are mainly absorbed as such, and pass in the blood to the tissues for which they are destined. If this is the case, the blood-proteins can no longer be looked upon as representing the main current of protein supply for the organs, but rather the 
store of protein material proper to the circulating tissue blood itself, and whicl confers on it certain chemical and physicochemical properties (e.g., the due degree of viscosity) necessary for its function. Slowly accumulated, under ordinary cunditions, and slowly consumed, this protein store may, of course, be at the disposal of the organs in an emergency-for instance, in starvation-or may be rapidly recruited from the organ-proteins, as after hamorrhage, just as in prolonged hunger the proteins of skeletal muscle may be utilized to feed the heart. It is impossible, with our present knowledge, to decicle definitely between these hypotheses. There is some evidence that serum-albumin is more directly related to the proteins of the food than serumglobulin. And it is said that during starvation the albumin is relatively diminished, and the globulin relatively increased. It is, of course, not at all improbable that the plasma-proteins have a double source-organ-proteins on the one hand, foodproteins on the other. That the plasma-protein mixture maintains a very constant composition in the face of wide variations in the composition of the food-proteins is indicated by the following experiment :

A horse fed mainly on hay and oats was bled to the amount of 6 litres, and in the total protein of the scrum the content of tyrosin and glutaminic acic was determined. In order to eliminate the influence of remains of the food in the digestive canal, nothing was given to the animal for a week. Then 6 litres of blood were again removed, and the tyrosin and glutaminic acid in the serum-protein again estimated. The horse was now fed with gliadin obtained from flour, a protein which contains $36^{\circ} 5$ per cent. glutaminic acid and 2.37 per cent. tyrosin-that is, about the same ameunt of tyrosin as the serum-protein, but about four times as much glutaminic acid. The serum-protein was again analyzed for the two amino-acids after this diet. The results of one experiment are shown in the table:

\begin{tabular}{|c|c|c|c|c|}
\hline Normal. & $\begin{array}{c}\text { After 8 Day's' } \\
\text { Hunger. }\end{array}$ & $\begin{array}{c}\text { After Feeding } \\
\text { with } 1,500 \\
\text { Girammes } \\
\text { Gliadin. }\end{array}$ & $\begin{array}{c}\text { After Fecding } \\
\text { again with 1,500 } \\
\text { Grammes } \\
\text { Gliadin. }\end{array}$ \\
\hline Tyrosin - - & 2.43 & 2.60 & 2.24 & 2.52 \\
Glutaminic acid - & 8.85 & 8.20 & 7.88 & 8.25
\end{tabular}

No increase in the glutaminic acid content of the serum-protein occurred, although, owing to the loss of blood, much new serum. protein must have been formed. If the amino-acids of the gliadin were used without cliange to build up the new serum-protein, three-quarters of the glutaminic acid must have becn superfluous, and the nitrogen of this portion may have been straightway changed into urea and excreted. But the possibility that one amino-acid may be changed into another in the body cannot be excluded, although there is no evidence that it occurs (Abderhalden). 
Living and Dead Proteins.-Carried to the tissues, the decomposition products of the food-proteins, or the regenerated proteins of the plasma, which in ordinary language are still to be regarded as dead material, are built up into the living protoplasm, at any rate to the cxtent necessary to make good its waste. In this form they sojourn for a time within the cells, and then they become dead material again. The nature of this tremendous transformation has, of course, been the subject of speculation, but the truth is that we do not understand wherein the difference between a living and a dead cell, between a living and a dead particle in one and the same cell, really consists. All we know is that now and again a living protein molecule in the whirl of flying atoms which we call a muscle-fibre, or a gland-cell, or a nerve-cell, must fall to pieces. Now and again a molecule of protein, hitherto dead, or a molecule of a particular amino-acid, or perhaps a polypeptid group intermediate in complexity between the simple amino-acid and the protein, coming within the grasp of the molecular forces of the living substance, is caught up by it, takes on its peculiar motions, acquires its special powers, and is, as we phrase it, made alive. Each cell has the power of selecting and, if necessary, further decomposing or further synthesizing the protein materials offered to it; so that a particle of serum-albumin or a mixture of amino-acids may chance to take its place in a liver-cell and help to form bile, while an exactly similar particle or mixture may furnish constituents to an endothelial scale of a capillary and assist in forming lymph, or to a muscular fibre of the heart and help to drive on the blood, or to a spermatozoon and aid in transferring the peculiarities of the father to the offspring. And just as a tomb and a lighthouse, a palace and a church, may be, and have been, built with the same kind of material, or even in succession with the very same stones, so every organ builds up its own characteristic structure from the common quarry of the blood.

It is not any difference in the kind of protein offered them which determines the difference in structure and action between one organ and another. In the case of the more highly developed tissues at least, no mere change of food will radically alter structure. A cell may be fed with different kinds of food, it may be over-fed, it may be ill-fed, it may be starved; but its essential peculiarities remain as long as it continues to live. Its organization dominates its nutrition and function.

The speculation of Pflüger, that the nitrogen of living protein exists in the form of cyanogen radicals, whilst in dead protein it is in the form of amides, and that the cause of the characteristic instability of the living substance-its prodigious power of dissociation and reconstruction - is the great intramolecular movement of the atoms of the cyanogen radicals, is interesting and ingenious, but it remains, and is likely to remain, a speculation. And the same is true of the suggestion of Loew and Bokorny, that the endowments of living protoplasm depend on the presence of the unstable aldehyde group $\mathrm{H}-\mathrm{C}=\mathrm{O}$. Nor do the known differences of chemical composition in dead organs give any insight into the peculiarities of organization and function which mark off one living tissue from another. In any case, the living protein molecule, whatever function it may have been fulfilling in the organized elements of the body, has certainly a much greater tendency to fall to pieces than the dead protein molecule. And it falls to pieces in a fairly definite way, 
the ultimate products, under the influence of oxygen, being carbon dioxicle, water, and comparatively simple nitrogen-containing substances, which after further changes appear in the urine as urea, kreatinin, uric acid, and other bodies. We shall sec later on that a great part of the urea excreted does not arise in the decomposition of living protein or protoplasm, but is the form in which 'surplus ' nitrogen is eliminated in the preparation of the foocl-protein for assimilation by the tissues. We have no definite information as to the production of water from the hydrogen of the tissues, except what can be theoretically deducer from the statistics of nutrition (p. 540). A few words will be said a little farther on about the production of carbon dioxide from proteins; we have now to consider the seat and manner of formation of the nitrogenous metabolites. And since in man and the other mammals urea contains, under ordinary conditions, by far the greater part of the excreter nitrogen, it will be well to take it first.

Formation of Urea.-The starting-point of all inquiries into the formation of urea is the fact that it occurs in the blood in small amount ( 4 to 6 parts per 10,000 in man; 3 to 15 parts per Io,ooo in the dog), the largest quantity being found when the food contains most protein and at the height of digestion, the smallest quantity in hunger (Schöndorff). Evidently, then, some, at least, of the urea excreted in the urine may be simply separated by the kidney from the blood; and analysis shows that this is actually the case, for the blood of the renal vein is poorer in urea than that of the renal artery, containing only one-third to one-half as much. If we knew the exact quantity of blood passing through the kidneys of an animal in twenty-four hours, and the average difference in the percentage of urea in the blood coming to and leaving them, we should at once be able to decide whether the whole of the urea in the urine reaches the kidneys ready made, or whether a portion of it is formed by the renal tissue. Although data of this kind are as yet inexact and incomplete, it is not difficult to see that all, or most of, the urea may be simply separated by the kidney.

If we take the weight of the kidneys of a dog of 35 kilos at I60 grammes ( 210 th of the body-weight is the mean result of a great number of observations in man), and the average quantity of blood in them at rather less than onc-fourth of their weight, or 35 grammes, and consider that this quantity of blood passes through them in the average time required to complete the circulation from renal artery to renal vein, or, say, ten seconds, we get about 300 liilos of blood as the flow through the kidneys in twenty-four hours. Even at $0^{\circ} 3$ per $\mathrm{I}, \mathrm{0o0}$, the urea in 300 kilos of blood would amount to 90 grammes. Now, Voit found that a dog of 35 kilos body-weight, on the minimum protein diet ( 450 to 500 grammes of lean meat per day) which sufficed to maintain its weight, excreted 35 to to grammes of urca in the twenty-four hours. If, then, the renal epithelium separated somewhat less than half of the 90 grammes urea offered to it in the circulating blood, the whole excretion in the urine conld be accounted for, and the blood of the renal vein would still contain more than 
half as much urea as that of the renal artery. So that the whole of the urea in the urine may be simply separated by the kidney from the ready-made urea of the blood.

Another line of eridence leads to the same conclusion: that the kidney is, at all events, not an important seat of ureaformation. When both renal arteries are tied, or both kicheys extirpated, in a dog, urea accumulates in the blood and tissues; and, upon the whole, as much urea is formed during the first twenty-four hours of the short period of life which remains to the animal as would under normal circumstances have been excreted in the urine.

Where, then, is urea chiefly formed? If the main source of urea were the decomposition of tissue-protein, we should naturally look first to the muscles, which contain three-fourths of the proteins of the body; but we should look there in vain for any great store of urea--only a trace is normally present. The liver contains a relatively large amount, and there is very strong evidence that it is the manufactory in which the greater part of the nitrogenous relics of broken-down proteins reach the final stage of urea. This evidence may be summed up as follows :

(I) An excised 'surviving' liver forms urea from ammonium carbonate mixed with the blood passed through its vessels, while no urea is formed when blood containing ammonium carbonate is sent through the kidney or through muscles. Other salts of ammonium, such as the lactate, the formate, and the carbamate, undergo a like transformation in the liver. It is difficult, in the light of this experiment, to resist the conclusion that the increase in the excretion of urea in man, when salts of ammonia are taken by the mouth, is due to a similar action of the hepatic cells.

(2) If blood from a dog killed during digestion is perfused through an excised liver, some urea is formed, which cannot be simply washed out of the liver-cells, because when the blood of a fasting animal is treated in the same way there is no apparent formation of urea (v. Schroeder). This suggests that during digestion certain substances which the liver is capable of changing into urea enter the blood in such amount that a surplus remains for a time unaltered. These substances may come directly from the intestine; or they may be products of general metabolism, which is increased while digestion is going on ; or they may arise both in the intestine and in the tissues. Leucin-which, as we have seen, is constantly, or, at least, very frequently, present in the intestine during digestion-can certainly be chariged into urea in the body. So can other amino-acids of the fatty series, like glycocoll or glycin, and aspartic acid, and it has been shown by perfusion experiments that this change 
takes place in the liver. Further, the blood of the portal rein during digestion contains several times as much ammonia as the arterial blood, and the excess disappears in the liver.

(3) Uric acid-which in birds is the chief end-product of protein metabolism, as urea is in mammals is formed in the goose largely, and almost exclusively; in the liver. This has been most clearly shown by the experiments of Minkowski, who took advantage of the communication between the portal and renal-portal veins (p. 356) to extirpate the liver in geese. IVhen the portal is ligatured the blood from the alimentary canal can still pass by the roundabout road of the kidney to the inferine cava, and the animals survive for six to twenty hours. While in the normal goose 50 to 60 per cent. of the total nitrogen is eliminated as uric acid in the urine, and only 9 to 18 per cent. as ammonia, in the operated goose uric acid represents only 3 to 6 per cent. of the total nitrogen, and ammonia 50 to 60 per cent. A quantity of lactic acid equivalent to the ammonia appears in the urine of the operated animal, none at all in the urine of the normal bird. The small amount of urea in the normal urine of the goose is not affected by extirpation of the liver. And while urea, when injected into the blood, is in the normal goose excreted as uric acid, it is in the animal that has lost its liver eliminated in the urine unchanged.

(4) After removal of the liver in frogs, or in dogs which have survived the previous connection of the portal vein with the inferior vena cara by an Eck's fistula (p. 356), the quantity of urea excreted is markedly diminished, and the ammonium salts in the urine are increased. When the Eck's fistula is established and the portal vein tied, without any further interference with the hepatic circulation, the amount of urea in the urine is not lessened to nearly the same extent, evidently because the substances from which urea is formed still, for the most part, gain access to the liver through the hepatic artery and by means of the back-flow which is known to take place through the hepatic vein. Yet while in normal dogs the proportion of ammonia to urea in the urine is only $I: 22$ to $I: 73$, in dogs with Eck's fistula it rises to $I: S$ to $I: 33$. If the animals are kept on a diet poor in proteins, no symptoms may develop for a considerable time. But if much protein is given, characteristic symptoms, including convulsions, alway's appear. These may be produced by the saturation of the organism with ammonia compounds, which are formed from the proteins as in the normal animal, but which the liver, with its circulation crippled, is unable to cope with, and to completely change into urea, although the statement has been made that when ammonia or ammonium salts are injected into the blood larger quantities must be present 
to produce these svmptoms than are found in animals with the Eck's fistula. Although the portal vein carries to the liver a much greater supply of blond than the hepatic artery, ligation of the latter causes a greater diminution in the ratio of the amount of urea to the total nitrogen in the urine than ligation of the former. This indicates that a good supply of oxygen is an important factor in the formation of urea in the liver (Doyon and Dufourt). But this is no proof that the process by which it is formed is an oxidation. The work of the liver, like that of other tissues, is no doubt deranged by lack of oxygen.

(5) In acute yellow atrophy, and in extensive fatty degeneration of the liver, urea may almost disappear from the urine, and leucin, tyrosin, and other amino-acids may appear in it along with a much larger amount of ammonia than normal. The amino-acids and ammonia formed in the intestine in the cligestion and absorption of proteins pass unchanged through the degenerated liver, and are excreted by the kidney.

Processes by which Urea is Formed.-If it be granted, as in the face of the evidence it must, that the liver plays an important part in the formation of urea, we have still to ask what the materials are upon which it works, in what organs they are produced before being brought to the liver, and by what process they are there changed into urea. To the last question it may be at once replied that wc know but little of the process by which urea is formed in the body. In the laboratory urea can be obtained from protein either by hydrolysis or by oxidation. We have already remarked that when a protein is split up by boiling with dilute acid under proper conditions, very much the same decomposition products appear as in tryptic digestion (p. 332). One of these, arginin $\left(\mathrm{C}_{6} \mathrm{H}_{14} \mathrm{~N}_{4} \mathrm{O}_{2}\right)$, on further hydrolytic cleavage by barium hydroxide, yields urea and ornithin (diamino-valerianic acid). half of the nitrogen of the arginin appearing in each. The amount of arginin, and therefore the amount of urea which can be artificially obtained in this way, varies extremely with the different proteins and protamins (p. 2). Thus, salmin, a substance prepared from the milt of salmon, yields $84^{\circ} 3$ per cent. of its weight of arginin, while the casein of cow's milk yields only $t^{-8}$ per cent., and gluten-fibrin, one of the proteins of wheat. only 3 per cent. In the body the hydrolysis of arginin to urea and ornithin is accomplished by the ferment arginase. This ferment is found in the liver, and also in many other organs. The urea formed in this way appears very rapidly in the urine. The ornithin itself is then more slowly transformed into urea. Since the ordinary food-proteins are poor in arginin, the amount of urea which can possibly be formed in mammalian metabolism by this process cannot be large, even if most of the arginin, as is the case when it is fed to an animal, is transformed into urea. Other amino-acids, as already mentioned, also cause an increased production of urea, corresponding to their nitrogen content, when administered by the mouth or subcutaneously. The same is truc when, instead of simple amino-acids, polypeptids, like glycyl-glycin, alanyl-alanin, or leucyl-leucin, are given.

Urea has also been artificially obtained from protein by oxidation 
with an ammoniacal solution of permanganate at body-temperature. When the protein is first split into its cleavage products and these are then oxidized. a very large amount of urea is produced-e.g., as much as 3 grammes of urea from to grammes of glycin.

While these facts suggest possible ways of formation of urca in the body, we cannot assume that what happens in the test-tube must happen in the tissues. It is now known that the greater part of the urea, at any rate, loes not come from tissue-protein, either by liyclrolysis or by oxiclation, but that much of it arises by the synthesis of decomposition products of the food-proteins simpler than itself viz., such ammonium compounds as have been alrearly mentioned as being transformed into urea when circulated through an excised liver (p. 50I). Ammonia in the form of carbonate or carbamate is constantly found in the blood, and the portal blood contains normally three to five times as much ammonia as arterial blood. ITnquestionably, then, a portion of the urea, and probably a large portion. is formed from such ammonia compounds, and if we still ask where these compounds arise, and what their immediate precursors are, the only reply which can be given is that ammonia is known to be one of the products of the digestion of proteins, and that there is some evidence that in certain ways the amide $\left(\mathrm{NH}_{2}\right)$ groups can be split off from amino-acids and then utilized as ammonia for the formation of urea. The decomposition products of the food-proteins absorbed from the intestine are thus clearly indicated as a source of urea-namely, of that large fraction which represents the surplus nitrogen eliminated without entering into the metabolism of the cells. It is of importance to remark that such hydrolytic cleavages as are associated with the splitting of protein into aminoacids, etc., only slightly reduce the available energy of the compounds. If, as is most probable, the liberation of the nitrogen from the amino-acids is also accomplished by hydrolytic cleavage. the residue, relatively rich in carbon, will still be available for yiclding to the body by its oxidation an amount of energy not much less than could be obtained from the original protein.

The combination of ammonia with carbon dioxide and the conversion of the carbonate into urea does not require any oxidation. But if, as there is every reason to believe, a part of the carbonaceous residuc is converted into carbo-hydrate. a certain amount of oxidation must occur in the transformation. It would be an error to suppose that all the ammonia or other forerunners of urea come from the intestine, or, indeed, that all the urea is manufactured in the liver. Urea does not entirely cease to be produced when the liver is removed. Some of the urea may be formed 'on the spot.' so to speak, in the endogenous metabolism of all the tissues, and perhaps by a different process from the hepatic urea and from different intermediate substances.

Such compounds as guanin, sarkin or hypoxanthin, xanthin, uric acid, and kreatin, used to be cited as among the possible intermediate substances. But while there is now complete evilence that the first three bodies can be and are converted into uric acid, there is no reason to believe that they are stages on the way to urea. ITric acicl is, indecd, very closely related to urea, and can be made to yicld it by oxidation outside the borly. Not only so, but it is, in part at least, excreted as urea when given to a mammal by the mouth, and it replaces urea as the great end-product of nitrogenous metabolism almost wholly in the urine of birds and reptiles, and partially 
in the human subject in leukiemia. But none of these things can be aclmitted as evidence that in the normal enclogenous metabolism of mammals uric acid lies on the elirect line from protein to urea. Freatin exists in the borly in greater amount than any of these, muscle contaning from $0^{\circ}$ to $0^{\circ}+$ per cent. of it; and the total fuantity of nitrogen present at any given time as kreatin is not only greater than that of the nitrogen present in urea, but greater than the whole excretion of nitrogen in twenty-four hours. But although in the laboratory kreatin can be changed into kreatinim, and kreatinin into wrea, there is no proof that in the body anything more than the first step in this process is accomplished. When kreatin is introduced into the intestine or injected into the blood, it appears in the urine, not as urea, but as kreatinin. We have already secn that kreatinin is the chief nitrogenous product of enclogenous protein metabolism.

Ammonia and amino-acids may also be produced from tissueprotein, or it may be from ' circulating' protein (p. 536), which has not been built up into protoplasm, for protcolytic ferments are everywhere found in the organs. And if ammonia and aminoacids are formed in the tissues, there is no reason to suppose that they will not yield mrea. just as if they were produced in the intestine.

Formation of Uric Acid.-Uric acid, like urea, is separated from the blood by the kidneys, not to any appreciable extent formed in them. In birds, and often in man, it can be detected in normal blood. It is present in increased amount in the blood and transudations of gouty patients, in whose joints and earcartilages it often forms concretions. 'Chalk-stones' may contain more than half their weight of sodium urate.

As to the place and manner of formation of uric acid, it has already been stated that in birds, after extirpation of the liver, the uric acid excretion is greatly diminished, and that ammonium lactate appears instead in the urine. It has been further shown that when blood containing ammonium lactate is circulated through the surviving liver of the goose, an increase in the uric acid content of the blood occurs. As demonstrated by control experiments, this increase is too great to be due merely to the sweeping out of previously formed uric acid from the hepatic cells. There can be no question, then, that the liver in birds is the seat of an extensive synthesis of uric acid from such simple materials as ammonia and lactic acid. A similar synthetic formation of uric acid from ammonia and a derivative of lactic acid may take place in mammals, and probably exclusively in the liver, but it is of much less importance. Another way in which uric acid arises both in mammals and in birds is by the splitting and oxidation of nucleins. This is by far the most important mode of formation in mammals, as synthesis is the chief mode of formation in birds. In both groups of animals the oxidative production of uric acid takes place, not in any particular organ, but 
in the tissues in general, including the liver. It has been shown that when air is blown through a mixture of splenic pulp and blood, uric acid is formed from purin bodies already present in the splcen. When the quantity of these is increased by the decomposition of nucleins induced by slight putrefaction, the yield of uric acid is also increased. Tric acid is also formed by the perfectly fresh surviving spleen, liver, and thymus in the presence of oxygen, and the quantity is increased when purin bodies are artificially added.

As to the source of the uric acid, it is well established that in the bird it arises both from the end-products of protein metabolism and from nuclein compounds and their derivatives in the food and tissues. In the mammal, the taking of food rich in nucleated cells, and therefore in nucleo-proteins and nucleins (thymus gland, pig's pancreas, and herring roe), or of food rich in purin bases (Liebig's meat extract), increases the quantity of uric acid in the urine. The increase is mainly due to the production of uric acid from the nuclein substances. But this is not the only source of the uric acid, since extracts of the thymus gland containing only traces of nucleins or nucleic acid cause, when injected, a characteristic increase in the uric acid excretion, just as the entire gland does when taken by the mouth. And during the period of increased nitrogen excretion occasioned by a meal containing protein the increase in the uric acid occurs particularly in the hours immediately following the ingestion of the food, and does not last so long as the increase in the urea. Now, the nucleins of the food are comparatively little affected during the earlier stages of digestion (Hopkins and Hope). WVe may conclude, therefore, that in the mammal, as well as in the bird, a portion of the uric acid, although certainly a far smaller portion in the mammal, is derived from hodies other than the nuclein substances of the food-that is to say, from the nuclein substances of the tissues contained particularly in the cell-nuclei, and from the ordinary proteins of both food and tissues. The portion derived from the proteins is that small fraction which has already been spoken of as synthetically formed.

Our knowledge of the metabolism of the nucleo-proteins and nucleins has been greatly augmented in recent years. When nucleo-protein is digested by gastric juice a certain amount of protein is easily split off, and hydrolysed to peptone and the other ordinary products of proteolysis. An insoluble residue of nuclein remains. This is acted upon with difficulty by gastric juice, although eventually an active juice will split it up also. $\mathrm{By}$ heating with dilute acids it is more easily hydrolysed, vielding a further quantity of protein along with nucleic acid. This second fraction of protein, which is split off with so much more 
difficulty than the first, undergoes proteolysis in the usual way. For the decomposition of the nucleic acid (or rather acids, since different nucleo-proteins contain different nucleic acids), still more drastic treatment is required-namely, heating with hydrochloric acid in a sealed tube. Thus treated, nucleic acid yields a number of products, among which phosphoric acid and purin bases (adenin, hypoxanthin, gnanin, xanthin) are always present, and probably a carbo-hydrate group also. Pyrimidin bases (uracil, cytosine, thymine) are also present, although, perhaps, not in all nucleic acids. The empirical formula for the purin bodies of greatest physiological interest are as follows:

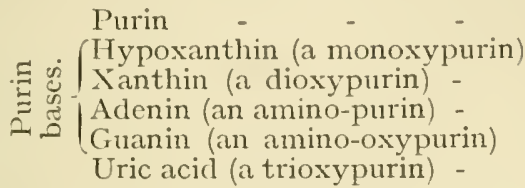

Purin has not been found in the body.

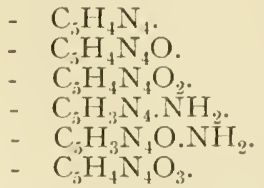

The purin bases and uric acid are widely spread in the tissues, although in very small amounts.

As to the manner in which uric acid arises from the nuclein substances in the tissues, we may picture the process as taking place by the following steps. Certain organs have been shown to contain ferments which split up nucleo-proteins into protein and nucleic acid. This nucleic acid, or nucleic acid arising in other ways in the metabolism of nuclein, and also the nucleic acid produced in the alimentary canal in the digestion of nucleincontaining substances, are then decomposed by another ferment, nuclease, which, along with phosphoric acid and the carbohydrate group, liberates purin (and pyrimidin) bases, especially adenin and guanin. Then follows the action of ferments (adenase and guanase), which remove the amino-group from these purin bases, transforming adenin into hypoxanthin and guanin into xanthin. By means of an oxidizing ferment or oxydase we may next imagine that hypoxanthin is oxidized into xanthin and xanthin into uric acid. Evidence of the existence of all these ferments, and of their wide distribution, has been obtained by making experiments on the various substances mentioned with extracts of different tissues.

The portion of the uric acid which comes from the food (mainly from the purin bodies in it) is sometimes denominated the exogenous portion, while that which arises from the tissues is called the endogenous portion. The latter moiety, which generally amounts to about 0.6 gramme in the twenty-four hours, can be estimated by restricting the diet to articles of food free from purin bodies, such as bread, milk, cheese, eggs, 
and butter. It is stated that the endogenous uric acid remains practically constant in the same individual under constant conditions, and is mnaffected by changes in the diet.

The total excretion of uric acid (and the other purin bodies) is by no means identical with the sum of the nric acict taken in as purin bases in the food and that produced in the body. A considerable destruction of uric acid (and other purin bodies) goes on in the body, and mainly in the liver. A ferment called the uricolytic ferment has been discovered in various organs, and it is believed that this is the active agent in the normal destruction of uric acid. There is reason to think that one of the factors in the production of gout may be a diminution in the amount or activity of this ferment. In some cases it is said to be entirely absent. The quantity of endogenous uric acid excreted by the kidneys bears a certain ratio to the total amount which has entered the circulation. This ratio varies much in different mammalian species. In man a full half is excreted and about a half destroyed. Some of the exogenous moiety is also broken down. When uric acid is heated in a sealed tube with strong hydrochloric acid, it is broken up into glycin, carbon dioxide, and ammonia. There are grounds for believing that a similar decomposition takes place in the body, and that the products are then synthesized to urea in the liver.

Hippuric acid can undoubtedly be produced in the kidney.

If an excised kidney is perfused with blood containing benzoic acid, or, better, benzoic acid and glycin, hippuric acid is formed. The kidney cells must be intact, for if a mixture of blood, glycin, and benzoic acid be added to a minced kidney immediately after its removal from the body, hippuric acid is produced, but not if the kidney has been crushed in a mortar. Nevertheless there is some evidence that a ferment is concerned in the reaction. In herbivora hippuric acid cannot normally be detected in the blood ; it is present in large quantities in the urine; it must therefore be manufactured in the kidney, not merely separated by it. In certain animals, as the dog, the kidney is the sole seat of the production of hippuric acid. But in the rabbit and the frog some of it may also be formed in other tissues, for after extirpation of the kidneys the administration of benzoic acid causes hippuric acid to appear in the blood. The benzoic acid comes mainly from substances of the aromatic groulp contained in vegetable food, but a small amount is produced in the body, since hippuric acid does not entirely disappear from the urine in starvation. It is not known in what form the nitrogenous glycin appears on the spot where it is wanted to form hippuric acid, since glycin has not been found anywhere in the tissues. But there is no doubt that it is a product of the metabolism of 
proteins (and gelatin). It is also at constituent of glycocholic acid, and may be derived in part from the bile which is reabsorbed.

Kreatinin can be so readily obtained from kreatin outside the body that it is tempting to suppose that the portion of the kreatinin of the mrine which is not formed from the kreatin in the food is derived from the kreatin of the muscles and other tissues. The constancy of the kreatinin elimination on a meatfree diet, and its complete independence of the changes in the total nitrogen excretion, show that it has a different significance in protein metabolism from the urea. There is some reason to suspect that the kreatinin may represent nitrogen given off in the constant wear and tear of the bodily machinery. The fact that the amount of kreatinin excreted by different persons seems to be related to the weight of active tissue in the body, excluding fat, is in favour of this suggestion. The statement that the content of the urine in kreatinin is increased by muscular work may indicate that the muscular machine wears out faster during activity than during rest, or perhaps only that already formed kreatin leaves the muscles in greater amount when the bloodflow is increased. As to the manner in which kreatin is changed into kreatinin in the body, a highly suggestive fact is the presence of ferments in various organs which possess this power. Ferments also exist which can decompose both kreatin and kreatinin.

Formation of Carbon Dioxide from Proteins. - We cannot say whether any carbon dioxide is normally produced at the moment when the nitrogenous portion of the protein molecule splits off, or whether a carbonaceous residue may not always hang. together for a time and pass through further stages before the carbon is fully oxidized. We shall see that under certain conditions some of the carbon of proteins may be retained in the body as glycogen or fat; and this suggests that in all cases it may run through intermediate products as yet unknown before being finally excreted as carbon dioxide.

Intracellular Ferments-Autolysis.-As to the agencies by which the decomposition of the proteins is carried out in the cells, we have already spoken of the oxidizing cell ferments, or oxydases (p. 264). Reducing ferments or reductases are also known, and can be extracted from most organs, if not all. Like oxydases, they act in a weakly alkaline medium, causing in the presence of hydrogen such reductions as the formation of nitrites from nitrates. There is some evidence that one and the same ferment may act as an oxydase or a reductase according to the conditions. Recent researches have brought to light in addition hydrolytic intracellular ferments, which split up proteins very much in the same way as the proteolytic ferments of the digestive juices. Not only do unicellular organisms, like leucocytes, 
yeast-cells, and bacteria possess such ferments, but their existence has been demonstrated in practically all the organs of the higher animals and man. When a piece of liver, e.g., is removed with aseptic precautions and kept at body-temperature, extensive auto-digestion occurs, and ammonia, and other basic substances, glycin, and the body which gives the tryptophane reaction (p. 430), appear among the products. Tyrosin appears so early that it is scarcely possible to doubt that it must be a product of protein decomposition in the liver-cells under normal conditions. Similar autolytic processes have been observed in the spleen, muscle, lymph-glands, kidneys, lungs, stomach wall (independently of pepsin), thymus, and placenta, also in pathological new growths like carcinoma, in the breaking down of which and in the removal of such exudations as occur in the alveoli in pneumonia, these proteolytic ferments seem to play a part. The ferments in certain cases have been obtained in extracts of the organs, and have been found still active. It is probable that the syntheses of the proteins or their products, which are scarcely less characteristic of the tissue cells than the decompositions effected by them, are also due to the action of separate intracellular ferments or upon the reversed activity of the proteolytic ferments. So many of the chemical reactions of the body have been found to depend upon enzymes that modern physiology may at first thought seem almost to have reverted to the position of van Helmont and his school in the seventeenth century, who resolved all difficulties by murmuring the magic word 'ferment.' No fewer than eleven ferments have been stated to be present and active in the liver aloneviz., a proteolytic and a nuclein-splitting ferment, a ferment which splits off ammonia from amino-acids, a milk-curdling ferment, a fibrin ferment, a bactericidal ferment, an oxydase, a lipase, a maltase, a ferment called glycogenase, which changes glycogen into dextrose, and an autolytic ferment. In the presence of such an array of enzymes the organs might seem to be little more than incubators in which the ferments do their work. It must not be supposed, however, that the intracellular ferments, whether they cause decomposition or synthesis, oxidation or reduction, work independently of what, for want of a better name, we must call the organization of the cell. We may be sure they are the servants and not the masters of the protoplasm, and that a drop of an extract containing intracellular ferments has very different powers from a living cell. "It is not in the existence of the ferments, but in their combined action at the proper time and in the proper intensity, that the riddle of metabolism lies' (Höber').

2. Metabolism of Carbo-hydrates-Glycogen.-The carbo- 
lydrates of the food, passing into the blood of the portal vein in the form of dextrose, are in part arresied in the liver, and stored up as glycogen in the hepatic cells, to be gradually given out again us sugar in the intervals of digestion. The proof of this statement is as follows:

Sugar is arrested in the liver, for during digestion, especially of a meal rich in carbo-hydrates, the blood of the portal contains more sugar than that of the hepatic vein. Popielski on the basis of experiments in which he fed with known quantities of sugar dogs whose inferior rena cava and portal vein had been united by an Eck's fistula, and determined the amount of sugar which passed into the urine, estimates the quantity of sugar kept back by the liver at from $\mathrm{I} 2$ to 20 per cent. of the whole. In the liver there exists a store of sugar-producing material from which sugar is gradually given off to the blood, for in the intervals of digestion the blood of the hepatic vein contains more dextrose (2 parts per $\mathrm{I}, 000$ ) than the mixed blood of the body or than that of the portal vein (about I part per $\mathrm{I}, 000$ ). When the circulation through the liver is cut off in the goose, the blood rapidly becomes free, or nearly free, from sugar (Minkowski). And a similar result follows such interference with the hepatic circulation as is caused by the ligation of the three chief arteries of the intestine in the dog, even when the animal has been previously made diabetic by excision of the pancreas (p. 5I8).

The nature of the sugar-forming substance is made clear by the following experiments: (I) A rabbit after a large carbohydrate meal, of carrots for instance, is killed and its liver rapidly excised, cut into small pieces, and thrown into acidulated boiling water. After being boiled for a few minutes, the pieces of liver are rubbed up in a mortar and again boiled in the same water. The opalescent aqueous extract is filtered off from the coagulated proteins. No sugar, or only traces of it, are found in this extract ; but another carbo-hydrate, glycogen, an isomer of starch giving a port-wine colour with iodine and capable of ready conversion into sugar by amylolytic ferments, is present in large amount. (See Practical Exercises, p. 608.)

(2) The liver after the death of the animal is left for a time in situ, or, if excised, is kept at a temperature of $35^{\circ}$ to $40^{\circ} \mathrm{C}$., or for a longer period at a lower temperature ; it is then treated exactly as before, but no glycogen, or comparatively little, can now be obtained from it, although sugar (dextrose) is abundant. The inference plainly is that after death the hepatic glycogen is converted into dextrose by some influence which is restrained or destroyed by boiling. This transformation might theoretically be due to an unformed ferment or to the direct action of the liver-cells, for both unformed ferments and living tissue elements 
are destroyed at the temperature of boiling water. It has been clearly shown that the action is brought about by a diastatic enzyme, which some writcrs call glycogenase, for it readily occurs when the minced liver is mixed with chloroform water, and chloroform kills all living tissues. Although blood contains a diastase in small amount, the change does not depend cssentially upon this, since the glycogen also undergoes hydroly'sis (glycogenoly'sis) to dextrose when all the blood has been washed ont of the organ. Lymph also contains a diastase, but there is evidence that the post-mortem glycogenolysis is chiefly due to an enzyme contained in the hepatic cells (an endo-enzyme) (IIacleod). The diastases in the blood and lymph seem to be 'discards' of the tissues which are on the way to destruction or elimination (Carlson). The post-mortem change is to be regarded as an index of a similar action which goes on during life: sugar in the intact body is changed into glycogen; glycogen is constantly being changed into sugar. There is no reason to doubt that here, too, the hydrolysis is effected by the endo-enzyme. But, as in the case of the intracellular protcolytic ferments, we may be certain that the vital action of the hepatic cells is a most important factor in controlling the rate of production of the ferment or the rate at which it works.

(3) With the microscope, glycogen, or at least a substance which is very nearly akin to it, which very readily yields it, and which gives the characteristic port-wine colour with iodine, can be actually seen in the liver-cells. The liver of a rabbit or dog which has been fed on a diet containing much carbo-hydrate is large, soft, and very easily torn. Its large size is due to the loading of the cells with a hyaline material, which gives the iodine reaction of glycogen, and is dissolved out by water, leaving empty spaces in a network of cell-substance. If the animal, after a period of starvation, has been fed on protein alone, less glycogen is found in the shrunken liver-cells; if the diet has been wholly fatty, little or no glycogen at all may be found. Glycogen can cven be formed by an excised liver when blood containing dextrose is circulated through it.

Formation of Glycogen from Protein.-In the liver-cells of the frog in winter-time a great deal of this hyaline material -this glycogen, or perhaps loose glycogen compound-is present ; in summer, much less. The difference is very remarkable if we consider that in winter frogs have no food for months, while summer is their feeding-time; and at first it seems inconsistent with the doctrine that the hepatic glycogen is a store laid up from surplus sugar, which might otherwise be swept into the general circulation and excreted by the kidneys. It has been found, however, that the quantity of glycogen is greatest in 
autumn at the beginning of the winter-sleep, and slowly diminishes as the winter passes on, to fall abruptly with the renewal of the activity of the animal in the spring. The glyongen present at any moment is, therefore, believed to be a residue, which represents the excess of glycogen formed over glycogen used up; and the amount is larger in winter, not because more is manufactured than in summer, but because less is consumed. It is possible, indeed, to produce the 'summer' condition of the hepatic cells merely by raising the temperature of the air in which a winter frog lives ; at $20^{\circ}$ or $25^{\circ} \mathrm{C}$., glycogen disappears from its liver. Conversely, if a summer frog is artificially cooled, a certain amount of glycogen accumulates in the liver. The meaning of this seems to be that at a low temperature, when the wheels of life are clogged and metabolism is slow, some substance, probably dextrose, is produced in the body from proteins in greater amount than can be used up, and that the surplus is stored as glycogen; just as in plants starch is put by as a reserve which can be drawn upon-which can be converted into sugarwhen the need arises. That carbo-hydrates may be produced from proteins has been shown by feeding dogs with almost pure protein (casein) after the production of permanent glycosuria by removal of the pancreas (p. 5I8). To induce the animal to take the casein it had to be mixed with a certain amount of butter, or serum, or meat extract. The amount of sugar excreted was much more than could possibly have come from the glycogen originally present in the animal's body, computing it on the most generous scale (4I grammes per kilogramme of body-weight, according to Pfliger), or from free carbo-hydrate present in traces in the food. or as prosthetic groups (p. 2) in the ingested protein. That the source of the sugar was protein and not fat was indicated by the fact that when the amount of protein food was increased, the dextrose and the nitrogen excreted increased proportionally.

Glycogen-formers. - As true glycogen-formers in the higher animals, only a few substances have been demonstrated, such as proteins (including gelatin), the fermentable sugars, and glycerin. The most interesting demonstration of the transformation of glycerin into glycogen, because the most direct, has been afforded by perfusing the liver of the tortoise with blood to which glycerin was added. The glycogen content of the liver was very distinctly increased. The monosaccharides dextrose, levulose, and galactose gave a similar result, while the disaccharides cane-sugar and lactose caused no increase in the glycogen of the perfused liver, since the liver contains no ferment capable of splitting them into monosaccharides. It is said that even such small molecules as those of formaldehyde $\left(\mathrm{CH}_{2} \mathrm{O}\right)$ can be condensed to glyco- 
gen in the liver (cirube) When given by the month heth cancsugar and lactose form glycogen, being hydrolysed in digestion. It has not hitherto been proved that the fatty acid component of neutral fats can be converted into glycogen. Manyother hodies are known to influence the formation of glycogen by 'sparing' substances which are true glycogen producers, but their carbon does not actually take its place in the glycogen molecule. Some writers deny that proteins call directly form giycogen or sugar apart from carbo-hydrate groups contained in the protein molecule. But the proteins of meat, gelatin. and cascin are capable of forming 60 per cent. of their own weight of dextrose in diabetic metabolism, and even the end products of pancreatic digestion of meat yield so much sugar that the greater part of it must have come from the amino-bodies, and not from a sugar-group in the protein. When given to dogs with total phloridzin glycosuria (p. 52I), giycin and alanin are completely, glutamic and aspartic acids in great part, converted into dextrose (Iusk, etc.).

Extra-hepatic Glycogen.-While the liver in the adult (containing as it does from 2 to ro per cent. of glycogen, or even, with a diet rich in sugar or starch, more than

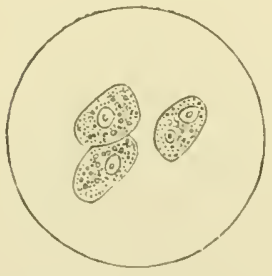

FIG, I83, - CELLS UF

PlaCleNTA CONTANING Gilicogex: IS per cent.) may be looked upon as the main storehouse of surplus carbo-hydrate, depots of glycogen are formed, both in adult and foetal life, in other situations where the strain of function or of growth is exceptionally heary-in the muscles of the adult $\left(0^{\circ} 3\right.$ to 0.5 per cent. of the moist skeletal muscle, or on a carbo-hydrate regimen $0^{\circ} 7$ to 3.7 per cent.), in the placenta, in many developing organs in the embryo (muscles, lungs, epithelium of the trachea, ossophagus, intestine, ureter, pelvis of kidney, and renal tubules). The fotus, however, is not, compared with the adult, especially rich in glycogen. In the adult under farourable circumstances the absolute amount of glycogen in the muscles may be several times greater than that in the liver, and usually the hepatic glycogen makes up considerably less than half the total glycogen of the body. That the muscles do not derive their glycogen by the migration of hepatic glycogen, but can themselves form it from dextrose, has been shown by injecting that sugar subcutaneously into frogs after excision of the liver. The muscle glycogen was found to be increased.

The glycogen store of the liver fulfils a different function from that of the muscles. This is indicated by the fact that when dogs,

* Such results, however, need confirmation in view of l'Hüger's recent analysis of possible errors in work with the tortoise liver. 
after being put on a given diet for two or three days, are starved for a time, and then put again on the original diet, the hepatic and the muscular glycogen behave differently at first during the period of re-alimentation. While glycogen accumulates in the liver in greater quantity than under normal conditions of nutrition, in the muscles it at first accumulates much less rapidly than normally.

Function and Fate of the Glycogen.-When a fasting dog is rllade to do severe muscular work, the greater part of the glycogen soon disappears from its liver. When a dog is starved, but allowed to remain at rest, the glycogen still markedly dininishes, although it takes a longer time; and at a period when there is still plenty of fat in the body, there may be only a trace of hepatic glycogen left. The glycogen which is usually contained in the skeletal nuuscles also diminishes very rapidly in the first days of hunger, but the heart contains the normal amount of glycogen at a time when the proportion in the skeletal muscles has sunk to $\frac{1}{10}$ to $\frac{1}{30}$ of the normal. These facts have been taken to indicate that glycogen and the sugar formed from it are the readiest resources of the starving and working organism, for the transformation of chemical energy into heat and mechanical work. To borrow a financial simile, the fat of the body has sometimes been compared to a good, but rather inactive security, which can only be gradually realized ; its organ-proteins to longdate bills, which will be discounted sparingly and almost with a grudge; its glycogen, its carbo-hydrate reserves, to consols, which can be turned into money at an hour's warning. Glycogen, on this view, is especially drawn upon for a sudden demand, fat for a steady drain, tissue-protein for a life-and-death struggle.

Although it cannot be doubted that much of the hepatic glycogen leaves the liver as sugar, there is no proof that it all does so. It is known that fat may be formed from carbo-hydrates (p. 524); and globules of oil are often conspicuous among the contents of liver-cells, side by side with glycogen. It is possible, therefore, that some of the glycogen may represent a half-way house between sugar and fat, or, since it is probable that fat can also be formed from protein, and a purely protein diet produces some glycogen, a half-way house between protein and fat.

Pavy has put forward the heterodox view that the glycogen formed in the liver from the sugar of the portal blood is never reconverted into sugar under normal conditions, but is changed into some other substance or substances, and he denies that the post-mortem formation of sugar in the hepatic tissue is a true picture of what takes place during life. But in spite of the brilliant manner in which he has defended this thesis, both by argument and by experiment, it must be said that the older doctrine of Bernard, which in the main we have followed above, 
is attested by such a cloud of modern witnesses that it seems to be firmly and finally established.

Fate of the Sugar-Glycolysis.-What, now, is the fate of the sugar which either passes right through the portal circulation from the intestine without undergoing any change in the liver, or is gradually produced from the hepatic glycogen? When the proportion of sugar in the blood rises above a certain low limit (about 1.5 or 2 to 3 parts per $I, 000$ ), some of it is excreted by the kidneys (Practical Exercises, p. 609).

A large meal of carbo-hydrates is frequently followed by a temporary glycosuria, but much depends upon the form in which the sugar-forming material is taken. Miura, for example, after an enormous meal of rice (equivalent to 6.4 grammes of ash- and water-free starch per kilo of body-weight), which, as he mentions, tasked even his Japanese powers of digestion for such food to dispose of, found not a trace of sugar in the urine. Dextrose, cane-sugar and lactose, on the other hand, when taken in large amount, were in part excreted by the kidneys, as was also the case with levulose and maltose in a dog (Practical Exercises, p. 6I0).* It has been suggested as an important practical rule that a person who can tolerate a certain amount of dextrose (say 2 grammes per kilo of body-weight taken not less than two hours after a meal) without excreting a portion of it is not the subject of incipient diabetes. Many healthy persons can tolerate much more.

Except as an occasional phenomenon, glycosuria other than alimentary is inconsistent with health; and therefore in the normal body the sugar of the blood must be either destroyed or transformed into some more or less permanent constituent of the tissues. The transformation of sugar into fat we have already mentioned, and shall have again to discuss; it only takes place under certain conditions of diet, and no more than a small proportion of the sugar which disappears from the body in twentyfour hours can ever, in the most favourable circumstances, be converted into fat. Accordingly, it is the destruction of sugar which concerns us here, and there is every reason to believe that this takes place, not in any particular organ, but in all active tissues, especially in the muscles, and to a less extent in glands.

* Twenty-four healthy students, whose urine had previously been shown to be tree from sugar, ate quantities of cane-sugar varying from 250 grammes to 750 grammes. The urine was collected in separate portions for twelve to twenty-four hours after the meal. In only three cases was reducing sugar found in the urine (by Fehling's and the phenylhydrazine test), and then merely in traces. In eight cases cane-sugar was found, and estimated by the polarimeter, and, after boiling with hydrochloric acid, by Fehling's solution. The greatest quantity of canesugar recovered from the urine was 8 grammes $\left(7^{\circ} 92\right.$ grammes by Fehling's method and 8.29 grammes by the polarimeter); the highest proportion of the quantity taken which appeared in the urine was $2{ }^{\prime} 5$ per cent. When dextrose was found, cane-sugar was always present as well. 
It has been asserted that the blood which leaves even a resting muscle, or an inactive salivary gland, is poorer in sugar than that coming to it; and the conclusion has been drawn that in the metabolism of resting muscle and gland sugar is oxidized, the carbon passing off as carbon clioxide in the venous blood. This is indeed extremely likely, for we know that when the skeletal muscles of a rabbit or guinea-pig are cut off from the central nervous system by curara, the production of carbon dioxide falls much below that of an intact animal at rest ; and the carbon given off by such an animal on its ordinary vegetable cliet can be shown, by a comparison of the chemical composition of the food and the cxcreta, to come largely from carbo-hydrates. But, considering the relatively feeble metabolism of muscles and glands when not functionally excited, the large volume of blood which passes through them, the difficulty of determining small differences in the proportion of sugar in such a liquid, the possibility that even in the blood itself sugar may be destroyed, or that it may pass from the blood, without being oxidized, into the lymph, too much weight may be easily given to the results of direct analysis of the in-coming and out-going blood. And although the results of Chauveau and Kaufmann, obtained in this way, fit in fairly well with what we have already learnt by less direct, but more trustworthy, methods, they cannot be accepted as yielding exact quantitative information. They found that in one of the muscles of the upper lip of the horse the quantity of dextrose used up during activity (feeding movements) was 3.5 times as much as in the same muscle at rest, and this corresponded with the deficit of oxygen in the blood entering the muscle, and with the excess of carbon dioxide in the blood leaving it. More dextrose was also destroyed in the active than in the passive parotid gland of the horse, but the excess per unit of weight of the organ was far less than in muscle.

Concerning the manner in which dextrose is destroyed in the tissues, we are in the same position as in the case of the proteins. It cannot be definitely stated at present what share is taken by cleavage and what by oxidation in the destruction of carbohydrates in the organism, although oxidation is known to play an important rôle. Normal blood has been credited with a ferment which has the power of destroying sugar (glycolysis). But with rigid aseptic precautions the loss of sugar, even in several hours, is small, and it is doubtful whether such a ferment exists. On the other hand, Cohnheim stated that while no glycolytic ferment can be demonstrated in the pancreas, and only an exceedingly weak glycolytic action in muscular tissue (Brunton), by combining pancreas and muscles distinct glycolysis, due to a ferment action, could be produced. He suggested that the glycolytic ferment is 
activated by another substance, as trypsinogen is activated by enterokinase $(p, 3+3)$. This announcement aroused great interest, since it is known that the pancreas is intimately concerned in the metabolism of sugar. Unfortunately, however, the accuracy of Cohnheim's observation is still disputed. Excision of the pancreas in dogs causes permanent glycosuria (pancreatic diabetes) (v. Mering and Minkowski), which is prevented if a portion of the pancreas be left (p. 553). Diabetes in man is known to be frequently associated with pancreatic lesions.

Diabetes. - In the disease known as diabetes mellitus, sugar accumulates in the blood, and is discharged by the kidneys, and it lias been supposed that a derangement in the glycogenic function of the liver is sometimes the cause of this accumulation and of this discharge. An artificial and temporary glycosuria, in which the sugar in the urine undoubtedly arises from the hepatic glycogen, can, indeed, be caused by puncturing the medulla oblongata in a rabbit at or near the region of the vasomotor centre. If the animal has been previously fed with a diet rich in carbo-hydrates-that is, if it has been put under conditions in which the liver contains much glycogen-the quantity of sugar excreted by the kidneys will be large. If, on the other hand, the animal has been starved before the operation, so that the liver is free, or almost free, from glycogen, the puncture will cause little or no sugar to appear in the urine. That nervous influences are in some way involved is shown by the absence of glycosuria if the splanchnic nerves, or the spinal cord above the third or fourth dorsal vertebra, be cut before the puncture is made. But sometimes these operations are themselves followed by temporary glycosuria. Section of the vagi has no effect either in causing glycosuria of itself or in preventing the 'puncture' glycosuria, although stimulation of the central ends of these and of other afferent nerves may cause sugar to appear in the urine, but not if precautions are taken to prevent any degree of asphyxia. Asphyria produces an increase in the sugar content of the blood (hyperglycæmia), an increase in the flow of urine and glycosuria. Under normal conditions the rate of transformation of the hepatic glycogen into dextrose is adjusted in some way to the dextrose content of the blood, so that when the latter tends to sink more dextrose is produced in the liver; when it tends to rise more glycogen is laid up in the hepatic cells. Thus, the great function of the glycogen store of the liver is to regulate the proportion of sugar in the blood. Although several of the operations which lead to temporary glycosuria undoubtedly bring about changes in the hepatic circulation, it is as yet impossible to say whether the whole phenomenon is at bottom a vaso-motor effect, or is due to direct 
merous stimulation of the liver-cells, or to witheliawal of such stimulation or control (see also p. $\left.47^{\circ}\right)$. There is some evidence that excitation of the nucut great splanchnic nerve (on the left side) in dogs may cause an increase in the hyperglycamia, diuresis, and glycosuria, eren nuder conditions in which as far as possible circulatory effects arc eliminated. But absolute proof of the existence of glycogenolytic nerve fibres has not yet been hronght forward (Macleod).

In the natural diabetes of man, as in all the forms of glycosuria mentioned, the immediate canse of the glycosuria is the increase of strgar in the blood. Instead of the I part per I,ooo, or a little more or less, which constitutes the normal proportion in a healthy man, in diabetes 3 or 4 parts, and in exceptional cases eren 7 to ro parts per r.ooo may be present. The riddle of diabetes is the cxplanation of this persistent hyperglycremia. It is possible that in some cases the sugar coming from the alimentary canal passes entirely or in too large amount through the liver, owing to a deficiency in its power of forming glycogen. But although in certain cases of diabetes specimens of the hepatic cells, obtained by plunging a trocar into the liver, have been found free from glycogen, in others glycogen has been present. The muscles also are usually much poorer in glycogen than normal muscles. The cause of this defect in glycogen-forming power has been supposed by some to be the absence of a glycogenforming ferment, or its production in too small an amount. But this has not been prored. In addition to an interference with the due and regulated storage of the surplus sugar as glycogen, it is necessary for a rational explanation of many of the facts of diabetes to assume that from some change in the tissues sugar has ceased to be a food for them, or is used up in smaller amount than in the healthy body. The change may be the loss or diminution of a glycolytic ferment or a substance necessary for the activation of such a ferment. And although the sugar-destroying power of blood from diabetic patients, of from animals in which glycosuria has been caused by phloridzin, is not at all inferior to that of healthy blood, it may be that the intracellular glycolytic ferments, if such really exist, are much less active, especially in the more severe forms of the disease. The actual primary production of sugar may be no gieater than in a normal person with the same diet. And there is no reason to suppose that an overproduction of dextrose is ever in pathological diabetes the proximate cause of the hyperglycremia and glycosuria. But a secondary overproduction of sugar unquestionably occurs in nany cases. The tissues, bathed as they are in liquids rich in dextrose, are nevertheless starving for sugar, since they cannot use what is offered to them, and the body labours to arert the famine 
by increasing its production of sugar, the sugar-forming tissues being stimulated to their task either through nervous influences or by chemical messengers circulating in the blood. Why the tissues cannot burn dextrose as they normally do is a question of great interest, but as yet no satisfactory answer can be given.

Another hypothesis, which endeavours to connect the impairment of the glycogenetic function with the impairment of the power to oxidize dextrose, is that it is only sugar which las been condensed or polymerized to glycogen which can he assimilated by the tissues, and therefore burned (v. Noorden).

It is remarkable that levulose may within limits be entirely used up in the tissues of a diabetic patient, or of a dog rendered diabetic by extirpation of the pancreas, while dextrose, which is so closely allied to it, is promptly cast out by the kidneys. Glycogen is also formed from levulose, though not from dextrose, in the depancreatized dog. This is not easily reconciled with the last-mentioned theory unless we suppose that the glycogen which levulose gives rise to is somewhat different from the glycogen produced by the condensation of dextrose. The opposite condition is also seen in a few individuals-namely, intolerance of levulose and its spontaneous appearance in the urine (levulosuria) - while other carbo-hydrates are normally used up. Like pentosuria (p. 450), the condition is not a serious one.

In dogs deprived of the pancreas, and in dogs under the influence of phloridzin, glycerin, given by the mouth, causes an increase in the excretion of sugar up to two or three times the original amount. The giving of fat does not increase the amount of sugar excreted, which, however, is increased by such substances as egg-yolk, which contain lecithin. These should accordingly be avoided in cases in which a strictly antidiabetic diet is desired. It is much more important to exclude carbohydrates largely or entirely from the food, although oatmeal and potatoes are said to occupy an exceptional position, and have even been recommended as beneficial. Calcium chloride las been stated to diminish the sugar excretion in diabetes (Boigey), and it has a similar effect in certain of the artificial glycosurias (Brown, Fischer).

In many cases even when carbo-hydrates are completely, or almost completely, omitted from the food, sugar, derived from the breaking-down of proteins, and possibly to some extent from fats, still continues to be excreted, although in smaller quantity. Other products of the deranged metabolism of proteins, and especially of fats, such as acetone, aceto-acetic acid, and oxybutyric acid, may also appear in the urine, or, accumulating in the blood, may, by uniting with its alkalies, serjously diminish the quantity of carbon dioxide which that liquid is capable of carry- 
ing, and thus lead to the condition known as diabetic coma. The small amount of carbon dioxide in the venous blood may also be partly due to the hyperjmoa, marked by increased depth of the respiratory movements produced by stimulation of the respiratory centre by other substances than carbon dioxide. The increased ventilation causes a fall in the carbon dioxicle pressure in the alveolar air, and therefore an increased elimination of that gas from the blood. This form of coma appears to be really in part an acid-poisoning comparable to the condition produced in animals by doses of mineral acids too large to be neutralized by the ammonia split off from the proteins. The administration of very large doses of alkalies (sodium bicarbonate, for instance, to the amount even of hundreds of grammes) has been recommended for the treatment of this serious complication, and in many cases it is successful in staving it off for a time. Often, however, in spite of a prolonged course of treatment, during which the urine has continued distinctly alkaline, fatal coma eventually occurs. The coma then is not merely a symptom of acidosis, but is also due to the specific toxic effects of the acids even when neutralized. Other toxic products may also be formed in the deranged metabolism.

Glycosuria can be caused in many other ways than those already mentioned-e.g., by concussion of the brain, occlusion and subsequent release of the arteries supplying the brain and cervical cord, acute hæmorrhage, injection of water or physiological salt solution into the bile-ducts, into the mesenteric veins, or, in considerable amount, into the general circulation. Carbon monoxide has a similar action owing to the deficiency of oxygen occasioned by it. Many drugs also cause glycosuria, including curara, morphine, phloridzin, adrenalin, and other substances. Of these the last two are the most interesting.

Phloridzin glycosuria agrees with pancreatic, but differs from 'puncture' diabetes in this, that it can be produced in an animal free from glycogen, and is accompanied by extensive destruction of proteins. It differs from other forms of diabetes in being associated, not with an increase, but with a diminution in the sugar of the blood. This is best explained by supposing that the phloridzin acts on the kidney in such a way as to increase the permeability of the glomerular epithelium for sugar, or (in terms of the secretion theory of urine formation) in such a way as to increase its sensitiveness to the stimulus of sugar circulating in the blood. The sugar is, therefore, rapidly swept out of the circulation, and this leads secondarily to an increased production of sugar to make good the loss. In addition, within certain limits there is a total inability on the part of the body to consume dextrose. 
After the preliminary sweeping out of the sugar already in the bodyat definite ratio is established betwen the dextrose and the nitrogen eliminated in the urine (dextrose: nitrogen $:: 3^{\circ}$ or $37:$ I). The sugar at this stage is produced entirely from proteins, and not at all from fat. The degree of intolerance for carbo-hydrates in pathological diabetes may he arrived at hy puting the patient on a diet of protein and fat (rich cream, meat, hutter, and eggs), and determining the ratio of dextrose to nitrogen excleted. If it is 3.6 to $377: 5$, intolerance is complete, none of the dextrose produced from protein being burned, and there will pobably be a quickly fatal issue (Insk and Landel). There is some evidence that, in addition to the increased permeability of the kidney to sugar and the diminished power of the tissues in general to destroy it, the renal epithelinm is actually an important seat of the sugar production (Brodie).

In adrenalin glycosuria the sugar-content of the blood is increased. Subcntaneous injection of adrenalin chloride causes a mild, intravenons injection a greater glycosuria, and intraperitoneal injection the greatest glycosuria of all (Herter). The best evidence is that the glycosuria is produced by some action on the liver, possibly through the excitation of sympathetic fibres controlling the production of dextrose from glycogen (Underhill and Closson), or by a direct effect on the hepatic cells, which lastens the normal transformation of glycogen into dextrose, or hinders the normal transformation of dextrose into glycogen. After repeated injections of adrenalin a tolerance for it is established, and glycosuria is no longer caused.

3. Metabolism of Fat.-The fat, passing along the thoracic duct into the blood-stream, is very soon removed from the circulation, for normal blood contains only traces, except during digestion. Where does it go? What is its fate?

The presence of adipose tissuc in the body might suggest a ready answer to these questions. The fat-cells of adipose tissue are ordinary fixed connective-tissue cclls which have become filled with fat, the protoplasm being reduced to a narrow ring, in which the nucleus is set like a stone. It would, at first thought, seem natural to suppose that the fat of the food is rapidly separated by these cells from the blook, and slowly given up again as the needs of the organism require, just as carbohydrate is stored in the liver for gradual use. And it has been found that a lean dog, fed with a diet containing much fat and little protein, puts on more fat, as estimated by direct analysis, or kecps back more carbon, as estimated by measurcments of the respiratory exchange, than can be accounted for on the supposition that even the whole of the carbon of the brokendown protein corresponding to the excreted nitrogen has been 
laid up in the form of fat. Even witl a diet of pure fat-and with such a dict digestion and absorption are carried on undrer unfavourable conditions-more carbon is retained than can liave come from the metabolism of the proteins of the body, as measured by the nitrogen given off in the urine and faces: the fat passes rapidly from the blood into the organs, and especially into the liver (Hofmann, Pettenkofer and Voit). It is thus certain that some of the absorbed fat may be stored up as fat in the body.

This is borne out by the careful experiments of Munk and I,ebedeff, who found that when logs are fed with excess of foreign fat (linseed oil, rape oil, mutton fat), a fat is laid down which is quite different from dog's fat, and has the greatest resemblance to the fat of the food. Thus, when rape oil, which contains a fat $y$ acid, erucic acid, not found in animal fat, was given, erucic acid could be detected in the fat laid on. When the dogs were fed with mutton fat, whose melting-point is much higher than that of dog's fat, the fat laid on did not melt till it was heated to $40^{\circ} \mathrm{C}$. or more. When they were fed with linseed oil, the body-fat was found liquid even at $0^{\circ} \mathrm{C}$. We have already referred (p. 4 $\mathrm{I}_{3}$ ) to the fact that neutral fat can be built up in the wall of the intestine from fatty acids given in the food. Munk has shown that fat formed in this way can also be laid down as body-fat. But besides the fat and fatty acids of the food, the fat of the body has other sources, and some of it is produced by more complex processes.

The fat of a dog consists of a mixture of palmitin, olein, and stearin. When a starved dog was fed on lean meat and a fat containing palmitin and olein, but no stearin, the fat put on contained all three, and did not sensibly differ in its composition from the normal fat of the dog (Subbotin). Stearin must, therefore, have been formed in some way or other in the body. If it was produced from the olein and palmitin of the food, the portion of these deposited in the cells of the adipose tissue must have undergone changes before reaching this comparatively fixed position. But there is conclusive evidence that fat may be derived from other sources, certainly from carbo-hydrates, and probably from proteins ; and the stearin may have been formed from the carbo-hydrates or proteins of the food or tissues, and not directly from fat. And if the stearin was produced from proteins or carbo-hydrates, it is evident that the olein and palmitin might have been formed in this way too, the portion of the carbohydrate or protein devoted to this purpose being sheltered from oxidation by the combustion of the fats of the food. It is well known that not only neutral fats, but also fatty acids, exert such a 'protein-sparing ' action. It is possible also that the fat which is normally excreted into the intestine (p. 4I5), and which is 
perhaps derived from broken-down proteins, may he reabsorbed, and take its place among the fat 'put on.'

Formation of Fat from other Sources than the Fat of the Food-(I) From Carbo-hydrates. - It has been found that the addition of protein to a diet of fat, and especially to a diet of carlonhydrate, in larger amount than is just necessary for nitrogenous equilibrium (p. 52(1), leads to a more rapid increase in the carbon deficit - that is, in the fat put on - than if the minimum quantity of protein required for nitrogenous equilibrium had been given. From this it is inferred that the carbonaceous residue of the broken-down protein is shielded from oxidation by the fat, and to a still greater extent by the carbo-hydrates, and so retained in the body as fat. And there is little doubt that the high repute of carbo-hydrates as fattening agents is in part due to their taking the place of proteins and fats in ordinary ' current ' metabolism, and so allowing body-fat to be laid down from these. Voit, indeed, has gone so far as to assert that this is the only sense in which carbo-hydrates can be said to form fat, and that, in carnivorous animals at least, a direct conversion never occurs. But the experiments of Rubner have shown that in a dog fed with a diet rich in carbo-hydrates, and containing but little fat and no proteins at all, the carbon deficit was greater than could be accounted for by the proteins being broken down in the body and the fat of the food. In the pig and goose, too, the direct formation of fat from carbo-hydrates has been demonstrated.

For cxample, in an experiment by Tscherwinsky two young pigs of the same litter were taken. They weighed respectively 7.300 grammes and 7,290 grammes. One was killed, and the amount of fat and nitrogen in its body dircctly estimated. From the nitrogen the maximum quantity of protein which could be present was calculated. The other pig was fed for four months with barler, which was analyzed. The excreta were also analyzed to determinc the amount of unabsorbed fat and protein. At the end of the four months the pig was killed. It now weighed $2+$ kilogrammes, and contained 2.52 kilogrammes protein and 9.25 kilogrammes fat. Subtracting the protein (0.96 kilogramme) and fat (0.69 kilogramme) originally present, $1{ }_{5} 6$ kilogrammes of protein and 8.56 kilogrammes of fat must liave been put on. The amount of protein taken in the food was 7.49 kilogrammes, and of fat 0.66 kilogramme. Therefore. 5.93 kilogrammes of protein must have been used up. and 7.90 kilogrammes of fat laid on. At least 5 kilogrammes of this fat must have come from the carbo-hydrate of the food. Only a small amount of the fat put on could possibly have come from the protein.

It is probable that in the formation of fats the carbo-hy'drates are first split up to some extent, and that the fats are then constructed from their decomposition products, oxygen being lost in the process, since fat is poorer in oxygen than carbo-hydrate. The production of wax by bees, which used to be given as a proof of the formation of fat from sugar, is not decisive, for in raw 
honey proteins are present; and even when bees fed on pure honey or sugar manufacture wax, it may be derived from the broken-clown proteins of their own bodies.

(2) From Protein.-Dry protein contains on the average I6 per cent. of nitrogen and 50 per cent. of carbon, and urea contains fo per cent. of nitrogen and 20 per cent. of carbon. Urea is therefore three times as rich in nitrogen as the protein from which it is derived, but two and a half times poorer in carbon ; and less than one-seventh of the carbon of protein will be eliminated in the urea, which carries off all the nitrogen. A carbonaceous residue is left, which, it is extremely probable, may under certain circumstances be converted into fat, as we know it may into carbo-hydrate; yet absolutely flawless experiments to prove the direct production of fat from protein seem still to be wanting.

In the experiments of Bauer, the amount of oxygen consumed and of carbon dioxide and nitrogen excreted was determined in starving dogs. Phosphorus, which, as is well known, causes extensive fatty changes in the organs, was then administered in small doses for several days. The excretion of nitrogen was doubled, the excretion of carbon dioxide and the consumption of oxygen diminished to one-half. When the animals died, in a few days, the organs were all found loaded with fat. In one case $+2^{\circ} 4$ per cent. of the solids of the muscles and 30 per cent. of the solids of the liver consisted of fat. This is much more than the normal amount. It was assumed that the fat could not have been simply transferred from the adipose tissue, since the dog had been starved for twelve days before the phosphorus was given. and died on the twentieth day of starvation. Now, after such a period of hunger the amount of fat in the adipose tissue is greatly reduced. It was therefore concluded that the source of the fat could only have been the broken-down protein. Since the nitrogen excretion was increased, while the carbon excretion was diminished, it was supposed that a residue rich in carbon must have been split off from the proteins, and, remaining unburnt in the body, must have been converted into fat. Experiments of this kind are open to criticism on several grounds, but especially on this : that unless the fat-content of the whole body before the administration of the poison is known, it is impossible to be sure that the fat in a particular tissue has not been increased simply by the transportation of fat from some other tissue. It has been conclusively shown that migration of pre-formed fat does occur, and on an extensive scale, in phosphorus poisoning. For example, a dog was fed for a time with sheep's tallow, and fat was laid down in its adipose tissue with the physical and chemical characters, not of dog's, but of sheep's fat. The animal was then poisoned with phosphorus, and the fat which accumulated in the liver examined. It also resembled sheep's fat, as it should have done had it migrated from the adipose tissue, and not dog's fat. as it might have been expected to do had it been formed in the hepatic cells from protein. The ease with which connective-tissue fat-i.e., food fat-migrates to the liver suggests, with other facts, that the liver has a special relation to the transformation of this 
fat into the list of the organs. This 'organized' intracellular fat differs in various way's from the fats of atlipose tissue. 1ts "iorline value' (1, . I) is ligher (leathes), and a large proportion of it consists of phosphatide lipoids (1).337).

The inost convincing evidence that fat is not produced in increased imount under the influence of phosphorus has been obtinined by determining by actual analysis the total fat in animals, and then poisoning similar animals with phosphorus and again estimating the total fat. Far from being increased, the fat may even be decreased in the poisoned animals (Taylor, ctc.). There is no ground, tlicn. for the assumption that phosphorus and other substances, like arsenic, antimony, etc., which bring about so-called ' fatty degeneration' of the organs, act by causing or accelerating the transformation of protein into fat. Yct there is good evidence that they do accelerate the decomposition of protein, or at least interfere with its normal metabolism, for after phosphorus poisoning amino-acids (leucin, tyrosin, glycin) appear in the urine. The observations of Lusk and his pupils indicate that phosphorus does not directly increase the amount of protein broken down, but does so indirectly, by favouring the conversion of the carbo-hydrate-like radicle of the protein molecule into leucin, tyrosin, and perhaps fat, and therely necessitating an increased consumption of protein.

A celebrated experiment, performed nearly forty years ago, was long supposed to furnish an absolute proof of the formation of fat from protein, under strictly physiological conditions, although in a humble form of animal life. Maggots were allowed to develop from the egg on blood containing a known amount of fat. 'The quantity of fat in the eggs was also known. After the maggots had grown, ten times as much fat was found in them as had been contained in the blood and eggs together. The trifling quantity of sugar in the blood was utterly inadequate to account for the fat, which, it was concluded, must therefore have come from the proteins of the blood (Hofmann). It can be objected to this experiment that no precautions were taken to prevent the growth of micro-organisms on the blood, and fat might have been formed by them from the proteins. Further, the fat estimations would scarcely pass muster according to the present standards.

The experiments of Pettenkofer and Voit, which were supposed to have demonstrated that in the higher animals also fat is formed from proteins under normal conditions, are in the same position. According to them, a dog fed for a time on a liberal diet of lean meat may go on excreting a quantity of nitrogen equal to that in the food, while there is a deficiency in the carbon given off. Or if the dog is not in nitrogenous equilibrium (p. 529), but putting on nitrogen in the form of 'flesh,' the deficiency in the carbon given off may be too great in proportion to the nitrogen deficit to warrant the assumption that all the retained carbon has been put on in the form of protein. In either case, carbon in large amount can only come from the proteins of the food, and can only be stored up in the body: in the form of fat. For lean meat contains but a trifling quantity of carbon in any other proximate principle than protein, and the nonprotein carbon of the animal body is only to a very small extent contained in carbo-liydrates or other substances than fat.

Pflüger has criticised these experiments, and has shown that lean meat contains more fat than was supposed, and this is now generally admitted. He has endeavoured to show that the fat and 
slycogen in the neat given to the animals fully accounts for the cirbon retaincel. Pfliiger, incleed, takes up the position that the fat of the body comes exclusively from the carbo-hydrates and fats of the food, and not at all from the proteins. But there is little doubt that in this he has gone too far, although his criticism hias rendered it impossible any longer to appeal to Pettenkofer and Voit's results as good evidence on the other side.

If none of the supposed quantitative proofs of the conversion of proteins into fat which have hitherto been brought forward are free from flaw, the same is true of the alleged qualitative indications of its possibility and of its actual occurrence. The accumnlation of fat between the hepatic cells caused by phloridzin is, at the best, no better evidence than the accumulation within the cells in phosphorus poisoning. The formation of adipocere (a cheesy substance, rich in fatty acids united with calcium or ammonium), sometimes seen in dead bodies which have remained a long time under water or in moist graveyards, is largely, if not entirely, due to the fat already present in the parts which have undergone the change, or to fat removed by the water from other parts of the body. If any portion of the adipocere represents fat formed from protein, this transformation may well be credited to the numerous micro-organisms present, and throws no light upon the question of fat formation in the normal organism. The fat in the cells of the sebaceous glands, and of the mammary glands, may be produced from protein by a transformation of the cell-substance. But absolutely convincing proof is wanting. The rule which experience has taught, that a woman during lactation requires an excess of proteins in her food corresponding not only to the proteins, but also to the fat given off in the milk, suggests such an origin for the milk-fat, but does not prove it.

As to the ultimate fate of the fat, from whatever source it may be derived, our knowledge may be compressed into a single sentence: Sooner or later it is split and oxidized to carbon dioxide and water, its cnergy being converted into heat or, directly or indirectly, into mechanical or chemical rork; some of the fat absorbed from the intestine rapidly undergoes this change without entering the fat-cells of the adipose tissue. A portion of the fat may be changed into carbo-hydrates. This has been proved for the glycerin component; its possibility must be admitted for the fatty acids, but complete proof has not yet been given.

The mechanism of the transformation of fats is no better understood than that of the carbo-hydrates or the proteins. Many of the tissues contain intracellular, soluble, fat-splitting ferments called lipases, especially the liver, the active mammary gland, and the intestinal mucosa. We have already seen that there is evidence that these lipases, like some other enzymes, have a reversible action. They are either fat-splitting or fat-forming 
ferments, according to the conditions (liastle and Loevenhart). The perfectly aseptic blood does not split ordinary neutral fats, although it contains a ferment which splits up monobutyrin (glycerin butyrate) into glycerin and butyric acid.

Summary. At this point let us sum up what we have learnt as to the relation between the proximate principles of the tissues and the proximate principles of the food. Inside the body we recognise representatives of the three groups of organic food-substances in a typical diet-proteins, carbo-hydrates, and fats. But we should greatly err if we were to imagine that the three streams of food-materials have flowed from the intestines into the tissues each in its separate channel, neither giving to nor taking from the others. The fats of the body may, indeed, in part be composed of molecules which were present as fat in the food; but they may also be formed from carbo-hydrates, and probably from proteins. The carbo-hydrates of the body-the glycogen of the liver and muscles, the sugar of the blood-may undoubtedly be derived from carbo-hydrates in the food, but they may also be derived from proteins, although probably not from fats (except from their glycerin constitucut). The proteins of the body arise solely from the proteins of the food; neither fats nor carbo-hydrates can form protcins, although both can cconomize them and shield them from an over-hasty metabolism.

\section{The Income and Expenditure of the Body-(I) Income and Expenditure of Nitrogen.-}

Preliminary Data.- The office of the food is to maintain the constituents of the body upon the whole in their normal proportions. A knowledge of the chemical composition of the body is, therefore. an important datum in the consideration of the statistics of its metabolism. The body of a man analyzed by Volkmann had the following composition :

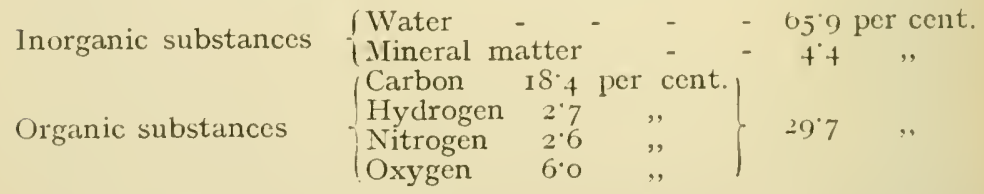

The muscles, the adipose tissue, and the skeleton form nearly four-fiftlis of the total body-weight in the adult. The following table shows the percentage amount of each of these tissues in a man, a woman, and a child (Bischoff) :

\begin{tabular}{|c|c|c|c|}
\hline & Man. & Woman. & $\begin{array}{l}\text { New joorn } \\
\text { Child. }\end{array}$ \\
\hline Voluntary muscles & $+1 \cdot 8$ & $35 \cdot S$ & $23 \cdot 5$ \\
\hline Adipose tissue - & I $S \cdot 2$ & $28 \cdot 2$ & I 3.5 \\
\hline Skeleton - - & I 5.9 & $15 \cdot 1$ & I $5 \cdot 7$ \\
\hline Rest of body & $24 \cdot I$ & $20 \cdot 9$ & $47 \cdot 3$ \\
\hline
\end{tabular}


The nitrogen is contained chiefly in the muscles, glands, and nervous system, and in the constituents of the connective tissues, whicl yicled gelatin, varions mucoids, and elastin. The ordinary proteins make up about 9 per cent. of the weight of the borly, or 22 per cent. of its solids; the albuminoids or sclero-proteins (gelatinvielcling material, etc.) (p. 2) abont 6 per cent. of the boxly-weight. Nitrogen exists in proteins to the extent of $\mathrm{I} 6$ per cent., so that the 6.5 kilos of protein of a $7^{0-k i l o ~ b o l l y ~ c o n t a i n ~ a b o u t ~} x$ kilo of nitrogen.

The carbon is contamed chicfly in the fat, which forms a very large proportion of the water-free substance of the bolly, and in the proteins. A small amount is present as calcium carbonate in the bones. In the body of a strong young man weighing 68 .6 kilos, Voit found the following quantities of dry fat in the various tissues :

Arlipose tissue

Skeleton

Muscles

Brain and spinal cord

Other organs

Total
- $8809 .+$ grammes.

- $2617 \cdot 2$..

- 636.8 ..

$226 \cdot 9$.

$73 \cdot 2 \quad$,

-12363.5,

equivalent to 18 per cent. of the whole body-weight, or 44 per cent. of the solids. In dry fat rather more than 75 per cent. of carbon is present, and in protein about $5^{\circ}$ to 55 per cent. ; so that while the fat of the body analyzed by Voit contained more than 9 kilos of carbon, only about a third of this amount would be found in the proteins.

In the fat there is, roughly speaking, 2 per cent. of hydrogen, in proteins only 7 per cent.; so that from three to four times as much hydrogen is contained in the fat of the body as in its proteins.

Oxygen forms about 12 per cent. of fat. and 20 to $2 .+$ per cent. of proteins; the protein constituents of the body, therefore, contain about as much of its oxygen as the fat.

Of the inorganic salts calcium phosphate, $\mathrm{Ca}_{3}\left(\mathrm{PO}_{4}\right)_{2}$, is much the most abundant orving to the large amount of it in bone, in the ash of which it is found to the cxtent of 83 per cent., along with 13 per cent. of calcium carbonate.

Nitrogenous Equilibrium.-It is a matter of common experience that the weight of the body of an adult may remain approximately constant for many months or years, even when the diet varies greatly in nature and amount. And not only may the weight remain constant, but the relative proportions of the various tissues of the body, so far as can be judged, may remain constant too. Here it is evident that the expenditure of the body must precisely balance its income: it must lose as much nitrogen as it takes in, otherwise it would put on flesh ; it must lose as much carbon as it takes in, otherwise it would put on fat. Or, again, the body may be losing or gaining fat, giving off more or less carbon than it receives, while its 'flesh' (its protein constituents) remains constant in amount, the expenditure of nitrogen being exactly equal to the 
income.* In both cases we say that the body is in nitrogenous equilibrium.

A starving animal or a fever patient, on the other hand, is living upon capital, the former entirely, the latter in part; the expenditure of nitrogen is greater than the income. A growing child is living below its income, is increasing its capital of flesh. In neither case is nitrogenous equilibrium present.

The starving animal, as long as life lasts, excretes kreatinin, urea and other nitrogenous substances, and gives off carbon dioxide; but its expenditure, and especially its expenditure of nitrogen, is pitched upon the lowest scale. It lives penuriously, it spins out its resources; its glycogen goes, its fat goes, a certain

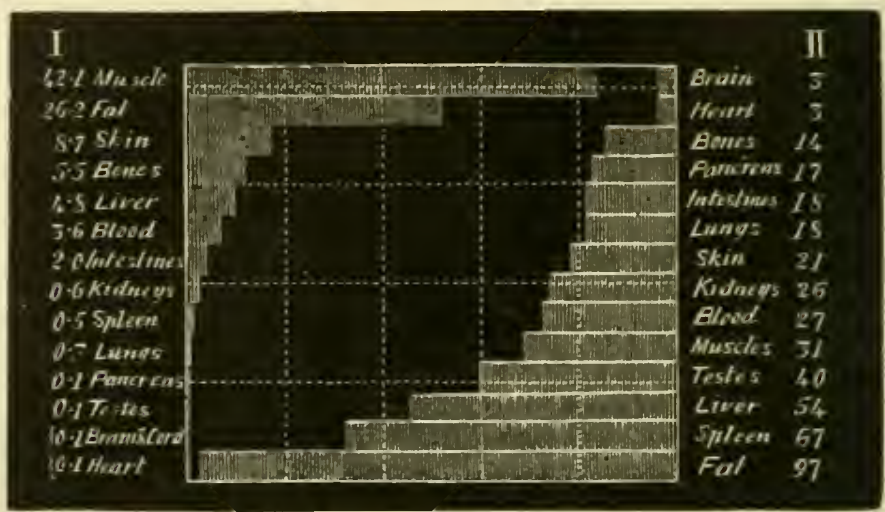

FIG. I84.-DIAGRAM SHOWTXG LOSS OF WVIGIIT OF TIIE ORGAIS IN STARVATIOS.

The numbers under I. are the percentages of the total loss of body-weight borne $b y$ the various organs and tissues. The numbers under II. give the percentage loss of weight of each organ calculated on its original weight as indicated by comparison with the organs of a similar animal killed in good condition.

part of its protein goes, and when its weight has fallen from 25 to 50 per cent., it dies. At death the heart and central nervous svstem are found to have scarcely lost in weight; the other organs have been sacrificed to feed them. Fig. I $S_{4}$ shows the percentage loss of weight and the proportion of the total loss which falls upon each of the organs of a cat in starvation (Voit).

For the first day of starvation the excretion of urea in a dog or cat is not diminished; it takes about twenty-four hours for

* For long experiments extending over many days the nitrogen balance may be considered as practically the same as the protein balance, but this is not necessarily true of short periods of time, since the stock of nitrogen present in the body in other forms than proteins, although relatively small, is subject to variations. 
all the nitrogen corresponding to the proteins of the last meal to be eliminated. On the second day the quantity of urea sinks abruptly; then begins the true starvation period, during which the daily output of urea remains constant or diminishes very slowly until a short time before death, when it rapidly falls, and soon ceases altogether. An increase in the excretion may precede the final abrupt decline (premortal increase). This seems to indicate the time at which all the available fat has been used 11p, and after which protein is no longer 'spared' by the fat.* If the animal has little fat in its body to begin with, the rise in the urea $\mathrm{cx}$ cretion takes place even after the first few days. So long as the fat lasts the rate at which it is destroyed as estimated from the amount of carbon given off minus the carbon corresponding to tlie broken-down proteins-remains very nearly constant after the first day. The fat to a certain extent economizes the proteins of the starving body, but however much fat may be present, a steady waste of the tissue-

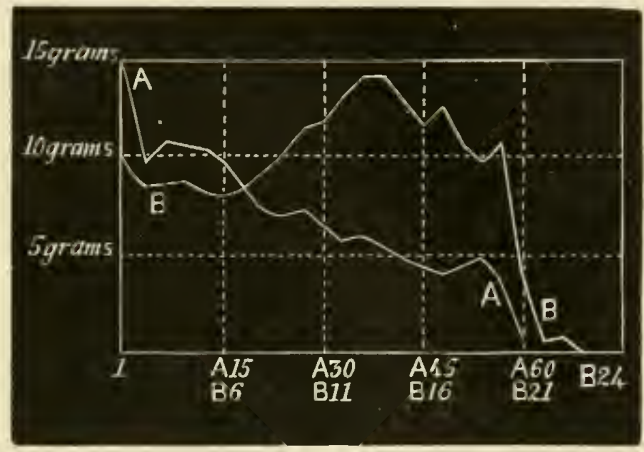

Fig, i85.-Excretion of Urea in Starvation.

$A$ is a curve representing the quantity of urea excreted daily by a fat dog in a starvation period of sixty days. $B$ is the curve of urea excre!ion in a lean young $d o g$ in a starvation period of twenty. four days. Both are constructed from Falck's numbers, but in A only every third day is put in, in order to save space. The numbers along the vertical axis represent grammes of urea; those along the horizontal axis days from the beginning of starvation.

proteins goes on. If non-nitrogenous tood in the form of sugar is supplied to an otherwise starving animal, the premortal rise in the nitrogen excretion does not occur. By giving a sufficient quantity of sugar, or of sugar and fat, but practically no protein (so-called nitrogen starvation), the excretion of nitrogen may be reduced to one-third of its amount when no food at all is given. This is true both in animals and man. In this way the daily excretion of nitrogen in a man has

* If the animal has been for some time on a diet containing an abundance of proteins, several days may elapse before the constant excretion of urea is reached; if the previous diet has been poor in protein. the constant starvation output may be at once established. 
been reduced to 4 grammes. It is a remarkable fact that while a mixture of carbo-hydrate and fat will act just as well as carbo-hydrate alone in bringing about this reduction in the nitrogen output, fat without carbo-hydrate is much less effective. The hypothesis has been suggested that the cells must have some sugar, and that when fat alone is given, a portion of the body-protein, which would otherwise have been saved, is broken down to supply the necessary sugar. (Landergren).

The results obtained on fasting men differ in some respects from those obtained on starving animals. In ten days of hunger, Cetti, a professional 'fasting man' of meagre habit, excreted II 2 grammes nitrogen, or an average of II grammes a day. The excretion was least on the eighth, ninth, and tenth daysnamely, about 9 grammes a day. On the third day it was higher than on the second, and almost as high on the fourth as on the third. A similar rise in the nitrogen excretion on the second day has been observed in other fasting men, but is either rare or absent in fasting dogs. The explanation apparently is that in the ordinary food of man there is a greater abundance of carbo-hydrates and fats, the protein-sparing action of which is most pronounced at the very beginning of the starvation period. The quantity of chlorine and alkalies in the urine was also diminished, while the phenol was increased. The respiratory quotient sank to 0.66 to 0.69 -even less than the quotient corresponding to oxidation of fats alone. The meaning of this, in all probability, is that some of the carbon of the broken-down proteins was laid up in the body as glycogen (Zuntz). In another professional fasting man (Succi) with a considerable amount of body-fat, the excretion of nitrogen was found to diminish continuously during a fast of thirty days, being less than 7 grammes on the tenth day. In another fast of 2 I days by the same person it was a little less than 3 grammes on the last day. The surprisingly small nitrogenous waste in this case is perhaps to be accounted for by the protein-sparing action of the abundant body-fat. The nitrogenous metabolism has also been investigated during long-continued hypnotic sleep (Hoover and Sollmann). The results were very much the same as in an ordinary starvation experiment.

It might be supposed that if an animal was given as much nitrogen in the food in the form of proteins as corresponded to its daily loss of nitrogen during starvation, this loss would be entirely prevented, and nitrogenous equilibrium restored. The supposition would be very far from the reality. If a dog of 30 kilos weight, which on the tenth day of starvation excreted I + grammes urea, had then received a daily quantity of protein 
equivalent to this amount - that is to say, about 34 grammes of dry protein, or 175 grammes of lean meat-the excretion of nitrogen would at once have leaped up to nearly double its starvation value. If the quantity of protein in the diet was progressively increased, the output of urea would increase along with it, but at an ever-slackening rate ; and at length a condition would be reached in which the income of nitrogen exactly balanced the expenditure, and the animal neither lost nor gained flesh.

In an experiment of Voit's, for instance, the calculated loss of flesh in a dog with no food at all was 190 grammes a day. The animal was now fed on a gradually increasing diet of lean meat, with the following result.

\begin{tabular}{|c|c|c}
$\begin{array}{c}\text { Flesh in the } \\
\text { Foid. }\end{array}$ & $\begin{array}{c}\text { Flesh used up in } \\
\text { the Body. }\end{array}$ & $\begin{array}{c}\text { Net Loss of } \\
\text { Body. Hesh. }\end{array}$ \\
\hline & 190 & 190 \\
250 & 341 & 91 \\
350 & 41 I & 61 \\
400 & 454 & 54 \\
450 & 471 & 21 \\
480 & 492 & 12 \\
\hline
\end{tabular}

The loss of nitrogen in the urine and fæees is what was measured. Knowing the average composition of 'body-flesh' (muscles, glands, etc.), it is possible to translate results stated in terms of nitrogen into results stated in terms of 'flesh.' Muscle contains approximately 3 ' 4 per cent. of nitrogen. Here, with a diet of 480 grammes of meat, the dog was still losing a little flesh; it would probably have required from 500 to 600 grammes for equilibrium. The results are graphically represented in Fig. I86, p. 535 .

The quantity of protein food necessary for nitrogenous equilibrium varies with the condition of the organism; an emaciated body requires less than a muscular and well-nourished body. The least quantity which would suffice to maintain in nitrogenous equilibrium the famous 35 kilo dog of Voit, even in very meagre condition, was 480 grammes of lean meat, corresponding to $\mathrm{I} 6$ grammes of nitrogen, or 35 grammes of urea-that is, about three times the daily loss during starvation. From this lower limit up to 2,500 grammes of meat a day nitrogenous equilibrium could always be attained, the animal putting on some flesh at each increase of diet, until at length the whole 2,500 grammes was regularly used up in the twenty-four hours. A further increase was only checked by digestive troubles. A man, or at least a civilized man, can consume a much smaller amount both absolutely and in proportion 
to the body-weight. Rubner, with a body-weight of 72 kilos, was able to digest and absorb over I, 400 grammes of lean meat; Ranke, with about the same body-weight, could only use up I,300 grammes on the first day of his experiment, and less than I, 000 grammes on the third.

So much for a purely protein diet. When fat is given in addition to protein, nitrogenous equilibrium is attained with a smaller quantity of the latter. A dog which, with protein food alone, is putting on flesh, will put on more of it before nitrogenous equilibrium is reached if a considerable quantity of fat be added to its diet. Fat, therefore, economizes protein to a certain extent, as we have already recognised in the case of the starving animal. On the other hand, when protein is given in large quantities to a fat animal, the consumption of fat is increased; and if the food contains little or none, the body-fat will diminish, while at the same time 'flesh' may be put on. The Banting cure for corpulence consists in putting the patient upon a diet containing much protein, but little fat or carbo-hydrate; and the fact just mentioned throws light upon its action.

All that we have here said of fat is true of carbo-hydrates. To a great extent these two kinds of food substances are complementary. Carbo-hydrates economize proteins as fat does, but to a greater extent, and they also economize fat, so that when a sufficient quantity of starch or sugar is given to an otherwise starving animal. all loss of carbon from the body, except that which goes off in the urea, kreatinin, etc.. still excreted, can be prevented. Of course, the animal ultimately dies, because the continuous, though diminished, loss of protein cannot be made good.

It is only necessary to add that peptone can, while gelatin cannot, replace the ordinary proteins in the food. When only enough protein is taken to prevent loss of nitrogen from the body, one-fifth of the necessary nitrogen can be supplied in the form of gelatin. When the food is much richer than this in ordinary protein, a correspondingly greater proportion of the protein can be replaced by gelatin. The surplus is not used in the endogenous metabolism of the cells (p. 497), but supplies energy to the body after the elimination of its nitrogen as urea, just as the surplus protein would do. Thus gelatin economizes protein in the same way that fat and carbo-hydrates do, but also to some extent in a different way by supplying 'building stones" for the protoplasm. Gelatin contains most of the amino-acids and other groups which compose the body-proteins, but tyrosin. tryptophane, and cystin are lacking in the gelatin molecule. It is therefore an interesting question whether gelatin can fully replace protein when these substances are given in addition. Kauffmann has shown that his own nitrogen requirement $\left(15^{*} 2\right.$ grammes) was almost completely covered by a mixture containing 93 per cent. of the nitrogen in the form of gelatin, 4 per cent. as tyrosin, 2 per cent. as cystin, and I per cent. as tryptophane. in addition to the same amounts of carbo-hydrate and fatty food as in the comparison diet, 
in which the nitrogen was supplied in the form of plasmon, a comninercial preparation of casein.

When, instead of protein, the cleavage prorlucts of pancrcatic cligestion are given to an animal, nitrogen

cquilibrium is also maintained. This has been shown for cascin. But if the casein has been hydrolysed by acid, the products will not preserve nitrogen equilibrium, perhaps because the acid has broken up all the polypepticles (p. 2), which the cells may need as the startingpoint of protein synthesis.

The Laws of Nitrogenous Metabolism.-Within the limits of nitrogenous equilibrium, which is the normal state of the healthy adult, the body lives up to its income of nitrogen; it lays by nothing for the future. In the actual pinch of starvation the organism becomes suddenly economical. When a plentiful supply of protein is presented to the starving body, it seems to pass at once from extreme frugality to luxury; some flesh may be put on for a short time, some nitrogen may be stored up; but the consumption is soon adjusted to the new scale of supply, and the protein income spent as freely as it is received. This is the first great law of nitrogenous metabolism, and we may formulate it thus: Consumption of protein is largely determined by supply (Practical Exercises, p. 612).

The explanation of this remarkable fact, or at least an essential part of the explanation, has already been given in defining the differences between exogenous and endogenous protein katabolism (p. 497). It is to the exogenous process, and practically to that alone, that the law applies. We have seen that a large proportion of the nitrogen of the food-proteins split off by hydrolysis in the lumen of the alimentary canal, the intestinal wall, and probably also in the liver, passes by a short-cut to the stage of urea without ever joining the protein of the blood, much less that of the organs.

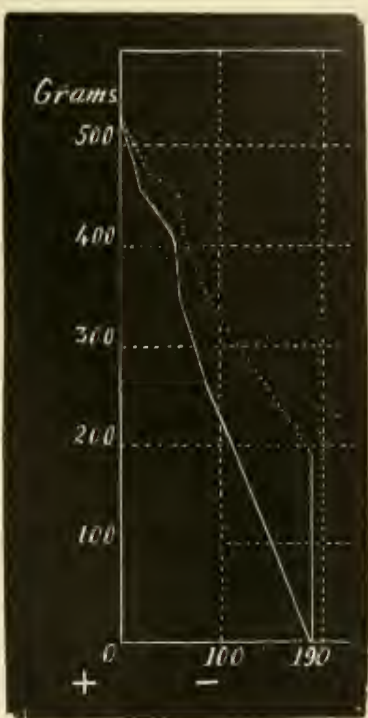

F1G. I86. - CURVES CONSTRUCTED TO ILLUSTRATE Nitrogenous Eouilib. RIUM (FROM AN EXPERIMENT OF VOIT'S).

The loss of flesh in grammes is laid off along the horizontal axis. The income and expenditure corresponding to a given loss are laid off (in grammes of 'flesh') along the vertical axis. The con. tinuous curve is the curve of income; the dotted curve, of expenditure. With no in. come at all the expenditure is I9o grammes; with an inconle of +80 grammes the expenditure is 492 and the loss I2 grammes. Nitrogenous equilibrium is represented as being reached witl an income of about 530 grammes ; here the two curves cut one another.

This is not a form of true 'luxus-consumption.' The expenditure is apparently but not 
really wasteful, since the greater pat of the energy of the protein molecule is still obtained by the oxidation of the carbon-rich residuc. The surplus nitrogen is shunterl out of the main metabolic current at its very source. Some writers conceive that in such a slort-cut from protein to urea we hate a kind of pliysiological safety-ralve to protect the tissues from the burden of an excessive motabolism. And if by this is meant that it is arlvantageous to the tissues that a special mechanism should exist to climinate at surplus of nitrogen which they do not require, and which they cannot store, and to present them with a residue which they can utilize, the conception is certainly correct. But there is no good evidence that in the presence of an over-abundant supply of protein the endogenous protein metabolism woukl be essentially morlified. And another interpretation of the preliminary splitting off and elimination of nitrogen, which must under ordinary conditions of diet account for a considerable formation of urea, has becn alluded to in discussing the influence of the food on the serum-proteins (p. 498). The surplus of those amino-acids which the fool-proteins contain in greater quantity than the boly-protcins cannot be utilizel for building protein in the tissues. Also at any moment the magnitude of this non-utilizable surplus will depend upon the quantity of that one of the indispensable amino-acids which is present in the smallest amount. For the proper proportion nust be preserved between the clifferent 'stones' out of which the moleculc is built. When a single amino-aciel is introduced into the body. it is at once changed into urea and excreted, since it cannot be utilized by itself for building up protein.

The relatively small and constant amount of the endogenous metabolism indicates that the actual protoplasmic substance, the living framework of the cell, is comparatively stable; that it clocs not break down rapidly; and that only a small and fairly constant amount of food- or circulating-protein, or of the decomposition products of protein, is required to supply the waste of the organprotein.

Other explanations of the relation between consumption and supply of protein have been put forwarl. Although some of these may now possess merely a historical interest, they must be mentioned, all the more as it has not been shown that the production of urea in the liver from decomposition products of proteins brought to it in the portal blood is the only form of exogenous protein katabolism. The famous theory of Voit assumes that the fooci-protcin after absorption (the so-called 'circulating-protein') is carried to the tissucs and taken up by the cells, where the greater part of it, without being incorporated with the protoplasm, is nevertheless acted upon, rendered unstable, shaken to pieces, as it were, by the whirl of life in the organized framework, the interstices of which it fills.

Pflüger, on the other hand, has maintained that we have no right to draw a distinction between the consumption of organ- and circulating-protein; that the whole of the latter ultimately rises to the height of organ-protein. and passes on to the rlownwarcl stage of metabolism only through the topmost step of organization. An increase in the supply of nitrogenous material in the blool must, on this riew: be accompanjed with an increased tendency to the break11), the dissociation, as Pfligger puts it, of the living substance. The actual organized clements, however, the existing cells, are not 
supposed to be clestroyed; the buileling remains, for although stones are constantly crumbling in its walls, others are being constantly built in.

A much less platusible view is that the tissue clements themselves are short-livel; that the old eclls disappear bolily and are replacel by new cells; and that the whole of the proteins of the food take pat in this process of total ruin and reconstruction. Histolngical evidence is strongly against this idea. Although the cells of certain glands, such as the mammary, and perhaps the mucous glands, exhibit changes which, hastily interpreted, might scem to inclicate that, like those of the sebacenus glands (1) 473), they break down bodily, as an incident of functional activity, there is no proof of the production of new cells on the immense scale which this theory would require.

A second law of nitrogenous metabolism is that within normal linits it is nearly independent of muscular work-that is to say, the quantity of nitrogen excreted by a man on a given diet is practically the same whether he rests or works. Before this was known it was maintained by Liebig that proteins alone could supply the energy of muscular contraction-that, in fact, proteins were solely used up in the nutrition and functional activity of the nitrogenous tissues, while the non-protein food yielded heat by its oxidation. As exact experiments multiplied, it was found that muscular work, the production of which is the function of by far the greatest mass of protein-containing tissue, had little or no effect upon the excretion of urea in the urine. More than this, it was shown that a certain anount of work accomplished (by Fick and Wislicenus in climbing a mountain) on a non-nitrogenous diet had double the heat equivalent of the whole of the protein consumed in the body, as estimated by the urea excreted during, and for a given time after, the work. On the assumption that all the urea corresponding to the protein broken down was eliminated during the time of this experiment, a part at least of the work must have been derived from the energy of non-nitrogenous material. And the increase in the carbon dioxide given off, which is as conspicuous an accompaniment of muscular work as the constancy of the urea excretion, showed that during muscular exertion carbonaceous substances other than proteins - that is to say, fats and carbohydrates-are oxidized in greater amount than during rest.

So the pendulum of physiological orthodoxy came full-swing to the other side. Liebig and his school had taught that proteins alone were consumed in functional activity; the majority of later physiologists have denied to the proteins any share whatever in the energy which appears as muscular contraction. The proteins, they say, "repair the slow waste of the framework of the muscular machine, replace a loose rivet, a worn-out belt, as occasion may require; the carbo-hydrates and fats are 
the fucl which feeds the furnaces of life, the material which, dead itself, is oxidized in the interstices of the living substance, and yields the energy for its work.'

Now, it is a singular circumstance, and full of instruction for the ingenuous student of science, that the facts which have been supposed absolutely to disprove the older theory, and absolutely to establish its modern rival, do neither the one thing nor the other. The fact--ind it is a fact-that the excretion of nitrogen is but little affected by muscular contraction, does not prove that none of the energy of muscular work comes from proteins ; the fact that, under certain conditions, some of the muscular energy must apparently come from non-nitrogenous materials, does not prove that these are the normal source of it all. The distinction has again been made too absolute. The pendulum must again swing back a little; and the experiments of Pflüger and others have actually set it moving.

In the first place, it is not perfectly correct to say that work causes no increase in the excretion of nitrogen; excessive work in man, and work, severe but not excessive, in a flesh-fed dog (Pfliiger), do cause somewhat more nitrogen to be given off. On the first day of work the increase is always much less than on the second and third; and on the first and second rest days, following work, the elimination of nitrogen is still increased. After excessive exercise in man not only is the urea increased, but also the ammonia, kreatinin, and if the subject is in poor training, the uric acid and purin bases (Paton, Stockman, etc.). Moderate exercise causes no increase on the first day, but a slight increase on the second.

In the second place, even if the excretion of nitrogen were entirely unaffected by work, this would not prove that none of the energy of the work comes from proteins. For, as we have seen, it is after the nitrogen has been split off and converted into urea that the energy of a great part of the food-protein is developed by oxidation. Further, since the animal body is a beautifully-balanced mechanism which constantly adapts itself to its conditions, it is conceivable that it may, when called upon to labour, save proteins from lower uses to devote them to nuscular contraction. In this case the excretion of nitrogen would not necessarily be altered; the proteins which, in the absence of work, would have been oxidized within the muscular substance or elsewhere, their energy appearing entirely as heat, nay, when the call for protein to take the place of that broken down in muscular contraction arises, be diverted to this purpose.

In any case, there is no doubt that a dog fed on lean meat may go on for a long time performing far more work than can be 
vielded by the energy of fat and carbo-hychates occurring in traces in the food, of taken from the stock in the animal's body at the beginning of the period of work. A large portion, and perhaps the whole, of the work, must in this case be derived from the energy of the proteins (Pfliiger). On the other hand, it is well established that when fats and carbo-hydrates are present in sufficient quantity in the tissues or the food, they constitute the main source of the energy of muscular contraction (p. 060$)$.

Experience has shown that the minimum quantity of nitrogen repuired in the food of a man whose daily work involves hard physical toil is higher than the minimum required by a person leading an easy, sedentary life. This is evidently in accordance with the view that protein is actually used up in muscular contraction; but it is not inconsistent with the opposite view. For the body of a man fit for continuous hard labour has a greater mizss of muscle to feed than the body of a man who is only fit to handle a composing-stick, or drive a quill, or ply a needle ; and the greater the muscular mass, the greater the muscular waste. But if an animal just in nitrogenous equilibrium on a diet of lean meat when doing no work is made to labour day after day, it will lose flesh unless the diet be increased. This must mean that some of the protein is being diverted to muscular work, and that the balance is not sufficient to keep up the original mass of 'flesh' (see p. 548).

(2) Income and Expenditure of Carbon.-This division of the subject has been necessarily referred to in treating of the nitrogen balance-sheet, and may now be formally completed.

Carbon Equilibrium. - A body in nitrogenous equilibrium may or may not be in carbon equilibrium. It has been repeatedly pointed out that the continued loss or gain of carbon by an organism in nitrogenous equilibrium means the loss or gain of fat; and, since the quantity of fat in the body may vary within wide limits without harm, carbon equilibrium is less important than nitrogen equilibrium. It is also less easily attained when the carbon of the food is increased, for, the consumption of fat is not necessarily increased with the supply of fat or fat-producing food, and there is by no nueans the same prompt adjustment of expenditure to income in the case of carbon as in the case of nitrogen.

Carbon equilibrium can be obtained in a flesh-eating animal, like a dog, with an exclusively protein diet; but a far higher minimum is required than for nitrogenous equilibrium alone. Voit's dog required at least I,500 grammes of meat in the twentyfour hours to prevent his body from losing carbon. For a man weighing 70 kilos, the daily excretion of carbon on an ordinary 
diet is 250 to 300 grammes. About 2,000 grammes of lean meat would be required to yield this quantity of carbon; and, 'ven if sucl a mass could he cligested and absorbed, more than three times the necessiry nitrogen would have to undergo preliminary cleavage and excretinn as urea or be thrown upon the tissues.

Not only may carbon equilibrium be maintained for a short time in a dog on a diet containing fat only, or fat and carbohydrates, but the expenditure of carbon may be less thas the income, and fat may be stored up. But, of course, if this diet is continued, the animal ultimately dies of nitrogen starvation.

So far we lave spoken only of the income and expenditure of carbon and nitrogen; and from these data alone it is possible to deduce many importailt facts in metabolism, since. knowing the elementary composition of proteins, fats, and carbo-hydrates, we can, on certain assumpticns, translate into terms of proteins or fat the gain or loss of an organism in nitrogen and carbon, or in carbon alone. But the bydrogen and oxygen contained in the solids and water of the food, and the oxygen taken in by the lungs, are just as important as the carbon and nitrogen; it is just as necessary to take account of them in drawing up a complete and accurate balance-sheet of nutrition. Fortunately, however, it is permissible to devote much less time to them here, for when we have determined the quantitative relations of the absorption and excretion of the carbon and nitrogen, we have also to a large extent determined those of the oxygen and hydrogen.

(3) Income and Expenditure of Oxygen and Hydrogen.The oxygen absorbed as gas and in the solids of the food is given off chiefly as carbon dioxide by the lungs; to a small extent as water by the lungs, kidneys, and skin; and as urea and other substances in the urine and faeces. The hydrogen of the solids of the food is excreted in part as urea, but in far larger amount as water. The hrdrogen and oxygen of the ingested water pass off as water, without, so far as we know, undergoing any chemical change, or existing in any other form within the body. But it is important to recognise that although none of the water taken in as such is broken up, some water is manufactured in the tissues by the oxidation of hydrogen. Ne have already considered (p. 242) the gaseous exchange in the lungs, and we have seen that all the oxygen taken in does not reappear as carbon dioxide. It was stated there that the missing oxygen goes to oxidize other elements than carbon, and especially to oxidize hydrogen. We have now to explain more fully the cause of this oxygen deficit. 
The Oxygen Deficit. - The carbo-hydrates contain in themselves enough oxygen to form water with all their hydrogen; they account for a part of the water-formation in the body, but for none of the oxvgen deficit.

The fats are very different; their hydrogen can be nothing like completely oxidized by their oxygen. A gramme of hydrogen is contained in 8.5 grammes of dry fat, and needs 8 grammes of oxygen for its complete combustion. Only I gramme of oxygen is yiclded by the fat itself ; so that if a man uses Ion granmes of fat in twentyfour hours, rather more than 80 grammes of the oxygen taken in must go to oxidize the hydrogen of the fat.

The proteins also contribute to the deficit. In 100 grammes of dry proteins there are 15 grammes of nitrogen, 7 grammes of hydrogen, and 2 I grammes of oxygen. The carbon does not concern us at present. The 33 grammes of urea, corresponding to 100 grammes of protein, contains 15 grammes of nitrogen, a little more than 2 grammes of hydrogen, and a little less than 9 grammes of oxygen. There remain 5 grammes of hydrogen and 12 grammes of oxygen. But 5 grammes of hydrogen needs for complete combustion 40 grammes of oxygen; therefore 28 grammes of the oxygen taken in must go to oxidize the hydrogen of 100 grammes of protein. Taking 140 grammes of protein as the amount in a libcral diet for a man, we get 39 grammes as the required quantity of oxygen. This, added to the 80 grammes needed for the hydrogen of the fat, makes a total of, say, 120 grammes, equivalent to about 85 litres of oxygen. A small amount of oxygen also goes to oxidize the sulphur of proteins.

With a dict containing less fat and protein and more carbohydrate, the oxygen deficit would of course be less.

The Production of Water in the Body.--One gramme of hydrogen corresponds to 9 grammes of water. In 140 grammes of proteins and Ioo grammes of fat there are, in round numbers, 22 grammes of hydrogen; in 350 grammes of starch, $2 I^{\prime} 5$ grammes. With this diet, 43.5 grammes of hydrogen is oxidized to water within the body in twenty-four hours, corresponding to a water production of $39 \mathrm{I}$ grammes, or 15 to 20 per cent. of the whole excretion of water. It has been observed that during starvation the tissues sometimes become richer in water, even when none is drunk. The only explanation is, that the elimination of water does not keep pace with the rate at which it is produced from the hydrogen of the broken-down tissue-substances, or set free from the solids with which it is (physically ?) united.

Inorganic Salts.-The inorganic salts of the excreta, like the water, are for the most part derived from the salts of the food, which do not in general undergo decomposition in the body. A portion of the chlorides, however, is broken up to yield the hydrochloric acid of the gastric juice. Within the body some of the salts are more or less intimately united to the proteins of the tissues and juices, some simply dissolved in the latter. The chlorides, phosphates and carbonates are the most important; the potassium salts belong especially to the organized tissue elements, the sodium salts to the liquids of the body; calcium phosphate and carbonate predominate in the bones. The amount and composition of the ash of each organ only change 
within narrow limits. In hunger the organism clings to its inorganic materials, as it clings to its tissue-proteins; the former are just as essential to life as the latter. In a starving animal chlorine almost disappears from the urine at a time when there is still mucl chlorine in the body; only the inorganic salts which have been united to the used-up) proteins are excreted, so that a starving animal never dies for want of salts.

W'hen sodium chloride is omitted as an addition to the food of man, the decomposition of protein seems to be slightly accelerated, but for a time, at least, there are no serious symptoms (Belli). The Hereros in Damaraland, who are physically one of the finest races in Africa, are said not to nse salt (Rérlns). On the other hand, when an animal is fed with a dict as far as possible artificially freed from salts, but otherwise sufficient. it dies of salt-hunger. The blood first loses inorganic material, then the organs. The total loss is very small in proportion to the quantity still retained in the body: but it is sufficient to canse the cleath of a pigeon in three weeks. and of a $\log$ in six, with marked symptoms of muscular and nervous weakness. A deficiency of lime salts causes changes particularly in the skeleton, although the nutrition of the rest of the body is also interfered with. These changes are of course most marked in young animals, in which the bones are growing rapidly. In pigeons on a diet containing very little calcium the bones of the skull and the sternmm become extremely thin and riddled with holes, while the bones concerned in movement scarcely suffer at all (E. Voit).

It is not indifferent in what form the calcium is talien, nor can it be replaced to any great cxtent by other earthy bases, as magnesium or strontium. Weislie fed five young rabbits of the same litter on oats, a food relatively poor in calcium. One of the rabbits received in addition calcium carbonate, another calcium sulphate, a third magnesium carbonate, and a fourth strontium carbonate. At the end of a certain time it was found that the slieleton of the rabbit fed with calcium carbonate was the heaviest and strongest of all. and contained the greatest proportion of mineral matter. Then came the rabbit fed with calcium sulphate. The animal which received only oats had the worst-developed skeleton: the condition of the animals fed with magnesium and strontium carbonates was but little better.

Milk is a food rich in calcium and also in phosphorus, a circumstance evidently related to the rapid development of the skeleton in the young child. As in the other natural foods, the ralcium and phosphorus are partly in the form of organic com. pounds, united with the proteins, the calcium especially with caseinogen, and partly in the form of inorganic salts. Both of these elements are more easily assimilated by the body in the 
organic than in the inorganic form. And the same is true of iron, which exists in organic combination in the bran of wheat, in the hemoglobin of the blood and of muscular fibres, in the nuclei of most cells, vegetable and animal, and conspicuously in the nuclein of the yolk of the egg. Attempts have been made to increase the amount of iron in hen's eggs by giving them food mixed with preparations of iron-e.g., iron citrate. An increase takes place, but only after a long time. Thus in one experiment Ioo grammes of egg-substance contained 4.4 milligrammes of $\mathrm{Fe}_{2} \mathrm{O}_{3}$ before the administration of the iron was begun; after feeding with iron for three and a half weeks the amount was 4.5 milligrammes, after more than two months 7.4 milligrammes; and after a year only $7 \cdot 3$ milligrammes. Although, as we have seen, inorganic iron can be absorbed, it is certainly the case that under ordinary conditions all the iron that the body receives or needs is taken in the form of organic compounds, since there is no inorganic iron in the natural food substances. Stockman, from careful estimations of the quantity of iron in a number of actual dictaries, finds that it only amounts to about 8 to ro milligrammes a day. He concludes that the greater part of it must be retained in the body and used over and over again.

Milk is poor in iron, but this does not hinder the development of the young child, except when it is weaned too late, when it is apt to become anæmic unless the milk is supplemented with a food rich in iron, such as yolk of egg. The explanation is that the foetus, especially in the last three months of intrauterine life, accumulates a store of iron in the liver and other organs; so that, in proportion to its body-weight, it is at birth several times richer in iron than the adult. This iron, of course, all comes from the mother, and the loss is not exactly balanced by the excess of iron in her food; certain of her organs, the spleen, for instance, though not apparently the liver, are impoverished as regards their content of iron.

\section{DIETETICS.}

There are two ways in which we can arrive at a knowledge of the amount of the various food substances necessary for an average man : (a) By considering the diet of large numbers of people doing fairly definite work, and sufficiently, but not extravagantly, fed-e.g., soldiers, gangs of navvies, or plantation labourers; $(b)$ by making special experiments on one or more individuals.

Voit, bringing together a large number of observations, concluded that an 'average workman,' weighing 70 to 75 kilos, and working ten hours a day, required in the twenty-four hours 
I 8 grammes protein, $5^{6}$ grammes fat, and 500 grammes carbohydrate, corresponding to about 18.8 grammes* nitrogen, and at least 328 grammes carbon.

Ranke found the following a sufficient dict for himself, with a body-weight of $7+$ kilos :

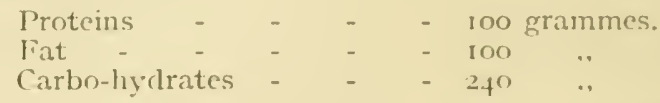

This corresponds to only I6 grammes nitrogen and, say, 230 grammes carbon.

A German soldier in the ficld receives on the average:

$\begin{array}{llllll}\text { Proteins } & - & - & - & \text { I } 5 \text { grammes. } \\ \text { Fat - } & - & - & - & f^{6} & ., \\ \text { Carbo-hydrates } & - & - & - & 522 & .,\end{array}$

representing about 24 grammes nitrogen and 340 grammes carbon. The average ration for four British regiments in peacetime contained I33 grammes protein, II 5 grammes fat, and $+2+$ grammes carbo-hydrate ( $=3,400$ calories). But in addition the soldiers constantly obtained at their own expense a supper, generally comprising meat (Pembrey). The Russian army war ration in the Manchurian campaign is said to have comprised I87 grammes protein and 775 grammes carbo-hydrate, but only 27 grammes fat ( $=4,900$ calories). The diet of certain miners (Steinheil) and lumberers (Licbig) contained respectively I 33 and II 2 grammes protein, II 3 and 309 grammes fat, and 634 and 60I grammes carbo-hydrates. The diet of prize-fighters and of athletes in training is richer in protein than any of thesc. The members of two college football teams are stated to have consumed on the average 225 grammes protein, $33+$ grammes fat, and 633 grammes carbo-hydrates $(=6,800$ calories). Caspari, from a study of the phenomena of training, concluded that continuous bodily work at a rate above the ordinary requires a large amount of protein (2 to 3 grammes a day per kilo of body-weight). But there seems to be a considerable difference between different individuals. So that a definite and typical diet for severe labour does not exist. And although perhaps the hardest physical work ever done in the world is to break athletic records, to cut and handle timber, to mine coal, and to make war, the dict on which these things are accomplished is very variable.

Recent observations tend to reduce the amount of protein considered necessary for a person under ordinary conditions. Sivén remained in nitrogen equilibrium, for a time at least, with an intake of only 0.07 to 0.08 gramme of nitrogen $(0.4$ to 0.5 gramme of protein) per kilo of body-weight, or not much more

* Taking the percentage of nitrogen in protein at 16. 
than one-third of the amount in Ranke's diet. It is obvious that the endogenous protein katabolism sets the limit below which it must be impossible permanently to reduce the allowance of protein. But it would be very hazardous to assume that this theoretical minimum limit corresponds with the permissible physiological limit. From experiments on men of various callings extending over many months, Chittenden has concluded that the average man eats at least twice as much protein as he really requires. We have already seen that the amount of nitrogen required to repair the actual waste of the tissues is comparatively small, and the with the ordinary amount of protein in the food a very large fraction of the total nitrogen is rapidly excreted as urea. There is no doubt, also, that many persons consume too much protein, at any rate in the form of animal food, and would feel better, work better, and probably live longer, if they restricted themselves in this regard. But there is no evidence that the digestion of such quantities of protein as the average healthy man eats, or the elaboration and excretion of the corresponding amounts of urea, 'strain' in the least the digestive apparatus, the liver, or the kidneys. And it may just as well be argued that it is advantageous that much more than the minimum protein requirement should be offered to the tissues, so that the appropriate amino-acids, even the scarcest of them, may be sure to be present in sufficient amount, rather than that the organs should be subjected to the unnecessary 'strain' of reconstructing some of the amino-acids themselves, supposing that they possess this power. In a question of this sort the immemorial experience and instinct of mankind cannot be lightly waved aside.

If we decide the matter merely on physiological grounds, we may say that for a man of 70 kilos, doing fairly hard, but not excessive, work, I5 grammes nitrogen and 250 grammes carbon are a sufficient allowance. The $5_{5}$ grammes nitrogen will be contained in 95 grammes dry protein, which will also yield 50 grammes of the required carbon. The balance of 200 grammes carbon could theoretically be supplied either in $45^{\circ}$ grammes starch or in 260 grammes fat. But it has been found by experiment and by experience (which is indeed a very complex and proverbially expensive form of experiment) that for civilized man a mixture of these is necessary for health, although the nomads of the Asian steppes, and the herdsmen of the Pampas, are said to subsist for long periods on flesh alone, and a dog can live very well on proteins and fat. The proportion of fat and carbo-hydrates in a diet may, however, be varied within wide limits. Probably no 'work' diet should contain much less

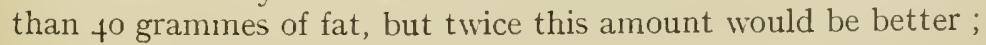


80 grammes fat give aloout 60 grammes carbon, so that from proteins and fat we have now got I Io grammes of the necessary 250 , leaving 1 fo grammes carbon to be taken in about 310 grammes starch, or an equivalent amount of cane-sugar or dextrose. Adding 30 grammes inorganic salts, we can put down as the solid portion of a normal diet sufficient from the physiological point of view for a man of 70 kilos :

\begin{tabular}{|c|c|c|c|c|}
\hline 95 & grammes & proteins & $=-100$ & $y-11$ \\
\hline So & ,, & fat & $=n \frac{1}{1}$ & , \\
\hline 310 & ," & carbo-hydrates & $=$ & ". \\
\hline 30 & , & salts. & & \\
\hline 525 & ,. & solid food & $=1 !: 0$ & . \\
\hline
\end{tabular}

Now, knowing the composition of the various food-stuffs, we can easily construct a diet containing the proper quantities of nitrogen and carbon, by using a table such as the following:

\begin{tabular}{|c|c|c|c|c|c|c|c|c|}
\hline & $\begin{array}{c}\text { Quantity } \\
\text { required } \\
\text { to yield } \\
15 \text { Grms. } \\
\mathrm{N} .\end{array}$ & $\begin{array}{l}\text { Quantity } \\
\text { required } \\
10 \text { yield } \\
250 \text { Grm. } \\
\text { C. }\end{array}$ & $\begin{array}{c}\mathrm{N} \text { in } \\
100 \\
\text { Grms. }\end{array}$ & $\begin{array}{l}\mathrm{C} \text { in } \\
100 \\
\text { Grms. }\end{array}$ & $\begin{array}{l}\text { Protein } \\
\text { in too } \\
\text { Grms. }\end{array}$ & $\begin{array}{l}\text { Fat in } \\
100 \\
\text { Grins. }\end{array}$ & $\begin{array}{l}\text { Carbo- } \\
\text { hydrate } \\
\text { in } 100 \\
\text { Grms. }\end{array}$ & $\begin{array}{l}\text { Water } \\
\text { in } 100 \\
\text { Grms. }\end{array}$ \\
\hline $\begin{array}{l}\text { Cheese* } \\
\text { (Gruvere) }\end{array}$ & 300 & & & & & & - & \\
\hline Peas (dried) & $\begin{array}{l}300 \\
430\end{array}$ & $\begin{array}{l}640 \\
700\end{array}$ & $\begin{array}{l}5 \\
3.5\end{array}$ & $\begin{array}{l}39 \\
35.7\end{array}$ & $\begin{array}{l}31 \\
22\end{array}$ & $\begin{array}{r}31 \\
2\end{array}$ & 55 & $\begin{array}{l}34 \\
15\end{array}$ \\
\hline Lean meat & 4.40 & I 860 & $3+1$ & 13.5 & 21 & $3 \cdot 5$ & - & 74 \\
\hline Wheat-flour & 650 & 625 & $2 \cdot 3$ & 39.8 & 12 & 2 & 70 & 15 \\
\hline Oatmeal & 580 & 620 & $2 \cdot 6$ & $40 \cdot 3$ & 13 & $5 \cdot 5$ & 65 & 15 \\
\hline Eggs & 790 & 1700 & $I^{\prime} 9$ & $1+7$ & II' 5 & 12 & 一 & 75 \\
\hline Maize - & SIO & 610 & $1 \cdot 85$ & $40^{\circ} 9$ & I0 5 & 7 & 65 & I 5 \\
\hline Wheat & $1=00$ & & & & $s$ & & & \\
\hline Rice - & $\begin{array}{l}1200 \\
1530\end{array}$ & $\begin{array}{r}\text { I I } 20 \\
685\end{array}$ & $\begin{array}{l}1.25 \\
0.9\end{array}$ & $\begin{array}{l}22.4 \\
36.6\end{array}$ & $\begin{array}{l}3 \\
5\end{array}$ & $\begin{array}{l}1 \\
1\end{array}$ & $\begin{array}{l}49 \\
8_{3}\end{array}$ & $\begin{array}{l}40 \\
10\end{array}$ \\
\hline Milk & 2380 & $35+0$ & 0.6 & 7 & 4 & 4 & 5 & $\mathrm{~S}_{5}$ \\
\hline Potators & 3750 & 2350 & 0.4 & 10.5 & 2 & $0: 15$ & 21 & 75 \\
\hline Good butter & 10000 & 360 & 0.15 & 69 & I & 90 & - & s \\
\hline
\end{tabular}

Economic and social influences-prices and habits-and not purely physiological rules, fix the cliet of populations. The Chinese labourer, for example, lives on a diet which no physiologist would commend. In order to obtain 15 grammes nitrogen or 95 grammes protein, he must consume more than I.500 gramme's rice, which will yield 700 grammes carbon, or twice as much as is required; but if the Chinese labourer could not live on rice, he could not live at all. The Irish peasant is even in worse case; he must consume nearly + kilos of potatoes to obtain his 15 grammes

* A cheese manufactured from whole milli, curelled before the cream has had time to rise, and therefore rich in tat. 
nitrogen, while little more than half this amount would furnish the necessary 250 grammes carbon. Of course a diet consisting, week in week out, entirely of potatoes or rice, would represent an extreme case. A certain amount of the necessary nitrogen is obtained even by the poorest populations, in the form of fish, milk, eggs, or bacon. A man attempting to live on flesh alone would be well fed as regards nitrogen with 500 grammes of meat, but nearly four times as much would be required to yield 250 grammes of carbon. Oatmeal and wheat-flour contain nitrogen and carbon in nearly the right proportions ( $\mathrm{N}: \mathrm{I}_{5} \mathrm{C}$ ), oatmeal being rather the better of the two in this respect; and the best-fed labouring populations of Europe still live largely on wheaten bread, while, one hundred years ago, the Scotch peasant still cultivated the soil, as the Scotch Reviewer the IIuses, 'on a little oatmeal.' But although bread may, and does, as a rule, form the great staple of diet, it is not of itself sufficient.

It is necessary to recognise that habit has much to do with the quantity as well as with the quality of the food used by an individual or a community: Some concession may be made to custom in what is, after all, not a purely physiological question, and in this country it is probable that 20 grammes of nitrogen and 300 grammes of carbon, while a liberal is not an excessive allowance, although it is certain that a man can maintain a normal body-weight and perform a normal amount of work on considerably less, in some cases even with advantage to his health.

We may take 500 grammes of bread and 250 grammes of lean meat as a fair quantity for a man fit for hard work. Adding 500 grammes milk, 75 grammes oatmeal (as porridge), 30 grammes butter, 30 grammes fat (with the meat, or in other ways), and 450 grammes potatoes, we get approximately 20 grammes nitrogen and 300 grammes carbon contained in I35 grammes protein, rather less than Ioo grammes fat, and somewhat over too grammes carbo-hydrates. Thus-

\begin{tabular}{|c|c|c|c|c|c|c|}
\hline & $x$ & c. & Proteins. & Fat. & $\begin{array}{c}\text { Carbo- } \\
\text { hydrates. }\end{array}$ & Salts. \\
\hline (9 oz.) 250 grms. lean meat & 8 & 33 & 55 & $8 \cdot 5$ & - & 4 \\
\hline (Is uz.) 500 grms. bread & 6 & II 2 & 40 & $7 \cdot 5$ & 245 & 6.5 \\
\hline ( 3 pint) 500 grms. milk & 3 & 35 & 20 & 20 & 25 & 3.5 \\
\hline (I oz.) 30 grins. butter - & - & 20 & - & 27 & - & 0.5 \\
\hline (I oz.) 30 grms. fat - & - & 22 & - & 30 & - & - \\
\hline (I 6 oz.) +50 grms. potatues & I. 5 & 47 & Io & - & 95 & 4.5 \\
\hline \multirow[t]{2}{*}{ (3 uz.) 75 grms. oatmeal } & $1 \cdot 7$ & 30 & Io & 4 & $4^{5}$ & 2 \\
\hline & $20 \cdot 2$ & 299 & 135 & 97 & 413 & $2 I$ \\
\hline
\end{tabular}

This would be a fair 'hard work' diet for a well-nourished labourer. But the great elasticity of dietetic formulæ is shown 
in the following tables, which give the ration of the German soldier in peace and war and the minimum allowance per " statute adult 'prescribed in the British regulations concerning passenger ships from Great Britain to America.

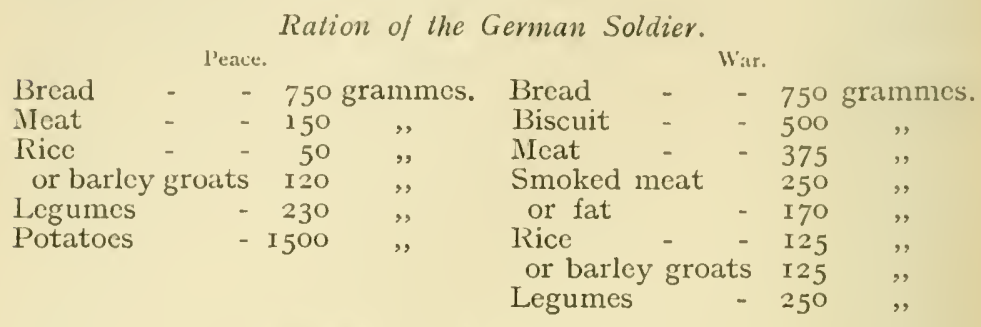

Minimum Ration for Passenger Ships.

\begin{tabular}{|c|c|c|c|c|c|c|}
\hline \multicolumn{2}{|c|}{ Bread or biscuit } & - & - & - & 227 & grammes \\
\hline \multicolumn{2}{|c|}{ Wheaten flour } & - & - & - & 65 & ", \\
\hline or bread & - & - & - & $\begin{array}{l}- \\
-\end{array}$ & SI & , \\
\hline Oatmeal & - & - & - & - & 97 & , \\
\hline Rice & - & - & - & - & 97 & , \\
\hline Peas - & - & - & - & - & 97 & , \\
\hline Potatoes & - & - & - & - & 130 &, \\
\hline Beef - & - & - & - & - & $S_{I}$ & , \\
\hline \multicolumn{4}{|c|}{ Pork or preserved meat } & - & 65 & , , \\
\hline \multirow{2}{*}{$\begin{array}{l}\text { Sugar - } \\
\quad \text { or trcacle }\end{array}$} & - & - & - & - & 05 & , \\
\hline & - & - & - & - & 97 & , \\
\hline \multirow{2}{*}{$\begin{array}{l}\text { Tea } \\
\text { or coffee }\end{array}$} & - & - & - & - & 8 & , \\
\hline & or coc & & - & - & I 4 & , \\
\hline Salt - & - & - & - & - & S & , \\
\hline Mustard & - & - & - & - & 2 & , \\
\hline \multirow{2}{*}{$\begin{array}{l}\text { Pepper- } \\
\text { Vinegar or }\end{array}$} & - & - & - & - & I & , \\
\hline & pickl & & - & - & 20 & c.c. \\
\hline
\end{tabular}

In prisons the object is to give the minimum amount of the plainest food which will suffice to maintain the prisoners in health. A 'hard work' prison diet in Munich was found to contain 104 grammes proteins, $3 \mathrm{~S}$ grammes fat, and $52 \mathrm{I}$ grammes carbohydrates ; a 'no-work' diet, only 87 grammes proteins, 22 grammes fat, and 305 grammes carbo-hydrates. Here we recognise the influence of price; carbon can be much more cheaply obtained in vegetable carbo-hydrates than in animal fats; the cleapest possible diet contains a minimum of animal fat and proteins.

Iany poor persons live on a dict which would not maintain a strong man, for an emaciated body has a smaller mass of flesh to licep up, and therefore needs less protein ; it can clo little work, and therefore needs less food of all kinds. A London needlewoman, according to Playfair, subsists, or did subsist thirty years ago, on 54 grammes protein, 29 grammes fat, and 292 grammes carbolnydrates. But this is the irreducible minimum of the decpest poverty, not so much in the protein content, perhaps, as in the very low heat equivalent ( $I, 600$ calories); and a woman, with a smaller mass of flesh and leading a less active life than a man, requires less food of all sorts. Even the Trappist monk, who has reduced asceticism to a science, and, instead of eating in order to 
live, lives in order not to eat, consumes, according to Vnit, 6.8 grammes protein, I I grammes fat, and 469 grammes carbo-hydrates ; but manual labour is a part of the discipline of the brotherhood, and this must be still above the lowest subsistence diet.

The question whether it is best to derive the proteins (and fats) of the food mainly from plants or mainly from animals is one which is never left to physiology alone to decide. But it has been definitely proved that vegetable proteins and vegetable fats are (when properly prepared) digested and absorbed as completely as those of animal origin, and play the same part in the metabolism of the body.

A growing child needs far more food than its weight alone would indicate; for, in the first place, its income must exceed its expenditure so that it may grow; and, in the second place, the expenditure of an organism is pretty nearly proportional, not to its mass, but to its surface. Now, speaking roughly, the cube of the surface of an animal varies as the square of the mass; when the weight is doubled, the surface only becomes $\sqrt[3]{4}$, or one and a half times as great. The surface of a boy of six to nine years, with a body-weight of I8 to $24 \mathrm{kilos}$, is two-fifths to one-half that of a man of 70 kilos; and he should have about half as much food as the man. A child of four months, weighing $5 \cdot 3$ kilos, consumed per diem food containing 0.6 gramme nitrogen per kilo of body-weight, or $3^{\circ}$ I 8 grammes nitrogen altogether, as against a daily consumption of only 0.275 gramme nitrogen per kilo in a man of 7 I kilos (Voit).

An infant for the first seven months should have nothing except milk. Up to this age vegetable food is unsuited to it ; it is a purely carnivorous animal. By careful observations on the amount of carbon dioxide and nitrogen excreted by a child nine weeks old, fed exclusively on its mother's milk, it has been shown that the absorption and assimilation of milk in the infant is very complete, over $9 I$ per cent. of the total energy being utilized; while an adult, taking as much milk as is necessary for the maintenance of nitrogenous equilibrium, does not utilize at most more than 84 per cent. Human milk contains about 2 per cent. of protein (mainly caseinogen), 3 per cent. of fat, 5 or 6 per cent of carbo-hydrate (lactose or milk-sugar), and from 0.2 to 0.3 per cent. of salts. Cow's milk contains about 4 per cent. of protein, 4 to 6 per cent. of fat, 4 per cent. of lactose, and $0^{\circ} 7$ per cent. of salts. When given to infants it should, as a general rule, be diluted with water, and some sugar should be added to it. Ass's milk has about the same amount of protein, lactose, and salts as human milk, but less than half as much fat. It is very well borne and very completely absorbed.

As to the place of water and inorganic salts in diet, it is neither necessary nor practicable to lay down precise rules. In most well-settled countries they cost little or nothing; very 
different quantities can be taken and excreted without harm; and both economics and physiology may well leave every man to his taste in the matter. Salt is indeed for the most part used, not as a special article of diet, but as a condiment to give a relish to the food, just as a great deal more water than is actually: needed is often drunk in the form of beverages. It is certain that the quantity of salt required, in addition to the salts of the fond, to keep the inorganic constituents of the body at their mormal amount, is rery small. A 30 -kiln dog ohtains in his diet of 500 grammes of lean meat only 0.6 gramme sodium chloride. and needs no more. An infant in a litre of its mother's milk, which is a sufficient diet for it at six tn nine months, gets only o. 8 gramme sodium chloride. Bunge, however, has shown that the proportion of potassium and sodium salts in the food is a factor in determining the quantitr of sodium chloride required. A double decomposition takes place in the body between potassium phosphate and sodium chloride, potassium chloride and sodium phosphate being formed and excreted; and the loss of sodium and chlorine in this way depends on the relative proportions of potassium and sodium in the food. In most regetables the proportion of potassium to sodium is much greater than in animal food, so that regetable-feeding animals and men as a rule desire and need relatively great quantities of sodium chloride. But it is stated that the inhabitants of a portion of the Soudan use potassium chloride instead of sodium chloride, obtaining the potassium salt by burning certain plants which leave an ash poor in carbonates, and then extracting the residue with water and evaporating (Drbowski). A beef-eating English soldier in India consumes about 7 grammes ( ${ }_{4}^{1}$ oz.), a regetarian Sepoy about IS grammes $\left(\frac{2}{3} \mathrm{oz}\right.$.), of common salt per day.

Wine, beer, tea, coffee, cocoa, etc., belong to the important class of stimulants. Some of them contain small quantities of food substances, but these are of secondary interest. In beer, for example, there are not inconsiderable amounts of proteins, dextrin, and sugar. But If litres of beer would be required to rield I5 grammes nitrogen, and Io litres to give 250 grammes carbon; and nobody, except a German corps student, could consume such quantities. The minimum nitrogen requirement, however, as well as the necessary heat value, could theoretically be covered by 6 or 7 litres of good German beer.

In some cocoas there is as much as 50 per cent. of fat, 4 per cent. of starch, and 13 per cent. of proteins; and in the cheaper cocoas much starch is added. Still, a large quantity of the ordinary infusion would be needed for a satisfying meal. Frederick the Great, indeed, in some of his famous marches 
clined off a cup of chocolate, and beat combined Europe on it ; but his ordinary menu was much more varied and substantial.

Alcohol.- The great social and hygienic evils connected with the abuse of alcohol, as well as its applications in therapeutics, render it necessary, or at least permissible, to state a little more fully, though only in the form of summary, some of the chief conclusions that may be drawn as to its action and uses.

(I) In small quantities alcohol is oxidized in the body, a little of it, however, being excreted unchanged in the breath and urine. A certain amount of protein is saved from decomposition when alcohol is taken, just as when fat or sugar is taken. For example, the addition of 130 grammes of sugar to the daily food of an individual caused a 'sparing' of 0.3 gramme nitrogen. The substitution of 72 grammes alcohol for the sugar caused 0.2 gramme nitrogen to be spared (Atwater and Benedict). Alcohol is therefore to some extent a food substance, although it is not, under ordinary circumstances, taken for the sake of the energy its oxidation can supply, but as a stimulant.

(2) There is no reason to suppose that this energy cannot be utilized as a source of work in the body. Indeed, a certain amount of alcohol seems to be normally formed in the tissues as one of the intermediate products in the oxidation of sugar. Heat can certainly be produced from it, but this is far more than counterbalanced by the increase in the heat loss which the dilatation of the cutaneous vessels caused by alcohol brings about.

(3) It is a valuable drug, when judiciously employed, in certain diseases-e.g., pneumonia and puerperal insanity (Clouston).

(4) Alcohol is occasionally of use in disorders not amounting to serious disease-e.g., in some cases of slow and difficult digestion. In these cases it may act by increasing the flow of certain of the digestive secretions, as saliva and gastric juice. This effect seems to more than counterbalance the retarding influence which, except when well diluted, it exerts on the chemical processes of digestion.

(5) Alcohol is of no use for healthy men.

(6) Alcohol in strictly moderate doses,* properly diluted and especially when taken with the food, is not harmful to healthy men, living and working under ordinary conditions.

(7) Modern experience goes to show that in severe and continuous exertion, coupled with exposure to all weathers, as in war and in exploring expeditions, alcohol is injurious, and it is well known that it must be avoided in mountain climbing.

Tea, coffee, and cocoa are more suitable stimulants for healthy persons, because they are less dangerous than alcohol, and they

* Not more than $\mathrm{r} \frac{1}{2} \mathrm{oz}$. of absolute alcohol, corresponding to about $4 \mathrm{oz}$. of whisky, or 2 to 3 wineglasses of sherry or port, or a pint of claret, or a couple of pints of light beer in 24 hours. 
leave no unpleasant effects behind them. But it should be remembered that there is no stimulant which is not liable to be abused. It has been shown by ergographic experiments (p. 649) that, like alcohol, tea, coffee, maté, and cola-nut, which all contain the alkaloid theine or caffeine, restore the power of performing muscular work after exhaustion, but only if food has been recently or is simultaneously taken.

Certain organic acids contained in fresh vegetables, although neither in the ordinary sense foods nor condiments, seem to be necessary for the maintenance of health, for in circumstances in which these cannot be obtained for long periods, scurvy is liable to break out. It is prevented by the use of lime or lemonjuice, in which citric and a trace of malic acid are contained.

\section{INTERNAL SECRETION.}

It is long since Caspar Friedrich Wolff expressed the idea that ' each single part of the body, in respect of its nutrition, stands to the whole body in the relation of an excreting organ,' and thus emphasized the importance of substances produced by the activity of one kind of cell for the normal metabolism of another. But it is only in recent years that it has become possible to illustrate this mutual relation by any large number of experimental facts.

Certain of the substances taken in from the blood by the liver find their way, after undergoing various changes, in to the biliary capillaries, and are excreted as bile; certain other substances, such as sugar and the precursors of urea, are taken up by the hepatic cells, transformed and sometimes stored for a time within them, and then given out again to the blood. Bile we may call the external secretion of the liver, glycogen and urea constituents of its internal secretion. In one sense it is evident that all tissues, whether glands in the morphological sense or not, may be considered as manufacturing an internal secretion. For everything that an organ absorbs from the blood and lympl it gives out to them again in some form or other except in so far as it forms or separates a secretion that passes away by special ducts. But it is usual to employ the term only in relation to organs of glandular build, whether provided with ducts or not. For convenience the action of extracts of some other tissues, such as nervous tissue, will also be considered here, although there is no reason to suppose that they form any sjecific internal secretion.

The capacity of manufacturing internal secretions of high importance can neither be attributed to all glands with ducts 
nor denied to all other organs. For the salivary, mammary, and gastric glands may be completely removed without causing iny serious effects, while death follows excision of the, so far as mere bulk is concerned, apparently insignificant masses of tissuc in the ductless thyroid, parathyroid, suprarenal or pituitary bodies.

$1 t$ is known that in the case of the liver the internal secretion is more important than the external, for an animal cannot survive without its liver, while it may be but little affected by the continuous escape of the bilc through a fistulous opening.

Pancreas. - The internal secretion of the pancreas is also indispensable. For when the pancreas is excised death follows in many species of animals, and especially in carnivorous animals ; and in man severe and ultimately fatal diabetes is often associated with pancreatic discase, while the mere loss of the pancreatic juice through a fistula does not necessarily shorten life, although the absorption of fat is seriously interfered with.

The ultimate cause of death seems to be a profound disturbance of metabolism, of which the most significant token is the increased proportion of sugar in the blood, and its speedy appearance in the mine-in dogs always within twenty-four hours following total removal of the organ. Associated with the glycosuria is an increase in the quantity of the urine (polyuria), excessive thirst (polydipsia), and a ravenous appetite (polyphagia), in spite of which the animal becomes more and more emaciated-in short, the classical symptoms of a severe type of pathological diabetes in man, but, of course, far more acute in their onset, and far more rapid in their progress towards the inevitable end. Dogs rarely survive more than two or three weeks, the immediate cause of the rapidly fatal result being perhaps the extensive suppuration which is apt to ensue on slight and practically unavoidable superficial injuries. The resistance of the tissues to bacterial invasion and their tendency to spontaneous healing are reduced by the overloading of the blood and tissue liquids with sugar. Even when carbo-hydrates are excluded from the food, or when no food at all is given, sugar continues to be excreted in large amounts. The destruction of proteins is increased. It is a significant fact that glycosuria does not appear or is only transient when the pancreas is partially removed, so long as a comparatively small fraction of the gland (one-quarter or one-fifth) is left. Even when such a remnant is transplanted from its original position, care being taken not to interfere with its circulation, and grafted in the peritoneal cavity or, indeed, under the skin, the animal remains in good health. In the dog this operation can be practised on the lowest part of the descending division of the pancreas, which is not united with the duodenum, but lies free in the mesentery. 
Removal of the fragment of pancreas is followed by the whole train of symptoms associated with total extirpation of the organ.

Although as yet we are igrorant of the precise manner in which the pancreas influences the metabolism of the body, it is impossible to cloubt, in view of the facts we have mentioned, that, like the liver, in addition to carrying on the exchanges necessary for the preparation of the ordinary or external secretion, the gland has other important relations with the circulating fluids, giving to them or taking from them substances on the manufacture or destruction of which the normal metabolic processes depend. It has been suggested that the pancreas neutralizes or renders harmless some toxic substance formed elsewhere in the body, the action of which produces glycosuria. But no evidence of the existence of any such substance has been obtained, and the transfusion into a normal dog of blood from a depancreatized animal, which ought to be laden with the hypothetical toxic material, does not cause glycosuria. It is much more probable that the hyperglycæmia on which the glycosuria depends is caused by the absence of something normally produced by the pancreas, and which is indispensable for the due regulation of the sugar-content of the blood. This something, as already pointed out in discussing pathological diabetes, may either be necessary to regulate the transformation of sugar into glycogen, so that too great a surplus of sugar does not remain unchanged, or to regulate the transformation of glycogen into dextrose and prevent too hasty and too extensive action by the glycogenase, or, finally, to regulate and to aid in the normal combustion of the sugar in the organs (p. $5 \mathrm{r} 7$ ). The seat of the internal secretion of the pancreas seems to be the very vascular epithelioid tissue which is peculiar to this gland, and occurs in islands between the alveoli (islands or islets of Langerhans) (Schäfer). For animals survive the complete atrophy of the ordinary secreting epithelium caused by the injection of paraffin into the ducts, and no sugar appears in the urine. The islets remain intact. When a portion of the pancreas is separated from the rest, and its duct ligated, it undergoes extensive atrophy, a tissue remaining which is apparently composed of enlarged islands of Langerhans and remains of pancreatic ducts. If the rest of the gland is now removed, no glycosuria occurs, even when considerable quantities of dextrose are injected. But when the atrophied remnant is also removed, typical pancreatic glycosuria at once ensues (IV. G. MacCallum).

As further evidence that the islets have a different function from the pancreatic alveoli may be cited the statement that in teleostean fishes, in which the islands are so large that they can 
be separated from the rest of the tissue, the cells of the islets, instead of containing an amylolytic ferment like the alveolar cells, contain a glycolvtic ferment, or at least possess t'.': power of destroving sugar. "Vet the question of the significance of the islets can hardly be considered settled, and there are good observers who believe that they do not differ essentially from the alreolar tissue, but are formed by certain changes in the arrangement and properties of the alvenlar clements. For example, after the gland has become exhausted under the influence of secretion, a great part of the alveoli are said to be converted into islet tissue, which after a period of rest is again altered, so as to rield the characteristic histological picture of the secretory alveoli (Dale). While, then, the importance of the pancreas in carbo-hydrate metabolism is certain, and the dependence of this function upon an internal secretion is highly probable, it is not ret definitely settled whether this secretion is formed in the organ as a whole or only in the islets. That lesions of the pancreas may be concerned in pathological diabetes is well established, and it is of interest in connection with the question we have just been discussing that in a certain number of cases the changes observed have been in the islands (Opie). And in diabetes accompanying cirrhosis of the liver, which has usually been considered to depend upon the hepatic changes, it has been shown that in many, if not all, of the cases the pancreas is also affected by a growth of connective tissue outside the acini (Steinhaus). Some authors, indeed, have gone so far as to say that in all cases of diabetes mellitus there is disease of the pancreas, but of this there is no evidence.

Ligation, or the establishment of a fistula, of the thoracic duct, causes glycosuria in dogs. It is possible that this is really a mild form of pancreatic diabetes, due to interference with the supply of the internal secretion of the pancreas, or of that part of it which reaches the blood by the lymph-stream (Tuckett).

Quite recently Pflüger has brought forward evidence which he considers to show that it is not the removal of the pancreas, as such, but the section of certain nerves running into or through it from the duoden um, which is the cause of the glycosuria. For when these nerves are divided or the duodenum removed while the pancreas remains untouched, the result is the same as if the pancreas itself had been excised. He imagines that these nerves are "antidiabetic" -that is, in some way oppose the production of sugar-while nerves coming from the so-called 'sugar centre' in the bulb (the centre assumed to be affected in the puncture experiment) favour sugar production. Between these the normal balance is struck in health ; it is the upsetting of this balance by the crippling of the duodenal fibres which is at the bottom of "pancreatic' diabetes. It is too early to appraise the value of this conception, especially as the facts upon which it is founded have only been clearly established for 
frogs, and it is doubtful whether they can be extended to mammals. But if these nerves end in the pancreas, and do not simply run through it, say, to the liver, it is possible that they act on the sugar metabolism by regulating the internal secretion of the pancreas.

Sexual Organs.-The influence of castration in preventing the phrsical and psychical changes that normally occur at puberty is no doubt also, in part at least, due to the loss of the internal secretion of the testes. In partially castrated cocks it was seen that so long as a portion of one testicle remained, the male characters were preserved, but after removal of this residue the comb and wattles withered in a few weeks (Hanau). At the breeding-time the muscles of the forearm of the brown land frog (Rana fusca) become hypertrophied in the male, so that it can more tightly hold the female. At the same time the balls of the toes increase in size, and become covered with a peculiar black growth. After the breeding season these secondary sexual characters disappear. If the male frog is castrated, the periodical return of these phenomena does not occur, but the presence of one testicle suffices for their development on both sides. When pieces of testicle from normal frogs are introduced under the skin of the castrated frogs, the phenomena occur just as if the animals had not been castrated (MI. Nussbaum). The exact experiments of Loewy and Richter on the metabolism of bitches before and after castration throw light upon the changes which follow that operation, and afford decisive proof that they are connected with the absence of substances specific to the ovary. They conclude that in the castrated animal the oxidative energy of the cells is lessened. The oxygen consumption sinks, even although protein is laid on and the total amount of active tissue thus increased. Under certain circumstances this specific diminution of metabolism may be balanced by conditions which cause an increase in the metabolism. The lessening of the oxidative power is due to the loss of ovarian substance, for the administration of an extract of the ovary (oophorin) not only neutralizes it, but actually causes an increase in the gaseous metabolism to far above the original amount, while it has no effect on the metabolism of the uncastrated animal. It is not the decomposition of proteins, but of nonnitrogenous substances, which is accelerated. Oophorin also brings about a notable increase in metabolism in the castrated male dog, while, curiously enough, extract of testicle causes only a small increase, due to a basic substance, spermin $\left(\mathrm{C}_{5} \mathrm{~N}_{2} \mathrm{H}_{14}\right)$. which can bc isolated from the testicle. But the orchitic extract is not without influence in other ways. It certainly increases the capacity for muscular work, as tested by the ergograph (p. 650), and this distinct physiological action is 
sufficient to encourage the hope that it may possess some therapeutic value, although far from what has been claimed for it by its more enthusiastic advocates. The only constituent of extracts of the testicle made with salt solution which causes any pronounced effect on the blood-pressure when injected into the circulation is a nucleo-protein, the most plentiful of the protein substances. The pressure falls, mainly owing to inhibition of the heart, but partly through vaso-dilatation in the splanchnic area (Dixon).

The testicles also influence the growth of the bones. In eunuchs and in young men with atrophy of the testicles a tendency has been observed for the long bones to go on growing far beyond the usual period. This has been shown by the Röngten rays to be due to delay in the ossification of the epiphyses. The same has been observed in animals, and is supposed to be caused by the loss of some substance normally formed in the testicle which influences the metabolism of the bones and the deposition of the bone salts.

A temporary diminution in the hæmoglobin and in the number of the erythrocytes has been observed in castrated bitches, an observation which, so far as it goes, is in favour of the view that an insufficient internal secretion of the ovaries is the cause of the form of anæmia known as chlorosis.

Evidence has recently been brought forward that the corpus luteum is a gland with an internal secretion, whose function is connected with menstruation and with the implantation of the ovum and the subsequent growth of both ovum and uterus in pregnancy (Born, Fraenkel) (Chap. XIV.).

Thymus.-It is well known that in castrated animals the thymus is larger and persists longer than in entire animals. In bulls and unspayed heifers the normal atrophy of the thymus, which begins after the period of puberty, is greatly accelerated when the bulls have been used for breeding, and when the heifers have been pregnant for several months. There is a reciprocal influence of the thymus on the testicles, and removal of the thymus before the time at which it naturally atrophies is followed by a more rapid growth of the testes (in guinea-pigs) (Paton). In young mammals the loss of the thymus causes transient disturbances of nutrition, a temporary decrease in the number of all varieties of leucocytes, and a diminished resistance to the pus-forming micrococci, probably connected with the relatively feeble leucocytosis (or increase in the number of leucocytes) by which the animals react to the infection. In the frog the thymus persists throughout life. Yet the removal of it is not fatal if precautions against infection be taken. In mammals (including man) the thymus does not completely 
disappear in the adult. Islands of thymus tissue are found at all ages among the fat by which the bulk of the organ is replaced. The chief effect of intravenous injection of extract of human or ox thymus is a lowering of blood-pressure. In this respect it resembles thyroid extract. The heart may be at the same time accelerated.

Thyroids and Parathyroids. - The thyroid consists of two lobes commected by an isthmus across the middle line in man and some animals, but often separate. In the neighbourhood

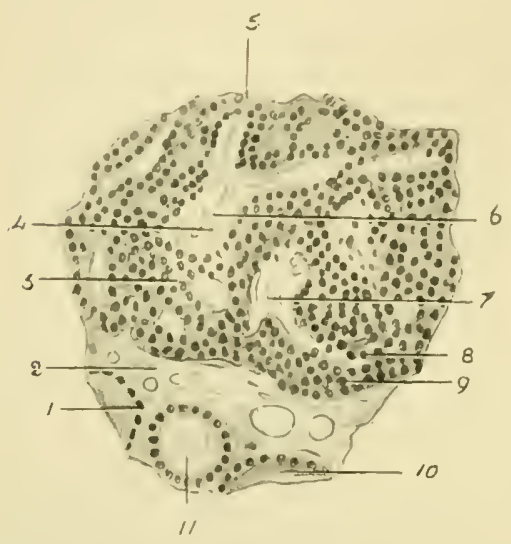

Fig. IS7.-PARATIITOHD (VINCENT AND JOLLY).

A sniall portion of parathyroid of cat embedded in thyroid tissue. It consists for the most part of solid columus of epithelial cells $(3,5,8)$ with strands of vascular connective tissue (6). A thyroid vesicle (I I) and portions of two others $(I, I 0)$ are seen in the lower part of the figure, separated from the parathyroid by a fibrous capsule (2). 4. 7 , bloodvessels ; 9 , lower boundary of the parathyroid tissue. $(\times 500$. animals the inferior, anterior, or external pair of parathyroids is more or less distinctly separated from the thyroid. The separation is especially evictent in the herbivora, in the monkey, and in man, and this pair of parathyroids is much larger than the other. In carnivorous animals, as the dog and cat, the anterior pair of parathyroids is closely adherent to the thyroid capsule. The superior, posterior, or internal pair, both in herbivora and carnivora, is always very closely associated with the capsule of the thyroid, and frequently embedded in the substance of the gland. The consequence of this arrangement is that in the older experiments the chief masses of parathyroid tissue were 
much nore likely to escape removal with the thyroid in the case of herbivorous than in the case of carnivorous anmals.

But even in one and the same species considerable variations may exist. It is easy to see, then, that in removing the thyroid the parathyroids would sometimes be completely removed as well, while at other times all or some of the parathyroid tissue would be spared. Add to this that sporadic masses of thyroid tissue (accessory thyroids), often existing as far down as the root of the aorta (always, indeed, in certain animals--e.g., the dog), must necessarily be spared in the most complete thyroidectomy, and it will cease to excite surprise that the symptoms and pathological changes described after that operation should have been so various and so contradictory. IVe know now that the parathyroids are perfectly distinct organs from the thyroid in histological structure, in function, and in the consequences of their removal. Nor do they show any compensatory hypertrophy when the thyroid alone is excised, or any changes which would indicate a definite relation to, still less an active participation in, the pathological processes occurring in the thyroid in goitre. The parathyroids contain no iodine, while iodine is a characteristic constituent of the thyroid.

Parathyroidectomy.--Total extirpation of the parathyroids is followed by a train of acute symptoms, ending fatally, as a rule, in from one to ten days. The typical nervous symptoms following the operation have been described as those of 'tetany,' and the tetany which used to be included among the consequences of removal of the thyroid is now known to be due to the simultaneous excision of the parathyroids (Kocher). A cat, after the combined operation, is perfectly well on the first day. On the second day a curious shaking of the paws is seen, tremors of central origin soon appear, and increase in severity until at length they culminate in general spasmodic attacks. Even when the animal is at rest the fore-legs tend to be flexed, while the hindlegs are extended, and this attitude is exaggerated in the convulsions. In the later stages unconsciousness is associated with the onset of the convulsions. Similar results follow excision of the parathyroids alone in dogs. Although the tetany is the most striking symptom, it is only one token of a profound general disturbance of nutrition. The pulse-rate and the rate of respiration are markedly increased. There is profuse salivation, with dilatation of the stomach and duodenum, and fever. The exact significance of these symptoms is unknown. The administration of calcium completely relieves them, and by its use death may be long or perhaps indefinitely postponed (WV. G. IIacCallum). The mode of action of the calcium has not been made clear as yet. It does not seem to be so efficacious in rabbits as in dogs (Arthus). 
Thyroidectomy. - The symptoms that follow remosal of the thyroid alone are perfectly different. The metabolic disturbance is eventually, in most animals, not less far-reaching than that which ensues when the parathyroids are alone excised. But it is far more chronic, reveals itself by totally distinct changes, is not amenable to calcium, but is completely corrected by the administration of thyroid substance. While no animals which have been examined survive the total removal of the parathyroids, certain species-e.g., the goat-are but slightly affected by thyroidectomy, and survive indefinitely. In man, before the consequences of thyroidectomy were known, the whole gland was not infrequently excised for goitre. If the parathyroids happened also to be completely involved in the operation, death quickly followed. But where only the thyroid itself, or the thyroid plus the small internal pair of parathyroids, was extirpated, the condition called cachexia strumipriva was observed to supervene. The symptoms resemble those of the disease known as myxœedema, in which the characteristic anatomical change is an increase (a hyperplasia) of the connective tissue in and under the true skin. Newly-formed connective tissue always contains an excess of mucin, and for this reason in the early stages of myxœedema there is somewhat more than the usual amount of that substance in the subcutaneous tissue. The skin is dry, and the hair falls off. The features are swollen and heary, the movements clumsy and trembling. As the disease progresses the mental powers deteriorate too; the patient becomes stupid and slow, and perhaps, at last, imbecile. When the gland is so affected in early life that extensive atrophy of the true secreting tissue occurs, a peculiar condition of idiocy (cretinism) results.

In animals there is a great difference in the results of total excision of the thyroids, both between different groups and between different individuals of the same group. In young animals the symptoms come on more rapidly, and are more severe than in old. Monkeys develop symptoms resembling those of myxœdema.

The older descriptions of the very acute onset of the symptoms and the quickly fatal result in carnivorous animals were vitiated by the circumstance that, for the anatomical reason already alluded to, the parathyroids were also involved in the operation. Nevertheless, the consequences of complete removal of the thyroid proper are in general more serious in the carnivora than in the herbivora. Muscular weakness soon becomes marked; the tissues waste, the temperature becomes subnormal, and this is associated with changes in the heat regulation (p. 593). If a portion of the thyroid be left, or a graft be made of some thyroid 
tissue from an animal of the same species, these effects are entirely obviated so long as the graft survives. It lias not been established that a hetero-throicl graft-i.e., a graft of thyroid tissue from an animal of a elifferent kind even temporarily succeeds. The alien throiel cells are destroved by cytolysins (p. 20) in the sermm and tissue lipuids of the animal. When a small part of a thyroid is left, it may mudergo great hypertrophy, and the same is true of the accessory thyouds. The atministration of extracts of the thyroid glands or the glands themselves by the mouth brings about a cure, permanent so long as the thyroid treatment is continued, in cases of myxodema in man, and prevents the development of the symptoms in anjmals or removes them when they have appeared. The same is true of a compound rich in jodine, the so-called thyroiodin, which has been extracted from the organ. Under this treatment the total metabolism, which in myxodema is below the normal, is markedly increased. This is partly due to an increase in the metabolism of protcin. An increase in the destruction of protein is also caused in normal persons. For this reason the use of thyroid preparations to reduce weight in cases of obesity, without evidence

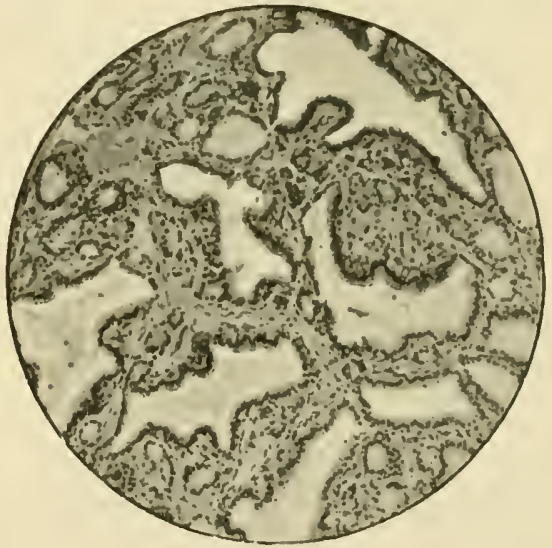

Fic. i88.-Microphotograpli of Active THYROID HYPERPLASIA FROM A CASE OF Exophthalmic Goitre (Marine).

The characteristic changes in the hyperplastic gland - the infoldings and plications of the alveolar epithelium, the great reduction in the colloid, and the increase in the stromaare shown.

of thyroid insufficiency, is a dangerous remedy. For while a fat man can very well spare a great deal of his fat, he cannot spare much of his tissue-protein. The question whether the thyroid or parathyroid is, in addition, concerned in the carbo-hydrate metabolism is at present the subject of lively discussion, but the data are so contradictory that it would not be advisable to enter into the matter here.

The relations of iodine to the gland itself, and the modifications in its structure and function determined by the giving or withholding of iodine, recently studied by Marine, are of great interest. In all animals, so far as examined, the normal thyroid contains iodine. The amount is variable, but the minimum 
percentage of iodine necessary, if the normal histological structure is to be maintained, is quite constant for a given species.

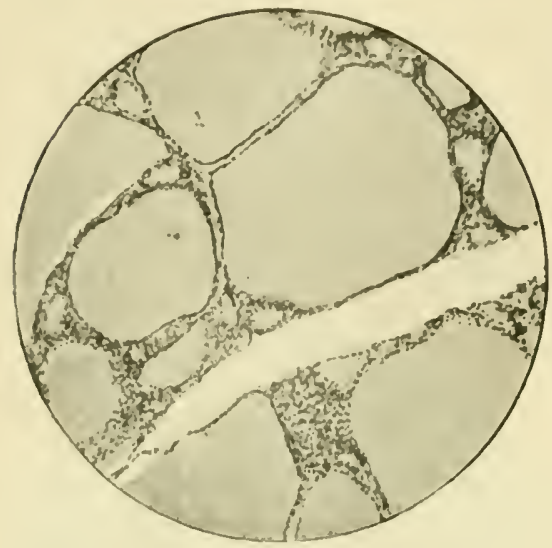

Fig. 189.-MICROPHOTOGRAPII OF A COLLOID Gland (GOITRE) (MIRINE).

The effect of administration of iodine is shown in the returu towards the normal structure from a preceding active hyperplasia. such as is shown in Fig. I 88 .

may be made that in the thyroid the iodine varies directly with the amonnt of colloid, and inversely with the degree of

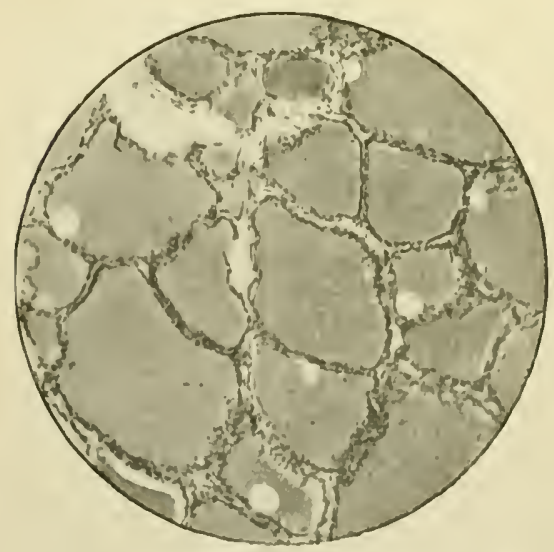

Fig. Igo.-Mickophotograph of Normal HUMAN TIYROID (MARINE). centage of incline associated with any degree of active hyperplasia (developing goitre) is always below the normal minimum, as shown by Marine in the dog, sheep, man, and other mammals. As active hyperplasia of the thyroid (goitre) (Fig. Is8) develops, the iodine content of the gland, both relative and absolute, decreases, until in extreme degrees of the condition there may be no demonstrable ioxdine present at all. Since the iodine is contained in the colloid as an iodine-protein compound, the generalization hyperplasia. The administration of any iodinecontaining substance to animals with actively hyperplastic thyroids (goitres) quickly (in two to three weeks in (logs) induces a listological change, the end stage of which is the so-called colloid goitre (Fig. IS9). This is a reversion to the normal histological structure (Fig. I 9o), so far as this is possible in a gland which has once undergone hyperplasia. The physiological influence of iodine

So also the highest peron the thyroid may be summed up as follows: Iodine is absolutely essential for the normal activity of the gland. It prevents spontaneous hyper- 
plasia (goitre), and also the compensatory hyperplasia which follow's partial removal of the thyroid. It exercises a curative effect on active hyperplasias. The physiological and therapentical"activity of thyroid substance varies directly with the amount of iodine in it in organic combination (thyroiodin).

As in the catse of other glands forming an internal secretion, it has been debated whether the function of the thyroid is to destroy toxic bodies or to form substances indispensable or advantageous to the organism. While the precise rôle played by the organ in the economy remains obscure, it is evident that in most animals and in man its secretion is of great importance, whether it be solely the quasi-external secretion of 'colloid,' containing the thyroiodin, that collects in its alveoli and slowly passes out of them by the lymphatics, or perhaps, in addition, some other substance, which, like the glycogen of the liver, never finds its way into the lumen of the gland-tubes at all. It may also be admitted that, by aiding in the maintenance of the normal level of general nutrition, particularly that of the central nervous system, the ability of the organism to cope with toxic substances introduced from the outside or manufactured in the body is favoured. There is, however, no evidence that an actual destruction or neutralization of toxic substances occurs in the gland itself.

Although no clear proof has yet been given that the secretion of the thyroid is influenced by nerves, it is probable that this is the case. Section of the superior and inferior thyroid nerves going to the gland is followed by degenerative changes in it.

Suprarenal Capsules.- It had been observed by Addison that the malady which now bears his name, and in which certain vascular changes, with muscular weakness, anæmia, and pigmentation or 'bronzing' of the skin, are prominent symptoms, was associated with disease, usually tuberculous, of the suprarenal or adrenal bodies. This clinical result was soon supplemented by the discovery that extirpation of the adrenals in animals is incompatible with life (Brown-Séquard). Our knowledge of the functions of these hitherto enigmatic organs was greatly extended by the experiments of Oliver and Schäfer, who investigated the action of extracts of the suprarenals (of calf, sheep, dog, guinea-pig, and man), when injected into the veins of animals. The arteries are greatly contracted, and this mainly through direct action on the vaso-motor nerve-endings or some structure intermediate between them and the smooth muscle of the vessels, but partly through the vaso-motor centre. The blood-pressure rises rapidly, although the heart may be inhibited through the vagus centre. The heart is at the same time directly stimulated, so that, although it beats slowly, the beats are 
stronger than before. When the vagi are cut the action of the heart is markedly augmented, and the arterial presunre rises enormously (it may be to four or five times its original amount). Stimulation of the depressor is of no avail in combating this increase of blood-pressure. The generalization may be made that suprarenal extract or adrenalin, its active principle, acts upon all plain muscle and gland-cells that are supplied with sympathetic nerve-fibres, and the result of the action, whether augmentation or inhibition, is the same as would be produced by stimulation of the srmpathetic fibres going to the muscle or gland in question. Fet it is not through excitation of these fibres that the adrenalin acts, for its effect is even more pronounced when the nerve-fibres have been caused to degenerite, in the case of the pupillo-dilator fibres, e.g., by excision of the superior cervical ganglion. Nor is the effect a direct one on the muscular fibres. For smooth muscle which is not, and never has been, in functional union with sympathetic nerve-fibres is indifferent to adrenalin (Elliott). It seems, then, to act on some structure intermediate between the nerve and the muscle, but so related to the latter that it continues to live so long as it is in connection with the muscle fibre. Instead of a definite listological structure, the seat of the action may be a special 'receptive' substance at the myoneural junction. Thus adrenalin causes marked diminution of tone in the small intestine, with disappearance of the peristalsis and pendulum movements. The same effect is produced on an isolated loop of intestine immersed in Locke's solution, and the action is therefore local. The drug is effective even in a dilution of $I$ : $I, 000,000$. A similar action has been observed on the stomacli. The vessels of the conjunctiva are constricted by local action when an extract of the capsules is dropped into the ere, a fact which has proved of value in ophthalmological practice. Inhibition of the contraction of the stomach, intestine, urinary bladder, and gallbladder; contraction of the uterus, ras deferens, and seminal vesicles; dilatation of the pupil and retraction of the nictitating membrane; stimulation of the salivary and lachrymal secretions, are among its actions (Langley). The curve of contraction of the skeletal nuscles is lengthened as in veratrine poisoning (p. 65t), though to a less extent. Adrenalin or suprarenin $\left(\mathrm{C}_{9} \mathrm{H}_{13} \mathrm{NO}_{3}\right)$ is solely contained in the medulla of the gland, and such is its extraordinary power, that a dose of one-millionth of a gramme per kilo of body-weight is sufficient to cause a distinct effect upon the heart and bloodvessels (a rise of pressure of If millimetres $\mathrm{Hg}$ ). Another delicate, and for certain purposes a convenient, reaction for the detection and the physiological assay of adrenalin is the 
dilatation of the pupil in the excised eyeball of the frog, first introduced by Meltzer, and afterwards develojed by Elırmann. The increase in the tone and the acceleration and strengthening of the rhythmical contractions of an isolated ring of rabbit's uterus have also been employed as a clinical test even for such minnte amounts of atrenalin as can exist in the circulating hlood (Iractical Exercises, Chap). XIV.). The alkaloid is used in medicine in the form of a dilute solution of adrenalin chloride, as a styptic, and for reducing congestion in accessible parts. The intense local anemia which it causes when given subcutaneously or by the mouth is one reason, perhaps the most important, for the slow absorption on which depends the absence of its general cffects, including that on the blood-pressure, when it is administered in this way. The function of the capsules, or rather of the so-called 'chromaffin' cells, which constitute the medulla and stain brown with chromic acid or chromates, is to secrete this substance, whicl is probably of great physiolegical importance for maintaining the tonicity of the muscular tissues in general, and especially of the heart and arteries. Adrenalin was entirely absent from the suprarenals of a person who had died of Addison's disease. There is some evidence that the active substance is really given off to the blood, and that normal plasma contains adrenalin in sufficient amount to be detected by that effect on the uterus which has just been mentioned. But statements which connect the increased bloodpressure in such conditions as chronic nephritis with hypertrophy of the chromaffin tissue, an increased adrenalin production, and an increased adrenalin content of the blood, must be received with caution. In a series of cases with high blood-pressure Fraenkel found no distinct difference in this regard between the pathological and normal sera. The so-called experimental arterio-sclerosis produced by repeated injections of adrenalin into the blood of rabbits throws little light upon the question, for the vascular changes, in so far as they have not been confounded with similar lesions occurring spontaneously in a considerable proportion of rabbits, differ from those observed in pathological arterio-sclerosis (M. C. Hill). An artificial adrenalin or suprarenin has been sintlictically prepared. Chemically it is identical with the natural adrenalin obtained from the suprarenal glands, but while the natural adrenalin rotates the plane of polarization to the left, the artificial substance is optically inactive. This is because it consists of equal parts of lacorotatory and dextro-rotatory adrenalin. The artificial adrenalim lias approximately half the effect of the natural on the bloodpressure, from which it may be inferred that the dextro-rotatory isomer has only a very slight pressor effect. Quite recently 
the left and right rotatory moieties have been separated. The former has exactly the same power of raising the blood-pressure as the natural adrenalin, the latter only $1_{1}^{1}$ to 1 as much. Practically the same proportion lolds when the power of the two isomers in producing glycosuria is compared. This constitutes important corroboration of the view already referred to (p. 522) that adrenalin glycosuria is caused by an action on the sympathetic system. For the effect on the blood-pressure is known to be thus produced (Cushny). The function of the cortex is unknown. It is stated that it contains cholin, a substance which lowers the blood-pressure, instead of raising it, as adrenalin does. It has been suggested that the adrenal glands have thus a double chemical grip upon the circulation, and can influence it in either direction, just as the bulb can influence it through its double nervous grip. But it is possible that the depressor substance of the cortex may be only a toxic body neutralized or destroyed in the glands. In any case the functional difference between cortex and medulla is easily understood when we reflect that the morphological history of the two tissues is quite different. The medulla is developed from cells which push their way into the gland from the rudiments of the sympathetic ganglia at that level, and is therefore of ectodermic origin. The cortex is derived from the same mesodermic structure which gives rise to the kidneys and genital organs.

The existence of secretory fibres for the adrenal glands in the splanchnic nerves has been rendered probable by the experiments of I)reyer, who finds that the amount of active substance in the blood of the suprarenal vein, as tested by its physiological effect when injected into an animal, is increased by stimulation of those nerves.

Pituitary Body.--In the pituitary body three parts may be distinguished: (I) The anterior lobe proper, or pars anterior, consisting of epithelial cells, many of which are filled with granules of the type seen in glandular epithelium, and abundantly provided witl bloodvessels; (2) the pars intermedia, consisting of epithelial cells, less granular and less richly supplied with hloodressels than those of the pars anterior; (3) the posterior lobe proper, or pars nervosa, consisting chiefiy of neuroglia closely invested by epithelial cells of the pars intermedia, and invaded by the colloid secreted by these cells. These differences in the structure of the anterior and posterior lobes of the pituitary body correspond to a difference in their dev lopment, the anterior lobe, witl the pars intermedia, being derived from an inpushing of the ectoderin of the buccal cavity, and the posterior lobe from an extension of the neural ectoderm, which grows backwards as the infundibular process till it meets and blends with that portion of the buccal invagination which gives rise to the pars 
intermedia. When the pituitary body is completely removed, death speedily and invariably ensues, in dogs, on the average within twenty-fonr to forty-eight hours. The much longer periods of survival occasionally witnessed are dne to failure to remove some small portion of the hypophyseal epithelium. On the day after the operation the animals may be able to walk about, to eat and drink, and may show an interest in their surroundings. The temperature, pulse, and respiration at this time may be normal. Soon, however, they become lethargic, then comatose, with characteristically incurved spine, slow respiration, with long-drawn inspiration, a feeble pulse, perfectly limp muscles, and often a subnormal temperature. This deep coma passes into death, with no perceptible transition, and without a struggle (Paulesco, Cushing). The ablation of a part of the

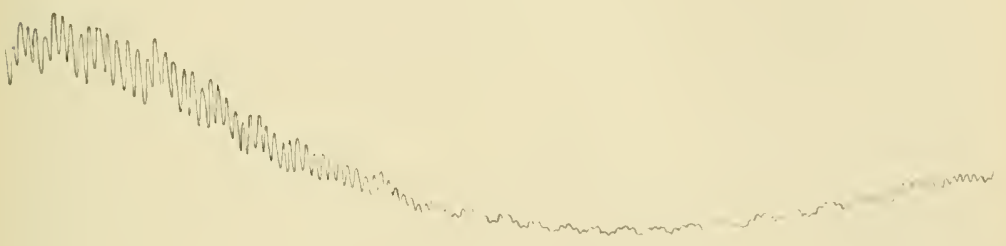

Fig. igi.-Action of Extract of Hypophyseal Lobe of Pituitary on the Blood-Pressure (WV. W. Hamburger).

The signal line at the top shows the time and length of injection of the saline extract into the blood. Time trace (at bottom) shows second intervals. The figure is to be read from left to right.

cortical substance of the anterior (epithelial) lobe of the hypophysis is compatible with permanent survival, and gives rise to no symptom of disorder. The same is true when only the posterior lobe is removed. On the other hand, complete removal of the anterior lobe causes death, just as if the whole gland had been taken away. Of all the structures included in the pituitary body, the most important from the functional point of view appears to be the superficial layer of the anterior lobe. It has been asserted that the pituitary undergoes (compensatory?) liypertrophy after thyroidectomy. Some observers have accordingly assumed a similarity of function for these organs. It has even been stated that the production of colloid material by the cells of the pars intermedia (lying between the anterior lobe and the nervous tissue of the posterior lobe, and investing the latter) is increased, and that colloid accumulates in the nervous 
portion of the posterior lobe. But this colloid, whatever its function may be, is very different from that of the thyroid alveoli, for the (sheep's) pituitary contains no indine after extirpation of the thyroid any more than before (Simpson and Hunter). And in man pathological changes (tumours) in the pituitary body are associated, not with myxoedema, or other disease commected with changes in the thyroid, but with another conclition, called acromegaly, in which the bones of the limbs and face, especially the hands and feet and the lower jaw, become hypertrophied.

Another condition often associated with tumours of the pituitary is gigantism - a condition occurring before the normal growth of the bones is completed, and resulting in a great increase in the length of the bones both in the limbs and the trunk.

The effects on the vascular system of intravenous injection of extracts of the pituitary gland are also very different from those caused by thyroid extracts. The posterior lobe, or infundibular body, including the pars intermedia, contains two active substances, one pressor and the other depressor. The former is soluble in salt solution, but insoluble in absolute alcohol and ether; while the latter is soluble in salt solution as well as in alcohol and ether. The pressor substance causes a great rise of blood-pressure, due partly to constriction of the arterioles and partly to an increase in the force of the heartbeat, both of which are brought about by direct action. This rise of pressure lasts for a considerable time, and is sometimes accompanied by a slowing of the heart. A second dose injected before the effect of the first has passed off is inactive; and this distinguishes the pituitary from the suprarenal extract. Associated with the pressor effect is an increase in the flow of the urine. Whether this is due to a separate diuretic substance, as some maintain, has not been definitely settled. The pressor substance, unlike adrenalin, directly stimulates smooth muscle fibres (especially the arteries, uterus, and spleen) irrespective of their innervation (Dale). The clepressor substance produces a marked fall of blood-pressure, even when it is injected during the rise of pressure caused by an injection of the pressor sub)stance. The anterior lobe, or hypophysis, also contains a depressor substance. Intravenous injection of a saline extract causes a distinct fall of blood-pressure, accompanied usually by acceleration and weakening of the heart (Fig. IOI). A secund injection immediately following the first produces no change in the pressure. But extracts of many organs, including the nervous tissues, produce a similar fall of pressure, and there is no evidence that the depressor substance of the anterior lobe is specific to the pituitary (WV. IV. Hamburger). 
It is not at present possible to deduce from such clinical and experimental observations as those described any colnerent theory of the function of the pituitary. That there is sone connection between the normal action of the gland, and in particular of its anterior lobe, and the normal glowth and nutrition of the skeleton is scarcely to be cloubted. The fact that administration of the dried gland substance to dogs causes an increased excretion of calcium on a diet rich in calcium is a further indication of its influence on the metabolism of bone (Malcolmu). But so far is the precise nature of this influence, if it exists, from being fully understood, that authorities of repute are still divided on the question whether the symptoms of acronegaly and gigantism are due to atrophy or to hypertropliy of the active elements of the gland, to loss of its internal secretion, or to its manufacture in excessive antount. There is evidence that the colloid secretion of the posterior lobe, probably formed by the epithelial cells of the pars intermedia, passes througl the nervous portion to enter the infundibulum and the third ventricle of the brain, where it breaks down in the cerebro-spinal fluid (Herring). And it has been suggested that in virtue of the action of the hormones ( $) .375$ ) in this secretion on the vascular system in general, and on the renal cells and the renal circulation in particular, the posterior lobe constitutes a mechanism for the control of the secretion of urine. But this suggestion is still in the realm of hypothesis.

Extracts of nervous tissue (sciatic nerve, white matter of brain, and spinal cord, but especially grey matter of brain) cause, on injection into the veins, a decided fall of arterial blood-pressure, which soon passes off, and can be renewed by a fresh injection. The fall of pressure is due to direct action upon the bloodvessels of a depressor substance in the extracts, and not to the action of vaso-motor nerves. It can be obtained after section of the vagi.

Extracts of misscular tissue also cause a distinct though transient fall of pressure, but not so great a fall as in the casc of extracts of nervous tissue. Saline decoctions of other tissues (testis, kidney, spleen, pancreas, liver, mucous membrane of stomach and intestine, lung, and mammary gland) all produce a fall of blood-pressure (Osborne and Vincent). The same is true of bone-marrow (Brown and Guthrie; Figs. I92, I93). It must be repeated that there is no evidence that these depressor substances are specific internal secretions in the same sense as adrenalin.

Kidney.- The experiments of Bradford, which seemed to inclicate that the kidney, in adclition to its function as an excretory organ. plays an important, and indeed indispensable, part in protein metabolism, possibly by forming something of the nature of all internal secretion, have not been confirmed. He stated that when the half or two-thirds of one kidney and the whole of the other have been removed from a $\log$ by successive operations, death ensues. although the quantity both of water and urea excreted by the fragment of renal substance that remains is far above the normal. In spite of the increased elimination of urea, that substance was sairl 
to accumulate in the tissues, showing that the destruction of protein was increased a conclusion which seemed to derive support forme the wasting of the inimal. It has since been shown that an increased nutput of nitrogen is not of constant occurrence, and only talies place muder the same conditions as in starvation (p. 531).

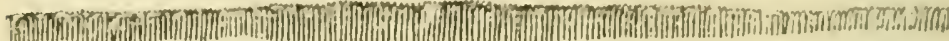

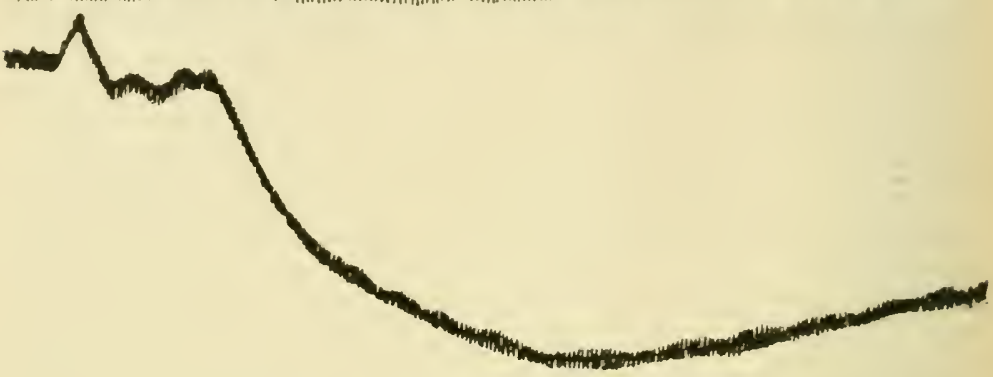

FIG. 192, EFFECT OF BONE-MARROW ON BLOOD-PRESSURE. INTRAlENOUS Injection of Salinf Extract. Vagi intact.

The uppermost line is it signal trace showing the time and length of injection. Below this is the record of the respiratory movements, and lowest the blond. pressure tracing. To be read from left to right.

As a matter of fact. the animals waste and die within a few days or weeks largely because they refuse to eat. Polyuria (increase of urine beyond the normal) does not necessarily occur. It is well known that when only one kiclney is extirpated the other hypertrophies, and no ill-effects ensue.

The statement that extracts of the kidney when injected into the veins of an animal cause a rise of arterial blood-pressurc, es-

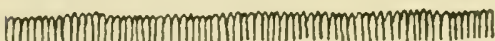

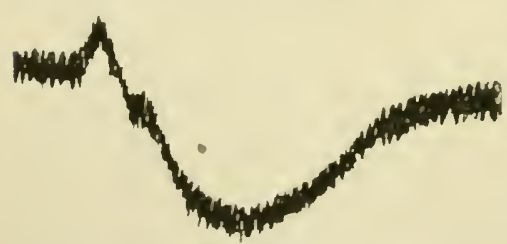

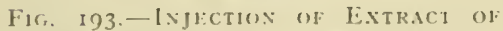
BONT-MARROW WITH THL V.MBI CLT.

To les read from left to right. sentially through direct action on the peripheral vaso-motor mechanism, is of considerable interest, for it may possibly have some bearing on the rise of pressure and consequent hypertrophy of the heart assocrated with certain renal diseases. But there is not as yet sufficient evilence that the hypothetical pressor substance, to whicl the name 'renin' has been given, in any sense represents an internal secretion of the kidney. The pressor substance (so-called " urohypertensine") which can be extracted by cther from normal luman urine (Abelous) is probably only excreter by the kidiney, and perhaps arises from the putrefaction of proteins in the intestine. For it has been shown that in the putrefaction of (horse-) meat bases are formed which, when injected intravenously. cause a rise of blood-pressure. The most active of these is a body 
known as p-hydroxyphenylethylamine, formed from tyrosin (Barger and Walpole).

The spleen does not proluce an internal secretion necessary to life. for it can be removerl both in animals and in man, not only witlsout causing (leath, but often without the development of any serious symptoms. It.s blood - forming and blood-destroying functions (p. 21) are taken on by other structures (particularly the red bone-marrow), but the formation of the bile-pigment is interfered with, and its amount rednced by more than 50 per cent. (P'ugliese). The production of trypsinogen by the pancreas is also said to be diminished, whereas if an cxtract of splecn be injected into the circulation of an animal deprived of its splecn, the amount of trypsinogen is increased. It has, therefore, been supposed that the splecen forms a substance (protrypsinogen) which, passing into the blood, is taken up by the pancreas and elaborated into trypsinogen (p. 392).

The salivary glands may be extirpated without any sensible change being produced in the normal metabolism. There is evidence, however, that the secretion of the gastric juice is diminished. It has been supposed that this may be dine to the absence of a hormone (p. 375) normally produced in the salivary glands. A temporary increase in the gastric secretion is causer when cxtracts of the glands of normal dogs are injected into the veins or into the peritoncal cavity of dogs deprived of their salivary glands (Hemmeter).

Extracts of the pineal gland injected into the circulation have no effect other than that due to the inorganic constituents of the 'brain sand.' 


\section{CHAPTER VIII}

\section{ANIMAL HEAT}

From the earliest ages it must have been noticed that the bodies of many animals, and particularly of men, are warmer than the air and than most objects around them. The 'vulgar opinion ' of Bacon's time, 'that fishes are the least warm internally, and birds the most,' if it does not imply a very extensive knowledge of animal temperature, at least shows that the fundamental distinction of warm and cold-blooded animals, which is to-day more accurately expressed as the distinction between animals of constant temperature (homoiothermal) and animals of variable temperature (poikilothermal), had been grasped. and was even popularly known. Since that time the accumulation of accurate numerical results, and the advance of physical and physiological doctrine. have given us definite ideas as to the relation of animal heat to the metabolic processes of the body. It is impossible to understand the present position of the subject without an elementary knowledge of the science of heat. For this the student is referred to a text-book of physics. All that can be done here is to preface the physiological portion of the subject by a few remarks on the physical methods and instruments employed .

Temperature.-Two bodies are at the same temperature if, when placed in contact, no exchange of heat takes place between them. They are at different temperatures if, on the whole, heat passes from one to the other, and that body from which the heat passes is at the higher temperature. It is known by experiment that if two bodies of different temperature are placed in contact. heat will pass from one to the other till they come to have the same temperature. If. then, we have the means of finding out the temperature of any one body, we can arrive at the temperature of any other by placing the two in contact for a sufficiently long time, under the proviso tli :t the quantity of heat necessary to bring the temperature of the first body. which nay be called the 'meisuring' body. to equality with that of the second, is so small as not to make a sensible difference in the latter. This is the principle on which thermometric measurements depend. A mercurial thermometer consists of a quantity of mercury 
ordinarily contained in a thin glass bulb, the cavity of which is continned into a tube of very fine bore in the stem. Like most uther substances, mercury expands when the temperature rises, and crontracts when it sinks, and the amount of expansion or contraction is shown by the rise or fall of the mercurial column in the stem of the thermometer. The point at which the meniscus stands when the bulb is immersed in melting ice or ice-cold water is, on the centigride scale, taken as zero; the point at which it stands when the thermometer is surrounded by the steam rising from a vessel of boiling water is taken as 100 degrees. The intermediate portion of the stem is divided into degrees and fractions of degrees. When, now, we measure the temperature of any part of an animal with such a thermometer. we place the bulb in contact with the part until the mercury has ceased to rise or fall. We know then that the mercury has ceased to expand or contract, and therefore that its temperature is stationary, and presumably the same as that of the part. It is to be noted that we have gained no information whatever as to the amount of heat in the body of the animal. We have only observed that the mercury of the thermometer when its temperature is the same as that of the given part expands to an extent marked by the division of the scale at which the column is stationary. And we know that if the mercury rises to the same point when the thermometer is applied to another part, the temperature of the latter is the same as that of the first part; if the mercury rises higher, the temperature is greater ; if not so high, it is less. The thermometer, then, only informs us whether heat would flow from or into the part with which it is in contact if the part were placed in thermal connection with any other body of which the temperature is known. In other words, the temperature is a measure of the heat 'tension,' so to speak : and difference of temperature between two bodies is analogous to difference of potential between the poles of a voltaic cell (p. 6i5), or to difference of level between the surface of a mill-pond and the race below the wheel.

The temperature of an animal is measured in one of the natural cavities, as the rectum, vagina, mouth, or external ear, or in the axilla, or at any part of the skin. For the cavities a mercury thermometer is nearly always used; the ordinary little maximum thermometer is most convenient for clinical purposes. The temperature of the slin may be measured by an ordinary mercury thermometer, the outer portion of the bulb of which is covered by some badly conducting material. An uncovered thermometer, heated nearly to the temperature expected, will also give results sufficiently accurate for most purposes, especially if the bulb is flat or in the form of a flat spiral, which can be easily applied to the surface. A theoretically better method, but more laborious in practice, is the use of a thermo-electric junction, or a resistance thermometer formed of a grating cut out of thin lead-paper or tinfoil (Fig. 194). This is especially useful for comparing the temperature of two portions of skin. The temperature of the solid tissues and liquids of the body may also be measured or compared by the insertion of mercurial or resistance thermometers or thermo-electric junctions (p. 664).

Calorimetry.-The quantity of heat given off by an animal is gencrally measured by the rise of temperature which it produces in a known mass of some standard substance. Sometimes, however, as in the ice-calorimeter of Lavoisier and Laplace and the ether calorimeter of Rosenthal, a physical change of state-in the one 
case liquefaction of ice, in the other evaporation of ether-is taken as token and measure of heat received by the measuring substance, the number of wnits of lieat corresponding to liquefaction of unit mass of ice or evaporation of unit mass of ether being known. The unit generally adopted in the measurement of heat is the quantity required to raise the temperature of a kilogramme of water $1^{\circ} \mathrm{C}$., which is called a calorie, or kilocalorie, or large calorie. The thousandth part of this, the quantity needed to raise the temperature of a gramme of water by $1^{\circ}$, is termed a small calorie or millicalorie.

In the calorimeters which have been chiefly used in physiology either water or air has been taken as the measuring substance. The simplest form of water calorimeter is a box with double walls, the

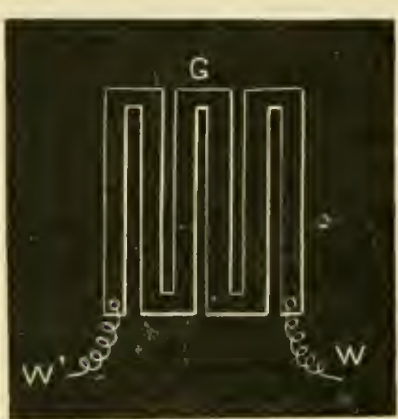

Fig. I94.-Resistance Ther. MOMETER FOR MEASURING Temperature of Skin.

G, grating of lead-paper, attached to a cover-slip, and mounted on a holder; W, W', wires to the Wheatstone's bridge. An increase of temperature causes an increase in the resistance of the lead. The balance of the bridge is thus disturbed. By experimental graduation the temperature value of the deflec. tion, or of the change of resist. ance that balances it, is known (p. 6I7).

actually present, and the sum is added to the quantity of water actually present, and the sum is multiplied by the rise of temperature. If the temperature of the room is constant, as will be approximately the case in a cellar, any error due to interchange of heat between the calorimeter and its surroundings may be eliminated by making the initial temperature of the water as much less than that of the air as the final temperature exceeds it. Then if the loss of heat by the animal is uniform, as much heat is gained during the first half of the experiment by the calorimeter from the air as is lost by it to the air during the last half. Or, without lowering the temperature of the water, the amount of heat lost by the calorimeter during an experiment may be previously determined by a special observation, and added to the cuantity calculated from the observed rise of temperature. Or, finally, two similar calorimeters may be 
used, one containing the animal and the other a hydrogen flame, or a coil of wire traversed by a voltaic current, which is regulated so as to keep the temperature the same in the two calorimeters. From the quantity of hydrogen burnt, or electricity passed, the heatproduction of the animal cin be cillculated.

In Atwater's great respiration calorimeter (Fig. I95) both the heat production and the respiratory cxchange are measured. The heat

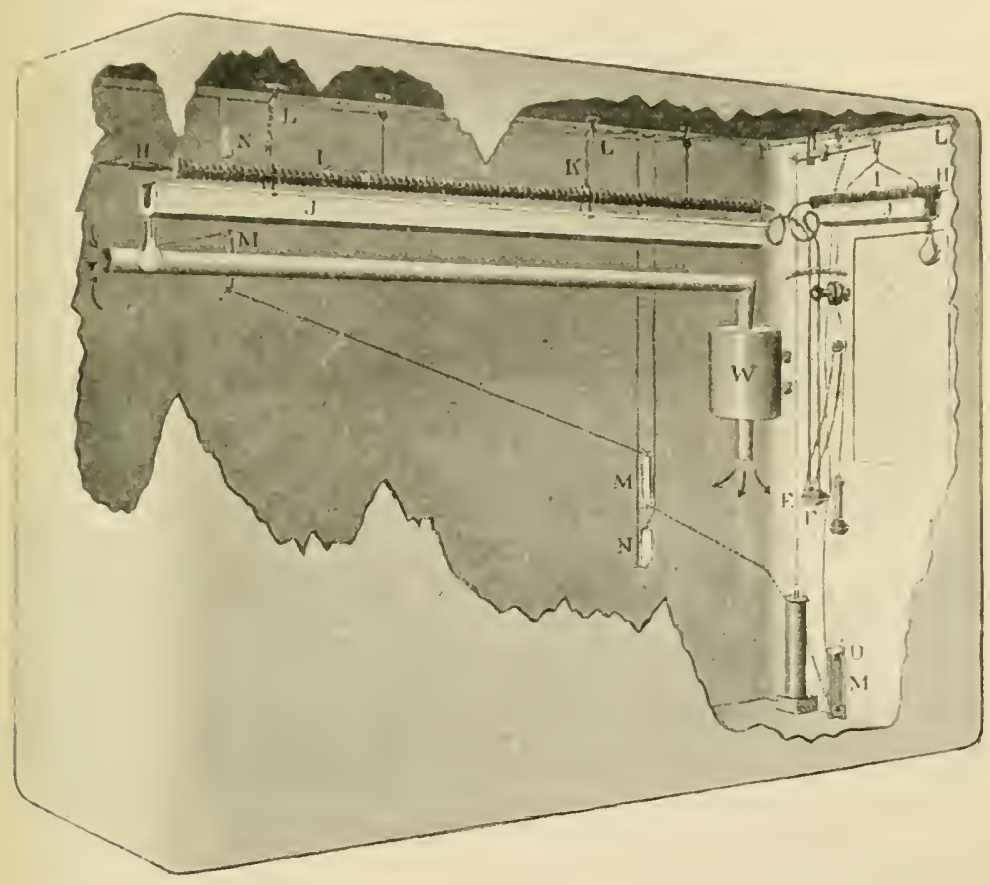

Fig. 195،-Respiration Calorimeter (Atwater).

Interior of chamber. A corner of the inner copper wall is supposed to be taken away. The ventilating air-current enters the chamber at the lower end of W, and leaves the chamber through the long tube fastened above W. The copper tubes $\mathrm{H}, \mathrm{H}$ are surrounded by copper discs I, I, fastened on them like a str.ng of beads to inrrease the surface. These tubes constitute the arrangement through which the stream of water flows which removes the heat formed in the chamber. J, J are copper troughs which receive the water dropping from H. H. M, MI, MI are electrical thermometers which show the temperature of the chamber; $\mathrm{N}, \mathrm{N}$, similar thermometers which show the temperature of the copper wall.

produced by the person in the calorimeter is carried away from it by a stream of water flowing through the chamber in a series of tubes, the temperature within the calorimeter being kept constant by regulating the temperature and velocity of the entering stream of water. The quantity of the escaping water and the increase in its 
temper..ture are measured. and the heat production can then be calculated. The apparatus consists of a chamber in which a human being can live for several days and nights. A stream of air is supplied and the chemical changes produced in this are investigated in the manner already described (p. 2.11).

Air calorimeters have sometimes been used for physiological purposes. A diagram of one is shown in Fig. 196. Such calorimeters are really thermometers with an immense radiating surface. for only a small proportion of the heat given off by the animal soes to heat the measuring substance. The heat required to ratise the

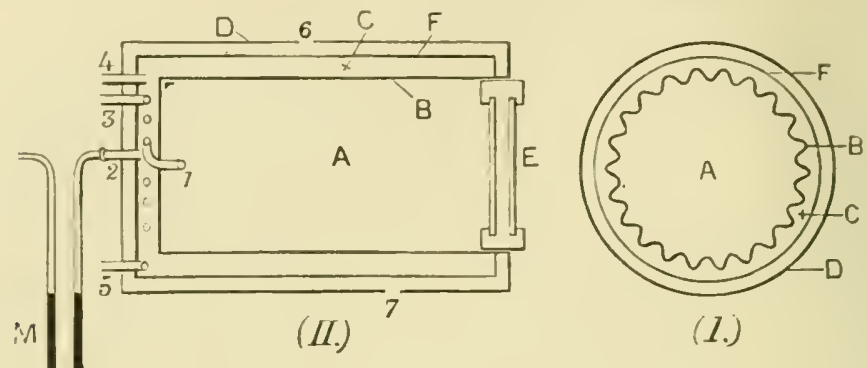

Fig. tg6.-Air Calorimeter.

(I.), cross-section; (II.), longitudinal section; A, cavity of calorimeter for animal : $\mathrm{B}$, copper cylinder corrugated so as to increase the radiating surface; $C$, air space enclosed between $B$ and a concentric copper cylinder $\mathrm{F}: \mathrm{C}$ is air-tight, and is connected by the tube 2 with the manometer M. The other end of the manometer is connected with an exactly similar calorimeter, in which a hydrogen flame is burnt in the space cor. responding to $A$, or in which the air in $A$ is heated by a coil of wire traversed by an electrical current. The flame or current is regulated so as to keep the coloured petroleum or mercury in the manometer. II at the same level in both limbs; the amount of heat given off to the one ealorimeter by the flame or current is then equal to that given off by the asimal to the other. $D$ is an external cylinder of copler or tin perforated by holes $(6,7)$ at intervals. The purpose of it is to prevent dranghts from affecting the loss of heat from $F ; 4,5$, are tubes through which thermometers can be introduced into $\mathrm{C}$; I is the terminal of a spiral tube. which is coiled in the end portion of the air space $C$. The sections of the coils are indicated by small eircles. The other end of the spiral tube is $3:$ through this tube air is sucked out. and so the proper ventilation of the animal is kept up. The object of the spiral arrangement is that the air aspirated out of A may give up its heat to the air in $C$ before passing out. $E$ is a door with double glass walls.

temperature of a litre of air by $\mathbf{I}^{\circ}$ is very small in comparison with that required to raise the temperature of a litre of water by the same amount. Hence a given quantity of heat raises the temperature of an air calorimeter much more than that of a water calorimeter of the same dimensions; and the loss of heat to the surroundings being proportional to the elevation of temperature. in the water calorimeter the chicf part of the heat is actually retained in the water. while in an air calorimeter the greater portion passes through the air space. and is radiated away. When the amount of heat lost by the calorimeter becomes equal to that gained from the animal, the 'steady' reading of the instrument is taken, and from this the heat production can be deduced by an experimental 
graduation of the apparatus. One alvantage of an air calorimeter is that it follows more closely rapicl variations in the heat-production of the animal, or, to speak more correctly, in the licat loss. It slinuld be carefully noted that in calorimetry what is directly measured is the quantity of heat given out by the animal, not the quantity produced. The two quantities are identical only when the temperature of the animal has remained unchanged throughout the experiment. If the temperature laas fallen, the quantity of heat produced is equal to the quantity measured by the calorimeter mimus the difference between the quantity in the animal at the beginning and at the end of the observation. This difference is equal to the average specific heat of the animal multiplied by its weight and by the fall of temperature. It can be approximately found by multiplying the weight (in kilogrammes or grammes) by the fall of rectal temperature (in (legrees). since the average specific heat of the body is not very lifferent from that of water, and the specific heat of water is taken as unity.

All the higher animals (mammals and birds) have a practically constant internal temperature (fowl $4 \mathrm{I}^{\circ}$ to $44^{\circ} \mathrm{C}$., mouse $37^{\circ}$ to $38^{\circ}, \operatorname{dog} 38^{\circ}$ to $39^{\circ}$, man $37^{\circ}$ in the rectum), but a few hibernating mammals, such as the marmot, are homoiothermal in summer, poikilothermal during their winter sleep. In the lower forms the body-temperature follows closely the temperature of the environment, and is never very much above it (frog $0.5^{\circ}$ to $2^{\circ}$ above external temperature). Both in a frog and in a pigeon heat is evolved as long as life lasts; but per unit of weight the amphibian produces far less than the bird, and loses far more readily what it does produce. The temperature of the frog may be $30^{\circ} \mathrm{C}$. in June and $5^{\circ}$ in January. The structure of its tissues is unaltered and their vitality unimpaired by such violent fluctuations. But it is necessary, not only for health, but even for life, that the internal temperature (the temperature of the blood) of a man should vary only within relatively narrow limits around the mean of $37^{\circ}$ to $38^{\circ} \mathrm{C}$.

Why it is that a comparatively high temperature should be needed for the full physiological activity of the tissues of a mammal, while the, in many respects, similar tissues of a fish work perfectly, although perhaps more sluggishly, at a much lower temperature, is not quite clear; nor do we know the precise significance of that relative constancy of temperature in the warm-blooded animal, which is as important and peculiar as its absolute height. The higher animals must possess a superior delicacy of organization, hardly revealed by structure, which makes it necessary that they should be shielded from the shocks and jars of varying temperature that less highly-endowed organisms endure with impunity. Leaving the discussion of the local differences and periodic variations of the temperature of warm-blooded animals to a future page, let us consider now 
the mechanism hy which the loss of heat is adjusted to its production, so that upon the whole the one balances the other.

Heat-loss.- Heat is lost (I) from the surfaces of the body. by radiation, conduction, and convection; (2) as latent heat in the watery vapour given off by the skin and lungs; and (3) in the excreta. Even in the bulky excrement of herbivora a comparatively trifling part of the total heat is lost. The second channel of elinination is much more important; the first is in general the most important of all.

The loss of heat by direct radiation from a portion of the slin or clothes, or from hair. fur, or feathers covering the skin, may be measured by means of a thermopile or a resistance racliometer (bolometer). The latter instrument is similar in principle and allied in construction to the resistance thermometer used in measuring superficial temperatures, and already describer (Fig. 194. p. 574). It may consist of a grating of lead-paper or tinfoil fixed vertically in a small box which protects it from draughts. The box has a sliding lid, which is kept closed till the moment of the observation. when it is withdrawn and the portion of skin applied to the opening at a fixed rlistance (5 to Io $\mathrm{cm}$.) from tlic grating. The intensity of radiation rlepends on the excess of temperature of the radiating surfacc over that of the surroundings, as well as on the nature of the surface. The uncovered parts of the skin (face and hands in man) radiate more per unit of area than the clothes or hair ; and the warm foreheal more than the comparatively cool lobe of the car or tip of the nose. When a man is sitting at rest in a still atmosphere, pure radiation plays a greater, and conduction and convection play a smaller, part in the total loss of heat from the skin than when he is walking about or sitting in a draught. The more rapidly the air in contact with the slin and clothes is renewed. the lower. other things being equal, the temperature of the radiating surfaces is kept, the grcater is the loss of heat by conduction to the adjacent portions of air, and the smaller the loss by radiation to the walls of the room, the furniture, and other surrounding objects. It is probable that. under the most farourable conditions, the amount of heat lost from the surface by true radiation does not exceed the amount lost by conduction and convection.

The loss of heat by evaporation of water from the skin call be calculated if we know the quantity of water so given off. For a gramme of water at the ordinary temperature (say $15^{\circ} \mathrm{C}$.) neerls 0.555 calories to convert it into aqueous vapour at the average temperature of the skin. If we take the average quantity of water cxcreted as sweat in twenty-four hours as $75^{\circ}$ c.c., this will be equivalent to a heat-loss of $+\mathrm{I}^{6} 25$ - say, in round numbers, 400 calories.

The quantity of heat given off by the lungs may be also dechucel from cenlculation, the data being (I) the weight, temperaturc, and specific heat of the expired air, and (2) the excess of water it contains in the form of aqueous vapour over that containerl in the inspircil air. I Ielmholtz calculated the quantity of heat nceded to warm the air expired by a man in twenty-four hours from an initial temperature of $20^{\circ}$ to body-temperature, at 70 calories, and that required to evaporate the water given off by the lungs at 397 . making the total heat-loss by the lungs in these processes from 400 to 500 calories. A certain amount of heat is also absorbed in connection with the escape 
of the carbon dioxide. The reason why a great deal more water and therefore more lecat is not given off by the lungs with their enormous surface, and the high degree of imbibition (p. 398) of the epithelium of the alveoli is that the air is already saturated with aqueous vapour, or nearly so, before it reaches the alveoli. By clirect calorimetric observations it was found that a man of 70 kilos weight gave off in normal breathing, with an air-temperature of $12^{\circ}$ to $15^{\circ} \mathrm{C}$., from $35^{\circ}$ to $+5^{\circ}$ calories. Forcel respiration, as might be expected, increased
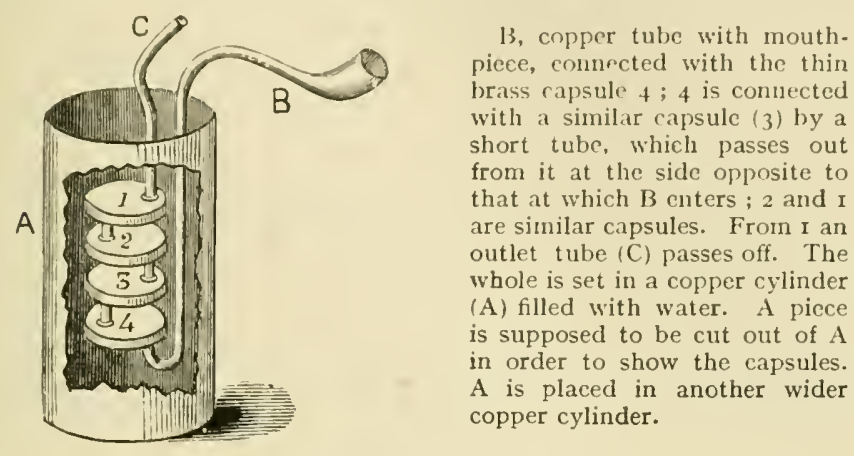

Fig. I97.-Respiration Calorimeter.

the amount often to double or even treble. A diagram of a respiration calorimeter (for measuring the heat given off in respiration) is shown in Fig. 197. (See Practical Excrcises, p. 613.)

The following table gives an analysis of the heat-loss of an average man. It must be understood that the figures are only approximate. In round numbers we may say that two-thirds of the heat-loss is due to radiation, conduction, and convection, and one-third to the evaporation of water.

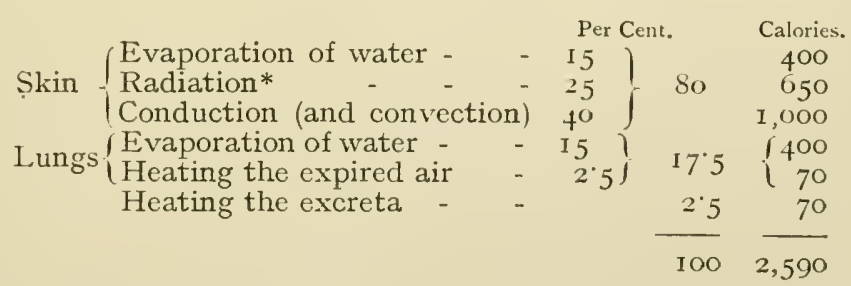

In the rabbit, according to Nebelthau, the heat lost by cvaporation of water is about 16 per cent. of the whole, or about half the proportion in man, according to the above calculation. This is not surprising when we reflect that the rabbit does not sweat, and drinks comparatively little water.

Sources of the Heat of the Body-Heat-production.-Some heat enters the body as such from without-in the food, and by radiation from the sun and from fires. The ultimate source

* The relative amounts lost by radiation and conduction cannot be accurately fixed. The proportion is extremely variable. 
of all the heat produced in the body is the chemical energy of the food substances. Whatever intermediate forms this energy may assume-whether the mechanical energy of muscular contraction; the energy of electrical separation by which the currents of the tissues are produced; the energy of the nerve impulse; or the energy, be it what it may, which enables the living cells to perform their chemical labours-it all ultimately, except so far as external mechanical work may be done, appears in the form of heat. We do not know at what precise stage of metabolism the chief outburst of heat takes place. But it is known, as already pointed out (p. 504), that the fraction of the total energy liberated in the processes of hydrolytic cleavage is comparatively small. Most of the heat is set free in the oxidative processes which accompany or follow the hydrolytic changes.

Thus the energy-value of a gramme-molecule (p. 398) of maltose, rane-sugar, or lactose is a little more than 1,350 calories; that of the two gramme-molecules of dextrose formed by hydrolysis of the maltose is $1347^{\circ}+$ calories; that of the gramme-molecule each of dextrose and levulose formed from the cane-sugar, $13+9^{\circ} 6$; and that of the gramme-molecule each of dextrose and galactose formed from the lactose, $13+3^{\circ} 6$ calories. That is to say, the hydrolysis of these disaccharides to monosaccharides, which is the first step in their metabolism, is accomplished with the liberation of very little heat. The same is true of the splitting of the fats and proteins. The (lried residue of a filtered pancreatic digest was found to yield. when burned in the calorimetric bomb, only 10 per cent. less heat than the same weight of dry meat. II uch the greater part of this deficiency was accounted for by the leucin and tyrosin which had crystallized out, and the derivatives of higher fatty acids in the meat, as these would be removed from the digest by filtration.

It has been shown that the law of the conservation of energy holds for the animal body ; in other words, there is a practically exact agreement between the potential energy of the food and the kinetic energy into which it is transformed in the body both during rest and during work. This kinetic energy is represented by the heat given off plus the heat-equivalent of any mechanical work done (Atwater). In other words, the food, whether it is burned in a calorimeter to simple end-products like carbon dioxide and water, or more slowly oxidized in the body, yields the same amount of heat, provided always that in both cases it is entirely consumed, and that no work is transferred to the outside. In the body the combustion of carbo-hydrates and fats is complete; but the nitrogenous residues of the proteins-urea, uric acid, etc. - can be further oxidized, and the remnant of energy which they yield must be taken into account in any calculation of the total heat-production founded on the heat of 
combustion of the food substances. From careful experiments, it lias been found that a gramme of dry protein (egg-albumin), when burned in a calorimeter, yields 5735 calories of heat, a gramme of dextrose $3.7+2$, and a gramme of animal fat 9.500 calories (Stohmann).

Heat-equivalent of I gramme of albumin

Calories.

Albumin (minus urea producerl from it)

5735

Cane-sugar

4949

Kreatin (water-frec)

Starch -

In applying such results to the calculation of the heat-production of the body, it is not sufficient to deduct from the heat of combustion of the proteins the heat which the residual urea would yield if fully oxidized. For other incompletely oxidized products arise from proteins when consumer! in the borly, and Rubner has shown, by actually determining the heat of combustion of the urine and frecs. that the real equivalent of a gramme of albumin is at most only $4+20$ calories. The heat-equivalent of our less liberal specimen diet (p. 5+5) will be approximately :

\begin{tabular}{|c|c|c|c|c|}
\hline Protein, 95 grammes & $x$ & 4420 & $=$ & $\begin{array}{l}\text { Calories } \\
419^{\circ} 9\end{array}$ \\
\hline Fat, So grammes & $x$ & $9^{\circ} 500$ & $=$ & $760^{\circ} 0$ \\
\hline $\begin{array}{l}\text { Carbo-hydrate (reckoned as } \\
\text { dextrose), } 320 \text { grammes }\end{array}$ & $x$ & $7+2$ & $=$ & I. IOT" \\
\hline
\end{tabular}

The heat-equivalent of the more generous specimen diet (P. 5 $4^{6}$ ) would be 2,873 calories.

But this is the diet of a man doing a fair day's work, and to get the quantity of energy which actually appears as heat, the heatequivalent of the mechanical work performed must be deducterl. A fair day's work is about I 50,000 kilogramme-metres - that is, an amount equal to the raising of 150.000 kilogrammes to the height of a metre. Now, a kilogramme-degree or calorie of heat is equivalent to 4255 kilogramme-metres of work, and a kilogrammemetre to $\frac{\mathrm{I}}{4255}$ calorie. The heat-equivalent of the day's work is, therefore, I $50,000 \times \frac{I}{425.5}=352$ calories. Deducting this from the heat-equivalent of the food. we get in round numbers 2,520 calories as the heat given off on the more liberal diet. This corresponds fairly well with the calculated heat-loss (p. 579).

The following table, based on the direct calorimetric observations of Atwater and Benedict, shows the average heat-production in a large number of experiments on several individuals at rest and doing measured amounts of work, with a stationary bicycle, for instance. This was connected with a small dynamo, which transformed the greater part of the work into electrical energy. The electrical energy in its turn was changed into heat, the current passing through a lamp : 


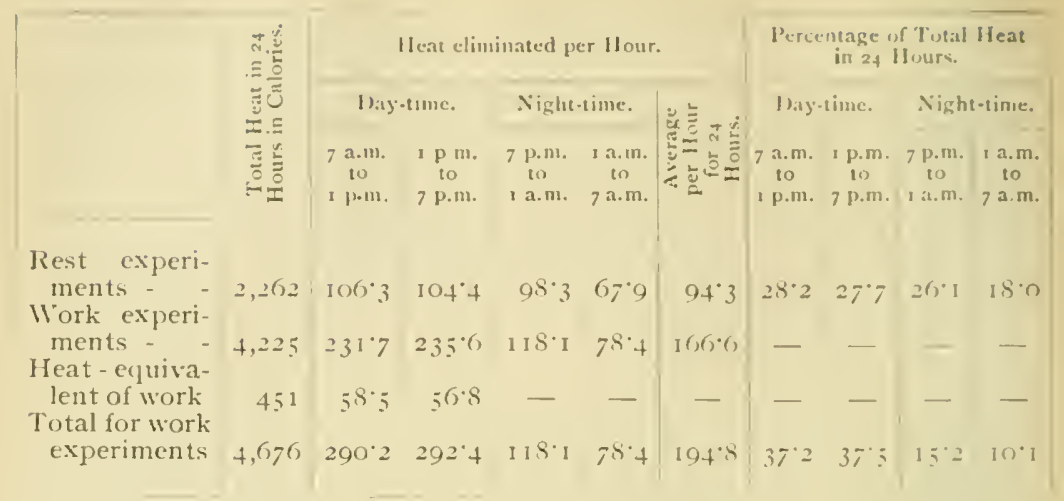

The heat-production during the hours of slecp, in the second niglat period, is much less than in the waking hours of rest, ancl of course enormously less than in the hours of work. After work the heatproduction in the period of sleep is only a little greater than after rest.

As already inclicated (p. 580 ). it is permissible to calculate the heatproduction from the diet, and Rubner has done this for various classes of men, reducing everything to the standard of a body-weight of 67 kilos. The fasting man, of 67 kilos body-weight, produces 2,303 calories in the twenty-four hours. The class of brain-workers, represented by physicians and officials, produce only a little more heat than the fasting man, viz., 2, $4+5$ calorics. The sccond class, represented by soldiers (presumably in time of peace) and (laylabourers (probably of a cautious and conservative type), work up to 2,868 calories. The third class, composed of men who work with machines and other skilled labourers, attain a Ineat-production of 3.362 calories. The fourth class, typified by miners (who are engaged, usually by the piece and not by the day, in severe and cxhausting toil), produce as much as 4.790 calories. In the fiftl and last class, represented by lumberers and other out-of-door labourers (who, in addition to excessive exertion, have often to face intense cold), the heat-production rises to 5,360 calories. The diet of ordinary prisoners in Scotland doing light work, chiefly of a sedentary character. was found to correspond to 3. II 5. and that of convicts on 'hard labour' to 3.707 calories. It is a fair presump)tion that in Scotch prisons the total heat value supplied is net excessive. From the general agreement of calculated results with actual measurements we can safely conclude that most healthy adults produce between 2,000 and 3,000 calories $(3,5$ to to per lilo of bodyweight) on a 'rest' day, or a day of light labour, and between 3.000 and 4,000 ( 45 to 60 per kilo of body-weight) on a day' of hard mannal work.

What has been already said in connection with standard dictaries (p. 545) indicates that the work of the world might possibly be accomplished as well with a smaller transformation of energy in the human machine, at least in the more prosperous countries, and that in the body, as in an engine, more careful 'stoking' might result in a saving of fuel. It is extremely improbable, however, that any argument of this sort will have much effect upon the deep-rooted dictetic habits of mankind. 
In any case it must be carefully remombered that the question of the minimum amount of protein necessary in a permanent diet is guite distinct from the furestion of the minimum lieat value of the clict for a man of given borly-woight doing a defmite amount of work under definite conditions. Whether the protein allowance be scanty or liberal, the total heat value camnot be permanently reduced below a certain minimum elepensling on the work done, the climate, and other conditions. McCay points ont that while Bengalis subsist on food containing only about onc-third the amount of protein in such a 'standard' diet as Voit's. and may therefore be supposed to be immune from the dangers of an excessive protein metabolism, the large intake of carbo-hydrate rendered necessary by the poverty of the food in protein is associated with perhaps greater evils, anong them a marked predisposition to diabetes and renal troubles. Their weight, chest measurement, and muscular development are inferior to those of other Asiatics living in the same climate but with dietetic habits which ensure them a larger supply of protein.

The Seats of Heat-production. - We have ahready recognised the skeletal muscles as important seats of heat-production. A frog's muscle, contracting under the most favourable conditions, does not convert at most more than one-fourth or one-fifth of the energy it expends into mechanical work; at least three-fourths or four-fifths of the energy appears as heat. The muscles of mammals and of man in the intact body work, upon the whole, more economically than the excised frog's muscles at their maximum efficiency. Under the best conditions - that is, when the work is moderate and not too rapidly done-about one-third of the chemical energy expended may be transformed into mechanical work, and only two-thirds into heat (Zuntz). In hard work three-quarters of the energy may be changed into heat; but even then the efficiency of the muscles far outstrips that of the best steam-engines, which convert only an eighth of the total energy into work.

Notwithstanding the splendid efficiency of the muscular machine, the gaseous metabolism easily rises during muscular work to five times, and in severe labour to nine times its resting value, although persons inured to toil work more economically than amateurs. In one of Atwater's 'severe work' experiments the work done in twenty-four hours had a heat-equivalent of 1,482 calories (equal to over 630,000 kilogramme-metres). The total heat-production (including the equivalent of the work) was $9,3 \mathrm{I} 4$ calories. It is not difficult to show that the greater part of the metabolism and heat-production of a man doing ordinary work is accounted for by the contraction of the voluntary and involuntary muscles.

Even in muscles completely at rest metabolism goes on, and some heat is produced. By analyzing the gases of the arterial and venous blood Zuntz compared the oxygen consumption and carbon dioxide production in the hind-legs of dogs when the sciatic and anterior 
crural nerves were clivikled and intact. In both cases the muscles were at rest in the orlinary sense. But in the second experiment the central 'tonus' (p. S13) was preserved. While in the first it was abolished. In one experiment in which the nerves were intact the oxygen consumed amounted to $1 \cdot 22$ c.c., and the carbon dioxide produced to 132 c.c., per kilo of tissue per minute. In the experiment in which the nerves were severed, the corresponding numbers were 0.68 c.c. for the oxygen and 0.63 c.c. for the carbon dioxide. Although it is probable, from the results of Chauveau and Kauffmann alrearly referred to (p. 263), that these figures are too low for the normal resting muscle, they still demonstrate that, even in th?

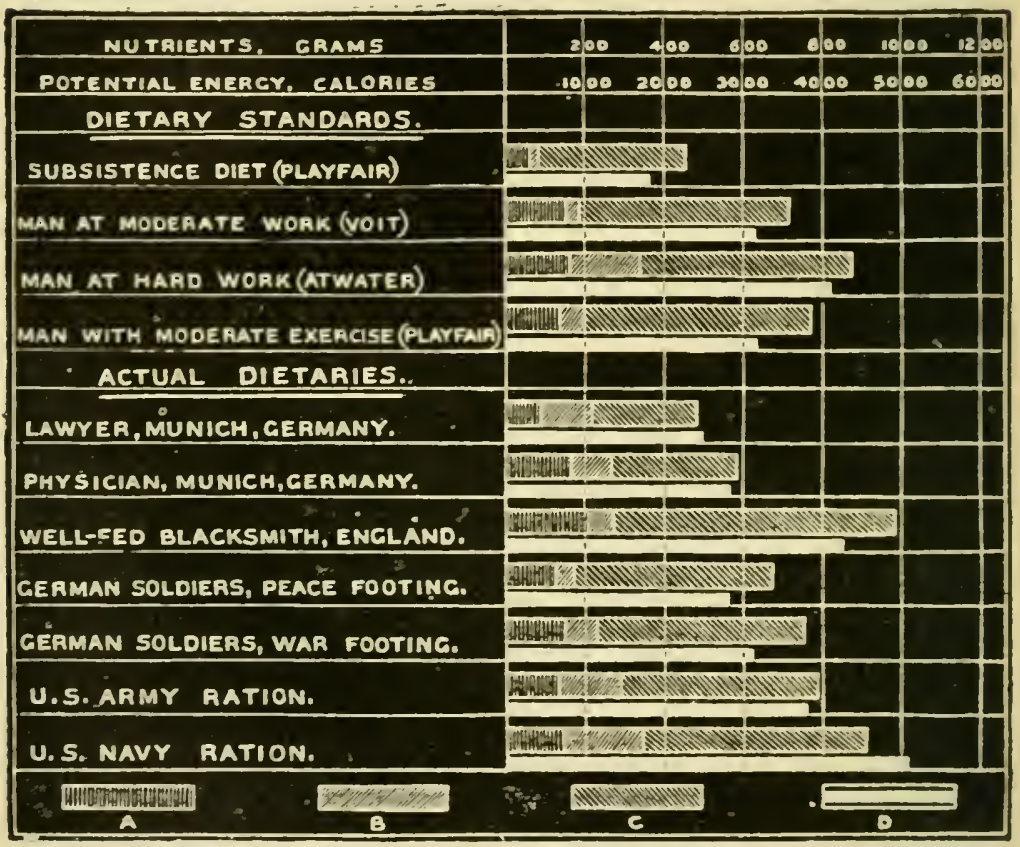

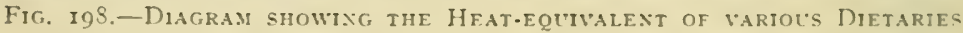

A, proteins ; B, fats ; C, carbo-hydrates ; D, heat-equivalent.

absence of innervation from the central nervous system, the metabolism, and therefore the heat-production of the muscles, are by no means negligible; 0.68 c.c. of oxygen per minute corresponds to 40.8 c.c. per hour, or more than one-tenth of the oxygen consumption per kilo per hour of a fasting dog lying at rest (Zuntz).

If the work of the heart is taken as 16,600 kilogramme-metres in twenty-four hours (p. I27), the total heat produced by this organ will be equivalent (on the assumption that it converts one-third of its energy into work) to about 50,000 kilogramme-metres, or not much less than 120 calories, since, practically, the whole work is expended in overcoming the friction of the vessels, and finally: 
appears as heat. Enough energy is transformed in twenty-four hours in the heart of the colonel of a regiment of 1,000 men to lift the whole regiment to the height of the mess-table, if it coukl be all changed into mechanical work. Barcroft and Dixon have calculated the energy of the heart's contraction on the assumption that it is derived from the oxidation of a carbo-hydrate by the oxygen absorbed by the organ. They concluded that the energy set free in the heart of a dog weighing 12 kilos corresponds on the average to 7.86 kilogramme-metres per minute, which is equivalent to 26.6 calories in twenty-fom hours. Allowing for the fact that the heart of a small animal pumps more blood in proportion to the boly-weight than the heart of a large animal (p. 1 27), this result agrecs very well with that decluced from the work of the heart. The work of the inspiratory muscles may be reckoned at 13,000 kilogramme-mctres, equal to $30^{\circ} 5$ calories, and the heat produced by them at, say, go calories. In sum, the muscular work cf the circulation and respiration is responsible for the production of about 210 calorics (without including the lieat produced by the smootl muscle of the bronchi and bloodvessels), or nearly one-twelfth of the total proluction of a man doing ordinary labour.

The glands, and then the central nervous system, rank after the muscles, though at a great distance, as seats of heat-production. The liver and brain (?) are the hottest organs in the body ; and that this is not altogether due to their being well protected against loss of heat is shown, in the case of the liver, by the excess of temperature of the blood of the hepatic over that of the portal rein. In view, however, of the exaggerated importance which some have given to these organs as foci of heatproduction, it may be well to point out that although many of the chemical changes in the animal body are undoubtedly associated with the setting free of heat (exothe:mic reactions), other, and not less weighty and characteristic, reactions may cause the absorption of heat (endothermic reactions); and it is possible that some of the syntheses which many of the tissues are capable of performing may be included in this latter category. For example, when urea is decomposed so as to yield ammonium carbonate (p. 438), heat is set free. We must assume that if ammonium carbonate were transformed into urea in the liver, an equal amount of heat would be, on the whole, absorbed. So that the heat-production of an organ may depend, not only upon the quantity, but also upon the quality, of its chemical activity. In all the tissues, including the muscles, it is necessary to assume that some of the energy transformed is expended in so-called 'restitution' processes - that is, in replenishing the store of nutritive material within the cells and in building up the protoplasm. Claude Bernard observed an excess of $0.6^{\circ} \mathrm{C}$. in the temperature of the blood of the hepatic vein over that of the portal during hunger, and as much as $1 \cdot 6^{\circ}$ at the height of digestion, although at the beginning of digestion the portal 
blond was the lotter by $04^{\circ}$. But such observations, like the corresponding ones on the salivary glands, are open to many errors, and when we consider the enormous tide of blood which during digestion sets through the portal system, we shall look with suspicion upon results that announce a difference of more than a small fraction of a degree in the temperature of the incoming and outgoing blood of the liver. Probably not less than 200 litres of blood pass in twenty-four hours through the liver of a 2-kilo rabbit. If the temperature of this blood is raised even one-tenth of a degree in its passage through the hepatic capillaries, this would correspond to a heat-production of 20,000 small calories, or one-tentl of the whole heat produced in the animal.

In the case of the brain there is some cridence, obtaincl by comparison of the gases of blood taken from the carotid and from the venous sinuses (torcula Herophili), that the metabolism is fecble as compared even with that of resting muscles (Hill). Nor is it possible to demonstrate any marked or constant increase when the cerebral cortex is roused to such an active discharge of impulses as leads to general epileptiform convulsions. The rise of temperature of certain regions, especially the occipital portion, of the scalp. which some obscrvers have stated to take place during mental activity, cannot be supposecl to be due to conduction of heat from the brain through the skull. It is perhaps caused by vaso-motor changes in the scalp. associated, it may be, with corresponding changes in related areas of the cortex. The increase observed by Mosso in the temperature of the brain during intense psychical activity. sometimes to $0.2^{\circ} \mathrm{C}$. or $0.3^{\circ} \mathrm{C}$. above the rectal temperature, may also have been due, in part at least, to vascular changes. And, indech, if we remember how large a proportion of the central nervous system is made up of nerve-fibres, in which. or at any rate in the fibres of periplieral nerves. no sensible production of heat has ever been demonstrated. it will not appear surprising if even a considerable increase in the metabolism of the really active elcments should fail to make itself felt.

With regard to the muscles, we are as yet in the dark as to the precise relation of the energy which appears as heat and of that which is converted into work. The original source of both is, of course, the oxidation (and cleavage) of the food substances, but it has been the subject of discussion whether in a muscle, as in a heat-engine, the chemical energy is first converted into leat, and part of the heat then transformed into work, or whether the chemical energy is immediately changed into work, or whether there is an intermediate form of energy other than heat. Some have supposed that the chemical energy is first converted into electrical energy (p. 640).

It has been very generally admitted that the chief seat of excessive metabolism in fever is the muscles; but U. Mosso luas stated that cocaine fever-the marked rise of temperature produced by injection of cocaine-can be obtained in animals 
paralyzed by curara. This, eren if true, would not support the conclusion that a 'nervous fever' - that is to say, a fever due solely to increased metabolism in the nervous system-exists; for in a curarized animal a large amount of 'active' tissuc (glands, heart, smooth muscle) still remains in physiological connection with the brain and cord. But, as a matter of fact, in an animal under a dose of curara sufficient to completely paralyze the skeletal muscle cocaine causes no appreciable rise of rectal temperature; and this is strongly in favour of the view that the fever produced in the non-curarized animal is connected with excessive muscular metabolism.

Regulation of Temperature or Thermotaxis. - What, now, is the mechanism by which the balance is maintained in the homoiothermal animal between heat-production and heat-loss? In answering this question we have to recognise that both of these quantities are variable, that a fall in the production of heat may be compensated by a diminution of heat-loss, and an increase in the loss of heat balanced by a greater heat-production.

The loss of heat from the surfaces of the body may be regulated both by involuntary and by voluntary means. It is greatly affected by the state of the cutaneous vessels, and these vessels are under the influence of nerves. A cold skin is pale, and its vessels are contracted. In a warm atmosphere the skin is flushed with blood, its vessels are dilated, its temperature is increased ; an effort, so to speak, is being made by the organism to maintain the difference of temperature between its surface and its surroundings on which the rate of heat-loss by radiation and conduction depends. A still more important factor in man, and in animals like the horse, which sweat over their whole surface, is the increase and decrease in the quantity of water evaporated and of heat rendered latent. It is owing to the wonderful elasticity of the sweat-secreting mechanism, and to the increase of respiratory activity and the consequent increase in the amount of watery vapour given off by the lungs, that men are able to endure for days an atmosphere hotter than the blood, and even for a short time a temperature above that of boiling water. The temperature of a Turkish bath may be as high as $65^{\circ}$ to $80^{\circ} \mathrm{C}$. Blagden and Fordyce exposed themselves for a few minutes to a temperature of nearly I $27^{\circ} \mathrm{C}$. Although meat was being cooked in the same chamber by the heat of the air, they experienced no ill effects, nor was their body-temperature even increased. But a far lower temperature than this, if long continued, is dangerous to life. During the 'hot waves,' not infrequently experienced in summer in the United States, hundreds of persons have died within a few days from the excessive heat. It is stated that during the unusually hot summer of ISIg the 
temperature at Bagdad ranged for a considerable time between $108^{\circ}$ and $120^{\circ} \mathrm{F}$. $\left(42^{\circ}\right.$ to $49^{\circ} \mathrm{C}$.), and there was great mortality. A much higher temperature may be borne in dry air than in air saturated with watery vapour. A shade temperature of $100^{\circ} \mathrm{F}$. $\left(377^{\circ}\right.$ C.) in the dry air of the South African plateaux is quite tolerable, while a temperature of $85^{\circ} \mathrm{F} .\left(29^{\circ}+{ }^{\circ} \mathrm{C}\right.$.) in the moistureladen atmosphere of Bombay may be oppressive. The reason is that in dry air the sweat evaporates freely and cools the skin, while in moist air, although according to Rubuer the loss of heat by radiation and conduction is increased, the loss of heat by evalporation of sweat is diminished in a still greater clegree. In saturated air at the body-temperature no loss of heat by perspiration or by exaporation from the pulmonary surface is possible ; the temperature of an animal in a saturated atmosphere at $35^{\circ}$ to $40^{\circ} \mathrm{C}$. soon rises, and the animal dies. In animals like the dog, which sweat little or not at all on the general surface, the regulation of the heat-loss by respiration is relatively. more important than in man.

The observations of Boycott and Haldane in a deep mine, in the incubating-room of a laboratory, and in a Turkish bath illustrate the important influence of the humidity of the air. In still air the body-temperature rose above normal when the wet-bulb thermometer rose above $31^{\circ} \mathrm{C} .\left(88^{\circ} \mathrm{F}\right.$.), and it remained normal whatever the external temperature might be so long as the reading of the wetbulb thermometer disl not excecl that level. The more the wet-bulb thermometer rose above $31^{\prime}$ the more rapicl was the increase in the borly-temperature. In moving air a greater degree of humidity could be borne without increase in the body-temperature, which diel not occur till the temperature shown by the wet-bulb thermometer excecled $35^{\circ} \mathrm{C}$. The great increase in the evaporation of sweat when the temperature of the air is high is shown by the obscration that on a warm day (dry bulb, $79^{\circ} \mathrm{F}$.; wet bulb. $67^{\circ} 5^{\circ} \mathrm{F}$ ) the average loss of moisture from the body was I,8I 6 grammes for four soldiers during a march of seven miles, while on a cold day (dry bulb. $+5^{\circ} \mathrm{F}$.: wet bulb, $38^{\circ} \mathrm{F}$.) it was only 419 grammes during the same march by the same men (Pembrey).

The winter fur of Aretic animals is a special device of Nature to meet the demands of a rigorous climate, and combat a tendency to excessive loss of heat. The experiments of Hösslin, and the experience of squatters in Australia, go to show that even domesticated animals have a certain power of responding to long-continued changes in external temperature by changes in the radiating surfaces which affect the loss of heat. It is said that in the hot plains of Queensland and New South Wales the fleeces of the sheep show a tendency to a progressive decrease in weight. And Hösslin found that a young dog exposed for eighty-eight days to a temperature of $5^{\circ} \mathrm{C}$. developed a thick coat of fine woolly hairs. Another dog of the same litter, exposed for the same length of time to a temperature of $3 I^{\prime} 5^{\circ}$ to 
$32^{\circ} \mathrm{C}$. , had a much scantier covering. The increased protection against heat-loss in the case of the cooled ' dog was not sufficient fully to compensate for the lowered external temperature. Tlie metabolism-that is to sity, the heat-production-was also increased. And although the food was exactly the same for both animals in quantity and quality, the $\log$ at $5^{\circ} \mathrm{C}$. put on less than half as much fat in the period of the experiment as the 'heated' dog, but the same amount of 'flesh.'

The voluntary factor in the regulation of the heat-loss is of great importance in man. Clothes, like hair and other natural coverings, retard the loss of heat from the skin chiefly by maintaining a zone of still air in contact with it, for air at rest is an exceedingly bad conductor of heat. A man clothed in the ordinary way has two or three concentric air-jackets around him. The air in the intervals between the inner and outer garments is of importance as well as that in the pores of the clothes themselves; and it is for this reason that two thin shirts put on one above the other are warmer than the same amount of material in the form of a single shirt of double thickness. When a man feels himself too hot and throws off his coat, he really removes one of the badly conducting layers of air, and increases the rate of heat-loss by radiation and conduction. At the same time the water-vapour, which practically saturates the layer of air next the skin, is allowed a freer access to the surface, and the loss of heat by the evaporation of the sweat becomes greater. The power of voluntarily influencing the heat-loss must be looked upon in man as one of the most important means by which the equilibrium of temperature is maintained. In the lower animals this power also exists, but to a much smaller extent. A dog on a hot day puts out its tongue and stretches its limbs so as to increase the surface from which heat is radiated and conducted. The mere placing of a rabbit on its back, with its legs apart, may cause in an hour or two a fall of $I^{\circ}$ to $2^{\circ} \mathrm{C}$. in the rectal temperature. The power of covering themselves with straw or leaves, of burrowing and of forming nests, may be included among the voluntary means of regulation of the heatloss possessed by animals. A man opens the window when he is too hot, and pokes the fire when he feels cold. Both actions are a tribute to his status as a homoiothermal animal, and illustrate the importance of the voluntary element in the mechanism by which his temperature is controlled.

The production of heat, like the loss, is to a certain extent under voluntary control. Rest, and especially sleep, lessens the production; work increases it. The inhabitants of the tropics, human and brute, often tide over the hottest part of the day by a siesta ; and it is as natural, and as much in accord- 
ance with physiological laws, that a man overpowered by the lieat should lie down, as it is that he should walk about and stamp his feet or clap his hands on a cold winter morning. In the one case a diminution, in the other an increase, in the heatproduction is amed at by a corresponding cliange in the amount of muscular contraction. The quantity and quality of the food also influence the production of heat. The Eskimo, who revels in train-oil and tallow-canclles, unconsciously illustrates the experimental fact that the heat of combustion of fat is high ; the rice diet of the ryot of the Carnatic, with its low heat-equivalent, seems peculiarly adapted to the dweller in tropical lands. But it would be easy to attach too much weight to considerations sirch as these. The Arctic hunter eats animal fat, and the Indian peasant vegetable carbo-hydrate, not only because fat has a high and carbo-hydrate a low heat-equivalent, but because in the climate of the Far North animals with a thick coating of badly-conducting fat are plentiful, and regetable food scarce; whereas in the river-valleys of India Nature favours the growth of rice, and religion forbids the killing of the sacred cow.

The production of heat is also controlled by an involuntary nervous mechanism, through which the 'chemical' regulation of the body-temperature is achieved, as the ' physical 'regulation is accomplished by the nervous mechanisms that control the circulation, the sweat-glands, and the respiratory movenents. It is a mat ter of everyday experience that cold causes involuntary shivering-involuntary inuscular contractions-the object of which seems a direct increase in the heat-production. But besides this visible mechanical effect, the application of cold to a warm-blooded animal, when not carried so far as to greatly reduce the rectal temperature, is accompanied by a marked increase in the metabolisn, as shown by an increased production of carbon dioxide and consumption of oxygen. In coldblooded animals like the frog the metabolism, on the other hand, rises and falls with the cxternal temperature; there is no automatic mechanism which answers an increased drain upon the stock of heat in the body by an increased supply. Or, in the light of recent experiments, we ought rather to say that, although the rudiments of a heat-regulating mechanism may exist in such animals as the frog, the newt, and even the earthworm (Vernon), it is only able to modify to a certain extent the effects of changes of external temperature, not to balance or even override them, as in the homoiothermal animal. The warmblooded animal loses its heat-regulating power when a dose of curara sufficient to paralyze the volumtary muscles is given. A curarized rabbit, kept alive by artificial respiration, reacts to changes of external temperature like the cold-blooded frog. Now, the only action of curara adequate to account for this 
effect is its power of paralyzing the motor innervation, and so cutting off from the skeletal muscles impulses which in the intact animal would have reached them. The excitation by cold of the cutaneous nerves, or some of them, which in the umpoisoned animal is reflected along the motor nerves to the muscles, and causes the increase of metabolism, is now blocked at the end of the motor path; and the muscles, the great heatproducing tissues, are abandoned to the direct influence of the external temperature (Pllüger).

How is it, then, that nervous impulses from the skin produce in the intact animal their effect upon the chemical processes in the muscles? $\mathrm{We}$ know that the heat-production of a muscle is greatly increased when it is caused to contract; but it has not hitherto been possible by artificial stimulation to demonstrate that any chemical or physical effect is produced in a muscle by excitation of its motor nerve unless as the accompaniment of a mechanical change. When the gastrocnemius of a frog poisoned with not too large a dose of curara is laid on a resistance thermometer (p. 664), and its nerve stimulated from time to time as the curara paralysis deepens, heating of the muscle is observed as long as, and only as long as, there is any visible contraction. The gaseous metabolism of a rabbit immersed in a bath of constant temperature may sink by as much as 30 to to per cent. when curara is given. One obvious cause of this is the complete muscular relaxation. And the whole secret of the regulation of the lieat-production might be plausibly supposed to lie in the bracing effect of cold upon the skeletal muscles and the relaxing effect of heat. Indeed, in man it has been observed that exposure to moderate cold causes no metabolic increase when shivering is prevented by a strong effort of the will (Loewy). Nevertheless, the explanation is inadequate in the case of small animals, such as guinea-pigs, rabbits, and cats; for very great changes in the metabolism may be brought about by external cold without any outward token of increased muscular activity. In a man also a fall in the external temperature from $23^{\circ}$ to $I 5^{\circ} \mathrm{C}$. caused a certain increase in the output of carbon dioxide (from $27^{\circ} 9$ to $32 \cdot 3$ grammes per hour), although no shivering was observed. As the temperature of the air is lowered, the point is soon reached at which shivering can no longer be suppressed, and then it is neither practicable nor perhaps very important to distinguish clearly the portion of the increased heat-production associated with the risible muscular contractions and the portion due to quickened muscular metabolism without contraction. Lefèvre found that in man a marked increase in the heat-loss, such as is caused by immersion for a considerable time (one to three hours) in cold water (at a temperature of $7^{\circ}$ to $I 5^{\circ} \mathrm{C}$.), was accompanied by a great increase 
in the production of heat, so that the axillary temperature fell comparatively little - e.g., only $\mathrm{I}^{\circ} \mathrm{C}$. during a stay of three hours in a bath at $15^{\circ} \mathrm{C}$. With short periods of immersion, a characteristic reaction occurs after the person comes out of the bath. The rectal temperature falls to a minimum, which is reached in twenty to thirty minutes after exit from the bath, and then gradually returns to normal. This fall of internal temperature is due to the heating of the superficial portions of the body at the expense of the central portions. By training, the fall of temperature is greatly lessened, the heat-regulating mechanism acquiring, so to speak, with practice, greater promptitude and precision of adjustment.

It must be admitted, then, that-especially in the smaller homoiothermal animals - the metabolic changes normally going on in the resting muscles may be reflexly increased without the usual accompaniment of mechanical contraction, and that such an increase of 'chemical tone' is an important means by which the temperature is regulated. It is possible that other organs besides the muscles may be concerned, though not to a sufficient extent to secure the due regulation of temperature during curara paralysis. It is obvious that in man, whose environment is so much under his own control, a mere automatic regulation is less required than in the inferior animals, and that a regulative power, if present in rudiment, would tend to 'atrophy' by disuse, or, at all events, to become less sensitive to slight changes of temperature. In the larger animals, again, mere bulk is an important safeguard against any sudden change of internal temperature. To reduce the temperature of a horse or an elephant by $\mathrm{I}^{\circ}$, a considerable quantity of heat must be lost, while a very slight loss would suffice to cool a mouse by that amount. Not only so, but the surface by which heat is lost is greater in proportion to the mass of the body in small than in large animals. The power of rapidly increasing the heat-production to meet a sudden demand is, therefore, far more important to the mouse than to the horse; and the fact (p. 5+9) that the metabolism of an animal varies approximately as its surface, and not as its mass, * is an illustration of the nice adjustment by which heat-equilibrium is maintained.

* The relation between mass (M) and surface (S) in man is approximately given by the equation $\frac{S I^{3} M}{M}=K$, and the relation between surface, mass, length of body (L), and circumference of chest (C) just above the nipples in the "mean " position of respiration, by the equation $\frac{\mathrm{S} \sqrt[6]{\mathrm{M}^{+} \cdot \mathrm{L}^{+} \cdot \mathrm{C}^{2}}}{\mathrm{MLC}}=\mathrm{K}^{\prime} . \quad$ II is expressed in grammes, $\mathrm{S}$ in square centimetres, $L$ and $C$ in centimetres. $K$ is a constant whose mean value is 12.3 , and $\mathrm{K}^{\prime}$ a constant whose mean value is $4^{\circ} 5$ (Meeh). 
The following table shows how close is the agreement in the heatproduction per unit of surface calculated by the formula for animals of different species and very different body-iveight.

N

The next table. calculated by Rubner from the quantity of tissue-protein and fat consumed, gives the relative intensity of heat. proluction in fasting dogs of different sizes :

\begin{tabular}{|cc|}
\hline Body.weight. & $\begin{array}{c}\text { Calories per Kilo } \\
\text { per Hour }\end{array}$ \\
\hline 3 I K & I. 58 \\
27 & I. 70 \\
20 & I. 87 \\
I 8 & I.92 \\
I0 & 2.55 \\
6 & 2.84 \\
3 & 3.78 \\
\hline
\end{tabular}

Rubner has found that animals abundantly fed do not show so much change in the production of heat when the external temperature is varied as starving animals, perhaps because the thicker coat of subcutaneous fat so steadies the rate at which heat is lost that it becomes easy for the vaso-motor mechanism alone to hold the balance between loss and production. In well-fed animals it is the heat-loss which is chiefly affected, and it may be that this has something to do with the explanation of Loewy's results on man (P. 59I).

Lorrain Smith discovered the interesting fact that after removal of the thyroid glands (in cats), the heat-production, as measured by the amount of carbon dioxide given off, is more sensitive to changes of external temperature than in the normal animal.

But it must not be imagined that the production of heat can be increased indefinitely to meet an increased heat-loss. The organism can make considerable efforts to protect itself, but the loss of heat may easily become so great that the increase of metabolism fails to keep pace with it. The internal temperature then falls, and if the fall be not checked, the animal dies. A mammal, when cooled artificially to the temperature of an ordinary room ( $5^{\circ}$ to $20^{\circ} \mathrm{C}$.), does not recover of itself, but may be revived by the employment of artificial respiration and hot baths, even when the rectal temperature has sunk to $5^{\circ}$ to $10^{\circ} \mathrm{C}$. If the skin of a rabbit be varnished, and 
the air which it is the function of the fur to maintain at rest around it be thus expelled, the animal dies of cold, unless the loss of heat is artificially prevented. If, without varnishing at all, the greater portion of the skin of a rabbit or guinea-pig be closely clipped or shaved, similar phenomena are observed. Prevented from covering itself with straw, the animal dies, sometimes in twenty-four hours. The racliation from the skin, as measured by the resistance-radiometer (p. 574), is greatly increased; the animal shivers constantly. and the rectal temperature falls. Placed in a warm chamber before the temperature in the rectum has fallen below $25^{\circ}$, the animal recovers perfectly. If the fall is allowed to go on, it rlies. If it is kept from the first in the warm chamber, no fall of temperature occurs. When the increased loss of heat is less perfectly compensated-when, for example, the animal is left at the ordinary temperature, but supplied with sufficient straw to cover itself, or allowed to crouch among other animals - a curious phenomenon may sometimes be seen. The rectal temperature, which has fallen sharply (luring the operation, remains subnormal (as much as $2^{\circ}$ to $3^{\circ}$ below the ordinary temperature) for a time (a weck or more), and then gradually rises as the coat again begins to grow. The meaning of this seems to be that the power of regulating the temperature by increasing the metabolism is overtasked by the removal of the natural protective covering, unless the escape of heat is artificially diminished. When the loss of the fur is entirely compensated, no fall of temperature occurs; when it is not compensated at all. the animal cools till it dies; when it is partially compensated, the increased metabolism may only suffice to maintain a temperature lower than the normal, although constant muscular contractions (shivering) are brought in to supplement the efforts of the regulative chemical processes.

Hitherto we have only spoken of a reflex regulation of the heat-production called into play by external cold. It might be supposed-and, indeed, has often been assumed-that heat would lessen the metabolism, as cold increases it ; and there are indications that in the smaller animals this is the case, although the influence of heat seems to be much smaller than the influence of cold. But neither experimental results nor general reasoning have as yet shown that in man, either in the tropics (Eykman) or in the north temperate zone (Loewy), the chemical tone is diminished by a rise of external temperature much above the mean of an ordinary English summer, apart from the effect of the muscular relaxation which heat induces. In a man, indeed, at rest in a hot atmosphere, the production of carbon dioxide and consumption of oxygen are, if anything, greater than at the ordinary temperature. The regulation of temperature in an environment warmer than the normal seems, in fact, to be brought about more by an increase in the loss than a decrease in the production of heat. Evaporation from the skin and lungs is an automatic check upon overheating as important as the involuntary increase of metabolism upon excessive cooling.

While it is known that the skeletal muscles, and perhaps the 
glands and other tissues, are at one end of the reflex are by which the impulses pass that regulate the temperature through the metabolism, we are as yet ignorant of the precise paths by which the afferent impulses travel, of the nerve-centres to which they go, and even of the end-organs in which they arise. There are nerves in the skin which minister to the sensation of temperature (Chap. XIII.). A change of temperature is their 'adequate' and sufficient stimulus; and it is a tempting hypothesis that these are the afferent nerves concerned in the reflex regulation of temperature-- that impulses carried up by them to some centre or centres in the brain or cord are reflected down the motor nerves to control the metabolism of the skeletal muscles, and down the vaso-motor nerves to control the loss of heat from the skin.

It is more than doubtful, however, whether the whole chemical regulation can be attributed to such stimuli. For it has been found that the relation between heat-production and cxtent of surface in animals (guinea-pigs) of different size is unaltered when the air temperature is made so nearly the same as that of the skin that the temperature nerves can hardly be supposed to be excited.

There is some evidence that the bioplasm - the living substance-of different animals, even when the external conditions are the same, may differ specifically in the average intensity of metabolism to which it is pitched. When exposed to a temperature about equal to that of warm-blooded animals, the green lizard (Lacerta viridis) and the bull-frog, which live in the temperate zone, and for which a temperature of $37^{\circ} \mathrm{C}$. is highly abnormal, double their heat-production, and soon die. Tropical poikilothermal animals, such as the alligator, also double their heat-production, but the highest values reached are only one-half that of the lizard at $25^{\circ} \mathrm{C}$. Apparently the bioplasm of the tropical animals has adapted itself to a high external temperature, and works very economically even at the highest temperatures (Krehl).

Heat Centres.- It is known that certain injuries of the central nervous system are related to disturbance of the heat-regulating mechanism. Puncture of the median portion of the corpus striatum in the rabbit by a needle thrust through a trephine hole in the skull is followed in a few hours by a rise of temperature in the rectum $\left(1^{\circ}\right.$ to $\left.2^{\circ}\right)$, and still more in the duodenum, which is normally the hottest part of the body in this animal. The heat-production, the respiratory exchange, and the nitrogen excretion are increased. These phenomena may last for several days (Ott, Richet, Aronsohn, and Sachs), and are due to stimulation of the portions of the brain in the immediate neighbourhood of the injury. Electrical stimulation of this region has a similar effect. When the temperature has returned to normal, a fresh puncture may again cause a rise.

Some observers hold that the chief seat of the increased metabolism is the skeletal muscles, others the liver. The question turns largely upon the success of the puncture experiment after the previous administration of curara on the one hand, and of strychnine on the other. For curara cuts out the motor innervation of the skeletal muscles, and strychnine convulsions exhaust the

$$
38-2
$$


store of hepatic glycogen. Certain investigators have found that after an adequate dose of curara no puncture fever can be obtained. and they locate the increased metabolism associated with the fever in the muscles. Others maintain that even after curara the puncture is followed by fever. but is not followed by fever if stryclinine has first been given. They accordingly conclude that the rapid combustion of the glycogen (or the dextrose derived from it) is the primary factor in the increased metabolism. It may be pointerl out, however, that neither experiment is a crucial test. For if stryclunine reduces the liver glycogen, it also reduces the glycogen of the muscles. And if in the puncture fever the liver glycogen is transformed into dextrose more rapidly than usual, the dextrose is probably in great part used up in the muscles more rapidly than usual, else it would appear in the urine. The effect of strychnine on the puncture fever. then, is no proof that the muscles are not essentially concerned in it. On the other hand, the alleged absence of the fever after curara is not sufficient to show that the muscles are alone concerned. For curara causes a lowering of the body-temperature, which, if it be not overcompensated, may mask the fever. The positive result of the puncture in curarized animals, which some observers say they have obtained, would, if confirmed, be important evidence that the primary effect is not on the muscles, or, at least, not solely on them. but would not prove that it is on the liver. That the liver is concerncd, however, is more directly indicated by the fact that during the puncture fever the liver continues to be what it is under normal conditions, the warmest organ in the body, warmer than the blood in the root of the aorta by about $I^{\circ} \mathrm{C}$. The most probable conclusion is that the increased production of leat in this form of experimental fever is due to an increased metabolism of carbo-hydrate (glycogen) both in the liver and in the muscles.

Injury to various portions of the cortex ccrebri in the dog and other animals, and lesions of the pons, medulla oblongata and cord in man may also be followed by increase of temperature. When the spinal cord is cut below the level of the vaso-motor centre, the increased loss of heat from the skin due to dilatation of the cutaneous vessels masks any increase of the heat-production which may possibly have taken place, and the internal temperature falls; but if the loss of heat is diminished by wrapping the animal in cottonwool the temperature may rise. From such phenomena it has been surmised that certain 'centres' in the brain have to do with the regulation of temperature by controlling the metabolism? tissues; that they cause increased metabolism when the internal tempcrature threatens to sink, diminished metabolism when it tends to rise. The cutting off, it is said, of the influence of the 'heat centres' by section iof the paths leading from them allows the metabolism of the tissues to run riot, and the temperature to increase.

The behaviour of hibernating mammals, such as the marmot, dormouse, hedgehog, and bat, is of interest in connection with the temperature regulation. In the active waking state these animals are homoiothermal, but in profound winter sleep they are poikilothermal, the body-temperature rising and falling with that of the air. The rectal temperature may be as low as $2^{\circ} \mathrm{C}$. There is an intermediate state in which the animal is partially awake, though inactive, and its temperature is much 
below the normal, but considerably above that of its environment. In this condition it has an imperfect thermotaxis, something like that of an ordinary mammal (including the human infant) in the period of immaturity, immediately after birth. When the hibernating mammal awakes the rise of temperature is enormous and abrupt. The temperature of a domouse rose in an hour from $13 \cdot 5^{\circ} \mathrm{C}$. to $35^{\circ} 7^{\circ} \mathrm{C}$., and that of a bat in fifteen minutes from $\mathrm{I} 7^{\circ} \mathrm{C}$. to $34^{\circ} \mathrm{C}$. (Pembrey).

Fever is a pathological process generally caused by the poisonous products of bacteria, and characterized by a rise of temperature above the limit of the daily variation (p. 605). It is further associated with an increase in the rate of the heart and the respiratory movements, and a diminution in the alkalies and carbon dioxide of the blood. The total excretion of nitrogen is increased, at least in proportion to the amount of protein ingested, indicating an increase in the consumption of tissueprotein. The distribution of the nitrogen among the urinary constituents is altered. The ammonia (in the form of ammonium salts of organic acids), the uric acid, and to a smaller extent the kreatinin (Leathes), are increased, while the urea is relatively decreased, even when its absolute amount is greater than normal. Ireatin, which is not normally present in urine, unless the food contains it, may also appear in fever (Shaffer). It has been suggested that the proximate cause of fever is the action of bacterial poisons or of other substances on the 'heat centres,' and that antiprretics, or drugs which reduce the temperature in fever, do so by restoring the centres to their normal state, by preventing the development of the poisons, aiding their elimination, or antagonizing their action. In favour of this view, it has been stated that when the basal ganglia are cut off, by section of the pons, from their lower nervous connections, fever is no longer produced by injection of cultures of bacteria which readily cause it in an intact animal, while antipyrin has no influence upon the temperature (Sawadowski). But some observers have been unable to find any clear evidence of the existence of 'heat centres' - that is, of localized portions of the central nervous system specially concerned in the regulation of the body-temperature. And while it is almost certain that some pyrogenic or fever-producing agents-cocaine, e.g. -act indirectly, through the brain or cord, it is quite possible that others affect directly the activity of the tissues in general, just as some antipyretics or fever-reducing agents, such as quinine, act immediately upon the heat-forming tissues, so as to diminish their metabolism, while others, like antipyrin, affect them through the nervous system. Quinine has no influence upon ' puncture' fever in rabbits. A still more important action of 
antipyrin, and the group of antipyretics to which it belongs, is the increase in the heat-loss which they bring about by the dilatition of the bloodressels of the skin. This effect is also produced through the nervous system.

Fever is a condition so interesting from a plyysiological point of view, and of such importance in practical medicine, that it will be well to consider a little more closely the possible ways in which a rise of temperature may occur. It must not be forgotten that the febrile increase of temperature is alwavs accom-

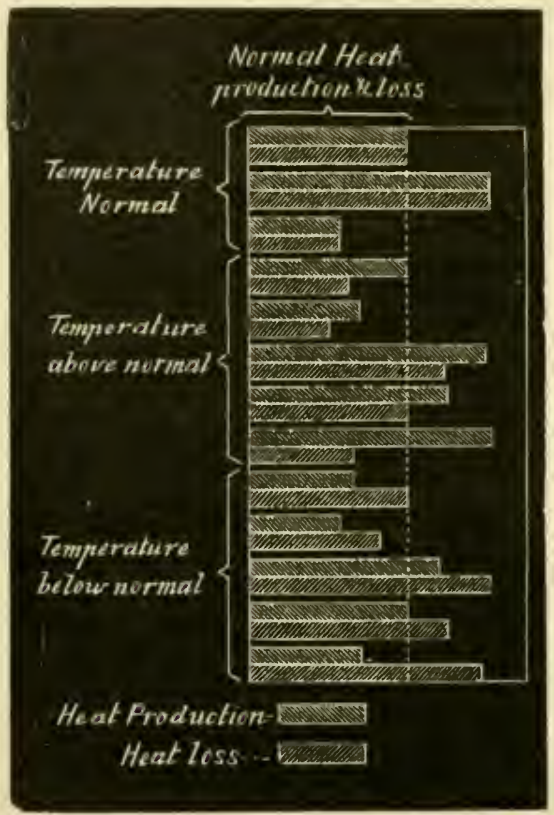

Fig. 199.-Diagram to SHOW the P'OSSIBLF. RELATIONS BETWEEN HEAT-PRODUCTION AND HEAT-LOSS IN FEVER. panied by other departures from the normal, and that all the fundamental febrile changes may even, in certain cases, be present without elevation, and even with diminution of temperature. But here 1 C have only to do with the disturbance of the normal equilibrium between the loss and the production of heat ; and it is evident that any of the five conditions illustrated in the diagram (Fig. I99) may give rise to an increase of temperature. It is not necessary to discuss whether cases of fever can actually be found to illustrate every one of these possibilities. It is probable that not infrequently diminished loss and increased production may be both involved; and it ought to be remembered that the healthy standard with which the heat-production of a fever patient should be compared is not that of a man doing hard work on a full diet, but that of a healthy person in bed, and on the meagre fare of the sick-room. When this is kept in view, the comparatively low heat-production and respiratory exchange which have sometimes been found in fever cease to excite surprise. But in any case, no mere change in the relative proportions of heat formed and lost is sufficient to explain the febrile rise of temperature. That an increase in heat-production is not of itself enough to produce fever is proved by the fact that severe 
muscular work, which increases the metabolism more than high fever, only causes in a healthy man a rise of about $I^{\circ} \mathrm{C}$. in the rectal temperature. When the work is over, the temperature comes rapidly back to normal. The essence of the change in fever is a derangement of the mechanism by which in the healthy body excess or defect of average metabolism, or of average heatloss, is at once compensated and the cquilibrium of temperature maintained.

This derangement only lasts as long as the temperature is rising. When it becomes stationary at its maximum we have again adjustment, again equality of production and escape of lieat; but the adjustment is now pitched for a higher scale of temperature. A rough analogy, so far as one part of the process is concerned, may be found in the behaviour of the ordinary gas-regulator of a water-bath. It can be ' set' for any temperature. That temperature, once reached, remains constant within narrow limits of oscillation; but the regulator can be equally well adjusted for a higher or a lower temperature. It is, however, important to note that the equilibrium is more unstable in fever than in health, so that changes of external temperature more easily depress or increase the temperature of a fever patient than of a healthy man.

Rosenthal has concluded from calorimetric observations that, in the first stage of fever, while the temperature is rising, there is always increased retention of heat. Maragliano actually found evidence, by means of the plethysmograph, that the cutaneous vessels are at this stage constricted, and that the constriction may even precede the rise of temperature. Both observations lend support to the famous 'retention' theory of Traube. In the great majority of cases the production of heat is also increased, on the average by 20 to 30 per cent. of the normal production of a resting man. The increase may be much greater during the chill which ushers in so many infections on account of the muscular contractions in shivering. During the period of rising temperature the production of heat is not necessarily increased. At the height of the fever there is often. though apparently not always, an increase in the heat-production. After the crisis, while the fever is subsiding, the rate at which heat is being lost rises sharply. As to the explanation of the increase of metabolism in fever, and especially of the increased metabolism of tissue-protein, various views have been held. Some have gone so far as to say that the increase is merely the consequence, not the cause, of the rise of temperature. But the rebutting evidence which has been brought against this view is strong and, indeed, overwhelming. It is perfectly true that, when the temperature of the body is artificially raised by pre- 
renting the free loss of heat for a sufficient time (so-called phesiological fever), the destruction of protein is angmented. A fasting dog whose temperature was increased to fo or f I C. for twelve lours eliminated 37 per cent. more nitrogen tlatn when the body-temperature was normal. But this increase in the protein metalolism could be entirely prevented by giving the animal a sufficient amount of carbo-hydrate. Similar results have been obtained in man. The carbon dioxide excretion and oxygen absorption are, of course, also markedly increased. But the increase in the nitrogen excretion is often much greater in fever than any increase which can be brought about by artificially raising the temperature of a healthy individual hy means of hot baths. A typhoid patient was found to lose io.S grammes of nitrogen a day (corresponding to $3 \mathrm{I} S$ grammes of muscle) during eight days of fever (F. Müller). A portion of the loss of nitrogen on the routine fever regimen may be due to the fact that the ordinary typhoid patient is really on a semi-starvation diet, the heat-cquivalent of which is not much more than half his heat-production. Iet it has not been found possible to completely prevent the loss of nitrogen by putting the ferer patient on a diet rich in protein, or on a diet containing a moderate anlount of protein with a large quantity of fat and carbohydrate, even when the total heat-ralue of the diet is much in excess of the 32 or 33 calorics per kilo of body-weight which corresponds to the heat-production of a resting man. Another suggestive fact is that the excessive excretion of nitrogen does not run parallel with the rise of temperature in fever, but is often most marked after the crisis, During the stage of deferrescence an enormous amount of urea is sometimes given off. In a case of typhus, in the mixed urine of the third and fourth days after the crisis, no less than I60 grammes of urea was found (Naunyn), or nearly three times the normal amount for a man on full diet. Again, when fever is caused by the injection of bacteria or their products, the increase in the carbon dioxide climinated and oxygen consumed occurs even when the temperature is prevented from rising by cold baths. It scems perfectly clear, then, that the increase of metabolism is, in many cases at least, a primary phenomenon of fever. Its course and incidence, falling as it does so largely upon the proteins, the steadr loss of tissue nitrogen. and the inability of the tissues to recoup their losses from the protein of the food or to shield their own protein hy burning more carbo-hydrate or fat, all suggest that the cells are poisoned by toxic products of the infective process. The poisoned bioplasm falls an easy prey to the hydrolyzing and oxidizing agents always present in the tissues. It bruaks down more rapidly and builds itsclf up more 
slowly than nommal bioplasm. This increased, and to soms extent perverted, metabolism, far from being occasioned by the febrile temperature, is quite probably the cause of the thermoregulative upset which we call fever. For Mandel las shown(I) that one of the purin bases (xanthin) causes fever in monkeys ; (2) that the purin bases in the urine are increased both in infective fevers and the so-called aseptic or surgical fever-that is, in cases where the temperature rises after such injuries as extensive crushing of tissues without infection. There is a constant relation between the lieight of the fever and the quantity of purin bases excreted. The source of the purin bases in aseptic fever is presumably the autolysis of the injured tissue, from which they pass into the blood without being oxidized to uric acid. The xanthin fever can be prevented by salicylates, though not by antipyrin.

It remains to ask whether the rise of temperature is anything more than a superficial and, so to speak, an accidental circumstance. The orthodox view for many ages has undoubtedly been that the increase of temperature is in itself a serious part of the pathological process, al symptom to be fought with and, if possible, removed. And, indeed, it is not denied by anyone that the excessive rise of temperature seen in some cases of febrile disease (to $43^{\circ} \mathrm{C}$., or even to $45^{\circ}$ ) is, apart from all other changes, a most imminent danger to life, unless, as is sometimes the case (in influenza, e.g., where a temperature of $44^{\circ}$ has been observed), the high temperature lasts only a short time. Experimental heat paralysis, a condition in which all voluntary and reflex movements are abolished, is produced in frogs by raising the internal temperature to about $34^{\circ} \mathrm{C}$. On cooling, the animal recovers. A similar condition can be induced in mammals, but, of course, at a higher temperature. The central nervous system succumbs before the peripheral structures. The superior cervical ganglion in the cat or rabbit loses the power of transmitting nerve impulses at $50^{\circ} \mathrm{C}$. But some evidence has been brought forward, mostly from the field of bacteriology, to support the idea that in infective processes the rise of temperature is of the nature of a protective mechanism, that the fever is, indeed, a consuming fire, but a fire that wastes the body, to destroy the bacteria. The streptococcus of erysipelas, for example, does not develop at $39^{\circ}$ to $40^{\circ} \mathrm{C}$., and is killed at $39^{\circ} 5^{\circ}$ to $4 \mathrm{I}^{\circ} \mathrm{C}$., and erysipelas infections in rabbits are less virulent if the body-temperature be artificially raised. Anthrax bacilli, kept at $42^{\circ}$ to $43^{\circ} \mathrm{C}$. for some time, are attenuated, and when injected into animals confer immunity to the disease. Heated for several days to $4 \mathrm{I}^{\circ}$ to $42^{\circ} \mathrm{C}$., pneumococci render rabbits immune to pneumonia, and in rabbits in which 'puncture' fever has been 
induced pneumococcus infections run a milker course. These bacteriological results are supported to a certain extent by clinical experience. For it has been olserved that a cholera patient with distinct fever has a better chance of recovery than a case which shows no fever. But too much weight ought not to be given to isolated facts of this sort, and adverse evidence can be produced both from the laboratory and the hospital. For alt lough hens are immune to anthrax under ordinary conditions, but can be infected by inoculation when artificially cooled, frogs, equally immune at the temperature of the air, become susceptible when artificially heated. And it is impossible to deny that the use of cold baths in typhoid fever is sometimes of remarkable benefit.

Distribution of Heat-Temperature Topography. - The great foci of heat-formation-the muscles and glands-would, if heat were not constantly leaving them, in a short time become much warmer than the rest of the body; while structures like the bones, skin, and adipose tissue, in which chemical change and heat-production are slow, would soon cool down to a temperature not much exceeding that of the air. The circulation of the blood insures that heat produced in any organ shall be carried away and speedily distributed over the whole body; while direct conduction also plays a considerable part in maintaining an approximately uniform temperature. The uniformity, however, is only approximate. The temperature of the liver is several degrees higher than that of the skin, and the temperature of the brain several degrees higher than that of the cornea. The blood of the superficial veins is colder than that of the corresponding arteries.

The crural vein, for cxample, carries colder blood than the crural artery, and the external jugular than the carotid. The heat produced in the elecper parts of the regions which they drain is more than counterbalaned by the heat lost in the more superficial parts. When loss of heat from the surface is sufficiently diminished by an artificial covering, or prevented by the protected situation of any organ with an active metabolism, the venous blond leaving it is warmer than the arterial blood coming to it. The temperature of the blood passing from the levator labii superioris muscle of the horse cluring mastication may be sensibly higher than that of the blood which feeds it; the blood in the vena profuncla femoris, and in the crural vein of a $\log$ with the leg wrapped in cotton-wool, is warmer by $0^{\circ} 1^{\circ}$ to $0^{\circ} 3^{\circ}$ than the blood of the crural artery. This difference of temperature is due to the heat produced in the muscles, and it is not difficult to show that the difference ought to be of this order of magnitude. The quantity of blood in a 7 -kilo clog is about $\frac{1}{2}$ kilo; $\frac{1}{4}$ of this, or $\frac{1}{2} \mathrm{kilo}$, is in the skeletal muscles, and the average circulation-time through them may be taken as ten seconds. Six times in the minute, or 360 times in the hour, $\frac{1}{k}$ kilo of blood passes through the muscles, and is heated on the average by $02^{\circ}$. If we 
take the speciflc heat of blood as about equal to that of water, this represents a heat-production of ${ }^{360} 8 \times \frac{2}{8}$, or 9 calories per hour. Now, the total heat-production of a 7 -kilo dog is about in calories per hour, of which somewhat less than onc-half is probably formed in the slieletal muscles.

The blood of the inferior vena cava at the level of the kidneys may be $0.1^{\circ}$ colder than that of the abdominal aorta, but is warmer than the blood of the supcrior cava. The right heart, therefore, receives two streams of blood at different temperatures, which mingle in its cavities. A controversy was long carried on as to the relative temperature of the blool of the two sides of the heart; but the researches of Heilenhain and Köner have shown that a thermometer passed into the right ventricle through the jugular vein stands, as a rule, slightly higher than a thermometer introduced through the carotid into the left ventricle. They consider that the method gives not so mucl the temperature of the blood in the two cavities as that of their walls. The thin-walled right ventricle, accorling to them, is heated by conduction from the warm liver, from which it is only separated by the diaphragm, while the left ventricle loses heat to the cooler lungs. They deny that the differcnce of temperature is caused by cooling of the blood in its passage through the pulmonary capillaries, for even when respiration is suspended, they find a difference of temperature between the two sides of the heart. Under ordinary circumstances, they say, the inspired air is already heated almost to body-temperature before it reaches the alveoli. But, while this is the case, a fall of less than $\mathbf{1}^{\circ}$ in the temperature of the blood passing through the lungs would account for all the heat lost by the expired air. If half of the loss took place in the upper air-passages, less than $1^{\circ}$ would be sufficient. A slight difference of temperature in the blood of the two ventricles might be caused, even in the absence of respiration, by the heat leveloped in the cardiac muscle itself during contraction, a large proportion of which must be conveyed by the blood of the coronary veins into the right side of the heart.

The surface temperature varies between rather wide limits with the temperature of the environment. The temperature of cavities like the rectum, vagina, and mouth, and of secretions like the urine, approximates to that of the blood in the great vessels or the heart, and undergoes only slight changes. An increase in the velocity of the blood causes the internal and surface temperatures to come nearer to each other, the former falling and the latter rising. When the loss of heat from a portion of the surface is prevented, the temperature of this portion approaches the internal temperature. For this reason a thermometer placed in the axilla approximately measures the internal temperature, and not that of the skin ; and a thermometer in the groin of a rabbit, and completely covered by the flexed thigh, may stand as high as, or, it is said, even higher than, a thermometer in the rectum (Hale White). The temperature in the mouth is not a very reliable index of the deep temperature of the body, especially in cold weather or after exercise, as it is apt to be influenced by the inspired air. The mouth must, of course, be kept closed during the measurement. On the average its temperature is about the same as that of the axilla, and $0^{\circ} 4^{\circ} \mathrm{C}$. below that of the rectum. The rectal temperature is $0^{\circ} 2^{\circ}$, or $0^{\circ} 3^{\circ}$ above that of the urine. In point of accuracy rectal observations are the best, and next to them determinations of the temperature of the stream of urine. The 
latter method, although subject to obvious limitations, is rapid and free from the (langer of conveying infection to the person (Pembrey).

The surface temperature is a rough inclex of the rate of heat-loss : the internal temperature, of the rate of heat-production. A normal skin temperature and a rising rectal temperature would probably indicate iucreased production of heat; an increased rectal temperature, in conjunction with a diminished surface temperature, as in the cold stage of algue. might be due either to diminished heat-loss while the heat-production remained normal, or to diminished heat-loss plus increased heat-production.

The following tables illustrate the differences of temperature found in the body. It should be remembered that the numbers are not strictly comparable with each other; the temperature of the mammals in which direct observations have been made on the blood is not exactly the same as that of man, the temperature of the dog. for example. being a little (abont I C.) higher. Then in the sime animal there is no very constant ratio between the temperature of the blood in two vessels or of the skin at two points. Even in the same vessel the temperature may vary with many circumstances, such as the velocity of the stream, and the state of activity of the organ from which it comes. Apart from physiological variations, experimental fallacies sometimes cause a want of constancy. cspecially in measurements of blood temperature. The insertion of a mercurial thermometer into a ressel is very likely to obstruct the passage of the blood; and if the blood lingers in a warm organ, it will be heated beyond the normal.

\section{I lood. (Dog.)}

\begin{tabular}{|c|c|c|c|c|}
\hline Right heart & - & - & - & $-\quad 3 S . C$. \\
\hline Left & - & - & - & -350 \\
\hline Aorta & - & - & - & $-\quad 38.7$ \\
\hline Superior vena & calval & - & - & -36.5 \\
\hline Inferior & .. & - & - & $-3 S^{\circ} 1$ \\
\hline Crural rein & - & - & - & $-\quad 37^{\prime} 2^{*}$ \\
\hline Crural artery & - & - & - & -350 \\
\hline Profunda femo & oris 1 & cin & - & $-38 \cdot 2$ \\
\hline Portal vein. & - & - & - & ( Varies with activit \\
\hline Hepatic vein & - & - & - & $354-397$ of digestive organs. \\
\hline
\end{tabular}

\section{Tissues.}

\begin{tabular}{|c|c|c|c|}
\hline Brain & - & - & $40^{\circ}$ \\
\hline Liver & - & - & to.6-40.9 \\
\hline \multicolumn{4}{|c|}{$\begin{array}{l}\text { Subcutancous tissue } 2 \cdot 1 \text { lower } \\
\text { than that of subjacent muscles } \\
\text { (man). } \\
\text { Anterior chamber of ere - }\end{array}$} \\
\hline Anterio & chamber of cyc & - & $319 ;$ (rabbit) \\
\hline Vitrcou & umour & - & $36.1\}$ \\
\hline
\end{tabular}

* The following numbers were obtained (in an andesthetized dog whose rectal temperature had fallen $2^{\circ}$ C.) for the temperature of the walls of the (rural artery and vein, as measured by an electrical resistance thrmometer.

l.cg of dog lightly urapped in wool.

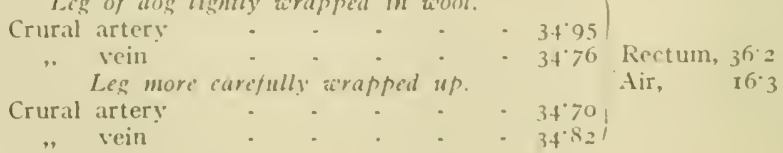




\section{Cavities. (Man.)}

$\begin{array}{llllll}\text { Axilla } & - & - & - & - & 36 \cdot 3-37 \cdot 5^{\circ} \mathrm{C} .\left(97 \cdot 3-99^{\circ} 5^{\circ} \mathrm{F} .\right) \\ \text { Rectum } & - & - & - & - & 36-37 \cdot 8 \\ \text { Mouth } & - & - & - & - & 37 \cdot 25 \\ \text { Vagina } & - & - & - & - & 37 \cdot 5-38 \\ \text { Uterus } & - & - & - & 37 \cdot 7 \cdot 38 \cdot 3 \\ \text { External auditory meatus } & 37 \cdot 3-37 \cdot 8 \\ \text { Bladlder (temperature of } \\ \text { the escaping urine) }\end{array}$

\section{Respiratory Passages. (Horse.)}

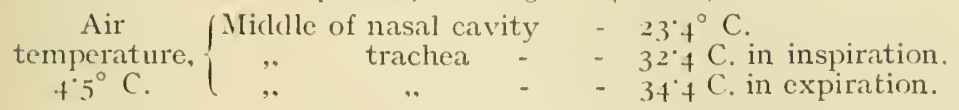

Natural Surfaces.

Check (boy, immediately after running) - $36 \cdot 25^{\circ}$

(Man) (l Anterior surface of forearm - $\quad-\quad$ - 33.5-3.4

Room Skin over biceps - " - - $34^{\circ} \mathrm{O}$

temperature,, , , head of tibia $\quad-\quad=35^{\circ} \mathrm{O}$

\begin{tabular}{l|ll}
$175^{\circ}$ &.. & immediately below xiphoid cartilage 347
\end{tabular}

,, over sternum - $\quad$ - $\quad-33^{\circ} 2$

On hair (boy) - - - - - 30.0

Under hair over sagittal suture (boy) - 33\%-34.0

Shaved skin of neck (rabbit) - - - 36.5

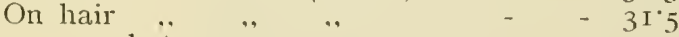

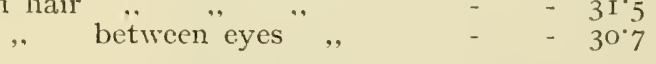

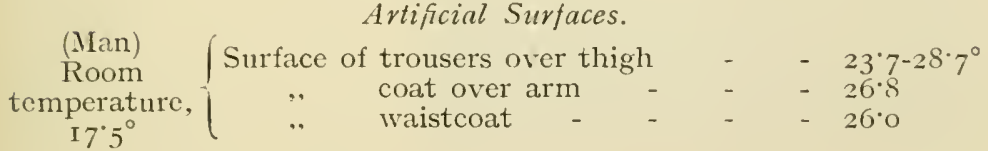

Normal Variations in the Temperature.-The internal temperature, as has been already said, is not strictly constant. It varies with the time of day; with the taking of food; with age ; to some extent with violent changes in the external temperature, such as those produced by hot or cold baths; and possibly with sex. On the average the range of variation in the temperature of the rectum or urine of a healthy man is from $36.0^{\circ} \mathrm{C} .\left(96.8^{\circ} \mathrm{F}\right.$.) to $37^{\circ} 8^{\circ} \mathrm{C}$. (100 $0^{\circ} \mathrm{F}$.).

In the monkey a very distinct and constant diurnal variation has been observed, and the range is much wider than in man, (as much as $54^{\circ} \mathrm{F}$.), the maximum falling between 6 and 8 p.m. and the minimum between 2 and 4 a.m. (Simpson).

The daily curve of temperature shows a minimum in the early morning, between two and six o'clock $\left(36 \cdot 3^{\circ} \mathrm{C}\right.$.), and a maximum in the evening, between five and eight $0^{\prime}$ clock $\left(375^{\circ} \mathrm{C}\right.$.) (Fig. 200). The daily range in health may be taken as a little over $\mathrm{I}^{\circ} \mathrm{C}$, , or about $2^{\circ} \mathrm{F}$. In fever it is generally greater, but the 
maximum and minimum fall at the same periods; and it is of scientific, and also of practical, interest that the early morning, when the temperature and pulse-rate are at their minimum, is often the time at which the flagging powers of the sick give way. From two to six o'clock in the morning the daily tide of life may be said to reach low-water mark. Even in a fasting man the diurnal temperature curve runs its course, but the variations are not so great. The taking of food of itself causes an increase of temperature, although in a healthy man this rarely amounts to more than half a degree. The rise of temperature is certainly due in part to the increased work of the alimentary canal, but it is also connected with the increase of metabolic activity which the entrance of the products of digestion into the blood brings about. The heat-production is especially increased

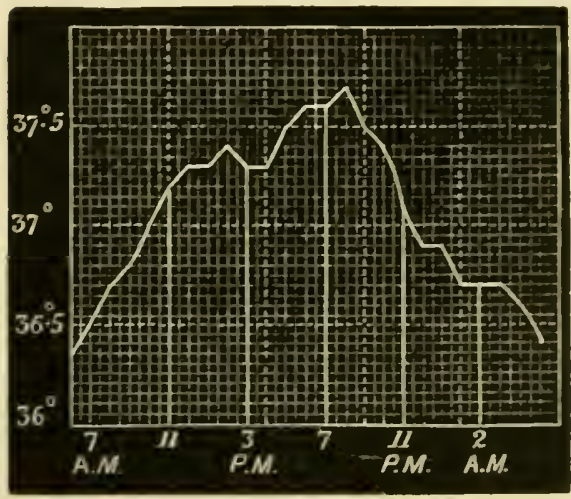

FIG. 200.-CURTE SHOWTAG THE DAILY VARIATION OF BODY-TEMPERATURE.

by proteins. The rise of temperature during digestion is gradual, the maximum being reached during the fourth hour, or even later. The greatest increase of heat-production takes place during the first hour after feeding (Reichert).

The cause of the daily variation of temperature has been much discussed. There is no doubt that several factors are concerned, among the most important being the variation in the amount of contraction of the skeletal muscles and the influence of food. Muscular exercise is capable of causing a considerable rise in the temperature of the rectum and urine, to $38.5^{\circ} \mathrm{C}$. (IOI. $3^{\circ} \mathrm{F}$.) or even $38.9^{\circ} \mathrm{C}$. (IO2 ${ }^{\circ} \mathrm{F}$.) without producing any feeling of distress. Other unknown influences seem also to be involved, as is shown by the fact that in persons who work at night and sleep during the day the curve of temperature, although greatly altered, is not reversed. Recent observations on this subject are those of Benedict. By means of a resistance thermometer in the rectum, readings were taken usually every four minutes. With such a thermometer no disturbance of the person's sleep is necessary to obtain a reading. He can sit without discomfort in any position, walk about the room (returning to the observer's table for the observations), and even ride a stationary bicycle. Even years of night-work do not eliminate the tendency to a 
fall of temperature at night, a minimum in the carly morning and a morning rise.

As to the relation of age and sex to temperature, it is only necessary to remark that the mean temperature both of the young cliild and of the old man is somewhat higher than that of the vigorous adult; but a point of more importance is the relative imperfection of the heat-regulation in infancy and age, and the greater effect of accidental circumstances on the mean temperature. Thus, old people and young children are specially liable to chills, and a fit of crying may be sufficient to send up the temperature of a baby. In infants an hour or two old the temperature may be as low as $34^{\circ} \mathrm{C}$. $\left(93^{\circ} 2^{\circ} \mathrm{F}\right.$.) or $33.0^{\circ} \mathrm{C} .\left(9 \mathrm{I}^{\circ} 4^{\circ} \mathrm{F}.\right)$ even when they are fully clothed in a room at I $5^{\circ} \mathrm{C} .\left(59^{\circ} \mathrm{F}\right.$.). It rises gradually during the first day or two, but shows marked variations. On the fifth day after birth, e.g., the rectal temperature ranged from $36 \cdot 2^{\circ}$ C. $\left(97^{1} 16^{\circ}\right.$ F.) to $33.5^{\circ}$ C. $\left(92.3^{\circ} \mathrm{F}\right.$.) in a child weighing $5 \frac{1}{2}$ pounds (Babak). The temperature of women is generally a little higher than that of men, and is also somewhat more variable.

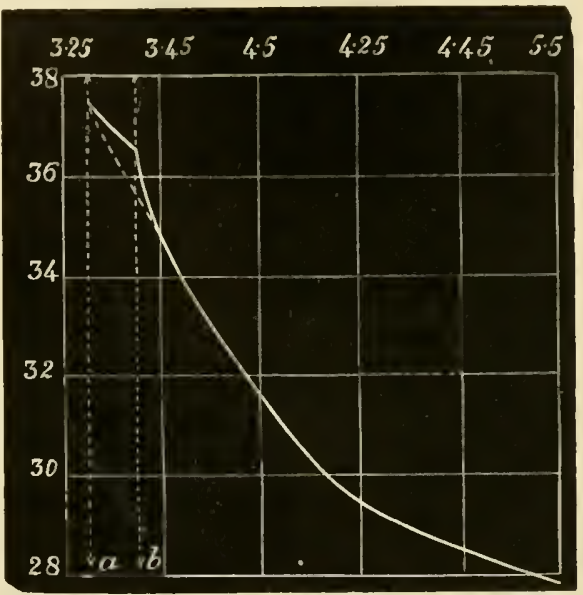

Fig. 201.-Curve of Cooling after Death : Guinea-PIG.

Time marked along horizontal, and tempera. ture along vertical axis. At $a$ ether and chloroform given to kill animal; death, as indicated by stoppage of the heart, took place at $b$. The dotted line shows the course the curve would have taken if death had occurred at the moment the anæsthetics were given. Air of room $17^{\circ} 6^{\circ}$.

After death the body cools at first rapidly, then more slowly (Fig. 20I). But occasionally a post-mortem rise of temperature may take place. In certain acute diseases (like tetanus) associated with excessive muscular contraction this has been especially noticed; in bodies wasted by prolonged illness it does not occur. Nearly an hour after death, in a case of tetanus, the temperature was found to be $45^{\circ} 3^{\circ}$, while before death it was $44^{\circ} 7^{\circ}$ (Wunderlich). In dogs a slight post-mortem rise may be demonstrated, especially when the body is wrapped up; but when an animal has been long under the influence of anæsthetics no indication whatever of the phenomenon may be obtained. The explanation of post-mortem rise of temperature is to be found: (I) In 
the continued metabolism of the tissues for some time after the heart has ceased to beat, for the cell dies harder than the body. (2) In the diminished loss of heat, clue to the stoppage of the circulation. (3) To a small extent in physical clianges (rigor mortis, coagulation of blond) in which heat is set free.

\section{PRACTICAL EXERCISES ON CHAPTERS VII. AND VIII.}

1. Glycogen* - (I) Preparation.-(a) Cut an oyster into two or threc pieces, throw it into boiling water, and boil for a minute or two. Rub up in a mortar with clean sand, and again boil. Filter. Precipitate any proteins which have not been coagulated. by adding alternately a drop or two of hydrochloric acid and a few drops of potassio-mercuric iodide so long as a precipitate is produced. Only a small quantity of these reagents will be required, as the greater part of the proteins has been already coagulated by boiling. Filter it aly precipitate has formed. The filtrate is opalescent. Precipitate the glycogen from the filtrate (after concentration on the water-bath if it exceeds a few c.c. in bulk) by the addition of four or five times its volume of alcohol. Filter off the precipitate, wash it on the filter with alcohol, and dissolve it in a little water. To some of the solution add a drop) or two of iodine; a reddish-brown (port wine) colour is produced, which disappears on heating, returns on cooling, is removed by an alkali. restored by an acid. Add saliva to some of the glycogen solution, and put in a bath at $4^{\circ} \mathrm{C}$. In a few minutes reducing sugar (maltose) will be found in it by Trommer's test (p. 10).

Note that dextrin (erythrodextrin) gives the same colour with iodine as glycogen does. Dextrin is also precipitated by alcohol. but a greater proportion must be added to cause complete precipitation. Glycogen is completely precipitated by saturation with magnesium sulphate or ammonium sulphate. so that the filtrate no longer gives the reddish colour with iodine. A pure solution of erythrodextrin is not precipitated. On the addition of a drop or two of a solution of basic lead acetate to a solution of glycogen in distilled water. a precipitate forms immediately. Wher the same reagent is added to a solution of dextrin in distilled water there is no immediate precipitate. Maltose is formed when dextrin is digested with saliva.

(b) Cut another oyster into pieces, throw it into boiling water acidulated with dilute acetic acid, and boil for a few minutes. Rub up in a mortar with sand, boil again, and filter. Test a portion of the filtrate with iodine for glycogen. Precipitate the rest with alcohol, filter, dissolve the precipitate in water, and test again for glycogen. On boiling some of the opalescent solution for a few minutes after the addition of a few drops of sulphuric acid the opalescence disappears, and when the solution has been neutralized with sodium hydroxide it gives Trommer's test, owing to the hydrolysis of the glycogen into dextrose.

* For the quantitative estimation of glycogen in organs, Pflüger's method is the best. The organ is minced and heated with strong $(60$ per cent.) potassium hydroxide. The glycogen is precipitated with alcoliol. and then, after hydrolysis with hydrochloric acid, estimated as dextrose. 
(2) Decply etherize a dog or rabbit fire hours after a meal rich in carbo-hydrates-e.g., rice and potatoes in the case of the dog. carrots in the case of the rabbit. Fasten it on a holder. Clip off the hair over the abdomen in the midclle line. Make a mesial incision through the skin and abdominal wall from the ensiform cartilage to the pubis. The liver will now be rapidly cut out (bv the (lemonstrator) and divided into two portions, one of which will be (distributcel among the class and) treated as in $(a)$ or $(b)$; the other will be kept for an hour at a temperature of $40^{\circ} \mathrm{C}$., and then subjecterl to process $(a)$ or $(b)$. Little, if any, sugar and much glycogen will be found in the portion which was boiled immerliately after excision. Abundance of sugar will be found in the portion kept at $40^{\circ} \mathrm{C}$. ; it may or may not contain glycogen.

2. Catheterism.-In many physiological experiments it is necessary to obtain urine from the bladder by means of a catheter. It is possible to pass a fine rubber catheter into the bladcler of a male dog. A larger one is easily passed in a male rabbit, and a still larger in a bitch, which is often used for cxpcriments on metabolism. Even in the bitch the opcning of the urethra lies entircly concealed within the vagina, much deeper than the cul-de-sac in the mucous membrane, into which the beginner usually tries to force the catheter. For a first attempt the animal should be etherized and fastened on a holder. The little or index finger of the left hand is passed into the vagina till the symphysis pubis can be felt. A little below this is the opening of the urethra. With the right hand the point of a catheter of suitable calibre is directed along the finger, and after a little 'guess and trial' it slips into the bladder, its entrance being announced by the escape of urine. A glass tube drawn out to a sufficiently small calibre and bent near the point is the easiest form of catheter to pass in a bitch. The point must, of course, be rounded in the flame.

When the bitch is to be used in a long series of experiments an operation is sometimes performed first of all to render the urethral orifice more accessible.

3. Glycosuria.-(I) (a) Weigh a dog (female by preference) or rabbit. Fasten on a holder, and etherize. Insert a glass cannula into the femoral or saphena vein of the dog, or into the jugular of the rabbit (p. 200). Fill a burette with a 2 per cent. solution of dextrose in physiological salt solution, connect it with the cannula by means of an indiarubber tube, taking care that there are no air-bubbles in the tube, and slowly inject as much of the solution as will amount to $\frac{1}{2}$ or $\frac{3}{4}$ grm. of sugar per kilo of body-weight. Tie the vein, remove the cannula, and in half an hour evacuate the bladder by passing a catheter, by pressure on the abdomen, or, if both of these methods fail, by tapping the bladder with a trocar pushed through the linea alba (supra-pubic puncture). In an hour again draw off the urine. Test both specimens for sugar.

In this experiment the opportunity may also be taken to demonstrate that egg-albumin, when injected into the blood, is excreted by the kidneys, a filtered solution containing the albumin of one egg and sugar in the quantity mentioned being injected.

The catheter may be inserted before the injection is begun, and the bladder evacuated. After the injection the urine that drops from the catheter may be collected in test-tubes, first every two minutes, and then, as soon as sugar is found, every ten minutes. Determine the interval between injection and the appearance of the 
first trace of sugar and albumin. If a sufficient amount of urine is obtained. the quantity of sugar in successive specimens may bre estimated and compared. The rate of flow of the urine as measured by the number of drops falling from the catheter may also be estimated from time to time, in order to determine whether diuresis is taking place.

If a rabbit is used for this experiment, the sugar solution may be injected into the ear vein. The vein is caused to swell up by pressing on it with the finger and thumb, and the liypolermic necdle is then inserted towards the heart.

(b) Instead of collecting the urine by a catheter in the bladder the abdomen of the dog may be opener, and a cannula tierl into each ureter. The two cannule are then connected by short rubber tubes with a glass $\mathbf{Y}$-piece, on the stem of which a test-tube is tied for collecting the urine. Replace the test-tube by a fresh one from time to time. The urine already in the bladder is removed by pressure or by a trocar, and tested for sugar. since the anasthetic itself may cause a certain amount of glycosuria. Test the samples of urine obtained from the ureters for sugar, and in those in which it is present estimate its amount. Note also any changes in the rate of secretion of urine, and any abnormal constituents, as albumin.

(2) Phloridzin Glycosuria.-Dissolve $\frac{\lambda}{\mathrm{grm}}$. of phloridzin in warm water, and inject it subcutaneously into a rabbit. Obtain a sample of the urine at the end of two hours, by pressure on the abdomen with the thumb or by passing a catheter, and test for sugar. If none is present, wait for another interval, and again test the urine.

This experiment can also be performed without risk on man. One grm. of phloridzin has been injected twice a day without disturbing the individual. Much sugar is found in the urine, but it disappears the day after the administration of phloridzin is stopped. The phloridzin may also be given by the mouth. but more is required, and it is not very easily absorbed, and often causes diarrhœea (v. Mering).

(3) Alimentary Glycosuria.-The urine having been tested for sugar for two successive days, and none being found,

(a) A large quantity of dextrose is to be taken in the form which is most agreeable to the student some hours after a meal. The urine of the next twenty-four hours is to be collected and measurerl. A sample of it is then to be tested for reducing sugar by Trommer's and the phenyl-hydrazine test. If any sugar is found, the reducing power of a definite quantity of the urine is to be determined by titration with Fehling's solution (p. 489).

(b) Instead of dextrose use cane-sugar and proceed as in (a). But cstimate the reducing power of the urine (a) before and ( 3 ) after boiling with hydrochloric acid (p. 433).

(c) A large meal of rice and arrowroot, sweetened with as much dextrose as the observer can induce himself to swallow, is to be taken, and the urine treated as in $(a)$.

(d) A large number of sweet oranges may be eaten.*

4. Milk.-(I) Examine a drop of fresh cow's milk with the microscope. Note the fat globules of various sizes.

(2) Determine the specific gravity of the milk with a hydrometer (lactometer). Then centrifugalize some of the milk to separate the cream, which rises to the top of the tubes. Remove the cream and

* These experiments may be distributed among the class so that each student does one. 
determine the specific gravity of the skimmed milk. It will be found to have increased since the fat is of lower specific gravity than the rest of the milk. Normal cow's milk has a specific gravity of $\mathrm{r}, 028$ to 1,034 . skimmed milk 1,033 to $\mathrm{I}, 037$.

(3) Test the reaction of the milk to litmus-paper. It is slightly alkaline.

(4) (a) Put ro c.c. of milk in a test-tubc, and nearly fill it up with water. Add strong acetic acil (lrop by drop. A precipitate of caseinogen is thrown down which entangles the fat, and carries it down mechanically along with it. Filter off the precipitate. Kecp the filtrate for $(b)$. Wash the precipitate with water, scrape a portion of it off the filter, and add to it some 2 per cent. sodium carbonate solution. The caseinogen dissolves, while the fat remains in suspension. The solution gives the colour reactions for proteins (p. 7).

(b) Test some of the filtrate by Trommer's test (p. ro) for lactose. Add dilute sodium carbonate solution to another portion till it is only slightly acid. Boil, and lactalbumin is coagulated. Remove the lactalbumin by filtcring, and test this filtrate for carthy (i.e., calcium and magnesium) phosphates by adding a few drops of ammonia. which precipitates them as a slight cloud.

(c) To 5 c.c. of milk add an equal volume of saturated ammonium sulphate solution. The caseinogen is precipitated, cntangling the fat. Filter off. The filtrate may be used to test for lactalbumin by boiling. The addition of water to the precipitate of caseinogen (and fat) on the filter causes the caseinogen to dissolve, as it is soluble in weak sait solutions. Caseinogen can also be precipitated by saturating milk with sodium chloride or magnesium sulphate.

(5) To 5 c.c. of milk add a couple of drops of 20 per cent. sodium or potassium hydroxide, and then a few c.c. of ether. Shake up. The ether dissolves the fat, and the opacity of the milk diminishes. Take off the ether with a pipette, evaporate away most of it on a water-bath, and place a drop or two of the remainder on a filterpaper. A greasy stain is left, showing the presence of the fat of the milk or butter.

(6) Clotting of Milk.-(a) To a few c.c. of milk in a test-tube add a few drops of rennet. Place the tube in a bath at $40^{\circ} \mathrm{C}$. In a short time a clot or curd is formed, consisting of casein, which is derived from the caseinogen. The fat is entangled in the clot. On standing some timc the clot contracts, and exudes the whey. Boil some of the whey after slight acidulation with acetic acid; the lactalbumin and whey-protein are coagulated. Test another portion of whey for proteins by one of the general protein tests (p. 7)-e.g., the xanthoproteic.

(b) Repeat $(a)$ but use rennet which has been previously boiled. The milk is not curdled, because the ferment has been inactivated by boiling.

(c) To ro c.c. of milk add 3 c.c. of I per cent. potassium oxalate. Divicle the oxalated milk into three portions- $\mathrm{A}, \mathrm{B}$, and $\mathrm{C}$. To $\mathrm{A}$ add a few drops of rennet, to B I c.c. of 2 per cent. calcium chloride solution and a little rennet, and to $C$ I c.c. of 2 per cent. calcium chloride solution alone. Put the tubes at $40^{\circ} \mathrm{C}$. Clotting will occur in B, but not in A or C.

5. Cheese.-(I) Rub up some finely-grated cheese in a mortar with 2 per cent. sodium carbonate solution. Filter. The filtrate contains casein, which can be precipitated by adding dilute acetic acid by drops to a portion of the filtrate. The precipitate is soluble in excess of the acid. With another portion of the filtrate perform some of the general protein tests (p. 7 ). 
(2) Shake up some fincly-grated cheese in a dry test-tube with ether. Take off the ether with a pipette, ancl evaporate on a waterbath till only a few (lrops remain. With a glass roul put a clrop of the ether on a piece of filter-paper. A greasy spot is left, showing that fat is present.

6. Flour.-(I) Mix some wheat-flour with a little water into a stiff clough. Let it stand for a few minutes at boly-temperature to facilitate the formation of gluten. Wrap a piece in cheese-cloth, forming a kind of bag, and linead it with the fingers in a capsule of water. The starch grains come through the cheese-cloth. Pour the water into a beaker. It is opaque, and on stancling the starch grains sink to the bottom. (a) Test for starch with the iodine test, and also examine microscopically. The grains are round, with a central hilum, and are smaller than those of potato starch $(p, 11)$. (b) Test for sugar by Trommer's test (p. 10). None is present unless the flour has been made from inferior grain in which some germination has taken place.

(2) Go on kneading the dough till no more starch comes through. The sticky mass which remains in the bag is a protein callerl gluten, which is formed from certain globulins and other proteins in the flour on addition of water. Oatmeal, ground rice, and other grains poor in gluten-forming globulins do not form dough when mixed with water. Suspend some of the gluten in water in a test-tube, and apply to it the general protein colour tests (p. 7)

7. Bread.-(I) Rub up a small piece of the crumb of a stale loaf in a mortar with water. Strain through cheese-cloth. The fluid which passes through contains starch grains. (a) Filter it, and test a portion of the filtrate for dextrose by Trommer's test. A positive result is obtained. Test another portion with iodine for erythrodextrin. (b) Test a portion of the residue of the bread which has not passed through the cheese-cloth for protein by the general protein tests-e.g., the xanthoproteic or Millon's tests.

(2) Repeat (I) using the crust of the bread. Both dextrose and erythrodextrin are present in the cold-water extract, but the dextrose is less plentiful than in the crumb, having been converted into caramel in the baking. The sugar and dextrin are formed from the starch of the flour by the ferments of the yeast employed to make the breacl rise.

8. Variations in the Total Nitrogen (p. 535) and in the Quantity of Urea Excreted, with Variations in the Amount of Proteins in the Food.-The student should put himseif, or somebody else if he can, for two days on a diet poor in proteins, then (after an interval of forty-eight hours on his or(linary food) for two days on a diet rich in proteins. A suitable table of diets will be supplied. The urine should be collected on the six days of the period of experiment, on the day before it begins, and on the day after it ends. Small samples of the mixed urine of the twenty-four hours for each of these eight days should be brought to the laboratory. and the quantity of urea determined by the hypoloromite method. The volume of the urine passed in each interval of twenty-four hours being known, the total excretion of urea for the tiventy-four hours can be calculated, and a curve plotted to show how it varies during the period of cxperiment.* If sufficient time is available, the cxperi-

* In 17 healthy students the average amount of urea excreted in twentyfour hours on the ordinary dict was $29^{\circ} 5^{1}$ grammes (minimum 19.35 grammes, maximum $46^{\circ} 007$ grammes) ; on a diet poor in protein, average 
ment will be male still more instructive by determining the total nitrogen in each sample in addition to the urea. A curve showing the variation in the total nitrogen can then be plotted on the same paper as the nrea curve, and a table calculated giving the percentage of the total nitrogen contained in the mea for each day of the cxperiment.

9. Measurement of the Quantity of Heat given off in Respiration. - This may be clone approximatcly as follows: Put in the inner copper vessel, $A$, of the respiration calorimeter (Fig. 197, P. 579) a measurel quantity of water sufficient to completely cover the series of brass discs. Place $\Lambda$ in the wide outer cylinder, the bottom of which it is prevented from touching by pieces of cork. The outer cylinder hinders loss of lieat to the air. Suspend a thermometer in the water throngh one of the holes in the licl. In the other hole place a glass rod to serve as a stirier. Read off the temperature of the water. Put the glass tube connected with the apparatus in the mouth, and breathe out through it as regularly and normally as possible, closing the opening of the tube with the tongue after each cxpiration and breathing in through the noss. Continue this for firc or ten minutes, taking care to stir the water frequently. Then read off the temperaturs again. If $I V$ be the quantity of wate: in c.c., and $t$ the observed rise of temperature in degrees Contigrade, Wt cquals the quantity of heat, cxpressed in small calories (p. 574), given off by the respiratory tract in the time of the experiment, on the assumptions (I) that all the heat has been absorbed by the water. (2) that none of it has been lost by radiation and conduction from the calorimeter to the surounding air. Calculate the loss in twenty-four hours on this basis; then repeat the experiment. brcathing as rapidly and cleeply as possiblc, so as to incrcase the amount of ventilation. The quantity of heat given off will be found to be increased.*

In an experiment of short duration (2) is approximately fulfilled. As to (I). it must be noted that in the first place the metal of the calorimeter is heated as well as the water, and the water-equivalent of the apparatus must be adcled to the weight of the water (p. 574). The water-cquivalent is determined by putting a definite weight of water at air temperature $\mathrm{T}$ into the calorimeter, and then allowing a quantity of hot water at known temperature $T^{\prime}$ to run into it, stirring well, and noting the temperature of the water when it has ceased to risc. Call this temperature T". Enough hot water should be added to raise the temperature of the calorimeter about $2^{\circ} \mathrm{C}$. The quantity run in is obtained by weighing the calorimeter before and after the hot water has been added. Suppose it is $m$. Let the mass of the cold water in the calorimeter at first be $\mathrm{M}$, and let $\mathrm{I}^{\prime}=$ the mass of water which would be raised $\mathrm{I}^{\circ} \mathrm{C}$. in temperature by a quantity of heat sufficient to increase the temperature of all the metal, etc., of the calorimeter by $\mathrm{I}^{\circ}$ - in other words, the waterequivalent of the calorimeter.

The mass $m$ of hot water has lost heat to the amount of $m\left(\mathrm{~T}^{\prime}-\mathrm{T}^{\prime \prime}\right)$, and this has gone to raise the temperature of a mass of water $\mathrm{II}$,

$20^{\circ} 75$ grammes (minimum 9*517 grammes, maximum 32.857 grammes) : on a diet rich in protein, average $38^{\circ} 83$ grammes (minimum 23.265 grammes. maximum 67.82 grammes).

* The average heat-loss by the lungs for 5 I men (calculated for the 24 hours) was 312 , o00 small calories for normal, 919,000 for the fastest, and 195,000 for the slowest breathing. 
and metal equivalent to a mass of water $\mathrm{M}^{\prime}$. by $\left(\mathrm{T}^{\prime \prime}-\mathrm{T}\right)$ ilegrees. $\therefore m\left(\mathrm{~T}^{\prime}-\mathrm{T}^{\prime \prime}\right)=\mathrm{M}\left(\mathrm{T}^{\prime \prime}-\mathrm{T}\right)+\mathrm{I}^{\prime}\left(\mathrm{T}^{\prime \prime}-\mathrm{T}\right)$. Evcrything in this cquation except $M^{\prime}$ is known, and $\therefore M^{\prime}$, the water-eqnivalent of the calorimeter, can be dedneed, and must be arlded in all exact experiments to the mass of water contained in it.

Secondly, all the excess of lieat in the expired over that in the inspired air is not given off to the calorimeter. for the air passes ont of it at a slightly higher temperature than that of the atmosphere. At the beginning of the experiment this excess of temperature is zero. If at the end it is $\mathrm{I}^{\circ} \mathrm{C}$. the mean excess is $0.5^{\circ} \mathrm{C}$. Now, when respiration is carried on in a room at a temperature of $10^{\circ} \mathrm{C}$. the expired air has its temperature increased by nearly $30^{\circ} \mathrm{C}$. About $\because$ of the heat given off by the respiratory tract in raising the

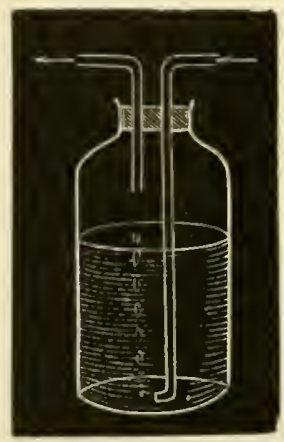

FIG. 202.- B Q T T L E AKRA N FED FOR WATER-VALVE. temperature of the air of respiration would accordingly be lost in such an experiment. But since the portion of the licat lost by the lungs which goes to leat the expired air is only is of the whole heat lost in respiration (p. 579 ), the error would only amount to :so of the whole. and this is negligible.

Thirdly, the air leaves the calorimeter satu. rated with watery rapour at, say, 105 , while the inspired air is not saturater for IO ${ }^{\circ} \mathrm{C}$. Now. the quantity of heat renclererl latent in the evapnration of water sufficient to saturate a given quantity of air at $f^{\circ} \mathrm{C}$. (the expired air is saturated for boly-temperature) is six times that requirerl to saturate the same quantity of air at Io. If, then, the inspired air is half saturated, the error under this head is $1_{1}^{1}$, or $\delta_{\frac{1}{2}}$ per cent. If the inspired air is threc-quarters saturated, the crror is $\ldots$ or about + per cent. If the air is fully saturated before inspiration, as is the case when it is drawn in through a water-valve (Fig. 202) by a tube fixed in one nostril, the only error is that due to the slight excess of temperature of the air leaving the calorimeter over that of the inspired air. The latent heat of the aqueous vapour in saturated air at $105^{\circ} \mathrm{C}$. is about $: 0$ more than the latent heat of the aqueous vapour in the same mass of saturated air at $10^{\circ} \mathrm{C}$.. or about $1: \pi$ of the latent heat in saturated air at $40^{\circ}$. The error in this case would therefore be under I per cent. The tubes must be wide and the bottle large. 


\section{CHAPTER IX}

\section{MUSCLE}

Ir is impossible to understand the general physiology of muscle and nerve without some acquaintance with electricity. It would be ont of place to give even a complete sketeh of this preliminary but essential knowledge here ; and the student is expressly warned that in this book the elementary facts and principles of physics are assumed to be part of his mental outfit. But in describing some of the electrical apparatus most commonly used in the study of this portion of our subject, it may be useful to recall the physical facts involved.

Batteries.--The Daniell cell is perhaps better suited for physiological work than any other voltaic element, although for special purposes Bunsen, Grove, Leclanché, and bichromate of potassium batteries may be employed. Dry batteries are very convenient for work in which the current does not need to be very constant, and where it is only closed for a short time.

The Daniell is a two-fluid cell. Saturated solution of sulphate of copper is contained in an outer vessel, and a dilute solution of sulphuric acid in a porous pot standing in the copper sulphate solution. The latter is kept saturated by a few crystals of copper sulphate.

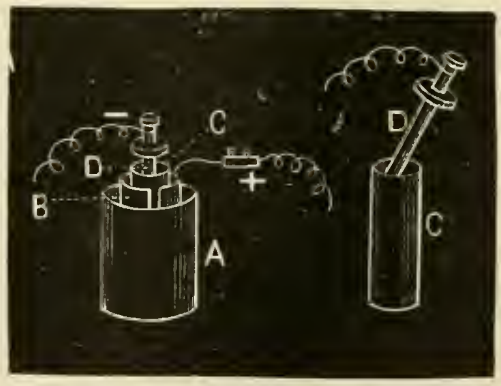

Fig. 203.-DANiell Cell.

$A$, outer vessel ; B, copper ; $C$, porous pot; D, zinc rod; $D$ is sipposed to be raised a ittle so as to be seen. A picce of sheet-copper, generally bent so as to form a hollow cylinder, dips into the sulphate of copper, and a piece of amalgamated zinc into the contents of the porous pot. Inside the cell the current (the positive electricity) passes from zinc to copper; outside, from copper to zinc. The copper is called the positive, the zinc the negative, pole. When the current is passed through a tissue, the electrode by which it enters is termed the anode, and that by which it leaves the tissue the kathode. The anode is, therefore, the electrode connected with the copper of the Dariell's cell ; the kathode is connected with the zinc.

Potential-Current Strength-Resistance.-We do not know what in reality electricity is, but we do know that when a current flows 
along a wire energy is expended, just as energy is expended when water flows from a higher to a lower level. Many of the phenomena of current electricity can, in fact, be illustrated by the laws of flow of an incompressible lipuid. The difference of level, in virtue of which the flow of licpuid is maintained, corresponds to the difference of electrical level, or potential, in virtue of which an electrical current is kept up. The positive pole of a voltaic cell is at a higher potential than the negative. When they are connected by a conductor, a flow of electricity takes place, which, if the difference of level or potential were not constantly restored, would soon equalize it, and the current would cease : just as the flow of water from a reservoil would ultimately stop if it was not replenished. If the reservoir was small, and the discharging-pipe large, the flow would only last a short time; but if water was constantly being pumped up into it, the flow would go on indefinitely. This is practically the case in the Daniell cell. Zinc is constantly being dissolved, and the chemical energy which thus diszppears goes to maintain a constant difference of potential between the poles. Electricity, so to speak, is continually ruaning down from the place of higher to the place of lower potential, but the cistern is always kept full.

The difference of electrical potential between two points is called the electromotive force; and from its analogy with difference of pressure in a liquid, it is easy to understand that the intensity or strength of the current-that is, the rate of flow of the electricity between two points of a conductor-does not depend upon the electromotive force alone, any more than the rate of discharge of water from the end of a long pipe depends alone on the difference of level between it and the reservoir. In both cases the resistance to the flow must also be taken account of. With a given difference of level, more water will pass per second through a wide than through a narrow pipe, for the resistance due to friction is greater in the latter. In the case of an electrical current, a wire connccting the two poles of a Daniell's cell will represent the pipe. A thick short wire has less resistance than a thin long wire; and for a given difference of potential, of electric level, a stronger current will flow along the former. But for a wire of given dimensions, the intensity of the current will vary with the electromotive force. The relation between electromotive force, strength of current, and resistance were experimentally determined by Ohm, and the formula $C=\frac{E}{R}$, which expresses it, is called Ohm's Law. It states that the current varies directly as the electromotive force, and inversely as the resistance.

For the measurement of electrical quantities a system of units is necessary. The common unit of resistance is the ohm, of current the ampire, of electromotive force the volt. The electromotive force of a Daniell's cell is about a volt. An electromotive force of a volt, acting through a resistance of an ohm, yields a current of one ampère; but the current produced by a Daniell's cell, with its poles connected by a wire of 1 ohm resistance, would be less than an ampere, because the internal resistance of the cell itself-that is, the resistance of the liquids between the zinc and the copper-must be added to the external resistance in order to get the total resistance, which is the quantity represented by $R$ in Ohm's I.aw.

Measurement of Resistance.-To find the resistance of a conductor, we compare it with known resistances, as a grocer finds the 
weight of a packet of tea by comparing it with known weights. The Wheatstone's briclge methol of measuring resistance depends on the fact that if four resistances, $\triangle B, \triangle D, B C, C D$, are connected, as in Fig. 20.4 with each other, and with a galvanometer, ( and a battery F, no current will flow througl the galvanometer when $A B$ BC $\mathrm{AD}=\overline{\mathrm{CD}}^{\text {. }}$

For when no current passes through the galvanometer, B and D are at the same potential. L.et the fall of potential from $C$ to $B$ or from ${ }^{-C}$ to $D$ be a ; then. since the total fall of potential from $C$ to $A$ must be the same along either of the paths CBA or CDA, the fall

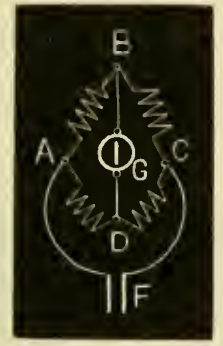

Fig. 204.-Wheat. STONE'S BRIDGE.

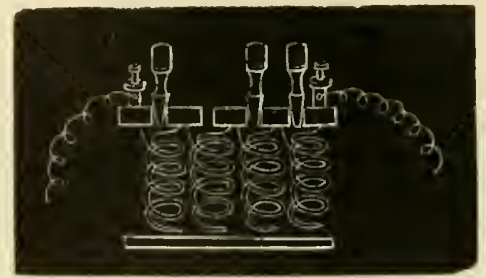

Fig. 205.-Diagram of Resistance Box.

from $B$ to A must be equal to that from D to A. Call this $B$. Now, the fall of potential which takes place in any given portion of a circuit is to the whole fall of potential in the circuit as the resistance of the given portion is to the whole resistance. That is,

$$
\begin{aligned}
\frac{u}{\alpha+\beta} & =\frac{\mathrm{BC}}{\mathrm{BC}+\mathrm{AB}}, \\
\frac{\beta}{\alpha+\beta} & =\frac{\mathrm{AB}}{\mathrm{BC}+\mathrm{AB}} ; \therefore \frac{\alpha}{\beta}=\frac{\mathrm{BC}}{\mathrm{AB}} . \\
\text { Similarly }: \frac{\alpha}{\beta} & =\frac{\mathrm{CD}}{\mathrm{AD}} ; \therefore \frac{\mathrm{BC}}{\mathrm{AB}}=\frac{\mathrm{CD}}{\mathrm{AD}} \cdot \text { or } \frac{\mathrm{AB}}{\mathrm{AD}}=\frac{\mathrm{BC}}{\mathrm{CD}}
\end{aligned}
$$

In making the measurement, a resistance box, containing a large number of coils of wire of different resistances, is used (Fig. 205). The resistances corresponding to $\mathrm{AB}$ and $\mathrm{AD}$, called the arms of the bridge, may be made equal, or may stand to each other in a ratio of I : IO, I : roo, etc. Then, the unknown resistance being CD, $\mathrm{BC}$ is adjusted by taking plugs out of the box till, on closing the current, there is either no deflection, or the deflection is as small as it is possible to make it with the given arrangement.

Galvanometer.-A galvanometer is an instrument used to detect a current, to determine its direction, and to measure its intensity. Since, by Ohm's law, electromotive force, resistance, and current strength are connected together, any one of them may be measured by the galvanometer. A galvanometer of the kind ordinarily used in physiology consists essentially of a small magnet suspended in the axis of a coil of wire, and free to rotate under the influence of a current passing through the coil. The most sensitive instruments possess a small mirror, to which the magnet is rigidly attached. A 
ray of light is allowed to fall on the mirror, from which it is reflected on to a scale; and the rotation of the mirror is magnified and measurecl by the cxcursion of the spot of light on the scale. In the Thomson galvanometers the magnet is very light. A strip or two of magnetized watch-spring does very well. The magnet is ' clamped '-that is, its terdency, when once rlisplacerl, to go on oscillating about its new position of equilibrium is overcome by cnclosing it in a narrow air space. In the Wielemann instrument the magnet is heavier (Fig. 206). It swings in a chamber wit? copper walls. Every movement of the magnet 'induces' currents in the copper ; these tend to oppose the movement, and so 'rlamping' is obtained. It is usual to read the deflections of the Wiedemann galvanometer by neans of a telescope. An inverted scale is placesl orer the telescope at a distance of. say. a metre from the mirror ; an upright image of the scale is formed in the telescope after reflection from the mirror, and with every movement of the latter the scale divisions appear to move correspondingly. The mothorl of rearling by a telescope can be applicd to any mirror galvanometer, ancl is

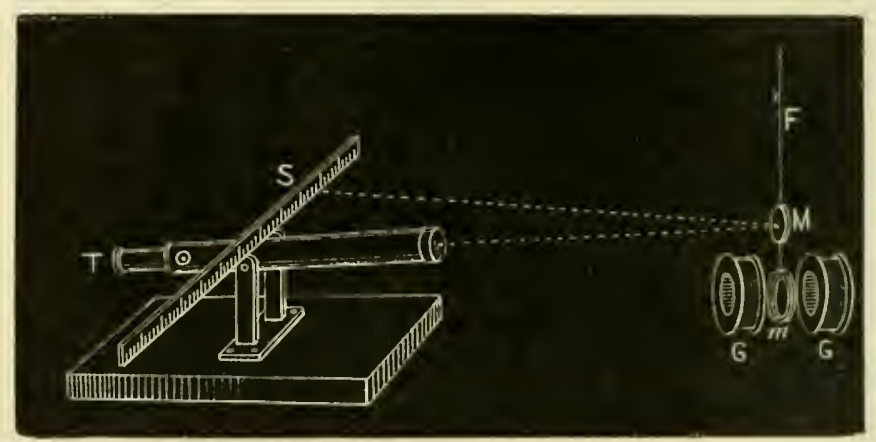

Fig. 206. - Scueme of Wiedemann's Galvanometer (Witu Telescope Reading).

$T$, telescope: $\mathrm{S}$, scale : $\mathrm{II}$, mirror: $m$, ring magnet suspended between the two galvanometer coils $F$, the distance of which from $m$ can be varied; $F$, fibre suspending mirror and magnet.

often extremely convenient in physiological work. Sometimes a small scale is fastened on the mirror itself, and observed directly through a low-power microscope.

A suspended magnet, if no other magnets are near, takes up a definite position under the influence of the earth's magnetisn ; its long axis, in the position of rest, lies in a vertical plane, called the plane of the magnetic meridian at the given place. The 'marked' or north pole points north, the soutl pole south. If the magnet is disturbed from this position, it tends to return to it as soon as the disturbing force ceases to act. If, for instance, the north pole is displaced in an eastward clirection, the carth's magnetism will produce a couple (a pair of parallel forces acting in opposite directions), one member of which may be considered to pull the north pole towarls the west, and the other to pull the south pole towards the east. Displacement of the magnet, then, is opposerl by this couple ; and where the displacing force is small - that is, the current passing through the galvanometer weak, as is usually the case in physio- 
logical observations--it becones important to recluce the effect of the magnetism of the earth, in other worls, the strength of the magnetic field, as much as possible. This can be done by bringing a magnet into the neighbonilhood of the galvanometer with its north pole pointing north. This pole, which is the one attracted by the eartlı's north pole, is magnetized in the opposite sense; and by properly arjusting its distance from the galvanometer magnet, the influence of the earth on the latter can be almost neutralized, and the system macle nearly. 'astatic.' In many galvanometers the magnets attacher to the mirror form an 'astatic' pair (Fig. 207). Two small magnets of nearly equal strength are connected to a light slip of horn or an aluminium wire, with their poles in oppositc clircetions. The earth's magnetism affects them oppositely, so that the resultant action is nearly zero. It is not possible to make the magnets exactly equal in strength, nor is it desirable, for then the system would not tend to come to rest in any definite position, and the zero point would be constantly shifting. Either one or both magnets may be surrounded by the galvanometer coils. If both are so surrounded, each must be within a separate coil, and the current must pass in opposite directions in the two coils, otherwise they would nentralize each other. In the d'Arsonval galvanometer the current passes through a small coil of fine wire suspended in the field of a strong magnet. When the current passes the coil is deflected. carrying with it a small mirror attached to the suspending filament. A great advantage of this galvanometer in many situations is that it is unaffected by neighbouring currents.

The cleflection of a magnet by a current of given strength is proportional to the number of turns of wire around it. Where an increase in the number of turns does not sensibly cut down the current, as in experiments on tissues like nerves, whose resistance is large in comparison

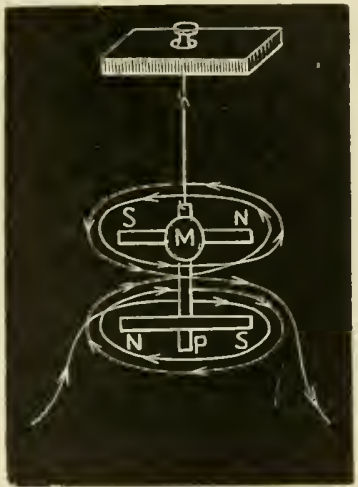

Fig. 207.-Astatic Pair of MAGNETS.

$\mathrm{SN}$ and $\mathrm{NS}$ are the magnets, fixed to the vertical piece $P$. $M$ is a mirror. The arrow-heads show the direction of a current which deflects both magnets in the same direction. with that of the galvanometer, an instrument with a great number of turns of wire-that is, a high-resistance galvanometer-is suitable. The resistance of the galvanometers generally used in electro-physiology varies from 3,000 or 4,000 ohms up to five times as much.

The string galvanometer of Einthoven has peculiar merits for certain physiological purposes. It consists of a silvered quartz-fibre stretched in a very strong magnetic field. When traversed by a current the fibre is deflected, and by means of a beam of light the deflection is greatly magnified. By photographing the beam a record of the swing of the fibre caused by a momentary current can be obtained. In this way the instrument may be employed to record the electrical changes occurring in the human heart with each beat (p. 732).

$A$ rheocord is an instrument by means of which a current may be divided, and a definite portion of it sent throngh a tissue (Fig. 208). 
A compensator is simply a rheocord from which a branch of a current is led off, to balance or 'compensate' any electricall difference

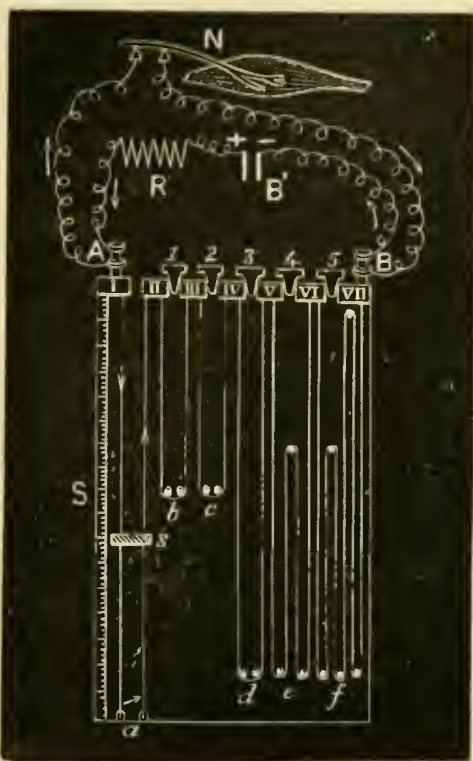

Fif: 208.-Diagran OF JHHetord (AFTER DU BOIS-REYMOND'S MODLL). in a tissue, like that which gives rise to the current of rest of a muscle, for example (lijg. 209).

In electrometer is an instrument for measuring electromotive force -that is, differences of electric potential. Lippmann's capillary electrometer is much employer in physiology. A convenient form of it is shown in ling. 210. A simple form, suitable for students worling in a class where a consiclerable number of copies? of the instrumont is neecled. can lo conveniemtly made as followis: it glas; tube is drawn out to a capilliry at onc

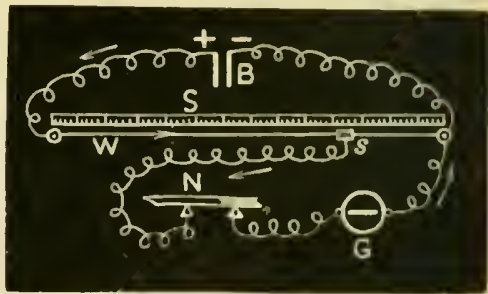

Fín. 209. COMENSATOR.

Description of Fig. 208: I. to VII. are pieces of brass comnected with the wires $a \mathrm{to} / \mathrm{in}$ such a way that by taking out any of the brass plugs $\mathrm{I} t(1) 5$, a greater or less resistance may be interposed between the binding-screws $A$ and 13 . The two wires $a$ are comected by a slider $s$, filled with mercury or otherwise making contact betweon the wires. The current from the battery $B$ divides at $A$ and $B$. part of it passing through the rheocord, part through $\mathrm{N}$, the nerve, muscle, or other conductor which forms the alternative circuit. When a sufficient resistance $R$ is interposed in the clief eireuit to make the total strength of the current independent of ehanges in the resistance of the rheocord, the strength of the current passing through $\mathrm{N}$ will vary inversely as the resistance of the rheocord. When all the plugs are in, and the slider chose up to A. there is practically no resistance in the rheocord, and all the eurrent passes across the brass pieces and plugs to 13, and thence back to the battery. As $s$ is moved farther away from A, the resistance of the rheocord is increased more and more, and the intensity of the current passing through $\mathrm{N}$ becomes greater and greater. The scale $S$ shows the length of wire interposed for any position of $s$, and this gives a rough measure of the fraction of the current passing through $\mathrm{N}$. When plug I or 2 is taken out, a resistance equal to that of the two wires $a$ is interposed ; plug 3 , twice that of $a$; plug 4 , five times; plug 5 , ten times.

lescription of Fig. $209: \mathbb{W}$ is a wire stretched alongside a seale $\mathbf{S}$. A battery $B$ is connected to the binding-serews at the ends of the wire. A pair of unpolariz. able electrodes are conneeted, one with a slider moving al a wire, the other through a galvanometer with one of the terminal binding-serews. In the figure a nerve is shown on the electrodes, wne of which is in contact with an uninjured portion, the other with an injured part. The slider is moved until the twig of the compensating current just balances the demarcation current of the nerve and the galvanometer sliows no deflection. 
end and filled with mercury. The tube is inserted into a small glass bottle, ${ }^{*}$ and fastened in its neck by a cork or a plug of sealing-wax which (loes not quite fill the opening, so that the interior of the bottle is still in communication with the external air. The upper end of the tube is comnected by a short piece of rubber-tuling with a glass $T$-tube as in Fig. 211 . The bottle is partially filled with 5 to Io per cent. sulphuric acid, under which the capilary dips. By means of a small reservoir made from a piece of glass-tubing filled with mereury, and connected with the stem of the T-tube, a little mercury is fored through the capillary

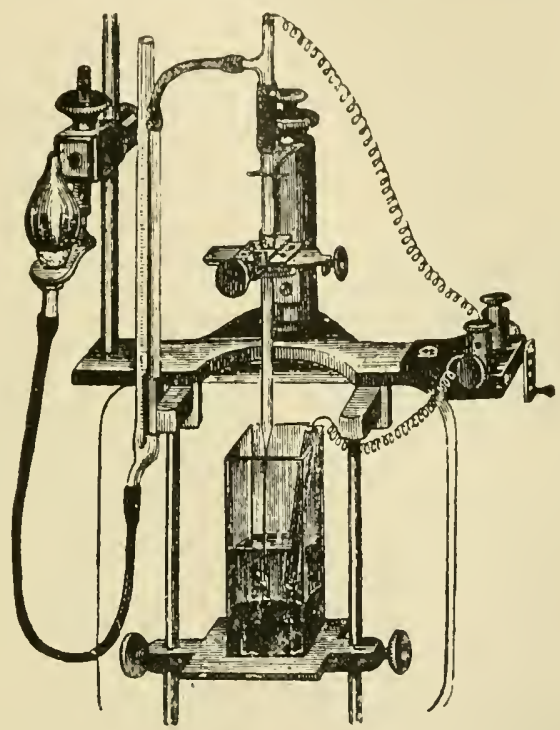

Fig. 210.-CApillary Electroneter (after Frey), as arranged for Mount ing on tile Microscope Stage.

The electrometer consists (I) of a small table carrying a parallel-sided glass vessel containing mercury and sulphuric acid. (2) The capillary tube, which can be moved in two directions at right angles to each other, and so adjusted in the field of the microscope. (3) A pressure-vessel, and a manometer connected with it for measuring the pressure. (4) Two binding-screws connected by wires to the mercury in the capillary tube and in the parallel-sided vessel. The binding-screws can be short-circuited by closing the friction-key shown at the right side of the figure, thus preventing any difference of electro-1notive force between two points connected with the screws from affecting the electrometer.

so as to expel the air in it. When the pressure is lowered again, sulphuric acid is drawn $u p$, and now lies in the capillary in contact with the meniscus of the mercury. A platinum wire fused through

* A parallel-sided bottle is best, as it gives the clearest image of the meniscus. But it is easiest to make a cylindrical bottle from a piece of wide glass-tubing, and to insert a platinum wire into it before closing it at the bottom in the blow-pipe flame. The tube can then be firmly fastened with sealing-wax in a depression in a piece of wood, the wire being brought out through a hole in the wood. Once the instrument is arranged, there is little chance of the capillary getting broken, and there is very little evaporation of the acid. 
the tube, or simply inserted through its upper end, dips into the mercury. Another, passing through the cork, or, better, fused through the botton of the bottle, makes contact with the sulphuric acid through some mercury. The bottle is fastened on the stage of a microscope, the capillary brought into focus, and the meniscus adjusted by raising or lowering the reservoir. When the platinum wires arc connected with points at different potential, a current begins to pass through the instrument, and the meniscus of the mercury in the capillary tube, where the current density is the greatest, becomes polarized by the ions separated from the sulphuric

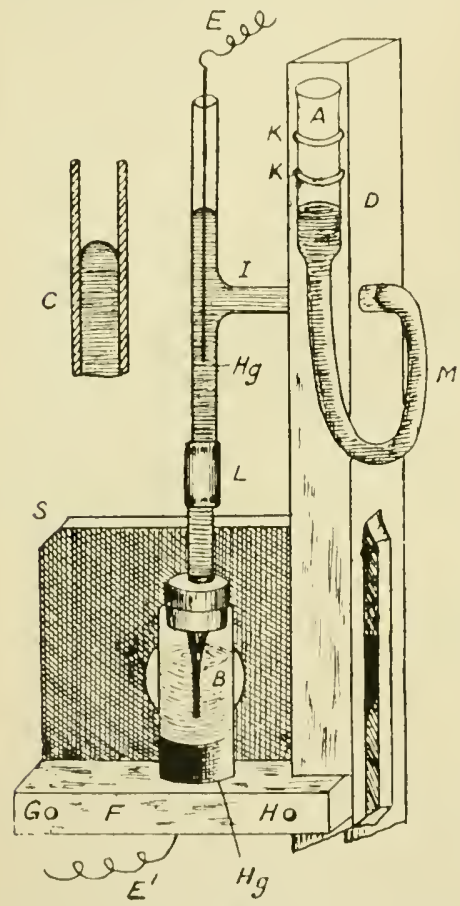

$B$, bottle containing sulphuric acid; $H g$, mercury ; $E, E^{\prime}$, platinum wires. $E$ dips into the mercury in the vertical tube, and $E^{\prime}$ is fused through the bottom of $B$, so as to make contact with the mercury in $B$, the other end of it passing out through a sinall hole in the wooden platform $F$, on which $B$ rests. $F$ is fastened to the stage of the microscope, $S$, by a pin, $G$, passing through one of the clip-holes, and to the wooden upright, $I$ ), by the pin, $H$. I) fits tightly over the microscope stage, but can be moved laterally a little so as to bring the capillary into the middle of the field. $I$, stcm of glass $T$-tube passing through a hole in $L$. $L$, rubber tube connecting the capillary point with the vertical portion of the T-tube. $A$ is a reservoir containing mercury connected by the rubber tube $I I$ to $I$. A can be raised or lowered by sliding it in the clips $k$. In the figure the capillary tube appears as if the mercury extended to the very point of it. This should not be the case; the sulphuric acid should rise for some distance in the capillary, so that the mercury shows a finely-bounded meniscus in the tube as is represented in $C$, the magnified inage of the capillary as seen with the microscope.

F:G. 2II.-A Simple CAPILlary

ELECTROMETER.

acid at the surface of contact between the acid and the mercury, so that the meniscus is no longer in cquilibrium in the tube. The surface tension is diminished when the direction of the current is from mercury to acid (mercury at a higher potential than acid), and is no longer able to counterbalance the hydrostatic pressurc of the mercury. The meniscus therefore moves down in the tube. With the opposite direction of current (mercury at a lower potential than acid) the surface tension is increased, and the meniscus moves up. The polarization develops itself almost instantaneously, and thus an electromotive force is at once established in the opposite dircction to that between the points connected with the electrometcr, and equal to it so long as the external electromotive force is not suff- 
ciently great to cause continuous electrolysis of the acid-that is, so long as it is below about 2 volts. The external current is therefore at once compensated, and after the first moment no current passes through the instrument, which is accordingly not a measurer of current, but of electromotive force. It is very suitable for detecting and measuring such small differences of potential as occur in animal tissues.

Induced Currents. -When a coil of wire in which a current is fowing is brouglit up suddenly to another coil, a momentary current is developed in the stationary coil in the opposite direction to that in the moving coil. Similarly, if instead of one of the coils being moved a current is sent through it, while the other coil remains at rest in its neighbouihood, a transient oppositely-directed current is set up in the latter. When the current in the first coil is broken, a current in the same direction is induced in the other coil.

Du Bois-Reymond's Sledge Inductorium (Fig. 2I2).- This consists

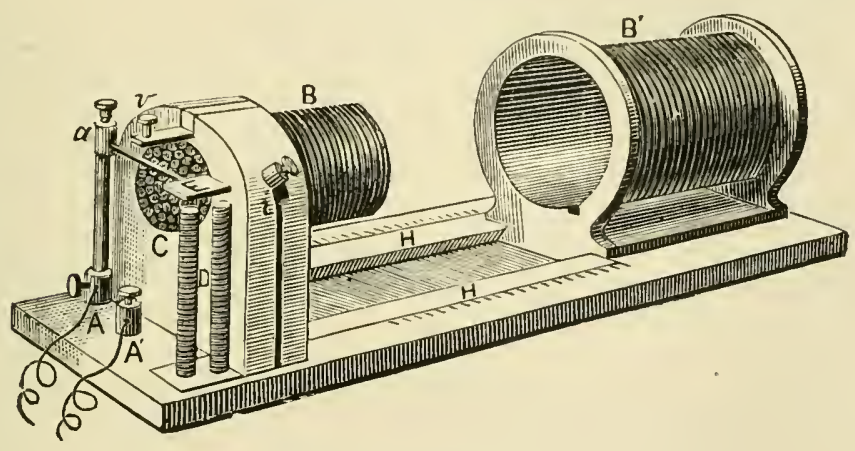

Fig. 212.-Du Bois-Reymond's Inductorium.

B, primary, $\mathrm{B}^{\prime}$, secondary, coil ; $\mathrm{H}$, guides in which $\mathrm{B}^{\prime}$ slides, with scale : $\mathrm{D}$, electro-magnet; E, vibrating spring; $i$, wire connecting wire of $\mathrm{D}$ to end of primary ; $v$, screw with platinum point, connected with other end of primary ; $A, A^{\prime}$, binding-screws, to which are attached the wires from battery. A' is connected with the wire of the electro-magnet $\mathrm{D}$, and through it and $i$ with the primary.

of two coils, the primary and the secondary, the former having a comparatively small number of turns of fairly thick copper wire, the latter a large number of turns of thin wire. The object of this is that the resistance of the primary, which is connected with one or more voltaic cells, may not cut down the current too much; while the currents induced in the secondary, having a high electromotive force, can readily pass through a high resistance, and are directly proportional in intensity to the number of turns of the wire.

By means of various binding-screws and the electro-magnetic interrupter, or Neef's hammer, shown in the figure and explained below it, the current can be made once in the primary or broken once, or a constant alternation of make and break can be kept up. We can thus get a single make or break shock in the secondary, or a 
series of sliccks, sometimes called an interrupted or faradic current. Such a series of stimuli can also be got by making and breaking a voltaic current at any given rate.

A ' self-induced ' current can also be obtained from a single coil ; for instance, from the primary coil alone of the induction apparatus. The reason of this is, that when a current begins to flow through any turn of a coil of wire, it induces in all the other turns a current in the opposite direction, and, when it ceases to flow, a current in the same direction as itself. The former current, ' the make extra shock,' being in the opposite direction to the inducing current, is retarded in its development, and reaches its maximum more slowly than 'the break extra shock.' But, as we shall see, the suddenness with which an electrical change is brought about is one of the most important factors in electrical stimulation, and therefore the break extra shock is a much more powerful stimulus than the make. Owing to these self-induced currents, the stimulating power of a voltaic stream may be much increased by putting into the circuit a coil of wire of not too great resistance.

The self-induction of the primary also affects the stimulating power of the currents induced in the secondary; the shock induced in the secondary by break of the primary current is a stronger stimulus than that caused at make of the primary. The reason is that with a given distance of primary and secondary, and a given intensity of the voltaic current in the primary, the abruptness with which the induced current in the secondary is developed depends upon the rapidity with which the primary current reaches its maximum at closing, or its minimum (zero) at opening. Now, the make extra current retards the development of the primary current, while in the opened circuit of the primary coil the current intensity falls at once to zero.

The inequality between the make and break shocks of the secondary coil can be greatly reduced by means of Helmholtz's wire. Connect one pole of the battery with $v$ (Fig. 212), and the other with $\mathrm{A}^{\prime}$. Join $\mathrm{A}$ and $\mathrm{A}^{\prime}$ by a short, thick wire. With this arrangement the primary circuit is never opened, but the current is alternately allowed to flow through the primary, and short-circuited when the spring touches $v$. The "make" now corresponds to the sudden increase of intensity of the current in the primary when the short-circuit is removed, and the 'break' to its sudden decrease when the short-circuit is established. In both cases self-induced currents are developed, and therefore both shocks are weakened. But the opening stimulus is now slightly the weaker of the two, because the opening extra shock has to pass through a smaller resistance (the short-circuit) than the closing extra shock (which passes by the battery), and therefore opposes the decline of current intensity on short-circuiting more than the closing shock opposes the increase of current intensity on long-circuiting through the primary.

By means of wires connected with the terminals of the secondary coil, and leading to electrodes, a nerve or muscle may be stimulated; and it is usual to connect the wires to a short-circuiting key (Fig. 2 I 5 , by opening which the induced current is thrown into the tissue to be stimulated. For some purposes the electrodes may be of platinum; but all metals in contact with moist tissues become polarized when currents pass through them-that is, have decomposition products of the electrolysis of the tissues deposited on them. 
And as any slight chemical difference, or even perhaps a difference of plysicial state, between the two electrodes will cause them and the tissues to form a battery evolving a continuous current, it is often desirable to use unpolavizable electrodes.

Unpolarizable Electrodes.--Some convenient forms of these are represented in Fig. 213. A piece of amalgamated zinc wire dips into silturated zinc sulphite solution contined in the upper part of a glass tube. The lower ez 1 of the tube may be straight, but drawn out so as to terminate in a not very large opening, or it may be bent into a hook, in the bend of which a hole is made. Before the tube is filled with the zinc sulphate solution, the lower purt of it is plugged witl china clay made (11) with physiological sait solution. The clay just projects through the opening, and thus comes in contact with

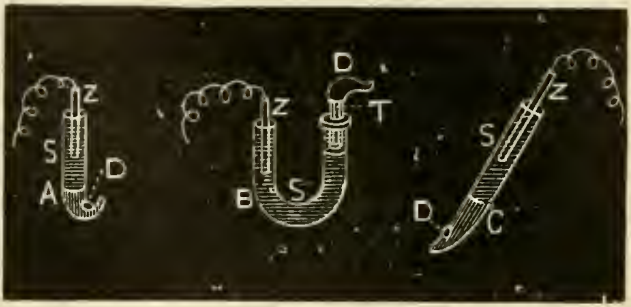

Fig. 2i3.-Unpolarizable Electrodes.

A, hook-shuped : B. U.tubes : C, straight. D, clay in contact with tissue: $\mathrm{S}$, saturated zinc sulphate solution ; $Z$, amalgamated zinc wire.

the tissue. When these

clectrodes are properly set up, there is very little polarization for several hours, but for long experiments, $U$-sh sped tubes, filled with saturated zinc sulphate solution, are better. The amalgamated zinc dips into one limb, and a small glass tube filled with clay, on which the tissue is laid, into the other.

Pohl's Commutator (Fig. 2I4) consists of a block of paraffin or wood with six mercury cups, each in connection with a binding-screw (not shown in the figure). Cups $\mathrm{I}$ and 6 and 2 and 5 are connected by copper wires, which cross each other without touching. The bridge consists of a glass or vulcanite cross-piece $a$, to which are attached two wires bent into semicircles, each connected with a straight wire dipping into the cups 3 and 4 respectively. With the bridge in the position shown in the figure, a current coming in at 4 would pass out by the wire connected with $I$, and back again by that connected with 2 , in the direction shown by the arrows. When the bridge is rocked to the other side so that the bent wires dip into 5 and 6 ,

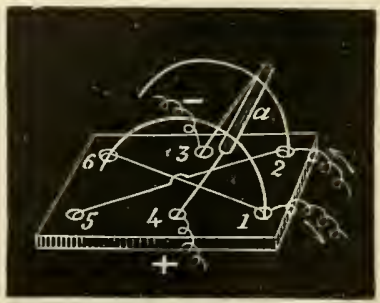

Fig. 214 .-POHL's COM. MUTATOR. the direction of the current is reversed. The cross-wires may be taken out altogether, and the commutator used to send a current at will through either of two circuits, one connected with I and 2 , and the other with 5 and 6.

Du Bois-Reymond's Short-circuiting Key.-A cheap and convenient form is shown in Fig. $2 \mathrm{I}_{5}$.

Time-markers-Electric Signal.-It is of importance to know the time relations of many physiological phenomena which are graphically recorded ; for example, the contraction of a skeletal muscle or 
the beat of a heart. For this purpose a tracing showing the speed of the travelling surface in a given time is often taken simultaneously with the record of the movement under investigation. For a slowlymoving surface it is sufficient to mark intervals of one or two seconds, and this is very readily done by connecting an electro-magnetic marker (such as the electric signal of Deprez) with a circuit which is

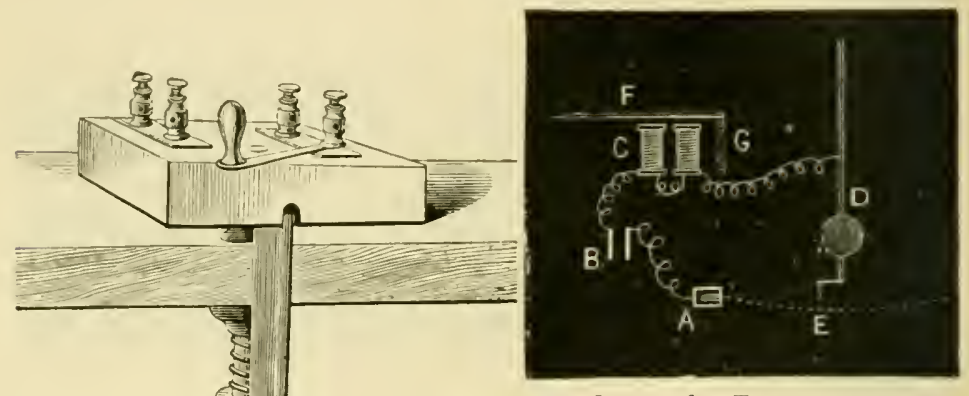

FIŨ. 2I6.-TIME.MARKER.

Arrangement for marking 2 second intervals. D, seconds pendulum, with platinum point $E$ soldered on ; A, mercury trough, into which $\mathrm{F}$ dips at end of its swing; B, Daniell cell; C, clectro-magnets which draw down writing-lever $F$ when the current is closed by $\mathrm{E}$ dipping into $\mathrm{A} ; \mathrm{G}$, spring (or piece of india.

FIG. $215-$ SHORT-CIRCU1TING rubber), which raises $F$ as soon as KEY. current is broken.

closed and broken by the seconds pendulum of an ordinary clock (Fig. 216) or a metronome (Fig. 76, p. 179). For shorter intervals a tuning-fork is used, which makes and breaks a circuit including an electromagnetic marker, or writes on the drum directly by means of a writing-point attached to one of the prongs.

In all the great functions of the body muscular movements play an essential part. The circulation and the respiration, the two functions most immediately essential to life, are kept up by the contraction and relaxation of muscles. The morements of the digestive canal, the regulation of the blood-supply to its glands and to all parts of the body, and that immense class of movements which we call voluntary, are all dependent upon muscular action, which, again, is indebted for its initiation, continuance, or control, to impulses passing along the nerves from the nerve-centres. Hitherto we have not gone below the surface fact, that muscular fibres have the power of contracting, either automatically, or in response to suitable stimuli. In this chapter and the two next we shall consider in detail the general properties of muscle, nerve, and the other excitable tissues. 
l.ying deeper than the peculiarities of individual musrles, muscular tissue has certain common properties-physical, chemical, and physiological. The biceps muscle flexes the arm upon the elbow, and the triceps extends it. The external rectus rotates the eyeball outwards. The intercostal muscles elevate the ribs. The sphineter ani seals up by a ring-like contraction the lower end of the alimentary canal. These actions are very different, but the muscles that carry them out are at bottom very similar. And it cannot be doubted that the functional differences are due entirely, or almost entirely, to differences of anatomical connection, on the one hand witlı bones and tendons, on the other with the nerve-cells of the spinal cord and brain. The common properties in which all the skeletal muscles agree are the subject-matter of the general physiology of striated muscle.

The cardiac muscle differs more, both in structure and in function. from the skeletal muscles than these do among themselves; the smooth muscle of the intestines and bloodvessels still more. But every muscular fibre, striped or unstriped, resembles every other muscular fibre more than it does a nervefibre or a gland-cell or an epithelial scale. The properties common to all muscle make up the general physiology of muscular tissue.

A nerve-fibre is at first sight very different from a muscular fibre. It has diverged more widely from the primitive type of undifferentiated protoplasm. It has lost the power of contraction, or contractility, but it retains, in common witil the muscle-fibre, susceptibility to stimulation, or excitability, the capacity for growth, and to a limited extent the capacity for reproduction. This inheritance of primitive properties, retained alike by both tissues, is the basis of the general physiology of muscle and nerve.

The electrical organ of the Torpedo or the Malapterurus is intermediate in some respects between muscle and nerve, and has properties common to both. In the gland-cell the chemical powers of native protoplasm have been specialized and developed. Contractility has been, in general, entirely lust; but excitability remains. The idea that certain comımon endowments find expression in the action of muscle, nerve, electrical organ, gland, etc., in the midst of all their apparent differences, is the basis of the general physiology of the excitable tissues.

Amœboid movement is the most primitive, the least elaborated form of contraction. An amœba may be seen under the microscope to send out pseudopodia, or processes, of its substance, and to retract them, and it is able by such movements 
to change its place and to take in and expel food and foreign bodies. The maximum velocity of the amoboid movement has been reckoned at 0.008 millimetre a second. Stimulation with the constant current or induction shocks causes the whole of the processes to be drawn in, and the amœba to gather itself into a ball. This illustrates a universal property of protoplasm, excitability, or the power of responding to certain influences, or stimuli, by manifestations of the peculiar kind which we distinguish as vital or physiological. Many other unicellular organisms and the chief varieties of the white blood-corpuscles b have like the amœba; and we have already dwelt upon some of the important functions fulfilled by such amcoboid movement in the higher animals and in man. But a great distinction between this kind of contraction and that of a muscular fibre is that it takes place in any direction.

Cilia.-Cilia possess a higher and more specialized grade of contractility. They are very widely distributed in the animal kingdom; and analogous structures are also found in many low plants, such as the motile bacteria.

In the human subject ciliater epithelium usually consists of several layers of cells, the most superficial of which are pearshaped, the broad end being next the surface, and covered with extremely fine processes, or cilia, about $8 \mu$ in length, which are planted on a clear band. It lines the respiratory passages, the middle ear and Eustachian tube, the Fallopian tubes, the uterus above the middle of the cervix, the epididymis, where the cilia are extremely long, and the central cavity of the brain and spinal cord.

Ciliary motion can be readily studied by placing a scraping from the palate of a frog, or a small portion of the gill of a freshwater mussel under the microscope in a drop of physiological salt solution. The motion of the cilia is at first so rapid that it is impossible to make out much, except that a stream of liquid, recognised by the solid particles in it, is seen to be driven by them in a constant direction along the ciliated edge. When the motion has become less quick, which it soon does if the tissue is deprived of oxygen, it is seen to consist in a swift bending of the cilia in the direction of the stream, followed by a slower recoil to the original position, which is not at right angles to the surface, but sloping streamwards. All the cilia on a tract of cells do not move at the same time; the motion spreads from cell to cell in a regular wave. The energy of ciliary motion may be considerable, although far inferior to that of muscular contraction. The work which cilia are capable of performing can be calculated by removing the membrane, fixing it on a plate of glass, cilia outwards, putting weights on the glass plate, 
and allowing the cilia, like an immense number of feet, to carry it up an inclined plane. Bowditch found in this way that the cilia on a square centimetre of mucous membrane did nearly 7 gramme-millimetres of work per minute (equal to the raising of 7 grammes to a height of a millimetre).

Since the cilia in the respiratory tract all lash upwards, they

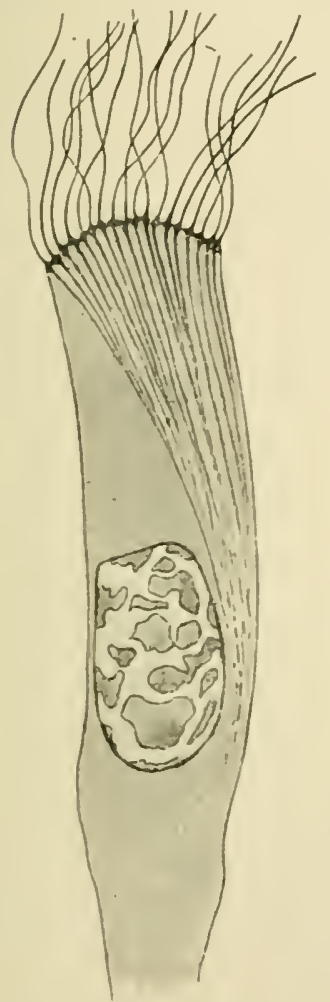

Fig. 2I7.-Ciliated Cell

(M. Heidenhais).

From a 'liver duct' of the garden snail $\times \mathbf{2 , 5 0 0}$. must play an important part in carrying up foreign particles taken in with the air, and the mucus in which they are entangled, as well as pathological products. Engelmann found that the energy of ciliary motion increases as the temperature is raised up to about $40^{\circ} \mathrm{C}$., after which it diminishes quickly. Over-heating causes cilia to come to rest, but if the temperature has

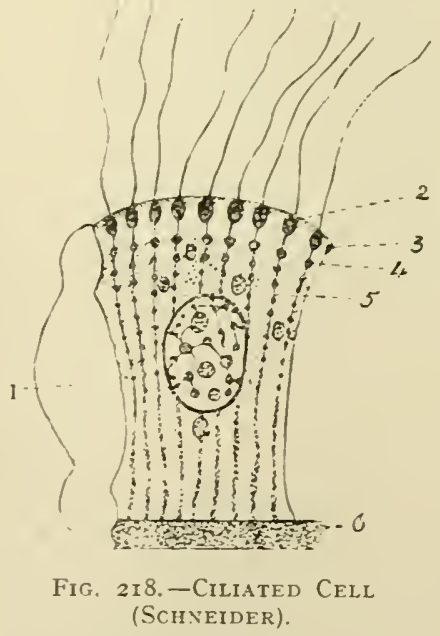

From a flatworm (Planocera folium). $\mathrm{x}$, space between two ad. joining ciliated cells : 2 , basal bodies ; 4, inner granule; 5, 'cilia ronts'; 6 . boundary layer.

not been too high, and has not acted too long, they recover on cooling.

It is not well understood in what way the contraction of the cilia depends upon their connection with the body of the ciliated cell. Very few cases occur in which cilia have the power of independent motion when severed from the cell-body. It has been 
observed in certain low forms of animals that cilia which have been broken off from the cell are still able to contract when a small portion of the substance of the cell-borly at the point where the cilinm is attached to the cell, the so-called basal piece. or basal borly (Jig. 2 I 8), has come off along with them. In other forms isolated cilia can contract in the absence of anything corresponcling to the basal picce. It cannot, therefore, be said that continuity with the basal piece is absolutely necessary. Nor is it known what significance for the ciliary movements is possessed by the long fibrillix, callerl the ' roots of the cilia,' which in some animals run down through the cell from the basal bodies (Figs. 2I7, 218). The theory has been put forward by Schäfer that the cilia are hollow processes of the cell-body, and that their contraction is caused by the passage of licuid into them from the cell. He believes that the direction of movement is determined by the investing membrane of the cilium being thickener (or less extensible) along one side or in a spiral line. In some worms and molluses ciliated cells are supplied with nerve-fibres, but this has not been demonstrated for the higher animals.

Muscle.-Since most of our knowledge of the general physiology of muscle has been gained from striped muscle, in what follows we always refer to ordinary skeletal muscle, unless it is otherwise stated. The sartorius and the gastrocnemius are the classical objects for experiments on striated muscle. For smooth muscle the adductor muscle of Anodon, the fresh-rater mussel, a ring cut from the middle portion of the frog's stomach, the rabbit's ureter and uterus, and the cat's bladder, have been most used.

Physical Properties of Muscle-Elasticity.-All bodics may have their shape or volume altered by the application of force. Some require a large force, others a small force, to produce a scnsible amount of distortion. The elasticity of a body is the property in virtue of which it tends to recover its original form or bulk when these have been altered. Liquids and gases have only clasticity of volume; solids have also elasticity of form. Most solids recover perfectly, or almost perfectly, from a slight deformation. The limits of clistortion within which this occurs are called the limits of elasticity, an.l they vary greatly for different substances. Living muscle has very wide limits of clasticity; it may be deformed-stretcherl, for example-to a very considerable extent, and yet recover its original length when the stretching force ceases to act.

The extensibility of a body is measured by the ratio of the increase of length, producerl by unit stretching force per unit of area of the cross-section, to the original length of a uniform rod of the substance.

If $e$ is the extensibility, $e={ }_{\mathrm{LF}}^{l s}$, where $l$ is the increase of length,

$\mathrm{L}$ the original length, $s$ the cross-section, and $\mathrm{F}$ the stretching force. Suppose we wish to compare the extensibility of two substances. Let $A$ and $B$ be strips or rods of the substances, the length of $A$ being $500 \mathrm{~mm}$., that of $\mathrm{Br}, 000 \mathrm{~mm}$.; the cross-section of $\mathrm{A}$, IOO $\mathrm{sq}$. mm., of $\mathrm{B}, 200 \mathrm{sq} . \mathrm{mm}$. Let the clongation produced by a weight of $\mathrm{I}$ kilo be $\mathrm{IO} \mathrm{mm}$. in each, then the extensibility of $\dot{A}$ is ${ }_{500 \times 1}^{10 \times 100}=2$; and that of $B$ is $\frac{10 \times 200}{1,000 \times I}=2$; that is, the substances are equally extensible. Young's modulus of clasticity, or the co- 
efficient of clasticity is the quotient of the deforming force acting on unit area of the given bolly by the deformation produced (within the limits of clasticity). In the above cxample it is $\frac{F}{s} \div \frac{l}{\mathrm{~L}}$, that is, $l s$,

the reciprocal of the extensibility $c$. For stecl the coefficient of clasticity is rery large, for muscle small. Or, as we may otherwise express it, living muscle within its limits of elasticity is very extensible : a small force per unit area of cross-section of a prism of it will produce a comparatively great elongation. The cxtensibility, however, diminishes continually with the clongation. so that equal increments of stretching force produce always less and less cxtension. If, for instance. the sartorius or semi-membranosus of a frog be connected with a lever writing on a blackened surface, and weights increasing by equal amounts be successively attacher to it, the recording surface being allowed to move the same clistance after the adclition of each weight, a series of vertical lines, representing the amount of each elongation, will be traced. When the lower ends of all the vertical lines are joinerl, a smooth curve with the concavity upwards is obtained (Fig. 219). This is a property common to living and dead muscle and to other animal structures, such as arteries. Marey's method. in which the weight is continuously increased from zero and then continuously decreased to zero again by the flow of mercury into and out of a ressel attached to the muscle, gives directly the curve of extensibility.

The elongation of a steel rod or other inorganic solid is proportional within limits to the extending force per unit of cross-section; and a curve plotted with the weights for abscissæ and the a.mounts of elongation for ordinates would be a straight line. But this is not a fundamental distinction between

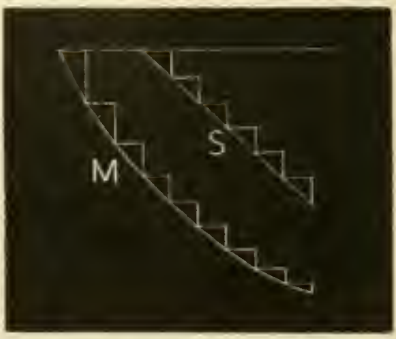

Fig. 219.-Curves of EXTEx. SIBILITY.

II, of muscle; S, of all ordinary inorganic solid. animal tissues, and the materials of unorganized nature, as some writers seem to suppose. For when the slow after-elongation which follows the first rapid increase in length in the loaded, excised muscle is waited for, the curve of extensibility comes out a straight line (Wundt), and within limits this is also the case for human muscles in the intact body. And although a steel rod much more quickly reaches its maximum elongation for a given weight when loaded, and its original length when the weight is removed, than does a muscle, time is required in both cases, and the difference is one of degree rather than of kind. WVen muscle (striated or smooth) is not stretched beyond the limit of physiological relaxation, the amount of stretching is proportional to the weight, and the same is true of all the simple tissues of the body (Haycraft).

Dead muscle is less extensible than living, and its limits of elasticity are much narrower. In the state of contraction the extensibility is increased in excised frog's muscle. When fatigue comes on after many excitations, the after-elongation becomes more pronounced. but the return after unloading is very incomplete. Donders and Van Mansveldt have found that contraction causes 
little difference in the muscles of a living man, although fatigue increases the extensibility.

The great extensibility and elasticity of muscle must play a considerable part in determining the calibre of the vessels, and in lessening the slocks and strains which the heart and the vascular system in general are called upon to bear, and must contribute much to the smoothness with which the movements of the skeleton are carried out, and immensely recluce the risk of injury to the bones as well as to the muscles themseives, the tendons and the other soft tissues. And not only is smoothness gained, but economy also ; for a portion of the energy of a sudden contraction. which, if the murscles were less extensible and elastic, might be wasted as heat in the jarring of bone against bone at the joints. is stored up in the stretcher muscle and again given out in its elastic recoil. The skeletal muscles, too, are even at rest kept slightly on the stretch, braced up, as it were, and ready to act at a moment's notice without taking in slack. This is shown by the fact that a transverse wound jin a muscle 'gapes,' the fibres being retracted, in virtue of their clasticity. towards the fixed points of origin and insertion. Smootl muscle, as we meet it in the hollow viscera, is highly distensible and
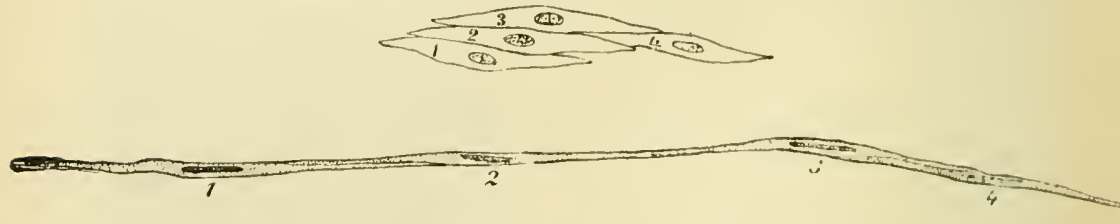

Fig. 220.-Extensibility of SMooth MUscle (CirützNer).

The upper group of four cells ( $I$ to f) is from a hollow organ. whose walls are contracted. and its lumen almost abolished; the under group represents the same fibres when the organ is full. The fibres are longer and somewhat darker. They are also displaced somewhat along each other.

elastic, as is suited to organs whose capacity is continually varying within wide limits (Fig. 220).

In the further study of muscle it is necessary first of all to consider the means we have of calling forth a contraction-in other words, the various kinds of stimuli.

Stimulation of Muscle.-A muscle may be excited or stimulated either directly or through its motor nerve. It is usual to classify stimuli as electrical, mechanical, chemical, or thermal. Electrical stimuli are by far the most commonly employed, and will be discussed in detail. A prick, a cut, or a blow are examples of mechanical stimuli. The action of a fairly strong solution of common salt or of a dilute solution of a mineral acid is usually described as chemical stimulation. But in considering the excitation of nerve (p. 680) we shall see that physical changes are often mixed up with so-called chemical stimulation. The contraction caused is not a single brief twitch, as is the case with a not too severe mechanical excitation, but a sustained contraction or a tetanus. Sudden cooling or heating acts as a stimulus for 
muscle, but thermal stimulation is somewhat uncertain. It is not quite settled whether the contraction which can be obtained from a muscle when it is subjected to bricf local leating to a 'thermic shock, as some writers prefer to say (e.g., by the momentary glow of a platinum wire below but not touching it) - is an ordinary muscular contraction, or a physical, although transient, contracture aralogous to that caused by crtain drugs (Waller). Smooth, like striped, muscle is susceptible to electrical, mechanical, thermal, and chemical stimulation. In addition, in certain situations it can be excited by light (photic stimulation), as in the case of the excised iris of fish and amphibia. In all artificial stimulation there is an element of sudden or abrupt change, of shock, in other words ; but we cannot tell in what the 'natural' or 'physiological' stimulus to muscular contraction in the intact body really consists, nor how it differs from artificial stimuli. All we know is that there must be a wide difference, and that our methods of excitation must be very crucle and inexact imitations of the natural process.

Direct Excitability of Muscle.-The famous controversy on the existence of independent 'muscular irritability' has long been forgotten, and has no further interest except for the antiquaries of science, if such exist. The direct excitability of muscle in the modern sense is not quite the same as the 'muscular irritability,' the discussion of which occupied Haller and his contemporaries. What the modern physiologists have been called upon to decide is whether muscular fibres can be caused to contract except by an excitation that reaches them through their nerves. In this sense there can exist no doubt that muscle is directly excitable, and some of the proofs are as follows :

(I) The ends of the frog's sartorius contain no nerves, yet they respond to direct stimulation. (2) Certain chemical stimuli-ammonia, for instance-excite muscle but not nerve. (3) When the motor nerves of a limb are cut they degenerate, and after a certain time stimulation of the nerve-trunk causes no muscular contraction, while the muscles, although atrophied, can be made to contract by direct stimulation. (4) Finally, there is the celebrated curara experiment of Claude Bernard, which is described in a somewhat modified form in the Practical Exercises, 1. 706. A ligature is tied firmly round one thigh of a frog, omitting the sciatic nerve; then curara is injected, and in a short time the skeletal muscles are paralyzed. That the seat of the paralysis is not the contractile substance of the muscles itself is shown by their vigorous response to direct stimulation. The 'block' is not in the nerve-trunk, nor above it in the central nervous system, for the ligated leg is often drawn up-that is, its muscles are contracted-although the poison has circulated 
freely in the sacral plexus and the spinal cord. Further, if the nerve of the ligated leg be prepared as high up above the ligature as possible, where the curara must undoubtedly have reached it (just above the ligature the nerve has been isolated and the circulation in it more or less interrupted), stimulation of it will cause contraction of the muscles of the limb; while excitation of the other sciatic is ineffective.

It can be also shown, by means of the negative variation or current of action (p. 7 I9), that a nerve-trunk on which curara has acted remains excitable, and capable of conducting the nerve-impulse. The conclusion, therefore, is that the curara paralyzes neither nerve-fibre nor the contractile substance of the

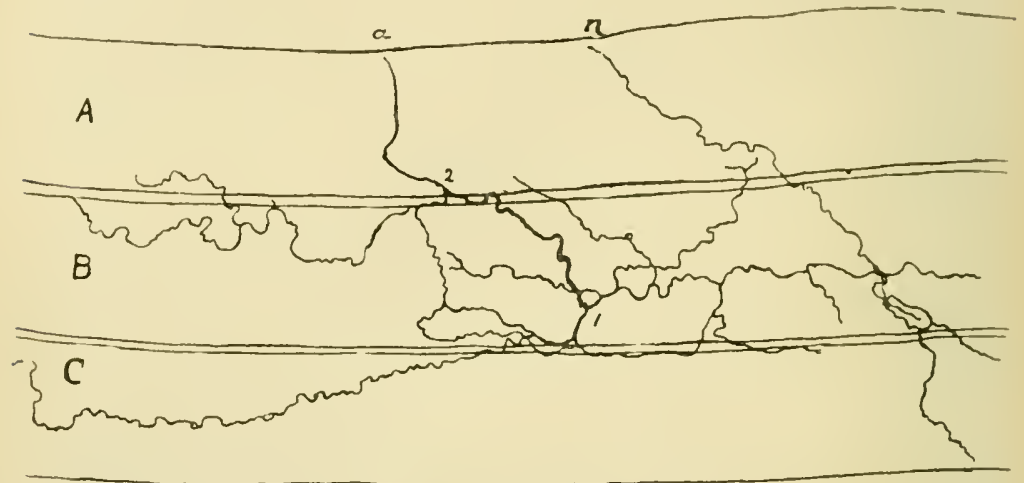

Fig. 221.-Frog's Motor NerVe-Ending (WiLson).

A, B, C, three muscle-fibres. The medullated nerve $a$ loses its medullary sheath and breaks up on B at $\mathrm{r}$. It gives off at 2 a large non-medullated branch, which also breaks up on B. The nerve-endings send ultraterminal fibrilla to $A, B$, and $C$, some of which were seen to end in small knobs. A separate nonmedullated nerve, $x$, is shown, which forms a small plexus on $B$, one fibre of which penetrates to a lower plane than the other, and ends by forming a knob) under the sarcolemma.

muscular fibre, but some link between the two. If the assumption be made that the efferent medullated nerve-fibres within the muscle, since they are anatomically similar to those in the nervetrunk till near their terminations, are similarly affected by curara -and it is a justifiable assumption-the seat of the curara paralysis must either be the nerve-ending or some mechanism, physiological if not anatomical, interposed between the nerveencling and the contractile substance. Now, Langley has shown that the contractions caused by the local application of dilute nicotine solution to points of the skeletal muscles of the frog, both in normal muscles and in muscles whose motor nerves and nerve-endings have degenerated after section of the nerves, 
are in either case prevented by curara. He therefore concludes that, since nicotine produces its effects by a direct action on muscle, and not by an action on nerve-endings or on any special structure (such as the protoplasmic mass or 'sole' at the nerveending in many animals) interposed between the nerve and the muscle, no such special structure existing in the frog (Fig. 22I), curara must also act directly on the muscle. But obviously curara does not paralyze the general contractile substance of the muscle, else the curarized muscle would not contract on direct stimulation. Langley accordingly assumes that, in addition to the contractile or 'general' substance, 'receptive' substances exist in the fibre, through which the excitation is transferred to the contractile substance when the motor nerve is stimulated. He pictures these receptive substances as 'side-chains' of the contractile molecule, in accordance with Ehrlich's theory of immunity (p. 29), and distinguishes those in the neighbourhood of the nerve-ending from those present throughout the muscle fibre. Both the slow local tonic contraction and the quick, brief conducted contractions or twitches set up in a muscle fibre by nicotine, but especially the latter, are much more easily elicited in that part of it which lies under the nerve-ending than elsewhere. Indeed, the position of the nerve-endings in the superficial fibres of a muscle can be ascertained by observing the points which respond most readily to nicotine. Nicotine and curara, etc., are supposed to combine with the receptive substance, which is then in both cases rendered incapable of being affected by nerve impulses. In the case of nicotine an additional action results from the combination with the receptive substance -viz, the change in the contractile substance which leads to contraction. Curara paralyzes the transmission of the excitation from the motor nerves to smooth muscle-the muscles of the bronchi, for instance-with much greater difficulty than to ordinary skeletal muscle, and the same is true of the inhibitory nerves of the heart.

The action of curara gives us the means of stimulating muscle directly; when electrical currents are sent through a non-curarized muscle, there is in general a mixture of direct and indirect stimulation, for the nerve-fibres within the muscle are also excited. Induced currents stimulate nerve more readily than muscle. Voltaic currents may excite a muscle whose nerves have degenerated, while induced currents are entirely without effect.

For direct stimulation, a curarized frog's sartorius or semimembranosus is generally used on account of their long parallel fibres. For indirect excitation, a muscle-nerve preparation, composed of a frog's gastrocnemius with the sciatic nerve attached to it, is commonly employed. as it is easy to isolate the muscle without hurting its nerve.

Stimulation by the Voltaic Current.-While the current continues 
to pass through a nerve without any sudden or great change in its intensity, there is no stimulation, and the muscle connected with the nerve remains at rest. The same is true of striated muscle when a weak current is passed directly through it. But in muscle the constancy of the rule is more and more frequently broken by cxceptional results as the current is strengthened, a state of permanent contraction being very apt to show itself during the whole time of flow (Wundt) (Fig. 222). Above a certain intensity of current a greater or less degree of permanent contraction is invariably produced. This is sometimes called the 'closing tetanus.' It is, however, not a true tetanus, but a tonic contraction, which is strongest in the neighbourhood of the kathode, and does not spread far from it. A similar condition, the so-callerl galvanotonus, is normally seen in human muscles when they or their motor nerves are traversed by a stream of considerable intensity. Under certain conditions, tooe.g., when a strong current is allowed to flow for a comparatively

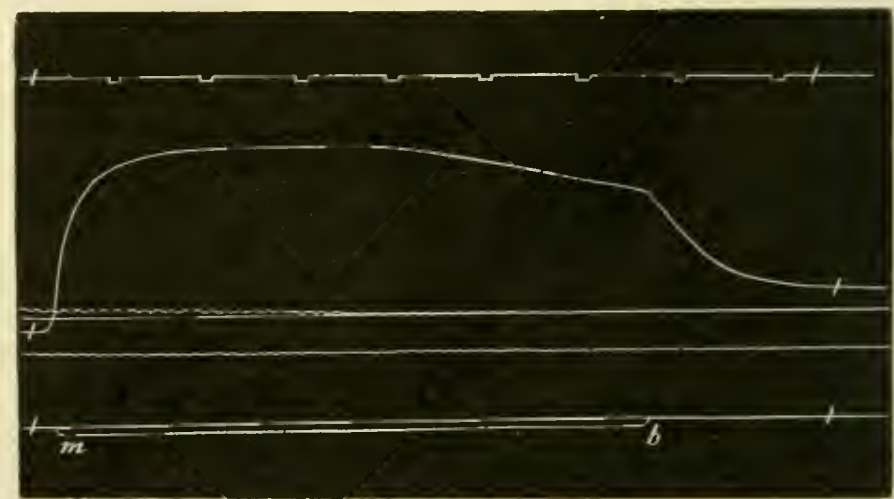

F1g. 222. - Tonic Contraction of Muscle during Passage of Constant Current.

Two sartorius muscles of frog connected by pelvic attachments. Current from I 2 small Daniell cells in series passed through their whole length. Current closed at $m$, opened at $b$. Time trace, two-second intervals.

long time through a muscle-the muscle remains contracted after the opening of the current (so-called 'opening or Ritter's tetanus '). Smooth muscle is excited to contraction even when a voltaic current is very gradually passed into it and slowly increased, and again when it is caused very gradually to disappear. But striped muscle is not stimulated under these conditions.

For nerve, and with these qualifications for muscle, too, we may lay down the law that the voltaic current stimulates at make and at break, but not during its passage. Or, gencralizing this a little, since it has been shown that a sudden increase or lecrease in the strength of a current already flowing also acts as a stimulus. wC may say that the voltaic current stimulates only when its intensity is suddenly and sufficiently increased or diminished, but not while it remains constant.*

* This law of du Bois-Reymond has been questioned by Hoorweg and others. It seems to need modification, but the subject cannot be discussed here. 
When a strong current is closed through a muscle there is an immediate sharp contraction (initial contraction). The muscle then promptly relaxes, but incompletely. When the current is openerl, there is another contraction (Fig. 223). The force of the initial contraction, as measured by the resistance necessary to prevent it, is greater than that of the tonic contraction which follows it.

A sccond law of great theoretical importance is that of polar stimulation. At make the stimulation occurs only at the kathode; at break only at the anole. This is true both for muscle and nerve, but it is most directly and simply demonstrated on muscle. A long parallel-fibred curarized muscle is smpported about its midcle ; the two ends, which hang clown, are connected with levers writing on a revolving drum, and a current is sent longitudinally through the muscle. It is not difficult to see from the tracings that at make the lever attached to the liathodic end moves first, and that the other lever only moves when the contraction started at the kathode has had time to reach it in its progress along the muscle. Similarly, at break the lever connected with the anodic encl moves first. The law of polar excitation holds both for striated and for smooth muscle. Not only is there no excitation of unstriped muscle at the anode on closure of the current, but a previously existing contraction dis a p pears. For

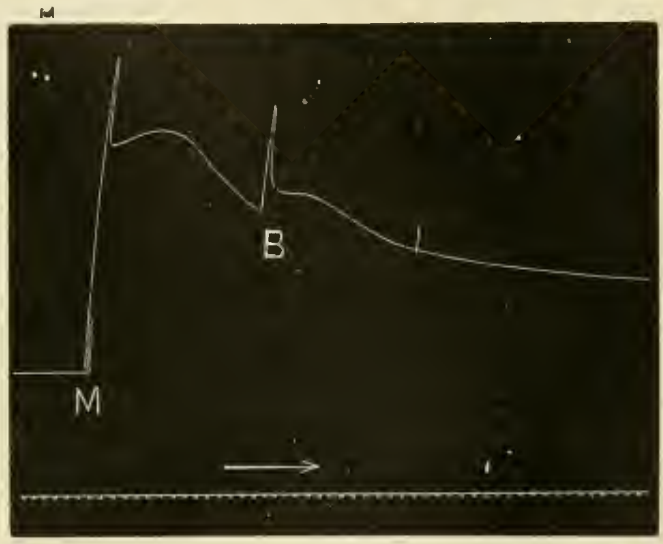

Fig. 223.-TONic Contraction DURing AND AFTER Flow of Voltaic CuRrent.

Curve from frog's gastrocnemius. At M constant current closed, at B broken. Contracture continues after opening of current. Time trace, two-second intervals.

skeletal muscle the

make is stronger than the break contraction. It has not been proved that this is the case for smooth muscle.

The Muscular Contraction.-.When a muscle contracts, its two points of attachment, or, if it be isolated, its two ends, come nearer to each other; and in exact proportion to this shortening is the increase in the average cross-section. The contraction is essentially a change of form, not a change of volume. The most delicate observations fail to detect the smallest alteration in bulk (Ewald). Living fibres kept contracted by successive stimuli can be examined under the microscope ; or fibres may be ' fixed' by reagents like osmic acid, and sometimes a very good opportunity of studying the microscopic changes in contraction is given by a group of fibres in which the 'fixing' reagent has 
caught a wave of contraction, and, so to speak, pinned it down. It is then seen that the process of contraction in the fibre is a miniature of that in the anatomical muscle. The individual fibres shorten and thicken, and the sum-total of this shortening and thickening is the nuscular contraction which we see with the naked eye. The phenomena of the muscular contraction may be classified thus: (I) Optical, (2) Mechanical, (3) Thermal, (4) Chemical, (5) Sonorous, (6) Electrical. (5) will be treated under 'Voluntary Contraction'; (6) in Chapter XI.

(1) Optical Phenomena-Microscopic Structure of Striped Muscle.The structure of striped muscle has long been the cuigma of histology ; and the labours of many distinguished men have not sufficed to make it clear. On the contrary, as investigations have multiplicel, new theories, new interpretations of what is to be seen, have multiplied in proportion, and a resolute brevity has becone the chicf duty of a writer on elementary physiology in regard to the whole question.

The muscle-fibre, the unit out of which the anatomical muscle is built $11 \mathrm{p}$, is surrounded by a structureless membrane, the sarcolemma. The length and breadth of a fibre vary greatly in different situations. The maximum length is about $4 \mathrm{~cm}$.; the breadth may be as much as $70 \mu$ and as little as Io $\mu$. When we come to analyze the musclefibre and to determine out of what units it is built up, the difficulty begins. The fibre shows alternate dim and clear transverse stripes, and can actually be split up into dises by certain reagents. It also shows a longitudinal striation, and can be separated into fibrils. Some have supposed that the discs are the real structural units which. piled end to end, make up the fibre. The fibrils they consider artificial. This view is crroneous. It seems certain that the fibres are built up from fibrils ranged side by side, and that the discs are artificial. The contents of the muscle-fibre appear to consist of two functionally different substances, a contractile substance, and an interstitial, perhaps nutritive, non-contractile material of more fluicl nature. The contractile substance is arranged as longitudinal fibrils cmbedded in interfibrillar matter (sarcoplasm). In a muscle impregnated with chloride of gold the interfibrillar matter appears as a network.

Schäfer has described the contractile elements of the muscle-fibre (Figs. 224, 225) as fine columns (sarcostyles), divided into segments (sarcomeres) by thin transverse discs (Krause's membranes), occupying the position of the midelle of each light stripe. Each sarcomere contains a sarcous element (a portion of the dark stripe) with a clear substance at its ends, filling up the space between the sarcous clement and Krause's membranc, and constituting a portion of the light stripe. The sarcous clement is itself double, and if the fibre be stretched, the two portions separate at a line which runs transversely across the middle of the dim stripe (Hensen's line). Schäfer considers that the appearance of longitudinal fibrillation in the sarcous elements is due to the presence in them of fine longitudinal canals or pores.

Rutherford has given a somewhat different account of the matter. According to him, each fibril is made up of a longitudinal row of segments of two kinds, alternating with each other (Fig. 226) : (I) 'Bowman's elements,' shaped like an clongated hour-glass, and 
containiner a substance reatily stained by various dyes; (2) an 'intermediate segunent' of cylindrical shape, the general substance of which does not readily stain. The intermediate segment contains in its centre a globule (J)bic's globule), which is easily stained.* the fibrits are regnlarly arranged in bundles within the fibre. The apposition of Bowman's elements gives rise to the dim stripe; the apposition of the intermediate segments to the clear stripe; the apposition of the Dobie's globules to a line in the middle of the clear stripe (Dobic's linc). Dobie's line has by some been consiclered to represent a membrane made up of the apposed Krause's membranes of all the fibrils. But the lixause's membrane of the individual fibrils is

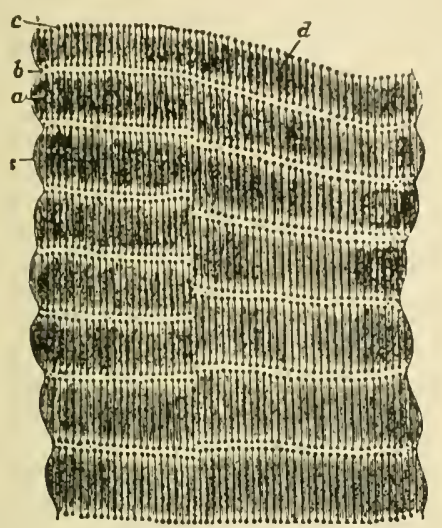

Fig. 224.-LIVING MUSCLE OF WATER-BEETLE (HIGHLY MAGNIFIED) (SCHÄFER).

s. sarcolemma; $a$, dim stripe : $b$, bright stripe; $c$, row of dots in bright stripe, which appear to be the enlarged ends of rod-shaped particles, $d$, but in reality represent expansions of the interstitial substance (sarcoplasin). scarcely ever visible in an intact mammalian fibre (Schäfer), and the apparent line in the clear stripe of an intact fibre is an optical appearance duc to interference of the light. Kühne, who was fortunate

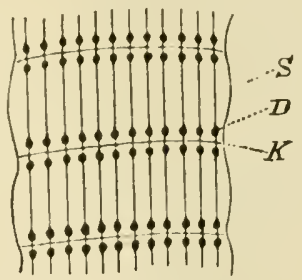

F1G. 225.-PORTION OF LEG MUSCLE OF INSECT, TREATED WITH DULUTE ACETIC ACid (Schäfer).

S, sarcolenma ; D, dot. like enlargement of sarcoplasm ; K, Krause's mem. brane. The sarcous ele. ments have been swollen and dissolved by the acid.

enough to find one day a small nematode worm moving in the interior of a fibre, saw it pass along the fibre with perfect freedom, ignoring Krause's membrane. Possibly, however, it was moving in the sarcoplasm, the fibrils being simply pushed aside.

Changes during Contraction. -When a muscle contracts, according to Schäfer, the clear substance between the Krause's membrane and the sarcous element passes into the canals, which are open towards Krause's membrane, but closed towards Hensen's line. The sarcous element therefore swells up, and the sarcomere is shortened. In the extended muscle the clear substance leaves the pores of the sarcous element, and accumulates in the space between it and Krause's

* In the muscles of certain invertebrate animals, though not in those of vertebrates, the intermediate segment contains, in addition to Dobie's globule, two pear-shaped bodies (Flögel's elements), each of which occupies an intermediate position between Dobie's globule and the end of the adjoining Bowman's element. Flögel's elements also stain well, and are doubly refracting. 


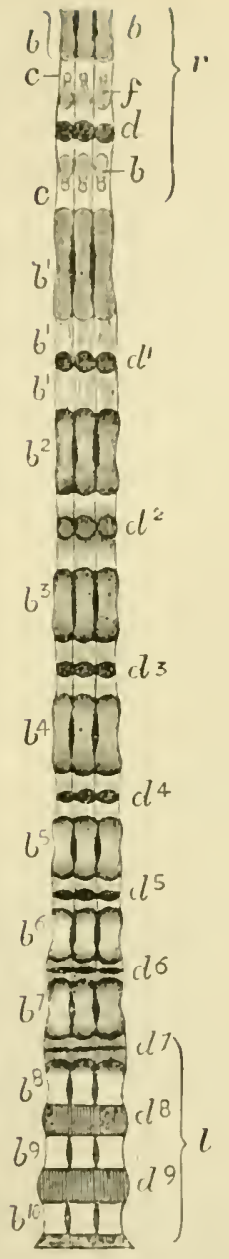

FIG. 226.-CrAB'S MLSCLE IN DIFFERENT STAGES OF CoNTRACTION (AFTEK RUTHERFORD).

Three fibrilla are shown: $r$, complete relaxation: $t$, complete contraction : $b-b^{10}$, sarcous elements; $d-d^{9}$, Dobie's granules ; $f$, Flögel's granules. such a transference of substance between
the light and dim stripes that the former becomes dim, and the latter light. This so-called reversal of the stripes is not really a reversal in the sense of being a bodily exchange of the whole of the

membrane. The sarcomere is thus lengthencel and narrowed. W"lile the existence of Schäfer's pores is not arlmitter! by all observers, there is a pretty general agreement that the sarcomere, like the cytoplasm of an amoboid cell. dloes consist of two substances, one of which (the hyaloplasm of the cell, the clcar material of the sarcomere) interpenetrates the other (spongioplasm of the cell, substance of the sarcous element); and that in relaxation the clear fluid passes from the sarcous element to the ends of the sarcomeres, whereas in contraction it passes in the reverse direction into the sarcous elements. Whether the fluid passes into and out of the meshes of an actual network, or along actual physical pores in the sarcous element, or whether it is transferred by some process like molecular imbibition (p. 398), need not be cliscussed here, since it is not definitely known The fundamental question by what process the transference is drtermined when the muscle is excited also remains unsettled. So far as is known at present, it is probable that the mechanical energy of the contracting muscle must be derived from the transformation of chemical energy into one of three forms: energy associated with osmotic processes, encrgy associated with imbibition, and energy associated with changes of surface tension. It is not difficult to sec that a sudden increase in the osmotic concentration in the sarcous element, duc to the breaking up of large molccules or colloid aggregates into small molccules, or the liberation of electrolytes from the colloids, might lead to the rapid passage of water into it from the bright bands. A sudden change of permeability of the sarcous elements for dissolved substances in the clear fluid would have a similar effect. The same is true of a change in their power of imbibition. But, according to Bernstein, it is scarcely to be supposed that the extraordinarily rapid movement of water molecules which must occur in contraction can be accounted for either by osmosis or by imbibition. A more plausible theory is that the surface tension-say betwcen the substance of the sarcous element and the clear fluid-is altered. Some writers assert that in contraction there is such a transference of substance between
that the former becomes clim, and the latter 
material of the chim and light stripes. Schäfer lias explained it as che to the spueczing of sarcoplatsm from between the sarcous elements into the position of the light stripe, when they bulge laterally in contraction. This accumulation of sarcoplasm in the stripes that were previously light makes them look darker in comparison with the true dim stripe.

Appearance of the Fibres in Polarized Light. - A ray of ordinary light consists of vibrations of the ether in all planes at right angles to the direction of the ray. In a ray of plane polarized light all the particles vibrate in one plane. A ray of light which has been polarized by a Nicol's prism cannot pass through another Nicol's prism with its principal plane at right angles to that of the first. If the sccond or analyzing prism be rotated so that the principal planes are no longer at right angles, some of the light will pass through. The same effect is produced if, without altering the original 'crossed' position of the nicols, a substance capable of rotating the polarized ray is introduced between the prisms. I rough illustration will perhaps tend to make this point clearer. Suppose that a string fixed at one end is set vibrating in various directions by a twisting movement. If the string lias to pass through a narrow vertical slit-e.g., between two fingers held vertically-all vibrations except those in the vertical plane will be extinguished; but vertical vibrations will be able to pass beyond the slit. The movement may be said to be plane polarized, and the effect of the slit corresponds to that of the first nicol. Now make the string pass also through a horizontal slit; the vertical vibrations will then be extinguished too; in otber words, none of the movements will pass beyond
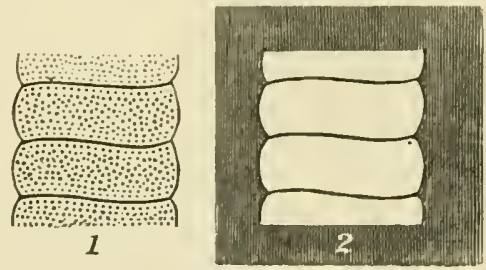

Fig. 227.-LIVING MUSCLLAR FIBRE

(FROM GEOTRCPES STERCORARIUS).

$\mathrm{I}$, in ordinary; 2, in polarized light. (Van Gehuchten.) In living muscle (at least in fibres which are not extended) in contrast to dead muscle after treatment with reagents, the doubly refracting or anisotropous substance is present in the greater part of the fibre; and with crossed nicols the position of the singly refracting or isotropous material is indicated only by narrow transverse black lines or row's of dark dots. the 'crossed' slits. 'This corresponds to the dark field of the crossed nicols. But if the vertical vibrations which have passed the first slit could be in any way changed into horizontal vibrations, they would no longer be extinguished by the second. This would correspond to rotation of the plane of polarization through $90^{\circ}$. A ray of light polarized by the first nicol will, if its plane of polarization be rotated through $90^{\circ}$, pass entirely (except for loss by ordinary reflection and absorption) through the second. If the angle of rotation is less than $90^{\circ}$, a portion will pass through.

The substance of the sarcous element which forms the dark stripe is doubly refracting, and therefore rotates the plane of polarization, but the clear substance of the light stripe is singly refracting. When an uncontracted fibre is viewed with crossed nicols, the dim stripe accordingly appears bright in the otherwise dark field. In the contracted fibre the doubly refractive material remains in the stripe 
which is dim in orelinary light. There is no transference of it, and no real reversil of the stripes.

Diffraction Spectrum of Muscle.-When a beam of white light passes througl a striped muscle, it is broken up into its constituent colours, and a series of diffraction spectra are produccel, just as happens when the light passes through a diffraction grating (a piece of glass on which are ruled a number of fine parallel equidistant lines). The nearer the lines are to cach other, the greater is the (lisplacement of a ray of light of any given wave-length. It hats accordingly been found that when a muscular fibre contracts, the amount of displacement of the diffraction spectra increases. At the same time the whole fibre becomes more transparent.

(2) Mechanical Phenomena.-The muscular contraction may be graphically recorded by connecting a muscle with a lever which is moved either by its shortening or by its thickening. The lever writes on a blackened surface, which must travel at a uniform rate if the form and time-relations of the muscle curve are to be studied, but may be at rest if only the height of the contraction is to be recorded. The whole arrangement for taking a muscle-tracing is called a myograph (Fig. 261, p. 707). The duration of a 'twitch' or single contraction (including the relaxation) of a frog's muscle is usually given as about one-tentl of a second, but it may vary considerably with temperature, fatigue, and other circumstances. It is measured by the vibrations of a tuning-fork written immediately below or above the muscle curve. When the muscle is only slightly weighted, it but very gradually reaches its original length after contraction, a period of rapid relaxation being followed by a period of 'residual contraction,' during which the descent of the lever towards the base-line becomes slower and slower, or stops altogether some distance above it. The duration of the contraction of smooth muscle evolked by a single momentary stimulus is much greater than that of striped muscle (two to seven seconds for the rabbit's ureter ; five to fifteen seconds for the cat's nictitating membrane; one to two minutes for the frog's stomach).

Latent Period.-If the time of stimulation is marked on the tracing, it is found that the contraction does not begin simultancously with it, but only after a certain interval, which is called the latent period.

This can be measured by means of the pendulum myograph (Fig. 229) or the spring myograpli (Fig. 228), in both of which the carrier of the recording plate opens, at a definite point in its passage, a key in the primary coil of an induction machine, and so causes a shock to be sent through the muscle or nerve, which is connected with the secondary. The precise point at which the stimulus is thrown in can be marked on the tracing by carefully bringing the plate to the position in which the key is just opened, and allowing the lever to trace here a vertical line (or, rather, an are of a circle). The portion of the time-tracing between this line and a parallel line drawn through the point at which the contraction begins gives the latent period.

Helmholtz measured the length of the latent period by means of the principle of Pouillet, that the deflection of a magnet by a current of given strength and of very short duration is proportional to the time cluring which the current acts on the magnet. He arranged that at the moment of stimulation of the muscle a current should be sent through a galvanometer, and should be broken by the 
contraction of the muscle the moment it began. In this way he obtained the value of 1 ii second for the latent period of frog's muscle. The tendency of later observations has been to make the latent period shorter. Burclon Sunderson found that the change of form begins in muscle with clirect stimulation in coin second after, and the electrical change (p. 721) simultancously with, the excitation. It is known that the apparent latent periol depends upon the resistance which the muscle has to overcome in beginning its contraction. A heavilyweighted muscle. for instance, cannot begin to shorten until as much energy has been developed as is necessary to raise the weight; and its latent periol will be listinctly longer thin that of unweighted or very slightly weighted muscles, sucl as those with which Sanderson worked.

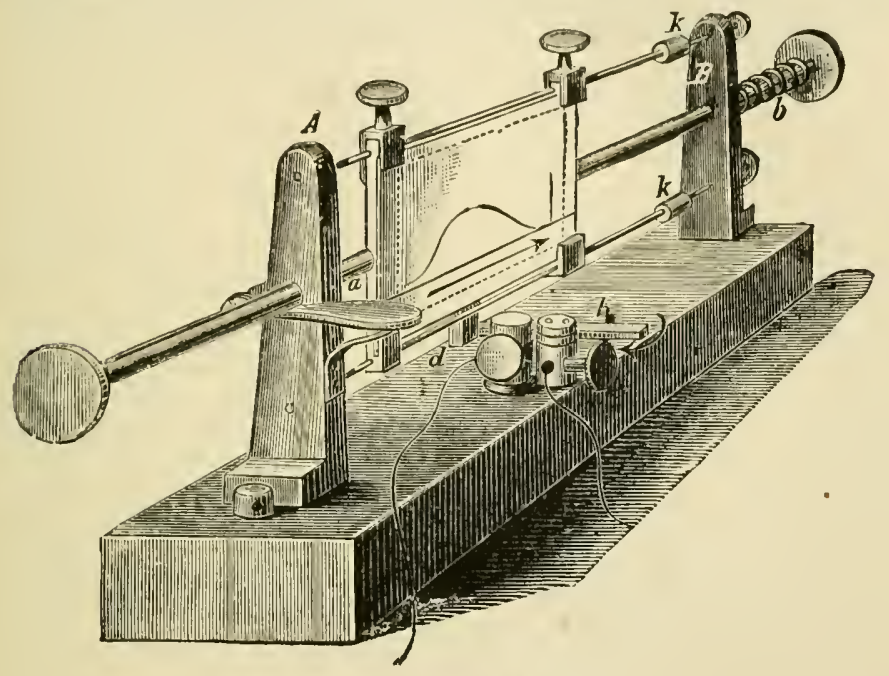

F1G. 228,-SPRING MYOGRAPH.

$A, B$, iron uprights, between which are stretched the guide-wires on which the travelling plate $a$ runs; $k$, pieces of cork on the guides to gradually check the plate at the end of its excursion, and prevent jarring; $b$, spring, the release of which shoots the plate along; $h$, trigger-key, which is opened by the pin $d$ on the frame of the plate.

The maximum shortening, or ' height of the lift,' depends upon the length of the muscle, the direction of the fibres, the strength of the stimulus, the excitability of the tissue, and the load it has to raise.

In a long muscle, other things being equal, the absolute shortening and therefore the maximum height of the curve, will be greater than in a short muscle; in a muscle with fibres parallel to its lengththe sartorius, for instance-it will be greater than in a muscle like the gastrocnemius, with the fibres clirected at various angles to the long axis. For stimuli less than maximal, the absolute contraction increases with the strength of stimulation, and a given stimulus will cause a greater contraction in a muscle with a given excitability than in a muscle which is less excitable. Under ordinary experimental conditions at least, weak stimuli cause a smaller contraction than strong, not only because each stimulated fibre contracts less, but 
because a smaller number of fibres are excited (P. 1. I). The objects used for the study of muscular contraction contain many fibres, and it is not in general possible to clistribute the stimulus equally to all. This is true for smooth muscle as well as for striped. Finally, increase

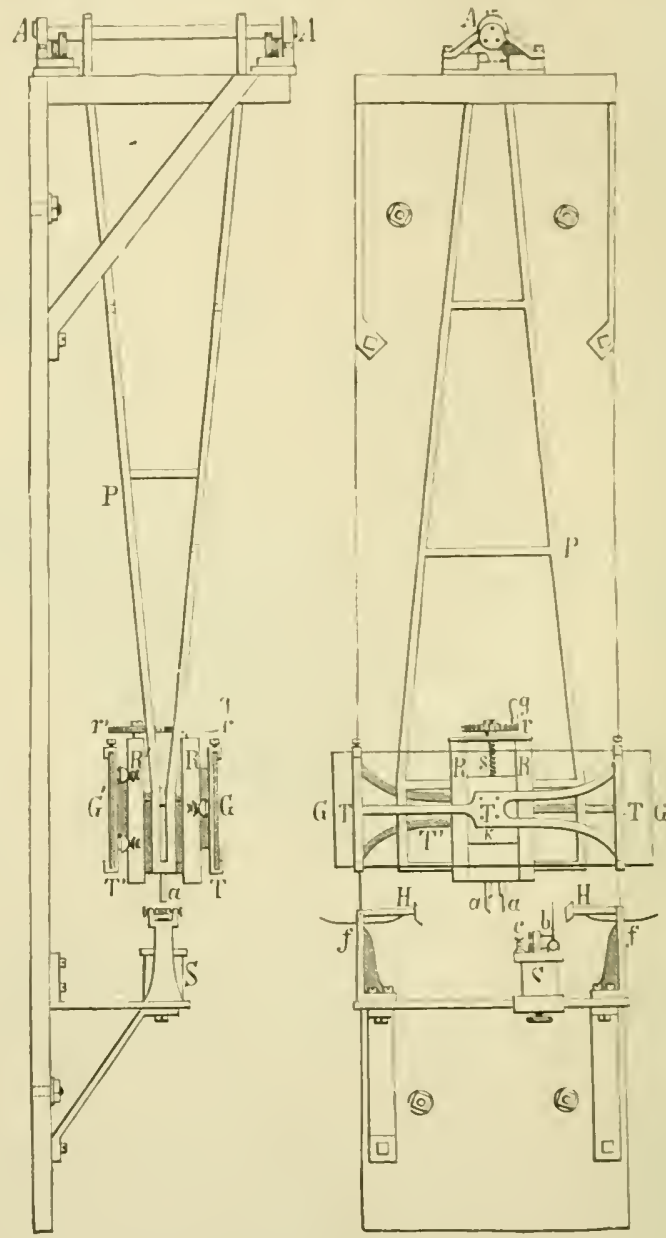

Fig. 229.-PENDLluis MYograpil.

At the left as seen from the side, at the right as seen from the front. $A$, bearings on which the pendulum swings: $P$, pendulum: $G, G^{\prime}$, glass plates carried in the frames $T, T^{\prime}$; $a$, pin which opens the trigger-key. The key, when closed. is in contact with $c$, and so completes the circuit of the primary coil.

of the load per unit of cross-section of the muscle diminishes above a certain limit the 'height of the lift,' although below that limit it may increase it.

Influences which affect the Time-relations of the Muscular Con- 
traction. - Many circumstances affect the form of the muscle curve and its time-relations.

(a) Influence of the I-oul.- The first effect of contraction is to suddenly stretch the muscle, and the more the muscle is loated the greater will this elongation be. So that at the beginning of the actual shortening part of the energy of contraction is alraaly

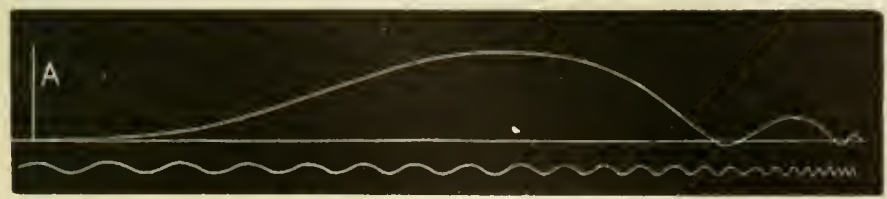

Fig. 23o.-Curve of a Sincle Muscular Contraction or Twitcil taken ox SMOKED (ilass WItI Spring Mrograph and Photographed.

Vertical line A marks the point at which the muscle was stimulated; time tracing shows i io of a second (reduced).

expended without visible effect, and has to be recovered from the elastic reaction luring the ascent of the lever.

Then the incrtia of the lever itself and of its load comes into play, and may carry the curve too high during the up-stroke and too low during the down-stroke. This can be minimized by making the lever very light, and attaching the weight close to the fulcrum, so that it has only a small range of mosement, and never acquires more than a small velocity. The contraction of a muscle loaderl by a weight which is not increased or liminished during the contraction is said to be iso-fonic, for here the tension of the muscle is the same throughout, and its length alters. When the muscle is attached very near tha fulcrum of the lever, so that it acts upon a short arm, while the long arm carrying the writingpoint is prevented from moving much by a spring, the muscle can only shorten itself very slightly; but the changes of tension in it will be related to those in the spring, and therefore to the curve traced by the writing-point. Such a curve is called iso-metric, since the length of the muscle remains almost unaltered. In the body muscles usually contract under conditions more nearly allicel to those of the isometric than to those of the iso-tonic contraction.

The maximum of the iso-metric curve (the maximum tension with practically constant

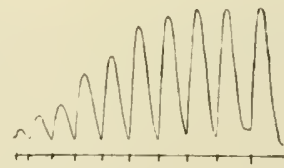

Fig. 23I.-CONTRAC. TIONS OF SHOOTII MUSCLE: CA T's BladDER (C. C. STEWA:T).

Stimulated with pro. gressively stronger induction shocks. The lowest line is the time trace (ro-second intervals). Immediately below the inuscular contractions are marked the points at which the stimuli were thrown in. length) is sooner reacherl than that of the isotonic (the maximum contraction with constant tension). From this it has been concluded that as the muscle shortens its coefficient of elasticity continuously diminishes (Fick), or, what comes to the same thing, its extensibility continuously increases. It follows that the tension of a muscle contracting against resistance, especially at the beginning of its contraction, is greater than would be the case when the muscle, contracting isotonically, had attained the same length. 
The work done by a muscle in raising a weight is equal to the product of the weight by the height to which it is raised. Beginning with no load at all, it is found that the weight can be increaser up to

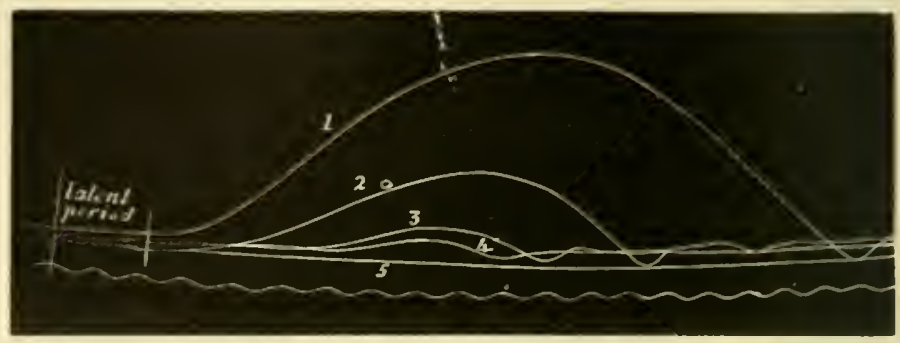

F1G. 232.-INFLIENCE OF LOAD OX THE FORN OF THF MUSCLF CTRST

I, curve taken with unloaded lever: $2,3,4$, weight surressively increased; 5, abscissa line: time trace, $\frac{1}{100}$ second (reduced).

a certain limit without rliminishing the height of the crntraction ; perhaps the height may eren increase. Up to this limit. then, the work evidently increases with the load. If the weight is marle still greater, the contraction becomes less and less, but up to another

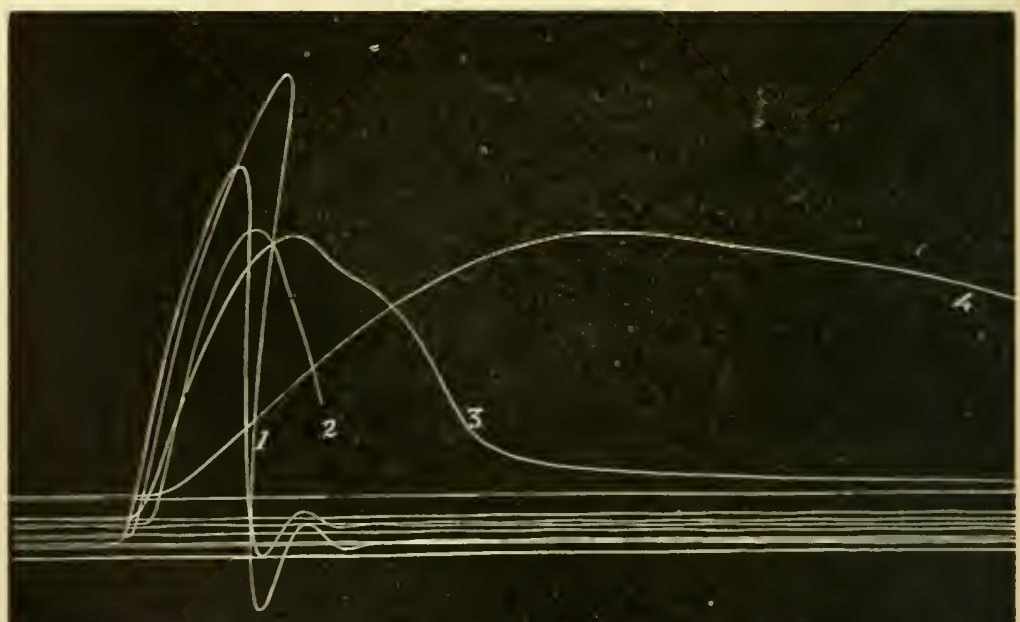

M.

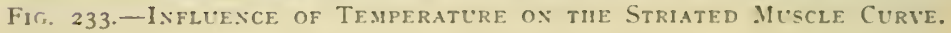
2 , air temperature; $1,25^{\circ}-30^{\circ} \mathrm{C} .: 3,7^{\circ}-10^{\circ} \mathrm{C}$; 4 , ice in contact with muscle. The fifth curve was taken at a little above air temperat ure.

limit the increase of weight more than compensates for the climinution of 'lift,' and the work still increases. Bevond this, further increase of weight can no longer male up for the lessening of the 
lift, and the work falls off till ultimately the muscle is unable to raise the weight at all.

The manner of application of the weight has an influence on the work done by the muscle. If it is applied before the contraction begins, so that the muscle is already stretched at the moment of stimulation, a cause of crror and uncertainty is introduced ; for it is known that mere stretching of muscle affects its metabolism, and therefore its functional power. So that it is usual in experiments of this kind to after-load the muscle-that is, to support the lever and its load in such a way that the weight cloes not come upon the muscle till contraction lias just begun. 'The 'absolute contractile force' of an active muscle may be measured

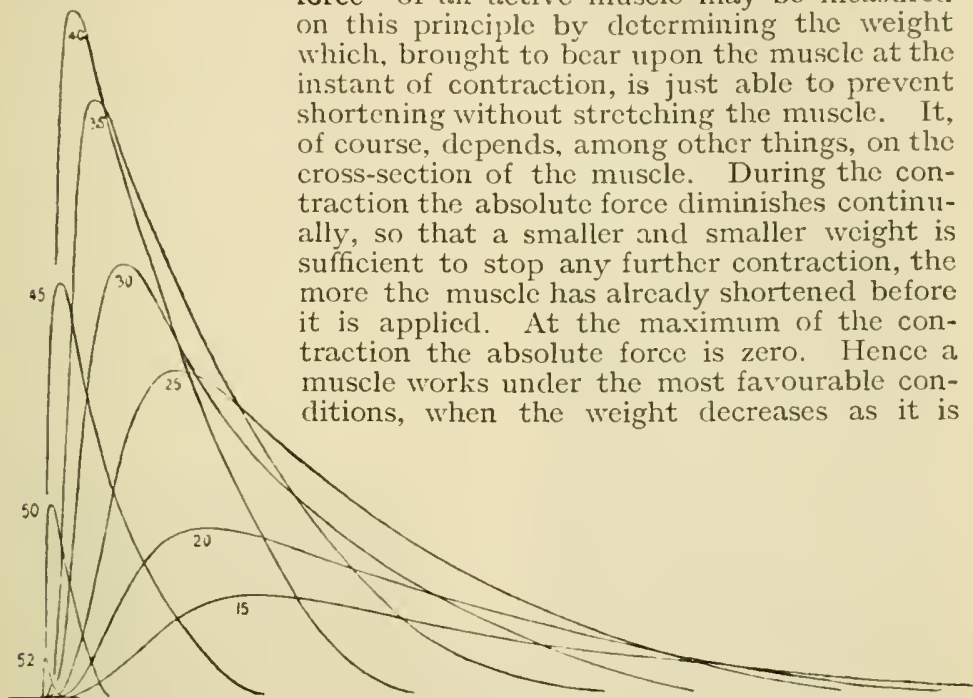
on this principle by etermining the weight instant of contraction, is just able to prevent shortening without stretching the muscle. It, of course, depends, among other things, on the cross-section of the muscle. During the conally, so that a smaller and smaller weight is sufficient to stop any further contraction, the more the muscle has alrcady shortened before it is applied. At the maximum of the contraction the absolute force is zero. Hence a muscle works uncler the most favourable conditions, when the weight decreases as it is

Fig. 234.-Influence of Temperature on the Smooth Muscle Curve Cat's Bladder (C. C. Stewart).

Contractions at different temperatures with the same strength of stimulus. The temperatures (Centigrade) are marked on the curves.

raised, and this is the case with many of the muscles of the body. During flexure of the forearm on the elbow, with the upper arm horizontal, a weight in the hand is felt less and less as it is raised, since its moment, which is proportional to its distance from a vertical line drawn through the lower end of the humerus, continually diminishes.

(b) Influence of Temperatuve on the Muscular Contraction.Increase of temperature of the muscle up to a ccrtain limit diminishes the latent period and the length of the curve, and increases the height of the contraction, but beyond this linit the contractions are lessened in height (Fig. 233). Narked diminution of temperature causes, in general, an increase in the latent period and length, and a decrease in the height of the contraction. It is evident that much depends upon the normal tempcrature which we start from, and moderate cooling may increase the height of the curve. In the heart 
the effect of cold in strengthening the beat is often very marked. Temperature affects the contraction "curve of smonth muscle much in the same way as that of striated milscle (Fig. 234). I?P to $f^{\circ} \mathrm{C}$.

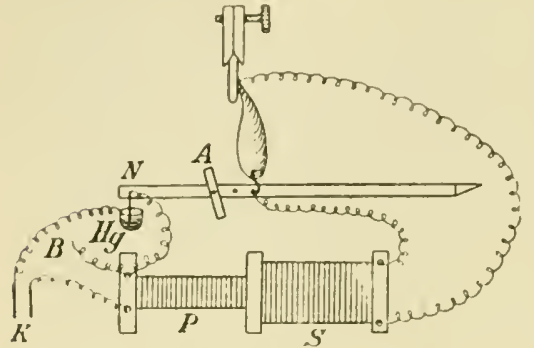

Fig. 235.-Automatic Muscle Interruptor.

K, battery ; P, primary ; S, secondary coil ; $A$, axis of lever: $\mathrm{N}$, needle: $\mathrm{Hg}$, mercury cup.

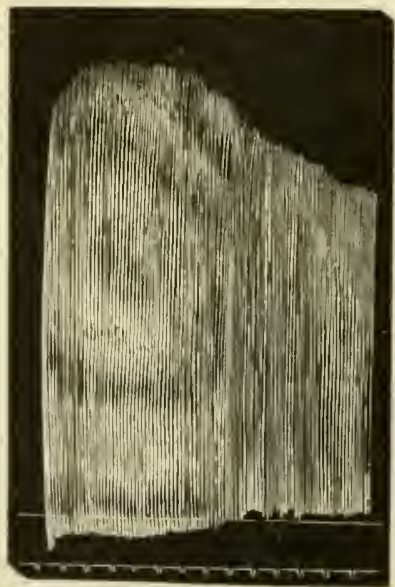

Fig. 236.-Fatigue Curve of Muscle: FroG's Gastrocnemus.

The arrangement with which the curve figured was obtained was a socalled automatic muscle interruptor (Fig. 235). A wire on the lever is made to close and open the primary circuit of an inductorium, the muscle or nerve being connected with the secondary. Every time the needle touches the mercury the muscle is stimulated auto. matically. Another arrangement is shown in Fig. 237. there is an increase in the height and a diminution in the length of the curve. Above that tenuperature the height is climinisherl. The latent perinel is markeclly diminished by heat (from 3.5 seconds at $\mathrm{I}^{\circ} \mathrm{C}$. to $0^{\circ} 2$ seconds at $10^{\circ} \mathrm{C}$ ). (C. C. Stewart).

(c) Influence of I'revious Stimulation - Fatigue. If a muscle is stimulated hy a series of equal shocks thrown in at regular intervals, and the contractions recorded, it is seen that at first each curve orertops its $\overrightarrow{p r e d e c e s s o r ~ b y ~ a ~ s m a l l ~ a m o u n t . ~}$ This phenomenon, which is regularly observed in fresh

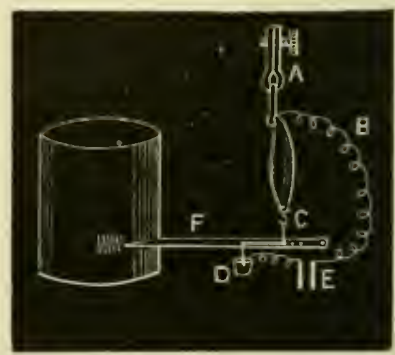

Ficr. 237.-Automatic Miscle: INTERRUPTOR.

A, femur with gastrocnemius at tached, supported in clamp; C, inetal ljook with fine wire attaclied to lever F. The wire is continued along the lever and connected with a sewing-needle, the point of which just dips into the mercury cup D. A wire from one pole of the Daniell cell E dips permanently into the mercury" : the wire $B$ from the other pole is attached to the upper end of the muscle or the clamp.

skeletal muscle (Fig. 238), although it was at one time supposed to be peculiarly a property of the muscle of the heart (Fig. 239), is called the 'staircase,' and seems to indicate that within limits 
the muscle is benefited hy contraction and its excitability increased for a new stimulus. Sonn, however, in an isolater preparation, the contractions begin to decline in height, till the muscle is at length utterly exhausted, and reacts no longer to even the strongest stimulation (Figs. $236,2+1,2+2$ ).

A conspicuous feature of the contraction-curves of fatigued muscle is the progressive lengthening, which is much more marked in the descending than in the ascending periods; in other words, relaxation becomes more and more difficult and imperfect (Fig. 2fI). In smooth muscle (cat's bladder or ring from frog's stomach) fatigue can be rery easily denonstrated in the same way, and the curves present

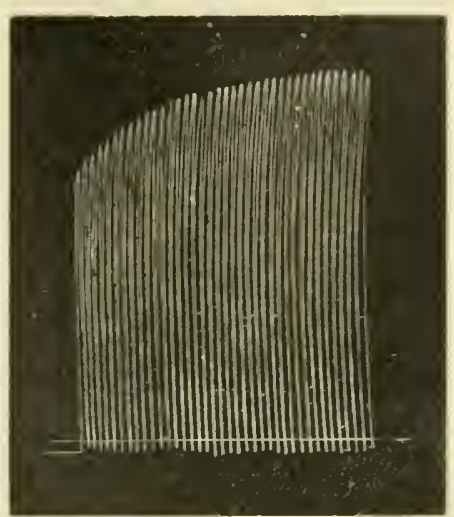

Fig. 238,- 'Staircase' ix Skeletal. Muscle : Frog.

Stimulation by arrangement shown in Fig. 237. similar features, with the exception that, instead of becoming longer in fatigue, the successive contractions become shorter.

It is by no means so easy to fatigue a muscle still in connection with the circulation as an isolated muscle. But even the latter, if left to itself, will to some extent recover, and be again

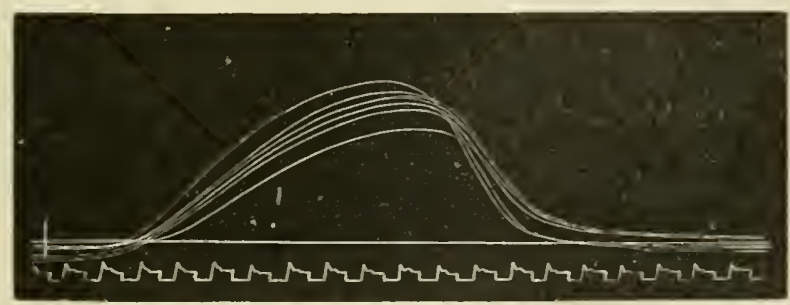

Fig. 239.--' Staircase' in Cardiac Muscle.

Contractions recorded on a much more quickly moving drum than in Fig. 238. The contractions were caused hy stimulating a heart reduced to standstill by the first Stannius' ligature (p. r5r). The contractions gradually increase in height.

able to contract, although exhaustion is now more readily induced than at first.

In man, muscular fatigue can be studied by means of an arrangement called an ergograph (Fig. 240). A record of successive contractions, say, of one of the flexor muscles of a finger, in raising a weight (iso-tonic method) or in deforming a spring (iso-metric metlod) is taken on a drum. When the contractions 
are repeated every second, or every half-second, distinct evidence of fatigne is seen on the tracing after a longer or shorter period, according to the conditions.

What is the cause of muscular fatigue? An exact answer is not possible in the present state of our knowledge, but we may fairly conclude that in an isolated preparation it is twofolel: (I) 'The material necessary for contraction is used up more guickly than it can be reproluced or brought to the jlace where it is required; (2) waste products are formed by the active muscle faster than they can be removed. That even an isolated muscle has a certain store of the materials needed for contraction which cannot be all cxhausted at once, or which can to a certain extent be replenished by processes going on in the muscle, is shown by the beneficial effect of mere rest. Among these

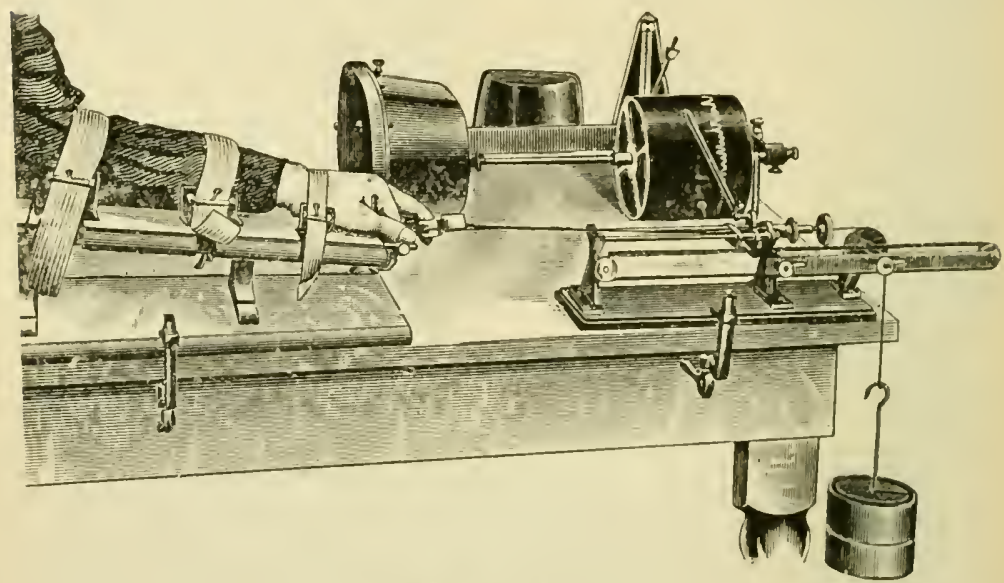

Fig. 240.-Ergograph (Mosso's, Modified by Lombard).

materials oxygen holds a conspicuous place. An isolated musclc is necessarily an asphyxiated muscle, and the favourable action of an atmosphere of oxygen on restoration of its contractile power after exhaustion (Fig. IIO, p. 26I) shows that asphyxia is itself an important condition in the onset of fatigue. Injection of arterial blond, or even of an oxidizing agent like potassium permanganate, into the ressels of an cxhausted muscle also causes restoration (Kronecker). The depletion of the available store of carbo-hydrate in the form of glycogen (and dextrose) scenus to be another factor. That the accumulation of fatiguc products lias something to do with the exhaustion is shown by the fact that the muscles of a frog, exhausted in spite of the continuance of the circulation, can be restored by blecling the animal, or washing out the vesscls with physiological salt solution, 
while injection of a watery extract of exhausted muscle into the bloodvessels of a curarized muscle renders it less excitable (Ranke). This observer supposed that it was specially the removal of the acid products of contraction which restored the muscle. Such acid products as carhon dioxide and lactic acid, when they act on muscle in more than a certain concentration, proluce the same effects on its power of contraction as are produced by fatigue. In smaller concentration, on the contrary, they increase the excitability of the muscle, and, according to I.ee, the phenomenon of the 'staircase' is due to the augmenting

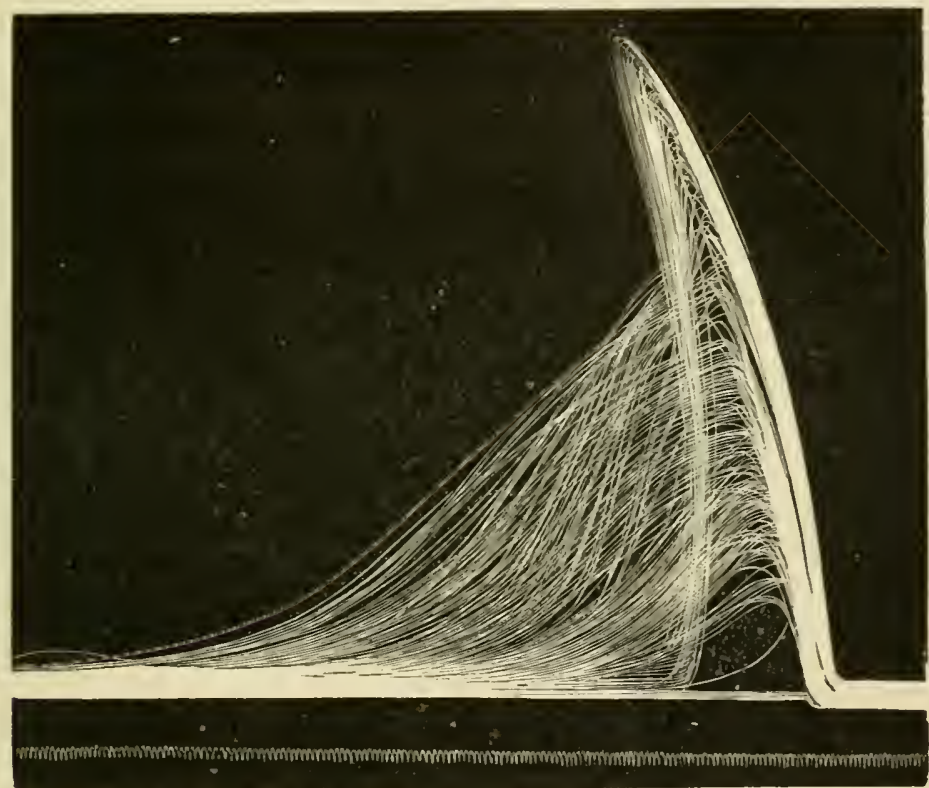

Fig, 24r.-Fatigte Curve of Skeletal Muscle: Gastrociemus of Frog.

Indirect stimulation; taken with arrangement shown in Fig. 26r (p. 707). Time tracing. $\frac{1}{10} \bar{v}$ of a second.

action of these, and perhaps other fatigue substances, before they have accumulated sufficiently to cause fatigue (p. 649).

Seat of Exhaustion in Fatigue.- When a fatigued muscle responds no longer to indirect stimulation, it can still be directly excited. The seat of exhaustion must therefore be either the nerve-trunk or the nerve-endings. It is not the nerve-trunk which is first fatigued, for this still shows the negative variation (p. 7Ig) on being excited. And if the two sciatic nerves of a frog or rabbit be stimulated continuously with interrupted currents of equal strength, while the excitation is prevented from reaching 
the muscles of one limb till those of the other cease to contract, it will be found that when the 'block' is removed the corresponding muscles contract vigorously on stimulation of their nerve. The passage of a constant current through a portion of the nerve or the application of ether between the point of stimulation and the muscles may be used to prevent the excitation from passing down (p. 70\$). Or a dose of curara just sufficient to paralyze the motor innervation may be given to a rabbit, and the animal kept alive hy artificial respiration. The sciatic is now stimulated for many hours. Nevertheless, as sonn at the influence of the curara begins to wear off, the muscles of the leg contract.

The possible seats of fatigue caused by voluntary muscular contraction are (I) the muscle; (2) the nerve-cndings (or the recep)-

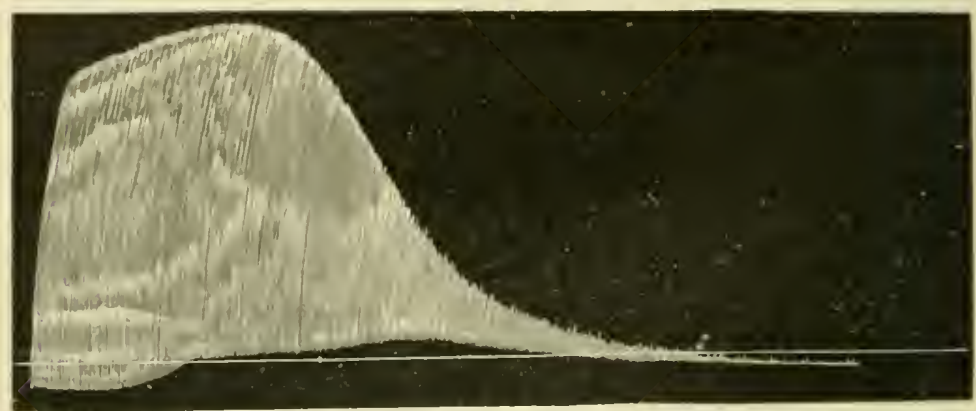

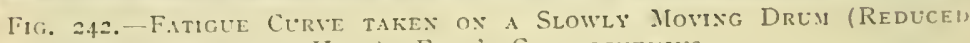
to HaLf) : Frog's (iastrocifmit's.

Excited through the sciatic nerve by maximal shocks unce in six seconds.

tive substances in the muscle, p. 63t); (3) the nerve-trunk : and (4) the path of the voluntary motor impulses in the central nervous sistem, which include's the pyramidal cells in the motor region of the cerebral cortex (p. 774 ), the fibres of the pyramidal tract, and the motor cells in the anterior horn of the spinal cord.

Althongh the matter cannot be considered definitely settled, ergographic experiments (Mosso and Mlaggiora, Lombard, etc.) (p. 700) have been interpreted as showing that fatigue after voluntary effort is due to central changes, and not entirely to changes in the muscles and nerves themselves. Thus, electrical stimulation, either of a 'tired' muscle or of its nerve, is readily responded to at a time when the weight cannot be raised by voluntary contraction. Now, it is argued, there is no reason to suppose that the nerve-fibres in the central nervous system differ cssentially from those of peripheral nerves, and thercfore no reason for placing the seat of the fatigue anywhere in the pyramidal fibres, 
except perhitps in their synapses (1). 775) in the cord, which correspond to the endings of the peripheral tibres in the muscles. That the synalpses easily lose their poiver of conducting nerve inpulses under the influence of repeated excitations is indicated by the experinents of Sherrington on fatigue of reflex mechanisms in which two or more afferent pathis can cause discharge along a common efferent path (p. Soo). When excitation of one of the afferent paths has ceased to be effective, the reflex contractions can still be obtained on exciting the other. In this case tle motor neuron from. cell-body to nerve-ending and the muscle are eliminated as the seats of the fatiguc block. Whether the temporary loss of conduction in this case is comparable to the fatigue of muscle, or is a perfectly different phenomenon ('pseudo-fatigue' of Lee), scarcely bears on our present question. For if 'pseudo-fatigue' of afferent synapses can cause a reflex to miss fire, this at least shows that the conductivity of the synapse is very easily affected by repeated excitation, just as it is known to be very easily affected by ancemia. The only other portions of the voluntary motor path besides these synapses that seem likely to become easily fatigued are the nerve-cells of the cortex and the

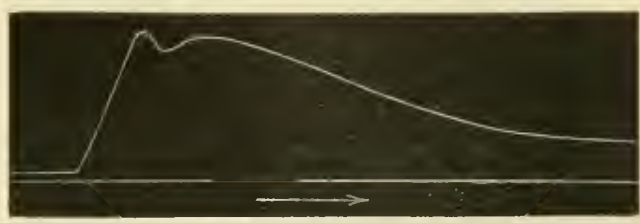

Fig. 243.-Veratrine CURve: Frog's Gastrocinemís.

The curve shows a peak, the lever falling a little before the sustained contraction begins.

cord. These central structures are usually considered the weakest links in the chain, the next weakest link being the motor endings in the muscles, and the strongest the nerve-fibres. The motor endings do not, in general, break down in voluntary contraction, because the central apparatus becomes sooner fatigued. It is not inconsistent with these facts that a muscle, fatigued by direct electrical stimulation, can still be voluntarily contracted. For the voluntary excitation may be more effective than any artificial stimulus.

It has been shown that the injection of the blood of an animal exhausted by running or other nuscular effort into the circulation of a normal animal produces in the latter all the symptoms of fatigue. Here the fatigue-producing substances will have the opportunity of acting on both the central and the peripheral mechanisms. There are reasons for believing that the fatigue process is fundamentally the same in different tissues. The fatigue substances produced in muscle, and not immediately eliminated or transformed during active muscular exertion, may 
therefore very well be a factor in inclucing fatigue of the central nervous mechanisms in addition to the formation of fatigue products, and the using up of necessary material in these mechanisms themselves. Contrariwise, active and long-continued mental exertion may occasion muscular fatigue (Fig. 244).

(d) The Influence of Drugs on the Contraction of Muscle. - The total work which a muscle can perform, its excitability and the absolute force of the contraction, may all be altered either in the plus or the minus sense by drugs. But in connection with our present subject those drugs which conspicuously alter the form and timerelations of the muscle-curve have most interest. Of these veratrine is especially important. When a small quantity of this substance
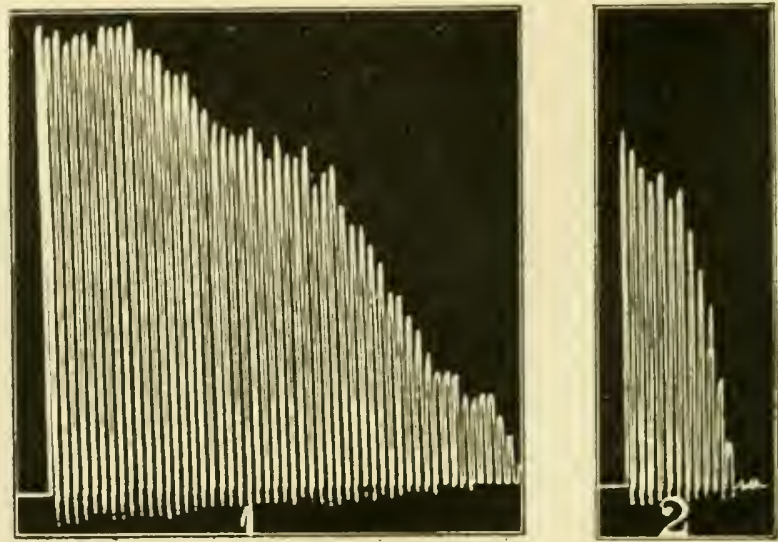

Fig. 244.-Influence of Mental Fatigue on Mescular Contraction.

I, series of contractions of flexors of middle finger before, and 2, series of contractions immediately after, a period of three and a half hours' hard mental work. In both cases the muscles werc stimulated directly every two seconds by an electrical current, and caused to raise a certain weight till temporary exhaustion occurred. In the first series fifty-three contractions were found possible, in the second only twelve (Maggiora).

is injected bclow the skin of a frog, spasms of the voluntary muscles, well marked in the limbs, come on in a few minutes. These are attended with great stiffness of movement, for while the aninal can contract the extensor muscles of its'legs so as to make a spring, they' relax very slowly, and some time clapses before it can spring again. If it be killed before the reflexes are completely gone, the peculiar alterations in the form of the muscle-curve caused by veratrine will be most marked. The poisoned muscle, stimulated directly or through its nerve, contracts as rapidly as a normal muscle, while the height of the curve is about the same, but the relaxation is enormously prolonged (Fig. 245). This effect scems to be to a considerable degree dependent on temperature, and it may temporarily disappear when the muscle is made to contract several times without pause. Adrenalin, barium salts, and, in a less degrec, those of strontium and 
calcium, have an action on muscle similar to that of veratrinc. Sometines the curve shows a peak (lig. 243), (lue to a rapid descent of the lever for a certain distance. This is followed by a slow relaxation. The peak appears to be analogous to the initial contraction when a strong voltaic current is passed througl a muscle, and the rest of the curve to the tonic contraction.

(c) The individuality of the muscle itself has an influence on the muscle-curve. Not only do the muscles of different animals vary in the rapiclity of contraction. but there are also differences in the slicletal muscles of the same animal.

In the rabbit there are two kinds of striped muscle, the red and the pale (the semitendinosus is a red. and the adeluctor magnus at pale muscle), and the contraction of the former is markedly slower than that of the latter. In many fishes and birds, and in some insects, a similar difference of colour and structure is present, although a physiological clistinction has not here been worker out.

Even where there is no distinct histological difference, there may be grcat variations in the length of contraction. In the frog, for

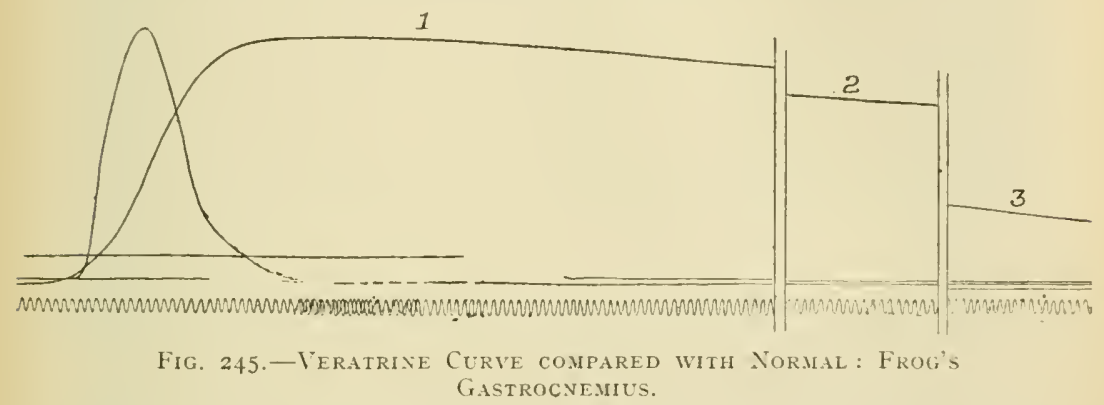

The tuning-fork narks hundredths of a second. Between I and 2 a portion of the tracing corresponding to one and a half seconds has been cut out, and between 2 and 3 a portion corresponding to one second. The veratrine curve does not show a peak. At 3 it has not yet fallen to the base-line.

instance, the hyoglossus muscle contracts much more slowly than the gastrocnemius. The wave of contraction, which in frogs' striped muscle lasts only about 0.07 sccond at any point, may last a second in the forceps muscle of the crayfish, though only half as long in the muscles of the tail. In the muscles of the tortoise the contraction is also very slow. The muscles of the arm of man contract more quickly than those of the leg.

Summation of Stimuli and Superposition of Contractions.Hitherto we have considered a single muscular contraction as arising from a single stimulus, and we have assumed that the muscle has completed its curve and come back to its original length before the next stimulus was thrown in. We have now to inquire what happens when a sccond stimulus acts upon the muscle during the contraction caused by a first stimulus, or during the latent period before the contraction has actually begun; and what happens when a whole series of rapidly-succeeding stimuli are thrown into the muscle.

First, let us take two stimuli separated by a smaller interval than the latent period (p. $6+2$ ). If they are both maximal-i.e., if each by itself would produce the greatest amount of contraction 
of which the muscle is capable when excited by a single stimulusthe sccond has no elfect whatever; the contraction is precisely the sime as if it hat never acted. But if they are less than maximal, the contraction. although it is a single contraction, is greater than would have been due to the first stimulus alone; in other words, the stimuli have been summed or adeled to each other during the latent period sor ats to produce a single result.

Next. let us consider the case of two stimuli separated by a greater interval than the latent period, so that the second falls into the muscle during the contraction produced by the first. The result here is very different: traces of two contractions appear upon the muscle-curve, the second curve being that which the second stimulus woukd have caused alone, but rising from the point which the first had reached at the moment of the second shock (Fig. 2.f6). Al-

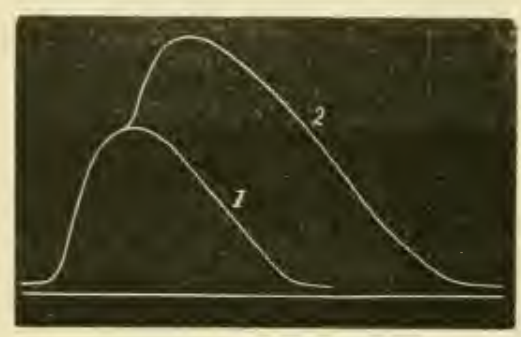

Fig. 246 .-StPerposition of Contrac. rioNs.

$I$ is the curve when only one stimulus is thrown in ; 2, when a second stimulus acts at the time when curve I has nearly reached its maximum height.

raction. No matter how rapidly they are thrown in. the muscle remains at rest. It is otherwise with smooth muscle. Stimuli which are singly ineficetive cause contraction when repeated.

Tetanus.-Not only may we have superposition or fusion of two contractions, but of an indefinite number; and a series of ripidly following stimuli causes complete tetamus of the muscle, which remains contracted during the stimulation, or till it is exhausted (Fig. 247).

The meaning of a complete tetanus is readily grasped if, beginning with a series of shocks of such rapidity that the muscle can just completely relax in the intervals between successive stimuli, we gradually increase the frequency (p. 7 I I). As this is done, the ripples on the curve become smaller and smaller, and at last fade out altogether. The maximum height of the contraction is greater than that produced by the strongest single stimulus ; and even after complete fusion has becu attained, a further increase of the frepuency of stimulation may cause the curve still to rise.

It is evident from what has been said that the frequency of 
stimmlation necessary for complete tetanus will depend upon the rapidity with which the muscle relaxes; and everything which diminishes this rapidity will lessen the necessary frequency of stimulation. A fatigued muscle may be tetanized by a smaller number of stimuli per second than a fresh muscle, and a cooled by a smaller number than a heated muscle. The striped muscles of insects, which can contract a million times in an hour, require joo stimuli per second for complete tetinus, those of hirds roo, of man fo, the torpid muscles of the tortoise only 3 . The pale muscles of the rabbit need 20 to fo excitations a second, the red muscles only io to 20 ; the tail muscles of the crayfish $f^{0}$, but the muscles of the claw only 6 in winter and 20 in summer.

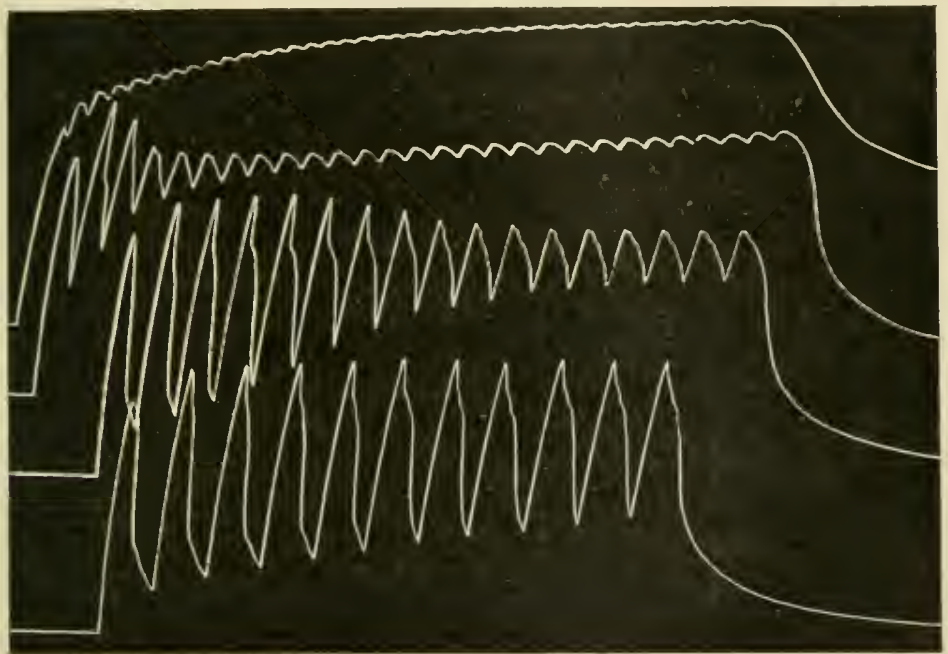

F1G. 247.- ANaly'sis of Electrical Tetanus (Reduced $10 \frac{2}{3}$ ).

Four curves showing the effect of increasing frequency of stimulation of the frog's gastrocnemius through its nerve. In the lowest curve the frequency is such that the muscle relaxes almost completely between the successive contractions. In the uppermost curve, with a frequency more than three times greater, the contractions are almost completely fused. In all the curves the fusion becomes more nearly complete as stimulation goes on, owing to the slower relaxation of the fatigued muscle.

The gastrocnemius of the frog requires 30 stimuli a second, the hyoglossus muscle only half that number (Richet). The frequency of stimulation necessary for complete tetanus of unstriped muscle is much less than for striped muscle. Smooth tetanus of a band of muscle from the frog's stomach was obtained with strong opening induction shocks at the rate of $I$ in 5 seconds.

We see, then, that there is a lower limit of frequency of stimulation below which a given muscle cannot be completely tetanized, 
and the guestion arises whether there is also an upper limit beyond which at series of stimuli becomes too rapiel to prosluce complete tetanus, or, indeed, to cause contraction at all. W'e may be ecrtain that every stimulus requires a finite time to produce an effeet, and it is possible that if the cluration of each shock were reducel below at cotan minimum, without lessening at the same time the interval between successive excitations, no contratetion would be camsed by iny or all of the stimuli in the series. But above this muimum there apparently lios a frequency of stimulation at least. when the interval between the stimuli is reluced exactly in the same proportion as the duration - at which an interupterl current comes to act like a comstamt current. causing it single twitch at its commencement or at its cond. but no contraction during its passage.

As to this last limit, on the fixing of which much labour has been expended. it undouletedly does not rlepend upon the frequency of stimulation alone; the intensity of the indivilual excitations, the temperature of the muscle, and probably other factors, affect it. for bermstein found that with moderate strength of stimulus

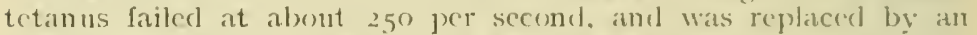
initial contraction; with strong stimuli at more thatu 1.700 per second. tetanus could still be obtaned. Kronecler and stirling, stimulating the muscle by induced currents set up in a coil by the longitulinal vibrations of a magnetized bar of iron. sall tcitanus even with the ntmost frepueney attainal)le, foroo shocks a second. according to koth; while 2 . Kries in a cooled muscle found tetanus replacer by the simple initial twitch at 100 stimuli per second. although in a muscle at $38^{\circ} \mathrm{C}$. stimulation of ten times this frequency still cinsed tetanus. Recently linthoven, exciting the nerve of a frog's nerve-muscle preparation with extremely frequent oscillatory condenser discharges, observed tetanus up to even al million vibrations a second, if the current intensity was at the same time very greatly increased (to more than 16,000 times the intensity needed with a constant current). These results are not really so cliscordant as they appear; for it is known that with elcctrical stimulation the number of excitations is not necessibrily the same as the nominal number of shocks. By applying a telephone to a muscle cxciter through its motor neric, it has been slown that the pitch of the nete produced by the tetanized muscle corresponds exactly to the: rate of excitation up to a certain frequency. "This frequency is about 200 per second for frog's and about 1,000 per sccond for mammalian muscle under the best conditions. If the rate of $\mathrm{cx}-$ citation is still further increased, there is no corresponding increase in the pitch. Therefore, some of the stimuli are now producing no effect - 'falling flat,' so to speak (Wedensky). (Mne reason for this is that even very brief currents leave alterations of conductivity and excitability behind them (Siwall). which we shall have to (liscuss in another chapter (p. 683). (Sec also p. 08, .)

It is only while the actual shortening is takmg place that a tetanized muscle can do cxternal work. But, although during the maintenance of the contraction no work is done, energy is nevertheless being expended. for the metaloolism of a muscle during tetanus is greater than during rest. Among other changes, the carbon dioxide given oft is increased. and lactic aciel produced. And upon the whole at muscle is more guickly cxhausted by tetanus than by successive single contractions, although there are great differences between different muscles. For cxample, the muscles which close the forceps of the crayfish or lobster have, as everyone kinows, the 
power of most obstimate contraction. lichet tetanized one for orer seventy minutes. and ancother for in lont and a half, before cxhaustion came on, while a tetanus of a single minute exhausted the muscles of the cribtish's tail. The gastrocnemius of a summer frog kept up for twelve minutes, and a tortoise muscle for forty minutes.

Contimunus stimulation is not always necessary for the production of continnons contration ; in some conclitions a singte stimulus is sufficient. A blow with a hard instrument may caluse a dying or cxhansted, and in thin persons eren a fairly normal, muscle to pass into lnng-continuel contraction. This so-called 'idio-muscular' contraction sccms to depend, in part at least, on the great intensity of the stimulus. It can semetimes be obtained in the frog's gastrocnemius, particularly in spring after the winter fast. It is not a tetanus and is not propagated along the muscular fibres, as an clectrical tetanus is. but remains localized at the spot where it arises. Similar non-tetanic contractions have already becn mentioned, such as the tonic contraction during the pissitge of a strong voltaic current and the sustained reritrine contraction. Ammonia causes also a long but non-tetanic contraction, and this, too, does not spread when the substance has acted only on a portion of the muscle. The contraction force of all these tonic contractions, as measured by the resistance necessury to overcome or prevent then, is less than the contraction force in clectrical tetanus (Schenck).

The rate at which the wave of muscular contraction tracls may be measured by stimuliting the muscle at ome end, and recording. by means of levers. the morements of two points of its surface as far apart from each other as possible. Time is marked on the tracing by means of a tuning-fork, and the distance between the points at which the two curves begin to rise from the basc-line divided by the time gives the velocity of the wave. Another method is fomded upon the measurement of the rate at which the negative rariation (p. $7(0)$ pisses over the muscle, this being the same as the velocity of the contraction-wave. In frog's muscle it is about three metres a second, or six miles an hour. Risc of temperature increases, ficll of temperature lessems it.

When a muscle is cxcited through its nerve, the contraction springs up first of all about the middle of each muscular fibre where the nerve-fibre cuters it, and then swecps ont in both directions towards the ends. But so long is the wave, that all parts of the fibre are at the same time involved in some phase or other of the contraction. And this is the case eren when the cnd of a long muscle like the sartorius is artificially stimulated.

The wave of contraction in unstriped muscle lasts a rclatively long time at any given point. and in tubes like the intestines and ureters, the walls of which are largely composed of smooth muscle arranged in rings, the wave shows itself as a gradually-adrancing constriction travelling from end to end of the organ. There is no evidence that the contraction of smooth muscular fibres is discontinuous - that is, composed of summated contractions like a tetanus ; it appears to be a greatly-prolonged simple contraction. An artificial stimulus, mechanical or clectrical, causes, after a long latent period, a very definitely-localized contraction in a rabbit's ureter, which slowly spreads in a peristaltic wave in one or both dircctions along the muscular tube. Here, as in the cardiac muscle. the cxcitation passes from fibre to fibre, while in striped skeletal muscle only the fibres excited directly or through their nerves contract. That the 
rlothmach contraction of the leart is not a tetanus has alrearly heen seen. It is a simple contraction, intermediate in its duration and other characters between the twitch of voluntary muscle and the contraction of smooth muscle. The contraction both of unstriped and of cardiate muscle is lengthenel anil made stronger by distension of the viscers in whose walls they uccur, just as a skeletal muscle contracts more powerfully against resistance.

Voluntary Contraction.-There is evirlence that the rolmmtarv contriction is a tetanus. One of the strongest buttresses of the thenry of natural tetanus has becin the muscle-sound, a lnw rumbling note which can be heard by listening with a stethoscupe ore's the contracting liceps, or, when all is still, by stopping the ears with the fingers and strongly contrating the masseter and the other muscles concerned in closing the jaws. Diserreded about ninety years agn, first h. Wollaston and then by Erman, llalf a century passed away before it was investigated more fully by Helmboltz. The later observer, contirming the results of his predecessors, put down the pitch of the sound at $3^{6}$ to fo vibrations per second. He found, lonwerere, that little vibrating reeds with a rate of oscillation of about Iy 5 per second were more affected when attached to muscle thrown into roluntare contraction. than these that vibrated at a smaller or a greater rate. He therefore concluclerl that the fundamental tone of the muscle corresponded to this frequency, although, since such a low mote is not easily appreciated, the somed actually heard was really its octave or first hamonic (p. 280).

The objection has been brought forwarel that the resonance tone of the ear also corresponds to a vibration frequency of 36 to to a second. In other words, this is the natural rate of swing of the elastic structures in the milllle ear. the rate they will most easily fall into if set moving by an irregular mixture of faint. low-pitched tones and noises. and not compelled to vibrate at some ot her rate by al distinct sommel of definite pitch. Now, this resonance tone might be elicited by a quivering muscle if. among many liverse rates of oscillation of flifferent portions of its substance, the rate of 36 to 40 a second anywhere appearel, and the note corresponding to the real rate of vibration of the muscle as a whole might be overpowered. Or. even if there were no regular rate of vibration of the whole muscle. but. insteal, it series of uregular tremors or pulls lue to irregularties in the contraction, connected with a want of co-ordination of all the fibres (Haycraft), the ear might from time to time pick out of the turmoil of fecble acrial waves those corresponding to its resonance tone. just as a tuning-fork or a piano-string attuned to a particular note would catch it up amid a thousand other sounds and strengthen it.

But while this renders it highly probable that the resonance of the ear contributes to the proluction of the muscle-sound, and shows that we camnot from the pitch of the muscle-sound alone

* In order that a muscular sound may be produced there must be a certain abruptness in the contraction. Thus, the slowly contracting smooth muscles do not produce a sound, nor the slowly-contracting heart-muscle of cold-blooded animals. 
derluce the rate at which the muscle-substance is vibrating, it loes not invalidate Ielmloltz's objective observations with the oscillating recds.

And several observers (Scläfer, Horsley, v. Kries) have noticed periodic oscillations, at the rate of ro or I $_{2}$ per second, in the

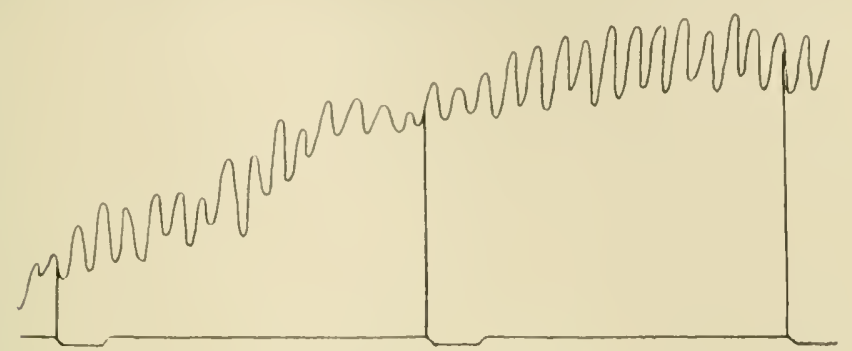

Fig. 248.-Vibrations of Contracted Arm Muscles (Griffitis).

The arm was stretched out, holding a weight of about 6 kilos.

curves taken from muscles (Fig. 248), contracted voluntarily against a snlall resistance. When the resistance is greater, the rate may be as mucl as $\mathrm{I} S$ or 20 a second, and in quick and rapidly repeated movements of the fingers even fo a second. In habitual movements, such as those employed by a man in his trade, the tremors are much less coarse than in unaccustomed movements. For this reason the trenors of the left hand are greater tlian those of the right in executing a morement usually made with the latter (Eshner). In disease these tremors are ofteu increased - e.g., in the clonic convulsions of epilepsy-but the

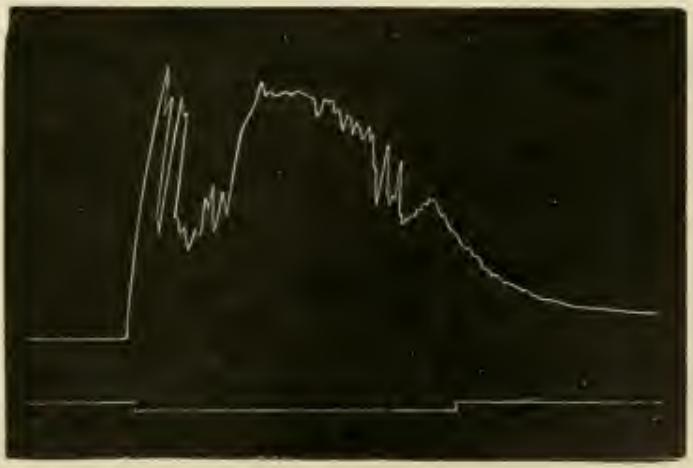

Fig. 249.-Contractions caused by Stimulation of TIIE SPINAL CORD.

frequency is the same. Similar ribrations, and at about the same rate, are seen in curves traced by muscles excited through stimulation of the motor areas of the surface of the brain. Since this rate remains the same whether the motor cortex, the corona radiata, or the spinal cord is excited, and, unlike the rate of response to excitation of peripheral nerves, is independent 
of the frequency of stimulation (so long as the rate of stimulation is greater than Io or I2 a second), it has been supposed to represent the rlythm with which impulses are clischarged from the motor cells of the cord (Fig. 249). It is probable that the cortical centres discharge at about the same rate, for not only is it impossible to articulate more rapidly than eleven syllables per second, but it is impossible to reproducs the act of articulation in thought at a greater rate than this (Riclict). But while this rate of ro or $\mathrm{I} 2$ a second does seem to represent a fundamental rhythm of the central discharge, there are facts which indicate that upon this relatively slow rlythm a quicker rlythm is superposed. In other words, each of tliese discliarges is itself discontinuous, and made up of a number of separate impulses.

Thus, according to Piper, the total number of simple discharges, each associated with an electrical change in the muscle, is 47 to 50 a second. The rhythm of strychnine tetanus in the frog is about 8 to 12 per second. By means of the capillary electrometer $(p, 021)$ large electrical oscillations at this rate can be ilemonstrated. each of which represents a short tetanic spasm, as is shown by the fact that a number of smaller electrical oscillations are superposed upon the large ones (Sanderson). The electrical changes suggest that cach discharge causes a simple contraction much more prolonged than the twitch of a directly stimulated muscle. This removes the difficulty of understanding how such a small number as io contractions per sccond could be smoothly fused, and indicates that even the shortest possible voluntary movement, which can be executed in I1 to til of a second, is not caused by a single impulse, but is a tetanus. For these brief movements the frequency of oscillation. as shown by the action currents, is the same as for sustained contractions. The electrical clianges in the voluntarily contracted muscle seem to differ in amplitude or abruptness from those proluced in experimental tetanus. For scondary tetanus (p. 730) is not caused by muscle in voluntary contraction. But this is also the case with the other prolongel contractions caused by continuous artificial stimulation-e.g.. Ritter's tetanus (p. 1136) and the contraction produced by sodim chlorile or ammoniat. We need not hesitate to conclude, then, that the voluntary contraction is discontinuous, in the sense that it is not a jerfectly smooth and uniform tonic contraction, althougl we still lack a lecisive proof that it is maintaied by a strictly intermittent outflow of nervous energy, and not by a contimuous outtlow causing a sustained contraction, which, it may be, remits and is reinforced at intervals. The apparent discrepancies as to the rate of discharge in the results obtained by different observers and by different methods, far from exciting distrust of them all, really lend support to the idea of a fundamental and fairly constant rlythm in the outflow as soon as it is recognised that the higher rates are approximately multiples of the lower. Thus, the number deduced by Helmholtz from the experiment of the springs is twice the lowest rate calculated from graphic records of the contraction. The rates corresponding to the muscle-sound and to the frequency of the electrical oscillations are about four times this number. Now, in a vibrating elastic body like a contracting muscle, a simple mathe- 
matical relation of this sort might be expected to appear when determinations of the rate of oscillation and of accompanying periodic changes are marle by methods varying in principle and in delicacy. For instince, an arrungement suited to record and to connt coarse ribration; could not be expecterl to give the sime result as an arrangement suited to record and count fine vibrations. But if both the coarse and the fine vibrations were related to a fundancintal rhythm, a simple proportion might be expected to cxist between the two sets of results.

\section{(3) Thermal Phenomena of the Muscular Contraction.-} When a muscle contracts its temperature rises; the production of heat in it is increased. This is most distinct when the muscle is tetanized, but lias also been proved for single contractions. The change of temperature can be detected by a delicate mercury or air thermometer ; and, indeed, a thermometer thrust among the thigh-muscles of a dog may rise as much as $\mathrm{I}^{\circ}$ to $2^{\circ} \mathrm{C}$. When the muscles are thrown into tetanus. In the isolated muscles of cold-blooded animals the increase of temperature is much less; and electrical methods, which are the most delicate at present known, have generally been used for its detection and measurement.

They depend either upon the fundamental fact of thermo-electricity, that in a circuit composed of two metals a current is set up if the junctions of the metals are at different temperatures; or upon the fact that the electrical resistance of a metallic conductor varies with its temperature. On the former principle the thermopile has been constructed (Fig. 250), on the latter the bolometer, or 'electricalresistance thermometer' (Fig. $25 \mathrm{I})$.

Where no very fune differences of temperature are to be measured. a single thermo-junction of German silver and iron, or copper and iron, is inserted into a muscle or between two muscles. But the electro-motive force, and therefore the strength of the thermoelectric current, is proportional for any given pair of metals to the number of junctions, and for delicate measurements it may be necessary to use several connected together in series. A thermopile of antimony-bismuth junctions gives a stronger current for a given difference of temperature than the same number of German silveriron couples, but from its brittle nature is otherwise less convenient.

The direction of the current in the circuit is such that it passes through the heated junction from bismuth to antimony and from copper or German silver to iron. Knowing this direction, we are aware of the changes of temperature which take place from the movements of the mirror of the galvanometer with which the pilc is connected. The galvanometer must be of low resistance, since the electromotive force of the thermo-electric currents is small. and a high resistance would cut down their intensity too much.

The muscle which is to be excited is brought into close contact with one junction or set of junctions, the other set being kept at constant temperature by immersing them in water, or covering them with muscle that is not to be stimulated. The image will now come to rest on the scale ; and excitation of the muscle will cause a morement indicating an increase of temperature in it, the amount of which can be calculated from the deflection. 
In this way Ifolmboltz observed a rise of temperature of $0.14^{\circ}$ to 0.18 C. in excised frogs' muscles when tetanized for a couple of minutes.

Heidembain, with a very delicalte pile, found a rise of $0.001^{\circ}$ to

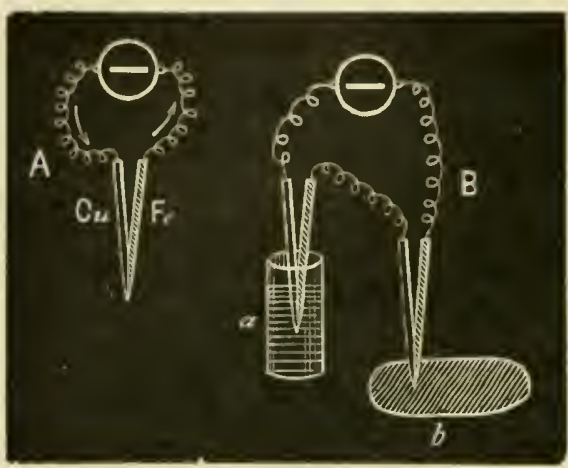

F1G. 250.

A, a single copper-iron therno-electric couple: $B, t w o$ pairs, one inserted into the tissue $b$, the other dipping into water in a beaker $u$. The lemperature of the water may be adjusted so that the galvanometer shows no deflertion. The temperature of the tissue is then the same as that of the waler.

It is certain that when work is clone by a muscle an equivalent amount is subtractel from its sum-totil of energy, and under proper conditions this can be actually demonstrated by the deficiency

As used for investigating heat. produrtion in mammalian nerses in silu. A, a piere of hard rubber in the hook-shaped part of which the fine platinum wire P' is lixed, and cowered with insulating sar. nish ; c, c, thick cepper wires connected with P, fastened in grooves, and covered with paraffin. Alrove they end in contact with the small binding posis, $\mathrm{B}_{1}, \mathrm{~B}_{2}$. $B$ is a hard rulber sliding piece. with a slot $s$. When $B$ is in position the screw, a, projerts through the slot. By a nut on this srrew $B$ is fixed on $A$ when the nerve has been arranged in the gromve. A similar larger arrange. ment ran be used for muscle. $0.005^{\circ} \mathrm{C}$. for a single contraction of a frog's muscle. On the assumption that the pile liad time to take on the temperature of the muscle before there was any appecialle losis of heat, this would he equal to the production by every gramme of muscle of a thousandth to five-tlousandths of at small calorie ( 1,574$)$ of leat. From lick's ol)servations we may take about three-thousandths of a small caloric as the maximum production of a gramme of frog's muscle in a single contraction.

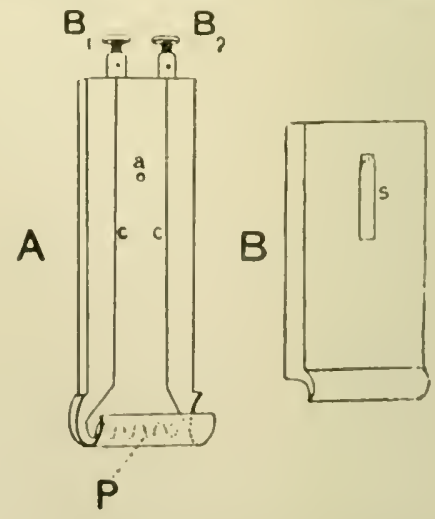

Fig. 25I.-Electrical-Rfistance Tuekmomfter (Natural Size).

in the heat-production. This is lone by means of a contrivance callew a work-adder. It consists of a wheel. the rotation of which raises a weight attached to a cord wound rouncl its axle. The 
muscle acts on the periplery of the wheel, and by rotating it raises 1he weight a little at ciuli contraction. At the end of the contraction the wheel is prevented from moving back by a catch The work clone in a series of contractions is calculated from the total height to which the weight has been raised. Suppose a frog's gastrocnemius is male to contract a certain number of times while attached to the work-adder, and that simultancously the heat procluction is measured by means of a thermopile. I et $H$ represent the licat actually produced. and $h$ the heat equivalent of the work clone. Now let the muscle be disconnected from the adder and made to raise the same weight, directly attached to it, by a series of contractions clicited in precisely the same way as the previous ones, except that the weight is allowed to fall with the muscle when it relaxes after each contraction. Here heat corresponding to the external work (lisappears from the muscle during the contraction just as in the furst experiment, but this licat is returned to the muscle during the relaxation, since on the whole no external work is done. The heat produced in the second experiment is found, as a matter of fact, allowing for unavoidable errors, to be equal to $I T+h$.

Here the assumption is made that the difference in the mechanical conditions during the relaxation (the muscle in the first experiment relaxing without load but being stretched by the weight as it relaxes in the second) cloes not affect the heat-production. This assumption has becn slown to be correct, althougl it was at one time supposed that clianges in the tension of the muscle produced during the relaxation dicl cause changes in the amount of heat produced. On the other hand, it has been clearly proved that the total energy transformed during the period of contraction, and the fraction of it which appears as extcrual muscular work, are greatly influenced by the mechanical conditions under which the contraction takes place. A stretched muscle, when caused to contract, produces more heat than if it had started withont tension, and still more heat when it is fixed so that it cannot shortcn during stimulation. A muscle, starting without tension, produces more heat when it contracts isometrically than when it contracts isotonically. This fact does not, however, prove that the heat-production is greater when $n 0$ work is clone, because the tension increases during excitation when contraction is prevented, and, as has been said, increase of tension alone causes more heat to be given out by an active muscle.

When a muscle, excited by maximal stimuli, is made to lift continuously increasing weights, both the work clone and the heat given out increase up to a certain limit. The muscle, as it were, burns the candle at both ends. This would be of itself enough to show that there is no fixed relation between the work and the heatproluction; although the latter reaches its maximum somewhat sooner than the former.

Although a loaded muscle kept in steady tetan ic contraction is doing no work, it proluces heat, but far less than would be produced if the muscle could fully contract and relax at each excitation. The amount of energy liberated by an excitation of given strength depends, therefore, on the mechanical conclition of the muscle into which it falls.

The fraction of the total energy transformed which appears as muscular work varies with the conditions of the contraction. The greater the resistance, so long as the muscle can overcome it so as to do its utmost amount of external work, * the larger

* This statement, based on experiments with excised frog's muscles, is not, of course, inconsistent with the fact mentioned on p. 583 , that in the 
is the proportion of energy which appears as work, the smaller the proportion which appears as heat. For every muscle, under given conditions, there is a certain load which can be raised more advantageously than any other; but even in the most favourable case, an excised frog's muscle never does work equal to more than $\frac{1}{\text { t }}$ of the heat given off. Generally the ratio is much less, and may sink as low as 's. In the intact mammalian body the muscles work somewhat more economically than the excised frog's muscles at their best; for both experiment and calculation show (p. $5^{83}$ ) that in a normal man under the most favourable conditions as much as one-third of the energy is converted into work. According to Zunt\% and hatzenstein, 35 per cent. of the total energy appeared as muscular work in climbing a mountain, and in bicycling only 25 per cent. Movements which have been much practised are more economically performed than unaccustomed ones, and this explains the superior efficiency of the muscles concerned in climbing, for no movements can possibly be more familiar than those concerned in locomotion. So far as this indication goes, it would seem that in the treatment of obesity unfamiliar, and therefore physiologically expensive, forms of exercise should be recommended, in so far, of course, as they do not injuriously react upon the general condition, especially upon the circulation.

As a muscle becomes fatigued it works more economically, the heat-production diminishing more rapidly than its working power. This is an illustration of the fact that the heat-producing mechanism and the work-producing mechanism of muscle are in some respects distinct, and a variation in the activity of the one is not necessarily associated with a corresponding variation in the activity of the other.

(4) Chemical Phenomena of the Muscular Contraction.-We know but little regarding the chemical composition of living muscle, since most chemical operations cause the immediate deatl of the tissue. The composition of dead mammaliun muscle of the striped variety may be stated, in round numbers, as follows, but there are considerable variations, even within the same species:

\begin{tabular}{|c|c|c|c|c|c|}
\hline ater & - & - & & & er cent \\
\hline $\begin{array}{l}\text { Protcins - } \\
\text { Fats, lecitlin, }\end{array}$ & - & - & 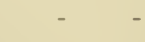 & 20 & , \\
\hline $\begin{array}{l}\text { Fats, lecitlin, } \\
\text { Nitrogenous }\end{array}$ & $\begin{array}{l}\text { and cholesterin } \\
\text { K'reatin }\left(\mathrm{O}_{2} 2\right.\end{array}$ & 2 to $0^{\circ}$ & & & \\
\hline $\begin{array}{l}\text { Nitrogenous } \\
\text { metabolites }\end{array}$ & $\left\{\begin{array}{l}\text { Janthin } \\
\text { Hypoxanthi }\end{array}\right.$ & & $\left\{\begin{array}{l}\text { Purin } \\
\text { bodies }\end{array}\right.$ & & \\
\hline Carbo-hydrate & s (glycogen, dext & trose, a & dimaltose) & & .. \\
\hline Lactic acid & - & - & . & & \\
\hline Inosit - & - & - & - & & \\
\hline
\end{tabular}

Salts, chicfly carbonate and pliosphate of potassium, less than I per cent. Potassium is absent from the nuclei (Frontispiece).

intact body the fraction of the energy transformed into heat is greater in hard than in moderate work. 
There is more water in the muscles of young than of old animals (v. Bibra), and more in tetanized than in rested muscle (Ranke). The fats belong to a small cxtent to the actual muscle-fibres. For even when the visible fat is separated with the utmost care, ncarly 1 per cent. of fat still remains (Stcil).

The glycogen content varies extremely in different muscles and in the same muscle under different nutritive and functional conditions. Thus, in one and the same log the biceps brachii contained $0^{\circ} 17$ and the quadriceps femoris $0^{\circ} 53$ per cent. In dogs on a dict rich in carbo-hydrate and protein the percentage in the wholc skcletal in usculature ranged from 0.7 to $3^{\circ} 7$, and in the heart from $0^{\circ}$ I to $I^{\prime} 2$. The average for human muscles has been given as $0^{+}+$per cent. In lean horse-flesli Pflüger found 0.3 .5 per cent. of glycogen, but no sugar. The total nitrogen was $3^{\circ}$ i per cent. of the moist tissuc. The lactic acid of muscle and other tissucs is the $d$-lactic acid, which rotates the plane of polarization to the right. By the action of certain bacteria on canc-sugar $l$-lactic acid is obtained, which is left rotatory. The optically inactive fermentation lactic acid is obtained by the fermentation of lactose.

Smooth muscle is somewhat richer in water than the striated variety from the same species, because skeletal muscle is richer in fat. Glycogen is either absent or present only in traces in the smooth muscle (of the stomach and bladder). Lactic acid, kreatin, and kreatinin are also found in much smaller amount than in striped muscle (Mendel and Saiki). As in striated muscle, hypoxanthin is the conspicuous purin base occurring in the free form-i.e., obtainable in muscle extracts. The most remarkable difference in the quantitative relations of the inorganic constituents is that in striated muscle potassium preponderates over sodium and magnesium over calciun, whereas in the smooth variety this relation is reversed.

It would be natural to expect that the proteins, which bulk so largely among the solids of the dead muscle, and which are so obviously important in the living muscle, should be affected by contraction. But up to the present time no quantitative difference in the proteins of resting and exhausted muscle has ever been made out. The quantity of kreatin (and kreatinin) is said by some authorities to be increased. The following chemical changes have been definitely established. In an active muscle-

(a) More carbon dioxide is produced.

(b) Morc oxygen is consumed.

(c) Lactic acid is formed.

(d) Glycogen is used up.

(e) The substances soluble in water diminish in amount; those soluble in alcohol increase.

That the carbon dioxide is not formed by direct oxidation, but by the splitting up of a substance or substances with which the oxygen has previously combined, is, as has already been shown (pp. 26r, 263), highly probable.

Formation of Lactic Acid-Reaction of Muscle.-To litmuspaper fresh muscle is amphicroic-that is, it turns red litmus blue and bluc litmus red. This is due, partly at least, to the phosphates. Monophosphate (tribasic phosphoric acid, $\mathrm{H}_{3} \mathrm{PO}_{t}$, in which one 
hyilrogen atom is replacerl, say, by sollium or potassium) redelens blue litmus, while diphosphate (where two hydrogen atoms are replaced) turns red litmus bluc. Litmoid (lacmoid) differs from litmus in not being affected by monophosphates. Diphosphates turn red litmoid blue, and so does fresh muscle, which has no effect on bluc litmoid. $\Delta$ cross-section of fresh muscle is about neutral (sometimes faintly acid) to turmeric paper. which is turned yellow by monophosphates. A muscle which has entered into rigor or has been fatigued by prolonged stimulation is distinctly acid to blue litmus and to brown turmeric, reddening the former and turning the latter yellow, but does not affect blue litmoid

Perfectly fresh resting muscle excised with aroidance of all unnecessary manipulation contains very little lactic acid (as little as 0.02 per cent. expressed as zinc lactate). Meclianical injury, heating, and chemical irritation cause a marked increase in the amount. Under anaerobic conditions-in an atmospliere of hydrogen, for instance-lactic acid is spontaneously dereloped in the resting muscle so long as irritability persists, but not longer. In air, which for even small excised muscles corresponds to a partial asphyxia, there is a small increase in the lactic acid, but its production is very slow in comparison with that in the hydrogen atmospliere. In pure oxygen not only is there no accumulation of lactic acid for a long time after excision, but is portion of the amount originally present in the resting excised muscle disappears. The same is true of the lactic acid formed in a muscle fatigued by stimulation when it is afterwards placed in an atmosphere of pure oxygen. There is no doubt that the production of lactic acid in functional activity and its transformation into other substances are processes that go on also in the muscles of the intact body. The formation of the acid in the excised muscle, far from being a sign of death, is an index of the 'survival' of a process by which it is normally formed, as the accumulation of it is an index of the crippling, in the absence of oxygen, of a mechanism by which it is normally transformed.

The lactic acid which accumulates in the excised muscle in rigor and activity does not remain free, since blue litmoid paper is not reddened as it would be by free lactic acid. It causes a repartition of the bases at the expense of the sodium carbonate and disodium phosphate, the latter being changed into monophosphate, which, in part at least, accounts for the acid reaction to turmeric (Röhmann). It is of great interest that this oxidative transformation of lactic acid only occurs in muscle whose structure is so far preserved that its irritability is not lost. In minced or triturated muscle it does not take place.

Glycogen is the one solid constituent of muscle which has been definitely proved to diminish during activity. It accumulates in a resting muscle, especially in a muscle whose motor 
nerve has been cut; but rapidly disappears from the muscles of an animal made to do work while fond is witheld; or from the muscles of an animal poisnned by strychnine, which causes violent muscular contractions.

What material is the lactic acid formed from? These are reasons for thinking that it is an intermediate substance which in metabolism may serve as a link between the products of protein decomposition and carbo-hydrates, and between carbohydrates and fat. From what we know of the production of lactic acid both outside the bodv and in the intestine from carbohydrates, it might seem a most plausible suggestion that in the active muscle it comes from glicogen. But the best evidence points the other way-c.g., in rigor mort is lactic acid is produced just as in muscular contraction. Nay, more, the amount of lactic acid (as much as $0^{\circ} 5$ per cent. expressed as zinc lactate) produced in full lieat rigor (at $40^{\circ}$ to $45^{\circ}$ C.) is constant for similar excised muscles. This 'acid-maximum' is the same when fresh muscle is at once put into rigor; or when fatigue is first induced, with formation of lactic acid, before rigor; or, finally, when the lactic acid of the fatigued muscle is caused to disappear under the influence of oxygen, and heat rigor is then brought about in the muscle (Fletcher and Hopkins). Yet in rigor mortis the quantity of glycogen is unaltered (Boehm). Further, under certain conditions an excised muscle is capable of producing a quantity of lactic acid much greater than could be derived from the glycogen contained in it. It is very possible that the lactic acid arises from protein in the transformation of the store of material whose decomposition is associated with the act of contraction. The facts just mentioned suggest that it is the same store which rields the lactic acid developed with the onset of rigor. But if this be so the transformation must be more complete in rigor than in the fatigue of excised muscles, since the amount of acid produced by severe direct stimulation of a muscle is not more than about one-half of that reached in full heat rigor.

Source of the Energy of Muscular Contraction.-The facts just mentioned show that glycogen may be one of the sources of muscular encrgy, but it cannot be the only source, for its amount is too small.

For example, the heart of an average man, which weighs 280 grammes, contains about 60 grammes of solids, and among these certainly not more than I gramme of glycogen. In twenty-four hours it produces 120 calories of heat (pp. $127,5 s_{4}$ ), equivalent to the complete combustion. of a little less than 30 grammes of glycogen. To supply this amount, the whole store of glycogen in the heart would have to be used and replaced every fifty minutes. But the accumulation of glycogen is immensely slower in the muscles of a 
rabbit inarle glycogen-free by strychnine, and therefore we have to lonk around for some other source of encrgy to supplement the glycogen. We have already brought forwarl evidence (p. 537) that, under ordinary circumstances, not a great deal, at any rate, of the cuergy of muscular contraction comes from the proteins. Of carbo-hydrates, the only one except glycogen which is at all arlequate to the task of supplying so unucl energy is the lextrose of the blond. The quantity of blood passing through the coronary circulation has been estimated at 30 c.c. per ron grammes of carliac muscle per minute (Bolar and Henriques), which would be equivalent for an average man to about i 20 litres in twenty-four hours. This quantity of blood will contain at least 120 grammes of dextrose, and about 32 grammes will suffice to supply all the heat produced by the heart. Of proteins a little less than 30 grammes would be necded. of fat a little more than 12 grammes. We sce. therefore, how intense must be the metabolism that goes on in an actively contracting muscle. On any probable assumption as to the source of muscular encrgy a quantity of material equal to half of its solids must be used up by the heart in twenty-four hours. Or, to put it in another way, the heart requires not less than two-fifths of its acight of ordinary solid food in a day. The body as a whole requires is to $:(1)$ of its weight.

To sum up: It is universally admitted that carbo-hyclrates can yicld energy for muscular work. It has been demonstrated by Zuntz and his pupils and by others that fat can do so. The experiments of Pflïger, to which we have already alluded (p. 538), have shown that when an animal is fed on lean meat, the muscular work done is far too great to have come from non-protein substances. We must conclude, therefore, that when carbohydrates and fats are plentiful in the food, the greater part of the energy of muscular contraction comes from them. It comes on the other hand from proteins, when the carbo-hydrates and the fats are restricted, and the proteins plentifully supplied. Not only so, but these three groups of fond substances yield muscular energy in isodynamic relation. In other words, a given amount of muscular work requires the expenditure of approximately the same quantity of chemical energy, whether it comes almost entirely from protein, or clicfly from carbolivdrates, or chiefly from fat. Some oloservers have stated that the taking of even a comparatively small quantity of sugar vastly increases the capacity for miscular work as measured by the ergograph (p. 6.49). The glycogen of the muscle is believed to be converted into dextrose during muscular activity. Dextrins and maltose, the intermediate products of this decomposition, liare becn detected in muscle, more maltose, indeed, than dextrose being present (Osborne), since the dextrose is rapidly oxidized. But although it is not to be doubted that sugar is under normal circumstances one of the most important substances used up in muscular contraction, the claim that 
sugar is, par cxcellence, the food for muscular exertion has not yet been made out.

Physico-chemical Conditions of Muscular Contraction.-For excised fresh muscle $\lrcorner\left(\right.$ p. 399) luas been estimaterl at $0.68^{\circ} \mathrm{C}$. But this is probably higher than in the living body, for after excision waste products, with their relatively small and numerous molecules. are still for a time prodnced. and are no longer removed by the blood. In salt solutions isotonic with the inuscle substance c.g., for the frog's gastrocnemius at room-temperature a $0^{\prime} 75$ per cent. solution of sodiun chloride-the resting muscle neither gains nor loses water for some hours. The active muscle behaves quite differently. When a muscle immersed in isotonic salt solution is tetanized, water enters it, leading to an increase in weight and a diminution in specific gravity (Ranke, Loeb, Barlow). The same occurs even when bloor is circulated throngh active muscles, the blood becoming poorer in water (Ranke). This may be explained by the increase of osmotic pressure in the muscle substance which must accompany the decomposition of large molecules into small. As fatigue progresses, a movement of water in the reverse direction occurs, and the muscle rapidly loses water. Exposure of the fatigued muscle for a sufficient time to an atmosphere of oxygen restores the osmotic properties of the resting muscle. Striking differences have also been demonstrated in the behaviour of resting and fatigned muscle to hypotonic solutions or water. Hales observed long ago that, on injecting large quantities of water into the bloodvessels of a dog, so as to replace the blood, marked swelling of the muscles occurred. This physiological fact is well known to the pork-butchers in China, who have given it a practical, if not a very praiseworthy, application in soplisticating their product by increasing its weight (MacGowan).

So long as the muscular fibres are uninjured they are permeable or impermeable for exactly the same compounds as other animal and vegetable cells. All substances casily soluble in media like ether or olive oil readily penetrate them (Overton). To most salts they are relatively impermeablc, as is shown by the fibres retaining their original volume in isotonic solutions of them. In particular, they cannot easily take up or retain the salts of the blood-plasma, otherwise the obscrved qualitative differences-e.g., the preponderance of potassium in the muscle and sodium in the plasma-could not be maintained. There are facts which indicate that temporary changes in the permeability to ions, not only of muscular fibres, but also of nerve fibres and other excitable structures, are concerned in their stimulation. Potassium salts after a time secm to produce an effect upon frog's muscle, which alters its permeability so that it takes up water from hypertonic solutions. Calcium salts have the opposite effect (Loeb). Sodium (and in a minor degree lithium) salts have a peculiar relation to the contraction of skeletal muscle, for which they appear to be indispensable. Yet sodium chloride produces a paralyzing action on the frog's motor nerve-endings, so that after perfusion with a solution of that salt stimulation of the motor nerve causes no contraction, or with a slighter degree of paralysis contraction only after a long interval. The effect can be counteracted by solutions containing calcium salts (Locke, Cushing).

Rigor Mortis.-When a muscle is dying, its excitability, after perhaps a temporary rise at the beginning, diminishes 
more and more motil it mlimatrly responds to mo stimulus. however strong. The loss of excitalility is not in itself a sure mark of death, for, as we have seen, an incxcilable muscle maty be partially or completely restored: but it is followed, or, where the death of the muscle tikes place very rapidly, perhaps accompanied, hy a more decisive crent, the appeitrance of rigor. The muscle, which was before soft and at the same time elastic to the tomch, becomes frrm; but its rasticity is gone. The fibres are no longer translucent, but oparpe and turbid. If shortening of the muscle has not been opposed, it may be somewhat contricted, although the absolute force of this contraction is small compared with that of a living muscle, and a slight resistance is enough to prevent it. The reaction is now distinctly acid to litmus. This is rigor mortis, the deatlestiffening of muscle.

An insight into the real meaning of this singular and sometimes sudden change was first given by the experiments of Kiilme. He took living frogr's muscle, freerl from blomel, froze it, and mincel it ju the frozen state. The pieces were then rubbed up in a mortar with snow contanining I pere cent. of commmom salt, and a thick neutral or alkaline lifuicl, the muscle-plasmat, was obtained hy filtration. This chotted into a jelly when the temperature was allowed to rise, lut at is ('. remained fludi. The clotting was accompanied by a change of reaction, the liquid becoming acid. An equally good, or better, methox is to use pressure for the extraction of the plasma from the froben fragments of muscle. A low temperature is essential, otherwise the plasma will coagulate rapidly within the injured muscle. A similan plasma can be expressed from the skedetal muscles of warmblooded animats (Halliburton), and witl greater difficulty from the heart.

When the muscle, after exhaustion with water. is covererl with a solution of a neutral salt, a 5 per cent. solution of magnesium sulphate or Io per cent. solution of ammonuim cliloride being the best. certain proteins are extracted which clot or are precipitated much in the same way as the muscle-plasma obtained by cold and pressure; and the process is hastened by liceping them at a temperature of to $\mathrm{C}$.

In the extracts of mammalian muscle three chicf proteins are present: paramyosinogen ( $v$. Fürth's myosin). coigulating by heat at $47^{\circ}$ to $50^{\circ} \mathrm{C}$.; myosinogen ( to $60^{\circ} \mathrm{C}$. (usually about $5^{\circ}$ ) ; and scrum-ilbumin, coagulating about $73^{\circ}$. There is reason to belicse that the sermm-albumin belongs to the blood and lymph, and is not a constituent of the muscle-fibre. In cxtracts of frog's muscle there is in acldition a substance which coagulates at about to ". Both the paramyosinogen and the myosinogen. but particularly the former. show a tendency, even at ordinary temperatures. to pass into an insoluble form myosin (v. Fürth's muscle fibrin). But whereas paramyosinogen passes directly into myosin, myosinogen is first clianged into a 
soluble modification (soluble inyosin). Which cragulates at fo C. and seems to be identical with the protein coigulating at that temperature which can be extracted from frog's muscles. At bodytemperature the transformation occurs more quickly. The myosin precipitate, which rapilly forms in muscle-plasma, is sometimes called the nascle-clot, and the liejuid which is left the muscle-serum. A similar myosin precipitate or clot seems to be formed in the interior of the muscular fibres in natural rigor and in the rapid rigor produced by leating a muscle to a little above the bodytemperature. But in natural rigor the whole of the paramyosinogen and myosinogen do not undergo the change. since a certain amount of these substances can as a rule be extracted from dead muscle by saline solutions. Thus, in rabbit's muscles, before the onset of rigor mortis, 87.3 per cent. of the total protein was found to be soluble in 10 per cent. ammonium chloride solution, and onły $12^{\circ} 7$ per cent. coagulated; while after rigor had occurred, $7 \mathrm{I}^{\prime} 5$ per cent. was coagulated, and only $2 S^{\circ} 5$ per cent. remained soluble (Saxl). It is not known whether in the living muscle paramyosinogen and myosinogen exist as such. Certain facts suggest that muscle contains only one protein (Mellanby). It has, indeed, been stated that if a tracing is taken from a muscle which is gradually heated, it first shortens at the temperature of coagulation of paramyosinogen. and then again at that of myosinogen, and that in frog's muscle there is an additional shortening at $40^{\circ}$. the temperature of coagulation of the soluble myosin. The conclusion has been drawn that these substances must be present as such in the living fibres, and that the successive shortenings are mechanical phenomena due to their heat coagulation. Similar shortenings have been described in nerve and liver tissue at about the temperatures at which the proteins in extracts of these tissues are coagulated by heat. But Meigs has shown that the supposed correspondence is far from being exact, and that muscles whose proteins have been already coagulated in a mixture of alcohol and salt solution still show the typical shortening on being heated. The heat shortening is, therefore, dependent on some other process than aggregation of the particles of coagulable protein.

It has been suggested that myosin (or its precursors), lactic acid, and carbon dioxide are all products of some complex body which breaks up both during contraction and at or before the death of the muscle, and that, indeed, contraction is only a transient and removable rigor (Hermann). But it cannot be admitted that there is any fundamental connection between rigor and contraction, although there are some superficial resemblances. In both there is (I) shortening; (2) heat-production; (3) formation of lactic acid and carbon dioxide; (4) electrical changes. Another analogy might be forced into the list by anyone who was determined to see only rigor in contraction: the rigor passes off as the contraction passes off. although the "resolution' of a rigid muscle takes days, the relaxation of an active muscle a fraction of a second. The disappearance of rigor is not dependent on putrefaction; it takes place when growth of bacteria is prevented'(Hermann). Possibly it is connected with autolytic processes "due to intracellular ferments (p. 509). 
Why does coagulation of myosin occur at the death of the muscle ? To this question no clear answer can be given. Some have looked on the process as analogous to the clotting of blood when it is shed, and it has even been suggesterl that just as a fibrin ferment is developed when the leucocytes and bloodplates begin to die, a myosin ferment, which aids coagulation. is developed in dead or dying muscle. But no clear profof has been given of the existence of such a ferment. And it is easy to make too much of the apparent analogy between the clotting of muscle and the clotting of blood, for there are differences as well as resemblances. For instance, the addition of potassium oxalate does not prevent coagulation of muscle extracts, as it does of blood and blood-plasma. The development of lactic acid in the muscle is not the primary cause of the coagulation which constitutes the essential feature of rigor mortis, although after rigor las occurred direct precipitation of hitherto unclotted muscle proteins may be induced by the acid, or the acid salts produced in its presence. Deficiency of oxygen is associated with the occurrence of rigor mortis, as it is with the accumulation of lactic acid, and a developing rigor can be abolished by oxygen. and its onset long or indefinitely delayed. W'hen strict asceptic technique is observed an excised sartorius muscle of the frog may remain irritable in sterile Ringer's solution, even without oxigenation, for as long as three weeks (Mines).

Various influences affect the onset of rigor. Fatigue hastens it ; heat has a similar effect ; the contact of caffeine, chloroform, and other drugs causes most pronounced and immediate rigor. Blood applied to the cross-section of a muscle first stimulates the fibres with which it is in contact. and then renders them rigid. But it is to be remembered that normally the blond does not come into direct contact even with the sarcolemma. much less with its contents.

The effect of heat is of special interest. A skeletal muscle of a frog, like the gastrocnemius, if dipped into physiological saline solution at $40^{\circ}$ or $4 \mathrm{I}^{\circ} \mathrm{C}$. goes into rigor at once ; the frog's heart requires a temperature $3^{\circ}$ or $4^{\circ}$ higher; the distended bulbus aorte can withstand even a temperature of $48^{\circ}$ for a short time. An excised mammalian muscle passes into immediate rigor at $45^{\circ}$ to $50^{\circ}$. In heat rigor the reaction of the muscle becomes strongly acid owing to the formation of lactic acid, and the production of carbon dioxide is also increased. Heat rigor resembles in these respects a greatly accelerated rigor mortis. A small quantity of heat is produced, and the temperature of the muscle may be raised as much as $?_{20}^{\circ} \mathrm{C}$. This is probably due chiefly to the increased chemical change, and only to a slight extent to the physical alteration in the myosin. 
When muscle is at once raised to a temperature of $75^{\circ}$ to $\mathrm{ro0}{ }^{\circ} \mathrm{C}$., and all the proteins thus coagulated by heat, there is also an increase in the discharge of carbon dioxide (Fletcher), and the reaction becomes distinctly acid to blue litmus, although still alkaline to red. This is the easiest way of obtaining a maximum evolution of carbon clioxicle from an excised nuscle. It is highly improbable that a marked production of carbon dioxice sliould take place in heat-coagulated proteins. We must therefore suppose that the characteristic decomposition associated with rigor mortis can complete itself in the brief space that elapses between the application of the heat and the heat-coagulation of the proteins. This decomposition is wanting in the so-called rigor caused by water, which is not a true rigor, and causes no increase in the carbon dioxide given off. Chloroform, on the other hand, produces a marked increase in the carbon dioxide production, and this is evidently related to its action in hastening the onset of rigor. The process is to some extent influenced by the nervous systen1, for section of its nerves retards the onset of rigor in the muscles of a limb. This and other facts have given rise to the idea that the rigor is initiated by something analogous to a muscular spasm. Ante-mortem stimulation of the peripheral ends of the vagi, even with currents too weak to cause a perceptible effect "pon the heart-beats, prolongs the period of spontaneous contraction and the irritability of the ventricles after death, and retards the onset of rigor (Joseph and Meltzer). Cold rigor is obtained when frog's muscles are cooled to $-15^{\circ} \mathrm{C}$. The muscles remain perfectly translucent. They do not recover their irritability on thawing, but if cooled only to $-7^{\circ} \mathrm{C}$. they recover (Folin).

In a human body rigor generally appears not earlier than an hour, and not later than four or five hours, after death. In exceptional cases, however, it may come on at once, and the annals of war and crime contain instances where a man has been found after death still holding with a firm grip the weapon with which he had fought, or which had been thrust into his hand by his murderer. It is related that after one of the battles of the American Revolutionary War some of the dead were found with one eye open and the other closed as in the act of taking aim. A high temperature favours a rapid onset ; a body wrapped up in bed will, other things being equal, become rigid sooner than a body lying stripped in a field. Muscular exhaustion, as we have said, is another favouring condition: hunted animals and the victims of wasting diseases go quickly into rigor. It is a rule, but not an invariable one, that rigor, when it comes on quickly, is short, and lasts longer when it comes on late. All the muscles of the body do not stiffen at the same time; the order 
is usually from above downwards, beginning at the jaws and neck, then reaching the arms, and finally the legs. After two or three days the rigor disappears in the same order. The position of the limbs in rigor is the same as at death ; the muscles stiffen without any marked contraction. This can be strikingly shown on a newly-killed animal by cutting the tendons of the extensors of one foot and the flexors of the other; when natural rigor comes on, the feet remain just as they were. If heat rigor, however, is caused, the one foot becomes rigid in flexion and the other in extension; and the contraction-force is considerable, although not so great as that of an electrical tetanus in a living mucsle.

The Removability of Rigor.-After interrupting the circulation in the hind-legs of rabbits by compression or ligation of the abdominal aorta (Stenson's experiment), and so causing the muscles to become rigid, Brown-Séquard saw them recover their irritability when the blood was again allowed to reach them. He performed a similar experiment with artifrcial circulation through the hand of an executed criminal, with a like result. But most writers have taken the view that rigor is the irrevocable end of excitability, and that the apparent recovery which Brown-Séquard saw was due to the muscles not having been completely rigid. Heubel has, however, stated that rhythmical contractions of the frog's heart can be restored by filling its cavity with blood, after rigor has been caused by heat and in other ways, and we have already seen that the same is true of the mammalian heart after the onset of rigor. Excised frog's muscles which have undergone rigor mortis become less stiff when exposed to an atmosphere of oxygen. Both mammalian and frog's skeletal muscles, after rigor mortis has come on, are said to regain their excitability in physiological salt solution (Mangold). 


\section{CHAPTER X}

\section{NERVE}

THE voluntary movements are originated by efferent or outgoin,, impulses from the brain, which reach the muscles along their motor nerves. The involuntary movements and the secretions are in many cases able to go on in the absence of central connections, but are normally under central control. Afferent impulses are continually ascending to the cord and brain from the skin, joints, bones, muscles, and organs of special sense like the eye and the ear. Everywhere the connection between the nervous centres and the peripheral organs, and between different parts of the central nervous system, is made by nerve-fibres. Those which run outside the brain and cord are called peripheral nerve-fibres to distinguish them from the intracentral fibres of the central nervous system itself.

In this chapter we propose to consider certain of the general properties of nerve-fibres. Most of our knowledge of these properties has been derived from experiments on the peripheral, and particularly the peripheral motor nerves; but there is every reason to believe that the main results are true of all nervefibres, afferent and efferent, peripheral and central.

What we call nerve-fibres were known and named, and many important facts in their physiology discovered, long before their true morphological significance was recognised. The researches of recent years have shown that every nerve-fibre is, as regards its essential constituent the axis-cylinder, a process of a nerve-cell. The nerve-cells, each of which, including all its processes, may be conveniently termed a neuron, are the essential elements of the nervous system. The cell-bodies of most of the neurons are situated in, or in close relation to, the spinal cord and the brain, and therefore the detailed description of them will be reserved till we come to treat of the central nervous system (see p. 748 and Figs. 300 to 3 I I ). It is enough to say here that in general a nerve-cell gives off two kinds of processes: (I) one or more dendrites or protoplasmic processes, which repeatedly bifurcate like the branches of a tree into thinner and thinner twigs, and extend only for a relatively short distance from the cell-body; (2) an axis-cylinder process or axon, 
which as a rule runs for a considerable rlistance withrut altering its calibre, and either gives off no branches (as in tine peripheral nerves) or only a comparatively small number of lateral twigs (collaterals). Ultimately the axis-cylinder process and its collaterals, if it has any, end by breaking up into a brush, a plexus or a feltwork or basketwork of fibrils. The axons of different nervecells vary great!y in length. Some terminate within the grey matter of the brain or spinal cord not far from their origin; others run in the white tracts of the central nervous system or in the peripher.l nerves for half the height of a man. All except the shortest axis-cylindrar processes become clothed at a little distance from the cell-body with a protective covering, which contiuues to invest them (and their ccllaterals) throughout the rest of their course, disippeiring only when they begin to break up at their terminttions. In axiscylinder process (spoken of simply as the axis-cylinder, when considered apart from the nerve-cell) constitutes, with its covering, at nerve-fibre.

An ordinary peripheral nerve like the sciatic is marle up of a number of bundles of nerve-fibres. Connestive tissuc surrouncl.s and separates the bundles, and also peantrates in fine septa within them and between the individual fibres, forming a framework for their support. and carrving the bloodvessels and lymphatics.

The great majority of the nerve-fibres of the sciatic consist of axiscylinders covered by two sheaths. The axis-cylinders are pronesses of nerve-cells in the anterior horn of the spinal cord in the case of the motor fibres, and of nerve-celts in the spinal ganglia in the ise of the sensory. The axis-cylinder is the essential conducting p.urt of the fibre, for it is present in every nerve-fibre, ruming from end to end of it without break, and towards the periphery it is alone present. It is made up of fine longiturlinal fibrils cmberlded in interstitial substance (Fig. 30I, p. 74 s). Such i fibrillar structure is best shown after treatment of the nervefibres with certitin reagents, although it is certain that it exists preformed in the living fibres. The innermost (Fig. 2,6), and by far the thickest, of the sherths is the medullary sheath, or white substance of Schwann, which is of fatty nature, and is blackened by osmic acicl. It undergoes a kind of coagulation at death, loses its homogeneity, and shows a double contour. This sheath is not continuous, but is broken by constrictions of the outer sheath, called nodes of Ranvier, into numerous serments. The outer sheath, or neurilemma, is a thin, structureless envelope immediately external to the medulla. It invests the nerve-fibre, as the sarcolemma docs the muscle-fibre. In eich internodal segment immediately under the neurilemma lies a nucleus surrounded by a little protoplasm. Fibres with a medulliry shoith such as those described are called medullated fibres. They are by far the most numerous in the cereoro-spinal nerves ; but they are mised with a few fibres which contain no white substance of Schwamn, and are, therefore, called non-medullated. In these the axis-cylinder is covered only by the neurilemm 2 . In the svmpathetic system the non-medullated varicty is present in greater abund ize thin the medullated. In the ce.itral nervolis system the medull ited fibres possess no neurilenm?.

So far as we know, the only function of nerve-fibres is to conduct impulses from nerve-centres to peripheral organs, or from peripheral organs to nerve-centres, or from one nerve- 
centre to another. And in the normal body these impulses never, or only very rarely, originate in the course of the nervefibres ; they are set up either at their peripheral or at their central endings. By artificial stimulation, however, a nerve-impulse may be started at any part of a fibre, just as a telegrani may be despatched by tapping any part of a telegraph wire, although it is usually sent from one fixed station to another.

\section{The Nerve-impulse : its Initiation and Conduction.}

What the nerve-impulse actually consists in we do not know. All we know is that a change of some kind, of which the only external token is an electrical change, passes over the nerve with a measurable velocity, and gives tidings of itself, if it is travelling along efferent fibres - that is, out from the central nervous system-by the contraction or inhibition of muscle or by secretion; if it is travelling along afferent fibres-that is, up to the central nervous system-by sensation, or by reflex muscular or glandular effects.

Whether the wave which passes along the nerve is a wave of chemical change (such, to take a very crude example, as runs along a train of gunpowder when it is fired at one end), or a wave of mechanical change, a peculiar and most delicate molecular shiver, if we mav so phrase it, or a shear in a definite direction along the colloidal substance of the axis-cylinder (Sutherland), there is no absolutely definite experimental evidence to decide. An electrical change accompanies the nerve-impulse travelling at the same rate, and although this is to be distinguished from the impulse itself, there is little doubt that the latter is essentially connected with a disturbance of the electrical equilibrium of the nerve-substance.

An attempt has been made to settle the question by determining the temperature coefficient of the velocity of conduction of the impulse-i.e.. the quantity which measures the change of velocity for a given change of temperature. For most physical processes the quotient velocity at $\mathrm{T} n+\mathrm{IO}$, where $\mathrm{T} n$ is any given temperature, is not greater than $\mathrm{I}^{\prime} 2$, while for frog's sciatic nerve the temperature coefficient for the most part lies between 2 and 3 (Snyder). The mean value of a large number of observations is $\mathrm{r}^{\cdot} 79$, with $\mathrm{T} n=8^{\circ}$ to $9^{\circ} \mathrm{C}$. (Lucas). For the pedal nerve of the giant slug the mean value of the temperature coefficient is $\mathrm{I}^{\prime} 78$ (Maxwell). In other words, while for most physical processes an increase of $10^{\circ} \mathrm{C}$. increases the velocity of the process by at most one-fifth, the same increase of temperature increases the velocity of conduction of the nerve-impulse by four-fifths, or even more. While it is true that it may not be entirely safe to apply such a criterion to a biological 
process which need not be either entirely chemical or entirely physical, and very likely is a complex one, the suggestiom, so far ats it eroes. is undoubtedly in farour of the chemical hypothesis.

That chemical changes go on in living nerve we need not liesitate to assume; and, indeed, if the circulation throngh a limb of a warm-blooded animal be stopped for a sloot time, the nerves lose their excitability. But the metabolism is very slight compared with that in muscle or gland. Even in active nerve no measurable production of carbon dioxide has ever been observed, nor, in fact. has any chemical difference between the excited and the resting state ever been unequivocally made out. Neither in cold-blooled nor in mammalian nerves is there any sensible rise of temperalure during stimulation. It has already been stated that, moler orelinary conditions, nerve-fibres are practically insusceptible of fatigue, and this lias been considered a strong support of the pliysical nature of the conduction process. Nevertheless, it is possible to show by special methods that nerve can be temporarily fatigued, although it recovers very rapidly. When a medullated nerve is stimulated, a brief period ensues during which it refuses to respond to a sceonel stimulus. This refractory period is normally very short - not more than $0^{\circ} 002$ second for the frog's sciatic. But it cin be creatly prolonged by cold, asphyxia, or anaesthesia, especially by the alkaloid yolimbine (Tait and Crunn), and when the refractory period is thus prolonged, fatigue phenomena are readily induced by stimulation.

Stimulation of Nerve.-With some differences, the sanc stimuli are effective for nerve as for muscle (1. 632) ; but chemical stimulation is not in general so easily obtained. The so-called thermal stimulation is not a real stimulation due to the sudden change of temperature. The irregular contractions of the muscle caused by the local application of heat to the nerve are dependent on desiccation of the nerve.

Chemical Stimulation.-When hyper- or hypotonic solutions are employed, the withdrawal or entrance of water may be an important factor:

For salts which penetrate the fibres with equal difficulty this factor can be climinated by applying them as isotonic solutions. There is evidence that chemical stimulation proper, as distinguished from the stimulation produced by changes in the water content of the fibres by osmosis, is connected with the electrical charges on the dissociated ions of the salts $(p .400)$. Electrical stimulation, indeed, may only be a variety of chemical stimulation (Locb. Nathews, etc.).

Mechanical stimulation may be applied to a nerve by allowing a small weight to fall on it from a definite height or permitting mercury to (lrop upon it from a vessel with a fine outflow tube. A regular tetanus may thus be obtained. Tigerstedt found that the smallest amount of work spent on a frog's nerve which would suffice to excite it was a little less than a gramme-millimetre-that is, the work clone by a gramme falling through a distance of a millimetre, or (taking an erg as equivalent to 1 ino gramme-centimetre) about 100 ergs. No doubt a great part of this is wasted. as a much smaller quantity of work done by a beam of light on the retina or by an electrical current on an isolated nerve, both of which may be supposed to act more directly on the excitable constituents, suffices to cause stimu- 
latum. Thus, the work lone by the minimal, nalural or specific, stimulus for the retina in the form of green light maty be ats little is

${ }_{10}^{1} \operatorname{erg}$ (S. P. Langley). or only one-ten-thousand-millionth part of

the minimmm work necessary for mechanical stimulation. Again, with electrical stimulation (closure of a voltaic current, or condenser

(lischarges) it has becn shown that an amount of work equal to 1 erg may be enongh to cause excitation of a frog's nerve. This is ten thousand times as great as the minimal luminous stimnlus, but a million times less than the minimal mechanical stimulus.

The laws of electrical stimulation for nerve are essentially the same as those we have already discissed for muscle (p. 636). The voltaic current stimulates a nerve, as it does a muscle, at closure and opening. During the flow of the current, so long as its intensity remains constant. there is, as a rule, no excitation, or at least none which is propagated along the nerve. so that the muscles supplied by it remain uncontracted. But under certain conditions-for cxample, when the nerve is more excitable than usual (as is the casc with nerves taken from frogs which have been long kept in the cold) -a closing tetanus may be seen while the current continues to pass through the nerve, and an opening tetanus after it has ceased to flow, just as when the current is led directly through the muscle. Sensory nerve-fibres, too, are stimulated by a ioltaic current during the whole time of flow. Induction shocks are relatively more powerful stimuli for nerve than the make or break of a voltaic current. The opposite. as we have seen, is true of muscle; and, upon the whole, we may say that muscle is more sluggish in its response to stimuli, and is excited less easily by very brief currents, than nerve is. An apparent illustration of this difference is the fact that the nervous excitation has no measurable latent period. while muscular excitation has. But it is quite possible that, if the conditions of experiment were as favourable in nerve as in muscle, a sensible latent period might be found here too.

In nerve as in muscle, strength of stimulus and intensity of response correspond within a fairly wide range, when we take the height of the muscular contraction or the amount of the negative variation (p. 719) as the measure of the nervous excitation. Summation of stimuli, superposition of contractions, and complete tetanus, are caused by stimulating a muscle through its nerve, just as by stimulating the muscle itself (p. 655).

Excitability of Nerve.-It has usually been stated that the excitability of frog's nerve, as measured by the muscular response to stimulation, is increased by rise of temperature, and diminished by fall of temperature. It has, however, been shown that this increase of excitability is only apparent, and due to the strengthening of the current by diminution of the resistance, since the resistance of all animal tissues, like that of electrolytic conductors in general, diminishes as the temperature rises (Gotch). When precautions are taken to keep the current intensity the same at the various temperatures compared, it is found that cooling of a (frog's) nerve, even to $5^{\circ} \mathrm{C}$., increases the excitability for currents of long duration (several hundredths of a second). It has, indeed. 
been shown both for nuscle and for nerve that the cooler tissue repuire's a smaller current strength for its excitation when the current is of long duration. With brief currents this effect is masked, either partially or completely, by the greater increase of current strength needed in the case of the cooler tirsue to corn-

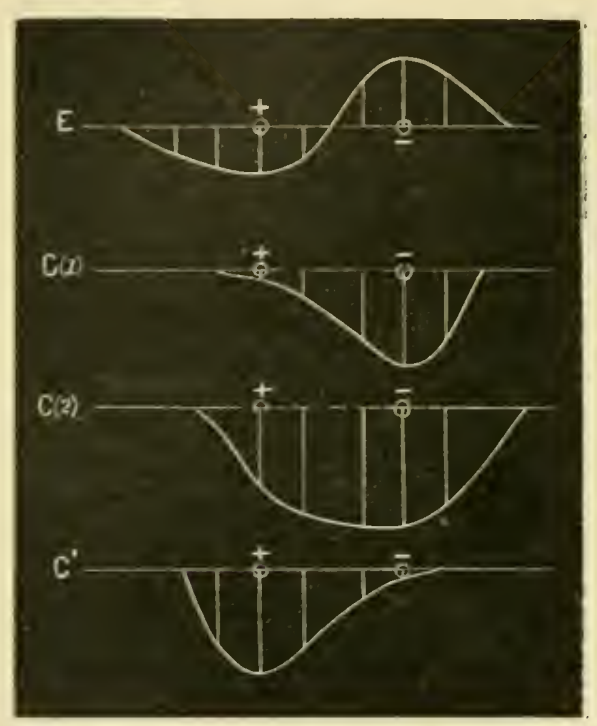

FIG, 252. IMAGRAM OF CHANGES OF IXCIT. ABILITY AND CONDICTIVITY PRODLCED IX A Nerie BY a Voltaic ClirRe.it.

E, rhanges of excitability during the flow if the current, arcording in Ptiger. The ordinates drawn from the abscissa axis to cut the curve represent the amount of the change. $C(r)$, changes of conductivity during the flow of a moderatrly strong current. Conductivitv greatly redured around kathode; little affected at annde. $\mathrm{C}(2)$, changes of conductivity during flow of a very strung current. Conductivity redured buth in anodic and kathodic regions, but less in the former. $C^{\prime}$, rhanges of cunductivity just afcer opening a moderately strong current. Conductivity greatly reduced in regun whirh was formerly anodic: little affected in region inrmerly kathodir. nearer to the muscle-it is applied. On this was based the now abandoned ' avalanche thenry,' according to which the impulse continually unlocked new energy as it passed along the nerve, and so gathered strength in its course like an avalanche. It is now known that no material change takes place in the intensity of the excitation while it is being propagated along a normal un- 
injured nerve. For instance, experiments on the plorenic nerve, in its naturat position, and with all its connections intact, have shown that with a given strength of stimulus the amount of contraction of the diaplnagm is the same whether the nerve be excited in the upper, middle, or lower portion of its course. In the above experiment on the isolitted, and therefore injured, nerve, the contraction varies in height with the distance of the point of stimulation from the muscle, not because the excitation grows as it travels, but becanse it is atready greater at the moment when it sets ont from a point near the central end of the nerve than at the moment when it sets out from a point near the muscle.

Electrotonus. - Although the constant current does not, unless it is very strong or the nerve very irritable, cause stimulation

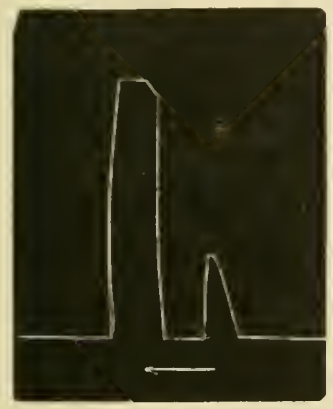

Fir. 253.-KATELECTROTONt:

Weak tetanus of muscle (the right-liand elevation), greatly in. tensified in katelectrotonus of the inotor nerve (the left-hand elevation).

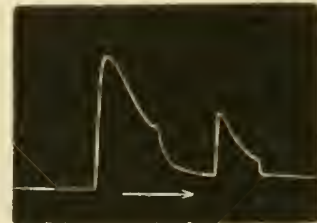

Fig, 25.-ANELECTROTONI'S.

Strong tetanus of muscle (lefthand elevation), lessened in strength by anelectrotonic con. dition of the notor nerve (righthand elevation).

during its passage, it modifies profoundly the excitability and conductivity of the nerve. In the neighbourhood of the kathode the excitability is increased (condition of katelectrotonus), while around the anode it is diminished (anelectrotonus). Immediately after the opening of the current these relations are for a brief time reversed, the excitability of the post-kathodic area (area which was at the kathode during the flow) being diminished, and that of the post-anodic increased. In the intrapolar area there is one point the excitability of which is not altered. This indifferent point, as it is called, shifts its position when the intensity of the current is varied, moving towards the kathode when the current is increased, towards the anode when it is diminished.

These statements have been made on the strength of experiments in which the height of the muscular contraction was taken as the index of the excitability of the nerve at any given point. But 
alterations of conductivity-i.e., of the power of a portion of the nerve to conduct an impulse set up elsewhere-are also produced by the constant current. which even outlast its flow. line all currents except the weakest the conductivity at the katlode and in its neighbourhood is diminished, and with currents still only moderately strong the block decpens in to utter impassability. The conductivity at the anorle is, lluring all this stage, but little affected, and is at any rate much higher than at the kathole, so that at the time of full kathodic block the nerve-impulse still freely passes through the region around the positive pole. With still stronger currents the conductivity here, too, begins to diminish, until at last the anode is also blocked ; but this is to be looked upon as merely an extension of the defect of conductivity which has been crecping along the intrapolar area from the kathode. After the opening of the current, the relation between kathodic and anodic conductivity is reversed, for now the post-kathodic region conducts the nerve-impulse relatively better than the post-anodic.

The above facts serve to explain the manner in which the effects of stimulation of a nerve with the constant current vary with the strength and direction of the stream. These effects, so far as the contraction of the muscles supplied by the nerve is concerned, have been formulated in what has been somewhat loosely termed the law of contraction. In this formula the direction of the current in the nerve is commonly distinguished by a thoroughly bad but now ingrained pliraseology, as ascending when the anode is next the muscle, and descending when the kathocle is next the muscle.

\section{Law of Contraction.}

\begin{tabular}{llcc|cc}
\multicolumn{1}{c|}{ Current. } & & \multicolumn{2}{c|}{ Ascendirg } & \multicolumn{2}{|c}{ Dexcending. } \\
& & M. & B. & N. & B. \\
\hline Weak - & - & C & - & C & - \\
Medium & - & C & C & C & C \\
Strong & - & - & C & C & -
\end{tabular}

Here II means 'make,' B, 'break,' of the current; C means 'contraction follows.'

The explanation generally given of the facts summed up in the "law of contraction" is as follows: Wherever there is an increase of excitability sufficiently rapid and sufficiently large, stimulation is supposed to take place; where there is a fall of excitability, stimulation does not occur. Accordingly, at closure the kathode stimulates - the anode does not; while at opening, the anode, at which the depressed excitability jumps up to normal or more, is the stimulating pole: the kathode, at which it declines to normal or under it, is inactive.

With a weak current. (I) contraction only occurs at make. and (2) the direction of the current is indifferent. The explanation of the first fact is that the make is a stronger stimulus than the break, and when the current is weak enough the break is less than a mini- 
mal stimulus. No sensible change of conductivity is causerl by weak currents, which suffices to explain (2).

With a 'medium' current, contraction occurs at make and break with both directions. Here the break excitation is effective as well as the make. With anode nest the muscle (ascending current), there is, of course, nothing to prevent the opening excitation, which starts at the anode, from passing down the nerve and causing contraction : and since there is no block around the anode or in the intrapolar region with ' medium ' currents, there is nothing to kcep the closing (kathodic) excitation from reaching the muscle too. With the kathode next the muscle (descending current), the closing excitation, which starts from the kathode, has no region of diminished conductivity to pass through, nor has the opening (anodic) excitation, for the kathodic block, caused by moderatcly strong currents, is removed as soon as the current is broken

With 'strong' currents there are only two cases of contraction out of the four, just as with 'weak,' but for very different reasons. There is a break-contraction with ascending, and a make-contraction with descending current. With ascending current the anode is next the muscle, and the break-excitation starting there has nothing to hinder its course. The make-excitation, although as strong or stronger, has to pass through the whole intrapolar region and over the anode, and here the conductivity is depressed and the nerveimpulse blocked. With descending current the kathode is next the muscle, and there is no hindrance to the passage of the make-excitation. The brcak-cxcitation, however, has to traverse the intrapolar region. and the anodic end of this area has a smaller conductivity immediately after opening than during the flow, while the kathodic end does not at once, after a strong current, become passable. The break-cxcitation, accordingly, cannot get through to the muscle.

In all these cases of complete or partial block, during or after the flow of a constant current, the progress of the nerve-impulse, its gradual weakening, and final extinction can be very well shown by means of the action stream (p. 7 I9).

The above formula can only be verified upon isolated nerves, and, even for these, exceptional results are apt to be obtained as soon as the nerves begin to die.

A formula similar to the law of contraction has been shown to hold for the inhibitory fibres of the vagus (Donders), 'inhibition' being substituted for 'contraction.' There is also some evidence that a similar law obtains for sensory nerves.

It is not difficult to see that with currents of brief duration the break follows so quickly on the make that interference of their opposed effects may occur. This is the reason-or, at least, one reason-why, above a certain frequency, a muscle or nerve ceases to respond to all of a series of rapidly recurring electrical stimuli (p. 658). It is also the reason why, with single very brief stimuli, a greater current intensity must be employed in order to cause excitation than when the duration of the stimulating current is greater (Woodworth, Lucas). Not enough weight has been given to this circumstance by some of the writers, who, in attacking du Bois-Reymond's law of the dependence of excitation upon variation in current density (p. 636), have sought to cstablish a relation between the excitatory effect and some such factor as current strength, the quantity of electricity passed, or the electrical energy expended. 
The Law of Contraction for Nerves 'in Situ.'-IVhen a nerve is stimulated without previous isolation-in the human body, for instance, through electroles laicl on the skin-the current will not enter and leave it througl definite small portions of its sheath, nor will it be possible to make the lines of flow nearly parallel to each other and to the long axis of the nerve, as is the case in at stencler strip) of tissue when there is a considerable distance between the clectrudes.

()n the contrary. when, as is usually the case in clectro-therapeutical treatment, a single electrode-say, the positive-is placed over the position of the nerve, and the other at a listance on some convenient part of the body, the current will enter the nerve by a broad fan of stream-lines cutting it more or less obliquely, and pass out again into the surrounding tissues; so that both an anode (surface of entrance) and a kathode (still larger surface of exit) will correspond to the single positive pole. Similarly, the single negative electrode will correspond to an anolic surface where the now narrowing sheaf of lines of flow enters the nerve, and a smaller kathodic surface, where they emerge. Even if the two electrodes were on the

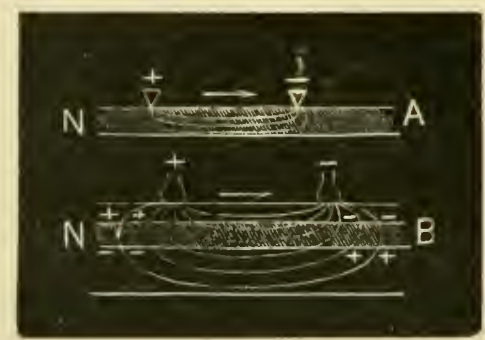

FiG. 255.-1)IAGRAM OF LINES OF FLOW OF A CURRENT PASSING THROUGil A Nervi:.

$A$, an isolated nerve; $B$, a nerve in situ. Secondary anodes $(+)$ are formed where the current re-enters the nerve below the negative electrode after passing throngh the tissues in which it is embedeled, and secondary kathodes $(-)$ where the current passes out of the nerve into the surrounding tissues below the positive electrode. spreading from the positive electrode passes out of the nerve. Now, this is much larger than the primary kathodic surface, through which the narrow wedge of stream-lines passes to reach the negative electrode, and the current density at the latter is accordingly much greater. The positive break-contraction is, for a similar reason, stronger than the negative.

With a 'weak' current, the only contraction is a closing one at the kathode; with a 'medium' current there are both opening and closing contractions at the positive pole, and a closing but no opening contraction at the negative (Practical Exercises, p. $7+3$ ).

The conductivity of the nerve, as we have seen in various examples, is not necessarily altered in the same sense as the 
excitability. In the neighlourhood of the kathode it is easier to cause excitation than in the normal nerve (increased excita bility), hut it is less easy for an excitation set up elsewhere to pass through (diminished comductivity). Change of temperature also, for cortain kinds of stimuli, at any rate, acts in the opposite way on these two properties of nerve. The excitability of frog's nerve is increased by conling (from $35^{\circ} \mathrm{C}$. to $2^{\circ}$ C.) for mechanical and chemical stimulation, and for stimulation by the opening or closure of a voltaic current, unless of very short duration (p. 6SI), but cooling diminishes and heat increases the conductivity. Carbon dioxide and monoxide depress the excitability without affecting the conductivity. Alcohol vapour rapidly impairs the conductivity without for a time affecting the excitability. On ceasing to apjly the vapour the conductivity is restored much sooner than the excitability (Gad and Sawyer. Piotrowski). Munk found that in a dying sciatic nerve certain points may be quite inexcitable to the strongest stimuli, while weak stimulation of points lying nearer the central end mav cause muscular contraction. These facts indicate that the process $b^{*}$ which the nerve-impulse is propagated nav not be the same as that by which it is originated, and therefore is not merely an excitation of each nerve-element by the one next it, as some have supposed.

Cocaine locally applied to a nerve diminishes or abolishes its conductivity, according to the dose. It exercises a selective action as regards nerve-fibres of different kinds, picking out and paralyzing sensory fibres before motor; vagus fibres conducting upwards before those conducting downwards, vaso-constrictors before vasodilators, and broncho-constrictors before broncho-dilators (Dixon). Pressure also abolishes the conductivity of sensory fibres sooner than that of motor fibres.

Double Conduction.-When a nerve (or muscle) is stimulated artificially, the excitation runs along it in both directions from the point of stimulation; so that nerve-fibres which in the intact body are afferent can conduct impulses towards the periphery, and efferent fibres can conduct impulses away from the periphery. In the normal state, however, double conduction must seldom occur, for efferent fibres are connected centrally, and afferent fibres peripherally, with the structures in which their natural stimuli arise. In general, too, an impulse, if it did pass centrifugally along an afferent fibre, would not give any token of its existence, for the peripheral organ would not be able to respond to it ; and we have no reason to believe that the central mechanisms connected with afferent fibres are better fitted to answer such foreign and unaccustomed calls as impulses reaching them along normally efferent nerves. There is good 
evidence that muscular excitation is not carried over to the motor nerve-fibres; in other words, the wave of action flows from the nerve to the muscle, but cannot be got to flow backwards. Excitation of the central end of an efferent (anterior) spinal root is not transferred to the corresponding afferent (posterior) root, the connection between the efferent and afferent nemons presenting the character of a physiological 'valve,' which per mits impulses to pass only in one direction. We have seen that vaso-dilator impulses possibly pass out to the limbs over fibres which, morphologically speaking, are afferent fibres (p. I 65 ). And we shall see that a nutritive influence is exerted over the afferent fibres of the spinal nerves by the ganglion cells of the posterior root ganglia (p. 693), an influence which must spread along these fibres in the opposite direction to that of the normal excitation.

The best proofs of double conduction in nerves, with artificial stimulation, are: (I) The propagation of the negative variation or action current in both directions. This holds for sensory as well as for motor fibres, as du Bois-Reymond showed on the posterior roots of the spinal nerves of the frog and the optic nerves of fishes. (2) Stimulation of the posterior free end of the electrical nerve of Malapterurus (p. 737) causes discharge of the electric organ, although the nerve-impulse travels normally in the opposite direction. (3) If the lower end of the frog's sartorius is split into two, gentle stimulation of one of the tongues causes contraction of individual fibres in the other. This is supposed to be due to conduction of the nerve-impulse up a twig of a nerve-fibre distributed to the one tongue, and down another twig of the same fibre going to the other tongue. A similar experiment can be done on the gracilis of the frog. This muscle is divided by a tendinous inscription into two parts, each supplied by a branch of a nerve which divides after entering the muscle. Stimulation of either twig is followed by contraction of both parts of the muscle (Kühne).

Bert's much-quoted experiment on the rat is valueless as a proof of double conduction. He caused union of the point of the tail with the tissues of the back, then divided the tail at the root, and found that stimulation of what was now the distal end caused pain, From this he concluded that the sensory fibres of the 'transposed ' tail conducted in the direction from root to tip. But the conclusion is not warranted, for sensation disappeared in the tail after the section, and did not return till some months later, when the nervefibres, after degenerating, would have been replaced by new sensory fibres growing down from the dorsal nerves (Ranvier). For a similar reason the so-called union of the peripheral end of the cut hypoglossal nerve (motor) with the central end of the cut lingual (sensory) proves nothing as to double conduction, nor as to the possibility of motor nerves taking on a sensory function. For while sensation is after a time restored in the affected portion of the tongue, this is due to the growth of sensory fibres from the central stump of the lingual down through the degenerated hypoglossal, and not to the conduction upwards of sensory impulses by the motor fibres of the latter. 
Every fibre of a nerve is physiologically isolated from the rest, so that an impulse set up in a fibre runs its course within it, and does not pass laterally into others (law of isolated conduction). In connection with this physiological fact there is the anatomical fact that nerve-fibres do not normally branch in the trunk of a peripheral nerve. (But see p. 697.) It has, however, been shown that bifurcation of nerve-fibres may occur in the spinal cord (Sherrington). The axis-cylinder of a peripheral nerve-fibre only begins to branch where complete isolation of function is no longer required, as within a muscle. The experiment of Külne on double conduction, mentioned above, shows that an excitation set up in one twig or one fibril of an axis-cylinder which has branched can spread to the rest.

Velocity of the Nerve-impulse.-We have said that the nerve-impulse travels with a measurable velocity. It is now time to describe how this has been ascertained (p. 7I3). For motor fibres the simplest method is to stimulate a nerve successively at two points, one near its muscle, the other as fat away from it as possible, and to record the contractions on a rapidly-moving surface (pendulum or spring myograph) (p. 643) The apparent latent period of the curve corresponding to the nearer point will be less than that of the curve corresponding to the point which is more remote, by the time which the impulse takes to pass between the two points. The distance between these points being measured, the velocity is known. Helmholtz found the velocity for frog's nerves at the ordinary temperature of the air to be a little under, and for human nerves, cooled so as to approximate to the ordinary temperature, a little over 30 metres per second. For observations on man the contraction curves of the flexors of one of the fingers or of the thumb may be recorded, first with stimulation of the brachial plexus at the axilla, and then with stimulation of the median or ulnar nerve at the elbow. Probably at the same temperature there is little difference in the rate of transmission in the nerves of warm-blooded and cold-blooded animals, but temperature has a considerable influence (p. 679).

By cooling a frog's nerve Helmholtz reduced the rate to $\frac{1}{10}$ of its value at the ordinary temperature. In the human arm he found a variation from 30 to 90 metres per second, according to the temperature, 50 metres being about the normal rate. This is greater than the speed of the fastest train in the world. According to Piper's recent measurements the velocity in human medullated nerve is even greater than Helmholtz concluded, about I 20 metres a second under ordinary conditions. The rate is independent of the intensity of the excitation.

The velocity with which the negative variation is propagated (p. 723) is the same as that of the nerve-impulse. 
In sonsory neves there is no reason to believe that the relocity of the nerve-impulse differs from that in motor nerves, but experiments on man really free from objection are as yet wanting.

The usual method is to stimulate the skin first at a point distant from the brain, and then at a much nearer point. The person experimented on, as soon as he feels the stimulation, makes a signal, say, by closing or opening with the hand a current connected with an electric time-marker, writing on a moving surface. There is, of course, a measurable interval between the excitation and the signal, and this being in general longer the more remote the point of stimulation is from the brain, it is assumed that the excess represents the time taken by the nerve-impulse to pass over a length of sensory nerve equal to the difference in the length of the path. But there is this difficulty, that the propagation of the impulse from the point of stimulation to the brain is only one link in the chain of events of which the signal marks the end. The impulse has first to be transformed into a sensation, and then the will has to be called into action, and an impulse sent down the motor nerves to the hand. And while the time taken by the excitation in travelling up and clown the peripheral nerve-fibres is probably fairly constant, the time spent in the intermediate psychical processes is very variable.

Chemistry of Nerve.-Our knowledge of this subject is still scanty; and most of what we do know has been obtained from analyses, not of the peripheral nerves, but of the white matter of the central nervous system.

I roteins are present, especially in the axis-cylinder. The proteins of nervous tissue include two globulins, one coagulater by heat at $47^{\circ} \mathrm{C}$, the other at $70^{\circ}$ to $75^{\circ} \mathrm{C}$., and a nucleo-protein coagulating at $56^{\circ}$ to $60^{\circ} \mathrm{C}$.

The lipoids of nervous tissue are very important constituents. They are substances soluble in organic solvents. like benzol and etlier, and comprise cholesterin, certain phosphatides (kephalin and lecithin), and ccrtain cerebrins on cercbrosides. The ccrebrins are glucosides containing nitrogen, but no phosphorus, and they yichl a reducing sugar (galactose) on hydrolysis. In the nervous tissue there is also present, according to some authorities, a compound called protagon. Others consider it a mere mixture of phosplaticles and cerebrosides. The lipoids of nerve-fibres belong largely to the medullary sheath, but they are not confined to it, since non-medullated nerves also yield a considerable quantity of lipoids $\left(\begin{array}{ll}1 & 5\end{array}\right)$ er cent. of the solids as against $4^{6.6}$ per cent. for medullated nerves). Non-medullated nerves (splenic nerves of the ox) are distinguished from medullated nerves (human sciatic) by the high proportion of their total lipoids constituted by the phosphatides (kephalin and lecithin) and cholesterin. Thus, in nonmedullated fibres 47 per cent. of the lipoid extract consisted of cholesterin, and 23.7 per cent. of kephalin; while in the merlullated fibres cholesterin made up only 25 jer cent. of the extract, and kephalin $12^{\prime} 4$ per cont. On the other hand. the cerebrosides are present, both relatively and absolutely, in much larger quantity in medullated than in non-medullated nerves. In both varicties of fibres kephalin, and not lecithin, is the chicf plosphorus-containing body (Falls). The medullary sheath further contains a kind of network of a peculiar resistant substance, neurokeratin. The neurilemma consists of substances insoluble in lilute sodium hydroxide. Gelatin 
is obtamed from the connective tissue which binds the nervefibres together. There may also be ordinary fat in the meshes of the epinemrium comnecting the bundles. Small quantities of xanthin, hypoxantlin, and other extractives, can also be obtained from nerve. Accorling to Halliburton's analyses, the water in sciatic nerves amounts to $65^{\circ}$ I per cent., and the solids to $34^{\circ} 9$ per cent. The proteins make up 29 per cent. of the solids.

For an analysis of the white matter of the brain, see p. $88 \mathrm{I}$.

Nerve-cells contain no potassium, according to Nacallum; and this is true both of the dendrites and the axons. In medullated nerves, however, potassium compounds are present external to the axons, chiefly at the nodes of Ranvier (Frontispiece) and in the neurokeratin framework of the sheath.

The only clienical difference between living and dead nervous tissue which has been made out with any degree of certainty is that the former is neutral or faintly alkaline, and the latter acid, in reaction to such indicators as litmus. This is especially true of the grey matter of the central nervous system, although the white matter also is often found acid. The change of reaction is duc to the accumulation of lactic acid. Such a change has not hitherto been clearly demonstrated in peripheral nerves, either after death or after prolonged stimulation. The (non-medullated) splenic nerves of the dog, even after stimulation for six hours, never became acid (Halliburton and Brodic).

Degeneration of Nerve.-Nerve-fibres are 'bound in the bundle of life ' with the nerve-cells from which their axis-cylinders arise; the connection between cell and axon once severed, the nerve-fibre dies inevitably. This is an illustration of a general law that no portion of a cell can live once it is separated from the nucleus. We shall see later on that changes also occur in the nerve-cell whose axon has been divided from it, although they are of a different nature (rather a slow atrophy than an acute degeneration), and do not necessarily lead to the destruction of the cell. We must regard the neuron not only as a morphological unit, a single cell from nucleus to remotest endbrush, but also as a functional and nutritive unit, the fortune of any portion of which is not indifferent to the rest. Thus, when a man's arm is amputated the arm fares worse than the man, for the arm dies. But the man is not unaffected. $\mathrm{He}$ lives, but he suffers much temporary disturbance and some permanent loss. What is left of him is not quite the same as it was. The acute changes that occur in severed nerve-fibres are most conveniently studied in the peripheral nerves, although essentially similar phenomena take place also in the fibres of the central nervous system.

A spinal nerve is composed of efferent fibres whose cells of origin are in the grey matter of the anterior horn, and afferent fibres whose cells of origin are in the posterior root ganglion. When such a nerve is cut below the junction of its roots, muscular paralysis and impairment of sensation at once follow in the 
region supplied by the nerve; but for a time the nerve remains excitable to direct stimulation. The excitability gradually diminishes, and in a few days is completely gone. If portions of the nerve distal to the lesion are examined at different periods after section, at remarkable process of degeneration (commonly

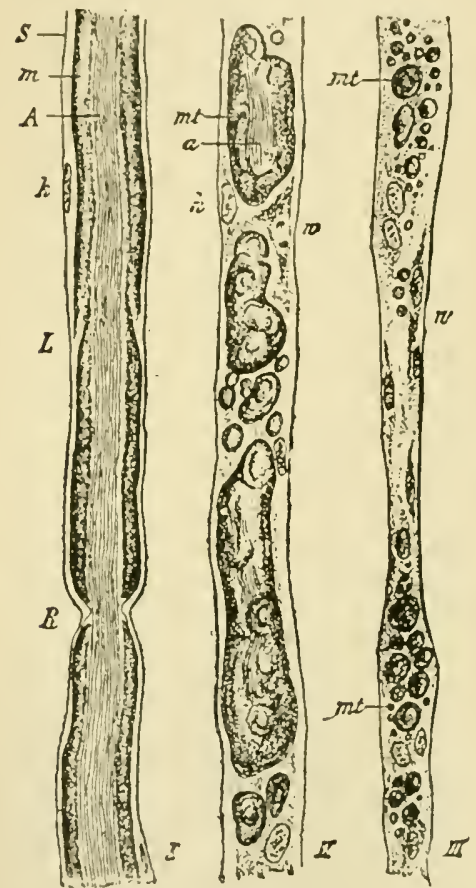

Fig. 256.-DEgeveratiox OF NerilFIBRESAFTER SECTION (BARKER, ATTER TIOMA).

1, normal fibre: II, degenerating fibre; III, further stage of degeneration; S. neurilemma: $m$, medullary sheath : A, axis-cylinder; I. Lantermann's line or cleft ; R, nod"; mt, drops of mvelin : $a$, remains of axis-rylinder ; $w$, proliferating cells of neurilemma. spoken of ats IVallerian legeneration) is seen to be going on. In the medullated fibres this begins on the second ${ }^{-}$ third day with a swelling of the axis-cylinder, which breaks up into detached picees (fragmentation), and assumes a granular appearance. The medullary sheatl also undergoes fragmentation at the lines of Lantermann, and a little later separates into clumps and droplets of myelin. The nuclei under the neurilenmma increase in size, proliferate by mitosis, and insinuate themselves between the fragments of the medullary sheath and axis-cylinder, which ultimately. disappear, leaving the nervefibre represented only by a kind of mummy of connective tissue, in which the neurilemma with its abnormally munerous nuclei can still be recognised. The protoplasm around the nuclei of the neurilemma also increases in amount, and undergoes other changes, which will be more particularly referred to in describing the regeneration of nerve. The degenerative process begins near the cut end, and extends gradually to the periphery, and more rapidly in warn than in coldblooded animals. At any rate, that is the interpretation generally given to the fact that at a given period after section the changes-especially the breaking-up of the myelin-are more pronounced near the proximal end of the peripheral stump. In a mammal degeneration is far advanced in a fortnight, although 
the last remnants of the myelin may not be absorbed for months. In the degenerated nerve (cat's sciatic) the percentage of phosphorus undergoes a diminution from about the third day. About the eighth day the loss of pliosphorts -i.e., of the phosphatides (lecithin, kephalin) -is markedly accelerated, coinciding with the appearance of a strong Marchi* staining reaction. By the twenty-ninth clay the degenerated nerve is practically devoid of phosphorus. A progressive increase in the water and a diminution in the total solids also culminate about the same time (Mott and Halliburton). In the portion of the nerve-fibre still connected with the nervecell the degeneration only extends as far back as the next node of Ranvier, and seems to be clue to the direct effect of the injury. In non - medullated fibres, sucli as the fibres arising from the cells of the superior cervical ganglion (Tuckett), the degeneration is confined to the axiscylinders. It begins in about twenty-four hours after section, and the loss of excitability and conductivity is complete by the fortieth hour.

It follows from what has been said

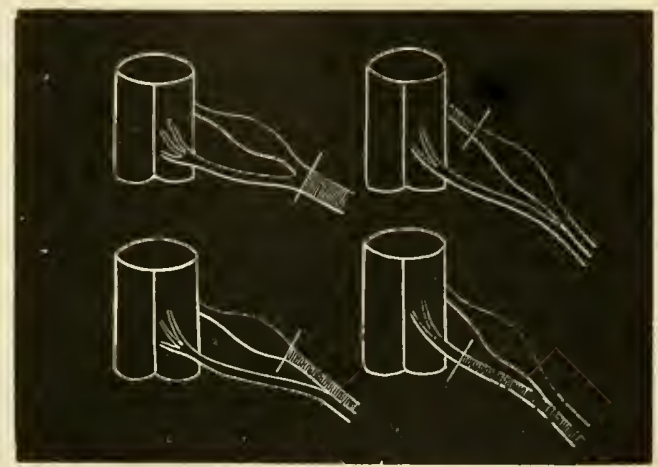

Fig. 257.-Degeneration of SPINAL Nerves and their Roots after Section.

The shading shows the degenerated portions. as to the position of the cells of origin of the root fibres of the spinal nerves that section of the anterior root causes degeneration on the peripheral, but not on the central side of the lesion. $\dagger$ Only the anterior root fibres in the mixed nerve degenerate. Section of the posterior root above the ganglion causes degeneration of the central stump, but not of the portion still connected with the ganglion, nor of the posterior root fibres below the ganglion or in the mixed nerve. Section of the posterior root below the ganglion causes degeneration of the fibres of the root below the section and in the mixed nerve, but not above it.

* The chief constituents of Marchi's solution are potassium bichromate and osmic acid. It stains mellullated nerve-fibres black in the earlier stages of degeneration.

$t$ A few fibres in the peripheral stump of the anterior root do not degenerate, and a few fibres in the central stump do. These are the 'recurrent fibres,' whose course is described on p. 791 . 
Regeneration of Nerve.-Degeneration of nerve is followed, if its divided ends are not kept artificially apart, by a process of regeneration, already distinct under favourable conditions in from three to four weeks after the section, and indeed in some cases commencing as early as the second week. This consists in the outgrowth of new axis-crlinders, in the form of fine fibres, from the ends of the divided axis-cylinders of the central stump of the nerve. These push their way into and along the degenerated fibres, ultimately acquire a medullary sheath, and develop into complete nerve-fibres, restoring first sensation, and later on voluntary motion, to the paralyzed part. Or they may possibly unite with imperfect fibres developed in the peripheral stump. The process needs sereral months for its completion, even in warm-blooded animals. It takes place under the influence of the nucleated portion of the neuron (the cell-body), and is never completed if the peripheral and central portions of the nerve are permanently separated by a substance through which the new axis-cylinders cannot grow or by a gap too wide for them to bridge over. When the cut ends of the nerve are carefully sutured together, the conditions for complete and speedy regeneration are rendered more farourable-a fact which finds its application in the surgical treatment of injured nerves. The cycle of chemical changes described in the degenerating nerve is retraced in the reverse order. In the cat's sciatic the first sign of the return of the phosphorus was seen with the beginning of the normal myelin reaction about the sixtieth day after section. At the one-hundredth day the phosphorus content was almost as great as that of the normal nerve (a little under I per cent. of the solids for the regenerated, as compared with a little over I per cent. for the normal nerve).

It is not as yet well understood how the regenerating fibres are directed in their growth, so that they join their centres to the appropriate end-organs without mistake. That they have a high capacity for finding their way is indicated by the results of cross-suturing such nerves as the median and ulnar-i.e., of uniting the central end of the one with the peripheral end of the other. Howell and Huber found that after this operation in the dog, both co-ordinated roluntary motion and sensation returned in large measure in the parts supplied by the nerves. Here the motor fibres of the median nerve must, of course, have made connection with muscles previously supplied by the ulnar, being guided to them along the nerve-sheaths of the latter. Doubtless the old nerve-sheaths serve to some extent as mechanical guides by offering to the new axons a path of least resistance. And when a nerve-trunk containing motor and sensory fibres is simply crushed so as to destroy all physiological 
continuity, but is not cut, no distortion of the motor and sensory 'patterns' of the nerve - in other words, no 'straying' of the fibres from their old patlis - can be detected on regeneration. When the nerve is cut and then sutured, a certain amount of distortion of the pattern is inevitable. The mechanical apposition of central and peripheral stumps is, of course, much more nearly perfect in the crushed nerve than in the cut nerve, lowerer cxact the suturing may be (Osborne and Kilvington). That, however, the degenerated peripheral stump directs the growth of the axons from the central stump in some other than a merely mechanical way is evident from the cxperiments of Langley on regeneration of the cervical sympathetic in the cat after section below the superior cervical ganglion. The nerve contains fibres of various functions which reach it from the upper thoracic nerves. The anterior roots of the first and third thoracic nerves supply the cervical sympathetic mainly with fibres which end in the ganglion around cells that give off dilator fibres for the pupil. The fibres connected with the cells in the ganglion which send vaso-motor fibres to the vessels of the ear are for the most part contained in the anterior roots of the second and fifth thoracic nerves; and the fibres connected with the cells that give origin to the pilo-motor fibres for the hairs of the face and neck in the anterior roots of the fourth to the seventh. Stimulation of any one of the upper thoracic roots accoldingly causes a specific effect, which, according to I.angley, is in general the same after regeneration as before section of the cervical sympathetic. We must assume, therefore, that each regenerating fibre seeks out either the ganglion cell with which it was originally connected, or one belonging to the same class. No mere mechanical guidance of the growing axons by the old neurilemmas will suffice to explain this selective growth. It is necessary to postulate, in addition, an attraction of a chemical or physicochemical nature (chemiotaxis), dependent upon a specific relation between the new axons and the scaffolding of the peripheral stump or the ganglion cells. Bit it is not possible at present to form any very precise conception of the properties on which the chemiotactic phenomena depend. And the specificity is not an absolute one. Under certain conditions these preganglionic nerve-fibres (that is to say, nerve-fibres running from the spinal cord to end around the sympathetic ganglion cells) can form connections with nerve-cells of a different class-e.g., pupillo-dilators with cells whose axons end in the erector muscles of the hairs. Further, after section of the sympathetic abore the superior cervical ganglion, the post-ganglionic nerve-fibres (i.e., the fibres coming off from the cells of the ganglion) may also, if the opportunity be favourable during regeneration, ex- 
change their old end-organs for new ones; pilo-motor fibres, for instance, finding their way into the iris and becoming pupillodilators. After excision of the superior cervical ganglion the cervical sympathetic does not recover its function. Accordingly the pre-ganglionic fibres cannot form direct functional connection with the post-ganglionic fibres, but can become connected with them only indirectly through the ganglion cells. Nor can efferent post-ganglionic fibres achieve regenerative union with a cerebro-spinal (somatic) motor nerve, although they can themselves regenerate, as has been shown, e.g., in the case of the vaso-constrictors of the limbs. On the other hand, union easily takes place between pre-ganglionic fibres and efterent somatic fibres, and vice versa. For example, the cervical sympathetic can unite with the phrenic nerve, and cause contraction of the diaphragm, or with the recurrent laryngeal nerve, and cause movement of the vocal cords, or with the spinal accessory; and cause contraction of the sterno-mastoid muscle. Conversely, the phrenic nerve, when united with the cerrical sympathetic, can, when stimulated, produce the usual effects observed on exciting the latter nerve (Langley and Anderson).

Although the establishment of connection with the central end of the cut nerve is necessary for complete regeneration, it must not be supposed that no share whatever is taken in the process by the peripheral stump. Even while it remains completely isolated from the central nervous system changes occur which are often described as the third or final stage of degeneration, but which are more correctly interpreted as forming a stage in the regenerative cycle. Spindle-shaped cells or fibres with elongated nuclei make their appearance, produced by the proliferation of the nuclei of the primitive sheath already described, and the increase of the protoplasm in which these nuclei are embedded. These so-called axial strand fibres or this fibrillated protoplasm may appear long before the remains of the degenerated axis-cylinder and myelin sheath have been completely removed. It is generally acknowledged that in the adult they do not derelop beyond this, so long as the peripheral portion of the nerve remains completely isolated, but neither do they disappear even after a very long interval. When strict precautions against union with other nerve-trunks were taken the radial nerve of an adult cat was found in this resting-stage nearly a year and a half after division, and the same was true after two years and a half in a nerve divided in a human being. The fibres are incapable of being excited or of conducting nerve impulses. The precise relation between these axial strand fibres of the peripheral stump and the myelinated fibres found 
there after regeneration has been much debated. All are agreed that nerve-fibrils sprout from the central stump, and the weight of evidence is in favour of the long-accepted view that it is by the growth of these fibrils along the peripheral stump that the new axons are formed, and that all the changes in the distal portion of the nerve, however important for directing and perhaps sustaining the growth of the central fibrils, are subsidiary to this. But some maintain that the outgrowing central fibrils meet and unite with corresponding fibrils sprouting from the peripheral stmmp, and that the new axis-cylinders arise from the fibrils of the axial strand. It is said that very shortly after being brought into connection with the central portion of the same or of another nerve by careful suturing the spindle cells begin to lengthen, and form non-medullated fibres, like those of the sympathetic. Four weeks after union the afferent fibres, although still non-medullated, are capable of heing stimulated mechanically and electrically, and of conducting impulses towards the centre. In about eight weeks they become medullated, but at first are of small calibre (Head and Ham). Bethe, the most strenuous defender of the inherent regenerative power of the isolated peripheral stump (autogenetic theory), has even stated that complete regeneration occurs in young animals in nerves entirely separated from their centres. The controversy turns largely upon the precautions judged necessary to prevent the ingrowth of central fibres. And while it is comparatively easy to make sure, by removing a large part of it, that the central end of the nerve under observation shall remain completely unconnected with the peripheral end, it is often a matter of the greatest difficulty to prevent the union of the distal stump with central fibres from other sources-e.g., from the nerves cut in the wound. There is no doubt that many of the results which seemed to favour the antogenetic theory were due to this cause.

A fact of great physiological interest, and also of practical importance, in connection with the anastomosis of nerves for the rclief of certain forms of paralysis, is the bifurcation of axons in regeneration, when the conditions are such that the axons of the central stump are offered more than one path along which to regenerate. If, for instance, a limb nerve-trunk containing motor fibres is cut, and its central end sutured both to its own distal end and to the distal end of an adjacent nerve-trunk, the sum of the nerve-fibres in the two distal trunks after regeneration has occurred is greater than the number of fibres in the central stump (Kilvington). That this is due to splitting of axons is shown by the fact that an axon reflex (p. 8og) can be elicited on dividing one of the distal trunks and stimulating its central end after complete separation of the proximal or parent stem from the central nervous system. Even when the second path offered to the regenerating motor axon 
is a sensory path, bifurcation of the axon occurs, one branch passing (lown along the previous motor path to its proper inuscular termination, and the other passing down the sensory path. Although there is no evidence that efferent fibres can unite with afferent fibres, a degenerated afferent path can therefore serxe as a chemiotactic scaffolding or guide for the growth of regenerating motor axons, though not such an efficient one as a degeneraterl motor path. Sensory fibres, however, cannot regenerate along motor patis or make functional union with the receptive substance of skeletal muscle.

It is a remarkable fact that regeneration of the fibres of the central nervous system either does not in general occur, or is exceedingly difficult to realize. This lends support to the doctrine of the importance of the neurilemma in regeneration, since the neurilemma is absent from the fibres of the brain and cord. It has, however, been shown that regeneration of the fibres which procecl from the cells of the spinal ganglia along the posterior roots into the cord may take place after the roots have been cut, so that the normal reflexes throngh the respiratory, carcliac, and vaso-motor centres may be once more obtained.

Degeneration of Muscle.-Experimental section or, in man, traumatic division or compression of a nerve leads not only to its degeneration, but ultimately, if regeneration of the nerve does not take place, to degeneration of the muscles supplied by it as well. The muscle-fibres dwindle to a quarter of their normal diameter; the stripes disappear; the lougitudinal fibrillation fades out; and at length only hyaline moulds of the fibres are left, filled, and separated by fatty granules and globules and surrounded by engorged capillaries. Amidst the general decay. the muscular fibres of the terminal 'spindles' with which the afferent nerves of muscles are connected, alone remain unchanged.(Sherrington). Certain diseases of the cord which interfere with the cells of the anterior lorn cause degeneration of motor nerves, and ultimately of muscles. The motor nerveendings degenerate sooner than the sensory. Both may, under suitable conditions, regenerate (Huber).

Reaction of Degeneration.-Muscles whose motor nerves have been separated from their trophic centres show, when a certain stage in degeneration has been reached, a peculiar behaviour to electrical stimulation, called the 'reaction of degeneration.' To the constant current the muscles are more excitable, and the contraction slower and more prolonged than normal. When a current, either constant or induced, is passed through a normal muscle, the muscular fibres may be stimulated either directly, or indirectly through the intramuscular nerves. Under ordinary conditions the nerves respond more reallily than the muscular fibres, especially to momentary stimuli like induction shocks, and therefore the so-called direct stimulation of uncurarized muscle is, as a rule, an indirect stimulation. When the musele is curarized and the nerves thus climinated, the excitability to induced currents is found to be diminisher. The same is the case in a muscle which exhibits the 
reaction of degeneration after section of its motor nerve, only the loss of excitability to induced currents is greater, and may even be complete. The closing anodic contraction is stronger than the closing kathodic-the opposite of the ordinary law. The nerves are inexcitable either to constant or induced currents. The rcaction of degeneration is only obtained from paralyzed muscles when the paralyzing lesion is situated in the cells of the anterior horn from which the motor nerves take origin, or below that level. Accordingly, it is sometimes of use in localizing the position of a lesion. For instance, a group of muscles might be paralyzed by a lesion in the grey matter of the brain or in the nerve-fibres connecting this with the grey matter of the anterior horn of the cord, or in the grey matter of the anterior horn itself, or in the peripheral nervefibres leading from this to the muscles. In the first two cases the reaction of degeneration would be absent, although the muscles, if the lesion was of long standing, would be atrophied to some extent; in the last two there would be acute atrophy of the muscles, and the reaction of degeneration would be obtained.

Trophic Nerves.--There is $n$ o question that nerves exert a very important influence upon the nutrition of the parts supplied by them, in influencing the specific function of those parts. So that in this sense all nerves are trophic nerves. The fact that the proper nutrition of nerve-fibres and striated muscular fibres is dependent on their connection with nervecells has been by some writers generalized into the doctrine that all tissues are provided with 'trophic' nerves, which, apart from any influence of functional activity, regulate the nutrition of the organs they supply. But the evidence for this view, when weighed in the balance, is found wanting ; and it may be said that up to the present no unequivocal proof, experimental or clinical, has ever been given of the existence of specific trophic fibres, anatomically distinct from other efferent or afferent nerves.

It is true that in various diseases and injuries of the nervous system nutritive changes in the skin, and sometimes in the bones and joints, are apt to appear. But it is very difficult in such cases to disentangle the effects produced by accidental injuries acting on structures whose normal sensibility is lost or lessened, or whose circulation is deranged, from true trophic changes. The most that can be said is that there is some evidence that the power of the skin to resist injury, and the capacity of recovering from it, are diminished by interference with its nervesupply, so that a large sore may result from a trifling lesion, and healing may be slow and difficult. Experimentally it has been found that division of the trigeminus nerve within the skull is sometimes followed by cloudiness of the cornea, going on to ulceration, and ultimately inflammation and destruction of the eyeball. Ulcers also form on the lips and on the mucous membrane of the mouth and gums; and the nasal mucous membrane 
on the side corresponding to the divided nerve becomes inflamed. But in this case the sensibility of the eye is lost, and reflex closure of the erelids ceases to prevent the entrance of foreign bodies. The animal is no longer aware of the contact of particles of dust or bits of straw or accumulated secretion with the conjunctiva, and makes no effort to remove them. The lips, being also without sensation, are hut by the teeth, particularly as the muscles of mastication on the side of the divided nerve are paralyzed, and decomposed food, collecting in the mouth, and inhaled dust in the nose, will tend still further to irritate the mucous membranes. There is thus no more need to assume the loss of unknown trophic influences in order to explain the occurrence of the ulcerative changes than there is to explain the production of ordinary bed-sores, bunions or corns on parts peculiarly liable to pressure. And, as a matter of fact, if the eye be artificially protected, after section of the trigeminal nerve. the oplithalmia either does not occur or is much delayed.

In man, too, a case has been recorded in which both the fifth and the third nerves were paralyzed. The eye was still shielded by the contraction of the orbicularis oculi supplied by the seventh nerve, as well as by the drooping of the upper eyelid that accompanies paralysis of the third. It remained perfectly sound for many months, till at length the tumour at the base of the brain which had affected the other nerves involved the seventh, too. The eve was now no longer completely closed; inflammation came on, and vision was soon permanently lost (Shaw). In another case a patient lived for seven years with complete paralysis of the fifth nerve, yet the eye remained free from disease and sight was unimpaired (Gowers).

The so-called 'trophic' effects following dlvision of both vagi we have already discussed (p. 236) so far as they are concerned with the respiratory system. The degenerative changes sometimes seen in the heart are perhaps dne to its being overworked in the absence of nervous restraint on its functional activity. The nutritive alterations in muscles and salivary glands after section of motor and secretory nerves seem to depend in part on functional and vaso-motor changes. In the paralyzed muscles mutrition is not only interfered with in consequence of their inactivity, as would be the case even if the paralysis were due to a lesion above the level of the anterior cornual cells, but the already poorly nourished fibres are continually pressed upon by the capillaries, which are dilated owing to the division of the vaso-motor nerves. The degeneration must also be in part ascribed to the loss of a tonic influence exerted on the muscles by the motor cells of the spinal corcl, through the ordinary motor 
nerves (p. Ṡij). When all allowance has been made for these fietors, the rapid and characteristic degeneration of the striated muscles, after their connection with the central nervous system is severed, is still inexplicable, except on the assumption that their nutrition is specially related to the integrity of their efferent nerves. In other words, it is necessary to suppose, not, indeed, that distinct trophic nerves exist for the muscles, but that an influence or impulses, which can be termed trophic or nutritive, do normally pass out to them from the spinal cord along their motor nerves.

Section of the cervical sympathetic in young rabbits and dogs increases the growth of the ear and of the hair on the same side. But it is impossible to separate these consequences from the vaso-motor paralysis; and the same is true of the hypertrophy following section of the vaso-motor nerves of the cock's comb and of the nerves of the bones. After section of the superior laryngeal the vocal cord on the side of the section is at once rendered motionless, and remains so, but the muscles, notwithstanding their inaction, do not degenerate. And Mott and Sherrington have found that, although section of the posterior roots in monkeys is followed after a time (three weeks to three months) by ulceration over certain portions of the foot, no corresponding lesions occur in the hand. They believe, therefore, that the lesions are not due to the withdrawal of a reflex trophic tone, but are accidental injuries in positions specially exposed to mechanical or microbic insults.

One of the best examples of interference with the proper nutrition of a part produced by a lesion in the nerves supplying it is an eruption (herpes zoster), limited to the skin supplied by the nerve-fibres coming from one or more spinal ganglia, and 'depending on an (infectious) inflammatory change in the ganglia. It has been suggested that the vesicles are formed either because the passage of afferent impulses normally concerned in the nutrition of the skin is interfered with, or because the skin is bombarded by antidromic (p. I65) impulses discharged from the inflamed ganglia. But an alternative hypothesis is that a toxine spreads out along the nerves from the ganglia, just as in traumatic tetanus the torine is known to pass in the opposite direction along the nerves from the seat of injury to the central nervous system.

Omitting the group of 'trophic' nerves, and the even more problematical 'thermogenic' fibres (which some have supposed to preside over the production of heat, and therefore to assist in the regulation of the temperature of the body, but of whose existence as distinct and specific nerve-fibres with no 
other function there is not the slightest proof), peripheral nerves m ry be classified as follows:

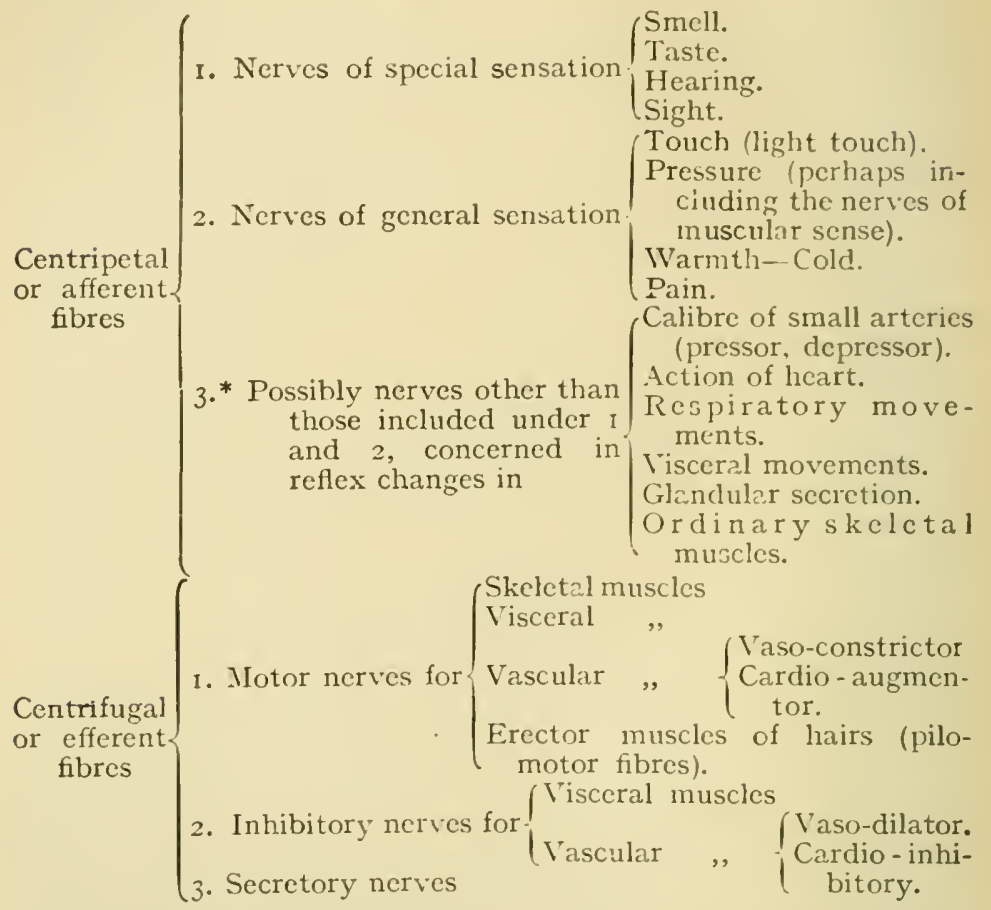

* It is not known whether the afferent portion of a reflex arc is always composed of fibres included in the first two categories, although undoubtedly in some cases it is.

\section{PRACTICAL EXERCISES ON CHAPTERS IX. AND X.}

1. Difference of Make and Break Shocks from an Induction Machine.-Connect a Daniell or other cell B (p. 615) with the two upper binding-screws of the primary coil $\mathrm{P}$, and interpose a spring key $\mathrm{K}$ in the circuit. Connect a pair of electrodes with the bindingscrews of the secondary coil (Fig. 258).

Electrodes can be very simply made by pushing copper wires through two glass tubes. filling the ends of the tubes with sealingwax, and binding them together with waxed thread. The projecting points may be filed, and the nerve laid directly on them, or they may be tipped with small pieces of platinum wire soldered on.

(a) Push the secondary away from the primary, until no shock can be felt on the tongue when the current from the battery is made or broken with the key. Then bring the secondary gradually up 
towards the primary, testing at every new position whether the shock is perceptible. It will be felt first at break. If the sccondary is puslied still furtlier up, a shock will be felt both at make and at break. From this we learn that for sensory nerves the break shock is stronger than the malie. The same can easily be demonstrated for motor nerves and for muscle.

(b) Smoke a drum and arrange a myograph, as shown in Fig. 26r. But omit the brass piece $F$, and do not connect the primary through the drum, as there shown, but connect it as in lig. 258. Pith a frog (brain and cord), and malic a muscle-nerve preparation.

To make a Muscle-nerve Preparation.-Hold the frog by the hind legs back upwards; the front part of the body will hang down, making an angle with the posterior portion. With strong scissors divide the backbone anterior to this angle, and cut away all the front portion of the body, which will fall down of its own weight. Make a circular incision at the level of the tendo Achillis, and another at the lower end of the femur, through the slin. The sciatic nerve must now be dissected out, as follows: Remove the skin from the thigh, and, holding the leg in the left hand, slit up the fascia which

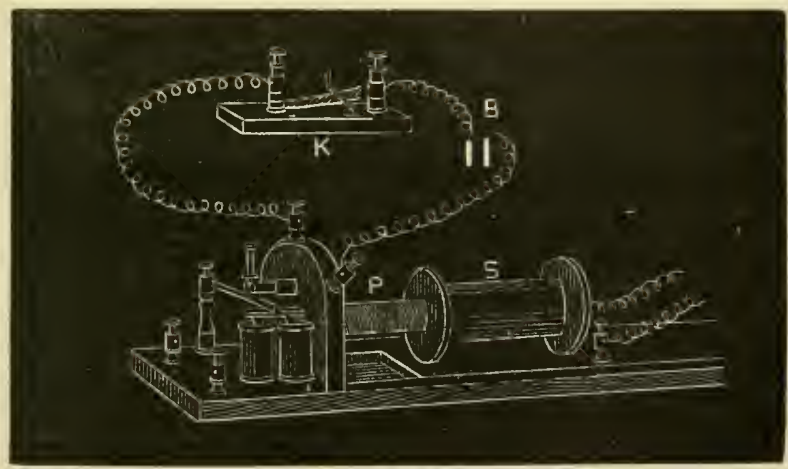

Fig, 258,-.-Arrangement of Coil for Single Shocks.

connects the external and internal groups of muscles on the back of the thigh. Complete the separation with the two thumbs. Cut through the iliac bone, taking care that the blade of the scissors is well pressed against the bonc, otherwise there is danger of severing the sciatic plexus. Now divide in the middle line the part of the spinal column which remains above the urostyle. A piece of bone is thus obtained by means of which the nerve can be manipulated without injury. Scize this piece of bone with the forceps, and carefully free the sciatic plexus and nerve from their attachments right down to the gastrocnemius muscle, taking care not to drag upon the nerve. The muscles of the thigh will contract, as the branches going to them are cut. 'This is an instance of mechanical stimulation. Now pass a thread under the tendo Achillis. tie it, and divide the tendon below it. Strip up the tube of skin tliat covers the gastrocnemius, as if the finger of a glove were being taken off. Tear through the loose connective tissue between the muscle and the bones of the leg, and divide the latter with scissors just below the knee. Cut across the thigh at its middle. 
Fix the preparation on the cork plate of the myograph by a pin passed througl tlie cartilaginous lower end of the femur, and attach the threal to the upright arm of the lever by one of the lioles in it. Hang not far from the axis by means of a hook a small learlen weight (5 to 10 grammes) on the arm of the lever which carries the writingpoint. and move the myograph plate or the muscle-nerve preparation until this arm is just horizontal. Fasten the electroles from the secondary coil on the cork plate with an indiarubber band ; lay the nerve on them; and cover both muscle and nerve with an arch of blotting-paper moistened with physiological silt solution, taking care that the blotting-paper does not touch the threal. (Or put the preparation in a moist chamber* (Fig. 29.4, p. 739). Adjust the writingpoint to the drum. Begin with such a distance between the coils that a break contraction is just obtained on opening the key in the primary circuit, but no make contraction. The lever will trace a vertical line on the stationary drum. Read off on the scale of the induction machine the distance between the coils, and inark this on the drum. Now allow the drum to move a little, still kecping the writing-point in contact with it; then push up the scconclary coil I centimetre nearer the primary, and close the key. If there is a contraction, let the drum move a little before opening the key again, so that the lines corresponding to make and break may be separated from each other. If there is still no contraction at makc, go on moving the secondary up, a centimetre (or less) at a time, till a make contraction appears. When the coils are still further approximated, the make may become equal in heiglit to the break contraction, both being maximal-i.e., as great as the muscle can give witl any single shock (Fig. 259).

(c) Attach a thin insulated copper wire to eacl terminal of the secondary. Loop the bared end of one of the wires through the tenclo Achillis, and coil the other rome the pin in the femur, so that the shocks will pass through the whole length of the muscle. Repeat the experiment of $(b)$, with direct stimulation of the muscle.

2. Stimulation of Nerve and Muscle by the Voltaic Current.- $(a)$ Connect a Daniell cell through a key with a pair of electrodes on which the nerve of a muscle-nerve preparation lies. Observe that the muscle contracts when the current is closed or broken, but not during its passage.

Connect the cell with a simple rheocord, as shown in Fig. 260 , so that a twig of the current of any desired strength may be sent through the nerve. As the strength of the current is decreased by moving the slider $\mathrm{S}$, it will be found that it first becomes impossible to obtain a contraction at break. The current must be still further reduced before the make contraction disappears, for the closing of a galvanic stream is a stronger stimulus than the breaking of it. Tlie break or make contraction obtained by stimulating a nerve with an in-

* P'orter's moist chamber is found in many laboratories, and is very convenient. It consists of a porcelain plate, around which rums a groove. 1 bell-shaped glass cover, which can be lifted off at will, rests in the groove. The femur of the muscle-nerve preparation is fixed in a small clamp, composerl of a split screw on which moves a nut. By means of the nut the clamp, is tightened on the femur. The gastrocnemius hangs vertically down, the thread on the tendo Achillis passing through a hole in the porcelain plate to a lever separately supported on the same stand as the moist chamber. A piece of wet blotting-paper fixed inside the cover keeps the air in the chamber saturaterl. 
duction-machine must not be confused with the break or make contraction caused by the voltaic current. In the care of the incluction machine, the break or make applies merely to what is clono in the primary circuit, not to what liappens to the current actually passing through the nerve. The current induced in the secondary at make of the primary circuit is, of course, both made and broken in the nerve-made when it begins to flow, broken when the flow is

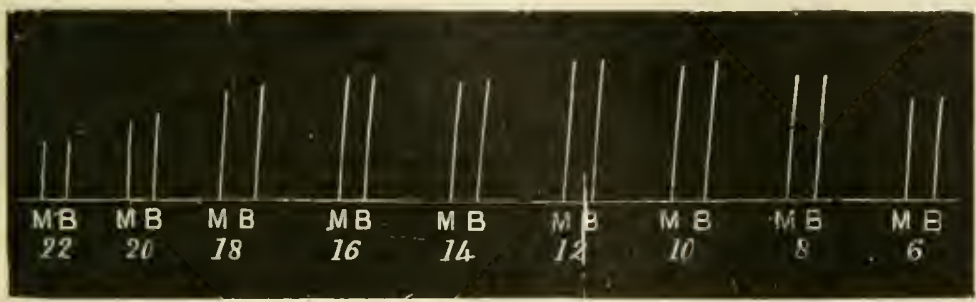

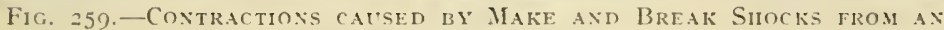
INDECTION MACHINE.

II, make, B, break, contractions. The numbers give the distance between ti.e primary and secondary coils in centimetres.

over; the shock induced at break of the primary is also made and broken in the nerve. And although make and break of the actual stimulating current come very close together, the real make, here, too, is a stronger stimulus than the real break.

(b) Repeat (a) with the muscle directly connected to the cell by thin copper wires, or. better. unpolarizable electrodes (p. 625).

3. Ciliary Mction.-Cut away the lower jaw of the same frog, and

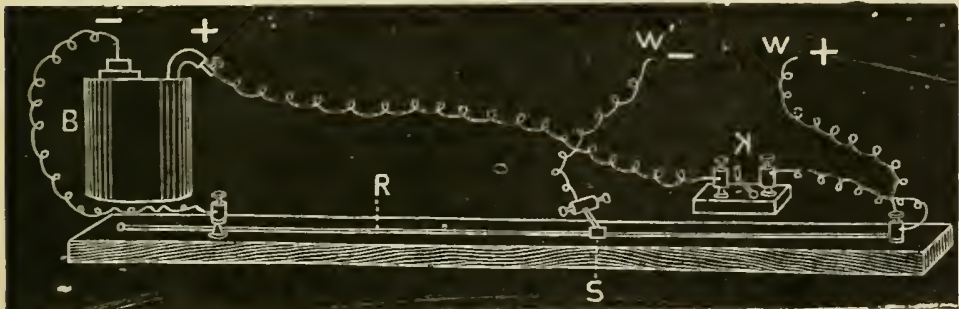

Fig. 260.-Simple Rheocord arranged to SENd a Tivig of a Currext THROtGH A MUSCLE OR NERIE.

$B$, battery ; R, rheocord wire (German silver) ; S, slider formed of a short piece of thick indiarubber tubing filled with merrury; $K$, spring kev; $W$, $\mathbb{I}^{\prime}$, wires connected with electrodes.

place a small piece of cork moistened with physiological salt solution ( 0.75 per cent.) on the ciliated surface of the mucous membrane covering the roof of the mouth. It will be moved by the cilia down towards the gullet. Lay a small rule, divided into millimetres, over the mucous membrane, and measure with a stop-watch the time the piece of cork takes to travel over ro millimetres. Then pour salt solution heated to $30^{\circ} \mathrm{C}$. on the ciliary surface, rapidly swab with blotting-paper, and repeat the observation. The piece of cork will 
now be moverl more (puickly than before, unless the salt solution has been so loot as to injure the cilia.

4. Direct Excitability of Muscle-Action of Curara.-Pith the brain of a frog. and prevent bleceling by inserting a picce of match. Expose the sciatic nerve in the thigh on one side. Carefully separate it, for a length of lialf an inch, from the tissues in which it lies. Pass a strong thread under the nerve, and tie it tightly round the limb, excluding the nerve. Now inject into the dorsal or ventral lymphsac a few drops of a I per ecint. curara solution. As soon as paralysis is complete, make two muscle-nerve preparations, isolating the sciatic nerves right up to the vertcloral column. Lay their upper ends on clectronles and stimulate; the muscle of the ligatured limb will contract. This proves that the nerve-trunks are not paralyzed by curara, since the poison has been circulating in them aloove the ligature. The muscle of the leg which was not ligatured will contract if it be stimulated directly, although stimulation of its nerve has no effect. The ordinary contractile substance of the muscular fibres, accorclingly, is not paralyzed. The seat of paralysis must therefore be some structure or substance physiologically intermediate between the nerve-trunk and the general contractile substance of the muscular fibres (1). 634).

5. Graphic Record of a Single Muscular Contraction or Twitch.Pith a frog (brain and cord), make a muscle-nerve preparation, and arrange it on the myograph plate, as in a (b). lay the nerve on electrodes connected with the secondary coil of an induction macline arranged for single shocks. Introduce a short-circuiting key (Fig. 2 I 5 , p. 626) between the electrodes and the secondary coil, and a spring key in the primary circuit. Close the short-circuiting key, and then press down the spring key with the finger. Let the drum off (fast speed); the writing-point will trace a horizontal abscissa line. Open the sliort-circniting key, and then remove the finger from the spring key. The nerve receives an opening shock, and the muscle traces a curve. Now adjust the writing-point of an electrical tuning-fork (Fig. 26r), vibrating. say, roo times a second, to the drum, and take a time-tracing below the muscle-curve. Stop the drum, or take off the writing-point, the moment the time-tracing has completed one circumference of the drum, so that the trace may not run over on itself. Cut off the drum-paper, write on it a brief description of the experiment, with the time-value of each vibration of the fork, the date, and the name of the maker of the tracing, and then varnish it. An exactly similar tracing can be obtained by directly stimulating the muscle (curarized or not).

6. Influence of Temperature on the Muscle-curve.-pith a frog (brain and cord), make a muscle-ncrve preparation, and arrange it on a myograpl. Ialy the nerve on electrodes connected through a short-circuiting key with the secondary coil of an induction-machine, or connect the muscle directly witl the key by thin copper wires. Take a Daniell cell, connect one pole through a simple key with one of the upper binding-screws of the primary coil, and the other pole with the metal of the drum. A wire, insulated from the drum, but clamped on the vertical part of its support, and with its bare cnd projecting so as to make contact with a strip of brass fastened on the spindle, is connected with the other upper terminal of the primary (Fig. 261). At each revolution of the clrum the primary circuit is made and broken once as the strip of brass brushes the projecting end of the wire. The object of this arrangement is to 
Now stop the elrum, mark with a pencil the position of the feet of the stand carrying the myograple plate, tiake the writing-point off the lrum, and surround the muscle with poundecl ice or snow. After a couple of minutes brush away any ice which could linder the movement of the muscle, rapilly replace the stand in exactly its original position, with the writing-point on the drum, and take another tracing. Again take off the writing-point, and remove all ummelter ice or snow. With a finc-pointed pipette irrigate the muscle with physiological salt solution at $30^{\circ} \mathrm{C}$.. and quickly take another tracing. Then put on a time-tracing with the clectrical tuningfork. lig. 233. P. 646. shows a series of curves obtained in this way.

7. Influence of Load on the Muscle-curve. Arrange everything as in 6 . Take a tracing first with the lever alone, then with a weight of to grammes, then with 50, 100 , 200, and 500 grammes (lig. 2.32 , 1). 646$)$

S. Influence of Fatigue on the Muscle-curve. Arrange as in $\%$. but leave on the same weight (say ro grammes) all the time. Place the nerve on the clectrodes. Leave the sliort-circuiting key open The nerve will be stimulated at each revolution of the lrum, and the

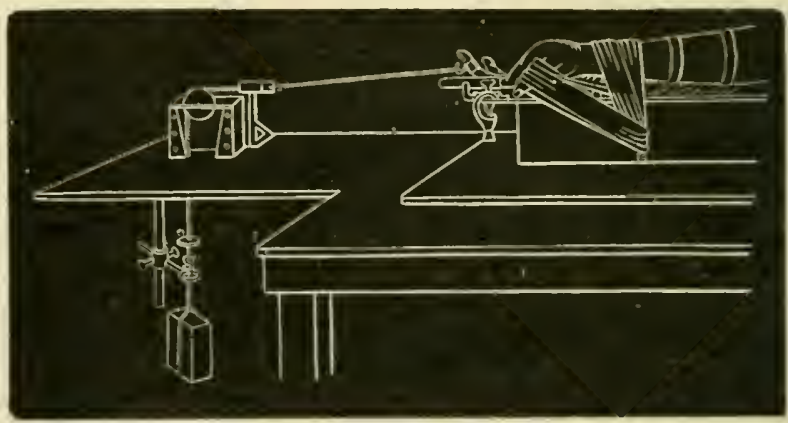

Fig, 262.-Arrangfuext for Studying Voluxtary IIUscular Fatigle

writing-point will trace a series of curves, which become lower, and especially longer, as the preparation is fittigued. Two or four curves can be taken at the same time, if both encls of one or of two brass slips be arranged so as to make contact with the projecting wire at an interval of a semicircumference or qualrant of the drum (Fig. 261). (For specimen curve, see lig. 2fI. p. 65I.)

9. Seat of Exhaustion in Fatigue of the Muscle-nerve Preparation for Indirect Stimulation.-When the nerve of a muscle-nerve preparation has been stimulated until contraction no longer occurs, the muscle can, under ordinary conditions. be made to contract by direct stimulation. The seat of exhaustion is, therefore, not the uceneral contractile substance of the muscular fibres themsclves. To letermine whether it is the nerve-fibres or some structure or substince intermediate between them and the orlinary contractile substance of the muscle, perform the following experiments:

(a) Pith a frog; malie two mnscle-neric preparations; arrange them both on a myograph plate, which has two levers connected with it. Attacl each of the muscles to a lever in the usual way, and lay both nerves side by sicle on the same pair of electroles. Cover with moist blotting-paper. The electrodes are connected with the 
sccondary of an induction machunc arranged for tetanus. With a camel's hair brush noisten one of the nerves between the electrodes and the muscle with a mixture of equal parts of ether and alcolnol, liluter with twice its volume of water, to abolisle the conductivity. ()r put the mixture in a small bottle, in whicl dips a piece of filterpaper. The projecting end of the filter-paper is pointed, and the nerve is ladid on the point. As soon as it is possible to stimulate the nerves witlout obtaining contraction in this muscle, procecl to tetanize both nerves till the contracting muscle is cxhansterl. If the other muscle begins to twitch during the stimulation, more of the etlier mixture must be pilinted on the nerve. As soon as the stimulation ccases to cause contraction in the non-ctherized preparation, wash off the mixture from the other nerve with physiological salt solution, and soon contraction may be secn to takc place in the muscle of this preparation. This shows that the nerve-trunk is still excitable. Now, both nerves have been equally stimulated, and therefore the exhaustion in the non-ctherized preparation was not due to fatigue of the nerve-fibres, but of something between them and the contractile substance of the muscle.

I0. Seat of Exhaustion in Fatigue for Voluntary Muscular Contraction.--Support the arm, extensor surface downwarls, on a rest such as that shown in Fig. 262. or Fig. $24^{\circ} .1 \% .65^{\circ}$, and connect the midlde finger of onc hand. by means of a string passing over a pulley on the erlge of a table, with a weight of 3 or + kilos. The string is attached to the finger by a leather collar surrouncting the second plialanx of the finger, but allowing free movements of the joints. Tlie extent of the vertical movements of the string fand therefore the work (lone) may be registered on a drum by a writingpoint connected with it, the whole arrangement forming what is called an ergograph. Two collar clectroles (strips of copper covered with cotton-wool soakerl in salt solution, and bent to a circular form) are placed on the forearm, and connected through a short-circuiting key with the sccondary coil of an induction machine arranged for tetanus (1). I $\left.S_{+}\right)$, and having a battery of two or three good lry cells or of four or five Danicll cells, compled in series, ${ }^{*}$ in its primary circuit. The middle finger is now made to raise the weight repeatedly by vigorous contractions of the flcxor muscles until at length a failure occurs. At this moment the short-circuiting key is opened, and the flexor muscles stimulated clectrically. They again contract, and raise the weight, therefore the seat of exhaustion in voluntary muscular effort is not usually in the ordinary contractile substance of the muscles. That it is not usually in the nerves may be shown by inducing fatigue of the finger for voluntary contraction in the same way, and then stimulating the meclian nerve at the bend of the elbow by sponge electrodes. The usual seat of fatigue for voluntary muscular contraction must therefore be in the spinal cord or brain.

II. Influence of Veratrine on Muscular Contraction.-Arrange a drum as in Fig. 261. Pith a frog (brain only), expose the sciatic nerve in one thigh, and isolate it for $\frac{1}{2}$ inch from the surrounding tissues. Pass under it a strong tliread, and ligature everything except the nerve. Now inject into the dorsal or ventral lymph-sac a few drops of $o^{\circ}$ i per cent. solution of sulphate of veratrine. In a few minutes malic two muscle-nerve preparations from the posterior limbs. First put the preparation from the unligatured limb on the

* I.e., the copper of one cell connected with the zinc of the next. 
myograph plate. Lay the nerve on electrodes connected through a short-circuiting key with the secondary of an induction machine arranged as in Fig. 2(1). Put the writing-point on the drum and set it off (fast speed). Open the short-circuiting key till the nerve has been once stimulaterl, then close it again. The curve obtained differs from a normal curve, in that the period of descent (relaxation) is exceclingly prolonged. Now connect the preparation from the ligatured limb with the lever, and take a tracing of a single contraction. Put on a time-tracing with the elcctrical tuning-fork (see Figs. 243, 245, pp. 653, 655).

12. Measurement of the Latent Period of Muscular Contraction.(1) For this the lrum must travel at a faster speed than usual. The arrangement for automatic stimulation clescribed in Experiment 6 (p. 706$)$ may be employed. Or an electro-magnetic signal may be connected in the primary circuit of the induction coil so that when the primary is closed or opened the writing-point of the signal moves. Arrange the writing-point of the signal on the drum in the same vertical line as the writing-point of the muscle lever, and in the same line place the writing-point of a vibrating electric tuning-fork. The coil is adjusted for single opening shocks as in Experiment 5 (p. 706). Pith a frog, and make a musclenerve preparation. Arrange it on the myograph plate. The muscle, or the nerve very near the muscle, is to be excited by a single opening shock while the drum is moving. When the curve has been traced the latent period is got by drawing a vertical line through the point at which the curve just begins to rise from the abscissa line, and another through the signal mark. The number of vibrations of the tuning-fork included between these two verticals gives the latent period.

Or (2) use the spring myograple (Fig. 228, p. 643), raising it on blocks of wood. Smolie the glass plate over a paraffin flame, or cover it with paper, and smoke the paper. Connect the knock-over key of the myograph with the primary circuit of an induction coil. Arrange a muscle-nerve preparation on the myograph plate. Place electrodes below the nerve as near the muscle as possible, and connect by a sloort-circuiting key with the secondary. Bring the writing-point in contact with the smoked surface of the spring myograph, so as to get the proper pressure. See that the writing-point of the tuningfork is in the right position for tracing time. Then push up the plate so as to compress the spring, 1 ill the rod connected with the frame which carries the plate is held by the catch.

With the short-circuiting key closed. press the release and allow an abscissa line to be traced. Again shove back the frame till it is caught. Push home the rod by means of which the prongs of the tuning-fork are separated, and rotate it through $90^{\circ}$. Close the knock-over kcy, open the short-circuiting key, shoot the plate again, and a muscle-curve and time-tracing will be recorded. Again close the short-circuiting key, withdraw the writing-point of the tuningfork, pusl back the plate, close the trigger key, then open the shortcircuiting key, and holding the travelling frame with the hand, allow it just to open the knock-over and stimulate the nerve. The writing-point now records a vertical line for, rather, an arc of a circle), which marks on the tracing the moment of stimulation. The latent period is obtained by drawing a parallel line (or arc) through the point of the muscle-curve where it just begins to diverge from the abscissa line. The value of the portion of the 
time-tracing between these two lines can be realily determinced, and is the latent periogl.

13. Summation of Stimuli. - Arrange 1 wo knock-over keys on the spring myograph at such a distince from cacle other that the plate travels from one to the other in a time less than the latent period. Connect each key with the prima"y circuit of a separate induction coil having a conple of Daniclls in it. Join two of the bincling-screws of the secondaries together; connect the other two throngh a shortcircuiting key with clectrodes, on which the nerve of a muscle-nerve preparation is arranged. I'ush 11) the scendarics till the break shocks obtained on opening the two knock-over keys are maximal. Then shoot the plate as llescribed in 12, first with one trigger key closed, and then with both. The curves olstained shoukl be of the same height in the two cases, as a second maximal stimulus falling within the latent period is ignored by the nerve or muscle. Repeat the experiment with submaximal stimuli-i.e., with such a distance of the coils that opening of either trigger key does not caluse as strong a contraction as is causcl when the coils are closer. The curve will now be higher when the two shocks are thrown in successively than when the nerve is only once stimulaterl. This shows that (submaximal) stimuli can be summed in the nerve. The same could be demonstrated for muscle (p. 65.5).

14. Superposition of Contractions.-Smoke a irum arranged for automatic stimulation as in Fig. 201. Adjust the brass points with a distance of, say, I centimetre between them, so that a second stimulus may be thrown into the nerve at an interval greater than the latent periol of muscle. Put two Daniells in the primary circuit. Lay the nerve of a muscle-ncrve preparation on electrodes connected through a short-circuiting key with the sccondary. Allow the drum to revolve (fast speed); open the short-circuiting key till botli brass points have passed the projecting wire, then close it. Now bend back the second brass point, and take a tracing in which the first curve is allowed to complete itself. This will not rise as high as the sccond curve obtained when the two stimuli were thrown in. Repeat the experiment with varying intervals between the brass points-that is, between the two successive stimuli. Put on a time-tracing with the clectrical tuning-fork. (For specimen curve, see Fig. 2.76, p. 656.)

I5. Composition of Tetanus. - ( $(\iota)$ Adjust a muscle-nerve preparation on a myograph plate, the nerve being laid on electrodes connected through a short-circuiting key with the secondary of an induction machine, the primary circuit of which contains a Daniell cell and is arranged for an interrupted current (Fig. 8I, p. I 84). The lever should be shorter than that used for the previous cxperiments, or the thread should be tied in a hole farther from the axis of rotation, so as to give less magnification of the contraction. Set the Neef's hammer going, let the drum revolve (slow speed), and open the key in the secondary. The writing-point at once rises, and traces a horizontal or perhaps slightly-ascending line. Close the shortcircuiting key, and the lever sinks down again to the abscissa line. If it does not quite return, it should be loaded with a small weight. This is an example of complete tetanus.

(b) Connect the spring shown in Fig. 263 with one of the upper terminals of the primary coil, and the mercury cup with the other. Fasten the end of the spring in one of the notches in the upright piecc of wood by means of a wedge, so that its whole length can be 
made to vibrate. let the drum off, set the spring vibrating by repressing it wh the linger, then open the key in the secondary. The"muscle is thrown into incomplete tetanus, and the writingpoint traces a wary curve at a higher level than the abscissal line. Close the short-circuiting key, and the lerer falts to the horizontal. Repeat the experiment with the spring fastened, so that only $3, \frac{1}{2}, \frac{1}{1}$. $\frac{1}{8}$ of its length is free to vibrate. The rate of interruption of the primary circuit increases in proportion to the shortening of the spring, and the tetanus becomes more and nore complete till ultimately the writing-point marks an unbroken straight line. Put on a time-tracing by means of an electro-magnetic marker connected with a metronome beating seconds or half-seconds (lig. 76,1 ). J 79). (For specimen curves, see Fig. 247, p. 657.)

16. Contraction of Smooth Muscles-(1) Spontancous Rhythmica! Contractions.-Immerse in oxygenated Ringer's solution a ring of

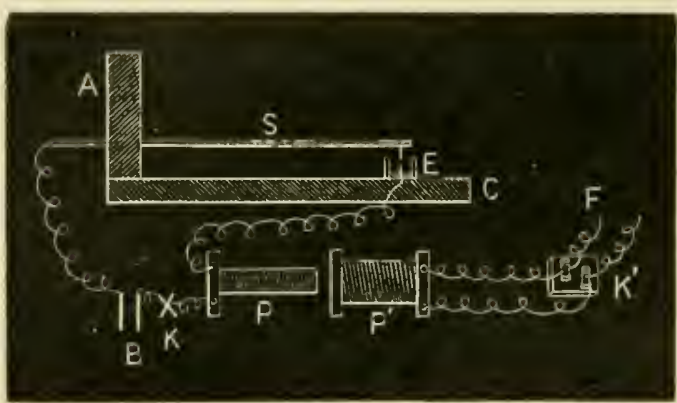

Fig. 263.-ARRANGEMENT FOR TETANI'S.

A, upright with notehes, in which the spring $S$ is fastened (shown in section); C, horizontal board to which $A$ is attached, and in a groove in which the mercury-rup E slides. The primary coil $P$ is connected with $E$, and through a simple key, $K$, with the battery $\mathrm{B}$, the other pole of which is connected with the end of the spring. The wires from the secondary coil, $P^{\prime}$, go to a short. circuiting key, $K^{\prime}$, from which the wires $F$ go off to the electrodes.

in a moist chamber, and the other end to a lever writing on a clrmm. Connect thin copper wires from the secondary coil of an inductorinm with the two encls of the piece of oesophagus. Take tracings to show. (a) the curve of a single contraction caused by a single make or break shock, with estimation of the latent period, as in Experiment 12. p. $710 ;(b)$ summation, as in Experiment 13, p. $711 ;(c)$ genesis of tetanus, as in Experiment I 5. p. 7 I $1 ;(d)$ the relations between strength of stimulus and amount of contraction. For this last expcriment the drum should be stationary while the contraction is being recorded, and should be allowed to move a little between successive contractions. Begin with the secondary at such a distance from the primary that a contraction is just caused by a break shock. Then gradually increase the strength of the stimulus (always using the break) till maximum contration is olstained. The gradual increase in the response is very clearly seen with the oesophageal preparation (Waller) 
17. Velocity of the Nerve-impulse. - l'se the spring myograph (lig. 228,1$) .643$ ) or a very rapielly rotating drum. Make a musclenerve preparation from a large frog (preferably a bull-frog), so that the sciatic nerve maty be as long as possible. Connect the knock-over key with the primary circuit of an induction machine, which should contain a single Daniell cell. Arrange two pairs of fine electrodes under the nerve on the nuyograph plate, one near the muscle, the other at the central end. Connect the electrodes with a Pohl's conmutator (without cross-uires), the side-cups of which are joined to the terminals of the secondary coil, as shown in Fig. 265. By tilting the bridge of the commutator the nerve may be stimulated at either point. Great care must be taken to keep the nerve in a moist atmosphere by means of wet blotting-paper or a moist chamber; but at the same time it must not lie in a pool of salt solution, as twigs of the stimulating current would"in this case spread down the nerve, and we could never be sure that the apparent was always the real point of stimulation. The writingpoints of the lever and tuning-fork having been adjusted to the smoked plate, as in 12 (p. 710), the bridge of the Pohl's commutator is arranged for stimulation of the distal point of the nerve, the plate is shot with the short-circuiting key in the secondary closerl, and an abscissa line and time-curve traced. Then the writing-point of the

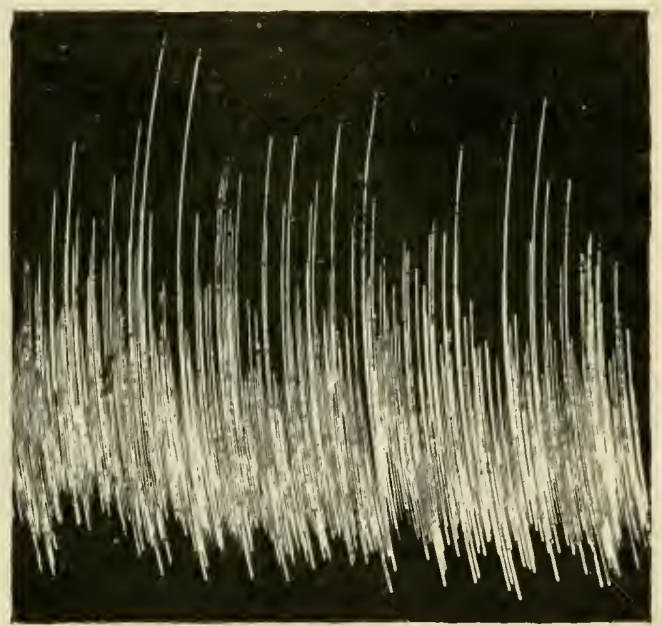

Fig. 264.--Rhithuical Contractions of G:sophagus of Chicken (Botazzi). fork is removed and

the plate again shot with the key in the secondary open, and a musclecurve is obtained. The commutator is now arranged for stimulation of the central end of the nerve, and another muscle-curve taken. Vertical lines are drawn through the points where the two curves just begin to separate out from the abscissa line. The interval between these lines corresponds to the time taken by the nerveimpulse to travel along the nerve from the central to the distal pair of electrodes. Its value in time is given by the tracing of the tuningfork. The length of the nerve between the two pairs of electrodes is now carefully measured with a scale divided in millimetres, and the velocity calculated (p. 689).

I8. Chemistry of Muscle.-Mince up some muscle from the hindlegs of a dog or rabbit (uscd in some of the other cxperiments), of which the bloodvessels have been washed out by injecting $0^{*} 9$ per cent. salt solution through a cannula tied into the abdominal aorta until the washings are no longer tinged with blood. To some of the minced muscle add twenty times its bulk of distilled water, to another 
portion ten times its bulk of a 5 per cent. solution of magnesium sulphate. Let stand, with frequent stirring. for twenty-four hours. Then strain through several folds of linen, press out the residue, and filter through paper. (I) With the filtrate of the watery cxtract make the following obscrvations:

(a) Reaction.-To litmus-paper acid.

(b) Determine the temperatures, at which coagulation of the various proteins in the extract takes place, accorling to the method described on p. 8.* Put some of the watery cxtract in the testtube, and heat the bath, stirring the water in the beakers occasionally with a feather. Note at what temperature a coagulum first forms. It will be about $47^{\circ} \mathrm{C}$. Filter this off, and again heat; another coagulum will form at $56^{\circ}$ to $58^{\circ}$. Filter, and heat the filtrate; a third slight coagulum may be formed at $60^{\circ}$ to $65^{\circ} \mathrm{C}$., but this represents merely a residue of the myosinogen which was left in

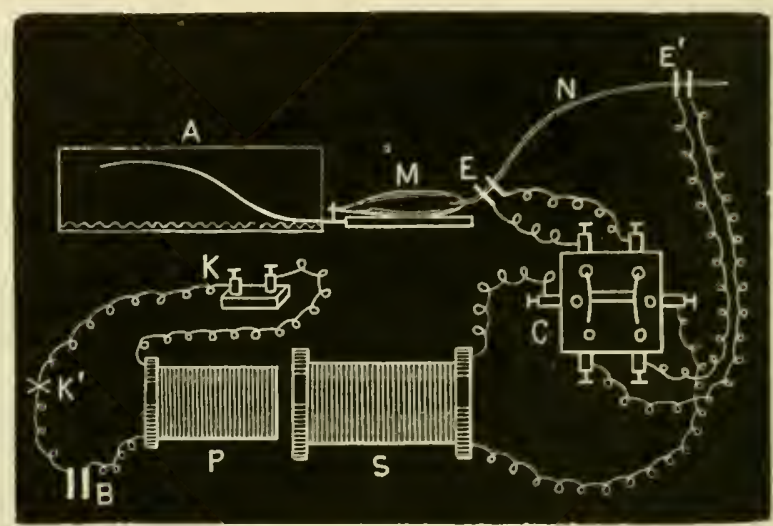

Fig. 265.-Arrangement for Measuring tue Velocity of tite Nerie. IMPL'LSE.

A, travelling plate of spring myograph; $\mathrm{M}$, inuscle lyiug on a myograph plate; $\mathrm{N}$, nerve, lying on two pairs of electrodes, $\mathrm{E}$ and $\mathrm{E}^{\prime} ; \mathrm{C}$, Pohl's commutator without cross wires; $k$, knork-over key of spring myograph (only the binding. screws shown) : $\mathbb{K}^{\prime}$, simple key in primary circuit ; $\mathrm{B}$, battery ; $\mathrm{P}$, primary coil ; $\mathrm{S}$, secondary coil.

solution at the previous heating. A fourth precipitate (of serumalbumin) will come down at $70^{\circ}$ to $73^{\circ}$. Saturate some of the watery extract with magnesium sulphate ; a large precipitate will be formed, showing the presence of a considerable amount of globulin. Filter off the precipitate and heat the filtrate ; coagulation will again occur at very much the same temperatures as before, although the total amount of precipitate will be less. Note in particular that there

* It should be remembered that the temperature of heat-coagulation of any substance is by no means an absolute constant. It depends on the reaction, the proportion and kind of neutral salts present, perhaps on the strength of the protein solution and the manner of heating. A solution of egg-albumin, e.g., can be coagulated at a temperature much below $70^{\circ}$ when it is heated for a week. Small differences in the temperature of heat-coagulation, unless supporter by well-marked chemical reactions, are not enough to characterize protein substances as chemical inclividuals. 
is still some precipitate at $+7^{\circ}$ to $50^{\circ}$. Paramyosinogen possesses some of the characters of both globulins and albumins, for it is partially but not entirely precipitated by saturation with magnesium sulphate, and is not precipitated by sodium chloride.

(2) (a) Test the reaction of the magnesium sulphate extract. It will usually be faintly acid to litmus.

(b) Heat some of it. Precipitates will be obtained at the same temperatures as in $(I)(b)$. but those at $47^{\circ}$ to $50^{\circ}$ and $56^{\circ}$ to $58^{\circ}$ will be more abundant. Of the two, that at $47^{\circ}$ to $50^{\circ}$ will usually be the larger when time is given for it to come down and the heating is gradual.

(c) Dilute some of the magnesium sulphate extract with three times, another portion with four times, and another with five times, its volume of water in a test-tube, and put in a bath, at $40^{\circ} \mathrm{C}$. Coagulation or precipitation will occur in one or all of these test-tubes. To another test-tube of the extract diluted in the proportion which has given the best ' muscle-clot' add a few drops of a dilute solution of potassium oxalate, and place in the bath at $40^{\circ}$. Coagulation occurs as before. Filter off the clot from all the test-tubes. The filtrate is the 'muscle-serum,' and vields a precipitate of serumalbumin at $70^{\circ}$ to $73^{\circ} \mathrm{C}$.

(3) Myosinogen, like other globulins, is insoluble in distilled water, but soluble in weak saline solutions. Saturation with neutral salts like sodium chloride and magnesium sulphate precipitates myosinogen, but not albumin, from its solutions; saturation with ammonium sulphate precipitates both. Mrosinogen is said to be dissolved without change in very weak acids. Stronger acids precipitate it. Verify the following reactions of myosinogen, using the original magnesium sulphate extract of the muscle.

(a) Dropped into water, it is precipitated in flakes, which can be redissolved by a weak solution of a neutral salt (say 5 per cent. niagnesium sulphate).

(b) When a solution of myosinogen is dialyzed, it is precipitated on the inside of the dialyzer as the salts pass out.

(c) If a piece of rock-salt is suspended in a solution, the myosin gradually gathers upon it, diffusion of the salt out through the precipitated myosin always keeping a saturated layer around it.

(d) Saturate a solution containing myosinogen with crystals of magnesium sulphate, stirring or shaking at frequent intervals. The myosinogen is precipitated.

(e) Without adding any salt, simply shake a myosinogen solution vigorously; a certain amount of the myosinogen will be precipitated, and the solution will become turbid. This reaction can also be obtained with solutions of other proteins, such as albumins(Ramsden).

Extracts qualitatively similar to those obtained from the muscles of a freshly-killed animal can be got from muscles that have entered into rigor, but the quantity of the various proteins going into solution is less.

19. Reaction of Muscle in Rest, Activity, and Rigor Mortis.(a) Take a frog's muscle, cut it across, and press a piece of red litmus-paper on the cut end; it is turned blue. Yellow turmeric paper is not affected.

(b) Immerse another muscle in physiological salt solution $(0.75$ per cent. for frog's tissues) at $40^{\circ}$ to $42^{\circ} \mathrm{C}$. It becomes rigid. The reaction becomes acid to litmus-paper, and also turns brown turmeric paper yellow. 
(c) Plunge another muscle into boiling physiological salt solution. It becones harder tlian in (b), and its reaction becomes acid to litmus. paper.

(d) Stimulate another muscle with an interrupted current from an induction machine (Fig. SI, p. 184), till it no longer contracts. The reaction is now acicl to litmus-paper. Brown turmeric paper may also be turned yellow.

(e) To demonstrate the formation of lactic acid in muscle in heat rigor or fatigue, perform the following experiment: Pith a frog, and afterwards leave it for half an hour at rest, so that the lactic acicl produced in the movements connected with the pithing operation may disappear from the muscles. See that the circulation in the hind-limbs is not interfered with by pressure or flexion. Then remove both hind-limbs. Carefully, but rapidly, remove the muscles of one from the bones with as little manipulation as possible. Immediately place them in a small mortar cooled in ice, and containing some sand and 20 or 30 c.c. of ice-cold 95 per cent. alcohol, and quickly grind them up). Produce heat rigor (p. 674) of the muscles of the other hind-limb, or fatigue them with induction shocks, and then grind them up under alcohol in the same way. Filter the alcoholic extracts, and then evaporate them to dryness on the water-bath. Rub wp the residues with a few c.c. of hot water. Add to each aqueous extract a small quantity (say a decigramme) of fincly powdered charcoal. Then heat cach extract to boiling in a testtube, and filter. Evaporate the filtrates to dryness, and apply

Hopkins's Reaction for Lactic Acid.-The reagents requirerl are (I) a very dilute alcoholic solution of thiophene (Io to 20 drops in I oo c.c.) ; (2) a saturated solution of copper sulphate; and (.3) ordinary strong sulphuric acid.

Have ready a glass beaker containing water briskly boiling. Place about 5 c.c. of strong sulphuric acid in a test-tube, with I drop of the copper sulphate solution.* Acll to the mixture a few drops of the solution to be tested, and shake well. $\dagger$

(In the case of the muscle extracts the dry resirlues are clissolved in the 5 c.c. of strong sulphuric acid, the acid transferred to test-tules, and the test proceeded with by the addition of the copper sulphate solution, etc.)

Now place the test-tube in the boiling water for one to two minutes. Then cool it well under the cold-water tap, and add 2 or 3 drops of the thiophene solution from a pipette. Replace the tube in the boiling water, and immediately observe the colour. If lactic acid is present the liquid rapidly takes on a bright cherry-red colour, which is only permanent if the test-tube be cooled immediately after its appearance. The tube should always be cooled, as describerl. before addition of the thiophene, as the gradual appearance of the colour cn re-warming malies the test more delicate.

(The extract of the resting limb generally gives a negative, that of the other a strongly positive, reaction.)

* The copper sulphate is added to hasten the oxidation that follows.

+ For practice use a I per cent. alcoholic solution of lactic acid. The test cannot be applied directly to material which chars with the strong sulphuric acid used. In this case preliminary extraction of the lactic acid is necessary. Alcohol should be used as the solvent, or if ether is employed it must first be well wasled to remove aldehyde-yielding products, since the colour change is due to an aldehyde reatction with thiophene. 


\section{CHAPTER XI}

\section{ELECTRO-PHYSIOLOGY}

A LitTle more than a hundred years ago the foundation both of clectro-physiology and of the vast science of voltaic electricity was laid by a chance observation of a professor of anatomy in an Italian garden. It is indeed true that long before this electrical fishes were not only popularly known, but the shock of the torpedo had been to a ccrtain extent scientifically studied. But it was with the discovery of Galvani of Bologna that the epoch of fruitful work in electrophysiology began. Engaged in experiments on the effect of static and atmospheric electricity in stimulating animal tissues, he happened one day to notice that some frogs' legs, suspended by copper hooks on an iron railing, twitched whenever the wind brought them into contact with one of the bars (p. 738). He concluded that eleetrical charges were developed in the animal tissues themselves. and discharged when the circuit was completed. Volta, professor of physics at Pavia, fixing his attention on the fact that in Galvani's experiment the metallic part of the circuit was composed of two metals, maintained that the contact of these was the real origin of the current, and that the tissues served merely as moist conductors to complete the circuit ; and after a controversy lasting for more than a decade, he finally clinched his argument by constructing the voltaic pile, a series of copper and zinc discs, every two pairs of which were separated by a disc of wet cloth, or paper moistened with salt solution. The pile yielded a continuous current of electricity. 'So,' said Volta, ' it is clear that the tissue in Galvani's experimcnt only acts the part of the cloth.' Galvani, however, had shown in the meantime that contraction without metals could be obtained by dropping the nerve of a preparation on to the muscle (p. 738) ; and it soon began to be recognised that both Galvani and Volta were in part right, that two brilliant discoveries had been made instead of one; in short, that the tissues produce electricity, and that the contact of different metals does so too. Although it is curious to note how completely the growth of that science of which Volta's discovery was the germ has overshadowed the parent tree planted by the hand of Galvani, yet animal electricity has been deeply studied by a large number of observers, and many interesting and important facts have been brought to light.

Since it is in muscle and nerve that the phenomena of electrophysiology are seen in their simplest expression, and have been chiefly studied, we shall develop the fundamental laws with 
reference to muscle and nerve alone, and afterwards apply them to other excitable tissues.

I. All points of an uninjured resting muscle or nerve are approximately at the same potential (or iso-electric). In other words, if any two points are connected with a galvanometer by means of unpolarizable electrodes, little or no current is indicated. (Although it is scarcely possible to isolate a muscle without its showing some current, the more carefully the isolation is performed the feebler is the current; and between two points of the inactive, uninjured ventricle of the frog's heart no electrical difference has been found. Frogs' nerves kept ten to twenty hours after excision in physiological salt solution to which a little calcium salt and frog's blood have been added, are absolutely iso-electric.)

2. Any uninjured point of a resting muscle or nerve is at a different potential from any injured point. The difference of

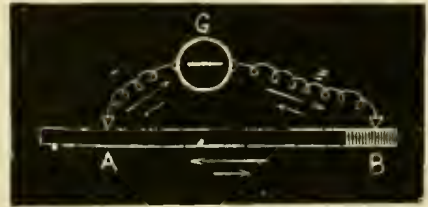

P1G. 266.-A, uninjured, B, injured, portion of nerve; $G$, galvanometer. The large arrows show direction of demarcation current or current of rest, the small arrows direction of negative variation or action current.

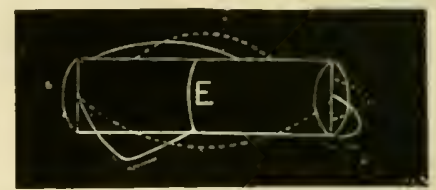

Fig. 267.-Diagras of Currents of Rest in a Rfgular Muscle, or Muscle Cylinder.

E, equator. The dotted lines join points at the same potential, between which there is no current.

potential is such that a current will pass through the galvanometer from uninjured to injured point and through the tissue from injured to uninjured point (current of rest, or demarcation current, or injury response) (Fig. 266).

3. Any unexcited point of a muscle or nerve is at a different potential from any excited point, and any less excited point is at a different potential from any more excited point. The difference of potential is such that a current will pass through the galvanometer from the excited to the unexcited or less excited point (action current, or negative variation, or excitatory electrical response).

It has been customary in physiological writings to speak of the electrical change in injured or active tissue as a negative one, because when the tissue is led off to a galvanometer the current passes from the galvanometer to the injured or excited portion of the tissue. It may be called with greater precision 'galvano- 
metrically negative.' 11 is in this sense that we shall employ the term.

The best object for experiments on the demarcation current is a straight-fibred muscle like the frog's sartorius. If this muscle be taken, and the ends cut off perpendicularly to the surface, a muscleprism or muscle-cylinder is obtained (Fig. 267). The strongest current is got when one electrode is placed on the middle of either cross-scetion and the other on the 'equator'-that is, on a line passing round the longitudinal surface midway between the ends. The direction of this current is from the cross-section towards the equator in the muscle. If the clectrodes are placed on symmetrica points on each sicle of the equator, there is no current.

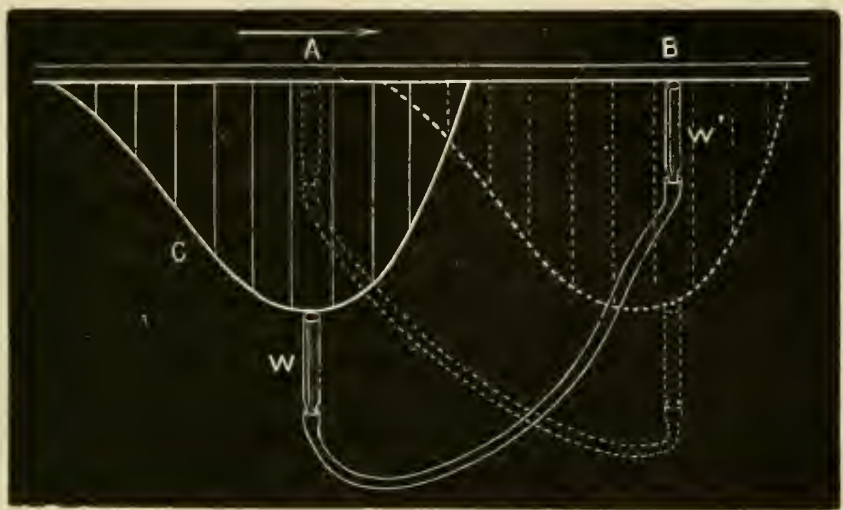

Fig. 268.-Diagrail to 1Llustrate Propagation of tile Electrical Change along an Active Muscle or Nerve.

Suppose AB to be a horizontal bar representing the muscle or nerve. Let C bc a curved piece of wood representing the curve of the electrical change at any point. Let $\mathrm{W}, \mathrm{W}^{\prime}$ be two glass cylinders connected by a flexible tube, the whole being filled with water. Suppose the rims of the cylinders originally to touch $\mathrm{AB}$ at the points $\mathrm{A}$ and $\mathrm{B}$, and let them be movable only in the vertical direction. The level of the water being the same in both, there is no tendency for it to flow from one to the other. This represents the resting state of the tissue when $A$ and $\mathrm{B}$ are symmetrical points. Now let $\mathrm{C}$ be moved along the bar at a uniform rate. The cylinder $\mathrm{W}$, being free to move down, but not horizontally, will be displaced by $\mathrm{C}$, and, if it is kept always in contact with its curved margin, will, after describing the curve of the electrical variation, come again to rest in its old position at $\mathrm{A}$. B will do the same when $\mathrm{C}$ reaches it. But since $\mathrm{C}$ reaches $\mathrm{A}$ before $\mathrm{B}$, the level of the water in $\mathrm{B}$ will at first be higher than that in $\mathrm{A}$, and water will flow from $B$ to $A$ as the current flows through the galvanometer. This will correspond to the time during which the point of the tissue represented by $A$ would be galvanometrically negative to a point represented by B. Later on, when $\mathrm{C}$ has reached the position shown by the dotted lines, the level of the water in $\mathrm{A}$ will be higher than that in $\mathrm{B}$, and a flow will take place in the opposite direction to the first flow. This corresponds to a second phase of the electrical variation.

Current of Action, or Negative Variation.-When a muscle or nerve is excited, an electrical change sweeps over it in the form of a wave. Suppose two points, A and B (Fig. 268), on the 
longitudinal surface of a muscle to be connected with a capillary electrometer (P. 62I), the movements of the mercury being photographed on a travelling surface, for example, a pendulum carrying a sensitive plate. Let the muscle be excited at the end, so that the wave of excitation will he propagated in the direction of the arrow. The wave will reach $A$ first, and while it has not yet reached $\mathrm{B}, \mathrm{A}$ will become negative to $\mathrm{B}$. It there is a resting difference of potential between $\mathrm{A}$ and 13 , this will be altered, the new and transitory difference adding itself algebraically to the old. When the wave reaches $1 B$, it may already have passed over $A$ altogether, and B now becoming negative to $\mathrm{A}$, there will be a morement of the meniscus of the electrometer in the opposite direction. This is called the diphasic current of action. If the wave has not passed over $A$ before it reaches $B$, as would in general be the case in an actual experiment, there will be first a period during which $A$ is relatively

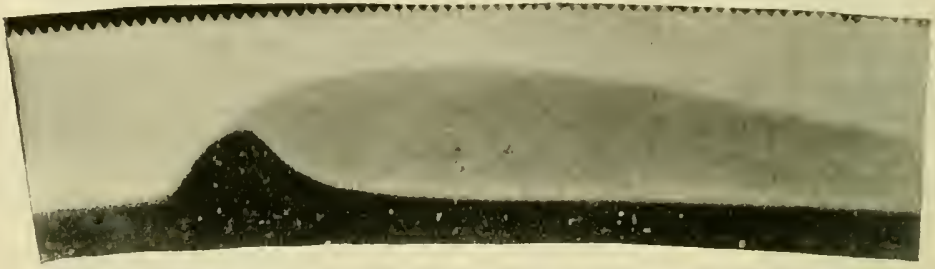

Fig. 269.-Photographic Electrometer Curves from Sartokius MUSCle (SANDERSON).

The darkly-shaded curve represents the diphasic variation of the uninjured muscle; the lightly-shaded curve the monophasic variation of the muscle after injury of one end. The toothed curve at the top is the time-tracing registered by photographing the prong of a tuning-fork vibrating five hundred times a second.

negative to $\mathrm{B}$ (first phase) ; this will end as soon as $\mathrm{B}$ has become iso-electric with $\mathrm{A}$, and will be succeeded by a period during which $B$ is relatively negative to $A$ (second phase). Since the wave takes time to reach its maximum, it is evident that a well-marked first phase will be favoured when the interval between its arrival at $\mathrm{A}$ and at $\mathrm{B}$ is long, for in this case $\mathrm{A}$ will have a chance of becoming strongly negative while $B$ is still normal. Similarly, if A has again become normal, or nearly normal, before the maximum negative change has passed over $B$, a strong second phase will be favonred. The heart-muscle, accordingly, where the wave of contraction, and its accompanying electrical change, move with comparative slowness, is better suited for showing a well-marked diphasic variation than skeletal muscle, and still better suited than nerve. In the gastrocnemius muscle of the frog, when excited through its nerve, the electrical 
response begins about $\frac{1}{100}$ second, and the change of form of the muscle about 1000 second after the stimulation. It is believed that in a muscle directly excited the electrical change begins in less than $\frac{1}{1000}$ second, and the mechanical change in 1000 second (Burdon Sanderson, Figs. 270-274).

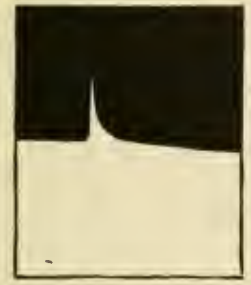

A.

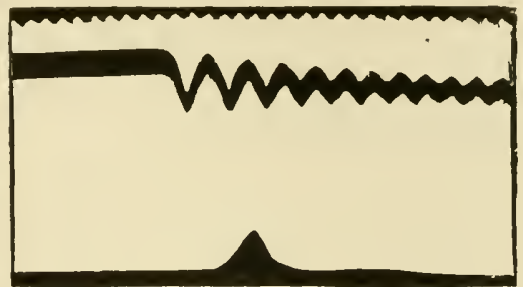

B.

Fig. 270.- 'Spike' (Diphasic Variation) of Uninjured Gastrocnemius (SANDERSON).

A photographed on slow, B on fast-moving plate.

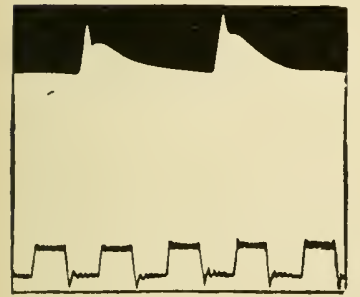

Fig. 271.-VARIATION OF InJUREd Gastrocnemius (SANDERSON).

A 'spike' followed by a 'hump.

\section{Numum}

FIG. 273.-VARIATION OF UNIN. JURed Muscle excited Eighty. Four Times a Second (Sander. SON).

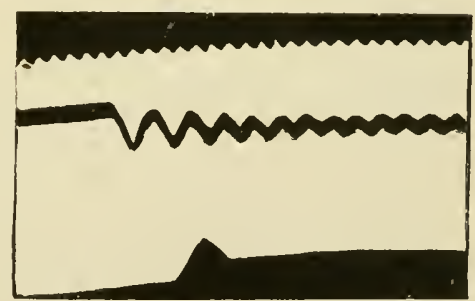

Fig. 272.-VARIATION OF INJURED Gastrocnemius (SANDERSON)

The plate was moving ten times faster than in Fig. $27 \mathrm{r}$.

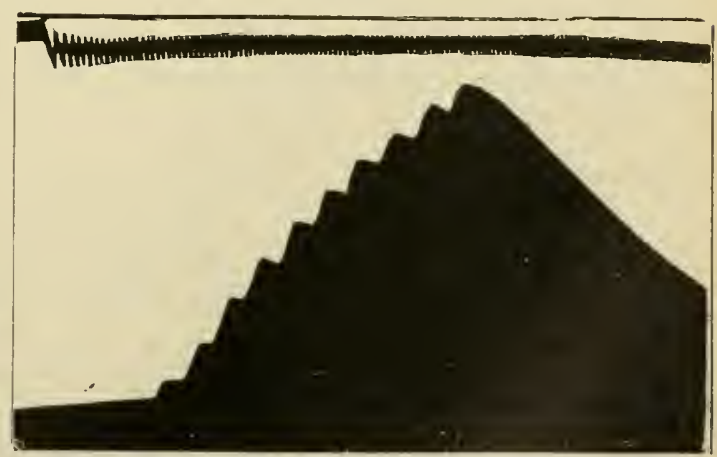

Fig. 274. - Curve of aN INJured MUSCle Excited Sixty Times a Second (Sanderson). 
When one electrode is placed on an injured part, the wave of action and of electrical change diminishes as it reaches the injured tissue; and if the tissue is killed at this part, it diminishes to zero; so that here the second phase may be greatly weakened or may disappear altogether, and we then have what is called a monophasic variation.

In this case the current of action can be demonstratel, even for a single cxcitation, but still better for a tetanus, with the galvanometer, which in general is not quick enough to analye a diphasic variation with cqual phases, and gives, therefore, only their algebraic sum-that is, zero. When the muscle or nerve is tetanized, the action current appears, while stimulation is kept up, as a permanent deflection representing the 'sum' of the separatc effects. It is in the opposite clirection to the current of rest, since the injureel tissue, being less affected by the excitation, and therefore undergoing a smaller negative change than the uninjured, becomes relatively to the latter less negative. Appearing as a diminution or reversal of the current of rest it was called the negative variation. The term negative is not used here in its electrical, but in its algebraic, sense, and merely as indicating the direction of the current with reference to that of the demarcation current. It is in this sense that "negative variation' and the converse term, 'positive variation,' are used (pp. 734.735) in speaking of the electrical changes produced in glands and in the retina by stimulation.

When the current of rest is compensated by a branch of an external current just sufficient to balance it and bring the galvanometer image back to zcro (Fig. 209, p. 620), the action current appears alone in uncliminished strength. This shows that the latter is not due to a change of clectrical resistance during excitation. since such a change would equally affect current of rest and compensating current, and they would still balance each other. The action current is really duc to a change of potential, which can be measurcd by determining what electromotive force is just required to balance it, and which may. actually exceed that of the current of rest. Thus, Sanderson and Gotch obtained an average of 0.08 of a Danicll cell (the clectromotive force of the Danicll would be about a volt) as the clectromotive force of the action current due to a single indirect excitation of a vigorous frog's gastrocncmius, and about $0^{\circ} \mathrm{O}_{4}$ Daniell as that of the current of rest. The elcctromotive force of the current of rest in the rabbit's nerve was found by du Bois-Reymond to be 0.026 ; (iotch and IIorsley found the average for the cat $0^{\circ} 01$, and for the monkcy only $0^{\circ} 005$.

That the fusion of the successive variations of a tetanizcd muscle, as seen with the galvanometer, is only apparent has been shown by means of the capillary clectrometer. Even with a frequency of stimulation far beyond what is necessary for complete tetanus, each stimulus is answered by a movement of the meniscus (Figs. 273, 274). In nerve, also, each of two successive stimuli causes its appropriatc electrical change when they are scparated by an interval longer than a certain small fraction of a sccond. The precise interval at which the sccond stimulus ccases to be effective depends on the temperature of the nerve, being markedly increased by cold (Gotch and Burch).":

Before Burdon Sanderson introduced the capillary electrometer for the study of the electrical phenomena of living tissucs, and Burch 
perfected a method for the measurement of the curves, the differential rheotome, originally constructed by Bernstein, was the most valuable instrument we possessed for experiments on the time relations of these phenomena. By its aid, for instance, it was shown that the rate of propagation of the clectrical change in muscle is the same as that of the meclanical $\mathrm{cl}^{-\prime}$ ' $\mathrm{ge}$, and in nerve the same as that of the nervous impulse. Observations on muscle made by the capillary clectrometer and the string galvanometer have confirmed those results. The velocity of propagation of the diphasic variation along a fresh sartorius at $\mathrm{I}^{\circ} \mathrm{C}$. Was in one experiment 2.8 metres, in another at $\mathrm{I}^{\circ}$ C., 3.5 metres (Sanderson). (Sec p. 659.) Lucas has pointed out that in strict accuracy what is observed is mercly that the time interval separating contraction at one point of the inuscle from contraction at another is equal to the time interval separating the electrical changes which occur at the same points. The facts observed do not formally prove that cither the contraction or the electrical clisturbance is propagated at all. So far as they go, some other perfectly distinct change may be propagated, which at all points of the fibre at which it arrives sets up both the contraction and the electrical change. Such direct evidence, however, as we possess goes to show that it is the clectrical disturbance which is the propagated one, and that this evolies the contractile disturbance.

The differential rheotome (Fig. 275) consists cssentially of a stationary metal ring, the whole or part of which is graduated, and of a portion which can be made to revolve at a known rate. The latter carrics two contacts: $a$, an obliquely - placed platinum wire which touches at every

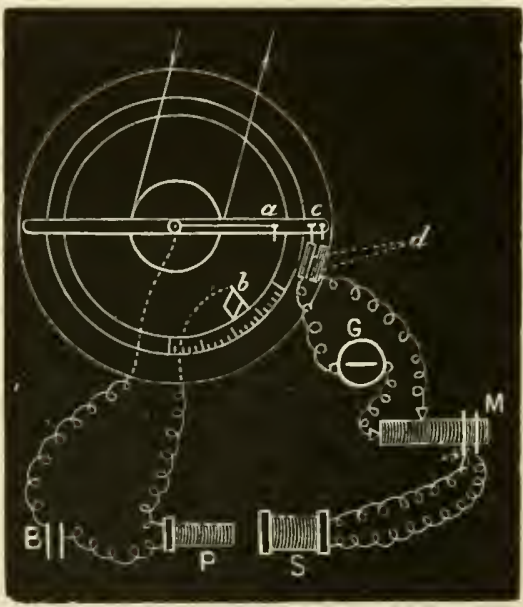

FIG. 275.-DIAGRAM OF DIFFERENTIAL RHEOTOME. revolution a horizontal wire $b$ on the fixed ring, thus making and breaking the primary circuit $P$ of an induction machine, and so causing stimulation of a muscle or nerve II connected with the secondary 'S; and, $c$, a double contact, either in the form of two platinum wires, which dip into two mercury troughs, or of two wire brushes rubbing on copper blocks $d$, at a certain part of the revolution. The troughs or blocks are connccted with a circuit containing a galvanometer $\mathrm{G}$, and a portion of the muscle or nerve arranged so as to give a strong action current. This circuit is completed by the wires or brushes, which are in metallic contact with each other; and the relative position of the fixed contact in the primary circuit and of the troughs or copper blocks can be altered so as to alter at will the interval between stimulation and closure of the galvanometer circuit. The proportion of the whole revolution during which this circuit is closed can be varied by changing the relative position of 
the two copper blocks. Suppose the tissue is stimulated at one end while the lealing-off electrodes are at the other. When the contact $a, b$ is made at the same time as $c, d$, no deflection will be shown by the galvanometer if the rheotome is revolving rapidly (the demarcation current being accurately compensated), because the circuit will be opened before the positive change has time to travel to the leaclingoff electrorles. But as the distance between $b$ and $d$ is increased, a small deflection will appear, which, with further increase of the distance, will become larger, reach a maximum, and then begin to fall off again. The first small deflection corresponds to the position in which the positive change has just had time to reach the learling-off electrodes before the galvanometer circuit is opened. The maximum leflection corresponds to a perind a little later than this, because the clectrical variation does not at once reach its maximum at any point.

There is ample evidence that the excitatory electrical response is a normal physiological phenomenon. In liuman skeletal muscles the current of action has been demonstrated by connecting a galvanometer with ring electrodes passing round the forearm, and throwing the muscles into contraction. A diphasic variation is thus obtained; and the electrical change travels with a velocity of as much as twelve metres per second, which is greater than the velocity in frogs' muscles. Electromotive changes are likewise associated with the beat of the heart. Action currents have also been detected in the phrenic nerves of living animals accompanying the respiratory discharge (Reid and Macdonald), in the vagi accompanying the movements of the lungs, in the oesophagus during swallowing, in the cutaneous sensory nerves in response to the 'adequate' stimulus of pressure (Steinach), in the retina in response to the adequate stimulus of light, in glands during secretion, in the central nervous system during the passage of impulses along its conducting paths. Some of these will be further considered a little later on.

As to the interpretation of the facts we have been describing, and which are summed up in the three propositions on p. 718 , two chicf loctrines long divided the physiological world: (I) the theory of du Bois-Reymond, the pionecr of clectro-physiology, and (2) the theory of Hermann. It was belicved by du Bois-Reymond that the current of rest seen in injured tissues is of eleep physiological import, and that the electrical difference which gives rise to it is not developed by the lesion as such, but only unmaskerl when the electrical balance is upset by injury. He looked upon the muscle or nerve as built up of electromotive particles, with lefinite positive and negative surfaces arranged in a regular manner in a sort of ground-substance which is electrically indifferent. The "negative variation' he supposed to depend on an actual diminution of previously cxisting clectromotive forces; and from this conception arose its historic name. Hermann and his school assumed that the uninjured muscle or nerve is 'streamless,' not because equal and opposite electromotive forces exactly balance each other in the substance of the tissue, but because electromotive forces are absent until they are called into existence (by chemical changes) at the 
boundary, or plane of elemarcation, between sound and injured tissue. For this reason du Bois-Reymond's current of rest is called in the terminology of Hermann the 'demarcation' current.

The newer theories, such as Macdonald's, liave sought to take acconnt of the recent (levelopments of physical chemistry, and it is unquestionable that it is here the real explanation is to be found. There is little (loubt that the electrical phenomena of the tissues are connected with the existence in them of $11 \mathrm{embranes,} \mathrm{envelopes,}$ or sheaths, pliysiological if not always anatomical, which are relittively impermeable to certain ions. When such a sheath is injured, these ions, carrying with them their electrical charges, may be supposecl to migrate with abnormal frecdom througl the injured part. A new distribution of electricity is thus established in the tissuc, and differences of potential depending upon differences in the concentration of the ions at different points are set up. Bernstein and Iscliermak, from an investigation of the thermodynamic relations of bio-electrical currents, have come to the conclusion that they are analogous to the currents produced by so-called concentration cells-i.e., arrangements of solutions of electrolytes of different concentration in contact with each other. Since the development of the new elcetrical condition depends upon the funclamental structure of the tissue, these modern views lead us back to du Bois-lieymond's doctrine of a pre-existing electrical equilibrium connected with the essential physiological properties of muscle or nerve. But instead of his electromotive elements and their definite arrangement, we have the ions and their definite relation to the semi-permeable membranes.

Relation between the Action Current and Functional Activity.Although the negative variation is so general an accompaniment of excitation, and is cven within tolerably wide limits, in muscle and nerve at least, pretty nearly proportional to the strength of the stimulus, it is at present impossible to say definitely what the chemical or pliysical changes are which underlie it. Unquestionably the electrical changes are closely related to the excitatory process and to the functional activity of the tissues.

Like the demarcation current, the action current and the excitation which accompanies it may be due to changes in the permeability of membranes or changes in the concentration of certain ions.

Although the electromotive changes caused by excitation are much more transient than those caused by in jury, everything suggests that there must be some deep analogy between the two conditions. Some have supposed that what may be called a subdued and more or less permanent excitation exists in the neighbourhood of the injured tissue, an excitation which, like some other forms, does not spread, and that this explains the similarity of electrical condition in activity and injury.

It is, of course, clear that cnergy must be transformed to produce an elcctromotive force capable of doing work. It may be assumed that this cnergy is ultimately derived from the stock of chemical energy in the tissue-substance. But whether in the final transformation the electrical plenomena are the expression of chemical changes or of physical (osmotic) changes, or of both, we do not know. Bernstein has supposed that in the chemical process, whose visible outcome is a muscular contraction, there are three stages: (I) The liberation of (intra-molecular) oxygen from the molecules of the living substance, and its appearance as active or atomic 
oxygen (p. 40I); (2) Oxidation of the contractile substance by this oxygen ; (3) The assimilation of oxygen by the contractile substance -i.e., its passage into the molecules of this substance (p. 26.4). According to him, it is the first of these stages which is associated with the abrupt development of the difference of potential between the excited and uncxcited portions of the muscle which we call the negative variation. This first stage he assumes to be completed before the visible contraction begins; and he originally asserted that the same was true of the negative variation. It is now known that the latter, although it begins before the contraction, and very rapidly reaches its maximum, declines more gradually, so that it overlaps the mechanical change of form. This is particularly well seen in veratrinized muscles (p. 65.), in which the electrical variation, like the contraction, is greatly prolonged (Garten). Nevertheless, Bernstein's theory, even with this limitation, agrees with what is known as to the influence exerted on the action current by the mechanical conclitions of the contraction. For the electrical cliange is little, if at all, affected by the tension of the muscle or the load it has to lift; and this is what we should expect it if depends on a process which is mainly completed before the contraction begins.

Polarization of Muscle and Nerve.-We have already spoken of electrical excitation and of the changes of excitability caused by the passage of a constant current (p. 683). We are now to see that these physiological effects are accompanied by, and indeed very closely related to, more physical changes which the galvanometer or electrometer reveals to us. Since these throw light on the physical, and therefore ultimately on the physiological structure of the tissues, they have been deeply studied, especially in nerve. There is no question that they depend upon the presence in the tissues of membranes presenting a relatively great resistance to the passage of ions. When a current is passed by means of unpolarizable electrodes (Fig. 2I3, p. 625) through a muscle or nerve for several seconds, and the tissue thrown on to the galvanometer immediately after this polarizing current is opened, a deflection is seen indicating a current (negative polarization current) in the opposite direction.

This (negative) polarization, like the polarization of the electrodes seen after passage of a current through any ordinary electrolytic conductor, dilute sulphuric acid, e.g., depends on the liberation of ions (p. 4OI) at the kathode and anode. It is seen not only in muscle, nerve, and other animal tissues. but also in vegetable structures, and indeed, to a certain extent. in unorganized porous bodics soaked with electrolytes. In muscle and nerve, however, it is particularly well marked; and although it is not bound up with the life of the tissue, and may be obtained when this has become quite inexcitable, it is nevertheless dependent on the preservation of the normal structure, for a boiled muscle shows but little negative polarization.

When the polarizing current is strong, and its time of closure short, we obtain. on connecting the tissue with the galvanometer after opening the current, not a negative, but a positive deflection, 
indicating a current in the sanne direction as that of the polarizing strean. This is really an action stream, due to the opening excitation set up at the anole (p. 637). It is only obtained when the tissuc is living, and is far more strongly marked in the anodic than in the kathodic region.

Suppose that the nerve in lig. 276 is stimulated by the opening of the battery $B$, and that, immediately after, the nerve is connected with the galvanometer $\mathrm{G}$ by the electrodes $\mathrm{E}, \mathrm{E}_{1}$. Suppose, further, that the shaded region near the anode remains more excited for a short time than the rest of the nerve, and we have seen $(p, 684)$ that after the opening of a strong current there is a defect of conductivity, especially in the neighbourhood of the anode, which would tend to

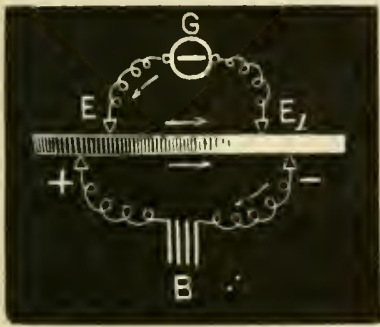

FIG. 276.-DIAGRAM TO SHOW Distribution of 'Positive Polarization' after open. ing Polarizing Current.

$B$, battery ; $G$, galvanometer. The dark shading signifies that the cxcitation to which the current causing the positive deflection after the opening of the polarizing current is due is greatest in the immediate neighbourhood of the anode, and fades away in the intrapolar region. + indicates the anode and - the kathode of the polarizing current.

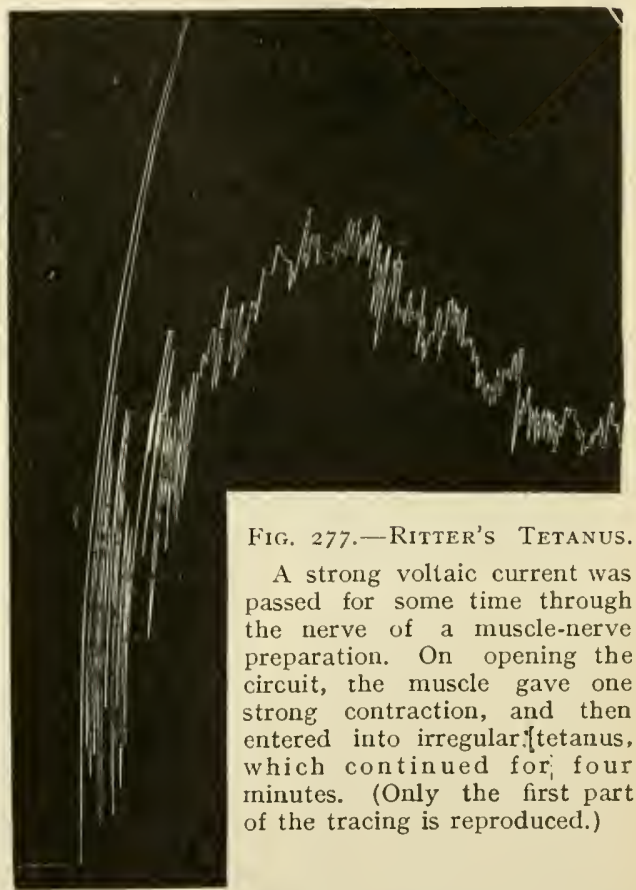

localize cxcitation. The portion of nerve at $\mathrm{E}$ bcing galvanometrically negative to that at $\mathrm{E}_{1}$, an action current will pass through the galvanometer from $\mathrm{E}_{1}$ to $\mathrm{E}$, and through the nerve in the same direction as the original stimulating stream.

Under certain conditions a state of continuous excitation in the anodic region of a nerve is shown by a tetanus of its muscle (Ritter's tetanus, p. 636, and Fig. 277).

Grützner and Tigerstedt liave put forward a different theory of the break contraction. They say it is really a closing contraction due to the closure of the negative polarization current through the tissue itself, as soon as the polarizing current is opened. But while stimulation does sometimes take place in this way, the contention 
of these observers that there is only one kind of electrical stimulus, the kathodic, or make, has not been clearly established.

Electrotonic Currents.-If a current be passed from the battery through a medullated nerve (Fig. 278) in the direction indicated by the arrows, while a galvanometer is connected with either of the extrapolar areas, as shown in the figure, a current will pass through the galvanometer, in the same direction in the nerve as the polarizing current, so long as the latter continues to flow.

These currents are called electrotonic (in the kathodic region katelectrotonic; in the anodic, anelectrotonic). The exact mode of their production is obscure. Similar currents can be detected in artificial models consisting of a good conducting core. and a badly

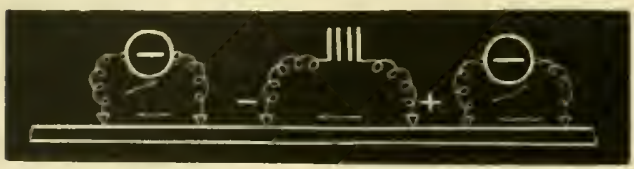

FIG. 278.-DIAGRAM SHOWING DIRECTION OF THE Extrapolar ElectrotoNic CURRENTS.

+ is the anode and - the kathode of the polarizing current.

(separation of ions) at the boundlary between the core and the sheath -i.e., between the wire and the liquid, where the current passes from the one to the other.

A current led into the sheath tries, so to speak, to pass mostly by the good conducting wire. If this is not polarizable-if it is, e.g., a zinc wire surrounded by saturated zinc sulphate solution-there is little or no spreading of the current outside the electrodes : it passes at once into the core, and so on to the other electrode. If, however, there is polarization when the current passes from the liquid into the wire, as is the case in the platinum-zinc sulphate, or the zinc-sodium chloride combinations, the stream spreads longitudinally in the sheath since the polarization introduces a virtual resistance at the surface of the wire, in comparison with which the difference in resistance of an oblique and a direct transverse path through the liquid becomes small. It has been supposed that in medullaterl nerve a similar polarization takes place at the bounclary between some part of the nerve-fibre which nay be called a core, and another part which may be called a sheath-for instance, between the axiscylinder and the medullary sheath, or between the latter and the neurilemma. It is known that the electrical resistance of nerve in the transverse direction is much greater (five to seven times)* than the longitudinal resistance. Since a rapidly-establishel polarization would, by the ordinary methods of measurement, appear as a resistance, this has been adduced as evidence of the great capacity of nerve for polarization by a current passing across the fibres. It is, however. probable, from what we know of the high electrical

* Since a part of the current is conducted by the connective tissue and other structures lying between the nerve-fibres, and the longitudinal and transverse resistance of these tissues may be supposed equal, the disproportion between the longitudinal and transverse resistance of the nerve-fibres themselves is probably much greater than this. 
resistance of the pliysiological envelopes of such cells as the red bloodcorpuscles (p. 25), that the great transverse resistance of nerve, and indeed the electrotonic currents, are lue in part if not wholly to the true resistance of one or more of its envelopes (perhaps the medullary sheath). Examples of such differences of resistance even in the fluid constituents of one and the same animal structure are not wanting. For instance, the specific resistance of the yolk of a hen's egg may be three times greater than that of the white.

The electrotonic currents cannot spread beyond a ligature; they are stopped by anything which destroys the structure of the tissue ; they are affected by various reagents. But this loes not prove that they are other than physical in origin, for what destroys the structure of the tissue or modifies its molecular condition may destroy or climinish its capacity for polarization,or alter its electrical resistance.

There are, however, certain facts which indicate that physiological factors, as well as physical, are concerned. While the currents obtained fron core-models show a general resemblance to the electrotonic currents of medullated nerve, there is one significant difference: in the former the katelectrotonic and anelectrotonic currents are of equal intensity; in the latter the anelectrotonic preponderates. The most probable explanation is that the anelectrotonic current of medullated nerve is made up of two distinct electrical effects, one physiological in nature, the other dependent merely on the structure and physical properties of the fibres, while the katelectrotonic current is wholly physical. It is in favour of this hypothesis that under the influence of ether, which abolishes the physiological functions of nerve, the anclectrotonic current diminishes till it becomes equal to the katelectrotonic. Non-

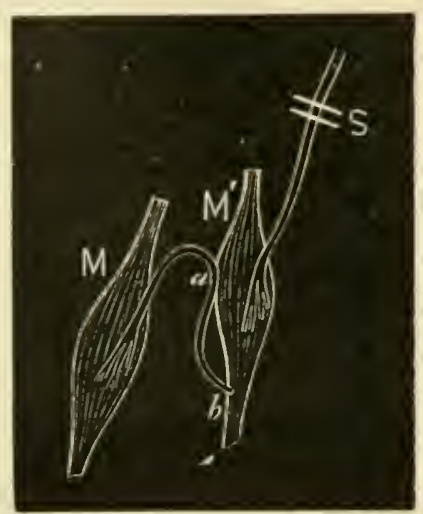

Fig. 279.-Secondary CoNtrac. TIOX.

The nerve of muscle $\mathrm{M}$ touches muscle $\mathrm{M}^{\prime}$ at $a$ and $b$. Stimulation of the nerve of $\mathrm{MI}^{\prime}$ at $\mathrm{S}$ causes contraction of $\mathrm{M}$. medullated nerves, in which the conditions for physical electrotonus, if present at all, are only feebly developed, and which exhibit no katelectrotonic current, or only a very weak onc, show an electrotonic current, which is abolished by ether, and seems to represent the physiological portion of the anclectrotonic current of medullated nerve.

A nerve may be stimulated by an electrotonic current produced in nerve-fibres lying in contact with it. A well-known illustration of this is the experiment known as the paradoxical contraction (Practical Exercises, p. 74I).

The current of action of a nerve can also stimulate another nerve when the excitability of both is greater than normal, as is the case in the nerves of frogs kept in the cold. This comes under the head of secondary contraction. But the best-known form of secondary contraction is where a nerve, placed on a muscle so as to touch it in two points (Fig. 279), is stimulated 
by the action-current of the muscle, and causes its own muscle to contract. A secondary tetanus can be obtained in this way by dropping a nerve on an artificially tetanized muscle. The beat of the heart causes usually only a single secondary contraction when the sciatic nerve of a frog is allowed to fall on it (1). ISS). But when the diphasic variation is well marked, as it is in an uninjured heart, there may be a secondary contraction for each phase--i.e., two for each heart-beat. Excitation of one muscle may in the same way cause secondary contraction of another with which it is in close contact.

The electromotive phenomena of the heart and of the central

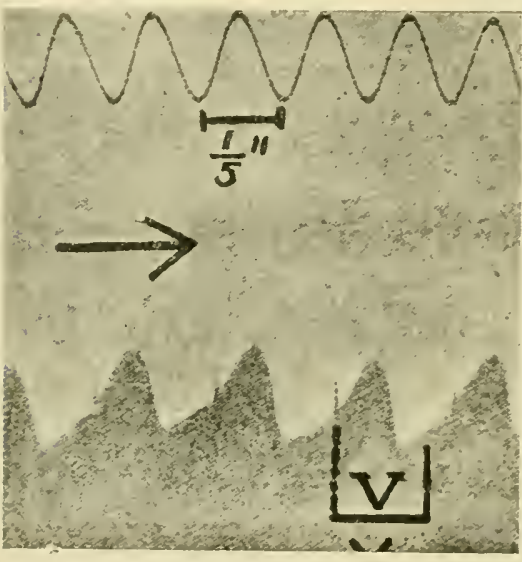

Fig. 2So. - Electrometer Record from Rabilt's Heart (Gotch).

The heart was exposed and beating in situ. Contacts, one on base of right ventricle, the other on right apex. The commencement of the beat is on the left-hand edge of the dark line $\mathrm{V}$. The length of the dark line shows the duration of the beat. Upward movement signifies relative negativity (activity) of the part at or near the base contact. Time-trace at top, one-fifth seconds. nervous system are naturally included under those of muscle and nerve.

Heart.-Records of the electrical changes obtained with the capillary electrometer from the exposed ventricles vary in certain details with the position of the two contacts. They indicate that in the mammalian ventricles the relative negativity corresponding to the contraction begins at the base, then derelops in the region of the apex, and finally involves the portion of the ventricles near the aorta and pulmonary artery; possibly extending even into the roots of these vessels. When one contact is on the base of the rentricles (in the rabbit) near the auriculo-rentricular groove, and the other on the apes, the record is quadriphasic-i.c., the sign of the electrical disturbance (as shown by the change of direction of movement of the mercury) changes four times (Fig. 280). For each beat of the ventricle the electrometer record show's (I) a sharp rise, indicatiug relative negativity (activity) of the base; (2) an equally sharp fall, indicating relative negativity at the apex; (3) a slower but marked rise, indicating an increase or a fresh development of relative negativity at the base; (4) a more rapid fall, which returns first slowly, 
then quickly, until (I) follows again (Gotch). The time between the beginning and the top) of rise ( $\mathrm{I}$ ) is believed to correspond to the time of transmission of the active state from the base to the apex. The rate of propagation on the rabbit's ventricle varies from I to 3 metres a second, according to the rate of the heartbeat. The fourth phase is due to the fact that the effect in the neighbourhood of the aorta (and pulmonary artery) is more transient than the apes effect. By altering the position of the contacts the record can be made diphasic, or even triphasic.

In the rentricle of the frog and tortoise the same order of derelopment of the negative change is seen, the base first becoming relatively negative, then the apex, and then the neighbourhood of the origin of the aorta (Fig. 2SI).

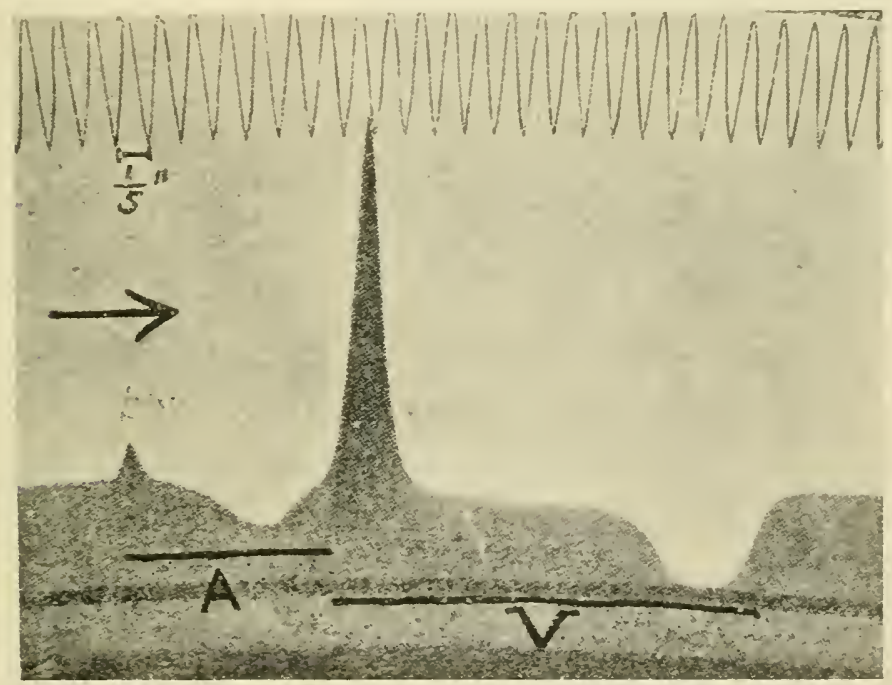

Fig. $2 S$ i.-Electroneter Record froy Tortoise Heart (Gotch).

One contact upon the sinus, the other on the apex of the ventricle. One complete beat shown. Upward movement signifies relative negativity of the sinus contact. The dark line A shows the auricular effect, and the dark line $\mathrm{V}$ the ventricular effect. Time-trace at top, one-fifth seconds.

Under certain conditions the action current of the heart may stimulate the pbrenic nerves, causing the diaphragm to contract synchronously with the heart.

The Human Electro-cardiogram.-An electrical change accompanies each beat of the human heart. Waller first showed how this may be demonstrated by means of the capillary electrometer.

Einthoven and Lint then investigated the phenomenon on a large number of persons. From the photographic records of the movements of the meniscus (Fig. 28z) they constructed the true 
electro-cardingraphic curves* (Fig. 283), which express the actual chinges in the potential difference between the two points led off. They distinguished in every one of these constructerl electro-cardingrams fise points or cusps, threc of which indicate relatise negativity of the bise of the heart to the atpex, and two negativity of the apex to the base. The capillary electrometer has now been largely superseded by the string galvanometer (p. Gio) for the investigation of the liuman electro-carliogram (ligs. $28+287$ ). Records are obtained by magnifying and photogritpling the movements of the quart\% fibre. The clectrocardiograms are listinctly affected by cxercise and by the position
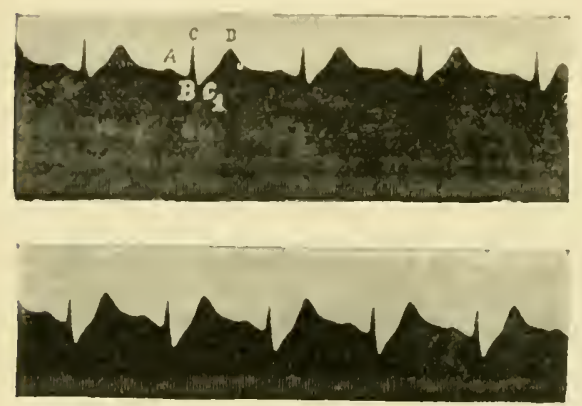

Fili. 282.- ELECTRO-CARDIOGRAMS FROM MAN (CAPILLARY ELECTROMETER), (ENNTIONEN AND LINT).

The image of the eapillary magnified eiglit hundred times was projected on a mosing photo. graphic plate. The figure is a reproduetion of the record (reduced to two-thirds of its original size) obtained from the same individual at rest (upper curve), and immediately after vigorous muscular exercise (lower curve). The novenents of a tuning-fork, inaking fifty complete vibrations a second, are shown below the cardiograms. The sulphuric acid pole of the electro. meter was connected with the thoracic wall in the neighbourhood of the apex of the heart, and the nercury with the right ann. The elevations A, C, D, indieate negativity of base to apex ; the notches $B$ and $C_{1}$, negativity of apex to base.
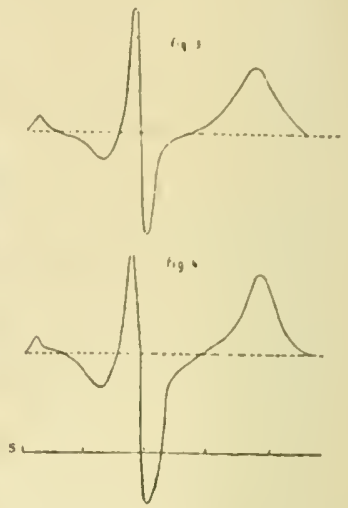

Fig. 283. - Constructed ELECTRO - CARDIOGRAMS FROM MAN (EINTIUTEN AN1) liNT).

The curves are coustructed from the photographic records shown un Fig. 282. These and not the photographs are the true expression of the actua changes of potential during the cardiac cycle. Time is laid off along the hurizontal, and electromotive furce along the vertical axis, the same: spice being allotted to ten millivolts (i.c., Ifo volt) as to one secund.

of the body, and very markedly in disease. They are being more and more employel in clinical investigations. The galvanometer may be connected with the two hands, or better, with the right hand and the left fool. Whe two feet are the most unfavinable combination.

Central Nervous System. - It was discovered by du Bois-Reymond that the spinal cord, like a nerve, shows a current of rest between

* In all accurate work with the capillary electrometer such curves must be obtainerl by construction from the direct photographic records, which do not themselves give an absolutely true picture of the variations. 
longitudinal surface and cross-section, and that a current of action is caused by excitation. Setschenow stated that when the meclulla oblongata of the frog was connected with a galvanometer, spontancous variations occurred which he supposed due to periodic

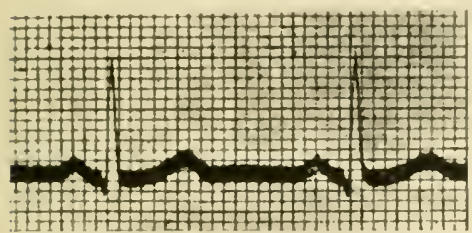

Fig. 2S +. - Human Electro cardo. GRAM (STRING GIEVNOMATER). (EINTIOIFE).

Led off from the two hands. I mm. df the absrissa correspouds to 0 or second.

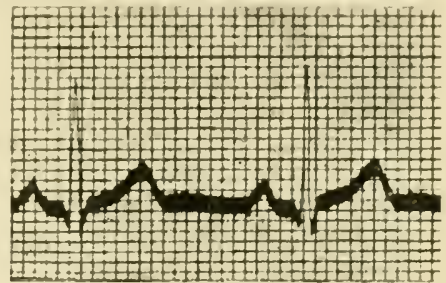

FIG, 285.-HUMAN EIECTRO•CARDIOGRAM (StRING GALYANOMETER). (EINTHOTEX). foot.

led off from right hand and left

0,1, Sec

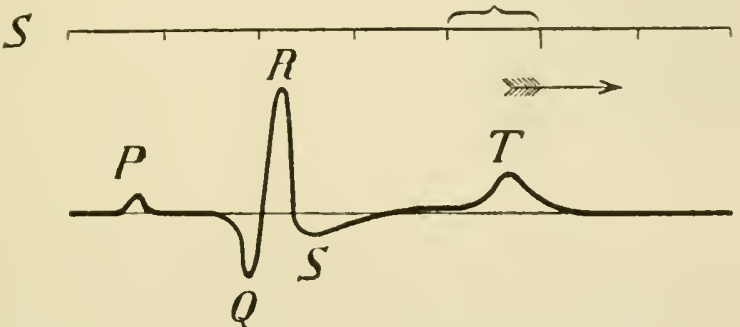

Fig. 2S6.-Schematic Represextation of Electro.cardiogram (String GalvaNometer), (EINTHOVEN).

Five points are lettered at which the curve changes sign. P. corresponds to the auricular contraction; the other four are included in the ventricular crcle.

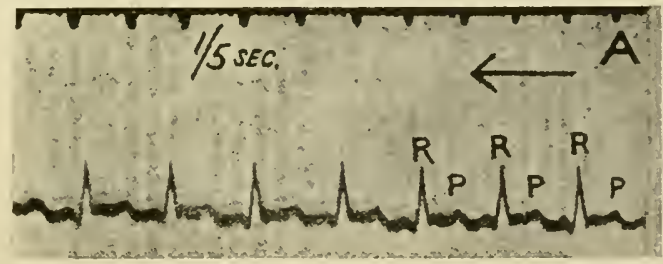

Fig. 287.-Electro-cardiogran from Man (String Galvanoneter), (Lewis).

From a case of paroxysmal tachycardia. The heart-rate was 200 a minute.

The upper notched line is the time-trace in one-fifth seconds.

functional changes in its grey matter. Gotch and Horsley have made elaborate experiments on the spinal cords of cats and monkeys. Leading off from an isolated portion of the dorsal cord to the capillary electrometer, and stimulating the " motor" region of the 
cortex cerebri, they obtained a persistent negative variation followed by a series of intermittent variations. This agrees remarkably with the muscular contractions in an epileptiform convulsion started by a similar excitation of the cortex. which consist of a tonic spasm followed by clonic or pliasic (interrupterl) contractions.

By means of the galvanometer the same observers have made investigations on the paths by which impulses set up at lifferent points travel along the cord. To these we shall have to refer again (1), 7(13).

Electrical Phenomena of Glands. - These have been studied with any care chicfly in the submaxillary gland and in the skin, although the liver, kiclney, spleen, and other organs also show currents when injured. In the submaxillary gland the hilus is galvanometrically positive to any point on the external surface of the gland : a current passes from hilus to surface through the galvanometer, and from surface to hilus through the gland (Fig. 288). When the chorda tympani is stimulated with rapidly-succeding shocks of

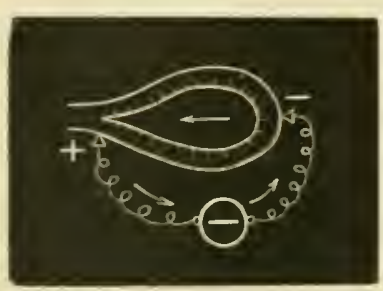

FIG. 2Mis.-CERREXIT OF SiBMAXILARY GLAXo. moderate strength, there is a positive variation -i.e.. the hilus becomes stiH more positive to the surface. This variation can be abolished by a small dose of atropine.

Skin Currents. - So far as has been investigated, the integument of all animals shorrs a permanent current passing in the shin from the external surface inwards. This is feebler in skin which possesses no glands. In skin containing glands the current is chicfly, but not altogether, secretory. As such. it is affected by influences which affect secretion, a positive variation being caused by excitation of secretory nerves - $\ell . g$. . in the parl of the cat's foct by stimulation of the sciatic. The deflection obtained when a finger of each hand is led off to the galvanometer. which was at one time looked upon as a proof of the existence of currents of rest in intact muscles, is duc to a secretion current.

()f more loubtful origin is the current of ciliated mucous membrane. which has the same direction as that of the skin cf the frog and the mucous membrane of the stomacli of the frog and rabbitviz.. from ciliated to under surface through the tissue, or from ciliated surface to cross-section. if that is the way in which it is led off. The current is strengthened by induction shocks, by heating. and in general by influences which increase the activity of the cilia. Some circumstances point to the goblet-cells in the membrane as the source of the current; but. On the whole, the balance of evidence is in farour of the cilia being the chief factor (Engelmann). although the mucin-secreting cells may be concerned, too. Electrical changes associated with secretion have been observed in the frog's tongue on excitation cf the glosso-pharyngeal nerve.

Eye-currents.-If two unpolarizable electrodes connected with a galvanometer are placed on the excised eye of a frog or rabbit. one on the cornea and the other on the cut optic nerve. or on the posterior surface of the eyeball. it is found that a current passes in the eye from optic nerve to cornea, the funclus of the eye being 
therefore negative as regards the cornea (Fig. 289). The current has the same direction if the anterior electrode is placed on the anterior surface of the retinil itself, the front of the eycball being cut away, or if one electrode is in contact with the anterior and the other with the posterior surface of the isolated retina. There is notbing of special interest in this; but the important point is that if light be now allowed to fall upon the eye, or upon the isolated retina. characteristic electrical changes are caused. These are spoken of ats the photo-electric reaction, and are best studied by means of the string galvanometer. The features of the curve representing the photo-electric reaction vary with the duration and intensity of the illumination and with the previous condition of the eye as regards illumination. A careful analysis of the curves obtained under different conditions supports the hypothesis that there occur in the eye three separate processes, which may for convenience

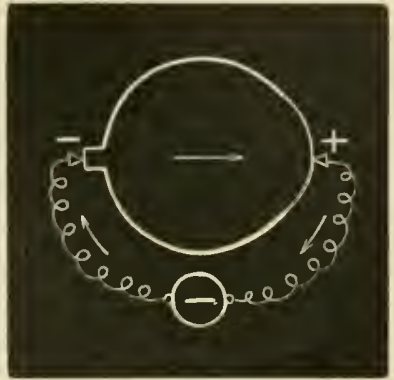

FIG. 2S9.-EYE.CURRENT. be considered to depend upon the existence in the retina of three separate photo-chemical substances (p. 934). When light of moderate intensity is allowed to act upon an eye which has not shortly before been exposed to strong light, a form of curve is obtained which seems to represent the combined reaction of the three substances (Einthoven and Jolly) (Fig. 290). After a latent

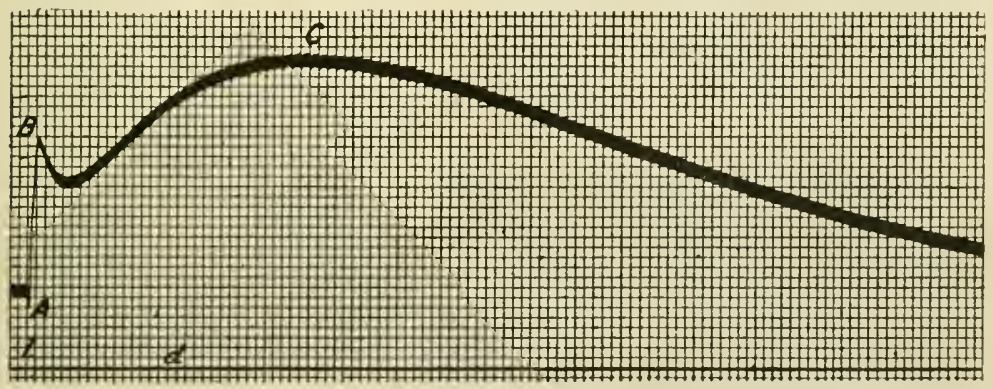

$$
\begin{aligned}
& \text { Fig. 290.-Photo-electric Reaction of Frog's Eye (Einthoven zand } \\
& \text { Jolly). }
\end{aligned}
$$

The duration of the flash (of green light) was o.o second. The eye had been previously in the dark. I millimetre of the abscissa corresponds to $0^{\circ} 5$ second, I millimetre of the ordinate to ro microvolts. Curve to be read from left to right.

period a small preliminary negative deflection A is observed (downward movement of the string). This is at once followed by a much larger upward movement (positive variation) in the same direction as the resting effect, the fundus becoming relatively more negative to the cornea than before. After the peak B has been reached, the curve sinks first rapidly, then more gradually, but soon mounts again, and reaches a second maximum $C$, which is higher than $B$ 
(second positive variation). Finally, the curve descends to its original level.* The photo-electric reaction is substantially the sane in all vertebrate eyes hitherto investigated. lin the cephalopod retina, too, the only important electrical change on illumination is in the same direction as the resting effect.

The reaction depends upon the retina alone, and does not occur when it is removed. Bleaching of the visual purple loes not much affect it, so that it is not connecterl with chemical changes in this

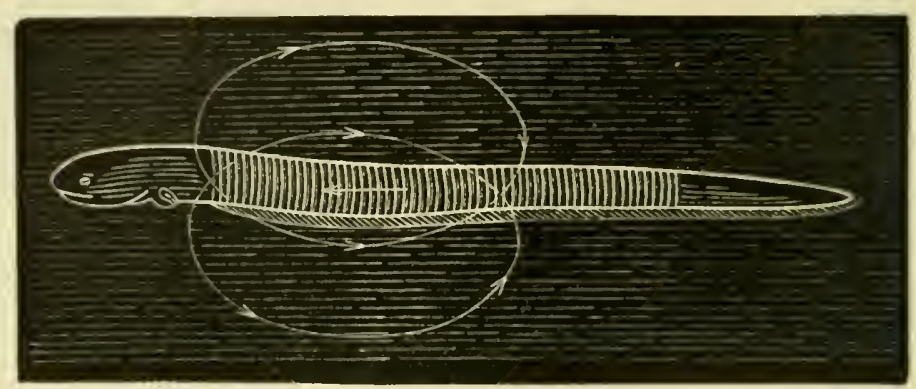

Fig. 291.-Diagram Showing Direction of Shock iN Gyanotus.

substance. Its seat must be the layer of rods and cones, since in the cephalopols the structure called the retina contains only this layer, the other layers of the vertebrate retina being represented in the optic nerve and ganglion (Beck). Of the spectral colours, yellow light causes the largest variation; blue, the least; but white light is more powerful than either (Dewar and Mckendrick). (For 'visual purple,' see p. 934.

Electric Fishes.-Except lightning, the shocks of these"fishes were probably the first manifestations of electricity observed by man.

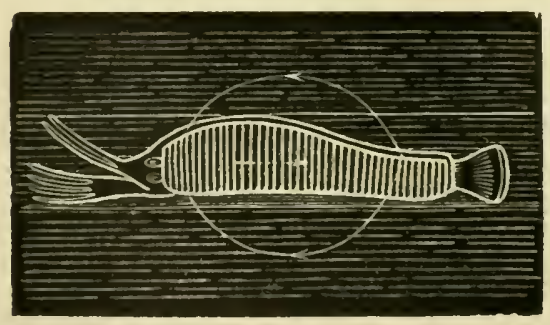

FIG. 292.-DIAGRAM SIIOWIXG DIRECTION OF SHOCK IX MaLAPTERIRUS. The Torpedo, or electrical ray, of the coasts of Europe was known to the Greeks and Romans. It is mentioned in the writings of Aristotle and Pliny, and had the honour of being described in verse $\mathrm{I}, 500$ years before Faraday made the first really exact investigation of the shock of the Gymnotus, or electric eel, of South America. Another of the electric fishes, Malapterurus electricus, although found in many of the African rivers, the Nile in particular, and known for ages, was scarcely investigated till fifty years ago.

In all these fishes there is a special bilateral organ immediately under the skin. called the electrical organ. It is in this that the shock is developed. It consists of a series of plates arranged parallel to each other. To one side of each plate a branch of the electrical

* In the figure the last portion of the curve while it is still slowly descending has not been reproduced. 
nerve supplying each lateral half of the organ is distributed, so that each half of the organ represents a bittery of many cells arrangerl in series.

In Gymnotus the plates are vertical, and at right angles to the long axis of the fish, and the nerves are distributed to their posterior surface; the shock passes in the animal from tail to head. In Malaptorurus, although the direction of the plates is the same, and the nerve-supply is also to the posterior surface, the shock passes from heal to tail.

In Torpedo, the plates or septa dividing the vertical hexagonal prisms of which each lateral half of the organ consists are horizontal : the nerve-supply is to the lower or ventral surface; and the shock passes from belly to back through the organ. In all electric fishes the lischarge is liscontinuous; an active fish may give as many as 200 shocks per second.

The electrical nerve of Malapterurus is very peculiar. It consists of a single gigantic nerve-fibre on each sidle, arising from a giant nerve-cell. The fibre has an enormously thick sheath, the axis-cylincler forming a relatively small part of the whole; and the branches which supply the plates of the organ are divisions of this single axis-cylinder.

The electromotive force of the shock of the Gymnotus may be very considerable; and even Torpado and Malapterurus are quite able to kill other fish, their enemies or their prey. Indeed, Gotch has estimated the electromotive force of $I \mathrm{~cm}$. of the organ of Torpedo at 5 volts. Schönlein finds that the electromotive force of the whole organ may be equal to that of 3 [ Danicll cells, or 0.08 volt for each plate, and it is one of

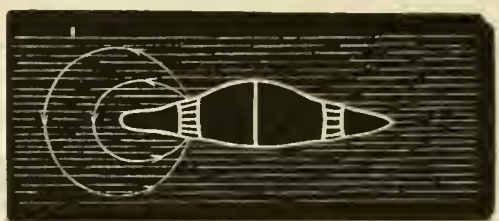

Fig. 293.-Ditgram Showisg DirecTION OF SHOCK IN TORPEDO.

the most interesting questions in the whole of electro-physiology. how they are protected from their own curzents. There is no doubt that the current density inside the fish must be at least as great as in any part of the water surrounding it, and probably much greater. The central nervous system and the great nerves must be struck by strong shocks, yet the fish itself is not injured; nay, more, the young in the uterus of the viviparous Torperlo are unharmed. The only explanation seems to be that the tissues of electric fishes are far less excitable to electrical stimuli than the tissues of other animals ; and this is found to be the case when their muscles or nerves are tested with galvanic or induction currents. It requires extremely strong currents to stimulate them; and the electrical nerves are more easily excited mechanically, as by ligaturing or pinching, than electrically. In general, too, the shock is more readily called forth by reflex mechanical stimulation of the skin than by electrical stimulation. But that the organ itself is excitable by electricity has been shown by Gotch. He proved that in Torpedo a current passed in the normal direction of the shock is strengthened, and a current passed in the opposite direction weakened, by the development of an action current in the direction of the shock. And, indeed, a single excitation of the electrical nerve is followed by a series of electrical oscillations in the organ. which gradually die away. The latent period of a single 
shock is about $\frac{1}{.20}$ second. The skate must be includer in the list of electric fishes. Although its organ is relatively small, and its electromotive force relatively feeble, yet it is in all respects a conplete electrical organ. It is situated on either side of the vertebral column in the tail. The plates or discs are placed transversely and in vertical planes. The nerves enter their anterior surfaces; the shock passes in the organ from anterior to posterior end. Gotch and Sanderson have estimated the maxinum electromotive force of a length of $\mathrm{I} \mathrm{cm}$. of the electrical organ of the skate at about half a rolt.

Whether the electrical organ is the homologue of muscle or of nerve-ending, or whether it is related to either, has been much discussed. Our surest guide in a question of this sort is the study of development; and researches along this line liave shown that there are two kinds of electrical organ, one being modified muscle (as in Gymnotus, Torpedo, and the skate) ; the other transformed skinglands (as in Malapterurus). The scanty blood-supply of the electrical organs in comparison with that of muscle is noteworthy. In no case (lo bloodvessels enter the substance of the plates.

\section{PRACTICAL EXERCISES ON CHAPTER XII.}

1. Galvani's Experiment.-Pith a frog (brain and cord). Cut through the backbone above the urostyle, and clear away the anterior portion of the body and the viscera. Pass a copper hook beneath the two sciatic plexuses, and hang the legs by the look on an iron triporl. If the tripod has been painted, the paint must be scraped away where the hook is in contact with it. Now tilt the tripod so that the legs come in contact with one of the iron feet. Whenever this happens, the circuit for the current set up by the contact of the copper and iron is completed, the nerves are stimulated, and the muscles contract ( $\mathrm{J} .7 \mathrm{r} 7$ ).

2. Make a muscle-nerve preparation from the same frog. Crush the muscle near the tendo Achillis, so as to cause a strong (lemarcation current. Cut off the end of the sciatic nerve. Then lift the nerve with a small brush or thin glass rod, and let its cross-section fall on or near the injured part of the muscle. Every time the nerve touches the muscle a part of the denarcation current passes through it, stimulates the nerve, and causes contraction of the muscle (p. 7I 7 ).

3. Secondary Contraction.-Make two muscle-nerve preparations. Lay the cross-section of one of the sciatic nerves on the inuscle of the other preparation (Fig. 279, p. 729). Place under the nerve near its cut end a small piece of glazed paper or of glass rod, and let the longitudinal surface of the nerve come in contact with the muscle beyond this. Lay the nerve of the other preparation on electrodes connected with an induction machine arranged for single shocks, with a Daniell cell and a spring key in the primary circuit (Fig. 258, p. 703). On closing or opening the key both muscles contract. Arrange the induction macline for an interrupted current. When it is thrown into one nerve, both muscles are tetanized; the nerve lying on the muscle whose nerve is directly stimulated is excited by the action current of the muscle. 
4. Demarcation Current and Current of Action with Capillary Electrometer. - (a) Stucly the construction of the capillary clectrometer (Fig. 210, p. 021). Raise the glass reservoir by the rack and pinion screw, so as to bring the meniscus of the mercury into the field. Llace two moistened fingers on the binding-screws of the electrometer, open the small key connecting them, and notice that the mercury moves. a difference of potential between the two bindingscrews being caused by the moistened fingers.

(b) Demarcalion Current. - Set up a pair of unpolarizable clectrodes (Fig. 213, p, -625). Fill the glass tubes about one-third iull of kaolin mixed witl physiological salt solution till it can be easily moulded. To do this, make a piece of the clay in to a little roll, which will slip down the tubc. Then with a match push it down until it forms a firm plug. Next put some saturated zinc sulphate solution in the tubes, above the clay, with a fine-pointed pipette. Fasten the tubes in the holder fixed in the moist chamber (Fig. 294). Now amalgamate the small pieces of zinc wire (p. I 8?), which $:$ are to be connected with the binding-screws of the chamber. (Or use Porter's 'boot' electrodes. These are made of unglazed potter's clay. In use the leg of the boot is half-filled with saturated zinc sulphate solution, into which dips a thick amalgamated zinc wire. In the foot of the

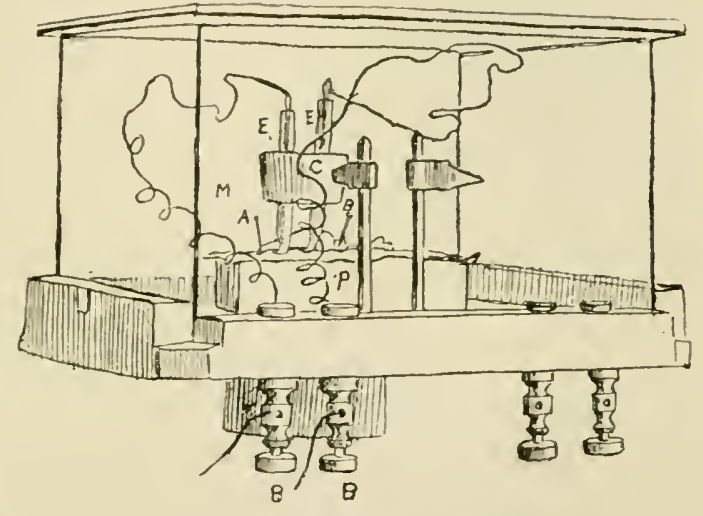

Fig. 294.-MOIST CHAMBER.

E, unpolarizable electrodes supported in the cork C; II, muscle stretched over the electrodes and lkept in position by the pins $A, B$, stuck in the cork plate $P$; $\mathrm{B}$, binding-screws connected with galvanometer or capillary electrometer. The other pair of binding. screws serves to connect a pair of stimulating electrodes inside the chamber with the secondary coil of an induc. tion machine.

boot is a hollow

(or well) which is filled with physiological salt solution and serves to keep the feet well moistened with the salt solution. The nerve is laid on the feet of the boots. When not in use the boots should be kept in physiological salt solution.)

The zincs are now placed in the tubes, dipping into the zinc sulphate. A piece of clay or blotting-paper moistened with physiological salt solution is laid across the elcctrodes to complete the circuit between their points, and they are connected with the electrometer to test whether they have been properly set up. There ought to be little. if any, movement of the mercury on opening the side-key of the electrometer. If the movement is large, the electrodes are 'polarized,' and must be set up again. The second pair of binding-screws in the chamber are connected with a pair of platinum-pointed electrodes on the one side, and on the other, through a short-cir- 
cuiting liey. with the secondiury coil of an induction machine arranged for tetanis.

Next pith a frog (cord and brain), and make a muscle-nerve preparation. Injure the muscle near the tenclo . Ichillis. I.ay the injured part over one unpolarizable clectrole, and an uninjured part over tie other. Put a wet sponge in the chamber to kecp) the air moist, and place the glats lid on it. Focus the meniscus of the mercury; and open the key of the electrometer; the mercury will move, perhaps right out of the ficld. Note the direction of movement, and remembering that the real direction is the opposite of the apparent direction, and that when the mercury in the capillary tube is comnected with a part of the muscle which is relatively positive to that connected with the sulphuric acisl. the movement is from capillary to acid, determine which is the galvanometrically positive and which the negative portion of the muscle (1). 7 r 8).

(c) Action Current.- Iow, without disturbing its position on the electroles, fasten the muscle to the cork or paraffin plate in the inoist chamber by pins thrust through the lower end of the fomur and the tendo Achillis. Lay the nerve on the platinum electrodes. Open the key of the electrometer, and let the menisciss come to rest. This happens very quickly, as the capillary electrometer has but little incrtia. If the meniscus has shot out of the field, it must be brought back by raising or lowering the rescrvoir. Stimulate the nerve by opening the key in the secondary circuit: the meniscus mores in the direction opposite to its former morement.

(d) Repeat $(b)$ and $(c)$ with the nerve alone, laying an injured part (crushed, cut, or overheated) on one electrode, and an mninjured part on the other. Of course, the nerve dloes not need to be pinned.

Clean the unpolarizable electrolles, and be sure to lower the reservoir of the electrometer; otherwise the mercury may reach the point of the capillary tube and run out.

In $f$ a galvanometer (p. 617) may be used with advantage by students, if one is available, instead of the clectrometer. the unpolarizable electroles being connected to it through a shortcircuiting key. The spot of light is brought (o) the milllle of the scale by moving the control-magnet; or if a telescope-realing (Fig. 206, p. 6IS) is being used, the zero of the scale is brought by the same means to coincicle with the vertical hair-line of the telescope. The short-circuiting key is then opened.

5. Action-current of Heart.-Pith a frog (brain and corl). Excise the heart, and lay the base on one unpolarizable clectrole, and the apex on the other, having a sufficiently large pad of clay on the tips of the electrolles to insure contact during the movements of the heart, or having little cups hollowerl in the clay and filled with pluvsiological salt solution, into which the organ dips. Connect the electrodes with the capillary electrometer and open its key. At each beat of the heart the mercury will move (1). 73(3).

6. Electrotonus. - Set up two pairs of unpolarizable electrodes in the moist chamber. Connect two of them with a capillary electrometer (or galvanometer), and two with a battery of three or four small Danicll cells, as in Fig. 278 . Lay a frog's nerve on the electroles. When the liey in the battery circuit is closed, the mercury (or the. needle of the galvanoneter) moves in such a direction as to indicate that in the extrapolar regions parts of the nerve nearer to the anode are relatively positive to parts more remote. and parts nearer to the kathode are relatively negative to parts more remote. 
The direction of movement of the mercury (or galvanometer needle) must be male out first for one direction of the polirizing current. Then the latter must be reversed, and the movement of the mercury (or necule) on closing it again noted (p. 728).

7. Paradoxical Contraction.-P'lth a frog (brain and corcl). 1) iss.ct ont the sciatic nerve Iown to the point whare it splits into two divisions, one for the galstrocnemius $b$, and the other for tha paroncill muscles a. Divide the proneal branch as low down as possible, and make a muscle-neric preparation in the usual way. Lay the centrat enel of the peroneal nerve on electrodes connected through a simpl: key with a battery of two Danicll cells. Whan the paroncal nerve is stimulated the gatstrocnemius muscle contracts. This result is not clue to the curront of action, for it is not obtained with mechanical stimulation of the nerve; but it is not the result of an escape of current, for if the peroncal nerve be ligatured between the point of stimulation and the bifurcation, no contraction is obtained. The contraction is really (lue to a part of the electrotonic current set up in the peroncal ncrve passing through the fibres for the gastrocnemins, where they lic side by sicle in the trunk of the sciatic.

8. Alterations in Excitability and Conduc-

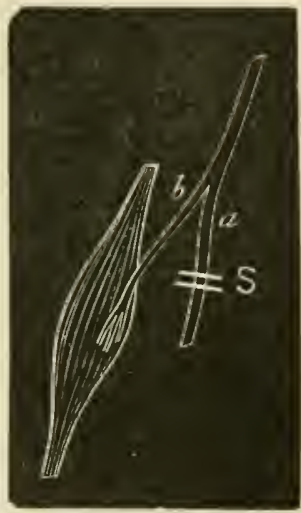

FIG. 295. - PARADOX1. CAL CONTRACTION.

tivity produced in Nerve by the Passage of a Voltaic Current through it.-(a) Set up two pairs of unpolarizable electrodes in the moist chamber. Conncet a battery of two or three Daniell cells, arranged in series through a simple key with the sicle-cups of a Pohl's commutator with cross-wires in. Connect the commutator to one pair of the

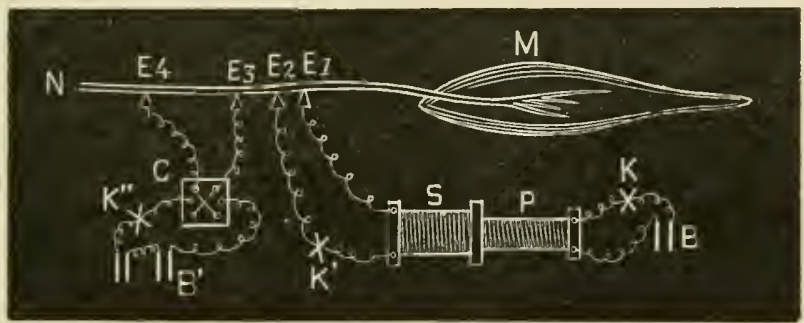

Fig. 296. - Arrangenent for Showing Changes of Excitability PRODUCED BY THE VOLTAIC CURRENT.

I, muscle ; $N$, nerve: $E_{1}, E_{2}$, electrodes connected with secondary coil S ; $E_{3}$, $\mathrm{E}_{4}$, unpolarizable electrodes connected with Pohl's commutator (with crosswires) C ; B', 'polarizing' battery ; $\mathrm{B}$, 'stimulating' battery in prinary' circuit $P$; $\mathbf{K}, \mathbf{K}^{\prime \prime}$, simple keys; $\mathbf{K}^{\prime}$, short-circuiting key.

unpolarizable electrodes (" the polarizing electrodes '), as in Fig. 296. The other pair of unpolarizablc clectrodes ("the stimulating electrodes ') are to be connected through a short-circuiting key with the secondary of an induction machine arranged for tetanus. A single Daniell is put in the primary coil. Pith a frog (brain and cord), make 
a muscle-nerve preparation, pin the lower end of the femur to the cork plate in the inoist chamber, attach the thread on the tenclo Achillis to the lever connected with the chamber through the hole in the glass provided for this purpose, and arrange the nerve on the electrodes so that the stimulating pair is between the muscle and the polarizing pair. By moving the sccondary, seek out such a strength of stimulus as just suffices to cause a weak tetanus when the polarizing current is not closed. Set the drum off (slow speed), and take a tracing of the contraction. Then close the polarizing current with a Pohl's commutator so arranged that the anode is next the stimulating elcetroles-i.e., the current ascending in the nerve. Again open the short-circuiting key in the secondary; the contraction will now be weaker than before, or no contraction at all may be obtained. Nllow the preparation two minutes to racover, then stimulate again, as a control, withont closing the polarizing current. If the contraction is of the same height as at first, close the polarizing current with the briclge of the conmutator reversed, so that the kathorle is now next the stimulating electrodes. On stimulating, the contraction will now be increased in height. (Sec Figs 253, 254, p. 683.)

(b) Arrange everything as in $(a)$, except that onc of the polarizing electrodes is placed at each end, and the two stimulating electrodes close together in the middle of the nerve. A large carbon resistance (say 500, 000 ohms) is introduced into the circuit of the secondary coil, to prevent more than a very small fraction of the polarizing current from passing through the coil. Seek out the strength of stimulation which just causes contraction when the polarizing current is not closed. Now clese the polarizing current in such a direction that the anode is between the stimulating electrodes and the muscle. If no contraction occurs on stimulation, push up the secondary towards the primary till the muscle contracts. Then stop the stimnlation, open the polarizing current, and allow an interval of two minutes. Now pass the polarizing current through the nerve in the opposite direction, so that the kathode is between the stimulating electroles and the muscies. No contraction will be obtained on exciting with the same strength of stimulus as caused contraction when the anole was next the muscle. The kathole has diminisherl the conductivity of the nerve; and if four or five small Daniell cells are put on in the polarizing circuit, no contraction may be obtained, even with the coils close together, while the excitation will still pass the anode and callse contraction.

9. Pfluger's Formula of Contraction (1). (184).-To ilemonstrate this, connect two minolarizable electrorles, through a spring key and a commutator, with a simple rheocord (Iiig. 200. P. 705). so as to lead off a twig of a current from a Danicll cell. The umpolarizable electrodes are placed in a moist chamber. A muscle-nerve preparation is arranged with the nerve on the electrodes and the muscle attached to a lever. The effects of make and break of a weak current, ascending and descending, can be worked out with the simple rheocord. The effects of a medium current will probably be obtained with a single Danicil connected directly with the electrodes throngh a key. The effects of a strong current will be got when three or four Daniells are connected with the electrodes. Care must be taken to licep the preparation in a moist atmosphere, and more than onc preparation may be necded to verify the whole formula. 
10. Formula of Contraction for (Human) Nerves in Situ. - Connect cight or ten dry cells in series. Connect one terminal of the battery to a l. rge plate electrode, and the other to a small electrocle, both cosered with cotton, flannel, or sponge, moistened with salt solution. 1 nclude in the circuit a simple key for making or breaking the current, and a commutator for changing its direction at will. Leave the key open. Place the large electrode behind the shoulder (or on the back of the neck), and the small electrode over the ulnar nerve at the clbow between the internal condyle and the olecranon. Arrange the commutator so that the small electrode shall be the kathode. Close, and then open the key. If no contraction occurs at closing, the battery is too weak, and more cells must be added. If contraction occurs at closing, but not at opening, reverse the commutator, making the small clectrode the anode, and observe whether contraction now occurs at closing, at opening, or at both. Note also the relative strength of the various contractions. If the current is 'weak' the only contraction will be a closing one when the kathode is over the nerve. If the current is of 'medium' strength, a closing kathodic contraction and both opening and closing anodic contractions will be obtained. With 'strong' currents contractions will occur at closing and at opening, whether the kathode or the anode is over the nerve. The contractions will vary in strength, as described on p. 686. To work out the different cases of the formula summarized in the table, the number of cells must be increased or diminished.

\begin{tabular}{cc|c} 
WeakCurrents. & Medium Currents. & Strong Currents. \\
KCC & KCC & KCC \\
- & ACC & ACC \\
- & AOC & AOC \\
\hline
\end{tabular}

The abbreviations $\mathrm{KCC}, \mathrm{ACC}$, are used respectively for liathodic closing contraction and anodic closing contraction; KOC, AOC, for kathodic opening contraction and anodic opening contraction. $\mathrm{KCC}$ is stronger than $\mathrm{KOC}$, and ACC than AOC. KCC is stronger than ACC, and AOC than KOC. Therefore, as the strength of the current is increased, in the case of normal tissues, $\mathrm{KCC}$ is first olstained, then $\mathrm{ACC}$, then $\mathrm{AOC}$, and finally KOC.

I r. Ritter's Tetanus. - Lay the nerve of a muscle-nerve preparation on a pair of unpolarizable electrodes connected through a simple key with a battery of three or four small Daniells. Connect the muscle with a lever. Pass an ascending current (anode next the muscle) for a few minutes through the nerve, and let the writingpoint trace on a slowly-moving drum. When the current is closed there may be a single momentary twitch, or the muscle may remain somewhat contracted (galvanotonus) as long as the current is allowed to pass, or it may continue to contract spasmodically (" closing tetanus"). When the current is opened the muscle will contract once, and then immediately relax, or there may be a more or less continued tetanus (Ritter's or 'opening tetanus'). If opening tetanus is obtained, divide the nerve between the electrodes : the tetanus continues. Divide it between the anode and the muscle : the tetan us at once disappears. This shows that the seat of the excitation which causes the tetanus is in the neighbourhood of the anode (p. 727). 


\section{CHAPTER XII}

\section{THE CENTRAL NERVOUS SYSTEM}

Ix other divisions of our subject we have been able to follow to a greater or less extent the processes which take place in the organs described. The chemistry and the physics of these processes have bulked more largely in our pages than the anatomy and histology of the tissues themselves. In dealing with the central nervous system we must adopt a method the very reverse of this. Its anatomical arrangement is excessively intricate. The events which take place in that tangle of fibre, cell, and fitril are, on the other hand, almost unknown. So that in the description of the physiology of the central nervous system we can as yet do little more than trace the paths by which impulses may pass between one portion of the system and another, and from the anatomical connections deduce, with more or less probability, the nature of the physiological nexus which its parts form with each other and the rest of the body. And here it may be well to remark that, although for convenience of treatment we have considered the general properties of nerves in a separate chapter, there is not only no fundamental distinction between the central nervous system and the outrunners which connect it with the periphery, but obviously a central nervous system would be meaningless and useless without afferent nerves to carry information to it from the outside, and efferent nerves along which its commands may be conducted to the peripheral organs.

\section{Structure of the Central Nervous System.}

In unravelling the complex structure of the central nervous system, we avail ourselves of information clerived (I) from its gross anatomy; (2) from its microscopical anatony; (3) from its development; (4) from what we may call, although the term is open to the criticism of eross-division, its physiological and pathological anatomy. 
Certain tracts of wlite or grey matter are lifferentiated from each other by the size of their fibres or cells. For cxample, the postero-median columu of the spinal cord lias small fibres, the direct cerebellar tract large fibres; the large pyramiclal cells (giant cells or cells of Betz), in what wc shall afterwards liave to distinguish as the 'nllotor area' (p. 8.t) of the cerebral cortex, are the cells of origin of fibres of the pyramidal tract subserving the volitional movements of the limbs and trunk. The pyramidal cells of the 'face area ' are comparatively small. In general, an efferent or motor nerve-cell is larger the longer its axon is-e.g.. the largest of all the pyramidal cells in the. 'motor' region are fonnd in the portion known as the 'leg area,' from which the pyramidal fibres have to pass all the way down the cord to the segments from which the spinal nerves going to the lower limbs arise.

The recent work of Brodman and of Campbell has shown that the ccrebral cortex may be histologically differentiated into regions which correspond to a great cxtent to the various functional regions mapped out by physiological methods (p. $85 \mathrm{r}$ ).

The study of development enables us not only to determine the homology, the morphological rank, of the various parts of the brain and cord. but also, by comparison of animals of different grades of organization, sometimes to decide the probable function and physiological importance of a strand of nerve-fibres or a column of nervecells. It is of special value in helping us to differentiate the various areas of grey matter on the surface of the brain. and to trace the various tracts or paths into which the white matter of the central ncrvous system may be divided. For the medullary sheath is not developed at the same time in all the tracts, and a strand of nervefibres in which it is wanting-e.g., the pyramidal tract (p. 776). which is the last of the spinal tracts to become myelinated-is readily distinguished under the microscope.

Then, again-and this is what we propose to include under the fourth head-experimental physiology and clinical and pathological observation throw light not only on the functions, but also on the structure, of the central nervous system. For instance, complete or partial section, or destruction by disease, of the white fibres of the cord or brain, or of the nerve-roots, or removal of portions of the grey matter, is followed by degeneration in definite tracts. And since, as we have already seen, degencration of a nerve-fibre is caused when it is cut off from the cell of which it is a process, the amount and distribution of such degeneration teaches us the extent and position of the central connections of the given tract. Conversely, the cells in which a tract of nerve-fibres arises may sometimes be identified by the alterations in the chromatin (p. 756$)$ and other changes which occur in them after scction of their axons. Particularly in young animals, removal of a peripheral organ-an eyc or a limb-or section of its nerves. may be followed by atrophy of portions of the central nervous system immediately related to it.

'Softening' of a definite portion of the white or grey matter may also in certain cases be caused by depriving it of its bloodsupply by the injection of artificial emboli, and the resulting degencrations may then be studied. For instance, fine particles like lycopodium spores are in jected in to the abclominal aorta between the origins of the renal and inferior mesenteric arteries. They are prevented by clamps from entering these vessels, and, passing through the lumbar arteries, stick in the branches of the anterior 
spinal artery, and cause softening mainly of the grey matter of the lumbar portion of the cord. When the abdominal aorta of a rabbit is temporarily compressed (for about an hour) below the origin of the renal arteries. the grey matter of the corresponding portion of the cord is so serionsly injured that it and the fibres that arise from it degenerate, while the fibres whose cclls of origin are not situated in this part of the grey matter are not affecterl, or at least completely recover.

Certain tracts may also be marked out by incaus of the electrical

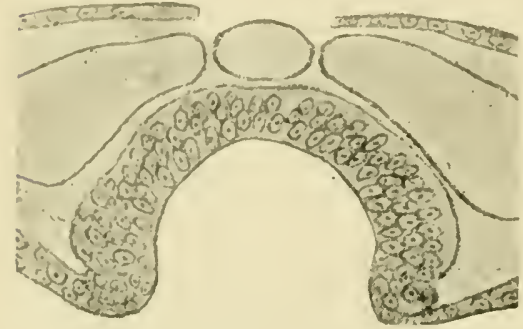

Fig. 297.-Formation OF tilf NeUral CANAL AT AN EARLY STAGE (AFTER BEARD). variation, which gives token of the passage of nervous impulses along them when portions of the central nervous system or peripheral nerves are stimulated (Horsley and Gotch).

Development of the Central Nervous System. - Very early in levelopment (Fig. 297) the kecl of the vertebrate embryo is laid down as a groove or gutter in the ectorlerm of the blastodermic area (Chap. $X I V$.). The walls of this 'medullary' or 'neural' gronve grow inwarls, and at length there is formed, by their coalescence. the 'newral canal' (Fig. 298), which expands at its anterior end to form four cerebral vesicles (Fig. 299). Thus there is a continuous tunnel from end to end of the primary cerebro-spinal axis ; and this persists as the central canal of the spinal cord and the ventricles of

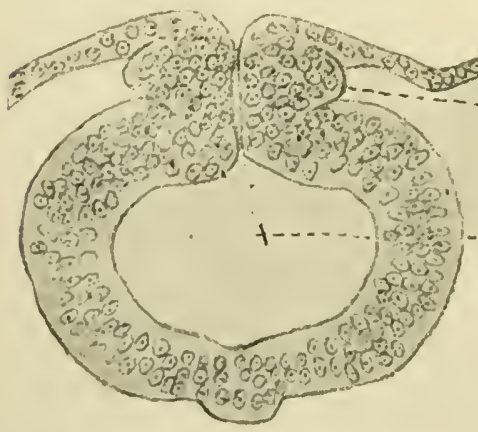

Frg. 298, -. NEURAL CAXAL AT A LATER STAGE (AFTER BEARD).

\section{मद्युल० \\ Porition of sfinal gangtion} neural camal

the brain, whose ciliated epithelium represents the cotodermic lining of the primitive neural canal In the adult portions of the canal may become obliterated from an overgrowth of the lining cells, and the cilia are, if present at all, less distinct than in the child, and far less distinct than in the lower animals. Fron the wall of this canal is formed the cerebro-spinal axis, in which developing nerve-cells or neuroblasts soon become differentiated from the supporting cells or spongioblasts, and wander outwards from the neighbourhood of the central canal (Fig. 309) till their further progress is checked by the barrier of the marginal veil, a closely-woven network or thicket, into which the processes of the spongioblasts break up at the outside of the primitive cercbro-spinal axis. Nlthougl the neuroblasts 
themselves are unable to penetrate the marginal veil, the axiscylinder processes of some of them do so, and form the motor root. of the spinal nerves. The neuroblasts from which the fibres of the white columns of the corl are developed are apparently unable to send their axons throngh the marginal veil. Tlicy are accordingly forced to assume a longitudinal direction, and in this way the central grey matter bccomes covered with a sheath of longitudinal white fibres. For a time only motor nerve-cells and the fibres connected with them are developerl in the cerebro-spinal axis. The ganglia on the posterior roots arise from a scries of ectolermic thickenings or sprouts from the newal crest which runs along the dorsal aspect of the neural canal. These sprouts contain the neuroblasts which develop into the spinal ganglion cells with the posterior root-fibres. lirom each pole of each neuroblast a process grows out, one towards the periphery, which forms a peripheral ncrve-fibre, the other centrally to connect the cell with the cord. lrom the afterbrain (or myclencephalon) is developed the medulla oblongata or spinal bulb, from the hind-brain (or metencephalon) the cerebelhm and pons, from the mid-brain (or mesencephalon) the corpora quadrigemina and crura cerebri. The fore-brain, or primary fore-brain (thalamencephalon) gives rise of itself only to the third ventricle and optic thalamus, but a secondary fore-brain (telencephalon) buds oft from it and soon tivides into two chambers, from the roof of which the cercbral hemispheres, and from the floor the corpora striata, are derived. Their cavities persist as the lateral ventricles, which conlmunicate with the third ventricle by the foramen of Monro. The olfactory tracts are formed as buls from the secondary fore-brain.

To complete the story of the development of the brain, it may bo added that the retina is really an

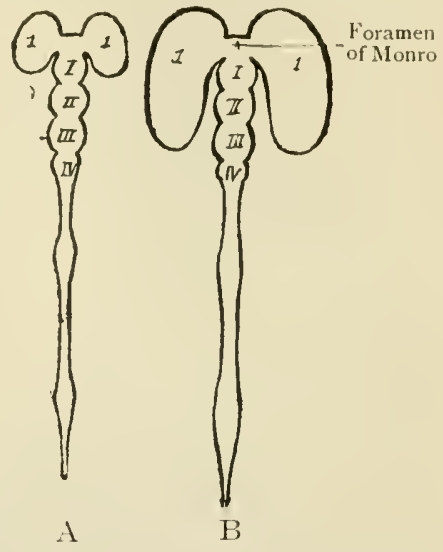

Fici, 209.-Diagran to illistrate THE FORMATION OF TIIE CEREBRAL VESICLES.

A. I indicates the cavity of the secondary fore-hrain, which eventually beeomes the lateral ventricles. In B the secondary fore-brain has grown backwards so as to overlap the wher vesicles. 1, first cerebral vesicle (primary for"-lorain or 'tween brain); 11, second cerebral vesicle (mid-brail) ; I11, third cerebral vesı. cle (hind-brain) ; IV, fourth cerebral vesicle (after-brain). expansion of its nervous substance. A hollow process, the optic vesicle, buds out on each side from the primary fore-brain. A button of ectoderm, which afterwards becomes the lens, grows against the vesicle and inclents it so that it becomes cup-shaped, the inner concave surface of the cup representing the retina proper, the outer convex surfacc the choroidal epithelium. The stalk becomes the optic nerve.

Histological Elements of the Central Nervous System.-The central nervous system is built up (I) of true nervous elements, (2) of supporting tissuc. The nervous elements have usually becn described as consisting of nerve-fibres and nerve-cells, but the antithesis of a 
time-honourerl dlistinction must not lead us to forget that the essential part of a nerve-fibre, the axis-cylinder, is a process of a nerve-cell, and the medullary sleath probably a product of the axis-cylinder.* In strictness, the term 'nerve-cell' ought to include not only the cell-boly. but all its processes, out to their last ramifications. But the habit of speaking of the position of the cell-bolyt as that of the nerve-cell is so ingrained. that it secms better to continuc the use of the latter term in its old signification, and to speak of the cell and branches together as a neuron (also spelled neurone).

The Neurons. - I typical nerve-cell (Figs. $300,3,2,3.4$ ) is a knot

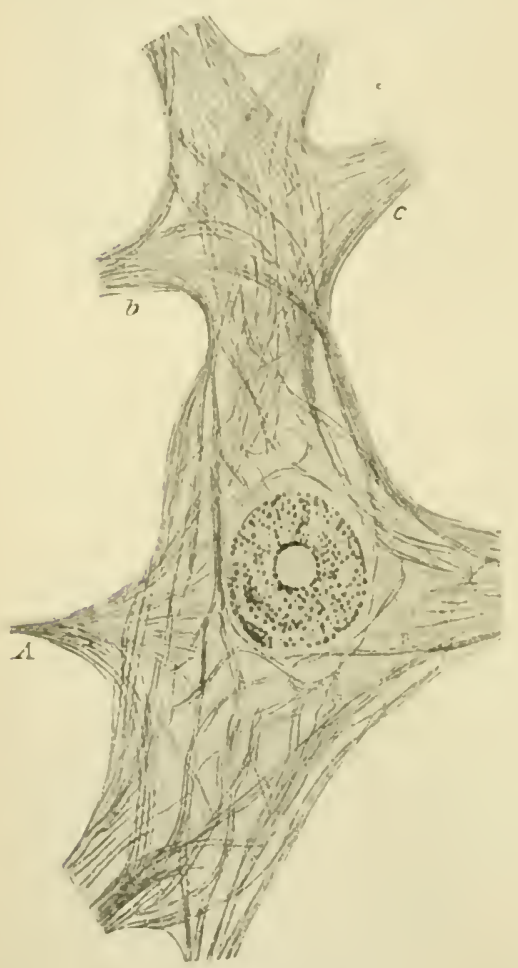

FiG. 300, - AxTERIOR HORN CELL FROM MAX SIOWIXG FIBRILS (BETIIE). of granular protoplasm, containing a large nucleus, insicle of which lics a highly refractive nucleolus. A centrosom c and attraction sphere (p. 5) have also been found in some nerve-cells, though not as vet demonstrated in all. Pigment may also be present, especially.

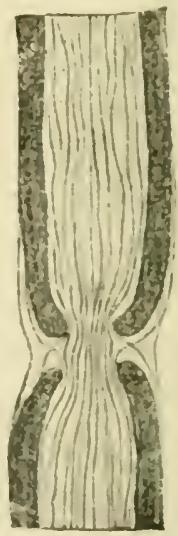

ViT, 301, - MEDLLLATED NERVIEFIBRE SHUWING FIBRILS OF Axis-Crltider (BETHi).

The tibrils are seen passing, withuul interruption, across a node of Ranvier.

in old age. By certain methods of staining it may be shown that fibrils (ncuro-fibrils) run through the protoplasm of the cell, forming a felt-Work in it. and entering the dendrites on the one hand and the

* The nuclei of the peripheral fibres belong to the neurilemma and not to the medullary sheath, and while the medullary sheath, like the axiscylincler, is as regards its nutrition under the control of the nerve-cell, and must therefore be looked upon as an integral portion of the neuron, the nenrilemma in respect both of its nutrition and its development appears to be an independent structure.

$\dagger$ Foster and sherrington call the cell-body the perikaryon. 
axis-cylinder process on the other (ligs. 300, 303. 307). In the axiscylinders of nerve-fibres the fibrils (ligg. 301) appear to preserve their identity down to the distribution of the fibre. ln the ground substance between the fibrils lie round, angular, or spindle-shaperl bodies (Nissl's bodies) which stain with basic dyes (Fig. 31 I).* These bodies vary in appearance in different kinds of nerve-cells, and in the same nerve-cell under different conditions. According to Macallum, they contain organically combined iron. In a mult ipolar cell, like those in the anterior liorn of the spinal cord, several processes - it may be five or six, or even more-pass off from the cell-body (Frontispiece). The most complete pictures of them are given by preparations impregnated according to the method of Crolgit (1igs. 302, 305). One of the processes of most nerve-cells is distinguished fron the rest by the fact that it maintains its original diameter for a comparatively great distance from the cell, and gives off comparatively few branches. This process, which in favourable preparations can be traced on till it becomes the axiscylinder of a nerve-fibre, is called the axis-cylinder process, or more shortly the axon. The few slender branches that come off from it, usually at right angles, are called collaterals. The collaterals consist essentially of one or more fibrils of the axon. Both the main thread of the axon and the collaterals end by breaking up into an arborescent system of fibrils or telodendrion. The telodendrions vary greatly in appearance from simple end-brushes to far-branching thickets, or such special end-organs as motor plates (Fig. 307) or muscular spindles (Fig. +33 , p. 983). The rest of the processes of the cell, which are termed dendrites or protoplasmic processes, very rapidly diminish in diameter, as they pass away from the cell, by breaking up into fibrils like the branches of a tree. The Nissl bodies extend for some distance into the dendrites, but not in to the axon. The dendrites of some cells, especially the pyramidal cells of the cerebral. and the Purkinje's cells of the cerebellar cortex, have small swellings, the so-called lateral buds or gemmules, on their course. Their significance is unknown. The dendrites terminate at a little distance from the cell, where they come into relation with the end-arborizations of the axons of other neurons. In this way two or more neurons are linked together to form a nervous path. According to the view most commonly held (neuron hypothesis), the relation is not one of actual anatomical continuity. but the processes come so close together that nerve impulses are able to pass across from the terminal brush of the axon of one nervous element to the (lendrites or cell-body of another. This kind of junction is called a synapse.

It has been suggested that the contact may be rendered more or less close through amoboid movements of the dendrites, and that in this way the nervous impulse may be switched like a railwaytrain from one path to another. But there is no experimental basis for this somewhat crude, if fascinating, hypothesis. Sherrington has suggested that the presence of a 'membrane' at the synapse

* In Nissl's method the sections are stained in a solution of methylene blue, and decolourized in anilin-alcohol.

$\dagger$ The method depends upon the deposition of mercury, or silver, in or around the cell-bodies and their processes in tissues which have been hardened in bichromate of potassium and then soaked in a solution of mercuric chloride or silver nitrate. In Pal's improvement of Golgi's method a solution of sodic sulphide follows the mercuric chloride. 


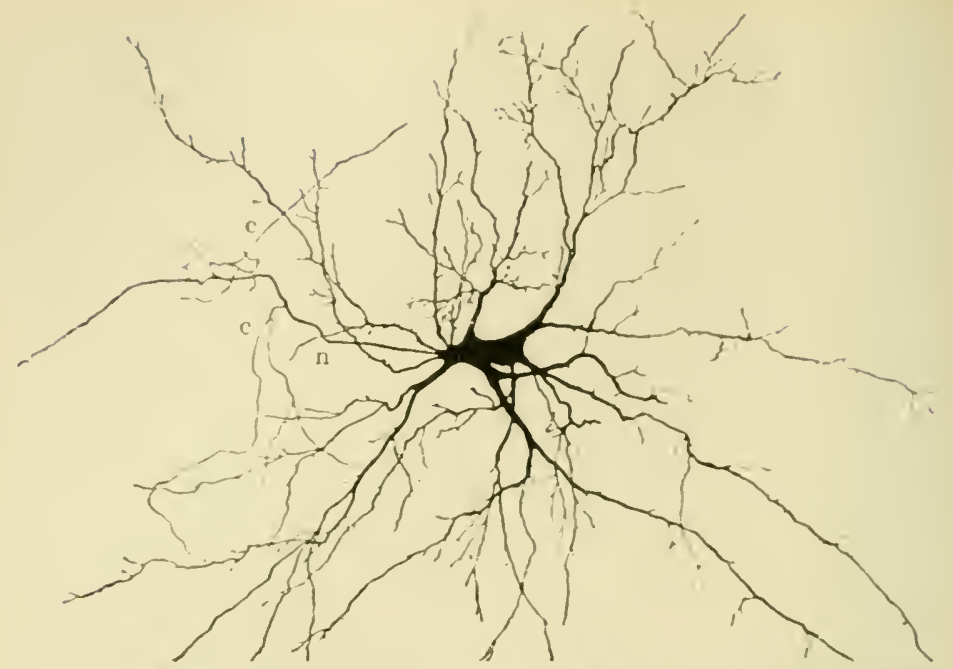

Fig. 302.-Multipolar Nerie-cell: Golgi Preparation (Barker, after KÖLLIKER).

n. axon : $c$, collaterals.

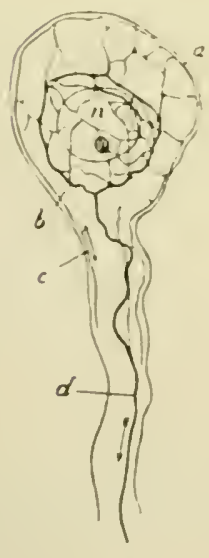

$\Delta$.

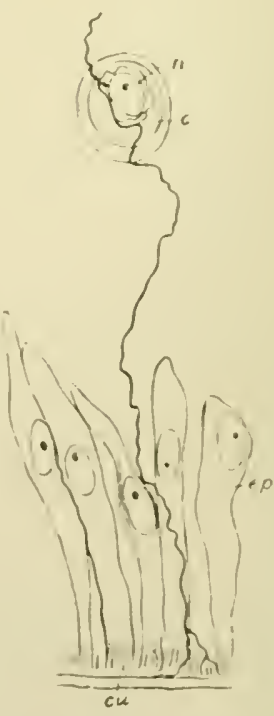

S.

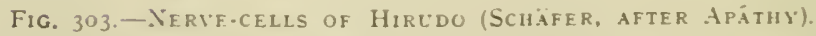

$A$, unipolar motor cell : $a$, network of neuro-fibrils near the surface of the cell $: b$, near the nucleus $n: c$, afferent $: d$, efferent neuro-fibril. B. bipolar sensory cell $a$ with its nucleus $n ; c u$, cuticle : ep, epidermis cells between which a neurofibril passes up from its branched ending near the surface of the shin to the nerve-cell, where it forms a network, which gives off a fibril passing towards the central nervius system. 
may limit the conluction and determine its direction. Some membranes, sllcli as frog's skin, are known to possess a so-called irreciprocal perneability for certain snbstances, permitting them to pass more easily in one direction than the other, and it is conccivable that a membrane at the synapse might have a similar action in respect to the movement of ions concerned in the propagition of the nervous excitation. Whatever the nature of the relation between two superposed neurons may be, it rlocs not permit the conduction of nerve-impulses indiscriminately in both directions. For instance, stimulation of the central end of the postcrior root of a spinal nerve causes an clectrical response (p. $7^{\text {I9) }}$ in the anterior root of the same segment. while no electrical cliange is produced in the posterior root by stimulation of the anterior. $\mathrm{WV}$ shall see later on (p. 770) that some of the fibres of the posterior root and their collaterals end by arborizing around the dendrites of the cells of the anterior horn. The excitation is, therefore, able to pass from the telodendrions of the posterior root-fibres through the dendrites of the anterior horn cells towards their cell-bodies, but not in the opposite direction, and in general the direction of conduction is from the dendrites towards the cell-body.

Some investigators believo that the fibrils already spoken of as forming a feltwork in the protoplasm of the nerve-cell may run right through from one cell to another, thus constituting an actual anatomical connection between the neurons, and that such a connection may be established also by fibrils which do not enter the cells at all, but run in

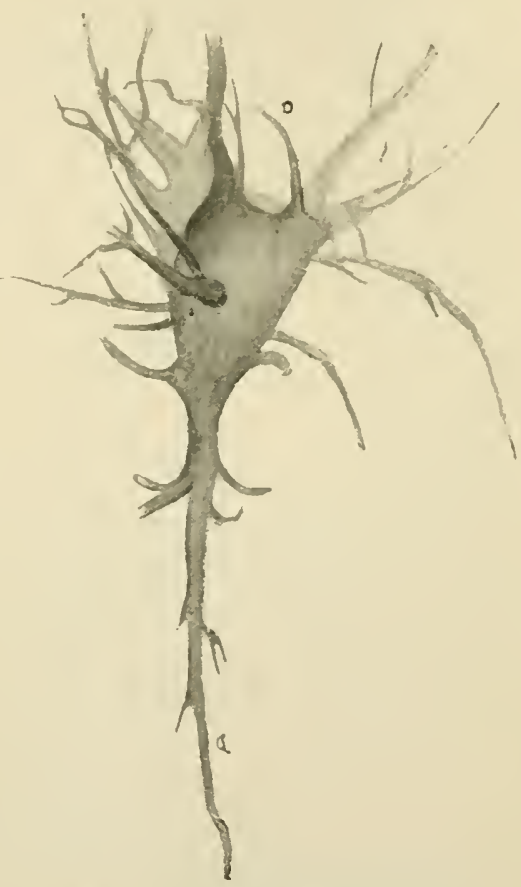

Fig. 304.-LARge Pyramidal Cell OF Cerebral Cortex (Barker, After Bech TEREW).

$a$, axon ; $b$, dendrite.

the intercellular substance of the grey matter. Such a continuity of fibrils from cell to cell lias been demonstrated in some of the invertebrates-e.g., in annclicls (Fig. 303)-where previously the best examples of strictly isolated neurons were supposed to be found (Apáthy). The supporters of the theory of continuity look upon the cell-body as merely necessary for the nutrition of the nerve-net, but deny that it is necessary for the conduction of nerve-impulses. If this is the case, it is obvious that the neurons can no longer be considered as functional units in which the law of isolated conduction of nerve-impulses (p. 689) holds good. Nor is 
it by any means so easy to understand as on the neuron hypothesis such facts as the strict limitation of Wallerian degeneration to the

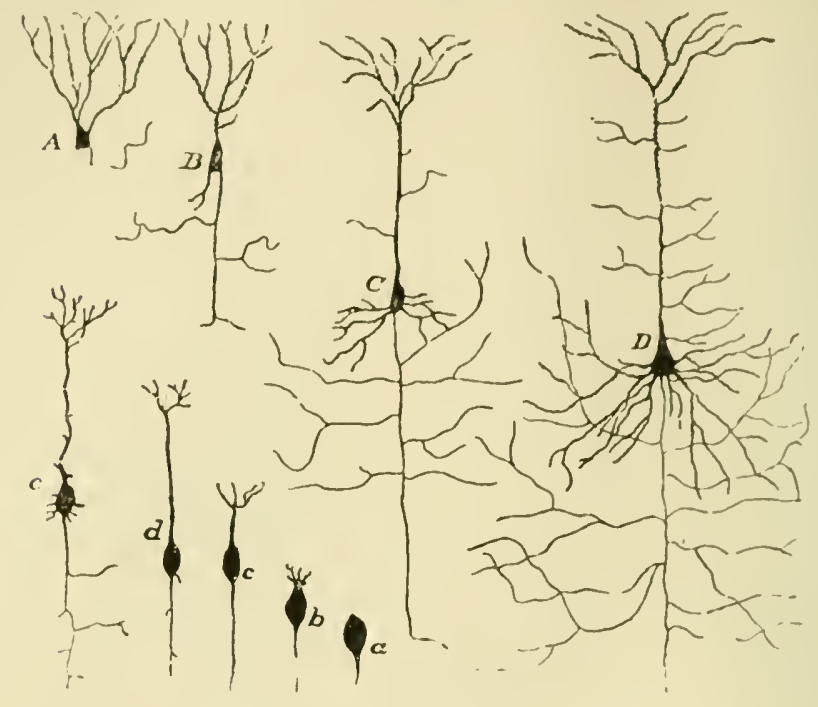

FIG, 305 .

$a--e$ shows the development of the pyramidal nerve-cells of the cerebral cortex in a typical manmal : $a$, neuroblast with commencing axon; $b$, dendrites ap. pearing; $d$, commencing collaterals. A-D shows the different degree of com plexity in the fully-developed pyramidal cells in different vertebrates : A, frog ; B, lizard ; C, rat : D, man (Donaldson, after Ramón y Cajal).

boundaries of the neurons directly affected, or the strict limitation of the silver reduction in Golgi preparations to single neurons. It is, of course, true that the simplicity and order introduced by the

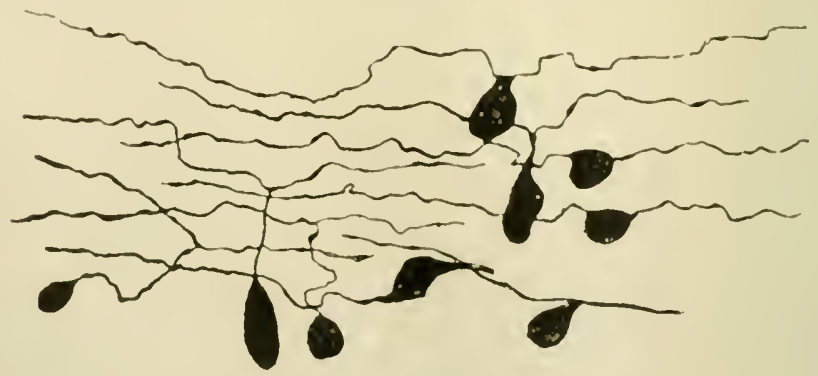

Fig. 306.

Cells from the Gasserian ganglion of a developing guinea-pig. The originally bipolar cells are seen changing into cells apparently unipolar. The same process occurs in the cells of the spinal ganglia (Van Gehuchten).

neuron hypothesis into our conceptions of the nervous conduction paths by no means prove its accuracy. Yet they are reasons for not lightly abandoning it. 
Varieties of Neurons. - Nearly all the nervecells of the cerebrospinal axis agree witl the cells of the anterior horn in the possession of an axon and one or more lendrites, a $\mathrm{t}$ ho $\mathrm{u} \mathrm{g}$ h sometimes the dendrites are scanty in number and insignificant in size. In the cerebral cortex the typical cells are of pyranidal shape. From the base comes off the axon, and from the angles dendritic processes, a particularly massive dendrite proceeding from the apex of the pyramid towards the surface of the brain.

Sometimes an axon, instead of ending in an arborization which comes into relation with the dendrites of another nerve-cell, or, as is more frequently the case, with the dendrites of more than one cell, breaks up into a sort of basketwork of fibrils surrounding the cell-body. The cells of Purkinje, for instance, in the cerebellum are surrounded by such pericellular baskets (Fig. 308). The cells of the spinal ganglia have two axons, which in the embryo arise one from each end of the bipolar cell, but in the adult, in all vertebrates except some fishes, are connected to the cell by a single process (Fig. 306). The great majority of them have no dendrites, unless. as some have supposed, the peripheral process really represents a dendrite. Another kind of cell which seems undoubtedly to be of

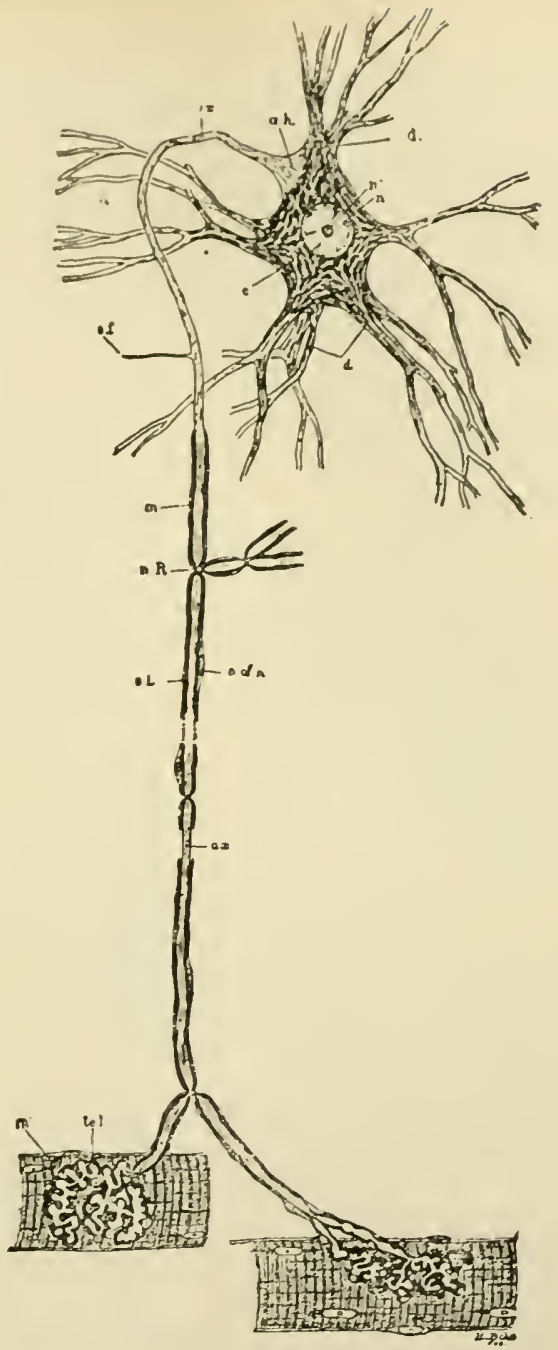

Fig. 307.-Scheme of Lower Motor Neurox (BARKER).

$a, h$, axon-hillock (the portion of the cell from which the axon comes off), containing 110 Nissl bodics, and showing fibrillation ; $a x$, axis.cylinder or axon; $m$, medullary sheath, outside of which is the neurilemma; $c$, cell-substance (cytoplasm), showing Nissl bodies in a lighter ground substance; $d$, protoplasmic processes or dendrites containing Nissl bodies; $n$, nucleus; $n^{\prime}$, nucleolus ; $n, k$, node of Ranvier; $s, f$, side fibril ; $n$ of $n$, nucleus of the neurilemma; tel., motor end plate; $m^{\prime}$, striped muscle-fibre $: s, L$, incisure. 
nervous nature is the 'granule-cell.' Granule-cells are much smaller than the nerve-cells we have been describing. Their processes are much less easily followed, but all appear to give off an axon and several dendrites. They contain a relatively large nucleus (5 to $S \mu$ in diameter), with only a mere fringe of cell-substance. The nucleus, unlike that of a large nerve-cell, stains deeply with hamatoxylin. Some parts of the grey matter are crowded with these granule-cells-e.g., the nuclear layer of the cerebellum and the substantia gelatinosa, or substance of Rolanclo, which caps the posterior horn in the cord. In other parts they are more thinly scattered. but probably they are as widely cliffused as the large nerve-cells proper, and no extensive area of the grey matter is wholly without them.

Although there are several varieties of granules (Hill), they all agree in this, that their axons run a comparatively short course,

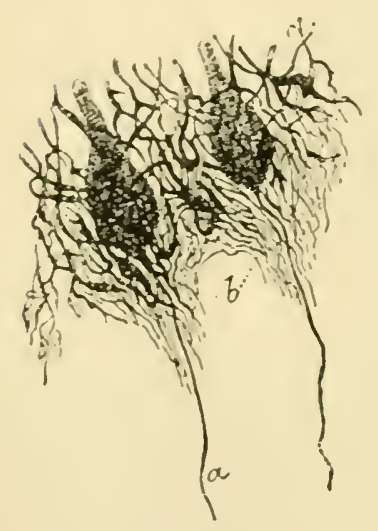

Fig. 309.-PERicelltLar Bas. KETS (SCḦ̈̈FR, AFTER CAJAL).

Two cells of Purkinje from the cerebellum are seen sur. rounded by end ramifications forming a baskel-work, $b$, derived from the branching of axons of small nerve-cells in the molecular layer; $a$, axon. and never, or rarely, pass beyond the grey matter. Another kind of neuron which is also confined to the grey matter, and is typically seen in the cortex of the cercbrum and cercbcllum, presents the peculiarity of an axon which branches into an intricate network immediately after coming off from the cell (cell of Golgi's second tÿpe). Lnlike the long axon of the typical large nerve-cell, the axis-cylinder process of this Golgi cell remains unmedullated.

The sympathetic ganglion cells are developed from immature ncuroblasts that migrate, in the course of development. from the rudiments of the spinal ganglia, and gathering in clumps form the ganglia of the sympathetic chain (His). They agree in general with the cells of the cerebro-spinal axis in possessing an axon and one or more, commonly several. dendrites, although a few of them are devoid of dendrites The great majority of the axons remain unmedullated, but a few acquire a very fine medullary sheath.

The epithelium lining the central canal of the cord and the ventricles of the brain has also been considered by some as of nervous nature. The fact that the deep ends of the cells are continued into processes which pierce far into the grey substance has been supposed to lend weight to this opinion. but there is no good ground for it.

Growth of Neurons. - The growth of a neuron is a comparatively slow process. Early in foetal life (about the third or fourth week in man) certain round germinal cells make their appearance amid the columnar ectodermic cells surrounding the neural canal. From their division are formed, in the first months of embryonic life, the primitive nerve-cells or neuroblasts. These soon elongate and push out processes, first the axon or axons, and then the dendrites (Fig. 305). As development goes on, the cell-body grows larger, and the processes 
longer ind more richly branched. The axon and its collaterals, when it has any, in the case of the great majority of the nervous clements of the brain ind cord, ultimately acquirc a medullary sheath, although. as we have said, the time at which medullation is completed varies in different groups of elements, and in some nervous tracts it is even wanting at birth. At birth, too, the branches of many of the cells are less numerous, and the connections between different nervous elements therefore less intimate than they will afterwards become. For many years the processes, and particularly the axons, continue not only to grow longer, but also to grow thicker. The cell-bolly also enlarges, and the quantity of material in it that stains with basic dyes increases. In the

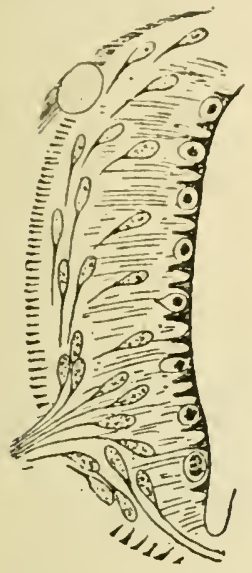

FIG. 309. - SECTION THROUGH HALF OF Neural Tube (BarKER, AFTER His).

The pear-shaped neuroblasts are seen migrating outwards. The axons of some of them are seen pushing their way out through the marginal veil as the anterior root of a spinal nerve.
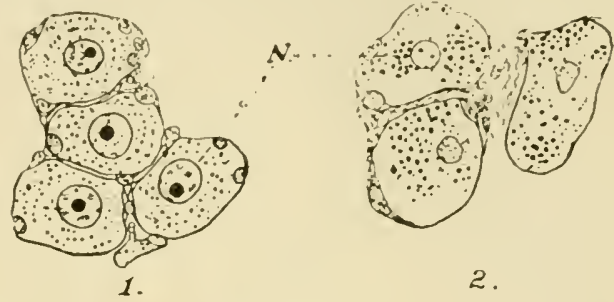

2.

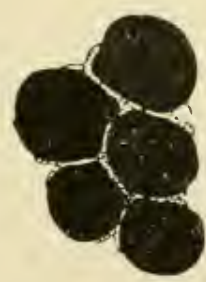

3.

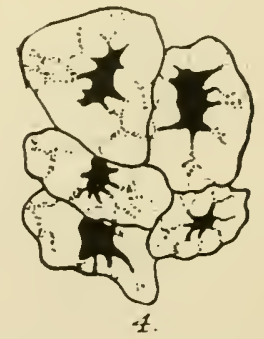

Fig. 3 I0.

I, spinal ganglion cells of a still-born male child; 2 , of a man ninety-two years old $(\lambda 250)-N$, nuclei ; 3 , nerve-cells from the antennary ganglion of a honey-bee just emerged in the perfect form; 4 , of an old honey-bee. The nucleus is black in the figure. In 3 it is very large, in 4 it is shrunken and the cell-substance contains vacuoles (Hodge).

growing (lumbar) spinal ganglia of the white rat the increase in volume of the largest cell-bodies is very closely correlated with the increasc in area of the cross-section of the nerve-fibres growing out of them. The cross-section of the axis-cylinder is, and remains, almost exactly equal to the area of the medullary sheath (Donaldson). Even after puberty is reached the anatomical organization of the nervous system may still continue to advance, although at an ever-slackening rate, and the finishing touches may only be given to its architecture in adult life. In old age the nervous elements decay as the body does. The cell-body diminishes in size; the stainable material lessens in amount; vacuoles form in the protoplasm and pigment accumulates; the 
nucleus shrinks; the nucleolus is obscured or may disappear altogether. At the same time the processes of the cell, and especially the dendrites, tend to atrophy (Fig. 3 Io).

Nutrition of the Neuron. - IVe have already scen that when an axon is cut off from its cell-body, it and its medullary sheath, when it possesses one, mudergo a rapid degeneration. It was long supposed that no cliange took place in the nerve-cell. The researches of recent years have shown that not only does loss of the specific function and troplic influcnce of the cell-body affect the nutrition of the axon, but loss of function of the axon reacts on the cellbody. In many cases at least, when a nerve-fibre is divided from its cell characteristic changes are produced in the latter and in its dendritic processes, and they are scarcely less rapid, although usually less profound, and far more transient than the degencration in the periplicral portion of the nerve-fibre. The cell-body and the

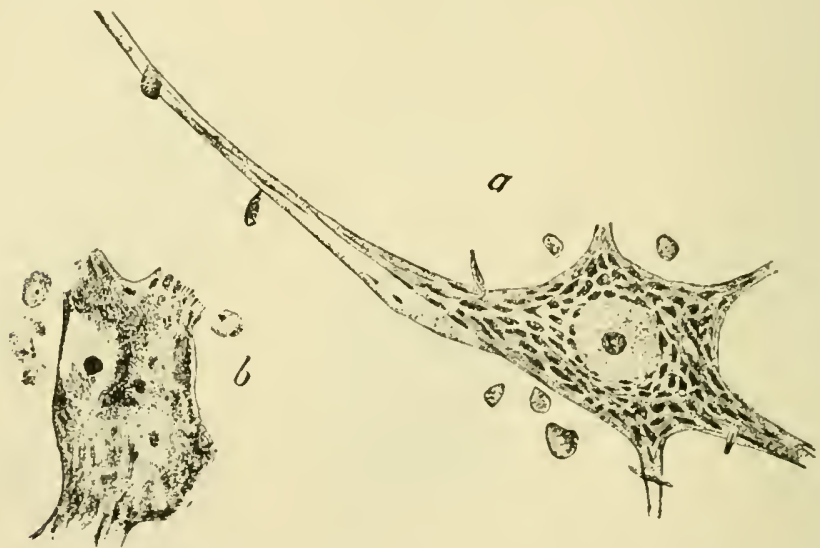

Fig. 3II.-Cells from the Nuclei of the Oculo-motor Nerves of tile Cat Thirteen Days after Division of tile Root-fibres on oNe Side: Nissl's Stain (Barker, after Flatau).

$a$, normal cell from side on which the roots were not cut; $b$, cell from side operated upon. Only a few Nissl bodies are present in $b$, and the nucleus is displaced to one side of the cell.

nucleus swell. Many of the Nissl bodies (Fig. 311) disintegrate, and are reduced to a finely granular condition. After a time much of the disintegrated chromatic substance disappears altogether. The nucleus may be displaced to onc side of the cell. Certain clianges in the neurofibrils of the cell may accompany the clianges in the chromatin. In rabbits after division of the facial nerve the alterations in its nucleus of origin have becn found to reach a maximum in about three wecks, after which there is a tendency to recovery on the part of the majority of the cells, even when regeneration of the nerve has becn prevented by cutting out a portion of it. Some of the cells may completcly atrophy and disappcar. Similar changes have been found by Warrington in the motor cells of the anterior horn after scction of the posterior (dorsal) spinal roots. Since in this case no anatomical injury has been inflicted on the motor 
neurons, it has been surmised that the cause of the alterations is the loss of impulses which normally reach them along their denclrites. In short. we may say with Marinesco, that the functional and anatomical integrity of the neuron depends on the integrity of all its constituent parts, and of the nenrons whicl carry to it functional excitations-i.e., excitations connected with its proper physiological work. The neuron, in fact, lives by its function, or, in common language, by loing its work. Yet the anatomical tokens of mere clisuse, as in the motor cells of the anterior horn after division of the cord at a higher level, are less listinct than those which follow section of the axon. Therefore it must be concluded that the latter, althougl not inclispensable for the nutrition of the cell as the cell is for the axon, exerts an influence upon it. Similar changes in the chromatin may also be produced in nerve-cells by a period of anzemia, in extensive superficial burns, in tetanus caused by the injection of bacterial cultures, in acute alcoholic poisoning, in fatigue, and in other ways. According to Wright, the inhalation of ether or chloroform (in dogs) so alters the chromatic substance, that it loses its affinity for aniline dyes. In long-continued anæsthesia the nucleus is also affected, while the nucleolus is the last part of the cell to suffer. A greater alteration occurs in the cells in the three hours between the sixth and ninth hours of anasthesia than in the five hours between the first and sixth. Although the changes are transitory, the cells, after a narcosis of nine hours, being practically normal in forty-eight hours, they indicate that the duration of safe surgical anæsthesia has a limit measured by hours.

It is probable that the alterations in the chromatic substance should not be looked upon as the token of any specific lesion; they are the common structural response of the cell to injurious influences of the most varied nature (p. 873).

Grey and White Matter.-Nerve-cells are the most distinctive histological feature of the grey nervous substance. Sown thickly in the cerebral cortex, the basal ganglia, the floor of the fourth ventricle, and the cervical and lumbar enlargements of the cord, they are scattered more sparingly wherever the grey matter extends. They also occur in the spinal ganglia and their cerebral homologues (such as the Gasserian ganglion), in the ganglia of the sympathetic system, and the sporadic ganglia in general. But wide as is their distribution, and great as is the size of the individual cells, some of which have a diameter of $\mathrm{I} 4 \mathrm{O} \mu$, or even more, they yet make up but a small portion of the whole of the central nervous substance, the total weight of the 9,000 millions of nerve-cell bodies in the human brain being less than 27 grammes (Donaldson). And although it is not to be wondered at that objects so notable when viewed under the microscope should have struck the imagination of physiologists, it is probable that the very high powers which it is so common to attribute exclusively to them are, in part at least, shared with the network or feltwork formed by their processes.

The grey matter, in addition to this exceedingly delicate network of non-medullated fibres and filaments representing the dendrites and such axons and collaterals as terminate within itself, contains also, as may be seen in preparations stained by Weigert's method,* great numbers of exceedingly fine medullated fibres, many of which are the collaterals of fibres that are passing out to the white matter.

* Weigert's is a special method of staining the medullary sheath with hrematoxylin. 
Only meclullated norve-fibres are met with in the white matter of the cerebro-spinal axis. They are alesoid of a nourilenma. In diameter they vary from $2 \mu$ to $20 \mu$. In . Malaplerurus electricus the fibre in the cord which supplies the electrical organ is of immense size: and in the anterior column of many fisleses maty also be seen a single gigantic fibre on each sicle with a diameter of nearly roos $\mu$. It cannot be saicl that any relation between the functions of neurons and the calibre of their axons has been definitely' established Many afferent fibres, it is true, are small this is notably the casc with the fibres of the posterior column, and many motor fibres are large. But the rlistinction can by no means be generalizel, for the fibres of the direct cercbellar tract $\left(p .7^{6} 4\right)$. Which certainly are afferent. are amongst the largest in the spinal corl ; and the vaso-motor fibres, which pass from the cord by the anterior (ventral) roots (1ijg. 3 12) into the sympathetic, are smaller than the fibres of the posterior column. Even the motor nerve-fibres of striated muscles

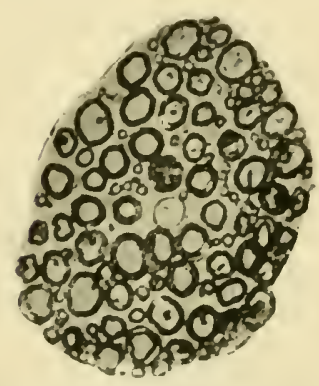

FIG. 312. - TRA NSVERSE SECTION OF A BUNDLE OF NERVE-FIBRES FROM THE ANTERIOR (VENTRAL) ROOT OF THE FIRST COCCYGeal Nerve of the Cat (i)ale).

The great difference in the diameter of the fibres is well shown. The: small fibres are vaso-motor.

sheaths: sheaths. Next the walls of the bony hollow in which it lies is the dura mater. Next the nervous substance itself, following the convolutions of the brain and the fissures of the corl. and giving off blookvessels to both, is the pia mater. Between the llura and the pia, separated from the latter by a jacket of cercbro-spinal fluid, is the double layer of the arachnoid. The comparatively coarse septa that run into the nervous substance as if coming off from the pia mater are the main beams in the scaffolding of non-nervous material with which that substance is interwoven, and by which it is supporterl. The interstices are fillerl in by a thick-set feltwork of interlacing neuroglia fibres, which lie close against the small glia cells, but accorcling to some authorities are, in the arlult at least, perfectly distinct from them, although originally formed from the cells. In preparations impregnated by the Golgi method many of the neuroglia fibres appear to be processes 
running out from the attenuated cell-boly like the arms of a microscopic crab or spicler. But accorling to IVeigert this is a leceptive appearance. as he has attempted to show by means of a special methol, in which the neuroglia fibres are alone statined. If this is the case, we must assume that in the embryo the fibres are fomed hy the cells, and afterwards become detached from them. The processes of the typical 'spicler' glia cells are mubanchel even when of great length in proportion to the rlameter of the cell-bolly. ()ther neuroglia cells have branched processes. The glia fibres are perfectly distinct from the nervous substance proper. but they are not ordinary connective tissue. Incleed, it would appear that no connective tissuc of mesodermic origin exists within the nervous substance: even the coarse septa, and particularly the one which constitutes the so-called posterior fissure, secm to consist of neuroglia, and not to be processes of pia mater. In the white matter nearly every medullated nerve-fibre is divided from its neighbours by glia fibres, which form a wide-meshed network. The network is denser in most parts of the grey substance. though not in all. The neuroglia is present in greatest abundance in the grey matter immediately surrounding the central canal of the cord and the ventricles of the brain (the ependyma, as it is called). from which long neuroglia fibres pass out radially, giving off branches on their course, and ending in little knobs or enlargements attached to the pia mater. Contrary to the common opinion, the substance of Rolando is poor in neuroglia (Weigert).

General Arrangement of the White and Grey Matter in the Central Nervous System.-(I) Around the central canal, as we have seen, a tube of grey matter sheathed with white fibres is developed. This tube, from optic thalamus to conus medullaris, may be conveniently referred to as the central grey axis or stem, which, in the lowest vertebrates-e.g., fishes-is much the most important part of the central nervous system.

(2) On the outer surface of the anterior portion of the neural axis, but not in the part corresponding to the spinal cord, is laid down a second sheet or mantle of cortical grey matter. Between this and the primitive grey stem are interposed $(a)$ the sheath of white fibres that clothes the latter, and connects its various parts, and $(b)$ a new development of white matter (corona radiata, cerebellar peduncles), which serves to bring the cortex into relation with the primitive axis, and through it with the rest of the body.

Although there are histological and developmental differences between the cerebral and the cerebellar cortex, we may, for some purposes, classify them together as cortical formations. And we may also include under this head the corpora striata, which, although for descriptive purposes generally grouped with the optic thalami and the other clumps of grey matter at the base of the brain, as the basal ganglia, are to be regarded as cortical in character. As we mount in the vertebrate scale the cortex formation of the secondary fore-brain and hind-brain acquires prominence. 
In other words, the grey matter developed in the roof of the cerebral vesicles I. and III. (Fig. 299) (the grey matter of the cerebral and cerebellar cortex) comes to overshadow the superficial grey matter hitherto present only in the roof of vesicle II. (in the corpora bigemina). And this cortex formation becones larger in amount, and, in the case of the cerebral grey matter, more richly convolnted, the higher we ascend, until it reaches its culmination in man. As the anterior cerebral vesicles develop, they spread continually backward, until at length the cerebral hemispheres cover over, and almost completely surround, the primary fore-brain and the midand hind-brains, so that the anterior portion of the primitive stem comes, as it were, to be invaginated into the second wider tube of cortical grey matter. This development of the cortical grey substance is accompanied with a corresponding development of nervefibres, for an isolated nerve-cell (apart, of course, from possible cmbryonic rudiments which have not undergone complete development) is no more conceivable than a railway-station the track from which learls nowhere in particular, or a harbour on the top of a hill.

But it is to be particularly observed that the new formation does not supplant the old, but works through and directs it. The neuroblasts of the cortex lo not throw out their axons to make direct junction with muscles and sensory surfaces. Such junction the cortex finds already established between the primitive cerebrospinal axis and the periphery. It joins itself on by nerve-fibres to the cells of the central sten; and we have reason to believe that no single axon in an ordinary spinal or cranial nerve* runs all the way from the peripliery to the cortex, and no axon of a cortical nerve-cell all the way from the cortex to the periphery, but that the connection is made by a chain of at least two neurons, the cellbolly of one of which is situate in this primitive grey tube.

The fibres from the cortex of each cerebral hemisphere (corona ra(liata), radiating out like a fan below the grey matter, are gathered together into a compact leash as they sweep lown through the isthmus of the brain in the internal capsule, to join the crura cercbri. The cortex of each cerebellar hemisphere, and the ribbed pouch of grey matter, known as the corpus dentatum, which is buried in its white core, are also connected by strands of fibres with the central stem and the cerebral mantle. The restiform body or inferior peduncle brings the cerebellum into communication with the spinal cord. The superior peduncle by one path, and the middle perluncle by another, connect it with the cerebral cortex. A great transverse commissure, the corpus callosum, unites the cerebral hemispheres across the middle line, while transverse fibres that break through the middle lobe or worm, form a similar though far less massive junction between the two hemispheres of the cerebellum.

The fibres of the nervous system may be divided into (I) fibres connecting the peripheral organs with nerve-cells in the central grey axis; (2) fibres connecting nerve-cells in this central axis with cells in the external or cortical grey tube; and (3) fibres

* The olfactory and possibly to some extent the optic nerves are exceptions to this statement. Their relation to the cortex, as is easily understood from the manner of their development (p. 747), is different from that of the other nerves. 
linking cortex with cortex, or central ganglia with each other. In the third group are included (a) fibres which connect portions of the cortex on the same side (association fibres); (b) fibres which connect portions on opposite sirles of the middle line (commissural fibres); (c) fibres which connect the central grey matter at different levels-c.g., the proprio-spinal or endogenous fibres of the cord. Our first task is, therefore, to trace the

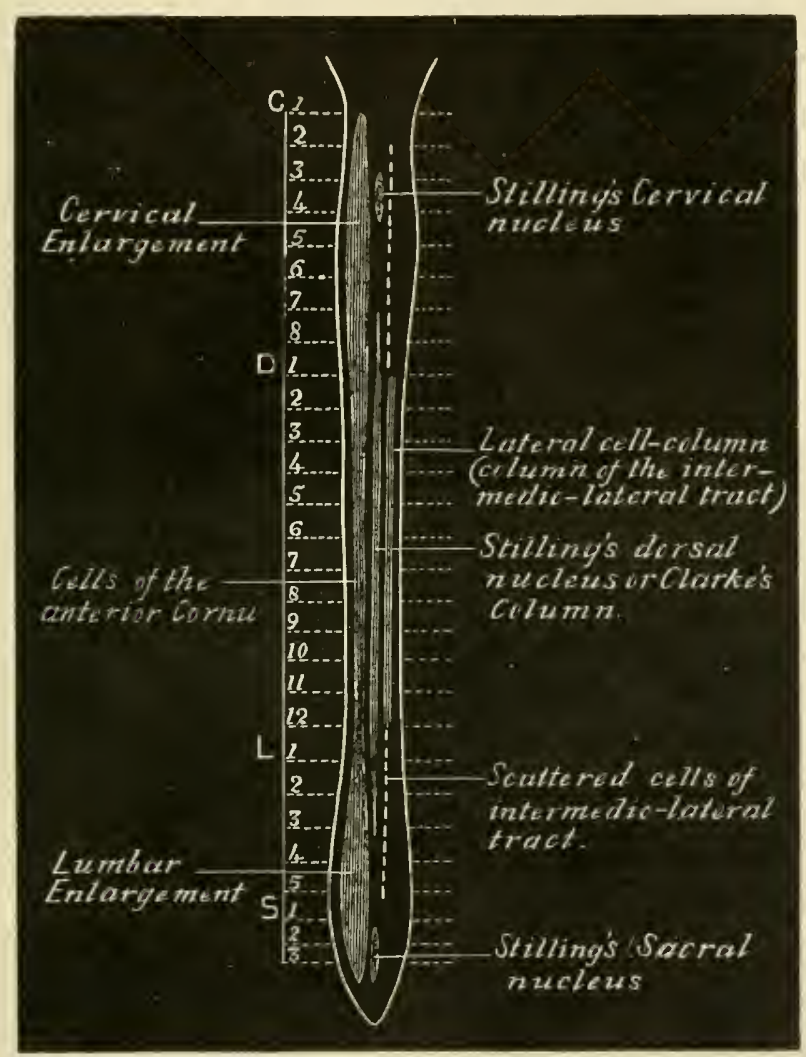

F1G. 3i3.-Diagrail of Grey Tracts of Cord.

peripheral nerves to their cells of origin or centres of reception* in the nervous stem. And although there is reason to believe that the whole of the peripheral nerves, cerebral and spinal (with the exception of the olfactory and optic, which are rather portions of the brain than true peripheral nerves), form a morpho-

* The centre or nucleus of reception of a nerve contains the nerve-cells around which its axons terminate; the nucleus of origin of a nerve contains the cells from which its axons arise. 
logical series, it will be well to begin with the spinal nerves, since their motor and sensory fibres are gathered into different and definite roots, whose course within the cord is, in general, more easily traced than the course of the cerebral root-bundles within the brain.

Arrangement of the Grey and White Matter in the Spinal Cord. - The grey matter of the spinal cord is arranged on eacl side in a great unbroken column of roughly crescentic section, joined with its fellow across the middle line by a grey bar or bridge, which springs from the convexity of the crescent, and is pierced from end to end by the central canal. The anterior horn of the crescent, although it varies in shape at different levels of the cord, is, in general, broad and massive, in comparison with the slender and tapering posterior horn. In the lower cervical and upper dorsal region a moulding or projection, forming a lateral horn, springs from the fluted outer side of the grey substance. Within the grey matter nervecells are found, sometimes so regularly arranged that they form veritable cellular or vesicular strands. Of these the best marked are: (I) The tract or tracts made up by the cells of the anterior horn (Fig. 313), which practically run from end to end of the cord, swell out in the cervical and lumbar enlargements, where the cells are very numerous and of great size (7o $\mu$ to I40 $\mu$ in diameter), and contract to a thin thread in the thoracic region, where they are relatively few, scattered, and small. In the enlargements there are several groups of these cells corresponding with the segments of the limbs, the movements of the hand, forearm, and upper arm being each represented by a group in the cervical, and those of the foot, leg, and thigh by groups in the lumbar swelling. In the rest of the cord only two wellmarked groups of cells are present in the anterior horn, a mesial and a lateral. (2) Clarke's column, whose cclls, mostly of good size and somewhat rounded in outline, are situated at the inner side of the root of the posterior horn just where it joins on to the grey cross-bar. It gradually increases in size from above downwards, usually appearing first at the level of the seventh or eighth cervical nerve, attaining its maximum development at the eleventh or twelfth dorsal and disappearing altogether, as a continuous strand, at the level of the second or third lumbar nerves. Scattered nerve-cells, however, constituting the socalled cervical and sacral nuclei of Stilling, are frequently found occupying the same position towards the upper and lower ends of the cord, and may be looked upon as isolated portions of Clarke's column. (3) A tract of small cells called the intermediolateral tract, lateral cell column, or lateral horn, situated at the outer edge of the grey matter, about midway between the anterior and 
posterior horns. It is best marked in the thoracic region, 11p to about the sccond thoracic segment, although in the corresponding situation there are scattered cells in the lumbar swelling and the cervical corl. There is reason to believe 1 liat the axons of cells of the intermedio-lateral tract, which pass out as small medullated fibres in the anterior roots, form the preganglionic segments of the efferent vascular and visceral nerves (p. I 60 ). (4) The cells of the posterior horn, which, although numerous, are smaller than those of the anterior horn. Throughout the whole cord, however, two small groups of cells may be distinguished, one on the lateral side of the horn, about its middle, and the other on the mesial side, a little in front of -i.e., ventral to-the edges of the substance of Rolando. Botli of these groups are broken up by the passage through them of bundles of fibres which form a network, and they are therefore called respectively the group of the lateral and the group of the posterior reticular formation.

The white matter of the cord is anatomically divided by the position of the nerve-roots and the anterior and posterior fissures into three columns on eacli side: the anterior, lateral, and posterior columns. The first two, since they are not separated by a perfectly definite boundary, are often grouped together as the antero-lateral column. In the cervical region it may be seen with the microscope that the posterior white column is almost bisected by a septum running in from the pia mater towards the grey commissure. The imner half is called the postero-median column, or column of Goll; the outer half the postero-external column, or column of Burdach (Fig. 3r4). No localization of any of the other conducting paths in the cord is possible by gross anatomical examination ; but by means of the developmental method and the method of degeneration the columns of Goll and Burdach can be followed throughout the cord, and several similar areas can be mapped out. We shall only mention those that are physiologically the most important.

When the spinal cord is divided, and the animal allowed to survive for a time, certain tracts are picked out by the degeneration of their fibres, although in every degenerated tract some fibres remain unaffected. We may distinguish the tracts that degenerate above the lesion (ascending degeneration) from those that degenerate below the lesion (descending degeneration).

Ascending Tracts.-Above the lesion degeneration is found both in the posterior and the antero-lateral columns. Immediately above the section nearly the whole of the posterior column is involved. Higher up the degeneration clears away from Burdach's tract, and, shifting inwards, comes to occupy a position in the column of Goll. In the antero-lateral column 
two degenerated regions are seen, both at the surface of the cord, one a compact, sickle-shaped area extending forwards from the neighbourhond of the line of entrance of the posterior roots, and the other an area of scattered degeneration, embracing many intact fibres, and completing the outer boundary of the column ahmost to the anterior median fissure. The compact area is called the dorsal or direct cerebellar tract, or tract of Flechsig, the diffuse area the antero-latcral ascending tract, or tract of Gozers, or ventral cerebellar tract.* The dorsal cerebellar tract is distinguished by the large size of its fibres. It is only distinct in the
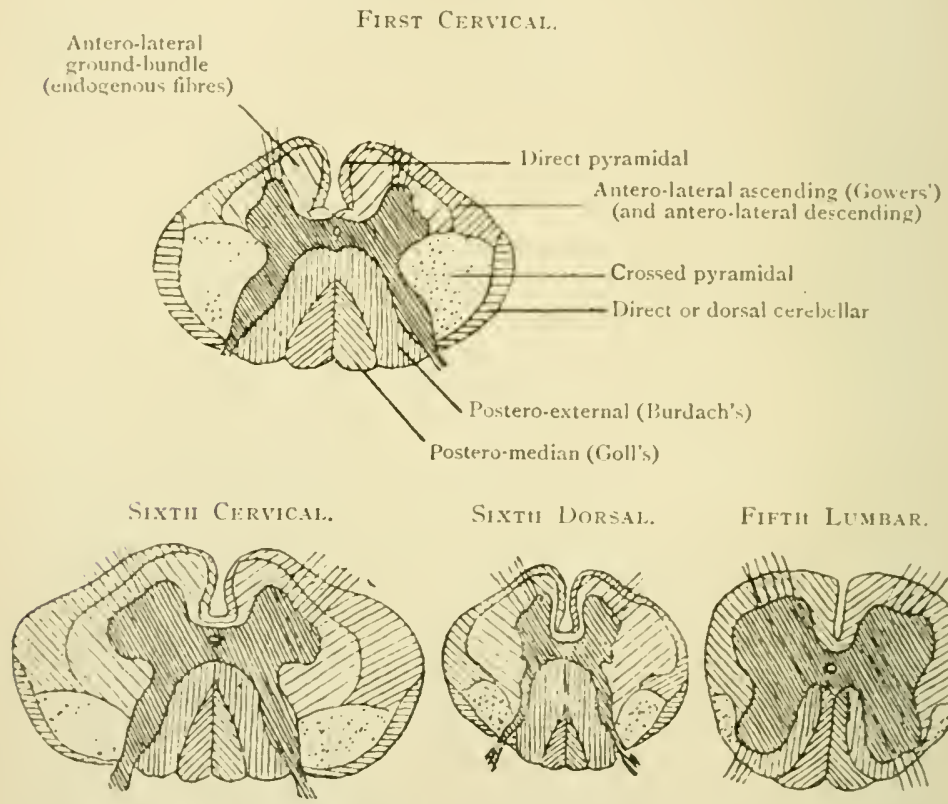

SIXTII DORSAL.

Fiftit LuMBar.

Fig. 3if.-Diagammatic Sections of the Spinal Cord to show the Tracts of White Matter at Different Levels.

dorsal and cervical regions of the cord. The truct of Lissauer, or posterior marginal zone, is another small ascending tract at the outer side of the tip of the posterior horn. It is made up of fine fibres from the posterior roots which soon pass into the posterior column.

Descending Tracts.-When the cord is clivided, say in the upper dorsal or cervical region, the following tracts degenerate below the lesion :

(I) A small group of fibres close to the antero-median fissure,

* Some writers employ the more precise terms, dorsal and ventral spino-cerebellar tracts. 
which has received the name of the direct pyramidal Iractpyramidal, because higher up in the medulla oblongata it forms part of the pyramid ; direct, because it does not cross over at the decussation of the pyramids, but continues down on the same side. The direct pyramidal tract is only present in man and the higher apes.

(2) A tract of degenerated fibres in the posterior part of the lateral column. This is the lateral or crossed pyramidal tract, and is much larger than the direct. In the medulla it also lies within the pyramid, but, unlike the direct pyranidal tract, it crosses to the opposite side of the cord at the decussation. The pyramidal tracts are also called cortico-spinal to indicate their origin and termination.

(3) A tract of scattered degeneration lying along the margin of the cord in the anterior portion of the antero-lateral column, and partly overlapping the tract of Gowers. It is called the antero-lateral descending tracl, or tract of Loewenthal.

(4) The prepyramidal (or rubro-spinal) tract, or Monakow's tract, lying immediatelyin front of the crossed pyramidal tract.

(5) A small, commashaped island of degeneration (comma tract) can

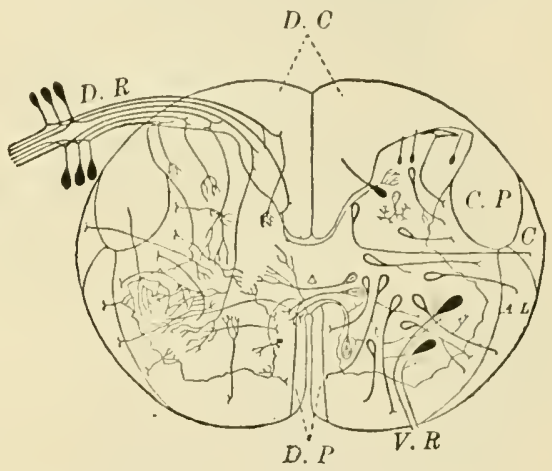

Fig. 315.-Scheme OF Cross-Section of SPINAL Cord (DONALDSON, AFTER LENHOSSEK).

On the left side only the afferent fibres are shown; the efferent fibres and the spinal cells on the right side. D.R., posterior (dorsal) root ; $V . R$, anterior (ventral) root; C.P, crossed pyramidal fibres; $C$, direct cerebellar tract ; A.L, antero-lateral tract; D.C, posterior columns.

be followed downwards for a short distance in the middle of Burdach's column. It is only seen in the cervical and upper thoracic regions.

When we have deducted the long ascending and descending tracts which have been described, there still remains in the antero-lateral column a balance of white matter unaccounted for. This white substance, which does not degencrate for any great distance either above or below a lesion, is called the anterolateral ground-bundle, and lies chiefly in the form of an incomplete ring around the anterior cornu. It is believed to consist of fibres (endogenous or proprio-spinal fibres) which run only a comparatively short course in the cord, and serve to connect nerve-cells at different levels. Some of these endogenous fibres 
are ascending, other descending. Some endogenous fibres may also be intermingled with the fibres of certain of the long tracts,

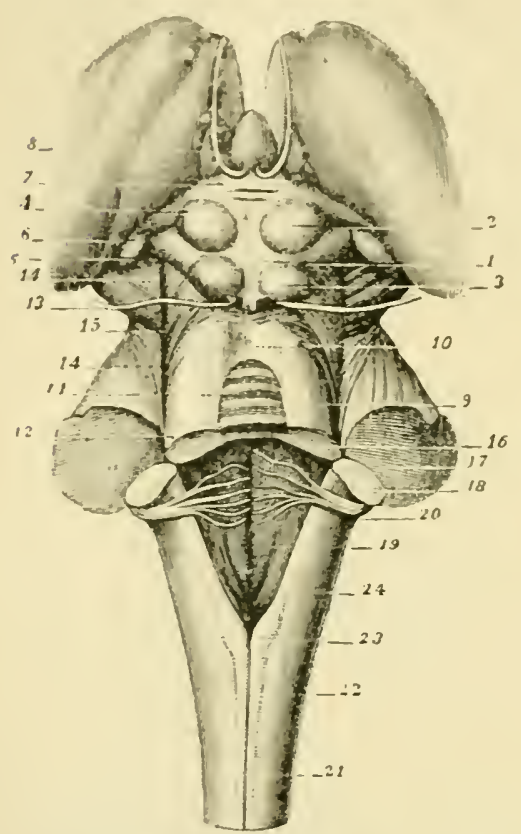

Fig. 316.-Miedulla Obloxgata, PoNs - axd Corpora Quadrigemixa (Dorsal or Posterior VIEW) (SAPPEY).

I, corpora quadrigemina; 2, nates; 3. testes: 4, anterior brachium uniting the nates to the lateral geniculate body ; 5, posterior brachium uniting the testes to the internal geniculate body 6 ; 7 , posterior commissure ; 8 , pineal gland pulled forward to show nates ; 9 , superior peduncle of the cerebellum; I0, I 1, I 2 , valve of Vieussens; I 3 , trochlear nerve ; I 4 , lateral sulcus : I5, fillet; 16 , superior, I 7, middle, and I5, inferior peduncle of the cerebellum: I 9 , foor of fourth ventricle; 20 , auditory nerve ; $2 \mathrm{I}$, spinal cord; 22, postero-median columu, continued in the medulla as the funiculus gracilis: 23 , the clava, the continuation of the iuniculus gracilis.

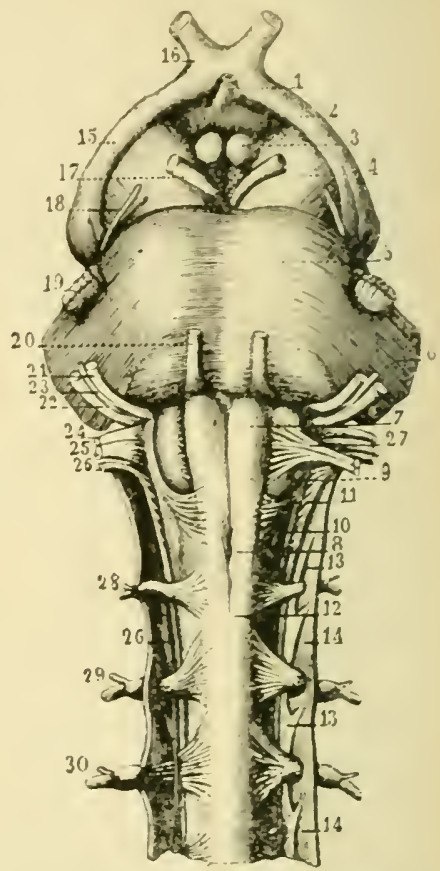

Fig. 3I\%.-Medilla Obloxgata, PoNs AND CRURA CEREBRI (VEX:TRAL OR ANTERIOR VIEW).

$I$, infundibulum; 2 , tuber cine. reum; 3 , corpus mammillare; 4 , cerebral peduucle or crus cerebri; 5. pons; 6 , middle peduncle of cerebellum ; 7 , pyramid ; 8, decussation of the pyramids; 9 , olive; Io, tubercle of Rolaudo; II, ex. ternal arcuate fibres; I2, upper end of spinal cord; 13 , ligamentum denticulatum : I 4 , dura mater of spinal cord; I5, optic tract; 16, chiasma ; I7, third or oculo-notor nerve : 18 . fuurth or trochlear nerve; 19 , fith or trigeminal nerve; 20 , sixth nerve or abducens ; 21 , seventh or facial nerve: 22 , eighth or auditury nerve ; 23. Herve of Wrisberg (portio interunedia), which unites with the facial : 24, glosso-pharyngeal nerve ; 25 , vagus nerve: 26 , spinal acces. sory nerve; 27 , hypoglossal nerve ; $28,29,30$, first, second, and third pairs of cervical spinal nerves. 
both in the antero-lateral and posterior columns, and Sherrington lias shown (in the dog) that long proprio-spinal fibres passing down in the lateral column connect the upper with the lower parts of the cord (p. 804$)$.

The next question which arises is: How are the long tracts connected below-i.e., witl the periphery-and above-i.e., with the higher parts of the central nervous system? The answer to this question, partly derived from clinical records and partly from experimental results, is in the case of some of the tracts unexpectedly full and minute, thougl meagre in regard to others. But to render it intelligible it is necessary, first of all, to describe briefly-

The Arrangement of the Grey and White Matter in the Upper Portion of the Cerebro-spinal Axis. - In the medulla oblongata the grey and white matter of the spinal cord is rearranged, and, in addition, new strands of fibres and new nuclei of grey substance make their appearance. Of these nuclei the most conspicuous is the dentate nucleus of the inferior olive, which, covered by a crust of white fibres, appears as a projection on the antero-lateral surface of the medulla. In front of the olive, be-

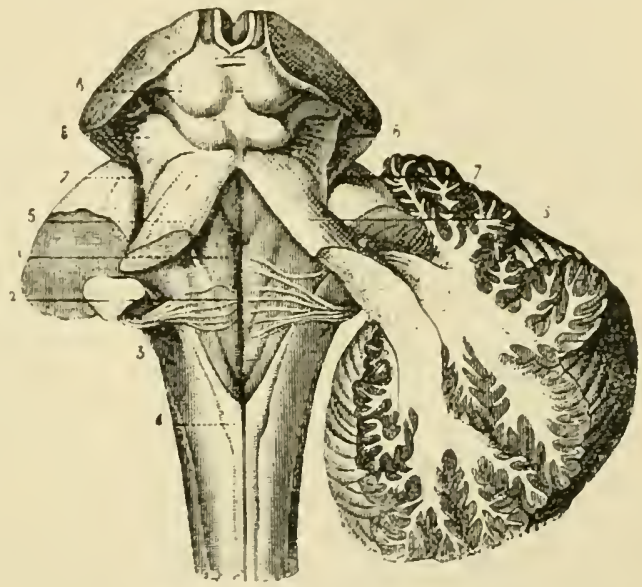

Fig. 3i 8. - Medulla Oblongata and Cerebellum, With Fourth Vextricle (Hirschfeld).

I, mesial groove of floor of ventricle running down to the calamus scriptorius; 2, striæ acusticæ; 3 , inferior peduncle of the cerebellum; 4, clava; 5 , superior peduncle crossing the inferior and passing to its internal side; 7,7 , lateral sulcus ; 8 , corpora quadrigemina.

tween it and the continuation of the anterior median fissure, is another projection, the pyramid, which looks like a prolongation of the anterior column of the cord, but is made up of very different constituents. Dorsal to the olive is the restiform body or inferior peduncle of the cerebellum, and behind the restiform body lie two thin columns, the funiculus cuneatus, which continues the postero-external column of the cord, and the funiculus gracilis, which continues the postero-internal column. In these funiculi are contained collections of small or medium-sized nervecells termed respectively the uucleus cumeatus and the nucleus gracilis. The rearrangement of the constituents of the cord is due mainly to two causes: (I) The opening up of the central canal to form the fourth ventricle, and the folding out, on either side, of the grey matter which lies posterior to it in the cord; (2) the breaking up of the grey matter of the anterior horn by 
strands of fibres as they sweep through it from the lateral pyramidal tract to take up a position in the pyramid of the opposite side (clecussation of the pyramids), and a little higher up by fibres passing across the middle line from the gracile and cuncate nuclei (sensory decussation or decussation of the fillet). The mosaic of grey and white matter formed in the medulla by the interlacing of longitudinal and transverse fibres with each other and with the relics of the anterior horn, is called the reticular formation (formatio reticularis). It occupics the anterior and lateral portions of the bulb behind the pyramids and olivary bodies, and is continued upwards in the dorsal portion of the pons and crura cercbri, and down wards for a little way into the upper part of the cervical cord.

The cercbro-spinal axis passes up from the medulla through the pons, encircled and traversed by the transwerse pontine fibres derived from the middle cerebellar peduncle or commissure, which enclose everywhere between them numerous collections of nervecells (nuclei pontis). Enlarged by the accession of many of these fibres which come from the cortex of the cercbellum on the opposite side, as well as of fibres from the nuclei of the cranial nerves that take origin in this neighbourhood (fifth and eighth). the central nervous stem bifurcates above the pons into the two divergent crura cerebri. From each crus a great sheet of fibres passes up between the optic thalamus and the caudate nucleus of the corpus striatum on the one hand, and the globus palliclus of the lenticular nucleus on the other, as the internal capsule, from which they are dispersed, in the corona racliata, to the cerebral cortex. Both in the upper part of the pons and in the crus a ventral portion. or crusta, containing the fibres of the pyramidal tract, and a dorsal portion, or tegmentum, can be distinguished, the line of separation being marked in the crus by a collection of grey matter, called from its usual, though not invariable. colour the substantia nigra (Fig. 324). A portion of the tegmentum is continucd below the optic thalamus.

Coming back now to our question as to the connections of the long tracts of the cord, let us consider, first of all,

The Connections of the Postero-median and Postero-external Columns.- When a single posterior root is divided, say in the dorsal region, between the cord and the ganglion, its fibres, as we have already seen (p. 603), degenerate above the section. Since the cell-bodies of these neurons lie in the ganglion, if a series of microscopic sections of the spinal cord be made, wellmarked degeneration will be found at the level of entrance of the root on the same side of the cord, while below that level there will be only a few degenerated fibres in the comma tract. Immediately above the plane of the divided root the degeneration will be confined to Burdach's column and to its external border. Higher up it will be found in the internal portion of Burdach's and the external rim of Goll's columu. Still higher up the degenerated fibres will be confined to the postero-median column; the postero-external will be entirely free from degeneration.

When a number of consecutive posterior roots are cut, the 
whole of the postero-external column in the sections immediately above the highest of the divided roots will be found occupied by degenerated fibres, while Goll's column may be free from degeneration, or degenerated only at its outer border. Higher up degeneration will be found to have involved the whole of the postero-median column, and to have cleared avay altogether from the postero-external. The degeneration in the column of Goll may be traced along the whole length of the cord to the medulla, although the number of degenerated fibres diminishes as we pass upward. The explanation of these appearances is as

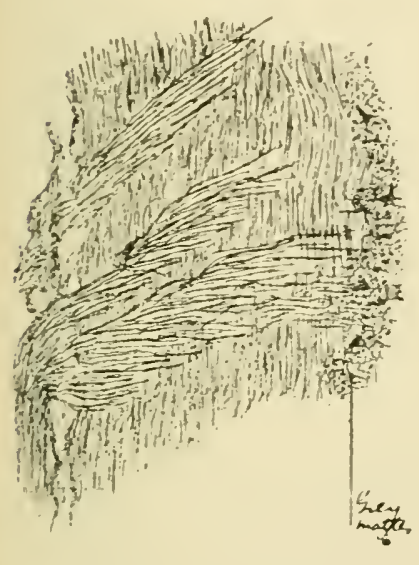

Fig. 3ig. - Posterior Roots entering Spinal Cord (at the LEFT OF THE Figure).

(From a preparation stained with aniline blue-black.)

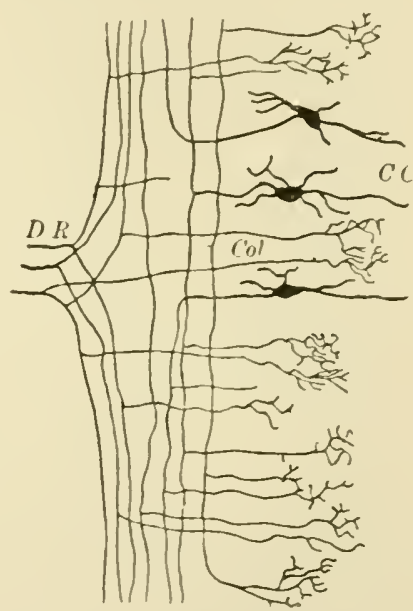

Fig. 320.-Braxchisg of Pos. TERIOR ROOT-FIBRES IN CORD (Doxaldsox, after Cajal).

Collaterals, Col, are seen coming off from the two main branches of the root-fibres, DR, and ending in arborizations. CC, cells in the grey matter of the cord, whose axons also give off collaterals.

follows : It may be seen in preparations of the cord impregnated by Golgi's method that the fibres of the posterior roots soon after their entrance into the cord divide into two processes, one of which runs up and the other down in the posterior column, or in the adjoining portion of the posterior horn. From both of these collaterals are given off at intervals to the grey matter. The descending branches run downwards only for a short distance, and the degeneration in the comma tract seen after section of the cord is due to the division of these branches. Many of the ascending branches pass up for a short distance in 
the postero-external column, sweeping obliquely through it to gain the tract of Goll. In this tract some of them run right on to the medulla oblongata, to end by arborizing among the cells of the mucleus gracilis. Other fibres, both of Goll's and of Burdach's tract, end at various levels in the cord, their collaterals, and ultimately the main branches themselves, coming into relation with nerve-cells in the grey matter. W'hen the cervical posterior roots are cut, many of the degencrated fibres remain in Burdach's column up to the medulla, where they terminate in the nucleus cuneatus. In the posterior coltumn, then, the numerous fibres of the posterior roots which do not end in the spinal cord are arranged in layers, the fibres from the lower roots being nearest the median fissure (in the postero-median column), and those from the higher roots farthest away from it (in the posteroexternal column). Other collaterals from the posterior rootfibres, and many of the main root-fibres themselves, run into the anterior horn and terminate in arborizations around its cells; some pass into the posterior horn, and doubtless come into relation with its scattered cells and, in the dorsal region, with the cells of Clarke's column. Some of the posterior root-fibres and their collaterals also form synapses with the cells of the intermedio-lateral tract. Other collaterals and probably some axons cross the middle line in the anterior and posterior commissures and end in the grey matter of the opposite side.

Connections of the Direct or Dorsal Cerebellar Tract.-Since the dorsal or direct cerebellar tract does not degenerate after section of the posterior nerve-roots, but does degenerate above the level of the lesion after section of the spinal cord, the nervecells from which its axons arise must be situated somewhere or other in the cord. Now, it has been observed that the vesicular column of Clarke first becomes prominent in the lower dorsal region, and that in this same region the direct cerebellar tract begins. Atrophy of the cells of Clarke's column has sometimes in disease been shown to accompany degeneration of the direct cerebellar fibres. After an experimental lesion of these fibres in animals, some of the cells of the vesicular column show the changes in the Nissl bodies and the other changes which we have already described as occurring in nerve-cells whose axons have been cut. After two or three months these cells may be found almost completely atrophied (Schäfer). Finally, axis-cylinder processes have been seen sweeping out from Clarke's column into the direct cerebellar tract (Mott). The evidence, then, is complete that the cells of origin of this tract are in Clarke's column. Clarke's cells are surrounded by arborizations, some of which, as previously stated, represent the terminations of posterior root-fibres and of their collaterals. The 
most; beneath these come fibres from the lowest thoracic segments, then fibres from the higher thoracic segments; and, internal to all, fibres from the topmost thoracic and lowest cervical segments (Sherrington and Laslett).

Connections of the Antero-lateral Ascending Tract.-Accorcling to Scliäfer, the axons of this tract are probably connected with cells situated in the middle and posterior parts of the grey crescent, mainly on the opposite side of the cord, although also on the same side. None of the fibres of the tract can come clirectly from the posterior nerve-roots, since no degeneration is seen in it on section of the roots alone.

The antero-lateral ascending tract passes up through the medulla, where some of its fibres perhaps form synapses with the cells of the lateral nucleus, a collection of grey matter in the lateral portion of the spinal bulb. But its main strand runs on unbroken through the medulla, in front of the restiform body, and behind the olive, and after reaching the upper part of the pons bends back over and in company

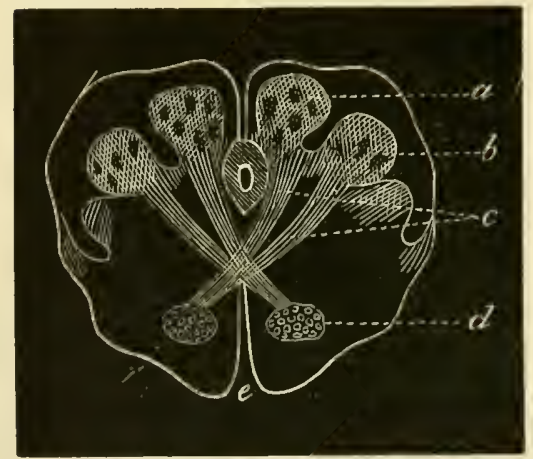

Fig. 323. - Diagram of Decussation or Fillet.

$a$, nucleus gracilis ; $b$, nucleus cuneatus ; $c$, internal arcuate fibres crossing the middle line from $a$ and $b$ to the fillet $d$, and forming the decussation of the fillet; $c$, anterior median fissure. with the superior peluncle as the ventral spino-cerebellar bundle, to end in the worm of the cerebellum (Fig. 330).

A few fibres of Gowers' tract may pass by the middle peduncle to the opposite cerebellar hemisphere. Some of its fibres do not go to the cerebelhum at all. One group can be followed to the corpora quadrigemina (spinotectal fibres), and another by way of the tegmentum of the crus cerebri to the optic thalamus (spino-thalamic fibres).

Through the relay of the gracile and cuneate nuclei, the postero-internal and postero-external columns of the cord are further connected on the one hand with the cerebrum, and on the other with the cerebellum. The cells of the nuclei give off fibres (internal arcuate fibres) which, sweeping in wide arches across the mesial raphe to the opposite side, take up a position behind the pyramid in the tract of the fillet, a bundle of fibres which becomes more compact, and therefore more distinct as it passes brainwards. Receiving fibres from other sources on its way, and also giving off fibres, it runs upwards through the dorsal or tegmental portion of the pons. In the mid-brain it divides into two portions, the latcral fillct, also called the lower fillet or fillet of Reil, and the intermediate, also called the upper 
fullet. The lateral fillet contains mainly fibres arising in the cochlear nucleus of the auditory nerve, and ends in grey matter of the posterior corpus quadrigeminum, and partly in the mesial geniculate body. It appears to be a path for the conduction of auditory impulses. The intermediate fillet contains chiefly the fibres that come off from the gracile and cuneate nuclei, but is enlarged by the accession of fibres from the sensory nuclei of the cranial nerves. It terminates in the lateral nucleus of the optic thalamus by forming synapses with nerve-cells, whose axons, passing through the posterior limb of the internal capsule

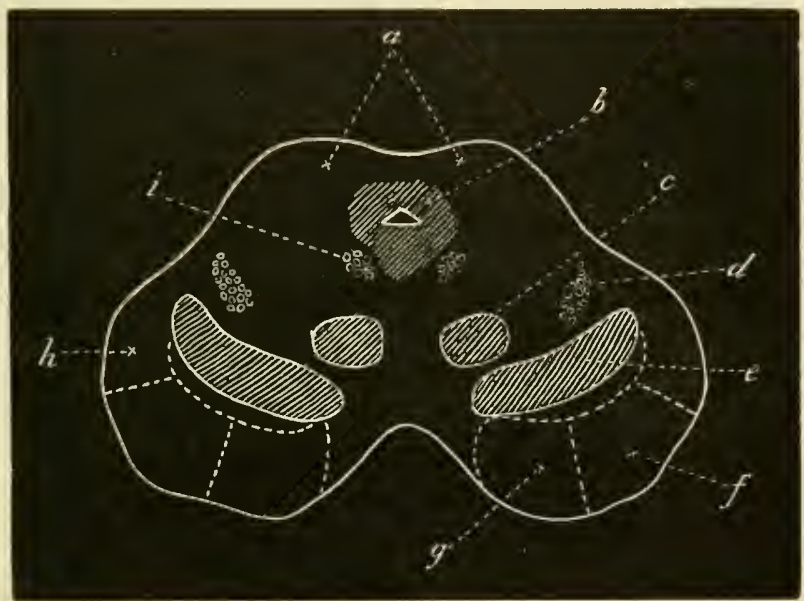

Fig. 324.-Diagrammatic Transverse Section of Crura Cerebri and Agueduct of Sylvius.

$a$, anterior corpora quadrigemina; $b$, aqueduct ; $c$, red nucleus ; $d$, fillet ; $e$, substantia nigra; $f$, pyramidal tract in the crusta of the crura cerebri : $g$, fibres from frontal lobe of cerebrum; $h$, fibres from temporo-occipital lobe ; $i$, posterior longitudinal bundle.

and the corona radiata, continue the afferent path to the cerebral cortex.

Besides the ascending fibres, the bundle anatomically described as the tract of the fillet contains some descencling fibres which on section of the tract degenerate below the lesion. They lie to the mesial side of the intermediate fillet, and since their cells of origin seem to be in the thalamus and their course is towards the bulb, they are spoken of as a thalamo-bulbar tract.

Not all of the axons from the cells of the cranial sensory nuclei run in the fillet. Many of then occupy a position in the reticular formation of the tegmentum dorsal to the fillet as they pass through the pons and mid-brain to end in the thalamus and the region below it (sub-thalamic region). From the sensory 
nucleus of the fifth nerve a separate bundle of fibres ascends to the thalamus, lying in the tegmentum of the mid-brain lateral to the posterior longitudinal bundle.

Connections of the Pyramidal Tracts. - When the cortex in and in front of the fissure of Rolando is destroyed by disease in man, or removed by operation in animals, it is found that in a short time degeneration has taken place in the fibres of the corona radiata which pass off from this area. The degeneration can bc followed down through the genu and the anterior two-thirds of the posterior limb of the internal capsule (Fig. 325) and the crusta of the cerebral peduncle of the corresponding side into the medulla oblongata. Below the decussation of the pyramids

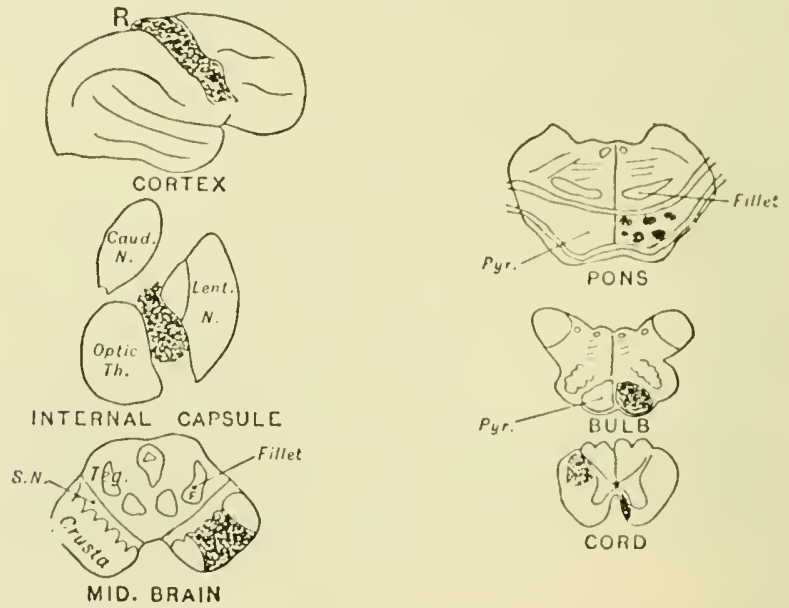

F1G. 325.-Pyranidal Patil (AFter Gowers).

Degeneration after destruction of the 'motor' area of the rijht cerebral hemisphere. The degenerated areas are indicated by the shading.

it is found that the degeneration has involved the two pyramidal tracts, and only these-the crossed pyramidal tract on the side opposite the cortical lesion, the direct pyramidal tract on the same side-and that the cross-section of the two degenerated tracts goes on continually diminishing as we pass down the cord. (We overlook, for the moment, in the interest of simplicity of statement, the fact that some degenerated fibres are found in the crossod pyramidal tract on the same side as the lesion.) This is proof positive that the cell-bodies of the neurons whose axons run in these tracts are situated in the cerebral cortex. They have indeed been identified with certain of the large pyramidal cells (the so-called giant cells or cells of Betz) in the cortex of the "motor" region in front of the Rolandic fissure (p. $85 \mathrm{I}$ ). For after division of the motor pyramidal fibres in the 
npper cervical region of the cord (in monkeys) clianges in the chromatin (so-called chromatolysis) and atrophy of these large cells occur. The same has been found to be true in man in cases where the cord was injured by fracture of the spine in such a way as to interrupt the tract (as well as other tracts) completely and permanently, without entailing death for a considerable time (Holmes and May). The fact that after destruction of the cortex or the path in its course the degeneration below the lesion does not spread to the anterior roots shows that at least one relay of nerve-cells intervenes between the pyramidal fibres and the root-fibres. The results both of normal and morbid histology enable us to identify the cells of the anterior horn as the cells of origin of the axons of the anterior root-fibres. For

(1) Axis-cylinder processes have been actually observed passing out from certain of the so-called motor cells of the anterior horn to become the axis-cylinders of the anterior root.

(2) In the pathological condition known as anterior poliomyelitis, the cells of the anterior horn degenerate, and so do the anterior roots of the affected region, the motor fibres of the spinal nerves, and the muscles supplied by them.

(3) As already mentioned (p. 756), comparatively transient but decided changes occur in the anterior horn cells on section of the corresponding anterior roots.

(4) An enumeration* has been made in a small animal (frog) of the cells of the anterior horn and of the anterior root-fibres, and it has been found that the numbers agree in a remarkable manner. From all this it cannot be doubted that most, at any rate, of the cells of the anterior horn are connected with fibres of the anterior root. But since the number of fibres in the pyramidal tracts (about So, ooo in each half of the human cord) falls far short of the number of fibres in the anterior roots (not less than 200,00o in man on each side), it is necessary to suppose either that one pyramidal fibre may be connected with several cells or that all the anterior root-fibres are not in functional connection with the pyramidal tract.

There is no reason to assume any such connection in the case of the fine medullated root-fibres arising in the lateral horm and going to the visceral and vascular muscles.

While there is no doubt that anterior root-fibres and pyramidal fibres of the brain and cord form segments of the same nervous path, the connection between the pyramidal fibres and the cells of the anterior horn has not yet been anatomically demonstrated. Many of the pyramidal fibres pass into the grey matter between the anterior and posterior horns or near the base of the posterior horn. The anterior horn cells are surrounded by arborizations. Some of these are probably the terminations of axons whose cell-bodies are situated in the posterior horn, others the terminations of posterior root-fibres or their collaterals. Many of them very likely represent the end arborizations of pyramidal fibres

* Such enumerations can be made with great accuracy from photographs of sections of the nerves (Hardesty, Dale). (See Fig. 312, p. 758.) 
or their collaterals. Some observers, however, supjose that the pyramidal fibres do not come into immediate relation with the anterior horn cells, but that another neuron is intercalated between them and the cells.

The pyramidal fibres are unquestionably paths for voluntary motor impulses passing down from the cortex to the cord. But they are not the only cortico-spinal efferent paths, and in many animals they are not even the most important paths for voluntary movements. It is the more skilled and delicate movements which the pyramidal tract subserves in man, and it is these movements which are permanently lost when the tract is destroyed. The size of the path is proportioned to the degree of development of the brain. Thus, it is larger in the monkey than in the dog, and larger in man than in the monkey. In the lower mammals it is exceedingly small. While in man the pyramidal tracts constitute nearly i 2 per cent. of the total cross-section of the cord, they make up little more than I per cent. in the mouse. In some mammals, as the rat, mouse, guinea-pig, and squirrel, the pyramidal tracts lie, not in the antero-lateral, but in the posterior columns. In vertebrates below the mammals the pyramidal system does not exist as a collection of neurons which send their axons without interruption down from the cortex to the cord. In birds, e.g., after the removal of a hemisphere, the degeneration does not extend below the mid-brain (Boyce).

Connections of the Antero-lateral Descending Tract.-The main origin of these fibres is the nuclcus of Deiters, a collection of large multipolar nerve-cells in the floor of the fourth ventricle near the inner auditory nucleus. These cells give off axons which pass into the posterior longitudinal bundle of the bulb and pons, mostly to the bundle of the same side, but partly into that of the opposite sicle. Here the fibres bifurcate into an ascending branch. which passes up to the oculo-motor nucleus, and a descending (vestibulo-spinal) branch, which passes downwards to the spinal cord and enters the antero-lateral descending tract. The fibres of this tract ultimately pass into the anterior horn, where most of them end by arborizing amongst the cells of the horn. Higher up corresponding fibres from the posterior longitudinal bundie arborize in the cranial motor nuclei.

Thus far, then, we have been able to map nut two great paths from the cerebral cortex to the periphery-one efferent, the other afferent.

(I) The great efferent or motor pyramidal path, which, starting in the cortex in front of the fissure of Rolando, where its axons give off numerous collaterals to the grey matter soon after emerging from the cells, and sweeping down the broad fan of the corona radiata, passes through the narrow isthmus of the internal capsule into the crusta of the crus cerebri, and thence into the pons (Figs. 326, 327). At this level, the fibres destined 
to make comnection with the motor muclei of the cranial nerves in the grey matter underlying the aqueduct of Sylvius and the fourth rentricle terminate. Most of these fibres decussate to make physiologrical connection with nuclei on the opposite side, but some join nuclei on the same side. The question whether they arborize directly around the cells of the motor nuclei or make junction with them through another intercalated neuron is precisely in the same position as the corresponding question

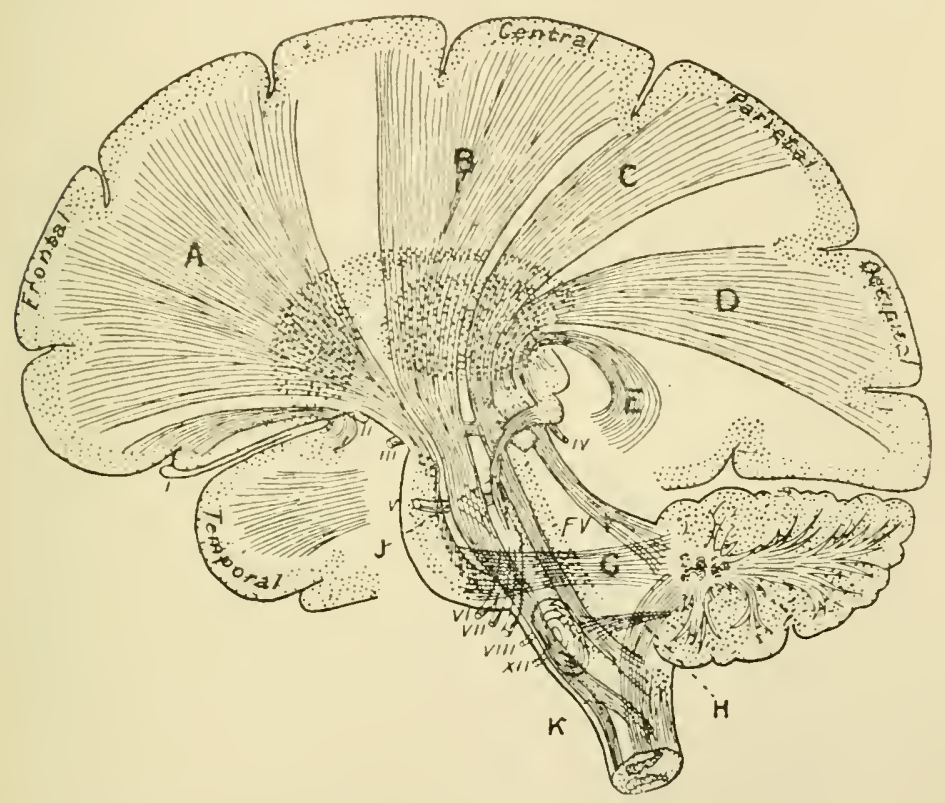

Fig. 326.-Patus from Cortex in Corona Radiata (Starr).

$A$, tract from frontal convolutions to nuclei of pons and so to cerebellum ; $B$, motor pyramidal tract; C, afferent tract for tactile sensations (represented in the diagram as separated from B by an interval for the sake of clearness); $\mathrm{D}$, visual tract; $\mathrm{E}$, auditory tract ; F, G, H, superior, middle, and inferior cerebellar peduncles; J, fibres from the auditory nucleus to the posterior corpus quadrigeminum; $K$, decussation of the pyramids in the bulb; $F V$, fourth ventricle. The Roman numerals indicate the cranial nerves.

for the spinal pyramidal path (p. 775). On their way through the pons they send off collaterals to the nuclei pontis, as they do higher up to the grey matter of the basal ganglia of the cerebrum and the substantia nigra, and the path may be continued to the motor nuclei by axons arising here. There is no proof, however, that this is the case. The rest of the pyramidal fibres run on into the pyramid of the bulb, where the greater part (usually about 90 per cent.) of the fibres decussate, appearing in the cervical 
cord as the massive crossed pyramidal tract of the opposite side. A few (usually about io per cent.) remain on the samo side as the slender direct pyramidal tract. The size of this tract varies much in different individuals, and it is occasionally absent. Its breadtl 1 constantly diminishes as it proceeds down the cord, and it disappears before the middle of the thoracic region is reached, its fibres continually decussating across the anterior white commissure and plunging into the opposite anterior horn.

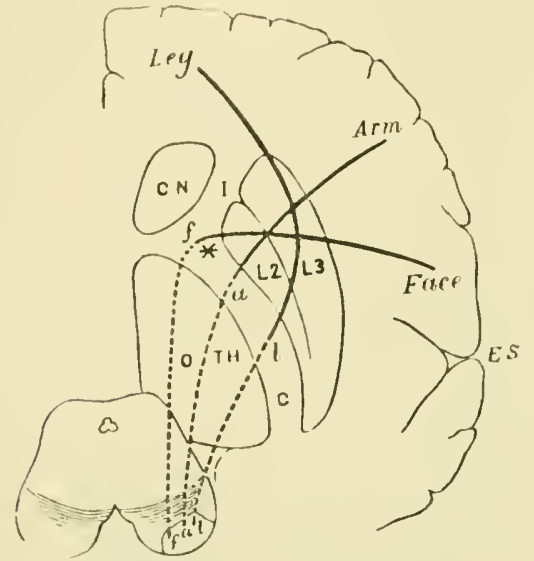

Fic. 327.-MIOTOR PYRAMIDAL Tracts (I)IAGRAMAATIC)(HALLIBURTON, AFTER GOWERS).

The convolutions are supposed to be cut in vertical transverse section, the internal capsule, I. C, and the crus in horizontal section. O, TH, optic thalamus; $C N$, caudate nucleus; $L_{2}$ and $L_{3}$, middle and $e x$. ternal portions of lenticular nucleus ; $f, a, l$, fibres from the face, arm, and leg areas of the cortex respectively : $\mathrm{E}, \mathrm{S}$, Sylvian fissure. The genu or linee of the internal capsule is indicated by the asterisk.

They either end among its cells, or, passing through it, reinforce the crossed pyramidal tract. The fibres of this crossed tract are, in their turn, continually passing off into the grey matter to make connection (p. 775) with the cells of the anterior horn, whose axis-cylinder processes enter the anterior roots of the spinal nerres. The losses which it suffers as it descends the cord may be in some slight degree compensated by the bifurcation of some of its fibres (geminal fibres), but ultimately the whole tract forms synapses with cells in the grey matter, and dwindles away as the lumbar region is reached (Fig. 3I f). It lias been asserted that on their way down the cord the two crossed pyramidal tracts exchange some fibres with each other (recrossed fibres); and it was supposed that this would explain the escape in hemiplegia (paralysis of one sicle of the body) of those muscles which are accustomed to work with the corresponding muscles on the opposite side-c.g., the respiratory muscles. But although there is no doubt that such muscles are innervated to some extent from both cerebral hemispheres, this is due not to recrossed, but to uncrossed (homolateral), fibres, which in the cord run down in the lateral pyramidal tract, and are represented by the fibres that degenerate in that tract after a lesion in the 'motor' area of the same side (p. 77t). 
(2) A great afferent or sensory path by which some at least of the impulses carried up through the posterior roots of the spinal nerves, after passing through various relays of nerve-cells, reach the cortex of the cerebellum; or the upper portions of the central grey tube, the corpora quadrigemina and optic thalamms; or, finally (through the tegmentum and the posterior limb of the internal capsule behind the motor fibres), the cerebral cortex itself.

The efferent pyramidal path from the cortex to the periphery is broken by at most two relays of nervecells - those intercalated cells to which reference has already been made ( $p .775)$, if they really exist, and the motor cells of the anterior horn. The afferent path to the cerebral cortex is interrupted by at least three relays with axons of considerable length. One of the cells is situated in the ganglion on the posterior root, another in the medulla oblongata, a third in the optic thalamus; and

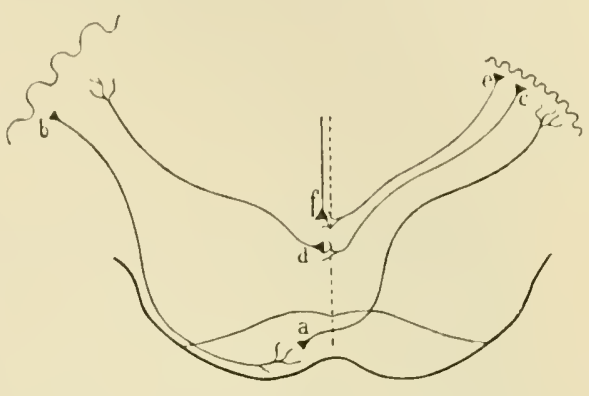

Fix. 328.-PAT1IS OF MiddLe CEREBLlLAR Peduncle (Mingazzini).

The scheme indicates the afferent and efferent paths which run through the middle cerebellar peduncle, connecting the cerebellum with the opposite side of the cerebrum. $a$, fibre coming from a cell in the nuclei pontis and going to the cerebellar cortex; $b$, fibre from a cell in the cortex of the opposite cerebral hemisphere making connection in the pons with $a$ ( $a$ and $b$ together constitute an afferent path to the cerebellum); $c$, a fibre springing from a Purkinje's cell in the cerebellar cortex and making connection in the pons with a cell $d$, which sends its axon to the cerebral cortex of the opposite side. $c$ and $d$ constitute an efferent path from the cerebellum to the opposite cerebral hemisphere; $\ell, f$, represent a path coming from the cerebellar cortex, which crosses the middle line in the pons, and then ascends till it loses itself in the formatio reticularis.

on some of the routes another, or even more than one, is intercalated between the medulla and the cortex.

Connections of the Grey Matter of the Cerebellum with the Periphery and other Parts of the Central Nervous System.-Numerous as are the nervous ties of the cerebral cortex, those of the grey matter of the cerebellum are, in proportion to its mass, still inore extensive, particularly as regards afferent fibres, and perhaps not less important.

Speaking broadly, we may say that the restiform body or inferior peduncle connects chicfly the dentate nucleus and the grey matter of the worm with the spinal cord and medulla oblongata, and through them with the periphery. The fibres which it receives from the direct cerebellar tract (dorsal spino-cerebellar tract) of its own side it carries to the worm. These fibres occupy the outer portion 
of the peduncle. The fibres which reach the restiform boly from the olivary nucleus of the opposite, and also in smaller numbers from that of the same side, run mainly to the hemisphere. All these fibres are afferent in relation to the cerebellum (cercbello-petal). An uncrossed afferent connection also exists between the cerebellum and the vestibular branch of the auditory nerve, through certain of its nuclei of reception, and also between it and the nuclei of other cranial nerves, snch as the trigeminus and the vagus. The fibres pass up in the inner portion of the inferior peduncle (direct sensory cercbellar path of Edinger, lig. 329) to the nucleus of the roof (nucleus tecti) and nucleus globosus. Some efferent fibres (cerebellofugal) also run lown from the cercbellum in the inferior pecluncle, including fibres from the nucleus tecti of the opposite side which are on their way to the merlulla oblongata.

The middle peduncle is in the main a link between the cerebellar

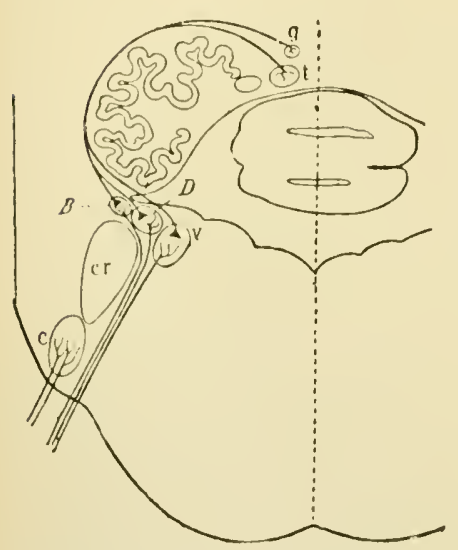

Fig. 329.-Direct Sensory CerebELLAR PATII OF EDINGER.

$D$, Deiters' nucleus ; $v$, median nucleus of auditory nerve ; $t$, nucleus of the roof ; g, nucleus globosus.

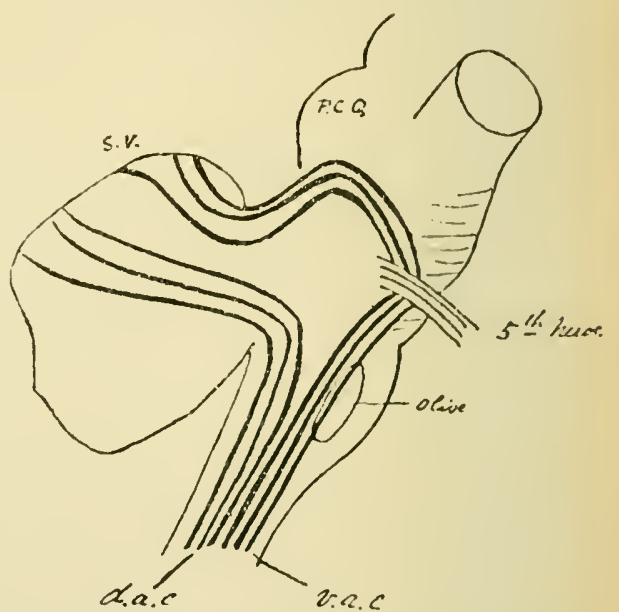

Fig. 330.-DIAGRAM OF DORSAL AND VENTRAL SPINO-CEREbellar Tracts entering Cere. BELLUM (MOTT).

P.C.Q., posterior corpora quadrigemina : s.v., superior vermis (worm) of cercbellum; d.a.c., v'.ct.c., dorsil and ventral ascending cercbellar tracts.

cortex and the cerebral cortex of the opposite side, through the relay of the pontine grey matter. Most of the fibres in it are afferent in relation to the cerebellım, their cells of origin being situated in the muclei of the pons, and sending their axons across the middle line to end in the cerebellar cortex.

The superior peduncle connects chicfly the dentate nucleus of one side with the cortex of the opposite cerebral hemisphere through the red nucleus of the tegmentum of the crus cerebri and the optic thatamus on the opposite sirle. The great majority, or perhaps all, of its fibres are efferent fibres as regards the cercbellum-i.e.. their cells of origin lie in the Ientate nucleus. Rumning upwards and forwards in the superior pecluncle towards the micl-brain they 
cross the middle line below the corpora quadrigemina, and then bifurcate into ascending and descendling branches. The ascending branches end mainly in connection with cells in the red nucleus, but some of them paiss on to the optic thalamus, with which cells of the red nucleus are also connected. The thalamus, as we have scen, is in its turn extensively connected with the cerebral cortex, and the red nucleus (by the efferent tract of Monakow) with the grey matter of the cord. The descending branches of the fibres of the superior peduncle, entering the reticular formation of the pons, pass down. it is said, to make connection with the motor nuclei of the cranial and spinal nerves. The tract of Gowers, as previously stated. comes into relation with the superior peduncle, passing backwards along its mesial border to the worm. Since the cortex of the

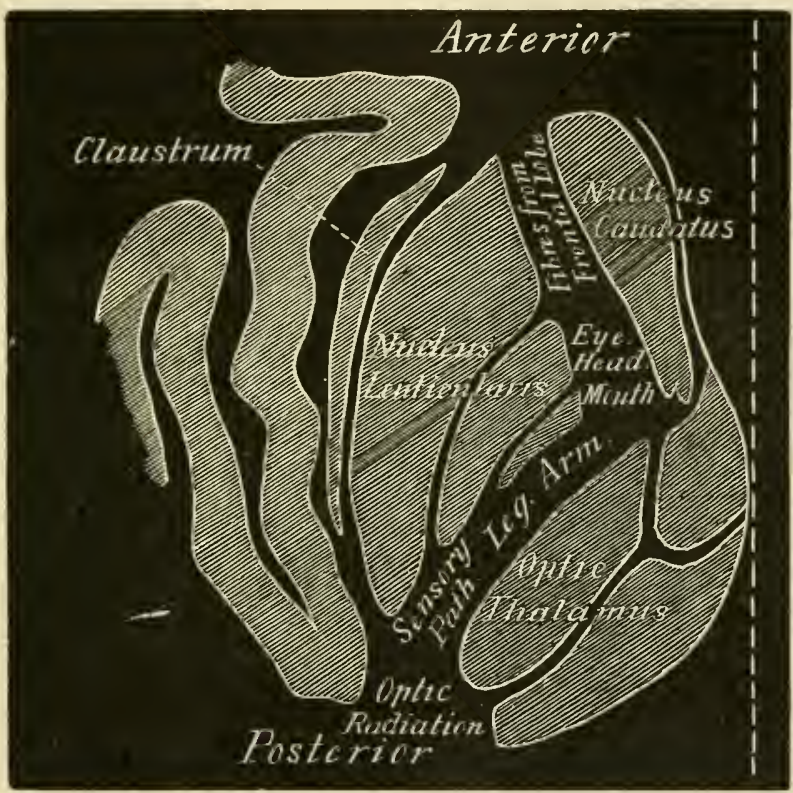

Fig. 33r.-Diagramiatic Horizontal Section of Left Half of Brain to show Internal Capsule.

cerebellum is linked to the dentate nucleus, the superior peduncle affords an indirect connection between it and the cerebral cortex. Through the restiform bolly afferent impulses pass up to the cerebellum. From the cerebellum they may proceed to the cerebrum. So that the path by the restiform body, dentate nucleus, and superior peduncle may form an alternative route for afferent impressions ascending from the periphery to the great brain - a path broken by at least four relays of nerve-cells. The cerebellar hemisphere may be connected by an efferent path through the nucleus of Deiters and the descending antero-lateral tract with the motor roots of the same side. Another efferent path (from the dentate nucleus) may be constituted by the fibres of the superior peduncle and Monakow's bundle. 
The Internal Capsule.-We have already recognised the pyramidal tract and the afferent tegmental path as constituents of the internal capsule. The cranial fibres of the pyramidal tract occupy mainly the genu or knee, the spinal fibres the posterior limb as far back as the posterior border of the lenticular nucleus. The fibres from the various motor areas are to a certain extent

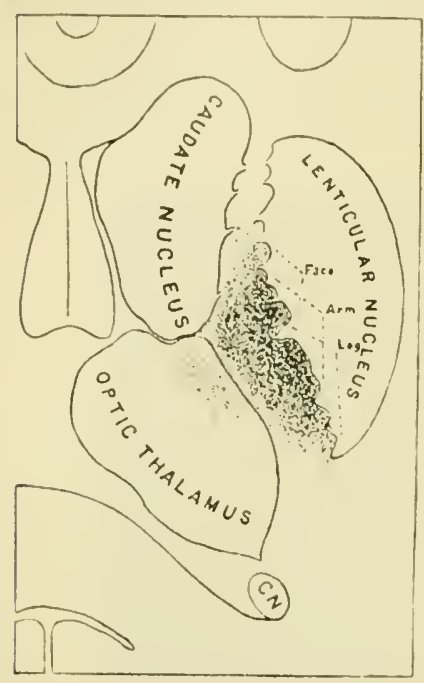

Fig, 332,-Prramidal Tract IX Internal Capstle (Simpson AND JoLLy).

Horizontal section through right cerebral hemisphere, cutting fibres of internal capsule transiersely at an upper level a little below the upper surface of the lenticular nucleus. The extent of the de. generation following destruction of the whole of the right 'motor' cortex, except the 'head and eyes' area (in one of the lower monkeys). is shown. Note overlapping of fibres from face, arm, and leg areas, as shown by experiments in which one or other of these areas was alone remosed. arranged in order in the capsule, those for the eres and head lying farthest forward, those for the leg farthest back, while the fibres going to the face, arm and trunk occupy intermediate positions (Fig. 33I). The separation, however, is far from complete, the fibres of neigluburing regions being considerably intermixed (Hoche). As the tracts pass downwards the intermingling

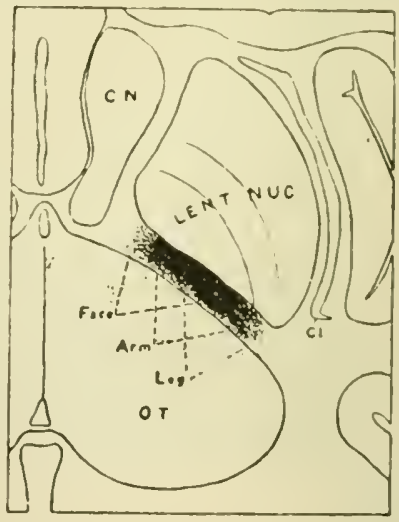

Fig. 333.-Pyramidal Tract in INter. Nal Capsule at Lower Level (Simp. SON AND JOLLY).

$\mathrm{CN}$, head of caudate nucleus : O.T, optic thalamus : $\mathrm{Cl}$, claustrum.

becomes continually greater (Simpson and Jolly) (Figs. 332, 333). The afferent fibres from the thalamus to the cortex, which we have described as the last segment of the afferent tegmental path, lie in the posterior part of the posterior limb. But here again there is no absolutely sharp line of demarcation. Some motor fibres are intermingled with the sensory in the posterior 
part of the capsule, for lesions of this region produce a certain degree of paralysis ats well as anesthesia on the opposite side of the body. A pure capsular liemianestlesia-that is, a loss of sensation on the opposite side due to a lesion in the internal capsule and muacompanied by motor defect- does not appear to exist. Accordingly the common statement that the efferent (motor) path occupies the anterior two-thircls, and the afferent (sensory) path the posterior third of the posterior limb of the internal capsule, while no doubt true in a general sense, is not strictly correct.

The destination of the afferent fibres of the internal capsule lias not been defnitely settled. There is mo doubt that they pass

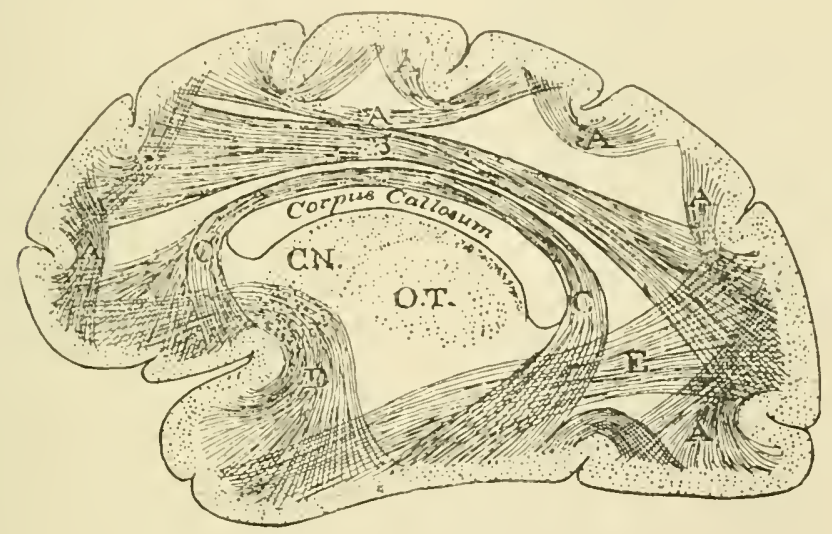

Fig. 334.-Association Fibres (After Starr).

Cerebral hemisphere seen from the side. A, A, association fibres between adjacent convolutions; B, between frontal and occipital lobes; $\mathrm{C}$, cingulum, connecting frontal and temporo-sphenoidal lobes : D, uncinate fasciculus between frontal and temporal regions: E, inferior longitudinal bundle between occipital and temporo-sphenoidal lobes; O.T., optic thalamus : C.N.. caudate nucleus.

up to the convolutions around the fissure of Rolando (central convolutions), and there is reason to believe that some of them terminate in the 'motor' region in front of that fissure.

But we have not yet exhausted the constituents of the internal capsule. Two great cones of fibres sweep down into it, one from the frontal, the other from the occipital and temporal portions of the cerebral cortex. The first passes through its anterior limb, the second behind the sensory path in its posterior limb. The cells of origin of the frontal fibres are known, and those of the occipital and temporal fibres are supposed, to be situated in the cortex. They are therefore efferent fibres as regards the cortex (cortifugal). Running on through the crusta of the cerebral peduncle (Fig. 324), the frontal tract internal, the 
occipito-temporal external, they end in the grey matter of the pons, and serve as one segment of an extensive commissural connection between the cerebral and the cerebellar cortex of the opposite sicle, the other segment being formed by neurons whose cell-bollies are situated in the pons, and whose axons,

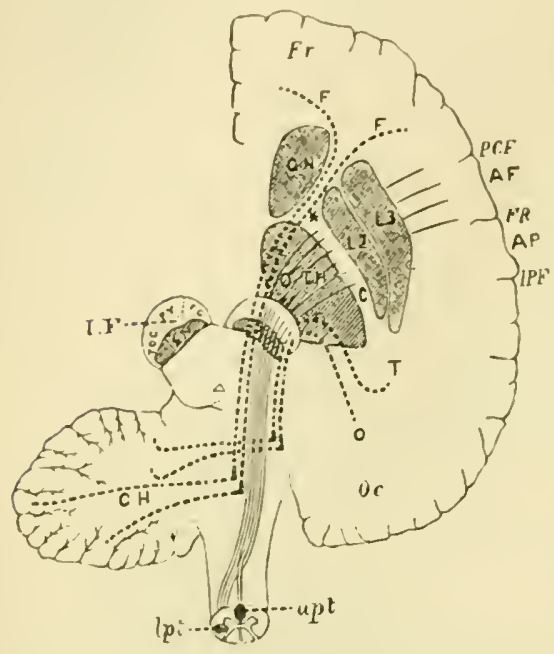

Fic: 335.-Fibres conNectixg Frontal AND TFMPORO - OCCIPJTAL LOBES WITH CEREBEllua, ETC. (Diagrai) (AFTER Gowirs).

Fr, frontal; $O c$, occipital lobe; the interrupted lines indicate the fibres (TOC) connecting the rerebellum and the temporooccipital cortex, and the fronto-cerebellar fibres $\left(l^{*} \mathrm{C}\right)$. On the left side the position of these two groups of fibres and of the motor (prramidal) tract, PY, in the crus, is indicated by letters. The pyramidal tract is seen on the right passing down from the Rolandic area througl the posterior limb of the internal capsule IC (the genu or knee of which is indicated by the astcrisk) to decussate in the bulb. AF, ascending frontal convolution; AP, ascending parietal convolution; FR, fissure of Rolando; IPF, intraparietal fissure; PCF, precentral fissure ; $l p t$, crossed pyramidal tract; apt. direct pyramidal tract.

ning in the anterior limb of the capsule to the pons; and from the thalamus to the occipital region through the extreme posterior border of the internal capsule, behind the occipital fibres that proceed to the pons. The fibres that connect the thalamus 
with the occipital cortex are spoken of as the optic radiation. Some of the fibres of the optic radiation, however, proceed, not from the thalamus, but from the anterior corpus quadrigeminum and the lateral geniculate body. The thalamus is also connectert with the cortex of the temporal lobe, with the cerebellum, and through the fillet with the posterior part of the tegmental system, the medulla oblongata and the spinal cord (p. 772). Fibres also pass from the inner and deeper part of the thalamus to the lenticular nucleus of the corpus striatum. The thalamus must be regarded as a great sensory centre through which afferent impulses stream on their way to all parts of the cortex.

We have purposely omitted to enumerate other paths by which the various tracts of grey matter in the brain are brought into communication with each other, and our knowledge of such connections is constantly augmenting. When we add that not only are the cerebral hemispheres united by many ties to the subordinate portions of the cerebro-spinal axis and to each other, but that cortical areas of one and the same hemisphere are in communication by short connecting loops of 'association' fibres (Fig. 334), it will be seen that the linkage of the various parts of the central nervous system is extremely complex; that an excitation, blocked out from one path, may have the choice of many alternative routes; and that the apparent simplicity and isolation of the pyramidal tracts must not be allowed too far to govern our views of the possibilities open to a nervous impulse once it has been set going in the labyrinth of the nervous network. Nor is it only by the main channel of the axis-cylinder that nervous impulses can be conducted. It cannot be doubted that they can also pass along the collaterals. And the actual route taken by a given impulse is, in all probability, determined not only by anatomical relations, but also by molecular conditions, particularly in the terminal fibrils of the axons, collaterals and dendrites, and in the substance, if such a substance there be, which intervenes between the end arborizations of a neuron and the dendrites or cellbodies of the neurons with which they lie in contact. So that a road open at one moment may be closed at another. We may suppose that the greater the number of connections between the cells of the central nervous system, the greater is the complexity of the processes which may be carried on within it. And, indeed, comparison of the brains of different an'mals shows that it is not so much by excess in the number of nerve-cells as by the increased complexity of linkage, that a highly-developed brain differs from a brain of lower type; the higher the brain. the more richly branched are the dendrites and the 
terminations of the axons and their collaterals, and, therefore, the greater is the number of possible paths between one nervecell and another.

\section{Functions of the Central Nervous System.}

Mucl of our knowledge of the functions of the central nervous system and of its divisions has been gained by the removal or destruction of more or less extensive tracts of nervous substance, or the cutting off of connection between one part and another. But it is well to warn the reader at the very outset that in no other part of physiology is such caution required in making deductions as to the working of the intact mechanism from the phenomena which manifest themselves after such lesions.

In the first place, every operation of any magnitude on the brain or cord is immediately followed by a depression of the functional power of the nervous tissue distal to the lesion, a depression which may extend far from the actual seat of injury and manifest itself by various phenomena, which are grouped together under the name of 'shock,' better termed spinal or cerebro-spinal shock. to distinguislı it from the cardiorascular or surgical shock already described (p. 175). Thus, when the spinal cord of a dog is divided. $e . g$. in the dorsal region, all power, all vitality, one might almost say. scems to be for ever gone from the portion of the body below the level of the section. The legs hang limp and useless. Pinching or tickling them calls forth no reflex movements. The vaso-motor tone is destroyed, and the vessels gorged with blood. The urine accumulates, orcrfills the paralyzed bladder, and continually dribbles away from it. The sphincter of the anus has lost its tone, and the faeces escape involuntarily. And if we were to continue our observations only for a short timc. a few lours or days, we should be apt to appraise at a very low value the functions of that part of the cord which still remains in connection with the paralyzed 2xtremities. But these symptoms are essentially temporary. They are the immediate results of the section; they are not permanent 'deficiency' phenomena. And if we wait for a time. we shall find that this torpor of the lower dorsal and lumbar cord is far from giving a true picture of its potentialities; that. cut off as it is from the influence of the brain. it is still endowed witl marvellous powers. If we wait long enough, we shall see that, although roluntary motion never returns, reflex movements of the hind-limbs. complex and co-ordinated to a high degrec, are readily induced. Vaso-motor tone comes back. The functions of defecation and micturition are normally performel. Erection of the penis and ejaculation of the semen take place in a dog. I man with complete paralysis bclow the loins and destitute of all sensation in the paralyzed region has been known to become a father (Brachet). Pregnancy carricd on to labour at full term lias been observed in a bitch whose cord was completely divided above the lumbar cnlargement. The scverity and duration of spinal shock are greater in the monkey than in the dog. in man than in the monker, and in the whole mammalian group than in the lower vertebrates. The mechanism of its production has been much discussed, and will be referred to on another page (p. Sos). 
We cannot doubt that the spinal cord takes an important share in the recovery of function after shock. But here again it would be crroncous to conclude that everything is due to the cord. For Goltz and Ewald have been able to keep dogs alive for long periods after preliminary section of the cord in the cervicial region and subsequent removal of large portions of it. They find that even after destruction of the lumbar and sacral regions of the cord the external sphincter of the anus, striped and even voluntary muscle though it be, regains its tone, although it is temporarily lost after the first cervical section. The bladder ultimately recovers the power of emptying itsclf spontancously and at regular intervals. A pregnant bitch in which the lumbar enlargement and the whole cord below it to the cauda equina had becn removed, and therefore all the nerve-roots supplying fibres to the uterus cut, whelped in a normal manner, and the corresponding mammary glands behaved exactly as the rest. Digestion went on as usual when practically nothing of the cord except its cervical portion was left. Certain vaso-motor phenomena were also observed which suggest that the sympathetic system, inclependently of the cerebro-spinal system, is itself possessed of regulative powers (p. 168).

Secondly, we must not run into the opposite error, and assume. without proof, that all the functions which the brain or cord is capable of manifesting under abnormal circumstances are actually exercised by either when, under ordinary conditions, it is working along with and guiding, or being guided by, the other. For example, in many animals certain of the reflex powers of the cord are, if not increased, at all events more freely exercised when the controlling influence of the higher centres has been cut off than when the central nervous system is intact.

Thirdly, there is an other class of phenomena which we must make allowance for, and perhaps more frequently in the case of pathological lesions in man than in experimental lesions in the lower animals. This is the class of 'irritative' phenomena. The irritation set up by a blood-clot or a collection of pus, or in any other way, in a wound of the grey or white matter, may cause a stimulation of nervous tracts by which, for a time, the 'deficiency' effects of the lesion may be masked.

In the fourth place, we must not hastily conclude that when no obvious deficiency seems to follow the removal of a portion of the central nervous system, the function of that portion must necessarily be of such a nature as to give rise to no objective signs. For there is reason to believe that, to a certain extent, the function of one part may, in its absence, be vicariously performed by another.

Bearing in mind the cautions we have just been emphasizing, we may broadly distinguish between tha functions of the cord (including the bulb) and those of the brain proper by saying that the cord is essentially the seat of reflex actions, the brain the seat of automatic actions and conscious prccesses. But neither of these conceptions is entirely correct. Both err by defect and by excess. The brain, it is true, is pre-eminently automatic. The movements which are started in the grey matter of the cerebral cortex are pre-eminently voluntary 
(p. 840), but we cannot deny to the brain the possession of reflex powers as well. The movements in which the only nerve centres concerned are those of the spinal cord are above all reflex (p. 796). But some of its centres, and especially those lying in the medulla oblongata- - .g., the respiratory centre - are, much as they are influenced by afferent impulses, capable of discharging automatic impulses too. And while consciousness is certainly bound up with integrity of the brain, and in all the higher mammals is probably associated with cerebral activity alone, it has been plausibly maintained that the spinal cord, even of such an animal as the frog, is also endowed with something which might be called a kind of luushed consciousness. If this is so for the frog, with its distinct though relatively ill-developed cerebral hemispheres, it must be still more likely in the case of fishes and animals below them, which are practically devoid of a cerebral cortex altogether.

\section{Functions of Spinal Cord (including Medulla Oblongata).}

The functions of the spinal cord may be classified thus :

I. The conduction of impulses set up elsewhere-either in the brain or at the periphery.

2. The modification of impulses set up elsewhere (reflex action).

3. The origination of impulses (?).

I. Conduction of Nervous Impulses by the Cord.-The old controversy as to whether the white fibres of the spinal cord are directly excitable may be considered as definitely settled in the affirmative. The inquiry was complicated by the presence of the spinal roots, which, since the experiments of Charles Bell, have been known to be capable of excitation by artificial stimuli. But at length the difficulty was overcome in this way. The posterior (dorsal) portion of several segments of the cord witl the attached posterior roots and the grey matter was excised. Long strands of the white matter of the anterior (ventral) portion of the cord were isolated, and laid on electrodes, and contractions of muscles were seen to follow stimulation, eren when the anterior roots nearest the stimulating electrodes had been cut, and cvery precaution taken to avoid escape of current on to the distant anterior roots of the nerves supplying the muscles. Indeed, apart from direct experimental evidence, the fact that the white fibres of the brain are universally admitted to be excitable by artificial means would be of itself almost sufficient to decide the question, for we know of no essential difference between the cerebral and the spinal fibres. But the conditions must rarely occur under whicli direct stimulation 
of white fibres in their course is possible in the intact body ; and the only impulses with which we need concern ourselves

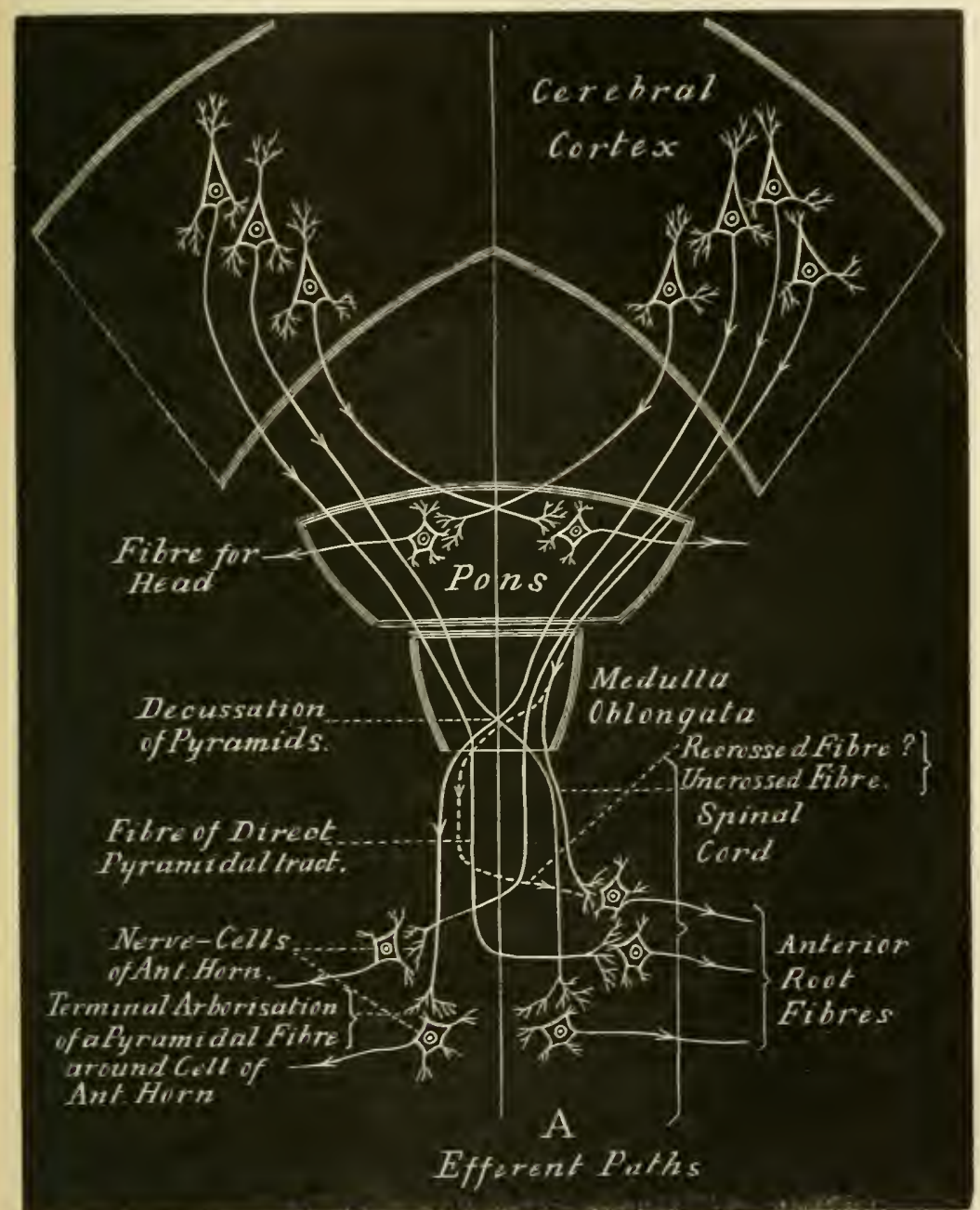

Fig. 336.-Some Possible Paths of Efferext Impulses ix the Cextral Nervols Systeil (Schematic).

Details are omitted from the scheme. For instance, each pyramidal fibre is represented as arborizing around one anterior cornual cell only, and no collaterals are shown. The hypothetical intercalated neurons between the pyramidal fibres and the anterior horn cells (p. 776) are not shown.

here are those that reach the conducting paths from grey matter in the cord itself or in the brain, or from the peripheral organs. 
What sort of impulses do the various tracts of the spinal cord conduct? For the dorsal or posterior roots this question was first fully answered by Magendie; for the ventral or anterior roots, although with a certain degree of ambiguity, by Sir Charles Bell. Bell observed that when, in an animal just killed, he mechanically stimulated the anterior roots, muscular contractions were obtained at each touch of the forceps. He concluded that the anterior roots are motor and sensory, while the posterior roots are 'vegetative'-i.e., connected with the functions of the viscera, the so-called 'vegetative' organs. But although he is often credited with the discovery of the functions of the posterior roots as well, he was not the first to make the decisive experiment necessary to show that they are the conductors of sensory impulses. It was after Magendie's discovery that only a portion of the nerves are sensitive, and that there are nerves "which are like tendons, aponeuroses, or cartilages in insensibility' that Bell formulated the law that the anterior roots are purely motor, the posterior purely sensory. This law, often termed Bell's Law, is more correctly denominated the Bell-Magendie Law.

When the posterior roots are divided, loss of sensation occurs in the region to which they are distributed. If only one root is cut, the loss of sensation is never complete in any part of the skin; and Sherrington has found that the cutaneous areas of distribution of consecutive nerve-roots are not perfectly independent, but to some extent overlap. Stimulation of the peripheral end of the divided posterior root has no effect. Stimulation of the central end gives rise, if the animal be conscious, to evidences of pain, and other signs of the passage of afferent impulses-e.g., a rise in blood-pressure. The latter may also be observed when the animal is anresthetized.

Referred Pain.- The posterior roots contain sensory fibres not only for the skin. but also for the decper structures and the viscera. The afferent fibres reach the viscera by the sympathetic, the vagus, and the pelvic nerves or nervi erigentes. Recent clinical observations have thrown much light upon the distribution of the visceral fibres and their relation to the cutaneous sensory nerves. It has long been known that in disease of an internal organ the pain is often referred to some superficial part. It has now been demonstrated that each organ is related to a more or less definite region. or more than one region, of the skin. In clisease of the organ there is in this area increased excitability (hyperalgesia) or tenderness to slight mechanical stimuli, and often also increased excitability for heat or cold, and the reflexes elicited by stimulation are exaggerated (Head, Dana).

The bond of connection appears to be the origin from the same spinal segments of the autonomic* sensory fibres of any viscus and

* Langley uses the term autonomic nervous system to include the nerve supply of heart muscle, all unstriated muscle, and all gland cells 
the sensory supply of the corresponding cutancous area. The common anatomical origin scems to carry with it a pliysiological correlation, either because the irritation of the visceral fibres spreads in the cord to the somatic afferent fibres which enter the corresponding seginents, or because of some action higher up in the cerebral centres, the nature of which will be best considered along with the general topic of the localization of sensory impressions (p. 982).

Recurrent Sensibility.-Although muscular contraction is the most conspicuous event that follows stimulation of the peripheral end of an anterior nerve-root, it is by no means the only one. It is frequently observed, though not in all kinds of animals, tliat here, too, pain is caused. 'That this pain is not due to the muscular contraction is proved by the fact that it can still be elicited when the nerve-trunk is divided between the junction of the roots and the periphery. The real explanation of the phenomenon is that certain fibres from the posterior roots ('recurrent fibres,' see footnote on p. 693) bend up for some distance into the anterior roots, and then turn round again and pursue their course to their peripheral distribution in the mixed nerve, or run on in the motor roots to supply the sheath surrounding them (nervi nervorum), and even the membranes of the spinal cord.

The afferent impulses that enter the cord along the posterior roots have the choice of many paths by which they may reach the brain. The following are a few of the routes which they may follow :

(I) They may pass directly up through the postero-median column. If they take this route, their course will be first interrupted by nervecells in the gracile or cuneate nuclei in the medulla oblongata. Thence they may find their way across the middle line by the arcuate fibres of the upper or sensory decussation, and sweeping along the fillet and the sensory path in the hinder part of the posterior limb of the internal capsule, finally arrive at the cerebral cortex. Between the gracile and cuneate nuclei and the cortex they pass through nerve-cells in the optic thalamus.

(2) They may pass up by the direct cerebellar tract and restiform body to the grey matter of the cerebellar worm. If they take this route their course will be interrupted very soon after their entrance into the cord in the cells of Clarke's column. Since the superficial grey matter of the vermis is connected by association fibres with the dentate nucleus, and the dentate nucleus by the superior peduncle with the opposite cerebral hemisphere, this is also a possible path to the great brain.

(3) They may reach the antero-lateral ascending tract of the same side through its cells of origin in the spinal grey matter, and passing through the medulla and pons to the superior pedincle of the cerebellum, enter the grey matter of the superior worm.

in the body. It embraces, in addition to the sympathetic, cranial autonomic fibres in several of the cranial nerves and sacral autonomic fibres in the nervi erigentes (see p. $88_{3}$ ). 
4) They may cross the middle line, attce entering the corrl, through axons or collaterals (p. 770 ) which run in the anterior and also in the posterior commissure, enter one of the ascending tracts on the other side - e.g., the tract of Gowers - and continue without further dccussation up to their central destination.

(5) They may spreal from neuron to neuron in the tangle of the grey matter itself, and pass out again at a different level inton onc of the white tracts on the same or on the opposite sicle of the corrl.

\section{Efferent Impulses from the brain may travel:}

1 Through the direct or crossed pyramidal tract

(2) From one side of the cerebral cortex to the other, and then down the pyramidal tracts corresponding to that side (?).

(3) From the frontal part of the cerebral cortex, through the interior limb of the internal capsule to the grey matter in the pons. and thence to the cerebellum by its middle peduncle.

(4) From the occipital or temporal cortex. in the hinder rim of the internal capsule, to the pontine grey matter and through the middle peduncle to the cercbellum. From the cerebellum they may possibly pass down to the nuclcus of Deiters and thence along the anterolateral descending tract to the anterior horn of the cord, and undirectly to the periphery.

All the paths enumerated, as well as other to which it would be tedious to formally refer, and which the ingenuity of the reader may profitably be employed in constructing for himself, from the data already given, are to be looked upon as possible channels for the passage of impulses between the brain and the periphery. But it must be distinctly pointed out that what is certain is in this case much more limited than what is possible. Among the efferent paths it is certain that the pyramidal tracts are conductors of voluntary motor impulses, and that in most individuals the great majority of such impulses decussate in the medulla oblongata, only a small minority in the corl. For a lesion involving the pyramidal tract above the decussation of the pyramids causes paralysis of the opposite side of the bodv, a lesion below the decussation paralysis of the same side. It is certain that when one pyramidal tract has been destroyed, in many animals at least, the resulting paralysis is soon recovered from, at any rate to a great extent, and it is possible that in this case the motor cortex on the side of the lesion has placed itself again in communication with the paralyzed muscles through it: commissural connections with the opposite lemisphere. Tlis, however, is not the only alternative, for, as already pointed out, the pyramidal tracts are not the only cortico-spinal paths which can subserve volitional movements, and division of the anterior portion of the antero-lateral column may cause deeper and more permanent paralysis than division of the pyramidal tract.

Decussation of the Sensory Paths.-On the other hand, it is certain that pathological or traumatic lesions, apparently 
involving the destruction of one lateral half of the cord in man and experimentil semisections in some mammals, are followed by symptoms which suggest that some kinds of sonsory impulies decussate chiefly in the sponal corel-vi\%, diminution or loss of sensibility to pain and to changes of temperature on the opposite sirle below the level of the lesion, with little or no impaiment, and often increase of sensibility (hyperesthesia) on the same side. Tactile sensibility is lost on the side of the lesion, and likewise the muscular sense.

The first general description of this symptom-complex was given by Brown-Séyuarl, although long after he saw cause to retract this interpretation of the facts. While it may be true that in man it has not been rigilly demonstrated that the symptoms are associated with a clean-cut lesion precisely linited to one-half of the corl, clinical observation has on the whole tended to confirm the view that an important portion of the sensory path decussates in the cord. But it is a curious circumstance that experimental physiologists have for the most part obtained contradictory results. Thus Mott, working witl monkeys, found that the different kinds of sensation, far from being abolished, are, as a rule, impaired in a smaller degree on the side opposite to the semisection than on the same side, while Ferrier and Turner obtained on the whole a contrary result, and one that corresponded closely with Brown-Séquard's original description. The discovery that no ascending degeneration, or only a trifling amount, is to be found on the opposite side of the cord, either after semisection or after division of posterior roots, cloes not of itself enable us to decide the question. For while this latter fact shows that few or none of the afferent fibres cross the middle line to enter the long conducting paths before being interrupted by nerve-cells, it by no means proves that afferent impulses do not decussate in the cord. The long paths of the posterior column, indeed, do not decussate below the level of the bulb. The dorsal and ventral spino-cerebellar tracts are also, in the main at least, uncrossed spinal paths. A portion of the afferent impulses must therefore be carriel up to the cerebrum and cercbellum without decussating in the cori. But nobody can tell how massive a link between the two halves of the cord may be formed by the grey matter and the endogenous fibres of the white columns and their collaterals. We know that some afferent impulses do decussate far below the level of the medulla. For, (I) A part of the action current (p. 7 I9) crosses the middle line and ascends in the opposite half of the cord when the central end of one sciatic is stimulated (Gotch and Horsley), (2) Crossed reflex movements are possible : and when excitation of the central end of the sciatic is followed by contraction of the muscles of the opposite fore-limb, the afferent impulses must either decussate in the lumbar cord, and then run up on the opposite sicle to the level of the brachial plexus, or must ascend on the same side and cross over somewhere between the plane of the sciatic and the brachial nerve-roots. The only other hypothesis on which crossed reflex action can be explained-but a hypothesis for which there is not a tittle of eviclence -is that the afferent impulse always acts on the few motor cells whose axis-cylinder processes pass over to the opposite sicle, and there enter anterior nerve-roots. But while, for these reasons, it cannot be denied that some afferent impulses decussate in the cord. 
it would be an error to conclude that all do so in any animal, or that all animals are in this respect alike. It is indeed extremely probable that in different species of animals, and even in individuals of the same species, there are considerable differences in the extent of the sensory decussation in the cord, just as there are in the extent of the motor decussation in the bulb. In some animals the greater part of the sensory path may decussate in the cord; in others the greater part may decussate in the bulb, or higher up. The lack of agreement in the experimental results may be lue partly to this cause. When it is further remembered how difficult it sometimes is to interpret the account which a man gives of his sensations and to recognise precisely the legree and nature of sensory defects produced by disease in the human subject, it will not be thought surprising that experiments on animals, from the time of Galen onwards, should have yielded evidence which, although perhaps now at length tending to a definite result, is still unfinished and in part conflicting.

If, leaving them out of account, not as valueless but as still difficult of interpretation, we attempt to draw any general conclusion from the clinical observations which, however imperfect, are in such questions our surest guide, it can only be this, that in man some of the sensory impulses, and particularly those connected with the cutaneous sensations of pain and temperature, decussate, in part at least, in the cord. But there is also evidence that tactile afferent impulses, including those coming from the muscles and related to the muscular sense, and, it may be, some of the impulses associated with pain, decussute, not in the cord, but in the bulb.

The Paths for Different Kinds of Sensory Impressions.-If this is the state of our knowledge where the problem is merely to determine the crossing-place of afferent impulses which are certainly known to cross, it is only to be expected that we should be still more in the dark as regards the routes by which different kinds of afferent impulses thread their way through the maze of conducting paths in the neural axis to reach their planes of clecussation and gain the 'sensory crossway' in the internal capsule. Some authors have indeed cut the Gordian knot by assuming that any kind of sensory impression may travel $11 \mathrm{p}$ any afferent path. Direct stimulation of a naked nerve-trunk, it has been argued in favour of this view, gives rise to a sensation of pain; stimulation of the skin in which the end-organs of the nerve lie gives rise to a sensation of touch or a sensation of temperature, according as the stimulus is a mild mechanical or a thermal one, the contact of a feather or of a hot test-tube. Why, it has been asked, should we imagine that the difference in the result of stimulation depends on a difference in the nerve-fibres excited, and not on a difference in the kind of impulses set up in the same nerve-fibres? This is a question which we shall have again to discuss (p. 968). But apropos of our present problem, we may say that there is very clear proof from the pathological side that a limited lesion in the conducting paths of the central nervous system may be associater with defect or total loss of one kind of sensation. while all the other kinds remain intact. 
And there secms no other tenable hypothesis than that in such cases the pathological change has picked out a particular group of fibres, either collected into a single strand or scatterel among unaltered fibres of different function. For example, in syringomyelia, a condition in which cavities are formed in the grey matter of the cord secondiry to a new growth of the neuroglia surrounding the central canal, a frequent symptom is the loss in a certain region of sensibility to pain and to changes of temperature, while tactile sensibility is unaffected (dissociation of sensations). Again, in locomotor ataxia, a disease in which inco-ordination of movement and derangement of the mechanism of equilibration are prominent symptoms, degeneration in the posterior column of the cord is a most constant lesion. And there is strong cvidence that afferent impulses from muscles and tendons, which either give rise to impressions belonging to the group of tactile sensations, or produce no effect in consciousness, and which, according to the most widely accepted (loctrine, serve as the basis of the muscular sense, and play an important part in the maintenance of equilibrium (p. 835), pass up in the posterior column. It may also conduct tactile impressions from the skin. A case has been observed where a man received a stab which divided the wholc of one side of the cord and the posterior column of the other side. Sensibility to touch was lost on both sides of the body below the level of the injury, sensibility to pain only on the side opposite to the main lesion. In another case, in which some small syphilitic tumours (gummata) in the lateral column on the left side caused marked degeneration in the left direct cerebellar tract, the tract of Gowers, and the crossed pyramidal tract, without affecting the posterior columns, tactile sensibility was only slightly impaired in the opposite leg, while the sensibility for pain and temperature was much enfeebled. In the left leg, which was paralyzed, there was slight hyperæsthesia. These observations indicate that impressions of pain and temperature pass up in the antero-lateral column, either in the tract of Gowers, or in the direct cerebellar tract, or in both (Déjerine and Thomas).

But it does not follow that they cannot ascend by other paths as well. It appears indeed that the grey matter of the cord, or, rather, short endogenous fibres arranged in series in the antero-lateral column so as to conncct the grey matter at different levels, constitute such a path, and that impulses which give rise to pain can be propagated along a cord in which hardly a vestige of white substance remains uncut. In man the path for pain and temperature impressions along these short endogenous fibres seems to be mainly or entirely a crossed path. The afferent paths for such vaso-motor reflexes as are elicited by stimulation of the central end of the sciatic ascend in the lateral column, and the impulses largely cross the middle line in the cord. The posterior columns have nothing to do with the conduction of painful impressions, for division of them causes not anæsthesia, but rather hyperasthesia, while if they arc left intact, and the rest of the cord, including the grey matter, divided, the animal is insensitive to pain below the level of the lesion. Just as man differs from lower animals in the completeness with which certain of the sensory impressions decussate in the cord, so differences exist in the degree of localization of the different kinds of impressions in particular tracts. One of the outstanding differences is that in animals it seems to be easier for a still intact path to be substitutecl for a severed path as a conductor of impulses which normally traverse 
the latter. The rapiclity with which sensation is restored below the lesion after semisection of the cord in animals is an illustration of this. Another difterence. which can be explained in the same way, is that a sharply-marked (lissuciation of sensations-retention of tactile seusibility, for example, witl loss of sensibility to pain or to pain and temperature clianges-either cannot be produced experimentally in animals, or is very difficult to realize.

The impulses which descend the cord give token of their arrival at the periplery by causing either contraction of voluntary muscles, or contraction of the smooth muscular fibres of arteries, or secretion in glands. They all pass down in the antero-lateral column, but the path of the voliuntary impulses in the pyramidal tracts is the best known and most sharply defined.

\section{Modification of Impulses set up elsewhere (Reflex} Action).- - The spinal cord, although it is a conductor of nerrous impulses originating elsewhere, is by no means a mere conductor. Many of the impulses which fall into the cord are interrupted in its grey matter. Some of the efferent impulses proceeding from the brain are perhaps modified in the cord, and then transmitted to the muscles. Some of the afferent impulses are modified, and then transmitted to the brain: some are modified, and deflected altogether into an efferent path. These last are the impulses which give rise to reflex effects. A reflex action has sometimes been defined as an action carried out in the absence of consciousness; not necessarily; however, in the absence of general consciousness, but in the absence of consciousness of the particular act itself. But the term is now more correctly used so as to embrace all kinds of actions which are not directly voluntary, whether the individual is conscious of them or not. For example. when the sole of the foot is tickled. the leg is irresistibly and involuntarily drawn up by reflex contraction of its muscles ; ret the person is perfectly cognizant buth of the movement and of the sensation which accompanies the afferent impulse. Many reflex actions usually associated with sensations proceed normally when consciousness is entirely in abeyance; during sleep most of the ordinary reflexes can be elicited.

Anatomical Basis of Reflex Action.-Since the essence of reflex action is that the arrival of afferent impulses in the spinal cord or brain causes the discharge of efferent impulses, there must be some connection between the incoming and the outgoing nerve-fibres. When the nervous system is still only a process of an epithelial (sensory) cell joining hands with a muscular cell, the distinction between afferent and efferent fibre does not exist. When development has gone a step further, and the neuro-muscular process is interrupted by a second epithelial cell transformed into a nerve-cell. the afferent fibre enters one pole and the efferent fibre leaves the other pole of the same cell. In a simple reflex action three events can be clistinguished: stimulation of a receiving mechanism. conluction of the excitation, and the consequent reaction or end-effect. The receiving inechanism or receptor may consist of ordinary sensory 
nerve-endings in the skin, or of special sense-cndings, as in the retina or internal car. The conducting mechanism or conductor is made up of at least two neurons, one the afferent portion of the reflex arc connected with the receptor, the other the efferent portion of the arc, connected with the organ, sometimes termed the effector organ-a muscle, e.g., or a gland-which accomplishes the end-effect. The transference of the excitation from the afferent to the efferent neuron takes place across the intervening synapse. The simple isolated reflex arc, as thus described, although a convenient abstraction, corresponds but little to anything which actually exists in one of the higher animals. With increasing complexity of organization the nervous impulse passing up an afferent fibre is in general offererl, instearl of a single efferent path, a choice of many potential routes when it reaches the spinal cord. WF have previously (p. 769$)$ describerl the course taken by the fibres of the posterior ronts on entering the cord. It is obvious that through the main fibres and their collaterals an extensive connection-partly direct, partly by the link of intermediate neurons - is established with the motor cells on both sides of the cord. But the facts of physiology demonstrate an even ampler connection than the mere anatomical study of the clistribution of the rootfibres would suggest. Indeed, the phenomena of strychninepoisoning seem to show that every afferent fibre is potentially connected with the motor mechanisms of the whole cord, or at least with a very large proportion of them. For in a frog under the influence of this drug.

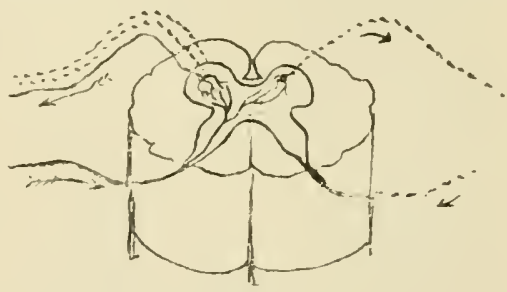

Fig. 337.-Diagrail of a Simple REFLEX ARC.

The arrows indicate the direction of the afferent and efferent impulses. stimulation of the smallest

portion of the skin will cause violent and general convulsions, which are unaffected by destruction of the brain, but cease at once on destruction of the cord (p. 886).

It is therefore a question of great interest how the isolated conduction of the impulses in a given reflex arc is normally achieved. The best answer which can at present be given is that it is not equally easy for a reflex excitation to pass across all the synapses which are potentially open to it. Following the path of least resistance, the excitation traverses the synapse or synapses which it is easiest for it to break through. What property of the synapse is associated with resistance to the passage of the impulse is unknown. But it is a variable property, and when a general reduction in the resistance is produced, as by strychnine or tetanus toxin, an exicitation impressed upon a single afferent path may force a great many synapses normally impervious to it.

While it is convenient in a prelininary survey to speak of the resistance to spreading of the excitation in the cord being diminished by strychnine or by tetanus toxin, we shall see presently that more than this is involved (p. 8or).

Principle of the Common Path.-In considering the architecture of the cerebro-spinal nervous system as a basis of reflex action, one feature is of such importance as to deserve special mention. The 
afferent neurons, running from the receptive surfaces to the centres, constitute each for its own receptive point a 'private' path which can only be used by impulses arising at that point, and not by impulses arising at any other point. Through its central connections an afferent neuron from a single point may be put into communication with numcrous efferent neurons, and thus with numerous and distant effector organs (muscles or glands). Conversely, the efferent portion of a single reflex arc can convey reflex excitations originating in numerous and distant receptive ficlds. It is the sole path which all efferent impulses-let them originate where they may-must use to reach the end-organ in question. It is therefore not a private but a public path, and may be termed in this relation the final common path (Sherrington).

The existence of the common path is of great importance in understanding the manner in which reflexes are compounded together, a problem absolutely fundamental in nervous coordination. One consequence of the existence of a common path is that when, among the receptors which may use it, two are simultaneously stimulated which, when separately excited, produce opposite effects upon the effector organ, only one of the effects is produced. In other words, impulses which produce the two opposed effects can be successively, but cannot be simultaneously, sent along the common path. Thus, 'excitation of the central end of the afferent root of the eighth or seventh cervical nerve of the monkey evokes reflexly in the same individual animal sometimes flexion at the elbow, sometimes extension. If the excitation be preceded by excitation of the first thoracic root, the result is usually extension; if by excitation of the sixth cervical root, it is usually flexion. Yet though the same root may thus be made to evoke reflex contraction of the flexors or of the extensors, it does not evoke contraction in both flexors and extensors in the same reflex response.... The flexor-reflex, when it occurs, seems, therefore, to exclude the extensor-reflex, and vice versa. Either the one or the other results, but not the two together' (Sherrington). It is obvious that this is an advantageous arrangement. An algebraical summation of the opposed effects by the common path would result in a useless action which was neither effective flexion nor effective extension, a compromise and not a co-ordination.

The rôle of the receptor in the reflex arc is above all to sift out from the various kinds of impressions impinging upon the receiving surface the particular kind to which the appropriate response is the reflex action in question. As will be pointed out in greater detail in the study of the special senses, each kind of afferent end-organ has become adapted to a special, or, as it is termed, an 'adequate ' stimulus, so that it is easily affected by this, and with difficulty or not at all by other modes of stimulation. Thus, light is the adequate stimulus of the end-organ of 
the optic nerve, heat that of the end-organs of the nerves by which we perceive the sensation of warmth, mechanical pressure that of the nerves by which we perceive the sensation of pressure. Other kinds of stimuli are either entirely inactive or much less effective in eroking the particular sensory response. There is every reason to believe that the receptor in the reflex arc occupies the same position in regard to adequate stimuli as it does when it functions as a sense-organ.

Sherrington has slown that the different kinds of nerveendings in one and the same area of the skin (in the dog) must be assumed to possess totally different spinal connections, since the movements elicited by stimuli suitable for one form of nerve-ending are quite different from those elicited by stimuli suitable for another.

The 'extensor-thrust' is a reflex obtained in the hind-leg of the dog, and characterized by a brief, strong extension at the hip, knee, and ankle. It is only elicited by a certain kind of mechanical stimulation, best in the spinal dog-i.e., in a dog whose brain has been destroyed or severed from the cord-by pushing the tip of the finger between the plantar cushion and the pads of the toes. It cannot be obtained by electrical stimulation or by any kind of direct stimulation of afferent nerve trunks. The same is true of the pinna-reflex in the cat-i.e., the backward crumpling of the ear elicited by squeezing or tickling its tip. The scratch-reflex, ${ }^{*}$ a scratching movement of the hindfoot, is much more easily elicited in the spinal dog by mechanical stimulation (rubbing, tickling, or tapping) applied to the skin of the back behind the shoulder than by electrical stimulation, which often fails to evoke it at all. The puzzling fact that, according to surgical experience, many of the internal organse.g., the ureters and bile-ducts-can be handled, cut, and sutured without pain, while the passage of a renal calculus or a gallstone may cause excruciating agony, becomes explicable in view of the apparently slight difference which sometimes distinguishes an adequate from an inadequate stimulus. Thus Sherrington has shown that very distinct reflex effects-e.g., a rise of bloodpressure-can be obtained by sudden distension of the bile-duct by the injection of salt solution into its lumen. Distension is here the adequate form of mechanical stimulation, and it is the form induced by the passage of a calculus, while nerve-cutting, although a mechanical stimulus, is not an adequate one.

Conduction in reflex arcs shows certain peculiarities when compared with the conduction in nerve-trunks already studied (p. 687): (I) The direction of the reflex conduction cannot be

* The scratch-reflex is very easily obtained in cats during resuscitation after a period of cerebral anæmia. 
reversed. (2) Its velocity over the whole reflex arc is much smaller than over a nerve-trunk of equal length. Both of these differences depend mainly on the fact that the impulses must be transmitted from one neuron to another, and very likely on a fundamental property of the symapse. (3) The reflex arc is easily fatigued, easily affected by deprivation of oxygen and by drugs, in comparison with the nerve-trunk. This difference is due to the portion of the arc in the grey matter, including the synapse or synapses. (4) The reflex end-effect may much outlast the stimulus -in other words, a marked 'after-discharge' is characteristic of reflexes. The more intense the stimulus which liberates the end-effect, the greater is the duration of the afterdischarge. For example, the 'crossed extension reflex' (extension at the knee, ankle, and hip, produced in the spinal dog by stimulation of the skin of the opposite or contralateral hindlimb), when provoked by a stimulus of more than a certain intensity, may outlast the stimulation by ten or fifteen seconds, and the after-discharge may be stronger than any other part of the reflex (Sherrington). (5) A succession of impulses mav easily pass along a reflex arc when one of the series would fail to pass (temporal summation). This does not occur in a nervetrunk. The first stimulus, though itself unable to produce the reflex effect, facilitates the action of succeeding stimuli, so that summation of the impulses occurs in the cord (Stirling). A stimulus-e.g., a make-indnction shock, far too weak to produce the scratch-reflex when applied once only to a point of that area of skin from which the reflex is normally elicited-has been seen to cause the reflex after more than forty shocks had been delivered at the rate of eighteen per second. (6) The rhythm and intensity of the reflex end-effect correspond much less closely with the rhythm and intensity of the stimulus than in nerve-trunks. (7) The phenomena of refractory period (p. I 4 ), inhibition and 'shock,' are much more conspicuous in the reflex arc than in nerve-trunks.

Inhibition in Reflex Action.-Special cmphasis must be laid upon the part played by inhibition in reflex actions. For the proper carrying out of many reflex movements it is necessary not only that the appropriate effector organ, the appropriate muscle, or group of muscles, should be caursed to contract at the proper time, but that their contraction, or that of other muscles, should be diminished or abolished by inhibition, or even rendered for a certain period impracticable by the establishment somewhere in the reflex arc of a refractory state, which is itself a phenomenon of inhibition. There is good evidence that this is a central inhibition-i.e., it depends on some process occurring in the spinal portion of the reflex arc. 
As an example of the numerons class of reflexes in which the excitation of certain muscles is atcompanied by the inhibition of their antagonists (reciprocal inhibition), we may take the 'tlexion retlex, the flexion at the knee, hip, and ankle of the hind-limb reaclily elicited in the spinal dog by 'nocuous' or harmful stimuli (such ats a prick, a strong squee\%, chemical agents, or excessive heat), or by electrical stimuli applied to the skin of the limb or of any afferent nerve of the limb.

sherrington has shown that when the legs of the animal are so prepared that only the flexors can act on one knee, and only the extensors on the otler, stimulation of symmetrical points on the $t w o$ sides in the area of skin (receptive field) from which the flexion reflex can be evoked causes contraction (excitation) of the flexors and simultaneous relaxation (inhibition) of the tone of the extensors. The same is true when corresponding afferent nerve-twigs are stimulated on the two sides. From this it is inferred that each of the nerve-fibres from the receptive field of the retlex divides in the cord into two sets of end-branches (e.g., collaterals) - a set which produces excitation when it is stimulated, and another set which produces inhibition.

The difference in action is specific in the sense that no mere change in the kind or intensity of stimulation affects it. Yet there are facts which show that the specificity is not absolutely immutable, and that a change of conclitions in the spinal cord may permit excitation of a given group of muscles to be produced by the stimulation of an afferent path which is primarily inhibitory for them. One of the most striking illustrations of this possibility is seen in the action of strychnine. Stimulation of the internal saphenous nerve below the knee-say in a dog after removal of the cerebrum - is known always to produce inhibition of the portion of the quadriceps extensor whose contraction causes the knee-jerk.

If now the animal be poisoned by a small dose of strychnine, stimulation of the nerve causes no longer reflex relaxation, but reflex contraction of the muscle. This fact indicates that the essential action of strychnine is something different from a mere reduction of the resistance to the spread of impulses in the cord (Sherrington). Tetanus toxin produces a similar effect, though more slowly.

Not only is the tone of the extensors diminished or abolished during the activity of the flexors, but the contraction of the knee extensors evoked by striking the patellar tendon, which is called the knee-jerk, either fails to appear, or appears but feebly, when the flexion reflex is simultaneously elicited, even when the mechanical antagonism of the flexor contraction has been eliminated by previously detaching the flexors from the knee. 
The Knee-jerk is sometimes termed a pseudo-reflex. For certain authorities believe that the mechanism by which it is produced is different from that concerned in the reflex blinking of the eyelid, or the reflex retraction of the testicle, or the drawing-up of the foot when the sole is tickled. The strongest objection to considering it an ordinary reflex is the fact that the interval which elapses between the tap and the jerk ${ }_{\frac{1}{1}} \frac{3}{0}$ to $\frac{1}{160}$ second) is distinctly shorter than the reflex time of the extremely rapid lid-reflex, and is not much greater than the latent period of the quadriceps muscle for direct electrical stimulation, as measured under the ordinary conditions of its contraction. The knee-jerk is obtained in undiminished strength when the nerves of the ligamentum patellae have been divided. It is therefore not a reflex movement caused by stimulation of afferent nerves coming from the tendon, and the name "tendonreflex' is clearly a misnomer. But that it is related in some way or other to afferent impulses is certain, for division of the posterior roots that enter into the anterior crural nerve abolishes the knee-jerk. The phenomenon, according to some authors, comes under the head of what is called myotatic irritability - that is, it depends on mechanical stimulation of the slightly-stretched muscle by the pull of the tendon when it is struck. It is necessary for this stimulation that the muscle should be to a certain extent tonically contracted. So that when the afferent fibres are interrupted, or the grey matter of the cord disorganized, and the reflex tone abolished, the knee-jerk disappears. It is admitted by all that, in addition to the direct stimulation of the muscle on the same side, the tendon-tap may cause also a true reflex knee-jerk on the opposite side, the interval between tap and contraction being about $\frac{1}{5}$ second.

Spread or Irradiation of Reflex Action.-As the strength of the stimulus which has been evoking a given reflex movement is increased the reflex effect becomes more and more extensive, spreading out or irradiating in various directions. If, for example, the reflex in question is the flexion reflex elicited by stimulation of the plantar surface of the hind-foot in the spinal animal, increase of the stimulus will cause, in addition to flexion of the same hind-foot, extension of the opposite hind-limb, then in the homonymous fore-limb (i.e., the limb on the same side) extension at the elbow and retraction at the shoulder, then certain definite movements, the details of which need not detain us here, in the opposite fore-limb, and ultimately also definite movements of the head and tail (Sherrington). Obviously there is a certain orderliness in the spread of the reflexes; they follow a certain regular march; the irradiation in the tangle of the spinal paths is not an indiscriminate one. The same fact emerges quite as 
clearly when other reflexes are studied in a similar way; and certain laws or rules which define the spread of the impulses in spinal reflexes have been deduced. For descriptive purposes, in dealing with reflex action, it is convenient to consider each lateral half of the cord as divisible into regions each related on the one hand to a certain area of the $r$-zeptive surface (skin), and on the other to certain muscles. Such regions are those of the neck, including the pinna (cervical), the fore-limb (brachial), the trunk (thoracic), the hind-limb (crural), and the tail (caudal). According to their relation to these regions the spinal reflexes can be classified as 'short ' or 'long.' The short spinal reflexes are those in which the muscular response takes place in the same region as the application of the stimulus. The long reflexes are those eroked when the stimulus is applied to the receptive field of one region, and the response occurs in the musculature of another region. For the short reflexes Sherrington has given a number of rules, which may be stated as follows: (I) The closer together their spinal segments, the easier is it for stimulation of a given afferent root to excite reflex contractions of muscles supplied by a given efferent root. (2) For each afferent root there exists in its own spinal segment (of course, on its own side of the cord) a reflex motor path of as low a threshold (i.e., as easily set into action) and of as high potency (i.e., producing as great a reflex effect) as any open to it anywhere. It has been shown that the afferent nerves of a skeletal muscle are derived from the spinal ganglion corresponding to the segment of the cord containing its motor cells. (3) Motor mechanisms for the skeletal musculature lying in the same region of the cord, and in the selfsame spinal segment, show markedly unequal accessibility to the local afferent channels as judged by the reflex contractions produced. For example, the reflex contraction of the flexors of the knee on the stimulated side, and of the extensors of the opposite knee, is in many animals much more easily elicited than contraction of the extensors of the homonymous and the flexors of the contralateral (i.e. opposite) side. This, however, is not because the last-named extensors and flexors are really incapable of being reflexly affected through the afferent fibres of the corresponding spinal segments, but because the reflex effect produced by them is in this case not contraction but inhibition. (4) The groups of motor cells contemporaneously discharged by spinal reflex action innervate synergic muscles (muscles which act in the same direction in effecting a harmonious movement), and not antergic muscles (which antagonize each other).

This disproves the old idea that the morements, caused by excitation of an efferent spinal root are co-ordinated synergic movements, since at many joints the flexors and extensors botl: 
receive motor fibres from onc and the same root, and stimulation of the root must simultaneously excite antagonistic muscles. 'The collection of fibres in a motor spinal root does not represent a reflex figure-i.e., a number of simple reflexes occurring simultaneously-nor does the receptive field of a reflex correspond with the distribution of an afferent root.'

(5) It follows from (I), (2), and (4) that the spinal reflex movement which can be elicited in and from any one spinal region will exhibit much uniformity even when the exciting stimulus is applied at different and distant points within the receptive field. The flexion reflex of the hind-limb, e.g., will have the same general character-i.e., flexion of each of the three main joints - whatever part of the surface of the limb is stimulated. Yet the flexion movement will be strongest at the joint whose flexors are innervated by motor cells situated in a spinal segment near the entrance of the afferent fibres from the stimulated skin area.

For the long spinal reflexes it is less easy to deduce definite rules, for they can be less easily and constantly evoked than the short reflexes. The so-called laws of spread formulated by Pfluger for the long spinal reflexes, and based mainly on observations made in the brainless frog and on clinical records in cases of spinal lesion in man, need not be stated here. For Sherrington has shown that they require serious modification. Especially is this true of Pfluger's fourth law that the reflex irradiation spreads always more easily up in the direction of the medulla oblongata, so that stimulation of a fore-limb does not cause reflex contraction of a hind-limb, although excitation of a hindlimb may cause movement of one or both fore-limbs. This law does not hold in the mammal. As a rule, indeed, irradiation takes place more easily down than up the cord. Excitation of the skin of the pinna easily causes reflex movements of the limbs, while the reverse is rare. Reflex movements of the hind-limb in the spinal animal are more easily evoked by stimulation of the fore-limb than movements of the fore-limb by stimulation of the hind. It is easier for the irradiation to cross the cord from hind-limb to hind-limb than to pass up from hind-to fore-limb ; but it is often easier for irradiation to occur down the cord from fore- to hind-limb than across the cord from one fore-limb to the other. Afferent channels from the skin of the shoulder, through which the scratch-reflex is discharged (in the dog), are freely connected with efferent paths to the muscles of the hip, knee, and ankle by an uncrossed path descending the lateral column (Sherrington). In cats, after temporary occlusion of the cerebral circulation, which throws the brain out of gear, it is easy to elicit movements of the hind-legs by pinching the fore-paws or the shin of the 
upper part of the body. The scratch-reflex can also be very readily evoked, and in great intensity, by stimulating the pinnat, and is not confined to the sicle stimulated. In anremia of the brain and (cervical) cord and subsequent resuscitation, homolateral reflexes (i.e., on the same side as the stimulus) are submerged later and emerge sooner than contralateral reflexes whose centres lie in the area which was rendered anemic (Pike, Guthrie, and Stewart).

Co-ordination of Reflexes.-The co-ordination or orderly combination of muscular actions for the production of appropriate and harmonious movements is one of the most important functions of the central nervous system. Both the brain and the cord take a share in this co-ordination. The rôle of the brain will be considered later on, but it is essential to recognise now that many of the movements which the brain directs represent spinal reflexes already synthesized, compounded, or co-ordinated in a very high degree. This is the reason why, in the spinal animal, the inexperienced observer may sometimes be startled by the apparently ' purposive character ' of a reflex movement-the scratch-reflex in the dog or cat, e.g., or the extensive reflex movements of the hind-legs of a brainless frog when the skin is pinched or painted with dilute acid, so plainly directed to the seat of irritation. When a drop of acid is applied to the flank of such a frog, it will attempt to wipe it off with the foot which is situated most conveniently. If this foot be held, it will use the other.

In the combining of reflexes we may distinguish between simultaneous combination-i.e., the combination of reflex actions taking place at the same time-and successive combination-i.e., the combination of reflexes in such a way that they follow each other in an orderly sequence. The facts already mentioned in speaking of irradiation afford a partial explanation of the co-ordination of reflexes by simultaneous combination. The movements are orderly and harmonious because the spread of the reflexes is not indiscriminate, but follows a definite ' march,' determined partly by the anatomical relations of afferent and efferent paths, partly by the varying resistance of the synapses or other structures whose properties fix the threshold value of the excitation by which an arc can be forced. In general it is not enough that the channel of the final common paths (p. 798) to the muscles whose contraction produces the reflex movement should be thus open to the afferent arcs that elicit the movement; they must be closed to other afferent arcs which might disturb the reflex. Not only so: there is evidence that very frequently the final common paths are, so to say, more widely opened to the afferent arcs in question by the ' reinforcing' or 'facilitating' influence of allied. though it may be distant, afferent arcs, which are simultaneously excited (p. So $)$ ). Further, 
the final common paths to antagonistic muscles must also be temporarily closed. The closing of these central connections, or rather the raising of their threshold sufficiently to har the impulses from passing through the door, is an inlibitory plecnomenon. Inhibition and excitation go hand-in-hand in the simultaneous combination of reflexes.

The successive combination of reflexes is well illustrated by the contraction of the wesophagus in deglutition. First one portion of the tube and then the next below are involved in the reflex action. The combination consists in the orderly sequence. The manner in which this is secured in this class of reflex actions has been luminously discussed by Sherrington,* but details cannot be given here. While only allied reflexesi.e., such as mutnally reinforce and therefore harmonize with each other-can be simultaneously combined, and antagonistic reflexes cannot, both allied and antagonistic reflexes can be successively combined. An example of the successive combination of allied reflexes is the series of scratch-reflexes caused by a parasite travelling across the receptive field of the reflex. An example of the successive combination of antagonistic reflexes is afforded when either the scratch-reflex or the flexion reflex is induced and caused to interrupt the other while it is proceeding. The transition-e.g., from flexion to scratch reflex -is made without any period of confusion. The same holds good for other antagonistic reflexes. In many cases the avoidance of confusion is due to the inhibition of the first reflex, or often to inhibition of the set of muscles which were active in the first reflex combined with excitation of their antagonists (so-called interference). It is obvious that this is an adaptation of great importance.

Influence of the Brain on the Spinal Reflexes.-The spinal reflexes can be influenced by impulses descending from the higher centres. For $(a)$ it is a matter of common experience that a reflex movement may be to a certain extent controlled, or prevented altogether by an effort of the will, and it is worthy of remark that only movements which can be voluntarily produced can be voluntarily inhibiterl. A scratching reflex in the normal dog may be seen to be modified in character or duration as compared with the same reflex in the spinal animal. (b) An animal like a frog responds to stimuli by reflex movements more readily after the medulla oblongata has been divided from the spinal cord. (c) Long-continned muscular contractions may be caused in animals after removal of the cerebral hemispheres by stimulation of afferent nerves-for example. by scratching the mucous membrane of the mouth in a 'brainless' frog or Necturus. (d) By stimulation of certain of the ligher centres reflex movements which would otherwise be elicited may be suppressed or greatly delayed. If the cerebral hemispheres are

* 'Integrative Action of the Nervous System,' to which work the advanced student is referred. 
removed from a frog. and one leg of the animal dipped into dilute acid, a certain interval, the (uncorrected) reflex time, will elapse before the foot is (lrawn up) (p. 886). If, now, a crystal of common salt be applied to the optic lobes or the upper part of the spinal cord, and the experiment repeaterl, it will be found either that the interval is much lengthened or that the reflex disappears altogether. Strong stimulation of an afferent nerve may abolish or delay a reflex movement which is being elicited through other receptors.

That the brain exerts more than a merely inhibitory influence on the production of reflex movements is suggested by many facts. The knee-jerk, for example, is increased or 'reinforced ' if an instant before the tendon is struck the patient makes a voluntary movement or is acted on by a sensory stimulus (Bowditch and Warren). In health it varies in strength with many circumstances which affect the activity of the central nervous system as a whole (Lombard, etc.). It often disappears in pathological lesions, situated high up in the cord in man, and is markedly impaired after high section of the cord in dogs. In hemiplegia (paralysis of one sicle of the body, caused by disease in the brain) the cutaneous reflexes on the paralyzed side may sometimes be absent for years. Some observers have even gone so far as to say that, under normal conditions, the so-called spinal reflexes are really cerebral-in other words, that the afferent impulses run up to the brain and there discharge efferent impulses, which pass down to the motor cells of the anterior horn and cause their discharge. It may be admitted that there is no physiological ground for supposing that the afferent impulses which have to do with the reflex contraction of the muscles of the leg when the sole is tickled. stop short at the motor cells of those spinal segments from which the efferent nerves come off, while the afferent impulses which have to do with the sensation of tickling pass up to the brain. The probability is that under ordinary circumstances such afferent impulses pass up the cord in long afferent paths, as well as directly towards the motor cells along those fibres of the posterior roots and their collaterals which bend forward into the anterior horn at the level of their entrance into the cord. And the only question is whether, as a matter of fact, the spinal motor cells are most easily discharged by the impulses that reach them directly, or by the impulses that come down to them by the roundabout way of the brain and the efferent fibres that connect it with the cord. It is evident that the answer to this question need not be the same for all kinds of animals. It may well be that in the higher animals, in which the cortex has undergone a relatively great development, the spinal motor mechanisms are more easily discharged from above than from below, while in lower animals the opposite may be the case. IVhen the cord is cut off from the brain, the afferent impulses may overflow more easily into the spinal motor cclls since their alternative path is blocked. In the frog, where there is already a beaten track between the posterior root-fibres and the cells of the anterior horn, this overflow may be established immediately after section of the cord, and may of itself lead to an exaggeration of the reflexes. In animals like the dog a longer time may be necessary before the unaccustomed route from the end arborizations of the afferent axons and their collaterals to the dendrites or the bodies of the motor cells becomes natural and easy ; in man a still longer interval may be required. Noore and Oertel have made a careful comparative study of reflex action after com- 
plete section of the cord in the cervical or upper dorsil region, and conclude that the spinal reflexes in the ligher animals are far more dependent on the upper portions of the central nervous system than in the frog.

The phenomena of spinal shock and its varying scverity in different animals may be accounted for by the rupture of the paths normally used in the reflexes. The theory that the shock is due to an inhibition set up by the mechanical injury is untenable. For shock affects only the portion of the central nervolis system distal (or aboral) to the lesion. When a dog is allowed to live after transection of the cord in the lower cervical region till shock has been recovered from, a second transection distal to the first is followed by only slight and very transient depression of the reflex power, although the direct effect of the second injury ought, of course, to be as great as that of the first. Finally, according to Sherrington, the condition of the spinal reflex ares in shock differs from the condition caused by inhibition, and resembles rather a gencral spinal fatigue in which conduction along the arc and especially across the synapses is difficult and uncertain. This condition is supposed to be due to the loss of a 'tonic 'influence of higher centres, assumed to be necessary for the maintenance of the normal conductivity of the arc. These cranial centres, if they exist, or, at least, the most efficient of them, must be assumed to be situated distal to the cerebral cortex, probably in the pons or midbrain. For section just behind the pons causes much more severe shock than remoral of the cerebral hemispheres.

Peripheral Reflex Centres.-The question whether any reflex centres cxist outside of the spinal cord and brain, and especially in the sympathetic ganglia, has been the subject of a lengtlyy controversy. That the spinal ganglia cannot act as reflex centres is generally acknowledged, and it is not difficult to sec that, for anatomical reasons, this must be so. A reflex arc must, so far as we know, in all highly-organized animals, include at least two neurons. There is no proof that an afferent impulse can ascend an axon to a cell-body and there excite an efferent impulse, which, descending the same axon in a separate set of fibrils, gives rise to a reflex contraction, or a reflex secretion. Now, the cells of a spinal ganglion represent the original neuroblasts from which the posterior rootfibres grew out as processes towards the cord on the one side and the periphery on the other. A sensory fibre passing into the ganglion makes connection with a cell by a $\mathrm{T}$-shaped junction and passes on its course again. No afferent fibres run from the nervetrunk into the gangtion, to end in arborizations around the ganglion cells, and no efferent fibres arise from nerve-cells in the ganglion to pass out into the trunk. For although a slightly greater number of inclullated fibres of small calibre is found in a spinal nerve-trunk immediately distal to the junction of the roots than in both roots taken together, this appears to be due to the passage into the nerve (from the grey ramus communicansi) of medullated fibres which end in the bloortvessels or other tissue of the ganglion (Dalc). Here it is evirlent that there is no possibility of a complete reflex arc. Indece, it is not certain that the normal afferent impulses pass through the bodies of the spinal ganglion cells at all. For (1) a negative variation can be observed in the posterior roots above the ganglia on stimulation of the trunk of a frog's sciatic nerve more than two day's after the death of the animal, when the ganglion 
cells may be supposed to Jate completely lost their vitality, and when 110 reflex 1 egative variation can be detected in the central stump of a severed anterior root on excitation of the sciatic or the corresponding posterior root. Such a reflex action current is normally obtainable from a fresh preparation. (2) When the bloodsupply of the posterior root-fibres and the ganglion is cut off without killing the frog. the nerve impulse is still conducted by the fibres, as is shown by the reflex movements eliciter on stimulation of the central end of the sciatic, at a time when the nerve-cells show marked histological alterations. (3) Prolonged excitation of the posterior roots or the mixed nerve causes no noticeable microscopical clianges in the ganglion cells (Steinach).* (4) The application of nicotine to a spinal ganglion does not hinder the passage of impulses through the corresponding afferent fibres. If it acts on spinal ganglion cells as it dloes on sympathetic ganglion cells (p. 165), this must be because the impulses do not require to traverse the ganglion.

Axon-reflexes.- In the ordinary sympathetic ganglia, $\uparrow$ also, it is doubtful whether the anatomical foundation for a reflex arc exists, and the most careful physiological experiments have failed to connect them with any reflex function. Sokownin, indecd, observed that stimulation of the central end of the hypogastric nerve caused contractions of the bladiler, and he considered these movements to be reflex, the centre being the inferior mesenteric ganglion. Langley and Anderson have also found that when all the nervous connections of the inferior mesenteric ganglion, except the hypogastric nerves, are cut, stimulation of the central end of one hypogastric causes contraction of the bladder, the efferent path being the other hypogastric. In addition, they have observed an apparent reflex excitation of the nerves which supply the erector muscles of the hairs (pilo-motor nerves) through other sympathetic ganglia. They belicve, however, that in neither case is the action truly reflex, but that it is caused by stimulation of the central ends of motor fibres, which come off from the spinal cord, and in passing through the ganglion give off collateral branches to some of its cells. In the case of the inferior mesenteric ganglion the spinal fibres passing down in the left hypogastric would send branches to arborize around ganglion cells which give origin to fibres of the right hypogastric, and vice versa. When the central end of the left hypogastric is stimulated the excitation is conducted up the spinal fibres, and so reaches their branches, and, through the ganglion cclls, the sympathetic fibres of the right hypogastric, which convey it to the muscles of the blaclder (sec sartorius or gracilis experiment of Kiilune, p. 685). Other examples of such axon-reflexes exist.

Reflex Time.-When a reflex movement is evoked, a measurable period elapses between the application of the stimulus and the commencement of the movement. This interval mav be called the uncorrected reflex time or the latent

* Hodge obtained changes. In such experiments it is necessary that the ganglion should not be directly excited by electrotonic currents or escape of the stimulating current.

$\dagger$ The ganglion cells of Anerbach's and Meissner's plexus in the intestine are not of ordinary sympathetic type, and, as has been previously pointed out, it is probable that they, or some of them, are true reflex centres for the stomach and intestines. 
period of the reflex. A part of the interval is taken up in the transmission of the afferent impulse to the reflex centre, a part in the transmission of the efferent impulse to the muscles, a part represents the latent period of muscular contraction, and the remainder is the time spent in the centre, or the true reflex time. Ordinarily this time, though absolutely short, is relatively so great that the total latent period of a reflex is much longer than when a similar length of nerve-trunk is interposed between the point of application of the stimulus and the muscle. When the conjunctiva or eyelid is stimulated on one side both evelids blink. This is a typical reflex action reduced to its simplest expression, and the true reflex time is correspondingly shortonly about $\frac{1}{2 \pi}$ second $\left(50 \sigma^{*}\right)$. An additional $\frac{1}{0} \pi$ second (Io $\sigma$ ) is consumed in the passage of the afferent impulse along the fifth nerve to the medulla oblongata, of the efferent impulse from the medulla to the orbicularis palpebrarum along the seventh nerve. and in the latent period of the muscle. When a naked nerve, like the sciatic, is stimulated, the true reflex time is reduced to 1, the uncorrected reflex time is greatly lengthened, it may be to several, or even many. seconds. For here it is evident that the time taken by the acid to soak through the skin and reach the nerve-endings in strength sufficient to stimulate them is included. But even when the peripleral factors remain constant, the central factor may vary. With strong stimulation, e.g.. the reflex time is shorter than with weak stimulation. With weak stimuli the latent period of the flexion reflex in the dog is usually $60 \sigma$ to I20 $\sigma$. It may even be as long as $200 \sigma$. Witll strong stimuli it may be as little as $30 \sigma$. Even $22 \sigma$ has been seen, which is little more than for nerve-trunk conduction. Fatigue of the nerve-centres delays the passage of impulses through them ; and strychnine, while it increases the excitability of the cord, also lengthens the reflex time.

Reflexes in Disease.-In order that a reflex action may take place, the reflex arc-afferent nerve, central mechanism, and efferent nerve-must be complete; and, in fact, a whole series of simple reflex movements exists, the suppression, diminution, or exaggeration of which can be used in diagnosis as tests of the condition of the reflex arc. It is customary to divide these into superficial reflexes, elicited from receptive fields on the surface of the borly (extero-ceptive fields), and deep reflexes, elicited from receptors in the depth of the organism (proprio-ceptive fields), especially in the muscles and the tendons and joints connected with them. The extero-ceptive reflexes are normally excited by extraneous stimuli acting on the surface from the environment. The proprio-ceptive reflexes are normally excited by changes (muscular contractions) occurring in the body itself, which changes are in turn usually initiated by excitation of surface receptors by the environment.

\footnotetext{
$* \sigma=0^{*}$ ou i second.
} 
Examples of superficial reflexes are the plantar reflex (the drawingup of the foot when the sole is ticlied), the cremasteric reflex (retraction of the testicle when the skin on the inside of the thigl just below Poupart's ligament is strokerl, especially in boys), the gluteal, abdominal. epigastric, and interscupular reflexes (contraction of the muscles in those regions when the skin covering them is tickled). The behaviour of the toes, especially of the great toe, is of considerable diagnostic importance. Normally, on tickling the sole, fthe toes are flexed towards the planta; but when a lesion of the pyramidal tract exists, as in liemiplegia, there is clorsal instead of plantar flexion, most marked in the case of the great toe, and the toe moves more slowly than in the healthy person (Babinski's sign). In children during the first few montlis of life stimulation of the sole causes normally a dorsal flexion of the big toe. Examples of deep reflexes are the knee-jerk (a sudden extension of the leg by the rectus femoris and vastus medialis components of the quadriceps muscle when the ligamentum patella is sharply struck), the heel-jerk or foot-jerk (a movement of the foot caused in most healthy persons, though not in all, by tapping the tendo Achillis), and the periosteal radial reflex (a movement of flexion and slight pronation of the forearm and hand elicited by tapping the lower end of the radius). The jaw-jerk (a movement of the lower jaw when, with the mouth open, the chin is smartly tapped) and ankle-clonus (a series of spasmodic movements of the foot, brought about by flexing it sharply on the leg) are phenomena of the same class, which can be elicited only in disease. Any condition which impairs the conducting power of the afferent or efferent fibres of the reflex arcinecessarily diminishes or abolishes the reflex movement, even if the cen-

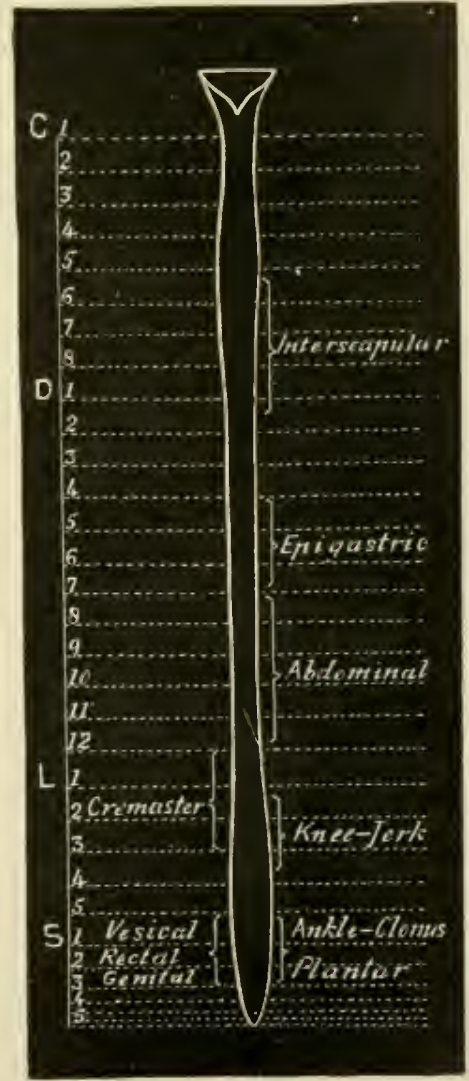

Fig. 338. - Diagram of Reflex Centres in Cord (after Hill). tral connections are intact. E.g.,

in locomotor ataxia the disappearance of the knee-jerk is one of the most important diagnostic signs. This clisease involves the posterior roots and the fibres that continue them in the posterior column. The anterior nerve-roots are perfectly healthy. The grey matter of the cord-at least, in the earlier stages of the disease-is unaffected. The weak link in the chain is the afferent path. Where the presence of the knec-jerk is doubtful, it is necessary 
to search for the most favourable position of the limb for eliciting it before determining that it is absent. The patient may be made to clasp his hands tightly at the moment of the tap to reinforce the jerk (p. 807). In anterior poliomyelitis (1). 775) the afferent link is intact, but the other two are broken, and the reflexes also disappear. Certain lesions which partially cut off the spinal cord from the higher centres without affecting the integrity of the spinal reflex ares increase the strength of reflex movements and the facility with which they are called forth. In primary spastic paraplegia (a paralysis of the legs and the lower portion of the bo(ly), which is associated with degenerative changes in the lateral columns, the deep reflexes are all exaggerated. But, according to the best authorities, a lesion amounting to total transection of the cord in man abolishes all reflexes below the lesion. In the monkey the knec-jerk may be tried for in vain for weeks after section of the cord in the midlle of the thoracic region, whereas in the rabbit it can be obtained ten to fifteen minutes after the transection. The position of the centres in the cord for the various reflex movements is shown in Fig. 338.

3. The Origination of Impulses in the Spinal Cord (Autonatism).-A physiological action is termed automatic when it depends upon a nervous outflow which seems to be spontaneous, in the sense that it is not brought about by any evident reflex mechanism, or, in other words, is not discharged by afferent impulses falling into the centre where it arises, although it may be determined by substances in the blood. An action known to be caused or conditioned by such afferent impulses is called a reflex action. Automatic actions being thus defined in a negative manner by the defect of a quality. there is always a possibility that some day or other it may be demonstrated that any given action which at present seems automatic in its origin depends on afferent impulses hitherto unnoticed. As a matter of fact, the supposed proofs of spinal antomatism have in more than one case vanished with the advance of knowledge, and as the domain of purely automatic action has been narrowed, that of reflex action has $\mathrm{ex}$ tended, until the controversy as to the boundaries between the two seems not unlikely to be ended by the absorption of the automatic in the reflex. And as we seem almost driven to conclucle that from the anatomical standpoint the nervous system is essentially a rast collection of looped conducting paths. each with an afferent portion, an efferent portion, and connections between them formed by the end arborizations of the axons and their collaterals, the dendrites and the cell-bodies, so it may be that no strict plyysiological automatism really exists either in cord or brain, that every form of physiological activitymuscular movement, secretion, intellectual labour, consciousness itself-would cease if all afferent impulses were cut off from the nervous centres. Assuredly no neuron is entirely 
isolated from other neurons. The more the nervous system is investigated, the deeper grows the conviction of its essential solidarity, the more clearly it displays itself as a single mechanism, the most distant parts of which are intricately knit together. But there are certain groups of actions so widely separated from the most typical reflex actions that, provisionally at least, they may be distinguished as automatic. Such are the voluntary movements, and certain involuntary movements, like the beat of the heart. And we may proceed to inquire whether the spinal cord has any power of originating movements or other actions of this high degree of automatism.

Muscular Tone.-So long as a muscle is connected with the spinal segment from which its nerves arise, it is never completely relaxed ; its fibres are in a condition of slight tonic contraction, and retract when cut. If a frog whose brain las been destroyed is suspended so that the legs hang down, and one sciatic nerve is cut, the corresponding limb may be observed to elongate a little as compared with the other. At one time this tone of the muscles was supposed to be due to the continual automatic discharge of feeble impulses from the grey matter of the cord along the motor nerves. But it has been proved that if the posterior roots be cut, or the skin removed from the leg, its tone is completely lost, although the anterior roots are intact. So that the tone of the skeletal muscles depends on the passage of afferent impulses to the cord, and must be removed from the group of automatic actions and included in the reflexes.

The 'rigidity' of the muscles, often observed in paralysis from lesions of the central system, and denominated 'early' or 'late' according as it comes on within a few days or a few weeks after the occurrence of the lesion, is also probably in part, at least, a reflex phenomenon, although, perhaps, possessing some of the characters of a tonic contraction due to automatic discharge from the spinal centres. For in such cases 'myotatic irritability ' is increased ; the knee-jerk is exaggerated ; a fingerjerk may be elicited by tapping the wrist, an arm-jerk by striking the skin over the insertion of the biceps or triceps, ankle-clonus by flexing the foot (Gowers). Now, myotatic irritability depends on reflex muscular tone (p. 8oz).

It is probable that the tone of such visceral muscles as the sphincters of the anus and bladder has also a reflex element, and possible that the same is true of the tone of the smooth muscular fibres of the bloodvessels on which the maintenance of the mean blood-pressure so largely depends (p. I69).

Trophic Tone. - The degenerative changes that occur in muscles, nerves, and other tissues when their connection with 
the central nervous system is intermpted have been already referred to (p. 699). It is possible to explain these clianges in some cases without the assumption that tonic impulses are constantly passing out from the brain and cord to control the nutrition of the peripheral organs; and we have seen that there is no real evidence of the existence of specific trophic fibres. But the degeneration of muscles after section of their motor nerves is difficult to understand except on the hypothesis that impulses from the cells of the anterior horn influence their mutrition. The only question is whether these are tlie impulses to which muscular tone is due, and therefore reflex, or different in nature and automatically discharged. Now, degeneration of a muscle is not usually caused, or at least not for a long time, by intermption of its afferent nerve-fibres, as in locomotor ataxia, or after section of the posterior nerve-roots (Mott and Sherrington). We can hardly suppose that in any case the troplic influence of the cells of the spinal or sympathetic ganglia to which all other reflex powers have been denied, is of reflex nature. And there is, indeed, more evidence in favour of trophic tone being an automatic action of the cord than for any of the other tonic functions hitherto considered.

The evidence for respiratory automatism upon which the spinal cord has been chiefly credited with true automatic action has previously been given (p. 233).

The 'Centres' of the Cord and Bulb.-We have frequently used the word 'centre' in describing the functions of the spinal cord, but the term, although a convenient one, is apt to convey the idea that our knowledge is far more minute and precise than it rcally is. When we say that a centre for a given physiological action exists in a definite portion of the spinal cord, all that is meant is that the action can be called out experimentally, or can normally go on, so long as this portion of the cord and the nerves coming to it and leaving it are intact, and that destruction of the 'centre' abolishes the action. For example, a part of the medulla oblongata on each side of the middle line in the floor of the fourth ventricle above the calamus scriptorius is so related to the function of respiration that when it is destroyed the animal ceases to breathe. But this respiratory centre-the 'noud vital' of Flourens-does not correspond in position witl any definite collection of grey matter, although it includes the nuclei of origin of several cranial nerves, and forms an important point of departure for efferent, and of arrival for afferent, fibres connected with the respiratory act. Its destruction involves the cutting off of the impulses constantly travelling up the vagus to modify the respiratory rhythm, and of the impulses constantly passing down the cord to the plurenics and the intercostal nerves. And just as the traffic of a wide region can be paralyzed at a single blow by severing the lines in the neighbourhood of a great railway junction, or morc laboriously, though not less cffectually, by separate section of the same traclis at a radius of a hundred miles, so destruction of the respiratory centre accomplishes 
by a single puncture what can be also performed by section of all the respinatory nerves at a distance from the medulla oblongata. But while nobody speaks of the destruction of a 'centre' when a reflex action is abolished by division of the peripheral nerves concerned in it, there is a tendency, when the same effect is brought about by a lesion in the brain or cord, to invoke that mysterious name, and to forget that the cerebro-spinal axis is at least as much a stretch of conducting paths as a collection of discharging nervous mechanisms.

It is, perhaps, a profitless task to cnumerate all the so-called centres in the bulb and cord with which the perverse ingenuity of investigators and systematic writers has encumbered the archives and text-books of physiology. In addition to the great vaso-motor, respiratory, cardio-inhibitory and cardio-augmentor centres in the bulb, which, perhaps, have more right than the rest to be regarded as distinct physiological mechanisms, if not as definitely bounded anatomical areas, there had been distinguished ano-spinal, vesicospinal, and genito-spinal centres in the lumbar cord, a cilio-spinal centre for dilatation of the pupil in the cervical cord, and in the medulla centres for sneezing, for coughing, for sweating, for sucking, for masticating, for swallowing, for salivating, for vomiting, for the production of general convulsions, for closure of the eyes, for the sccretion of tears, and even a 'diabetes' or 'sugar' centre (p. 5 I8). It would be just as correct, and more practically useful (for it would perhaps encourage the student who has lost his way amidst these interminable distinctions), to say that the cerebral cortex contains a centre for learning sense, and another for forgetting nonsense, and that in a healthy brain it is the latter which is generally thrown into activity in the study of a list like this.

\section{The Cranial Nerves.}

Unlike the spinal nerves, which arise at not very unequal intervals from the cord, the nuclei of the cranial nerves, with the exception of the olfactory and optic, are crowded together in the inch or two of grey matter of the primitive neural axis in the immediate neighbourhood of the fourth ventricle and the Sylvian aqueduct. Of these nuclei some are the end nuclei or 'nuclei of reception' of sensory fibres - that is to say, collections of nerve-cells around which the sensory fibres break up into terminal arborizations. Such are the sensory nuclei of the fifth, the nuclei of the eighth, and the sensory nuclei of the glossopharyngeal and vagus nerves (Figs. 339, 340). The nuclei of origin of the motor fibres lie, upon the whole, in two longitudinal rows - a median row, which consists of the nuclei of the third and fourth nerves in the floor of the aqueduct, and those of the sixth and twelfth nerves in the floor of the fourth ventricle; and a lateral row comprising the motor nuclei of the fifth, seventh, tenth, and eleventh nerves. The clumps of grey matter which make up these nuclei may be considered as homologous with the grey matter of the ventral or anterior 
(including the lateral) liorn of the spinal cord ; and the motor fibres of the nerves themselves as homologous with the anterior spinal roots. Without going further into the thorny subject of the homologies of the cranial and spinal nerves, we may point out that while all the spinal nerves contain botl efferent and afferent filores, some of the cranial nerves are purely efferent, some purely afferent, and others mixed. So that if we are to look upon the motor nerves as the homologues of the rentral roots, the dorsal (posterior) rout-fibres corresponding

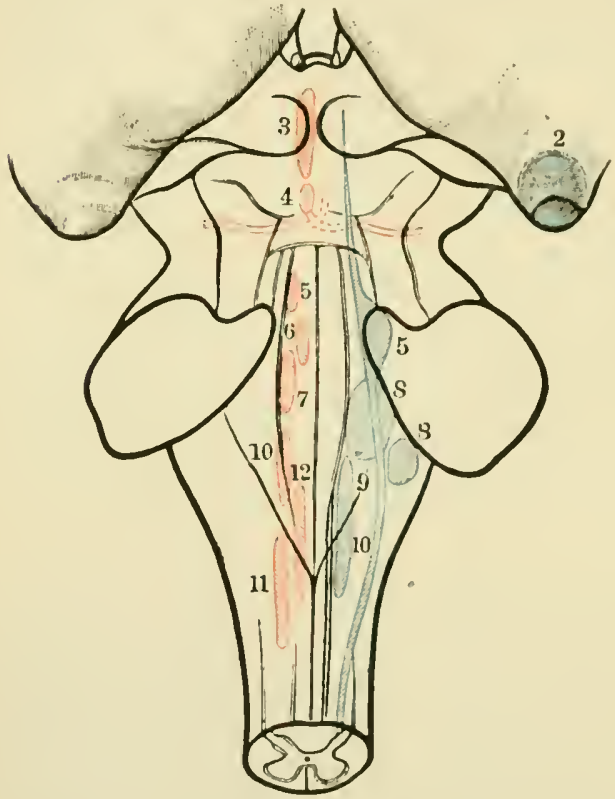

Fig. 339.-Nuclei of Cranial Nerves (Tolnt).

Motor red, sensory blue. The numbers correspond to the cranial nerves. representing a dendrite, which runs ont to the surface of the mucous membrane, and a longer but more slender process, representing an axon, which as a fibre of the olfactory nerve pierces the cribriform plate of the ethmoid bone, and plunges in to the olfactory bulb.

In the olfactory bulb at least four layers can be distinguished (1) on the surface, beneath the pia mater, the layer of entering olfactory nerve-fibres; (2) the layer of olfactory glomeruli, peculiar structures, each of which is made up of an intricatc basket-like arborization formed by an olfactory nerve-fibre, or, it may be, more than one, and a brush-like arborization belonging to a dendrite of one of the mitral cells of the next layer; (3) the molecular or mitral layer, which contains a number of large nerve-cells called, from their 
most common shape, milval cells, along with smaller nerve-cells ('granules') and neuroglia; (t) the nuclear layer, containing numerous small nerve-cells or 'granules' intermingled with white fibres. The mitral cells give off axons, which pass through the fourth layer, and then as fibres of the olfactory tract to the grey matter of the hippocampal region of the brain. The course of the impulses from the olfactory mucous membrane to the brain is shown in Fig. $3+1$. The olfactory tract, as it runs back, divides into portions called its 'roots.' 'Of these the lateral is the most

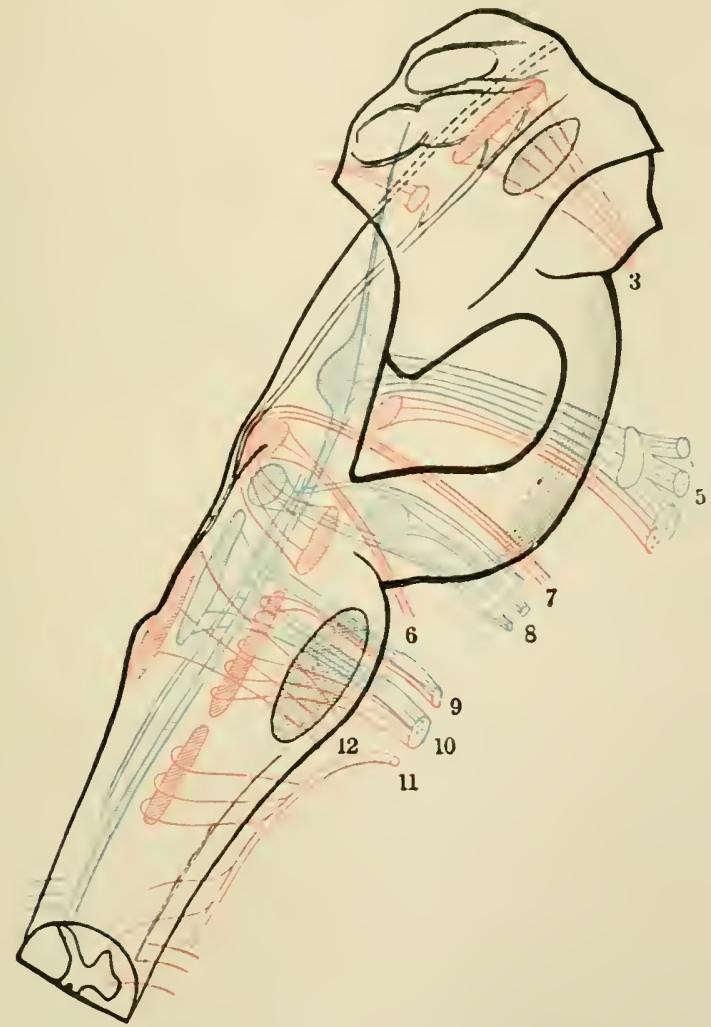

Fig. 340,-Nuclei axd Roots of Cranial Nerves (Toldt).

Lateral view. Motor red, sensory blue.

important, and it terminates in the hippocampal and uncinate gyri of the same side. Fibres of the olfactory tract are also connected either directly or through the relay of another neuron with the opposite side of the brain, especially the opposite uncinate gyrus. The anterior commissure contains numerous fibres, which connect the hippocampal regions of the two sides. Other central connections of the olfactory tract exist, but some are imperfectly known. The name 'rhinencephalon' is given to the portions of the brain concerned with the sense of smell. Disturbances of smell sensation may 
be caused by lesions in any part of the rhinencephalon, and also by changes in the ollactory mucous membrane and olfactory fibres; but the symptoms (lo not obtrude themselves, and are cloubtless often overlooked. Excessive stimulation of the olfactory nerve by exposure to a strong oflour has been said to cause complete and permancint loss of smell.

The second or optic nerve contains mainly afferent fibres, which arise from the ganglion cells of the retina, and terminate by forming synapses with nerve-cells in the lateral or external geniculate bocly, the pulvinar (or posterior portion) of the optic thalamus, and the anterior corpus quadrigeninum. In young animals all these structures undergo atrophy after extirpation of the eycball. The visual path is continued from the pulvinar and the external corpus geniculatum by the axons of these nerve-cells, which proceed in

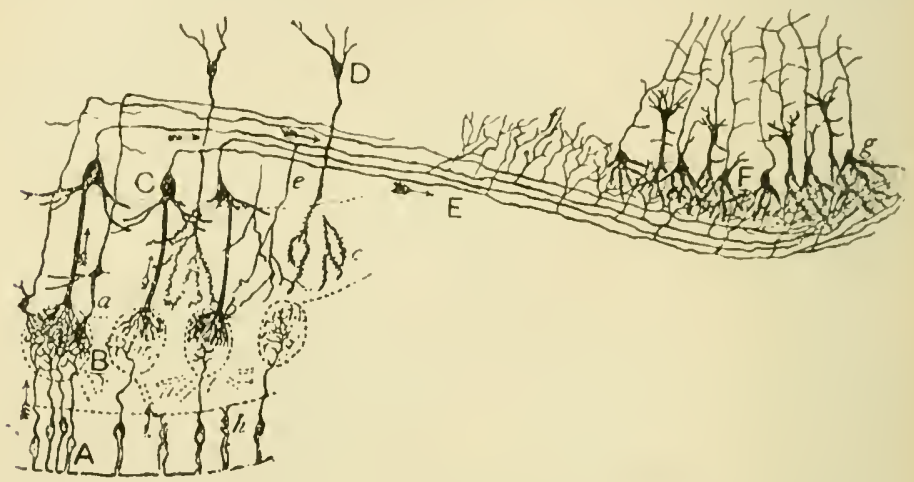

Fif. 341. - Schene of the Olfactory Nervous apparates (Halli. BURTON, AFTER CAJAL).

A, olfactory cells; B, glomeruli ; C, mitral cells; D, olfactory granule cell; $F$, lateral root of olfactory tract ; $F$, cortex of brain in the region of the uncinate gyrus ; $a$. small cell of mitral layer : $b$, brush of dendrite of a mitral cell ending in a glomerulus; $c$, thorns or spines on the processes of an olfactory granule; $e$, collateral coming off from the axon of a mitral cell; $t$, collaterals ending in the molecular layer of the uncinate gyrus : $g$, pyramidal cells of the cortex : $h$, supporting epithelial cells of the olfactory mucous membrane.

the optic radiation (p. 785) to the occipital cortex. The fibres which pass from the retina to the anterior corpus quadrigeminum are distinguished by their small size, and probably constitute the path of the impulses which cause contraction of the pupil when light falls on the retina. The reflex are is schematically shown in Fig. $3+2$. where optic nerve-fibres are represented as forming synapses with cells in the anterior corpus quadrigeminum whose axons pass to the nucleus of the third nerve and arborize around some of its cells (Figs. 326, 331, and 35.). At the chiasma the fibres of the optic nerve decussate, partially in man and some mammals, as the rabbit. dog, cat, and monkey, completely in animals whose visual field is entirely independent for the two eyes, as in fishes ancl birls. In man the fibres for the nasal halves of both retina cross the midclle line at the chiasma, those for the temporal halves do not. This does not mean, however, that exactly half of the optic nerve-fibres 
decussate. The number of uncrossed fibres is smaller than that of crossed. The cliasma also contitins fibres in its posterior portion, which extend from one optic tratet to the other, but are not connected with the retina or the optic nerves. They are commissural fibres which connect the two mesial geniculate bodies across the middle line, and are called Gudden's commissure. A sufficiently extensive lesion involving the occipital cortex on one side, or the posterior portion of the optic thalamus, or the optic tract, causes hemianopia* or defect of the risual ficld on the side opposite to the lesion, with blindness of the corresponding halves of the two retinæ. Thus, a lesion equivalent to complete section of the right optic tract would cause blindness of the nasal half of the left, and of the temporal half of the right eyc, and the left half of the field of vision would be blotted out-the patient would be unable, with his eyes directed forwards, to sce an object at his left. Such a complete hemianopia is much rarer in disease of the cortex tluan in clisease of the optic tract. A lesion -e.g., a tumour of the pituitary boly -- involving the whole of the optic nerve in front of the chiasma, would cause complete blindness in the corresponding eye. Sometimes in disease of the optic nerve vision is not totally destroyed in the eye to which it belongs, but the field is narrowed by a circumference of blindness. In this case the pathological change involves the circumferential fibres of the nerve. When the chiasma is affected by disease, a very frequent symptom is bitemporal hemianopia, blindness

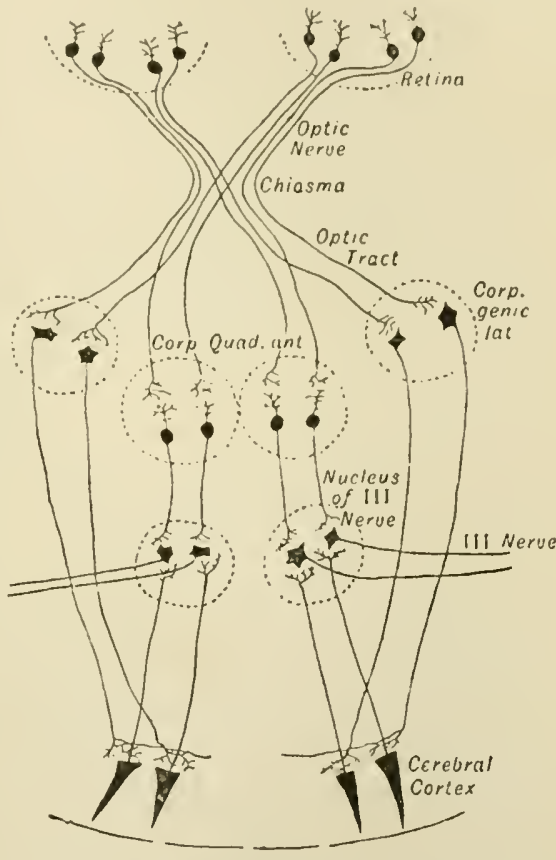

Fig. 342.-Scheme of the Visual Patil (Halliburton, after Schäfer). of the nasal halves of the retinx, with loss of the outer or temporal half of each field of vision. The optic nerve and tract contain a few efferent fibres for the retina, whose cell-bodies have not yet been certainly located.

The third nerve, or oculo-motor, arises from an elongated nucleus, or a series of nuclei, containing large nerve-cells in the floor of the Sylvian aqueduct below the anterior corpora quadrigemina. The root-bundles coming off from the most anterior of the nuclei carry fibres that innervate the ciliary muscle, and thus have to do with

* The terms 'hemiopia,' 'hemianopia,' 'hemianopsia,' are used with reference sometimes to the blind side of the retinæ, but ordinarily to the half of the visual field which is deficient. We shall always use the word 'hemianopia' in the latter sense. 
the mechanism of accommolation, and also fibres that innervate the sphincter muscle of the iris, and thus cause contraction of the pupil when light falls on the retina. Both groups of fibres terminate by arborescing around sympathetic cells in the ciliary ganglion, from which the path to the (unstriated) ciliary and sphincter muscles is continued by post-ganglionic fibres. Further back in the oculo-motor nucleus arise the motor fibres for four of the extrinsic muscles of the eycball and the elevator of the upper eyelicl. In the $\log$ these fibres come off in the following order, from before backwards: internal rectus, superior rectus, levator palpebra superioris, inferior rectus, inferior oblique. Nost of the fibres of the third nerve arise from nerve-cells on their own side of the middle line. but a certain number decussate to enter the nerve of the opposite side. Complete paralysis of the third nerve causes loss of the power of accommodation of the corresponding eye, dilatation of the pupil by the unopposed action of the sympathetic fibres, diminution of the power of moving the eyeball, ptosis, or drooping of the upper licl. external squint, and consequent diplopia, or double vision.

The fourth or trochlear nerve arises from the posterior part of the same tract of grey matter which gives origin to the third nerve. It supplies the superior oblique muscle. Paralysis of the nerve causes internal squint when an object below the horizontal plane is looked at, owing to the unopposed action of the inferior rectus. There is also diplopia on looking down. Unlike the other cranial nerves, the two trochlear nerves decussate completely after they emerge from their nuclei of origin.

The fifth or trigeminus nerve appears on the surface of the pons as a large sensory root and a smaller motor root. Its deep origin is more extensive than that of any of the other cerebral nerves, stretching as it does from the level of the anterior corpus quadrigeminum above to the upper part of the spinal cord below. Its sensory root, in fact, seems to include the sensory divisions of several motor cranial nerves.

The motor root arises partly from a nucleus (principal motor nucleus) in the floor of the fourth rentricle below the pons, partly from large round nerve-cells lying at the side of the grey matter bounding the aqueduct of Sylvius all the way from the anterior quadrigeminate body to the point at which the motor root is given off (accessory or superior motor mucleus).

The fibres of the sensory root have their cells of origin in the Gasserian ganglion, whence they pass into the pons. Here they bifurcate into ascending and descending branches. The ascending branches end in the principal sensory mucleus, a collection of grey matter at the side of the principal motor nuclens. The descending branches, turning downwards into the medulla oblongata, terminate in a long tract of scattered cells, constituting with the fibres the so-called spinal root, and extending from the level of the second cervical nerve through the meclulla oblongata and the pons, where it is continued into the principal sensory nucleus. The afferent path is continued by the axons of cells of the sensory nuclei (or nuclei of reception) of the nerve, many of which cross the middle line and enter the intermediate fillet of the opposite side, and also the special ascending bundle going to the thalamus. Some of the axons do not decussate, but ascend in the fillet of the same side.

The motor fibres of the fifth nerve supply the muscles of mastication and the tensor tympani. The sensory fibres confer common 
sensation on the face, conjunctiva, the mucous membranes of the month and nose, and the structures contained in them, and, according to Gowers, special sensation, through branches given off to the facial and glosso-pharyngeal nerves, on the organs of taste.* Complete paralysis of the nerve causes loss of movement in the muscles of mastication, sometimes impaired hearing, and loss of common sensation in the area supplied by it. Loss or impairment of taste in the corresponding half of the tongue is also often seen in cliscase involving the sensory root, although not in affections of the trunk of the nerve, since the taste fibres leave it near its origin (Gowers). Both taste and touch are lost in the monkey in the an-

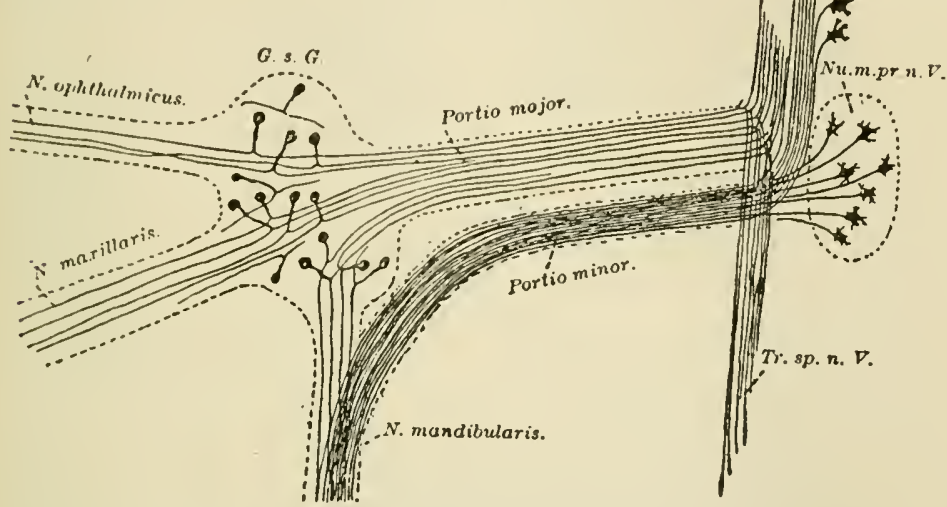

Fig. 343,-Scheale of Motor and Sensori Neurons of Trigeninus

(GEHUCHTEN).

G. s. G., Gasserian ganglion; $N u, m . m, n, V$, nucleus of the descending rout; Nu. m. pr. n. V., chief motor nucleus of the fifth nerve; Rad. desc. mes, $n$. V., accessory motor nucleus, sometimes called the descending root; Tr. sp. n. V.. tractus spinalis, or spinal root of the fifth.

terior two-thirds of the tongue after intracranial section of the trigeminus (Sherrington).

Vaso-motor changes are occasionally, and 'trophic' changes frequently, observed in clisease of the fifth nerve. The trophic

* It should be stated that some physiologists believe that the glossopharyngeal is the nerve of taste, and that none of the taste fibres go to the sensory nuclei of the fifth nerve. The majority hold that the glossopharyngeal supplies the posterior third, and the chorda tympani and lingual the anterior two-thirds of the tongue with gustatory fibres. The removal of the Gasserian ganglion and the adjacent portion of the fifth nerve for severe and persistent neuralgia, has afforded opportunities to test this question. But, unfortunately, the results described by various observers do not agree, some finding that taste is unimpaired, others that it is abolished. Gowers states that the gustatory sensations may persist for some time after the operation, although ultimately (in two or three weeks) they disappear. It may be, however, that this disappearance is due to secondary changes produced in the end-organs of the true taste fibres, the taste buds, by degeneration of the supporting cells consequent on section of the 
(listurbance is most conspicuous in the eychall (ulceration of the cornea, going on, it may be, to complete disorganization of the eyc). These effects are partly due to the loss of sensation in the eye, and the consequent risk of (lamage from without, and the unregarderl presence of foreign bodies and accumulation of secretion within the licls (p. 6gy)).

The sixth or abducens nerve takes origin from a nucleus in the florer of the fourth ventricle at the level of the posterior portion of the pons. It is a purcly efferent nerve, and supplies the external rectus muscle of the eyciball. Paralysis of it causes internal squint.

The motor fibres of the seventh or facial nerve arise from a nucleus in the reticular formation of the medulla otslongata, and running up some distance inte the prons. They supply the muscles of the face; and when these are greatly developerl, as in the trunk of the elephant, the nerve reaches very large proportions. Since the fibres which connect the cerchral cortex witl the nuclews rlecussate about the micldle of the prons, a lesion abrove this level which causes hemiplegia paralyzes the face on the same sirle as the rest of the borly-i.e., on the side opposite the lesion. But the paralysis is confined to the muscles of the lower portion of the face, ancl affects especially the muscles about the mouth. Sometimes the pyramirlal tract and the facial nerve, or nucleus, are involved in a common lesion. In this case paralysis of the face is on the sicle of the lesion, and is total. while the rest of the body is paralyzed on the opposite sicle. Paralysis of the seventh nerve is more common than that of any other nerve in the body. It is often causerl by an inflammatory process in the nerve itself (ncuritis). The symptoms of complete facial palsy are very characteristic. The face and forehearl on the paralyzerl side are smonth, motionless, and devoid of expression. The eye remains open even in sleep, owing to paralysis of the orbicularis palpebrarum. A smile becomes a grimace. Ar attempt to wink with both eyes results in a grotesque contortion. The mouth appears like a rliagonal slit in the face, its angle being (Irawn up on the sound side, and the patient cannet bring the lips sufficiently close together to be able to blow out a candle or to whistle. Licjuirls escape from the mouth, ancl food collects between the paralyzed buccinator and the teeth. The labial consonants are not properly pronouncel. Taste may be lost in the anterior two-thirds of the tongue when the nerve is injured above the exit of the gustatory fibres in the chorla tympani, but not when the lesion is in the nucleus of origin, or anywhere above it. Hearing is sometimes impaired because the auclitrory and facial nerves, lying close together for part of their course, are apt to suffer together, but perhaps also becalise the staperlius muscle is supplied by the seventh.

The seventh nerve is not purely motor. From the cells of a ganglion on it corresponcling to a spinal ganglion (the geniculate ganglion) afferent fibres arise, which pass in the pars intermedia or nerve of Wrisberg into the pons between the seventh and eighth nerves, and there bifurcate into ascending and descendling branches, like other afferent fibres originating in ganglia of the spinal type. The desceneling branches enter the fasciculus solitarius, and end by arborizing around

trigeminus, or to degeneration and swelling of the trigeminal fibres in the lingual nerve and consequent interference with the conluctivity of the intermingled chorda tympani fibres. Cushing believes that the fifth nerve supplies no taste fibres, but that the taste fibres for the anterior twothirds of the tongue have their cells of origin in the geniculate ganglion of the pars intermerlia of the seventh nerve, and those for the posterior third in the ganglion petrosum of the ninth nerve. 
nerve-cells in the upper part of that bundle. The peripleral axons of the nerve-cells in the geniculate gangtion enter the large superficial petrosal nerve and the chorda tympani, in which they, or some of them, perhaps represent taste fibres.

The eighth or auditory nerve enters the medulla oblongata by two roots (a lorsal and a ventral), one of which passes in on each sicle of the restiform body. The cells of origin, both of the dorsal and of the ventral root, are situated in the internal ear, the former in the ganglion spirale, or ganglion of Corti, which is embedded in the bony spiral of the cochlea, the latter in the ganglion vestibulare, or ganglion of Scarpa, which lies in the vestibule. These cells correspond to the ganglion cells on the posterior root of a spinal

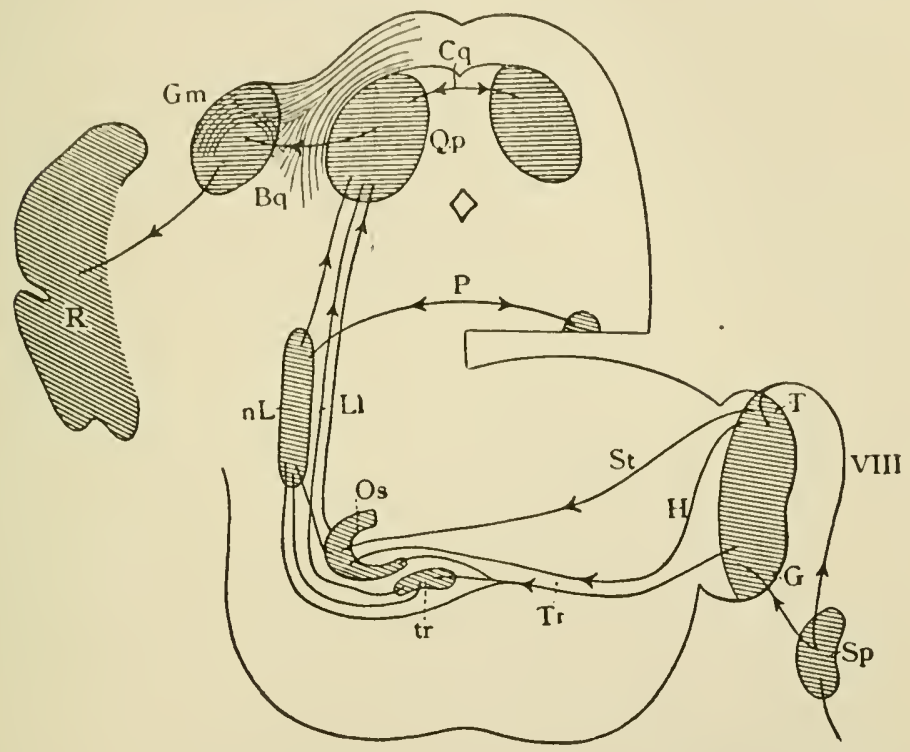

Fig. 34. - Scheme of Patil of Auditory hmules (Lewandowsky).

Sp. ganglion spirale ; G, accessory nucleus ; T, acoustic tubercle ; Tr, trapezium : $H$, Held's fibres ; St, striæ acustice : tr, trapezoid nucleus ; Os, upper olive ; LI. lateral fillet, with its nucleus, $n \mathrm{~L}$; P, commissure of the lateral fillets ; Qp, pos terior corpora quadrigemina; with $\mathrm{Cq}$, their commissure, and $\mathrm{Bq}$, the brachia; Gm, mesial or internal geniculate body ; R, cerebral cortex.

nerve, but, unlike them, they remain, even in mammals, bipolar throughout life. Their central processes form the axons of the eighth nerve. Their peripheral processes are distributed in the case of the dorsal root to the organ of Corti, in the case of the ventral root to the semicircular canals and the vestibule. For this reason the dorsal root is often called the cochlear division, and the ventral root the vestibular division of the auditory nerve. And the cochlear and vestibular roots are physiologically as well as anatomically distinct. For (p. 958) the cochlea subserves the function of hearing, the semicircular canals and vestibule the function of equilibration. As they enter the medulla oblongata, the fibres of the dorsal root bifurcate. Of the two branches, one is considerably 
thicker than the other. Many of the thicker branches terminate by arborizing around the cells of the accessory auditory nuclcus. whose position is inclicated by a swelling on the ventral surface of the restiform body at the junction of the dorsal and ventral roots; but some pass over the restiform body to end in another nucleus (lateral nucleus), alsc indicated by a swelling (tuberculum acusticum) lying $0 \mathrm{ve}^{-}$the restiform bolly. The nerve-cells of the accessory nucleus and the acoustic tubercle, therefore, constitute nuclei of reception for the dorsal root-fibres. The more slender branches of the cochlear root-fibres run clownwards for some distance before breaking up into fibrils.

The path to the high parts of the brain is continued by the axons of nerve-cells in the accessory nucleus and the acoustic tubercle. The fibres from the accessory nucleus pass into the trapezium, a mass of transverse fibres lying in the pons behind the pyramidal fibres. In their course through the trapezium some of the fibres terminate around the cells of the nucleus of the trapezium, others run into the superior olive of the same side, and end there; but most of them cross the middle line, and enter the trapezoid nucleus and superior olive of the opposite side, where many of them terminate. Others, however, run through those nuclei and pass into the lateral fillet, to end in its nucleus or in the posterior corpora quadrigemina. The path of the fibres which terminate in the nuclei of the trapezium, superior olive, and lateral fillet, is continued by another relay of fibres, which link them also to the posterior corpora quadrigemina. The axons of the cells of the acoustic tubercle enter for the most part the strice acustice, a series of prominent strands that run transversely across the floor of the fourth ventricle. Passing across the raphe, they join the fibres from the accessory nucleus on their way to the superior olive, and accompany them into the lateral fillet, which terminates in the grey matter of the posterior corpus quadrigeminum. We must assume, from clinical and experimental data, that the dorsal root is ultimately connected with the first, or first and second temporo-sphenoidal conrolutions on the opposite side. From the posterior corpora guadrigemina the auditory path to the convolutions secms to run in the brachium to the internal or mesial geniculate body, whence it is continued in the posterior extremity of the internal capsule.

The fibres of the ventral root of the eighth nerve, better termed the vestibular nerve, after entering the medulla oblongata, pass to a nucleus called the principal nucleus of the vestibular division. Here each bifurcates into a descencling and an ascending branch. The descending branches running down in the medulla terminate at different levels around cells in the principal nucleus, and the grey matter continued down from it (descending vestibular nucleus). The ascencling branches run up on the inner sicle of the restiform body towards the nucleus of the roof (mucleus tecti) in the cercbellar worm. On their course they enter into relation through their collaterals with the nuclei of Deiters and Bechterew. The nucleus of Deiters, as already stated, sends fibres into the posterior longitudinal bundles. Through ascending branches of these fibres a communication is established with the nuclei of the third and sixth nerves, and through descending branches that pass into the anterolateral descending tract of the cord with the anterior horn cells. It is obvious that througl these connections which link the restibule with the cerebellum, the nuclei of the motor nerves of the eycball and the motor cells of the cord, the nucleus of Deiters has an 
important relation to the co-ordination of those movements mainly concerned in equilibration. Nothing is known of the connections of the vestibular nerve with the cercbrum. Two prominent symptoms may be associated with discase of the auditory nerve-- $(a)$ disturbance or loss of hearing ; $(b)$ loss or impairment of equilibration.

The ninth or glosso-pharyngeal nerve comprises both sensory and motor fibres-sensory for the posterior third of the tongue and the mucous membrane of the back of the mouth, motor for the middle constrictor of the pharynx and the stylo-pharyngeus. It also contains the nerves of taste for the posterior third of the tongue. The efferent fibres arise from a nucleus (motor nucleus of the glossopharyngeal) a little posterior to the facial nucleus. The afferent fibres take origin from unipolar cells in ganglia of spinal type connected with the nerve (ganglion petrosum and ganglion superius). Entering the medulla oblongata, the central processes of these cells bifurcate into ascending and descending branches. Their peripheral processes pursue their course as the axons of sensory fibres to the strictures to which the nerve is distributed. The ascending branches terminate in a nucleus (principal nucleus of the glossopharyngeal) beneath the floor of the fourth ventricle. The descending branches, as well as similar branches from the pars intermedia of the seventh nerve and from the afferent fibres of the vagus, form a bundle called the fasciculus solitarius (sometimes termed the descending root of the facial, vagus, and glosso-pharyngeal). It can be traced to the lower boundary of the spinal bulb. Along the mesial border of the fasciculus solitarius are strung out the somewhat scattered nerve-cells (descending nucleus of facial, vagus, and glosso-pharyngeal), around which the descending branches arborize. At its upper end the grey matter of the fasciculus solitarius is continuous with the principal nuclei of the glosso-pharyngeal and vagus.

The tenth nerve, or vagus, also contains both motor and sensory fibres. The efferent fibres arise partly from the nucleus ambigutus or ventral nucleus of the vagus, a collection of large nerve-colls situated in the reticular formation, and extending from a point a little below the facial nucleus to a point a little above the lower limit of the medulla oblongata, where it becomes continuous with the column of cells from which the spinal fibres of the eleventh nerve take origin. A second nucleus of origin for efferent vagus fibres is constituted by the upper part of the dorsal accessory-vagus mucleus, a collection of rather small cells extending from a little below the lower margin of the pons to nearly the level of the first cervical nerve.

The afferent fibres of the vagus arise from unipolar cells in ganglia connected with the nerve (ganglion jugulare, ganglion nodosum). In the medulla oblongata they bifurcate, like other fibres coming off from the cells of ganglia of spinal type. The ascending branches, which are short, terminate in the upper sensory or principal nucleus, and the descending branches, which are long, in the cells of the fasciculus solitarius, just as in the case of the glosso-pharyngeus.

The motor fibres of the vagus are partly derived from the accessory, whose internal branch joins the vagus not far from its origin. The distribution of the nerve is more extensive than that of any other in the body. The œsophagus receives both motor and sensory branches from the œsophageal plexus. The pharyngeal branch of the vagus is the chief motor nerve of the pharynx and soft palate (including the tensor palati). The superior laryngeal branch is the nerve of common sensation for the larynx above the vocal cords, and the motor nerve of the crico-thyriod muscle. The inferior or recurrent 
laryngeal supplies the rest of the laryngeal muscles, and the sensory fibres for the mucous membrane of the trachea and the larynx below the glottis. The superior laryngeal contains afferent fibres, stimulation of which gives rise to coughing, slows respiration, or stops it in expiration. Reflex movements of deglutition are also caused. The vagus supplies the lung both with motor and sensory filaments through the pulmonary plexus. The motor fibres when stimulated cause constriction of the bronchi; excitation of the afferent fibres causes reflex changes in the rate or deptl of respiration. The cardiac branches contain inhibitory fibres probably clerived from the spinal accessory, and depressor fibres which pass up in the vagus trunk ( $\log )$, or as a separate nerve to join the vagus or its superior laryngeal branch or both (rabbit). The gastric and intestinal branches contain both motor and sensory nerves for the stomach and intestines. The sensory are probably large medullated fibres $(7 \mu$ to $9 \mu)$. The afferent vagus fibres from the stomach carry 11 ) impulses which excite the action of vomiting. Lesions of the vagus, its nuclei of origin, or its branches, are associated with many interesting forms of paralysis and other symptoms. Paralysis of the pharynx is generally caused by disease of the nucleus in the medulla. From its anatomical relation to the nuclei of the glossopharyngeal and hypoglossal, it will be easily understood that these nerves are often involved in localized central lesions along with the vagus. But the fact that in progressive bulbar palsy (glosso-labiolaryngeal paralysis)-a condition characterized by progressive paralysis and atrophy of the muscles of the tongue, lips, larynx. and pharynx-the orbicularis oris and other muscles of the mouth and chin are paralyzed, while the rest of the muscles supplied by the facial remain intact, might seem to indicate that in system diseases it is not so much anatomical groups of nerve-cells which are liable to simultaneous degeneration and failure, as physiologicai groups normally associated in particular functions. Such functional groups of cells, occupied with the same kincls of labour at the same: times and under the same conditions, might be supposel to take on a similar bias or tendency to degeneration-a tendency not indicated, it may bc, by any structural peculiarity, but traced deep in the molecular activity of the cells. There is no founclation for the view that the lips are involved in progressive bulbar palsy because the fibres of the facial which supply them arise from the hypoglossal nucleus, any more than for the idea that the upper part of the face escapes because its motor fibres, while reaching it in the seventh nerve, really arise from the oculo-motor nucleus (Bruce). Difficulty in swallowing is the chief symptom of pharyngeal paralysis. The symptoms of laryngeal paralysis have becn already described under "Voice' (p. 286). Tachycardia, or a permanent increase in the rate of the heart, has been stated to occur in certain cases of paralysis of the vagus, caused by disease or accidental interference; and a persistent slowing of the respiration has been occasionally attributed to the same cause. But it is difficult to reconcile many of these cases with expcrimental results, for in most of them the lesion only involved one vagus; and in animals section of one vagus has no permanent effect on the rate of the heart or of the respiratory movements.

Destruction of the nerve ncar its origin has been sometimes found associated with disappearance of the food-appetites, hunger and thirst, and it has been assumed that this was due to loss of afferent 
impulses from the stomach. But clinical testimony is by no means unanimous on this point, and experiments on animals show that other factors are involved in these sensations.

The eleventh or spinal-accessory nerve contains only efferent fibres. The cells of origin of its spinal portion lie in the lateral horn of the cord, from about the level of the first to the fifth or sixth cervical nerves. The bulbar portion, sometimes called the bulbar accessory, arises from the lower two-thirds of the dorsal accessory-vagus nucleus, from about the level of the first cervical nerve up to the level of the tip of the calamus scriptorius. Tho accessory portion of the nucleus lies behind and to the side of -i.e.. dorso-lateral to-the central canal; the upper or vagus portion is more laterally placed in the floor of the fourth ventricle. Soon after the junction of its bulbar and spinal portions, the nerve divicles into two branches, an internal and an cxternal. The external branch, coutaining the spinal fibres, passes out to supply the trapezius and sterno-mastoid muscles with motor fibres. The internal brancli, containing the bulbar fibres, passes bodily into the vagus.

The twelfth or hypoglossal nerve is exclusively an efferent nerve. Its nucleus of origin is an elongated collection of large nerve-cells extending throughout approximately the lower two-thirds of the bulb close to the median line and parallel to it. It contains the motor supply of the intrinsic and extrinsic muscles of the tongue and of the thyro- and genio-hyoid. Paralysis of it causes deficient movement of the corresponding half of the tongue. When the tongue is put out, it deviates towards the paralyzed side, being pushed over by the unparalyzed genio-hyoglossus of the opposite side, which is thrown into action in protruding the tongue.

\section{The Functions of the Brain.}

The paths by which the various parts of the central nervous system are connected with each other and with the periphery have been already described, and we have completed the examination of the functions of the spinal cord and medulla oblongata. The events that take place in the upper part of the central nervous stem and in the cortex of the cerebellum and cerebrum now claim our attention.

From very early times the brain has been popularly believerl to be the seat of all that we mean by consciousness - sensation, ideation, emotion, volition. And he who loves to trace the roots of things back into the past may see, if he choose, running through the whole texture of the older speculations a belief that the brain does not act as a whole, but is divided into mechanisms, each with its special work-a forcshadowing, often in grotesque outlines, of the doctrine of localization so widely held to-day. But until comparatively recent times, cerebral physiology remained a kind of scientific terra incognita ; and no notable additions were made for a thousand years to the doctrines of Galen. Even to-day the utmost limit of our knowledge is reached when in certain cases we have connected a particular movement or sensation with a more or less sharplydefined anatomical area. How the cerebral processes that lead to sensations and movements, to emotions and intellectual acts, arise 
and die out; what molecular changes are associated with them ; above all, how the molecular changes are translated into consciousness-how, for example, it is that a series of nerve-impulses from the optic radiation flickering across the labyrinth of the occipital cortex should light up) there a visual sensation-these are questions to which we can as yet give no answer, and the answers to some of which must for ever remain hidclen from us.

Functions of the Upper Part of the Central Stem and Basal Ganglia.-The function of the pons is sufficiently indicated by its name. The grey matter so plentifully scattered, especially in its ventral portion, may excrcise a not unimportant influence on the impulses that traverse it. But on the whole its main office is to provide a bridge along which impulses may travel between other portions of the nervous system. IVe have already scen that many of its transverse fibres arising from the cells of the pontine grey

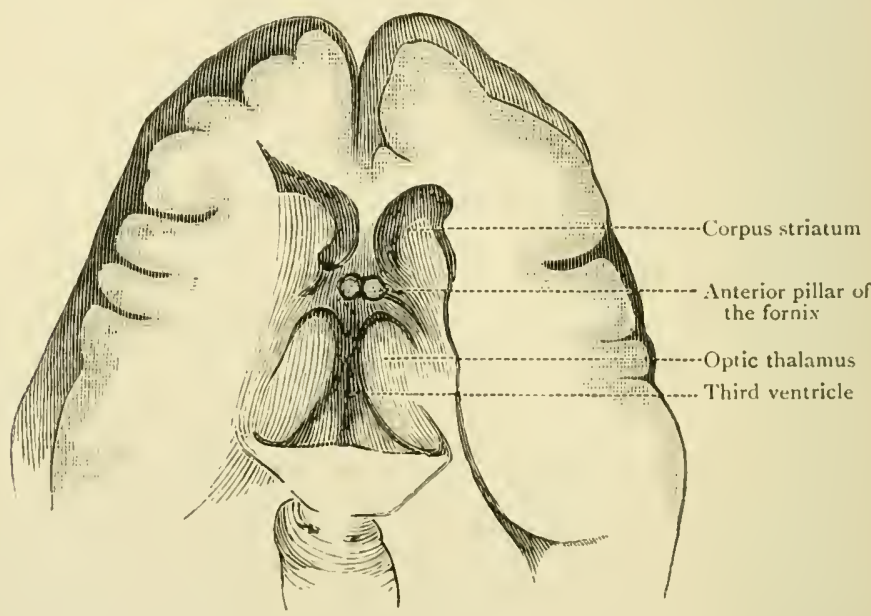

Fig. 345.-Horizontal Section throvgh Brain to show the Basal Ganglia and Third Ventricle (Human).

matter, and then crossing the middle line to the opposite midclle perluncle, are the cerebellar segments of commissural ares connecting the cerebral with the opposite cercbellar hemispleres. The cercbral segments of these ares are the cortico-pontine fibres originating in the prefrontal. temporal, and occipital portions of the cerebral cortex, and passing through the corona radiata, internal capsule, and crura cerebri, to end in the nuclei pontis. Many fibres and collaterals of the pyramidal tract also terminate here. On the dorsal aspect of the pons in the floor of the fourth ventricle are the nuclei of origin (or reception) of the fifth, sixth, and seventh cranial nerves. Various reflex centres are situated in this region-e.g., that for the closure of the eyclids, when the conjunctiva is stimulated.

The posterior corpora quadrigemina and internal geniculate bodies are connected with the coclilear division of the auditory nerves, and form important stations on the auditory path to the cortex. 
The anterior corpora qualrigemina and the lateral corpora geniculala are connected with the optic tracts. Their development is arrested after cxtirpation of the eycball in young animals, and they may therefore be assumed to be concerned in vision, although the size of their homologues, the optic lobes or corpora bigemina, in animals below the rank of mammals (birds, reptiles, amphibians), (loes not seem to be related to the development of the organs of sight. Proteus and the Hag-fish, e.g., have large optic lobes, rudimentary eyes and optic tracts. The optic nerve, the anterior corpus quadrigeminum, the nucleus of the oculo-motor nerve in the wall of the Sylvian aqueduct, and the fibres which it carrics to the iris, form a reflex arc for the contraction of the pupil to light, as represented in Fig. 342, p. 8 I9.

The functions of the optic thalami have not been fully defined cither by experiment or pathological observation, except so far as they can ba deduced from their connections. Lying as they do in the isthmus of the brain, begirt by the great motor and sensory paths, it is to be expected that lesions of the thalami should affect also the internal capsule, and give rise to the symptoms of motor and sensory paralysis. But it is questionable whether any definite defect of motor power or common sensation has ever becn uncquivocally associated with a lesion restricted to the thalami. The most constant features of the so-called thalamic syndrome (or symptomcomplex) are partial loss of sensibility, especially to tactile impressions, and of the muscular sense on the opposite side, with some degree of inco-ordination and disorder, though little, if any, actual paralysis of voluntary movements. These phenomena are accounted for by the extensive connections of the thalami. Each of the thalamic nuclei is linked with a definite cortical region in such a way that destruction of the cortical area in young animals or human beings leads to degeneration of the corresponding nucleus. Some of the fibres connecting the cortex (and the corpus striatum) with the thalamus end in the thalamic grey matter, and are therefore efferent with respect to the cortex (corticofugal). It is, however, the afferent paths to the cortex with which the thalami are specially related as centres of relay. The fibres of the upper fillet carrying afferent impulses up from the opposite posterior column of the cord to the cerebrum end in the grey matter of the thalamus, as does the central path of the afferent fibres of the opposite fifth nerve. The posterior portion of the thalamus, or pulvinar, forms part of the central visual apparatus; for $(a)$ it is found to be undeveloped in animals from which the eyeballs have been removed soon after birth ; (b) a portion of the optic tract is certainly connected with it ; (c) in some cases of atrophy of the occipital cortex, which, as we shall see, is undoubtedly a central area for visual sensations, atrophy of the pulvinar has also been noticed; $(d)$ a lesion of the pulvinar may give rise to hemianopia (p. 819).

Hæmorrhage into the caudate or lenticular nucleus of the corpus striatum often causes hemiplegia, but this is frequently due to implication of the internalcapsule. It is said, however, that lesions presumably confined to the lenticular nucleus cause paralysis or paresis of the limbs or face, which is less severe than that produced by lesions in the internal capsule. Experimental lesions in dogs and rabbits are stated to be followed by disturbances of the heat-regulating mechanism and rise of temperature.

Certain structures belonging to the primary fore-brain which have now lost some or all of their functional importance, may neverthe- 
less be mentionerl as milestones in the march of rlevelopment. The pineal body is made up of the vestiges of the unpaired mesial eye of such animals as the ancient labyrinthodonts, which resembled the eyc of invertebrates in having the retinal rods directed towards the cavity instead of towards the circumference of the eyeball. In many living forms, especially in certain lizards, this pineal or parietal eye is found in a more perfect condition, though corered by a thin membrane. The ganglia habenula, two small collections of nerve-cells, one of which is situated at the posterior part of each thalamus, are supposed by some authorities to represent the optic

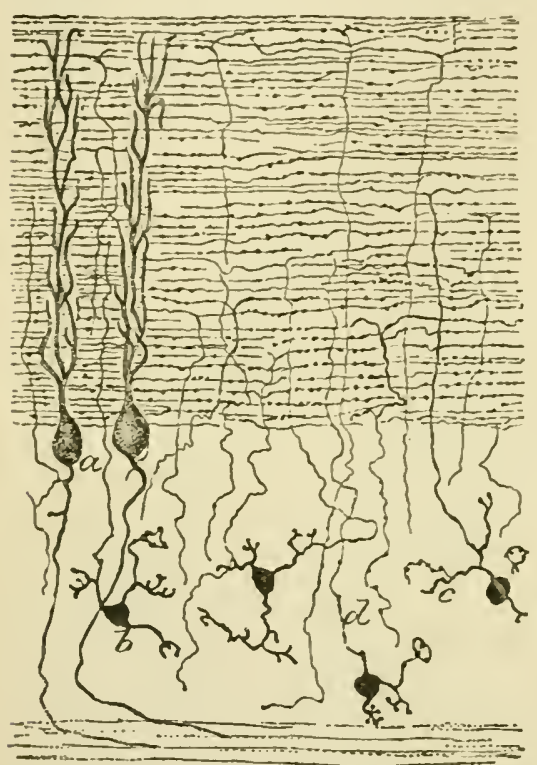

Fig. 346.-CEREBEllar Cortex: Section IX DiReCtION OF LAMINA (CAJAL).

$a$, Purkinje's cell ; $b$, granule cell in inner layer ; $c$, dendrite of a granule cell ; $d$, axon of a granule passing into the molecular layer, where it bifurcates into two fine longitudinal branches (Golgi's method). ganglia of this cyclopean eyc. They are less prominent in man than in many of the lower animals. The infundibulum is probably what remains of the gullet of the ancestors of the vertebrates. The pituitary body is in a different category. It is now known that, far from being a useless vestigial remnant. it has a highly important function (p. 566). It con. sists of two portions, the anterior lobe, or hypophysis. clerived from the buccal cavity, the posterior lobe. or infundibular body, from the primary forc-brain.

\section{Functions of the Cere-} bellum. - The elaborate pattern of the arbor ritæ, the appearance given by the branched laminæ in a section of the cerebellum, excited the speculation of the old anatomists. A structure so marvellous must be matched, they thought, with functions as unique. At a time when the discoveries of Galvani and Volta were fresh, and the world ran mad on electricity, the hypothesis of Rolando, that 'nerve-force' was generated by the lamellæ of the cerebellum as electrical energy is generated by the plates of the voltaic pile, ridiculous as it now appears, was not unnatural. The speculation of Gall, who connected the cerebellum with the derelopment of sexual emotions and the action of the generative mechanisms, was based on no fact. It has been definitely disproved by the observations of Luciani, who found that a bitcl deprived of 
its cerebellum showed all the phenomena of heat or ' rut,' was impregnated, whelped at full term in an entirely normal manner, and manifested the maternal instincts in their full intensity. Flourens put forward the doctrine that the cerebellum is an organ concerned in the co-ordination of movements and especially the maintenance of equilibrium, supporting his conclusions by an elaborate scries of experiments. Notwithstanding the very large anount of experimental and clinical study which has been devoted to the cerebellum since the time of Flourens, our actual knowledge of its functions has not greatly advanced beyond the point then reached. Some of the more modern authorities restrict its influence entirely to the actions on which equilibration depends; others extend it to all volitional movements. Luciani looks upon it as 'an organ which by processes that do not awaken consciousness exerts a continual strengthening (reinforcing) action upon the activity of all other nerve-centres.' Sherrington conceives of the cerebellum as the head ganglion of the proprio-ceptive system-i.e., of the system of neurons whose receptors lie not on the surface, but in the deeper parts of the body (labyrinth of ear, muscles, tendons, joints, viscera, etc.) (p. 8Io). After removal of the whole cerebellum (in the dog or monkey), there is at first rigidity and tonic spasm of certain muscles, which contribute to the difficulty of coordinating their movements. When this stage has passed, the muscles all over the body, but especially those of

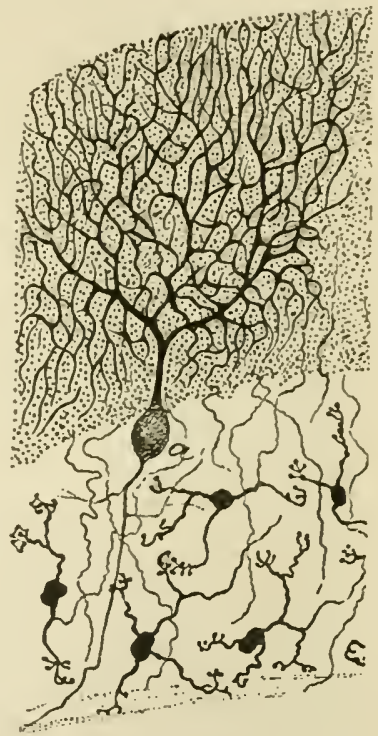

Fig. 347.-CEREbellar Cortex: SECtION ACROSS A LAMIIA (CAJAL).

$a$, Purkinje's cell; the numerous dots in the molecular layer represent cross-sections of the bifurcated axons of the granule cells (Golgi's method).

the loins and hind-limbs, and those which fix the head, are weaker than normal, are deficient in tone, and contract with a peculiar want of steadiness (Luciani). When one lateral half of the cerebellum is removed, the symptoms affect especially the muscles on the same side. In extensive lesions of the cerebellum in man what has been noticed is a marked inability to maintain the upright posture, giddiness, a staggering gait, twitching movements of the eyes (nystagmus), tremor accompanying voluntary movements - in a word, a general breakdown 
of the co-ordinating machinery, and especially of the part of it concerned in the movements necessary for locomotion, and for the maintenance of the equilibrium of the body-the so-called cerebellar ataxia. There is no sensory paralysis and none of voluntary movement, such as lesions of the cerebral cortex produce, nor is there any psychical disturbance. In cáses of congenital defect of the cerebellum, the power of walking, and even of standing, may be late in being acquired, and imperfect. But it is remarkable what great deficiencies in the cerebellar substance are often compensated for when established early in life, so that even cases of marked atropliy or lack of development liave sometimes been recognised for the first time at the autopsy.

The connections of the cerebellum with other parts of the central nervous system and with the periphery corroborate the direct results of experiment. For, in addition to the visual impressions, the most important afferent impulses concerned in equilibration are those from the semicircular canals and vestibule of the internal ear, the muscles, tendons, joints, etc., and certain portions of the skin, such as that of the soles of the feet. And the cerebellum, as we have seen (p. 779), is linked with all of these, and has besides an extensive crossed connection through the middle and superior peduncles with the opposite cerebral hemisphere. The importance and extent of this crossed connection with the great brain is illustrated by the facts that in disease atrophy or deficient development of one cerebellar hemisphere is associated with a similar condition of the opposite cerebral hemisphere, and that a lesion in one-half of the cerebellum affects chiefly the co-ordination of the movements of the same side of the body - that is to say, of the side connected with the opposite cerebral hemisphere.

We do not as yet know the full significance of this extraordinarily free communicaticn of the grey matter of the cerebellum with every part of the central nervous system. But it is eviclent that by the broad lighway of the restiform body, or the cross-country routes from cerebral cortex to cerebellum, impulses may reach it from every quarter; while impulses passing out from it along its peciuncles may influence the motor discharge either indirectly through the Rolandic cortex and the pyramidal tract, or more directly through the antero-lateral descending spinal path that brings it into relation with the nuclei of origin of the motor nerves. It is an organ so connected that is suited to take cognizance of the multitudes of afferent impressions concerned in the co-ordination of movements and the maintenance of equilibrium, and to regulate the outflow of efferent impulses in correspondence with the inflow of afferent.

Sherrington points out that all the modern theories of cerebellar function harmonize with his conception of the cercbellum as the head ganglion of the proprio-ceptive system (p. 83 I). The most influential 
of the proprio-ecptive organs being the labyrinth, the central organ of the whole proprio-ceptive mechanism is built "1p over the central connections of the labyrinth. Thither converge connceting (internuncial) paths from the central endings of proprio-ceptive neurons in all segurents of the body (from joints, muscles, tendons, ligaments, viscera, etc.). Thus a central organ is developed, which varies in size and complexity in different kinds of animals according to the complexity of their liabitual movements. This is a convenient place to consider a little more in detail the nature and peripheral sources of some of the most important afferent impressions concerned in equilibration.

(I) Afferent Impulses from the Semicircular Canals.-The semicircular canals are three in number, and lie ncarly in three mutually rectangular planes : the cxternal canal in the horizontal plane, the supcrior canal in a vertical longitudinal plane, and the posterior canal in a vertical transverse plane. Each canal bulges out at one end into a swelling, or ampulla, which opens into the utricular division of the vestibule (Figs. 348,421 ). The other extremities of the superior and posterior canals jo in together, and have a common aperture into the utricle, but the undilated end of the external or horizontal canal opens separately. The utricle and the semicircular canals are thus

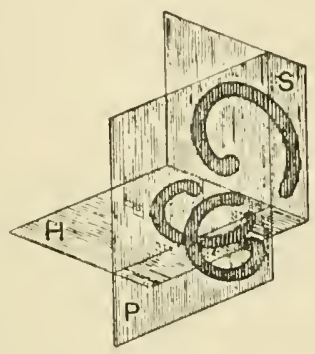

Fig. 348.-The Semicircular Canals (AFTER EWALD).

$\mathrm{H}$, horizontal or external ; $\mathrm{S}$, superior; $\mathrm{P}$, posterior. The two horizontal canals lie in the same plane. The plane of the superior vertical eanal of one side is parallel to the plane of the posterior vertical canal of the opposite side.

\section{connected by}

five distinct orifices. The greater part of the internal surface of the membranous canals, utricle and saccule, is lined by a single layer of flattened epithelium. But at one part of each ampulla projects a transverse ridge, the crista acustica, covered not with squamous, but with long columnar epithelium. Hair-like processes (auditory hairs) are borne by some of the columnar cells, between which lie more elongated fibre-like supporting cells. The harrs project into a mucus-like mass, sometimes containing otoconia, or crystals of calcium carbonate. The ampullæ, like the rest of the membranous labyrinth, is filled with a watery fluid called endolymph. The utricle and saccule have each a somewhat similar but broader elevation, the macula acustica, covered with epithelium and haircells of the same character, and the hairs project into a similar mass in which otoconia are constantly present. In some animals, as fishes, the calcareous matter in the utricle and saccule forms masses of considerable size (otoliths). Fibres of the auditory nerve end in arborizations around the bodies of the hair-cells of the macula and cristæ acusticæ. We have already seen that it is the ventral or 
vestibular division of the nerve which is especially related to the vestibule (p. 823).

There is very strong evidence that the semicircular canals are concerned, not in hearing, but in equilibration. A pigeon from which the membranous canals have been removed still hears perfectly well so long as the cochlea is intact, but exhibits the most profound disturbance of equilibrium. If the horizontal canal is clestroyed or divided the pigeon moves its head continually from sicle to side around a vertical axis; if the superior canal is divided, the liead moves up and down around a horizontal axis. The power of co-ordination of movements is diminished, but not to the same extent in all kinds of animals. Thrown into the air, the pigeon is helpless; it cannot fly ; but a goose with divided semicircular canals can still swim. The condition is only temporary, even when the injury involves the three canals on one side; but if the canals on both sides are destroyed, recovery is tardy, and often incomplete. In mammals the loss of co-ordination is much less than in birds; and movements of the eyes, the direction of which depends on the canal destroyed, take to a large extent the place of movements of the head. The effects of destructive lesions have their counterpart in the phenomena caused by stimulation; excitation of a posterior canal, for example, in the pigeon causes movements of the head from side to side.

Lee's results in fishes are, on the whole, of similar tenor. Mechanical stimulation of the ampullæ in the dogfish, by pressing on them with a blunt needle, calls forth characteristic movements of the eyes and fins, and clectrical stimulation of the auditory nerve causes movements compounded of the separate movements obtained by stimulation of the ampullæe one by one. Lee concludes that the semicircular canals are the sense-organs for dynamical equilibrium (i.e., equilibrium of an animal in motion), and the utricle and saccule for statical equilibrium (i.e., equilibrium of an animal at rest).

The evidence from all sources points strongly to the conclusion that afferent impulses are actually set up in the fibres of the auditory nerve, through the hair-cells, by alterations of pressure or by streaming movements of the endolymph when the position of the head is changed. Rotation of the head to the right may be supposed to cause the endolymph in the right external canal, in virtue of its inertia, to lag behind the movement, and to press upon the anterior surface of the ampulla. The disorders of movement after lesions of the canals may be explained as the result of the withdrawal of certain of these afferent impulses, and the consequent overthrow of that equipoise of excitation necessary for the maintenance of equilibrium. Even in man there is evidence of the existence of some mechanism not depending on the muscular sense or on impressions passing up the channels of ordinary or special sensation. by which orientation (the determination of the position of the body in space) is rendered possible. For a man lying perfectly still. witl eyes shut, on a horizontal table which is made to rotate uniformly, can not only judge whether, but also in what direction. and approximately through what angle, he is moved (Crum Brown). The phenomena of pathology afford weighty additional testimony in favour of the equilibratory function of the semicircular canals. For many cases of vertigo are associated with changes in the internal ear (Ménière's disease). And while nearly every normal individual becomes dizzy when rapidly rotated, 35 per cent. of deaf-mutes are entirely un. 
affected (James), and the proportion scems to be much higher amoug congenitil deaf-mutes. Kreidl and Bruck, too, have found that abnormalities of locomotion and equilibration are much more common in deif-and-dumb children than in others. Now, in these cases the defect is usually in the internal ear. We must conclude, then, that the co-ordination of muscular movements necessary for equilibrium is achieved in some centre, to which afferent impulses pass from the internal ear by the vestibular branch of the auditory nerve, and from which efferent impulses pass out to the muscles. If, as there is strong reason to believe, this centre is situated in the cercbellum, the efferent path is, as already suggested (p. 832), partly an indirect onc (perhaps by commissural fibres to the Rolandic area, and then out along the pyramidal tract). or more probably to lower centres, perhaps in the posterior portion of the optic thalamus, which control such massive co-ordinated movements as those concerned in walking and the maintenance of the norma! attitude, and thence out along certain tracts that connect the thalamus to the spinal cord (p. $78 \mathrm{I}$ ).

Ewald has made an observation which illustrates the peculiar relation of the semicircular canals to the muscular system-namely, that the labyrinth (in rabbits) influences the course of rigor mortis in the striped muscles. Rigor loes not come on so soon on the side from which the labyrintl has been removed (p. 675). He attributes to the labyrinth, as one of its functions, the maintenance of a certain tonus in the entire skeletal musculature.

(2) Afferent Impressions from the Muscles.-Muscles are riclly supplied with afferent fibres, for about half of the fibres in the nerves of sleletal muscles degenerate after section of the posterior roots beyond the ganglia (Sherrington). Various kinds of impressions may pass up these nerves: (a) Impressions giving rise to pain, as in muscular cramp and in experimental excitation of even the finest muscular nerve-filament ; $(b)$ impulses causing a rise of bloodpressure; $(c)$ impulses which arc not associated with a distinct impression in consciousness, but which enable us to localize the position of the limbs, head, eyes, and other parts of the body ; $(d)$ impulses which inform us as to the extent and force of muscular contraction, and seem to underlie the so-called muscular sense. It is the last two kinds-if, indeed, they are distinct-which must be concerned in equilibration. In locomotor ataxia such impressions are blacked

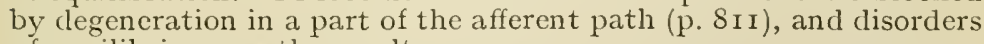
of equilibrium are the result.

(3) Afferent Impressions from the Skin.-Of the various kinds of impulses that arise in the nerve-endings of the skin, only those of touch and pressure seem to be concerned in the maintenance of equilibrium. When the soles of the feet are rendered insensitive by local anæs thesia or by cold, and the person is directed to close his eyes, he staggers and sways from side to side. The disturbance of equilibrium in locomotor ataxia must be partly attributed to the loss of these tactile sensations, for numbness of the feet is a frequent symptom, and the patient asserts that he does not feel the ground. An interesting illustration of the importance of afferent impulses from the skin in the maintenance of equilibrium is afforded by the behaviour of a frog deprived of its cerebral hemispheres. Such a frog will balance itself on the edge of a board like a normal animal, but if the skin be removed from the hind-legs, it will fall like a log.

In birds and lower vertebrates the cerebellum is only represented by the worm. Yet in many of these animals the same characteristic 
disturbances follow its removal as in the higher animals where the cerebellar hemispheres have become so prominent. Indeed, it was mainly on the pigeon that filourens made his classical experiments. At first the pigeon can neither fly nor feed itself. When it attempts to walk extensor spasms of the legs come on, and it falls, wildly struggling and apparently panic-stricken, to the ground. The power of flight is soon regained, but for a long time the animal is unable to perch, the legs and talons stiffening in rigid extension as it attempts to alight.

In the higher animals stimulation of certain parts of the worm and lateral lobe causes conjugate movements of the cyes towards the same side, both eyes being turned to the right. e.g., when the cerebellum is stimulated to the right of the middle line. Inhibition of movement can also be clicited from the organ. Excitation of the cerebellar cortex for some distance outwards from the line of junction of the superior worm with the lateral lobe in animals which exhibit tonic contraction of cxtensor muscles after excision of the cerebral hemispheres (decerebrate rigidity or acerebral tonus, as it is called) causes immediate relaxation of the rigid muscles of the neck, tail, and especially the anterior limb, particularly on the same side. The relaxation of the cxtensors may be accompanied by contraction of the antagonistic flexors-for example, relaxation of the triceps and contraction of the biceps (Horsley and Löwenthal). But this can scarcely be considered a reaction specific to the cerebellum. For Sherrington, who finds that the tonus or spasm is largely due to centripetal impulses coming from the rigid limb, has been able to inhibit it by stimulation of various other regions, including the portion of the cerebral cortex in front of the fissure of Rolando (p. 847).

Forced Movements. - IVe have incidentally mentioned that in fishes injuries to the semicircular canals may give rise to movements which seem to be beyond the control of the animal. and which have consequently received the name of 'foreed movements.' It may be added that when the internal ear of a Necturus (one of the tailed amphibia) is destroyed on one side, rapid movements of rotation around a longitudinal axis are observed. The animal spins round and round apparently without voluntary control, purpose, or fatigue. The direction of rotation is towards the side of the lesion, the observer being supposed to look down upon the animal as it lies in its normal position. After a time it becomes quiescent; but the forced movements can be again produced by pinching or exciting it in other ways.* In man, too, during the passage of a galvanic current through the head by clectrodes applied just bchind the cars, a tendency to move the head towards the anode is experienced. The person may resist the tendency, but if the current be strong enough his resistance will be overcome; he will execute a forced movement. When the head turns towards the anode the eres move in the same direction, and then undergo jerking movements towards the kathode. There is at the same time a feeling of vertigo. Complex as such an experiment is, involving as it does stimulation of so many structures within the cranium. there is reason to believe that it is the excitation of the semicircular canals, or their cerebellar connections, that is responsible for these forced movements. For when the experinent is performed on a pigeon, forced movements are caused so long as the inembranous canals are intact, but not after they have been

* Personal observation. 
(lestroyed (Evalel). The observation of Rawitz, that the peculiar rotatory movements of the so-called Japanese dancing mice are associated with markel imatomical peculiarities in the labyrinth, is another fact in farour of the connection of the canals with the mintenance of equilibrium and the sense of rotation. So is the relation between the degree of development of the canals in different species of birds and the degree of agility in the co-ordination of their movements (Liuldenbach).

But fored movements may also follow injuries (especially uni. lateral) to many portions of the brain-e.g., the pons, crus cercbri, posterior corpora quadrigemina, corpus striatum, even the ccrebral cortex, and above all the cerebellum. The movements are of the most various kinds. The animal may run round and round in a circle (circus movement); or, with the tip of its tail as centre and the length of its body as radius, it may describe a circle with its head, as the hand of a clock does (clock-hand movement); or it may rush forward, turning endless somersaults as it gocs. Intervals of rest alternate with paroxysms of excitement, and the latter may be brought on by stimulation. In man forced movements associated witl vertigo have been sometimes seen in cases of tumour of the cerebellum -e.g., involuntary rotation of the body in tumour of the middle peduncle. No entircly satisfactory explanation of these forcel movements has been given. They are cvidently connected with disturbance of the mechanism of co-ordination, leading to a loss of proportion in the amount of the motor discharge to muscles or groups of muscles accustomed to act together in executing definite movements. For instance, in circus movements the muscles of the outer sides of the body contract more powerfully than those of the inner side, and the animal is therefore constrained to tracc a circle instead of a straight linc, the excess cf contraction on the outer sicle being analogous to the acceleration along the raclius in the case of a point moving in a circle.

Co-ordination of Movements.-The capacity of executing some co-ordinated movements, occasionally of considerable complexity, seems to be inborn in man, and to a still greater extent in many of the lower animals. The new-born child brings with it into the world a ccrtain endowment of co-ordinative powers; it has inherited, for example, from a long line of mammalian ancestors the power of performing those movements of the cheeks, lips, and tongue, on which sucking depends; perhaps from a long, though somewhat shadowy, race of arboreal ancestors the power of clinging with hands ancl feet, and thus suspending itself in the air. Many movements, such as walking and the co-ordinated muscular contractions involver in standing, and even in sitting, which, once acquired, appear so natural and spontaneous, have to be learnt by painful effort in tlic hard school of (infantile) experience. Most people learn, and are willing to confess that they have learnt, to execute a considerable number of co-ordinated movements with the arms, and especially with the fingers ; but few have considered that the extreme dexterity of jaws, tongue, and teeth displayed by a hungry mouse or schoolboy is the result of the much practice which maketh perfect. The exquisite co-ordination of the muscles of the eycball, which we shall afterwards have to speak of, and the no less wonderful balance of effort and resistance, of power put forth and work to be done. of which we have already had glimpses in studying the mechanism of voice and speech, become to a great extent the common property 
of all fully-developed persons. But the technique of the finished singer or musician, of the swordsman or acrobat. and even the operative skill of the surgeon, are in large part the outcome of a special and acquired agility of mind or body, in virtuc of which highly-complicated co-ordinated movements are promptly determined on and immediately executed.

With such special and elaborate movements it is inpossible to occupy ourselves in a book like this. Their number may be almost indefinitely extended, and their nature almost infinitely varied, by the needs and training of special trades and professions. It will be sufficient for our purpose to sketch in a few words the mechanism of one or two of the most common and fundamental co-ordinations of muscular effort, passing over the rest with the general statement that the more refined and complex movements are in gencral brought about not by the abrupt contraction of crude anatomical groups of muscles, but by the contraction of portions of muscles, perhaps cven single fibres or small bundles of fibres, while the rest remain relaxed. The excitation may gradually wax and wane as the different stages of the movement require. Antagonistic muscles may be called into play to balance and tonc down a contraction which might otherwise be too abrupt.

Many interesting illustrations of this process of 'give and take' between opposing muscles have been reported, especially by Sherrington. Some have been already alluded to in discussing reflex movements (p 8or). One or two additional observations may be given here. In the cortex cerebri, as we shall see (pp. 845.859), there is an area in the frontal region, and another in the occipital region, stimulation of which gives rise to conjugate deviation of the eyes - that is, rotation of both eyes-to the opposite side. Sherrington divided the third and fourth cranial nerves in monkeys-say. on the left sicle. The external rectus, which is supplied by the sixth nerve, caused now by its unopposed contraction external squint of the left eye. When either of the cortical areas referred to, or even the subjacent portion of the corona radiata, was stimulated on the left side, both eyes moved towards the right, the left eye, however, only reaching the middle line-that is, the position in which it looked straight forward. The same thing was observed when the animal, after complete recovery from the operation, was caused to voluntarily turn its eyes to the right by the sight of food. Here an inhibitory influence must have descended the fibres of the abducens, the only nervous path connecterl with the extrinsic muscles of the left eye, and the relaxation of the left external rectus must have kept accurate step with the contraction of the right internal rectus. Hering has made an exhaustive analysis of the co-ordinated movements concerned in opening and closing the hand in monkeys. These movements can be produced by stimulation of the cortex or the internal capsule, but not by stimulation of the anterior spinal roots. When the hand is opened the muscles that open it are excited, and those which close it are inhibited from the cortex.

Standing.--In the upright posture the body is supported chiefly by non-muscular structures, the bones and ligaments. But muscles also play an essential part, for it is only peculiarly-gifted individuals. like some of the fishermen of the North Sea. who can go to sleep on their feet, and a dead body cannot be made to stand erect. The condition of equilibrium is that the perpendicular clropped from the centre of gravity to the ground should fall within the base of support 
-that is, within the area enclosed by the outer borders of the feet and lines joining the toes and heels respectively. The centre of gravity alters its position with the position of the body, which tends to fall whenever the perpendicular cuts the ground beyond the base of support.

In the comfortable and natural erect position the centre of gravity of the head is a little in front of the vertical plane passing through the occipital conclyles, and as much as + centimetres in front of the vertical plane passing througl the ankle-joints. A certain degrec of contraction of the muscles of the nape of the neck is required to balance it. When these muscles are relaxed, as in sleep, the hearl must fall forward, and this is the reason why Homer or any lesser inclividual nods. In animals which go upon all-fours none of the weight of the head bears directly upon the occipito-atloid articulation; its support by muscular action alone would be an intolerable fatigue, and the ligamentum nuchre is specially strengthened to hold it 1 p.

The vertebral column is kept erect by the ligaments and muscles of the back. The centre of gravity of the trunk lies almost vertically over the horizontal line joining the two acetabula, but the centre of gravity of the whole bolly is about the level of the third sacral vertebra, and a little more than 4 centimetres in front of the vertical plane passing through the ankle-joints. Equilibrium is maintained by contraction of the muscles of the back and of the legs. By means of the muscular sense, and the tactile sensations set up by the pressure of the soles on the ground, alterations in the position of the centre of gravity, and consequent deviations of the perpendicular passing through it, are detected, and adjustment of the amount of contraction of this or the other muscular group is promptly made.

In standing at 'attention' the heels are close together, the legs and back straightened to the utmost, and the head erect ; the weight falls equally upon both legs, but the advantage may be more than counterbalanced by the muscular exertion associated with this more ornamental than useful position. In 'standing at ease,' practically the whole weight is supported by one leg, the perpendicular from the centre of gravity passing through the knee and ankle-joints. The centre of gravity is brought over the supporting leg by flexure of the body to the corresponding side, and comparatively little muscular effort is required. The other foot rests lightly on the ground, the weight of the leg itself being almost balanced by the atmospheric pressure acting upon the air-tight and air-free cavity of the hipjoint. The light touch of this foot varies slightly from time to time, so as to maintain equilibrium.

When the head or arms are moved, or the body swayed, the centre of gravity is correspondingly displaced, and it is by such movements that tight-rope dancers continue to keep the perpendicular passing through it always within the narrow base of support.

In sitting, the base of support is larger than in standing. and the equilibrium therefore more stable. The easiest posture in sitting without support to the back or feet is that in which the perpendicular from the centre of gravity passes through the horizontal line joining the two tubera ischii.

Locomotion.--In walking, the legs are alternately swung forward and rested on the ground. In military marching, it is directed that toe and heel be simultaneously set down. But with most persons the swinging foot first strikes the ground by the heel; then the sole 
comes down, the heel rises, the leg is extended, and, with a parting push from the toe, the leg again swings free. By this manouvre the body is raised vertically, tilted to the opposite side, and also pushed in advance.

The forward swing of the leg is only slightly, if at all, due to muscular action; it is more like the oscillation of a pendulum displaced behind its position of equilibrium, and swinging through that position, and in front of it, under the influence of gravity. For this reason the natural pace of a tall man is longer and slower than that of a short man; but it may be modified by voluntary effort, as when a rank of soldicrs of different lieight keeps step.

The lateral swing of the body is illustrated by the everyday experience that two persons knock against each other when they try to walk close together without keeping step. In step both swing their bodies to the same side at the same moment, ancl there is no jarring.

Even in the fastest walking on level ground there is a short time during which both feet touch the ground together, the one leg not beginning its swing until the other foot has begun to be set down. In running. on the other hand, there is an interval during which the body is completely in the air, while in walking uphill or in carrying a load the one foot is not raised until the other has been firmly planted.

Functions of the Cerebral Cortex.-When an animal, like a frog, is deprived of its cerebral hemispheres, the power of automatic voluntary movement appears to be definitively and entirely lost. The animal, as soon as the effects of the anæsthetic and the shock of the operation have passed away, draws up its legs, erects its head, and assumes the cliaracteristic position of the normal frog at rest. So close may be the resemblance, that if all external signs of the operation have been concealed, it may not be possible for a casual observer to tell merely by inspection which is the intact and which the 'brainless' frog. The latter will jump if it be touched or otherwise stimulated. It will croak if its flanks be stroked or gently squeezed together. It will swim if thrown into water. If placed on its back, it will promptly recover its normal position. But it will do all these things as a machine would do them. without purpose, without regard to its environment, with a kind of 'fatal' regularity. Every time it is stimulated it will jump, every time its flanks are squeezed it will croak, and, in the absence of all stimulation, it will sit still till it withers to a mummy, even by the side of the water that might for a while preserve it.

A Necturus, without its cerebral hemispheres, will, like the frog, refuse to lie on its back. On stimulation it moves its feet or tail, or its whole body; but if not interfered with, it lies for an indefinite time in the same position. Its gills are seen to execute rhythmic movements, which never stop, and rarely slacken, except for an instant, when some part of the 
skin, particularly in the region of the head, is mechanically or electrically stimulated. The normal Necturus, on the other hand, lies for long periods with its gills at perfect rest, and when stimulated, moves for a considerable distance.* After a time, -two months or more-it is true the brainless frog, if it be kept alive, as may be done by careful attention, will recover a certain portion of the powers which it has lost by removal of the cerebral hemispheres; and, indeed, the longer it lives, the nearer it approximates to the condition of a normal frog. A brainless frog has been seen to catch flies and to bury itself as winter drew on. A fish even three days after the destruction of its cerebrum has been seen to dart upon a worm, seize it before it had time to sink to the bottom of the aquarium, and swallow it. Even in the pigeon the loss of the hemispheres, which at first induces a state of profound and seemingly permanent lethargy, is to a great extent compensated for, as time passes on, by the unfolding in the lower centres of capabilities previously dormant or suppressed. A brainless pigeon has been known to come at the whistle of the attendant and follow him through the whole house.

In the mammal the removal of the whole or the greater part of the cerebral hemispheres at a single operation is uniformly and speedily fatal; even rabbits or rats, which bear the operation best, survive but a few hours. During those hours they manifest phenomena similar to those observed in the bird and the frog. In the dog the entire cortex has been remored piecemeal by successive operations. In this case, of course, the change in the condition of the animal is more gradually produced, and an opportunity is afforded for a certain recovery of function in the intervals between the operations. On the whole, however, as might be expected from its greater intellectual derelopment, recovery is more imperfect in the dog than in the bird, much more imperfect than in the frog. But even in the dog wonderful resources lie hidden in the grey matter of the central neural axis, and are called forth by degrees to replace the lost powers of the cerebral cortex. It is true that a brainless dog is a less efficient animal than a brainless fish, or even than a brainless frog; but in farourable cases, even in the dog, the morements of walking may still be carried out with tolerable precision in the absence of the cerebral hemispheres. The animal can swallow food pushed well back into the mouth, although it cannot feed itself. Stupid and listless as it is compared with the normal dog, it seems to be by no means deroid of the porver of experiencing sensations as the result of impressions from without, nor of carrying on mental operations of a low intel-

$$
\text { * Personal observations. }
$$


lectual grade. Goltz liad a clog which lived more than a year and a half practically without its cerebral hemispheres, and another which lived thirteen weeks. He believes that they had lost understanding, reflection, and memory, but not sensation, special or general, nor emotions and voluntary power. Their condition may be best described as one of general imbecility. Hunger and thirst are present. They experience satisfaction when fed, become angry when attacked, see a very bright light, avoid obstacles, hear loud sounds, such as those produced by a fog-horn, and can be awakened by them. They are not completely deprived of sensations of taste and touch. But it ought to be remembered that the interpretation of the objective signs of sensation in animals is beset with difficulties; and although everybody admits the accuracy of Goltz's description of what is to be seen, his interpretation of the facts has been severely criticised, particularly by H. Munk.

To the monkey there can be no doubt that the loss of the cerebral hemispheres would be a still heavier and more irremediable blow than to the dog. But nobody has yet succeeded in keeping a monkey alive after complete removal of even one hemisphere.

In man the destruction of considerable masses of brain-substance, particularly if gradual, is not necessarily fatal. How great a loss is compatible with life cannot be exactly stated. It depends to a large extent on the position of the lesion. But it is possible that one cerebral hemisphere may be rendered functionally.useless without immediately putting a term to existence. In the fœtus, however, no portion of the great brain is absolutely indispensable for life and movement. An anencephalous fœt us (in which the brain has remained undeveloped) may be born alive, and live for a short time.

We see, then, that homologous organs are not necessarily, nor indeed usually, of the same physiological value in different kinds of animals. A loss which perhaps hardly narrows the range of the psychical, and certainly restricts only to a slight extent the physical powers of a fish, impairs in a marked degree the voluntary movements of a dog, in addition to cutting off from it a great part of its intellectual life, and is in man incompatible with life altogether.

The results of the removal of the entire cerebral henispheres help us to fix their position as a whole in the physiological hierarchy. A more minute analysis shows us that the cerebral cortex itself is not homogeneous in function, that certain regions of it have been set aside for special labours. Our knowledge of this localization of function in the cerebral cortex has been derived partly from clinical, coupled with pathological observa- 
tions on man, and partly from the results of the removal or stimulation of definite areas in animals. And so varied and extensive have been the contributions from both of these sources, that it is difficult to decide to which we owe most. In addition, the study of the development of the myelin sheath, and especially in recent years the minute study of the histology of the various regions, have aided materially in mapping out the cortex.

It is a fact which might appear strange and almost inexplicable (lid the history of science not constantly present us with the like, that forty years ago the universal opinion among physiologists, pathologists, and physicians was that the cerebral cortex is inexcitable to artificial stimuli, that no visible response can be obtained from it. The great names of Flourens and Magendic stood sponsors for this error, and repressed research. In $187^{\circ}$, however, Hitzig and Fritsch showed that not only was it possible to elicit muscular contractions by stimulation of the cortex of the brain in the dog with voltaic currents, but that the excitable area occupied a definite region in the neigh bourhood of the crucial sulcus or sulcus centralis, which runs out over the convexity of the hemispheres nearly at right angles to the longitudinal fissure. In this region they were further able to isolate several distinct

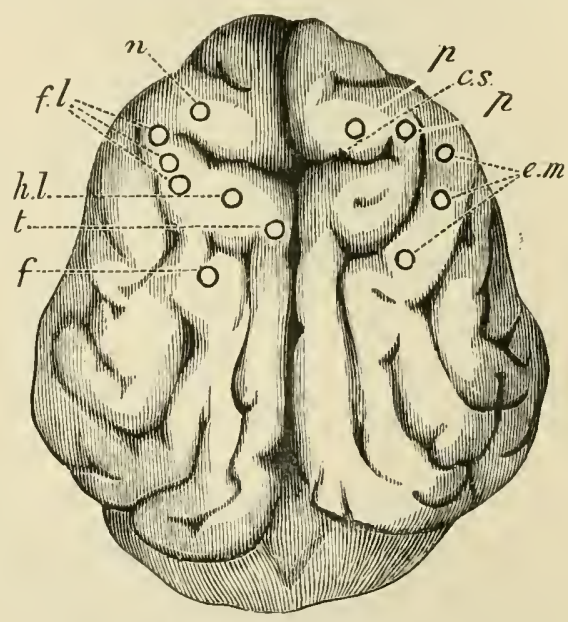

Fig. 349.-Motor Areas of Dog's Brain.

h, neck; f.l., fore-limb; h.l., hind - limb; $t$, tail ; $f$, face; c.s., crucial sulcus ; e.m., eye movements; $p$, dilatation of the pupil in both eyes, but especially in the opposite eye. All the areas are marked in the figure only on the left side except the eye areas, whose position, to avoid confusion, is indicated on the right hemisphere. areas, stimulation of which was followed by movements respectively of the head, face, neck, hind-leg, and fore-leg (Fig. 349). This was the starting-point of a long series of researches by Ferrier, Munk, Horsley, Schäfer, Heidenhain, and many others, on the brains of monkeys as well as dogs-researches which have formed the basis of an exact cortical localization in the brain of man, and have enriched surgery with a new province. In these later experiments the interrupted current from an induction machine has been found the most suitable form of stimulus (see Practical Exercises, p. 889), especially when one electrode only is placed on the cortex and the other on some indifferent part of the body-e.g., in the rectum, (unipolar stimulation), a procedure which permits of finer localization than when both electrodes are applied to the brain (bipolar 
stimulation). For certain purposes the method of Ewald has advantages. He fixes in a trephine hole in the skull an ivory plug, through which pass the electrodes. When the animal has recovered from the operation, the region of the brain in contact with the electrodes can be stimulated without fastening the animal.

'Motor' Areas.*-These have been recently localized with great care (both by stimulation and by removal of portions of the cortex) in the brains of the higher apes (gorilla, orang, and

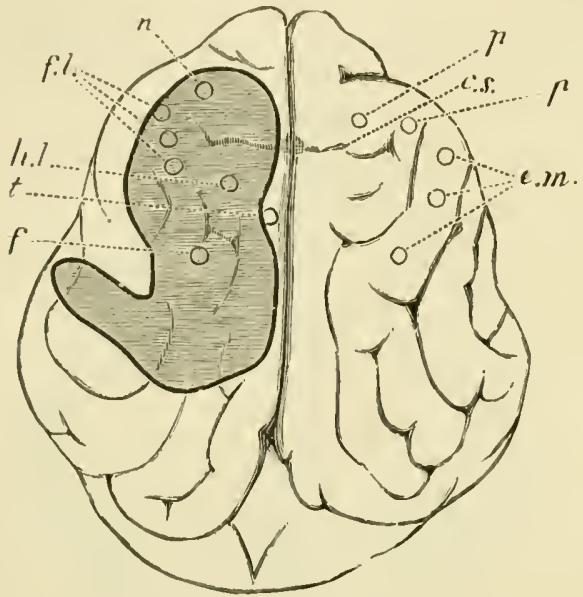

Fic. 350.-Dog's Brain with LESion.

A portion of the cortex indicated by the shaded area was destroyed by callerization. The symp. touns were complete blindness of the opposite ey'e (in this case the right); weakness of the muscles of the limbs and of the neck on the right side; slight weakness of the limbs on the left side. When the animal walked, there was a tendency to turn to the left in a circle. In eating or drinking, the head was turned to the left, so that the mouth was oblique, and the right angle of the mouth was lower than the left. The tail movements were normal, and there was no deviation of the tail to one side. quite sharp, but shades off somewhat gradually into inexcitable contex. The sulci in this region cannot be considered to repre-

* Since the so called 'motor' area, as is now well known, is really sensori-motor, and a region having to do purely with the discliarge of motor impulses cloes not exist, it would be better to call it the sensorimotor, or, following Bastian's suggestion, the kinxesthetic area. Probably, however, the alteration of a term so long sanctioned by custom in physological writings would lead to confusion. Accordingly, in what follows the worl 'motor' will be retained, but to show that it is used in a special sense it will be enclosed in quotation marks. 
sent physiological boundaries, and they vary so much in these higher brains, that they can easily prove fallacious landmarks. On the mesial surface of the hemisphere the 'motor' area does not extend quite to the calloso-marginal fissure.

Within this area are localized movements of the leg and arm and their various joints, of the head, face, moutl, tongue, ear, nostril, and vocal cords, of the neck, chest, and abdominal wall, of the pelvic floor, and the anal and vaginal orifices.

The arrangement of the various regions follows very closely the order of the cranio-spinal nerves, which supply them, but

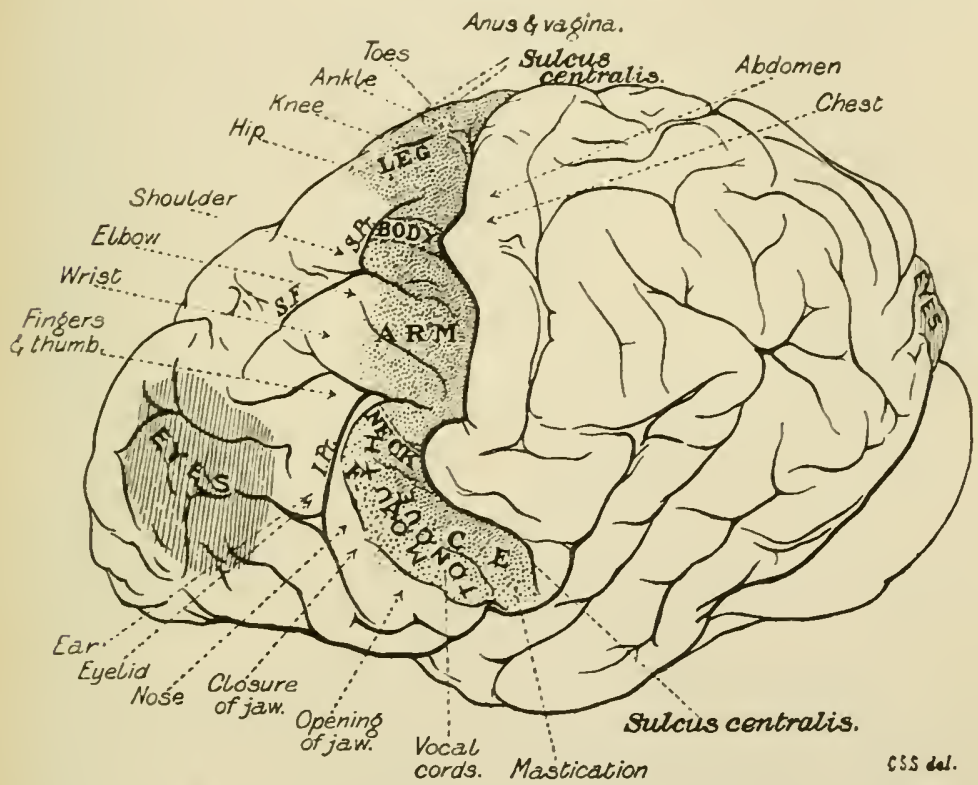

Fig. 351.- 'Motor' Area of Cortex of Chimpanzee (Grünbaum and SHERRINGTON).

Lateral aspect of the hemisphere.

the organs whose nerves come off lowest down are represented lighest up in the 'motor' area. Figs. 35I, 352 will make this clear. In the frontal region, isolated from the motor area by a strait of inexcitable cortex, lies an area the stimulation of which causes conjugate deviation of the eyes. But the reaction differs from that obtained on excitation of the 'motor' area proper in front of the Rolandic fissure.

It is to be particularly noted (I) that within the larger areas, such as those of the arm and leg, smaller foci can be mapped off which are related to movements of the separate joints-thus, 
in the leg area, the hip, knee, and ankle joints, and the great toe, are represented by separate and special centres; (2) that stimulation of any one of these areas leads, not to contraction of individual muscles, but to contraction of muscular groups which have to do with the execution of definite movements.

Inhibition from the Cortex.-Contraction is not the only effect

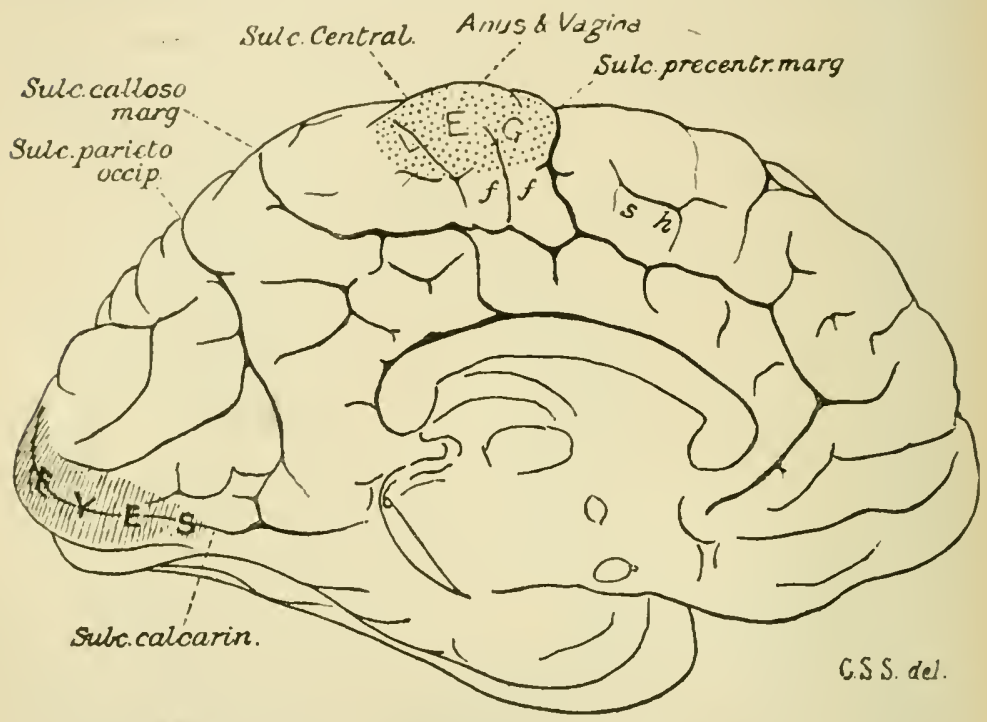

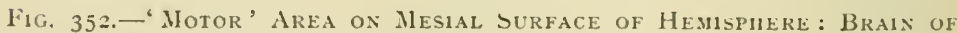
a Chimpanzee (Troglodytes Niger) (Grünbaum and Silerrington).

Left hemisphere : inesial surface.

The extent of the 'motor' area on the free surface of the bcmispliere is indicatcd by the black stippling. On the stippled area ' LEG' indicates that the movenents of the lower limb are represented in all the regions of the 'motor' area visible from this aspect. The minuter subdivisions in this area overlap each other so nutucl that no at tempt is made to distinguish them in the diagram. "Anus and vagina" indicates the position from which perineal movements can be primarily elicitcd. Sulc. central.=central fissure; Sulc. calcarin.=calcarine fissure; Sulc. paricto occip. = parieto.occipital fissure; Sulc. calloso marg.=calloso.marginal fissure ; Sulc. precentr. marg.=precentral marginal fissure. The single italic letters mark spots whence, occasionally and irregularly, snovenents of the foot and leg (f), of the shoulder and chest $(s)$, and of the thumb and fingers $(h)$ have been evoked by strong faradization. The shaded area niarked 'FYES' indicates a field of frce surface of cortex which, under faradization, yields conjugate move. nents of the eyeballs. The conditions under which these reactions are obtained separates thein from those characterizing the 'motor' area.

on the muscles which can be elicited by stimulating the cortex. Cortical inhibition of tonus and of active contraction is just as characteristic, though not so obvious a result. There is abundant evidence, some of which has previously been alluded to (p. 838), of reciprocal innervation of volitional movements from the 
cortex. When, c.g., the part of the arm area which presides over extension of the elbow is stimulated (in the monkey), it can be shown that the biceps relaxes as the triceps contracts. In like manner, stimulation of the appropriate part of the leg area will cause along with contraction of the extensors of the hip relaxation of such flexors as the psoas-iliacus and the tensor fascia femoris. Such observations are most easily made when, in a certain stage of narcosis, the limbs, instead of hanging limp, assume a position of tonic flexion, especially at the elbow and hip. Under other conditions the position of tonic extension of a joint may be assumed, and then it can be shown that excitation of the appropriate focus for flexion of that joint will cause simultaneous contraction of the flexors and relaxation of the extensors.

The observer cannot fail to be struck with the general resemblance between these cortical reactions and their co-ordination and the co-ordinated bulbo-spinal reflex movements previously studied. There are, however, certain differences which place the cortical reactions upon a higher level. One of the most important is the part played by visual, auditory, and pure 'touch' stimuli in eliciting cortical motor responses-e.g., "the closure of the hand, pricking of the ear, opening of the eyes, and turning of the head in the direction of the gaze" (Sherrington). The facility of response to stimuli acting from a distance through the distancereceptors, such as those of the retina and the labyrinth, is one of the great characteristics of the cerebrum as an organ concerned in movements, and helps to place the 'motor' cortex at the helm, since these distance-receptors control more than others the skeletal musculature as a whole. Spinal reflex movements are mainly such as are elicited by harmful (nocuous) stimuli (protective reflexes), or through the sexual shin nerves, or from the visceral afferent fibres, or such as are concerned in the chief movements of locomotion.

Decerebrate Rigidity is a phenomenon closely related to the inhibitory function of the cerebral cortex. It is a condition of prolonged spasm of certain groups of skeletal muscles (especially the retractor muscles of the head and neck, the elevators of the jaw and tail, and the extensors of the elbow, knee, shoulder, and hip), supervening on removal of the cerebral hemispheres by transection anywhere in the mid-brain or in the posterior part of the thalamus, and favoured by suspending the animal in the vertical posture. If the afferent roots belonging to one of the rigid limbs are severed, it at once becomes flaccid, while the other limbs remain rigid. The tonus is therefore reflex through the local afferent nerves, and, to be more precise, through those that supply the deep structures (joints, muscles, etc.). The 
centre must be situated somewhere between cerebrum and spinal bulb, since section of the bulb abolishes the rigidity. It is not apparently in the cerebellum. It is noteworthy that the muscles mainly involved in decerebrate rigidity are those which are much more easily inhibited than excited from the ' motor ' cortex, and also in the local spinal reflexes. After removal of the cerebrum, the mechanism which maintains their tonic contraction has free play. Sherrington points out that this mechanism sustains the steady muscular tension necessary to preserve against the force of gravity the attitude or posture of the body. When the transient spinal reflex or the transient cortical effect breaks in upon this tonic contraction-e.g., in locomotion -inhibition of the contracted extensors accompanies contraction of the flexors (see also p. 836).

Removal of a single 'motor' region leads to paralysis of the corresponding limb, or part of a limb, on the opposite side. For example, after extirpation of the hand area the hand is for a few days practically useless and apparently powerless. In a few weeks, however, it recovers remarkably, so that it is once more used in climbing or in conveying food to the moutl. It is an important question in what way this recovery is brought about. If the whole of the corresponding area in the opposite hemisphere is now removed, a similar paralysis occurs in the other hand, but the hand whose motor area was first extirpated remains entirely unaffected by the second lesion. On the contrary, the first hand is used more freely and more adroitly than before the second operation, probably because the animal needs to use it more. The second hand recovers eventually, like the first. If when this has taken place the remaining part of the arm area from which the hand area was first excised be removed, neither hand is apparently affected, although there is severe paralysis of the shoulder and slighter paralysis of the elbow on the side opposite to the lesion, which is again largely recovered from. The recovery of the hand movement camnot therefore be attributed to the taking on of the function of the corresponding motor area either by the opposite hand area or by the adjacent ' motor' cortex of the same hemisphere. According to some authorities, the recovery is due to the representation of the upper limb in the post-central gyrus (ascending parietal convolution in man) acting through fibres that descend from this gyrus to the optic thalamus, and thence through the rubro-spinal tract, which runs to the spinal cord (p. 765$)$.

Removal of the whole of the 'motor' cortex of one hemisphere, in such animals as this operation lias been performed on, causes paralysis of movement on the opposite side of the body. The paralysis is less marked in the case of bilateral muscles that 
habitually act together than in the case of those which ordinarily act alone. Thus the muscles of respiration and the muscles of the trunk in general are, although perhaps weakened, never completely paralyzed. This is an indication that each member of such functional pairs of muscles is innervated from both hemisplieres; and this plyysiological deduction is supported by the anatomical fact already referred to, that after removal
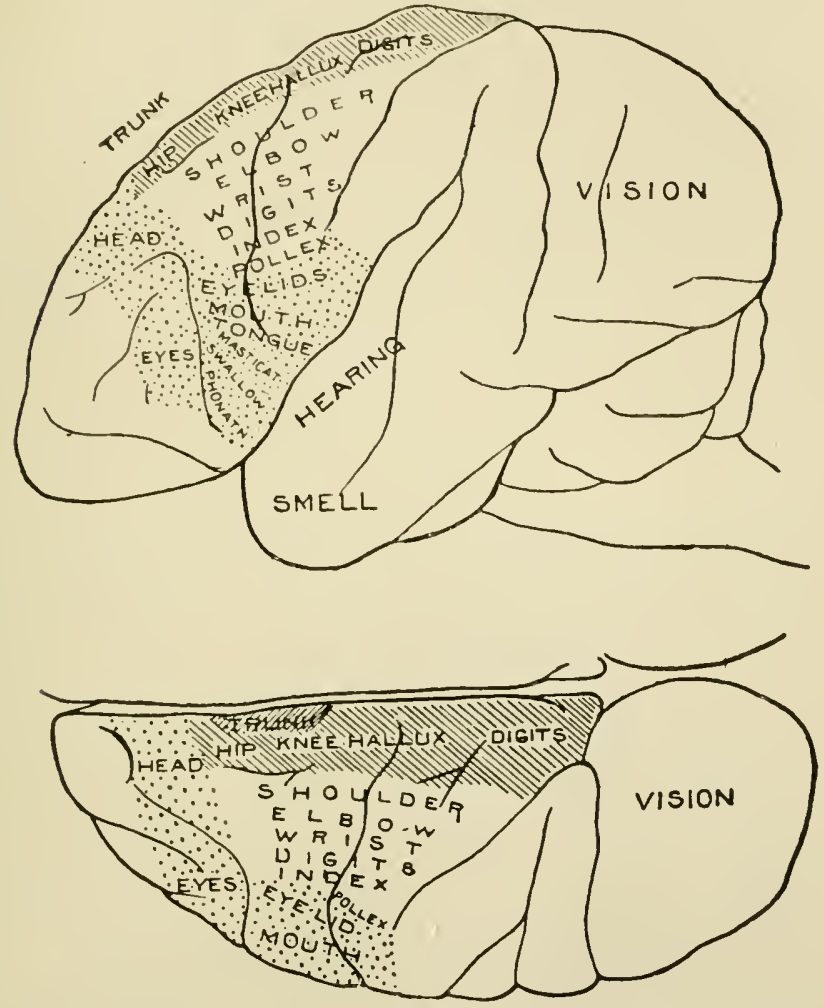

Fig. 353.-Brain of Macaque Monkey (Beevor and Horsley).

The upper figure shows the lateral aspect of the left hemispliere, and the lower figure its upper (or dorsal) surface. The 'motor' and sensory areas are indicated. It is questionable whether the 'motor' region is as extensive as represented. Some observers do not admit that it extends behind the central sulcus in these lower monkeys any more than in the higher apes.

of the 'motor' cortex, or injury to the pyramidal tracts in the internal capsule or crus, some degenerated fibres (homolateral fibres) are found in the crossed pyramidal tract on the side of the lesion (p. 778 ).

In the dog after a time the paralysis may more or less completely disappear. In the monkey restoration is less complete. 
Some interesting observations have been made on a monkey, which was carefully watched for eleven years after the removal

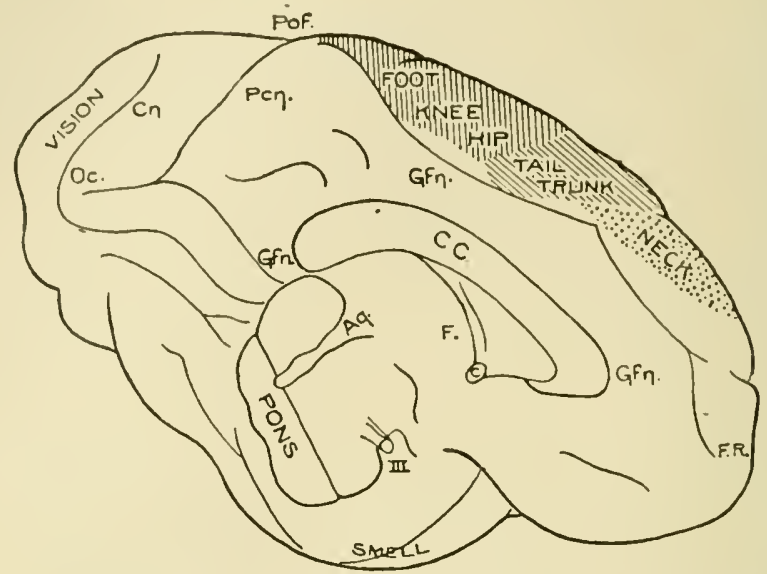

lig. 354. -Mesial Sirface of Left Hemisphere of Macagee Monkey (HORSLEY).

by two operations of the cortex of the greater portion of the frontal and parietal lobes on the left side. The character of the animal, which had been studied for months before the

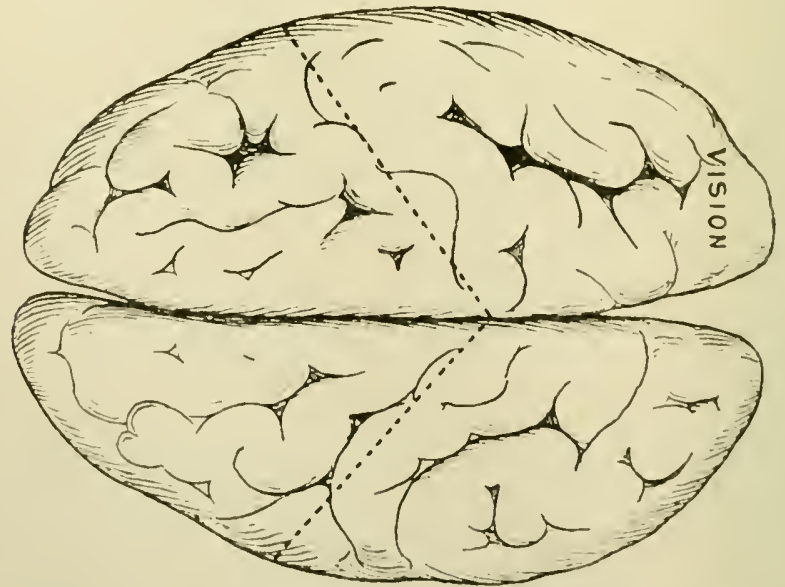

Fig. 355.-Clerlibral Cortex: Max (Seen from Above).

The front of the brain is towards the right. The dotted line shows the position of the fissure of Rolando, as fixed by. Thane's rule (p. 856).

operations, was entirely unaffected. All its traits remained unaltered. There was no loss of memory or intelligence. On the 
other hand, disturbances of movement on the right side were very noticeable 11) till its death. It learned again to use the right limbs in locomotion; but, although they were not markedly weaker than those of the left sicle, their movements had a certain chumsiness, which was associated with a permanent diminution in the sensibility of the skin of these limbs. Nuscular sensibility was also lessened. In acts requiring the use only of one hand, the right was never willingly employed, and it evidently cost the animal a great effort to use it in such movements, but by special training it learnt again to give the right hand when asked for it, and to make use of it for other purposes. The movements with which the motor areas are concerned are essentially skilled movements, and we may suppose that it is more difficult for a monkey to educate again a centre for sucl complex and elaborate manceures as are performed by its hand than for a dog to regain noimal control of the comparatively simple movements of its paw. In man in cases of hemiplegia, when the patient lives for some time, a certain amount of recovery usnally takes place, especially in young persons, in the paralyzed leg, but much less in the paralyzed arm.

In the lower monkey's the 'motor' area was formerly stated to extend behind the sulcus centralis into what in man would be called the ascending parietal convolution (postcentral gyrus), and also to be more extensively represented on the mesial surface of the hemisphere than in the higher apes (Figs. 353, 354). Such observations, hovever, require to be reinterpreted in view of the results of Sherrington and Grünbaum, especially as they were carried out by the bipolar method of stimulation, with both electrodes on the cortex. This method does not admit of such strict localization of the stimulus as the unipolar method. The most recent work with the unipolar method has indicated that in the lower apes also excitation of the gyrus postcentralis does not cause morements (C. and O. Vogt).

It is in the light of the results obtained in monkeys, and by the aid of histological, embryological, clinical, and pathological observations, that the 'motor' areas in man have to a great extent been mapped out.

The histological differentiation of the various cortical regions recently (lemonstrated by Brodmann and by Campbell are of cspecial interesi. (Figs. 356-360). It has long been customary to divide the cortex into layers, although the number and the boundaries of these layers are somewhat arbitrarily fixed. Brodmann distinguishes six layers: (1) A zonal or peripheral layer, containing many nerve-fibres and neuroglia cells, but fer nerve-cells ; (2) a layer containing 'granules' and small pyramidal cells (external granular layer); (3) a layer of medium and large pyramidal cells (pyramidal layer); (4) a layer of small irregular cells (internal granular or stellate layer); (5) a 


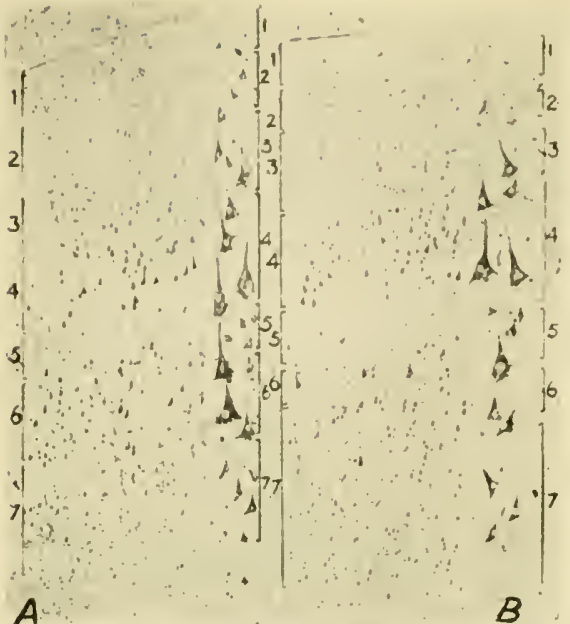

F1G. 356.-Cell-lamination of Girl's POSTCENTRALIS (CAMPBELL).

A. just behind upper end of fissure of Ro. lando; $B$, from the posterior edge of the gyrus (intermediate postcentral area of Campbell).

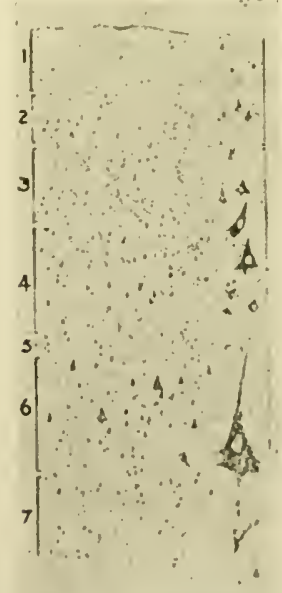

Ficr. 357.-Cell-Lamixation of GYrus Precentralis (CAMPBell).

From the portion of the grrus im. mediately in front of the central sulcus (Campbell's jrecentral area in Figs. 359,360 ). 'ganglionic' layer, con. taining the largest pyramillatl cells (deep large pyramids); (6) at layer (lamina multiformis) of spindle-shaped or polymorphous cells. These layers vary in their structural letails, and especially in their relative development in animals of different rank in the mammalian scale, in one and the same animal at rlifferent periods in its cmbryonic and extra-nterine growth, and also in clifferent parts of the cortex in an adult animal of given species. The region in front of the central sulcus (fissure of Rolando), e.g., is characterized by the presence of the giant pyramids of Betz, which give origin to the pyramidal fibres going to the trunk and limbs (Fig. 357).

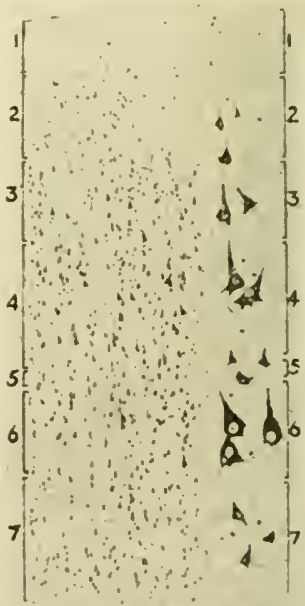

Fig. 358. - CELL - LAMINATION OF Gýrus Precentralis (Camplell).

from anterior part of the gyrus (Campbell's intermediate precentral area in Figs. 359,360 ).

Although the results are less definite, the work of Flcclusig on the time of development of the medullary sheath of the fibres in the various cerebral convolutions has also contributed to our knowledge 


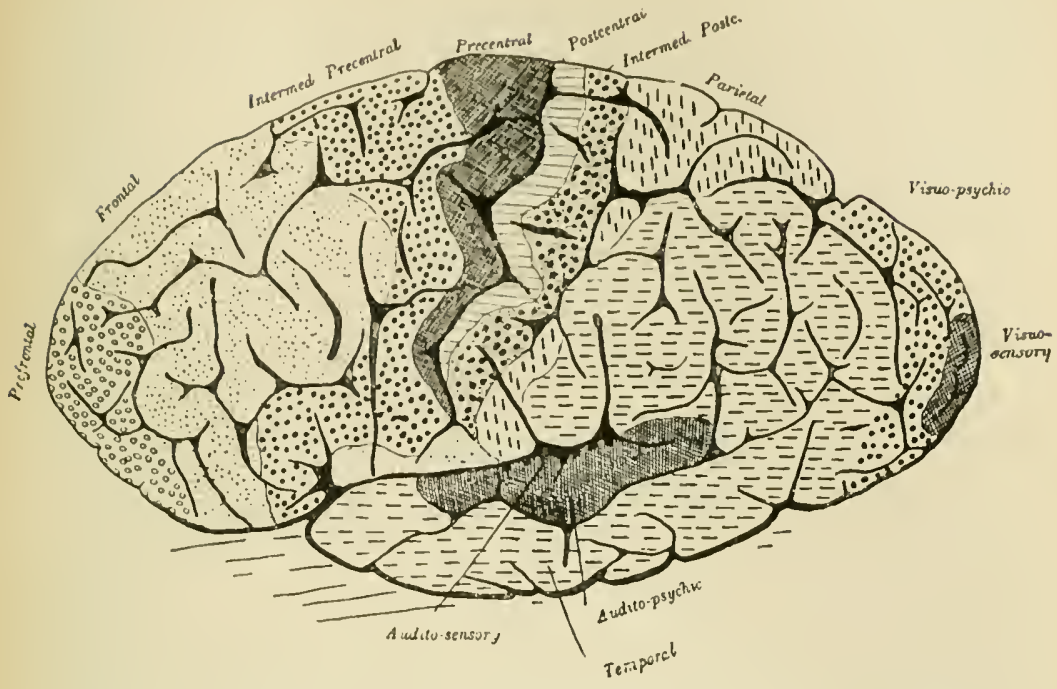

Fig. 359.-Structurali.y Differentiated Cortical Areas (Campbell). External surface of hemisphere (human brain).

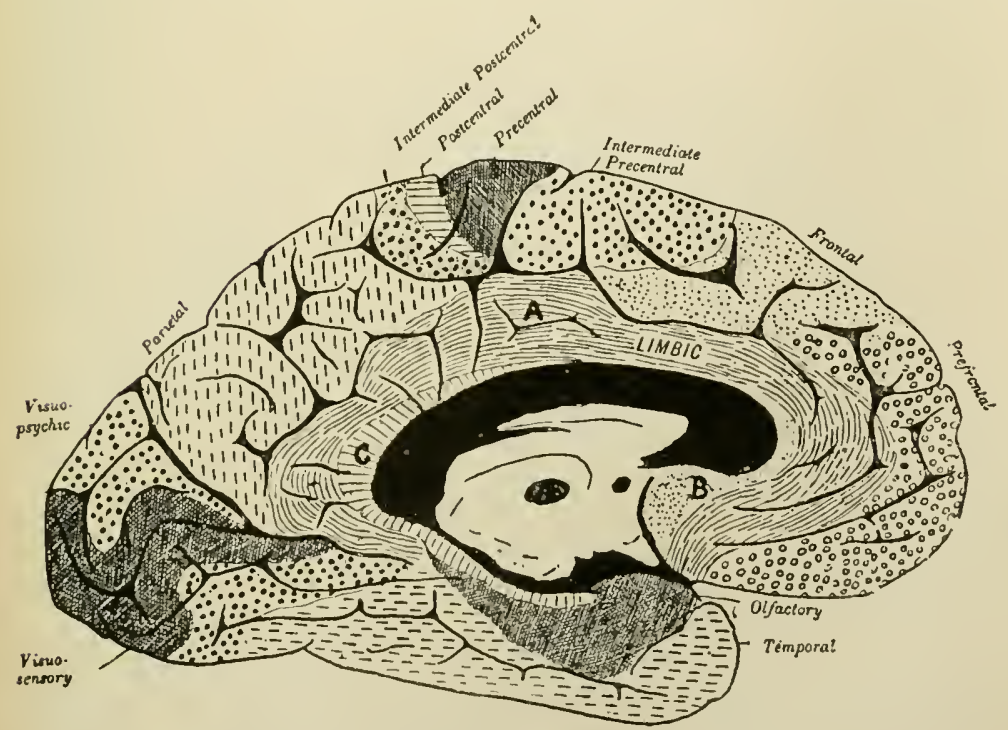

Fig. 360.-Structurally Differentiated Cortical Areas (Campbell). Mesial surface of hemisphere (human brain). 
of localization in the cortex. In the clevelopment of a neuron four stages cin be clistinguished: (1) Cells without processes; (2) the appearance of processes, first the axon and then the dendrites;

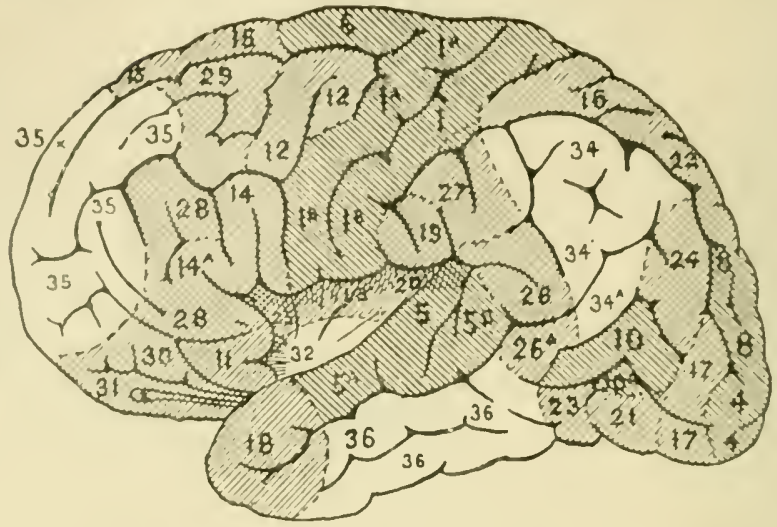

Fig. 3rit.-Fifchisig's Developmentai. Zoxifs (after Flecusig).

Outer surface of human cerebral hemisphere. Primary zones (I-IO), darkly shaded : intermediate zones (II-3i), less deeply shaded; terminal zones (32-36), unshaded.

(3) the formation of collaterals; (4) myclination or the formation of the melullary sheath (Fig. 305, p. 752 ).

Myclination occurs in the cerebral convolutions in a regular oreler. In sume areas the fibres mat be medullated three months before

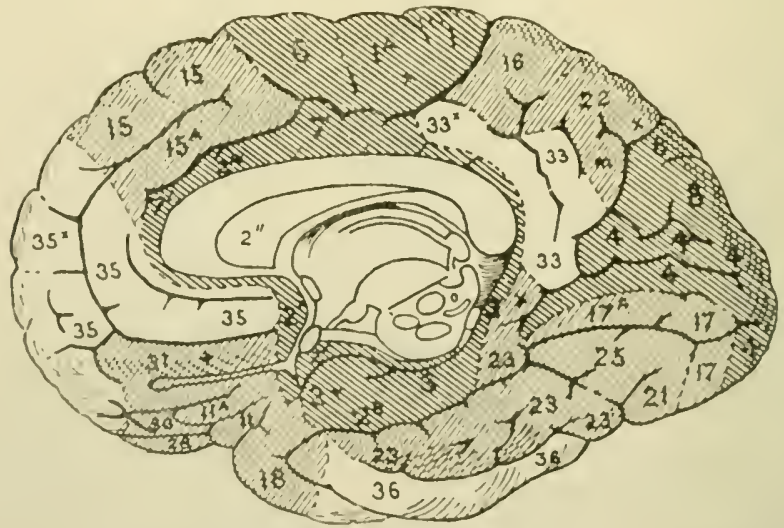

Fig. 362.-Flechigig's Developmental Zones (after Flecisig). Inner surface of human cereloral hemisphere.

birth, in others not till six months later. lior instance, the Rolandic and olfactory regions, the calcarine portion of the occipital lobe associated with vision, and the portion of the temporal lobe associated with hearing, are plentifully provided with medullated fibres a short time after birth, at any rate before the first month, whereas the remaining regions of the cortex are completely, or almost completely, 
free from such fibres. In this waty lilechsig has distinguished thirty-six cortical fields (ligs. 361,362 ), which he divides according to the tiune of myclination into three groups :

1. Primary fields, ten in number, which are well proviled with myelinated fibres at birth. They inclurle the cortical centres for the various sensations and also the 'motor' area. They are connected especially with the so-called projection fibres. Thus, the cutaneons and mnscular sense is assumed to be represented in field 1 , the sense of smell in field 2, of vision in 4 , and of hearing in 5. Fron field 1 arise the fibres of the pyranidal tract. chicfly from the ascending frontal convolution, while the sensory fibres from the skin and muscles cncl mainly in the ascending parictal. This is an illustration of what Flechsig considers a general rule for these primary fields-viz., that each primordial sensory region is connected both with an afferent (cortici-petal) and with an efferent (cortici-fugal) tract. From the visual area (4), e.g.. arises a tract which proceeds mainly to the anterior corpus quadrigeminum.

2. Terminal fields ( 32 to 36 in the figures) which become myelinated late, the process not beginning until at least a month after birth.

3. Intermediate fields (1 1 to $3 \mathbf{I}$ ) which become myelinated earlier than the terminal, but later than the primary. They and the terminal fields constitute par excellence association centres, which furnish fibres (association fibres) connecting the centres representel in the primary fields-e.g.. such fibres as must be continually conveying impressions from the visual centre to the notor cortex when the hand is sketching a landscape. It may also be considered a function of these association centres to store up the memories of previous sense impressions. Flechsig divides the association centres represented in the terminal fields into: (I) The great anterior association centre in the frontal lobe in front of the 'motor' area: (2) the great posterior association centre in the parietotemporal region; (3) the smaller midllle or insular association centre which coincides with the island of Reil, an area which, according to Sherrington and Grünbaum, is totally. 'inexcitable' as regards the production of movement in the anthropoid apes. These association centres are foci, from which issue and to which come the long association paths. The reader must bear in mind that Flechsig's conclusions as to the functions of his very numerous areas are in many cases hypothetical, and can only be accepted when corroborated by other methods. We are far from being able at present to subdivide the functions of the cortex so minutely as is suggested by his map.

Clinical and Pathological Observations in man agree, upon the whole, with wonderful precision with the results of experiments on animals; and, indeed, before any experimental proof of the ininute and elaborate subdivision of the cortex had been obtained, Broca had already, from the phenomena of the sick-bed and the post-mortem room, located a centre for speech in the left inferior frontal convolution (but see p. 863), and Hughlings Jackson had associated pathological lesions of the Rolandic area with certain cases of epileptiform convulsions.

An extensive hæmorrhage involving the Rolandic area of the cerebral cortex or an embolus blocking the middle cerebral artery, causes paraly'sis of the opposite side of the body. An embolus of a branch of the middle cerebral artery causes para- 
lysis of the muscles, or rather movements, represented in the area supplied by it. A tumour causes symptoms of irritation, notor or sensory convulsions beginning in, or sensations referred to, the parts represented in the regions on which it presses. In comnction with the localization of lesions in the 'motor' area of the cortex, and operative interference for their cure, the cortex las been frequently stimulated in man. There is no doubt that the 'motor' region corresponds closely in position to that of the higher apes. It cloes not include the postcentral gyrus, for stimulation of this conrolution witl such strengtlis of current as are permissible cvokes no movements, while movements are readily elicited from the precentral gyrus (Horsley, etc.). In exposing the 'motor' region, or any particular part of it, the exact position of the fissure of Rolando becomes important; and Thane has given the following simple metlod for fixing it: The point midway between the point of the rose and the occipital protulerance is fixed by measuring the clistance with a tape. The upper end of the fissure of Rolando lies half an inch behind this middle point. The fissure makes an angle of $67^{\circ}$ with the longitudinal fissure (Fig. 355). The minor fissures are so inconstant as to afford no safe guidance in the localization of a given area. This must be delimited by stimulation.

Sensory Functions of the Rolandic Area.-There are many proofs that the 'motor' region is not a purely motor, but a sensori-motor, or Fincesthetic, area. Histological and cmbryological studies on the course of the sensory paths, as already pointed out, support this conclusion. It has also been mentioned that, according to Goltz's observations (p. 850), removal of the Rolandic cortex causes defects of sensation as well as of movement. In man, in connection with operations on the brain, still better evidence has been obtained. In two cases Cusling was able to elicit tactile sensations by clectrical stimulation of the gyrus postcentralis (ascending parietal convolution), and the scuse of muscular movement by electrical stimulation of the gyrus precentralis. In a very careful study of a case in which he removed the upper limb area of the right hemisphere in a boy for violent convulsive movements of the whole of the left arm, Horsley came to the conclusion that the precentral gyrus in man is the seat of representation of (I) slight tactile sensation (after the operation appreciation of the lightest tactile stimuli was lost) ; (2) topognosis-i.e., appreciation of the localization in space of the point touched; (3) muscular sense ; (4) stereognosis, or the power of recognising the form of objects touched and handled; (5) pain -e.g., that caused by a pin-prick; (6) volitional movement. The postcentral gyrus in man appears to be the seat of a similar sensory representation, but as its relation to the efferent impulses 
concerned in volitional movements is less decided than that of the precentral gyrus, so its relation to afferent impulses, both from the skin and the deeper structures, is better marked. From the field of experiment further evidence of the sensori-motor nature of the ' motor' region is forthcoming.

(I) It has been found that if the posterior roots of the nerves supplying one of the limbs be cut in a monkey, all the most delicate and skilled movements of the limb are either greatly impaired or totally abolished (Mott and Sherrington). The limb is not used for progression or for climbing, but hangs limp, and apparently helpless, by the sicle of the animal. That this condition is not due $t o$ any loss of functionc] power by the peripheral portion of the motor path may be assumed, since the anterior roots remain intact. Tliat it is not clue to any want of capacity on the part of the 'motor' centres to discliarge impulses when stimulated may be shown by exciting the cortical area of the limb-either electrically or by inducing epileptic convulsions by intravenous injection of absinthe - when movements of the affected limb take place just as readily as movements of the sound limb. The cause of the impairment of rol-

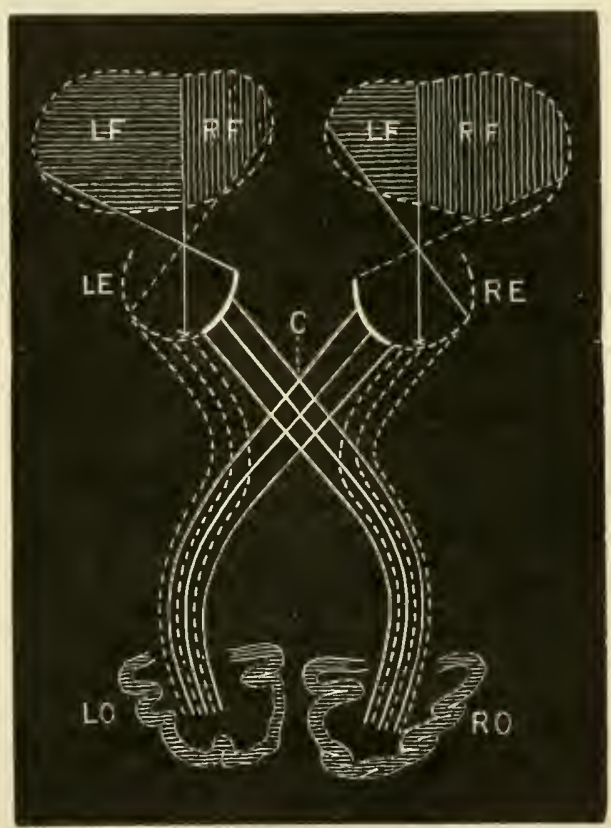

Fig. 363.-Diagrail of Relations of Occi pital Cortex to the RetiNe.

RO, LO, right and left occipital cortex: RE, LE, right and left retina; $C$, optic chiasma; RF, LF, right and left risual fields. The continuous lines passing back from the retina to the accipital cortex represent the crossed, the broken lines the vincrossed. fibres of the optic nerves and tracts. For the sake of simplicity the intermediate stations on the visual path in the anterior corpora quall rigemina, lateral geniculate bodies, and pulvinar are not represented in the diagram. For these ronnections see Fig. 3+2, p. 8 I . untary motion, then, can only be the loss of the afferent impulses which normally, pass up to the brain, and presumably to the 'motor' cortex. W'hen only one sensory nerve-root is cut, no defect of movement can be seen; and this is evidently in accordance with the fact previously 
mentioned (p. 790), that complete anesthesia of even the smallest patch of skin is never caused by section of a single posterior root. And that it is the loss of impulses from the skin which plays the chief part is shown by the fact that after division of the posterior roots supplying the muscles of the hand or foot, which only partially interferes with the sensory supply of the skin, joints, sheaths of tendons, etc., movement is unimpaired ; while section of the nerve-roots supplying the skin, those of the muscles being left intact, causes extreme loss of motor power.

(2) If a strength of stimulus be sought which will just fail to cause contraction of the muscular group related to a given motor area, and a sensory nerve, or, better, a sensory surface (best of all, the skin over the corresponding muscles), be now stimulated, contraction may occur-that is to say, the excitability of the motor centres may be increased. This shows that the "motor' region is en rapport not only with efferent, but also with afferent fibres, that it receives impulses as well as discharges them.

The same experiment is a proof that the results of excitation of the motor cortex are due to stimulation of the grey matter, ancl not, as might be objected, of the white fibres of the corona radiata. It is undoubtedly possible to excite these fibres by electroles clirectly applied to the motor cortex, but in the latter case the current lias to be made stronger than is sufficient to excite the grey matter alone. Further eviclence is afforded by the following facts: ( $\alpha$ ) The 'period of delay' - that is, the period which clapses between stimulation and contraction -is greater by nearly 50 per cent. when the cortex is stimulated than when the white fibres are directly cxcited. (b) Morphine greatly increases the period of delay for stimulation of the cortex, and at the same time renders the resulting contractions more prolongel than normal, while the results of direct stimulation of the white fibres are much less, if at all, affecterl. (c) Stimulation of the grey matter, when separated from the subjacent white matter by the knife, but left in position, is without effect unless the strength of stimulus be increased, although twigs of the current ought, of course, to pass into the corona racliata as casily as before. Perfectly definite movements can, however, be excited or inluibited by stimulating definite spots in the corona raliata, and even in the internal capsule. This simply means that in these positions the fibres representing these movements are not yet intermingled with fibres representing other movements.

Sensory Areas-Visual Centres.-In the occipital lobe in animals an area of considerable extent has been found, destruction of which causes hemianopia (p. SIg). Thus, if the right occipital cortex is destroyed, the right halves of the two retina are paralyzed, and the left half of the field of vision is a blank. There is conjugate deviation of the liead and eyes to the same side as the lesion - in other words, the animal turns its head and eyes to the right. Destruction of this region on both sides causes complete blindness. When the same region is stimulated, the 
eyes and head are turned to the left-that is, there is conjugate deviation to the opposite side. In the higher monkeys the eyo movements can be elicited only from the extreme posterior apex of the occipital lobe and from its calcarine region, and then not easily. The movements differ from those produced by stimulation of the area for eye movements in the frontal lobe. They are not so certain, their latent period is longer, and a stronger stimulus is required to eroke them. It camnot be doubted that the occipital region is concerned in vision, and it is a very natural suggestion that the movements are the result of visual sensations in the excited occipital cortex. The right occipital lobe is concerned with vision in the right halves of the two retina (Figs. $3+2$ and 363). Now, under normal conditions, a visual image would be cast on the two right retinal halves by an object placed towards the left of the field. The movements of the head and eyes to the left may therefore be plausibly explained as an attempt to look at, and a rotation towards, the supposed object.

The pathological evidence is very clear that disease of the occipital lobe, especially of the cuneus, a triangular area on its mesial surface, causes hemianopia in man. A limited lesion may even be associated with an incomplete hemianopia, and cases have been recorded in which colour hemianopia (blindness of the corresponding halves of the two retinæ for coloured objects) co-existed with normal vision for white light. The precise limits of the occipital visual area are still clisputed. It probably occupies, in addition to the cuneus, the lingual lobule and a portion of the external aspect of the occipital lobe. The question of the projection of the retina upon the risual cortex-i.e., the question whether each retinal area is represented in a definite cortical area-has given rise to much debate. The representation of the fovea centralis, the area of most distinct vision, has aroused especial interest. It has been asserted that a circumscribed area in the region of the calcarine fissure is the centre for the forea (Henschen). But it is totally opposed to this view that extensive lesions of the occipital cortex, even on both sides, do not. except in rare cases, cause total blindness in the foreal region, although peripheral vision is destroyed. On the other hand, in no case has a purely cortical lesion been found associated with blindness confined to the fovea (Monakow). The fibres of the optic radiation which are on the path from the fovea are accordingly distributed liffusely to the risual cortex. Sometimes dimness of vision in the whole of the opposite eye (crossed amblyopia), and not hemianopia, is caused by a lesion of the occipital cortex. It seems impossible to explain this and other facts without postulating the existence of more tlian one visual centre ; and it has been supposed that in the angular gyrus and the neighbouring region a higher visual centre exists which is connected with the lower occipital centres for the two halves of the opposite eye. Thus, the right angular gyrus would be in connection with the part of the right occipital cortex which has to do with vision in the nasal half of the left eye, and with the part of the left occipital cortex which has to do with vision in the temporal half of that eye. This higlier centre, which perhaps functions as a storehouse of visual memories, probably corresponds 
to the structurally differentiated area (visuo-psychic area of Campbell), as the lower centre corresponds to his structurally differentiated visuo-sensory area (Figs. 359, 360).

Auditory Centre.-On the outer surface of the temporosphenoidal Jobe, mainly in the first temporal convolution, lies an area associated with the sense of hearing. Stimulation in the region of the first temporal convolution may cause the animal to
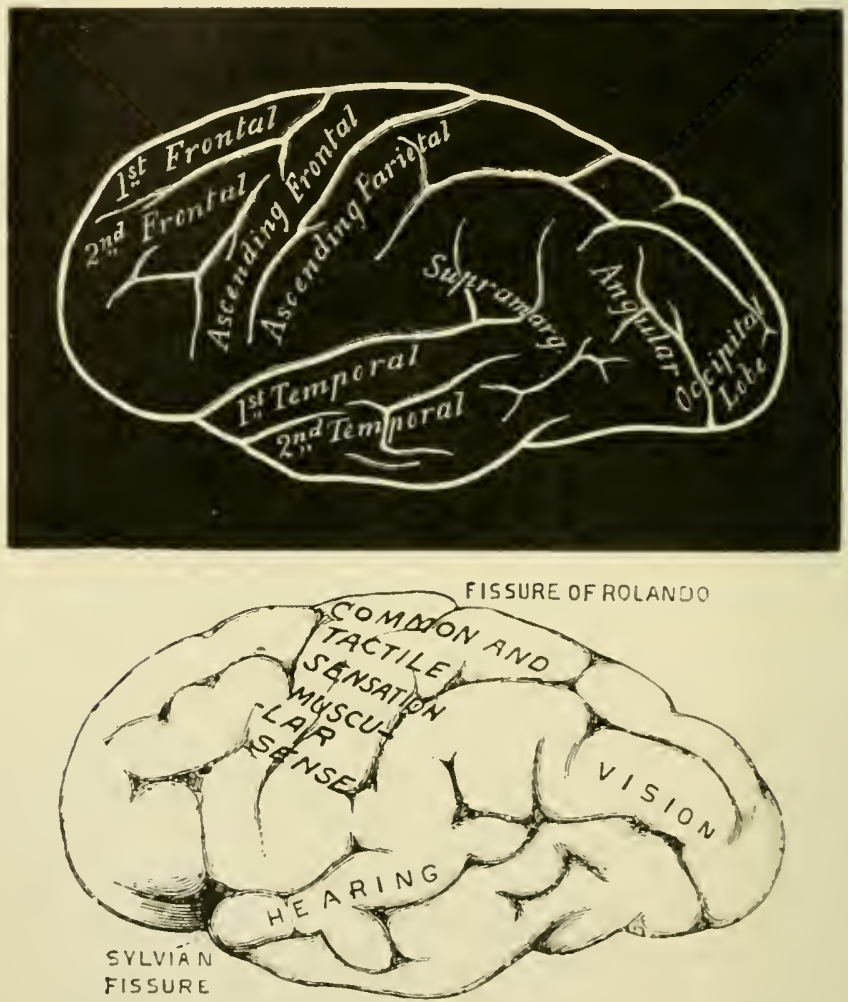

Fig. 364.-Lateral View of Left Hrmispinere witil Sensory Areas : Man.

The front of the brain is tuwards the left.

prick up its ear on the opposite side. Destruction of this area on botls sides is followed by complete and irremediable loss of liearing. If it is destroyed only on one side, there is partial deafness of the opposite ear, and also to some extent of the ear on the same side. This is gradually recovered from. If it is destroyed on the left side there is also the peculiar condition called 'word-deafness,' which will be referred to directly (p. S64). In deaf-mutes the first temporal convolution mat be atroplied. 
There is evidence that the posterior corpora puadrigemina and the mesial geniculate body form an inferior relay on the route between the fibres of the auditory nerve and the temporal cortex. There are indications that within the auditory area so-called 'musical centres' exist - that is, an orderly' arrangement of the cell-bodies of the neurons that fiave to do with the perception of pitch, so that a limited lesion may causc deafness to notes of a particular pitch when it is situated on one part of the area, and deafness to notes of a different pitch when it is situated elsewhere (Larionow).

Centre for Smell.- As to the position of the centre for smell, direct experiment on animals cannot teach us much, for if the outward tokens of visual and auditory sensations are dubious and fluctuating, still more is this the case with the signs of sensations of smell. A further source of fallacy is the fact that

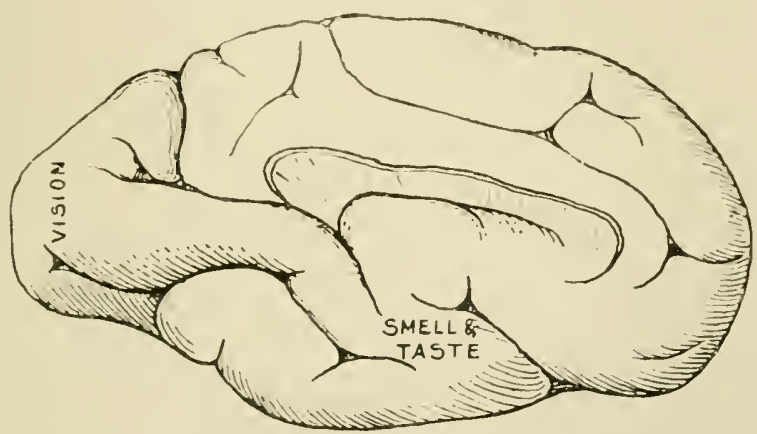

Fig. 365.-Sexsory Areas of Mesial surface of Human Brain.

The front of the brain is towards the right.

other sensations than those of smell are cansed by stimulation of the mucous membrane of the nose. Substances like ammonia, for example, affect entirely the endings of the trigeminus, which is the nerve of common sensation for the nostrils. Pathological and clinical eridence would be of great value, but it is as yet scanty, and of itself indecisive. Some cases of epilepsy liave been reported in which the attack was heralded by smells for which there was no objective cause. At autopsy the uncinate gyrus was found diseased. So far as it goes, such eridence supports the view derived from the anatomical connections of the olfactory tracts, that the centre for smell is situated in the uncinate gyrus on the mesial aspect of the temporal lobe, for the olfactory tract may be traced into this region. In animals with a very acute sense of smell, this gyrus is magnified into a 
veritable lobe, called from its shape the pyrilom lobe; from its supposed function, the rhinencephalon. The centre for taste is supposed to be situated in the same region as the centre for smell (in the hippocampal convolution posterior to the uncinate gyrus).

Ordinary and Tactile Sensations, including the muscular sense, have been located in the Rolandic area (p. 856); and there are sood srounds for believing that afferent fibres from the joints, the muscles and their accessory structures and the skin terminate here in arborizations which come into contact either with the motor pyramidal cells, or with intermediate cells which link tlem to the pyramidal cells.

Aphasia.- Mords arc, at bottom, arbitrary signs by which certain icleas are expressed. The power of intelligent communication by spoken or written language may be lost: (1) by paralysis of the muscles of articulation or the muscles which guide the pen ; (2) by inability to hear or sec the spoken or written word-i.e., by leafness or blindiness; (3) by inability to comprehend the meaning of spoken or written language, although sensations of hearing and sight may' not be abolished - that is to say, by inability to interpret the auditory or visual symbols by which icleas are conveyed ; $(4)$ by inibility to clothe ideas in words, although the words may be present in the paticnt's consciousness. and the ideas conveyed by spech or writing may be comprehended. Neither (I) nor (2) is considered to constitute the condition of aplasia ; (3) represents what is called amnesia, or sensory aphasia; $(f)$ is aphasia in the ordinary restricted sense, or motor aphasia.

Motor aphasia may be diviled into two varieties-subcortical or pure motor aphasia, and cortical, or Broca's aphasia. In the subcortical type the patient understands speech and writing perfectly. and is able to write normally; but he cannot speak spontancously or read aloud, or repeat words when requested to do so. He may know quite well what to reply in answer to a guestion. but the words necessary to express his meaning do not come to him. In Broca's type of aphasia, which is the most common form, the patient may understand spolien and written worls - often imperfectly, it is true-but he is unable to speak spontancously, to repeat worls spoken to him. and to read aloud. Lnlike the subcortical type of motor aphasic, he has difficulty in reading by the eye without articulation, and in writing spontaneously or to dictation. There is often or always a certain amount of intellectual deficiency. The gradations in the loss of the cxpressive factor in spech may bc infinite. A paticnt may sometimes sing a song without a single slip in works or measure, and yet be unable to speak or write it. In a casc recorled by Larionow an aphasic could speak only onc syllable, 'tam,' but could sing the 'Marscillaisc.' In certain cases the cliange is confined to loss of the power of spontaneons specelt, and the patient may be able to read intelligently. Sometimes he can cxpress his ideas in speech, but not in writing (agraphia). Sometimes the loss is restricted to certain sets of ideas. For example, a boy was injured by falling on his head. Typical symptoms of motor aphasia developed, but the power of dealing with ideas of number was not interfered with, and the boy continued to learn arithmetic as if nothing harl happened. Proper names and nouns are more 
casily lost thin adjectives and verbs. Motor aphasia is generally accompanicel by paralysis, frequently transicnt, of volumtary movement on the right side, sometimes amount ing to conplete hemiplegia, but more often involving the right arm alone. This association is generally cxplained by the proximity of the inferior frontal convolution to the motor area of the arm, and their common blood-supply. It has alreatly been stated that since Broca it has been generally assumed that in most persons the inferior frontal convolution on the left sicle is concerned in the expression of ideas in spoken or written language. It is even saicl that oratorical powers have been found associated with marked development of this convolution (as in the case of Cambetta, the French statesman). It is the cortical or Broca's type of motor aphasia which has been supposed to be associaterl with a lesion in the left inferior frontal convolution. The portion of the convolution concerned is the posterior extremity, where it borders on the fissure of Sylvius, and it either completely coincides with or largely overlaps the centre for the movements of the tongte, lips, and larymx concerned in articulation. The failure, lowever, does not lic in the articulatory mechanism. The patient uses the same muscles of articulation, without any marked impairment of function. for chewing and swallowing his food. It is only when the corresponcling area in the right inferior frontal convolution, or the path from it to the internal capsule, is also destroyed, that articulation is greatly and permanently interfered with.

The question obviously presents itself why it is that motor aphasia is commonly due to a lesion in the left hemisphere alone. The answer to this question is supposed to be partly supplied by the important and curious observation that in left-handed individuals lamage to the right inferior frontal convolution may cause aphasia. In the right-handed man the motor areas of the left hemisphere may be supposed to be more highly educated than those of the right hemispherc. The movements of the right side which they initiate or control are stronger and more delicate and precise than those of the left side. It is only necessary to assume that this process of specialization, of selective training, has been carried on to a still greater extent in the left frontal convolution, that in most men the speech-centre there has taken upon itself the whole, or the greater part, of the labour of clothing ideas in words, leaving to the right centre only its primitive but undeveloped powers. In left-handecl persons the speech-centre on the right side may be supposed to share in the gencral functional development of the right hemisphere. That great capabilities are lying dormant in the right speech-centre of the ordinary right-handed individual is indicated by the fact that after complete destruction of the left inferior frontal convolution the power of speech may be to a considerable extent, though slowly and laboriously, regained; and it is said that this second accumulation may be swept away, and without remedy, by a second lesion in the right inferior frontal convolution. But frail is the tenure of life in a person who has twice suffered from such a lesion; and we do not know whether recovery might not take place to somc extent even after destruction of both inferior frontal convolutions, if the patient only lived long enough.

Recently Marie has reopened the whole question of the relation of aphasia to lesions of the inferior frontal convolution. He believes that the so-called Broca's area has nothing to do with aphasia in the proper sense of the term-i.e., it is not a cortical area concerned 
in 'internal' specels processcs, or in which motor or kinesthetic 'specch memories' are stored-but simply a 'motor' area for the movements of articulation. IIe maintains that there is but one form of truc aphasia - the apliasia of Wernicke-which has for its basis a lesion of the so-called zone of Wernicke (the supramarginal and angular gyri, and the posterior portions of the first and second temporal convolutions). Tlis, according to him, is the true speechcentre. The symptom-complex known as Broca's aphasia, which cveryborly arlmits (o) exist as a distinctly characterizerl clinical conclition. is due. lie says, to it double lesion. ()ne lesion canses aphemia (loss of the power of co-r)rlinating the inwements neerlerl in the articulation of worls without actual paralysis of the muscles), and the other the disturbance of internal speech, and the difficulty of reading and of writing, which constitute the true aphasia. According to . Narie. the lesion which causes the aphemia is not even situated in Broca's convolution, but somewhere in a rather badly defined region, which he renominates the lenticular zone, since it inclurles the lenticular as well as the caudate nucleus, in addition to the cxternal and internal capsules and the cortex of the island of Reil. It would be out of place to enter more minutely here upon sucl controversial matters. The conclusion which emerges most definitely from the discussion is that Broca's localization was based upon a very narrow foundation, and must probably be modified.

A so-called temporary aphasia may occur without any structural change in the speech-centre-for example, luring an attack of migraine. In children it may even be caused by some comparatively slight irritation in the digestive tract, such as that due to the presence of a tape-worm.

In the anthropoid apes no evidence of the existence of any ' speechcentre,' even distantly foreshadowing the human, has been obtained by stimulating the inferior frontal convolution on either side. No movements, and particularly no movements connected with vocalization, are elicited.

Sensory Aphasia.-In typical motor aphasia spoken and written words convey to the patient their ordinary meaning. They call up in his mind the usual sequence of ideas, but the chain is broken at the spech-centre, and the outgoing idcas cannot be clothed in words. The expressive factor in specch is deranged. In sensory a phasia the perceptive factor in specch is deranged. In ordinary sensory aphasia (IVernicke's, or cortical sensory aphasia) the patient cannot understand spoken or written language. but, far from being unable to speak, he often babbles incessantly. He may string together a series of words, each correctly articulated, but having no meaning. or may utter a jargon not composerl of known worls at all. Instead of the words whicls he desires to use to express his meaning. he may use others having a similar somel (paraphasia). Damage to two regions of the left licmisphere of the Lrain lias been found associaterl with this strange condition: (1) the upper portion of the temporo-sphenoidal lobe, (2) the angular gyrus and the occipital lobe. Ithen the temporal region is alone affected, it is the spoken word that is missed, the written that is understood (word-deafness). When, as occasionally happens. the lesion is confined to the occipital region, spoken language is perfectly understood, written language not at all (word-blindness). Sensory, like motor aphasia, may exist in any degrec of completeness, from absolute word-deafness or wordblindness, in which no spoken or printed word calls up any mental 
image. to a condition not amounting to much more than a marked absence of mind or unusual obtuseness. Motor and sensory aphasia may be present together. In well-marked cortical word-cleafness speech is always interfered with to some extent. In so-called pure word-deafness (subcortical sensory aphasia) the patient may be perfectly capable of rational speech. He may talk to himself or on a set topic with fluency and sense, may write intelligently, and understand what he reads; but he may be unable to understand a single word spoken to him, or to repeat words when asked to do so.

Cortical Epilepsy.-While it was still believed that the cortex was inexcitable, epilepsy was supposed to be exclusively due to morbid conditions, structural or functional, of the medulla oblongata (Kussmaul and Tenner). Some more recent writers have put forward precisely the opposite opinion, that the discase is always cortical in origin (Unverricht, etc.). What we know for certain is that some cases of epilepsy, but only a minority, are associated with cortical lesions. Among these are the cases of so-called Jacksonian epilepsy-a condition characterized by the fact that the scizure does not begin by general, but by local, convulsions. They inay remain confined to a single limb, or to one side of the face, or to one side of the body. So long as the convulsions are not general, consciousness need not be lost. Or a seizure beginning as Jacksonian may spread so as to involve the whole body, in which case the symptoms become identical with those of ordinary epilepsy, including the loss of consciousness. It has been found possible in some cases to localize the position of the lesion from the part of the body in which the fit, or the aura (the sensation or group of sensations peculiar to each case, which precedes and announces the attack) begins. For example, if the convulsions commence with a twitching of the right thumb and extend over the arm, or if the aura consists of sensations beginning in the thumb, there is a strong presumption that the seat of the lesion is the part of the arm-area known as the 'thumb-centre' in the left cerebral hemisphere. It is the seat of the convulsion at its commencement, not the regions to which it may afterwards spread, that is important in diagnosing the position of the lesion. For just as strong or long-continued electrical stimulation of a given 'centre' of the 'motor' cortex may give rise to contractions of muscles associated with other 'centres,' so the excitation set up by localized disease may spread far and wide from its original focus, involving area after area of the "motor' region first in the one hemisphere and then in the other. The part of the body to which a sensory aura is referred is as significant an indication of the seat of the discharging lesion as is the part of the body which first begins to twitch. This is one of the proofs that the 'motor' region is not a purely motor area.

Seat of Intellectual Processes.-When we have deducted from the cortex of the hemisphere the whole Rolandic region and the sensory centres, there still remains a large territory unaccounted for. Considerable portions of the occipital, parietal, and temporal lobes, nearly the whole of the island of Reil and the greater part of the frontal lobe anterior to the ascending frontal convolution are ' silent areas,' and respond to stimulation by neither motor nor sensory sign. They correspond to the association centres previously referred to. By a process of 
exclusion it las been supposed that, in addition to, om partly in virtue of their associative function, they are the seat of intellectual and psychical operations. The intellectual function has been more particularly assigned to the frontal lobes, and witl great probability, although we have little real knowledge to gunde us to a decision. Extensive destruction and loss of substance of the pre-frontal region may sometimes occur without any marked symptoms. But usually there is restriction of mental power or it may be loss of moral restraint. Thus in lle famous 'American crowbar case,' an iron bar completely transfixed the left frontal lobe of a man engaged in blasting. Although stumned for the moment, he was able in an hour to climb a long flight of stairs, and to answer the inquiries of the surgeon. Finally, he recovered, and lived for nearly thirteen years without either sensory or motor deficiency, except that he suflered occasionally from epileptic convulsions. But his intellect was impaired; le became fitful and vacillating, profane in his language and inefficient in his work, altlough previously decent in conversation and a diligent and capable workman.

lilechsig supposes that his great anterior association contre in the frontal lobe is concerned in the retention of the memory of all conscious bodily experiences, especially those connected with voluntary acts. The great posterior association centre he imagines to be engaged in the formation and collection of ideas of external objects and of the 'word pictures' which represent them, and with the preparation of specen in respect of the thoughts to be expressed and the form of expression, the office of the Broca's area (but see p. 804 ) being to exccute the mechanical part of the process by transforming these thoughts into actual spoken words. This posterior association centre may be looked upon as the seat of intellect in the narrower sense, as the anterior is of will and feeling.

The experiments of Franz on the relation of the cerebral association areas, and especially the frontal area, to certain acquired habits are of interest. Cats were allowed to acpuire certain habits involving simple mental processes, and then it was seen how these were aftected by cortical lesions. After bilateral extirpation of the frontal lobes (the area anterior to the crucial sulcus) newly-formed, but not long-standing, habits are lost. This camnot be due to shock, since other brain lesions are not followed by loss of the habits. Extirpation of onc frontal area usually causes a partial loss of newly-acquired habits, or, rather, a slowing of the association process icading to unusual delay in the execution of the movements connected witl the habit. Ifabits once lost after removal of the frontals may be relcarned.

The influence of psychical events upon bodily functions is well linown, and has becn more than once illustrated in preceding pages. The converse question of the influence of bodily states upon psychical events has also been raised, especially in connection with the genesis of emotions. Some psychologists assume that the bodily changes associated with such cinotions as grief, fcar, rage, or love, are not evoked as a consequence of the emotinns, but that the bodily changes follow directly the perception of the exciting fact-e.g., a spectacle 
which canses fear or rage, "and that our feeling of the same changes as they occur is the emotion' (Jimes). Sherrington, however, has shown that in dogs in which, hy trinsection of the vagi and the spinal cord, all sensation of viscera, skin, and muscles belind the level of the shoulder was eliminated, no obvious emotional defect was caused. Notwithstanding the immense abridgment of the field of sensation, anger, joy, fear, disgust (as on being offered /log's flesl, which most (logs refuse to eat), were as nuthed as ever, and were cyoked by the same objects as before the operition. When the afferent field is still more restricted, as in the head of a dog grafted on the circulation of another dog by anastomosis of the blook vessels, with precatutions to avoid intermption of the bloof-flow, not only does the respiratory centre continue to discliarge itself with a regular rhythm, but cortical volitional movements persist (Cutluric, Pike and Stewart), and, so far as can be jutged, sense perception, cmotional, and even intellectual, processes continue. In one case the picture presented by the engrafted head was essentially the same as that presented by the liead of the 'host' for over two hours. In a transplanted hear from a younger dog in which the circulation had been internupted for twenty-nine minutes, a renalrable return of cerebral function was observed (Cuthrie).

Localization of Function in the Central Nervous System. -Let us now consider a little more closely the real meaning of this localization of function. Scattered all over the grey matter of the primitive neural axis, and, as we have seen, wer the grey mantle of the brain as well, are numerous ' centres' which seem to be related in a special way to special mechanisms, sensory, secretory, or motor. The question may fitly be asked whether those centres are really distinct from each other in quality of structure or action, or whether they owe their peculiar properties solely to differences in situation and anatomical connection. It is clear at the outset that the nature of the work in which a centre is cngaged must be largely determined by its connections. The kind of activity which goes on in the vaso-motor centre in the bulb, for example, may in no essential respect differ from that which goes on in the respiratory centre. The calibre of the bloodvessels will alter in response to a change of activity in the one because it is anatomically connected with the muscular coat of the bloodvessels. The rate or depth of the respiratory movements will alter in response to a cliange of activity in the other because it is connected with muscles which can act upon the cliest-walls.

Recent experiments afford a very interesting illustration of the determining influence of their peripheral connections on the function of nerve-fibres. It has, in fact, been shown that the central end of any efferent sonatic fibre-i.e., any fibre running from the central nervous system and ending in striated muscle-can make functional connection with the peripheral end of any other efferent fibre of the same class, whaterer be the normal actions produced by the two fibres. Advantage has 
been taken of this in surgery. For instance, in a case of severe facial (motor) tic the facial nerve was divided and its peripheral end united with a portion of the fibres of the spinal accessory. The voluntary movements of the face, after regeneration lad occurred, were normally carried out througl impulses descending the spinal accessory. In cases of local paralysis, due to destruction of anterins horn-cells (anterior poliomyelitis), restoration of movement has also been obtained by connecting the motor nerve of the paralyzed inuscles to a portion of a nerve coming off from an uninjured region of the cord.

The central end of any efferent somatic fibre can also make functional union with the peripheral end of any of the efferent fibres which run from the central nervous system and end in ganglion cells (pre-ganglionic fibres), and the central end of any pre-ganglionic fibre can do the same with the periplieral end of any efferent somatic fibre (Langley and Anderson). For instance, Langley divided (in cats) the vagus nerve and the cervical sympathetic. The peripheral end of the former degenerated, of course, below the section, and the peripheral (cephalic) end of the latter degenerated above the section, up to the terminations of its axons in the superior cervical ganglion. The central end of the cut vagus was subsequently sutured to the peripheral end of the cut sympathetic. After a time the vagus-fibres grew along the course of tlie degenerated sympathetic up to the ganglion, where some of them formed arborizations around the ganglion cells. It was now found that stimulation of the vagus produced the effects usually caused by stimulation of the cervical sympathetic-for example, dilatation of the pupil and constriction of the bloodvessels of the head and neck. From these experiments it follows that the functions of the various groups of fibres in the cervical sympathetic do not depend on anytling peculiar to the fibres; any fibre which can make connection with one of the ganglion-cells tliat send axoms to the dilator muscle of the iris will, when stimulated, act as a pupillo-dilator fibre, just as well as a cervical sympathetic fibre. Other instances of the same law have already heen given in connection with the regeneration of nerves $(p ., o(f))$.

Functional union does not take place between efferent somatic fibres (or pre-ganglionic fibres) and post-ganglionic fibres-i.e., fibres arising in peripheral ganglia, and ending in smootl muscle and glandular tissue; e.g., the cervical sympathetic after excision of the superior cervical ganglion does not unite with the fibres leaving the anterior end of the ganglion in sucl a way that stimulation of it can produce any of the effects normally produced through these fibres. No proof has been given that afferent fibres can unite with efferent fibres or efferent with afferent 
Afferent fibres of one nerve can unite with afferent fibres of another nerve, but there is not sufficient evidence to show whether fibres concerned in one sensation can unite with fibres concerned in another.

The localization of function in the ccrebral cortex has been likened to the localization of industries in the multiplex commercial life of the molern world. The barbarian houschold in which cloth is woven and worked into garments; sandals, or moccasins cobbled together; rough pottery baked in the kitchen fire, and all the rude furniture of the lodge fashioned by the hands which built it, and which rest beneatll its roof at night-this state of things wherc centralization lias not yet begun, it has been sairl, is a picture of what goes on in the undeveloped brains of the frog, the pigeon. and the rabbit. The "diffusion' of indlustries which is characteristic of a primitive state has given place among the most highly civilized men to extreme centralization and concentration. Manchester spins cotton and Liverpool ships it. Chicago handles wheat and pork that have been produced on the prairies of Minnesota and Illinois. Amsterdam cuts diamonds. IIunich brews beer. Lyons weaves silk. New York and London are centres of finance. This, it is said, is the picture of the highly specialized brain of a monkey or a man. But ingenious and alluring though such analogies are, they do not rest upon a sufficient basis of fact. Indeed, the more deeply the structure and function of the central nervous system are studiecl, the more clearly does its essential solidarity appear, the more clearly does it emerge as an organized co-ordinated system, not an aggregate of separate mechanisms jumbled together for convenience of storage in the protected cranio-spinal cavity.

It has never been shown-nor is it likely that the proof will soon be forthcoming-that there is any difference whatever in the physical, chemical, or psychical processes which go on in the various centres of the "motor' cortex. It may be supposed, indeed, that the so-called sensory areas of the cortex differ more widely in their internal activity from the 'motor' areas than the latter do among themselves, and that the activity of the anterior portion of the brain, the portion which has been crellited par excellence with psychical functions, differs in kind, not merely in degree, from that of all the rest. But, as we have just seen, even the 'motor' areas have sensory functions. A cast-iron physiology may explain this by the assumption of 'sensory' as well as 'notor' cells in the Rolandic area, and may find support for such an assumption in the well-known fact that the large pyramidal cells whose axons form the pyramidal tract make up but a small proportion of the total number of pyramidal cells in this region, which, besides, contains numerous cells of Golgi's second type (p. $75 \mathrm{f}$ ). Yet there is absolutely nothing to contradict the supposition that the discharge of energy from the most circumscribed motor area or element may be accompanied not only with consciousness, but with a high degree of psychical activity. And, incleed, some writers liave supposed that such a consciousness of, or:even conscious measurement of, the discharge from the ' motor' areas is the basis of the muscular sense (Bain, Wundt).

So far, at least, as the 'motor' region and the grey matter immediately around the neural canal are concerned, the analogy of an electrical switch-board connected with machines of various kinds might be more correct. Touch one key or another, and an engine 
is set in motion to grind corn, or to saw wood, or to light a town. The clifference in result lies not in any difterence of material or workmanship in tlic switches, but solely in the difference in their connections.

Grey matter in the upper part of the precentral convolution is excited, and the muscles of the leg contract. Grey matter on the lower part of the convolution is excited, and there are movements of the face and moutl. Grey matter in the medulla oblongata is excited, and the salivary glands pour forth a thin, watery fluid, poor in proteins, and containing an amylolytic ferment. Another portion of grey (?) matter in the medulla is thrown into activity, and the pancreatic ducts become flushed with a thiclicr secretion, relatively rich in proteins and in ferments which act on proteins, starch, ancl fat. Here, too, there is a varicty in result according as one or another nervous switch is closed ; here, too, the variety is due, not to essential differences in the structure or the activity of the nervous centres, but to their connection, by nervous paths, with peripheral organs of clifferent kinds. There is, indecd, a specialization, a localization, of function, but the localization is at the periphery, the specialization is in the peripheral organs.

It may be asked whether, if this is the case for the peripheral organs of efferent nerves, the converse does not hold true for the afferent nerves-in other words, whether the localization here is not at the centre. And that there is in some degree a central localization of sensation may be considered proved by the wellknown clinical fact, already referred to, that sensations of various kinds may be produced by pathological changes in the cortex. For example, a tumour involving the upper part of the temporal lobe may give rise to epileptiform convulsions preceded by an auditory aura, a sound, it may be, resembling the ringing of bells; a tumour involving the occipital region may canse a visual aura, and so on. Central sensory localization is the fundamental idea of the old doctrine of the specific energy of nerves, which, in modern phraseology, expresses the fact that excitation of the central end of a sensory nerve by various kinds of stimuli causes always-or at least very often-the particular lind of sensation appropriate to the nerve. The observation so frequently made in surgery before the lays of ancesthetics, that when the optic nerve was cut in removing the cyeball the patient experienced the sensation of a flash of light,* was long looked upon as the strongest prop of the law of specific energy, and well illustrates the meaning of the term. Here a mechanical excitation of the optic fibres in their conrse gives rise to the same sensation as excitation of the retina by the natural or homologous or adequate stimulus of light. Since a similar mechanical stimulus applied to the aulitory nerve gives rise to a sensation of sound, and, applied to the trigeminal nerve, to a sensation of pain, many physiologists have assumed that the impulses set up in the auditory nerve when sound impinges on the tympanic membrane do not liffer essentially from those set up in the optic nerve when a ray of light falls upon the retina. or from those set up in the fifth nerve by the irritation of a carious looth, or from those set up in certain fibres of the cutancous nerves when a warm body comes in contact with the skin. Since the results in consciousness are very different, this assumption lias necessitated the further conclusion that somer-here or other in the central nervous system there exist

* It is said that this is not always the case. 
organs that are differently affected by the same kinds of afferent impulses-in other worls, that sensory localization is at the centre. On this view, the visual arcas in the cortex respond to all kinds of stinuli by visual sensations; the auditory areas by sensations of sound, and so on.

But while it camont be loubterl that special sensory regions exist in the grey matter of the brain, where the afferent paths concerned in the different kincls of sensation end, there is no reason to suppose that the nerve-impulses which travel up the various paths are absolutely similar until they have reached the centres, and there suddenly become, or produce, sensations absolutely different. There is, indeed, evidence of a certain amount of sensory specialization at the periphery. For example, when an orlinary nerve-trunk is touchel, the resultant sensation is not one of touch. If there is any sensation at all, it is one of pain. Heating or cooling a naked ncrvetrunk gives rise to no sensations of temperature. When the ulnar nerve is artificially cooled at the elbow, the first effect is severe pain in the parts of the hand supplied by the nerve. The pain disappears somewhat abruptly as cooling goes on, and is succeeded by gradual loss of all sensation in the uInar area of the hand; but the cooling of the nerve-trunk does not give rise to any sensation of cold (Weir Mitchell). Stimulation of the receptors or encl-organs is normally essential in order that scnsations of touch and temperature should be experienced (but see p. 980). Although, as previously stated, one great function of the receptor is to lower the threshold of the adequate stimulus, and thus to render the afferent newon more easily excited by an adequate stimulus than by any other, it may also serve to impress a particular rhythm or other character upon the nerve impulse, so that the afferent impulses may be to some extent differentiated before they reach their centres. One reason, then, why excitation of the temporal cortex by impulses falling into it along the auditory nervefibres causes a sensation different from that caused by impulses reaching the occipital cortex through the fibres of the optic nerve may be a difference in the nature of the impulses. If this were the only reason, it would follow that were it possible to pliysiologically connect the fibres of the optic radiation with the temporal cortex, and those of the temporal radiation with the occipital cortex, sights and sounds would still be perceived and discriminated in a normal manner, although now the integrity of the occipital lobe would be bound up with the perception of sound, the integrity of the temporal lobe with visual sensation. This state of affairs would correspond to complete specialization for sensation in the peripheral organs, complete absence of specialization in the centres. On the other hand, it is conceivable that, after such an ideal experiment. sound-waves falling on the auditory apparatus might cause visual sensations, and luminous impressions falling on the retina sensations of sound. This would correspond to complete specialization of sensation in the centres, complete absence of specialization at the periphery. A third possibility would be that the 'transposed' centres, responding at first feebly or not at all to the new impulses, might, by slow degrees. become more and more excitable to them. This would correspond to a peripheral specialization, combined with a tendency to development of central specialization. And, incleed, it is not easy to conceive in what way, except as the result of differences in the nature of impulses coming from the periphery. specialization of sensory areas in the central nervous system could have at first arisen. 
Degree of Localization in Different Animals. - Before leaving this subject, two points ought to be made clear: (I) The degree of localization of function in the cortex goes hand in hand with the general development of the brain. In man and the monkey, the motor localization is more elaborate than in the dog-that is to say, a greater number of movements can be associated with definite cortical areas. In the rabbit, whose 'motor' centres have been particularly studied in recent years by Mann and Mills, localization is still less advanced than in the dog. Towards the bottom of the mammalian group certain 'motor' areas can still be demonstrated, though they are rather ill-defined, for instance in the hedgehog (Mann), opossum (Cunningham), and ornithorhynchus (Martin). In general the movements of the anterior limb are easier to obtain than those of the posterior. In birds Mills found no evidence of the existence of any' 'motor' centres.

(2) Areas of the same name (homologous areas) in different groups of animals do not necessarily have the same functionthat is, in the case of the 'motor' areas, are not necessarily associated with the same morements. Taking the position of the centre for the orbicularis oculi as a test, Ziehen has come to the conclusion that in the anthropoid apes and in man, this centre has been pushed forward by the encroachment of the centres behind it, and especially of the visual centre, the arm centre, and the speech centre, which have undergone a great functional development.

Reaction Time.-Just as in a reflex act a certain measureable time (reflex time) is taken up by the changes that occur in the lower nervous centres, so we may assume that in all psrchical processes the element of time is inrolved. And, indeed, when the interval that elapses between the application of a stimulus and the signal which announces that it has been felt (reaction time) is measured, it is found that for the cerebral processes associated with the perception of the simplest sensation and the prodaction of the simplest roluntary contraction it is longer than the time which the spinal centres require for the elaboration of even complex and co-ordinated reflex movements. Suppose, e.g., that the stimulus is an induction shock applied to a given point of the skin, and that the signal is the closing of the circuit of an electro-magnet, then, if both events are automatically recorded on a revolving drum, the interval can be readily determined. It is evident that this includes, not only the time actually consumed in the central processes, but also the time required for the afferent impulse to reach the brain, and the efferent impulse the hand, along with the latent period of the muscles. The time taken up in these three events can be 
approximately calculaterl, and when it is subtracted, the remainder represents the reduced or corrected reaction timethat is, the interval actually spent in the centres themselves. This is by no means a constant. It is influenced not only by the degree of complexity of the psychical acts involved, and the mental attitude of the person (whether he expects the stimulus or is taken by surprise, whether he has to choose between several possible kinds of stimuli and respond to only one, etc.), but it varies also for different kinds of sensation, for the same sensation at different times, and as is recognised in the personal equation of astronomers, in different individuals. For sensations of touch and pain it may be taken as one-nintl to one-fifth, for hearing one-eighth to one-sixth, and for sight one-eighth to one-fifth of a second. So that the proverbial quickness of thought is br no means great, even in comparison with that of such a gross process as the contraction of a muscle (one-tenth of a second). Nor is it the case that the man 'of quick apprehension' has always a short reaction time, or the dullard alway's a long one, although in all kinds of persons practice will reduce it.

Sleep and Fatigue.-Certain gland-cells, certain muscular fibres, and the epithelial cells of ciliated membranes, never rest, and perhaps hardly ever even slacken their activity. But in most organs periods of action alternate at more or less frequent intervals with periods of relative repose. In all the higher animals the central nervous srstem enters once at least in the twenty-four hours into the condition of rest which we call sleep. What the cause of this regular periodicity is we do not know. It is accompanied by changes in the microscopical appearance of the nerve-cells. Thus, Hodge found differences between the cells of certain portions of the cerebral cortex in bircls, and of certain ganglia in the honey-bee after a long day of work and after a night's rest. Mann, Lugaro, and other observers, found similar differences in the cells of the cerebral cortex and the anterior horn, and Dolley in the Purkinje's cells of the cerebellum in dogs fatigued by muscular exercise as compared with rested dogs (Fig. 366).

According to Dolley, who has marle the most recent observations on this subject, there is, as a result of continued activity, at first a steady increase of the basic chromatic material. This increase affects first the extra-nuclear chromatin. the Nissl substance. which. according to the most morlern view, is really nuclear substance distributerl through the cytoplasm, and functions as such (Goldschmidt). The size and number of the granules are increased, and some of the chromatic material is diffused throughout the cytoplasm. as indicated by diffuse staining. Then the intranuclear chromatin also undergoes an increase, and the size of the cell is increased too. 
In mollerate activity the change goes no farther. At this stage the cell is hyperchromatic-i.e., as comparel with a normal resting cell it contains an excess of chromatin. The production of chromatin having reaclicel the maximum of which the nucleus is capable. and functional activity, which entails the using up of the extranuclear chromatin still continuing. the total chromatin content begins to (lmminish. furst in the nuclens, through the passage of its chromatin into the cytoplasm to recruit the Nissl substance, then in the cytoplasm as well. Accompanying the disappearance of the chromatic material there is diminution in the size of both cell

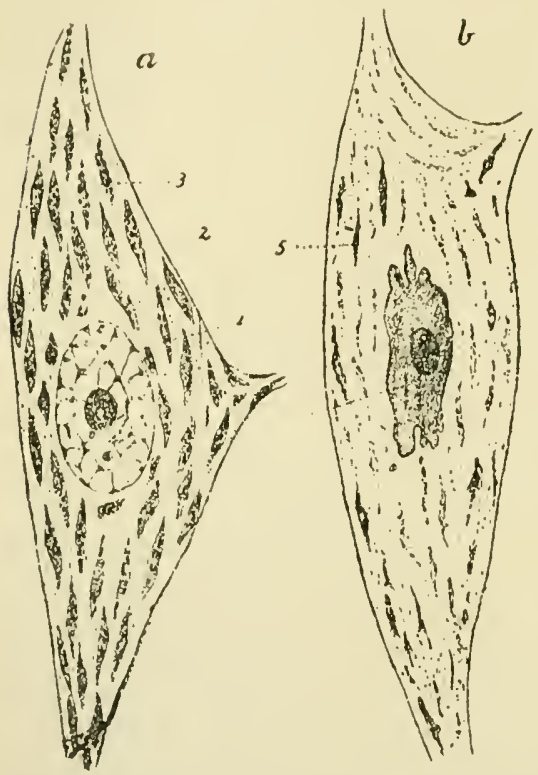

lici, 366.-EFHECT OF Fatigule ON NERVECLLLS (BARKFR, AFTER MANN).

Two inotor cells from lumbar cord of dog fixed in sublimate and stained with toluiclin blue. $a$, from rested $\log : \mathbf{r}$, pale uncleus: 2 , dark Nissl spinclles: 3 , bundles of nerve fibrils. $b$, from the fatigued $\log : 4$, dark shrivelled nurleus : 5 , pale spindles. and nucleus, but especially of the nucleus, so that the normal proportion between volume of cell and volume of nucleus (nucleus-plasma relation of Hertwig) is disturbel in favour of the cytoplasm. Both cell and nucleus become irregular in outline or crenatel. Later on, and, it would seem, rather ab. ruptly. swelling of the nucleus and, after some time, of the cytoplasm occurs. This is llue to eedema, and may be taken to inclicate an upset of their normal osmotic rela. tions. The earlier occurrence of oedema in the nucleus leads to another change in the mu. cleus-plasma relation, which is now disturbed in farour of the nucleus. In the measure in which fatigue progresses the extranuclear chromatic material continues to be used up, and, in spite of its replenishment from the nucleus, it almost or entirclyvanishes from the cytoplasm. Then follows what is perhaps a 'last effort' on the part of the nucleus to supply the cytoplasm, in the form of a discharge of chromatic substance, which first masses itself around the outside of the nuclear membrane, and thence grarlually liffuses into the cytoplasm. With the using up of this supply all the basic cliromatic material of the cell. except that in the karyosome (nucleolus), is exhausterl. linally, this too is yielded up to the cytoplasm, and with its consumption there remains a totally exhausted cell, devoid of basic cluromatin and incapable of recuperation.

Accorling to Pugnet, exen in extreme fatigue, as when llogs were caused to run forty to nearly sixty miles in a special apparatus. the changes varied greatly in legree in different cortical cells, from 
mere rliminution of the chromatic substance to complete disappearance of it and such disintegration of the cell as must lave precluded its recovery hat the aninial been allowed to live. Many, and indeel most, of the cortical cells were quite unaffected. Histological alterations may also be caused in sympathetic ganglion cells by prolonged artificial stimulation of the nerves connected with the ganglia. Experiments on fatigne changes in the cells of the spinal ganglia after electrical excitation of the posterior root-fibres are less decisive, some observers having obtained positive, others negative, results (p. Sog).

Theories of the Causation of Sleep.--(1) Some lawe suggested that slecp is induced by the nsing np of substances necessary for the functional activity of the neurons e.g.e the stored-up or intramolecular oxygen-or by the action of the waste products of the tissues, and especially lactic acis, when they accumulate beyond a certain amount in the blood, or in the nervous elements themselves.

(2) Others have looked for an explanation to vascular changes in the brain, but so far are the possible causes of such changes from being understood, that it is even yct a question whether in sleep tho brain is congested or anæmic. Certain writers have settled this question by the summary statement that when the brain rests the quantity of blood in it must be supposed to be dininished, as in other resting organs. But this is a fallacious argument. For when the whole body rests, as it does in slcep, it has as much blood in it as when it works; in sleep, therefore, if some resting organs have less blood than in waking life, other resting organs must have more ; and it is the province of experiment to decide which are congested and which anæmic. In ccma, a pathological condition which in some respects has analogies to profound and long-continued sleep, the brain is congested, and the proper elements of the nervous tissue presumably compressed. And artificial pressure (applied by means of a distensible bag introdnced through a trepline hole into the cranial cavity) may cause not only unconsciousness, but absolute anæsthesia. But it is possible that this artificial increase of intracranial pressure may produce its effects by rendering the brain anamic, and it has been actually observed that the retinal vesscls. as seen with the ophthalmoscope, and the ressels of the pia mater exposed to direct observation in man by discase of the bones of the skull, or in animals by operation, shrink lluring sleep. Statements to the contrary may be due to negleeting the influence of difference of position in the sleeping and waking states. In sleeping children the fontanelle sinks in, an indication that the intracranial pressure is reduced. Observations with the pletlysmograph have shown that the arm swells in sleep, and shrinlss when the slecper awakes. or even when he is subjected to sensory stimuli not sufficient to arouse him-e.g., a tune played by a musical-box (Howell). The tone of the vaso-motor eentre is therefore diminished. and the arterial pressure falls during sleep. But a fall of general arterial pressure is usually accompanied by a diminution of the quantity of blood passing through the brain. So that the balance of evidence is in favour of the view that sleep is associated with a certain degree of cerebral ancemia.

As to the nature of the relation between the two conditions, it has been suggested that the anæmia is produeed by fatigue of the vaso-motor centre, which causes it to relax its grip upon the peripheral bloodressels, and that the conclition of the cortical nerve- 
cells which we call slecp is directly produced by the lack of blood. But there does not appear to be any good reason for believing that the vaso-motor centre is more susceptible of fatigue than the higher cercbral centres. On the contrary, it is probable that the bulbar centres are less delicately organized and more resistant than the higher centres. In any case, if the cerebral nerve-cells 'go to sleep, because their blood-supply is diminished, ought we not to look for a similar cause for diminished activity of the vaso-motor centre? Or if the answer is made that the activity of the vaso-motor cells is directly lessened by fatigue, or by the cessation of external stimuli, why should not this be the case also for the cortical cells? It can be shown by means of the sphygmomanometer (p. ro6) that the fall of arterial pressure is not essentially connected with sleep, but is produced by the bodily rest and warmth which accompany it. Further, even a great dimimution in the supply of blook going to the brain is not necessarily followed by sleep. For example, both carotids and both vertebral arteries may frequently be tied in dogs at the same time without producing any symptoms, the anastomosis of the superior intercostal arteries with the anterior spinal artery providing a sufficient channel for the blood absolutely required by the brain. Monkeys after ligation of both carotids may be most alert and active. To produce sopor in animals the cortical circulation must be reduced almost to the vanishing-point, and to a far greater degree than ever occurs in sleep (Hill). We must, therefore, conclude that although sleep is normally associated with some ancemia of the brain, it is not directly caused by it. The cortical centres go to slecp because they are 'tired,' or because the stimuli which usually excite them have ceased, and not because their bloodsupply is diminished.

(3) The idea that the dendrites are contractile, and by pulling themselves in, and thus breaking certain nervous chains, cause slecp, is a mere theory, mnsupported by any real evidence. The same is true of the notion that the fibrils of the nemroglia insinuate themselves into the ' joints,' by which one nemron comes into contact with another, and acting as insulating material, block the nerveimpulses.

In general, the depth of sleep, as measured by the intensity of sound needed to awaken the slecper, increases rapilly in the first hour, falls abruptly in the sccond, and then slowly crecps (lown to its minimum, which it reaches just before the person awakens. As to the amount of slecp requirecl, no precise rules can be laid down. It varies with age, occupation, and perhaps climate. An infant, whose main business is to grow, spends, or ought to spenil, if mothers were wise and feeling-bottles clean, the greater part of its time in sleep. The man, whose main business it is to work with his hands or brain, repuires his full tale of eight hours' slcep, but not usually more. The dry and exhilarating air of some of the inland portions of North America, and perhaps the platins of Victoria and New South WVales, incites, and possibly enables a new-comer to live for a considerable period with less than his ordinary amount of slecp. Idiosyncrasy, and perhaps to a still greater extent habit, liave also a marked influence. The great Napoleon, in his heyclay, never slept more than four or five hours in the twenty-four. Five or six hours or less was the usual allowance of Frederick of Prussia throughout the greater part of his long and active life.

Hypnosis is a condition in some respects allied to natural slumber ; 
but insteibl of the atceivity of the whole bratu or perhitps we should rather say, the whole alcivity of the brain-being in abeyance, the susceptibility to external impressions remains as great ats in waking life, or may be even increased. While the critical faculty, which normally sits in judgnent on them, is lulled to sleep. The condition can be indlucel in many ways-by asking the subject to look fixedly at a bright object, by closing his eyes, by occupying his attention. by a sudelen loud souncl or a flash of light, etc. The essential condition is that the person should have the idea of going to slecp, and that he should surrender his will to the operator. In the hypnotic condition the subject is extremely open to suggestions made by the operator with whom he is en rapport. He adopts and acts upon them without criticism, If, for cxample, the hypnotizer raises the subject's arm above his liead, and suggests that he camnot bring it down again, it stays fixed in that position for a long time without any appearance of fatigue; or the whole borly may be thrown, on a mere hint, into sone unnatural pose, in which it remains rigicl as a statue. Suggested hemiplegia or hemianasthesia, or paralysis of motion and sensation together or apart in limited areas, can also be realized; and surgical operations have been actually performed on hypnotized persons without any appearance of suffering. If, on the other hand, the operator suggests that the subject is undergoing intense pain. he will instantly take his cue, writhing his body. pressing his hands upon his head or breast. and in all respects behaving as if the suggestion were in accord with the facts. If he is told that he is blind or deaf, lie will act as if this were the case. If it is suggested that a person actually present is in Timbnctoo, the subject will entirely ignore him, will leave him out if told to ccunt the persons in the room, or try to walk through him if asked to move in that direction. What is even morc curious is that the organic functions of the body are also liable to be influenced by suggestion. A postagc-stamp was placed on the skin of a hypnotized person, and it was suggested that it would raise a blister. Next day a blister was actually found beneath it. The letter $\mathrm{K}$, embroidered on a piece of cloth, was suggested to be reclhot. The left shoulder was then 'branded' with it, and on the right shoulder appeared a facsimile of the $\mathrm{I}$ as if burnt with a hot iron. The secretions can be increased or diminisherl, subcutaneous hæmorrhages, veritable stigmata, ${ }^{*}$ can be caused, and many of the 'miracles' of Lourdes and other shrines, ancient and modern, repeated or surpassed by the aid of hypnotic suggestion. Hypnotism has also been practically employed in the treatment of various diseases, and particularly in functional derangements of the nervous system. But care and judgment are necessary on the part of the operator, and although as a rule there is no difficulty in putting an end to the condition by a suitable suggestion. it is said that in rare instances grave mischances have occurred. There seems to be no ground for the opinion that women are more easily hypnotized than men. Out of more than a thousand persons, I.iébault found only seventeen absolutcly refractory.

I.e., bleeding spots on the skin generally corresponding to the wounds of Christ. In the well-known case of Louise Latour, which excited great interest in France in 1868, blisters first appeared; they burst and then there was bleeding from the true skin. The probable explanation is that she concentrated her attention on these parts of her body and so influenced them, perhaps by causing congestion through the vaso-motor centre. 
Relation of Size of Brain to Intelligence.-Wlils it is the catse thit some: men of greal ability have had remarkably heavy and richly comolnted brans, it would seem that in general neither great size nor any other obvious anatomical peculiarity of the cerebrum is comstantly associated with exceptional intellectual perser. In the animal kingdom, as a whole, there is undoubtedly some: relation between the status of a group and the arerage hain deredopment within the group. But that this is a relation which is complicated by other circumstances than the mere legree of intelligence is sufficiently shown by the fact that a mouse has more brain, in proportion to its size, than a man, and thirteen times more than a horse; while both in the ribbit and sleep the ratio of brain-weight to bodyweight is mearly twice as great as in the horse, in the dog only lialf as great as in the cat, and not very much more than in the donkev. The following tables, too, which illustrate the weight of the brain in man at different ages, show that, although we miglit give 'the infant phenomenon' an anatomical basis, we should greitly werrate the intellectual acuteness of the arerage baby if we were to measure it by the ratio of brain to body-neight alone.

\begin{tabular}{|c|c|c|c|c|}
\hline \multicolumn{3}{|c|}{ face. } & \multicolumn{2}{|c|}{ lirain-weisht. } \\
\hline 2 & rear.; & . & gog & .. \\
\hline 3 & ,, & . & $1,07 \mathrm{I}$ & , \\
\hline 4 & .. & $\ldots$ & I. (100) & ,, \\
\hline .5 & , & . & 1,033 & ". \\
\hline 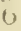 & ,, & $\ldots$ & $1,1+7$ & ,. \\
\hline 7 & ., & . & $1,20 \mathrm{I}$ & ,, \\
\hline
\end{tabular}

\begin{tabular}{|c|c|c|}
\hline $\begin{array}{l}\text { lirain-we } \\
\text { Mlen }\end{array}$ & & $\begin{array}{l}\text { lirain-weight } \\
\text { Women. }\end{array}$ \\
\hline 1,111 & rin. & I. $219 \mathrm{grl}$ \\
\hline $20-21, \ldots 1, f 10$ & ,. & 1.260 \\
\hline $30-31) \ldots 1,12-1$ & , , & .. 1.272 \\
\hline $\left.40-f^{1}\right) \ldots 1, f 00$ & ,. & 1,272 \\
\hline
\end{tabular}

\begin{tabular}{|c|c|c|c|c|}
\hline \multicolumn{3}{|c|}{ Ase. } & \multicolumn{2}{|c|}{ I rain-werght. } \\
\hline 8 & vears & . & 1,045 & grm. \\
\hline IO & ,. & $\ldots$ & 1,315 & ,. \\
\hline I I & ,. & $\ldots$ & 1,108 & ," \\
\hline 12 & .. & . & I , 286 & ., \\
\hline 13 & ., & . & 1,505 & , \\
\hline If & , & $\ldots$ & 1,330 & ,. \\
\hline 15 & , , & . & $\begin{array}{r}1,4^{1}+4 \\
-B 15\end{array}$ & त̈ \\
\hline
\end{tabular}

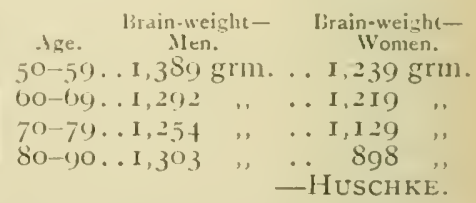

In some small birds the ratio is as high as $\mathrm{I}: \mathrm{I} 2$, in large bircls as lnw as 1 : I, 200; in certain fislies a gramme of brain lati to serve for over 5 kilos of body: As a rule, especially within a giren species, the brain is proportionally of greater size in small than in large animals. It is to be supposed that quality as well as quantity of brain substance is a potent factor in determining the degree of mental capacity.

The Cerebral Circulation.- The arrangement of the cercbral bloodvessels has certam peculiarities which it is of importance to remember in connection with the study of the diseases of the brain, many of which are causel by lesions in the vascular system-hæmorrhage or cmbolism. Four great arterial trunks carry blood to the 


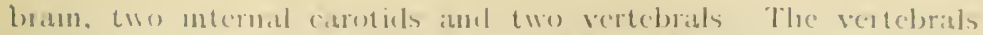
umite at the bise of the skull to form the single mesial basilar artery, which, rumning forwarel in at groose in the occipital bone, splits into the two pesterior cerebral arteries. Each carotirl. patssing in through the carotid foranen, divides into a midlle and an anterior cerebral artery; the lat ter runs forward in the great fongiturinal fissure. the former lies in tle fissure of Sylvius. A communicating branch joins the midllle and pesterior cerobrals on each sirle, and it short loop comects the two anterior cerebrals in front. In this way a liexagnn is formed at the batse of the buan, the so-called circle of Willis. While the andstomosis between the large arteries is thus very free, the opposite is truc of their branches. Nll the arteries in the substance of the bram and cord are 'end-arteries' - that is to say, each terminates within its area of clistribution without sencling communicating branclies to make junction with its neighbours. The consequence of thesc two anatomical facts is (1) that interference with the blond-supply of the brain between the licart and the circle of 1 illis does not restlily produce symptoms of cerebral anamia; (2) that the blocking of any of the arteries which arise from the circle or any of their branclics lcats to elestruction of the area supplied by it. Nearly all $\log$ secover after ligation in one operation of both carotids and botli vertcbral arterics. In monkeys both carotids may, as a rulc, be safely tied, and onc carotid in man. If, in culdition to the two caroticls, onc vertebral be ligated at the same time in the monkey, sopor results, and this is generally followed by cxtensor rigidity, coma, and death in twenty-four liours. In one case a monkey survivel this triple ligation, but became demented. The motor paralysis and rigidity were much greater than in the dog. In the condition of partial anremia the cortex is more excitable than normal, but the cxcitability clisappears at once when the anremia is rendered complete (Hill).

The basal ganglia are fed by twigs from the circle of Willis and the beginning of the posterior, middle, and anterior cercbral arteries. Of thesc the most important are the lenticulo-striate and lenticulooptic branches of the middle cercbral, which are given off near its origin, and run through the lenticular nucleus into the internal capsule. and thence to the caudate nucleus and optic thalamus respectively. The chief part of the blood from the circle of Willis is carrical by the three great cercbral arteries over the cortex of the brain. The white matter, with the exception of that in the immeliatc ncighbourhood of the basal ganglia, is nourished by straight arteries which penetrate the cortex. The middle cerebral supplies the whole of the parietal as well as that portion of the frontal lobe which lies immediately in front of the fissure of Rolando and the upper part of the temporal lobe. The rest of the frontal lobe is supplied by the anterior cercbral, and the occipital lobe, with the lower part of the temporal lobe, by the posterior cercbral. The medulla oblongata. cerebcllum, and pons are fed from the vertebrals and the basilar artery before the circle of Willis has been formed.

Resuscitation of the Central Nervous System after Total Anæmia.-Complete temporary anamia of tlie brain and upper cervical cord can be produced in most cats by passing temporary ligatures around the innominate artery and left subclavian proximal to the origin of the vertebral artery. Artificial respira- 
tion is maintained through a tube passed through the glottis. The eye reflexes disappear very quickly, and a period of high blood-pressure immediately follow's the occlusion. A fall of pressure succeeds, due to vagus inhibition of the licart, and this is followed by a second rise after the vagus centre succumbs to the ancmia. Respiration stops temporarily (in twenty to sixty seconds) after occlusion; then follows a series of strong gasps, and finally cessation of all respiratory movements. The bloodpressure slowly falls to a level which is then maintained approximately constant for the remainder of the occlusion period. The anterior part of the cord and the enceplialon lose all function : no reflexes can be elicited from this part of the central nerrous system. The intra-ocular tension is much reduced, and the cornea is characteristically wrinkled.

When the cerebral circulation is restored by releasing the vessels, the general arterial pressure soon begins to rise if the period of occlusion lias not overstepped the limit of successful cardio-rascular resuscitation. The respiration returns suddenly, the time of return depending on the length of the occlusion and on otler factors. The respiratory rate, at first slow, soon becomes normal, and then more rapid than normal. The eye-refleses reappear more gradually ; the intra-ocular tension increases, and the shrunken cornea becomes smooth and hard. The anterior part of the cord recovers its functions gradually; the reflexes connected with it return, first the homonymous, then the crossed. A short period of quiet follows; then spasms of the skeletal muscles appear, gradually increase in severity and extent, and terminate in $(a)$ death, $(b)$ partial, or $(c)$ complete recovery. In partial recovery, disturbances of locomotion, sucl as walking in a circle, paralysis, apparent dementia or loss of intelligence, and loss of sight or hearing, may be observed. Voluntary movements of the head, neck, sloulders, and fore-limbs have been seen eiglit minutes after release from an occlusion of six minutes. Blindness lias been observed without loss of the pupillary light reflex. In this case the visual cortex would seem to have suffered more than the lower centres, an illustration of a general rule. Complete recovery is rare after total ancmia lasting as much as fifteen minutes, and has not been observed after an anæmia of twenty minutes. Ten to fifteen minutes of total anremia represent the limit beyond which recovery of the brain, and therefore successful resuscitation of the animal, cannot be expected.

Chemistry of Nervous Activity.-Of this we are practically ignorant. The percentage composition of the solids and the percentage of water in the brains of three persons of different ages are exhibited in the following table ( $\mathrm{V}$. Koch) : 


\begin{tabular}{|c|c|c|c|c|c|c|c|}
\hline & \multirow{2}{*}{$\begin{array}{c}\text { ( hild } 6 \text { Weeks } \\
\text { (1rain ófoGrms.) } \\
\text { Whole Brain. }\end{array}$} & \multicolumn{3}{|c|}{$\begin{array}{c}\text { Clikld 2 Vears } \\
\text { (Brain } 1,100 \text { (irms.). }\end{array}$} & \multicolumn{3}{|c|}{$\begin{array}{l}\text {. Iduht to Vears } \\
\text { (lirain 1,670 (irmu.). }\end{array}$} \\
\hline & & lirey. & White. & $\begin{array}{l}\text { Whole } \\
\text { Brain. }\end{array}$ & Grey. & White. & $\begin{array}{l}\text { Whole } \\
\text { lirain.t }\end{array}$ \\
\hline Proteins & +00 & $4^{5}+$ & 319 & $f(0.1$ & $+7 \cdot 1$ & $27 \cdot 1$ & $37^{\cdot 1}$ \\
\hline Extractives .. & $1 \geq 0$ & in 0 & $5 \cdot 9$ & $8 \cdot 0$ & 45 & $3^{\circ 13}$ & (1) \\
\hline $\begin{array}{l}\text { Ash } \\
\text { l.ecithins and }\end{array}$ & $8 \cdot 3$ & $j S$ & $3 \cdot 2$ & +3 & 50 & $2+$ & +1 \\
\hline $\begin{array}{l}\text { 1.ecithins and } \\
\text { kephalins . }\end{array}$ & $2+2$ & $\therefore 47$ & 20.3 & 25 & 237 & 310 & -7 \\
\hline Cerebrins $\quad \ldots$ & $6 \cdot 0$ & 56 & 172 & 12.9 & $8 \cdot 8$ & $\mathrm{~J} 6 \cdot 6$ & 12 \\
\hline l.ipoid $\mathrm{S}_{\text {as }} \mathrm{SO}_{4}$ ) & 0.1 & 0.1 & 0.5 & $0 \cdot 3$ & $0 \cdot 1$ & $0 \% \overline{5}$ & $O$ \\
\hline Cholesterin $\dagger$ & I 9 & 24 & $15^{\circ} \mathrm{O}$ & $8 \cdot 7$ & +9 & I $S \cdot \overline{5}$ & $11 \%$ \\
\hline$\cdots$ & $S S \cdot 7 S$ & 49 & $76 \cdot 45$ & $80+7$ & 3.17 & $69 \cdot 67$ & 76 \\
\hline
\end{tabular}

The next table shows the variations in the content of water, solids, and protein in different parts of the nervous system (Halliburton) :

\begin{tabular}{|c|c|c|c|c|}
\hline & & Water. & Solide. & $\begin{array}{l}\text { l'ercentage of l'ro. } \\
\text { teins in solids. }\end{array}$ \\
\hline Cerebral grev matter . & . & $83 \cdot \overline{3}$ & 165 & jI \\
\hline Cerebral white matter. & . & $69 \cdot 9$ & $30 \cdot 1$ & 33 \\
\hline Cerebellum $\quad \ldots$ & . & 79.8 & $20 \cdot 2$ & 42 \\
\hline Spinal cord as a whole & . & $71 \cdot 6$ & $28+$ & $3 I$ \\
\hline Cervical cord .. & . & 72.5 & 27.5 & 31 \\
\hline Dorsal cord & . & $69 \cdot 8$ & $30 \cdot 2$ & 28 \\
\hline I.umbar cord .. & . & $72 \cdot 6$ & $27+$ & 33. \\
\hline Sciatic nerves .. & . & $6.5 \cdot 1$ & $3+9$ & 29 \\
\hline
\end{tabular}

The grey matter of the cerebrum in the adult contains 8I to 86 per cent. of water, the white matter 68 to 72 per cent., the brain as a whole $8 \mathrm{I}$ per cent., the spinal cord 68 to 76 per cent., and the peripheral nerves 57 to 64 per cent. In the fotus more water is present $(92$ per cent. in the grey and 87 per cent. in the white matter).

The superior richness of the grey matter in proteins and the preponderance of water in it, are the chief chemical peculiarities which distinguish it from the white matter. That it should have a high protein content is easily understood, for the protoplasmic structures, the nerve-cells, are situated in the grey matter. But that the most important functions should have their seat in a tissue containing only I $_{4}$ to I9 per cent. of solids is surprising, and should warn us that the water is no less signifi.

* Calculated.

$\dagger$ Calculated by difference. 
rant a constituent of living matter than the solid;, and ilat it is not the mass of the solid substances in a tissue which is the essential thing, but the whole colloid complex. which cannot be constituted witlont the water.

Fresli nervous tissues are alkaline to litmus, but become acid soon after death. No change of reaction has been detected during activity.

That oxygen is used up during cerebral activity is certain, and when the brain is coloured with methylene blue, by injecting it into the circulation, any spot of it which is stimulated loses the blue colour, the pigment being reduced. But if the animal is so deeply narcotized that it does not respond to stimulation, the change of colour does not occur.

Cholin, a substance which can be derived from lecithin, is believed to represent one of the waste products of nervous activity. Exceedingly small traces of it are present in normal cerebro-spinal fluid, and in certain diseased conditions of the brain, as in general paralysis, the quantity is said to be notably increased. Some writers assert that this increase in the cliolin can be used as a test to distinguish organic nervous diseasc from that which is purely functional. But the matter is in dispute.

Cerebro-spinal Fluid.-The cerebro-spinal fluid, which fills the ventricles of the brain and the central canal of the cord, is continuous with that contained in the subarachnoid space through the foramen of Magendie, an opening in the piece of pia mater that helps to roof in the fourth ventricle. It is secreted in part by the cubical cells covering the choroid plexus, a fold of pia mater which projects into each lateral ventricle. Extracts of choroid plexus increase the rate of secretion. Cerebro-spinal fluid can easily be obtained in man by lumbar puncture with a hypodermic needle sufficiently long to enter the subarachnoid space in the spinal canal. The point usually selected for the puncture is between the fourth and fifth lumbar vertebre. The normal pressure of the fluid is such that it trickles out by drops, but in disease it is sometimes so high that it spurts out in a steady stream. An examination of the fluid, especially for leucocytes or bacteria, is of great diagnostic value in certain conditions. Normally it is a thin, clear, watery fluid, faintly alkaline in reaction to litmus, and witl a specific gravity of about ioof to I007. It contains the ordinary salts, but more potassium than sodium, unlike other body fluids ; a very small amount of protein (globulin)-usually about $0 . \mathrm{I}$ per cent.-and a little dextrose (Nawratzki). Its composition is evidently different from ihat of ordinary lympli. Only a few lymphocytes are present in health, but in some diseases (as in general paralysis of the insane, tabes, and cerebro-spinal syplilis) a marked increase occurs. In acute 
cerebro-spinal meningitis mumerous polymorphonuclear leucocytes are found, which are absent from the normal fluid.

The depression of the freezing-point $(\Delta)$ usually lies between $-0.60^{\circ}$ and $0.65^{\circ} \mathrm{C}$. In a case of hydrocephalus it was $-0.65^{\circ} \mathrm{C}$. Normally, cerebro-spinal fluid is somewhat hypertonic to the blood-serum. In injury of the cribriform plate of the ethmoid bone and also in some cases where there is no traumatic injury, the fluid escapes from the nose, and the rate of its formation can thus be ascertained. In one case it was found to be as much as 2 c.c. to nearly + c.c. in ten minutes.

The Autonomic Nervous System (the Sympathetic and Allied Nerves). - The efferent fibres of the body can be divided into two classes: (I) Those which supply multinuclear striated muscle (skcletal muscle); (2) those which supply. other structures (smooth muscle, heart muscle, glands). The second group is called 'autonomic,' to indicate that it possesses a certain independence of the central nervous system, although this independence is far from absolute. The autonomic fibres arise from four regions of the central nervous system : (I) The mid-brain; (2) the bulb; (3) the thoracic and upper lumbar cord; (4) the sacral portion of the cord. All autonomic fibres after issuing from the central nervous system end sooner or later by forming synapses around nerve-cells of sympathetic type, by whose axons the path is continued to the peripheral distribution. The autonomic path accorlingly comprises two neurons, the fibre which arises from the brain or cord being termed the 'preganglionic,' and that which arises from the sympathetic ganglion the "postganglionic fibre.'

The autonomic fibres originating in the midbrain emerge in the oeulo-motor nerve, and form synapses with eclls in the ciliary ganglion, which in turn send fibres to the ciliary muscle and the constrictor muscle of the iris (pp. 819,909$)$. The bulbar autonomic fibres emerge in the seventh. ninth, and tenth cranial nerves. Those in the vagus include inhibitory fibres for the heart muscle, motor and inlibitory fibres for the smooth muscle of the alimentary canal from

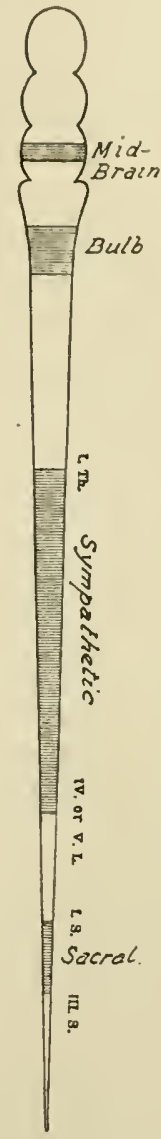

FIG. 367.-DIAGRAM SHOWING THL CEN. TRAL ORIGIN OF THE AUTONOMIC FibRES (LANGLEY). the osophagus to the descending colon, and for the muscles of the trachea and lungs, and secretory fibres for the gastric glands and the pancreas. The sympathetic ganglion cells with which these preganglionic fibres form symapses have not always been definitely located, but lie near or in the tissue supplied (p. I65). The autonomic fibres in the seventh and ninth nerves supply the mucous

$$
56-2
$$


membranes of the mouth and nose with vaso-clilator and secretory fibres. The preganglionic portion of the path terminates in such ganglia as the submaxillary and sublingual (p. 362) and the sphenopalatine and otic ganglia.

The part of the autonomic system which originates in the middle region of the spinal cord (in the cat from the first thoracic to the fourth or fifth lumbar nerves) is the sympathetic proper. The course of the fibres has already been described in connection with the vaso-motor neries (p. I65). Among the fibres may be mentionerl the dilators of the pupil, the augmenters of the heart. motor (viscero-motor), and inluibitory fibres for the smooth muscle of the alimentary canal, sweat-sccretory, pilc-motor and vaso-constrictor fibres. The preganglionic fibres issue from the corl in the anterior roots, and leave the corresponding spinal nerve in the white ramus communicans, which conncets it with the corresponding ganglion of the lateral sympathetic chain. A fibre nay cither end in this ganglion by forming a synapse, or it may run up or down in the chain for some distance before terminating. Some of the preganglionic fibrc.s, particularly the vaso-constrictors for the abrlominal and pelvic viscera, (lo not end in the lateral chain at all. but issuing from it still as medullated fibres, terminate in one of the prevertcbral ganglia-e.g.. coliac ganglion, inferior mesenteric ganglion-from which postganglionic fibres procecl to the viscera, as previously described (p. 310). The postganglionic fibres arising from cells of the lateral ganglia return as non-medullated fibres in grev rami communicantes to the spinal nerves, and are clistributed with them to the head. limbs, and the superficial parts of the trunk.

The autononic fibres arising from the sacral region of the cord emerge as preganglionic fibres in the anterior roots of the second to the feurth sacral nerves, from which they pass to the pelvic nerve (ncrvus erigens) (pp. 163,310). They comprise vaso-dilator fibres for the rectunl. anus, and cxternal genitals, moter (viscero-motor) fibres for the smooth muscle of the rlescending colon. rectum, and anus. inhibitory fibres for the smooth muscle of the anus. and the muscles of the external genitals, motor fibres for the bladder, etc. The preganglionic fibres terminate by forming synapses with sympathetic ganglion cells in the pelvic plexus, or in the neighbourhood of the organs which they supply. From these ganglion cells the postganglionic fibres arise.

\section{PIRACTICAI, EXERCISES ON CHAPTEIR XII.}

I. Section and Stimulation of the Spinal Nerve-roots in the Frog. - (a) Selcct a largc frog (a bull-frog, if possible). Pith the brain. Fasten the frog. belly lown. on a plate of cork. Make an incision in the middle line over the spinous processes of the lowest three or four vertebra, separate the muscles from the vertebral arches, and with strong scissors open the spinal canal. taking care not to injure the corcl by passing the blade of the scissors too deeply. Extend the opening upwarls till two or threc postcrior roots conic into view. Pass fine silk ligatures under two of them, tic, and clivide onc root central to the ligature, the other peripheral to it. Stimulate the central encl, and reflex movements will occur. Stimulate the peripheral end : no effect is producer. Now cut away the exposed posterior roots and isolate and ligature two of the anterior 
roots, which are smiller than the posterior. Stimulate the central end of one: there is no effect. Stimulation of the peripheral end of the other causes contractions of the corresponding muscles.

(b) Stimulation of the roots mily be repeated on the mammal, using the dog employed for the experiment on the motor areas (p. 889). Place the animal, belly down, and insert a good-sized block of wood between it and the board at the level of the lumbar vertebra of the spinc. Divide the skin and muscles on cither side of this region till the lamina of the vertebra are exposed. Snip through them with strong forceps, and open the spinal canal, exposing a length of cord corresponding to three or four rertebra. Ligate and stimulate the roots as in $(a)$.

2. Reflex Action in the 'Spinal' Frog.--I'ith the brain of a frog, destroying it down to the posterior third of the medulla oblongata. (t) Note the position of the limbs immecliately after the operation, and again thirty to forty minutes later. Its hind-legs possess tone, and are drawn up against the flanks. The animal can still execute certain co-ordinated movements-e.g., pulling away its $\mathrm{leg}$ if a toe is pinched. The power of maintaining equilibrium is lost. If placerl on its back, it lies there. When thrown into water it sinks usually without any attempt at swimming. Verify the following facts, using mechanical stimulation (pinching the toes or skin of the leg): (a) If the stimulus provokes nuscular movements only on one sicle of the bolly, this is usually on the same side as the stimulated point. (b) When the stimulus causes reflex movements on botli sirles of the bolly. the stronger contractions are on the side to which the stimulus was applied.

Determine whether it is easier to obtain movement of a portion of the body innervated from a region of the cord above the level of the stimulated nerves or below that level.

(2) With electrical stimuli (using a coil arranged for single shocks) determine whether reflex movements are elicited by a single induced shock applied to the skin. Verify the fact that a series of shocks is more efficient, the effects of the separate stimuli being summated in the reflex centres.

(3) To test the effect of thermal stimuli, (lip the leg into a beaker of warm water. Vary the temperature of the water. using a series of beakers with water at $10^{\circ} \mathrm{C} ., 20^{\circ} \mathrm{C}$., etc., above the temperature of the room. Place the leg for a moment in each, and determinc which is the most effecient stimulus. Immecliatcly on withdrawing the leg from each of the hot-water beakers immerse it in a beaker of water at room temperature. Finally, dip the leg into a beaker of cold water, and heat it gradually to a temperature at which a reflex was previously obtained. Probably it will not be elicited by the gradual warming.

(4) 'Purposive' Movements.-Touch the skin of one thigh with blotting-paper soaked in strong acetic acid. The leg is drawn up, and the foot moved as if to get rid of the irritant. If the leg is held, the other is brought into action. Immerse the frog in water to wash away the acid.

(5) Spread (Irradiation) of Reflexes.-Gently stimulate a toe or a small spot on the flank with weak induction shocks or weak mechanical stimuli, and note the reflex effect obtained. Then go on gradually increasing the strength of stimulation without increasing the arca of the field stimulated. and observe the extent and order of spread of the reflex movements. 
3. Reflex Time. l'atss a hook through the jaws. Hohling the frog by the hook, dip one legr into a dilute solntion of sulphuric acid $\left(0^{\circ} 2\right.$ to $0^{\circ} .5 \mathrm{per}^{\circ}$ cent.), and note with the stop-watch the interval which clapses before the frog draws up its leg (Türck's method of determining the reflex time). Wash the acid off with water.

Determine how the reflex time varies with the strength of the stimulus. This can be done by using various strengths of acid. The reflex time will be shorter the stronger the stimulus up to at certain point. Compare the reflex time of movements on the same side of the body as the point of application of the stimulus and on the opposite side.

4. Inhibition of the Reflexes.-(1) Destroy the cerebrum of a frog. Dip one leg into dilute sulphuric acid as in 3 , and estimate the reflex time. Then apply a crystal of common salt to the upper part of the spinal cord. If the opcning marle for pithing the frog is not large enough to enable the cord to be clearly secn, enlarge it. Again dip the leg in the dilnte acirl. It will either not be drawn up at all, or the interval will be distinctly longer than before.

(2) Expose the viscera, inclutling the heart, taking care not to injure the cardiac nerves. Tap the intestines sharply with the handle of a scalpel many times in succession. The heart is inhibited.

(3) Tie strings tightly around both fore-legs of a normal frog. Place the animal on its back; it does not turn over. The hindlegs may be pulled about in various ways without the frog turning over into its normal position. The reactions concerned in the maintenance of equilibrium are inhibited. Remove the strings. The animal cannot be made to lie on its back except by force.

5. Spinal Cord and Muscular Tonus.--Destroy the brain of a frog. Isolate the gastrocnemius, and cut away the bone below the knee. lsolate the sciatic nerve without injuring it. Remove the muscles from the femur, cut the bone and fix it in a clamp for graphic recording. Connect the tendon with a lever. weighted with 5 to Io grammes. Take a base line. Destroy the spinal cord, or cut the sciatic and again take a base line. The length of the muscle is slightly altered.

6. Spinal Cord and Tonus of the Bloodvessels.-Destroy the brain of a frog. Arrange the web of the foot on the stage of a microscope, and note the calibre of the bloodressels in the field. Destroy the cord, and observe the change in their calibre. They will dilate.

7. Action of Strychnine.-Pith a frog (brain only). Inject into one of the lymph-sacs three or four drops of a $0^{*} 1$ per cent. solution of strychnine. In a few minutes general spasms come on, which lave intermissions, but are excited by the slightest stimulus. The extensor muscles of the trunk and limbs overcome the flexors. Destroy the spinal cord; the spasms at once cease, and cannot again be excited.

S. Mammalian Spinal Preparation (Sherrington).* - Decply aniesthetize a cat with ether. Insert a cannula into the trachea (p. I86), and continne the anesthesia through this. Expose and ligate both common carotids. Make a transverse incision through the skin over the occiput, and cxtend it laterally behind the ears. Pull back the skin so as to expose the neck muscles at the level of the axis vertebra. lieel for the ends of the transverse processes of

* A similar preparation can be used for certain experiments on the circulation (Crile, Gnthrie). For these, as well as for the studly of many reflexes, a good preparation is obtained by occlusion of the cerebral bloodsupply in cats (without decapitation). 
the atlas, and divicle the muscles down to the bone just behind these processes. Now start artificial respiration ( $P$. 187), or sooner if necessary. Notch the spinons process of the axis with bone forceps. Pass a strong thick ligature by a sharp-ended aneurism needle close under the bolly of the axis and tic it tightly in the groove left by the incision behind the transierse processes of the atlas and the notch made in the spinous process of the axis. This compresses the vertebral arteries where they pass from transverse process of axis to transverse process of atlas. Pass a second strong ligature under the trachea at the level of the cricoid cartilage and include in it the whole neck, except the trachea, but at present only tie a single loop on it. Now decapitate the animal with a large knife (an amputating knife) passed from the ventral aspect of the neck through the occipito-atlantal space. severing the cord just behind its junction with the bulb. At the moment of decapitation tighten the ligature round the neck, and complete the knot. Destroy the head. If there is oozing of blood from the vertebral canal, arrest it by raising the neck somewhat above the level of the body. The carcass must be kept warm by placing it on a metal box or tabie containing hot water, and the air used for artificial respiration must also be warmed, as by passing it through a coil of rubber-tubing inmersed in a water-bath which is kept hot. Stitch the skin-flaps together so as to cover the cut end of the spinal cord and the other structures cut in decapitation. By this procedure the spinal cord is usually severed about + millimetres behind the point of the calamus scriptorius. Although the blood-pressure remains low, reflexes employing the skeletal muscles can be fairly well clicited for hours. Study on the preparation the reflexes describer in the text (pp. 799, 801)-e.g., the flexion reflex of the hind and fore limb, as elicited from the skin, or one of the afferent nerves of the limbthe crossed extension reflex of hind and fore limb, the scratch reflex.

(1) Scratch Reflex.-(a) Evoke the reflex by rubbing the skin of the neck behind the pinna. The scratching movements are in the hindleg of the same side. Record them on a drum, on which is also written a time-tracing in seconds. The record can be obtained by tying a piece of tape, not too tightly, round the foot, leg, or thigh, and connecting this by a thread with a lever. The thread is passecl over a pulley below the lever, so that its pull may be exerted at right angles to the axis of rotation of the lever. The lever is attached to a light spring or a rubber band, which is stretched when it moves in one direction, and in recoiling brings it back again to its position of rest at the end of the contraction. If the reflex is not easily evolied, it can be facilitated by producing a slight degree of asphyxia by temporarily clamping the respiration tube. Some time must elapse after the decapitation before a fair scratch reflex can be expected. It is usually sufficiently well marked within an hour.

(b) While the reflex is occurring. stimulate with an interrupted current the central stump of the popliteal nerve of the opposite hindlimb. The scratch reflex may be cut short by inhibition. Also, during the stimulation of this nerve the reflex may be incapable of being elicited till the excitation of the inhibitory afferent nerve is stopped.

(2) Flexion Reflex.-(a) Stimulate with a weak interrupted current the skin of some part of the hind-limb-say one of the toes. The flexion reflex of the hind-limb on the same side may be evokedi.e., a flexion movement at the linee, hip, and ankle. Recorl the movements of one of the joints or of flexor muscles after severing them from their insertion. 
(b) Stimulate witl a wcik interrupted (faradic) current the cent ral stump of one of the nerves of a hind-limb saty, the peroneal nerve. The flexion reflex of the same limb may be elicited. Record the movements. Now proluce temporary asphyxia by clamping the respiration tube, and repeat the stimulation at lialf-minute intervals. The reflex will be increased by the asphyxia. Do not interrupt the respiration for more than two or three minutes, and immediately start it if the heart, which can be felt through the chest, begins to weaken.

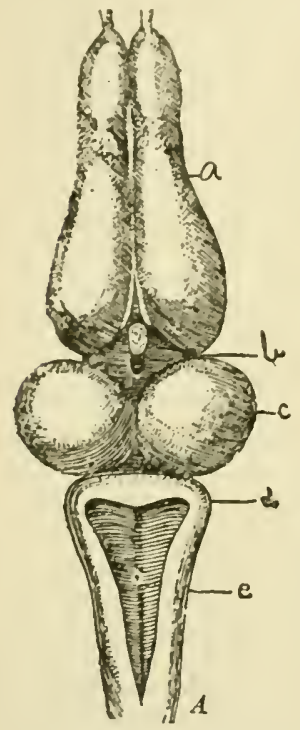

Fir. 358. - Prats or

F R $O G$ (A T T K

Steiner).

a, rereloral hemi. sphrese ; b, position of (1) tic thalami; $c$, optic lobes; $d$, rerebellum ; $c$ merlullat oblongata: A. upper end of spinill cord. ctermine the reflex time by Türch's method; apply a crystal of common salt to the optic lobes, and repeat the observation. The reflex movements will be completely inhibiterl or delayed. Remove the salt, wash with physinlogical salt solution, excise the optic lobes, and see whether the frog will now swim.

11. Excision of the Cerebral Hemispheres in a Pigeon.-.Fecrl a pigeon for two or threc days on dry food, etherize it by holding a piece of cotton-wool sprinkled with ether over its beak, or inject into the rectum f gramme chloral hydrate. The pigeon being wrapped up in a cloth, and the head held steady by an assistant, the feathers are clipped off the head, an incision made in the middle line through the slin, and the flaps reflected so as to expose the 
skull. (ut through the bunes with scissors, and make a sulficiently large opening to bring the cencbral hemispheres into view. They are now rapielly divisled from the corporat bigemina and lifted out witl the liandle of a scalpel. The blecting is very free, but may be partially controlled by stuftung the cavity with the vegetable tibre known as Pengavar Diambi, which should be removed in a few minutes, the wound cleansed with iodoforn gauze wrung out of physiological salt solution at $50^{\circ} \mathrm{C}$., and sewed $11 \mathrm{p}$. Study the phenomena describerl on p. $S_{f}$

12. Stimulation of the Motor Areas in the Dog.--(a) Study a hardened brain of a dog, noting especially the crucial sulcus (Fig. $3 f^{\prime}$ ). 1). $\left.S_{\uparrow 3}\right)$, the convolutions in relation to it, and the areas napped out around it by Hitzig and litsch and others. (b) Inject morphine under the skin of a dog. Set up an induction-coil arranged for tetanus. with a single Daniell in the primary circuit. Connect a pair of fine but not sliarp-pointed electrodes through a short-circuiting key with the sccondary. Fasten the dog on the holder, belly down, and put a large pail under the neck to support the head. Clip the hair over the scalp. Feel for the conrlyles of the lower jaw. and join them by a string across the top of the head. Connect the outer canthi of the eres by another thread. The crucial sulcus lies a little behind the mid-point between thess two lines. Now give the $\log$ ether. make a mesial incision through the skin down to the bose, and reflect the flaps on either sicle. Drtach as much of the temporal muscle from the bone as is necessary to get room for two trephine holes, the internal borders of which nust be not less than 1 inch from the midrlle line, so as to avoid wouncing the longitudinal sinus. Carefully work the trephine through the skull, taking care not to press heavily on it at the last. Raise up the two picces of bone with forceps, connect the holes with bone forceps. and cnlarge the opening as much as may be necessary to reach all the 'motor' areas. At this stage only cnough cther should be given to prevent suftering. Now unbind the hind and fore linbs on the side opposite to that on which the brain has been exposed, apply blunt electrodes successively to the areas for the fore and hind limbs. and stimulate.* The 'unipolar' method of stimulation (p. S $\$ 3$ ) may also be employed. Contraction of the corresponding groups of muscles will be secn if the narcosis is not too decp. IIorements of the head, ncck. and cyclids may also be called forth by stimulating the 'motor' areas for these regions. Stimulation in front of the crucial sulcus may also cause great dilatation of the pupil, the iris almost disappearing. The dilatation takes place most promptly, and is greatest on the opposite side, but the pupil on the same sicle is also widened. Even after section of botli vago-sympathetic nerves in the neck. a slow and slight dilatation. greatest perhaps on the same side, may be caused by cortical stimulation. Repeat the whole experiment on the opposite side of the brain. In the course of his observations the student will perhaps have the opportunity of seeing general epileptiform convulsions set up by a localized excitation. They begin in the group of muscles represented in the portion of the cortex directly stimulated. After the convulsions have been sufficiently sturlied. they should be again induced, and the stimulated 'motor' area rapidly excised during their course. In some cases this will be followed by immediate cessation of the spasms. (c) The same animal can be used for stimulation of the spinal nerve-roots, as described in Experiment I (p. $\left.8 S_{5}\right)$.

* It is not necessary to remove the dura mater. 


\section{CHAPTER YIII}

\section{THE SENSES}

Hitherto we have been considering from a purely objective standpoint the organs that compose the body, and their work. The student has been assumed to be in the little world - the ' microcosm' - of organization which he has becn studying, but not of it. He has listened to the sounds of the heart, scen its contraction. felt it hardening under his fingers; but we have not inquired as to the meaning or the mechanism of this hearing, seeing, and feeling. IVc have now to recognise that all our knowledge of external things comes to us by the channels of the senses, and, like the light that falls through coloured windows on the floor of a church, is tinged, and perhaps clistorted, in the act of reaching us.

The Senses in General.-The old and orthodox enumeration of 'the five senses' of sight, hearing, touch, taste, and smell, must be augmented by at least two more, the senses of pressure and temperature. The so-called temperature sensations are themselves divisible into two groups of quite distinctive quality, sensations of warmth and sensations of cold. The power of appreciating the amount of a muscular effort ; the power of localizing the various portions of the body in space ; the sensations of pain, tickling, itching, hunger, and thirst ; the sensations accompanying the generative act, etc., can certainly be no longer lumped together in the omnium gatherum of 'common sensibility.' They are more appropriately regarded as separate senses subserved by special nerves, and perhaps connected with definite centres. In the development of a simple sensation we may distinguish three stages: the stimulation of a peripheral end-organ, the propagation of the impulses thus set up along an afferent nerve, and their reception and elaboration in a central organ.

We do not know in what manner a scries of transverse vibrations in the ether when it falls upon the eyc, or a series of longitudinal vibrations in the air when it strikes the ear, excites a sensation of light or sound. We can trace the ray of light through the refractive media of the cycball, sec it focussed on the retina, lead off the current of action set "np in that membrane, which, (loubtless, gives token of the passage of nervous impulses into and up the optic nerve. We can even follow the nervous impulses to a definite portion of the cortex of the occipital lobe, and eleterminc that if this is removed no sensation of sight will result from any excitation of retina or optic 
nerve. And it is fair to conclude that in some manner this part of the cerebral contex is essential to the production of visual sensitions. But in what waty the chemical or physical processes in the axiscylinders or nerve-cells are related to the psychical change. the interruption of the smooth and unregarded flow of consciousness which we call a sensation of light, we lo not know. To our reasoning, and even to our imagination, there is a great gulf fixed between the physical stimulus and its psychical consequence : they secm incommensurable quantities : the transition fronl light to sensation of light is certain, but unthinkible.

Each kind of peripheral end-organ is peculiarly suited to respond to a certain kind of stimulus. 'The law of "adequate' or 'homologous' stimuli is an expression of this fact. The 'adequate' stimuli of the organs of special sense may be divided into ( $\mathrm{I}$ ) vibrations set $\mathrm{up}$ at a distance without the actual contact of the object- $\mathcal{E} . g$., light, sound, radiant heat; (2) changes produced by the contact of the object-e.g., in the production of sensations of taste, touch, pressure, alteration of temperature (by conduction). Midway between (I) and (2) lies the adequate stimulus of the olfactory end-organs, which are excited by material particles given off from the odoriferous body and borne by the air into the upper part of the nostrils.

The end-organs of the special senses all agree in consisting essentially of modified ectodermic cells, but they occupy areas by no means proportioned to their importance and to the amount of information we acquire through them. The extent of surface which can be affected by light in a man is not more than $20 \mathrm{sq} . \mathrm{cm}$.; the endings of both nerves of hearing taken together do not at most expand to more than $5 \mathrm{sq}$. $\mathrm{cm}$. : the olfactory portion of the mucous membrane of the nose lias an area of not more than $10 \mathrm{sq} . \mathrm{cm}$.; the sensations of taste are ministered to by an area of less than $50 \mathrm{sq} . \mathrm{cm}$.; the end-organs of the senses of pressure. touch, and temperature are distributed over a surface reckoned by square metres. As the physiological status of the sensory end-organs rises, their anatomical distribution tends to shrink. The organs of comparatively coarse and common sensations are widely spread, intermingled with cach other, and seated in tissues whose primary function may not be sensory at all. Even the nerve-endings of the sense of taste are not confined to one definite and circumscribed patch. but scattered over the tongue and palate : and both tongue and palate are at least as much concerned in mastication and deglutition as in taste. The olfactory portion of the nasal micous membrane, although a continuous area with fairly distinct boundaries, is still a part of the general lining of the nostril. The epithelial surfaces which minister to the supreme sensations of sight and hearing - the retina and the sensitive structures of the cochleaare the most sequestered of all the sensory areas, as the organs of which they form a part are, of all the organs of sense, the most. highly specialized in function, and anatomically the most linited. But although hidden in protected hollows. they are endowed, either in virtue of their own movements or of those of the head, with the power of receiving impressions from every side, and their actual size is thus indefinitely multiplied. 


\section{VISION.}

Physical Introduction.- l'hysically, a ray of light is a series of disturbances or vibrations in the luminiferous ether, which radiates out from a luminous borly in what is practically a straight line. The ether is supposed to fill all space, including the interstices between the molecules of matter and the atoms of which those molecules are composed. Suppose a bar of iron to be gradially heated in a dark room. In the cold iron the molecules are moving on the average at a relitively slow rate, and the waves set up in the ether by their vibrations are comparatively long. Now, the long ethereal vibrations do not excite the retina, because it is only fitted to respond to the impact of the shorter waves; and, indeed, the long waves are larely absorbed by the watery media of the eye. As the temperature of the iron bar is increased, the molecules begin to move more quickly, and waves of smaller and smaller length, of greater and greater frequency, are set up, until at last some of them are just able to stimulate the retina, and the iron begins to glow a dull red. As the heating goes on the molecules move more quickly still, and, in

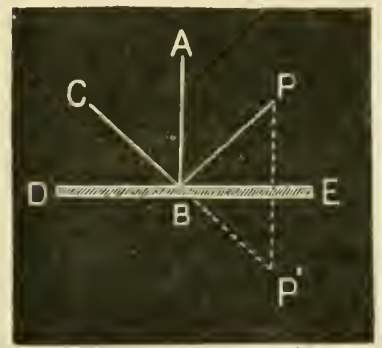

lic. 36ig. -- Rtriection From a M'LANI: MIRROR. addition to waves which cause the sensation of red, shorter waves that give the sensation of yellow appear. Final!y, when a high temperature has been reached, the very shortest vibrations which can affect the ere at all mingle with the medium and long waves, and the sensation is one of intense white light.

We have said that a ray of light travels in a straiglt line, and the direction of the straight line loes not change as long as the medium is homogrencous. But when a ray reaches the boundary of tlie medium through which it is passing, a part of it is in general turned bick or reflected. If the second medium is transparent (water or glass, e.g.). the greater part of the ray passes on through it, a smaller portion is reflected. If the second medium is opalue, the ray does not penetrate it for any great distance; if it is a piece of polished metal, c.g., nearly the whole of the light is reffected : if it is a lityer of lampblack, very little of the light is reflecterl, most of it is absorbed.

Reflection. - The first law of refiection is that the reflected ray, the ray which falls upon the reflecting surface (incident ray), and the normal to the surface, are in one plane. The second law is that the reflected ray makes with the perpendicular (nomal) to the reflecting surface the same angle as the incident ray. A corollary to this is that a ray perpendicular to the surface is reflected along its own patli.

Reflection from a Plane Mirror.-Let a ray of light coming from the point P' (Fig. 369) meet the surface Dl: at B, making an angle P'ISA with the normal to the surface. The reflected ray $\mathrm{BC}$ will make an equal angle $\triangle B C$ with the normal; and the eye at $C$ will see the image of $]^{\prime}$ as if it were placed at $\mathrm{P}^{\prime}$, the point where the prolongaton of $\mathrm{BC}$ cuts the straight line drawn from $P$ perpendicular to $\mathrm{DE}$. This is the position of an ordinary looking-glass image. 
Reflection from a Concave Spherical Mirror. - A spherical surface may be supposed to be mate up of an infinite number of infinitely small plane surfaces. The normal to each of these plane surfaces is the radius of the spliere, and the reflected ray makes with the radius at the point of incidence the same angle as the incident rity. Lot D (Fig. 370) be the midelle point of the mirror, and C its centre

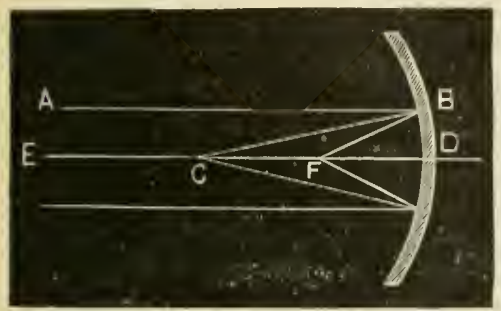

Fig. 370.-Reflection from a CONCAVE lil SPIIERICAL MIIRROR.

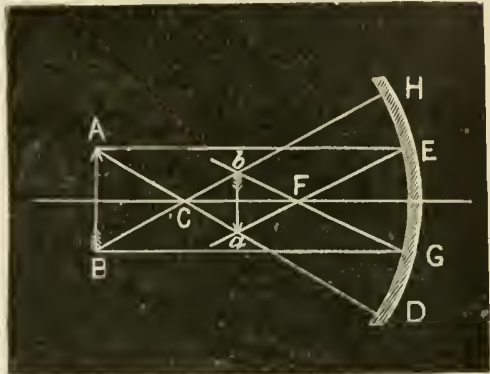

Iig. 371. - FORMATION OF REAL IŇVERTED IMAGE BY A CONCAVE SPIIERICAL MIRROR.

of curvature-i.e., the centre of the spliere of which it is a segment. Then CD is the principal axis, and any other line through $\mathrm{C}$ which cuts the mirror is a secondary axis. When the mirror is a small portion of a sphere, rays parallel to the principal axis are focussed at the principal focus $F$ midway between $C$ and $D$; rays parallel to any secondary axis are focussed in a point lying on that axis; and rays diverging from a point on any axis are focussed in a point on the same axis.

These facts afford a simple construction for finding the position of the image of an object formed by a concave mirror. Let $\mathrm{AB}$ be the object (Fig. 371). Then the image of $A$ is the point in which all rays proceeding from $A$ and falling on the mirror, including rays parallel to the principal axis, are focussed.

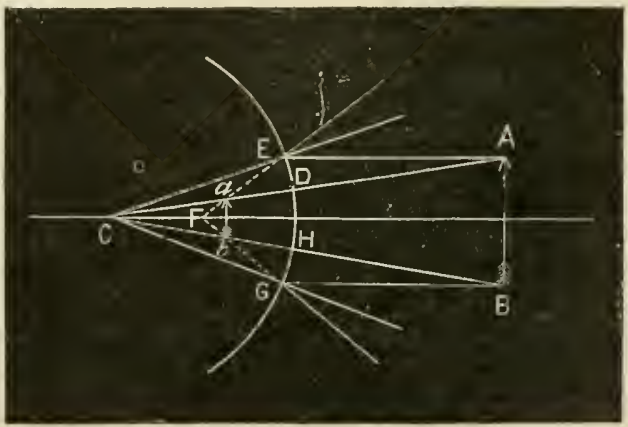

Fig. 372.-Formation of IMAGE BY A CoNvex MIRROR.

\section{But the ray $\mathrm{AE}$,}

parallel to the principal axis, passes after reflection through the principal focus $\mathrm{F}$, therefore the image of A must lie on the straight line EF. If any secondary axis $A C D$ be drawn, the image of $A$ must lie on ACD. It must therefore be the point of intersection, $a$, of EF and ACD. Similarly, the image of B must be the point of intersection, $b$, of $\mathrm{GF}$ and $\mathrm{BCH}$. The image $a \dot{b}$ of an object $\mathrm{AB}$ farther 
from the nirror than the principal focus is real and inverted. The Purkinje-Sanson image reflected from the concave anterior surface of the vitreous lumour (Fig. 387) is an cxample.

After reflection from a convex mirror, rays of light always diverge, and only erect, virtual images are formed-i.e., images which do not really exist in space, but which, from the direction of the rays of light, we judge to exist. The position of the image of an object $\mathrm{AB}$ (Fig. 372) may be found by a construction similar to that for reflection from a concave mirror. The image of a flane reflected from the anterior surface of the cormea or lens is erect and virtual. It diminishes in size with increase in the curvature or convexity of the reflecting surface (liig. 387 ).

Refraction. - A ray of ligh passing from one medium into another has its veloeity. and consequently its direction, altered. It is said to be refracted. The first law of refraction is that the refracted ray is

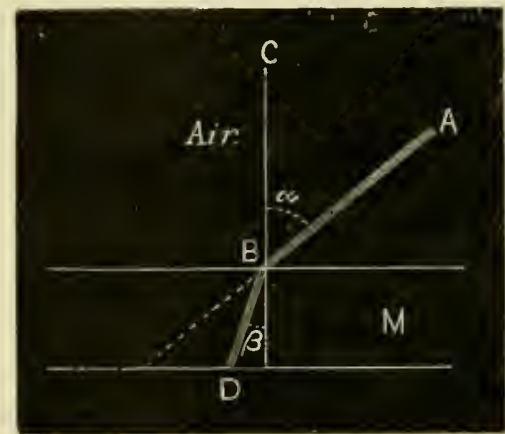

Fig. 373.-Refraction at a Plaxe SURFACE.

$A B$ is the incident: $B D$, the refracted ray; $C B$, the normal to the surface. When the ray passes from air into another medium, the refractive index of the latter is the fraction $\frac{\sin \alpha}{\sin \beta}$.

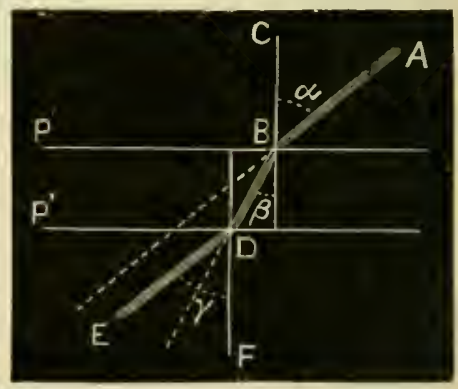

FIG. $374 .-$ REFRACTION BY A MEDIT:M bounded by Parallel Pianes, $P$ AND $P^{\prime}$.

The ray ABDE issues parallel to its original di ection: $\mathrm{CB}, \mathrm{FD}$, noimals to $\mathrm{P}^{\mathrm{P}}$ and $\mathrm{P}^{\prime} ; \boldsymbol{\omega}$, angle of incidence ; $\beta, \gamma$, angles of refraction.

In the same plane as the incident ray and the normal to the surface. The second law is that the sine of the angle of incidence has a constant ratio (for any given pair of media) to the sine of the angle of refraction. The angle of incidence is the angle which the ray makes with the normal to the surface, separating the two media ; the angle of refraction is the angle made with the normal in the second medium. This ratio is called the index of refraction between the two media. For purposes of comparison, the refractive index of a substance is usually taken as the ratio of the sine of the angle of incidence to the sine of the angle of refraction of a ray passing from air into the substance.

When a ray strikes a surface at right angles, it passes through without suffering refraction. When a ray passes from a less dense to a denser medium (e.g., from air to water), it is bent towards the perpendicular. When it passes from a more dense to a less dense medium (as from water to air), it is bent away from the perpendicular.

When a ray passes across a medium bounded by parallel planes, it 
issues parallel to itself ; in other words, it undergoes no refraction (Fig. $37+$ ).

Refraction and Dispersion by a Prism.-The beam of light is bent towards the normal $N$ as it passes across $B A$ and away from the normal $N^{\prime}$ as it passes across BC (Irig. 375); at both surfaces it is bent towards the base of the prism AC. At the same time the light suffers dispersion-that is, the rays of shorter wave-length are more refracted than those of greater wave-length. The deviation of any given ray is measured by the angle which the refracted ray makes with its original direction. The amount of dispersion produced by a prism is measured by the difference in the deviation of the extreme rays

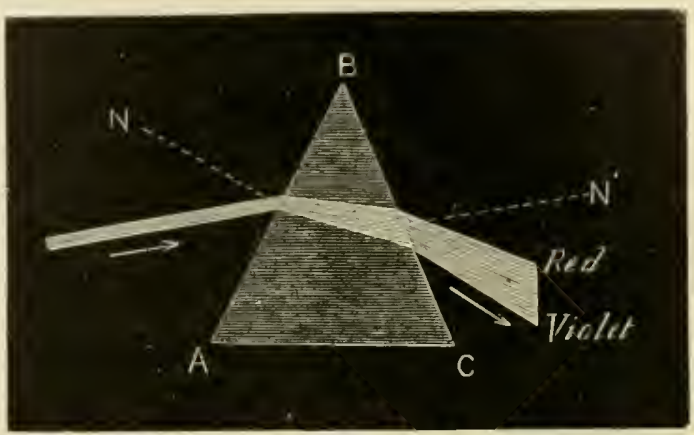

Fig. 375.-Refraction AND Dispersion bY a Prisi. of the spectrum.

The dispersion produced by a given substance is proportional to the difference of its refractive indices for the extreme rays.

Refraction by a Biconvex Lens.-A straight line ACB passing through the centres of curvature of the two surfaces of the lens is called the principal axis. A point $C$ lying on the principal axis between the two centres of curvature, and possessing the property

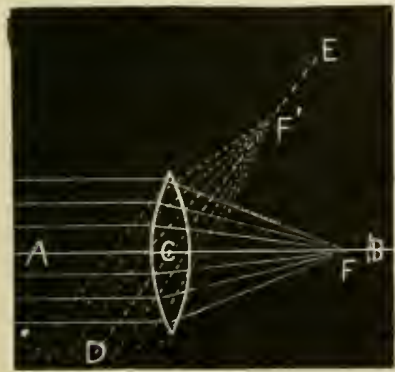

Fig. 376. - REFRACTION BY A Biconvex Lens.

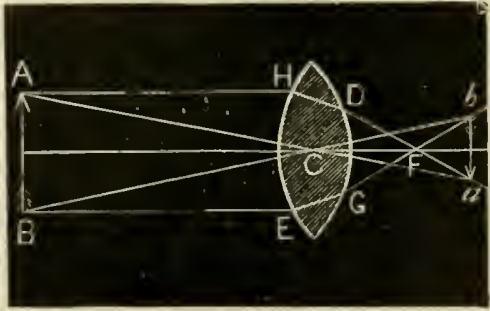

Fig. 377.--ForMation OF IMAGE BY BiconveX LENS.

that rays passing through it do not suffer refraction, is called the optical centre of the lens. Any straight line, DCE, passing through the optical centre is a secondary axis. Rays of light proceeding from a point in the principal axis are focussed in a point on that axis. When the rays proceed from an infinitely distant point in the principal axis-i.e., when they are parallel to it-they are focussed in $\mathrm{F}$, the principal focus. Similarly, rays parallel to, or proceeding from, a point in a secondary axis are focussed in a point on that axis : 
but if the focus is to be sharp, the angle between the seconclary and the principal axis must not be so large as is indicated in ligig. 376 .

Formation of Image by Biconvex Lens (Fig. 377).-Let $\Lambda \mathrm{B}$ be the object; then if $A H D$ be the path of a ray from $A$ parallel to the principal axis, the image of $\Lambda$ will be the intersection of the straight line DF and the secondary axis passing through $A$. Similarly, the image of $B$ will be the intersection of $\mathrm{GF}$ and the secondary axis BC. Where $A B$ is farther from the lens than the principal focus, the image $a b$ is real and inverted. This is the case with the image of an external object formed on the retina. When the object is nearer than the principal focus, the image is virtual and erect. The image formed by the objective of a microsenpe when the olject is in focus is real and inverted ; the ocular forms a virtual erect image of this real image.

Refraction by a Biconcave Lens (Fig. 378).-Parallel rays are rendered divergent by the lens; there is no real focus : but if the rays are prolonged backwards they meet in the virtual focus $F$, from which they appear to come when received by the eye through the !ens.

Formation of Image by Biconcave Lens (Fig. 379). - Let AB be the object. Let AHDI be the path of a ray from any point $A$ of

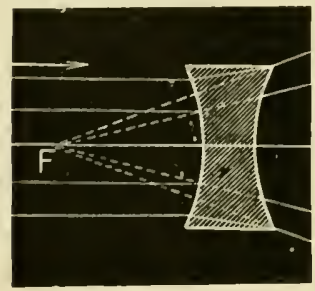

Fir. 378. -REFR.iCTION BY a Biconcave Less.

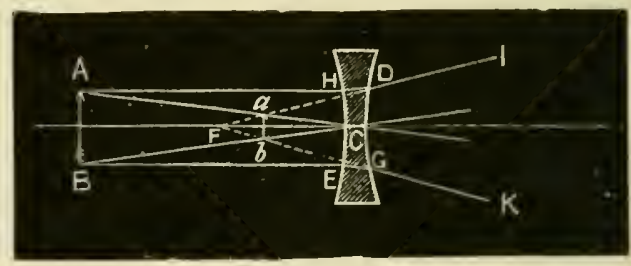

Fig. 379.-Formation of Image By Biconcall LENS.

the object parallel to the principal axis. Produce DI backwards (dotted line); it will pass through the principal focus F. Through A draw the secondary axis $\mathrm{AC}$. The image of A must lie both on $\mathrm{AC}$ and on IDF-i.e., it must be the intersection, $a$, of these straight lines. Similarly, the image of $B$ is $b$, the intersection of KGF and BC. The image is virtual and erect.

Absorption.- No substance is perfectly transparent; in addition to what is reflected, some light is always absorbed. In other words, in passing through a body some of the light is transformed into heat. a portion of the energy of the short, luminous waves going to increase the vibrations of the molecules of the medium, just as a wave passing under a row of barges or fishing-boats sets them swinging and pitching, and so imparts to them a certain amount of energy, which is ultimately changed into heat by friction against the water, and against each other, and by the straining and rubbing of the chains at their points of attacliment. Some bodies absorb all the rays in the proportion in which they occur in white light; whether looked at or looked through, they appear colourless or white. Other substances absorb certain rays by preference, and the amount of absorption is proportional to the thickness of the layer. The colours of most natural bodies are due to this selective absorption. 
Even when looked at in reflected light, they are seen by rays that have penetrated a certain way into the substince and have then been reflected : and, of course, a smuller number of the rays which the body specially absorbs are rélected than of the rays which it readily transmits, for more of the latter than of the former reach any given depth. This is called 'body colour'; and such substances have the same colour when seen by reflected and by transmitted light. The colour of hamoglobin is due to the absorption of the violet and many of the yellow and green rays, as is shown by the position of the absorption bands in its spectrum (p. 44). In Fig. 380 the violet rays are represented as being totally absorbed before passing through the silbstance. Some of the green rays are reflected, some transmitted, some absorbed. The red ralys are supposed to be mostly reflected and transmitted, only to a slight extent absorbed. The colour of such a substance, both when looked at and when looked through, would therefore be that due to a mixture of red light with a smaller quantity of green. Then there is another class of substances which owe their colour to selective reflection. Certain rays only are reflected from their surface, and the light transmitted through a thin layer is complementary to the reflected lightthat is, the reflected and transmitted rays together would make up white light. These bodies have what is called 'surface colour,' and include metals, various aniline dyes, and other substances.

Comparative.-Mlany invertebrate animals possess rudimentary sense-organs, by means of which they may receive certain luminous impressions It is truo that the mere sensation of light is not in itself sufficient for the exact appreciation of the form and situation of surrounding objects. But even the closure of the eyelids does not prevent a person of normai eyesight from distinguishing differences in the intensity of illumination. And it is possible that many of the humiler animals may, through the pigment spot:

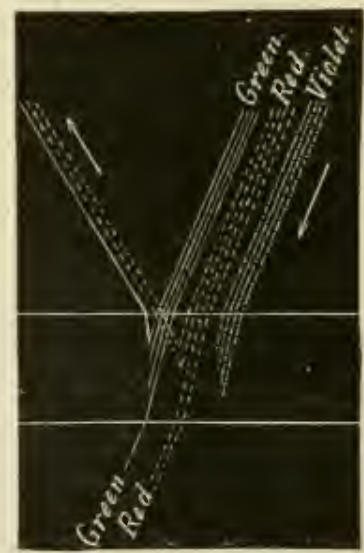

Fig. $3^{80 .--D i a t r a l i ~ t o ~ S h o w ~}$ CONNECTION OF BODY Colour with Selective ABSORPTION. which are often called eves, or perhaps, as in the earthworm, by means of end-organs more generally diffused in the skin, attain to some such dim consciousness of light and shadow as will enable them to avoid an obstacle or an enemy, to seek the sunny side of a boulder or the obscurity of an overhanging ledge of rock. But the indispensable condition of distinct vision is that an unage of each part of an object should be formed upon a separate portion of the receiving or sensitive surface. This condition is, to a certiin extent, fulfilled by the compound eyes of some of the higher invertebrates (inserts, e.g.). Here rays from one point of the object pass through one of the funnelshaped elements of the compound eve, and rays from another point through another. Rays striking obliquely on the facets are stopped by the opaque partitions between them. In the Cephalopods we find that this compound type of eye has already been abandoned; the single system of curved refracting surfaces so characteristic of the vertebrate eye has made its appearance; and the formation of a 
clean-cut image of the object on the retina, with the excitation of a sharply-bounded area of that membrane, follows as a geometrical consequence from the theory of lenses.

We have to consider (I) the mechanism by which an image is formed on the retina, and (2) the events that follow the formation of such an image and their relations to the stimulus that calls them forth.

Structure of the Eye.-The eye may be described with sufficient accuracy as a spherical shel!, transparent in front, but opaque over the posterior five-sixths of its surface, and filled up with a series of

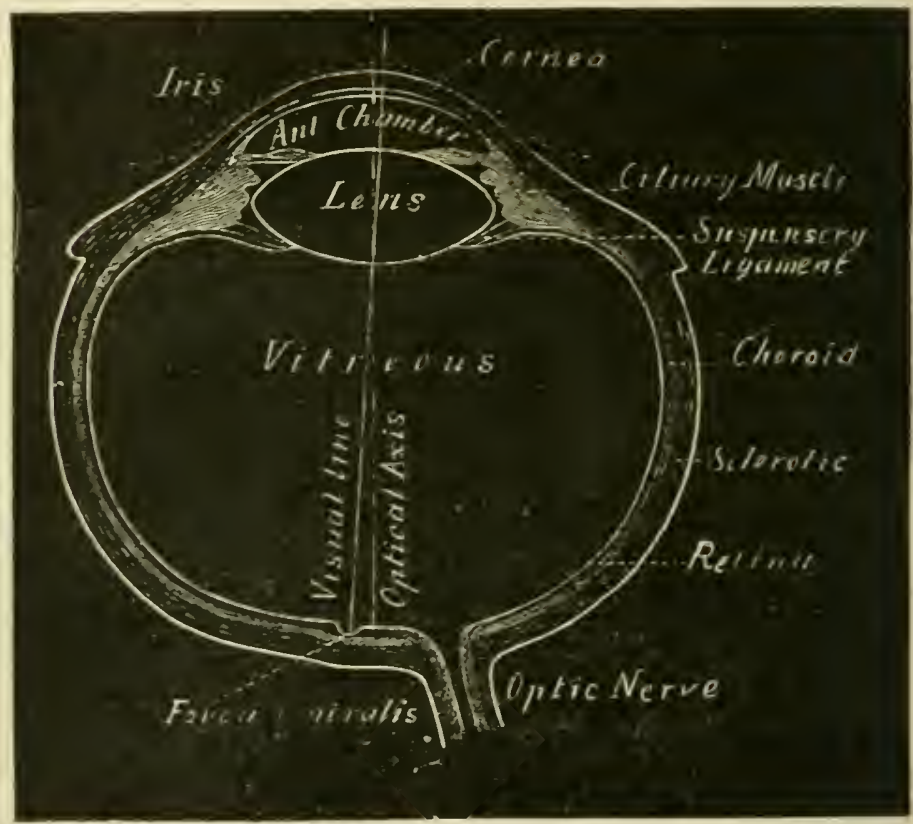

Fig. 39i,-Diagramatic ilorizontal. Section of the Left Eye.

transparent liquids and solids. The shell consists of three layers concentrically arranged, like the coats of an onion : (1) An external tough, fibrowis coat. the sclerotic, the anterior portion of which appears as the white of the eve. In front this external layer is completed by the transparent coriea. (2) A vascular layer, the choroid, which, in the restricted sense of the term, ends in front in a series of folds or plaits, the ciliary processes. The choroid contains a greater or smaller quantity of the black pigment melanin. The ciliary processes abut on the outer boundary of the iris, which may be looked upon as an anterior continuation of the choroidal or middle coat of the eyeball. Between the corneo-sclerotic junction and the anterior portion of the chorcid is interposed a ring of unstriped muscular fibres, the ciliary muscle. (3) The inner or sensitive coat, termed the retini 
(Figs. 382, 383 ). This covers the choroid as a delicate membrane, crtending to the cilizry processes, where it ends in a toothed margin, the ora serratit. The optic nevve forms a kind of stalk to which the creball is attached. Its point of entrance at the optic disc is a little nearer the median line than the antero-posterior axis, which nearly passes through the centre of a small depression, the fovea centralis, situated in the mirldle of the macula lutea, or vellow spot. From the optic disc (sometimes called the optic papilla) the optic nerve

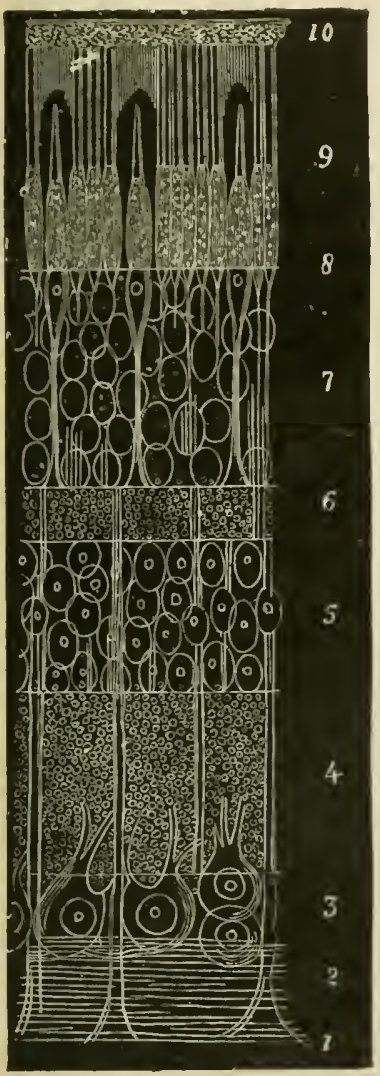

FIG. 382,-TIIE REIINA.

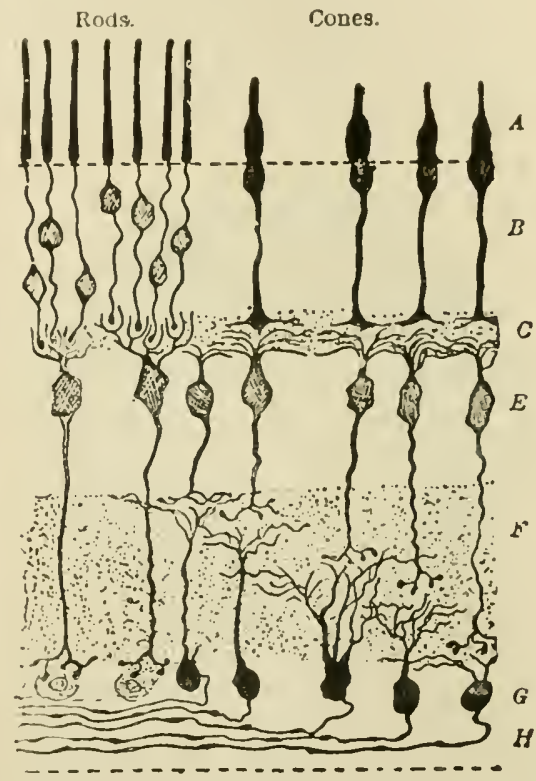

IIG. 383.-DIAGRAY OF Structure OF Retina (After Cajal).

Figs. 3\$2, 383.-I, internal limiting mell. brane; $2, H$, layer of nerve-filses ; $3, G$, laver of ganglion cells; $4, F$, internal molecular layer; $5, E$, internal nuclear layer ; $6, C$, external molecular layer ; $7, B$, externaI nuclear laver; 8 , external limiting membrane; $9, A$, layer of rods and cones; Io, pigmented epithelium.

spreads vver the retina as a layer of non-medullated fibres, scparated from the interior of the eycball only by the internal limiting membrane. This so-called membrane is formed by the expanded feet of the fibres of Müller, which run like a scaffolding or framework through nearly the whole thickness of the retina, terminating at the outer limiting membrane. External to the layer of nerve-fibres is the stratum of large ganglion cells, whose axons they are ; next to this the inner molccular layer, or inner synapse layer, made up largely of the branching dendrites of these cells. The fifth layer is the inner 
granular or nuclear laycr, containing many fusiform (bipolar) ' granule' cells which send out axons into the fourth, and dendrites into the sixth, or outer molecular layer, and are thus connected with the ganglion cells of the third layer on the one hand, and with the terminations of the rod and cone fibres of the seventh or outer nuclear layer on the other. The arborizations of the axons of these bipolar cells are situate at different levels in the internal molecular layer. The bipolar cells connected with the rod fibres send their axons right through the internal molecular layer to arborize around the bodies of the ganglion cells, whereas the axons of the bipolar cells connected with the cone fibres ramify about the middle of the layer (Fig. 383). The seventh stratım receives its name from the large number of nuclei which it contains. These belong to structures continuous with the rods and cones of the ninth layer, which is

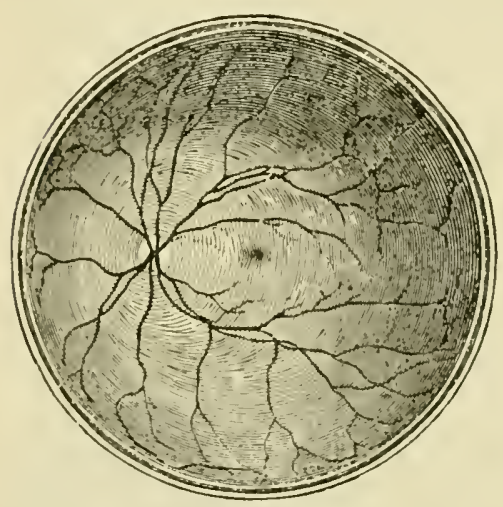

Fig. 384.-Retinal Bloodvessels (HENLE).

The arteria centralis is seen issuing from the optic disc and branching over the retina. The shaded area in the middle of the figure represents the yellow spot with the fovea centralis in its centre.

The disc is pierced by the retinal blood vessels (Fig. 384 ).

External to the rods and cones is a sheet of pigmented epithelial cells of hexagonal shape, belonging to the choroid, but remaining attached to the retina when the latter is separated, and therefore often reckoned as its most external layer.

A little behind the cornca and anterior to the retina is the lens, enclosed in a capsule, and attached to the choroid by the suspensory ligament, or zonule of Zinn. The iris hangs down in front of the lens like a diaphragm, with a central hole, the pupil. Incorporated in the stroma or framework of the iris are two arrangements of smooth muscular fibres. which confer on it the power of adjusting the size of the pupil. One of these-the sphincter pupilla-consists of a well-defined band of concentric fibres surrounding the margin of the pupil. The other-the dilator pupilax-is less sharply 
differentiateal. It is represented by radial bundles of elongated, spindle-shaped cells running in from the ciliary border of the iris towards the pupil. Between the iris and the posterior surface of the cornea is the anterior chamber of the eye. filled with the aqueous humour. Between the iris and the anterior surface of the lens lies the posterior chamber. which is rather a potential than an actual cavity. The space between the lens and the retina is accurately occupied by an almost structureless semi-fluid mass, the vitreous humour. cuclosed by the delicate hyaloid membranc, which in front is reflected over the folcls of the ciliary processes, and blends with the suspensory ligament of the lens. The attachment of the suspensory ligament is rendered firmer by the connection of this part of the hyaloid membrane to a circular fibrous portion of the vitreous. Around the edge of the lens is left a space, the canal of Petit.

Chemistry of the Refractive Media.-The aqueous humour is a perfectly colourless, watery liquicl, of slightly alkaline reaction to litmus. The specific gravity is about 1008 , and the total solicls about I per cent. Of the solicls the inorganic salts (mainly sodium chloride) constitute much the largest portion. A very small amount of protcin (o*or to $0^{\circ} \mathrm{o}_{4}$ per cent.) is present, also a little dextrose ( $0_{0} 0_{5}$ per cent.), and minute traces of urea and other substances. The liquid of the vitreous humour has a very similar composition, except that it contains a mucin-like body, hyalomucoicl, to the amount of 0.06 to 0.1 per cent. A similar mucin-like substance is present in the cornea. The freezing-point of both liquirls is a little lower than that of blood-serum, $\rightarrow$ being about $06^{\circ}$.

The lens is far richer in solids than the aqueous and vitreous humours with which it is in contact ( 30 to 35 per cent. of sclids, 60 to 65 per cent. of water). The salts, with small quantities of lecithin and cholesterin, make up about I per cent.; the balance of the solids consists of proteins. The physical alterations, with production of turbidity, which occur in the lens, and presumably in its proteins, when water enters or leaves it in too great amount through imbibition or osmosis, are of importance in connection with the etiology of cataract. The anatomical and physiological integrity of its capsule is a prime factor in the maintenance of that high degrce of transparency which is necessary for the function of the lens. Cataract can be experimentally incluced by injuring the capsule. In like manner the cornea is protected against injurious changes in its water-content (normally about 80 per cent.) and consequent turbidity by the epithelium, which separates it from the tears, and the endothelium, which scparates it from the aqueous humour.

Secretion of the Intra-ocular Liquids.-The aqueous humour is secreted by the uveal epithelium covering the ciliary processes. and to some extent by that covering the iris. As it is contin ually secreted, so it is continually absorbed, the absorbed constituents finding their way eventually into the vein or venous sinus called the camal of Schlemm and the bloodvessels of the iris and ciliary processes. The source of the liquid of the vitreous body is also the uvea. While the intra-ocular liquids differ from ordinary lymph, there is no reason to doubt that they are secretions which contribute to the nutrition of those transparent structures of the eye which are not, and, on account of their function, cannot be supplied with bloodvessels. Their most obvious use is to maintain the proper intra-ocular pressure 
on which the geometrical figure of the eycball, and therefore its efficiency as an optical instrument, depend. The bälance between secretion and absorption is accurately adjusted in health, but in disease it may be 1 pset, as in glaucoma, where the intra-ocular tension is so much increased as to interfere with the circulation, and injuriously affect the nutrition and function of the retina. Experimentally, occlusion of all the arteries supplying the head causes a rapid fall of tension, and the cornea becomes wrinkled and slack to the touch. On restoring the circulation after not too long an interval, the tension gradually returns to normal, and then becomes markedly hypernormal, even when the general arterial pressure is still low. This is probably (lue to the crippling of the clements which secrete and absorls the intra-ocular fluids, or of the capillary walls, so that a proper adjustment can no longer be attained. as happens in a tissue renlered oelematous by temporary anxmia. Where asplnyxia of the eyeball is avoiled or is brief the intra-ocular pressure varies directly as the blood-pressure in the ocnlar vessels within a wide range (Henderson and Starling).

Refraction in the Eye-Formation of the Retinal Image. -The amount of refraction which a ray of light unclergoes at a curved surface depends upon two factors--the radius of curvature of the surface, and the difference between the refractive indices of the media from which the ray comes and into which it passes. The smaller the radius of curvature, and the greater the difference of refractive index, the more is the ray bent from its original direction. A ray of light passing into the eye meets first the approximately spherical anterior surface of the cornea, covered with a thin layer of tears. Since the refractive index of the tears is much greater than that of air, the ray is strongly refracted here. The anterior and posterior surfaces of the cornea being practically parallel, and the refractive indices of the tears and aqueous humour being nearly equal, but little refraction takes place in the cornea itself. At the anterior and posterior surfaces of the lens the ray is again refracted, since the refractive index of the aqueous and vitreous humours is less than that of the lens. The following tables show the radii of curvature of the refracting surfaces and the refractive indices of the dioptric media, as well as some other data which are of use in studying the problems of refraction in the eye :

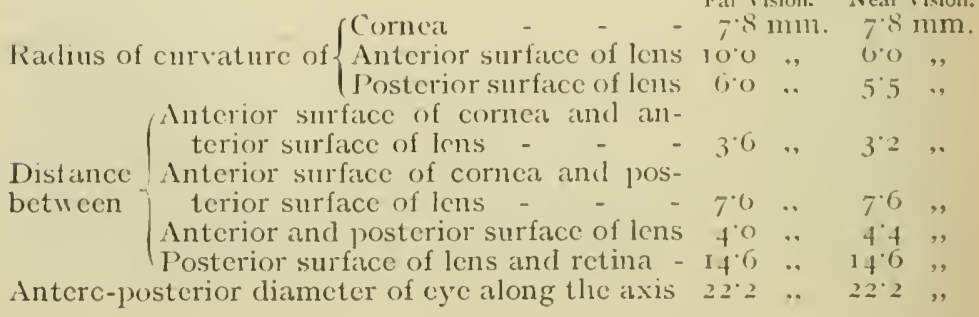

$\frac{\text { In accommodation for }}{\text { Far Vision. }} \cdot \frac{\text { Near Vision. }}{7 \text { Int }}$ 7.8 mill. $7.8 \mathrm{~mm}$. $10 \%$, $10^{\circ} \mathrm{O}$, i. $0 \quad 5 \%$, $7^{\circ}, \cdots \quad 7^{\circ}$,

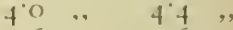
,$\quad 14^{\circ}$, 


\section{Refractive Indices -}

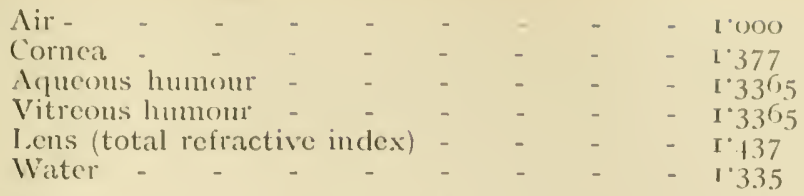

It will be seen that the refractive indices of the aqueous and vitreous humours are nearly the same as that of water. That of the lens differs for its various layers, the central core having a higher refractive index ( $\left.{ }^{\circ}+\mathrm{Ir}\right)$ than the more superficial portions $(1 ; 88)$. Although such calculations are open to error, it has been computed that the lens acts as a homogeneous lens of the same curvatures, and with a refractive index of $\mathrm{I}+37$ would do. This is called the total refractive index of the lens. The apparent paradox that it is greater than the refractive index even of the core is explained by the consideration that the core taken by itself has a greater curvature than the entire lens, and therefore causes a greater amount of refraction in proportion to its refractive index.

The optical problems connected with the formation of the retinal inage are complicated by the existence in the eye of several media, with different refractive indices, bounded by surfaces of different and, in certain cases, of variable curva-

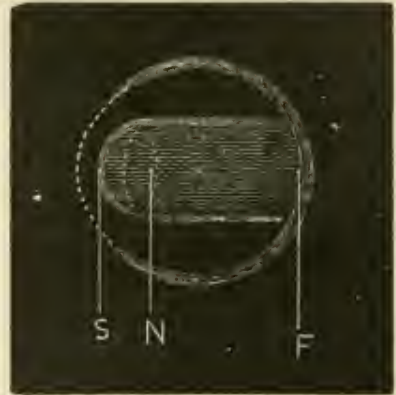

Fig. 385.-The Reduced Eye.

$S$, the single spherical refract. ing surface, $2.2 \mathrm{~mm}$. behind the anterior surface of the cornea; $\mathrm{N}$, the nodal point, $5 \mathrm{~mm}$. behind $S$ : F, the principal focus (on the retina), $20 \mathrm{~mm}$. behind $\mathrm{S}$. The cornea and lens are put in in dotted lines in the position which they occupy in the normal eye. ture. For many purposes, however, the matter can be greatly simplified, and a close enough approximation yet arrived at, by considering a single homogeneous medium, of definite refractive index, and bounded in front by a spherical surface of definite curvature, to replace the transparent solids and liquids of the eye. The principal focus being supposed to lie on the retina, the position of the nodal point-i.e., the point through which rays pass without refraction - of such a 'reduced ' or 'schematic' or ' simplified' eye, and other constants, are show'n in the following table. The single refracting surface would be situated behind the cormea and in front of the lens, at a rather smaller distance from the anterior surface of the latter than from the anterior surface of the former. The nodal point would be less than half a millimetre in front of the posterior surface of the lens (Fig. 385). The refractive index of the single transparent medinm would be a little greater than that of water. 


\section{Reduced Eye-}

Radius of curvature of the single refracting surface Index of refraction of the single refracting medium -

Antero-posterior diameter of recluced eye (distance of principal focus from the single refracting surface) -

Distance of the single refracting surface behind the anterior surface of the cornea

Distance of the nodal point of the reduced eye from its anterior surface

Distance of the nodal point from the principal focus (retina)

$$
\begin{aligned}
& 5 \cdot 1 \quad 11113 . \\
& 1.35^{*} \text {, } \\
& 20 \% \text {, } \\
& 2 \cdot 2 \quad, \\
& 5^{\circ} \mathrm{O}
\end{aligned}
$$

I $5^{\circ} \mathrm{O}$

Knowing the position of the centre of curvature of the single ideal refracting surface-i.e., the nodal point of the resluced eye-all that is necessary in order to determine the position of the image of an object on the retina is to draw straight lines from its circumference through the nodal point. Each of these lines cuts the refracting surface at right angles, and therefore passes through without any deviation. The retinal image is accordingly inverted and its size is proportional to the solid angle contained between the lines drawn from the boundary of the object to the nodal point, or the equal angle contained by the prolongations of the same lines towards the retina. This angle is called the visual angle, and evidently varies directly as the size of the object, and inversely as its distance. Thus the visual angle under which the moon is seen is much larger than that under which we view any of the fixed stars, because

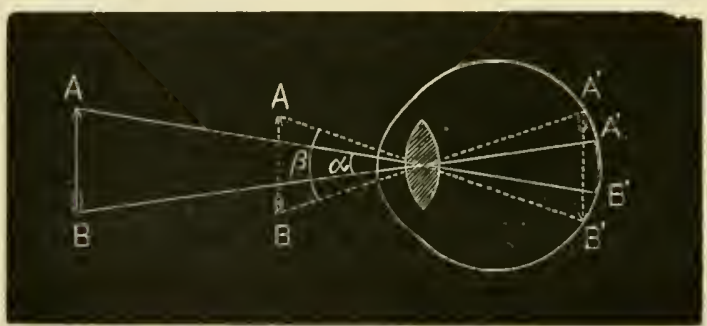

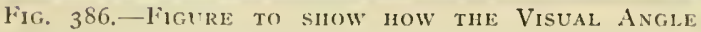
AND SIZE OF RETINAL IMAGE VARIES WITH THE JIS. TANCE OF AN OBJECT OF GIVEN SIZE.

For the distant position of $A B$ the visual angle is $\alpha$, for the near position (dotted lines) $\beta$.

$A B$ in Fig 386 represent 2 AB in Fig. 386 represent one diancter of in object, A'B the image of this diameter, and let $\mathrm{AB}^{\prime}, \mathrm{BA}^{\prime}$, be straight lines passing through the nodal point. Then $A B$ and $A^{\prime} B^{\prime}$ may be considered as parallel lines, and the triangles of which they form the bases, and the nodal point the common apex, as similar triangles. Accordingly, if $D$ is the clistance of the nodal point from $A$, and the comparative nearness of the earth's satellite more than makes up) for its relatively small size.

The dimensions of the retinal image of an object are casily calculated when the size of the object and its distance are known. For let

(n)

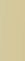




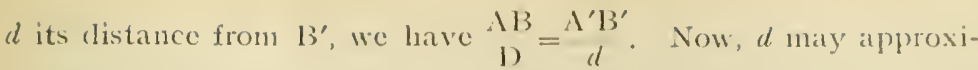
mately be taken as $15 \mathrm{~mm}$. Suppose, then, that the size of the moon's image on the retina is required. Here $D:=238$, 000 miles, and $A B$ (the diameter of the moon) $=2, x 60$ miles. 'Thus we get $\frac{2,160}{238,000}=\frac{A^{\prime} B^{\prime}}{I_{5}}$, or (say) $\frac{1}{I_{10}}=\frac{A^{\prime} B^{\prime}}{I_{5}}$, from which $A^{\prime} B^{\prime}$ (the diameter of the retinal image $)=\frac{15}{110}$, or about $1 \mathrm{~mm}$.

A ship's mast 120 feet high, seen at a distance of 25 miles, will throw on the retina an inage whose height is $\frac{120 \text { feet }}{25 \text { miles }} \times 1_{5} \mathrm{~mm}$., i.e., $\frac{120 \text { feet }}{5,280 \times 25 \text { feet }} \times 15 \mathrm{~mm}$, or $\frac{1}{1,100} \times 15 \mathrm{~mm}$., equal to $0.013 \mathrm{~mm}$. or $13 \mu$ in size. This is not much larger than a red blood-corpuscle, and only four times the diameter of a cone in the forea centralis, where the cones are most slender. In this calculation the effect of aberration (p. 912) in enlarging the image has been neglected. This effect is, of course, proportionately greater for small and distant than for large and near objects ; and it is doubtful whether the smallest possible image can be confined to an area of the retina of the size of a single cone.

Accommodation.-A lens adjusted to focus upon a screen the rays coming from a luminous point at a given distance will not be in the proper position for focussing rays from a point which is nearer or more remote. Now, it is evident that a normal eye possesses a great range of vision. The image of a mountain at a distance of 30 miles, and of a printed page at a distance of $30 \mathrm{~cm}$., can be focussed with equal sharpness upon the retina. In an opera-glass or a telescope accommodation is brought about by altering the relative position of the lenses ; in a photographic camera and in the eyes of fishes and cephalopods, by altering the distance between lens and sensitive surface ; in the eye of man, by altering the curvature, and therefore the refractive power of the lens. That the cornea is not alone concerned in accommodation, as was at one time widely held, is shown by the fact that under water the power of accommodation is not wholly lost. Now, the refractive index of the cornea being practically the same as that of water, no changes of curvature in it could affect refraction under these circumstances. That the sole cffective change is in the lens can be most easily and decisively shown by studying the behaviour of the mirror images of a luminous object reflected from the bounding surfaces of the various refractive media when the degree of accommodation of the eye is altered. Three images are clearly recognised: the brightest an erect virtual image, from the anterior (convex) surface of the cornea ; an erect virtual image, larger, but less bright, from the anterior (convex) surface 
of the lens; and a small inverted real image from the (concave) posterior boundary of the lens (Purkinje-Sanson images). The second image is intermediate in position between the other two. It is possible with special care to make out a fourth image; but since it is reflected from the posterior surface of the cornea. at which only a slight change in the refractive index occurs, it is less brilliant than the first three. When the eye is accommodated for near vision, as in focussing the ivory point of the phakoscope (Fig. 434), the corneal image is entirely unchanged in size. brightness, and position. The middle image diminishes in size, comes forward, and moves nearer to the corneal image.

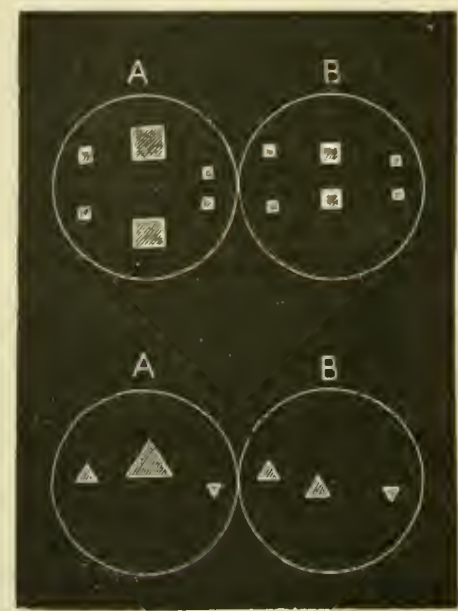

Fig. 387.-PURKINJE-SANSOX IMAGES.

$A$. in the absence of accommodation ; 13, during accommodation for a near object. The upper pair of circles en. close the images as seen when the light falls on the eve through a double slit on a pair of prisms; the lower pair show the images seen when the slit is single and triangular in shape.

This shows that the curvature of the anterior surface of the lens has been increased--that is to say, its radius of curvature diminished-for the size of the image of an object reflected from a convex mirror varies directly as the radius of curvature. A slight change takes place in the image from the posterior surface of the lens, indicating a small increase of its curvature too. Bymeans of a method founded on the observation of the changes in these images, and a special instrument called an oplithalmometer which allows of their measurement, Helmholtz has calculated that, during maximum accommodation, the radius of curvature of the anterior surface of the lens is only $6 \mathrm{~mm}$., as compared with io $\mathrm{mm}$. when the eye is directed to a distant object and there is no accommodation. When the lens has been removed for cataract, fairly distinct vision may still be obtained by compensating for its loss by convex spectacles of suitable refractive power (Io diopters* for distant vision, and I 5 diopters

* I liopter (1 D).) is the unit of refractive power generally adopted in neasuring the strength of lenses, and corresponds to a lens of 1 metre focal length. A lens of 2 cliopters (2 1).) has a focal length of $\frac{1}{2}$ metre, a lens of 4 diopters $(4 \mathrm{D}$.) a focill length of 1 metre, and so on. The diverging power of concave lenses is similarly expressed in diopters with the negative sign prefixed. "Thus, a concave lens of 1 metre focal length has a strength of -1 D., and will just neutralize a convex lens of 1 b. 
for the distance at which a book is uswally held), hut no power of accommodation remains. The person does indeed contract the pupil in regarding a near object, just as happens in the intact eye; the most divergent rays are thus cut off and the image made somewhat sharper. and there may appear to be some faculty of accommodation left. But the loss of the whole iris by operation does not affect accommodation in the least; the iris, therefore, takes no part in it. That no change in the antero-posterior diameter of the eyeball, caused by its deformation by the contraction of the extrinsic muscles, can have any share in accommodation, as has been suggested. is clearly proved by the fact that atropine, which does not affect the action of these muscles, paralyses the mechanism of accommodation. To the consideration of that mechanism we now turn.

The Mechanism of Accommodation... While everybody is agreed that the main factor in accommodation is the alteration in the curvature of the lens. there is by no means the same unanimity as to the manner in which this is brought about. Helmholtz's explanation, which has long been the most popular. is as follows: In the unaccommodated eye the suspensory ligament and the capsule of the lens are tense and taut. the anterior surface of the lens is flattened by their pressule, and parallel rays (or, what is the same thing. rays from a distant object) are focussed on the retina without any sense of effort. In accommodation for a near object, the meridional or anteroposterior fibres of the ciliary muscle by their contraction pull forward the choroid and relax the suspensory ligament. The elasticits of the lens at once causes it to bulge forwards till it is again checked by the tension of the capsule.

The explanation of Helmholtz, although wiclely adopted in the text-books, has not escaped question in the archives. Tscherning has put forward the view that when the ciliary muscle contracts, the suspensory ligament is pulled backwards and outwards. Its tension is thus increased, and the soft external layers of the lens are in consequence moulded upon the harder nucleus, so as to increase the curvature especially around the anterior pole. And Schoen, reviving a similar theory originated fifty rears ago by Mannhardt, believes that the ciliary muscle, in contracting, exerts pressure on the anterior portion of the lens, and so increases its curvature. He likens the process to the bulging of an indiarubber ball when it is held in both hands and compressed by the fingers a little behind one of the poles. It will he observed that in loth of these theories the suspensory ligament is supposed to be stretched during accommodation, not relaxed as Helunholtz supposed. While they live certain advantages over the theory of Helmholtz, particularly in taking account of the presence of radial and circular as well as meridional fibres in the ciliary muscle, they do not agree so well with such experimental tests as have been applied, and therefore Helmholtz's explanation must still be regarded as the best.

It is supported by the observation of Hess that when the ciliary 
muscle has been very strongly contracted by eserine the lens can be observed to move about with each slight movement of the eye. The suspensory ligament must therefore be slackened by the contraction of the ciliary muscle. When atropine is applied the movability of the lens soon clisappears, owing to paralysis of the ciliary muscle. These facts were first established in patients after iridectomy, but have also been demonstrated in the normal eye. Even under the influence of gravity alone, without any movements of the eye, the lens sinks about $\frac{1}{4}$ to $\frac{1}{3} \mathrm{~mm}$. in strong accommodation. An additional proof that the suspensory ligament is perfectly slack during accommodation is derived from the result of simultaneous measurements in animals of the pressure in the anterior chamber and in the vitreous. Even in strong accommodation no alteration occurs, although even slight contact with the outer surface of the eyeball or contraction of the external eye muscles causes a distinct effect. In two cavities separated by a slack inembrane no differences of pressure would be expected.

Anderson Stuart lays stress upon the function of those fibres of the suspensory ligament which are attached to the vitreous body, and are put under tension by the contraction of the ciliary muscle, in anchoring the lens during strong accommodation. He believes that the liquid contents of the hyaloid canal move from its anterior to its posterior end in accommodation, and in the opposite direction when accommodation is relaxed, and that this movement tends to prevent strains in the vitreous.

In cephalopods and fishes, which are normally short-sighted, accommodation for objects at a distance is effected by a movement of the lens towards the retina. In the fish's eve this is accomplished by the contraction of a special muscle, the retractor lentis. In amphibia and most snakes the lens is moved towards the cornca and away from the retina by changes of intra-ocular pressure (Beer).

Innervation of the Ciliary Muscle and the Muscles of the Iris.-Tho ciliary muscle and the splineter pupillac are supplied by autonomic fibres (p. 883), reaching them through the short ciliary nerves arising from the ciliary ganglion (Fig. 388). The preganglionic fibres take origin from cells in the anterior part of the oculo-motor nucleus in the mid-brain. Passing to the orbit in the third nerve, they reach the ciliary ganglion. and end there by forming synapses with some of its cells. The axons of these cells continue the path as postganglionic fibres in the short ciliary nerves. The dilator pupilla is supplied by the long ciliary neries coming from the ophthalmic branch of the fifth nerve.

The preganglionic dilator fibres pass out by the anterior roots of the first three thoracic nerves (dlog. cat, rabbit). accompanied by vaso-constrictor fibres for the iris. Reaching the sympathetic chain through the corresponding rami communicantes, they traverse the first thoracic ganglion, the annulus of Vieussens, the inferior cervical ganglion. and the cervical sympathetic. They end by arborizing around some of the cells of the superior cervical ganglion. whose axons eventually arrive at the Gasscrian ganglion. and running along the ophthalmic division of the trigeminal to the eye, reach the iris by its long ciliary branches.

The exact origin of the dilator path in the brain has not been definitely settled. Some place it in the mid-brain, others in the bulb. There must be at least one neuron on the path central to the spinal neuron whose axon cmerges from the cord as a preganglionic fibre. The lower cervical and upper thoracic portion of the spinal cord has 
received the name of the cilio-spinal region from its rclation to the pupillo-dilator fibres. It must not be looked upon as a centre in any proper sense of the term, but rather as the pathway by which these fibres pass down from the bulb, and where they may accordingly be tapped by stimulation.

Stimulation of certain areas on the cortex of the frontal lobe of the cerebrum (p. 889) causes slight dilatation of the pupil cven after the sympathetic has been divided. This is che to inhibition of the pupilloconstrictor fibres in the third nerve.

Changes in the Pupil during Accommodation.-It has been already mentioned that along with the alteration in the curvature of the lens a cliange in the clianeter of the pupil takes place in accommodation. When a distant object is looked at, the pupil

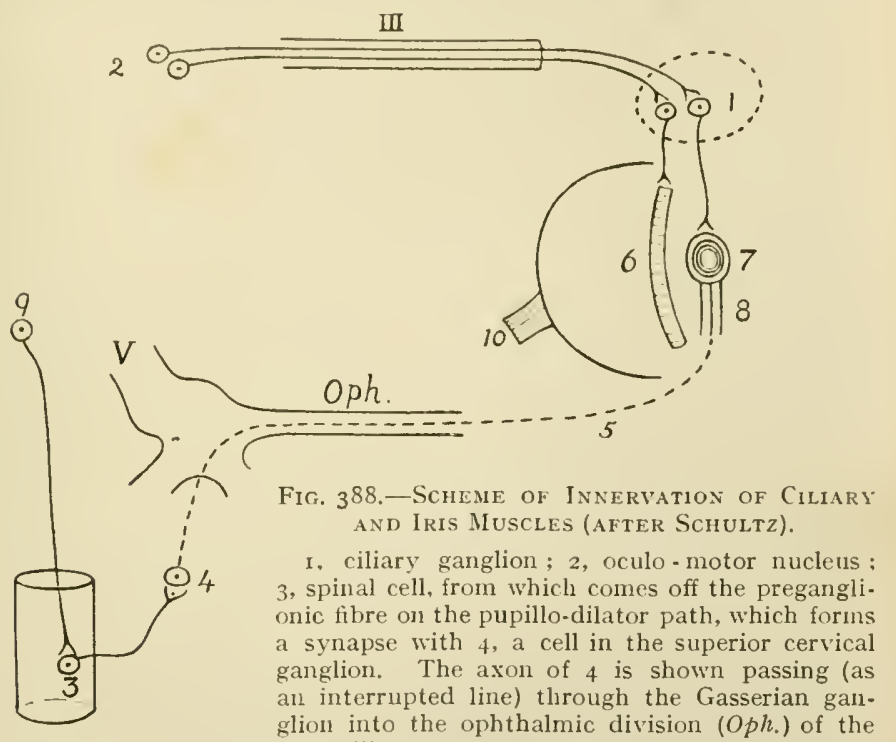

fifth nerve, $\mathrm{V}$, and thence in a long ciliary nerve, 5 , to the dilator of the iris, 8 . From I axons are shown passing by short ciliary nerves to the ciliary muscle, 6 , and the constrictor pupille, $7 ; 9$, cell of origin (in mid-brain ?) of fibre which constitutes the central neuron of the pupillo-dilator path; Io, optic nerve ; III, third nerve; V, fifth nerve with Gasserian ganglion.

becomes larger; when a near object is looked at, it becomes smaller. Narrowing of the pupil is thus associated with contraction of the ciliary muscle, and widening of the pupil with its relaxation.

This physiological correlation has its anatomical counterpart; for the third nerve supplies both the iris and the ciliary muscle. Stimulation of the nerve within the cranium causes contraction of the pupil, while stimulation of certain portions of its nucleus in the floor of the third ventricle and the Sylvian aqueduct or of the short ciliary nerves (Fig. 388), which receive branches from the third nerve, or of the ganglion itself, is followed by that change in the anterior surface of the lens which constitutes accommodation (Hensen and 
Voelckers). This can be observed either through a window in the sclerotic in a $\log$ or by following the movements of a needle thrust into the eyeball. By carefully localized stimulation near the junctim of the aqueduct with the third ventricle, it is possible to bring about the forward bulging of the lens without any change in the iris; but the normal and voluntary act of accommodation cannot be lisjoined from the corresponding alterations in the size of the pupil. Inward rotation of the eyes accompanies contraction of the pupil in accommodation, and the question may be raised whether the pupillary change is associated with the action of the extrinsic muscles of the eycball which cause convergence or with the action of the intrinsic muscles which determine the changes in the curvature of the lens. It is usually considered to be associated with both. In any case, actnal convergence is not necessary for the reaction, since it may still be obtained on accommolation when convergence is impossible on account of paralysis of the internal recti.

Changes in the Pupil produced by Light.-It is not only by accommodation that the size of the pupil may be affected. In the dark it dilates, at first rapidly; then gradually, and it maintains the width it has reached for several hours. This lias been shown by taking photograplis of the eye witl the magnesimm flashlight. In this way the width of the pupil is recorded before it has time to alter. Or a longer exposure to ultra-violet light, which affects the pupil but little, may be employed. When ordinary light falls upon the retina the pupil contracts, and the amount of contraction is rouglily proportional to the intensity of the light. Contraction of the pupil to light is brought about by a reflex meclianism, of which the optic nerve forms the afferent and the oculo-motor the efferent path, while the centre is situated in the floor of the aqueduct of Sylvius. The relation of this centre to that which controls the changes in the pupil during accommodation has not as yet been sufficiently elucidated; but this we do know, that one of the paths may be interrupted by disease, while the other is intact. For in tabes (locomotor ataxia), and in dementia paralytica (general paralysis), the light-reflex sometimes disappears, while the constriction of the pupil in accommodation and convergence still takes place (Argyll-Robertson pupil). Artificial stimulation of the optic nerve has the same effect on the pupil as the "adequate' stimulus of light; and in many animals (including man), though not in those whose optic nerves completely decussate, there is a consensual light-reflex-i.e., both pupils contract when one retina or optic nerve is excited. This should be remembered in using the pupil-reaction as a test of the condition of the retina. For although the absence of contraction may show that the retina of the eye on which the light is allowed to fall is insensible (unless there is some physical hindrance to its passage, such as opacity of the lens or cataract), the occurrence of contraction does not exclude insensibility of the retina unless the other eye has been protected from the light. 
Stimulation of the cervical sympathetic causes marked dilatation of the pupil, even when the third nerve is excited at the same time. The pupillo-dilator fibres do not act by constricting the bloodressels of the iris. For dilatation of the pupil can be caused in a bloodless animal by stimmlating the sympathetic. And even when the circulation is going on, a short stimulation of the sympathetic canses rilatation of the pupil without vasoconstriction, while with longer excitation the dilatation of the pupil begins before the narrowing of the hloodressels. Nor does it seem possible to accept the view that the sympathetic fibres are inhibitory for the sphincter muscle of the iris. They act directly upon dilator muscular fibres. It las, indeed, long been known that in the iris of the otter and of birds a radial dilator muscle exists; and it has been shown by Langley and Anderson that in the iris of the rabbit, cat, and dog, the presence of radially arranged contractile substance, different it may be in some respects from ordinary smooth muscle, must be assumed. Both the constrictor and the dilator muscles of the iris are normally in a condition of greater or less tonic contraction, so that the size of the pupil at any given moment depends on the play of two nicely balanced forces. Reflex dilatation of the pupil through the srmpathetic fibres is caused in man by painful stimulation of the skin, by dyspnœea, by muscular exertion, and in some individuals even by tickling of the palms. In animals the stimulation of naked sensory nerves has the same effect. The 'starting of the eyeballs from their sockets,' which the records of torture so often note, is due to a similar reflex excitation of the sympathetic fibres supplying the smooth muscle of the orbits and eyelids.

Action of Drugs on the Function of the Intrinsic Eye Muscles.The local application of atropine causes temporary paralysis of accommodation and dilatation of the pupil. W'hen the third nerve is divided, the pupil dilates; it dilates still more when atropine is administered after the operation. Dropped into one cye in small quantity, atropine only produces a local effect ; the pupil of the other eye remains of normal size, or somewhat constricted on account of the greater reflex stimulation of its third nerve by the greater quantity of light now entering the widely-dilated pupil of the atropinized eye. Even in the excised eye the effect of the drug is the same. Introduced into the blood atropine canses both pupils to dilate. Its action is to paralyze the endings of the oculo-motor fibres to the sphincter pupilla and ciliary muscle. Other mydriatic, or pupil-dilating drugs, are cocaine, daturine, and hyoscyamine. Phy'sostigmine or eserine. pilocarpine, and muscarine are the chief miotics, or pupil-constricting substances. They also cause spasm of the ciliary muscle, and inability to accommodate for distant objects. They act by stimulating the structures (nerve-endings) (see pp. I66, 635) which atropine paralyzes. The work of the mydriatics can bc undone by the miotics. Thus the dilatation produced by atropine is removed by pilocarpine. Adrenalin, when in jected intravenously, causes 
a flecting rlilatation of the pupil, clistinct in cats, less marked in rabbits. Subcutaneous injection has no effect. Instillation of the drug into the conjunctival sac is withont effect in the normal rabbit's eye, but causes dilatation if the superior cervical ganglion lats been removed.

Functions of the Iris.-In vision the iris performs two chief functions: (I) It regulates the quantity of light allowed to fall upon the retina. The larger the aperture of a lens, the greater is its collecting power, the more light does it gather in its focus. In the eye, the area of the pupil determines the breadth of the pencil of light that falls upon the lens. If this area was invariable, the retina would either be " dark from excess of light ' in bright sumshine, or dark from defect of light in dull weather or at dusk. In order that the iris may act as an efficient diaphragm it must be pigmented, and it is the pigment in it which gives the colour to the normal eye. The vision of albinos, in whose eyes this pigment is wanting, is often, though not

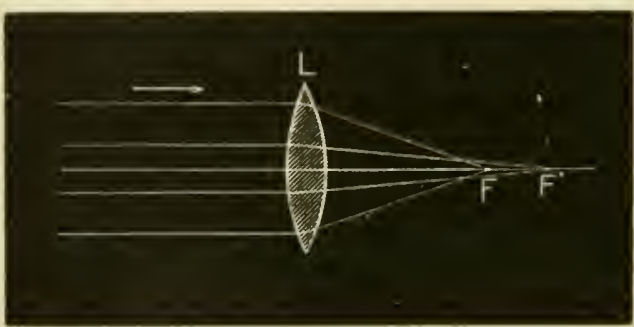

Fig. 389.-Spilerical Aberration.

Rays passing through the more peripheral parts of a biconvex lens $L$ are brought to a focus $F$ nearer the lens than $F^{\prime}$, the focus of rays passing through the central portions of the lens. invariably, deficient in sharpness. There is always intolerance of bright light; and the same is true in the condition known as irideremia, or congenital absence or clefect of the iris.

(2) Another, and perhaps equally inportant, function of the iris is to cut off the more divergent rays of a pencil of light falling upon the eye, and thus to increase the sharpness of the image. This leads us to the consideration of certain defects in the dioptric arrangements of the eye.

Defects of the Eye as an Optical Instrument.--(I) Spherical Aberration.-It is a property of a spherical refracting surface that rays of light passing through the peripheral portions are more strongly refracted than rays passing near the principal axis. Hence a luminous point is not focussed accurately in a single point by a spherical lens; the image is surrounded by fainter circles of light, the sG-called circles of diffusion representing the rays which liave not yet come to a focus, or having been already focussed have crossed and are now diverging. In the eye this spherical aberration is partly corrected by the interposition of the iris. which cuts off the more peripheral rays, especially in accommodation for a near object, when they are most divergent. In addition, the anterior surfaces of the cornea and lens are not segments of spheres, but of ellipsoids, so that the curvature dininishes somewhat with the distance from the optic axis, and, therefore, the refracting power as we 
pass away from the axis cloes not increase so rapidly as it would rlo if the surfaces were truly splecical. Further, the refractive index of the periplieral parts of the lens is less thun that of its central portions.

(2) Chromatic Aberration. - All the rays of the spectrum (lo not travel with the same velocity through a lens, and are, therefore, unequallv refracted $W$ it, the short violet rays being focussed nearer the lens than tlie long red rays. It was at one time supposed that this clromatic aberration, as it is called, is compensated in the eye; and it is said that this mistake gave the first hint that Newton's dictum as to the proportionality between deviation and dispersion was erroneous, and led to the discovery of achromatic lenses. But in reality the eve is not an achromatic combination; and the violet rays are focusserl abont $\& \mathrm{~mm}$. in front of the red. Thus, in Fig. 390 the white light passing through the lens is broken up into its constituents : the violet focus is at $V$, and the red at $R$, behind it. A screen placed at $R$ would show not a point image, but a central point surrounded by concentric circles of the spectral colours, with violet outside. If the screen was placed at $Y$, the centre would be

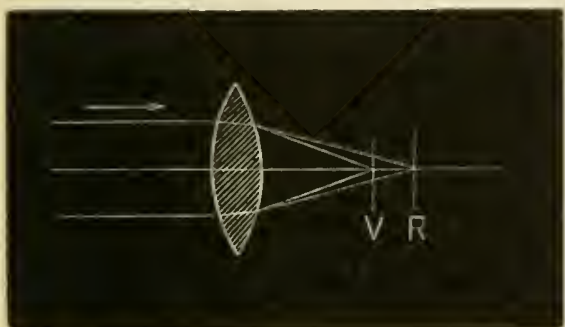

Fig. 390.-CHROMATIC ABERRATION.

The violet rays are brought to a focus $\mathrm{V}$ nearer the lens than $R$, the focus of the red rays.

violet and the red would be external. For this reason it is impossible to focus at the same time and with perfect sharpness objects of different colours: a red light on a railway track appears nearer than a blue light, partly perhaps for the reason that it is necessary to accommodate more strongly for the red than for the blue, and we associate stronger accommodation with shorter distance of the object, although other data are also involved in such a visua judgment. When we look at a white gas-flame through a cobalt glass, which allows only red and violet to pass, we see either a red flame, surrounded by a riolet ring, or a violet flame surrounded by a red ring, according as we focus for the red or for the violet rays. But the dispersive power of the eye is so small, and the capacity of rapidly altering its accommodation so great, that no practical inconvenience results from the lack of achromatism, which, however, mar be easily demonstrated by looking at a pattern such as that in Fig. $39 \mathrm{I}$ at a distance too small for exact accommodation.

It is also reckoned among the optical imperfections of the eye (3) that the curved surfaces of the cornea and lens do not form a centred' sristem-that is to say, their apices and their centres of 
curvature do not all lie in the same straight line; (4) that the pupil is eccentric, being situated not exactly opposite the middle of the lens and cornea, but nearer the nasal side, and that in consequence the optic axis, or straight line jcining the centres of curvature of the lens and cornea, does not coincide with the visual axis, or straight line joining the fovea centralis with the centre of the pupil, which is also the straight line joining the centre of the pupil and any point to which the eye is directed in vision. The angle between the optic and visual axis is about $5^{\circ}$ (Fig. 38I). (5) Musca volitantes, the curious bead-like or fibrillar forms that so often flit in the visual field when one is looking through a microscope, are the token that the refractive media of the eye are not perfectly transparent at all parts : they seem to be due to floating opacities in the vitreous humour. probably the remains of the embryonic cells from which the vitreous body was developed. (6) Lastly, it may be mentioned that slight irregularities in the curvature of the lens exist in all cyes, so that a point of light, like a star or a distant street-lamp, is not seen as a point, but as a point surrounded by rays (irregular astigmatism). In bringing this review of the imperfections of the dioptric media of the

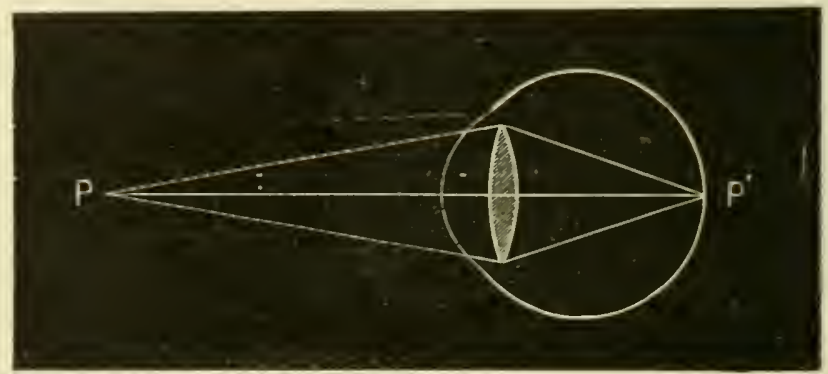

Fig. 392.-Refraction in the (Noraral) Emmetropic Eye.

The image $\mathrm{P}^{\prime}$ of a distant point $\mathrm{P}$ falls on the retina when the eye is not accommo. dated. To save space, $\mathrm{P}$ is placed mich too near the eye in Figs. $392,393$.

normal eyc to a close, it may be well to explain that what are defects from the point of view of the student of pure optics are not necessarily defects from the freer standpoint of the physiologist, who surveys the mechanism of vision as a whole, the relations of its various parts to one another and to the needs of the organism it has to serve, the long series of developmental changes through which it has come to be what it is, and the possibilities, so far as we can limit them, that were open to evolution in the making of an eye. The optician may perhaps assert, and with justice, that he could easily have made a better lens than Nature has furnished, but the physiologist will not readily admit that he could have made as good an eye.

While the defects hitherto mentioned are shared in greater or less degree by every normal eye, there are certain other defects which either occur in such a comparatively small number of eyes, or lead to such grave disturbances of vision when they do occur, that they must be reckoned as abnormal conditions. In the normal or emmetropic eye, parallel rays-and for this 
purpose all rays coming from an object at a distance greater than 65 metres may be considered parallel-are brought to a focus on the retina without any effort of accommodation. The distance at which objects can be distinctly seen is only limited by their size, the clearness of the atmosphere, and the curvature of the eartls; in other words, the punctum remotum, or far-point of rision, the most distant point at which it is possible to see with distinctness, is practically at an infinite distance. When accommodation is paralyzed by atropine, only remote objects can be clearly seen. On the other hand, the normal eye, or, to be more precise, the normal eye of a middle-aged adult, can be adjusted for an object at a distance of not more than $12 \mathrm{~cm}$. (or 5 inches). Nearer than this it is not possible to see distinctly; this point is accordingly called the punctum proximum

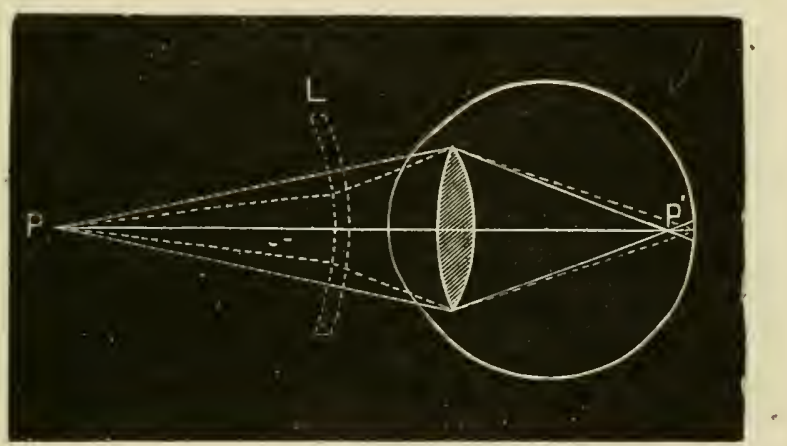

Fig. 393.-IIYoric EyE.

The image $\mathrm{P}^{\prime}$ of a distant poirt $\mathrm{P}$ falls in front of the retiza, even without accommodation. By means of a concave lens $L$ the image may be made to fall on the retina (dotted lines).

or near-point. The range of accommodation for distinct vision in the emmetropic eye is from $\mathrm{I} 2 \mathrm{~cm}$. to infinity.

Myopia, or short-sightedness, is generally due to the excessive length of the antero-posterior diameter of the eyeball in relation to the converging power of the cornea and the lens. Even in the absence of accommodation, parallel rays are not focussed on the retina, but in front of it ; and in order that a sharp image may be formed on the retina the object must be so near that the rays proceeding from it to the eye are sensibly divergent-that is to say, it must be at least nearer than 65 metres-but as a rule an object at a distance of more than 2 to 3 metres cannot be distinctly seen. With the strongest accommodation the near point may be as little as $3 \mathrm{~cm}$. from the eye. The range of vision in the myopic eye is therefore very small. The defect 
may be corrected by concave glasses, which render the rays more divergent. It is to be noted that many cases of internal squint in children are connected with myopia, the eyes necessarily rotating inwards as they are made to fix an abnormally near object. The treatment both of the squint and the myopia in these cases is the use of concave spectacles (Fig. 393). Myopia, althougl a condition that shows a distinct hereditary tendency, is rarely present at birth; the elongation of the anteroposterior diameter of the eyeball develops gradually as the child grows.

In hypermetropia, or long-sightedness, the eye is, as a rule, too short in relation to its converging power; and with the lens in the position of rest, parallel rays would be focussed bchind the retina. Accordingly, the hypermetropic eye must accommodate even for distant objects, while even with maximum accom-

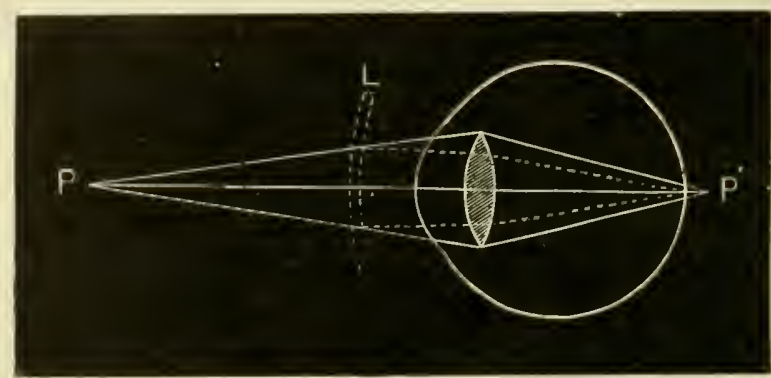

Fig. 394.-HyPermetropic Eye.

The image $\mathrm{P}^{\prime}$ of a point $\mathrm{P}$ falls behind the retina in the unaccommodated eye. By means of a convex lens L it may be focussed on the retina without accoin. modation (dotted lines).

modation an object cannot be distinctly seen unless it is farther away than the near-point of the emmetropic eye. The farpoint of distinct vision is at the same distance as in the emmetropic eye-viz., at infinity-the near-point is farther from the eye. The defect is corrected by convex glasses (Fig. 394). Hypermetropia, unlike myopia, is present at birth.

Presbyopia, or the long-sightedness of old age, is not to be confounded with hypermetropia. It is essentially due to failure in the power of accommodation, chiefly through weakness of the ciliary muscle, but partly owing to increased rigidity and loss of elasticity of the lens. Images of distant objec s are still formed on the retina of the unaccommodated eye with perfect sharpness-i.e., the far-point of $\mathrm{v}$ sion is not affected. But the eye is unable to accommodate sufficiently for the rays diverging from an object at the ordinary near-point; in other 
words, the near-point is farther away than normal. Convex glasses are again the remedy.

The near-point of distinct vision can be fixed in various ways -among others, by means of Scheiner's experiment (Practical Exercises, p. 987). Two pin-holes are pricked in a card at a distance less than the diameter of the pupil. A needle viewed through the holes appears single when it is accommodated for, double if it is out of focus. The near-point of vision is the nearest point at which the needle can still, by the strongest effort of accommodation, be seen single.

Astigmatism.-It has been mentioned that slight differences of curvature along different meridians of the refracting surfaces exist in all eyes. But in some cases the difference in two meridians at right angles to each other is so great as to amount to a serious defect of vision. To this condition the name of ' astigmatism_' or ' regular astigmatism' has been given. It is usually due to an excess of curvature in the vertical meridians of the cornea, less fre-

quently in the horizontal meridians ; occasionally the defect is in the lens. Rays proceeding from a point are not focussed in a point, but along two lines, a horizontil and a vertical, the horizontal linear focus being in front of the other when the vertical curva-

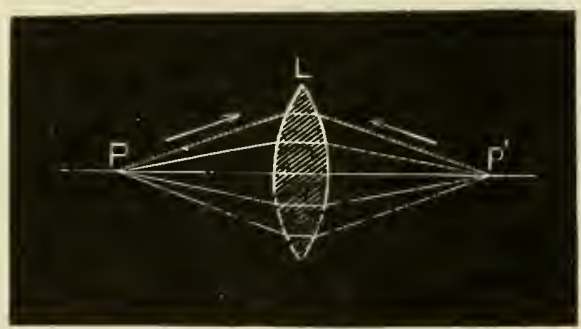

FIG. 395. ture is too great, behind it when the horizontal curvature is excessive. The two limbs of a cross or the two hands of a clock when they are at right angles to each other cannot be seen distinctly at the same time, although they can be successively focussed. The condition may be corrected by glasses which are segments of cylinders cut parallel to the axis (Practical Exercises, p. 989).

The Ophthalmoscope.-The pupil of the normal eye is dark, and the interior of the eye invisible, without special means of illuminating it. But this is not because all the light that falls upon the fundus is absorbed by the pigment of the choroid, for even the pupil of an albino appears dark when the eye is covered by a piece of black cloth with a hole in frent of the pupil. The explanation is as follows :

Let the rays from a luminous point, $\mathrm{P}$, be focussed by the lens, $\mathrm{L}$, at $\mathrm{P}^{\prime}$ (Fig. 395). It is plain that rays proceeding from $\mathrm{P}^{\prime}$ will exactly retrace the path of those from $\mathrm{P}$ and be focussed at 
P. Now, the eye receives rays from all directions, and, when it is sufficiently well illuminated, sends rays out in all directions. The moment, however, that the observing eye is placed in front of the obscrved eye, the latter ceases to receive light from the part of the field occupied by the pupil of the former, and therefore ceases to reflect light in to it.

This difficulty is avoided by the use of an ophthalmoscopic mirror. The original, and theoretically the most perfect, form of such a mirror is a plate or several superposed. plates, of glass, from which a beam of light from a laterally placed candle or lamp is refiected into the observel eye, and through which the eye of the observer looks (Fig. 395). But the illumination thus obtained is comparatively faint; and a concave mirror is now

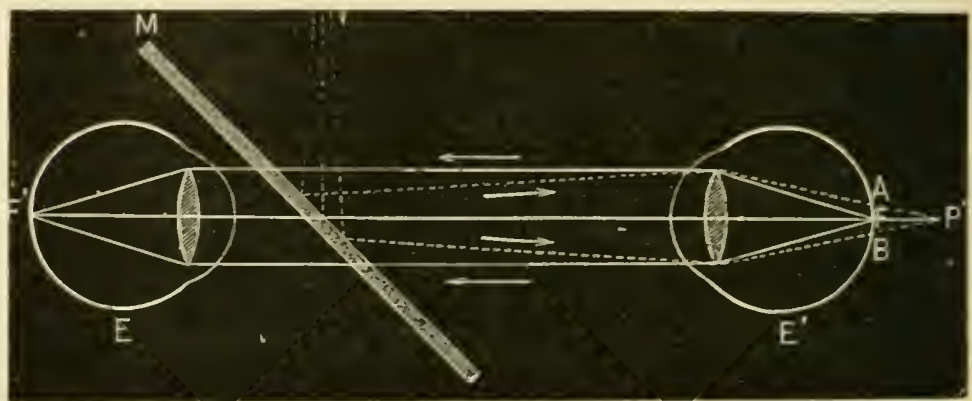

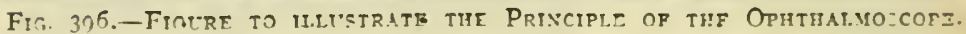

Rays of light from a point $y$ are reflected by a glass plate II (several plates together in Helmholtz's original form) into the observed eve $E^{\prime}$. Their focus would fall, as shown in the figure. at P', a little belind the rctina of $E$. The portion of the retina $A B$ is therefore illuninated by diffusion circles; and the rays from a point of it $\mathrm{F}$ will, if $\mathrm{F}^{\prime}$ is emmetropic and unaccommodated, issue parallel from $E^{\prime}$ and be brought $t$ a focus at $F^{\prime}$ on the retina of the (emmetropic and unaccommodated) observing eye E.

generally used. In the centre is a small hole or a small unsilvered portion of the mirror for the observer's eye. In the direct method of examination (Fig. 397), the mirror is held close to the observed eye, and an erect virtual image of the fundus is seen. When the eye of the observer and of the patient are both emmetropic, and both eyes are unaccommodated, the rays of light proceeding from a point of the retina of the observed eye are rendered parallel by its dioptric media, and are again brought to a focus on the observer's retina.

If the observed eye is myopic, the rays of light coming from a point of the retina leave the eve, even when it is unaccommoclated, as a convergent pencil: and the emmetropic nonaccommodated cye of the observer must have a concave lens 
placed before it in order that the funclus may be distinctly seen.

When the observed eye is hypermetropic, the rays emerging from the unaccommodated eye are divergent, and a conves

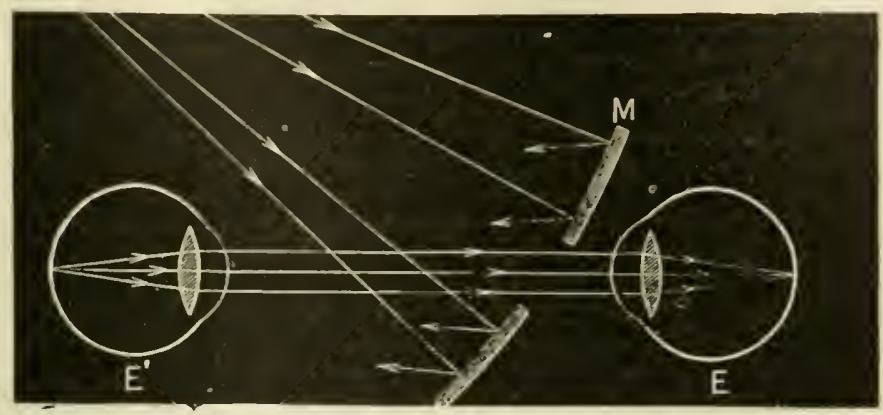

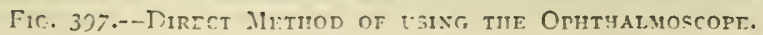

Light faling on the perforated concave mirror II passes into the observed eye $F^{\prime}:$ and, both $E^{\prime}$ and the observing eye $E$ being supposed emmetropic and unaccomniodated, an erect virtual image of the illuminated retina of $\mathrm{F}^{\prime}$ is seen by $\mathrm{E}$.

lens, the strength of which is proportional to the amount of hypernetropia, must be placed befoce the observer's unaccommodated eye if he is to see the fundus distinctly. By ascommo.

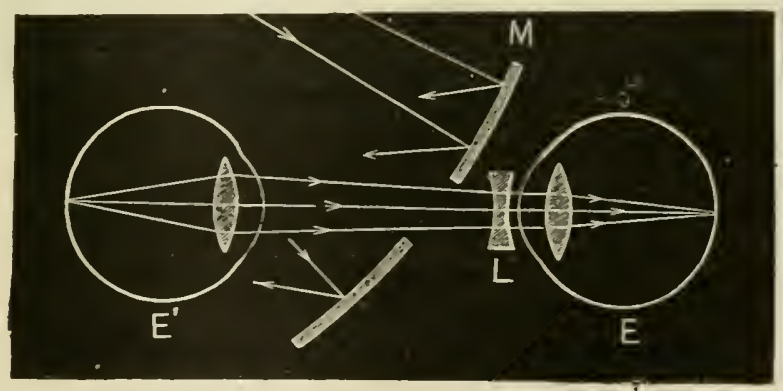

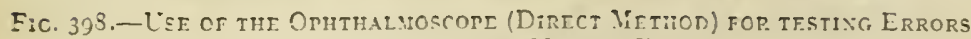
of RFFraction in MYYopic EYe.

Rays issuing from a point of the retina of F', the observed (myopic and un. accommodated) eyc, pass out, not parallel, but convergent. They will therefore be focussed in front of the retina of the observing (unaccommodated) ere $\mathrm{E}$ if the latter is emmetrosic. By introducing a concave lens $\mathrm{L}$ cf suitable strength, however, a clear view of the retina of $E^{\prime}$ will be obtained, and the strength of this lens is the measure of the amount of myopia.

dating, the observer can see the fundus clearly without a convex lens.

By this method errors of refraction in the eye may be detected 
and measurect. The olserver must always keep his eye unaccommodated, and if it is not emmetropic, he must know the amount of his short- or long-sightedness-i.e., the strength and sign of the lens needed to correct his defect of refraction, and must allow for this in calculating the defect of his patient. Non-accommodation of the eye of the latter can always be secured by the use of atropine.

By the direct method of ophthalmoscopic examination, only a small portion of the retina can be seen at a time, and this is highly magnified. A larger, though less magnified, view can be got by the indirect method. The observed eye is illuminated as before, but the mirror and the observer's eye are at a greater distance (Fig. foo). Here the rays from a considerable portion of the retina are brought to a focus by a convex lens held near

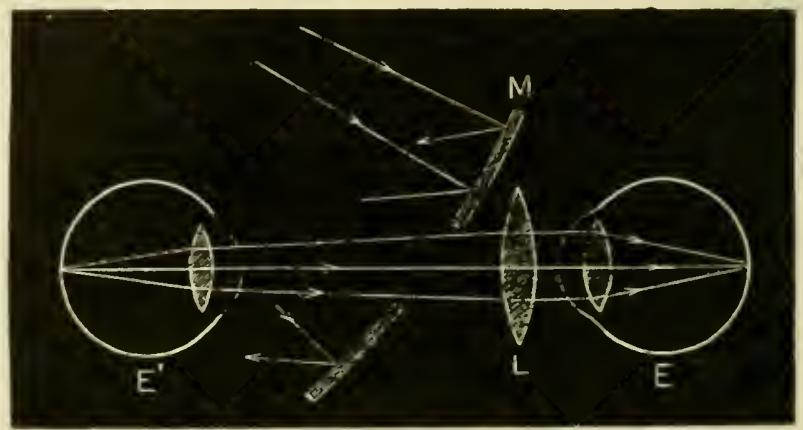

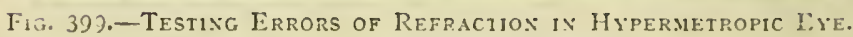

Rays from a point of the retina of $\mathrm{F}$, the observed eye, issue divergent, and are focussed behind the retina of the observing (unacconimodated and emmetropic) eve $E$. The strength of the convex lens L, which must be introduced in front of $\mathrm{E}$ to give clear vision of the retina of $\mathrm{E}^{\prime}$, measures the degree of hypermetropia.

the eye of the patient. so as to form a real and inverted aerial image of the retina. This image is viewed by the observer at his ordinary visual distance. It is not necessary in this method that the observed eye should be non-accomnodated, although it is convenient as in the direct method to cause dilatation of the pupil by atropine, which also relaxes the accommodation (Practical Exercises, p. 994).

Skiascopy.-To a great extent the ophthalmoscopic method of measuring errors of refraction has been replaced by the more modern method of skiascopy (shadow test). It depends upon the following observation: When one throws light from a little distance with a concave mirror into an observed eye and then rotates the mirror slowly around the long axis of the handle, one sees that the pupil, which at first was completely illuminated, 
becomes dark from one side as if covered by a shadow. This shadow will move in the same direction in which the mirror is rotated or in the opposite direction, according to whether the observer is farther from the observed eye than its far-point, or between the eye and the far-point. If the observer is exactly at the far-point, no direction of movement of the shadow can be made out, but the pupil in its whole extent is either illuminated or altogether dark. In this way the distance of the far-point of a myopic eye can be easily determined by a metre rule, and from this the degree of myopia. If the far-point is either too near, as in strong myopia, or too distant, as in weak myopia.

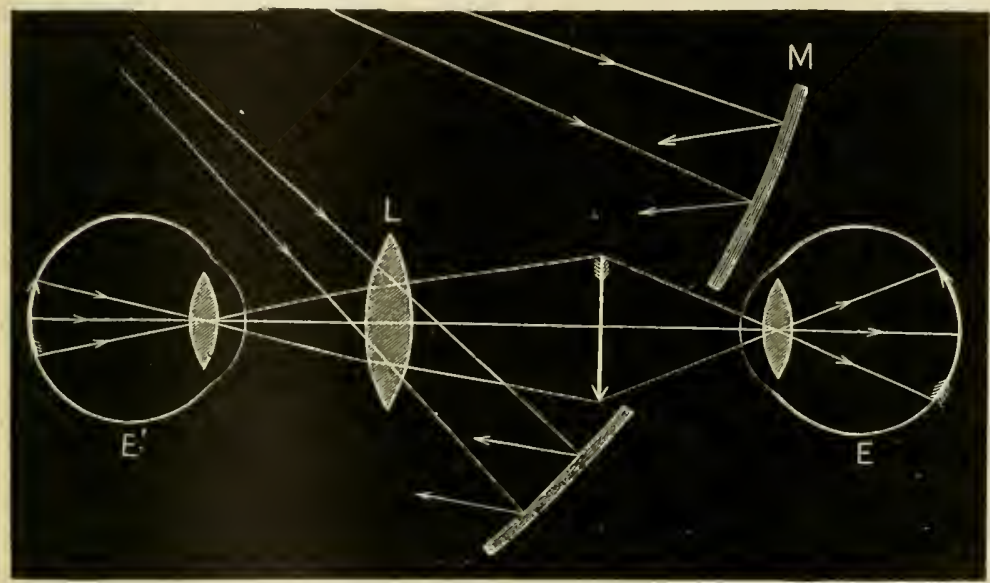

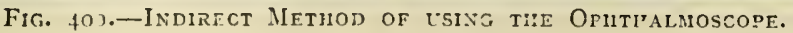

The rays of light issuing from $\mathrm{F}^{\prime}$, the coserved eye, are focussed by the biconvex lens $\mathrm{L}$, and a real inverted image of a portion of the retina of $\mathrm{E}^{\prime}$, magnified four or five tines, is formed in the air between the lens and the observing eye $\mathrm{E}$. This image is viewed by $\mathrm{E}$ at the ordinary distance of distinct vision (Io to I 2 inches). (The exaggeration of the size of the mirror makes it appear as if some of the rays from the lamp passed through the lens before being reflected from the mirror. This would not be the case in an actual observation.)

and emmetropia, or behind the observed eye, as in hypermetropia, it can be brought to a convenient distance by interposing suitable lenses. The observer then determines the far-point exactly by moving his eye nearer to or farther from the observed eye, or, keeping his own eye fixed, by bringing the far-point of the observed eye to coincide with it by inserting lenses (Practical Exercises, pp. 994, 995).

The phenomenon depends upon the interruption which the light proceeding from the observed retina experiences first at the margin of the pupil of the observed eye, and then at the margin of the hole 
in the mirror or of the observer's pupil. When the mirror is rotated, an illuminated point of the observed retina will move in the opposite direction over the retina.* The light procecding from this point when the observed eyc is emmetropic is so refracted by the lens and cornea that it leaves the eye as a bundle of parallel rays in the direction of the image of the source of light (I') (Fig. +o1). If the image of the flame reflected by the mirror is situated on the principal axis of the observer's eye, and if the pupils of observed and observer are of equal size, all the rays coming from the observed retina will fall on the observer's retina, and therefore the whole pupil of the observed eye will appear light. If the mirror is now rotated so that the image of the source of light moves away from the principal axis, and the illuminating rays are no longer in that axis, the illuminated point will move in the opposite direction from the principal axis, and the light returning from the pupil of the observed eye will again issue in the direction of the imige of the source of light. It can then happen that none of the rays hit the observer's pupil, and the observed pupil wil! appear entirely dark. Or the direction of the

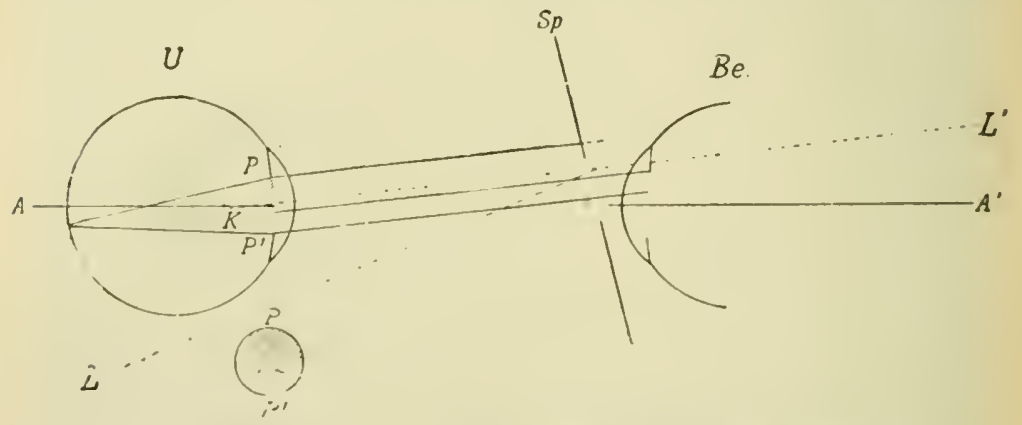

Fig. 401.-Path of Rays in Skiascopy (SNellen).

$U$, observed eve : $B e$, eye of observer ; $S p$, mirror ; $L$, source of light ; $L$, image of the source of light ; $A, A^{\prime}$, principal axis ; $I^{\prime}, P^{\prime}$, pupils.

rays may be such that a portion of them enters the observer's pupil. the rest being interrupted by its border. In this case the part of the observed pupil from which rays enter the observer's pupil will appear light, while the rest is dark. From Fig. for it can be seen that the light part of the observed pupil is on the opposite side of the principal axis from the image of the source of light. If, therefore. the image of the source of light moves to the right (by rotation of a concave mirror to the right, or rotation of a plane mirror to the left) the skiascopic appearance in the observed pupil moves to the left-i.e., in the opposite direction to the image of the source of light.

If the observed pupil is myopic-i.e., if its far-point is between the observer and the observed eye, rotation of the mirror so far from the principal axis that only a part of the rays issuing from the observed pupil enter the observer's eye, will cause the pupil to appear

* When a concave mirror is rotated to the right, the inverted real mirror image also moves to the right, and the illuninated point to the left. When a plane mirror is rotated to the riglit, the virtual mirror image moves to the left, and the illuminated point on the retina therefore to the right. 
light only on one side, and on account of the crossing of the rays this illuminated portion will be on the same siele of the principal axis as the image of the source of light (Fig. 402). When the image of the source of light is moved to the right the light area of the observed pupil will also move to the 1 ight i.e., with rotation of a concave mirror in the same direction as the image of the source of light. and with rotation of a plane mirror in the opposite direction (Snellen).

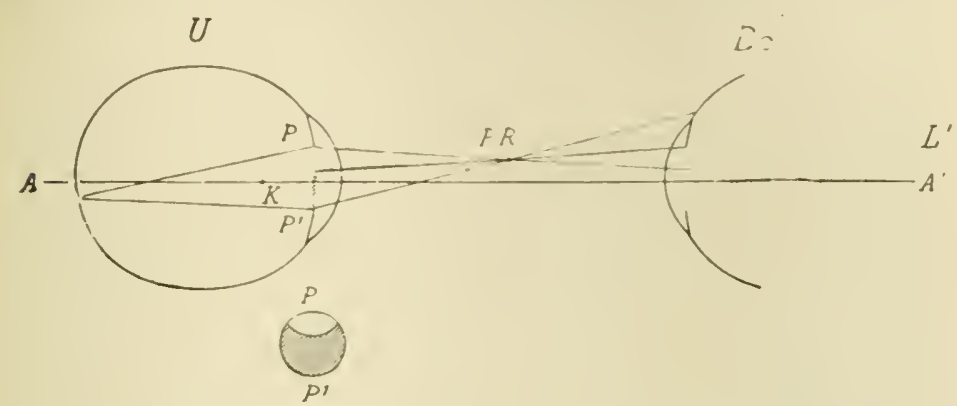

Fig. 402,- Path of RaYs IN SkiAscopy (MYopic EYe) (S.IEllex).

$P R$, far-point of observed eye. The other references are as in Fig. $40 \mathrm{r}$.

A method of photographing the retina in the living eye which has recently been employed with success bids fair to become an important supplementary means of investigating the funclus (Dogiel).

Single Vision with Both Eyes-Diplopia.-Scheiner's experiment shows that it is possible to have double vision, or diplopia, with a single eye when two separate images of the same object fall upon different parts of the retina. In vision with both eyes, or binocular vision, an image of every object looked at is, of course, formed on each retina. and we have to inquire how it is that as a rule thes? images are blended in consciousness so as to produce the perception of a single object: and how it is that under certain conditions this blending does not take place, and cliplopia results. Two chief theories have been involied in the attempt to answer these questions : (I) the theory of identical points, (2) the theory of projection.

In regard to the sccond theory, we shall merely say that it assumes that in some way or other the retina, or, rather, the retino-cerebral apparatus, has the power of appreciating not only the shape and size of an image, but also the direction of the rays of light which form it, and that the position of the object is arrived at by a process of mental projection of the image into space along these directive lines. Where the directive lines of the two eyes cut each other the two images coincide, and the object is seen single in the position of the point of intersection. The first theory we shall examine in some detail.

The Theory of Identical Points. - This theory assumes that cvery point of one retina 'corresponds' to a definite point of the other retina. and that in virtuc of this correspondence, either by an inborn necessity or from experience, the mind refers simultaneous impressions upon two corresponding or identical points to a single point in external space. If we imagine the two retinz in the position which the cyes occupy when fixing an infinitely distant object-that is, with the visual axes parallel-to be superposed, with fovea over 
fovea, every point of the one retina will be covered by the corresponding point of the other retina, so that identical points could be pricked threugh with a needle. But since the actual centre of the retina does not correspond with the forea centralis (Fig. 38r), but lies nearer the nasal side, the nasal edge of the left retina will cverlap the temporal edge of the right, and the nasal edge of the right will overlap the temporal edge of the left; so that a part of each retina has no corresponding points in the other.

The adherents of this theory claim, and with justice, that a small object so situated that its image must be formed on corresponding points of the two retina does, as a rule, appear single, and, what is even more striking, that a phosphene, or luminous ring produced by pressing the blunt end of a pencil or the finger-nail on a point of the globe of one eye (which Newton compared to the circles on a peacock's tail), is not doubled by pressure over the corresponding point of the other eyc, although two circles are seen when pressure is made upon points which do not correspond. If in rotating the eyes one eve is prevented by pressure with the finger from following the movement of the other, there is double vision. When strabismus or squinting is produced by paralysis of the third (p. 819 ) or the sixth cranial nerve (p. 822), it is accompanied by diplopia, until in course of time the mind learns to disregard one of the images. In some cases of squint the double images are never ccmpletely suppressed, but a new abnormal form of visual localization is developed, which, however, very seldom permits any accurate judgment of depth. In strabismus it is obvious that the two images of an object cannot fall on correspondling points.

But it is also a fact that, under certain conditions, images situated on corresponding points may not, and that images not situated on corresponding points may. give rise to a single impression. For example, if one of the closed eycs be held slightly out of its ordinary position by the finger, pressure on identical points of the two eyes gives rise to two separate phosphenes. And some of the phenomena of stercoscopic vision (p. 925) show clearly that images falling on points not strictly corresponding may give a single impression ; while we do not habitually see double, although it is certain that the images of multitudes of objects are constantly falling on points of the retinæ not anatomically 'identical.'*

The question therefore arises, How is it that we lo not see these double images? This is one of the difficulties of the theory of identical points. The following is a partial explanation : (I) The images of objects in the portion of the field most distinctly seenthat is, the portion in the immediate neighbourhood of the intersection of the visual lines, or the part to which the gaze is directed-

* In every fixel position of the eyes, the objects whose images fall on corresponding points will be arranged on certain definite lines or surfaces which vary with the direction of the visual axis and to which the name of horopter, or point-horopter, has been given. For most eyes when directed to the horizon-that is, with the visual axes parallel-the horopter is practically the horizontal plane of the ground, so that all objects within the field of vision, and resting on the ground, fall upon corresponding points, and are seen single. When the eyes are directed to a point at such a distance that the lines of vision are sensibly convergent, the horopter consists ( 1 ) of a straight line drawn through the fixing-point and at right angles to a plane passing through the fixing-point and the two visual lines (visual plane); (2) of a circle passing through the fixing-point and the nodal points of the two eyes (the famous horopteric circle of Nïller). 
are formed on identical points; and by rapid movements the eyes fix successively different parts of the field of view. (2) Vision grows less distinct as we pass out from the centre of the retina, and we are accustomed to neglect the blurred peripheral images in comparison with those formed on the fovea. (3) When the images of an object lo not fall on iclentical points, one of the points on which they do fall may be occupied with the images of other objects, some of which may be so boldly markel as to enter into conflict with the extra image and to suppress it. (.f) Lastly, the physiological 'identical point' is not a geonetrical point, but an area which increases in size in the more peripheral zones of the retina and can also be increased by practice; and images which lic wholly or in chief part within two corresponding arcas practically coincide.

Stereoscopic Vision.-Although the retinal image is a projection of external objects on a surface, we perceive not only the length and breadth, but also the depth or solidity of the things we look at. When we look directly at the front of a building. the impression as to its form is the same whether one or both eyes be used, although with a single eye its distance cannot be judged so accurately. But when we view the building from such a position that one of the corners is visible, we obtain a more correct impression of its depth with the two eyes. This is partly due to the fact that to fix points at different distances from the eyes the visual lines must be made to converge more or less, and of the amount of this convergence we are conscious through the contraction of the muscles which regulate it. But there is another elcment involved. When the two eyes look at a uniformly-coloured plane surface, the retinal image is precisely the same in both. But when the two eyes are directed to a solid object (say a book lying on a

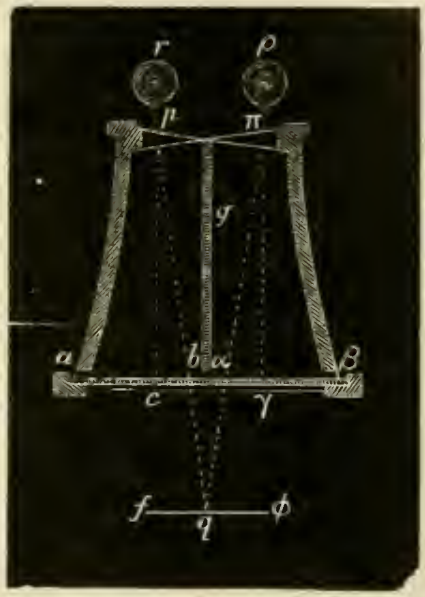

Fig. 403.-Brewster's Stereo. SCOPE.

$p$ and $\pi$ are prisms, with their re. fracting angles turned towards each other. The prisms refract the rays coming from the points $c, \gamma$ of the pictures $a b$ and $a, 3$ so that they appear to come from a single point $q$. Similarly, the points $a$ and $\alpha$ appear to be situated at $f$, and the points $b$ and $\beta$ at $\phi$. table) the picture formed on the left retina differs slightly from that formed on the right, for the left eye sees more of the left side of the book, and the right eye more of the right side.

That there is a close connection between uniformity of retinal images and impression of a plane surface on the one hand, and difference of retinal images and impression of solidity on the other, is proved by the facts of stercoscopy. It is evident that if an exact picture of the solid object as it is seen by each eye can be thrown on the retina, the impression produced will be the same, whether these images are really formed by the object or not. Now, two such pictures can be producad with a near approach to accuracy by 
photographing the object from the point of view of each eye. It only remains to cast the image of each picture on the corresponding retina, while the eyes are converged to the same extent as would be the case if they were viewing the actual object. This is accomplished by means of a stereoscope (lig. to3).

It is formel that the resultant impression is that of the solid object. It is impossible to reconcile this with the doctrine of strictly iclentical geometrical points. A pair of identical pictures gives with the stereoscope not the inpression of a solid, but of a plane surface. If the relative position of any two points differs in the two pictures, the blended picture has a correspondling point in relief. So great is the delicacy of this test that a gool and a bad banknote will not blend minter the stereoscope to a flat surface, and the method may be actually used for the detection of forgery.

When the pictures are interchanged in the stereoscope so that the image which ought to be formed on the right retina falls on the left, and that which is intended for the left eye falls on the right, what were projections before become hollows, and what were hollows stand out in relicf. The pseudoscope of Wheatstone is an arrangement by which each eye sees an object by reflection, so that the images which would be formed on the two retinæ, if the object were looked at directly, are interchanged, with the same reversal of our juclgments of relief.

Visual Judgments. - We say judgments of relief; for what we call secing is essentially an act that involves intellectual processes. As the retina is anatomically and developmentally a projection of the brain pushed out to catch the waves of light which beat in upon the organism from every side, so, physiologically, retina, optic nerve, and visual nervous centre are bound together in an indissoluble chain. We cannot say that the retina sees, we cannot say that the optic nerve sees-the optic nerve in itself is blind-we cannot say that the visual centre sees. The ethereal waves falling on the retina set up impulses in it which ascend the optic nerve; certain portions of the brain are stirred to action, and the resulting sensations of light springing up, we know not where, are claborated, we know not how (by processes of which we have not the faintest guess), into the perception of what we call external objects-trees, houses, men, parts of our own bodies, and into judgments of the relations of these things anıong themselves, of their distance and movements.

A child learns to see, as it learns to speak, by a process, often unconscious or subconscious. of 'putting two and two together.' The musical sounds united and terminated by noises which make up the spoken word 'apple' are gradually associated in its mind with the visual sensation of a red or grecn object, the tactile sensation of a smooth and round object. and the gustatory and olfactory sensations which we call the taste or flavour of an apple. And as it is by experience that the child learns to label this bundle of sensations with a spoken, and afterwarls with a written, name, so it is by experience that it learns to group the single sensations together, and to make the induction that if the hand be stretched out to a certain clistance and in a certain direction-i.e., if various muscular movements, also associated with sensations. be made-the tactile sensation of grasping a smooth round body will be felt, and that if the further muscular movements involved in conveying it to the mouth be carried out, a sensation agreeable to the youthful palate will follow. At length the child comes to belicve, and, unless he happens to be 
specially instructerl, carries his belief with him to his grave, that when lie looks at an apple he sees a round, smooth, tolerably harel body, of defunite size and colour ; while in reality all that the sense of sight can inform him of is the difference in the intensity and colour of the light falling on his retina when he turns his head in a particular directicn.

An interesting illustration of the rôle of experience in shaping our visual judgments is found in the sensations of persons born blind and relieved in after-life by operation. A boy between thirteen and fourteen years of age, operated on by Cheselden, thought all the objects he looked at touched his eyes. 'He forgot which was the dog and which the cat, but catching the cat (which he knew by feeling), he looked at her steadfastly and said, "So, puss, I shall know you another time." Pictures seemed to him only parti-coloured planes; but all at once, two months after the operation, he discovered they represented solids.' Numnely, perhaps remembering the dictum of Diderot, true as it is in the main, though tinged with the exaggeration

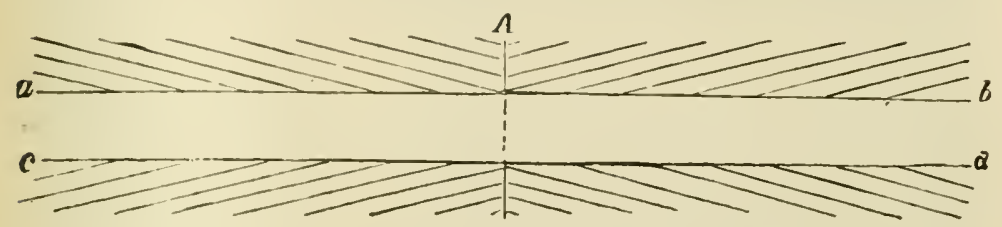

Fig. 404--Illésion of Parallel lines (Hering).

of the Encyclopédie, that 'to prepare and interrogate a person born blind would not have been an occupation unworthy of the united talents of Newton, Des Cartes, Locke, and Leibnitz,' made an elaborate investigation in the case of a boy nine years old, on whom he operated for congenital cataract of both eyes, and, what is of special importance, instituted a set of careful experiments and interrogations before the operation, so as to gain data for comparison. Objects (cubes and spheres) which before the operation he could easily recognise by touch were shown him afterwards, but although ' he could at once perceive a difference in their shapes, he could not in the least say which was the cube and which the sphere.' It took several days, and the objects had to be placed many times in his hands before he could tell them by the eye. 'He said everything touched his eyes, and walked most carefully about, with his hands held out before him to prevent things hurting his eyes by touching them.'

Many other illustrations might be given of the fact that 'seeing' is largely an act of reasoning from data which may sometimes mislead. Thus in Figs. 404 and 405 the long hori- 
zontal lines are really parallel, but do not appear so owing to the confusion of judgment produced by the short sloping lines. In Fig. 406 the spaces covered by $\mathrm{A}, \mathrm{B}$, and $\mathrm{C}$ are equal squares, but $A$ appears taller than $B$, and $C$ smaller than either $A$ or $B$. In the same figure the lines $D$ and $E$ are of the same length, but E seems considerably longer than D.

Illusions of movement are among the most interesting optical illusions. If two similar objects are momentarily shown to the

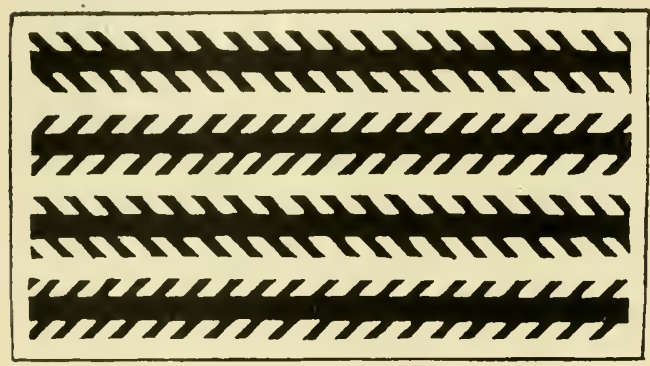

Fig. 405.-Illusion of Parallel Lines (Zöllaner). eye in rapid succession and at points in space not separated by too great a distance, the illusion is produced that the first object has moved to the position of the second. Such illusions are the basis of the so-called 'moving pictures'

shown by the cinematograph. A series of instantaneous photographs of a movement are taken, recording the successive positions assumed by the moving body. When these are thrown on the retina in the same order and in rapid succession, an illusion of the original movement is produced.

The apparent size and form of an object is intimately related to the size, form, and sharpness of its image on the retina. We a re, therefore, able to discriminate with great precision the unstimulated from the excited portions of that membrane, especially in the fovea centralis, and also
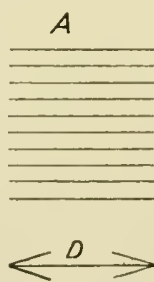

$B$

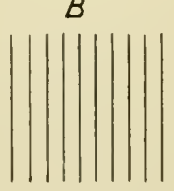

Fifr. 406.-Illusions of Space-perception. the degree of excitation of neighbouring excited parts. But instead of localizing the image on the retina as we localize on the skin the pressure of an object in contact with it, we project the retinal image into space, and see everything outside the eye.

In vision, in fact, we have no conception of the existence of either retina or retinal image; and even the shadows of objects within the eyc are referred to points outside it. . Thus, for instance, an opacity 
or a foreign boly in iny of the refractive melia-mind no cye is cntirely free from relatively opilque spots-caln be detected, ind its position determincel by the shablow which it casts on the retina when the cye is cxamined by a pencil of light proceeling from a very small point. Let a liaplarigm with a small hole in it be placed in front of the eye at such in distance that a pencil diverging from the hole will pass thromgh the vitreous humour as a parallel beam, equal in cross-section to the pupil (ligg. f07), and let the aperture be illuminated by focussing on it the light of a lamp placed behind a sereen. The puoper position of the hole will obviously be that of the anteriur principal focus of the cyc-i.e., the point at which parallel

Fili, 707.-In A the oparine body $O$ is in the plane of the pupil. The position of the shadow relativels to the bright field is not altered when the illuminating pencil is focussed at $\mathrm{P}^{\prime}$ instead of $\mathrm{P}^{\prime}$. In 13 the opaque body is in front of the plane of the pupil. When P' is Iowered to l', the shadow moves towards the upper edge of the bright field, and appears to mose downwards in the risual field. When P is raised, the shadow moves towards the lower edge of the bright field, and appears to move upwards. In $\mathrm{C}$ the opaque body is behind the plane of the pupil. When P' is moved downwards to $\mathrm{P}^{\prime}$, the shadow moves towards the lower edge of the bright field, and appears to the person under observation to move upwards, and vice versit when $\mathrm{P}$ is moved upwards. The farther the opaque body is from the pupil, the greater is the apparent movement, or parallax, of its shadow for a given movement of the source of light.

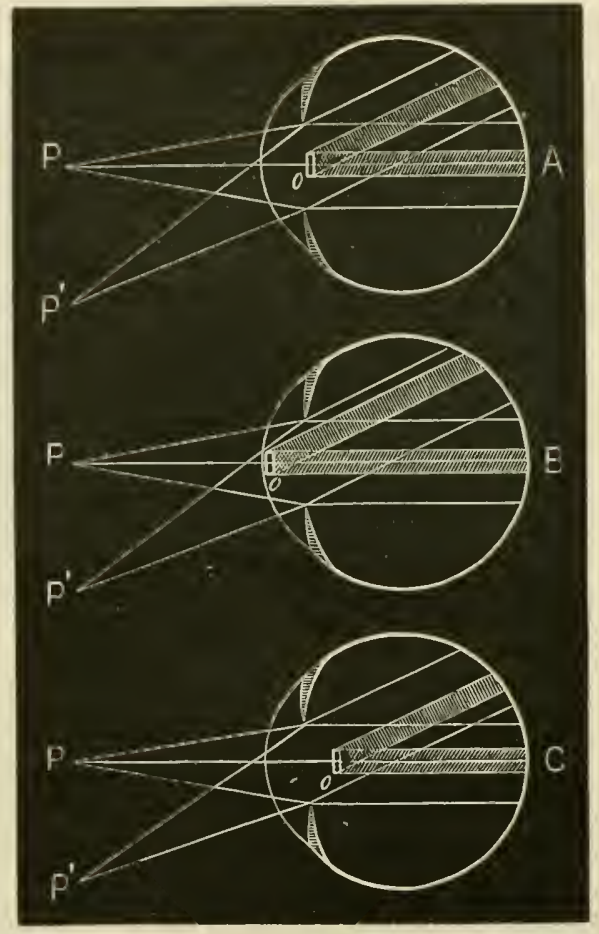

rays passing from the vitreous into the lens, and then out of the eye would be focussed. This method of cxamination of the eye is. therefore, called focal illumination. Opaque bodies in the vitreous humour will cast shadows on the retina equal in area to themselves, The shadows of opacities in the lens and in front of it will be somewhat larger than the bodies themselves, since the latter intercept rays which are still diverging; but since the greater part of the refraction of the eyc occurs at the anterior surface of the cornea, it is only the shadows of objects on the front of the cornea, such as drops of mucus, which will be much magnified. Fig. 407 shows cliagrammatically how the shadows shift their position within the 
bright ficld when the direction of the illuminating beam is altered. Generally opacities in the itreous humour are movable, in the lens not

Purkinje's Figures.-As was first pointed out by Purkinje, the shadow's of the bloodressels in the retina itself, and even of the corpuscles circulating in them, althougl neglected in ordinary vision, may be recognised under suitable conditions, a conclusive proof that the sensitive layer must lie belind the vessels (j). 932).

If a beam of sunlight is concentrated on the sclerotic as far as possible from the margin of the cornea, and the eye directed to at

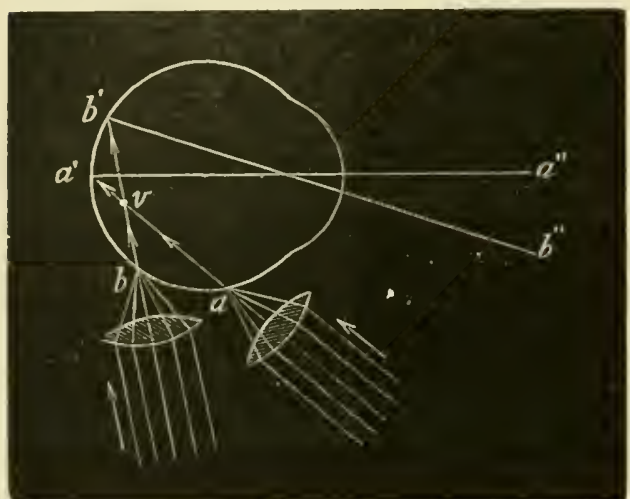

FIG. 408.-METHOD OF RENDERING TII: RETINAL. BLOODVESSELS VISIBLE BY CONCENTRATING A BEAM OF LIGHT ON THE SCLEROTIC.

From the brightly-illuminated point of the scle. rotic, $a$, ray's issuc, and a shadow of a vessel, $v$, is cast at $a^{\prime}$. It is referred to an external point, $a^{\prime \prime}$, in the direction of the straight line joining $a^{\prime}$ with the nodal point. When the light is shifted so as to be focussed at $b$, the shadow cast at $b^{\prime}$ is referred to $b^{\prime \prime}-i, c_{\text {. }}$, it appears to move in the same direction as the illuminated point of the sclerotie. by interrupting the rays from which the retinal vessels form sliadows. The distance of the sensitive from the vascular layer may be approximately calculated by meatsuring the amount by which the shitelows cliange their position. when the position of the illuminated point of the sclerotic is altered. The nearer a vessel lies to the sensitive layer. the smaller must be the angle through which the apparent position of its shadow moves for a given movement of the spot of light. In this way it has been calculated that the sensitive layer is about $0^{\circ} 2$ to $0^{\circ} 3 \mathrm{~mm}$. behind the stratum which contains the bloodressels. This corresponds sufficiently well with the position of the layer of rols and concs, which all other evidence shows to be the portion of the retina actually stimulated by light. The shadows of the blood-corpuscles in the retinal vessels may be rendered visible by looking at a bright and uniformly illuminated ground, like the 
milk glass shate of a lamp or the blue sky, and moving the slightly separated fingers or a perforated card rapidly before the cye. From the rate of their apparent movement. Vierordt calculated the velocity of the blook in the retinal capillaries at 0.5 to $0.9 \mathrm{~mm}$. per second. One reason why the shadows of these intrib-retinal structures do not appear in ordinary vision seems to be their small size. The retinal vessels are in reality only vascular threads; the thickest branch of the central vein is not $1.5 \mathrm{~mm}$. in diameter. The apex of the cone of complete shadow (umbra) cast by a disc of this size, at a (listance of $20 \mathrm{~mm}$. from a pupil $+\mathrm{mm}$. Wide, would lie only! mm. behind the disc--that is to say. the umbra of the retinal vessels would not reach the layer of the rods and cones at all, and only the penumbra, or region of relative darkness, would fall upon it.

When the eyes, after boing closed for some time, are suddenly opened, the branches of the retinal vessels may be secn for a moment. This is especially the case after sleep; and a good view of the phenomenon may be obtained by looking at a white pillow or the ceiling immediatcly on awaking. If the eyes are kept open for a few seconds, the branching pattern facles away; if they are only allowed to remain open for an instant. it may be seen many times in succession. The main vessels appear to radiate out from a central point. But their actual junction there is not seen, since it lies in the optic lisc or blind spot.

The Blind Spot.-The fibres of the optic nerve are insensible to light; light only stimulates them through their end-organs. This can be proved by direct-

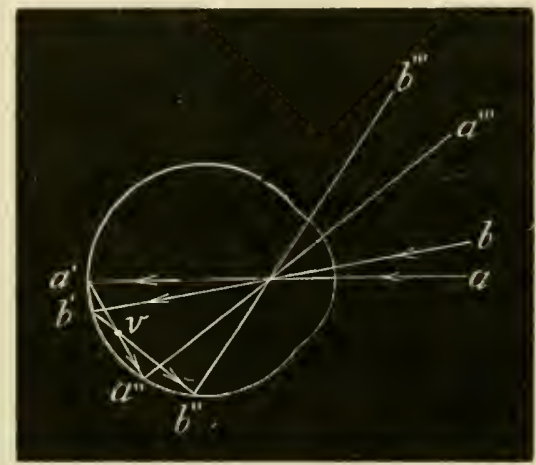

FIG. 409.- METHOD OF RENDERING THE Bloodvessels of the Retina visible by Obliove Illumination through THE CORNEA.

Light from a candle at $a$ illuminates $a^{\prime}$, and rays proceeding from $a^{\prime}$ cast a shadow of the bloodvessel, $v$, at $a^{\prime \prime}$, which is referred to $a^{\prime \prime \prime}$. When $a$ is moved to $b$, the shadow on the retina moves to $b^{\prime \prime}$, and the shadow in the visual field of the illuminated eyc to) $b^{\prime \prime \prime}$. ing by neans of an ophtlialmoscope a beam of light upon the optic disc, where the true retinal layers do not exist. 'The person experimented on lias no sensation of light when the beam falls entirely upon the disc; when its direction is shifted so that it impinges upon any other portion of the retina, a sensation of light is at once experienced. The blind spot is not recognised in ordinary vision, for (I) the two optic discs do not correspond. The left disc has its corresponding points on a sensitive part of the right retina, and the right disc on a sensitive part of the left retina; and the consequence is that in binocular vision the objects whose images are formed on the corresponding points fill up the blind spots. 
(2) The optic disc does not lie in the line of direct, and therefore distinct, vision. The eye is constantly moving so as to bring the surrounding objects successively on the fovea centralis; and the gap which the blind spot makes in the visual field of a single eye is thus more easily neglected. In any case we ought not to see it as a clark spot, for darkness is only associated with the absence of excitation in parts of the retina capable of being excited by light. There is no more reason why the optic discs should appear dark than there is for our liaving a sensation of darkness behind us when we are looking straight in front. And since the experience of our other senses - the sense of touch, for example - tells us that the objects we look at do not in general have a gap in the position corresponding to the part of the image that falls on the blind spot, we see, so to speak, across the spot.

By Mariotte's experiment, however, the existence of the blind spot can not only be demonstrated, but its size determined and its botinclaries mapped out. Let the left cye be closed, and fix with the

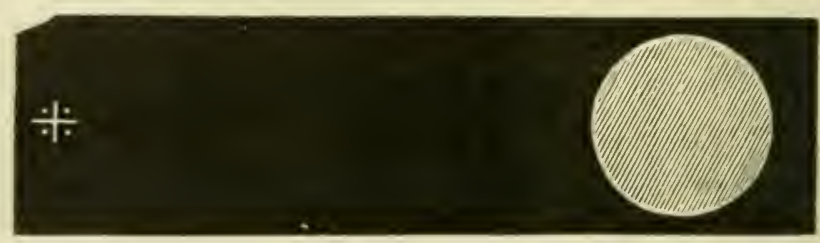

Fig. 4TO.-MARIOTTE'S EXPERIMENT.

right the small cross; then, if the eye be moved towarls or away from the paper, keeping the cross fixed all the time, a position will be found in which the white disc disappears altogether. In this position its image falls on the blind spot.

Relation of the Rods and Cones to Vision. - We liave more than once referred to the rods and cones as the sensitive layer of the retina. It is now necessary to develop a little more the evidence in favour of this statement. And at the outset, since the sensitive layer has been shown to lie behind the plane of the retinal bloodvessels, the only competitors of the rods and cones are the external nuclear layer and the pigmented epithelium. The nuclear layer may be at once excluded as a separate mechanism, since, as we liare seen (p. goo), the portions of the rod and cone elements in it are continuous with the portions in the layer of the rods and cones proper. In the forea centralis, where vision is most distinct, the nuclear layer becomes very thin and inconspicuous.

The layer of pigmented liexagonal cells, or at least their pigment, cannot be essential to vision, for albino rats, rabbits, 
and men, in whose eyes pigment is absent, can see. In man and most mammals there are cones, but no rods in the yellow. spot and forea centralis; the relative proportion of rods increases as we pass out from the fovea tomards the ora serrata. But this does not enable us to analyze the bacillary layer into sensitive cones and non-sensitive rods, for on the rim of the retina, which is still sensitive to light, there are only rocls; in the bat and mole there are said to be no cones even in the vellow spot, in the rabbil very few. Reptiles possess only cones over the whole retinal surface, and birds, true to their reptilian affinities, have everywhere more cones than rods, as have also flshes.

One of the difficulties in the way of understanding how a ray of light can set up an excitation in a rod or cone is the transparency of these structures. An absolutely transparent substance-that is, a substance which would allow light to traverse it without the least absorption - would, after the passage of a ray, remain in precisely the same state as before; its condition could not be altered by the passage of the light unless some of the energy of the ethereal vibrations was transferred to it. But an absolutely transparent body does not exist in Nature; and it is not necessary to suppose that all the energ! required to stimulate the end-organs of the optic nerve comes from the luminous ribrations. These may, and probably do, act by setting free energy stored up in the retina, just as the touch of a child's hand could be made to fire a mine, or launch a ship, or flood a province. Some have looked upon the transverse lamellæe into which the outer members of the rods and cones can be made to split as an arrangement for reflecting back the light to the inner members, and have compared them to a pile of plates of glass, which, transparent as it is, is a most efficient reflector. It is even possible, although here we are already treading the thin air of pure speculation, that the light may be polarized in the process of reflection, and that the rods and cones niay be less transparent to light polarized in certain planes than to unpolarized light.

As to the nature of the transformation undergone by the ethereal vibrations in the rods and cones, various theories have been formulated. Some have supposed that the absorbed lightwaves are transformed into long heat-waves, and that the endings of the optic nerve are thus excited by thermal stimuli. This hypothesis has so little evidence in its farour that it is perhaps an unjustifiable waste of time even to mention it. It is ruled out of court by the mere fact that the long radiations of the ultra red, filtered from luminous rays by being passed through a solution of iodine, and focussed on the eye by a lens 
of rock-salt, produce not the slightest sensation of light, although they are by no means all absorbed in their passage through the dioptric media. Again, it has been suggested that the energy of the waves of light is first transformed into electrical energy, and that the visual stimulus is really electrical. In support of this view it has been urged that the passage of a voltaic current through the eye canses sensations of light and that light, undonbtedly, causes (p. 735) an electrical change in the retina and optic nerve. But, as has more than once been pointed out, an electrical change is the token and accompaniment of the activity of the excitable tissues in general; and all that the currents of action of the retina show is that light excites the retina-a proposition which nobody who can see requires an objective proof of, and which does not carry us very far towards the solution of the problem hor that excitation is brought about. Then there is the photo-mechanical theory, according to which the pigmented epithelial cells of the retina, altering their shape and volume under the stimulus of light, press upon the rods and cones, and thus mechanically stimulate them. Lastly, there is the photo-chemical theory, which supposes that some chemical change produced in the rods and cones under the influence of light sets up impulses in them which ascend the optic nerve. This is the most probable of all the theories, notwithstanding the fact that the discovery by Boll of the famous visual purple or rhodopsin, which at first seemed likely to place it upon a sure foundation, has lost its significance in this regard. But although the visual purple is not a photo-chemical substance through which the retinal elements are excited by luminous stimuli, it seems to fulfil an important function in adapting the retina-i.e., rendering it more sensitive-for vision in dim light. In any case, its discovery is in itself so interesting and so suggestive as a basis for future work, that a short account of the properties of the substance cannot be omitted here.

Visual Purple.--If the eye of a frog or rabbit, which has been kept in the dark, be cut out in a dimly-lighted chamber or in a chamber illuminated only by red light, and the retina removed, it is secn. when viewed in ordinary light, to be of a beautiful red or purple colour. Exposed to bright light, the colour soon fades, passing through red and orange to yellow, and then disappearing altogether. The yellow colour is due to the formation of another pigment, visual yellow; the preceding stages are due to the intermixture of this visual yellow with the unchanged visual purple in different proportions. With the microscope it may be seen that the pigment is entirely confined to the outer segment of the rods, whicre it exists in most vertebrate animals. It may be extracted by a watery solution of bile-salts, and the properties of the pigment in solution are very much the same as its properties in situ ; light bleaches the solution as it does the retina. Examined with the spectroscope, the solution 
shows no definite bands, but only a general absorption, which is very slight in the red, and reaches its maximum in the yellowish-green. [n accordance with this, it is fomel that of all kinds of monochromatic light the yellowish-green rays bleach the purple most rapidly, the red rays most slowly.

If a portion of the retina is kept (lark while the rest is exposed to light, only the latter portion is bleached. And when the image of an object possessing well-marked contrasts of light and shadow (e.g., a glass plate with strips of black paper pasted on it at intervals, or a window with (litk bars) is allowed to fall on an eye otherwise protected from light, the pattern of the object is picked out on the retina in purple and white. A veritable photograph or 'optogram' may thus be formed even on the retina of a living rabbit; and if the eyc be rapidly excised, the picture may be 'fixed' by a solution of alum. and thus rendered permanent.

These facts certainly suggest that light falling on the retina may cause in some sensitive substance or substances chemical changes, the products of which stimulate the endings of the optic nerve, and set up the impulses that result in visual sensations.

The visual purple cannot itself be such a substance, for it is absent from the cones of all animals and the rods of some. Frogs and rabbits can undoubtedly see at a time when, by continued exposure to bright sunlight, the

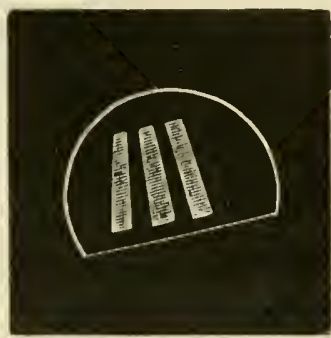

Fig. 4II.-Optogram.

Part of retina"of rabbit, the eye of which had been directed to an illuminated plate of glass covered with strips of black paper. purple must have been completely bleached. And although the alleged absence of the pigment in the eye of the bat might seem to afford a ready explanation of the proverbial 'blindness' of that animal, such a hasty deduction would be at once corrected by the fact that birds with as sharp vision as the pigeon are equally devoid of visual purple, while in other nocturnal animals, like the owl, it is plentifully found. The most probable hypothesis of the function of the visual purple is indeed that which attributes to it the property, in virtue of its capacity for regeneration in the dark, of adapting the eye for night or twilight visionin other words, of increasing the sensitiveness of the retina for faint light, especially of the shorter wave-lengths. If this is the case, it is precisely in nocturnal animals that we should expect to find it in large amount ; and recently visual purple has been obtained from more than one species of bat (Trendelenburg). The fact that central vision (p. 946) in which the rodless fovea is concerned is but little, if at all, susceptible of dark-adaptation, while peripheral vision shows a marked capacity of adaptation, agrees well with this liypothesis. We shall see later that there is some evidence that it is the mere perception of luminous im- 
pressions as sucl and of their intensity, without any distinction of quality or colour, with which the rods have to do. They are, then, on the hypothesis under discussion, elements concerned in achromatic sensations under conditions of feeble illumination (twilight vision). The cones are supposed on this theory to be more highly developed elements than the rods, their function being connected especially with the perception of colour, lut also with the perception of achromatic sensations under daylight conditions.

The pigmented retinal epithelium is undoubtedly sensitive to light. and has important relations to the formation of the visual purple. When the eye is exposed to light, black pigment migrates along the processes of the epithelial cells between the rods, even as far as the cxternal limiting membrane. In the dark the pigment moves back again. and gathers around the outer portions of the rods, where the visual purple is being regenerated. The precise meaning of the changes in the pigmented cells is obscure.

The pigmented epithelium is known to be concerned in the regeneration of the visual purple. When a frog is curarized, odema occurs between the retina and the choroid, so that the former membranc is separated from the hexagonal epithelium. If the frog is now exposed to sunlight till the visual purple is bleached, and the retina then taken out and placed in the dark. no regeneration of the purple takes place. When the same experiment is repeated on a non-curarized frog. the visual purple is restored in the dark, and may be seen under the microscope in the rods. The only difference in the two experiments is that in the latter the pigmented epithelium adheres to the retina, and it must therefore have a hand in the regeneration of the pigment. Even the risual purple of a retina from which the epithelium has been detached will, after being bleached, be restored if the retina is simply laid again on the epithelial surface. And it does not seem to be the black pigment of the hexagonal cells which is the agent in this restoration. for it takes place in the pigment-free retine of albino rabbits or rats. Even a retina isolated from the pigmented epithelium, and then bleached, may, to a certain extent. develep new visual purple in the dark. This is even true when it has been kept in the dark in a saturated solution of sodium chloride, and is then. after washing with physiological salt solution. bleached by light. Here the regeneration of the pigment cannot be the result of vital processes, but must be due to chemical changes in products formed from the original pigment by the action of light. No such regeneration takes place in a retina which, after having been bleached in situ, is remored without the pigmented epithelium and placed in the lark; and the only probable explanation of the difference is that in this case the photo-chemical substances frem which visual purple can be formed have been absorbed into the circulation. and have so escaped.

The inner segments of the cones of certain animals (birds, reptiles. amphibia, and some fishes) contain globules of various colours, ranging over almost the whol spectrum, and including, besides, the non-spectral colour, purple. The globules are composed chiefly of fat with the pigments (chromophanes, as they have been called) rlissolved in it. The function of these globules is unknown. They 
cinnot be concerned in colnur vision, or, at least, they cannot be cssential to it, for in the luman retina they do not c'xist.

The yellow pigment of the macula lutea does not breng to the layer of rods and cones ; it only cxists in tle cxternal molecular layer and the lavers in front of it; in the forea centralis it is absent.

Time necessary for Excitation of the Retina by Light Fusion of Stimuli. - Whatever the exact nature of retinal excitation may be, it is called forth by cxcecdingly slight stimuli. A lightning flash. althougl it may last only ${ }_{I, 000,000}$ th of a sccond, lasts long enough to be scen. A beam of liglit thrown from a rotating mirror on the eyc stimulates when it only acts for $\frac{1}{8,000,000}$ th of a second. The minimum stimulus in the form of grecn light corresponds, as we have already scen (p. 68I), to a quantity of work cquivalent to no morc than $\frac{\mathrm{I}}{1 \mathrm{O}^{8}}$ erg--that is, about $\frac{1}{\mathrm{IO}^{10}}$ gramme-millimetrc, or ${ }_{\mathrm{IO}^{7}}^{\mathrm{I}} \mathrm{milli-}$ gramme-millimetre, which is the work done by $\frac{1}{10.000 .000}$ th of a milligramme in falling through a millimetre; and it cannot be doubted that a portion cven of this Lilliputian bombardment is wasted as heat. So quickly; too, is the stimulus followed by the response that no latent period has as yet ever been mcasured. It is certain. however, that there is a latent period. as surcly as there is a latent period in the excitation of a naked nerve-trunk. although this also has never been experimentally detected. The analogies, in fact. betwcen a muscular contraction and a retinal excitation are numerous and close. Like the muscle. the retina seems to possess a store of explosive material which the stimulus serves cnly to fire off. The retina. like the muscle, is exhausted by its activity, and recovers during rest. Like the muscle curve, the curve of retinal excitation rises not abruptly, but "ith a measurable slowness to its height, and when stimulation is stopped. takes a sensible time to fall again, the retinal impression outlasting the luminous stimulus by about one-eighth of a second. With comparatively slow intermittent stimuli the retinal. like the muscle curve. flickers up and down. When the rate of stimulation is increased. the steady contraction of the tetanized muscle is analogous to the fusion of the individual stimuli by the tetanized retina (or retinoccrebral apparatus) into a continuous sensation of light, such, e.g., as the bright 'trail' of a falling star, or the fiery circle traced in the air when a firebrand is rapidly whirled round. But the maximum retinal excitation which a stimulus of given strength can call forth depends much more closely upon the time during which the stimulus acts than the maximum contraction does upon the length of the muscular stimulus.

As the strength of the light increases in geometrical progression, the time during which it must act in order to produce its maximum effect decreases approximately in arithmetical progression (Exner). For light of moderate intensity this time is about $\frac{1}{4}$ second. Since for complete fusion the stimuli must follow each other at a much more rapid rate than four in the second, the intensity of the resultant sensation is always less when a succession of similar stimuli are fused than when one of the stimuli is allowed to produce its maximum effect.

If the time of each stimulus is equal to the interval during which 
there is no stimulation, the sersation. when complete fusion has been reached, is the same as would be produced by a constant light of half the strength employed. Ancl, in general, if $m$ be the proportion of the time during which the eye is stimulated by a light of intensity $I$. and $n$ the proportion of the time during which it is not stimulated, the resultant impression is the same as that which would be producer by an uninterrupted light of intensity $\left(\frac{m}{m+n}\right) I$. This is Talbot's law, which may be expressed without the aid of symbols thus: When a light of given intensity is allowed to act on the eye at intervals so short

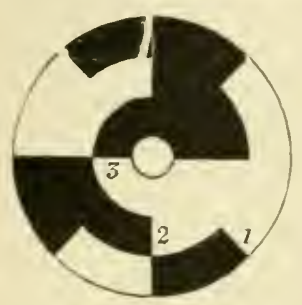

FIG. 4I2.-DISC FOR DE. IONSTRATING TALBOT'S I.AW. that the impressions are completely fused, the resultant sensation is independent of the absolute length of each flash, and is proportional only to the fraction of the whole time which is occupied by flashes and to the intensity of the light. Talbot's law may be readily demonstrated by means of a rotating (lisc with alternate white and black sectors (Fig. 412). so arranged that the same proportion of the circumference of each of the three concentric zones is black.

When the rotation is sufficiently rapid to give complete fusion (say 20 to 30 times a second), the whole disc appears equally bright. However much the rate of rotation is now increased, no further change occurs. It has been shown that even for stimuli as short as the $\frac{\pi 00000}{1}$ th of a second, repeated at intervals of $\frac{1}{1}$ th second. Talbot's law holds good. So that not only does a flash so inconceivably brief affect the retina, but it sets up changes whicl last for a measurable time. For intense stimuli Talbot's law ceases to be true: the field appears brighter than it should be (Crünbaum)

Two chicf theories have been proposed to account for the fusion of intermittent retinal stimuli : (I) The persistence theory, according to which the excitatory process in the retina remains for a short time at the maximum reached when the light ceases to act. Steady fusion is supposed to be obtained when the interval between successive stimuli does not exceed this time. (2) The theory of Fick, who maintains that as soon as the light is withdrawn the retinal excitation begins to sink, at first rapidly, then more gradually. As the rate of stimulation is increased the time allowed for the decline of the excitation is, of course, correspondingly shortened, and ultimately the oscillations become so small that a continuous smooth sensation results. Fick's theory appears to explain the phenomena best.

The experiments of Charpentier have shown that the retina when stimulated has a natural tendency to enter into oscillations at the rate of about 36 in the second. So that the effect of a flash of light when it falls on a retinal area is not a single excitation which rises smoothly to its maximum and then declines smoothly to zero, but a series of swings which die away like the vibrations of an elastic body. This may be demonstrated by slowly rotating a well-illuminated disc, one quadrant of which is white and the rest black, while the cye is kept fixed on the centre. A black band, or rather sector, running out from centre to circumference, will be scen in the white quadrant a little behind the border of it which first passes the eye. This band may be succeelerl by one or more fainter black bands placed at 
regular intervals in the white portion of the disc. The explanation is this. At the moment when the image of the advancing edge of the white quadrant falls upon the retina it is excited, and we get the sensation of white. Then comes a swing in the opposite direction which gives rise to the first black band, and succeeding swings cause the other bands. The period of the oscillatory process can be calculated from the speed of the disc, and the distance of the first band from the edge of the white quadrant. The well-known fact that a single flash of lightning, or other intense stimulus, may appear as two flashes, finds its explanation in these retinal oscillations.

Colour Vision.-Besides differences in the distance, size, shape, and briglitness of objects, the eye recognises differences in their colonr ; and we liave now to consider the pliysical and physiological differences on which these depend.

Colours may differ from each other-(I) In tone or hue, e.g., red, yellow, green. (2) In degrec of saturation or fulness or purity, i.e., in the degree in which they are free from admixture with white light, e.g., a 'pale' or 'light ' blue is a blue mixed with much white light, a 'deep' or 'full' blue with little or none. (3) In brightness or intensity, i.e., in the amount of the light coming from unit area of the coloured object. Thus, a ' lark' red cloth sends comparatively little light to the eye, a 'bright' red cloth sends a great deal.

When a beam of sunlight falls into the eye, a sensation of ' white light' results. When a prism is placed before the eye, the sensation is entirely different; we see a spectrum running up from red through green to violet, with a multitude of intermediate shades, the eye being able to distinguish in the solar spectrum at least one thousand different hues (Aubert). What, then, has happened? Physically, nothing more has taken place than a rearrangement of the rays in the beam of white light. A few of them may have been lost by reflection, but upon the whole the beam is made up of exactly the same constituents as before; only the rays are now arranged in the precise order of their refrangibility, the more refrangible, which are also those of shortest wave-length, being displaced more towards the base of the prism than the longer and less refrangible rays. Instead of the long and short rays falling together on the same elements of the retina, as they did in the absence of the prism, they now fall, if proper precautions have been taken to secure a pure spectrum, in regular order from one side to the other of the portion of retina on which the image is formed. The physical condition, then, of our sensations of the prismatic colours is, that rays of approximately the same wave-length should fall unmixed with other rays upon the retinal elements. Rays of a wave-length of $760 \mu \mu^{*}$ to $650 \mu \mu$ give the sensation of red; from $650 \mu \mu$ to $590 \mu \mu$, the sensation of orange; from $430 \mu \mu$ to $400 \mu \mu$, the sensation of violet, and so on. When rays of

* $\mu \mu$ is a symbol representing one-millionth of a millimetre. 
all these wave-lengths fall together, in the proportion in which they are present in sunlight, upon the same part of the retina, the resultant physiological effect is very different; we are no longer able to distinguish red, blue, green, etc.; we receive the single sensation of white light. The sensation is a simple one; in consciousnesis we have no hint that it has a multiple physical cause.

But we find furtler that it is not necessary for the sensation of white light that waves of every length present in the solar spectrum should be mixed. If rays of wave-lengths $675 \mu \mu$ (which acting alone produce the sensation of red) be mixed in certain proportions-i.e., be allowed to fall on the same part of the retina-with rays of wave-length $496 \mu \mu$ (which give the sensation of bluish-green), the resultant sensation is also that of white light. And an indefinite number of sets can be combined, two and two, so as to give the same sensation of white. Such colours are called complementary. The following are pairs of complementary colours :

\section{Red and bluish-green. Yellow and ultramarine-blue. \\ Orange and cyan-blue.* Greenish-yellow and riolet.}

The green of the spectrum has no simple complementary colour ; purple, a colour not present in the spectrum, but obtained by mixing light from the two spectral extremes-i.e., by mixing red and violet-may be considered complementary to it. Suppose now that one of a pair of complementary colours is added to the other in greater intensity than is required to give white, the resultant sensation is a colour which has a certain amount of resemblance both to white and to the colour present in excess. Thus, if the two colours are orange and blue, and the blue is present in greater intensity than is necessary to give white, the resultant colour is a whitish or pale blue, or, to use the technical phrase, an unsaturated blue. The more nearly the intensity of the blue rays in the mixed light approaches the proportion necessary to give white, the less saturated is the resultant colour ; the greater the excess of blue, the more nearly does the resultant sensation approach that of the saturated blue of the spectrum. But any non-saturated spectral colour produced by the mixture of two complementary colours may be equally well produced by the mixture of the corresponding spectral colour with a certain quantity of ordinary white light. And it is found that when two spectral colours which are not complementary are mixed together the resultant is not white, but a colour which may be matched by some spectral colour lying between the two (or by purple), either without addition or plus a larger or smaller

* Cyan-blue is a greenish-blue. 
quantity of ordinary white light. From all this it follows that the retina may be excited by an infinite number of clifferent physical stimuli, and yet the resultant sensation may be the same. This leads straight to the conclusion that somewhere or other in the retino-cerebral apparatus simplification, or synthesis, of impressions must take place; and we have to inquire what the simplest assumptions are which will explain all the phenomena. Now, it is not possible, from two spectral colours alone, to produce a sensation corresponding to all the others. By mixing three standard spectral colours, however, in various proportions, we can produce not only the sensation of white light, but that of every colour of the spectrom (and of purple). These statements are based on demonstrated facts obtained by very numerous experiments on colour mixtures. The hypotheses framed to explain the facts are to be carefully discriminated from the facts themselves.

Primary Colours. - The simplest assumption we can make, then, is that there are three standard sensations, and that either the retina itself can respond by no more than three distinct modes of excitation to the multiplex stimuli of the luminous vibrations, or that complex impulses set up in the retina are reduced to simplicity because the central apparatus is capable of responding by only three distinct kinds of sensation. W'hich three sensations we select as fundamental or primary is, to a certain extent, arbitrary. Fick chose red, green, and blue; most commonly red, green, and violet are accepted as the primary colours. Red, yellow, and blue, although so long considered the primary colours, from data yielded by the mixture of pigments, will not do; for no possible combination of them will produce either a pure green or white light.

The Young-Helmholtz Theory.-The theory which has been most widely accepted is that of Young, generally called, on account of its adoption and extension by Helmholtz, the Young-Helmholtz theory. Red, green, and violet are taken as the fundamental or elementary colour sensations. In its more modern form it assumes that in the retina, or in the retino-cerebral apparatus, there are three kinds of elements - (I) a substance or a component chiefly affected by light of comparatively long wave-length (red), to a less extent by light of medium wave-length (green), and to a still less extent by the shortest visible waves (violet); (2) a component mainly affected by medium, but also to a certain extent by long and short waves ; (3) a component cliefly affected by the short vibrations, less by the medium, and still less by the long waves. The curves in Fig. 4 I3 illustrate these relations.

The theory explains as follows the phenomena of colour- 
mixture referred to above. When all the rays of the spectrum act upon the retina togetler, the three components are about equally affected, and this equal effect is supposed to be tlic condition of the sensation of white light. When the green of the spectrum alone falls on the retina, the 'green' component is strongly excited, the other two only slightly; this is the relation

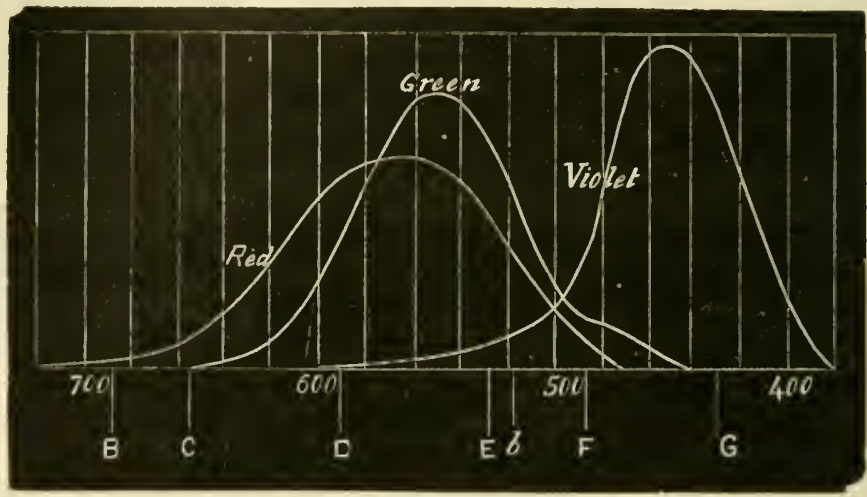

Fig. 4i3.-Curves of Excitability of Primary Sexsations from Obserlations on Colour Mixtures (KöNig).

The numbers give wave-lengths of the spectrum in millionths of a millinctre.

between the amount of excitation in the three components which is associated with a sensation of spectral green. When two complementary colours, such as red and bluish-green, fall together on the same portion of the retina, the three components are excited in the relative proportions associated with the sensation of white light.

The colour triangle is a graplic method of representing various facts in colour-mixturc (Fig. 414 ).

The chief points to be noted are the following : ( I) On the curve the

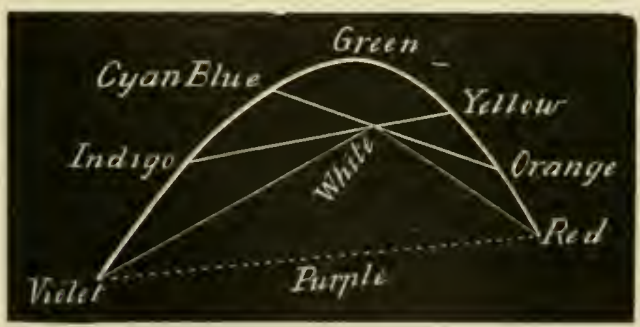

Fig. 4I4.-Colour Triangli: spectral colours arc arrangerl at sucl distances that the angle contained between straight lines drawn from the point inarked 'white,' and intersecting the curve at the positions corresponding to any two colours is proportional to their difference in tone. (2) The distance of any point of the curve from the point marked 'white' is proportional to the stimulation intensity of the colour corresponding to it. (If the stimulation intensities of all the colours be represented by propor- 
tional weights lying at the corresponding points on the curve, the point 'white' will be the centre of gravity of the system.) (3) The position of a colour produced by the mixture of any patir of spectral colours is found by joining the corresponding points by a straight line. The mixed colour lies on this line at distances from the two points inversely proportional to the stimulation intensity of the two colours-i.c., it lies in the centre of gravity of the weights representing the two colours. (4) It is a particular case of (3) that the complementary colours are situated at the points where straight lines drawn through 'white' intersect the curve, since the point marked 'white' is the centre of gravity corresponding to a pair of colours only when it lies on the stratight line joining them. Thus the orange and yellow lying between the red and green are mixtures of the red and green sensations in different proportions the cyan-blue and indigo-blue are mixtures of the green and violet sensations. The purples, represented by a broken linc, are not present in the spectrum, and wre mixtures of red and violet.

It is a point of great theoretical interest that on the YoungHelmholtz theory the pure spectral colours, although physically saturated (i.e., cluc to ethereal vibrations of a definite wave-length for each colour), ought not to be physiologically saturated, since they all affect the three components, although in different degrees. In other words, the red, let us say, of the spectrum ought not to be the purest or fullest red which it is possible to perceive. Now, it is found that this is really the case. If, for example, we look first at the bluish-green, and then at the red of the spectrum, the sensation of red is fuller or more saturated than if we had looked at the red directly. Similarly, if we look first at a small bluish-green square on a black ground, and then at a red ground, we see a more fully saturated square in the michlle of the latter. The explanation, on the Young-Helinholtz theory, is that the ' green' component, being fatigued before the eye is tumed upon the red, the latter colour no longer affects it, or affects it less than it would otherwise clo, and therefore the excitation is almost entirely confmed to the red component in the area fatigued for grcen. This brings us to the subject of retinal fatigue, and the related phenomena of after-images and contrast.

After-Images.-We have seen that the retinal excitation always takes time to dic away after the stimulus is removed. If a white object is looked at, especially when the cyc is fresh, for a time not long enough to cause fatiguc, and the eyc is then closed, an imagce of the object remains for a short time, diminishing in brightness at first rapidly, then more slowly. This is a positive after-image, and by careful obscrvation it may, under certain conditions, be secn that the positive after-image of a white object, of a slit illuminated by sunlight, for cximple, undergoes changes of colour as it fades, passing through greenish-blue, incligo, violet, or rose, to dirty orange. On the Young-Helmholtz theory this is explained by the supposition that the cxcitation does not decline with the same rapidity in the three hypothetical components. If the object is looked at for a longer time, or if the cyc is fatigued, a dark or negative image may be seen upon the faintly-illuminated ground of the closed cyes; but negative after-images may be more easily obtained when the eye, after being made to fix a small white object on a black ground, is suddenly turned upon a white or neutral tint surface.

Here Helmholtz supposed the portion of the retina on which the 
image of the object is formed to be more or less fatigned. And this fatigac will extend to all threc kinds of fibres; so that white light of a given intensity will now cause less excitation in this part than in the rest of the retina. It is easy to understand that the negative after-image of a coloured object will be seen, upon a white ground, in the complementary colour, for the components chicfly excited by the latter will have been least fatigued. The negative afterimages scen when the eyc, after receiving the positive impression, is turnel mpon a coloured gromud, vary with the colour of the object and ground in a manner which luas been explained as (lue to fatiguc of one or other component. It is lifficult, howerer, to reconcile the fitigue hypothesis of the after-image with all the facts. Hering supposis that the retina is not passively fatigued, but that a metabolic change is set 11p in it which is of the opposite kind to that catused by the original excitation (sce p. 945).

The plienomena of negative after-images are often included together as cxamples of successive contrast, the name inplying mutual influence of the portions of the retina (or retino-cercbial apparatus) successively stimulated. We lave now to consicler simultancous contrast, often spoken of simply as contrast.

Contrast. - A small white disc in a black ficld appears whiter, and a small black disc in a white ficld darker, than a large surface of exactly the same objective brightness. A clisc with alternate sectors of white and black, so arranged that the proportion of white to black increases in each zone from centre to circumference, when set in rotation, ought, by Talbot's law, to show sharply narked and uniform rings, of which each is brighter than that internal to it. But cach zone appears briglitest at its inner edge, where it borders on a zone darker than itself, and darkest at its outer edge. where it borders on a brighter zone. A plausible explanation of this is based on the assumption that in the neighbourhood of an excited area of the retina, as well as within the area itself, the excitability is rliminished ; and the same explanation has been extended to the contrast phenomena of coloured objects. A small piece of grey paper, e.g., is placed on a green shect. The grey patch appcars in the complementary colour of the ground-viz., pink or rose-red (Meycr). The red colour is much stronger if the whole is covered with translucent tracingpaper. Here we may suppose that the fatigue of the substance or component chicfly affected by the ground colour spreals into the portion of the retina occupied by the image of the grey paper; the white light coming from the latter, therefore, affects mainly the component connected with the sensation of the complementary colour.

The curious phenomenon of coloured sharlows is also an illustration of contrast. They may' be produced in various ways. lor cxample, wlicn a lamp is lit in a room in the twilight, before it has yet grown too dark, the shadows cast by opaque objects on a white windowblind are coloured blue. The yellow light of the lamp overpowers the fecble daylight which passes through the blind, and the generat ground is yellowish; but wherever a sladow is thrown it appears of a bluisl tint in contrast to the vellow ground. Here the only illumination the cye receives from the region occupied by the sliadow is the fecble caylight. lialling upon an area in which the component chiefly affected by yellow rays is more or less fatigued, it causes a sensation of the complementary colour. As clarliness comes on, the shadows become black, for now practically no light at all comes from them. 
Helmholtz looked upon simultineous contrast as a result of false julgment, and not of a change of excitibility in parts of the retinat borlering on the actually excited parts. For the sake of perspective, it will be worth while to apply this theory by way of illustrating it, to the explanation of the cisc of contrist we hatve just been considering. from the other point of view, in Meyer's experiment. IJelmholtz's explanation of this experinent is as follows: When a coloured surface is corered with translucent paljer, the latter appears ats at coloured covering spreat oser the tickl. The mind does not recognise that at the grey patch there is any breach of continuity in this covering; it is therefore assumed that the greenish veil extends over this spot too. Now, the grey seen through the transluent white paper is objectively white - i.e., sencls to the eye the vibrations which together would give the sensation of white light. But with a green veil in front of it, this could only happen if the really grey patch was the colour complementary to green-that is, rose-recl. The mind, therefore, judges falsely that the patch is red. Hering has severely criticised this theory of Helmholtz as to false judgments; and the weight of evidence certainly scems to be in favour of the view that simultaneous, like successive, contrast is due to the influence of one portion of the retina, or retino-cerebral apparatus, on another.

Hering's Theory of Colour Vision.-The Young-Helmholtz theory of colour vision has not met with universal acceptance. The best-known rival theory is that of Hering, who takes his stand upon the fact that certain visual sensations (red, yellow, green, blue, white, black) do appear to us to be fundamentally distinct from each other, while all the rest are obviously mixtures of these. Accepting these six as primary sensations, he assumes the existence in the visual nervous apparatus of substances of three different kinds, which may be called the black-white, the green-red, and the blue-yellow. Like all other constituents of the body, these substances are broken down and built up again - in other words, undergo disassimilation and assimilation, destructive and constructive metabolism. The sensations of black, of green, and of blue he supposes to be associated with the constructive, and the sensations of white, of red, and of yellow with the destructive, processes in the three substances. The black-white substance is used up under the influence of all the rays of the spectrum, but in different degrees; the smaller the quantity of light falling on the retina, the more rapidly is it restored, and the more intense is the sensation of black. The green-red substance is built up by green rays, broken down by red. The blue-yellow substance is destroyed by yellow rays, restored by blue. A prominent difference between this and the Young-Helmholtz theory, and, so far as it goes, an advantage, is that Hering's theory attempts to assign a direct objective cause for the risual sensations of white, black, and yellow, as well as for red, green, and blue, instead of making the sensations depend upon the magnitude of the stimulation process. When any of the risual substances are consumed at one part of the 
retina, they are supposed to be more rapidly built up in the surrounding parts, and in this way many of the phenomena of simultaneous contrast receive an easy and natural explanation. The same is true of the simpler phenomena of after images or successive contrast. But in applying the theory to the more complicated phenomena difficulties soon emerge, which, to say the least, are not less formidable than those connected with the Young-Helmholtz theory. Neither theory, in short, can be considered more than a partially successful attempt to grapple with a very complex mass of facts. Each, however, has been fruitful in leading to the discovery of new facts-a great merit in a scientific lyppothesis.

Sensibility of Different Parts of the Retina-Perimetry.-

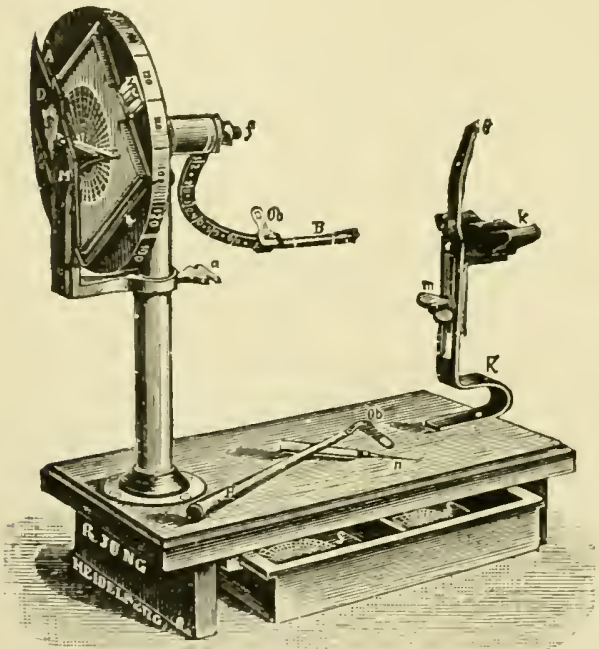

Fig. 4I5.-Priestley Simth's Perimeter.

$K$, rest for chin ; $O$, position of eye ; $O b$, object, white or coloured, which slides on the graduated $\operatorname{arc} B ; t$, point fixed by the eve.
The perception of colours, like the perception of white light, is not equally distinct over the whole retina. We have repeatedly had occasion to refer to the forea centralis as the region of most distinct vision; but it would be a mistake to suppose that it is therefore necessarily, more sensitive than the rest of the retina. As a matter of fact, when the minimum intensity of white light which will cause an impression at all is determined for each portion of the retina, it is found that the fovea centralis requires a somewhat stronger stimulus than the zone immediately surrounding it. Objects only a little brighter than the general ground on which they lie-e.g., very faint starsare best seen when the eye is directed a little to one side. This has been attributed to the absence of visual purple from the fovea, in accordance with the theory previously alluded to that the visual purple acts as a mechanism which 'adapts 'the retina for the perception of light of varying intensity. But, with this exception, the sensibility of the retina diminishes steadily from centre to periphery, both for white and for coloured light. 
When the eye is fixed, and the visual field-that is, the whole space from which light can reach the retina in the given position, ro, what comes to the same thing, the projection of the visual field on the retina by straight lines passing through the nodal point-explored by means of a perimeter (Figs. 4 I5, 4I6), it is found that, under ordinary conditions, a white object is seen over a wider field than any coloured object, a blue object over a wider field than a red, and a red over a wider field than a green

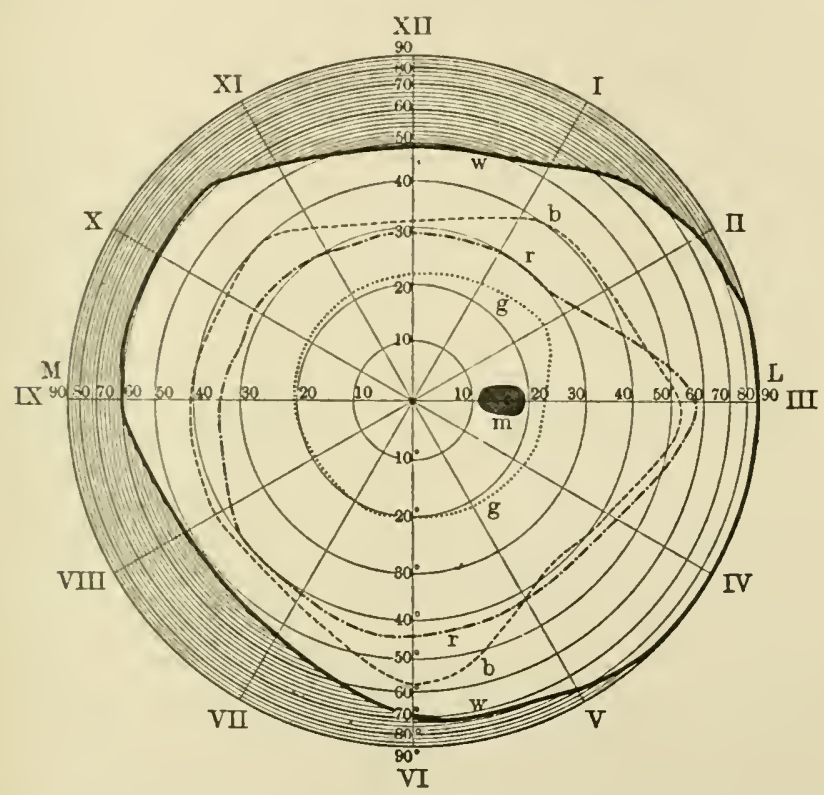

Fig. +16.-Perimetric Chart of Right Eye (after Hirschierg).

The numbers represent degrees of the visual field measured on the graduated arc of the perimeter. W, boundary of field for white object ; $b$, for blue; $r$, for red ; $\mathrm{g}$, for green ; $\mathrm{m}$, blind spot ; M, medial, and L, lateral side of the field of vision. The Roman numbers represent twelve meridians of the retina, each making an angle of $30^{c}$ with the next. They fix the 'longitude' of any point in the field. The concentric circles indicated by Arabic numbers represent angular distances from the fixation point in the planes of these meridians. They give the 'latitude' of any point.

object. The exact shape, as well as size, of the visual field also differs somewhat for different colours. In disease of the retina, or of the visual path between it and the cortex, or of the visual cortex itself, the abridgment of the field for white and for monochromatic light as mapped out by observations with the perimeter is often of value in diagnosis. Although it has been shown by Aubert and others that monochromatic light of considerable intensity can be perceived over the whole retina, yet 
it may be said that the retinal rim is even then relatively and, under ordinary conditions, absolutely, colour-blind. This and other facts have given rise to the theory (p. 935) that the rods, which are alone present at the ora serrata, are concerned in achromatic vision (under conditions of dark adaptation), the cones in colour vision as well as in achromatic vision (under daylight conditions).

This brings us to the subject of colour-blindness, a plenomenon of great interest in its theoretical as well as in its practical bearings.

Colour-blindness.-A considerable number of persons (about 4 per cent. of all males, but only one-tenth of this proportion of females) are deficient in the power of distinguishing between certain colours. They are said to be colour-blind; but the term must not be taken to signify that they are absolutely devoid of colour-sensations. A very small minority of the colour-blind appear to have but one sensation of colour tone, everything appearing as white, grey, or black (total colourblindness, sometimes called monochromatic vision). All colours are confused by them, but differences of brightness are correctly appreciated. Probably the totally colour-blind person receives somewhat the same impressions from a coloured picture as the normal person does from a reproduction of the same picture in black-and-white. There are close resemblances between the vision of the totally colour-blind eye and that of the normal eye adapted by resting in the dark for twilight vision. The fovea is relatively, and in some cases absolutely, insensitive to light, while the peripheral portion of the retina is normal, or nearly normal, in this regard. This is the foundation of the theory that in total colour-blindness the cones are devoid of their normal functions, and that the hypothetical mechanism for twilight vision (the rods) is functioning alone. In another condition (night-blindness, or hemeralopia) it is sometimes assumed that the other mechanism (that of the cones) which is adapted for daylight vision, and has little power of dark-adaptation, is alone acting. But it cannot be said that this has been proved.

The rest of the colour-blind are dichromatic-i.e., their colour reactions seem to correspond only to two of the fundamental colour sensations of the normal person and their combinations, in addition to white. Of the dichromates a very few confuse blue with yellow. The great majority are unable to distinguish between red and green. The condition will be most easily understood by considering some of the extraordinary mistakes which may be made by the colour-blind, without necessarily leading them to suspect that there is anything abnormal in their vision. Thus, to quote the words of a distinguished writer on 
this subject, limself a sufferer from the deficiency: 'A naval officer purchases red breeches to match his blue uniform; a tailor repairs a black article of dress with crimson clotli; a painter colours trees red, the sky pink, and human checks blue.' The shoemaker, Harris, the discoverer of colour-blindness, picked np a stocking, and was surprised to hear other people describe it as a red stocking ; it seemed to him only a stocking. The celebrated Dalton was twenty-six years of age before he knew that he was colour-blind. He matched samples of red, pink, orange, and brown silk with green of different shades; blue both with pink and with violet; lilac with grey.

When the condition of vision in clichromates is tested by means of the spectrum, it is found that they fall into two classes: (1) A class (of green-blind) by whom the whole of the spectrum from red to ycllow is described as y cllow of different degrees of brightness (intensity); the green appears as a palc yellow with a grey or white band in its midst: while the violet end is seen as different shades of blue. (2) A class (of red-blind) whose whole spectrum, from red to green. is seen as green of different intensities, the extreme red being entirely invisible. The violet encl is blue, as in (I), and there is a band of white or grey near the blue end of the green.

Sir John Herschell explained Dalton's peculiarity of vision on the hypothesis that he only possessed two, instead of three, primary sensations.

On the Young-Helmholtz theory, the missing sensation is supposed to be either red or green. At the intersection of the curves tliat represent the violet and green sensations (Fig. 4I3), the red-blind indiviclual will see what he describes as white-viz., the sensation produced by the stimulation of the only two components he possesses. Similarly, at the intersection of the red and violet curves the green-blind person will see what is white to him.

Those who have attempted to explain colour-blindness on Hering's theory have usually assumed that the colour-blind possess the blueyellow, but lack the green-red visual substance. So that on this theory there should be no difterence between red-blindness and greenblindness. But $v$. Kries, in a study of twenty cases of congenital partial colour-blindness, brings forward strong evidence that the red-green blind can be divided, as regards the comparison of red (lithium) and orange (sodium) light, into two sharply-separated groups - a result which is, so far as it goes, in favour of the YoungHelmholtz theory, and against the theory of Hering.

The observations of Burch on temporary colour-blindness proluced by placing the eye behind a transparent coloured screen and focussing a beam of strong sunlight on it, lend additional support to the former theory. Thus, if a spectrum is looked at after greenblindness has been induced by exposure of the eye to green light, the red portion of the spectrum seems to pass into the blue, and no intermediate green band is seen. If the eye is exposed to yellow light it becomes temporarily blind not only for ycllow, but also for red and green. This is in favour of the assumption of the YoungHelmholtz theory that the sensation of yellow is caused when the retinal elements concerned in the production of the sensations of red and green are simultancously stimulated. It is, however, 
equally difficult to reconcile some of the phenomena of colourblindness with the Young-Helmholtz theory. Anomalies and defects of colour-sensation are common accompaniments of pathological lesions of the visual apparatus, and can be produced by various drugs, as by abuse of tobacco. But colour-blindness, in its true sense, is congenital, often hereditary ; the cnlour-blind are 'born, not made.' And although the condition cannot be cured, it is of great importance that it should be recognised in the case of persons occupying positions such as those of engine-drivers, railway-guards, and sailors, in which coloured lights have to be distinguished. For, while it is true that the sensations which red and green lights give the colour-blind are far from being identical (Pole) under favourable conditions, it is preciscly when the conditions are unfavourable-as in a fog or a snow-storm-that the capacity of distinguishing them becomes invaluable (Practical Exercises, p. 998).

Irradiation.- The phenomenon known as irradiation was first described by Kepler, who gave as an example the appearance linown as the 'new moon in the old moon's arms,' where the crescent of the new moon scems to overlap and embrace the unilluminated portion of the lunar disc. A white circle on a black ground (Fig. 4I 7) appears, in a good light, to be larger than an cxactly equal black circle on a white ground. The explanation is as follows: Owing to the aberra-

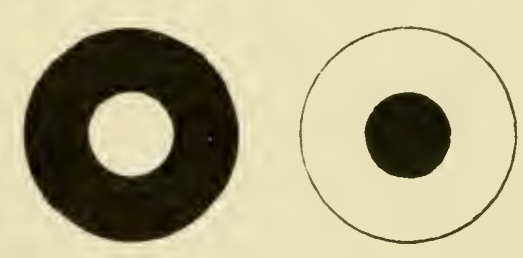

FIG. 4 I 7 . tion of the refractive media of the eye (p. 912), all the rays procecding from the luminous object are not brought accurately to a focus on the retina. and the image is surrounded by diffusion circles (p. 912) which encroach upon the unilluminated boundary. Physically these represent a weaker illumination than that of the image proper, and therefore the latter ought to stand out in its real size as a brighter area surrounded by weaker haloes. That this is not the case, and that the image is projected in its full brightness for a certain distance over its dark boundary, is due to the fact that the eye does not recognise very small differences of brightness. When the accommodation is not perfect, the diffusion circles are, of course, much wider, and irradiation is better marked when the object is a little out of focus.

Visibility of Radium and Röntgen Rays.-It is a question of interest whether the retina can be cxcited by any other disturbances in the ether than ordinary light waves. The Röntgen rays scem to bc capable of cxciting visual sensations, and it is certain that this is true of the rays or the emanation given off by radium. If in the rlark a vessel containing a raclium salt is brought into the neighbourhood of the closed eyelids or the temple, a sensation of briglitness is experienced. This, however, is not due to direct excitation of the retina by the radium rays, but to the plosphorescence set up in the media of the eye by the radium emanation. Of all the tissues the lens is most strongly phosphorescent after exposure to radium. In blindness clue to opacity of the media, where the retina is still sensitive, the radium action is perceived, but not where the retina is totally insensitive to ordinary liglit. The radium rays do not cause bleaching of the visual purple 
The Movements of the Eyes.--That the eyes may be efficient instruments of vision, it is necessary that they should have the power of moving independently of the head. An eye which could not move, though certainly better than an eye which conld not see, would yet be as imperfect after its kind as a ship which could run before the wind, but could not tack. The mere fact that the angle between the visual axes must be adapted to the distance of the object looked at renders this obvious; and the beauty of the intrinsic mechanism of the eyeball has its fitting complement in the precision, delicacy, and range of movement conferred upon it by its extrinsic muscles.

Not only are movements of convergence and divergence of the eyeballs necessary in accommodating for objects at different distances, but without compensatory movements of the eyes it would be impossible to avoid diplopia with every movement of the head; for the images of an object fixed in one position of the head would not continue to fall on corresponding points of the retinæ in another position.

All the complicated movements of the eyeball may be looked upon as rotations round axes passing through a single point, which to a near approximation always remains fixed, and is situated about $\mathrm{I} \cdot 77 \mathrm{~mm}$. behind the centre of the eye.

The position which the eycballs take up when the gaze is directed to the horizon, or to any distant point at the level of the cyes, is callecl the primary position. Here the visual axes are parallel, and the plane passing through them horizontal. While the head remains fixed in this position, the eycballs can rotate up or down around a horizontal axis, or from side to side around a vertical axis; or upwards and inwards, downwards and outwards, downwards and inwards, and upwards and outwards around oblique axes, which always lie in the same plane as the vertical and horizontal axes of rotation-i.e., in the vertical plane passing through the fixed centre of rotation. These facts, spolen of collectively as Listing's law, and first deduced by him from theoretical considerations, wero afterwards proved experimentally by Helmholtz and Donders. It necessarily follow's from Listing's law (and this is, indeed, another way of stating it) that in moving from the primary position into any other, there is no rotation of the eyeball round the visual axis-no wheel-movement, as it is called.

A true rotation of the cyc round the visual axis does, however, occur when the eyes are converged as in accommodation for a near object, each eyeball rotating towards the temporal side. This is especially the case when the cyes are at the same time converged and directed downwards ; and the rotation may amount to as much as $5^{\circ}$. When the head is rolled from side to side, while the eyes are kept fixed on an object, a slight compensatory rotation of the cyeballs takes place against the direction of rotation of the head. The amount of rotation of the eyes is relatively greater for small than for large movements of the head (eye $5^{\circ}$ for head $20^{\circ}$; eye $10^{\circ}$ for hearl So $0^{\circ}$ Kïister). 
The Extrinsic Muscles of the Eyes.- The eveball is acted upon by six muscles arranged in three pairs, which nuay be considered, roughly speaking, as antagonistic sets. These are the internal and external recti, the superior and inferior recti, and the superior and inferior obliqui.

Although the movements of the eye have been very fully stuclied, and are, upon the whole, well understood, our knowledge of the manner in which any given movement is brought about, and of the exact action of the muscles which take part in it, is by no means as copious and jrecise. And from the nature of the case, the grcater part of what we do know has been inlerred from the anatomical relations of the muscles as revealed by dissection in the dead body rather than gained from actual

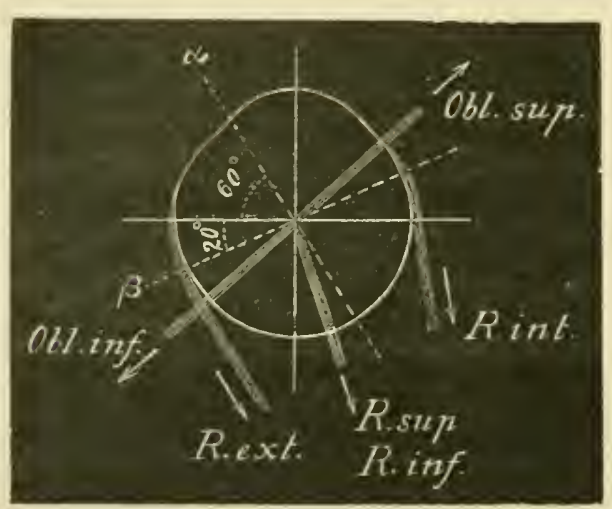

Fig, fy 8 - Horizontal. Section of l.fFt livi

Arrows show direction of pull of the muscles. The axis of rotation of the external and internal recti would pass through the intersection of $\alpha$ and $\beta$ at right angles to the plane of the paper. observation of the living ere. A plane, called the plane of traction, is supposed to pass through the middle points of the origin and insertion of the muscle whose action is to be investigated, and through the centre of rotation of the eyeball. A straight line drawn at right angles to this plane through the centre of rotation is evidently the axis round which the muscle when it contracts will cause the eye to rotate, provided that the fibres of the muscle are symmetrically distributed on each side of the plane of traction. The axes of rotation of the antagonistic pairs almost, but not completely, coincide with each other. The common axis of the external and internal recti practically coincides with the vertical axis of the eyeball (Fig. 4IS) in the primary position. The eye is turned towards the temple when the external rectus alone contracts, towards the nose when the internal rectus alone contracts. The common axis of the superior and inferior recti, $\beta$, lies in the horizontal risual plane in the primary position, but makes an angle of about $20^{\circ}$ with the transverse axis, its inner end being tilted forwards. The consequence is that contraction of the superior rectus turns the eye up, and contraction of the inferior rectus turns it down, 
but both movements are also combined with a slight inward rotation. The common axis of the oblique muscles, $a$, makes an angle of $60^{\circ}$ with the transverse axis, the outer end of it being the most anterior. The direction of traction of the superior oblique is, of course, given not by the line joining its bony origin and its insertion, but by the direction of the portion reflected over the pulley. When the superior oblique contracts alone, the eyeball is rotated outwards and downwards; the inferior oblique causes an outward and upward rotation. None of the common axes of rotation of the pairs of muscles, except that of the external and internal recti, lies in Listing's plane. Now, as we have seen that every movement which the eye, supposed to be originally in the primary position, can execute may be considered as a rotation round an axis in this plane, it is clear that every movement, except truly transverse rotation, must be brought about by more than one pair of muscles. For vertical rotation, the inward pull of the superior rectus is antagonized by a simultaneous outward pull of the inferior oblique ; for downward rotation, the inferior rectus and superior oblique act together. In oblique movements, a muscle of each of the three pairs is concerned. The effect on the eyeball of simultaneous contraction of certain pairs of muscles may be summarized thus :

Extcrnal rectus (outward) + internal rectus (inward) $=$ none.

Superior rectus (upward and inward) + inferior oblique (upward and outward) = upward.

Inferior rectus (downward and inward) + superior oblique (downward and outward) $=$ downward.

HEARING.

The transverse vibrations of the ether fall upon all parts of the surface of the body, but only find nerve-cndings capable of giving the sensation of light in the little discs, which we call the retinæ. so the much longer and slower longitudinal waves of condensation and rarefaction which are being constantly originated in the air or imparted to it by solid or liquid bodies that have been themselves sct vibrating fall upon all parts of the surface, but only produce the sensation of sound when they strike upon the tiny mechanism of the internal ear.

But just as the ethereal vibrations, and especially those of greater wave-length, are able to excite certain end-organs in the skin whicl have to do with the sensation of temperature, so the sound-waves, when sufficiently large, are also capable of stimulating certain cutancous nerves and of giving rise to a sensation of intermittent pressure or thrill. This is readily perceived when the finger is immerserl in a vessel of water into which dips a tube connecter with a source of sound, or when a vibrating bell or tuning-fork is touched. 
So far as we know, what takes place in the ear is essentially similarthat is to say. a mechanical stimulation of the ends of the auditory ncrve, but a stimulation which acts through, and is graduated and controlled by, a special intermediate mechanism.

As the visual apparatus consists of a sensitive surface, the retina, which contains the end-organs of the optic nerve and of dioptric arrangements which receive and focus the rays of light, the auditory apparatus consists of the sensitive end-organs of the cochlear division of the eighth nerve and of a mechanism which receives the sound-waves and communicates them to these.

Physiological Anatomy of the Ear.--At the bottom of the external auditory meatus lics the membrana tympani. a nearly circular membrane set like a drum-skin in a ring of bone, and separating

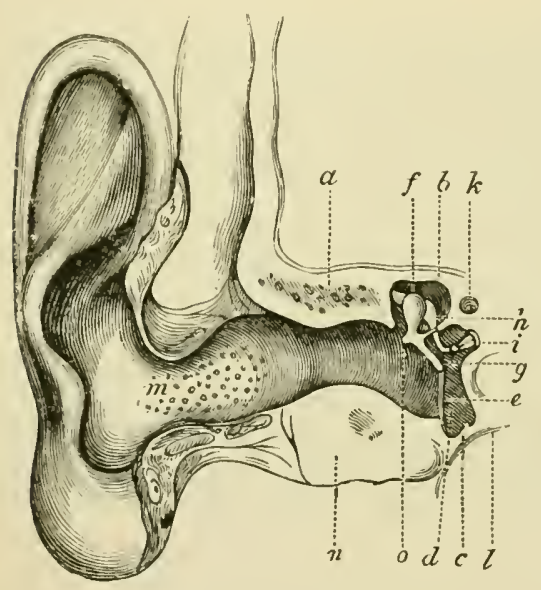

FIG. 4I9.-THE EAR.

$m$, external meatus ; $f$, head of malleus ; $o$, short process of inalleus; $g$, handle of malleus ; $h$, incus ; $i$, font of stapes in oval foramen : $e$, tympanic membrane. the meatus from the tympanum or middle ear. Its external surface looks obliquely downwards, and at the same time somewhat forwaris, so that if prolonged the menbranes of the two cars would cut each other in front of, and also below. the horizontal line passing through the centre of each (Figs. 419, 420).

The tympanum contains a chain of little bones stretching right across it from outer to inner wall. Of these the malleus, or hammer, is the most cxternal. Its manubritim, or handle, is inserted into the membrana tympani, which is not stretched taut within its bony ring, but bulges inwards at the centre, where the handle of the malleus is attached. The stapes, or stirrup, is the most internal of the chain of ossicles, and is inserted by its foot-plate into a small oval opening-the foramen ovale-on the inner wall of the tympanic cavity. A membranons ring - the orbicular membrane-surrounds the foot of the stapes, helping to fill $\dddot{0}$, the foramen and attaching the bone to its edges. The inner surface of the foot of the stapes is in contact with the perilymph of the internal ear. The incus, or anvil. forms a link between the malleus and the stapes. The auditory ossicles, as well as the whole cavity of the tympanum, are covered by pavement epithelium.

The tympanum is not an absolutely closed chamber; it has one channel of communication with the external air--the Eustaclian tube-which opens into the pharynx. By the action of the cilia lining this tube the scanty secretion of the middle ear is moved towards its pharyngeal opening, which, usually closed. is opened 
when a swallowing movement occurs. Its function is to keep the pressure in the miclelle ear appoximately that of the atmospliere. In a balloon aseent in excess of pressure is established on the internal surface of the tympanic membrane. In the air-lock of a caisson when the air is being compressed the excess of pressure is on the external surface of the membrane. The feeling of uncomfortable tension is relieved in both cases by swallowing movements which allow the pressure in the tympanmm to arjust itself to that in the pharynx. In catarrh of the naso-pharynx the orifice may be occluded, and this is accompinied by impairment of hearing and a disagrecable sensation of tension in the car, owing to absorption and consequent rarefaction of the air in the tympan um." patient instinctively makes efforts which increase the pharyngeal pressure from time to time so as to open the tube.

The loosely-jointed chain of ossicles is steadicd and its movements directed by ligaments and by the tension of its terminal membranes. It forms a kind of bent lever, by which the oscillations of the membrana tympani are transferred to the membrane covering the oval foramen, and at the same time reduced in size. Two slender muscles, the tensor tympani and stapedius, contained in the tympanic cavity, are also connected with and may act upon the ossicles. The former lies in a groove above the

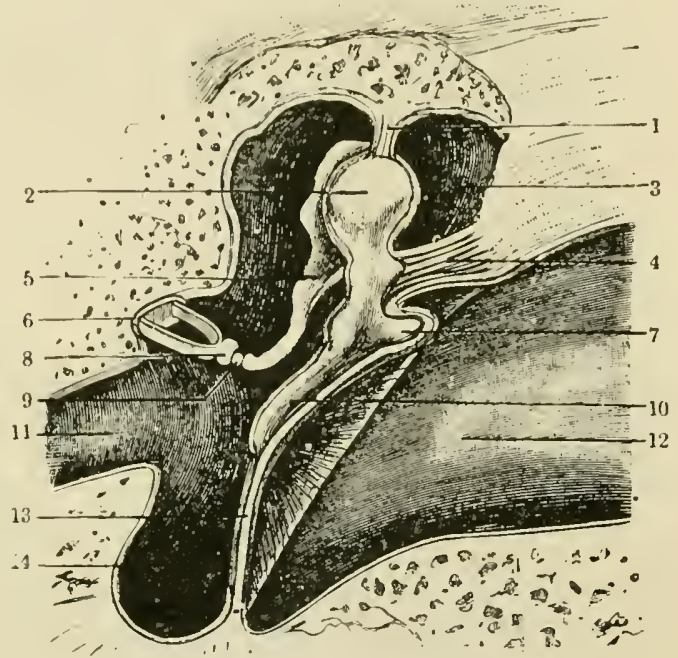

Fig. 420.-Tympanum of Left Ear, showing the OSSICles (MIORRIS).

I, superior, and 4, external, ligament of malleus ; 2, head: 7 , short process, and Io, manubrium or handle, of malleus ; 5 , long process of incus, terminating in 9 , the os orbiculare; 6 , base, and 8 , head, of stapes ; I , Eustachian tube; I2, external auditory neatus; I3, membrana tympani ; 3 , upper, and I4, lower, part of tympanum.

Eustachicun tube, and

its tendon, passing round a kind of osseons pulley (processus cochleariformis), is inserted into the handle of the mallens; the stapedius is lodged in a hollow of the inner bony wall of the tympanum. Its tendon is attached to the neck of the stapes near its articulation with the incus. This inner wall is pierced not only by the oval foramen, but also by a round opening, the fenestra rotunda, which is closed by a membrane to which the name of secondary membrana tympani is sometimes given.

The internal ear consists of the bony labyrinth, a series of curiously cxcavated and communicating spaces in the substance of the petrous 
portion of the temporal bone, filled with a linuid called the perilymph, in which, anchored by strands of connective tissue, floats a corresponding series of membranous canals (the membranous labyrinth). filled with a liquid called enclolymph. The labyrinth of the internal ear is divided into three well-marked parts: the cochlea, the vestibulc, and the semicircular canals (Fig. $f_{21}$ ). The cochlea, the mest anterior of the three, consists of a convoluterl tube which coils round a central pillar, the columella or modiolus, like a spiral staircase. The lamina spiralis projects from the modiolus and divides the tube inte an upper compartment, the scala vestibuli, and a lower. the scala tympani ( 1 ig. 422 ). The part of the lamina next the modiolus is of bone, but it is completed at its outer elge by a membrane, the lamina spiralis membranacea, or basilar menbrane. The scala tympani abuts on the fenestra rotunda, and its perilympl is only separated from the air of the tympanic cavity by the membrane which closes that opening. At the apex of the coclilea the lamina spiralis is inconplete, ending in a crescentic border, so that the scala

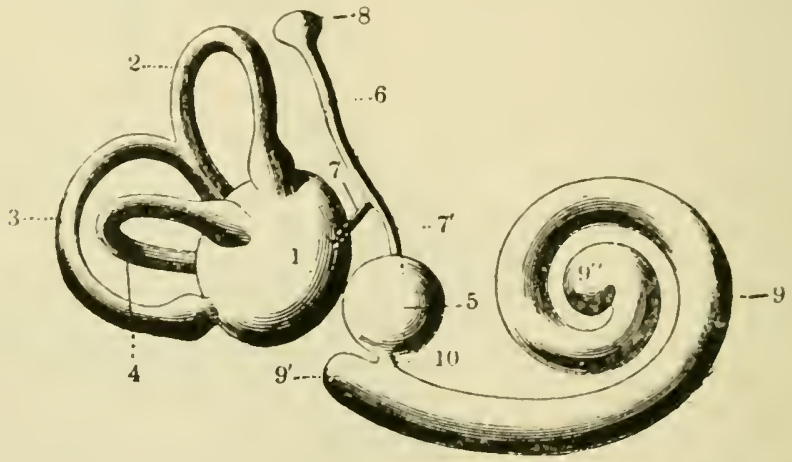

Fig. 421.-Diagran of Right Membranoes Labyrinth (AFter TESTUT).

I, utricle: 2, 3, 4, superior, posterior, and horizontal semicircular cantils : 5. saccule: 6 , ductus endolymphaticus arising by two branches, $7.7^{\prime}: 8$, saccus endolymphaticus : 9. canalis cochlearis (canal of the cochlea) ending at $9^{\prime}$, and 9" : Io, canalis reuniens.

tympani and the scala vestibuli here communicate by a small opening, the helicotrema. The scala restibuli communicates with the vestibule, and the vestibule with the semicircular canals, so that the perilymph of the entire labyrinth forms a continuous sheet separated from the cavity of the middle ear by the structures that fill up the round and oval foramina. In the membranous labyrinth. and in it alone, are contained the end-organs of the auditory nerve. The membranous portion of the cochlea is a small canal of triangular section, cut off from the scala vestibuli by the membrane of Reissner, which stretches from near the elge of the bony spiral lamina to the outer wall (Fig. +23). to which it is attached by the spiral liganent. The canal lias received the name of the scala media. or canal of the cochlea. The membrane of Reissner forms its roof. Its floor is composed ( 1 ) of the projecting edge of the spiral lamina. called the limbus, and (2) of the basilar nembranc. The most conspicuous constituent of the basilar membrane is a layer of stiff. parallel. trans- 
parent fibres arranged rallially--i.e., in the direction from limbus to spiral ligament. They are embedelel in a homogeneons material. Jelow the cochleat ciulal ends blindly, but communicates by a sicle-channel with the portion of the membranous vestibule called the saccule, which in its tmm communicates with the utricle by the $Y$-shaped origin of the ductus enclolymphaticus. Into the utricle opentice three semicircular canals, the cndolymph of which has,

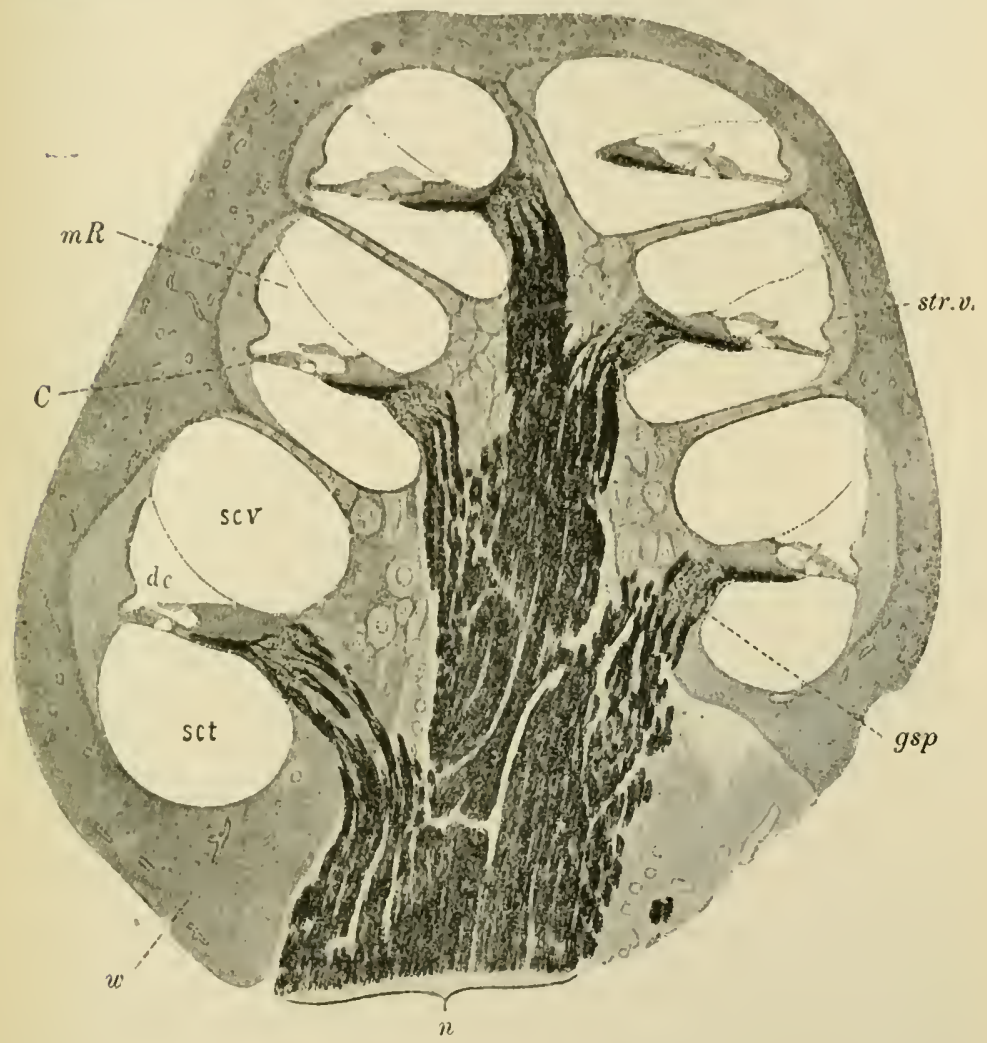

Fig, 422.-Longitudinal Section throvgit the Cochlea of a Cat (Schäfer, AFter Sobotta). $\times 25$.

$d c$, canal or duct of cochlea; $s c v$, scala vestibuli ; sct, scala tympani; w, bony wall of cochlea ; $C$, organ of Corti : $m R$, Reissner's membrane ; $n$, fibres of cochlear nerve : gsp, ganglion spirale : str.v., stria vascularis.

therefore, free communication with that of the vestibule and cochlea. But although the semicircular canals and vestibule belong anatomically to the internal ear, and are supplied by branches of the auditory nerve, we have no positive proof that in the higher animals, at least, they are in any way concerned in hearing; and since experiment has assigned them a definite function of another kind (p. 834), we shall not consider them further in this connection. The scala media 
contains the organ of Corti, which (Fig. 42.4 ) consists of a series of modified epithelial cells planted npon the basilar membrane. The epithelial cells are of thrce kinds: (I) supporting epithelial cells ; (2) the pillars or rorls of Corti, in two series (inner and outer), sloped against each other like the rafters of a roof, and covering in a vault or tumnel which runs along the whole of the scala mediu from the base to the apex of the cochlea; (3) the hair-cells, around which the fibres of the atuditory nerve arborize. These last are columnar epithelial cells, surmounted by haiss. They are arranged in several rows, one row lying just internal to the inner line of pillars, and several rows external to the outer line of pillars. Between the outer

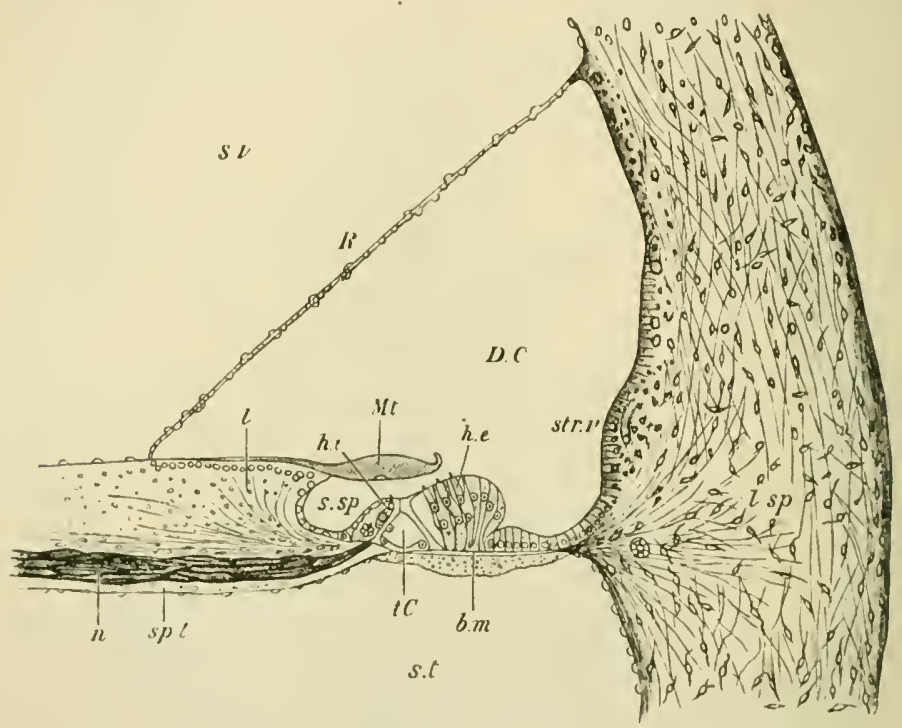

Fig. 423. - Vertical Section of the First Turn of the Cocilea (after Retzius).

D.C, canal of cochlea ; $t C$, tumnel of Corti; $b . m$, basilar membrane; h.i, h.e, internal and external hair-cells;.$M$, membrana tectoria; s.sp, spiral grome: $s t r . v$, stria vascularis; $s p . l$, spiral lamina: $n$, fibres of the enchlear nerve; $l$, limbus lianina spiralis ; $R$, Reissner's membrane : s.v, scala vestibuli ; s.t, scala tympani : l.sp. spiral ligament.

hair-cells are supporting cells (cells of Deiters). A thin membrane, the reticular lamina or membrana reticularis, composed of fiddleshaped rings or phalanges, covers the hair-cells, and through openings in it the hairs project. A thicker membrane, the membrana tectoria, springing from the edge of the osscous spiral lamina near the attachment of Reissner's membrane. forms a kind of canopy over both pillars and hair-cells. "The outer wall of the canal of the cochlea is clad by cubical epithelium covering a membranc rishly supplicd with bloodvessels (stria vascularis). The fact that the hair-cells of Corti's organ are connecterl with the fibres of the cochlear division of the auditory nerve, and its elaborate structure, suggest that it must play a peculiar part in auditory sensation. Comparative 
anatomy shows us that the cochlea is the most highly-rleveloped portion of the internal car, the last to appear in its evolution, and the most specialized. It is absent in fishes, which possess only a vestibule and one to three semicireular canals. It first aequires importance in reptiles. Dut ittions its higlost development in mitmmals: and there is every reatson to believe that it is the terminal appatritus jof the sense of locitring.

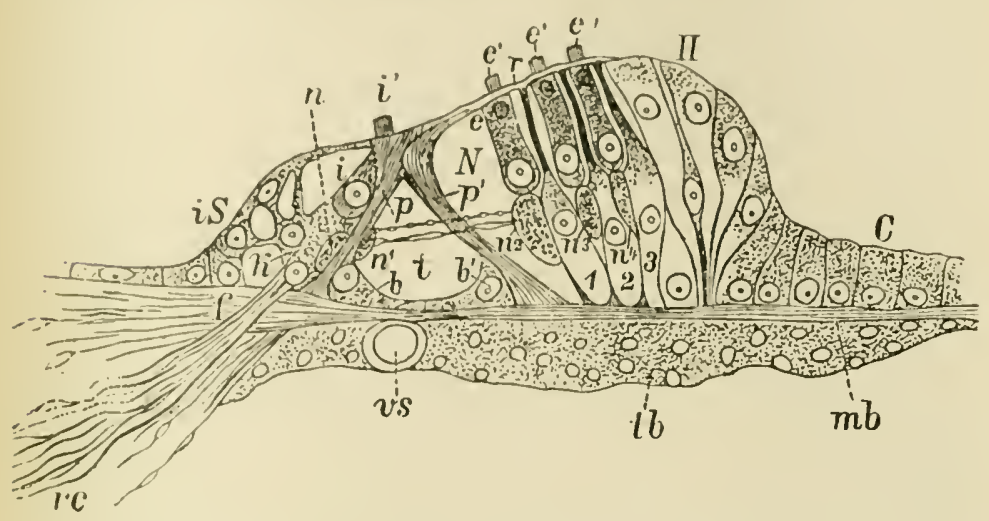

Fig. 424.-Organ of Corti (Barker, AFter RetziUs).

$m b$, basilar membrale ; $t b$, its tympanal covering ; $v s$, bloodvessel (vas spirale); $r c$, inedullated distal processes of bipolar nerve-cells in the ganglion spirale, passing in to arborize around the hair-cells ; iS, epithelial cells continuous with the epithelium of basal cell, $b ; p^{\prime}$, outer pillar with its basal cell, $b^{\prime} ; \mathbf{I}, 2,3$, supporting cells of Deiters, whose processes run up to be attached to the lamina reticularis, $r ; \mathrm{H}$, Hensen's supporting cells ; $\mathrm{C}$, cells of Claudius ; $i$, internal hair-cell with its hairs, $i^{\prime}$ (the upper part of the hair-cell is concealed by the head of the inner pillar of Corti) : $e$. external hair-cell ; $e^{\prime}$, hairs of three external hair-cells; $n, n^{1}$, to $n^{4}$, cross-sections of the spiral strand of cochlear nerve-fibres.

Functions of the Auditory Ossicles.-The anatomical arrangements of the middle ear suggest that the tympanic membrane and the chain of ossicles liave the function of transmitting the sound-raves to the liquids of the labyrinth; and observation and experiment fully confirm this idea. Tracings of the movements of the ossicles have been obtained by attaching very small levers to them, and their movements have ibeen directly observed with the microscope. Even in man it may be shown, by viewing the membrane through a series of slits in a rapidly-revolving disc (stroboscope), that it vibrates when sound-waves fall on it.

When the handle of the malleus moves inwards, rotating around an axis which may be supposed to pass through its neck, its head moves in the opposite direction. The joint between that bone and the incus is thus locked, on account of the shape of the articular surfaces. The long process of the incus, consti- 
tuting the second portion of the bent lever, passes inwards, carrving with it the stapes, which is attached to it by an almost rigid joint, and the stapes is pressed into the oval foramen. Since the long process of the incus is about one-third shorter than the liandle of the malleus, the excursion of the point of the former is correspondingly smaller than that of the latter, but at the same time more powerful. When the tympanic membrane passes ontwards, the handle of the malleus and foot of the stapes do the same. But the joint now unlocks, and excessive outward movement of the stapes, which might result in its being torn from its orbicular attachment, is prevented. The ossicles vibrate en masse. It is only to a trifling extent that sound can be conducted through them to the labyrinth as a molecular vibration ; for when they are anchylosed, and the foot of the stapes fixed immovably in the foramen ovale, as sometimes occurs in disease, hearing is greatly impaired.

Of course, every vibration of the tympanic membrane must cause a corresponding condensation and rarefaction of the air in the middle ear ; and this may act on the membrane closing the fenestra rotunda, and set up oscillations in the perilymph of the scala tympani. That this is a possible method of conduction of sound is shown by the fact that, even after closure of the oval foramen, a slight power of hearing may remain. But under ordinary conditions by far the most important part of the conduction takes place viâ the ossicles. And when it is remembered that the tympanic membrane is about thirty times larger than that which fills the oval foramen, it will be seen that the force acting on unit area of the foot of the stapes may be much greater than that acting on unit area of the membrana tympani, and that the mode of transmission by the ossicles is a very advantageous method of transforming the feeble but comparatively large excursions of the tympanic membrane into the smaller but more powerful movements of the stapes. The average excursion of the membrane of the oval foramen does not at most amount to more than 0.04 millimetre. Even the so-called cranial conduction of sound when a tuning-fork is held between the teeth or put in contact with the head, which was at one time supposed to be due solely to direct transmission of the vibrations through the bones of the skull to the liquids of the labyrinth or the end-organs of the auditory nerve, has been shown to take place, in great part, through the membrana tympani and ossicles; the vibrations travel through the bones to the tympanic membrane, and set it oscillating. So that this test, when applied to distinguish deafness caused by disease of the middle ear from deafness due to disease of the labyrinth or the central nervous system may easily mislead, although it 
enables 14 s to say whether the auditory meatus is blocked-by wax, $c . g$. beyond the tympanic numbrane.

A nembranc like a drum-head has a note of its own, which it gives ont when struck, and it vibrates more readily to this note than to any other. It would rvilently be a serions disarlvantage if the tympanic membranc, whose office it is to receive all kinds of vibrations, and respond to all, had a marked fundamental tone which would be continually obtrucling itself among other notes. The Iifficulty is obviated by the damping action of the ossicles and the liquids of the labyrintli on the movements of the membrane, which in acklition is not stretcherl, but lies slackly in its bony frame, so that when the landle of the malleus is detached from it, it retains its shape and position.

The tensor tympani, when it contracts, pulls inwards the hanclle of the malleus, and thus increases the tension of the tympanic membrane. The precise object of this is obscure. It has been suggested that clamping of the movements of the auditory ossicles is thus secured. Another theory is that the increased tension of the membrane renders it more capable of responding to higher tones, and that the muscle thus acts as a kind of accommodating mechanism. But Hensen las observed that the tensor only contracts at the beginning of a sound, and relaxes again when the sound is continued; and this is difficult to reconcile with either of these hypotheses. The muscle is normally excited refiexly through the vibrations of the membrana tympani, but some individuals have the power of throwing it into voluntary contraction, which is accompanied by a feeling of pressure in the ear and a harsh sound. The function of the stapedius is unknown. Its contraction would tend to press the posterior end of the foot-plate of the stapes decper into the foramen ovale, and cause the anterior end to move in the opposite direction; but it is not easy to sec how this would affect the action of the auclitory mechanism.

The tensor tympani is supplied by the fifth nerve through a branch from the otic ganglion; the stapedius is supplied by the seventh. Paralysis of the fifth nerve may be accompanied with difficulty of hearing, especially for faint sounds. When the seventh nerve is paralyzed, increased sensitiveness to loud sounds has been observed.

We have already recognised the organ of Corti, particularly the hair-cells, as a sensory epithelium which constitutes the terminal apparatus of the cochlear nerve. The adequate stimulus of the auditory receptors is the periodic changes of pressure in the endolympli. But there are various opinions as to how these vibrations are transmitted to the hair-cells, and as to how the vibrations of the hair-cells are translated into nerve impulses in the auditory fibres. The pillars of Corti, the basilar membrane, and the membrana tectoria, have in turn been regarded as the structures immediately set into vibration by the changes in the endolymph. The case for the tectorial membrane is perhaps the most plausible, for its position renders it most capable of acting on the hairs. Others have supposed that the hairs of the hair-cells are directly affected by the endolymph. Some, 
despairing of further analysis, content themselves with the conclusion that the organ of Corti vibrates as a whole. Some of these theories will be again referred to in considering what is the greatest problem of the physiology of hearing, viz.:

The Perception of Pitch-Analysis of Complex Sounds. As the eye, or, rather, the retina plus the brain, can perceive colour, so the labyrinth plus the brain can perceive pitch. The colour-sensation produced by ethereal waves of definite frequency depends on that frequency; and upon the frequency of the aerial vibrations depends also the pitch of a musical note. But there is this difference between the eye and the ear: that while the sensation produced by a mixture of rays of light of different wave-length is always a simple sensation-that is, a sensation which we do not perceive to be built up of a number of sensations, which, in other words, we do not analyze-the ear can perceive at the same time, and distinguish from cach other, the components of a complex sound. When a number of notes of different pitch are sounded together at the same distance from the ear the disturbance which reaches the membrana tympani is the physical resultant of all the disturbances produced by the individual notes, and it strikes upon the membrane as a single wave. 'A single curve describes all that the ear can possibly hear as the result of the most complicated musical performance. . . . In the complicated sound the variations of the pressure of the air are more abrupt, more sudden, less smooth, and less distinctly periodic than they are in softer, purer, and simpler sound. But the superposition of the different effects is really a marvel of marvels' (Kelvin). The ear or brain must, therefore, possess the power of resolving the complex vibrations into their constituents, else we should have a mixed or blended sensation, and not a sensation in which it is possible to distinguish the constituents of which it is made up. Several hypotheses have been proposed to explain this physiological analysis of sound, on the assumption that the analysis takes place in the labyrinth. The most important, in spite of certain defects, is still that of Helmholtz.

Helmholtz attempted to explain the perception of pitch on the assumption that in the internal ear there exists a series of resonators, each of which is fitted to respond by sympathetic vibration to a particular note, while the others are unaffected; just as when a note is sung before an open piano it is taken up by the string which is attuned to the same pitch and ignored by the rest. Let us suppose that a given fibre of the auditory nerve ends in an organ which is only set vibrating by waves impinging on it at the rate of 100 a second, and that the end-organ of another fibre is only influenced by waves with a frequency of 200 a second. 
Then, on the doctrine of 'specitic energy' (according to which the sensation caused by stimulation of a nerve depends not on the particular kind of stimulus but on the anatomical connection of the nerve with certain nerve centres), in whatever way the first fibre is excited, a sensation corresponding to a note with a pitch of 100 a second will be perceived. Whenever the second fibre is excited, the sensation will be that of a note of 200 a second, or the octare of the first. If both fibres are excited at the same time the two notes will be heard together. Now, Hensen actually observed that in the auditory organs of some crustaceans, the hair-like processes of certain epithelial cells can be set swinging by waves of sound, and, further, that they do not all vibrate to the same note unless the sound is very loud. In the lobster there are between four and five hundred of these hairs, varying in length from $I+\mu$ to $740 \mu$; and in some insects, such as the locust, similar hairs, also graduated in length, exist.

To gain an anatomical basis for his theory, Helmholtz supposed first of all that the pillars of Corti were the vibrating structures, and that, directly or through the hair-cells, their mechanical ibrations were translated into impulses in the auditory nerve-fibres. But apart from the fact that their number is too small (about 3,000) to allow us to assign one rod to each perceptible difference of pitch, and their dimensions too similar to permit of the requisite range of vibration frequency, it was pointed out that birds do not possess pillars of Corti-a fact which was decisive against the assumption of Helmholtz. since nobody denies to singing-birds the power of appreciating pitch. Helmholtz accordingly, choosing between the remaining possibilities, gave up the pillars of Corti, and adopting a suggestion of Hensen, substituted the radial fibres of the basilar membrane as his hypothetical resonators. These are more adequate to the task imposed on them, since their range of length is far greater ( $4 \mathrm{I} \mu$ at the base to $495 \mu$ at the apex of the cochlea-Hensen); and the elaborate structure of Corti's organ certainly suggests that some one or other of its elements may be endowed with such a function. Experimentally, too, it has been shown that destruction of the apex of the cochlea causes loss of appreciation of low notes, and destruction of the base loss of appreciation of high notes, which agrees with Helmholtz's view. But while the theory of peripheral analysis of pitch tends upon the whole to be strengthened as evidence gathers, it is possible that the analysis is accomplished in some other way than by sympathetic resonance.

Ewald has developed a theory according to which each note causes the basilar membrane to vibrate throughout its whole extent in

$$
6 I-2
$$


such a waty that stationary waves are produced in it, like the Chladni's figures seen on a metal plate strewed with sand when it is set into vibration. The pattern of the movement, the 'sound-picture,' will be different for each tone, since the interval between the waves will be different. The hair-cells and auditory fibres of particular parts of the organ of Corti will therefore be stimulated by the pressure of the menbranc, or escape stimulation, accorling to the position of the stationary waves with reference to them for each note. In this way each souncl-picture will be printed. so to speak, upun the sensitive terminal apparatus of the atuditory nerve, as a letter is printed upon is piece of paper by a type. The corresponding excitation pattern-i.c., the particular distribution of cochlear fibres stimulated - is supposed to be associated in consciousness with the appreciation of the pitch of the particular note. Ervald has endeavoured to support his theory by showing that fine membranes of the dimensions of the basilar membrane do vield very distinct sound-pictures for

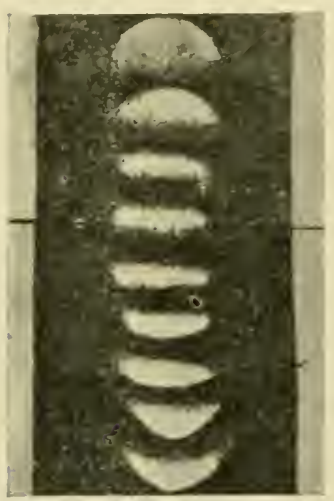

FIG. 425.-PHOTOGRAPH OF A SOUND-PICTURE (EWALD). stamp the impress of its form on the auditory cxcitation-wave; just as in a telephone every wave in the air causes a swing of the vibrating plate. and thus sets up a current of corresponding intensity and clura. ticn in the wires. This theory evidently abandons the doctrine of specific energy for the particular case of the analysis of pitch. for it assunes that differences of auditory sensation are related to differences in the nature of the impulses travelling up the auditory nerve, and not merely to clifferences in the anatomical connections (peripleral and central) of the auditory nerve-fibres. It is unsatisfactory because it takes no account of the remarkable and suggestive structurc of the telcphone plate-i.e. of the organ of Corti-and gives no hint of how the analysis is accomplished in the central organ.

The range of hearing is very great. The highest audible tone corresponds to 30,000 to 40,000 vibrations a second, the lowest to about 30. Between these limits as many as 6,000 variations of pitch can be perceived. 
Wrien has elalonately investigated the fuestion how the sensitiveness of the car viries for tones of different pitch. A tone of $5^{\circ}$ vibrations a second, in orier to be just heard, must have an intensity corresponcling to abut ron million times as much energy as is necied for a tone of 2,000 vibrations. It is only on the extraordinary sensibility of the ear for the range of tones used in ordinary speech that the possibility of understanding speech depends when the circumstances are unfavourable-e.g., at a great distance, or in the presence of much stronger accompanying noises.

\section{Smell and Taste.}

Smell was defined by Kant as 'taste at a distance'; and it is obvious that these two senses not only form a natural group when the quality of the sensations is considered, but are closely associated in their physiological action, especially in connection with the perception of the flavour of the food. The olfactory endnigans in the unucous membrane of the upper part of the nostrils, the so-called regio olfactoria, have been already described (p. SI6). In cases of anosmia, in which the olfactory nerve is absent or paralyzed, smell is abolished; but substances such as ammonia and acetic acid, which stimulate the ordinary sensory nerves (nasal branch of fifth) of the olfactory mucous membrane, are still perceived, though not distinguished from each other. In fact, the so-called pungent odour of these substances is no more a true smell than the sense of smarting they produce when their vapour comes in contact witl a sensory surface like the conjunctiva, or a piece of skin devoid of epidermis.

It was at one time believed that odoriferous particles could not be appreciated unless they were borne by the air into the nostrils ; but this appears not to be the case, for the smell of substances dissolved in physiological salt solution is distinctly perceived when the nostrils are filled with the liquid; and fish, as every linefisherman knows, have no difficulty in finding a bait in the dark.

The substances which can affect the olfactory mucous membrane can be livided into four groups :

I. Those which act only on the olfactory nerves, the odours proper.

2. Substances which act at the same time on olfactory nerves, and on nerves of common sensation (tactile nerves)-e.g.. acetic acid.

3. Substances which act at the same time on the gustatory ncrves.

4. Substances which act only on the nerves of common sensation (tactile nerves) - e.g., carbon dioxide.

Zwaardemaker has classified the pure odlours as follows :

(I) Ethereal odours, as those of fruits; (2) aromatic odours, as of camphor or bitter almonds; (3) fragrant olours. as of flowers ; (4) ambrosial odours, as of amber or musk; (5) F́garlic odours, as 
of onion, garlic, asaf(xticla; (0) empyreumatic, or burning odours, as of burnt coffec or tobacco smoke: (7) caprylic or goat odours, as of sweat; (8) repulsive odours, as the odour of the disease ozma : (9) nauseating odours, as of freces or putrefying material.

The most interesting form of inadequate stimulation is electrical excitation of the olfactory mucous membrane. which causes a sensation like the smell of phosphorus. The sensation is experienced at the kathode on clesure and the anode on opening. As to the manner in which the multitudincus adequate stimuli excite the olfactory nerves, we can only suppose that they act as chemical stimuli. Smell and taste are pre-eminently the 'chemical' senses, as sight and hearing are pre-eminently 'physical' senses. But little is known of the relation between the chemical constitution or physical properties of substances and the quality of the odoriferous sensation which they excite, although Haycraft has pointed out some interesting relations between the atomic weights of certain elements and their power of exciting odlours. The number of distinct oclours which can be perceived is so great that it is scarcely conccivable that each is subserved by special olfactory fibres. Marked changes occur in discase, and all odours need not be affected to the saine extent. Some may be almost normally perceived, while relative or complete loss of smell exists as regards others. These and other facts have given rise to the idea that there are several groups of olfactory fibres, each concerned in the appreciation of a particular oclour or group of ollours. Yet it has not proved possible to reduce them to a limited number of fundamental odours and their combinations.

Acuteness of smell may be measured by arrangements called olfactometers. Zwaardemaker's olfactoneter consists of a piece of indiarubber tubing fitted inside a glass tube, through which air is clrawn into the nostrils. Another glass tube just fitting the rubber tube is pushed inside it, so as to cover a portion of it. The minimum amount of surface of the indiarubber tube which must be left exposed so that the smell of the rubber may be perceived is a measure of the acuteness of smell. To investigate other odours tubes of the corresponding odorous substances can be constructed.

Taste.-The sense of taste is not so strictly localized as the sense of smell. The tip and sides of the tongue, its root, the neighbouring portions of the soft palate, and a strip in the centre of the dorsum, are certainly endowed with the sense of taste ; but the exact limits of the sensitive areas have not been defined, and, indeed, vary in different individuals.

The nerves of taste are the glosso-pharyngeal. which innervates the posterior part of the tongue, and the lingual. which supplies its tip) (see p. $82 \mathrm{r}$ ). The encl-organs of the gustatory nerves are the taste-buds or taste-bulbs, which stud the fungiform and circumvallate papilla, and are most characteristically seen in the moats surrounding the latter. They are barrel-like bodies, the staves of the barrel being represented by supporting cells; each bud encloses a number of gustatory cells with fine processes at their free ends projecting through the superficial end of the barrel. They are surrounded by the end arborizations of the fibres of the gustatory nerves. Taste-buds are also found on the posterior surface of the epiglottis and in the larynx. It has been suggested that these form the afferent end-organs of a reflex apparatus which guards the glottis 
against the ontrince of food in deglutition (WVilson). Epithelial bucls, different from the olfactory clements, also occur in the nlfactory region of the natsal mucous membrane. It is possible that the so-called nasal taste-er.g. the sweet taste caused by chloroform when aspiratid in not tor small an amount through the nose $\rightarrow$ depends upon these buts.

As to the properties in virtue of which sapid substances are enabled to stimulate the gustatory nerve-endings, we know that they must be soluble in the liquids of the mouth, and there our knowledge ends. An attempt has been made by various authors to connect the taste of suclı bodies with their chemical composition, but researches of this kind lave not hitherto yielded much fruit. The number of distinct qualities of taste sensation is considerable, but by no means so great as the number of qualities of olfactory sensations, and they are more easily reduced to a few primary or fundamental sensations. Sapid substances have generally been divided into four classes, as regards the fundamental sensations produced by then-viz. : (I) Sweet, (2) acid, (3) bitter, (4) saline. All taste sensations seem to be combinations of these, or combinations of one or more of them with olfactory sensations, or with sensations due to excitation of the ordinary sensory nerves of the tongue.

Sweet and acid tastes are best appreciated by the tip, and bitter tastes by the base, of the tongue. Differences have been detected between individual papilla in their power of reaction to sapid substances which produce one or other of the fundamental sensations. Of I25 fungiform papillae tested with solutions of tartaric acid, sugar, and quinine, 27 gave no sensation of taste. Tartaric acid evoked its acid taste in $9 \mathrm{I}$ of the remaining 98, sugar its sweet taste in 79, and quinine its bitter taste in $7 \mathrm{r}$; $\mathrm{r} 2$ reacted only to tartaric acid, and 3 only to sugar (Ölirwall). Such facts indicate, although they do not definitely prove, the existence of specific receptors for each of the fundamental taste sensations-i.e., gustatory end-organs, which are easily excited by an adequate stimulus (acid, e.g., in the case of an 'acid' taste-bud), with difficulty or not at all by an inadequate stimulus.

The form of inadequate stimulation most investigated is that produced when a constant current is passed through the tongue. An acid taste is expericnced at the positive, and an alkaline or bitter taste at the negative, pole; and this is the case even when the current is conclucted to and from the tongue by unpolarizable combinations, which prevent the deposition of electrolytic products on the mucous membrane (p. 625). The sensations are due to stimulation of the gustatory encl-organs and not of the nerve-trunks.

Normal lymph, which bathes these end-organs, does not excite any sensation of taste, but when the composition of the blood is altered in discase or by the introduction of foreign substances, 
tastes of varions linds may be perceived. Sometimes this may be due to the stimulation of substances excreted in the saliva; but in other cases it seems that, without passing beyond the blood and lympl, foreign substances may excite the gustatory nerves.

Flavour cmbraces a group of mixed sensations in which smell and taste are both concerned, as is shown by the common observation that a person suffering from a cold in the head. which blunts his sense of smell. loses the proper flarnur of his fookl. and that some nauscous medicines do not taste so badly when the nostrils are held.

In common spech, the two sensations are frequently confouncled with each other and with tactile sensations. Thus the 'bouquet' of wines, which most people imagine to be a sensation of taste, is in reality a sensation of smell ; the astringent 'taste' of tannic acill is not a taste at all, but a tactile sensation; the 'hot' taste of mustarcl is no more a true sensation of taste than the sensation producel by the same substance when applied in the form of a mustard poultice to the skin.

\section{Tactile and Common Sensations.}

Under the sense of touch it is usual to include a group of sensations which differ in quality-and that in some instances to as

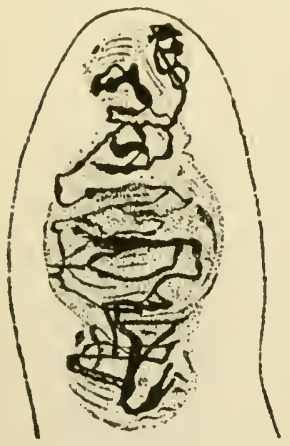

Fin. 426.-T A C T I L E CORPUSCLE FROM SKIX OF FIXGER (SMIRNOW).

(Golgi preparation.) The winding and intersecting black lines are. the non-medullated endings of the one or more nerve-fibres that enter the corpuscle. great an extent as any of the sensations which are universally considered as separate and distinct-but agree in this, that the end-organs by which they are perceived are all situated in the skin, the mucous membranes, or the subcutaneous tissue. Such are the common tactile sensations-including pressure, tickling, and itchingand the sensations of temperature, or, more correctly, of change of temperature, or of warmth and cold. The sensation of pain, although it cannot be absolutely separated from these, ouglit not to be grouped along with them. It is called forth by the stimulation of afferent nervefibres in their course; and it may originate, under certain conditions, in internal organs which are devoid of tactile sensibility, and the functional activity of which in their normal state gives rise to no special sensation at all. The peculiar sensation associated with voluntary muscular effort, to which tlie name of the muscular sense has been given, also deserves a separate place; for although it may in part depend on tactile sensations set up through the medium of end-organs situated in muscle, tendon, or the structures which enter into the formation of the joints, other elements are, in all probability, involved. 
The simplest form of tactile sensation is that of mere contact, as when the skin is lightly touched with the blunt end of a pencil. This soon deepens into the sensition of pressure if the contact is made closer; and eventually the sense of pressure merges into a feeling of pain. Most physiolngists agree that in the skin itself four fundamental qualities of sensation are represented-touch in the restricted sense (the sensation elicited by light contact), warmth, cold, and pain. Pressure is mainly a sensation connected with the stimulation of structures deeper than the skine.g., the sensation of contact is abolished in cicatrices where the true skin has been clestroyed, while semsibility to pressure persists - although the sensation of light pressure may be to some extent represented in the skin itself in association with touch. In a sonewhat diagrammatic sense it may be said that the surface of the skin is divided into a great number of very small areas, each of which is related especially to one or other of the four fundamental sensations. Areas concerned in one sensation are everywhere mingled with areas concerned in the others. By appropriate methods it has been found possible to determine the existence on the shin of the trunk and limbs of not less than 30,000 'warm-spots,' which always react to stimulation by a sensation of warmth; 250,000 'cold-spots,' which react by a sensation of cold; and half a million touch-spots, whose specific reaction is a sensation of touch. It is more difficult to localize definitely bounded 'pain-spots,' partly because of the very rich supply of pain-fibres to the skin. Yet there is reason to believe that pain, like touch, warmth, and cold, is subserved by separate receptors. The simplest assumption which will satisfactorily account for the distribution of the four fundamental cutaneous sensations is that the skin is supplied with four kinds of nervefibres, anatomically as well as functionally distinct. Some fibres minister to the sensation of cold, others to that of warmth, others to that of touch, and others still to pain. And just as stimulation of the optic nerve gives rise to a sensation of light, so stimulation of any one of the cutaneous nerves gives rise to the specific sensation proper to the group to which it belongs. The existence of different forms of sensory end-organs in the skin and other tissues (tactile or toucli-corpuscles, corpuscles of Pacini, end-bulbs of Krause, etc.) points in the same direction. The end-organs of the touch sensations are believed to be the ring-like arrangements of non-medullated nerve-fibres encircling the hair-follicles, and in parts of the skin devoid of hairs the corpuscles of Meissner (v. Frey).

Touch-spots can easily bc demenstrated by touching the skin lightly with some small object such as a hair. The most exact quantitative observations have becn made by means of v. Frey's 
hair xestlesiometer. This consists of a handle in which hairs of different diameters can be fixed. The area of the cross section of each hair is measured under the microscope, and the pressurc necessary to bend it is determined by pressing it upon the scale-pan of a balance. The pressure in milligrammes, divided by the cross section in square millinutres, gives the pressure per square millimetre, which, accorcling to $v$. Frey, permits hairs to be chosen so as to give a uniform intensity of stimulation or a variable intensity, accorrling to the object of the in vestigation. Many observers, however, belicve that it is more accurate to take ne account of the pressure per unit of area, but to graduate the hairs according to the total pressure needed to bend them. When touch-spots ascertained in this way are excited by an inalequate stimulus-e.g., an alternating current of minimal strength, applied by the unipolar method through the head of a pin as an electrode-they still respond by their characteristic or specific reaction-namely, a sensation of touch-in the case supposed, a vibrating sensation like that caused by a tuning-fork in contact with the skin. In the spaces between the touch-spots the sensation produced by the same strength of current. or even by a weaker current, is not one of touch, but a painful pricking sensation which has no vibratory character. but is permanent as long as the current lasts.

The spots most sensitive to touch lie close to the hairs on their 'windward' side-i.e., on the side away from which they slope. The minimum pressure necessary to evoke a sensation of contact is not the same for every portion of the skin. The forchead and palm of the hand are most sensitive.

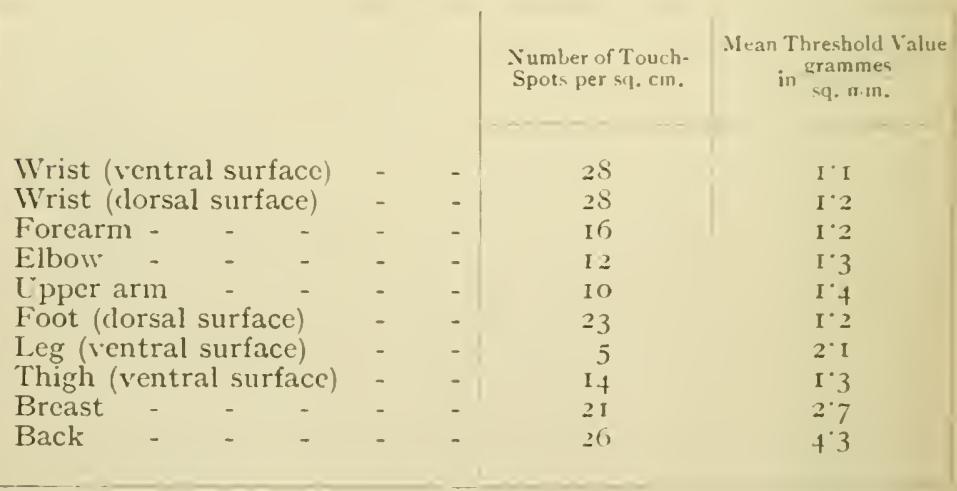

(Kiesow).

If two points of the skin are touched at the same time there is a double sensation when tlis distance between the points exceeds a certain minimum, which varies for different parts of the sensitive surface.

Practice increases the acuity of touch for the two points test. Even in a few hours it may be temporarily quadrupled on some parts of the skin. Since at the same time it is incrcased in the corresponding part of the opposite side of the body, it is argued that the modification takes place in the central nervous system, not in the end-organs themsclves. 


\begin{tabular}{|c|c|}
\hline & $\begin{array}{l}\text { Distance at which Two loint } \\
\text { can be distinctly felt, in nim. }\end{array}$ \\
\hline Point of tongue - & $I^{\cdot} \cdot I$ \\
\hline Palmar surface of thire & \\
\hline $\begin{array}{l}\text { phalanx of finger } \\
\text { Dorsal surface of thirrl }\end{array}$ & $2 \cdot 2$ \\
\hline phalins of finger & $6 \cdot 7$ \\
\hline Tip of nose - & $6 \cdot 7$ \\
\hline Back - - & $1 I^{\circ} \cdot 2$ \\
\hline Eyclids - & I I 2 \\
\hline Skin over sacrum & $40^{\circ} 5$ \\
\hline Upper arm - & $67 \cdot 6$ \\
\hline
\end{tabular}

Few of the internal organs are supplied with tactile nerves. The movements of a tapeworm in the intestines are not recognised as tactile sensations, nor the movements of the alimentary canal during digestion, nor the rubbing of one muscle on another during its contraction.

Pressure is only perceived when it affects two neighbouring areas to a different degree. Thus, the atmospheric pressure, bearing uniformly on the whole surface of the body, causes no sensation ; we are so entirely unconscious of it that it needed the inspiration of genius to discover it, and the persistence of genius to force the discovery on the world. When the finger is dipped in a trough of mercury at its own temperature, no sensation is perceived except a fecling of constriction at the surface of the liquid. The perception of light pressure and of the form and size of objects in contact with the skin is believed to be due to the touch-spots. Deep pressure. however, is appreciated, not by the skin, but through sensory endorgans in deeper structures-probably, e.g., Pacini's corpuscles and the muscle-spindles (Fig. 433, p. 983).

Sensations of Temperature.-When a body colder or hotter than the skin is placed on it, or when heat is in any other way withdrawn from or imparted to the cutaneous tissues with sufficient abruptness, a sensation of cold or warmth is experienced. And when two portions of the skin at different temperatures are put in contact, we feel that, relatively to one another, one is warm and the other cold. But it is worthy of remark that it is only difference of temperature (or, perhaps, rather the rate at which heat is being gained or lost by the skin), and not absolute height, which we are able to estimate by our sensations. Thus, a hand which has been working in ice-cold water will feel water at $10^{\circ} \mathrm{C}$. as warm; whereas it would appear cold to a warm hand.

Blix, Goldscheider, and others have shown that the whole skin is not endowed with the capacity of distinguishing temperature, but that the temperature sensations are confined to minute scattered areas over the cutaneous surface. The great majority of these are 'cold' spots-i.e., respond to stimulation only by a sensation of cold-while a smaller number are 'warm' spots, and 
respond only by a sensation of warmth (Fig. 427). These spots can be inapped out by bringing into contact with the skin small pieces of wire at a temperature a few degrees above or below that of the skin. Witl such mild st imuli a response can generally be obtained only from one kind of spot - that is, the cold wire stimulates only the cold and not the warm spots, and vice versâ-but with
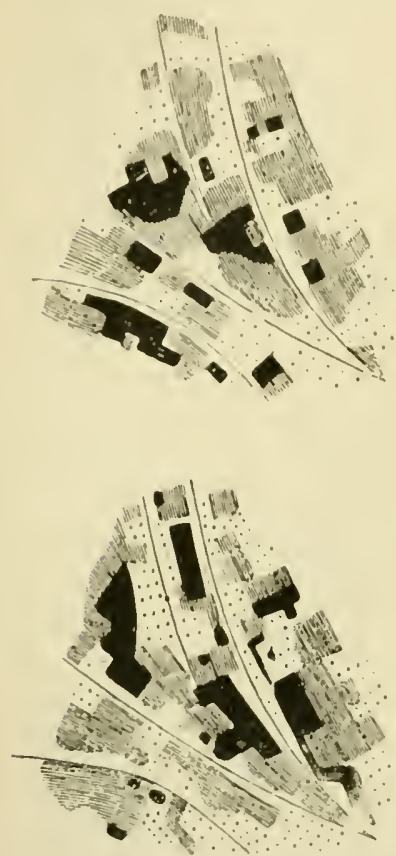

Fic. 427. ' W A R M' A N D

"COLD' AREAS ON SkiN (COLDSCIEIDER).

The areas are mapped out on the palm of the left hand. In the upper figure the relative sensitiveness to warmth is represented by the depth of the shading, the black areas being unst seusitive, then the lined areas, then the lutted, and last of all the white areas. In the low'r figure the relative sensitiveness to cold stimuli is shown in the same way. much more intense thermal stimuli - say, temperatures of $45^{\circ}$ to $50^{\circ} \mathrm{C}$. - not only do the warm spots respond with the appropriate sensation, but the cold spots respond with a sensation of cold. This is well seen when a beam of sunlight is focussed successively on a warm and a cold spot. Inadequate stimuli (mechanical and electrical) also evoke the specific response of warmth from warm spots, and of cold from cold spots.

When the hand is put into water at the temperature of the skin, and the water slowly heated, the warm spots are at first alone stimulated, and the sensations of lukewarm and then of warm are experienced. When the temperature of the water reaches $45^{\circ} \mathrm{C}$. the quality of the sensation changes to 'hot.' At a still higher temperature the sensation becomes painful or burning. The most probable explanation of these facts is mentioned below (p. 974).

It is not only of physiological interest, but of practical importance, that most mucous membranes are in comparison with the skin but slightly. sensitive to changes of temperature. Only towards the ends of the alimen. tary canal, in the month, pharynx, and rectum, and to some extent in the stomach, dloes a blunted sensibility appear. The uterus, too, is quite insensible to moderate heat: and hot liquids may be in jected into its cavity at a temperature higher than that which can be borne by the hand, without causing inconvenience-a fact which finds its application in the practice of gynæcology, and obstetrics. It is, indeed, obvious that in the greater 
number of the internal orgatns the conditions necessitry fer stimulation of temperature nerves, even if such were present, could hardly ever exist.

It has alrearly been mentionerl that clianges of external temperature excrt a remarkable influence on the intensity of metabolism (1). 590), and it has been supposed that this is brought about by afferent impulses travelling 11$)$ the cutancons nerves. We have also seen that for certain kinds of stimuli the excitability of nerve-fibres is increascel by cooling (1). 681). It is possible that this is the case for the fibres in the skin which are concened in the regulation of the froluction of heat, and it has been suggested that this fact may have a bearing on the reflex regulation of temperature (Lorrain Sinith).

\section{Pain.}

While the cold and the warm th spots are irregularly distributed over the skin in more or less compact groups, and the touch sensations are intimately associated with the hair follicles, the pain spots are more uniformly spread, and at the same time set closer together. In parts of the body where but one of these elementary forms of general sensibility is present, as in the central parts of the cornea and in the dentine and pulp of the teeth, it is always pain.

In certain situations pain and temperature sensibility are found together, but not touch-e.g.. at the margin of the cornea and on the conjunctiva.

In general, the skin is far more sensitive to pain than the deeper structures. The most painful part of an operation is generally the stitching of the wound. The cutting of healthy muscle causes no pain. In an operation in which an artificial connection was established between the stomach and the small intestine (gastro-enterostomy), and in which no anæsthetic was administered, the only pain of which the patient complained was produced by the incision in the skin (Senn). This, however. does not prove that the abdominal viscera are devoid of pain nerves, for it has been shown in animals that exposure of the intestines, etc., as in laparotomy, leads to a rapid depression (exhaustion ?) of the sensibility for pain (Kast and NIeltzer). In the intact animal and human being painful impressions can unquestionably be excited in the riscera by adequate stimuli (p. 799). Thus, the spasmodic contraction of the intestines and stomach causes the intense pain of colic and gastralgia. Labour is an example of a strictly physiological function which is the occasion of severe pain. Tissues normally insensible, or, rather, but slightly sensible, to pain may become acutely painful when inflamed.

The question has been raised whether the sensation of pain can be caused by excessive stimulation of the nerves of common tactile sensibility, or of the nerves that subserve the sensations 
of coo'ness and warmtle. It is true that when the skin is lightly tonched in the region of a touch-spot with a small object at its own temperature the sensation is one of pure touch. As the pressure is increased, a sensation of pressure, quite distinct from that of contact, may be felt ; and if the pressure is still further increased, a sensation of pain may be elicited. It seems to be quite clearly made out that the pressure sensation in this case is due not to excessive stimulation of the touch-nerves, but to stimulation of the specific pressure-nerves when the threshold is reached. The most natural explanation of the pain sensation is that it, too, is due to excitation of the nervous apparatus for pain. Similarly (as was stated on p. 972). if the skin is raised to higher and higher temperatures, the response is at first a pure sensation of warmth, increasing in intensity without changing its quality. When a certain temperature (about $45^{\circ}$ C.) is exceeded, the sensation changes to 'hot,' either because a pain element is now added to the pure thermal sensation, or because the cold spots are now stimulated as well as the warm spots, and mingle their specific response (cold sensation) with that of the warm spots. Further increase of the temperature will cause distinct pain, the sensation assuming a burning character. When a cold spot is tested with decreasing temperatures, an analogous series of sensations is run through, the pure sensation of coolness eventually giving place to cold, intense cold, and finally pain. Here, also, it is simplest to assume that the pain sensation is caused not by excessive stimulation of warm or cold spots, but by excitation of the specific pain-spots. In any case, there is no doubt that afferent ' pain ' fibres exist which are anatomically distinct from the fibres of tactile and of temperature sensations. For the conducting paths in the spinal cord are not the same for tactile and for painful impressions. And in certain cases of disease sensibility to pain may be lost, while tactile sensations are still perceived; or, on the other hand, pain may be felt in cases where tactile sensibility is abolished. Loss of temperature sensation, however, is usually accompanied by loss of sensibility to pain. When a nerve is compressed, the sensibility of the tract supplied by it disappears for cold sooner than for warmth.

Pain has been defined as 'the prayer of a nerve for pure blood.' The idea is not only true as poetry, but, with certain deductions and limitations, true as physiology ; that is to say, pain, as a rule, is a sign that something has gone wrong with the bodily mashinery; freedom from pain is the normal state of the healthy body. Physiologically, pain acts as a danger-signal. It points out the szat of the mischief, and even, in certain cases, by compelling rest, favours the process of repair. Thus, the surgeon has sometimes looked upon 
pain ats 'Nature's splint.' But, ats a matter of fact, a certain amount of pain occurring at intervals is not incompatible with high health ; and probably noboly, even when accidents and indiscretions of all kinds are avorled, is entirely free from pain for any considerable time. Sometimes, indeel, the mere fixing of the attention on a particular part of the body is sufficient to bring out or to detect a slight sensation of pain in it ; and it is matter of common experience that a dull continuous pain, like that of some forms of toothache, is aggravated by thinking of $i t$ and relieved when the attention is cliverted.

As to the sensations of tickling and itching, it is enough to say that plysiologists are not agreed whether they represent specific sensibilities subserved by special nerves distinct from those of touch and pain, or merely modifications or mixtures of these sensations.

Phenomena observed after Section of Cutaneous Nerves.The innervation of the skin can be explored not only by appropriate stimulation of the normal skin. but by study of the defects or alterations of sensibility which follow section of a cutaneous nerve, and which may be observed at different stages in its regeneration. In recent years this has proved a fruitful method, especially in experiments made by skilled observers in whom one or more cutaneous nerves were intentionally divided.

Quite recently a very elaborate investigation has been made by Trotter and Davies. They divided at different times, extending over more than a year, no fewer than seren of their own cutaneous nerves, including the internal saphenous at the knee, the great auricular, three divisions or branches of the internal cutaneous of the arm just below the elbow. and a branch of the middle cutaneous of the thigh. The operations were purposely done at such intervals as would allow the experience gained in investigating one area to be applied to others. About a quarter of an inch was cut out of each nerve, and the ends then sutured togethel. 'In each case the area of skin supplied by the nerve showed defects in seren distinct functions : four sensory-namely, sensibility to touch, cold, heat, pain-and three motor-namely, vaso-moto: pilo-motor. sudo-motor (sweat-secretory). The sensory changes showed a central area of profound loss, an area of moderate extent surrounding this of partial loss, and a large area in which a qualitative change could be alone detected.' The maximal extent of change, and therefore the outer boundary of this third area. can be mapped out by getting the subject to determine by Jight, stroking touches the area which feels in any way unnatural when he touches it himself. The most common feeling is that the skin has become smoother at the boundary as the stroking finger crosses it, coming from the normal skin. This area is always much larger than the area included in it, in which by quantitative methods-e.g., the use of a very fine camel's- 
hair brush, or more exactly he the b. Frey hairs - the sensibility to touch can les showin to be diminished (region of hypoasthesia to touch) (Fig. 428).

For a variable clistance within the 'stroking outline' the hyporesthesia for tactile stimuli is so slight that it camnot be

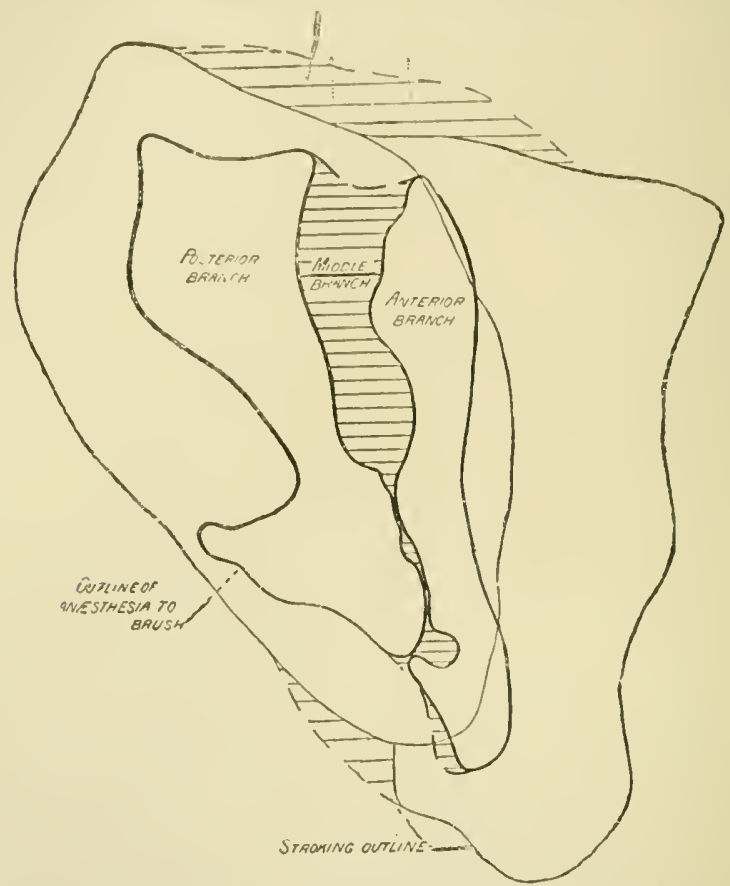

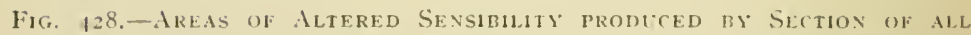

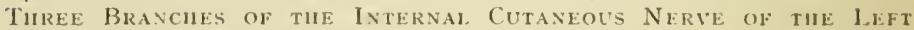
forearu (Trotter axd Davies). (REDUCED bY TWO-THIRDs.)

The thick lines show the areas of anxesthesia to the brush. The thick continuous lines enclose the areas of the anterior and posterior branches. The thick broken line and heavy stading mark the areal of the increase in anesthesia which followed section of the middle branch. The thin lines show the areas of minimal hypoasthesia-i.e., the 'stroking outline.' The complete oval outline is the "struking outline" which followed section of the posterior branch. The large addition to the oval on the right of the diagram shows the increase in the 'stroking outline' which followed section of the anterior brunch. The thin broken line and fine shading show the additions to the "stroking ontline" produced by division of the middle branch.

detected with the brush or with cotton-wool, or even with the v. Frey hairs. Like those of normal skin, 90 per cent. of its hair-bulbs respond to a hair exerting a pressure of 70 milligrammes, and the remaining ro per cent. to hairs exerting a pressure of $\mathrm{I} f \mathrm{o}$ or 280 milligrammes. Inside this zone of minimal hyporesthesia the defect of sensibility rapidly increases as we 
pass inwarks, each line of hair-bulbs requiring a heavier pressure than the line external to it, till at last $3 \frac{1}{2}$ or + grammes' pressure is needed to cause a sensation of touch, and inside of this line of hairs the skin does not respond at all. When a bristle of this pressure fails to elicit touch sensiation, no greater pressure will in general (l) so (Fig. +29).

For thermal sensibility there is also a region of complete

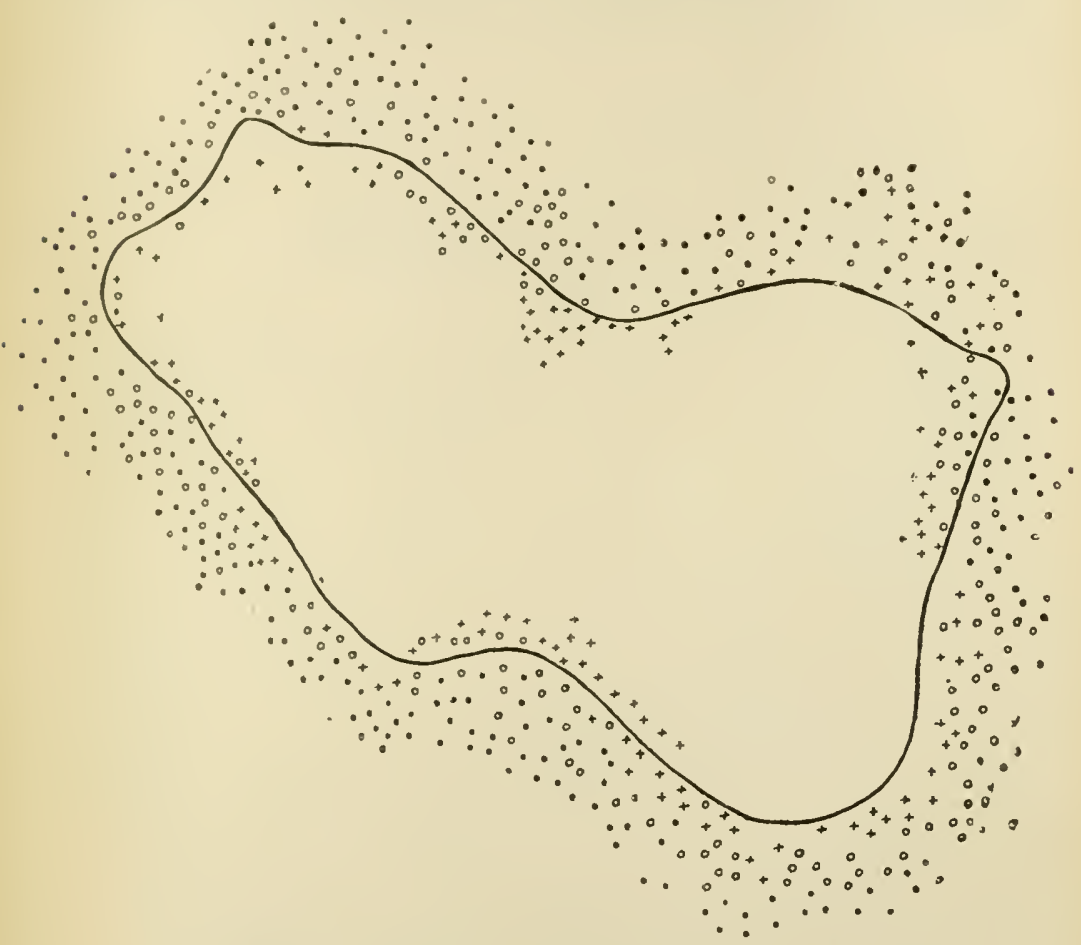

lig. 429.-Mindef. Citaneous: Left THigh (Trotter AND Davies) (REDLCED BY ONE-THIRD LINEAR).

lwenty-six days after section. Results of examination with $\because$ Frey hairs. Touch spots marked ' responded to hair of 280 milligrammes' pressure ; those marked o to hair of Soo milligrammes; and those unarked + to hair of 2,280 milli. grammes. The continuous line marks the limit within which there was anæsthesia to the camel's-hair brush.

anæsthesia and a region of partial anæsthesia. The best way of outlining these is the use of a temperature of $0^{\circ} \mathrm{C}$. as the stimulus (Fig. 430).

Outside the zone of complete thermal anæsthesia there is a region in which temperature sensations are distinctly elicited, but do not possess the normal intensity, the temperature of 
$0^{\circ} \mathrm{C}$., for example, being felt only as cool, and not as cold. The outer limit of this region is the line at which the temperature of $0^{\circ} \mathrm{C}$. is first felt as we work inwards from the normal skin to yick the sensation of cool instead of cold. Similarly, the outer limit of thermo-hypoasthesia can be determined by using a high temperature $\left(50^{\circ} \mathrm{C}\right.$. $)$. It is the line at which the sensation of hot vielded by the normal skin gives place to the sensation of

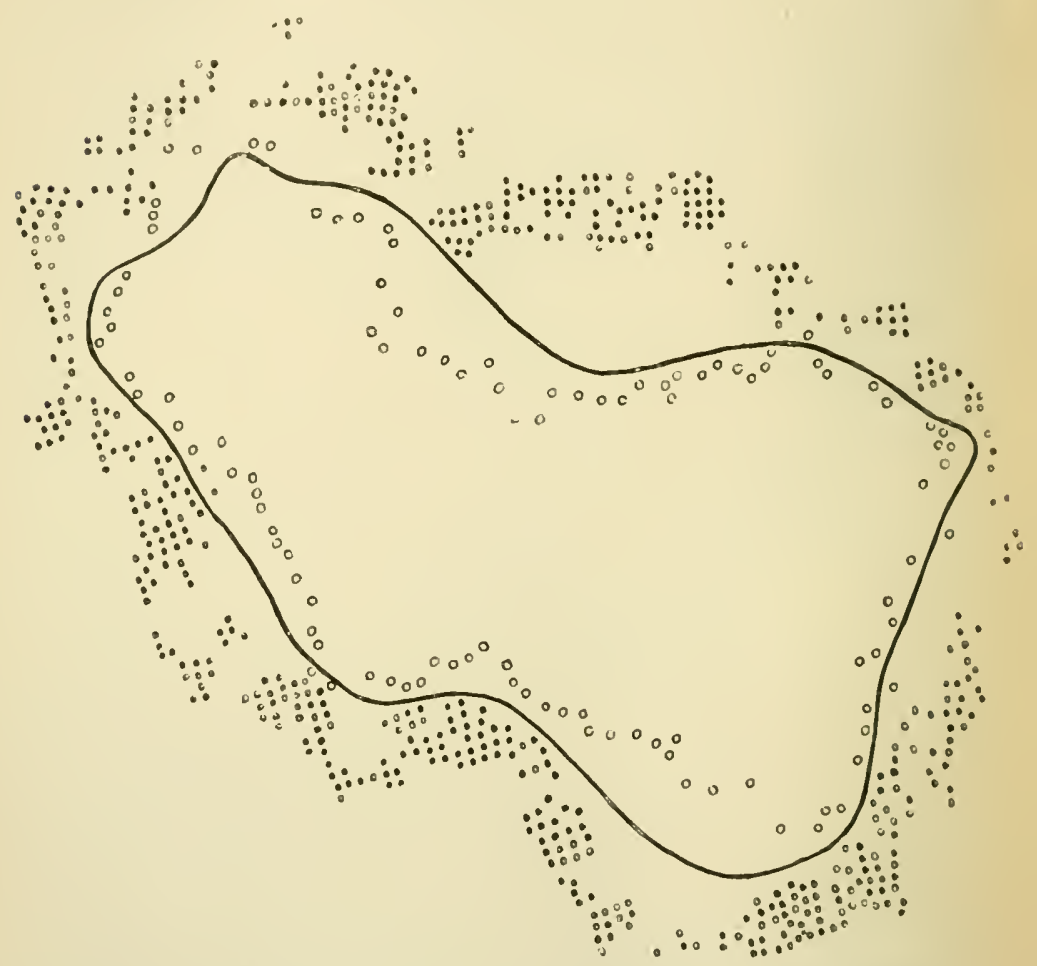

Fig. 430.-Minder Cutaneous: Left THifil (Trotter and Dailes).

Twenty-one days after section. Results of examination with temperature of $o^{\circ}$ C. On spots marked - stimulus was felt as cold; on spots marked o it was felt as cool. The blank area is that of thermal anxsthesia. The continuous outline marks the limit within which there was anasthesia to the camel's-hair brush.

warm. The two boundaries correspond closely when allowance is made for the separate grouping of cold and warmth spots on the normal skin.

The investigation of the sensibility of the skin areas for painful stimuli is complicated by the fact that during a certain period, from about the second to the sixth week after division of the 
nerve, hyperalgesia (increased sensitiveness to painful impressions) may appear. This, however, does not seem to be a consequence of any sensory loss, but rather a complication due to an irritative change. When this is taken account of, it is found that the defect of sensibility to pain after nerve section resembles the defects of sensibility to touch and temperature, showing a central area of absolute ansesthesia surrounded by a zone of

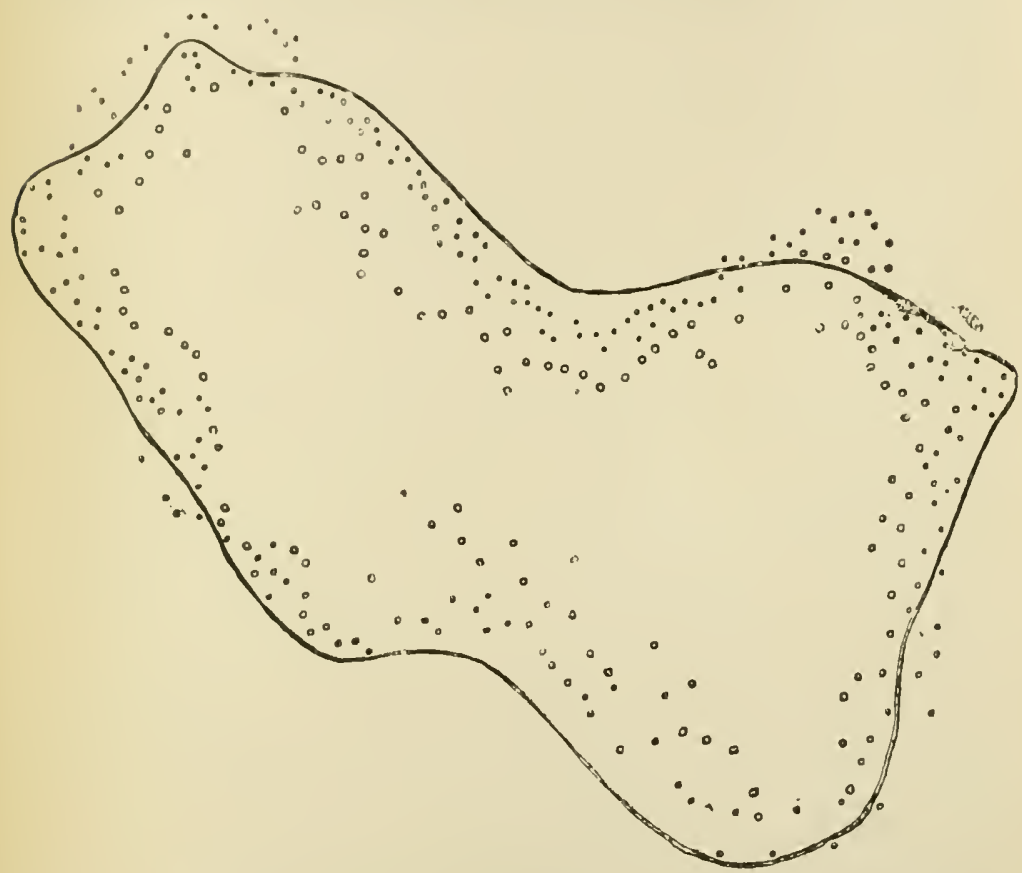

Fig. 431.-Midde (uraneots (External Branch) : Left Thigh (Trotter and Davies).

Twenty-threc days after section. Results of examination with algometer (an arrangement by which a needle is pressed against the skin by a hair whose pressure value has been determined). Spots marked - rearted by sensation of pain to pressure of I,860 milligrammes (normal threshold); Spots marked o required 2,280 milligrammes. The continuous line marks the area within which there was anæsthesia to the camel's-hair brush.

partial loss, which is slight towards the outer boundary, but increases as we pass inwards (Fig. 43I).

After section of a nerve function is recovered only as a result of regeneration. This is true of all the sensory functions of the skin and of the pilo-motor and sudo-motor functions. Vasomotor tone in the affected area is restored much sooner than the other functions. This rapid recovery probably depends upon 
a local compensatory mechanism, and not upon regeneration of the vaso-motor fibres. Recovery of all the functions dependent upon regeneration begins about the same time, and this recovery progresses over the area at about the same rate for all, although the rate at which they progress towards normal acuity is different.

Sensibility to touch probably appears a little earlier than sensibility to cold and pain. Yet the recovery of touch does not progress so fast, and for a while a given zone of the recovering area remains hypoæsthetic (less sensitive than normal) to touch, while to cold and pain it soon becomes even hypersensitive. The most remarkable peculiarities of a recovering area are: (I) This qualitative change, in virtue of which cold, pain, and the pain element of heat are intensified, while touch is little altered, although more difficult to elicit; (2) the reference of sensations, not to the point stimulated, but to distant parts of the area.

"When a spot which has dereloped this peripheral reference is touched, one of two possibilities may occur: either the touch is felt locally, and is referred as well. or nothing is felt locally, and the touch is felt in the area of peripheral reference. The region in which the referred touch is felt is always at the edge of the most peripheral part of the anzesthesia,' perhaps more than a foot away from the spot actually touched. The peripheral reference of cold is even more striking. particularly in the remarkable intensity of the referred sensation.

Peripheral reference occurs also with pain. 'The referred pain shows three well-marked qualities: it is, proportionately to the stimulus, very intense; it does not reproduce a normal sensation with the exactitude found in the case of touch or cold, but has a special quality of strangeness and unpleasantness. such as no pin-prick on normal skin can give; finally, it produces an almost irresistible desire on the part of the subject to rub or scratch the region in which it is felt.' As recovery proceeds the local sensory response becomes more distinct, and the abnormal quality of both local and referred sensations fades. But 'while peripheral reference is the earliest phenomenon of recovery, it persists until recovery is so far advanced that hypoxsthesia is scarcely detectable by any quantitative methods.'

It is a remarkable circumstance that during regeneration stimulation of the nerve-trunk itself below the section, by the application of touch, cold. or pain stimuli to the skin over its course, produces peripherally referred sensations of the corresponding kind. This is the case even when the nerve is stimulated outside the formerly anasthetic area, and suggests that the nerve-trunk itself has acquired the specific sensibility normally 
associated with the terminal organs of its afferent fibres through the lowering of the threshold for the fibres themselves.

The work of Head, who was the pioncer in this method of investigation, must also be mentioned. He found that when the median nerve was livided in his own arm, total loss of sensation was caused over the greater part of the index and middle fingers, and over a portion of the thumb in its palmar aspect. In addition, sensation was partially lost over a larger area, where there was complete insensibility to certain stinuli, such as light touch, moderate heat and coll, and where the contact of the two points of a pair of compasses conld not be discriminated. Recovery of sensation after complete division of a peripheral nerve began with the restoration of sensibility to pain and to extreme degrees of lieat and cold ; but the hand still remained for a time as insensitive as before to such stimuli a slight touch. In the parts which had regained their sinsibility to severe stimuli, like pricking and extremes of heat and cold, the sensation raliated widely, was referred to remote parts, and could not be accurately localized. This form of sensibility Head calls protopathic. As the nerve recovered further, a second form of sensibility appearerl, associated with accurate localization of cutaneous stimuli and discrimination of two compass points. light touch and molerate (legrces of heat and cold could now be again appreciated. This form of sensibility he terms epicritic. A third form of sensibility (deep sensibility) was investigated after complete division of the radial and external cutaneous nerves at the clbow. The radial half of the arm and back of the hand became totally insensitive to cutaneous stimuli, but retained their sensibility to pressure or to any stimulus which deformed the subcutaneous structures, as well as their power of localization of such stimuli. The afferent fibres upon which this deep sensibility depends must run with the motor nerves. According to Head, the other two forms of sensibility (protopathic and epicritic) also depend on two separate systems of nerves. It is assumed that the protopathic fibres regencrate more casily and speedily than the epicritic or than the motor nerves of voluntary muscle. The protopathic fibres are supposed by Head to exert a trophic influence. A part deprived of its nerve-supply is liable to injuries, and the sores so produced heal slowly. But as soon as 'protopathic' sensibility returns to the part, they heal rapidly, even in the absence of all epicritic sensation. The intestine is described as possessing 'protopathic,' but not 'epicritic,' sensibility-i.e., it reacts to extremes of heat and cold, but not to moderate heat and cold or light touch.

Head's experimental results must be sharply distinguished from his interpretation of them, and the student is warned that the dis. tinction of protopathic and epicritic sensibility has met with adverse criticism. There does not seem to be any real necessity in the observed facts for introducing so revolutionary a conception of the nervous system. Nor is it possible to uphold the distinction in any thoroughgoing fashion for all structures. For instance, in abdominal operations performed under local anæsthesia it has been seen that the parietal peritoneum is quite insensitive to touch, pressure, and temperature stimuli, including extreme temperatures (Ramström), while pain is caused by traction on it. Its sensibility is therefore neither purely epicritic nor purely protopathic in Head's sense. In like manner the mucous membrane of the mouth, in 
Which sensibility only to touch and temperature is present, conforms entirely to neither type. Its sensibility is not alone epicritic. since it responels to extreme temperatures, nor is it purely protopathic, since a pin-prick produces no painful sensation.

Localization of Cutaneous Sensations.-We not only perceive the yuality and estimate the intensity of sensations of touch, temperatture, pain, etc., lut are able, more or less accurately, to localize the part of the body from which the sensory impressions come. In other words, two impressions from different parts of the body, although identical in quality and intensity, are nevertheless stamper with a distinctive something, which may be called the local sign. This power of localization is not equal for all portions of the body nor for all kinds of sensations. It is best developed for touch (in the restricted sense), and all the varieties of common sensation are better localized on the skin than in any of the decper structures. The precise mechanism of the localization is unknown. But we must suppose that each peripheral area is 'representer]' in the brain, so that the arrival of afferent impulses from it affects particularly the related cerebral area. The brain, therefore, so to speak, associates cxcitation of a given cerebral area with stimulation of the corresponding peripheral area, and thus not only recognises the quality and quantity of the resultant sensation, but also localizes it ; just as a waiter who watches the bell-indicator not only learns how a bell has been rung, whether once or twice, peremptorily or languidly. but also in which room it has been rung. If, to pursuc the illustration a little farther, he is aware that two rooms are connected with one bell, but that one of the rooms is scarcely ever occupied, he associates the ringing of the bell with a summons from the other room even when it happens to be rung from the usually vacant room. In like manner the brain seems to connect the arrival of sensory impulses from the internal organs, which have few sensory fibres, and tliese perliaps not often stimulated, with excitation in a related cutaneous region, from which it is constantly recciving sensory impressions. The fact already mentioned (p. 790), that in discase of internal organs the pain is referred to some portion of the skin, may be thus explained.

It is through the localization of touch sensations that the size and form of objects in contact with the slin are perceived in the absence of other than the cutaneous sensations, and especially in the absence of visual and muscular sensations (stereognosis).

\section{Muscular Sensations (Muscular Sense), etc.}

Sometimes, although rather loosely, grouped together as muscular sensations, are a 11 mber of forms of sensation of which our knowledge is much less accurate than it is in the case of the fundamental skin sensations. Among these may be mentioned especially (I) the sensations by which the position in space of the body as a whole or of particular parts is recognised in the absence of visual sensations ; (2) the sensations associated with movements, passive as well as active; (3) the sensations associated with resistance to movement. In none of these groups are we dealing with purely muscular sensations; cutancous tactile sensations and pressure sensations elicited from other structures than muscles are also involved.

Voluntary muscular movements are accompanied with a peculiar sansation of effort, graduated according to the strength of the con- 
traction, and affording lata from which a judgment as to its amount and direction may be formed.

Some writers have supposed that this so-called muscular sense does not depend upon afferent impulses at all, but that the nervous centres from which the voluntary impulses depart take cognizance, retain a recorl, so to speak, of the yuantity of outgoing nervous force; that the eftort which we feel in lifting a heary weight is an effort of the cells of the motor centres from which the groups of muscles are innervated, and not of the muscles themselves.

But although this fecling of central effort or outflow (we can hardly say of central fatiguc) may be a factor, it cannot be doubted that the brain is kept in touch with the contracting muscle by impulses of various linels which reach it by different afferint channels.

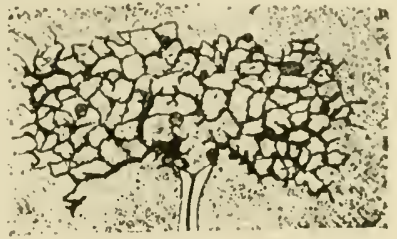

FIG, 432. - NERVE-ENDING IN TENDON NEAR THE IXSERTION OF THE MUSCULAK Fibres (Golgi).

The corpuscles of Pacini, which exist in consilerable numbers in the neighbourhood of joints and ligaments, and in the periosteum of bones, would scem well fitted to play the part of end-organs for the tactile sensations caused by the movements of flcxion, extension, or rotation of one bone on another, which form so large a portion of all voluntary muscular movements. And it has been stated that paralysis of these bollies in the limbs of a cat by section of the nerves going to them causes a characteristic uncertainty of move-

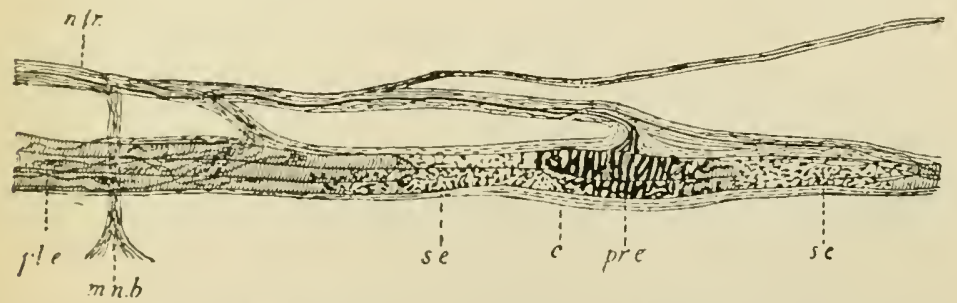

Fig. 433.-Miscle Spinde (Halliburton, after Ruffixi).

$c$, sheath of the spindle; $n . t r$., trunk of nerve, which sends fibres through the sheath into the spindle, where they form endings (pr.e., s.e., pl.e.) of various kinds; $m . n . b$. , bundle of motor fibres.

ment which suggests that something necessary to normal co-ordination has been taken away. Tendons also possess afferent nervefibres, which terminate by breaking up into reticulated end-plates (Fig. 432). We have already seen that the skeletal muscles possess numerous afferent fibres (p. 835). Some of these must be nerves of ordinary sensation. For although, when a muscle is laid bare in man and stimulated electrically, the sensation does not in general amount to actual pain, it is capable. under the influence of strong stimuli, of taking on a painful character. And nobody who has felt the severe and sometimes almost intolerable pain of muscular cramp would be likely to deny the existence of sensory muscular nerves. But after deducting these, we must assume that a large proportion 
of the afferent nerves of muscle have other functions, and among then may be the conveyance of impulses connected with the muscular sense. The unuscle-spindles or neuro-muscular spindles (Fig. 433), peculiar structures which occur in large number in most of the slieletal muscles, and have been carefully studied by Huber, Sihler, Ruffini, and other observers, are the terminations of many of the sensory fibres. They are long narrow bodies, with a thick sheath of connective tissue enclosing fine striped muscular fibres. Medullated nerve-fibres cnter the spindle, and there, dividing into branches and losing their medullary sheath, form endings of various kinds around and between the muscular fibres. It is possible that in contraction of the muscles the nerve-fibres in the spindles are compressed, and thus mechanically stimulated.

In the spinal cord these impulses are conclucted up through the posterior column; and, although less is linown as to the paths they follow in the higher parts of the central nervous system, it is certain that there is some afferent bond of connection between the cortical motor areas and the muscles which they control (p. 856).

Tactile sensations set up in the skin or mucous membrane lying over contracting muscles may also help the nervous motor mechanism in appreciating and regulating the amount of contraction; but the fact that, in anæsthesia of the mucous membrane covering the vocal cords produced by cocaine, the voice is not at all impaired, shows that muscular contractions of extreme nicety can be carried on without any such aid.

\section{Relation of Stimulus to Sensation.}

It is impossible to measure sensation in terms of stimulus. All that we can do is to compare differences in the intensity of stimuli and differences in the resultant sensations, or, in other worcls, to compare stimuli together and to compare sensations together. And when we letermine the amount by which a given stimulus must be increased or diminished in order that there may be a just perceptible increase or diminution in the sensation, it is found that (witl certain limitations) the two are connected by a simple law: If hatcver the absolute strength of a stimulus of given kind may be, it must be increased by the same fraction of its amount in order that a difference in the sensation may be perceived (sometimes called I'cber's law). Thus, a light of the strength of one standard candle must be increased by 100 th candle, a light of io candles by 100 ths, and a light of 100 candles by a candle, in order that the eye may perceive that an increase has taken place, just as the weight necessary to turn it balance increases with the amount already in the pans. The fraction varies for the different senses. It is about $\frac{1}{10}$ for light, $\frac{1}{3}$ for sound. But it would appear that Weber's law does not hold for the pressure sense, nor for the other senses above and below certain limits. Fechner, making various assumptions, has thrown Weber's law into the form $y=k \frac{\log x}{x_{0}}$, where $y$ is the intensity of sensation, $x$ the intensity of stimulation, $x_{0}$ the smallest intensity of stimulus which can be perceived (liminal intensity), and $k$, a constant. This so-called psyclio-physical law of Fechner states that the sensation varies as the logarithm of the stimulus. But liechner's law has been subjected to serious criticism, and the subject cannot be furtlier pursued here. 


\section{PRACTICAI, EXERCISES ON CHAPTER XIII.}

\section{VISION}

1. Dissection of the Eye.-The student may profitably refresh his menory on the anatonly of the eye by dissecting a fresh evethat of a large animal like an ox is preferable, but the eye of a sheep or dog may also be used. The eye is removed from the orbit by cutting through the conjunctiva where it is reflected on to the eyelids, carefully severing the extrinsic muscles and scooping the eyeball out of the mass of loose connective tissue and fat in which it is embedded, and which serves as a cushion to protect it from injury during its movements. Observe the transparent cornea in front, blending at its posterior border with the opaque sclerotic, which is covered by a layer of conjunctiva refleated from the lids. On clearing the fat cautiously away, the tendinous insertions of the external or cxtrinsic muscles of the eveball into the anterior part of the sclerotic will be secn. Identify the various muscles (p. 952 ).

Inmerse the eye in water in a small glas; dish, with the cornea uppermost. The interior cən now be seen, because the refractive index of the cornea being nearly the same as that of water, the light is only very slightly refracted there. The same effect is produced when a cover-slip is placed over the cornea in the air; a plane surfare being substituted for the curved anterior surface of the cornea, its refraction is abolished. Observe in the fundus of the eye the optic disc, eccentrically placed in the retina, and the retinal vessels radiating out from it. A portion of the fundus shows brilliant iridescent colours in many animals (the tapetum lucidum). This portion is abruptly bounded by a line a little above the optic disc. The appearance is due to a peculiar arrangement of the connective-tissue (including elastic) fibres in this part of the choroid.

Pinch up with forceps a small portion of the sclerotic a little posterior to its junction with the cornea, and clip it away with fine, blunt-pointed scissors, being careful not to penetrate the choroid layer, which lies immediately beneath the sclerotic. Extend the incision through the sclerotic backwards, and then transversely, and peel off strips of the sclerotic from behind forwards. The lower surface of the sclerotic (the so-called lamina fusca) is dark, owing to the presence in it of the same pigment which is so abundant in the choroid coat. Go on removing the sclerotic piecemeal until a considerable area of the dark choroid layer is exposed with the ciliary nerves passing forward on its surface towards the iris. One. or other of the long ciliary arteries may also be seen coursing between the sclerotic and choroid if the sclerotic happens to have been removed at its position. On the anterior part of the choroid may be observed some pale fibres passing backwards from the corneosclerotic junction. They are the meridional fibres of the ciliary muscle (p. 907).

The eye bcing immersed in water, remove cautiously with the forceps and scissors the portion of the choroid exposed. The retina is now scen as a pale membrane, transparent when quite fresh, but becoming whitish soon after death. Cut through sclerotic, choroid, and retina about half-way round the eyeball, a little posterior to the corneo-sclerotic junction. The vitreous humour will bulge 
out. Since its refrictive index is nearly the same as that of water, it is scarcely observed when immersed, and the interior of the eye can be easily seen through it.

The optic disc can now be again studied, with the stump of the optic nerse entering it and the retinal vessels piercing the disc. In the centre of the retina is the yellow spot.

In the anterior portion of the eyeball note the crystalline lens, and at its circumference the radiating folds of the choroid called the ciliary processes. Closely covering the ciliary processes, the anterior border of the retina forms the ora serrata, a plaited arrangement like an old-time ruff.

Now complete the separation of the anterior and posterior portions of the eyeball. Remore the vitreous humour. noting that it is attached to the ciliary processes and the posterior surface of the capsule of the lens by its enveloping membrane, the hyaloid membrane. With scissors snip through the corneo-sclerotic junction at one point down to the border of the lens, and observe the suspensory ligament passing from the ciliary body chiefly towards the anterior surface of the lens, where it blends with the lens capsule. Open the anterior chamber of the eye by an incision through the corne. in front of its junction with the sclerotic. It is filled with the clear. watery, aqueous humour. Note the pigmented iris projecting in front of the lens.

Remove the sclerotic and cornea for some distance along their line of junction, using gentle pressure with the edge of a fine knife to separate the junction from the attached border of the iris. The ciliary muscle, forming a pale, narrow ring around the eye at the corneo-sclerotic junction will be thus exposed. Its external surface is closely adherent to the sclerotic, and its internal blends with the ciliary body. The circumference of the iris is attached at its anterior border. Posteriorly it passes into the choroid.

Take out the lens and observe the curvature of its anterior and posterior surfaces. Determine which has the greater curvature. In the excised eye the lens will, of course, be in the condition of relaxed accommodation.

2. Formation of Inverted Image on the Retina.-Fix the eyc of an ox or of a dog or rabbit, after careful removal of part of the posterior surface of the sclerotic, in one end of a blackened tube, with the cornea in front. A tube made by rolling up a piece of thick brown paper will do. Place a candle in front of the eyc. Look through the other end of the tube, and observe the inverted image of the candle formed on the retina. Nove the candle until the image is as sharp as possible. Now bring between the candle and the ere a concave lens. The image becomes blurred, the candle must be put farther away to rencler it distinct. and perhaps no position of the candle can be found which will give a sharp image. If the lens is convex, the candle must be brought nearer, and a sharp image can always be formed by bringing it near enough. If both a convex and a concave glass be placed in front of the eye, they will partially or wholly neutralize each other. Instead of the candle a window may be looked at. If the eye of an albino rabbit can be obtained, it is not necessary to remove a part of the sclerotic.

3. Helmholtz's Phakoscope (Fig. 4.34). - This instrument is employed in studying the clianges that take place in the curvature of the lens during accommodition. It is to be used in a dark room. A candle is placed in front of the two prisms $\mathrm{P}, \mathrm{P}^{\prime}$. The observer 
lookis thromgli the lonle 13 ; the observed eye is placed at at hole opposite the hole $A$. The canclle or the observed eye is moved till the observer sees three patirs of images, onc pair, the brightest of all, reflected from the anterior surface of the cornea; another, the largest of the three, but dim, reflected from the anterior surface of the lens: and it thirel pair, the smallest of all, reflected from the posterior surface of the lens (liig. $387, p .90(3)$. The last two pairs can, of course, only be seen within the pupil. The observed eye is now focussed first for a distant object (it is cnough that the person should simply leare his cye at rest, or imagine he is looking far away), and then for a near object (an ivory pin at $A$ ). During accommodation for a neitr object no change takes place in the size. brightness, or position of the first or thirel pair of images; therefore the cornea and the posterior surfice of the lens are not altered. The middle images become smaller, som what brighter, approach each other, and also come nearer to the corneal images. This proves $(a)$ that the anterior surface of the lens undergoes a change; (b) that the change is increase of curvature (diminution of the raclius of curvature), for the virtual image reflectel from a convex mirror is smaller the smaller is its radius of curvature. (The thircl pair of images really undergc

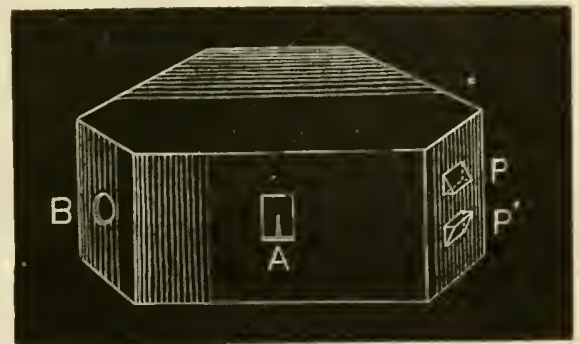

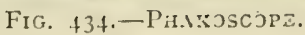
a slight change, such as would be caused by a small increase in the curvature of the posterior surface of the lens; but tlis student need not attempt to make this out ;

4. Scheiner's Experiment.-Two small holes are pricked with a needle in a card, the rlistance between them being less than the diameter of the pupil. The card is nailed on a wooden holder, and a needle stuck into a picce of wood is looked at with one eye through the holes. When the eye is accommodated for the needle, it appears single; when it is accommodated for a more distant object, or not accommodated at all, the needle appears double. The two images approach each other when the needle is moved away from the eye, and separate out from each other when it is moved towards the eye. When the eye is accommodated for a point nearer than the needle, the image is also double; the images approach each other when the needle is brought closer to the eye, and move away from each other when it is moved away from the eye. If while the needle is in focus one of the holes be stopped by the finger, the image is not affected. When the cye is focussed for a greater distance than that of the needle, stopping one of the holes causes the image on the other side of the field of vision to disappear; if the cye is focussed for a smaller distance, the image on the same side as the blocked hole disappears (Fig. 435). To determine the near-point of distinct vision (p. 9 I 5) the card may be mounted vertically on a cork, and this fastened by a rubber band to the end of a foot-rule. Hove a nectle. also inserted vertically into a cork, along the rule, beginning at the end farthest from the eyc, until with the strongest effort of accommoda. 
tion it is seen cluuble. Then push it back slightly to the point at which, again with maximum accommodation, it is just seen single. liepeat the measurement with a needle mounted horizontally. If regular astignatism is present, the distances will not be the same. Jost cyces liare slight regular astigmatism.

In myopic persons the far-point of distinct vision can also be determined by Scheiner's experiment. The needle heing left on a shelf at the level of the eye, the person walks away from it backwards, regarding it all the time through the perforated card, till it is no longer seen single.

5. Kuhne's Artificial Eye. - This is an elongated box provided with a glass lens to represent the crystalline, and a ground-glass plate to represent the retina. The box is filled with water to which a little eosin has been added. The water must be perfectly clear. If the tap-water is turbid it should be filtered or allowed to settle, or dis-
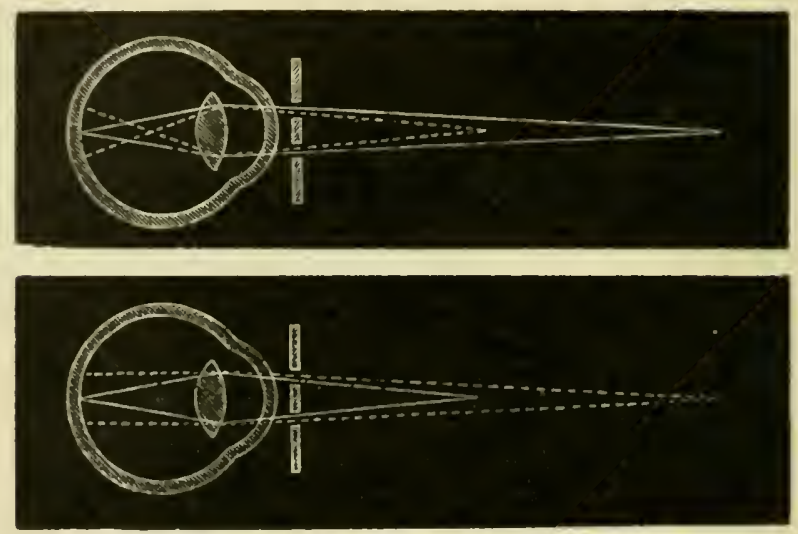

Fig. 435.-SCHEINER'S EXPERIMENT.

In the upper figure the eye is focussed for a point farther away than the need:e, in the lower for a nearer point. The continuous lines represent rays from the needle, the interrupted lines rays from the point in focus. But the lines inside the eye, which by an error in engraving are drawn as continuous lines, ought to be interrupted, and vice versa.

tilled water should be used. A beam of sunlight or electric light, or, in case these are not available, a beam from an oil stereopticon, is made to pass through the box. Many of the facts of vision can be illustrated by means of this piece of apparatus. The modification of it introduced by Lyon is very convenient.

(a) Let the rays of light pass through an arrow-sliaped slit in a picec of cardboard. An inverted image of the arrow is formed on the retina. Nove the retina nearer to or farther from the lens to make the image sliarp. In the eye of man and of most animals. accommodation is not brought about by a change in the distance of retina and leus, but by a change of curvature in the lens.

(b) Remove the lens. The focus is now far behind the retina. This illistrates the state of matters after the lens has been removed for cataract. The arrow can again be sharply focussed on the retina by putting a convex lens in front of the artificial eyc. But 
this must be much weaker than the lens which has becn removerl, for if the latter be placed in front of the eye, the image is formed a little behind the cornea.

(c) Replace the Iens. More the retina sa far back that the image is focussel in front of it. This is the condition in the myopic eye. Put a weak concave lens in front of the eye; the image now falls more ncarly on the retina. Move the retina forward so that the focus is behind it. This corresponds to the hypermetropic eye. Put a wak convex lens in front of the eve to correct the defect.

(d) Observe that a plate with a hole in it. placed in front of the eye, renders an indistinctly focussed image somewhat sharper by cutting oft the more divergent peripheral rays.

(e) Fill with water the chamber in front of the curvel glass that represents the cornca. The focus is now behind the back of the eyc altogether. Refraction by the cornea is here abolished, as is the case in vision uncler water. An additional lens inside the eye, or a weaker one in front of it. corrects the defect. Fishes have a much nore nearly spherical lens than land animais, and a flat cornea.

(f) Fill the hollow cylindrical lens with water, and place it in front of the artificial eye. The eye is now astigmatic. A point of light is focussed on the retina, not as a point, but as a line. The vertical and horizontal limbs of a cross cut out of a piece of cardboard and placed in the path of the beam of light cannot be both focussed at the same time.

6. Astigmatism (Regular).- - (I) Look at a figure showing a number of lines radiating horizontally, vertically, and in intermediate directions from a common centre. First fix the figure at such a

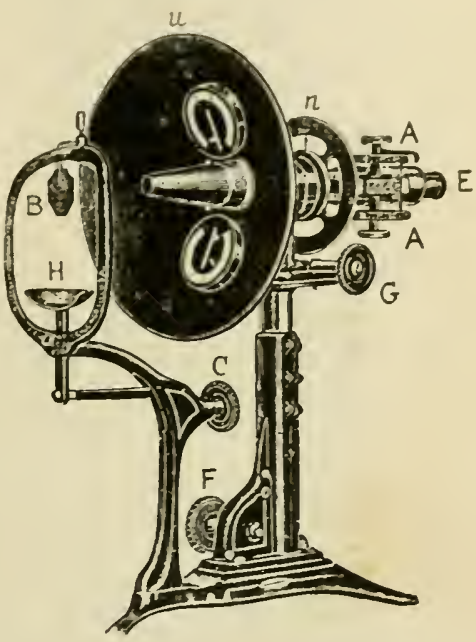

Fig. +36.-OPHTHALMOMETER, AS SEEX FRON BEHIND THE PATIENT.

$B$, blind for covering the eve not being examined: $H$, chin-rest ; A, A, graduated discs on which radii of curvature of the cornea in various meridians are read off or their equivalent in diopters; E, eye-piece of telescope; C, milled head for raising and lowering chin-rest ; F, milled head for adjusting height of the ophthalmo. meter, and $\mathrm{G}$ for moving it horizontally back and forth: $u$, graduated disc for giving the rotation of the outer tube of the telescope and the black discu. In $u$ are seen the two illuminated mires. distance that one can comfortably accommodate. If astigmatism is present, all the lines cannot be seen with equal distinctness at the same time, but they can all be successively accommodated for. Next, bring the figure to the near-point of distinct vision for the horizontal and neighbouring lines. Probably the vertical lines will be blurred and cannot be made as distinct as the horizontal by any effort of accommodation. If the eye is distinctly astigmatic, the difference will be marked.

(2) Use the Ophthalmometer. - A convenient form is shown in Figs. 436 and +37 . 
Raise or lower the chin-rest till the upper bar of the hear-rest is just above the patient's cyebrows, his head being exactly vertical. The eye not to be examined is covered with the blind. The patient looks steadily into the opening of the tube with his eye wide open. The height of the instrument having been adjusted, a clear image of the mires is obtained by focussing. The tube is then turned horizontally slightly to right or left until the two images of the mires are close together and equally distinct. Rotate the outer tube

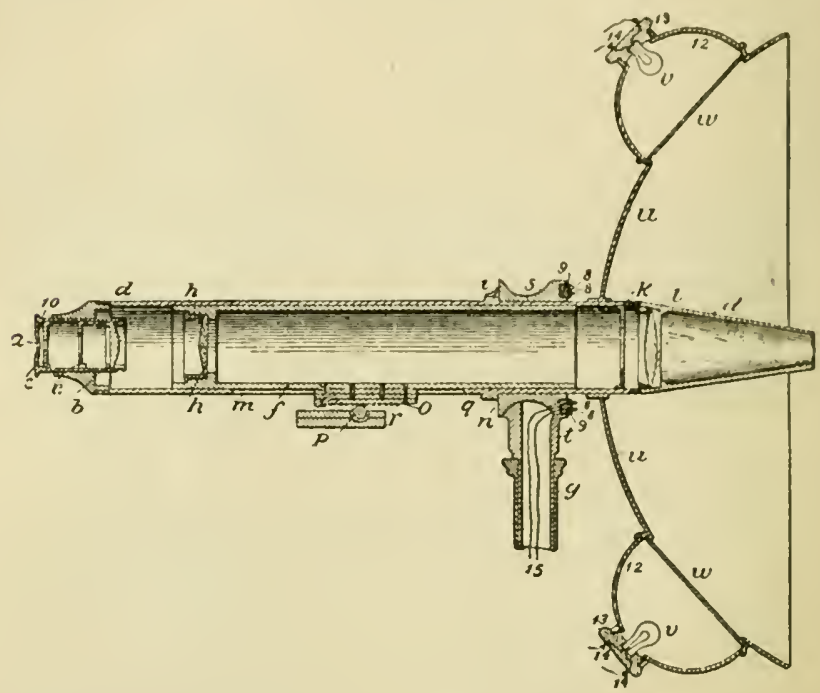

Fig. 437.-Vertical Sectiox of OpHTHALMometer.

$d$, outer tube of the telescope rotating in sleeve or collar $s$ (supported by standard $t$, which is swivelled in tubular support, $g$ ) ; $k$, diaphragm : Io, eye-piece with lenses $a$ and $b ; n$, a stationary disc, borne on collar $s$, graduated to indicate angle of rotation of $u$, a black concave disc rotating with tube $d$, and having fixed in it two illuminated figures (or mires). $w$, wh, whe images reflected from the cornea are observed; $i$ is a pointer carried on the tube $d$ which shows on the graduated arc the amount of rotation; I2, I2, hemispherical shells containing small incandescent lamps for illuminating the translucent mires. The lamps are ronnected with wires running in the hollow stem $t ; f$ is the inner tube of the telescope carrying the double prisu, $h, h$. By means of the rack o. projecting through the slot $m$, and engaged by the pinion $p, f$ is moved back and forth in the outer tule, thus approximating or separating the corneal images of the mires. On the axis of $p$ is a milled head for turning $\mathrm{it}$, and two duplicate dises graduated with a scale showing the radii of curvature of the cornea in millimetres, and another scale showing their equivalent in diopters.

(Fig. $437, d$ ) until the long meridian lines of the images are exactly in line with each other. If there is no astigmatism, this will be seen at all axial positions; if there is astigmatism, at only two positions. An axis having thus been obtained, the graduated disc (Fig. $436, A$ ) on either side of the tube is rotated until the sliorter lines or spurs of the images also unite, forming a perfect cross with the longer ones (Fig. 435 ), and the adjustable pointer on the left-hand disc is made to coincicle with the stationary one 
and a realing talken. Now rotate d through 90 rlegrees; the fong axial lines of the images will be in aligmment without further adjustment. But if the eye is astigmatic, the short lines will not (Fig. 439). By rotating $d$, the short lines are inacle to coincide, so that a perfect cross is atgain formed, ambl the graluation is read. The difference

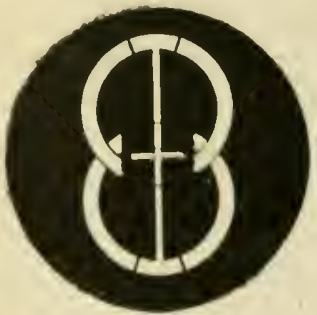

FIfr, $43^{8}$.

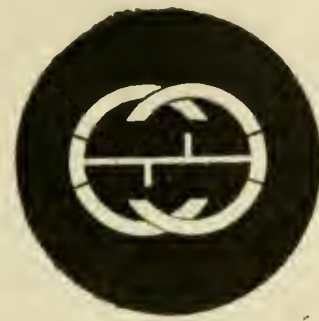

FIG. 439.

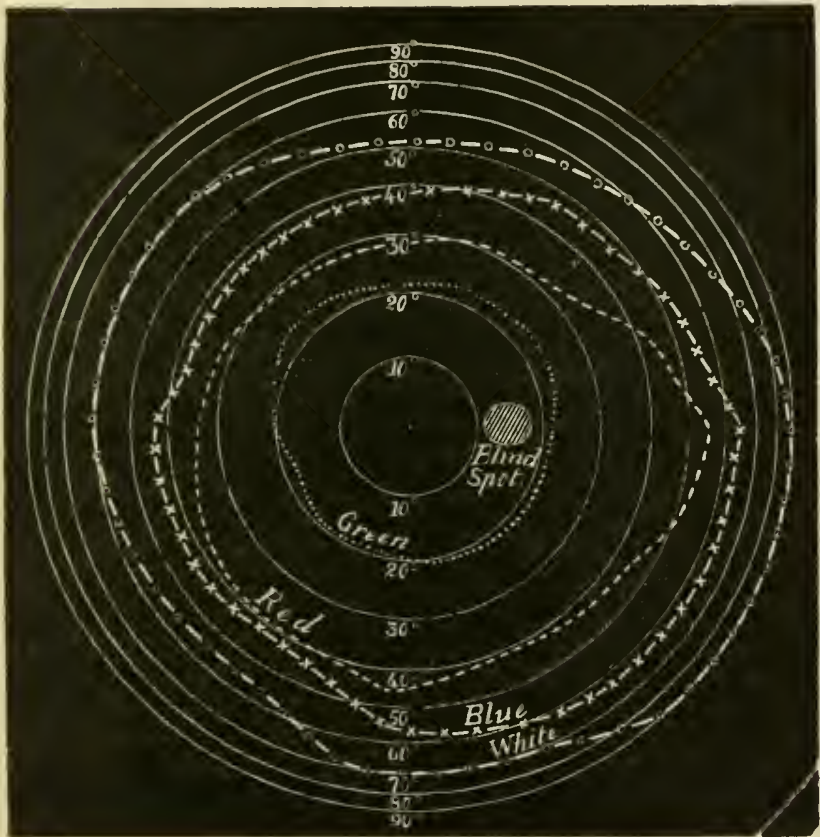

Fig. 4fo.-Perimetric Chart of Right Eye.

Obtained with the perimeter shown in Fig. $4^{15}$ (p. 946). The numbers represent degrees of the visual field measured on the graduated arc of the perimeter.

between this and the previous reading-i.e., the difference between the two pointers-gives the difference in the curvature of the cornea in the two meridians. The images of circles which form the outer portion of the mires are oval in ordinary astigmatism.

7. Spherical Aberration.-Close one eye, and bring a small object 
(a pin or the point of a pencil) towards the other cye till it becomes blurrel. Interpose between the object and the eye a card perforated by a small hole. The object becomes more distinct owing to the cutting off of the peripheral rays (1). 912).

8. Chromatic Aberration.-Look at Fig. 39 r (p. 913) from a distance too small for perfect accommolation, and verify the facts given in the description of the figure.

9. Measurement of the Extent of the Field of Vision.-I'se the perimeter shown in Fig. fI5 (p. 946).

(1) For IIhite Light.-Fix in the holder, Ob, on the graduated arc, a small piece of white paper, and put one of the charts supplied with the instrument at the back of the wheel which revolves with the arc. The observations can be recorded on this chart. The patient rests his chin on $H^{*}$ and adjusts one eye against $O$. This eye is liept fixed on the mark at $f$ during the whole period of observation, and the other eyc is covered. The arc is placed in a definite position, and the white object gradually moved from the encl of the arc until the person announces that he can just see it. The

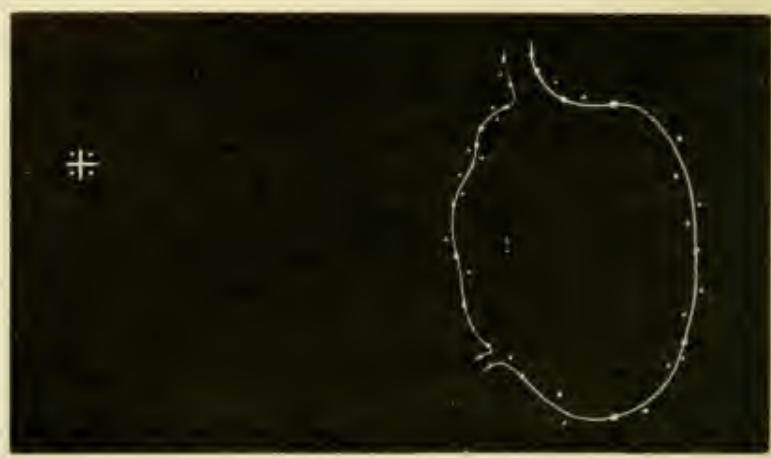

Fig. 441.- Map of Blixd SPOT (REDUCED BY ONE-IIALF).

Right eye. Distance of eye from paper, i 2 inches.

angle at which this occurs is read off and recorded on the chart. The arc is then rotated into a new position and the observation repeated. A line is (lrawn through all the points thus obtained. and this constitutes the boundary of the ficld of vision (Fig. 4 fo).

If the position of each point is inserted on the clart, a point above the horizontal plane passing through the visual axis being placel below it, and a point to the right of the vertical plane being moverl to the left, we obtain a map of the sensitive portion of the retina. Usually perimeters are arranged to do this automatically.

(2) Repeat the mapping of the field, using colnurecl papers (red, green, and bluc) instead of white.

ro. Mapping the Blind Spot.-Make a black cross on a piece of white paper attached to the wall, the centre of the cross being at the height of the eye in the erect position. Stand about 12 inches from the wall, the chin supported on a projecting piece of wood. Fix the centre of the cross with one eye, the other being closed, and move over the paper a pencil covered, except at the point, with white paper, until the point just clisappears. Make a mark on the paper at this point, and repeat the observation for all diameters of the 
fielel. The blond spot is thus marked out (Fig. +41 ). Its shape is not the sume in all eves (Fig. +12 ). Its size and distance from the forea centralis cin be calculated from the construction given in lig. $380(1) .(1)$ ).

11. The Macula Lutea, or Yellow Spot.-(I) After closing the cyes

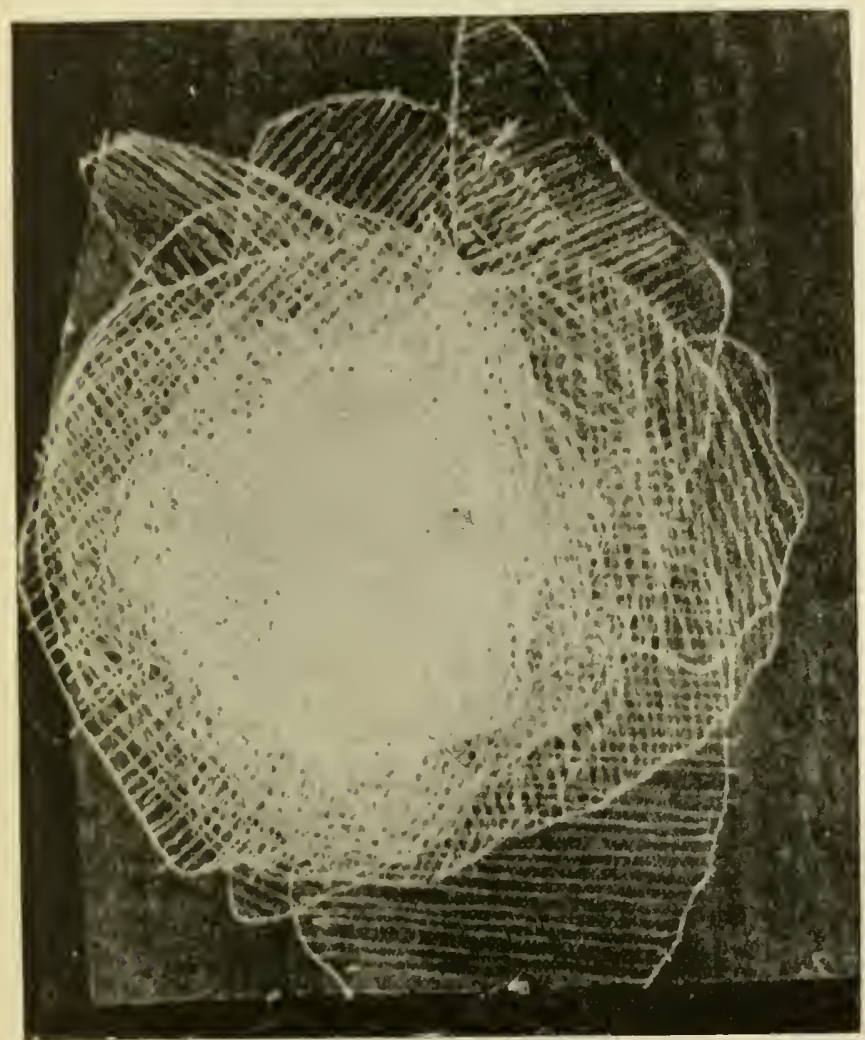

Fig. 442.-Composite Picture of Blind spot (not reduced).

The blind sput of the right eye was mapped by 31 men. the eye being always at a distance of 12 inches from the paper. The maps were then superposed. The amount of white at any point of the figure is intended to correspond to the number of maps which nverlapped at that point. Although the mechanical process of reproduction gives rather an imperfect view of the composite map, the area in the centre of the figure where the white is most continuous, and which represents the shape of the majority of the blind spots, evidently bears a general resemblance to the outline in Fig. $+4 x$.

for a minute or two, look with one eyc through a strong solution of chrome alum in a clear glass bottle with parallel sides. Hold the bottle between the eyc and a white screen or a white cloud. An oval rose-coloured spot will be seen in a greenish field. The pigment of the yellow spot absorbs the blue and green rays. 
(2) licep the eye closed for a short time. Then direct it to at surface illuminated by a weak blice light. A dark blue or almost black spot (Maxwell's spot), corresponding to the macula, is secn in the risual field, owing to the absorption of the blue rays.

12. Ophthalmoscope-(I) Human Eye (p. 918). - - Iet A be the observer, and $B$ the person whose eye is to be examined. A and $B$ are seated facing each other. Suppose that the right eye of $\mathrm{B}$ is $t 0$ be examined. Close to the left car of $B$ is a lamp on a level with his eyes: the room is otherwise dark. For a clinical cxamination, the pupil should be dilated by putting into the eye a drop of a 0.5 per cent. solution of atropine sulphate, but this is not indispensable for the experiment.

(a) Direct Method.-A takes the mirror in his right hand, and, holding it close to his own eyc, looks through the central hole, and throws a beam of light into B's eyc. A red glare, the so-called 'reflex' from the choroidal vessels, is now seen. A then brings the mirror to within 2 or 3 inches of B's eyc, kecping his own eye always at the aperture. $A$ and $B$ both relax their accommodation. as if they were looking away to a distance. If both cyes are emmetropic, the retinal ressels will be seen. B should now look away past the little finger of $A$ 's right hand. This causes slight inward rotation of B's eyc, and brings into view the white optic disc with the central artery and rein of the retina crossing it.

(b) Indirect Method.-A takes the mirror in his right hand to examine $B^{\prime}$ 's right eye. places his own cyc behind the aperture ats before at a distance of about i 8 inches from $B$, and throws a beam of light into B's eye. Then A takes a small biconvex lens in his left hand, and places it 2 or 3 inches in front of B's eye, keeping it steady by resting his little finger on B's temple. A now moves the mirror until he sees the optic disc.

(2) Examine a rabbit's cye by the direct and indirect method. Dilate the pupil by a cliop or two of atropine solution.

For practice. before loing (1) and (2) the student should examinc an artificial ' eye' by both methods, so as to get a clear view of what represents the retina. A substitute for the artificial eyc may be made by unscrewing the lower lens of the cyepiece of a microscope. and fastening in its place a piece of paper with some printed matter on it. The letters must be made out with the ophthalmoscope.

The opportunity should also be taken to observe the eye of all andesthetized animal by the simple cover-glass method mentioned in 1 (p. 985). A round cover-glass is slipped under both eyclids and so held in position on the cornea. The fundus of the eye can now be clearly scen, including the optic disc and retinal vessels. The instillation of a little cocaine into the eyc of a rabbit will produce local ancesthesia sufficient to permit the experiment.

13. Skiascopy or Retinoscopy.- The simplest methorl is as follows : The observer places himself at a distance of a metre from the observed eve, which he illuminates by a beam reflected from a concave ophthalmoscopic mirror held in front of his eye. The accommodation of the observed eye is relaxed. If, now, when the mirror is rotated no direction of movement of the shadow or the light area (p. 920) can be made out, the pupil becoming all at once dark throughout its whole extent when the mirror is rotated in one direction, and all at once light throughout its whole extent when the mirror is rotated in the opposite direction. the observer is in the far-point of the observed eye sinec the far-point is at the clistance 
of a metre, there is 111 this case myopia amounting to one cliopter. If. hewerer. the light area moves in the same direction as the rotation of the concive mirror, the far-point of the observed eye

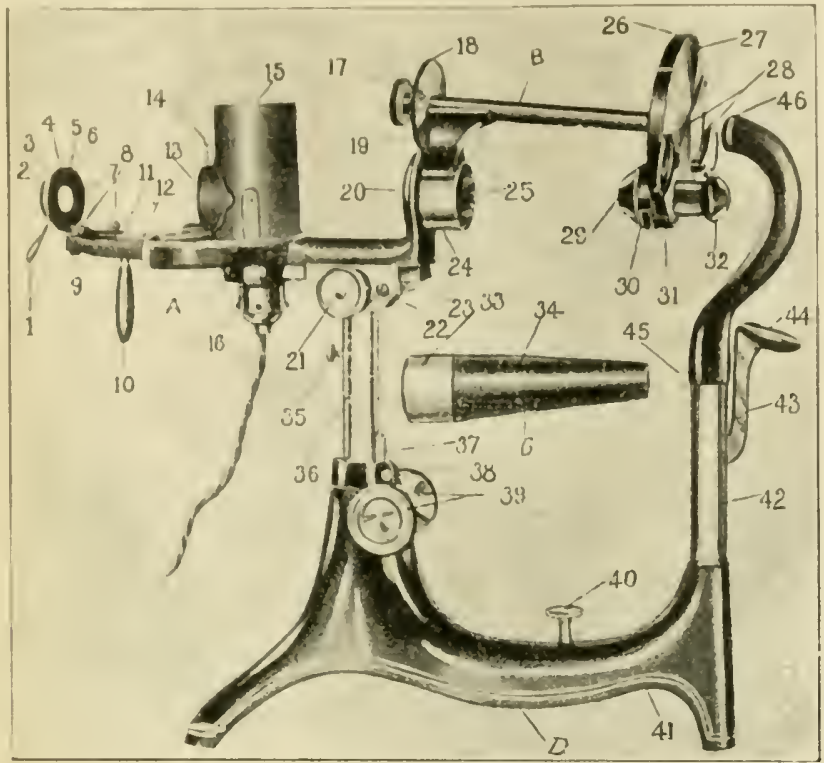

Fig. 443.-Gexeva Retinoscope axd Ophthalmoscope.

A. trame of instrument; B, retinoscope attachment: C, ophthalnoscope attachment : 1), base : 1 , mirror handle : 2 , clip to hold the proper lens to correct the abnormality of refraction of observer or patient when viewing the retina with the ophthalmoscope; 3 , scale indicating the meridian of handle and pointer ; 4 . ring in which mirror cup rotates ; 6 , mirror : 7 , mirror spring for reflecting the light to a given point: 8, screws for adjusting mirror; 9, screw for holding light and ring 4 in position : $I_{0}$, handle for swinging $A$ from side to side ; $I_{3}$, opening in iris diaphragm, controlled by handle I $_{4}$ : I 5 , lamp hood : I 7 , knurled handle for rotating disc containing the full diopter lenses; 18 , handle for rotating the disc containing the fractional lenses (white numbers indicate plus lenses, and red minus lenses); 20. opening throngh which observer looks when adjusting the retinoscope to the patient's eve ; 21 , pinion for advancing or retracting instrument; 24 , bracket ring of retinoscope attachment B, which is slipped over ring 25 when putting retinoscope attachınent into place; $2 S$, clips for 'fogging ' lenses through which the patient looks to 1 elix accommodation ; 29, opening throngh which the pupil is viewed in retinoscopy : 30 , opening containing clip in which cxtra lenses naay be inserted when required, or the defect is over 8 diopters; 32 , patient's evecup : 33. ring of ophthalmoscope attachment C, which telescopes over 25: 34. ophthalmoscope tnbe: 35 . binding-screw which holds the instrument in at fixed position when retinoscope is being used : 37 , rack to raise and lower the instrument : 40 , handle controlling height of chin-rest $4 t ; 46$, forehead-rest.

lies between the observer and the observed eye, so that the myopia amounts to more than one diopter. The precise degree of myopia can be estimated by interposing biconcave lenses of different strength until the far-point is made just I metre. 
If the light area moves in the opposite direction to the rotation of the mirror, the far-point is more than a metre distant, and therefore the observed eye is emmetropic or hypermetropic, or myopic to a degree less than a diopter. The lens, convex or concave, can now be sought out which will just bring the far-point to a metre, and from the strength of it, minus one diopter, the refraction can be estimated. Suppose, for instance, that a convex lens of two diopters is required, then hypermetropia of one diopter exists.

In order to facilitate the introduction of the various lenses. instruments called skiascopes or retinoscopes may be used, one of which is shown in I.ig. +43

14. Pupillo-dilator and Constrictor Fibres.-(a) Set up an incluction machine arranged for tetanus, and connect a pair of electrodes through a short-circuiting key with the secondary. Etherize a cat by putting it into a large vessel with a licl, slipping into the vessel a piece of cotton-wool soaked with ether, and waiting till the movements of the animal inside the vessel have ceased. Then quickly put the cat on a holder and maintain anæsthesia with ether. Expose the vago-sympathetic in the neck (pp. I 48,202$)$; the carotid is taken as the guide to it. Ligature the nerve and cut below the ligature. On stimulating the upper (cephalic) end, the pupil of the corre. sponding eye dilates.

Carcfully separate the sympathetic from the vagus, and repeat the obscrvation on the former. The result on the pupil is the same.

(b) Observe in the eye of a fellow-student, or, by means of a looking-glass, in your own cye, that when light falls on one cye both pupils contract.

(c) Observe that when the eyc is accommodated for a near object the pupil contracts, and that it dilates when a distant object is looked at.

15. Colour-mixing.-(a) Arrange a red and a bluish-green disc on onc of the steel discs of the colour-mixing apparatus shown in Fig. $4+4$, so that a part of each is scen. On another arrange a violet and a yellow disc. and on the third an orange and a blue disc. By adjustment of the proportions of the two colours a uniform grey can be obtained from each of these combinations (complementary colours) when the discs are rapidly rotated.

(b) IIx two colours that are not complementary-e.g., bluc and red-grey or white cannot be obtained by any adjustment of proportions; the result is always a mixed colour, the precise hue depending on the amount of each ingredient.

(c) Take papers of any three colours from widely-separated parts of the spectrum-e.g.. blue. green, and red-and arrange them on one of the rotating discs. By varying the proportions, white (grey) can be produced, and any other coloured paper fastened on another of the rotating discs can be matched by adding white to the three colours.

16. After-images - (1) Positive.-(a) Rest the eyes for two or three minutes by closing them, or by going into a dirk room. Then look for an instant at a bright object. a window or an incandescent lamp, and at once close the cyes again. I bright positive afterimage of the object looked at will be seen.

(b) Look at an incandescent lamp through a coloured gliass as in $(a)$. The positive after-image will appcar in the same colour as the glass.

(2) Negatice After-image.-(a) Look at an incandescent lamp for thirty seconds, and then clirect the eyes to a white surface. The after-image of the filament will appear dark. 
(b) Look at the lamp through a coloured glass for thirty or forty seconds, and then close the eye or look at a white ground. The after-image of the filament will appear in the complementary colour of the glass. If the glass was red, for instance, the after-image will be greenish.

(c) look at a white syuare on a dark gromml for thirty seconds, then quickly cover the field with white paper. A dark square will be seen on the white ground.

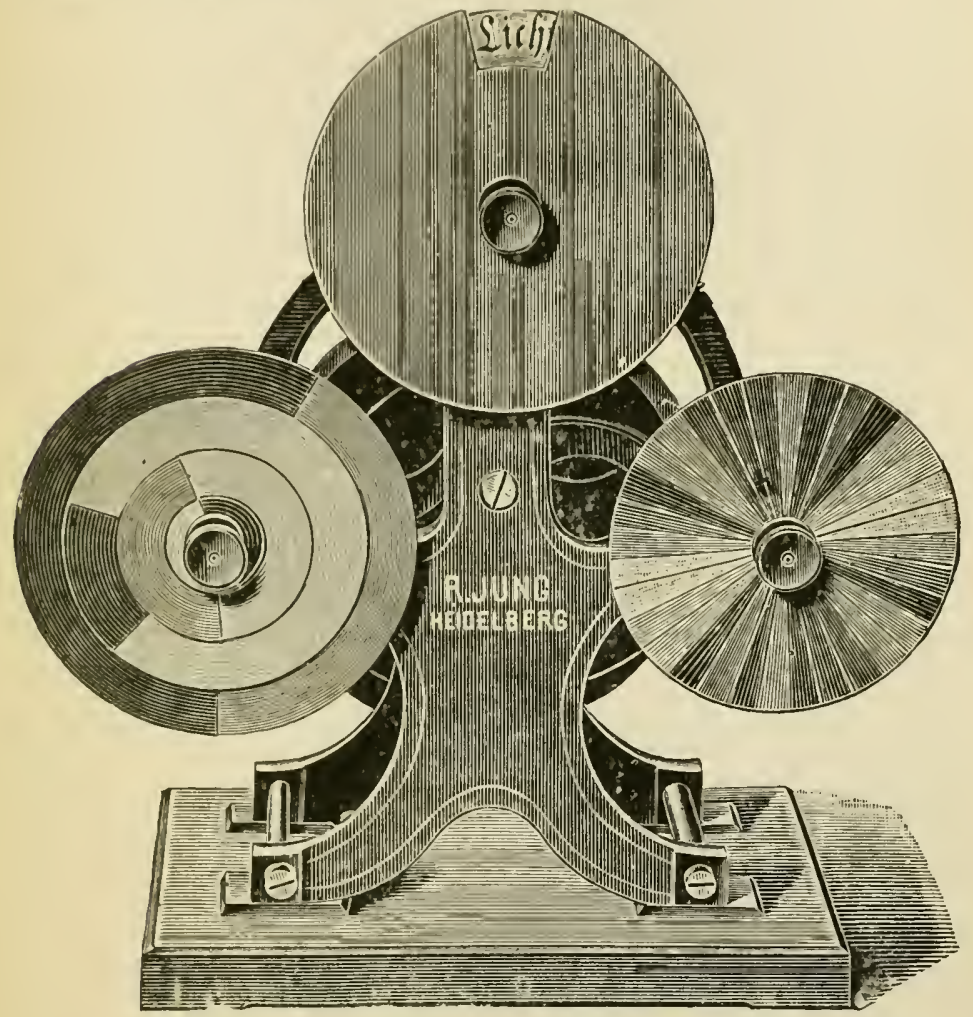

Fig. 444.-APParatus FOR COLOUR-MixiNg.

(d) Repeat (c) with coloured squares. The after-image of the square will be in the complementary colour.

Contrast.-Perform Meyer's experiment (p. 944).

I 7. Retinal Fatigue.-Fix the eye steadily on a portion of a printed page a considerable distance away. Note that the print soon becomes blurred. Wink the eye; the short rest causes a notable recovery of the retina.

IS. Visual Acuity.-Draw on a white card a series of vertical black lines I millimetre thick, and separated from each other by a distance of a millimetre. Set the card up in a good light, and walk backwards from it till the individual lines just fail to be discriminated. 
Measure the distance from the carcl at which this occurs, and calculate the size of the retinal image (p. 90.4).

I9. Colour-blindness. - Spread out Holmgren's coloured wools on a sheet of white filter-paper in a good light. Do not mention the colours of any of the wools, but (I) ask the person who is being tested to pick out all the wools which seem to him to match a pale pure green wool (neitlier yellow green nor bluc green), which is handed to him. He is not to inake an exact match, but to pick out the skeins which seem to have the same colour. If he makes any mistakes, by selecting, e.g., in addition to the green skeins any of the 'confusion colours,' such as grey, greyish yellow, or bluc wools, there is some defect of colour discrimination. To determine whether the person is red or green blind, tests (2) and (3) are then made. (2) Give him a medium purple (magenta) wool, and ask him to pick out matches for it. If he is red-blind, he will select as matches to it only blues and violets, as well as other purples. If he is green-blind, he will select only greens and greys. (3) The third test is a red wool. In selecting matches for this, the red-blind will choose (with reds) greens, greys, or browns less bright than the test. The green-blind will choose (with reds) greens, greys, or browns which are brighter than the test.

It must be remembered that the results of tests with the coloured wools need not be precisely the same as those with coloured lights, and that when there is a discrepancy between the two the test with the coloured lights should be accepted; for it is usually the normal perception and discrimination of coloured lights which has practical importance.

20. Talbot's Law. - Rotate a disc one sector of which is black and the rest white, or a disc like that in Fig. +12(p.938). A uniform shade is produced as soon as a speed of about 25 revolutions a sccond has been attained, and this is not altered by further increase in the speed.

2I. Purkinje's Figures.-(a) Concentrate a beam of sunlight by a lens on the sclerotic at a point as far as possible from the corneal margin, passing the beam through a parallel-sicled glass trough filled with a solution of alum to sift out the long heat-rays. The eyc is turned towards a dark ground. The ficld of vision takes on a bronzed appcarance, and the retinal bloodvessels stand out on it as a dark network, which appears to move in the same direction as the spot of light on the sclerotic. A portion of the ficld corresponding to the yellow spot is devoid of shadows (p. 930).

(b) Direct the eyes to a dark ground while a flame held at the side of the eye, and at a distance from the visual line, is moved slightly to and fro. A picture of branching bloodressels appears. This experiment is performed in a dark room.

(c) Immediately on awaking look at a white ceiling for an instant; a pattern of branched bloodvessels is secn. If the eye be at once closed, and then opened with a blinking morement, this may be observed again and again. Ultimatcly the appearance fades away.

\section{HEARING, TASTE, SMELL, TOUCH, ETC.}

22. Monochord.-Study by means of the monochord, a stretched string with a movable stop, the relation between the pitch of the note given out by a vibrating string, and its length and tension. 
23. Beats.-Cause two tuming-forks of nearly equal pitch to vibrate at the same time. Make out the beats, and count their number per second.

24. Sympathetic Vibration. - Take three tuning-forks mounted on resonators. L.et two of them be of the same pitch. Strike one of these ; the other is thrown into sympathetic ribration, and continues to give out a note after the first is quickly stopped by touching it. The third fork is unaffected.

25. Determine by means of (ialton's whistle the pitch of the higliest anclible tonc.

26. Cranial Conduction of Sound.-Wlien a tuning-fork is held between the teeth, a part of the sound passes out of the ear from the vibrating membrana tympani; if one ear is closed, the sound is hearl better in this than in the open ear. If the tuning-fork is held between the teeth, till, with both ears open, it becomes inaudible, it will be heard for a short time if one or both ears be stopped; and when in this position the sound again becomes inappreciable, it can still be caught if the handle be introduced in to the auditory meatus.

27. Taste.-(I) Apply to the tongue by means of a camel's-hair brush a solution of quinine ( $\mathrm{I}$ to $\mathrm{r}, \mathrm{O00}$ ), sodium chloride ( $\mathrm{I}$ to 200 ), cane-sugar ( $r$ to 50 ), and sulphuric acid ( $\mathrm{r}$ to $\mathrm{r}, 000$ ). Determine at what part of the tongue the strongest sensations are elicited by each. (2) Prepare a series of solutions of sulphuric acid of gradually increasing strength, beginning with a $\frac{M}{2,000}$ solution (a two-thousandth grammc-molecular solution) (p. 398). Put into the mouth, after previous rinsing with distilled water, 4 or 5 c.c. of one of the solutions of the acid, beginning with the weakest, and determine at what concentration of the $\mathrm{H}$ ions the acid taste first appears, rinsing out the mouth after each observation. Repeat the experiment with solutions of hydrochloric acid, and determine whether the threshold value is the same.

A similar comparison of the necessary concentration of the $\mathrm{OH}$ ions can be made with solutions of sodium hydroxide and potassium hydroxide.

(3) Connect two short pieces of platinum wire with the copper wire from the poles of a Daniell or dry cell. Apply one platinum wire to the inner surface of the lip and the other to the tip of the tongue. Reverse the poles. Note the difference in the sensation according to whether the anode or the kathode is on the tongue.

28. Smell.-(r) Pass a current through the olfactory mucous membrane by connecting one electrode with the forehead and the other by means of a small piece of sponge or cotton-wool soaked in physiological salt solution with one nostril. An odour like that of phosphorus will be perceived.

(2) To distinguish between Taste and Smell.-Use a solution of clove-oil in water which can just be distinguished from water when it is placed on the tongue by means of a camel's-hair brush. Close the nostrils. and determine whether the clove-oil can now be detected.

29. Touch and Pressure.--(I) Prepare a number of hair æsthesiometers by fastening hairs of different thicknesses to small wooden handles about 3 inches long by means of sealing-wax. Hairs as straight as possible should be chosel, or straight portions of hairs. The hair is to be fastened on one end of the piece of wood at right angles to the long axis of the handle, so that about an inch of the hair projects to onc side. Determin the pressure value of each 
hair by pressing it down upon the scalc of a balance till it is slightly bent, and observing the greatest weight in the other scalc which it will lift. Mark the number in inilligrammes on the handle. In this way, when a hair is placerl at right angles to a point of the skin. and pressure exerted on it till it begins to bend. the intensity of the touch stimulus i.e., the pressure exerted on the skin is clefmitely measured, and by using hatirs of different pressure values the thresholil value of the stimulus for any touch area-i.e., the pressurc which just gives the sensation of light touch-can be determined (p 970).

(a) Using the back of the hand, note how light a touch of the aesthesiometer applied to the end of a hair suffices to elicit a sensation of toucli, as compared with a part free from hairs. The hairs diminish the threshold of the stimulation by acting as levers, whose short arm presses against the nerve-endings surrounding the hairfollicles, while the stimulating weight acts on the long arm. When the skin is shaved the threshold is always raised.

(b) Shave an area on the back of the hand, and make out the relation of the touch-spots to the hair follicles. Each hair has an especially sensitive touch-spot just on the 'windward' side of the follicle (p. 970). Using aesthesiometers of different pressure values, determine the threshold value for the shaved area. Outline an area of a square centimetre on the skin, and determine the number of touch-spots, using first a hair of the threshold value, and then going over the area again with a hair of a decidedly higher pressure value. The threshold value for many parts of the hairy skin is obtained with a hair which bends at 70 milligrammes. Repeat the determinations for other skin areas, such as the back of the upper arm, the palm of the hand, the anterior surface of the leg, the chest. the back, and the cheek, forehead, and lips.

It is well that the subject should be blindfolded during the examination of the skin areas. He should understand by preliminary practice what the sensation of light touch is, the perception of which he is to indicate. With strong asthesiometer hairs the pricking sensation due to stimulation of pain-spots must be discriminated from touch sensation. When the two sensations are elicited together, the touch sensation is momentary, and the subject must be alert to detect it immediately on stimulation. The pain sensation develops more slowly, but lasts longer and becomes much more conspicuous than the touch sensation. which accordingly is apt to be submerged by it in consciousness.

(2) Touch the skin with a blunt point (at or about skin temperature). With light contact the sensation is that of simple touch. On increasing the pressure, the quite distinct sensation of deep pressure is perceived.

(3) Touch a portion of skin with a cancl's-hair brush of ordinary size, pressing on it till the hairs of the brush begin to bend. The first sensation of simple contact gives place to a sensation of pressure. Repeat with a camel's-hair brush of the finest liairs half a centimetre in length, cut away till its cross section is only half a millimetre in diameter at the base. Probably a pure sensation of torch, without any pressure element, will be obtained when the brush is applicel so as just to bend the hairs.

(4) Find the least distance apart at which the points of the esthesiometer compasses can be recognised as two when applied to the back of the hand, the forearm. upper arm, forcheat. fingertips, or tip of the tongue. Both points of the compasses must be 
placed on the skin at the same time, atud the sane pressure applied to both. The subject nuust not see the points.

(5) Time Discrimination of Touch.-Touch the prong of it vibrating tuning-fork lightly with the tip of the finger. The taps of the prong on the skin do not blend into a continuous sensition eren when the fork vibrates several hundred times per second.

30. Temperature Sensations. - For the investigation of these, piece's of thick copper wire, filed at one end to a blunt point, and fixed by the other in a small wooden handle, may be used. 'They' can be heated in a sand-batl or in a beaker of water to the desirecl temperature, or couled in cold water or in ice. Or a metal tube drawn out at one end, through which water at the required temperature can be passed before use, may be cinployed. Another device is a metal cylinder ending in a point, and filled with water at the given temperature.

(I) On the dorsal side of the hand outlinc an area of skin with a pen or a coloured pencil. Divide this into areas of + square milliinctres. Go over the area with a wre or cylinder at a temperature of about $+0^{\circ} \mathrm{C}$, and determine the extent and position of the spots which on contact yield a sensation of warmth, marking them on the skin by ink-dots, or mapping them on ruled paper. Then repeat the exploration with points at a temperature of about $15^{\circ} \mathrm{C}$., and map the spots which yicld a sensation of coolness. Now note whether a warm spot touclied with a point at $15^{\circ} \mathrm{C}$., or a cold spot touched with a point at $40^{\circ} \mathrm{C}$. yiclds any temperature sensation.

(2) Touch the skin with a test-tube containing water at $50^{\circ} \mathrm{C}$, and again with a test-tube containing ice. Do the sensations differ in any way from those of purc warmth and coolness? Repeat (I) with temperatures of $50^{\circ}$ and $0^{\circ}$, and note whether there is any difference in the quality of the sensations yiclded by the warm and cold spots. When a cold spot is touched with a point at a temperature of $50^{\circ}$. or a warm spot with a point at a temperature of $0^{\circ}$, is any sensation obtained? If so, what?

(3) Apply successively to one and the same portion of the skin test-tubes containing water at $50^{\circ}, 45^{\circ}, 40^{\circ}, 35^{\circ}, 30^{\circ}, 25^{\circ}, 20^{\circ}, 15^{\circ}$, $\mathrm{IO}^{\circ}, 5^{\circ}$, and $\mathrm{O}^{\circ}$ (ice), and determine the sensations excited in each case. The contact should only be momentary, so as not to cause extensive and lasting change of temperature of the skin. Note that there is a certain range of temperature above and below that of the skin within which no sensation of heat or cold is given.

(4) Take three beakers of water at $20^{\circ} \cdot 30^{\circ}$, and $40^{\circ} \mathrm{C}$. respectively. Place a finger of one hand in the coldest beaker, a finger of the other hand in the warmest, until no definite temperature sensations are felt by either finger. Plunge both fingers into the beaker at $30^{\circ} \mathrm{C}$., and temperature sensations will be perceived.

(5) Temperature Discrimination.--Find the least perceptible difference in temperature between two beakers of water at about $0^{\circ} \mathrm{C}$. Repeat the experiment with two beakers of water at about $30^{\circ} \mathrm{C}$. and again with two beakers of water at about $55^{\circ} \mathrm{C}$. Use the same hand. Expose the same amount of surface to the water.

(6) Compare the acuteness of the temperature sensations of the skin and the mucous membrane of the mouth, touching a given portion of skin and then a portion of mucous membrane with tubes containing water at various temperatures.

31. Pain.-(1) Using a pin, explore a cutaneous arca to rletermine whether every point of the skin yields the painful sensation of 
pricking. Especially compare the result of stimulating the region in the immediate neighbourhood of the hairs with the spaces between hairs. Discriminate the touch sensation given by the light contact of the pin-point from the painful impression caused when the pressure is increaser. Note that the touch element is more cranescent than the pain element.

(2) With strong $v$. lirey hairs determine the pressure at which the sensation of touch passes into that of pain.

(3) Compare the sensibility to pin-pricks of the mucous membrane within the month with that of the skin. 


\section{REP RODUCTION}

Regeneration of Tissues.-Sincc cells are constantly dying within the body, they must be constantly reproduced. In some tissues the process by which this is accomplished is more cviclent, and therefore bettel known, than in others. The most highly-organized tissues are with difficulty repaired, or not at all. The epidermis is always wearing away at its surface, and is being constantly replaced by the multiplication of the cells of the stratum Malpighii. In the corneous layer we have only dead cells; in the Malpighian layer we have every histological gradation from squames to columns, and every physiological gradation from cells which arc about to dic to cells that have just been born. The corpuscles of the blood undoubtedly arise at first, and are recruited throughout life, by the proliferation of mother-cells. The gravid uterus grows by the formation of new fibres from the old, and by the enlargement of both old and new. A severed muscle is gencrally united only by connective or scar tissuc, but under favourable conditions a complete muscular 'splice' may be formed. A broken bone is regenerated by the proliferation of cells of the periosteum, which become bonecorpuscles. We do not know whether there is any new formation of nerve-cells in the adult organism, but peripheral nerve-fibres which have been destroyed by accident or operation are readily regenerated, and the end-organs of efferent nerves may share in this regeneration.

In lower forms of animals, and in all or most vegetables, the power of regeneration is much greater than in man. The starfish can not only repair the loss of an arm, but from a severed arm a complete animal can be developed. A newt can reproduce an amputated toe, and evcry tissuc-skin, muscle, nerves, bone-will be in its place. After cxtraction of the crystalline lens in triton larvæ, a new lens is formed from the iris epithelium. Artificial mouths surrounded by tentacles can be formed in Cerianthus, an animal belonging to the same group as the sca-anemones, merely by making a cut in the body-wall and preventing it from closing. In an Ascidian, too (the Cynone intestinalis), artificial openings in the branchial sac, surrounded by numerous pigmented points similar to the cye-spots around the natural mouth and anus, have been produced (Loeb).

Thus, in a sense, reproduction is constantly going on within the bodies even of the higher animals. But since the whole organism cventually dies, as well as its constituent cells, a reproduction of the whole, a regencration en masse, is required.

A cell of the stratum Malpighii can only, so far as we know, reproduce a similar cell, and this is characteristic of cells that have 
undergone a certain amount of differentiation, especially in the higher animals. The fertilized ovum, on the other hand, has the power of reproducing not only ova like itself, but the counterparts of every cell in the bodly. And this is only the highest development of a power which is in a smaller degree inherent in other cells in lower forms. Plants and the lowest animals are far less dependent i1pon reproduction by means of special cells. A piece of a IIydra separated off artificially or by simple fission becomes a complete llyilra, as was shown by Trembley a century and a half ago. A cutting from a branch, a root, a tuber, or even a leaf of a plant, may reproduce the whole plant. It is as if each cell in these lowly forms carricl within it the plan of the complete organism, from which it built up the perfect plant or animal.

Reproduction in the Higher Animals.-In all the higher animals reproduction is sexual, and the sexes are separate.

In regard to the secretions of the reproductive glands, all that is necessary to be said here is that, unlike other secretions, their essential constituents are living cells. The spermatozoa in the male have, indeed, diverged far from the primitive type. Certain cells (spermocytes) in the tubules of the testicle divide, each forming two daughter spermocytes. Each of the daughter spermocytes in turn divides, so that four cells (spermatids) are formed from each spermocyte. In the final division which produces the spermatids a reduction of the chromosomes $(p .5)$ occurs, so that the spermatid possesses only one-half the number characteristic of the somatic cells of the species. The spermatids elongate and become spermatozoa, the head of the latter representing the nucleus of the former; and it is this nucleus (with the middle piece originally containing the male centrosome and attraction sphere) which is the essential contribution of the male to the reproductive process. The tail of the spermatozon is sinply, from the physiological point of viev, a motile arrangenent. whose function it is to carry the nucleus of the male element. freighted with all that the father can transmit to the offspring, into the neighbourhood of the female reproductive element or ovum. After the spermatozoon has penetrated the ovum its tail disappears, being probably absorbed.

The ovum also begins as a typical cell with nucleus (germinal vesicle), nucleolus (germinal spot), centrosome and attraction sphere (p. 5), and it forms, by its repeated subclivision, all the cells of the fotal body. But, except in some (parthenogenetic) forms, it never awakens to this reproductive activity till fecundation has occurred; and fecunclation essentially consists in the union of the male with the female element, or rather in the union of the male and female nucleus.

From time to time a Graafian follicle, overdistended by its liquor folliculi, bursts on the surface of the ovary and discharges an orum. It was formerly believed that the frayed or fimbriated end of the Fallopian tube, rising up finger-like from the dilatation of its bloodvessels, grasps the orum. But it is more than doubtful whether this occurs. It is more probable that the ovum is first discharged into the pelvic cavity, and is guided to the orifice of the Fallopian tube, not necessarily that of its own side, by the movements of the cilia around the orifice, and then passed slowly along the tube by the downward lashing cilia which line it. If not impregnated, it soon perishes amid the secretions of the uterus-how soon has been matter of discussion, and can hardly be considered as settled. If, however, impregnation occurs, the orum penetrating the superficial 
epithelium mto the subepithelial connective tissue becomes fixed in one of the crypts or pouclies of the uterine mucous membrane (decidua scrotina). which grows round it as the decidua reflexa.

Menstruation.- In the mature female, from puberty, the age at which the reproductive power begins (thirteenth to fiftecnth year), on till the time of the menopause (fortieth to fifticth year), at which it ceases, all ovum - or it may be in some cases more than one-is discharged at regular intervals of about four weeks. This discharge is accompanicel by certain constitutional symptoms and local signs that last for a variable number of days. The genital organs are congesterl, and a quantity of blood, which varies in different indivicluals, but is usually not over 50 c.c., is shed. If more than 6o c.c. is lost the flow is copious. Over 100 c.c. it is abnormally great ( 3. Hoppe-Seyler). At the same time, the whole or a portion of the mucous membrane of the uterus is cast off.

As to the pliysiological meaning of this mensiruation, as it is called, opinion is clivided. Two chief theories have been proposed to account for it, both of which agree in considering the phenomenon to be connected with a preparation of the uterus for the reception of the ovim. But according to the theory of P'fliger the mucous membrane is stripped off (by a process analogous to the "freshening, or paring of the indurated edges of a wound by the surgeon, in order that union may occur when they are brought together) on the chance, so to speak, that an impregnated ovum may arrive. On the alternative theory, this change takes place because the ovum has not becn impregnated, and the bed prepared for it not being required, the swollen and congested uterine mucous membrane undergoes (legeneration, and is in part cast off (Reichert, Williams, etc.).

The process of menstruation, and the nutrition of the genital organs. especially the uterus, are intimately dependent upon the ovaries. There is good evidence that the influence is exerted through an internal secretion formed by some portion of the ovarian substance. When, for instance. the ovarics of young animals (guincapigs) are removed from their normal situation and transplanted to a distant part of the body, the external genitals, vagina, and uterus undergo the normal development instead of being arrested in their growth, as is the case when the ovaries are removed altogether. The removal of the ovaries in adult animals leads to fibrous degeneration of uterus and Fallopian tubes. On the other hand, removal of the uterus has no effect on the development of the ovaries in a young animal, and does not cause degeneration of the ovaries of an adult animal. In monkeys, in which a menstrual flow comparable to that in the human female occurs, it was found that menstruation took place atter the ovaries had been transplanted from their original seat, and the flow stopped when the transplanted ovaries were removed. It has been stated. too, that in a young woman suffering from amenorrhoea (lack of menstruation) a regular flow appeared after the transplantation of an ovary from another woman into her uterus. Recently the view has been put forward that the important part of the ovary for these functions is the corpus luteum. which is by some investigators considered to be a gland with an internal secretion (Born). This secretion seems to be connected with the implantation of the ovum and the subsequent growth of both orum and uterus. According to Fraenkel, the absence of the corpus luteum prevents implantation. When the ovum has not been fertilized the corpus luteum brings about menstruation. Where fertilization has occurred 
it prepares the uterus for the implantation of the ovum. IIe considers that there is 110 difference between the true and the false corpora lutea. 'Lutein,' the dried extract of the corpora lutea of cow's, is recommended for the treatment of suppressed menstruation, and the troublesome symptoms arising from the premature production of the menopause by removal of the ovaries.

The mode of origin of the corpus luteum has given rise to much discussion. Two chief views have been put forward: (I) That it is a structure derivel from the connective-tissue wall (theca) of the (lischarged follicle (v. Baer, etc.): (2) that it is developed from the follicular epitlelium (membrana granulosa) (Sobotta, etc.). The second vicw seems to be best established. The granulosa cells enlarge, it is said, without becoming more numerous. In certain animals (guinea-pig), however, mitotic division of cells of the membrana granulosa has been observed (L. Locb).

The influence of the ovary on the formation of the decidua has been illustrated in a very interesting way by the investigations of 1. Loeb on the artificial production of deciduomata. He has shown that if a number of incisions are made into the uterus of a rabbit or guinea-pig within a certain interval after the ostral period (period of heat), a structure with the histological characters of the decidua develops at each wound. Impregnation does not appear to be a necessary factor, nor even contact of the ovum with the uterine mucous membrane. On the other hand, ovulation, the discharge of an ovum or ova, or at any rate the condition of the ovary associated with this discharge, seems to be indispensable. For extirpation of the ovaries in a large number of guinea-pigs prevented the formation of deciduomata from wounds of the uterus made at the most favourable period after copulation. The uterus then appears to have an inherent power of responding to such a stimulus as a mechanica injury by the production of a decidual structure, but only under the influence of the ovary. The ovarian factor is probably not nervous but chemical, some specific substance which acts on the uterus being liberated periodically in connection with the sexual rhythm.

Development of the Ovum.-Before fecundation, and apparently as a preparation for it, the orum is the seat of remarkable changes, similar upon the whole to those seen in the mitotic or indirect division of ordinary cells.* They have been most fully studied in the eggs of certain invertebrate animals. The division of the cell is initiated by changes in the centrosome and attraction sphere. The centrosome divides into two daughter centrosomes. These take up a position one at each pole of the nucleus. Each daughter-centrosome is surrounded by a system of radiating lines or filaments, which are less conspicuous than the cliromatin filaments of the nucleus, since they do not stain as these do. Meanwhile the nuclear membrane and the nucleoli (lisappear, or at any rate become indistinguishable from the rest of the chromatin skein. The skein breaks up into chromosomes, the number of which is constant for a given species, but is not the same in all species of animals.

The daughter centrosomes or astrospheres are united by meridional achromatic fibres. which form a spindle running througli the nucleus from one pole to the other. The chromosomes arrange themselves at right angles (equatorially) to the spindle, and then each chromosome divides longitudinally into two. The halves of the chromosomes now pass toward their respective centrosomes, being perhaps guided

* For figures illustrating the changes see any good textbook of $\mathrm{Histology.}$ 
by the fibres of the spinclle. It results, from this that two daughter nuclei are formed, each with the same number of chromosomes as the original nucleus, although with only half the amount of chromatin. The cytoplasm divides also, so that the parent cell is now represented by two daughter cells. In ordinary cell division the two (liughter cells are of equal size, but in the division of the ovum which occurs before fertilization the two resulting cells are very uncepual. The large cell continues to be known as the ovum; the small one is the first polar body. After extrusion of the first polar body the orum again divides uncqually. A new spindle forms, and a second polar body, again much the smaller of the two daughter cells, is cist off. There is a clifference, however, between the process of division which gives rise to the first and that which gives rise to the second polar borly. In the case of the latter a so-called reduction-division occurs; the chromosomes do not split longitudinally, but lialf of the original number pass into each daughter nucleus. As to the significance of these changes there has been much discussion. It is agreed that the result of the process is the expulsion of a portion of the chromatin, the ovum now possessing only half the original number of chromosomes, although nearly all the original cytoplasm. In fertilization the original number is restored by the male element when it arrives and penetrates the orum. For in the final cell-division by which the mature spermatozoon is formed the chromosomes of its nucleus are also, after two divisions essentially similar to those occurring in maturation of the ovum, reduced to lialf the normal number.

The two reduced nuclei in the fertilized ovum are spoken of as the male and female pronuclei. By their union a single nucleus is formed with the number of chromosomes normal to the species.

An enormous amount of interesting work has been done with the view of illustrating the connection of the complicated phenomena described with the structure of the ovum. Only a bare reference to one or two of the experiments is possible liere. Driesch and Hertwig find that the nucleus can be made artificially to change its place with reference to the yolk, without hindering the levelopment of a normal animal. Lillie has shown that centrifugalization of the eggs of annelids, although it markedly alters the distribution of the yolk and other substances, does not affect the form of cleavage. The polar bodies appear in the position which they would normally occupy. In other words, no redistribution of the granules or nucleus affects the polarity of the egg, which therefore is a function or property of the ground substance of the protoplasm. The whole of the protoplasm, however, is not necessary for complete development. Even in Amphioxus, the lowest of the vertebrates, the eggs have been broken up by shaking, and a complete animal cvolved from as little as one-eighth of an ovum. If the separation was incomplete a kind of Siamese twins, or even triplets, could be obtained (Wilson and Mathews). Nor is it always indispensable that both pronuclei should be present.

Whatever it is that the spermatozoon supplies, the process of fertilization can in certain forms be started artificially. The studies of Loeb and his pupils on artificially induced parthenogenesis are of special importance. When the unfertilized eggs of the sea-urchin are cxposed for one or two minutes to 50 c.c. of sea-water, to which 3 or 4 c.c. of decinormal acetic acid has been added, the majority of the eggs form the membrane characteristic of the entrance of the 
spermatozonn. When these eggs are afterwards exposed for thirty to forty minutes to 100 c.c. of sea-water, to which 14 or 15 C.C. of it strong solution of sodium chlorisle (two and a half times the strength of a normal solution, or about if 0 per cent.) has been arlded, those of the eggs which hate formed membranes develop into swimming larvas that rise to the surface. These larvas develop into perfect sea-urchin larvas or 'plutei' as fast as the larvas of eggs fertilized with sperm.

The facts of parthenogenesis show that it is not absolutely necessary for development that the ovum should have the normal number of chromosomes restored. It can develop with half the number, the chromosomes of the female pronucleus being sufficient for growth, although, of course, in this case for a growth uninfluenced by the propertics of the male clement. In like manner it is stated that portions of the maturated ovum devoid of a nucleus can undergo development if penetrated by a spermatozoon, the chromosomes of the male pronucleus being sufficient for growth.

Not till all these events have taken place-extrusion of the two polar bodies, or maturation; penctration of the spermatozoon, and blending of its head (the male pronucleus) with the remnant of the nucleus of the ovum (female pronucleus), or fecundation-not till then does the ovum begin the process of repeated division by which the whole body is reproduced. The fused or segmentation nucleus divides into two, each containing the normal number of chromosomes derived from the splitting of those contributed by both the malc and female elements. It is belicved that the division takes place in such a way that both male and female chromosomes are represented in each nucleus. The cytoplasm being also cleft by a corresponding furrow, two complete nucleated cells make their appearance. These divide in turn, till at length (in the mammal) the embryo is represented by a hollow sphere or vesicle, with a cellular crust. During division the upper or outer cells have always been larger than the inner and lower, and have multiplicd more rapidly; and thus it comes about that the hollow sphere of large cells encloses a mass of smaller cells, along with remnants of broken-down yolk and of fluid derived by absorption from the contents of the uterus. The smaller cells continue to multiply and arrange themselves as a lining to the sphere already formed. so that in a short time it becomes louble. and we have already differentiated two of the primary embryonic layers-the ectoderm. also called the epiblust. or superficial, and the cndoderm, also called the hypoblast, or deep layer. The whole sphere is called the blastoderm, or the blastodermic vesicle.

While this inner shell of endedermic cells is gradually creeping on to completion, there appears at a part where it is alrealy fully formed a small opaque whitish disc, the germinal area or embryona! shield. This represents the stocks on which the framework of the embryo is to be laid down. The area elongates; at its posterior end appears at thickened line, the primitive streak, soon furruwed by at Ingitudinal growe, the primitive groove, that marks the direction in which the long axis of the futume embryo will lic, but is not itself a permanent line in the building. and ultimately vanishes. The appearance of the primitive streak is the signal that a rapicl proliferation of the cells of the germinal area, and especially of the cetoderm, has begun ; and this goes on until a third layer is formed. intermediate in position to the original two, and therefore naned the mesoderm. While this is pushing its way over the germinal 
area and into the rest of the blastodermic vesicle, the ectoderm in front of the primitive streak rises 11 p in two lateral ridges, enclosing between them the medullary groove. The medullary groove is the beginning of the cerebro-spinal axis ; its walls first come to overhang the furrow, and then to ccalesce; and the melullary groove has now become the neural canal. Immediately uncler it the mesoderm forms a rod of cells, the notochord. which is the forerunner of the vertebral column; around this the bolies of the vertebra are afterwarts developel from cubical masses of mesodermic cells, arranged in pairs along the notochord, and called the protovertebre. The rest of the mesoderm, rumning out on each side from the protovertebrx, splits into two lavers. an upper or somatic layer, which unites with the ectoderm, and a lozer or splanchnic layer, which unites with the endoderm. Between the two layers is a space called the coelom, or plenro-peritoncal cavity (Fig. +45).

Up to the present, apart from the enclosure of the neural canal, all this formative activity is buried beneath the surface of the blastoderm, and has not showed itself by any external token; the cmbryo still appears as a portion of the germinal area, and lies in its planc. But now a pocket, or crease, or moat, beginning at the head as the head-fold, then pushing under the tail, gradually creeps round and undermines the whole embryo, which is raised above the general level, and, as it were. scooped out from the rest of the blastoderm; till at length it lies on the latter, something like an upturned canoc, enclosing a tube, complete in front and behind, but still open in the middle. where it communicates with the interior of the yolk-vesicle. Since this tube has been formed by the tucking in of the three ancestral layers of the blastoderm, it follows that it is lined by endoclerm, supported externally by the splanchnic sheet of mesoderm. So that now the body consists of a dorsal tube (the neural canal), essentially of ectodermic origin, a ventral tube (the alimentary canal), essentially of endodermic origin, and between the two a massive double layer of mesodermic tissue, which contributes supporting elements to both. At this point it may be well to emphasize the fact that this embryological distinction of the three primitive layers has a deep and fundamental meaning, and corresponds to a physiological distinction that endures throughout life. The endoderm, the lowest layer in position, may also be described as the lowest in the physiological hierarchy. It furnishes the epithelial lining of the alimentary canal from the beginning of the œsophagus to near the end of the rectum, as well as the epithelium of the organs which arise from diverticula of the primitive intestine - viz., the digestive glands (with the exception of the salivary glands), the lungs. and the passages leading to them, the thyroid, and the greater part of the thymus gland in its primitive condition before the lymphoid tissue derived from the mesoderm has as yet grown into it. According to some authorities, the notochord is also derived from the endoderm.

Upon the whole, it may be said that the tissues of endodermic origin are essentially concerned in chemical labours, in the absorption of food material and excretion of waste products. The meso dermic tissues are essentially concerned in mechanical labour; they are the tissues of movement and of passive support. The ectodermic tissues are at the top of the pyramid ; they govern the rest.

From the mesoderm arise the muscles, the entire vascular system, with its blood-and lymph-corpuscles, the bones and connective 
tissues; and the Wolftian body and its appendages, which are the predecessors of the genital glands and ducts, and of the chief portion of the renal apparatus.

The ectoderm forms the epidermis and its appendages, the cpithelial encl-organs of the nerves of special sense, and the nervous system, cerebro-spinal and sympathetic. The salivary glands and the mucous lining of the mouth and anus are developed from the ectoderm, which is indented to meet the intestinal canal and give it access to the exterior at either end.

It is not possible here to trace in detail the development of all the organs of the cmbryo. Its nutrition and metabolism not only distinctly belong to the physiological domain, but. carried on as they are under conditions that seem so strange, and even so bizarre. to one acquainted only with adult pliysiology, are calculated to throw light on the metabolic processes of the tully-developed body. And they cannot be understood without reference to the peculiarities of the vascular system in fotal life. These we shall accordingly describe. but for further details as to the anatomy of the embryo the student is referred to some standard anatomical text-book, such as Quain's ' Anatomy.

Physiology of the Embryo.-In the first period of its development the ovum, nestling in the pouch formed by the decidua serotina and reflexa, is fed from the maternal blood and tissues directly, without the mediation of fotal bloodvessels, through the finger-like processes or villi with which its external layer, the zona pellucida, becomes studded. At the earliest stage at which a human ovum has been studied after implantation it is already enveloped by a thick ectodermic covering (the trophoblastic envelope), consisting of two layers of cells, one unquestionably of fœtal origin, the so-called cells of Langhans, and the other the syncytium, the origin of which is assigned by some authorities to the ovum, by others to the maternal tissues. The trophoblastic covering is every where in contact with the maternal blood, which, pushing its way into the trophoblast at intervals, divides it into columns. Later on the foetal mesoderm grows into these, and so the primary villi are formed. It is not till after the first three weeks that bloodvessels make their way into these villi, although the mesoderm of the foetus begins to enter the villi about the end of the first, or the beginning of the second, week. The scanty yolk of the human orum is totally inadequate to supply it with nutriment for the time that elapses before the bloodvessels are developed, and food substances must be obtained from the maternal liquids by imbibition, osmosis, diffusion, or filtration, aided, perhaps, by more special absorptive processes on the part of the fotal tissues. Soon the heart appears as a tube (at first double), formed by cells belonging to the splanchnic layer of the mesoderm. It begins to pulsate in the chick as early as the middle of the second day, although it as yet contains neither nerve-cells nor fully-formed muscular fibres. In the mammal pulsation is late in making its appearance, in man about the beginning of the third week. A bloodvessel grows out from the anterior end of the heart and divides into two primitive aortic arches. from each of which a vessel (omphalo-mesenteric or vitelline artery) runs out in the mesoderm covering the umbilical vesicle, or yolk-sac. The blood is returned to the heart by the vitelline veins coursing in on the walls of the vitelline duct. In this way the store of nutriment in the umbilical vesicle of the chick. which is the only solid or liquid food it receives or needs during the 
whole periol of ilevelopment, is tapped, and a regular channcl of supply establisleel. Oxygen is at the same time absorbel through the porous shell ; but later on this respiratory function is taken over by the second or allantoic circulation. In the mammal the circulation on the umbilical vesicle is of much less consequence. for the quantity of material left over after the formation of the blastoderm is exceclingly small : it is only with a few days' provision in its haversack that the embryo starts out on its developmental march. . Ind the vitelline vessels deriving their further supply of food and oxygen from the tissues of the mother in contact with the ovum cease to be of use as soun as the second and more perfect placental circulation is cs-

tablished. and soon shrivel up and clisappear. ats the umbilical resicle slurinks.

The second circulation of the cmaryo is alcveloped in conrection with a remarkable offshoot from the hind-gut called the allantuis. which, before the fifth day in the chick and d uring the second weck in man, pushes its way out between tlie so$\mathrm{matic}$ and $\mathrm{splanchn} \mathrm{ic}$ layers of the mesoderm-i.e., in the pleuroperitoneal cavity-and grows through the um-

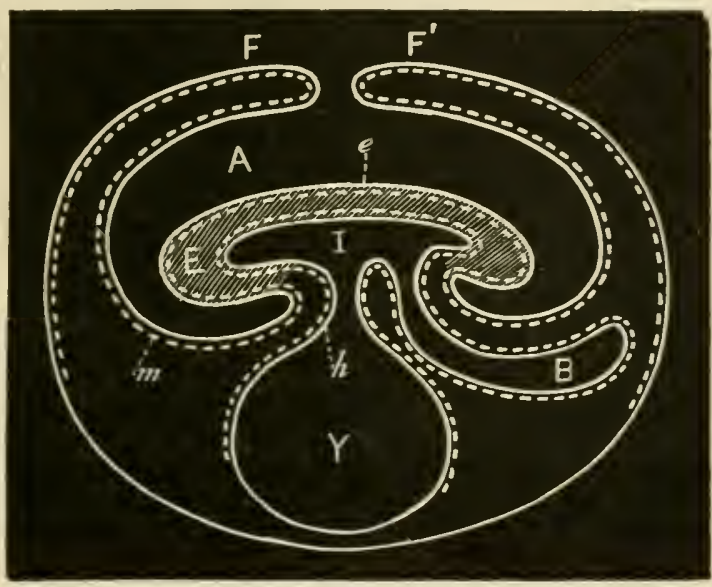

Fig. 445.-Diagran to illestrate Formation of Ansiox.

A, cavity of true amnion; $F, F^{\prime}$, folds about to coalesce and complete the amniotic cavity : $m$, mesodermic layer of amnion; $\mathrm{B}$, allantois; $\mathrm{I}$, intestinal cavity of embryo; I, yolk-sac; $h$, endodermic layer; $c$, ectodermic laver of embryo. The embryo is the shaded portion in the iniddle of the figure. $E$ is placed orer the head region. No attempt is made to delineate its actual form. The mesoderm is represented by the ir terrupted line.

bilicus, carrying

bloolvessels along with it in its mesodermic layer. Still earlicr. and, indeed, while the embryo is being separated off from and raised above the level of the rest of the blastoderm by the deepening of the ditch around it. the further banks of this furrow. formed of ectoderm and somatic mesoderm. have risen up on every side. and, growing over the back of the embryo, have finallycoalesced and enclosed it in a double-walled pouch (Fig. +45$)$. The superficial layer of the pouch is called the false amnion; it soon blends with the tufted chorion or common outer envelope of the orum. The inner layer persists as the true amnion; a liquid, the ammiotic fluid, is secreted in the cavity which it encloses; and the embryo, loosely anchored for the rest of its intrauterine life by the umbilical cord alone, floats freely within it. The

$$
64-2
$$


amniotic fluid acts as a watcr-jacket or cushion. to break the force of the incvitable shocks and jars transmitted from tlic mother to the fœetus and from the foctus to the mother. To some extent, in addition, it may serve as a nutritive fluid, for substances can pass from the blood of the mother into the amniotic fluid, and the amniotic fluid can be swallowed by the foetus. This is shown by the fact that sodium sulphindigotate, when in jected into the maternal circulation, is found in the amniotic fluid and in the alimentary canal of the foetus, although not in any of the foetal tissues. Fine lanugo hairs from the foetal skin have also been found in the meconium.

The precise origin and mamner of formation of the amniotic fluid have not been settlecl. It is probably in the main a maternal secretion or transudation. But something is contributed by the foetus in the form of renal, and perhaps of skin, secretions. The fluid is poor in solids. Its maximum content of protein. reached during the first half of pregnancy, is only 0.7 per cent. Later on it diminishes, and at full term is only one-tenth of this amount. The specific gravity is 1006 to 1009 . Its osmotic concentration, as measured by the clepression of the freczing-point, is less than that of the mother's blood-serum.

The allantois, growing out at the umbilicus, in the manner described, insinuates itsclf between the true and false amnion, and soon blends with the latter. For a time the secretion of the primitive kidneys continues to be poured into the cavity of the allantois, so that it serves in part as an excretory organ, while in the bird it also performs the function of respiration; and in the mammal both food and oxygen are carried by its ressels to the foctus during the greater part of intra-uterine life. But later on the outgrowth atrophies and disappears, all except its origin from the alimentary canal, which dilates and persists as the urinary bladder, and its bloodvessels, which grow in the form of tufts or loops into the chorionic villi. The vessels are fed by two umbilical arteries which arise from the hypogastric arteries and run out at the umbilicus on the allantois. The blood is returned by an umbilical rein, whose further course we shall have soon to trace. The shrivelled stalk of the allantois, projecting through the umbilicus, takes part, with its bloodvessels, in the formation of the $n$ mbilical cord, which contains also the remains of the yolk-sac and is clothed externally by a layer of the amnion. Continuous with the umbilical cord, and stretching from the umbilicus to the urinary bladder, is a portion of the allantois which is represented in extra-uterine life by a thin cord-like structure, the urachus. The vascular tufts of the chorion, which at first cover the whole surface of the ovum and suck up food and oxygen from decidua serotina and reflexa alike, disappear in the region of the reflexa, hypertropliy all over the serotinathat is, where the ovum is in actual contact with the uterine walland this part of the chorion is now distinguished as the chorion frondosum. The giant villi of the chorion frondosum push their way into the thickened decidua serotina, and ultimately penetrate into the great capillaries or sinuses of the uterine mucous membrane. At the same time the tissue of the villi external to the vessels becomes reduced to a mere film, so that, except for a thin covering of decidual cells, the fotal vessels are bathed in maternal blood. By this interweaving of decidua and chorion frondosum is formed the placenta, which for the rest of intra-uterine life acts as the great respiratory, alimentary, and excretory organ of the fotus. 
Exchange of Materials in the Placenta.-The maternal blood, as it streams through the colossal capillaries of the decidua, gives up to the fortal blood oxygen and food substances and receives from it carbon dioxicle and in all probability urea. It is true that the blood in the uterine sinuses is not itself fully oxygenated; it is not bright red arterial blood. But it yet contains more oxygen, and oxygen at a higher partial pressure (p. 248), than the purest blood of the fotus, and is, therefore, able to part with some of the surplus to the dark stream of oxygen-imporerished blood brought by the umbilical arteries to the placenta. Thus, it has been found that while the blood of the umbilical artery of the foetus of a shcep had +7 volumes per cent. of carbon clioxide, and only $2 \cdot 3$ of oxygen, that of the 11 mbilical reins had 6.3 volumes of oxygen, and only $40^{\circ} 5$ of carbon dioxide (Zunt\% and Cohnstein). In the exchange of gases between the placental and the fortal blood the same general features present themselves as in the external and internal respiration of the mother, with this difference, that the exchange of oxygen is neither between air and hamoglobin, as in the lungs, nor between hamoglobin and tissue elements, as in the organs; but between maternal and fotal hamoglobin, of course, through the mediation of the maternal and fortal plasma. There is no reason to suppose that the mechanism of the exchange is essentially different from that of the more familiar forms of respiration. Diffusion of the gases from places of higher to places of lower tension unquestionably plays an important part. But this does not exclude the possibility of a more active process of some other kind, although there is at present no direct evidence of such a gaseous secretion as has been previously discussed in connection with pulmonary respiration (p. 258). The presence of oxydases in the placenta does not throw any light on the question. For there is no proof that they act in transferring oxygen from the one circulation to the other, and oxydases are found in the most cliverse tissues. Their significance for the combustion processes of the body has already been alluded to (p. 264).

Salts soluble in water, including not only those necessary for nutrition, like sodium chloride, but many foreign salts, pass readily from the placenta to the fotus, and in general more easily the lower their molecular weight. Such salts as potassium iodide, e.g., when injected into the maternal circulation, appear in the foetus in a very short time. On the other hand, colloidal solutions-e.g., of silver or silicic acid-do not pass over at all. It is of practical importance that substances like chloroform, ether, and other narcotics, and alkaloids like morphine and scopolamine, when administered in obstetrica practice, may find their way from the mother to the child, although more slowly and more capriciously than the salts. While diffusion and osmosis assuredly take part in the passage of materials from the placenta to the fœetus, there is no more reason to conclude that the whole exchange, even for the salts, depends upon such simple physical processes than there is in the case of the exchange between any one of the maternal tissues and the maternal blood. The essential similarity of placental and intestinal absorption, to take one instance, is seen in the mechanism by which the foetus gains the iron required for the development of its hæmoglobin. The hæmoglobin of the mother appears to be the most important source of this iron. Erytinrocytes in all stages of decomposition can be found in contact with the chorionic villi, and even in the epithelium covering the villi. These corpuscles come partly from extravasations in 
the maternal portion of the placenta, but it is possible that the villi also possess the power of hamolyzing intact corpuscles in the circulating placental blood. Iron can be demonstrated by microchemical reactions in the epithelial cells of the chorionic villi as fine gramules, which increase in size towards the base of the cells. As we pass leeper into the villus towards its central bloodvessel, the granules again diminish in size. The picture is very like that seen in the absorption of iron from the intestine. And if the microcliemical picture is practically the same, the process by which the iron is absorbed is not likely to be fundamentally difterent in the two cases $(p .+17)$.

The same is true of the passage of fat across the placenta. Fat can always be demonstrated microchemically in the chorionic villi. The most superficial layer of the villi is free from visible fat chroplets. They increase in number towards the base of the epithelial cells. As in the case of the intestine, these appearances agree well with the view that the fat is split before being absorbed by the villi, and undergoes resynthesis in the epithelium. That, as a matter of fact. fat passes from the mother to the fotus is shown by the observation that when pregnant guinea-pigs were fel witl a foreign fat (from cocoanuts), the characteristic fatty acid (lauric acid) was found in the fotus. This, however, (locs not exclude the possibility that the foctus may form fat in its own tissues from carbo-hydrates, and perhaps from proteins, as it is clestined to do in extra-nterine life.

Among the carbo-hydrates the passage of dextrose from the maternal to the fotal blood has been experimentally demonstrated. A specially interesting proof is afforded in cases wherc the mother suffers from diabetes mellitus. In one case in which the mother, during diabetic coma, was delivered of a stillborn clild, the blood of the child contained $2^{\circ} 2$ per cent. of sugar, its urine $5^{\circ} 2+$ per cent., and the amniotic fluid $0^{\circ}+7$ per cent. The blood of the mother had a sugar content of 0.8 per cent., and her urine a content of 6.94 per cent. The sugar of the maternal blood is not the only source of the carbo-hydrates of the fotus. The glycogen store of the placenta is to be regarded as a second source, which is rendered available on conversion into dextrose by the placental diastatic ferment. This store of easily available food material is especially important in the early stages of development of the ovum before a circulation has been establishel in the villi. In the youngest ova investigated the decidual covering has been found rich in glyengen.

While it is to be supposed that the products of the hyolrolytic lecomposition of proteins can be absorbed by the fotal blood in its luassage through the placenta, to be synthesized to the appropriate tissue proteins in the fortal organs, there is evidence that certain proteins can be taken up without change. In this connection it must be remembered that the mother is much more closely related to the foetus as regaris her protein composition than any ordinary protein fool can be to an animal in extra-nterine life. In some respects, indeed, the foetus may be considered, especially, perhaps, in the first stages of its levelopment, as a part of the mother, an additional, although very complex, organ rather than an inclependent organism.

The blood of the umbilical artery, although far from the level of the ordinary arterial blool of the mother as regards its gaseous content, is yet the best the fotus ever gets; and by a series of contrivances it is assured that this best should go first to the most important parts-the liver. the heart, and the head-while the legs 
and most of the abdominal organs have to put up with an inferior supply. This is brought about mainly by the existence of three short-cut.s for the blood, which disappear in the adult circulation, the ductus venosus, the ductus arteriosus, and the foramen ovale.

The bloof of the umbilical vein, rich in oxygen for fontal blood, passes partly through the circulation of the liver, but a part takes the route of the ductus venosus, and empties itself into the inferior venal cavil. The litter gathers up the more or less vitiated blood from the inferior cxtremities and the renal and hepatic veins, and pours its mixcl, but still fairly oxygenated, contents into the right atricle. By means of the Eustachian valve, the jet coming from the moutl of the inferior vena cava is directed into the left auricle througle the foramen ovale in the inter-auricular septum. There it is joincel by the trickle of bloos which is creeping through the uncxpanderl lungs. The left ventricle propels its contents througl the aorta, ancl thus a large part of this comparatively pure or scconcl-best blool is sent to the head and upper extremitics. It returns in a vitiated state by the superior vena cava into the right auricle, and owing to the position of the Eustachian valve and the lirection of the current, it flows now, not through the foramen ovalc, but into tluc right ventricle. Thence it is clriven through the pulmonary artery, but only a small quantity of it finds its way through the lungs; the main stream is short-circuited through the ductus arteriosus, and mingles with the contents of the thoracic aorta below the origin of the cephalic and brachial vessels.

We may now give something more of precision to the statements that different parts of the bocly receive blood of different quality; and it is possible roughly to divide the organs in this respect into four categories: (r) The liver, which partakes both of the best and the worst, the purified blood of the umbilical veins and the vitiated blood of the intestines and spleen; (2) the heart, head, and upper limbs, which receive the blood frcm the inferior extremitics and kidneys, mixed with the pure blood of the venous luct; (3) the lcgs, trunk, intestines, and kidneys, which are fed chiefly by the off-scourings of the cephalic end, mitigated, however, by a proportion of mixed blood from the inferior cava; (4) the lungs, which receirc only a feeble stream of unmixed venous blood.

These peculiaritics of the embryonic circulation are in obvious correspondence with the physiological events taking place in the fotal body. The liver is not only the greatest gland in the embryo, as it continues to be in the adult, but its activity seems to dwarf that of all the other glands put together, and is in striking contrast with the functional torpor of the lungs. From the third month of intra-uterine life the secretion of bile begins and the intestines gradually fill with meconium, of which the principal constituent is bile. Accordingly the liver is most lavishly supplied with blood, while the lungs are stinted. And since the liver has, as we have already learnt, other and, in the adult at least, even more important labours than excretion, a large portion of the blood it receives is of the best quality: it enters the gland comparatively rich in oxygen, and passes out comparatively poor ; while the lungs, which have to be nourished only for their own sake, and are of no use whatever till the child is born and respiration has begun, must be content with the poorest fare-with the crumbs that fall from the table of fotal nutrition. The full-fed cephalic end of the embryo grows far more rapidly than the half-starved inferior extremities, 
and the head of the new-born clild is large in proportion to the rest of the body.

There are some other points in the physiology of intra-uterine life which call for remark : and, to sum up in a few words the grand distinction between fotal and adult life, we may say that growth is the keynote of the former, work (functional activity) of the latter. Thus, the muscles at an early period in their development, long before any glycogen can be found in the liver, become the seat of an accumulation of glycogen, which, since it cannot be used up in contraction as in the adult muscles, seems to be intimately connected with their own growth, and perhaps also with the growth of other tissues. It is true that the foetal tissucs as a whole, including the muscles, are not richer, as userl to be taught, but poorer in glycogen than adult tissues, and therefore the old doctrine that the foetal glycogen fulfils a special 'formative' function in the development of the tissues, has lost its experimental basis. Nevertheless, there is a parallclism between the growth of the fotus and its glycogen content. In cases where the growth of the fotus has been spontaneously arrested. the percentage amount of glycogen in its organs lias been found to be diminished out of proportion to the diminution in weight. A similar retardation of development can be produced by repeatedly injecting phloridzin into the mother, and thus reducing the glycogen store of the fotus (Lochead and Cramer). Probably, then, the fotal glycogen assists the growth of the embryo, which is known to be accompanied by an intense carbo-hydrate metabolism, by furnishing a store of easily oxidized material for the nutrition of the developing tissues. When the muscles have been formed, their glycogen is still consumed in growth, and their functional powers lic dormant, but for the infrequent and feeble movements, generally regarded as reflex, but possibly to some extent originated in the cerebral cortex. which give the mother the sensation of 'quickening.' It is only late in development that the embryonic liver takes on its glycogenic function. In the earlier stages it is entirely free from glycogen. It is an interesting illustration of that exact adaptation of means to ends which so constantly impresses the investigator of the animal mechanism that the ferment which converts glycogen into dextrose (glycogenase) is also either entirely absent from the liver early in gestation, or present only in traces; and that as the glycogen-forming and glycogen-storing functions of the organ increase in importance, it becomes richer in glycogenolytic ferment. It cannot be doubted that the glycogen found in the placenta is also deposited there in the interest of the rapilly growing fotal tissues, perhaps as a kind of current account on which they can operate at any moment of emergency, when the more distant matemal rescrves cannot be drawn upon in time. The glycogen is formed in the placenta, probably from the lextrose of the maternal blood. By means of a glycogen-splitting ferment, which can be extracted by glycerin from the placenta, the glycogen appears to be reconverted into dextrose for absorption by the foetus. In the earlier period of gestation the placenta seems to perform vicariously the glycogenic function of the liver, and as the glycogen content of the liver increases in the later stages of intrauterine life, that of the placenta diminishes proportionally.

The excretory glands of the embryo, except the liver, scarcely awaken to activity during fotal life. Urine may indeed be sometimes found in the bladder at birth, but it is often absent. It is a dilute urine, with a molecular concentration only about half as great 
as that of the blool, and although a portion of the amniotic fluid, which contains traces of urea and salts, in addition to small quantities of albumin, may be secreted by the renal tubules, and find its way through the still open urachus into the amniotic sac, this contribution cannot imply more than a slight degree of glandular action. Under certain experimental conditions, however, it can be largely increased. Thus, extirpation of the kidneys in a pregnant animal causes an incicase in the amount of amniotic fluid (hydramnios) through the stimulation of the foetal kidneys to increased activity by the passage of the uncxcreted urinary constituents of the mother's blood into that of the fatus. After the injection of pliloridzin into the fotus sugiu luas been found in abundance in the amniotic fluid, although the injection of that drug into the mother caused no such effect. On the other hanel, after injection of sodium sulphindigotate into the circulation of the fotus in the shecp, the fotal kirlneys contained particles of the pigment, while the amniotic fluid remained uncoloured. Long before full term the sebaceous glands have begun their work by the secretion of the vernix caseosa, an oily material which covers the skin and serves to protect it from the continual irritation of the fluid in which the embryo floats.

The nervous system is even less active than the glandular tissues, and not more active than the muscles. There is evidently no scope for the exercise of the special senses. Psychical activity of every kincl must be at its lowest ebb. Consciousness, if it exists at all, must be dull and muffled. And if motor impulses are discharged from the cortex, the psychical accompaniments of such discharge are doubtless widely different from those which we associate with voluntary effort.

It is a remarkable fact that this functional calm, broken only by the beat of the heart, is accompanied by a relatively intense metabolism of the same order of magnitude as that of the adult. In the hen's egg at all stages of development the consumption of oxygen and production of heat (per kilogramme and hour) are the same as in the adult hen. The oxygen consumption and carbon dioxicle production of pregnant guinea-pigs were determined before and rluring compression of the umbilical cord of a fœetus, and a distinct (liminution was observed when the respiratory exchange of the foetus was climinated. Fron the results of a number of cbservations it was calculated that the carbon dioxide produced by the mother was 462 c.c., and by the foetus 509 c.c. per kilogramme of bodyweight per hour (Bohr and Hasselbach). A similar comparison between women before and during pregnancy never showed any liminution in the respiratory exchange reckoned on the unit of bodyweight in the pregnant condition. In one case, inleed, and that the most exactly observed, there was an increase in pregnancy. Now, in the pregnant woman a considerable part of the increase of body-weight is due to the amniotic fluid, in which, of course, metabolism does not go on. It is evident, then, that in the human fœtus also the intensity of metabolism is at any rate not of a lesser order of magnitude than in the mother, in spite of the much smaller amount of muscular contraction taking place. The heat production of mot:-er and child together has been directly estimated in several cases in a respiration calorimeter provided with a bed just before parturition and just after it. After parturition the heat production of the mother was also separately determined. From the difference it was concluded that the heat production of the child per kilogramme of 
boly-weight per hour is approximately two and a half times that of the mother under the same conditions. (Carpenter and Murlin.)

The foetal heart beats at the rate of about 1 fo times a minute at full term.* The blookl-pressure in the umbilical artery of the mature cmbryo (sheep) varies from 60 to $80 \mathrm{~mm}$. of mercury ; but at the beginning of the aorta it will be more. The pressure in the pulmonary trunk must be about equal to that in the aorta, since the comparatively short and easy circuit through the. lungs does not as yet exist; and in accordance with this equality of pressure (of work to be done) is tlie equality of thickness (of working power) in the walls of the two sides of the heart

Suppose, now, that the embryo contains 60 grammes of blood for every kilo of body-weight, and that the whole of the blood passes through the circulation in twenty seconds. Then in twenty-four hours $259^{\circ} 2$ kilos of blood will be forcel through the heart for every kilo of bolly-weight against a pressure of, say, $80 \mathrm{~mm}$. of mercury, or 1 metre of blood. This is equivalent, in round numbers, to 260 kilogramme-metres of work, or $0^{\cdot} 6$ calories. Now, taking the total heat-production of the heart at three times the equivalent of its mechanical work, we get 1.8 calories per kilo of body-weight in twenty-four hours (see p. 585 ), or about $\frac{1}{20}$ of the heat-production of a resting adult.

Such movements of the skeletal muscles as occur cannot account for any large proportion of the total metabolism, since they are executed in a medium (the amniotic fluid) of nearly the same specific gravity as that of the body, and therefore require the expenditure of a very limited amount of energy. The ordinary functional activity of the cmbryo, then, is quite incapable of accounting for the intensity of the fotal metabolic processes. Still less can it be due to an active combustion in the tissues to compensate for a rapid loss of heat, for the fotus lies sheltered in the uterus as in a thermostat at its own temperature, and can lose practically no heat unless its temperature be kept a little above that of the maternal blood. The only remaining explanation of the magnitude of the fotal metabolism is that the growth processes are associated with a large amount of oxiclation (and cleavage).

Notwithstancling the intensity of metabolism in the cmbryo, not only is even the purest blood, as has already been stated, far from saturated with oxygen, but the relative proportion of hamoglobin. the oxygen-carrier, is less than in the adult; and although constantly increasing in amount from the moment of its first appearance, it is still somewhat deficient, even at full term. but leaps sharply up at birth. At an early period of development the embryo also contains much more water than the adult; the specific gravity of its tissues increases as development goes on.

The remarkable vitality of the fotus, and its resistance to asphyxia. are related not to the fecbleness of its metabolism, but to the comparatively slight cxcitability and high endurance of nervous centres

* It has not been finally determined whether the rate of the lieart varies with the size or, what probably comes to the same thing, with the sex of the fœtus. As we have seen, the variation of the rate in the adult with the size of the body is associated with a corresponding variation in the metabolism and heat-loss, which are proportionally greater in a small than in a large animal. If this is a causal connection we should not expect that in the embryo in wiero, where the conditions as regards heatloss are entirely different, such a relation should exist, at any rate within the same species. 
like the respiratory, vaso-motor, and carlio-inhibitory. Even when totally deprived of oxygen, is by pressure on the umbilical cord during delivery, the child does not perish in the two or threc minutes which clecile the fate of the aspliyxiated adult; nor are the conrulsions, rise of blood-pressure, and slowing of the heart-beat, associated with aspliyxia in the latter, so reaclily inducerl, nor premature and latal efforts at respiration casily cxcited in utero. But although in such a case the embryo belaves as a separate nrganism, governerl by its own laws, there are circumstances in which it becomes mercly a part of the mother and participates in her fate. Thus, the stream of oxygen which normally passes from the maternal to the foetal blood is turned back if asphyxia threatens the mother: the blool of the nmbilical arteries, instead of being purifiel in the placenta, loses the little oxygen it holds to the blood of the uterine sinuses, and the tissues of the embryo are impoverished to support the metabolism of the maternal organs. In the same way, the phenomena of starvation have taught us that the mutrition of the organism is not subject to the rules of red tape. In normal circumstances the flow of nutriment follows definite lines: the blood feeds the tissues through its intermediary, the lymph, and recoups itself from the contents of the alimentary canal. But when the normal sources of nutrient material fail, the body falls back upon its stores. The organs immediately necessary to life are kept, as far as possible, on full diet ; organs of secondary importance have to be content with half-rations; organs less important still are drawn upon for supplies.

Parturition.- The pcriod of gestation is abruptly closed about 2 So days after the last menstruation, usually in what would have been the tenth intermenstrual period had menstruation been occurring. There is necessarily a considerable variation in the time when reckoned in this way, since the cessation of the menses merely announces that conception has occurred some time after the last period. It may even be disputed whether the fertilized ovum corresponds to the last menstruation or to the first absent period. Parturition, or the expulsion of the fotus, is accomplished by periodical contractions, the 'pains' of labour, at first confined to the nterus. Soon the os uteri begins to soften and dilate, the walls of the vagina become congested, and its secretions are augmented. The uterine contractions increase in frequency and force, and are now accompanied by reflex contractions of the abdominal muscles, and, if the woman is not anzesthetized, also by voluntary contractions of these and of other muscles, which can increase the intra-abdominal pressure. The uterine contractions can be initiated and modified by impulses coming from the central nervous system by way of the extrinsic nerves of the organ. It is known, e.g.. that the gravid uterus can be excited to contraction by the stimulation of various sensory nerves. Powerful mental impressions, such as fright, may bring on premature labour. Conversely, sudden cessation of labour pains during parturition is not uncommonly observed to be produced by emotional disturbances-for instance, the entrance of a stranger into the room. Yet the contractions of the uterus are not essentially dependent upon extrinsic impulses. For not only do rhythmical contractions occur, but the whole process of parturition has been seen to take place in a uterus whose nerves have all been cut. Even the excised uterus may be kept alive for as long as forty-eight hours, and may go on executing 
periodical contractions when its bloolvessels are perfused with such an artificial fluid as Locke's solution, or, indecl, when it is simply immersed in the oxygenated solution (Kurdinowski) (Practical Exercises, p. 1025).

It is a question of great interest how the uterine contractions are started so abruptly at full term after so long a periol of quicscence. It can lardly be that the increasing mechanical distension of the uterus, tolerated for so many months, should suddenly, in an hour, become intolerable. For if the foetus dies before full term it is expelled without reference to the bulk which the werus has reached. It is more likely that some chemical change associated with the completion of intra-uterine development, a change which leads, perhaps, to the production of some specific substance in the placenta or the foctus, is the letermining event. The placenta is a structure whose function is strictly limited to the term of intra-uterine development. The foetus is to live on, and so is the mother. May it not be that the placenta or essential elements in it are timed to die, or to begin to die, at full term, and that in their death or degencration the substance or substances are produced which start, and later sustain, the uterine contractions? And may not the contractions of the uterus, by exciting its afferent nerves, or through the pressure of the foetus the afferent nerves of the vagina, in turn croke the associated reflex contractions of the abdominal walls? These are questions which have been asked, but not as yet satisfactorily answered.

At birth, great changes take place in the foetal circulation, and these are intimatcly connected with the commencement of the respiratory activity of the lungs. The causes of the first respiration are : (I) The increasing venosity of the blood circulating in the bulb, which stimulates the respiratory centre when the umbilical cord has been cut or tied and the placental circulation thus interfered with; (2) the stimulation of the skin by the air, which, as we have seen, acts reflexly upon the respiratory centre. That both of these factors may be involved is shown by the fact that cither compression of the umbilical cord alone, or exposure of the fotus by opening the uterus of an animal without interference with the circulation, has been observed to be followed by attempts at breathing. Once distended, the lungs never again completely collapse-not even after death, nor when the chest is opened. The aspiration caused by the clevation of the chest-walls in inspiration (for the respiration of the newborn child is mainly costal) suclis blood into the thorax, and cxpands the vessels of the lungs for its reception ; and in the measure in which the blood passing through the pulmonary trunk finds an easy way through the lungs, the quantity which takes the route of the ductus arteriosus diminishes. The pulmonary veins, and consequently the left auricle, are better filled; and the increasing pressure on this side of the septum tends to oppose the passage of the blood through the foramen ovale, to approximate its valve, and to close its orifice.

By the second or third day the ductus arteriosus has usually become obliterated. The umbilical arteries and veins and the ductus venosus become impervious soon after the interruption of the placental circulation. The vein and venous luct remain in the adult as the round ligament of the liver, the arteries as the lateral ligaments of the bladder.

Although from birth onwards the young manmal obtains its oxygen and gets rid of its carbon dioxide through its own pulmonary surface instead of through the placenta, it still lives, as regards its 
fond proper, on the lissues of the mother, and that in as literal a sense as when it drew its supplies directly from the maternal blood.

Milk. - The milk secreted luring the first few days of each lactiltion, the colostrum, as it is called, indeed maly represent in part the fragnents of cells lining the alveoli of the mammary glands, which have undergone a fatty cliange and been bodily broken down. The colostrum corpuscles are leucocytes filled with fat globules taken "up from the contents of the alveoli. The chief chemical difference between colostrum and ordinary milk is the greater richness of the former in protein. It has been supposed that it is of special importance for the nutrition of the suckling, perhaps in virtue of the enzimes containel in it, and it is said that young animals bear artificial feeding much batter if they have been allowed to suckle the mother for the colostrum. In addition to the fat, which when milk is allowerl to stand rises to the top as cream, milk contains a considerable quantity of caseinogen, to whose coagulation, under the influence of the lactic acil produced from the lactose, or milk-sugar, by certain bacteria, spontancouscurdling is due. Another protein, lact-albumin (Halliburton), a large amount of water, and some inorganic salts, are the most important of its remaining constituents. The molecular concentration $(1,308)$ of milk, as measured by its freezing-point, is almost cxactly the same as that of blood-serum. Its electrical conductivity varies extremely, since it depends on the quantity of fat present, the fat globules, like the blood-corpuscles, being practically non-conductors.

The inorganic composition of milk is particularly interesting when compared with that of the blood on the one hand and that of the suckling on the other. Thus, Ioo grammes of ash from each source gave the following values for the rabbit (Abderhalden) :

\begin{tabular}{|c|c|c|c|c|c|}
\hline & & $\begin{array}{l}\text { Rabbi's (14 Days } \\
\text { old). }\end{array}$ & Rabbit's Milk. & Rabbit's Blood. & $\begin{array}{l}\text { Rabbit's Blood } \\
\text { serum. }\end{array}$ \\
\hline $\mathrm{K}_{2} \mathrm{O}$ & . & 10.84 & 10.06 & 2375 & 3.19 \\
\hline $\mathrm{Na}_{2} \mathrm{O}$ & .. & 5.96 & 7.92 & 3138 & 54.72 \\
\hline $\mathrm{CaO}$ & . & $35 \circ 02$ & $35 \cdot 65$ & 0.81 & 142 \\
\hline $\mathrm{MlgO}$ & .. & $2 \cdot 19$ & $2 \cdot 20$ & $0 \cdot 64$ & 0.56 \\
\hline $\mathrm{Fe}_{2} \mathrm{O}_{3}$ & .. & 0.23 & 0.08 & 6.93 & 0.00 \\
\hline $\mathrm{P}_{2} \mathrm{O}_{5}$ & . & +1.94 & $39 \cdot 86$ & $11^{\circ} 11$ & $2 \cdot 98$ \\
\hline $\mathrm{Cl} \ldots$ & .. & $4^{\circ} 94$ & $5^{\circ} 4^{2}$ & $32 \cdot 66$ & $47 \cdot 83$ \\
\hline
\end{tabular}

The richness of the milk (and of the suckling) in calcium, phosphorus. and magnesium, as compared with the serum, is to be especially remarked. This is, of course, essential for the development of the bones. Whereas sodium predominates greatly over potassium in the scrum, the opposite is the case in the nilk (and the suckling). This is connected with the development of the tissue cells, which are richer in potassium than in sodium. The high chlorine content of the serum is in sharp contrast with the relative poverty of the milk in that clement, which preponderates in the tissue liquids and is relatively scanty in the cells.

In addition to substances susceptible of chemical analysis, milk contains enzymes like those present in blood-serum, including oxydases and various hydrolytic ferments (proteolytic, diastatic, and perhaps lipolytic). It is now universally acknowledged that 
motlier's milk is incomparably superior for the feeding of the intant to any artificial substitute, and one factor in this superiority nay be the presence of ferments specifically arlapted for the cligestion of the luman suckling. Jore important is the practical sterility of the human milk and the necessarily finer alaptation of its quantitative and gualitative composition, particularly the closer relationship of its proteins with those of the child. In adclition, there is some evidence that the maternat milk contains immune bodies (antibodies) which may increase tlıc resistance of the suckling to infections.

However this ma. be, there is no question that much of the high infant mortality associated with the industrial conclitions of our great cities could be prevented if breast-feeding were carriel out by crery mother physically capable of it.

As to the manner in which milk is szcretal, thore is no doubt that its chief constituents are formel in the gland-cells. Caseinogen and litctose do not exist in the blood or lymph. The former is probably produccl by an alteration in one or other of the serum protems, the latter by a change in the elcxtrose of the blood. The fat of the milk nuav come partly from the fat of the blook, but it may also be formed in the gland-cells from proteins and carbohyrirates. The precise manner in which the fat globules are cxtruded from the cells into the lumen of the alveoli is not clear, but there is no good ground for belicving that the cells or their free cnds break up bodily in the process.

Little is known as to the influence of the nervous system on the secretion of milk, and no definite secretory fibres have as yet been clearly demonstrated, although the fact that marked changes may be produced in the milk of nursing women as the result of cmotional disturbances indicates that such nerves do exist.

Pregnancy is accompanied with vascular clilatation and hyper. trophy of the mammary glands, but the mechanism by which these changes are produced is unknown. It is probable that they depend upon some internal secretion of the ovary or some other of the organs of reproduction. Pregnancy is not an absolutely inclispensable condition, and therefore it would seem that the cxciting substance, if any spccific substance cxists, is not a product of the foetus or of the placenta. Precisely similar plienomena arc occasionally seen in animals which have not been impregnated and even in men. Humboldt relates the casz of an Indian father. who so well understood the responsibilities of paternity, and was so capable of fulfilling them, that he suckled his child for five months on the death of the mother. Virgin bitclies are frequently known to produce milk, occasionally cven in quantity sufficient to rear pups. the flow occurring about the timc when they would have whelped harl they conceived (luring the previous ostrus (period of heat). Bitches which after copulation have 'missed' having pups have becn known to preduce so mucl milk, beginning at the time they were duc to whelp, that they were able to rcar litters of puppies belonging to other bitches. Inles, which are themselves sterile, may have enough milk to suckle a foal. The nipples of certain monkeys becom? swollen and congested at each menstruation (Heape), and in women som 2 development of the mammary glands is often associated with the menstrual period. The stimulus to the development of the gland in these cases appears to be some change corrclated with ostrus, and cannot be a change correlated with pregnancy. 
Transplantation of Tissues. - Besides the growtl and regeneration of tissues or organs, the simple displacement of them from their normal situation and their implantation in a ncw environment have been studied. Normitly', a migration of tissue clements is only witnessed in the alult in the casc of cells moving with the circulating liquids, or endowed with the power of amœboid movement. Under pathological conclitions fragnents of tissuc, such as tumour cells, may be carried by the blood or lymph to distant parts, and, settling there, may undergo development (forming metastases). In the cmbryo the slow migration of tissue elements is a process which is responsible for sone of the anatomical peculiaritics of the adult. The migration of the orum from the ovary is the starting-point of the process of reproduction. The artificial clisplacement of tissues within the body of one and the same animal (auto-or homo-trans-

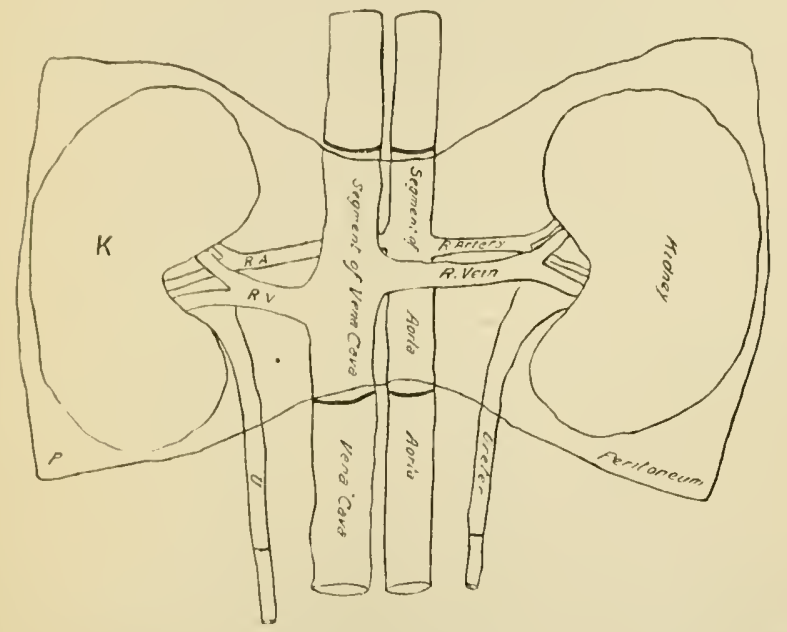

Fig. 4f6.-Method of Transplantation (of both Ridneys) in Mass. (After Guthrie.)

Segments of the inferior vena cava and abdominal aorta are removed with the kidney's and renal vessels, and interposed in the course of the vena cava and aorta of another animal, according to the method of Carrel and Guthrie.

plantation, or graft), or from onc animal to another of the same species (iso-transplantation, or graft) has been successfully accomplished in many cases. But hetero-transplantation, or grafting between animals of different species, is in general not permanently successful, the graft undergoing cytolysis (p. 29) in the alien environment.

Transplantation, or engrafting, may be done either with or without anastomosis of bloodvessels. In the second method a portion of tissue, usually small, or a small organ, is simply inserted in its new situation without provision for the immediate establishment of a circulation in it. Strips of cuticle may casily be grafted in this way to restore deficiencics in the skin after burns or extensive operations. The ovary can also be grafted by simple implantation with success. Guthrie has thus shown that hens whose ovaries have been 
interchangerl are capable of laying eggs. When the hens were impregnated and the eggs hatched out the colour characters of the resulting offspring seemed to have been influenced, not only by the hen to which the ovary originally belonged, but also by the hen to which it had been transferred. Grafts of the thyroid and parathyroid have also been shown to 'take.'

In transplantation with anastomosis of bloorlvessels the main vessels of the engrafted organ are sutured to suitable arteries and veins in the 'host,' so that the cireulation is at once effective. Consequently there is practically no limit to the size of the grafts. The kidncy, spleen, and even a limb, have been successfully transplanted in this way from one dog to another. Segments of arteries preserver in cold storage for a few days or even weeks, and even portions of arteries fixed by formaldehyde, have been transplanted so as to take the place of segments removed from arteries of living animals, and have continued to function perfectly for long periols. Portions of veins have also been used to fill up gaps in arteries. Even heteroplastic vascular grafts have been found to succeed, portions of dog's

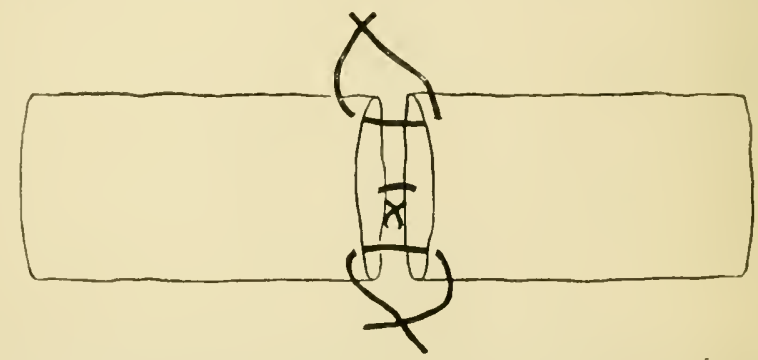

Fig. 447.-Suturini; Bloodiessels. Preliminary Fixition of Exds of Divided Vessels (After Guturie).

Three fixing ligatures are placed at equidistant points on the circumference of the cut ends, each ligature being passed through corresponding points of the two vessels. The ends of the ressels are approximated by drawing on the ligatures, which are then tied, and the margins of the vessels sewed together by continuous stitches in the intervals between the fixing ligatures, as in Fig. 449. (Carrel's inethod.)

arteries, e.g., grafted into a cat, and portions of rabbit's, cat's, or human arteries grafted into a dog. Doubtless the favourable result is largely due to the fact that the function of the large arteries is mainly a passive, mechanical one, which can be discharged even by a dead tube of the requisite strength, and with the smooth interior presented by a dead endothelial lining (Carrel, Guthrie).

Parabiosis. - Not only may an organ or a portion of tissue from one individual be engrafted on another, but two indivicluals may be so united that a greater or smaller degree of physiological intimacy is produced between them. Occasionally, as in the famous Siamese twins, an anomaly of development results in such close anatomical union of the circulatory and other systems that in certain respects the two individuals constitute almost a single organism, and cannot be separated by surgical interference. A less intimate union can be established experimentally by opening the peritoneal cavities of the two animals, and suturing the skin and connective tissuc together so as to permit of permanent communication. Pairs of 
animals living in this condition (so-called parabiosis) have bəen utilized for the study of certain questions in immunity. White rats have been kept alive in parabiosis for as long as thirtv-four clays in order to test the question whether destructive antiborlies for cancer are present in the circulation (Rous), since it has been shown that circulating antibodies easily pass from one to the other of such a

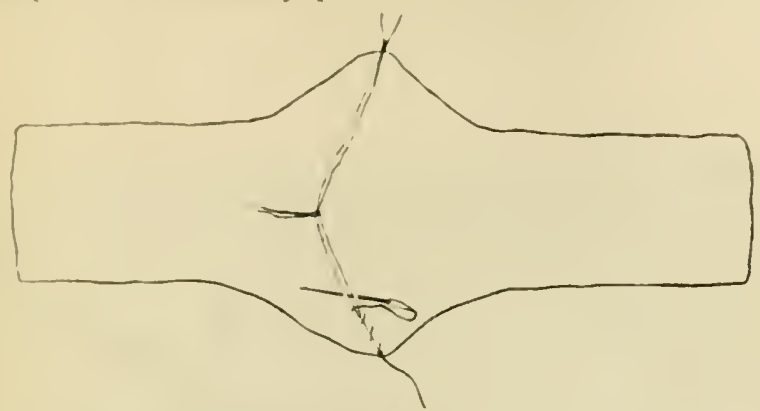

Fig. 44. -SLturing Bloodvessels. Method of Approximating Edges and putting in Continuous Surure (AFter Guthrie).

The needles are very fine cambric sewing-needles, and the threads single strands of Chinese twist silk or human hair. Needles and threads are sterilized in parattin-oil. (Method of Carrel and Guthrie.)

pair of animals (Ehrlich). One of each pair of rats had a growing tumour produced by transplantation, while the other had been proved resistant to the same type of tumour. No evidence of the passage of an antibody was found in this casc.

\section{PRACTICAL EXERCISE.}

Contractions of Isolated Uterine Rings.-Kill a female aduit rabbit by striking it at the back of the neck. A rabbit which is not pregnant, or only at the beginning of pregnancy, should be sclected. Open the abdomen, and carefully remove the uterus. While separating the organ from the broad ligament and ragina, support the horns of the uterus on soft threads. Ligature the vaguma before cutting through it, and cut below the ligature, which can then be used to manipulate the uterus. Do not pinch the uterus with forceps, and handle it as little as possible. At once place it in Ringer's solution (p. I86), kept at body temperature $\left(3 \mathrm{~S}^{\circ} \mathrm{C}\right.$.) in a small beaker immersed in a water-bath. Cut a ring of tissue about I $\frac{1}{2}$ centimetres in width from one of the horns. Tie a loop with a fine silk thread at each end of a diamster of the ring, pinching up a little of the cxternal coat to do so with fine forceps. Nake the arrangements nccessary for recording contractions of the ring while it is immersed in a very small beaker or a glass cylinder in the bath, as in Experiment 12, p. I $8_{5}$, but do not divide the ring. A narrow glass tube connected by a rubber tube with a cylinder of oxygen must be arranged to dip down to near the bottom of the beaker. The valve of the cylinder is turned cautiously, so as to permit oxygen to bubble slowly through the solution. After a longer or shorter interval spontancous rhythmical contractions of the uterus ring 
commence. As soon as they are well established, and while the contractions are being recorded on a very slow clrum, adrenalin solution should be rum into the beaker from a graduated capillary pipette in such amount as will make the concentration of it in the beaker $I$ : $1,000,000$. Run the adrenalin solution in at a point as far as possible from the uterus ring, so that it does not reach it till mixture has occurred. Nix carefully with a thin glass rod without disturbing the preparation. Note whether the tone of the ring (as shown by its permanent shortening) or the rate and strength of the contractions are increased. If not, remove the solution from

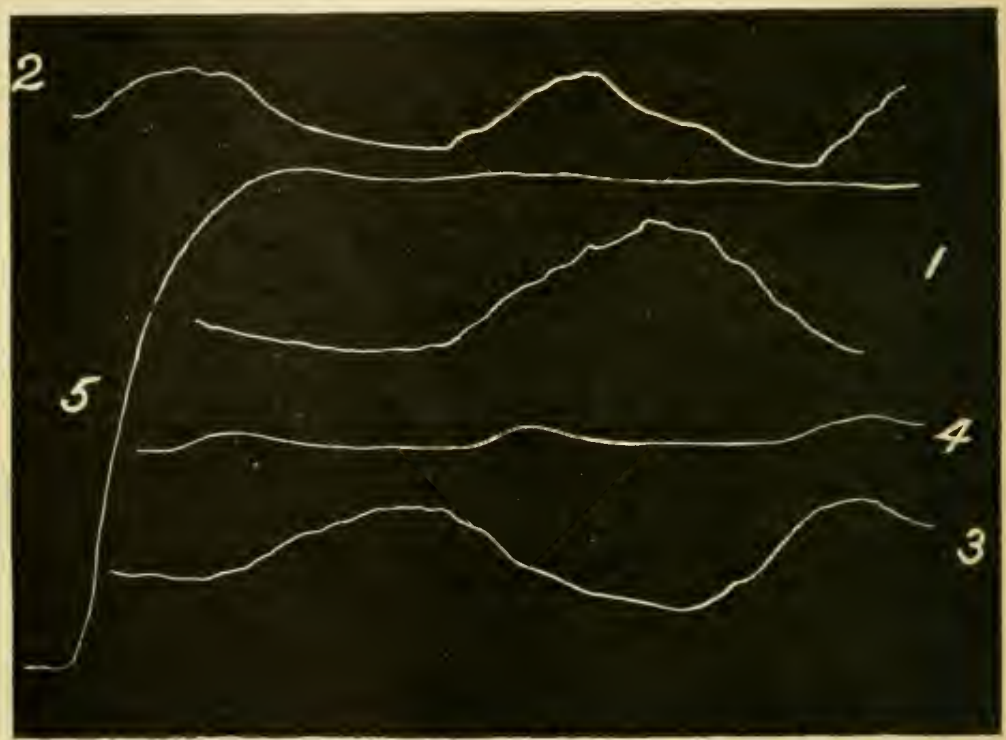

Fig. 4.49.-Contractions of RabBit's Uterl's Ring.

I, Spontaneous contractions in Ringer's solution; 2, contractions in human serum from a case of persistent high arterial pressure; 3 , contractions in the same serum diluted with its own volume of Ringer's solution; 4, contractions in Ringer's solution after washing away the serum. Tracings $I$ to 4 were obtained from the same ring ; 5 , increase of tone produced in another ring by the addition of a little adrenalin solution to the Ringer's solution, in which the ring had been executing spontaneous contractions. These ceased for a time after addition of the adrenalin, but later recommenced.

the beaker with a pipette or siphon, replace it by fresh warm Ringer's solution, and start the oxygen current again. While a tracing is being taken repeat the observation, adding a larger proportion of adrenalin. Determine in what concentration a distinct effect is produced. This is the basis of a method saicl to be capable of detecting such minute quantities of adrenalin as can be supposed to be present in blood-serum (Fraenkel). A sufficient number of uterus rings can be obtained from one animal for a considerable number of experiments. 


\section{A P P E N D I X}

CUMPARISON OF METRICAL WITH ENGLISH MEASURES

\section{Measures of Length.}

1 millimetre $=0.03937$ inch.

I centimetre $=0.3937 \mathrm{I}$

I decimetre $=3.93708$ inches.

I inetre $=39.37079$,.

I inch $=25.3995$ millimetres.

Measures of Weight.

I gramme $=\mathbf{I} 5+323+9$ grains.

I kilogramme $=2 \cdot 2046213$ pounds.

I ounce $\quad=28.3495$ grammes.

I pound $=453^{\circ} 5926$,".

Measures of Volume.

I cubic centimetre $=0.061027$ cubic inch.

I litre ( $\mathrm{I}, 000$ cubic centimetres $)=6 \mathrm{I}^{\circ} 027052$ cubic inches.

$$
\begin{aligned}
& =\mathrm{I}^{\prime} 760773 \text { English or } 2^{\circ} \mathrm{II} \\
& \text { American pints. } \\
& =0.22009668 \text { gallon. }
\end{aligned}
$$

I cubic inch $=\mathbf{I} 6 \cdot 3861759$ cubic centimetres.

I cubic foot $=28.31531$ I9 cubic decimetres (or litres).

1 pint $=0.567932$ litre.

I gallon $=45434579$ litres.

\section{Measures of W'ork.}

I kilogrammetre =about $7 \cdot 24$ foot-pounds.

I foot-pound $\quad=0$ I3 38 I kilogrammetre.

I (kilo)calorie of heat $=425^{\circ} 5$ kilogrammetres of work.

Temperature Scales. - To convert degrees Fahrenheit into degrees Centigrade, subtract 32 , and multiply the remainder by 5. To convert degrees $\mathrm{C}$. into degrees $\mathrm{F}$., multiply by result. 



\section{INDEX}

** References to the Practical Exercises are in black figures.

ABDOMINAL breathing, 2 I 4

luuscles in expiration, 2 I 3

Abelicens or sixth nerve, 822 inhibition by, $83^{8}$

Aberration, chromatic, 913, 992 spherical, 912,99 I

Absint he convuisions, 857

Absurption, 398 from the peritoneal cavity, 406 from the stomach, 39I, f03, 433 in different animals, 402 intrit-and inter-epithelial, 4 I 7 of bile-constituents in jaundice. 355

of cane-sugar, $405,416,433$

of carbo-hydrates, +16

of fat. $+12.432,433$

of light, 897

of proteins, 418

of the fond. for

physical introduction to, $39^{8}$ theories of, $404-406$

of water and salts, 395, 417 parenteral, 4 I 8

Acapnia and blood-pressure, 168 and inountain sickness, 275 and shock, 175

Acceleration of heart by sipping water, 155,195

Accelerator nerves of heart, 143,147 , I 84

Accessory auditory nucleus, 824 vagus nucleus, 825

Accommodation, $905,987,988$ mechanism of, 907 pupil in, 909

A.C.E. mixture, 55,47 I

Acerebral tonus, 836,847

Aceto-acetic acid in diabetes, 520

Acetone in diabetes, 520, 492

Acid albumin, 2, 9, 325, 426

Acidity of gastric juice, $323,390,39$ I

Acidosis, 521

Acrolein, 12

Acromegaly and pituitary, 568
Action currents, $718,719,720,726$, 739

diphasic, 720

double conduction of, 688

electromotive force of, 722

in polarized nerves, 727

monophasic, 722

of eye. 735

of glands, 734

of heart, $78,720,730,733,740$

of human muscles, 662,724

of phrenic nerves, 724

of spinal cord, $688,733,746,793$

of vagus, 225

of veratrinized muscles, 726

reflex, 809

theories of, 725

velocity of propagation of variation, 723

Adaptation of digestive juices to food, $340,369,376,381,387$

of retina, 935,946

Adenase, 507

Adenin, 4tI, 507

Adequate stimuli, $798,870,891$

Adipocere, 527

Adrenal glands. See Suprarenal cap. sules

Adrenalin, I 57, 20I, 564 action of, on heart, I 56, 564 on pupil, 9 I 1,9 I 2 on uterus, 563 , 1025

on vasomotors, I57, I 63,201 , 563

artificial, 565

glycosuria, 522, 566

Aerotonometer, 256

Esthesiometer compasses, I000 hair, 970, 999

Afferent impulses, decussation of, 792 paths of, $779,791,794$

After-images, 943,997

Agglutination, 29, 63

Agglutininogens, 30

Agraphia, 862 
Alanin, 2, 332

formation of dextrose from, 5 I.

Albinus, intravascular clotting in, 38

Albumius, 2, 8

heat-coagulation of, 8

in urine, $443,451,4(10,462,486$ 488

Albuninates or derived albumins, 9

Albuminoids, 2. 529

Albuminous glands, 3 I0

Albumoses, 3

artion of, on blond-pressure, 157. $20 I$

un coagulation, $35,40,55$

in peptic digestion, 325

tests for, 10, 426

in urinte, 486

Alcohol, action of, on respiratory centre, 175,236

on gastric secretion, 388

in diet, 551

poisoning, blond-pressure curve in, I 75

precipitation of proteins by, 8

Alcohols, relation of, to rarbuhydrates, 3

Aldehydase, 265

Aldehyde groups in living protein, 499

Aldehydes, relation of, to carbohydrates, 3

Alexins, 53

Algometer, 979

Alimentary canal, auatomy of, 207 length nf, 297

time of passage through, 31 I glycusuria, 5i 6,610

Alkali-albumin, 9, 333, 429

Alkalinity of blood. ete., titratable, 2.1

Alkaptonuria, 444

Allantois, formation of, ror 1

'All or nothing ' law, I 4 I

Alloxurir bodies. $44 \mathrm{I}, 507$

Amblyopia after uccipital lesion. 859

Ambocepturs, 27, 63

Anide-nitrogen in proteulysis, 332

Amino-acetic acid, 2

Amino-acids, $1,325,332$

absorption of, 420

ronversion of, into glycogen and dextrose, 514

formation of urea from, 501. 503. 505,536

in liver diseases, 503

in phosphorirs-poisoning. 526

in irine, $44 \mathrm{r}, 450$

reaction for, +50

Amino-valerianic acid, 3.32

Ammonia, action of, on muscle and nerve, 633,659

inspermeability of lungs for, 242

in protenlysis, 332
Ammonia in urine, $44^{\circ}$

after Eck's fistula, 502

reflex inhibition of heart by, 154 , I95

Ammonium salts, formation of ureal from, 501, 50.4, 505

sulphate, precipitation of proteins by, 8, 486

Amnion, Ior I

Amniotic fluid, 1011, 1012, 1017

Amoeba, 6, 14, 359, 627

Amoboid movement, I7, I 8, 53, 627

Ampère, 616

Anylase, 32 I

Anvl nitrite, action on pulse, 97, 192 formation of methemriglobin by, $4^{6}$

Amylolytic stage of gastric cligestion, $322,390,403$

Amylopsin, 33I, 333, 429 influence of bile on, 340

Analolic changes in living matter, 6

Anacrotic pulse, 97

Anasthesia by A.C.li. mixture, 55

l)y chloral, 204

1) y cliloroform (Grithant's method), I 86

l) y inorphia, 55, I86

l) pressure on brain, 875

Anelectrotonus, 683,742

Angular gyrus and vision, 859

Animal heat, 572

Anions, tor

Ankle-elonus, 8 I I 8 I $3_{3}$

Innulus of Vieussens, I43, $1+7,162$, 163,190

Anode, 401,615

Anteriur conmissure, 8 I 7

horn, cells of, 748,762

connections of, 775

roots, 775

Antern-lateral ascending trart, 764. 772. 781

connections of, 773

deseending tract, 765,776

connections of. 776

ground bundle, 765

Anti-bodies, 30,3 I 8

'Antidromic 'nerve-impulses, 165,685 708

Antifrements, $318,36 \mathrm{r}$

Antigens, 30

Antikinase, $36,39,361$

Antilytic secretion, 360

Antimony and protein metabolisin, 526

Antiperistalsis, 308,394

Antipyretics. 507

Antiseptirs for operations, 202

Antithrombin, $35,36,40,4$ I

Antitrypsin. 318,361

Autrum pyluri, 304, 305 
Aurta, effect of compression of, 188

Aortic insutficience, effret of, on pulse, 98

uotch, So, 96

struosis, "flect uf, on pulse, os

villors, 7(), S(1), I1) I and dierotic ware, no

Apex-lusit, 52, I91, 193

Ap"x preparation of lieart, 131,178

Aphasia, untutor, snz

seusory, $\$ 6.4$

temporary, 86.4

Wernicke's, 86.4

Aphemia, 86,4

Apnxa, 23r, 238,288

vagi, 232

vera, 232

Apocodeine, action of, nil vaso-motors, 157

A pumorpline as cmetic, 3 I 3. 427, 432

Aepueduct of Sylvius. Bing

Aqueous humour. gor

Arachnoid, $75^{8}$

Araclinulysin, action of, on erythro. cytes, 27

Arcuate fibres, intermal, 772

Arginase, 503

Arginin, 33 I, 332, 503

Argvll-Robertson pupil, 9 Io

Arhythmia, respiratory, 270

Aromatic sulphates in urine, +45.479

Arsenic and protein netabolism, 526

Arteries, structure of, 74

to insert cannula into, 55

tolle of. $169,8{ }_{3} 3$

Arterioles, resistance in, I I 0 , I 9

Arteriosclerosis, velocity of pulse in, 99

Articulation, positions of, 283

Artificial respiration, 187,214 with oxygen, I 89

Ascending degeneration, 763

Aspartic acid, 332 formation of dextrose from, $+x_{4}$

Asphyia, 23I, 269, 274

condition of hamoglobin in, 44

effect of, on circulation, I 7 I, I 72 , 189. 198

glycosuria caused by, 5 I 8

in the foetus. Io I 9

Association fibres, $761,783.785$ centres, $855,865,866$

Astatic system of magnets, 6 I0

Asthma, spasmodic, and bronchial muscles, 238

Astignatism, irregular, 9 I 4 regular, 917,989

Astrospheres, roo6

Atelectasis, 222

Atheroma, effect on pilse, 97,98

Atrio-ventricular bundle. See Auriculo-ventricular bundle
Atropine, action of, on lieart, 149,183

wn digestive secretions, 337

oli pupil, 9 I I

(un salivary secretion, 363. 367,425

Aetriction sphere, 5, Iool

in nerve-cells, $74^{8}$

Auditory reutre, 824,860

nerve, 823,828

vestibular branch of, 824

ossicles, 954, 955, 959

path, scheme of, 823

Auerbach's plexus, 298, 307, 308

Augmentation of heart-beat, I $+3, \quad I+5$, I 48, I 56, I 84

nature of, 15 I

primary, $\mathbf{I} 45$

secondary, $I+5, I+7$

Alugmentor nerves, effect of, on

quicscent lieart, I 52

Aura, 865

Auricular canal, 72, 73

pressure curve, go

Auriculs-ventricular bundle, 73. I34I 37

pulse tracings in disease of, 137

duriculo-ventricular junction, stimula. tion of, 183

node. 73

valves. 80, 190

moment of opening of, 89

Auscultation of heart-sounds, I9 of breath-sounds, 29 I

Auto-digestion of stomach, 360,434

Autogenetic theory of nerve regenera. tion, 697

Autolysis, 509

Automatic actions of spinal cord, 812$8 \mathrm{I}+$

Autonomic nervous system, $790,88_{3}$. 884

Avalanche theory, 682

Axial strand fibrils, 696

Axis-cylinder or axon, $677,748,749$, 755

bifurcation of, 697

fibrils in, $7+8$

Axoll-reflexes, $372,697,809$

Babinski's sign, 8 I I

Bacteria in freces, 397

in intestine. 3 $28,393,394,395$

Bactericidal action of gastric juice, $32 \mathrm{~S}$ ferments, 5 ro

Balloon ascents, deaths in, 275

Banting cure for obesity, 534

Baryta, absorption of carbon dioxide by, 24 I

Basal ganglia, 777,828

Basilar membrane, 956, 958, 963

Bat's wing, contractile vessels of, 73 , I 6 
Batteries. I 82, 615

Beats, 999

Beaunont (s) digestion, 323

Bechterew's nucleus, 82.4

Beckmanu's apparatus, 399,492

Bellon's recorder, 290

Bell's experiments on nerve-roots, 790

Benzoic acid, 44I, 508

Bert on double condurtion in nerves, 688

on effects of oxygen at high pressure, 274

Betz rells in the cortex, 774.852

Bichromate cell, 182

13idder's ganglia, I 29

Bile, $33 t-341,430$

absorption uf, $35.5 \cdot 383$

acids. $33^{6}, 430$

formation of, 355

Hay's test for, 430,49 I

adaptation of, to food, 3 \&6

as an excretion, 396

circulation of, 383

romposition of, 335

curve of secretion of, $3 \delta_{3}, 3 \delta_{5}$

digestive action of, $34 \mathrm{I}$

freezing-point of, $35^{8}$

gases of, 260,337

in emulsification of fats, $33^{8}$

influence of nerves on serretion of,

$3^{8} 3$

mucin, 335.430

pigments, $335,355,43$ I

relation of, to spleen, 57 I

precipitation of gastric digest by, $3+1$

quantity of, $33^{8}$

rate of flow of, into gut, 385.392

reactions of, 430

salts, artion of, on blond. 27.62

decomposition of, 337

secretory pressure of, 386

spectrum of, 336

Biliary fistula, 339,383

Bioplasm. See Protoplasm and Living matter

Bipolar ganglion cells, 747,753

Bird's blond, coagulation of, 34.39

Biuret reartion, 7,426

Bladder, +72

Blastoderm, roos

Blind spot, 931, 992

Blnod, carbon dioxide in, 24 coagulation of, $30.36,54$ composition of, 4 I conductivity of, 25,60 distribution of, $46.49,172$ flow through organs, 173 freezing-point of, 26,358 infuence of carbon diuxide on, 255
Blood, functions of, 5 I

gases of, 246

analysis of, $25 \mathrm{r}$

distribution of, 252

in embryo, 1013, 1017

tension of, 256

guaiacum test for, 68

kinetic and pritential energy of circulating, I I

laking of, 26,6 I

opacity of, $26,6 \mathrm{I}$

oxygen dissociation curve of, 255

precipitin test for, 30

quantity of, $47,+8$

in lungs, 208

reaction of, $23,54,255$

regeneration of, 2 I

specific gravity of, 25,54

stains, examination of, 71

sugar in, 4I, 462, $5 \mathrm{II}, 516,5 \mathrm{In}$

velucity of, Io8.I I 9

in arteries, 116

in capillaries, 77, 119,177

in veins, 122

measurement of, I I I - I I 3, 204

viscosity of, 22,464

volume of corpuscles and plasma. 26,59

why it does not clot in the vessels, 40

Blood-corpuscles, coloured, 15

composition of, 42

crenation of, $I 6$

destruction of. 21,29

enumeratiou of, r 8,58

formation of, in embryo, 20

gaseous metabolism of, 252

life-history of, I9

osmotic resistance of, 64

potassium in, 255

rouleaux formation of, I 6

shadows or ghosts of, 6 I

size of, I 5

structure of, I 5

white, I 6

enumeration of, Io

Blood-plates, 18

in coagulation, 36

Blood-pressure and acapnia, I 68

curves with elastic manometers, 85,102

with mercurial manometer. IOI, IO3, I 95

effect of extracts of bone-marrow on, 570

of kidney on, 570

of nervous tissue on, 569

uf pituitary on, 568

of suprarenal on, 201, 563

of testicle on, 557

of thymus on, $55^{8}$ 
Bloud-pressure, effect of freezing the cord un, 168

of hiemorrhage on, 174,200

of muscular ixercise on, Io6, I 98

of peptolle oll, I 57,201

of posture o11, 106. I 73, I99

of transfusion on, 174, 200

fictors which maintain, ro7

firll of, in sleep, 876

hydrostatic and hydrodymamic factors ill, 173

in capillaries, I 8,120

in pulmonary artery, I 08

in right and left ventricles, 85, Io8, I 27

nlean arterial, 100-I07

ineasurennent of, Ioo, 195

in nian, 104, 173, 198

permanent element in, IO3

respiratory variations in, 104. 265272

systolic and diastolic, ro5, 106, I98

Blood-pump, 250

Blood-serum, freezing-point of, 357

Blood-supply, regulation of, 172

Bloodvessels, anastomosis of, 1024

rhythmically contractile, 73,160 , I 64

structure of, 74

tone of, I 69,886

Body, composition of, 529

Bohr, on tension of blood-gases, 256

Bone-marrow and blood-formation, o, 22

action of extracts of, 570

Bones, composition of, 529

effect of deficiency of lime salts on, 542

of various salts on, $5+2$

influence of pituitary on, 569

of testicles on, 557

'Boot ' electrodes, 739

Brain, anæmia of, 876

chemistry of, $880,88 \mathrm{r}$

circulation in, 878

condition of, isolated, 867

in sleep, 874

development of, 746

functions of, $827-878$

heat-production in, 586

respiratory changes in volume of, $27 \mathrm{I}$

resuscitation of, 879,880

size of, at different ages, 878 and intelligence, 878

Bread, chemistry of, 612

Break-contraction, Tigerstedt's theory of, 727

Breast-feeding, superiority of, 1022

Breath, holding, 2 I 9
Brora's area. $855,863,866$

aphasia, 862,864

Bromine, reflex inhibition by inhala. tion of, 235

Bronchi, 207

movements of, in respiration, 2 I 6 nerves of, 237

13ronchial breathing, 2I6, 291

Brown-Séquard's syndrome, 793

Brunner's glands, $298,343,345,354$

'Buffy' coat, 29

Bulbus arteriosus, 72

'Bull-dog' forceps, 55

Burdach's column. See Postero-inc. dian column

Burdon-Sanderson on negative variation, 720-722

Burns, superficial, death from, 276

Caffeine, 44 I, 552 diuretic action of, 470

Caisson disease, 274

Calcium salts, action of, on heart strips, 185

in milk-curdling, 327

and bone formation, $5+2$

and glycosuria, 520

deficiency of, 542

in bone, 529

relation of, to heart-beat, 139 , 183

Calorie, definition of, 574

Calorimeter, air, 576 respiration, $579,6 I_{3}$

Atwater's, 575

water equivalent of, $6 \mathrm{I}_{3}$

Calorimetry, 573, 6I 3

Cancer, autolytic ferments in, 5 ro gastric juice in, 324

Cane-sugar, 3 , ro absorption of, 405, 4I 6,433 inversion of, IO, 3 I 5

by gastric juice, 328

Cannula, three-way, I96 to put into artery, 55 trachea, I 86 vein, 200

gastric, 427

Capillaries, blood-pressure in, 120 changes in lumen of, I09, I57 circulation in, I09, III, I77 pulse in, 120

resistance in, I I 1 , I I

structure of, 74

total cross-section of, I I 9

velocity of blood in, I 19

Capillary electroneter, 62 I, 622, 739 records, $720,721,730$ tubes, flow of liquid through, 77

Carbo-hydrates, absorption of, 403,416 classification of, 3 
Carbo-hydrates, composition of, I, 3 constitution of, 3

iil urine, 442 metabolism of, 5 I I

passage of, througl placeuta, ro 4 proteill-sparing atction of, 534 reictions of, ro

scheme for testing for, 13

Carbon balance. 539 distribution of, in luxdy. 520 cquilibrium. 530)

dioxide, action of. on respiratory rentre. 230

and blood-finw in heart, I63

rstimation of, 24 r, 25 I, 293. 294

formation of, from proteins, 509

in lungs. 240

in alveolar air. 242, 257

iu hloud. 2., 25.3

in fortal blowd. I0I 3

iil sfrum. 253. 255

partial pressure of, in blood, 257

in tissues and liquids. 260

production of, in different animals, $24^{6}$

iil inuscular work, $2+3$. 294

in relation to boly. weight, 245

in rigor mortis, 263,673 . 674

washing out of, 242,246

monoxide himoglobin, 45,66

metholl for partial pressure of oxygen, 258

for quantity of blood, $4^{8}$

Cardiac rycle, 78

rhanges in enducardiar pres. surc during. 90

death, I56, 235

impulse, 82, I9I, I93

nerves, I.3, I.t6. I $47, I 82, I 84$. I87, I90

normal excitation of. I 52

sound (Chauveall and Marey's), 87 sphincter, 302, 300, 3 r 3

Cardiogram, 83 , I9I

inversion of. 84

Cardiograph, 82 , I9I

Cardis - inbibitory and angmentor centres, 1.53

tome of, r 53, I 5 t

Cardio-pnemmatir movements, II 7.29I

Cardio-vascular nerves, I 28

Casein, 327, 503, 53.5

Caseinogen, 2, 327, 549, 6 I I

Castration, effects of, 556
Catalases, 26.4

in blood-corpuscles, 68

Catalysers, 3 I 5

Cataract and physical chemistry of lens, got

Catheter, pulmonary, 257

Catheterism, 609

Cells, structure of, 4

Cellulose, digestion of, 396

Central canal of $\operatorname{cord}, 7+6,753$

Central nervous sistem, development of $74^{6}$

electromotive phenomena of. 732,733

functions of, 786

generai arrangement of. 753

histology of, 747-758

localization of function in, 867,872

methods of study of, 745

resuscitation of, $879, \times 80$

grey axis, 759

Centre uf gravity of body, 839

Centres of cord and bulb, 8 It

cardio-inhibitory and augmentor.

I 53 , I 54

heat -, 595, 597

'motor,' of cortex, $846-858$

inusical, 86 I

sensory, of cortex, $858-861$

vaso-motor, I 66-I 69

Centrifuge, 56

Centrosome, 5

in nerve-cells, $7+8$

in the orum, roof, roof

Cerebellar ataxia. 832,836

Cerebellum. conllections of, 779,835 , 832

with auditory nerve, 780

development of, 747

cfferts of removal of. 830.836

functions of. $830,8.35$

inferior peduncles of, $760,77 \mathrm{r}$. 779.832

inhibition from. 836

middle peduncles of, 760,768 , 780,832

struct ure of, 830

superior pedmales of, 760,772 . 7 So

worm of, $771,772,779,829.835$. 836

Cerebral anxinia, $172,174.876$ resuscitation after, 879 , 8.80

circulation, 878,879

rortex, and respiration, 225 developmental differentiation of. 854.855

functions of, 840

histological differentiation of, $85 \mathrm{I}-853$ 
Cereluril cortex, inhibitinn from. s, 6

$$
\text { lityers of, } 851
$$

'motor' areas of. 8. 1 selsory , Ire.15 of, 85,5

vesicles. 7.17

Cerebrins, erebrosides, (m) I

(ireloru-spillal thuil, 758,882 displacement of, 272

Cirelurum, excision of. 840.888

Cervical srompathetic. Se' Siympathetic corvical

Chalk stomes, 505

Cheese, chemistry of, $5 f^{6}, 6$ II

Chendiotaxis, $5+$

in nerve regeneration, 605

Cherne-Stokes respiration, $238,27.5$. 288

Cliasma, 8 I 8

(hild. food requirement of, 5.10) gaseous exchange in, 24.1

Clloral, andesthesial by, I 74, I99

Chlorides, estimation of, 477

Clikroform, alosorption of, by erythrorytes, 23.5

action of, on cardio-inhibitory centre, 235

on respiratory centre, 234

()n urine secretion, $47 \mathrm{I}$

oll vaso-motor centre. I7.

I 99, 234, 235

anasthesia, dangers of. 234

laking action of, $6 \mathbf{r}$

passage of, through placenta. 1o[3 reflex inhibition of heart by, 154

Chlorusis, absorption of iron in, 396 , $+18$

blood-count in. $23 \cdot 25$

quantity of blood in, 49

Cholagogues, $385,3^{88}$

Cholesterin, $4 \cdot+2 \cdot+7 \cdot 335 \cdot 337 \cdot 431$ in hamolysis, 28

in serum. i $\mathrm{I}$

Cholic acid, 337

Cholin, 4, 566, 882

Cholohamatin, 336

Chorda tympani, $362,363,424$ antagonism of synpathetic with, $365,368,425$ hypothetical fibres in, 366 taste fibres in, 821,822

Chorda tendinea. 79

Chorion, IOI 2

Choroidal epithelium, noo. $33+$

Choroid plexus, 882 and visual purple, 036

Chromaffin tissue of adrenals, 565

Chromatin, 5, 749,873 changes in, in nerve-cells, 360 .

$745,756,770,775,874$

extranuclear, 874

Chromogens, $336,4+3$
Cliromophanes, 936

Chromo-proteins, 2

Chromosomes, 5, 1006

Clirysotoxin, artion of, in blumet. pressiure, 157

Chyle, composition uf, $\mathrm{I}+50,+1+$

fat in, $34^{\circ}$

fistula, 50

Chỵme, $305,3^{80}$

to obtain, 427

Chymosin. See Renuin

Cilia, 628, 705

Ciliary ganglion, 820 muscle, 8I9,898,907,908 nerves, 8 I9, 820, yo8, 985 processes, 898,986 and secretion of aqueous liumour, 901

Ciliated membranes, rectromotive phenomena of, 734

Cinematograph, 928

Circulating liquids, the, 14

Circulation, artificial, 262

changes in, at birth, I020

comparative, 72

cross, through brain, 232,867

general view of, 73

iil brain, 878

in the capillaries, I 09 , I I I

in the embryo, IOIO, IOII. IOI 5

in the frog's web, I 5. I77

in the lungs, 207

in the veins, I 09 , I I I, I 2 I

influence of posture on, I 73 of respiration o11, 265

of $1 \mathrm{ymph}$, i 76

time, I 23, 203, 208 electrical method, 123, 203 Hering's method, 123 methylene blue method, I25, 203

Circus movements, 837

Clarke's column, 762 connections of, 770

Climate and food, 590

Coagulated proteins, reactions of, 9

Coagulation of blood, 30,54

factors in, 38

influences restraining, 39

intravascular, 38,39

of crayfish, 36

of Limulus, 36

prevention of. 3 I. 54

of lymph, 49

temperature, to determine, 8

Coagulins. 33

Coal-gas poisoning, 45, 66

Cobra-venom and coagulation, 39

Cocaine, action of, on intestinal nusve ments, 307

on nerve-fibres, 687 
Cocaiue, action of, on the pupil, o I $\mathrm{r}$ fever, 586

Cochlea, $823,956.957 .958$

Cochlear ront of eightli nerve, 773, 823

Cucoa, 4+I, 550, $55 \mathrm{I}$

Cirlom, roor)

Coffee, $44 \mathrm{I}, 550,55 \mathrm{I}$

Cold sensations, $068,969,971,972$, IOOI

after section of cutaneous nerves, 975, 977 patlis for, 795

Collaterals, $678,749,785$

of posterior ront fibres, 769,792 , 797

Colloids of Grimaux, effert of, on coagulation, 39

Cislon, movements of, 308 innervation of, $3 \mathrm{~J} 0$

Colostrum, I 02 I

Colnur, body - and surface-, 897 blindness, 94\%, 998 temporars, 949

mixing, 940, 996

triangle, 942

vision, 939

Hering's theory of, 245

Young-Helmholtz, thenry of, 94 I

Coloured shadows, 944

Colours, complementary, 940, 996

primary, 94 I

Coma, congestion of brain in, 875 diabetic, $52 \mathrm{I}$

Comma tract, 765,769

Commissural fibres, $76 \mathrm{I}$

Common path, principle of the, 797 , $79 \mathrm{~S}$

Commutator, Pohl's, 625

Compensator, 620

Compensatory pulse of heart, $I_{4} \mathrm{I}, \mathrm{I}_{4} \mathrm{Z}$

Complement, 27,63

Complemental air, 220, 29I

Complementary colours, 940, 996

Condensed air, effects of breathing, 272-274

Condensor discharges, hamolytic action of, 27

as stimuli for nerves, 658

Conduction, double, 687

irreciprocal, 688

isolated, 680

loss of heat by, 573,579

Conductivity, molecular, $f 0 r$

of nerve, effect of temperature on, 687

effect of electrical rurrents on.

specific, 40 $658,74^{1}$

Congo-red as test for acjds, 324

Conjugate deviation, $838,8_{45}, 858$
Conservation of energy, law of, in body; 580

Consonants, 282

Contraction, idio-muscular, 659

law of, 684,742

for nerves in situ, 686, 743

páradoxical, 729,74 I

secondary, 729,738

without metals, 717

Contrast, $944,996,997$

Co-ordination of movements, 778,831 , 837

Core-models and electrotonic currents, 728,729

Cornea, radius of curvature of, 902

Corona radiata, $768,774,776$

Corpora Arantii, 79

quadrigemina, 772, 773

and respiration, 225

anterior, 818,829

posterior, $824,828,86 \mathrm{I}$

striata, $747,759,768,785,892$ and temperature regulation, 595

Corpus callosum, 760,784

luteum, and menstruation, rao5

hiematoidin in, 356

internal secretion of, 557 , roo6

origin of, 1006

Cortex of brain, functions of, $8+0$. See also Cerebral cortex

'motor' areas of, 844 sensory areas of, 85.8

Corti, ganglion of, 823

organ of, $958,961,963$

Costal breathing, 214

Coughing, 239

Cranial conduction of sound, 960,999

nerves, 8 I 5

bifurcation of afferent fibres, of, $820,822,823,825$

homologies of, 8 I 6

nuclei of, 816,817

Crayfish blood, clotting of, 36

Cream, 6r I

Crista acustica, 833

Cross-circulation through brain, 232

Crossed pyranidal tract, $765,774,777$ conllections of, 774,778

Crura cerebri, 768,783

Crusta, 768

Cuneate funiculus, 767 nucleus, 767 relation of, to fillet, 772

Cuneus and vision, 859

Curara, action of, on skeletal inuscle. 633,706

on gaseous exchange, 245,517 on heat-production, 590, 596 oll vomiting, 313 
Curdling of milk by renuim, 320,327 . 427

Current intensity and stimulatiom, 1,36 , $68_{5}$

of artion. Sié Action current

ef rest. Sic Demarcation current

Cutaneous burns, death from, 270

excretion, 473

nerves. phenomena after section of, 975

respiration. 275

Cyanogen groups in living protein, 499

Cybulski's method for velucity of blood. I I 4

Cystill, 332, 337, +50, $53+$

Cytolysins, 29

Cytoplasin, 5

Cytosine, 507

1) ancing mice. labyrinth in, 837

Daniell cell, I 82, 6 I 5

Duphnia, Metschnikoff's researches on, 52

action of un uscarine on heart of, I 5 I

Dark-adipted eye, 934, 936, 946

L) aturine, action of, on heart, 150 on pupil, 91 I

'Dead space,' respiratury, 22 I

Deaf-mutes, equilibration in, 835 atrophy of temporal convolutions in, 860

Decerebrate rigidit $y, 8_{3} 6,8_{47}$

Decidua. I005, I IO absorption of, by leucocytes, 52 artificial production of, 1006

Decinormal solutions, 439,483

Decussation of afferent inpulses, 792 of efferent impulses, 792

of fillet, 772

of optic nerve, $8 \mathrm{~s} 8, \$ 59$

of pyramids, $768,774,777$

Deficcation, 310,311

Deficiency phenomena. 786

Degeneration of muscles, 698 of nerves, 691 chemistry of, 693

reaction of, 698

Deglutition, 30 I

centre, 303

nerves of, 303

sounds, 303

Deiters' nucleus, $780,781,792,824$

Demarcation current, 7 1 8, 739 electromotive force of, 722 theories of, 724

Dendrites, $7+9$

anıboid movements of, $7+9$ and sleep, 876

Dentate nucleus of cerebellum, 760 , 771,779

of olive, 767
Depressor nerve, I 54, I6y

pressor action ot, 172

Descending degeneration. 764,774

Deutero-protcose, 3, ro, 325

Development of embry:0, 1006

Dextrin, 3, I I , 442, 608

formed in salivary digestiull, 321 , 423

1)extrose, $3,315,316,4+2,4+3$ estimation of, in urine, 489 in blood, $+1,4$ I $7,462,5$ I

in lyanph, 49,417

Trominer's test for, IO, 45 I

Diabetes, 5 I 8

dextrose-nitrogen ratio in, 522

duodenal, 555

levulose used up in, 520

oxygen consumption in, 243

pancreatic, 520, 553

phloridzin. 52 I, 6 I 0

reaction of blood in, 23

respiratory quotient in, $2+3$

sugar-destruying power of bluod iil. 519

1)iabetic coma, 521

Diapedesis. 53,177

Diaphragm in respiration, 2 I I, 223

recording novements of, 2 I 8

Diastases, $32 \mathrm{I}, 355,5 \mathrm{I} 2$

Diastole of heart, 80

Dichromatic vision, $948,949.998$

Dicrotic wave, 94, IOI

Dietaries, standard, $5+3-5+\mathrm{S}$

Dietetics, 543

Differential rheotome, 723

Diffusion, $39^{8}$

circles, 9 I 2,950

of gases, 247

Digestion as a whule, 389

bacteria and, 318,395

chemical phenomena of, 319-3+t

comparative, 296

gaseous exchange during, 245

in intestine, 391

in stomach, 389

mechanical phenomena of, 300

of carbo-hydrates, 322, 38v

of fats, $328,334,338,427,43^{2}$

of proteins, $390,39 \mathrm{I}$

significance of, 299

time required for, 391,432

Digestive glands, structure of, 345

juices, action of drugs on. 387 adaptation of, to food, 3 fo, $369,376,3^{81}, 3^{87}$

protection of gut against, 359 secretion of, $344,35+$ summary, 388,389

organs in different animals, 296

Digitalis, diuretic action of, 470

Dilatur of pupil, $91 \mathrm{I}$ 
Diopter, 906

Diphtheria toxin, atctin of enzynes on. 342

1)iplopia, $820,023,024$

1)irect cerebellar trac:t, 704 connections of, 770,779

l'yramidal tract, $765,774,778$

1)isaccharides, 3,342 absorption of, $f$ I 6,5 I

Jischarge of ventriele, period of, 79, go 0

Dispersion in eye, gr 3

luy a prism, 875

Jiuresis by salts, 103

Dimretics, 470

1)oremus' ureoneter. 482

1) unble conduction in nerve, $6 \times 7$ inniges, neglect of, $y 24$

1) rumugraph, II 3

Drum, to smoke a, I79

Dry cells, I 82

Ductus arteriosus and dluctus venosus, 1015

J) ulcite, relation of, to galactose, 3

1) uodenum, glycosuria after removal of. 555

J )ura mater, 758

1)ysprinua, 23I heat, 290

respiratery quotient in, $24^{2}$

Lar, anatorny of, 954

ussicles of, 954,955

functions of, 950

resullance tone of, so, gou

Echidnase, 46

Eick's tistula, 356, 502

Ectuderin, 6, 1008, 1009

Ectoplasm, 4

Effector of reflex are, 7y7

Efferent impulses, decussdtion of, 708 , $774,777,792$ paths of, 792

bigg-albumin, absorption of, fis innino-acids in, I (x)retion of , $419,162,609$ reartions of, 8

ligg-yolk, I010, IOI 7

Ehrlich's triarid stain, 17

Eiglitl nerve. See Auclitory nerve:

Jilasticity of muscle, 630

Electrical conductivity, of blood, 25,60 of gastric juice, $35^{5}$

of milk, IOL2I

of seruni, 60,357

organ, develnpillent of, 738

response. Sec Action current

Electric fishes, 736

Electrucardiogram, human, 730,731 , 732,734

Electrodes, unpolarizable, 625,739
Electrolytes, 400

Electrometer, capillary, 621, 622, 740

Eilectromotive force, 616

Electrons, for

Elcctrotonic alterations of excita. bility and conductivity, 683,684

currents, 728,740

Electrotonus, $683,740,741$

Eleventh nerve, 827

Emboli, artificial, $7+5$

Limbryo, aspliyxia in, IOI8

circulation in, IOIU, IOII, IOI.5, I 020

development of, 1006, 1010

gases of bloud in, I U 3

glycogen in, 514,1016

lieat-production in, 1017,1018

inverting enzymes in, 3.12, 1014 , I016

jiver in, 1015

metabolism of, IOIT

physiulogy of, ro

Emeties, $313,427,432$

Enmuetropif eye, 914,115

Emotions, genesis of, 866

Emulsification of fats, I2. 338.431

Emulsin, 315. 3 I 6

End-brush, 740

Endocardiac pressure, 84 , 84

anlount of, 85

curves of, $84,87,88$

ineasurement of, $\delta_{5}$

llegative, 92

Lindodern, 6, roo8, I voy

Eudogenous fibres of curd, 761,765 , 795

Endolyumph, 833, 956

Endoplasm, 4

Endothermic reactions, 585

Enenata, 308,421

linergy of foud, influence of hydrolysis ()I1, 580

law of conservation of, in body, 580

Enterokinase, 343,387

Enzymes. Sie Ferments

Ependyma, 759

Epiblast. Sce Ectoderm

Epicritic sensibility, g\$1

Epiglot tis, 301, 302

Epilepsy, cortical, 865,889

produced by absintlue, is

Eijuilibration, cercbellum and, 831

1)eiters 'nucleus and, 825

in dog-fisll, 834

in pigeon, 834

muscular nerves and, 835

sernicircular canals and, 823, \$25, 833

skin and, 835

Lrection centre, 167 
Lirepsin, 342, 110

lirgograph, $550,049,650,052,708$

lirgot and blood-pressure, I 57

Erucic acid, 523

Ervthroblasts, 2 I

lirythrocytes, I 5

enumeration of, 18.58

gases of, 252, 253

life-histury of, ry

lirvthrudextrin, I I, 321, 423

Eshach's albuminimeter and reagent, 488

Liserine, action of, on accommodation, gos

()In pupil, 9I I

litler, ation of, on bloud-corpuscles, $27,28,6$ I

on urine secretion, $47 \mathrm{I}$

Etlul butyrate, synthesis of, by lipase, 4 I 5

Euclioneter, 215

Euglohulin, $\$ 2$

Eustachian tube, 274,954

villee, 10 I 5

Evaporation, los of heat by. 578,579

Excitability, a property of living inatter, 6

direct, of muscle, 633,706

of nerve, effect of temperature on. $6 \mathbf{S}_{1}, 687$

effect of electrical currents on, $658,74^{\mathrm{I}}$

Excitiale tissues, the, 627

Excretion, 435

Exothermic reactions, $5 \mathrm{~s}_{5}$

Expecturation, +35

Expiration, 213

duration of, 2 Is

forced, $21+$

Expired air, composition of, 2 \&, 293

Lixtensibility of muscle, 630

Extension refex, crossed, Soo, 887

Eixtensor reflex, the, 798

thrust, the, 799

Lixteroceptive reflexes, 8 io

Extra contraction of heart, I \& I systoles, in man, $1+1,1+2$

Exudation, inflammatory, 53

liyc, chemistry of refractive media of, 90 I

compound, of insects, 897

currents of, $73+, 735$

defects of, 912

development of, $7+7$

li iihne's, 988

movements of, 951

muscles of, 952

nerves of, $818,908,909$

optical constants of, 902, 903

reduced, 903, 904

refraction in, 902 liyc, structure of, \$os, 985

Eyes, conjugate deviation of, $x_{3} 8,8+5$, 858

primary position of. 951

wheel movements uf, 95 I

Facial nerve. 822

umion of, with accessory, 868

palsy, 822

Fices, bacteria in, 397

compusition of, 396,432

odour of, 3$)^{6}$

storage of, in sigmoid flexure, 309

Fainting, 175

Fallopian tubes, 100.4

Falset to voice, $28 \mathrm{r}$

Faradic current, 624

Far-point of vision, 91 5,988

Fasciculus solitarius, 822,825

Fasting men, metabolism in, 532

Fat, absurption of, $+12,432,433$

function of bile ill, 414

conposition of, I, 3, I I, 523, 529

digestion of, $328,334,338$

emulsification of, I 2,338

excretion of, into intestine. 35 , $+15$

formation of, from carbo-hydrates, 524

frum fat $y$ acids, 523

from proteins, 525

in faces in jaundice, 3 to

influence of, on pancreatic secre-

tion. 3 so

iodine value of, t, 526

melting-points of, + , I 2

metabolism of, 522,527

in phosphorus-poisoning, 525, 526

migration of, $522,525,526,527$

organized, 526

passage of, through placenta, Ior 4

protein-sparing action of, 523, 53t

saponifieation of, I I

solvents of, +

sources of, in body, 523, 524

stained, absorption of, $41+432$

synthesis of, in intestine, +15

Fatigue, muscular, $648,650.653 .708$

cause of, 650

of nerve-cells, $873,87+$

uf reflex arcs, soo

seat of exhaustion in, 651, 652,

708, 709

Fat-sphitting ferment of gastric juice, 323,325

of intestinal juice. 342

of pancreatic juice, 331,334 .

339,429

of tissues, 527

bacteria of intestine, 393. 395 
Fatty acids, absorption of, $113,+15$ tests for, I1, 12

Fechuer's latw, os 4

Fehling's solution, 489

Fenestra rotunda, 95.5

Ferments, 3 I 4

glycolytic, 517

intracellular, 314, 35\%, 50\%, 510, 527

in urine, 4.4

mode of action of, 316

oxidizing, 42, 68, 264. 295. 314 $507,509,1013$

quantitative estimation of, 317,422 reducing. 509

reversible action of, $317,509.528$

Ferricyanide of potassium, action of, on hamoglobill, 46

estimation of oxigen in blood by, $25 \mathrm{I}$

Fertilization of ovum, 1007. 1005

Fever, 597

effect of, on pulse, yy

metabolism in, 507, 600, 601

produced by eocaine, $5>6$

by puncture. 595

retention theory of, 599

significance of, oo I

Fibrillar contraction of heart, I38, I90

Fibrin-ferment, 32

formation of, 33

Ilature of, 32. 38

precursors of, 33

preparation of, 33,57

source of, 36

Fibrin, formation of, 30

protein reactions of, 9 quantity of, in blood, +1

Fibrinogen, 32, 33, 49

Fick and Wislicenus' experiment, 537

Fifth nerve. See Trigeminus

Fillet, 772

decussation of, $772,70 \mathrm{I}$

descending fibres of, 773

lower or lateral, 772,824

upper or intermediate, 772,321

Fish-sperm, proteins of. 2

Fistula, biliary, 339,383

double gastric and cesuphageal, 257,374

lick's. 356

gastric, 373

intestiral, 341

pantreatic, 378

salivary, 370

Flavour, 965

Flechsig's cortical helds, 854, 055

tract. See l)irect cerebellar tract

Flour, chemistry of, 612

Flow of liruuids, 75

with intermittent pressure. 77
Finorides, infuence of, "n coagulation, 35

Fucal illumination of eve, 929

Fu:tus, See Embryon

Folin's method of estinating indican.

\section{0}

kreatinis, 485

uric acid, 485

Fontanelle, sinking of, in sleep, 875

Food and climate, 590 relation of, to surface, $54 \%, 503$ time of passage uf, along gut, 3 I I

Fuods, cumposition of, $546,611,612$ isodvnamic, 670

Foot-jerk, 8 I I

Foramen of Magendie, 882 of Monro, 747 ovale, 954, 955, 960

Forced movements, 836

Fure-brain, 747

Formaldehyde reaction for pruteins. 8 reflex inhibition by inhaling. 235

Formatio reticularis. 768

Formic acid in Saliva' of Octopus, $36 \mathrm{I}$

Fourth or truchlear nerve, 820

Fovea centralis, $899,900,935,946$

representation of, on cortex, 859

lireezing and thawing, hamolysis by, 27

Freezing-point and osmotic pressure. 398

determination of, 399,492

of solid tissues, 4 I

of urine $+46,492$

Frontal lobes, functiun of, $865, \$ 66$

Fundus of stomach, in digestion, 304. 305

Funiculus gracilis and cuneatus, 707

Furfuraldehyde in Pettentiofer's test, 337.430

Furunculosis, opsonius and treatment of, 53

Galactose, 3. 316

Gall-bladder, action of peptone on, 4 I I nerves of. $38+4$

Galvăuic rotation, 836

Galvani's experinent, 717,738

Galvanometer, 617,740

'string, 619

(i.llianutonus, 636

(i.lnglion-cells, changes in, with age, 755

bipolar, 753

Ganglion jugulare, 825

nodosuni, 825

petrosum, 822,825

spirale, $8 \geq 3$

superius, 825

restibulare, $8 \geq 3$ 
Gasenus exchange, 2.11, 293

circumst,unes atfecting, 244

relution of, to extermal tempera-

ture, $2+5$

Giases of blund, $2+6$

diffusion of. 247

partial pressure of, $2,8,249,256$

solution of, 2.48

Gas-pump, 250

Gasserian ganglion, 821

developing. 752

Castric digestion, amylulytic stage of, 322. 390

testing for products of, 426

glauds, changes in, during secretion, 347,349

influence of nerves on, 372

structure of, 345,350

juice, 322,427

acidity of, $323,324,428$

adaptation of, to food, 376

altiseptic function of, 328

artificial, 426

Beaumont's researches on, 323

composition of, 323

formation of hydrochloric acid of. $35 \mathrm{I}$

freezing-point and conduc. tivity of, $357,35^{8}$

in cancer, 324

lactic acid in, 324

organic and inorganic acids iil, 324

psychical secretion of. 374

quantity of. 324

secretion of. 350

to obtain. $323,374,427$

lipase, 323,328

secretin, 374

Gastro-enterostomy, assimilation of protein after, 330

Gelatin, 2, 690

cleavage products of, 2,534

protein-sparing action of, 534

reactions of, 9

(ielatose, 3, 4 I6

Gemmules, 749

Geniculate bodies, lateral, 818,829 mesial or internal, 773. 819. $828,86 \mathrm{I}$

and auditory nerve, 773 .

ganglion, 822 824,828

Germinal area, 1008

cells, 754

vesicle, 1004

Ghosts of erythrocytes, 6 I

Giant pyramidal cells, 774, 852

Gianuzzi, crescents of, 345,353

Gigantism and disease of pituitary, 568
Glands, electromotive phenomena of. 734

heat-production in, 585

racemose, 345

serous and mucous, 3 in

Glaucoma, intra-ocular tension in, 902

Gliadin, influence of, on serum proteins, $49^{8}$

Globin, 2, 47

Globulins, 2, 47

in urine, 486

reactions of. 9

Globulose, 3

Glomeruli, 452, 455, 458, 465, 469 olfactory, 816,818

Glosso-labio-laryngeal palsy, 825

Glosso-pharyngeal nerve, 821,825 and taste, $821,822,825$ in deglutition, 304

Glottis, $216,277,282,302$

movements of, in respiration. 2 I 5

Gluco-proteins, 3

Glucose. See Dextrose

Glutamic acid, 332

formation of dextrose from, 514

in serum proteins, $49^{8}$

Gluten, $6 \mathrm{Iz}_{2}$

Gluten-fibrin, 503

Glycerin, formation of glycogen from, 513

in blood, 4 i I

test for, I2

Glycin or glycocoll, 2, 332, 337, 44I, 510,526

formation of dextrose from, $5 I_{4}$ of hippuric acid from, 508

of urea from, 5or

hydrolysis of, 322

Glycocholic acid, 336

Glycogen, 3, I3. 5 I I, 527

disappearance $\mathrm{f}$, in fasting, 5 I 5

extra-hepatic, 5 I 4

formation of, 512

formers, 5 I 3

function of, 5 I 5

in diabetes, 519

in embryo, 514 , I016

in leucocytes, 47

in liver-cells, 5 I 2

in muscles, 514, 515, 667

in placenta, 514 , IOI4, IOI6

in strychnine -poisoning, 595, 596

preparation of, 511,608

used up in muscular contraction, 5 Is

Glycogenase, 510, 554, 10I6

Glycogenolysis, 5I2, 515 , I 16

splanchnic nerves and, 519

Glycolysis, 516, 554, 555

by pancreas and muscle, 5I\%, 554 
Glycosuria, 516

adrenalin, 522

after injection of sugar into blond, 609

alimentary, 516,610

duodenal, 555

in diabetes, 5 I 8

pincreatic, 520,553

phloridzin, 521, 609

puncture, 5 I 8,555

Glycuronic acid, $4+2$

Glycyl-glycin, 503

Glyoxylic acid in Adamkiewicz's reaction, 8

Gmelin's test for bile-pigments, 43I

Golgi's method. $7+9$ second type, cells of, 754, 869

Goll's column. Se' Postern-median columin

Ciultz on dog's brain, 842 (m) monkey's brain, 850 (in removal of spinal cord, 787

Fout, uric acid in, 449, 505 uricolytic ferment in, 508

Gowers' tract. See Antero-lateral ascending tract

Graafian follicle, $\mathrm{roo}_{4}$

Gracile and cuneate nuclei, 767,772

Gracilis experiment, Kühne's, 688

Grafting of tissues, 1023

Gramme-molecular weight, 398

' Granule-cell,' 754, 8I7

Gravity, centre of, in standing, 839 influence of, on circulation, I73. I99

Gréhant's method of anæsthesia, I 86

Ground-buridle, antero-lateral, 765

Guaiacum test for blood, 68, 264

Guanase, 507

Guanin, 441, 504, 507

Gudden's commissure, $\delta 19$

Günzburg's reagent, 428

Gymnotus, 737

Habits, effect of cortical lesions on, 866

Hæmatachometer, I I 3

Hamatin, 47,67

Hamatoblasts, 2 I

Hxmatocrite, 25, 59

Hæmatoidin, 356

Hæmatoporphyrin. 47,67 in urine, $4+3$

Hamautographic tracing, I02

Hæmin, 47 test for blood, 7 I

Hxmochrome, 16, 28, 252

Hæmochromogen, 47, 67

Hæmocytometer, 58

Hæmoglobin, 42

composition of, $2,4.3$

crystals of, $3,44,65$
IIxmolobin, derivatives of, $45 \cdot 47.66$ 67

dissociation of, $43,248,252,254$

in foetus, IoI 8

intracorpuscular crystallization of, 44. 62

iron and sulphur in, 3, 43

quantitative estimation of, 69

relation of, to bile pigment, 388

spectrum of, 44,65

Hamoglobinometer, Gowers-Haldane, 68

Hæmoglobinuria, 46, 444

Hxmolysis, $26,61,63$

in placenta. IOI 4

mechanism of, 28

Hæmolysinogens, 30

Hæmometer, Fleischl's, 69

Hamophilia, 34

Hamorrhage, effect of, on hlood. pressure, I74, 200

Hair æsthesiometer, 970,999

Hair-cells of vestibule, 833 of Corti, 958, 961, 963

Haldane and Smith's method for quantity of blood, 48

Harmonics or overtones, 280

Hay's test for bile-salts, 49 I

Hayem's solution, I 8

Head, grafting of the, 867

Head on referred pain, 790 on sensory nerves, $98 \mathrm{I}$

Hearing, 953

centre for, 824,860

impairment of, in facial palsy, 822 range of, 964

Heart, action current of, $78,730,740$ action of drugs on, I52, I 83 ' all or nothing' law in, I 4 I anatomy of frog's, I 29,177 automatism of, I 2 \%, 130

beat. 78, I 78, I 86

cause of, 129

chemical conditions of, I39. 185

voluntary acceleration of, 156 conduction and co-ordination in, 129, 134

discharge of, 90

embryonic, I32, IoIO, IOI 5,1020

excitability of, 130

extra contraction of, 141

fibrillar contraction of, 138, I90

filling of, 90

ganglion-cells of, 129

gaseous metabolism of, 264

glycogen in, 515

hamorrhage from, I 89

heat produced by, 585,$6 ; 0$, I0I 8

heat standstill of, I 52,178

inpulse of, 82 , I9I 
Inart. intluence of temperature on, I7 $8,18 \mathrm{I}$

I11.umualinu, artinn of, 186

Inuscle, 74

action of salts on, I $39, \mathbf{1} 85$

nature of contraction of, $\mathbf{1 . 1 2}$

nerves of, distribution in heart, 148,149

a ugnientor, $143,146,147,184$. I90

extrinsic, $1+3$

inhibitory, I43, I $47,182,187$

intrinsic, I 29

normal excitation of, I 52

output of, 127

pressure in, 84,90

primitive vertebrate, 70,72

refractory period of, $1+4$

respiratory, 72

resuscitation of, 139. I49

rlythuicity of, 129,133

suinds of, $80,19 r$

source of energy of, 670

suction action of, 92, 12 I

tonicity of, I 30

tracings, I $45.147, I 51,152$, I 55 , I $78, \mathrm{I} 80, \mathrm{I} 85, \mathrm{I} 88$

simultaneous, from auricle and ventricle, $\mathbf{I} 80$

valves of, 78 , I 90 , I 9 I

insufficiency of, $\mathbf{x} 9 \mathrm{I}$

moment of closure of, 89

work of, I 27

in fœtus, ro 8

Heart-strips, contraction of, I 39,185

Heat-centres, 595, 597

Heat-dyspnoea, 290

Heat, distribution of, 602

equivalent of food-substances, 58 I

of cleavage products of food, 580

of work of heart, 585

given off in respiration, 579, $6 \mathrm{r}_{3}$

involuntary regulation of, 587

voluntary regulation of, 589

loss from body, 578

after varnishing skin, 276 , 476,594

by evaporation, 578,579

mechanical equivalent of, $58 \mathrm{r}$, 1027

Heat-production, effect of curara on, 590

and size of body, 593

in brain, 586

in fever, 598, 599

in glands, 585

in heart, 585

in muscles, 583

in sleep, 582
IIeat-production, involuntary regulation of, 590

of different classes, 58 ?

of 111ail, 58 I

relation to muscular work, 583 , 66.4

to surface of body, 593, 595

seats of. 583

sources of, 580

voluntary regulation of, 589

Heat-rigor, $263,674,715$

Ieat-sensations, 972,974 , roor

after section of cutaneous nerves, 975,978

Heat-units, 574

Heidenhain's experiments on renal secretion, 458

Heller's test for albumin, 486

Helmholtz's wire, 624

Hemeralopia, $94^{8}$

Hemianæsthesia, capsular, 783

Hemianopia, 8I9, 829,858 nasal, 8I9

Hemiplegia after removal of motor cortex, 848

and motor aphasia, 863

cutaneous reflexes in, 807

Hemisection of cord, 793

Hering's theory of colour vision, $9+5$

Herpes zoster and trophic nerves, 701

Hetero-proteose, 3, ro

Hexone bases, 331,332

Hexoses, 3

Hibernation, respiratory quotient in, 242

temperature regulation in, 596

Hiccup, 239

Hippocampal convolution and olfactory tract, 8 I 7,862

Hippuric acid, 44I, 486

Hirudin, 36

synthesis of, $44 \mathrm{I}, 508$

Histidin in tryptic digestion, 331, 332

Histones, 2

Holder for animal, I 86

Holmgren's wools, 998

Homogentisinic acid, 444

Homoiothermal animals, 572, 577, 587

Homolateral fibres of pyramidal tracts, 774,778

Hopkins's method of estimating uric acid, 484

test for lactic acid, 7 I 6

Hormones, 375, 57 I

Horopter, 924

Humidity of air, and body temperat ure, 588

Hunger, sensation of, 826

Hyalomucoid, $70 \mathrm{I}$

Hydra, structures of, 6

Hydramic pletliora, +62 
Hydramnios, IOI 7

Hyclrobilirubin, 336

Hydrocele fluid, clothing of, 33

Hydrochloric acid, action of, on proteins, 326

in gastric juice, $323,326,428$ formation of, 35 I

Hydrogen balance, 540

in expired air, 242

ions in blnod, etc., 23

percentage of. in tissues, 529

Hydrolysis by acids, I, 322

by ferments, $31+$

and energy value of food, 580

Hydrostatic and hydrodynamic ele. ments in blood-pressure. 173

Hydrostomia, 372

Hydroxylions in blood, etc,, 23

Hyoscyamine, action of, on pupil, 9 I I

Hyperalgesia after nerve section, 793. 979

Hyperchromatism, 874

Hyperglycamia, 518.519

Hypermetropia, 916

Hyperpncea, 23I

in mountain sickness, 275

Hypnosis, 877

Hypoblast. See Endodern

Hypobromite method of estimating urea, 440,480

Hypogastric nerves, 310,3 II

Hypoglossal nerve, 827 and lingual, union of, 685

Hypoisotonic solutions, 400

Hypophysis, 830 action of extracts of, 566

Hypoxanthin, 441, 504, 507, 691

Identical points, theory of, 923

Idio-muscular contraction, 659

Ileo-cacal valve, $307,308,394$

Ileo-colic sphincter, $308,310,394,42$ I

Illusions, visual, 928

Image on retina, formation of, 902.903 , 986

size of, 904

Imbibition, $398,405 \cdot 474$

Immunity, 30

Income and expenditure of body, 528

Incus, 954, 955, 959

Indicators, 24, 392, 393

Indigo-carmine, excretion of, ly kidney, $45^{8}$

Indol, 333

formation of, in intestine, 395

Indophenyloxydase, 260,265

Indox $y^{*}$ in urine, $445,449,479,480$

Induced currents, 623

Induction machine, 623

arranged for single shocks, 703 for tetanus, 184
Induction machine, make and break shorks from, 702

Infant, food requircment of. 549

Inferior peduncle of cerebellum. See Restiform body

Inflammation, diapedesis in, 53, I77

In fra-proteins, 2

Infundibuluin, 830

Inhibition in reflex action, 800

from the cortex, 846

Inhibition of heart, I43, I 44, 182, I87 nature of, 151

reflex, 154

by vapours, I 54 , I 55 ,

\section{5}

Injury-current. See Demarcation current

Inorganic salts. See Salts

Inspiration, 210

duration of, 2 I 8

forced, 214

Insufficiency of cardiac valves, 81,96. I9I

Intellectual processes, seat of, 865

Intercostal muscles, 2 I 2

Intermediary body, 27

Intermedio-lateral tract, 762

Internal capsule, $760,768,776,779$ arrangement of fibres in, 782 respiration, 206, 259

Internal secretion. See Secretion

Intestinal contents, reaction of, 392 , 393

juice, 34 I

action of, in digestion, 342344

adaptation of, to food, 387

influence of nerves on, 386

Intestine, large, absorption in, $42 \mathrm{I}$

Intestines, bacteria in, $318,328,393$, 394,395

digestion in, $39 \mathrm{I}$

movements of, 306.308

nerves of, 309

reartion of contents of, 392,393

relative length in different animals, 297

resection of, $42 \mathrm{I}$

Intracranial pressure, effects of increasc of, 875

Intra-ocular tension, 880,902

Intrathoracic pressure, 209, 22 I

in foetus. 222

Intravascular clotting, 38

influences restraining, 39,40

Inversion of carbo-hydrates, 328,342 , 433

lodine in thyroid, 56r

Ions, 25, 400

Iris, centre for movements of, 8 I 5,820 , 909 
Irs. effect of stimulation of sympathe tic o11, 911,996

functions of, $7 \mathrm{I}^{2}$

in accommodation, 900

lual nuechanisun of, 9 II

Herves of, $819,820,908,909$

sphincter of, 820,008

Ir'ul, absorption of, 417

in bile, 337

in brall, $5+3$

in fictus, 543 , 1013

in liver, 21, 355, 432

in milk. $5+3$

in ordinary dietary, 543

in placenta, ror 4

in spleen, 21

Iron-ammonia alum as indicator, 477

Irradiation, 950

of reflexes, 802,885

Isliund of Reil, 855,865

lsodynamic relation of foods, 670

Isomaltose, $315,320,442$

Isotonic solutions, foo

and isonetric contraction, 645

ltching, 968, 975

Jacksonian epilepsy, 865

J acobson's nerve, 363

Japanese dancing mice, internal ear of, $8_{37}$

Jaundice, absorption of bile in, 355

fat in faces in, $34^{\circ}$

hiematogenic and obstructive, 356 , 386

Jaw-jerk, 8I I

J udgment, false, as explaining contrast, 345

J udgments, visual, 926

J ugular pulse, $9 \mathbb{I}$

tracings, 137,193

Karyokinesis, 5

liaryosome, See Nucleolus

Katabolic changes in living matter, 6

Kathode, 401, 615

Kations, $40 \mathrm{I}$

Kephalin, 690

Keratin, 2

Ketones, relation of, to carbo-hydrates. 3

Key, short-circuiting, 625

Kidney, absorption in, 457

bloodvessels of, 452,468

execretion of pigments by, 458

formation of hippuric acid in, 508 gaseous metabolism of, 264

'internal secretion' of, 569

nerves of, $16 \mathrm{I}, 468,469$

removal of only, 447

greater part of renal tissue, 569
Kicluey, sccretory pressure in, $4^{66}$

tubules of, $453,+54$

transplantation of, 1023

Kinasthetic function of Rolandic area, 856

Kjeldahl's method for total nitrogen, 482

Kince-jerk, $801,802,8$ I I, 8 I 3,888

reinforcement of, 807

lireatin, 4 I, 442,504, 666

Kireatinin, $437,442,485,497,505,509$ excretion of, after inuscular york, $53^{8}$

in starvation, 530

Fresol in urine, 445

liuhne's eye, 988

kynograph, IOI

Labyrinth of ear, $833,956,957,960$ as a proprio-ceptive organ, 833 extirpation of, 835

Laccase, 264, 314

Lachrymal glaıds, 435

Lactase, 316, 342, 4 I6

Lactation, relation of, to ovary, 1022

Lacteals and fat absorption, $4 \mathrm{I}, 433$

and sugar absorption, 417

Lactic acid, action of, on bloodvessels, 162

on respiratory centre, 230. 275

Hopkins's test for, 7 I 6

in gastric juice, 324,329

in intestine, 393

in muscle, $162,658,667,668$, 7 I 6

Uffelmann's test for, 428

L.actose, 3, 3I6, 549

absorption of, 405,416

supposed adaptation of pancreas to, 382

Laking of blood, $26,61,63$

of nucleated corpuscles, 62

Langerhans, islets of, $347,368,554$

Langley's experiments on union of vagus and cervical sympathetic, 868

Lanolin, absorption of, 4 I 3

Lanugo, IoI 2

Laryngoscope, 280

Larynx, anatomy of, 277

abductors and adductors of, 277 , 285

movements of, in respiration, 2 I 5

nerves of, 285,286

paralysis of, 286,826

Lateral horn, 762

nucleus of bulb, 772

Lauric acid, passage of, through placenta, roI 4

Lavoisier and carbon dioxide, 240

Law of contraction, $684,656,742,743$ 
Lecithin, 4, 41, 42, $17,335,337$

in bile, 337

in lianolysis, 28

in nerves, bono

Leclanché rell, 182

Leech extract and coigulation, 35

Legal's test for acetrne, 492

L.ens, 900, 986

alteration of, in accommodation, 005, 906

chemistry of, no

radii of curvature of, 902

refractive inclices of, 903

regeneration of, 1003

Lenses, refraction by, 895,806

Leucin, 2, 526

formatiou of urea froll, 501

and tyrosin formed in tryptic digestion, 332,430

in urinary sediments, 450,452 , 495

Leucocytes, 16

and absorption of fat, 414

of peptone, 420

and coagulation, 36

classification of, 17

coinposition of, 47

destruction of, 22

emigration of, 54,177

enumeration of. I9, 58

ferments in, $47,355,359,510$

formation of, 22

glyengeu in, 47

Leucocytosis, In

Leucyl-leucin, 503

Leukæmia, blood-corpuscles in, I0 uric acid in, 449

Levatores costarum, actiun of, in respiration, $21 \mathrm{I}$

Levulose, 3, 315, 516,520

Levulosuria, 5 I6, 520

Liben's test for arretone, 492

Lieberkuihn's crypts, 345,42 I

Light bath, artion of, on blood, 275 reflex, path of, 8 I 8,829 consensual, 9 I0

Lime-juice as antiscorbutic, 552

Limulus blood, coagulation of, 36 heart, cause of beat of, I 30 action of drugs on, I 51

Lipases, 42, 315, 334, 510, 527

gastric, 323,328

in succus entericus, $3+2$ pancreatic, 331

Lipoids, 4, 690

of erythrocytes in hamolysis, 28

Lissauer, tract of, 764

Listing's law, 95 I

Litmus as indicator, $2.1,393$ paper. glazed, 54

Liver and coagulation of blend, fo
Liver and destruction of erytlirocytes, 21

ferments ill, 5 jo

formation of bile-pigments and acids in, 355

of glyeogen in, $511,512,513$. 519,608

of sugar inl, 5 I I, 5 I 5

of urea iu, 501,505

heat-produrtion in, 585

internal secretion of, 552

iron in, 2I, 355, 432

Minkowski's experiments orı, 502

structure of, 14. 345

living and dead proteins, 499

Living matter, composition, I

functions, 6

structure, 4

'1.iving test-tube' experiment, 3 I

Localization of function in brain, 867 , 872

degree of, in different animals, 872

of sensations, 980,982

Locke's solution, I 39

I.ocomotion, 839

Loconnotor ataxia, rquilibration in, 835

knee-jerk ill, 81 I

pupil in, 9 ro

tactile sensations in, 795

Loeb on artificial parthenogenesis, rooz

Loewenthal's tract. See Antero-lateral descending tract

Lungs, circulation in, 208

rlastic tension of, 209

influence of, on coagulation, to

quantity of blood in, 208, 268

secretory action of, 258

structure of, 207

vaso-motur nerves of, $16_{3}$

Lutein, roo6

L.uxus-consumption, 535

Lymph, circulation of, $1 ; 6$

composition of, 49

dextrose in, $49,+17$

formation of, +06

and activity of organs, $f 10$

and blood-pressure, 408,409

functions of, $5 \mathrm{I}$

gases of, 260

osmotic pressure of, $410,+$ II

post-mortem flow of, $4 \mathrm{I}_{2}$

Lyinphagogues, 406

Lymphatic glands, formation of lympl. ocytes in, 22

Lymphatics, 407

absurption of bile by, 355

Lymph-hearts, 177

Limphocytes, $17,22,47$

Lysin, 331,332 
Iavul.t acustic.1, $\$ 3.3$ Iute.1, s่no, 237,993

Magendic, foramen of, 882

pxperinents on nerve.routs, 790

Maggots, formation of fat ju, 526

M.uguesinu sulphate solution for blood. pressure tracings, 195

Make and break shocks, 702

Malapterurus, 736,737

clectrical nerve of, 737,758

double conduction in, 688

Malleus, 954. 955. 959

Maltase. $315,334,342,410,5$ I0

Maltose, 3. 320 absorption of, 416

Mammary glands, 537, I02 I

Manganese in bile, 337 absorption of, +17

Mannite, relation of, to levulose, 3

Manometer, differential, 89

Fick's C-spring, 85

Fick's elastic, 86

Hirthel selastic, 86

naximum and mininum, $\S_{5}$

mercury, IOI, I95

solutions for filling tubes of, 195

Marchi's solution, 693

Marginal veil, 746

Mariotte's experiment, 932

Massage of muscles, effect of, on blood. pressure, I 7 I

' II ast cells, I 8

Mastication, 300

Maturation of orum, I oos

Maxwell's spot. 994

Meconium, 396, IOI2, IOI 5

Mediastinum, 200

Medulla oblongata. anatomy of, 767 centres of, 8 I $4,815,816$ derelopment of, 748

Medullary groove, 746 , I0o9 sheaih of nerve, 677 developinent of, $74^{8}$

Megaloblasts, 2 I

Meissner's plexus, $29^{8}$

Membrana tectoria, $958,96 \mathrm{I}$

Memories, storage of. 866

Menière's disease, 834

Menopause, Ioo5

Menstruation, roo5

Mental processes, seat of, 865,866

Mesenteric ganglion, inferior, 473

Mesoblast, mesoderm, I008, Ioog

Metabolism, endogenous, 497, 504, 505, 534

exogenous, 497

in fever, $530,596,597,600$

in muscular work, $537,583,584$

in plants, 6

nitrngenous. laws of, 535-539

of carbo-hydrates, 5 I I
Metabolism of embryo, IOI 7

of fat, 522

of living matter, 6

of proteins, $\$ 96$

Metiumorphosis of larva, phagocytosis

in, 52

Meta-proteins, 2, 3, 9

Methaunoglobin, 46, 67 in urine, $4+4$

Methylene blue, reduction of, in tissues, 205

Mcthyl orange as indicator, 24, 393

Methyl violet as test for acids, 324

Metronome, 179

Metschnikoff's theory of phagocytosis, 52

Mett's tubes, 318,422

Microblasts, 2 I

Micturition, 471 centre, 472

Mid-brain, 747

Milk, assimilation of, 549 calciun and phosphorus in, $5+2$ carbon and nitrogen in, $5+6$ chemistry of, 6ro, 6ri composition of, $5+6,549$, IO2 I formation of fat in, 527 iroll ill, $5+3$, I O 2 I

salts of, 102 I

secretion of, I022

without pregnaney, 1022

Milk-curdling ferment, 323, 327, 6II

Millon's reagent 8

Miniature stomach, 373

Miotics, gI I

Mirrors, reflection from, 892, 893

Mitosis, 5, 1006

Mitral cells of olfactory bulb, 816 valve, 78,190

Moist chamber. 704

Molecular concentration, 398 layer of cerebellum, 830 of olfactory bulb, 8 I 6

Molisch's test for carbo-hydrate, I I

Monakow's tract, $765,78 \mathrm{I}, 848$

Monobutyrin, splitting of, by blood, 528

Monochord. 998

Mono-saccharides, 3 absorption of, 410 as glycogen-formers, $5 \mathrm{I}_{3}$

Moreau's experiment on intestinal juice, 386

Norphine, action of, on motor centres, 858

quantity of, for dog, 55, I 86

Mother-substances of ferments, 328 , $33 \mathrm{I}, 353,354$

'Motor-areas,' $8+3.8+4$ in dog. 843.889

in hedgehog. 872 
'Motor-areas' in man, s..4., $851,855$. 860

in monkey, 844

in opossum1, 872

in ornithorhyuchus, 872

in rabbit, 872

path, $774,776,792$

recovery after ablation of, 848 , $850,85 \mathrm{I}$

sensory functions of, 8.4, 856

Mountain sickness, 275

Lovements, co-ordination of, 798,831 , 837

Mucin, 3

forced, $8_{3} 6,837$

of bile, 335

Mucous glands, 319,345 changes in artivity. 352,425 nembranes, sensibility of, 981 , I 002

Müller's experiment, 273

Murexide test for uric acid, 484

Musca volitantes, 914

Muscarine, action of, on digestive secretions, 387

on heart, 150,183

on pupil, 9 I 1

Muscle, afferent impressions from, 835 , 983 path for, 984

composition of, 666,713

degeneration of, $698,700,8 x_{4}$

diffraction spectrum of, 642

direct excitaluility of, 633.706

clasticity and extensibility of, 630

gases of, 260

general physiology of, 627

glycogen in, 5 I 4 , 5 I 5

heat-production in, 583

heat-shortening of, 673

lactic acid production in, 667,668 , 716

oxygen consumption and carbon

dioxide production of, 263

permeability of, 67 I

polarization of, 726

proteins of, 672,714

reaction of, 667,716

respiration of, $26 \mathrm{I}$

rigor of, 263,715

smooth, contraction of, 645,647 , 713

sound, 660

stimulation of, 632, 704,707

by voltaic current, 636,704

structure of, 638

in polarized light, 64 I

Muscles, antagonistic, co-ordination of, 838

Muscle-interruptor, automatic, 648

Muscle-ncrve preparation, to make, 703
Muscle spindles, 229, 983, 984

Muscular contraction, chenical phe. unmena of, 666 ,

cluration of. 642

formula of, $684,686,742,743$

heat produced in, 583,663

idio-muscular, 659

in absence of oxygen, 26I, 295

influence of fatigue on, 6.98 . $652,708,709$

of load on, 645,708

of mental fatigue on. 654

of temperature on. 647. 706

of veratrine on, 654,709

isometric and isotonic, 645

lactic acid formed in, 667,668 , 7 I 6

latent period of, 642,710

mechanical phenomena of. 643

mechanism of, 640

of smooth inuscle, 645,647 . 713

1) ptical phenomena of. 638

pluysico-chemical conditions of, $6>1$

recording of, 706, 707

reversal of stripes in, 640

seat of fatigue in, 651,652 ,

708. 709

source of energy of, 537,586 , 669

superposition of, 656,7 I I

velocity of wave of, 659.719

voluntary, 660

seat of fatigue in, 652

work done in, 646

Muscular fatigue, 650

influence of stimulants on.

552

seat of exhaustion in, 651,652 cxercise, effect of, on pulse, 97. 192

sensations, $835,856,862,869,968$. 982

scuse, 983

tetanus, $656,657,7$ II

tissue, action of extracts of, 569

tone, 8I 3,886

work. nitrogen metabolism in, 537 relation of, to energy expended, $583,665,666$

Musical centres, 86 I

Mvdratics, 9 I I

Myclination, time of, in cortex, 854 . 855

Myocardiogram, I 55

Myocardiograph, I 88

Iyogenic hypothesis of heart-beat, I32 
IIser.uph, pendulum, 044

sisiple, 879.707

spring, 043.713

Mir pha, ujs

Mvosin, 072, 073.715

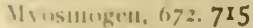

I1vot.tic irritatsilits, 802, 813

Mrxudema and thyroidectomy; 560

\issul secretion, 435

Ve.ur-point of vision, 9) $15,917,987$

Aecturus, ablation of cerebral hemi. spheres in, 84 I

equilibration in, after destruction uf interual ear, 836

intracorpuscular crystallization in erythrucytes of, 62

Vegative phase in coagulation, 38

Negative variation. See Action current

Nerve-cells, 677

changes in, after section of axoti, 601

in fatigue, 874

with age, 755

growth of, 754

number of, in brain, 757

Nerre, chemical changes in, 680, $88 \mathrm{I}$

composition of, 690,88 I

conductivity of, 686

crussing, 694, 867,868

degeneration of, $69 \mathrm{I}$

chemistry of, 692

double conduction in, 687

effect of temperature on excita.

bility and conductivity of, 681

effect of voltaic current on, 683 ,

\section{$74 I$}

fatigue of, 680

isolated conduction in, 68 ?

ininimum stimulus of, 681

pattern in regenetation, 695

polarization of, 726

refractory period of, 680

regeneration of, 694

chemistry of, 693

stimulation of, 680

structure of, 677

Nerve-ending, motor, 634, 635

Nerve-fibres, enumeration of, 775

size of, 745,758

Nerve impulses, ' antidromic,' I 65,688 , 7OI

nature of, 679

velocity of, 689,713

in reflex arcs, 800

temperature co-efficient of, 679

Nerve-muscle preparation, to make, 703

Nerves, classification of, 702

cutaneous, phenomena after sec.

tion of, $975-98$ I
Nerves in silu, law of contraction for 686,743

trophic, 690

Nervous activity, chemistry of, 880,88 I tissue, effects of extracts of, 560 reaction of, 6y I

Nervus erigens, 164, I65, 167, 301, 473 , 884

pudendus, vaso-motors in, 164, I 67

Neuril axis, primitive, 759

canal, development of, 746

Neuroblasts, 746,754

Neurofibrils, 748,750

theory of continuity of, 751

Neurogenic hypothesis of heart-beat, I 30

Neuroglia, 758, 817

Neurokeratin. 690

Neuro11s, 677, 748-757

development of, $747,752,754$

fibrils in, 748

itutrition of, 756

processes of, 749,752

scheme of inotor, 753

varieties of, 753

Nicotine, action of, on ganglion cells of heart, 150

of salivary glands, $36_{3}$. 365

on intestinal movements, 307

on skeletal muscle, 634,635

on sympathetic cells, 165

Night-blindness, 948

Ninth nerve. See Glosso-pharyngeal

Nissl's bodies in nerve-cells, 749,755 . 873

chromatolysis of, $369,745$. $756,873,874$

method of staining, 749

Nitrogen, execretion of, in starvation. 532

after muscular work, 538

variation of, with protein in food, 535,612

estinuation of total, 482

in proteins, 1,529

of body, 528

requirement, minimum, 533, 544, 539

starvation, $53 \mathrm{I}$

Nitrogenous equilibrium, 529, 533

metab lism, 496

influence of fat and carbo-

hydrates on, 523, 534

of muscular work on, 537 .

538

in starvation, 530,532

laws of, 535,537

Noud vital, 8 I 4

Normal solutions, 439

Normoblasts, 2 I 
Notochord, 1009

Nuclease. 507, 510

Nucleic acid. I, 2. 5

Nucleins, 1, 2, 506

formation of uric acid from, 506

Nuclei pontis, $768,777,784.828$

Nucleolus, 874. 1004

Nucleo-proteins, 1, 2. 5, $42,47,506$ hydrolysis of, 507 influence of, on coagulation, $34.3^{8}$

Nucleus ambiguus, 825 cuneatus and gracilis, 767,772 dentatus, 760,780 globosus, 780 of Deiters, $780,781,792,824$ tecti, $780, S_{24}$

Nucleus, function of. 5 daughter, 5, 1007 influence of, on oxidation, 260 structure of. 5

Nucleus-plasma relation, 874

Nussbaum's experiments on renal excretion, 460

Nystagmus, 831

Oatmeal as a food, 546,547

Obermayer's reagent, 479

Obesity, Banting cure for, 534

Occipital cortex and vision. $8_{5} \&, 8_{59}$

Octopus macropus, saliva of, $36 \mathrm{I}$

Oculo-motor or third nerve, 8 I 9

Odours, classification of, 965

Edena, 40S

Esophagus, contraction of, 302, 303, 713

successive combination of reflexes in, 506

(Estrus, 1006, 1022

Ohm. 6 I 6 reciprocal, 26

Ohm's, law, 6I6

Oleic acid, 12

Olein, 4, 12

Olfactometer, 906

Olfactory bulb, structure of, 816,818 glomerulus, 816

nerve, 816

sensations, nhis

tract, $S_{17}$

development of, $7+7$

Olive, 767,771 connections of cerebellum with, 780 superior, 824

Oncometer, 117,467

Opacities inthe eye, 914.929

Ophthalmometer, 906. 990

Ophthalmoscope, direct method, 919. 920,994

indirect method. 919.994

testing errurs of refraction by; sof. 919. 994
Opitz on velocity of blood in veins, $12 \mathbf{I}$. I 22

Opsonic index, 53

Opsonins, 53

Optical constants of the eye, 902 of reduced eye, 904

Optic axis, 898,9 I 4

disc, $900,902,932$

lobes, 829

nerve, 818 efferent fibres of. 819

radiation, 785.818

thalami, $747,768,772,781,784$. 818,820

and tegmental path, 773. 779 . 784

functions of, 829

Optimum temperature, 315

Optogram, 935

Orbicularis oculi, displacement of centre for, 872

Orcin reaction for pentoses, 491

Ornithin, 503

Osmic acid test for fat, I 2

Osmosis, $39^{8}$

Osmotic pressure, 398,492

of proteins, 406

of solid tissues, 4 II

resistance of erythrocytes, 64

Otoconia, $\$_{33}$

Otoliths, 833

Output of heart. 127

Ovary, grafting of, 1023

influence of uterus on. 1005. 1006 internal secretion of, 556,1005

Overtones, 280

Orulation, I004, 1006

Orum, development of, 1006

Oxalates and coagulation, 34, 55 in urinary sediments, 442,495

Oxidation, seats of, 259 nature of, in body, 264

Oxidizing ferments, +2, 68, 26.4. 295 $314,507,509,510$

Oxvalanin, 332

Oxybutyric acid in diabetes, 520

Oxydases. Sce Oxidizing ferments

Oxygen, amount consumed, 241, 243 . 293

artificial respiration with, 189

balance, 540

deficit. 242,541

dissociation curve of blood, 255

estimation, 25 I

in blood, 252

inhalation, 46

in heart, 585

in resting muscle. 584

partial pressure of, in blood and alvcolar air, 258

toxic effects of, 274 
Oxigen, used up in nuscular work, $26 \mathrm{I}, 263$

Oxyutic or acid-forming cells, $3.5,3+9$. 350

()xyphenvlalinin, 2

Oxiproliu, 332

Oysters, glyougen in, 608

Pacinian corpuscles, 960,983

1'ait1, 960, 1968, 973. 1000, I00I

centre for. 856

reftrred, $700,982,080$

seusatious after section of cu.

taneous nerves, 975, 979

Painful impressions, paths of, 795

decussation of, 793

frow interual organs, 799,973

Palinitiı, 4

l'ancreas, changes in, during secretion, 346

internal secretion of, 553

nerres of, 378

relation of spleen to, $38_{2}$

Pducreatic juice, artificial, 428 adaptation of, to food, $38 \mathrm{I}$

to lactose. 382

composition of, 330

ferments of, 33 I, 428

freezing-point of, 357

quantity of, 331

rate of secretion of, $380,38 \mathrm{I}$, ${ }_{3} \mathrm{~S}_{2}$

secretory pressure of, 382

to obtain, 330,429

Papillary muscles, 78,79

Parabiosis, 1024

Paradoxical contraction, 729, 74 I

Paralysis, crossed, 822

Paralytic secretion of intestinal juice, 386

of saliv:a, 369

Parauvosiuogeu, 672, 7 I5

Paraphasia, 864

Paraplegia, reflex movements in, 8 I 2

Parasternal line, 82

Parathyroids, $55^{8}$

effect of excision of, 559

l'arenteral absorption, 418

Parotid, changes in. during secretion, 346

Pars intermedia of seventh nerve, 821 , 822

of pituitary, 566

Parthenogenesis, 1004 artificial, 1007

Partial pressure, $24^{8}$ measurement of, 249,256 of air of alveoli, 242, 257 of blood-gases, 256

Parturition, 1019

Pause of heart, 79,80 leduncle, inferior cerebellar. See Restiforin body

middle cerebellar, $760,768,780$, 832

superior cerebcllar, $760,772,780$

l'elvic nerve, $164,165,310,884$

l'enclulum myograph, 644

movements of intestine, 306

l'entoses, tests for, 490

Pentosuria, 450, 520

Pepsin, 323, 326, 426

sccretion of, 349,350

rate of, 377,378

Pepsinogen, 353

Peptones, 2, 3

absorption of, 419

effect of, on coagulation, 35, 40, 55

on blood-pressure, $20 I$

in diet, 534

reactions of, 10,487

Percussion of lungs, 217

Pericellular basket, 754

Perikaryon or cell-body, 748

Perilymph, 954, 956

Perimeter, 946

Perimetric chart, 947,991

Periodic breathing, $238,275,288$

Peripheral nervous centres, 808 reference of sensations, 980

Peristalsis, 302, 303, 306, 659

Peristaltic rush, 308

Peritoneal cavity, absorption from, 406

Peritoneum, sensibility of, $973,98 \mathrm{I}$

Pernicious anæmia, blood-count in, 19 quantity of blood iir, 49

Peroxydase in blood-corpuscles, 68

Personal equation, 873

Perspiration. See Siveat

Pettenkofer's test for bile-acids, 377 , 430

Peyer's patcles, formation of lympho. cytes in, 22, 298

Phagocytosis, $5 \mathrm{I}$

Phakoscope, 906, 987

Phenol, formation of, in intestine, 395 in urine, 445.532

Phenolphthalcin as indicator, 24, 393

Phenyl-alanin, 1, 332

Phenyl-hydrazine test for sugar, 320 , 488

Phlorizidin and fat migration, 527

diabetes, 52I, 609

effects of, on foctus, ror6, ror 7

Phloroglucin reaction for pentoses, 490

Phonograph, analysis of vowel sounds by, 284

Phosphates in urinary sediments, 439 , 494

in urine, $444 \cdot 486$

Phosphatides, $337,526,690$ 
Phosphewes, 924

Plosphorescence, oxidation in, 260

Phosphoric acid, estimation of, 478

Phospho-proteius, 2

l'hosphorus in milk, 542

influence of, on protein metabolism, 526

poisoning, fat metabolism in, 525

Photo.chemical substances, 735. 934

Photo-electric reactions, 735

Phrenic nerves, 223. 224. 289

action current of, 724

nuion of, with sympathetic, 696

nuclei, connections of, 223

Plysiological salt solution, $\mathbf{1 7} 8$

Physostigmine, action of, on digestive secretions, 387

on pupil, 911

Pia mater, $75^{8}$

Pigmented epithelium of retina, goo, 034

and risual purple, 936

l'igments, excretion of, by kidney, 458 Pigment spots and light sensation, 897 Pilocarpine, action of, on digestive secretion, 387

un heart nerves, 150,183

on lymph formation, 408

on pupil, 9 I I

on salivary secretion, 425

Pilu-motor ne-ves, $165,695,884,975$, 979

Pineal body, 571,830

liutrowski's reaction for proteins, 7

Pitch, 280

appreciation of, $y, 62$

sensitiveness of ear for sounds of different, 965

Pithing a frog, 177

Pitot's tubes, I 3

Pituitary body, 819,830

action of extracts of, 568

effects of removal of, 567

internal secretion of, 566

Placenta, autolysis in, 510

bile-pigment in, 356

exchanges in, 1013

glycogen in, 514, 1014, 1016

passage of blood - substances through, IoI 4

Plants and animals compared, 6

Plasma, blood., I 5, 25, 31, 4 I

Plasmine of Denis, 3 I

Plasmolysis, 400

Plasmon, 535

Plethora, hydramic, 462

Plethysmograph, II 7, 159,194

l'leural cavity, 209

Pneumonia after section ol vagi, 237

Pneumothorax, 209 l'oikilothermal animals, 572, 577

l'oiseuille's space, го9, 177

laws for capillary flow, 77

lolar bodies, 1007

I'olarineter, 489

I'olarization of light, 64 I

of musele and nerve, 726 positive, 727

Pole-changer, 625

poliomyelitis, anterior, degeneration in, 775

knee-jerk in, 812

nerve anastomusis in, 868

Polycythamia, 19, 23

Polygraph, 94, I93

Polypeptides, 2, 3, 535

l'olysaccharides, 3

absorption of, 416

l'ons, $747,768,777,784$

functiuns of, 828

l'ortal vein, dextrose in, 417 vaso-motors of, $16 . t$

P'osterior corpora quadrigemind and auditory nerve, 824,828

horn, cells of, 762

longitudinal bundle, 773,824

root-fibres, branching of, 769

overlapping of, 790,857

roots, degeneration after section of, $693,768,769$

loss of movement after section of, 857

loss of sensation after section of, 790

I'ostero-external and postero-median columns, 763,768

Post-ganglionic fibres, 868,883

l'ost-sphygmic period of cardiac cycle. 90

l'osture, influence of, on blnod-pres. sure, 173,199

(1) pulse-rate, 99, I95

I'otassium in nerves, 961

in erythrocytes, 255

in nuclei, 5

microchemical test for. 5

relation of, to heart-beat, 139,185 .

186

Potassium ferrocyanide test for pro-

teins, 8

Potential, 6 I 5

differences of, in tissues, 718

Precipitins, 30, 418

Predicrotic wave, 95

l'refrontal region of cortex, function of, 866

P'reganglionic fibres, $868,88_{3}, 88_{4}$

'repyramidal tract. See Monakow's tract

I'resbyopia, 9 I6

['resphygnic period of cardiac cycle 90 
Previour and depresser nerves, 1.57, 170. $1>1$

P'rensure, arterial, 100, I1)3. 195. 198

endiveardiac, $S_{4}, 20$

intracr.titisl, 875

intrathoracic, 210,221

negative in heare-. 92,96

respiratury, 222

secretors: of saliva. 363

sensitions, 968, 971, 98x, 1000

l'runary colours, $9+1$

position of eyes, 951

Primitive streak and groove, roos

Prochromatiu, 5

Projection of inage into space. 923.928

Prolin. 332

Proulucleus, I007

Proprio-ceptive reflexes, 810 system and cerebellum, 831,832

Proprio-spinal fibres, 761,765

Pro-secretiı, 380

Prosthetic groups, 2

P'rotagon, 47.69 I

Protamins, 2

influence of, on coagulation, 4 I

Proteins, absorption of, 4 I 8

circulating. 536

classification of, 2

rleavage products of, I, 332

in diet, 535

composition of, I, 525

conjugated, 2

derivatives of, 3

formation of carbon dioxide from, 509

of fat from, 5 I 5

of glycogen from, $5 \mathrm{I} 2$

in urine, $4+3,486$

living and dead, 499

metabolism of, 496

endogenous, $497,534,536,545$

exogenous, 497,535

influence of poisons on. 526

minimum requirement in food,

533, $544,545,583$

inuscular energy from, 538

passage of, through placenta, ror 4 reactions of, 7

scheme for testing for, I3

specificity of, $3,+18$

synthesis of, $2,420,497,510$

Protein-sparing action of other food substances, 523, 534. $53 \mathrm{I}$

Proteolysis, 332

difference between acid and ferment, 535

Proteoses, 2, 3, 325, 421 action of, on blood-pressure, I 57. 201

influence of, on coagulation, 35,40 , l'rutenses, tests for, 10. 486

Protopathic somsibility, gis

l'rutoplasm, composition uf, I functions of, 6,628

structure of. 4

Prothrombin, 33

l'rotovertebrie, 1009

Pseudo-fatigue, 653

Pseudo-globulin, +2

Pseudopodia, 16, 627

Pseudo-reflexes, Soz

Psychical secretion of gastric juice, 374 of saliva, $37 \mathrm{I}$

processes. seat of, 865,866

Ptosis, 820

Ptyalin, 320, 422

Pulmonary catheter, 257

Pulse, the, 92

anacrotic, 97

aortic, 95

rharacters of, 94,192

dicrotic wave of, 94,95

frequency of, 98,195

influence of posture on, 99. 156 .

\section{I92}

in faetus, Ior 7

respiratory variation in 269

of swallowing on, 155, I 95

jugular, 9 I, I93

venous, 91, I20, 122, I93

Pulse-tracings, 94, I 92

effect of amyl nitrite on, 97, I92

of muscular exercise on, 97. I 92

from different arteries, 97, I93

from jugular vein, I37, I93

secondary waves of oscillation in, 95

Pulse-wave, disappearance of, in capil. lary region, 103

reflexion of, 95,96

velocity of, 99

Pulsus alternans, $I_{42}$ bigeminus, 142

Pulvinar, 818, 829

Puncture fever, 595. 596, 597 glycosuria, 5 r 8, 555

Pupil, Argyll-Robertson, 9ro changes in, during accommoda. tion, 909

constrictor nerves of, $88_{3}, 908$

dilator nerves of, 908,996

eccentricity of, $9 \mathrm{I} 4$

influence of drugs on, 9 I I of adrenalin on, 563 of light on, 818,829

photography of, 9 ro

Purin bases, 2, 397,44I, 538, 507 in fever. $60 \mathrm{r}$

Purkinje's cells in cerebellum, 753,754 figure, 930,998 
Purkinje-Sanson inuges, $n \cap 6,987$

Pus cells, origin of, 54 glycogen in, 47

Putrefaction, and crystallization of hisuoglobin, 45

hamolytic effect of, 27,62

products of proteins, action of, in blood-pressure, 570

Pycnometer, 57

Pyloric sphincter, 305. 309 regulation of opening of, 305 . 380,392

Pyramidal cells, 751 giant, 774

tracts, 765,777 connections of, $774,775,852$ in different animals, 776 relations of, to pons, 828

Pyramids, 767

connections of, 774

decussation of, $768,774,777,792$

Pyrimidin bases from nuclein sub. stances, 507

l'yrocaterhin in urine, $436,+45$

Pyrogallic acid, absorption of oxygen by, 251

Quadratus lumborum, action of, in respiration, 2 II

Radiation, loss of heat by, 578

Radium rays, visibility of, 950

Raffinose, 3 I 6

Reaction, in physico-chemical sense, 23. 439

of blood, $23,54,57$

of degeneration, 698

of intestine, 392,393

of tissue liquids, 2.3

of urine, 439

regulation of, $2+$

time, 872

Receptive substances, I 50, I66, 635 field of reflexes, 801

Receptor of reflex arc, $796,798,87 \mathrm{I}$

Reciprocal innervation, in reflex inovements, 801

in volitional movements, $83^{8}$, 846

of bloodvessels, I 7 I

Recurrent fibres, 372,693, $79 \mathrm{t}$

sensibility, 79 I

Red nucleus, 781,784

Reduced eye, 903

Reductases, 509

Reference of scnsations, peripheral, 980

Referred pain, 790, 980, 982

Reflection of light, 892,893

Reflex action, 796, 885, 887

anatomical basis of. 797

inhibition in, 800 , 801
Reflex ares, irreciprosal conduction in, 688,799

peruliarities of coudurtion in. 799, 800

refractory state in, 800

summation of stimuli in, 8oo

'cardiac death,' 156, 235

centres in rord, 811

peripheral, 808

figure, 804

tiine, 809,886

Reflexes, action of strychnine on, 80 I of tetanus toxine oll, 801

after-discharge of, 800

axon-, 372, 809

combination of, 805

common path of, 797,798

compounding of, 798

ro-ordination of, so5

rrossed, 793

differtices between cortica

reactions and, $8+7$

extero-and proprio-reptive, 8 ro

facilitation of, 805, 807

from sympathetic ganglia, 371 , 809

in disease, 8 10

inhibition of, $800,801,806,886$

irradiation of, 802,885

'purposive' character of, 805

reinforcement of, 805.807

resuscitation of, 880

spinal relation of, to brain, 806

superficial and deep, 8 Io, 8 II

Refraction of light, $89 \uparrow-896$

in eye, 902

Refractive index, 894 of media of eye, 903

Refractory period of heart, I 4 I in reflex arc, 800

Regeneration of nerve, $69+-698$ autogenetic theory of, 697 chemistry of, 694

of nerves after anastomosis, 807

of tissues, $\mathrm{IOO}_{3}$

Reil, island of, 855,865

Renal nerves, I6 I, 468, 469

secretion, theories of, 455

tubules, 453, 454

vein, ligation of, 468

Renin, 570

Rmnin, 323, 326, 327, 353,427

Reproduction, a property of living matter, 6

se'xual, 1004

Reserve air, 220, $29 \mathrm{r}$

Residual air, 220, 29r

Resistance, electrical, 615 incasurement of, 60,617 osmotic, of erytlirocytes, 64 thermoneter, 574, 664 
Resoniance, 999

tone of ear, 8n, 660

liespiration, accessory phenomena of, 215

afferent nerves of, 223,289

and pulse, relation in frequency of, 219

apparatus, $2+1$

artificial, 187, 21.4

intluence of, on blood-pressure, 269

with oxygen, I 89

calorimeter, $2+1,579,613$

chemistry of, 240, 292-295

Cherne-Stokes, 238

comparative physiology of, 206

cutaneous, 206, 275

efferent nerves of, 223

external and internal, 206

forced, $214,219,246$

frequency of, 218

gaseous changes in, 24I, 25 I

heat lost in, 579. 578, 61 3

in condensed and rarefied air, $272-275$

influence of ragi on, $224-229$. 289

of cutaneous nerves on, 229

of 'higher paths' on, 224

of muscular exercise on, 229

on blood-pressure, 265

on capacity of pulmonary vessels, 266

on pulse-rate, 269

internal, 206, 257, 259

mechanical phenomena of, 209

of muscle, $26 \mathrm{I}-264$

of tissues, 264

reflex inhibition of, 229

regulation of, 230

types of, 213

Respiratory arhythmia, 270

automatism, 233, 814

capacity, 220, 29I

centre, 223

action of alcohol on, I 75, 236

of carbon dioxide on,

230,242

of chloroform on, 234

of deficiency of oxygen

on, 230,275

of venous blood on, 230232

initial rate of discharge of, in resuscitation, 234

centres, spinal, 236

'dead space,' 22 I

exchange, $24 \mathrm{I}$

impurity, permissible, 243

movements, 2 II-2I 3

duration of, 218
Respiratory organs, anatoiny of, 207

pressure, 222

pump, I 2 I

quotient, 242, 295

in different animals, 246

in muscular work, 242

sounds, $216,29 I$

tracings, $218,226,227,228,287$. 288

Restiform body, $760,767,771,779$, 780,823

and direct cerebcllar tract, $77 \mathrm{I}$

and olive, 780

constituents of, 779,780

Restitution processes, 585

Resuscitation of central nervous system, 879, 880

of heart, I30, I59

of reflexes, 880

of respiratory inechanism, 233. 880

scratch-reflex in, sos

Reticular formation, 768 posterior, 763

Retina, adaptation of, 935.946

curves of excitation of, 942

development of, 747

electromotive phenomena of, 734 .

735,934

fatigue of, 944,997

intermittent stimulation of, 937 $93^{8}, 998$

photography of, 910

pigmented epithelium of, 90o, 932.

934,936

sensibility of different parts of, 946 of, for colours, 947

structure of, 899

time necessary for excitaton of, 937

Retinal bloodvessels, shadows of, 930 , 932,998

image, formation of, 902,986 size of, 904

Retinoscopy, 920, 994

Rheocord, 619, 620

simple, 620,705

Rheotome, differential, 723

Rhinencephalon, 817,862

Rhodopsin. See Visual purple

Ribs in respiration, 2 I 2

Rigor mortis, 6o8, 671

analogies of, to muscular contraction, 673

influence of labyrinth on, 835 of nerves on, 675

production of carbon dioxide in, 263,673

of lactic acid in, 669, 7 I I

removability of, 676

time of onset of, 675 
Rigor, heat., 263,67t

production of carbon dioxid. in, 263

Ringer's solution, 186

Ritter's tetauus, 636, 662, 727.743

Ritter-V'alli law, 682

Rods and cones in vision, 932, 935.936. $9+8$

Rolaudo, fissure of, 844,856

substance of, 759,763

Rontgen rays, for study of gastric movernents, 306,3 II

of lungs, 217

of vomiting. 3 I 2

visibility of. 950

Root-fibres, posterior, course of, in cord, 769,79 I

Roots of spinal nerves, functions of, $790,792,884$

section and stimulation of 884.885

Rosolic acid as indicator, 24

Rubro-spinal tract. See Monakow's tract.

Saliva, action of, in the stomach, 322 adaptation of, to food, 369 anylolytic action of, 319.423

chemistry of, 3 I 9,422

freezing-poin of. $35^{8}$

functions of, 320

influence of nerves on secretion of, $363-37 \mathrm{I}$

paralytic secretion of, 369

reflex secretion of, 360

in vomiting, 312, 369

secretory pressure of, $36_{3}$

Salivary centre, 37 I

corpuscles, 320

fistula, 370

glands, 3 I9. 345

action currents of. 734

blood-flow in, during activity, 364

changes in, during secretion, $346,347,352$

cranial nerves of, 362,424

heat production in, 364, 586

removal of, $57 \mathrm{I}$

sympathetic nerves of, 363 . 425

'trophic-secretory' fibres of. 366

Salmin, 2, 503

Salol, action of, on bile secretion, 388

Salt-hunger, 542

gastric secretion in, 35 I

Salt solution, phy'siological, 178

Salts, absorption of, 417 action of, on heart muscle, 185 in diet, 549
S.les in netabolism, 518

of hile, 337

of bune, 529

of erythrocytes, 43

of leucocytes, 47

of living matter, I

of lymph, 50

of inilk, 542, 550

of muscle, 667

of serum, +2

of urine, $\$ 36,4+4,4+9$

passage of, through placenta, IOI 3

Saponification of fats, I1, 12, 338

Saponin, action of, on bloud-corpuscles, $27,28,62$

Sarcolactic acid. See Lactic acid

Sarkin. See Hypoxanthin

Scalene muscles, in inspiration, 211

Scarpa's ganglion, $8 \mathbf{2 3}$

Scheiner's experiment, 9 I $\% 987$

Schmidt's fibrin-ferment, 32, 57

Schïtz's law of ferment artion, 317

Sciatic nerve, to expose, 198

Scleroproteins, 2, 529

Scopolamine, passage of, through placenta, Ior 3

Scratch-reflex, the, 799, 800, 804, 805. 806,887

Scurvy, prevention of, by vegetable acids, 552

Sebaceous glands, sebum, 435. 473 . 527.737

Secondary contraction, 729,738 with heart. 188

Secretin, $347,379,384,429$

gastric, 375

Secretion, electromotive changes in, 734 internal, 552

of corpus luteum, 557

of kidney, 569

of liver, 552

of ovary. 556

of pancreas, 553

of parathyroid, 559

of pineal gland. 571

of pituitary body, 566

of spleen, 57 I

of suprarcnals, 563,201

of testes, 556

of thymus, 557

of thyroid. 558,560

paralytic, 369

psychical, 371,374

Secretory pressure of bile, 386

of pancreatic juice, 382

of saliva, 363

of urine, $\$ 66$

Segmentation of food in intestine, 307

Self-digestion of stomach, 360,434

'Self-steering' of respiratory' move. ments, 226 
Semularcular eidulals. $823,833,456,057$ andel equilitoretton, $83.4,837$ and forced mesements. 830

Simulunar vilve's, 71), 19 I and (licrotic witve, y) 6 moment of clusture: of (S. C [)(int). Bंy

Sernisecetiun of cord. 79) 3

Sunsations, dissociation of. 795. 70) lecelization of, 1982 after section of cutameous nerves, y8o

relation of, to stimulus, yst

Se'llses, the, 8y,

Sensibility of interual organs, y73, 9fir sensori-motor functions if "motor" cortex, 856

Sensory areas. 857

patlis to brain, 791, 794. 779 decussation (if, 703

Serin. 332

Serous glands, 319,346

Serum, 24, 26, 27, 30, 41, 57

composition of, 41,57

conductivity of, 317

ferments in, 42

freezing-point of, 26,357

specific gravity of, 25.57

reaction of, 57

Serum-albumin, $41,42,49,57,487$. 498

amino-acids in, I

crystallization of, 3

Serum-globulin, $32,4 \mathrm{I}, 42,49,57,487$

Serum-proteins, in starvation, 498 source of, 498

Serratus posticus, action of, in respira. tion, 2 II

Seventh nerve, $\$ 22$

Sexual organs, internal secretion of, 556

Shadow test. See Skiascopy

Sham feeding, 324, 352, 374

in puppies, 375

Shivering and temperature regulation, 59 I

Shock, spinal, $796,800,803$ surgical, $1>5$ acapnia as a factor in, 169,175

Side-chains, 635

Sighing, 239

Sigmoid flexure, 309, 3 I I

Signal, electric, 626

Silent areas of cortex, 865

Single vision, theories of, 923

Sino-auricular junction, stimulation of, 183

node, 72

Sinus venosus, 72 stinulation of, 144 sixth nerve or ablucetus, $\mathrm{x} 2 \mathrm{z}$

Skitte, clectrical organ wf, 738

Sikitul, 33.3. 3195

Skituxyl in urint. $\$ 3(),+15$

Skill, currents of. 734

excretion by, 473

impulses from, in enpilibration, 835

respiratiou by, 275

varnishing $1 f, 276,474$

Sleep. 873

annount necessury, 876

cerebral circulation in, 875

depth of, 376

effect of, on pulse, 99

gaseutus exrhange in, 245

intracranial pressure in, 875

plethysmographic tracings frum arth in. 875

theories of causation of, 875

Sinell, 965, 999

centre for, $8 I 7,86 I$

Sirake venom, effect of. un coagulation, 39

on blood-corpuscles, 27

Sileezing, 239

Soda-lime, absorption of carbon dioxide by, 24I, 294

Sudiun, relation of, to heart-beat, I 39 chloride, action of, on heart. strips, I 85

amount needed in food, 550 influence of potassium salts on, 550

citrate solution for blood-pressure tracings, I95

lyydrosulphite, absorption of oxygen by, 25 I

Solidity, judgment of, 925, 926

Sorbite, relation of, to dextrose, 3

Soret's hamoglobin band in violet, 45 .

47

Sound, cranial conduction of, 960, 999 pictures, 964

Sounds, complex, analysis of, 962

Specific energy, 870, 930 sensibility; 980

Spectroscope, 43,65

Speech, 282

centre for, $86 z-864,872$

Spermaceti, absorption of, 414

Spermatozoa, development of, Ioo4

Spermin, 556

Spherical aberration, 9 I 2, 99 I

and irradiation, 950

Sphincter ani, 3 II, 8 I 3

cardiac, 302,309

ileo-colic, 308,310

pylori, 305, 309

Sphygmic periud of cardiac cycle, go 


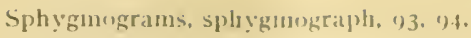
192

Sphygmomanometer of Erlanger, 104. I98

of Riva-Rocci, 198

Sphygmometer of Hill and Barnard, 106

Spider-puison, action is, on bloodcorpuscles, 27

Spinal canal, $7+6,754$

Spinal curd, action currents of, 733.793 action of strychuine on, 797. 801

of tetanus toxine on, 797. SoI

allatomy of, 762

ascending tracts of, 763

automatic functions of, 813

centres of, 811,814

complete section of, 786

conduction of impulses by, 788

descending tracts of, 764

endogenous fibres of, $76 \mathrm{~s}, 765$, 795

excitability of tibres of, 788

functions of, 788

grev inat ter of, 762

reinoval of, 787

semisection of. 793

white inatter of, 763

Spinal frog, experiments on, 885,886

gauglion, cells of, 747,753

fatigue of, 809,875

fibres, bifurcation of, 760

relation of, to posterior root.

fibres, 693, 808

preparation, mammalian.

887

reflexes, 796, 799

centres for, 811

inhibition of, soo, 8o1, 806 , 886

long, ios

relation of, to brain, sut

sliort, boz

respiratory centres, 236

roots, functions of. 790 section and stimulation of,

\section{4}

shock, -86

Spindle, nuclear, son 7

Spino-tectal fibres, $77 z$

Spino-thalamie fibres, 772

Spirometer, 219, 29I

Splanchnic nerves, I61, 309, 470,518 and gastro-intestinal move. Incuts, 300 and glycogenulysis, 51'; and ilen-colic sphincter, 3 I0
Spleen and blood-formation, 2I, 571 and blood-destruction, 21, 57 I and formation of bile-pigment, 571 and formation of trypsin. $3^{8} 3$ proteolytic ferment of, 383 relation of, $t \omega$ pancreas, $382,57 \mathrm{I}$ relinvial of, $57 \mathrm{I}$

Spungioblasts. $7+6$

Spring myograph, 643

'Staircase ' or 'treppe,' I 43.64\%.65I

Standard dietaries, 543-549 solution of ammonium sulpho. cyanide, 478

of silver nitrate, 478 of uranium nitrate, 478

Standing, 837

Stannius' experiment, 132, I5 I, 183

Stapedius, 822,955,96I

Stapes. 95\%. 955.959

Starch, 3 action of acids on, I I digestion by saliva, 320,423 tests for, Io

Starvation, excretion of salts in. $5+2$ loss of weight of organs in. 530 unetabolism in, 530, 532 premortal rise in urea excretion in. $53 \mathrm{I}$ respiratory quotient in, 243 serum proteins in, $49^{8}$

Stasis, 53. 177

Stationary air, 220

Steapsin, 33 I, 334

Stearin, 4

Stenson's experiment, 670

Stercobilin, 336. 396

Stereognosis, 856

Stereoseope, 925

Stereoscopic vision, 1/24

Stethograph, 287, 289

Stethoscope, I9I

Stilling's sacral and cervical nuclei, 762

Stimulants, 550

Stimulation, law of polar, 637 chemical, of nerve, oro electrical, $638,681,685$

Stimuli, adequate, $798,870,891$ summation of, 655,7 I I

Stukes-Adans discase and auriculu.

ventricular bundle, I 37

Stonach, absorjtion irom, 3') I auto-digestion of, 360,434 excision of, 329

glands of, $3+5$ changes in, during secretion. $3+7$

movements of, 304

nerves of, 309

protection of, frum gastric juice, 359 
Stomach, transverse biund of, 3n5, 312 Strubismus, 8200, \$22, 721

Sitr.wherrecestrot, effect of, on lymple. flow, 12 2

Stri.e acustica, 821

Sitring galvinometer, Gis)

Strom.t of inlutired (norpuscles, is

Sitrumulur, 112, 122, 173

Strontium and bulle formation, 5.t2

sirverluine, artion of, en icurd, 797, sor. 886

Silurin, 2

(c):intus, rhvthus of, b6,

Sublingual ganglion. 36,3

Submaxillary glambl, gatseons metalle ulism uf. 26,4

Sulstance of Rulando, 750. 703

Sulstintiat nigral. 76 s., 777

Sucous entericus, $3+1$

action of, in digestion. 342$34+$

alaptation of, to food, 387

influence of nerves on, 386

Suckling. food requirement of, 54 ?

suerase. Sic Invertase

Surrose. See Cane-sugar

Sudo-motor nerves. Se? Sweat-nerves

Singar, absorption of, $405,+16,433$

and muscular contraction. 5 I 7

'centre' in bulb, 5 I 8.555

destruction of, in blood, 517

estimation of. by Fehling's solution. 489

by polarimeter, 490

exrretiun of, by kidneys. 5 I6, 609

fate of, in organism, $5 \mathrm{I} 6$

firmation of, in liver, 515

in blood, 4 I, 462,5 I I, 5I6, 5 I0

regulation of, 554

in urine, $451,488,516$

phenvl-hydrazine, test for, 488

Tronmer's test for. Io. 488

yeast, test for, 489

Sulphates in urine, $437,4+4,4+5$

estimation of, 479

Silphoryanide in saliva, 319, 422

in urine, +45

Sulphur, 'neutral,' 497

Summation in reflex are, 800

of stimuli, 655, 7 II

Superior laryngeal nerve, 825

and deglutition, 30.

and respiration, 227,289

Superposition of contractions, 656, 7 II

Supplemental air, 220, 29 I

Suprarenal capsules, secretion of, 563 cholin in cortex of. 556

extract, action of, I57, I63, 20 I, 563,564

serretion of, 563
Suprarenin, 56f. Sie alsu Aelrenalim

Surfare of body, relation to mass, 502. 5.49

tonsion, influenre of bile: (1), 340 in Intusenlar intrartion, 6.10

Suspernstry ligament, gon

in accommodition, 907,908

Sintures, 203

Sinturing of bloodressels, roz.t

uf 11 irves. 867

Swallowing, effert of, on pulse-rate.

I 55,195

Sivialt. 473

rentres. +75

nerves, $47.1 .+75,979,975$

fluantity (if. 47.4588

Swim-lyladder, ghases of, 250

Sivian aplueduct. 810

Simpathetic, ahdominal, reflex in.

hibition through, I 5 t

cardiar fibres of, in frog, 143, 146, I 84

in manmals. $I+7, \quad 148$. I90

cervical, vaso-motor fibres in, I.59. 202

dissection of, in dog, I9o

in frog, I 8 ?

fibres for salivary glands, 367 , I $60,363.424$

pilo-notor fibre's in, 69.5

pupillo-dilator, fibres of. 695

regeneration of, 693.695

union of, with phrenic. 606

course of vaso-motor fibres in.

I 65

ganglia, action of nicotine on. I 65

development of. 754

supposed reflexes from. 8o9

ganglion cells, 754

vibration, 999

Synapse, 749, 785, 797, 800

membrane theory of, 749,75 I

Syncope, 173

Syncytium, 6, 10I0

Synergic muscles innervated in re. flexes, $80_{3}$

Srringonyelia, dissociation of sensations in, 795

Systole of heart, 78

Tachograph gas, I I 5

Tachycardia in disease, 826

Tactile impressions, path of, in cord, 795

sensations, 968 centres for, 856,842

Trenia terminalis, 78

Talbot's law, 938,998 
Tallyuist's metlind of estimating hamoglobin, 70

Tambour receiving, 83,217 recording, $x_{3}, 192$

Taste, 966. 999 nerves of, $821,822,825,966$ sensations, rlassification of, $9 f_{1}$. 999

Taste-buds, 966

Taurin, 337

Taurocholic acid, 336

Tea, 44 I, 550, 55 I

Tears, 435, 902

Teeth, 300

Tegmental afferent path. 772,779

Tegmentum, 768

Teloxlendrion, 749

Temperature in axilla, 603 in cavities of heart, 503 in different animals, 577 influence of age on, 607 of humidity on, 588

in mouth, 603

in rest um, 577,603

of blood, $577,602,604$

of body, daily variation of, 605

of brain, 585, 604

of skin, 574,605

post-mortem rise of, 607

regulation of, $5^{8} 7$

"chemical" and 'physical, 590

effect of thyroidectomy on, 593

in hibernating animals, 596 sensations, paths for. 793.795 topography, 602

Temporo-sphenoidal convolutions and hearing, 824,860

'Tendon-reflex,' 802

Tendons, nerve-endings in, $99_{3}$

Tension of blood-gases, 256 of oxygen in human blood, 258

Tensor tympani, 820, 955, 961

Testicles, action of extracts of, 557 effect of removal of, 556

Tetanolysin, hæmolytic artion of, 27

Tetanus, composition of, 657.71I electrical, 656, 7II

frequency of stimulation necessary for, 657,658

negative variation in, 721,722

Ritter's, 636, 662, 727, 743

secondary, 730,738

toxin, action of, on spinal rord, 797,801

Tetany after parathyroidectomy, 559

Thalainencephalon, 747

Thalamo-bulbar tract, 773

Thalamus, See Optic thalamus
Theobromine, $4 f$ I

Theophyllin. 4 I

Thermo-electrir junctions, 573. $6_{1} 6_{3}$

'Thermogenic' nerves, 70I

Thermometers, 572 resistance, $66_{3}, 6,6$

Thermopilı, 663

Thermotaxis, 537.590

Thiosulphuric arid in urine. $\$+5$

Third nerve, 8Iy

Thirst, sensation of, 826 ,

Thiry's fistula, 3.1 I

Thoracir, duct, 176 absorption o proteicls liy, 418

and jaundire. 355

glycosuria after ligation of. 555

Iespiration, $2 I_{4}$

Thrombin, 32 specificity of, 37

Thrombogen, 33, 36, 37

Thrombokinase, $33,37.57$

sources of, 34,36

Thymine, 507

Thymus, feeding with, 506 formation of lymphocytes in. 22 nucleo-proteins of, and rragula. tion, 38

remnval of, 557

Thyroid, effects of excision of, 560 on heat-regulation, 593

feeding and metabolism, $56 \mathrm{r}$

grafting, 56I

iodine in, 561,562

Thyroiodin, $56 \mathrm{I}$

Tickling. 968, 975

Tidal air, 220, 29 I

Timbre, 280

Time-markers, I79, 625

Tissue liquid, 407,408 respiration. 264

Titratable acidity of urine, 438,439 . 477

alkalinity of blood, 24

Tone, muscular, $632,813,886$ trophir, $8 \mathbf{I}_{3}, \mathrm{XI}_{4}$

Tonsils. formation of. Iymphorytes in. 22

Tonus, acerebral, $8_{3} 6,847$

Topognosis, 856

Torpedo, 736, 737

Torricelli's thenrem. 75

Touch, acuity of, 970, I000

after section of cutaneous nerve. 975.976

corpuscles, 968

spots, $969,970,1000$

Trachea, to put a cannula in, I 86

Tracheal cannula, to make, I 86 
Tracings, to varmish, I79

Trats in cord. 70.4

Transfusiun, $40,174,200$

Trumsplantation of tissues, 1023

Transverse band uf stomarl. 305. 312

Tr.lpezilum, \& 21

Traube-Hlering curves, 270,271

Triruspid volve. $7 \mathrm{~S}$

Trigemiuns n.rve, 820

special astencling bundle uf, $\$ 20.829$

'trupluic' effects of lesions uf. (01)1). 822

Triple phosphate. +39

Tristeariu, I

Truclulear or funth urve. $\$ 20$

Trommer's test for reduring sugar. Io

Trophic nerves. 697. $>22$

tolle. SI 3. SI 4

Trophoblast, roro

Trypsin, 33 r

influence of bile on, 340

relation of, to spleen, 382.571

Trypsinogen. 33 I. $343,353.57$ I

Tryptic digestion, 331, 340, 343, 391, 428

products of, 332

Tryptophane, 2, 332, 430, 5 I0, 534

and Adam Kiewicz's reaction, 8

and the formaldehvde reaction for proteins, 8

as a precursor of indol, 449

Tubercle bacilli, absorption of, from intestine, 413

Tuberculum acusticum, 824

Twelfh nerve, 827

'Twitch,' the, 706

Tympanic membrane, 954, 960, 761 fundamental tone of, 961

Tympanum. 954

Tirosin, 2, 430, 526, 534

and Millon's reaction, 8

in pancreatic digestion, 332, 430

in serum proteins, 498

in urinary sediments, 450,495 production of, in liver, 5 to

Tyrosinase, 265

Uffelmann's test for lactic acid, 428

Umbilical cord. I012, 1020 vesicle, roro

Uncinate gyrus, 817,861

Unicellular organisms. 6

Unipolar stimulation, $\$ 43$

Unpolarizable electrodes, 625, 739

Urachus, 1012

Uracil, 507

Uramia, 447

Urates in urinary sediments, 438,494 [rean, 4 I, 40, 436, 440, 407

action of, in blood-corpusrles, 62

after Eck's fistula. 502

decomposition of. $410,480,585$

diuretic action of, 47 I

estiuation of, 48 I

furmation of. 500. 503

by "xidation, 504

in liver. 501

ill bloud. 462.500

in fever, $44^{\circ}$ )

in starvation. 531

premortal rise in excretion of, 5.31

substances which frorm. 501

variations with proteins in food.

$437,440,497.500,612$

'“remmeter, 1)orenus', 482

'reter, contractions of, 659

Urir acid, 437. 440, 449. 50.4. 50\%. $53^{8}$

destruction of, 50.5

endogenous, 441, 507

estimation of. $484 \cdot 485$

exogenous, 507

formation of, in birds, 502. 505

in mammals. 505

from nuclein substances. 504, 506, 507

from nucleo-proteids, 506

hydrolysis of, $50 \mathrm{~s}$

in blood, 505

in gout, $4+49,505$

in leukremia, 449.505

in urinary sediments. 438,494

Uricolytic ferment, 508

Urine, acidity of, $437,+38,439,477$

acelone in, 492,520

aceto-acetic acid in, 520

acid fermentation of, 438

alkaline fermentation of. 439

amino-acids in, 441,450

in liver diseases, 505

ammonia in, $\$ 40$

after Eck's fistula. 502

aromatic bodies in, $4+5.449 .479$

bile in, 451,49 I

carbohydrates in, 442

chlorides in. 444,477

collection of, 476,609

composition of, 436,437

cystin in, 450

ethereal sulphates in, 445.479

examination of, 494

ferments in, 443,444

freezing-point of, $446,447,465$. 492

hæmatoporphyrin in, $4+3$

hippuric acid in, 441, 486

incontinence of, 473 
Urine in disease, 447 indoxyl in, 436, 43\%, 4 15, 449, 479

in totus, 1016, 1017 ill starvation. 530, 532 kreatinin in, $4+2,485$ lencin and tyrusin in, 450, 495 meth.tnoglobin in. +4 . $15 \mathrm{I}$ nemotice pressure of, fo.5 ixalic acicl in, +11 pentoses in, 450,49 I phenol in. 445 plosphoric acid in, $44 \%, 478$ physicoerhemical analysis of. 4.6 pigunents uf. $4+3$ proteins in. $+43,45$ I, 486 proteoses in, 451,486 purin bases in, $4+1$ quantity of, $\$ 36,437$ reabsorption of, 457 reaction of, $436,43^{8}, 439,477$ secretion of, +5 I action of glomeruli in, $455.45 \delta$, $+65$

of 'rodded' epithclium in, $458,460,466$

Beddard's experiments on, 460

Heidenhain's experiments on, $45^{8}$

Nussbaum's experinents on. 460

influence of circulation on, 467

of drugs on, 470

of nerves on, $467 \cdot 470$

relation to blood-pressure, $f^{60}$ theories of, 455

work done by kidney in, 465

secretory pressure of, 466

sediments of, $+38,+39,494$

skatuxyl in, 445,480

specific gravity of, $436,+45,477$

sugar in, 45 I, 488

sulphuric acid in, 444,479

total nitrogen in, 482

urates in, 438, 439.440, 449.

494

urea in, $436,440,480$

uric arid in, $440,449.480$

xanthin bases in. 44 I

Urinometer, 477

Urobilin, 336, 396, 4.3

Urochrome, ++3

Uroerythrin, $4+3$

Urohypertensine, 570

Urorosein, 443

Urea and secretion of aqueous humour, gor

Utricle, 833, 957
Vagi, section of looth, 23 r, 700 effect of, on respiration, 224 $23 \mathrm{I}$

Vagus, cardiac filores of, in frog, $1+3$ I $8 \mathrm{I}, \mathrm{I} 82$

centre, effoct of suprarenal extract on, 20 I

in mannmals, 147, 187. 197

iil tortoise. I 8x

negative vitriation of, 225

relation of, to respiration, 224,227 . 289

to deglutition, 304

to gastric secretion, 374

to gastro-intestinal move. ments, 300

to pancreatic secretion, $37^{\text {. }}$. 380

tracings, I 82, I97

Vagus nerve, 825

and cervical sympathetic, union of. 868

Valsalva's experiment, 273

sinuses, I 9 I

Valves of heart, action of, I90, I 91

moment of opening and closure of, 88,80

of veins, $7 t$

Valvula conniventes, 403

Varnishing skin. 276,474

tracings, I 79

Vaso-constrictors and dilators, differ. ences between, I 59

Vaso-dilator fibres, 163.164 of chorda trmpani, 163 of limbs, I 65 nervi erigentes, 163,165

Vaso-motor cells in the cord, 169

Vaso-motor centres, I 66 in sleep, 875 peripheral, 168,979 spinal, $x 67,763$

nerves, 1 57-172, 763, 975, 479 methods of investigating. 158 nerves of brain. 160 rervical sympathetic, 159 202

course of. 165,884 in splanchuics, $16 \mathrm{r}$ in trigeminus, $16 \mathrm{I}$ of ear, I6I, I5?, 202 of heart, 162 of kidney, 161,467 of limbs, I6I of lungs. 163 of muscles, 162 of veins, $157,16.4$ reflexes, $169,171,198$ tone, wature of, 168

Vein, to put a cannula in, 200 
Virit in rilblut's resr, injection mito. 610

Venus, citculation in, IU4, I 1 I, I 21 pulso at, 01, I 20

structure of, 7.4

vallies of, 7.4

viso-motor nerve's of, 16.1

velecity of blond in, I 21

leellal's tistula, $3+1$

lelowits ol blowel. sos

in irteries, 110

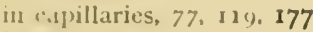

i) voius, 122,121

ine,asuriment of, III - I I 3 , 204

Velocity of the nerve-impulse, 689. 7 I3

V(elecity-pulse, curves of, II 4,115

Vénums pulse, i) I, I 20 tracings uf, I 37,193

lintilation, 243

Ventricles of braiu, 747

Veratrine, action of, un muscle, 654

Virmix cilseosa, 1017

Vertigo, 830,837

Vesicular murınur, 2 I6, 29 I

lestibular branch of auditory nerve, 823,824

Vestibule, 823, 956 and equilibration, 83.3

Vieussens, anmulus of, 147 , I90

Villi, $408,+13$

Viscosity of blood, 22

V'ision, central and peripheral, 925, 935, 946

colour, 939

far-point of, 9 I 5.988

in congenitally blind, after opera.

tion. 927

1sear-point of, 915, 9I 7

physical introduction to, 892

stereoscopjc, 925

Visual acuity, 997

angle, 904

axis, 808,914

(entres, 819, $857,855^{\circ}$

field, 819, 946, 992

illusions, 928

judgment, 926

patli, scheme of, 859,857

purple, 736,934

regeneration of, 935, 936

visuo-psychic and visuo-sensory areas, 853,860

Vital capacity, 220, 29 I

Vitellin, 2

Vitelline a tery and veiss, Ioru

Vitreous humolr, 901, 902, $9^{85}$ opacities in, 929, 914

Yocal cords, 277,282
Vinesl corcls, movennents int, in respiration, 21.5

in voice proluction, 27y parialysis of, 286

Voice, production of, 276,278

pressure: in traclica in, 270

falsetto, 28 I

in children, 270

viskmann's method for bloud velucity, I I I

Volt, 616

Volune of corpuscles and plasma in bloud, 26, 59

Volume-pulse, I 6

Voluntary contraction, fatigue in, 652 . 709

11ature of, 660

Voniting, 312

calsed by aponorpline, 312,313 . 427,432

centre, 3 I 3

Vowel cavities, 28.4

Vowels, Helmholt $z$ 's theory of, 283

Hermann's theory of, 28 ,

Wallerian degesteration, 692

Waruth sensations, $968,969,791,972$, IOOI

after section of cutaneous nerves, $975,977,975$

t)aths for, 795

Water, absorption of, 395, 417 equivalent of calorimeter, $6 \mathrm{r}_{3}$

in diet, 549

production of, in body, $5+1$ valse, 6 I $4_{4}$

IVeber's law, 984

Weigert's method, 757

Welcker's method for quantity of bloud, 47

IVeyl's test for kreatinin, 485

IVhartun's duct, $362,363,424$

Wheatstone's bridge, 6 i 7

Wheat flour, $546,547,6 \mathrm{r} 2$

Wheel-movements of eyes, 95I

Whey-protein, 327

Whispering voice, 283

White blood-corpuseles, I 6

Wolfian body, ro Io

Wuoldridge's tissue extracts and coagu-

lation, 38

Word-blindness, 864

deafness, 866

pictures, 866

IVork-adder, 664

Work, Imuscular, 583, 664, 640 relation of, to heat-produc. tion, 582, 583, 665 source of energy uf, 660 of heart, 137 
Wirnil (1) cercluellu11, 771, 772, 779.

Yawning, 23n

$8 \geq 0,835,830$

Mrisberg, nerve of, $\$ 21,822$

Xinthin, 511. 109 ?

fever prodisced by,

Xintluin-bases in urine, $4+1$

Xinthro-potcic reaction, 7

Nerustomia, 372

Yollow-sput, s(1), 1) 37, 993

Yeast-test for sugar, 489

Yohimbine, action of, on nerve, 68̈'s

Yolk.sac, Io Io

Zollner's illusion of parallel lines, yzh Zonule of Zinn, you

Zymugens, $328,331,353,354,3^{60}$

THE END 
St4

GP 34

1910

stewart 
